Nevada

Environmental

Restoration

Project

\title{
Corrective Action Decision Document for Corrective Action Unit 224: Decon Pad and Septic Systems Nevada Test Site, Nevada
}

Controlled Copy No::

Revision No.: 0

May 2005

Approved for public release; further dissemination unlimited.

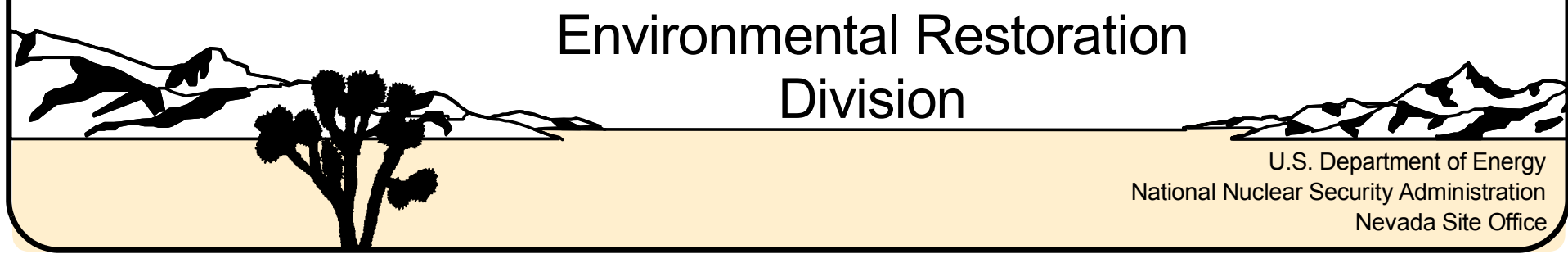


Available for public sale, in paper, from:

U.S. Department of Commerce

National Technical Information Service

5285 Port Royal Road

Springfield, VA 22161

Phone: 800.553 .6847

Fax: 703.605.6900

Email: orders@ntis.gov

Online ordering: http://www.ntis.gov/ordering.htm

Available electronically at http://www.osti.gov/bridge

Available for a processing fee to U.S. Department of Energy and its contractors, in paper, from:

\section{U.S. Department of Energy}

Office of Scientific and Technical Information

P.O. Box 62

Oak Ridge, TN 37831-0062

Phone: 865.576 .8401

Fax: 865.576.5728

Email: reports@adonis.osti.gov

Reference herein to any specific commercial product, process, or service by trade name, trademark, manufacturer, or otherwise, does not necessarily constitute or imply its endorsement, recommendation, or favoring by the United States Government or any agency thereof or its contractors or subcontractors. 


\title{
CORRECTIVE ACTION DECISION DOCUMENT FOR CORRECTIVE ACTION UNIT 224: DECON PAD AND SEPTIC SYSTEMS NEVADA TEST SITE, NEVADA
}

\author{
U.S. Department of Energy \\ National Nuclear Security Administration \\ Nevada Site Office \\ Las Vegas, Nevada
}

Controlled Copy No.:

Revision No.: 0

May 2005

Approved for public release; further dissemination unlimited. 


\section{CORRECTIVE ACTION DECISION DOCUMENT FOR CORRECTIVE ACTION UNIT 224: DECON PAD AND SEPTIC SYSTEMS NEVADA TEST SITE, NEVADA}

\begin{tabular}{|c|c|c|c|}
\hline \multirow{2}{*}{ Approved by: } & APPROVED SIGNATURE & \multirow{2}{*}{ Date: } & $5 / 2 / 05$ \\
\hline & $\begin{array}{l}\text { Janet Appenzeller-Wing, Project Manager } \\
\text { Industrial Sites Project }\end{array}$ & & \\
\hline & APPROVED SIGNATURE & Date & $5 / 2 / 05$ \\
\hline
\end{tabular}

Robert M. Bangerter, Jr., Acting Division Director Environmental Restoration Division 


\section{Table of Contents}

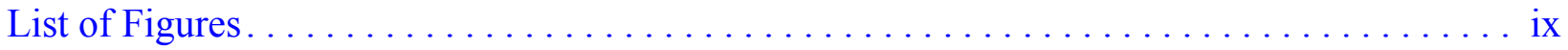

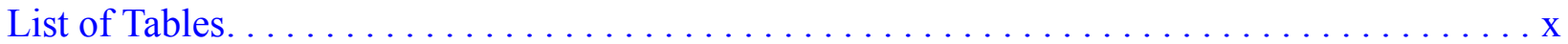

List of Acronyms and Abbreviations $\ldots \ldots \ldots \ldots \ldots \ldots \ldots \ldots \ldots \ldots \ldots \ldots \ldots \ldots \ldots$

Executive Summary . . . . . . . . . . . . . . . . . . . . . . . ES-1

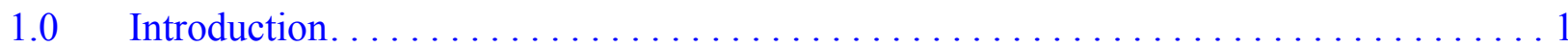

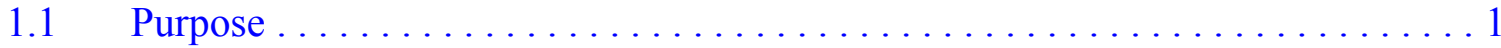

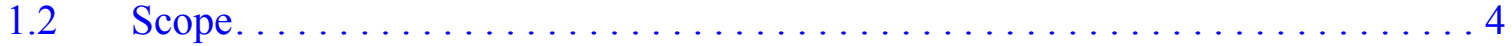

1.3 Corrective Action Decision Document Contents . . . . . . . . . . . . . . . . . 4

2.0 Corrective Action Investigation Summary $\ldots \ldots \ldots \ldots \ldots \ldots \ldots \ldots \ldots \ldots \ldots \ldots \ldots$

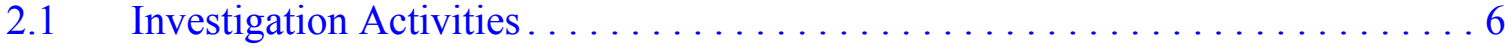

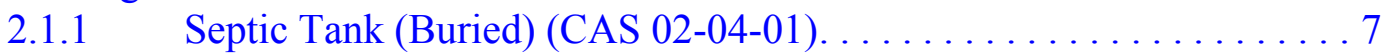

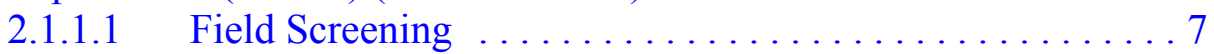

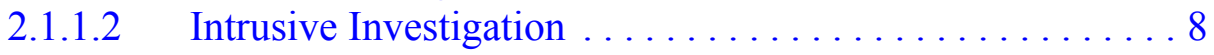

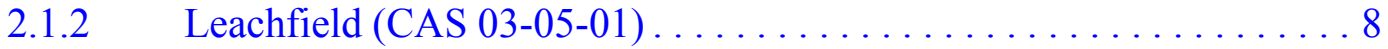

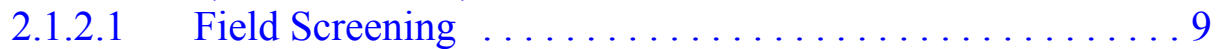

2.1.2.2 Intrusive Investigation $\ldots \ldots \ldots \ldots \ldots \ldots \ldots$

2.1.3 Septic Tanks (4)/Discharge Area (CAS 05-04-01) . . . . . . . . . . 9

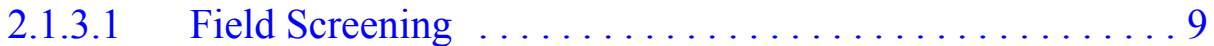

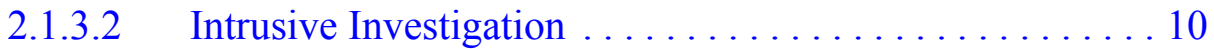

2.1.4 Sewage Lagoons (3) (CAS 06-03-01) . . . . . . . . . . . . . 10

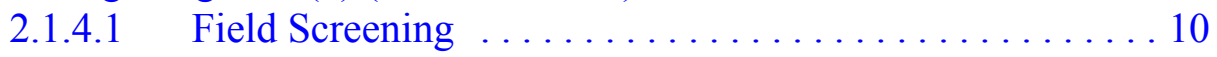

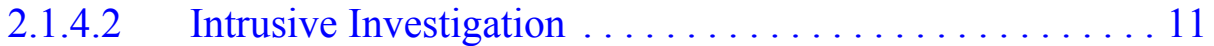

2.1.5 Leachfield (CAS 06-05-01), Decon Pad and Wastewater

Catch (CAS 06-17-04), and Decon Pad Discharge

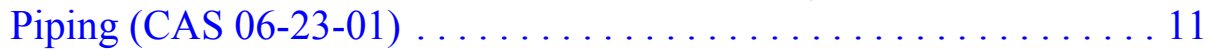

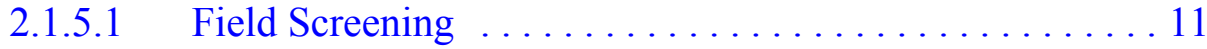

2.1.5.2 Intrusive Investigation $\ldots \ldots \ldots \ldots \ldots \ldots \ldots \ldots \ldots$

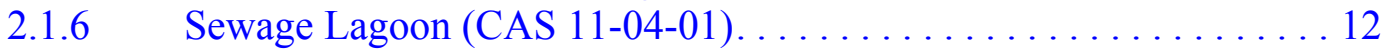

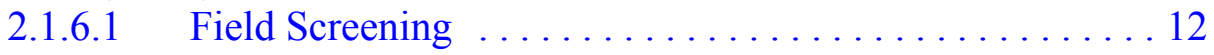

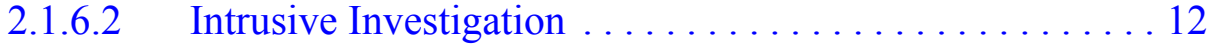

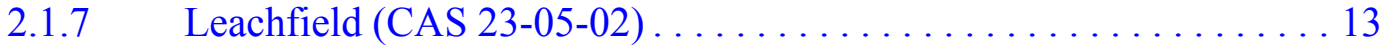

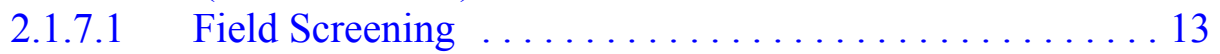

2.1.7.2 Intrusive Investigation $\ldots \ldots \ldots \ldots \ldots \ldots \ldots \ldots$

$2.1 .8 \quad$ Waste Characterization . . . . . . . . . . . . . . . . . 14

2.1.9 Conceptual Site Model Validation. . . . . . . . . . . . . . . . . 14

$2.2 \quad$ Results. . . . . . . . . . . . . . . . . . . . . . . . . . . 15

2.2.1 Summary of Analytical Data . . . . . . . . . . . . . . 15

2.2.1.1 Septic Tank (Buried) (CAS 02-04-01) . . . . . . . . 15 


\section{Table of Contents (Continued)}

2.2.1.2 Leachfield (CAS 03-05-01) ................ 16

2.2.1.3 Septic Tanks (4)/Discharge Area (CAS 05-04-01) ..... 16

2.2.1.4 Sewage Lagoons (3) (CAS 06-03-01) ............. 17

2.2.1.5 Leachfield (CAS 06-05-01), Decon Pad and

Wastewater Catch (CAS 06-17-04), and Decon Pad

Discharge Piping (CAS 06-23-01) . ............ 17

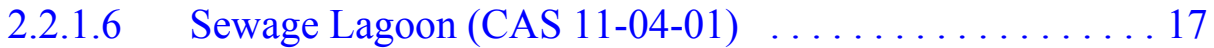

2.2.1.7 Leachfield (CAS 23-05-02) . . . . . . . . . . . . . . . 17

2.2.2 Data Assessment Summary ...................... 18

$2.3 \quad$ Need for Corrective Action. ............................. 18

2.3.1 Septic Tank (Buried) (CAS 02-04-01) . . . . . . . . . . . . . 19

2.3.2 Leachfield (CAS 03-05-01) . . . . . . . . . . . . . . . . 19

2.3.3 Septic Tanks (4)/Discharge Area (CAS 05-04-01) . . . . . . . . . 19

2.3.4 Sewage Lagoons (3) (CAS 06-03-01) .................. 20

2.3.5 Leachfield (CAS 06-05-01), Decon Pad and Wastewater

Catch (06-17-04), and Decon Pad Discharge Piping (06-23-01). . . 20

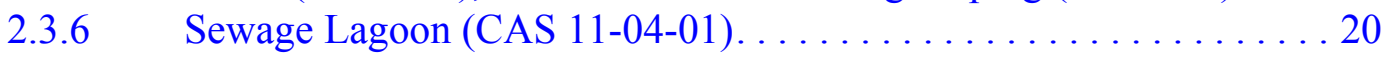

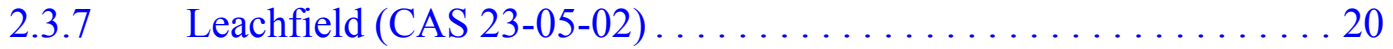

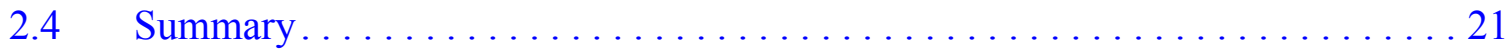

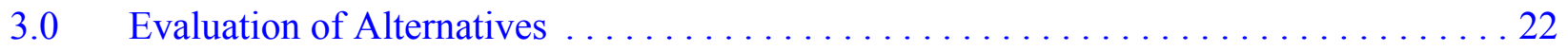

$3.1 \quad$ Corrective Action Objectives . . . . . . . . . . . . . . . . . . . 22

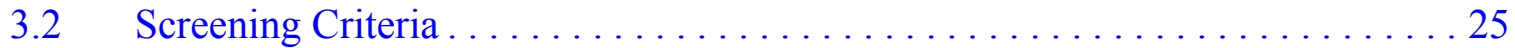

3.2.1 Corrective Action Standards . . . . . . . . . . . . . . 26

3.2.2 Remedy Selection Decision Factors . . . . . . . . . . . . 27

3.3 Development of Corrective Action Alternatives . . . . . . . . . . . . . . . . . 29

3.3.1 Alternative 1 - No Further Action ..................... 29

3.3.2 Alternative 2 - Clean Closure .................... 30

3.3.2.1 CAS 05-04-01, Septic Tanks (4)/Discharge Area ...... 30

3.3.2.2 CASs 06-05-01, Leachfield, 06-17-04, Decon

Pad and Wastewater Catch, and 06-23-01, Decon Pad

Discharge Piping $\ldots \ldots \ldots \ldots \ldots \ldots \ldots \ldots \ldots \ldots \ldots$

3.3.3 Alternative 3 - Close in Place with Administrative Controls ...... 31

3.4 Evaluation and Comparison of Alternatives.................... 31

3.4.1 Evaluation of Alternative 1 - No Further Action .............. 31

3.4.2 Alternative 2 - Clean Closure ..................... 32

3.4.3 Alternative 3 - Close in Place with Administrative Controls . . . . . . 33

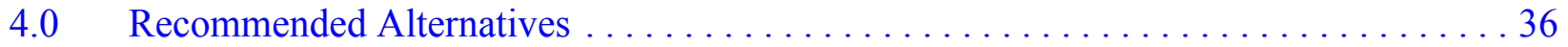

$5.0 \quad$ References......................................... 38 


\section{Table of Contents (Continued)}

\section{Appendix A - Corrective Action Investigation Report for CAU 224}

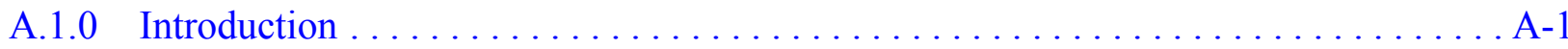

A.1.1 Project Objectives. . . . . . . . . . . . . . . . . . . . . A-2

A.1.2 Content ................................. A -2

A.2.0 Investigation Overview $\ldots \ldots \ldots \ldots \ldots \ldots \ldots \ldots \ldots \ldots \ldots \ldots \ldots \ldots \ldots \ldots$

A.2.1 Preliminary Conceptual Models . . . . . . . . . . . . . . . . . A-5

A.2.2 Sample Locations . . . . . . . . . . . . . . . . . . . . . . A-6

A.2.3 Investigation Activities . . . . . . . . . . . . . . . . . . . . . . .

A.2.3.1 Site Walkovers......................... A-6

A.2.3.2 Field Screening ........................ A

A.2.3.3 Surface and Subsurface Sampling . . . . . . . . . . . . . . . A-7

A.2.3.3.1 Waste Characterization and Sampling ........... A-8

A.2.3.3.2 Sample Location Documentation . . . . . . . . . . . . . A-9

A.2.4 Laboratory Analytical Information. . . . . . . . . . . . . . . . . A-9

A.2.5 Comparison to Action Levels . . . . . . . . . . . . . . . . . . A-10

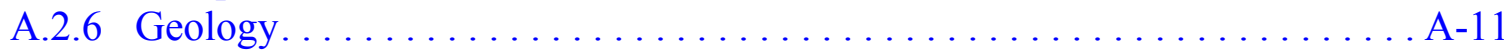

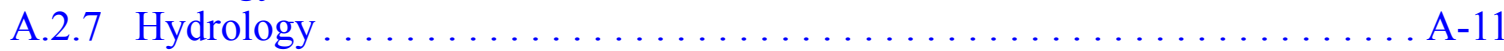

A.3.0 CAS 02-04-01, Septic Tank (Buried) . . . . . . . . . . . . . . . . . A-13

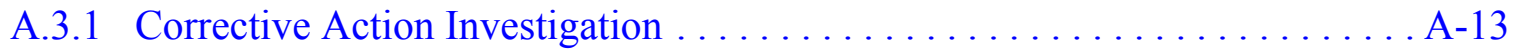

A.3.1.1 Field Screening . . . . . . . . . . . . . . . . . A-13

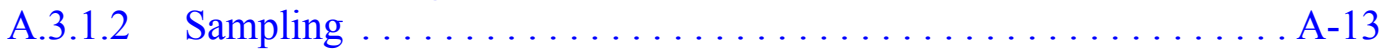

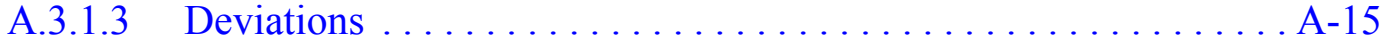

A.3.2 Investigation Results. . . . . . . . . . . . . . . . . . A-15

A.3.2.1 Total Volatile Organic Compounds.... . . . . . . . . . . . A -17

A.3.2.2 Total Semivolatile Organic Compounds . . . . . . . . . . . . . A A-17

A.3.2.3 Total Metals . . . . . . . . . . . . . . . . . . . . . . A-19

A.3.2.4 Polychlorinated Biphenyls. ...................... A-19

A.3.2.5 Total Petroleum Hydrocarbons-Diesel-Range Organics . . . . . . . . . A-19

A.3.2.6 Gamma-Emitting Radionuclides . . . . . . . . . . . . . . . A-19

A.3.2.7 Isotopes . . . . . . . . . . . . . . . . . . . . A-19

A.3.2.8 Septic Tank Liquid Results ... . . . . . . . . . . . . . . . . . A-19

A.3.3 Nature and Extent of Contamination . . . . . . . . . . . . . . . . . A-19

A.3.4 Revised Conceptual Site Model . . . . . . . . . . . . . . . . A-22

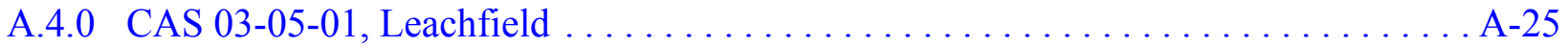

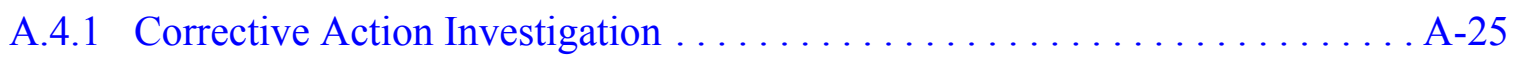

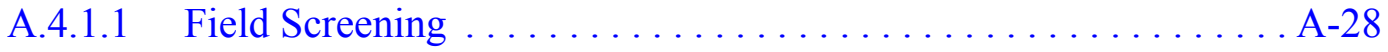




\section{Table of Contents (Continued)}

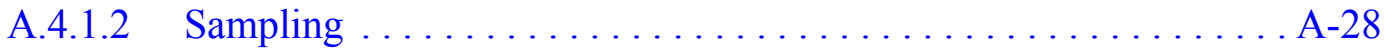

A.4.1.3 Deviations ........................... A-28

A.4.2 Investigation Results. . . . . . . . . . . . . . . . . . . . . . . . A-29

A.4.2.1 Total Volatile Organic Compounds.................. A-29

A.4.2.2 Total Semivolatile Organic Compounds ................... A-29

A.4.2.3 Total Metals ............................ A-30

A.4.2.4 Polychlorinated Biphenyls................... A-33

A.4.2.5 Total Petroleum Hydrocarbons-Diesel-Range Organics . . . . . . . . A-33

A.4.2.6 Gamma-Emitting Radionuclides ................... A-34

A.4.2.6.1 Isotopes ......................... A-34

A.4.3 Nature and Extent of Contamination $\ldots \ldots \ldots \ldots \ldots \ldots \ldots \ldots \ldots$ A-34

A.4.4 Revised Conceptual Site Model . . . . . . . . . . . . . . . . . . . . . A-34

A.5.0 CAS 05-04-01, Septic Tanks (4)/Discharge Area ................... A-37

A.5.1 Corrective Action Investigation . . . . . . . . . . . . . . . . . A-37

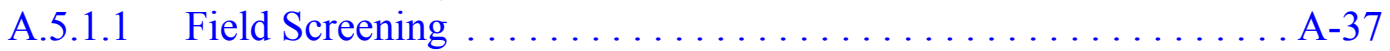

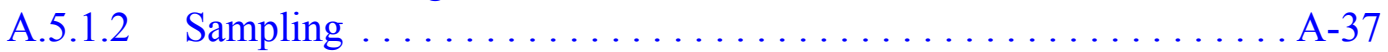

A.5.1.3 Deviations ........................... A-40

A.5.2 Investigation Results. .......................... A-40

A.5.2.1 Total Volatile Organic Compounds................. A-40

A.5.2.2 Total Semivolatile Organic Compounds ................ A-40

A.5.2.3 Total Metals . . . . . . . . . . . . . . . . . . . . . . A-41

A.5.2.4 Polychlorinated Biphenyls.................... A-41

A.5.2.5 Total Petroleum Hydrocarbon-Diesel-Range Organics. . . . . . . . A-41

A.5.2.6 Gamma-Emitting Radionuclides . . . . . . . . . . . . . . A-41

A.5.2.7 Isotopes ................................ A-41

A.5.2.8 Pesticides . . . . . . . . . . . . . . . . . . . . A-41

A.5.2.9 Liquid and Sludge Sample Results ................ A-45

A.5.3 Nature and Extent of Contamination . . . . . . . . . . . . . . . . A-45

A.5.4 Revised Conceptual Site Model . . . . . . . . . . . . . . . . . . . . . . . A-45

A.6.0 CAS 06-03-01, Sewage Lagoons (3) . . . . . . . . . . . . . . . . . A-46

A.6.1 Corrective Action Investigation . . . . . . . . . . . . . . . . . A-46

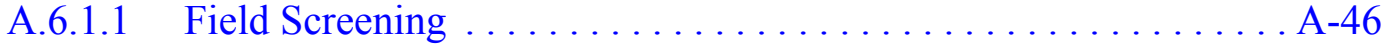

A.6.1.2 Sampling ............................ A-46

A.6.1.3 Deviations .............................. A-46

A.6.2 Investigation Results. ........................ A-50

A.6.2.1 Total Volatile Organic Compounds................. A-50

A.6.2.2 Total Semivolatile Organic Compounds . . . . . . . . . . . . A-51

A.6.2.3 Total Metals . . . . . . . . . . . . . . . . . . . . . . . A A-51

A.6.2.4 Polychlorinated Biphenyls................... A-51 


\section{Table of Contents (Continued)}

A.6.2.5 Total Petroleum Hydrocarbons-Diesel-Range Organics . . . . . . . . A-51

A.6.2.6 Gamma-Emitting Radionuclides .................. A-51

A.6.2.7 Isotopes . . . . . . . . . . . . . . . . . . . A-51

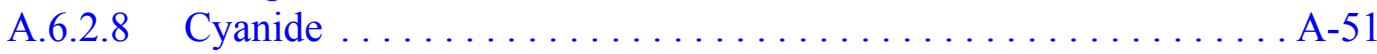

A.6.2.9 Herbicides ......................... A -51

A.6.3 Nature and Extent of Contamination . . . . . . . . . . . . . . . A-56

A.6.4 Revised Conceptual Site Model . . . . . . . . . . . . . . . . . . . . A-56

A.7.0 CASs 06-05-01, Leachfield, 06-17-04, Decon Pad and Wastewater Catch, and 06-23-01, Decon Pad Discharge Piping. . . . . . . . . . . . . 57

A.7.1 Corrective Action Investigation . . . . . . . . . . . . . . . . . A-57

A.7.1.1 Field Screening . . . . . . . . . . . . . . . . . . A-57

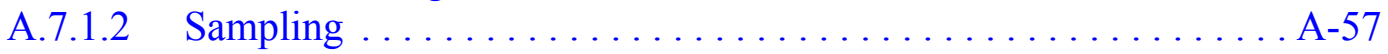

A.7.1.3 Deviations ........................... A-68

A.7.2 Investigation Results. . . . . . . . . . . . . . . . . . A-68

A.7.2.1 Total Volatile Organic Compounds . . . . . . . . . . . . . . A-70

A.7.2.2 Total Semivolatile Organic Compounds . . . . . . . . . . . A-71

A.7.2.3 Total Metals . . . . . . . . . . . . . . . . . . . . . . A-72

A.7.2.4 Polychlorinated Biphenyls. . . . . . . . . . . . . . . A-72

A.7.2.5 Total Petroleum Hydrocarbons-Diesel-Range Organics . . . . . . . . A-72

A.7.2.6 Gamma-Emitting Radionuclides . . . . . . . . . . . . . A-85

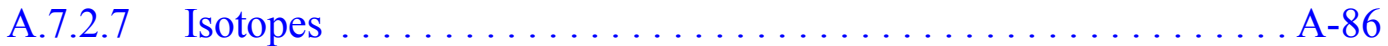

A.7.2.8 Pesticides . . . . . . . . . . . . . . . . . . A-86

A.7.2.9 Sludge and Concrete Results . . . . . . . . . . . . . . . . A-86

A.7.3 Nature and Extent of Contamination . . . . . . . . . . . . . . A-116

A.7.4 Revised Conceptual Site Model . . . . . . . . . . . . . . . . . A-116

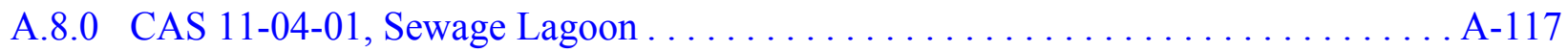

A.8.1 Corrective Action Investigation $\ldots \ldots \ldots \ldots \ldots \ldots \ldots \ldots \ldots \ldots \ldots \ldots \ldots \ldots \ldots \ldots \ldots$

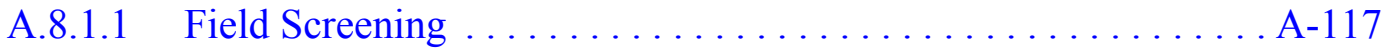

A.8.1.2 Sampling ........................ A 117

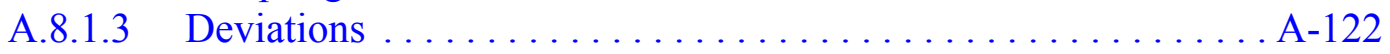

A.8.2 Investigation Results. . . . . . . . . . . . . . . . . . .

A.8.2.1 Total Volatile Organic Compounds . . . . . . . . . . . . . . . . . A-122

A.8.2.2 Total Semivolatile Organic Compounds . . . . . . . . . . . . . . . A-122

A.8.2.3 Total Metals . . . . . . . . . . . . . . . . . . . A-122

A.8.2.4 Polychlorinated Biphenyls. . . . . . . . . . . . . . . A-122

A.8.2.5 Total Petroleum Hydrocarbons-Diesel-Range Organics . . . . . . . A A-128

A.8.2.6 Gamma-Emitting Radionuclides . . . . . . . . . . . . . . . A-128

A.8.2.7 Isotopes . . . . . . . . . . . . . . . . . . . A-128

A.8.2.8 Liquid and Sludge Sample Results . . . . . . . . . . . . A A-128 


\section{Table of Contents (Continued)}

A.8.3 Nature and Extent of Contamination . . . . . . . . . . . . . A-128

A.8.4 Revised Conceptual Site Model . . . . . . . . . . . . . . . . . . . A-132

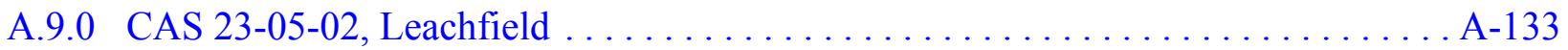

A.9.1 Corrective Action Investigation $\ldots \ldots \ldots \ldots \ldots \ldots \ldots \ldots \ldots \ldots \ldots \ldots \ldots \ldots$

A.9.1.1 Field Screening . . . . . . . . . . . . . . . . . A-133

A.9.1.2 Sampling ......................... A-133

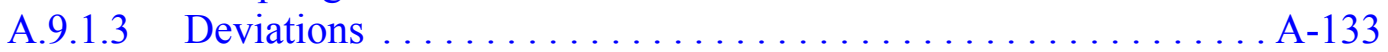

A.9.2 Investigation Results. . . . . . . . . . . . . . . . . . . . A-137

A.9.2.1 Total Volatile Organic Compounds . . . . . . . . . . . . . . . . . . A A-137

A.9.2.2 Total Semivolatile Organic Compounds . . . . . . . . . . . . . . . A-137

A.9.2.3 Total Metals . . . . . . . . . . . . . . . . . . . . . A-137

A.9.2.4 Polychlorinated Biphenyls................... A-138

A.9.2.5 Total Petroleum Hydrocarbons-Diesel-Range Organics . . . . . . . A A-138

A.9.2.6 Gamma-Emitting Radionuclides . . . . . . . . . . . . . . . . . . . A-140

A.9.2.7 Isotopes . . . . . . . . . . . . . . . . . . . . . . A-140

A.9.3 Nature and Extent of Contamination . . . . . . . . . . . . . . A-140

A.9.4 Revised Conceptual Site Model . . . . . . . . . . . . . . . . . . . . A-140

A.10.0 Waste Management. . . . . . . . . . . . . . . . . . . . . . . . . . . . A-148

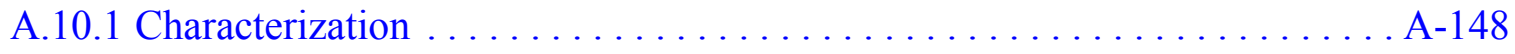

A.10.2 Waste Streams. . . . . . . . . . . . . . . . . . . . . . . . . . A-148

A.10.3 Investigation-Derived Waste Generated . . . . . . . . . . . . . . . . . A-149

A.10.4 Waste Characterization Samples . . . . . . . . . . . . . . . . . . . . A-149

A.10.4.1 CAS 02-04-01, Septic Tank (Buried) . . . . . . . . . . . . . . . A-149

A.10.4.2 CAS 05-04-01, Septic Tanks (4)/Discharge Area . . . . . . . . . . . A-149

A.10.4.3 CAS 06-17-04, Decon Pad and Wastewater Catch . . . . . . . . . A-150

A.10.4.4 CAS 11-04-01, Sewage Lagoon . . . . . . . . . . . . . . A-150

A.11.0 Quality Assurance. . . . . . . . . . . . . . . . . . . . . A-151

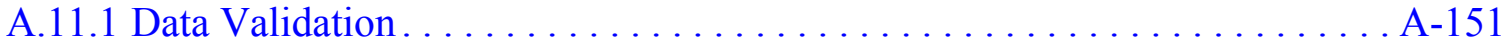

A.11.1.1 Tier I Evaluation . . . . . . . . . . . . . . . . . A-151

A.11.1.2 Tier II Evaluation. . . . . . . . . . . . . . . . . A 152

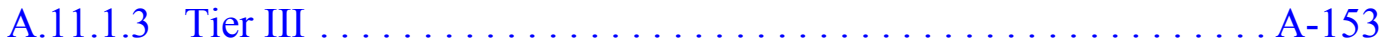

A.11.2 Field Quality Control Samples . . . . . . . . . . . . . . . . . A-154

A.11.2.1 Field Quality Control Samples . . . . . . . . . . . . . . . A-154

A.11.2.2 Laboratory Quality Control Samples.................. A-155

A.11.3 Field Nonconformances . . . . . . . . . . . . . . . . . . . A-155

A.11.4 Laboratory Nonconformances . . . . . . . . . . . . . . . . . A-155

A.12.0 Summary. . . . . . . . . . . . . . . . . . . . . . . . . . . . 


\section{Table of Contents (Continued)}

A.13.0 References.

A-158

\section{Appendix B - Data Assessment}

B.1.0 Data Assessment. . . . . . . . . . . . . . . . . . . . . . . . . B-1

B.1.1 Review DQOs and Sampling Design . . . . . . . . . . . . . . . B-2

B.1.1.1 Decision I. . . . . . . . . . . . . . . . . . . B-2

B.1.1.1.1 DQO Provisions To Limit False Negative

Decision Error .......................

B.1.1.1.2 DQO Provisions To Limit False Positive

Decision Error . . . . . . . . . . . . . . . B-21

B.1.1.2 Decision II . . . . . . . . . . . . . . . . . . B-25

B.1.1.2.1 DQO Provisions To Limit False Negative

Decision Error .......................

B.1.1.2.2 DQO Provisions To Limit False Positive

Decision Error . . . . . . . . . . . . . . . B-30

B.1.2 Conduct a Preliminary Data Review . . . . . . . . . . . . . . B-30

B.1.3 Select the Test and Identify Key Assumptions ................ B-30

B.1.4 Verify the Assumptions . . . . . . . . . . . . . . . . . . . B-30

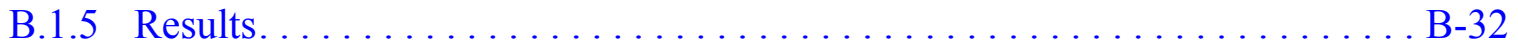

B.1.5.1 Decision I. . . . . . . . . . . . . . . . . . . . . . . . . . . .

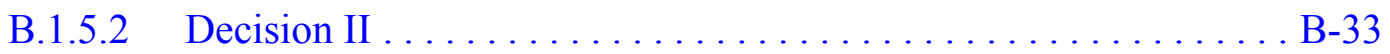

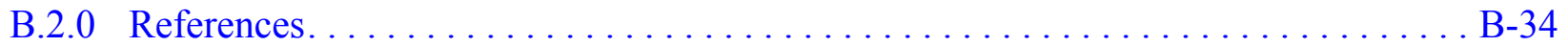

\section{Appendix C - Cost Estimates for CAU 224}

\section{Appendix D - Sample Location Coordinates for CAU 224}

D.1.0 Sample Location Coordinates. . . . . . . . . . . . . . . . . . . D-1

D.1.1 CAS 02-04-01, Septic Tank (Buried) . . . . . . . . . . . . . . . . . D-1

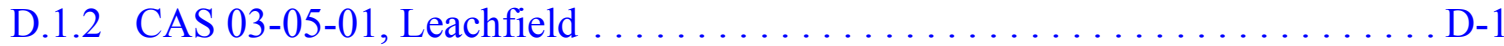

D.1.3 CAS 05-04-01, Septic Tanks (4)/Discharge Area ................ D-2

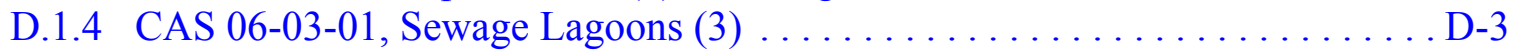

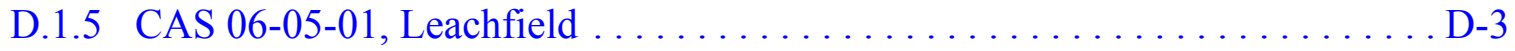

D.1.6 CAS 06-17-04, Decon Pad and Wastewater Catch. . . . . . . . . . . . . . D-4

D.1.7 CAS 06-23-01, Decon Pad Discharge Piping . . . . . . . . . . . . . D-8

D.1.8 CAS 11-04-01, Sewage Lagoon . . . . . . . . . . . . . . . . . . D-9

D.1.9 CAS 23-05-02, Leachfield ...................... . 


\section{Table of Contents (Continued)}

Appendix E - Evaluation of Risk

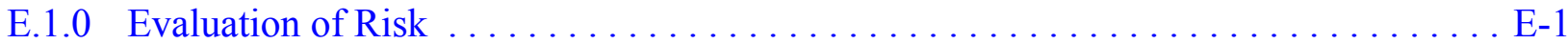

Appendix F - Project Organization for CAU 224

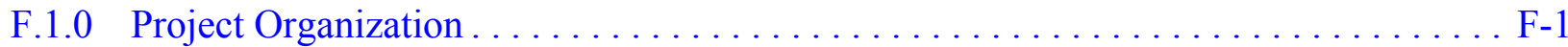

Appendix G - Derivation of Residual Radioactive Material Guidelines for Radionuclides in Soil at CAU 224

Appendix H - NDEP Comments 


\section{List of Figures}

Number

Title

Page

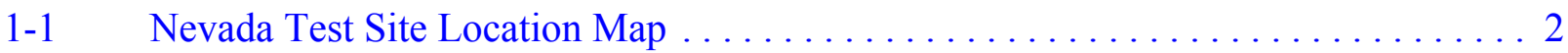

1-2 CAU 224, CAS Location Map .............................. 3

A.3-1 Sample Location Map CAS 02-04-01, Septic Tank (Buried) ............. A-16

A.4-1 Sample Location Map, CAS 03-05-01, Leachfield................... A-27

A.5-1 Sample Location Map, CAS 05-04-01, Septic Tanks (4)/Discharge Area ...... A-39

A.6-1 Sample Location Map, CAS 06-03-01, Sewage Lagoons (3).............. A-49

A.7-1 Sample Location Map, CAS 06-05-01 (E), CAS 06-17-04 (F), and

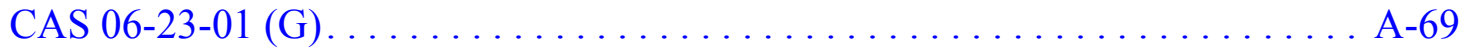

A.8-1 Sample Location Map, CAS 11-04-01, Sewage Lagoon .............. A-121

A.9-1 Sample Location Map, CAS 23-05-02, Leachfield ............... A-136

B.1-1 Extent of Contamination, CAS 05-04-01, Septic Tanks (4)/Discharge Area .... B B-27

B.1-2 Extent of TPH-DRO Contamination, CASs 06-05-01, Leachfield, 06-17-04, Decon Pad and Wastewater Catch, and 06-23-01, Decon Pad

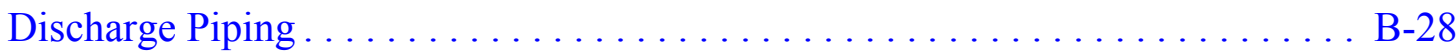

B.1-3 Extent of Pu-239 Contamination, CASs 06-05-01, Leachfield, 06-17-04,

Decon Pad and Wastewater Catch, and 06-23-01, Decon Pad

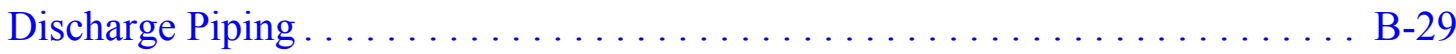




\section{List of Tables}

Number

Title

Page

3-1 Total Petroleum Hydrocarbons Constituent Final Action Levels

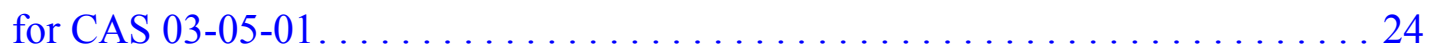

3-2 Evaluation of Alternatives for CAS, 05-04-01, 06-05-01, 06-17-04,

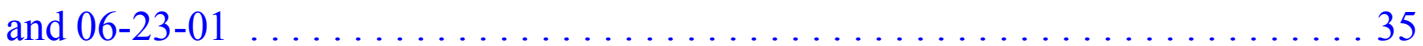

A.2-1 Corrective Action Investigation Activities Conducted at Each Corrective Action Site To Meet Corrective Action Investigation Plan Requirements . . . . A A-4

A.2-2 Laboratory Analytical Parameters and Methods, CAU 224

Investigation Samples $\ldots \ldots \ldots \ldots \ldots \ldots \ldots \ldots \ldots \ldots . \ldots \ldots$

A.3-1 Samples Collected at CAS 02-04-01, Septic Tank (Buried) . . . . . . . . . . A-14

A.3-2 Soil Sample Results for Total VOCs Detected Above Minimum

Reporting Limits at CAS 02-04-01, Septic Tank (Buried) . . . . . . . . . . . A-17

A.3-3 Soil Sample Results for Total SVOCs Detected Above Minimum

Reporting Limits at CAS 02-04-01, Septic Tank (Buried) . . . . . . . . . . . A-18

A.3-4 Soil Sample Results for Metals Detected Above Minimum Reporting Limits at CAS 02-04-01, Septic Tank (Buried). . . . . . . . . . . . . A-20

A.3-5 Soil Sample Results for PCBs Detected Above Minimum

Reporting Limits at CAS 02-04-01, Septic Tank (Buried)

A.3-6 Soil Sample Results for TPH-DRO Detected Above Minimum

Reporting Limits at CAS 02-04-01, Septic Tank (Buried) . . . . . . . . . . . . A-22

A.3-7 Soil Sample Results for Gamma-Emitting Radionuclides Detected

Above Minimum Detectable Concentrations at CAS 02-04-01,

Septic Tank (Buried)

A.3-8 Soil Sample Results for Isotopes Detected Above Minimum

Detectable Concentrations at CAS 02-04-01, Septic Tank (Buried) . . .

A.3-9 Liquid Sample Results Detected Above Minimum

Reporting Limits at CAS 02-04-01, Septic Tank (Buried) 


\section{List of Tables (Continued)}

Number

A.4-1 Samples Collected at CAS 03-05-01, Leachfield................. A-25

A.4-2 Soil Sample Results for Total VOCs Detected Above Minimum Reporting Limits at CAS 03-05-01, Leachfield ........... A-30

A.4-3 Soil Sample Results for Total SVOCs Detected Above Minimum Reporting Limits at CAS 03-05-01, Septic Tanks (4)/Discharge Area . ...... . A-30

A.4-4 Soil Sample Results for Metals Detected Above Minimum Reporting Limits at CAS 03-05-01, Leachfield . . . . . . . . . . . . . . . . . . . . A-31

A.4-5 Soil Sample Results for TPH-DRO Detected Above Minimum Reporting Limits at CAS 03-05-01, Leachfield................. A-33

A.4-6 Soil Sample Results for Gamma-Emitting Radionuclides Detected Above Minimum Detectable Concentrations at CAS 03-05-01, Leachfield. . . . . . . . A-35

A.4-7 Soil Sample Results for Isotopes Detected Above Minimum Detectable Concentrations at CAS 03-05-01, Leachfield ............. A-36

A.5-1 Samples Collected at CAS 05-04-01, Septic Tanks (4)/Discharge Area..... . A-38

A.5-2 Soil Sample Results for Metals Detected Above Minimum Reporting Limits at CAS 05-04-01, Septic Tanks (4)/Discharge Area. ............ A-42

A.5-3 Soil Sample Results for Gamma-Emitting Radionuclides Detected Above Minimum Detectable Concentrations at CAS 05-04-01, Septic Tanks (4)/Discharge Area ......................... A-43

A.5-4 Soil Sample Results for Isotopes Detected Above Minimum Detectable Concentrations at CAS 05-04-01, Septic Tanks (4)/Discharge Area. ........ A-43

A.5-5 Soil Sample Results for Pesticides Detected Above Minimum Reporting Limits at CAS 05-04-01, Septic Tanks (4)/Discharge Area ...... . . A-44

A.5-6 Liquid Sample Results Detected Above Minimum Reporting Limits at CAS 05-04-01, Septic Tanks (4)/Discharge Area .............. A-45 


\section{List of Tables (Continued)}

Number

A.6-1 Samples Collected at CAS 06-03-01, Sewage Lagoons (3)............. A-47

A.6-2 Soil Sample Results for Total VOCs Detected Above Minimum Reporting Limits at CAS 06-03-01, Sewage Lagoons (3) . .............. A-50

A.6-3 Soil Sample Results for Metals Detected Above Minimum Reporting Limits at CAS 06-03-01, Sewage Lagoons (3) ................. A-52

A.6-4 Soil Sample Results for Gamma-Emitting Radionuclides Detected Above Minimum Detectable Concentrations at CAS 06-03-01,

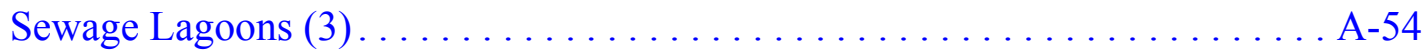

A.6-5 Soil Sample Results for Isotopes Detected Above Minimum Detectable Concentrations at CAS 06-03-01, Sewage Lagoons (3) ............. A-55

A.6-6 Soil Sample Results for Cyanide Detected Above Minimum Reporting Limits at CAS 06-03-01, Sewage Lagoons (3) ................. A-55

A.7-1 Samples Collected at CASs $06-05-01$, Leachfield . . . . . . . . . . . . . . A-58

A.7-2 Samples Collected at CAS 06-17-04, Decon Pad and Wastewater Catch...... A-62

A.7-3 Samples Collected at CAS 06-23-01, Decon Pad Discharge Piping ......... A-68

A.7-4 Soil Sample Results for Total VOCs Detected Above Minimum Reporting Limits at CAS 06-05-01, Leachfield.................. A-70

A.7-5 Soil Sample Results for Total SVOCs Detected Above Minimum Reporting Limits at CAS 06-05-01, Leachfield................ A-71

A.7-6 Soil Sample Results for Total SVOCs Detected Above Minimum Reporting Limits at CAS 06-17-04, Decon Pad and Wastewater Catch ...... . A-71

A.7-7 Soil Sample Results for Total SVOCs Detected Above Minimum Reporting Limits at CAS 06-23-01, Decon Pad Discharge Piping ......... A-72

A.7-8 Soil Sample Results for Metals Detected Above Minimum Reporting Limits at CAS 06-05-01, Leachfield ....................... A-73 


\section{List of Tables (Continued)}

Number

Title

Page

A.7-9 Soil Sample Results for Metals Detected Above Minimum Reporting Limits at CAS 06-17-04, Decon Pad and Wastewater Catch. . . . . . . . . . . . A-79

A.7-10 Soil Sample Results for Metals Detected Above Minimum Reporting Limits at CAS 06-23-01, Decon Pad Discharge Piping . . . . . . . . . . . A-82

A.7-11 Soil Sample Results for PCBs Detected Above Minimum Reporting Limits at CAS 06-05-01, Leachfield . . . . . . . . . . . . . . . A-83

A.7-12 Soil Sample Results for PCBs Detected Above Minimum Reporting Limits at CAS 06-17-04, Decon Pad and Wastewater Catch. .

A.7-13 Soil Sample Results for PCBs Detected Above Minimum Reporting Limits at CAS 06-23-01, Decon Pad Discharge Piping . . . . . . . . . . . . A-84

A.7-14 Soil Sample Results for TPH-DRO Detected Above Minimum Reporting

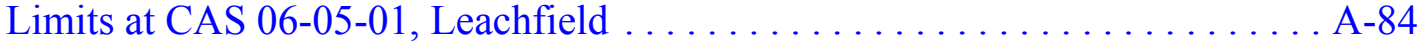

A.7-15 Soil Sample Results for TPH-DRO Detected Above Minimum Reporting Limits at CAS 06-17-04, Decon Pad and Wastewater Catch . . . . . . A-85

A.7-16 Soil Sample Results for TPH-DRO Detected Above Minimum Reporting Limits at CAS 06-23-01, Decon Pad Discharge Piping . . . . . . . . . . A-86

A.7-17 Soil Sample Results for Gamma-Emitting Radionuclides Detected Above Minimum Detectable Concentrations at CAS 06-05-01, Leachfield . . . . . . . . A-87

A.7-18 Soil Sample Results for Gamma-Emitting Radionuclides Detected Above Minimum Detectable Concentrations at CAS 06-17-04, Decon Pad and Wastewater Catch . .

A.7-19 Soil Sample Results for Gamma-Emitting Radionuclides Detected Above Minimum Detectable Concentrations at CAS 06-23-01, Decon Pad Discharge Piping

A.7-20 Soil Sample Results for Isotopes Detected Above Minimum Detectable Concentrations at CAS 06-05-01, Leachfield . 


\section{List of Tables (Continued)}

Number

Title

Page

A.7-21 Soil Sample Results for Isotopes Detected Above Minimum Detectable Concentrations at CAS 06-17-04, Decon Pad and Wastewater Catch. . . . . . . A-107

A.7-22 Soil Sample Results for Isotopes Detected Above Minimum Detectable Concentrations at CAS 06-23-01, Decon Pad Discharge Piping . . . . . . . . . A-111

A.7-23 Soil Sample Results for Pesticides Detected Above Minimum Reporting Limits at CAS 06-23-01, Decon Pad Discharge Piping.

A.7-24 Sludge and Concrete Samples Detected Above MRLs or MDCs at CAS 06-17-04, Decon Pad and Wastewater Catch . . . . . . . . . . . . A-112

A.8-1 Samples Collected at CAS 11-04-01, Sewage Lagoon . . . . . . . . . . A-118

A.8-2 Soil Sample Results for Metals Detected Above Minimum Reporting Limits at CAS 11-04-01, Sewage Lagoon . . . . . . . . . . . . . . . A-123

A.8-3 Soil Sample Results for TPH-DRO Detected Above Minimum Reporting Limits at CAS 11-04-01, Sewage Lagoon . . . . . . . . . . . . . . . . . A-128

A.8-4 Soil Sample Results for Gamma-Emitting Radionuclides Detected Above Minimum Detectable Concentrations at CAS 11-04-01, Sewage Lagoon . . . . . . . . . . . . . . . . . . . . . . . . . . A-129

A.8-5 Soil Sample Results for Isotopes Detected Above Minimum Detectable Concentrations at CAS 11-04-01, Sewage Lagoon . . . . . . . . . . . . . . A-131

A.8-6 Liquid Sample Results Detected Above MRLs or MDCs at CAS 11-04-01, Sewage Lagoon. . . . . . . . . . . . . . . . . . . . A-132

A.9-1 Samples Collected at CAS 23-05-02, Leachfield. . . . . . . . . . . . . A-134

A.9-2 Soil Sample Results for Total VOCs Detected Above Minimum Reporting Limits at CAS 23-05-02, Leachfield. . . . . . . . . . . . A-138

A.9-3 Soil Sample Results for Total SVOCs Detected Above Minimum Reporting Limits at CAS 23-05-02, Leachfield. . 


\section{List of Tables (Continued)}

Number

Title

Page

A.9-4 Soil Sample Results for Metals Detected Above Minimum Reporting Limits at CAS 23-05-02, Leachfield . . . . . . . . . . . . . . . . . . A-141

A.9-5 Soil Sample Results for PCBs Detected Above Minimum Reporting Limits at CAS 23-05-02, Leachfield .

A.9-6 Soil Sample Results for TPH-DRO Detected Above Minimum Reporting Limits at CAS 23-05-02, Leachfield

A.9-7 Soil Sample Results for Gamma-Emitting Radionuclides Detected Above Minimum Detectable Concentrations at CAS 23-05-02, Leachfield . . A-144

A.9-8 Soil Sample Results for Isotopes Detected Above Minimum Detectable Concentrations at CAS 23-05-02, Leachfield . . . . . . . . . . . . . . A-146

B.1-1 Decision I Sample Location Justification. . . . . . . . . . . . . . B-4

B.1-2 CAU 224 Analytical Program . . . . . . . . . . . . . . . . . . B-5

B.1-3 Chemical Analytes Failing Sensitivity Criteria . . . . . . . . . . . . . B-6

B.1-4 Radiological Analytes Failing Sensitivity Criteria. . . . . . . . . . . . . . . . B-9

B.1-5 Chemical Precision Measurements . . . . . . . . . . . . . . . . . B-10

B.1-6 Radiological Precision . . . . . . . . . . . . . . . . . . . . . B-11

B.1-7 Chemical Accuracy Measurements . . . . . . . . . . . . . . . . . . . . B-12

B.1-8 Radiological Accuracy Measurements for CAU 224. . . . . . . . . . . . . . B-13

B.1-9 CAU 224 Rejected Data for CAS 02-04-01, Septic Tank (Buried) . . . . . . . . . B-14

B.1-10 CAU 224 Rejected Data for CAS 03-05-01, Leachfield . . . . . . . . . . . . B-14

B.1-11 CAU 224 Rejected Data for CAS 06-05-01, Leachfield . . . . . . . . . . . B-16

B.1-12 CAU 224 Rejected Data for CAS 06-17-04, Decon Pad and Wastewater Catch. . . . . . . . . . . . . . . . . . . . . . B-17

B.1-13 CAU 224 Rejected Data for CAS 06-23-01, Decon Pad Discharge Piping. . . . B-19 


\section{List of Tables (Continued)}

B.1-14 CAU 224 Rejected Data for CAS 11-04-01, Sewage Lagoons ............. B-19

B.1-15 CAU 224 Rejected Data at CAS 23-05-02, Leachfield................. B-22

B.1-16 Chemical Completeness Measurements........................ B-24

B.1-17 Radiological Completeness Measurements for CAU $224 \ldots \ldots \ldots \ldots \ldots$. . . 25

B.1-18 Key Assumptions ................................... B

D.1-1 Sample Location Coordinates and Locations of Interest for CAS 02-04-01 . . . . D-1

D.1-2 Sample Location Coordinates and Locations of Interest for CAS 03-05-01 . . . . D-2

D.1-3 Sample Location Coordinates and Locations of Interest for CAS 05-04-01 . . . . D-3

D.1-4 Sample Location Coordinates and Locations of Interest for CAS 06-03-01 . . . . D-4

D.1-5 Sample Location Coordinates and Locations of Interest for CAS 06-05-01 . . . . D-5

D.1-6 Sample Location Coordinates and Locations of Interest for CAS 06-17-04 . . . . D-6

D.1-7 Sample Location Coordinates and Locations of Interest for CAS 06-23-01 . . . . D-9

D.1-8 Sample Location Coordinates and Locations of Interest for CAS 11-04-01 . . . . D-9

D.1-9 Sample Location Coordinates and Locations of Interest for CAS 23-05-02 . . . D-11 


\section{List of Acronyms and Abbreviations}

ALARA As-low-as-reasonably-achievable

Am

Americium

ASTM

American Society for Testing and Materials

bgs

Below ground surface

CAA

Corrective Action Alternative

CADD

Corrective Action Decision Document

CAI

Corrective Action Investigation

CAIP

Corrective Action Investigation Plan

CAS

Corrective Action Site

CAU Corrective Action Unit

CFR

Code of Federal Regulations

CLP

Contract Laboratory Program

$\mathrm{cm}$

Centimeter

$\mathrm{COC}$

Contaminant of concern

COPC

Contaminant of potential concern

$\mathrm{CP}$

Control Point

Cs

Cesium

CSM

Conceptual site model

DDD

Dichlorodiphenyl-dichloroethane

DDE

Dichlorodiphenyl-dichlorethylene

DDT

Dichlorodiphenyl-trichloroethane

DOE

U.S. Department of Energy

DQA

Data quality assessment

DQI

Data quality indicator

DQO

Data quality objective

DRO

Diesel-range organics 


\section{List of Acronyms and Abbreviations (Continued)}

EPA U.S. Environmental Protection Agency

FADL Field activity daily log

FAL Final action level

FD Field duplicate

FFACO Federal Facility Agreement and Consent Order

FID Flame-ionization detector

FSL Field-screening level

FSR Field-screening result

$\mathrm{ft} \quad$ Foot

GPS Global positioning system

HWAA Hazardous Waste Accumulation Area

ID Identification number

IDW Investigation-derived waste

in. Inch

K Potassium

Lab-Dup Laboratory Sample Duplicate

LCS Laboratory control sample

LCSD Laboratory control sample duplicate

MDC Minimum detectable concentration

$\mathrm{mg} / \mathrm{kg} \quad$ Milligrams per kilogram

$\mathrm{mg} / \mathrm{L} \quad$ Milligrams per liter

mi Mile

$\mathrm{mrem} / \mathrm{yr} \quad$ Millirems per year

MRL Minimum reporting limit

MS Matrix spike

MSD Matrix spike duplicate

NA Not applicable 


\section{List of Acronyms and Abbreviations (Continued)}

NAC Nevada Administrative Code

NAD North American Datum

NCRP National Council on Radiation Protection and Measurement

ND Normalized difference

NDEP Nevada Division of Environmental Protection

NI Not identified

NIST National Institute for Standards and Technology

NNSA/NSO U.S. Department of Energy, National Nuclear Security Administration Nevada Site Office

NTS Nevada Test Site

PAH Polyaromatic hydrocarbon

PAL Preliminary action level

PB Preparation blank

PCB Polychlorinated biphenyl

$\mathrm{pCi} / \mathrm{g} \quad$ Picocuries per gram

POC Performance objective criteria

PPE Personal protective equipment

ppm Parts per million

PRG Preliminary Remediation Goal

$\mathrm{Pu} \quad$ Plutonium

QA Quality assurance

QAPP Quality Assurance Project Plan

QC Quality control

RCRA Resource Conservation and Recovery Act

RESRAD Residual Radiation

ROTC Record of Technical Change

RPD Relative percent difference 


\section{List of Acronyms and Abbreviations (Continued)}

$\mathrm{SC}$

SCL

SDG

SVOC

TaDD

TCLP

$\mathrm{TPH}$

TSCA

UTM

VOC

WM

$\mu \mathrm{g} / \mathrm{kg}$

$\% \mathrm{R}$
Site Characterization

Sample collection log

Sample delivery group

Semivolatile organic compound

Tactical Demilitarization Development

Toxicity characteristic leaching procedure

Total petroleum hydrocarbons

Toxic Substance Control Act

Universal Transverse Mercator

Volatile organic compound

Waste Management

Micrograms per kilogram

Percent recovery 


\section{Executive Summary}

This Corrective Action Decision Document has been prepared for Corrective Action Unit (CAU) 224, Decon Pad and Septic Systems, in Areas 2, 3, 5, 6, 11, and 23 of the Nevada Test Site, Nevada, in accordance with the Federal Facility Agreement and Consent Order (1996). Corrective Action Unit 224 is comprised of the following corrective action sites (CASs):

- 02-04-01, Septic Tank (Buried)

- 03-05-01, Leachfield

- 05-04-01, Septic Tanks (4)/Discharge Area

- 06-03-01, Sewage Lagoons (3)

- 06-05-01, Leachfield

- 06-17-04, Decon Pad and Wastewater Catch

- 06-23-01, Decon Pad Discharge Piping

- 11-04-01, Sewage Lagoon

- 23-05-02, Leachfield

The purpose of this Corrective Action Decision Document is to identify and provide the rationale for the recommendation of a corrective action alternative for the nine CASs within CAU 224. Corrective action investigation activities were performed from August 10, 2004, through January 18, 2005, as set forth in the CAU 224 Corrective Action Investigation Plan.

The results of the corrective action investigation identified contaminants of concern at four of the nine CASs in CAU 224 and required the evaluation of corrective action alternatives.

Analytes detected during the corrective action investigation were evaluated against appropriate final action levels to identify the contaminants of concern for each CAS. Assessment of the data generated from investigation activities conducted at CAU 224 revealed the following:

- CASs 02-04-01, 03-05-01, 06-03-01, 11-04-01, and 23-05-02 do not contain contaminants of concern.

- CAS 05-04-01, Septic Tanks (4)/Discharge Area, contains the pesticides chlordane, dichlorodiphenyl-dichloroethylene (DDE), and dichlorodiphenyl-trichloroethane (DDT) above action levels in soil in the discharge area.

- CASs 06-05-01, Leachfield, 06-17-04, Decon Pad and Wastewater Catch, and 06-23-01, Decon Pad Discharge Piping, comprise a system formerly used for radiological decontamination of personnel and equipment at Control Point Hill in Area 6 of the Nevada 
Test Site. Total petroleum hydrocarbons (TPH)-diesel-range organics (DRO) and plutonium-239 were detected above final action levels in the surrounding soil. Material that upon generation would become a mixed waste was found in the residual media of the wastewater sump containing several solvents, polychlorinated biphenyls, TPH-DRO, and radionuclides above action levels.

Based on the evaluation of analytical data from the corrective action investigation, review of future and current operations at the nine CASs, and the detailed and comparative analysis of the potential corrective action alternatives, the following recommended corrective actions were selected for CAU 224.

No Further Action is the preferred corrective action for CASs 02-04-01, 03-05-01, 06-03-01, 11-04-01, and 23-05-02, with the following provision:

- The septic tanks and distribution box (if applicable) will be removed from CASs 02-04-01 and 11-04-01 and closed in accordance with Nevada Administrative Code 444.818. The piping leading to the tanks will be plugged and any accessible manholes will be filled in order to prevent future dumping.

Clean Closure is the preferred corrective action for CASs 05-04-01, 06-05-01, 06-17-04, and 06-23-01:

- The pesticide-contaminated soil will be removed from CAS 05-04-01. The four septic tanks will be closed in accordance with Nevada Administrative Code 444-818. The overflow pipes and distribution box will be removed, along with the concrete slab supporting the tanks. Piping leading to the distribution box will be capped and upstream manholes will be filled to prevent future dumping.

- The wastewater sump at CAS 06-17-04 and its contents will be removed and disposed of as mixed waste. Several soil locations at CASs 06-05-01, 06-17-04, and 06-23-01 will be excavated in order to remove TPH-DRO and radiological contamination. The radiologically contaminated soil will be handled as Low Level Waste, once generated. In addition, the concrete decon pad will be removed. All excavations will be filled with clean soil.

The preferred corrective action alternatives were evaluated on technical merit focusing on performance, reliability, feasibility, safety, and cost. The alternatives were judged to meet all requirements for the technical components evaluated. The alternatives meet all applicable federal and state regulations for closure of the site and will reduce potential exposure pathways to the contaminated media to an acceptable level at CAU 224. 


\subsection{Introduction}

This Corrective Action Decision Document (CADD) has been prepared for Corrective Action Unit (CAU) 224, Decon Pad and Septic Systems, Nevada Test Site (NTS), Nevada. The corrective actions proposed in this document are in accordance with the Federal Facility Agreement and Consent Order (FFACO) that was agreed to by the State of Nevada, U.S. Department of Energy (DOE), and the U.S. Department of Defense (FFACO, 1996). The NTS is approximately 65 miles (mi) northwest of Las Vegas, Nevada (Figure 1-1).

Corrective Action Unit 224 is comprised of the nine Corrective Action Sites (CASs) that are shown on Figure 1-2 and listed below:

- 02-04-01, Septic Tank (Buried)

- 03-05-01, Leachfield

- 05-04-01, Septic Tanks (4)/Discharge Area

- 06-03-01, Sewage Lagoons, (3)

- 06-05-01, Leachfield

- 06-17-04, Decon Pad and Wastewater Catch

- 06-23-01, Decon Pad Discharge Piping

- 11-04-01, Sewage Lagoon

- 23-05-02, Leachfield

A detailed discussion of the history of this CAU is presented in the Corrective Action Investigation Plan (CAIP) for Corrective Action Unit 224: Decon Pad and Septic Systems (NNSA/NSO, 2004).

\subsection{Purpose}

Corrective Action Unit 224, Decon Pad and Septic Systems, consists of nine inactive sites located in Areas 2, 3, 5, 6, 11, and 23 of the NTS. The nine CAU 224 sites consist of septic tanks, leachfields, sewage lagoons, piping, a leaching pit, and a decon pad. The CAU 224 sites were used for sanitary and industrial waste management including radiological decontamination activities.

This CADD develops and evaluates potential corrective action alternatives and provides the rationale for the selection of recommended corrective action alternatives for the CASs in CAU 224, Decon Pad and Septic Systems. The need for evaluation of corrective action alternatives is based on process knowledge and the results of investigative activities conducted in accordance with the CAIP 


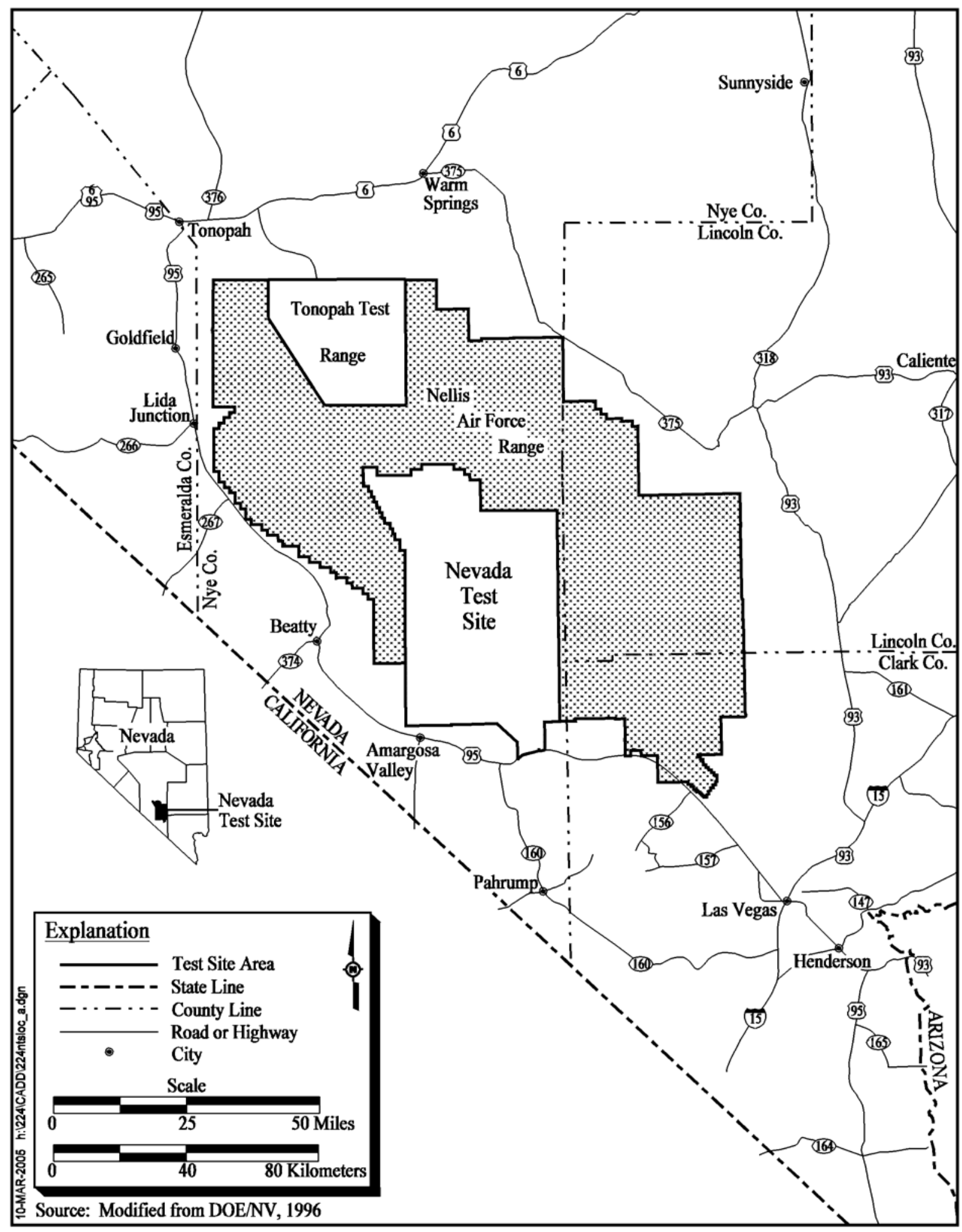

Figure 1-1

Nevada Test Site Location Map 


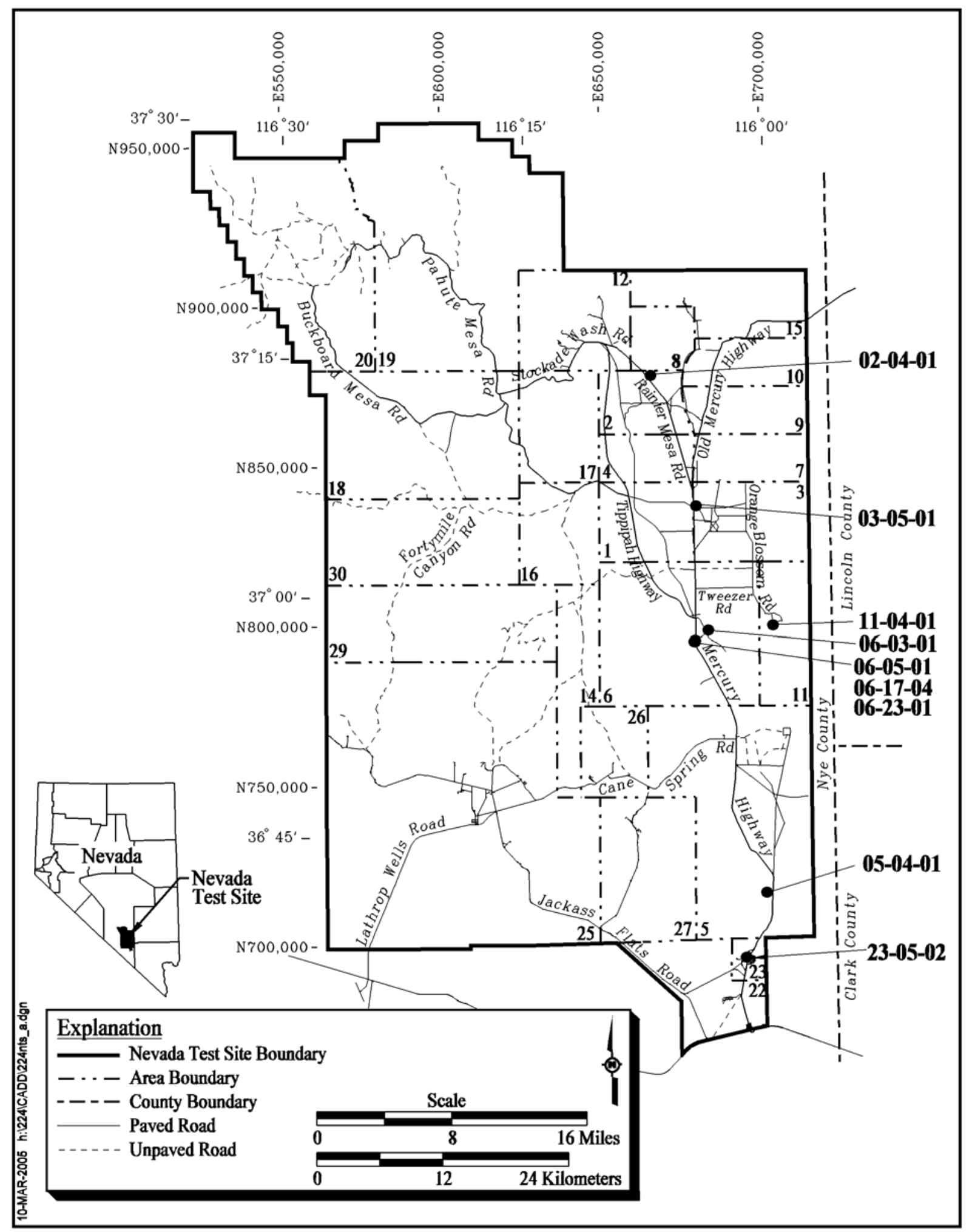

Figure 1-2

CAU 224, CAS Location Map 
(NNSA/NSO, 2004). The CAIP provides information relating to the history, planning, and scope of the investigation; therefore, this information will not be repeated in this CADD.

\subsection{Scope}

The scope of the activities used to identify, evaluate, and recommend preferred corrective action alternatives for CAU 224 included the following:

- Evaluation of current site conditions, including the concentrations and extent of contaminants of concern (COCs).

- Development of corrective action objectives commensurate with the complexity of the CAS.

- Identification of corrective action alternative screening criteria.

- Performance of detailed and comparative evaluations of corrective action alternatives in relation to corrective action objectives and screening criteria.

\subsection{Corrective Action Decision Document Contents}

This CADD is divided into the following sections and appendices:

Section 1.0 - Introduction: Summarizes the purpose, scope, and contents of this CADD.

Section 2.0 - Corrective Action Investigation Summary: Summarizes the investigation field activities, the results of the investigation, and the need for corrective action.

Section 3.0 - Evaluation of Alternatives: Describes, identifies, and evaluates the steps taken to determine preferred corrective action alternatives.

Section 4.0 - Recommended Alternatives: Presents the preferred corrective action alternatives for each CAS and the rationale based on the corrective action objectives and screening criteria.

Section 5.0 - References: Provides a list of all referenced documents used in the preparation of this CADD. 
Appendix A - Corrective Action Investigation Report for CAU 224: Provides a description of the project objectives, field investigation and sampling activities, investigation results, waste management, and quality assurance. Section A.3.0 through Section A.9.0 provide specific information regarding field activities, sampling methods, and laboratory analytical results from the investigation.

Appendix B - Data Assessment: Provides a data quality assessment (DQA) that reconciles investigation results to the requirements set forth during the data quality objective (DQO) process.

Appendix C - Cost Estimates for CAU 224: Presents cost estimates for the construction, operation, and maintenance of the corrective action alternatives evaluated for each CAS.

Appendix D - Sample Location Coordinates for CAU 224: Provides investigation sample locations coordinates.

Appendix E - Evaluation of Risk.

Appendix F - Project Organization for CAU 224: Identifies the DOE Project Manager and other appropriate personnel involved with the CAU 224 characterization and closure activities.

Appendix G - Residual Radiation (RESRAD) Derivation of Residual Radioactive Material Guidelines for Radionuclides in Soil at the Corrective Action Unit 224 Site, Nevada Test Site.

Appendix H - NDEP Comment Responses for CAU 224: Contains responses to Nevada Division of Environmental Protection (NDEP) comments on the draft CADD.

To ensure all project objectives, health and safety requirements, and quality control procedures were adhered to, all investigation activities were performed in accordance with the following documents:

- CAIP for CAU 224, Decon Pad and Septic Systems (NNSA/NSO, 2004)

- $\quad$ Record of Technical Change (ROTC) No. 1 for the CAIP for CAU 224, Decon Pad and Septic Systems

- Industrial Sites Quality Assurance Project Plan (QAPP) (NNSA/NV, 2002a)

- $\quad$ FFACO (1996)

- Approved standard quality practices and detailed operating procedures 


\subsection{Corrective Action Investigation Summary}

The following sections summarize the investigation activities and investigation results, and identify the need for corrective action at CAU 224. Detailed investigation activities and results for individual CAU 224 CASs are presented in Appendix A of this document.

\section{$2.1 \quad$ Investigation Activities}

Corrective action investigation activities were performed as set forth in the CAU 224 CAIP (NNSA/NSO, 2004) from August 10, 2004, through January 18, 2005. The purpose of the CAU 224 CAI was to address the decision statements in the project-specific DQOs by:

- Determining whether COCs are present in the soils and liquids associated with CAU 224.

- Determining the lateral and vertical extent of identified COCs.

- Ensuring adequate data have been collected to close the sites under the Nevada Division of Environmental Protection (NDEP), Resource Conservations and Recovery Act (RCRA) (CFR, 2003a), Toxic Substance Control Act (TSCA) (CFR, 2003b), and DOE requirements.

The scope of the CAI included the following activities:

- Field screening soil samples for volatile organic compounds (VOCs) and alpha and beta/gamma radiation.

- Field screening septic tank and contents for fecal coliform.

- Collecting environmental soil and residual waste media samples for laboratory analyses to determine the presence of COCs.

- Collecting environmental soil samples for laboratory analyses to define the lateral and vertical extent of COCs, if present.

- Collecting quality control (QC) samples for laboratory analyses to ensure that the data generated from the analysis of investigation samples meet the requirements of the data quality indicators (DQIs).

A judgmental (nonprobabilistic) sampling scheme was implemented to select sample locations and evaluate analytical results. Judgmental sampling allows the methodical selection of sample locations 
that target the populations of interest (defined in the DQOs) rather than non-selective random locations. Random sample locations are used to generate average contaminant concentrations that estimate the true average ("characteristic") contaminant concentration of the site to some specified degree of confidence.

Because individual sample results, rather than an average concentration, will be used to compare to action levels, statistical methods to generate site characteristics (averages) will not be necessary. Section 0.4.4 of the U.S. Environmental Protection Agency Data Quality Objectives for Hazardous Waste Site Investigations (EPA, 2002) guidance states that the use of statistical methods may not be warranted by program guidelines or site-specific sampling objectives. The need for statistical methods is dependent upon the decisions being made. Section 7.1 of the EPA guidance document (QA/G-4HW) states that a nonprobabilistic (judgmental) sampling design is developed when there is sufficient information on the contamination sources and history to develop a valid conceptual site model and to select specific sampling locations. This design was used to confirm the existence of contamination at specific locations and provide information (such as extent of contamination) about specific areas of the site.

Confidence in judgmental sampling results will be established qualitatively by the validation of the conceptual site model developed and concurred to by stakeholder participants (NNSA/NSO and NDEP) during the DQO process based on investigation results, and by performing a DQA to determine whether DQOs have been satisfied.

The following sections describe specific investigation activities conducted at each CAS.

\subsubsection{Septic Tank (Buried) (CAS 02-04-01)}

This CAS consists of an underground septic tank, associated piping and surrounding soil in Area 2 of the NTS. The following sections summarize the field screening and intrusive investigation activities conducted at CAS 02-04-01.

\subsubsection{Field Screening}

Field screening was conducted on soil samples using a flame-ionization detector (FID) with the headspace method for volatiles, handheld instrument surveys for alpha and beta/gamma radiation, 
and gas chromatography for TPH-DRO. The field-screening results (FSRs) were compared to field-screening levels (FSLs) to guide subsequent soil sampling decisions and to determine which samples were submitted for laboratory analysis. Fecal coliform screening was performed on the sludge collected from the septic tank. The screening for fecal coliform was negative and no soil samples exceeded FSLs.

\subsubsection{Intrusive Investigation}

A total of 20 soil and 1 liquid samples from 9 soil locations and the septic tank were collected and submitted to the laboratory for the analyses listed in Appendix A (Table A.3-1). Surface and shallow subsurface soil sampling activities were conducted at CAS 02-04-01. Soil samples were collected using grab sampling and backhoe methods.

Surface soil samples were collected from 0.0 to 0.5 feet (ft) below ground surface (bgs) at biased locations based on criteria listed in the CAIP.

Subsurface soil samples were collected from various depth intervals as deep as $8 \mathrm{ft}$ bgs at selected sample locations using the backhoe method. The backhoe was used to excavate the soil around the septic tank and samples were collected from the backhoe bucket once the appropriate depth was reached.

Residual liquid and sludge samples were collected from the septic tank using disposable plastic scoops. One liquid sample was collected from the septic tank for laboratory analysis. The amount of sludge at the bottom of the tank was too small for a laboratory sample to be collected; however, an aliquot of sludge was collected for fecal coliform screening.

\subsubsection{Leachfield (CAS 03-05-01)}

This CAS consists of a former leach pit associated with acetylene generation in the Area 3 Subdock on the NTS. The following sections summarize field screening and intrusive investigation activities conducted at CAS 03-05-01. 


\subsubsection{Field Screening}

Field screening was conducted on soil samples using an FID with the headspace method for volatiles, handheld instrument surveys for alpha and beta/gamma radiation, and gas chromatography for TPH-DRO. The FSRs were compared to FSLs to guide subsequent soil sampling decisions and to determine which samples were submitted for laboratory analysis. The TPH-DRO FSL was exceeded in several samples.

\subsubsection{Intrusive Investigation}

A total of 31 soil samples from 15 locations were collected and submitted to the laboratory for the analyses listed in Appendix A (Table A.4-1). Surface and shallow subsurface soil sampling activities were conducted at CAS 03-05-01. Soil samples were collected using grab sampling and hand-auger methods.

Surface soil samples were collected from 0.0 to $0.5 \mathrm{ft}$ bgs at biased locations based on criteria listed in the CAIP.

Subsurface soil samples were collected from various depth intervals as deep as $6 \mathrm{ft}$ bgs at selected sample locations using the hand-auger method.

\subsubsection{Septic Tanks (4)/Discharge Area (CAS 05-04-01)}

This CAS consists of four septic tanks, associated piping, and a potential discharge area associated with the former trailer park in Area 5 of the NTS. The following sections summarize the field screening and intrusive investigation activities conducted at CAS 05-04-01.

\subsubsection{Field Screening}

Field screening was conducted on soil samples using an FID with the headspace method for volatiles, handheld instrument surveys for alpha and beta/gamma radiation, and gas chromatography for TPH-DRO. The FSRs were compared to FSLs to guide subsequent soil sampling decisions and to determine which samples were submitted for laboratory analysis. No FSLs were exceeded in soil samples. Fecal coliform screening was performed on the contents of all four tanks. The sludge 
collected from Tank 2 (Sample 224C506) contained two positive readings out of five tubes tested, resulting in 51,000 colonies per 100 milliliter.

\subsubsection{Intrusive Investigation}

A total of 12 soil, 4 liquid, and 4 sludge samples from 13 locations, including the four tanks, were collected and submitted to the laboratory for the analyses listed in Appendix A (Table A.5-1). Surface and shallow subsurface soil sampling activities were conducted at CAS 05-04-01. Soil samples were collected using grab sampling, and hand-auger methods.

Surface soil samples were collected from 0.0 to $0.5 \mathrm{ft} \mathrm{bgs}$ at biased locations based on criteria listed in the CAIP.

Subsurface soil samples were collected from a 2-3 ft bgs interval at two Decision II sample locations using the hand-auger method.

Residual liquid and sludge samples were collected using disposable plastic scoops.

\subsubsection{Sewage Lagoons (3) (CAS 06-03-01)}

This CAS consists of three former sewage lagoons, comprising the former Yucca Lake sewage lagoon systems in Area 6 of the NTS. The following sections summarize the field screening and intrusive investigation activities conducted at CAS 06-03-01.

\subsubsection{Field Screening}

Field screening was conducted on soil samples using an FID with the headspace method for volatiles, handheld instrument surveys for alpha and beta/gamma radiation, and gas chromatography for TPH-DRO. The FSRs were compared to FSLs to guide subsequent soil sampling decisions and to determine which samples were submitted for laboratory analysis. No FSLs were exceeded at this CAS. 


\subsubsection{Intrusive Investigation}

A total of 35 soil samples from 15 locations were collected and submitted to the laboratory for the analyses listed in Appendix A (Table A.6-1). Surface and shallow subsurface soil sampling activities were conducted at CAS 06-03-01. Soil samples were collected using grab sampling and backhoe methods.

Surface soil samples were collected from 0.0 to $0.5 \mathrm{ft}$ bgs at biased locations based on criteria listed in the CAIP.

Subsurface soil samples were collected from various depth intervals at biased sample locations using the backhoe method. Soil was excavated up to approximately $12 \mathrm{ft}$ bgs in order to find any features associated with sewage lagoons (e.g., piping, distribution boxes). No such features were found.

\subsubsection{Leachfield (CAS 06-05-01), Decon Pad and Wastewater Catch (CAS 06-17-04), and Decon Pad Discharge Piping (CAS 06-23-01)}

These three CASs comprise one system on Control Point (CP) Hill in Area 6 of the NTS. Corrective Action Site 06-05-01 consists of a leachfield and lagoons that received wastewater from the decon pad (CAS 06-17-04) via the associated piping (CAS 06-23-01). The following sections summarize the field screening and intrusive investigation activities conducted at these three CASs.

\subsubsection{Field Screening}

Field screening was conducted on soil samples using an FID with the headspace method for volatiles, handheld instrument surveys for alpha and beta/gamma radiation, and gas chromatography for TPH-DRO. The FSRs were compared to FSLs to guide subsequent soil sampling decisions and to determine which samples were submitted for laboratory analysis. Several samples in this area exceeded the FSLs for alpha and beta/gamma radiation.

\subsubsection{Intrusive Investigation}

A total of 241 soil, 2 sludge, and 1 concrete samples from 112 locations were collected and submitted to the laboratory for the analyses listed in Appendix A (Table A.7-1). Surface and shallow 
subsurface soil sampling activities were conducted at CASs 06-05-01, 06-17-04, and 06-23-01. Soil samples were collected using grab sampling, hand-auger, drilling, Geoprobe ${ }^{\circledR}$, and backhoe methods.

Surface soil samples were collected from 0.0 to $0.5 \mathrm{ft}$ bgs, or directly beneath the concrete on the decon pad, at biased locations based on criteria listed in the CAIP.

Subsurface soil samples were collected from depths up to $11 \mathrm{ft}$ bgs at biased sample locations using the backhoe method at CAS 06-05-01, the hand-auger method at CAS 06-23-01, and drilling, Geoprobe $^{\circledR}$, and hand-auger at CAS 06-17-04. Several underground utilities, including a pair of high-voltage lines, are located near the area utility surveys indicated as the distribution box and piping at CAS 06-23-01. Due to the proximity of the high-voltage lines no intrusive activities were performed at these locations.

\subsubsection{Sewage Lagoon (CAS 11-04-01)}

This CAS consists of a septic system that formerly serviced the Tactical Demilitarization Development (TaDD) facility in Area 11 of the NTS. The system consists of a two chamber septic tank, an evapotranspiration bed (shallow leachfield), piping, and a former sewage lagoon. The following sections summarize the field screening and intrusive investigation activities conducted at CAS 11-04-01.

\subsubsection{Field Screening}

Field screening was conducted on soil samples using an FID with the headspace method for volatiles, and handheld instrument surveys for alpha and beta/gamma radiation. The FSRs were compared to FSLs to guide subsequent soil sampling decisions and to determine which samples were submitted for laboratory analysis.

\subsubsection{Intrusive Investigation}

A total of 75 soil, 3 liquid, and 1 sludge samples from 26 locations, including two septic tanks and one distribution box, were collected and submitted to the laboratory for the analyses listed in Appendix A (Table A.8-1). Surface and shallow subsurface soil sampling activities were conducted 
at CAS 11-04-01. Soil samples were collected using "scoop and trowel," grab sampling, and backhoe methods.

Surface soil samples were collected from 0.0 to $0.5 \mathrm{ft}$ bgs at biased locations based on criteria listed in the CAIP.

Subsurface soil samples were collected from depths up to $11 \mathrm{ft}$ bgs at biased sample locations using the backhoe method.

Residual liquid and sludge samples were collected using disposable plastic scoops. Liquids were collected inside both tank chambers and the distribution box, while sludge was only found and sampled in the most upstream tank chamber.

\subsubsection{Leachfield (CAS 23-05-02)}

This CAS consists of a former leachfield and associated piping that serviced former Building 155 in Mercury, Area 23 of the NTS. The site is currently used as a parking lot and is covered with asphalt. The following sections summarize the field screening and intrusive investigation activities conducted at CAS 23-05-02.

\subsubsection{Field Screening}

Field screening was conducted on soil samples using an FID with the headspace method for volatiles, handheld instrument surveys for alpha and beta/gamma radiation, and gas chromatography for TPH-DRO. The FSRs were compared to FSLs to guide subsequent soil sampling decisions and to determine which samples were submitted for laboratory analysis. The TPH-DRO FSL was exceeded in one sample at CAS 23-05-02.

\subsubsection{Intrusive Investigation}

A total of 41 soil samples from 16 locations were collected and submitted to the laboratory for the analyses listed in Appendix A (Table A.9-1). Surface and shallow subsurface soil sampling activities were conducted at CAS 23-05-02. Soil samples were collected using Geoprobe ${ }^{\circledR}$ drilling methods. 
Surface soil samples were collected directly beneath the asphalt (approximately 0.5 to $1 \mathrm{ft}$ bgs) at biased locations identified in the CAIP and the Field Instructions.

Subsurface soil samples were collected from various depth intervals up to $8 \mathrm{ft}$ bgs at biased sample locations using the Geoprobe ${ }^{\circledR}$ method. No physical evidence of a leachfield was uncovered at any of the sample locations. Several locations were drilled to $12 \mathrm{ft}$ bgs to confirm the absence of leachfield features (e.g., piping, leaching rock).

\subsubsection{Waste Characterization}

Waste characterization activities were conducted to gather sufficient information and data to support waste disposal decisions. One concrete sample was collected from the decon pad at CAS 06-17-04 for waste characterization, as well as a total of 8 tank liquid samples from CASs 02-04-01, 05-04-01, and 11-04-01, and 5 tank sludge samples from CASs 05-04-01 and 11-04-01. Two additional sludge samples were collected from the two-chamber sump attached to the decon pad (CAS 06-17-04). In addition, the analytical results from the environmental soil sampling and radiological screening were used to determine the proper disposition of the investigation-derived waste (IDW). Additional information regarding this investigation activity is presented in Appendix A (Section A.4.0). Laboratory analysis for the waste characterization samples was performed in accordance with the CAIP.

\subsubsection{Conceptual Site Model Validation}

A conceptual site model (CSM) was developed to represent the release mechanisms and potential migration pathways for contaminant releases at CAU 224 CASs. The CSM and associated discussion for this CAS are provided in Figure A.1-9 and Section A.1.2.3 of the CAIP. The CSM addressed surface and shallow subsurface soils potentially impacted by releases of contaminants. The release mechanisms include spills/leaks from tanks and sumps, direct dumping of contaminants into leach pits and lagoons, and release from mixing during earthmoving activities as well as limited leaching and lateral physical dispersal during precipitation events.

The CSM assumed that all contaminant migration would be minimal based on the affinity of the contaminants of potential concern (COPCs) for soil particles, and the low precipitation and high 
potential evapotranspiration rates typical of the NTS environment. The extent of underlying soil impact was expected to be variable and dependent upon the concentration of contaminants released, physical, and chemical properties of the surrounding media, geological conditions, and physical and chemical properties of the COPCs.

The migration pathways and release mechanisms identified during the CAI were consistent with the CSM and all information gathered during the CAI support and validate the CSM as presented in the CAIP.

\subsection{Results}

The summary of data from the CAI provided in Section 2.2.1 defines the extent of contamination and identifies the areas within the CAU 224 CASs where the COCs exceeded final action levels (FALs). These data also fulfill the waste characterization and IDW disposal requirements. Section 2.2.2 summarizes the assessment made in Appendix B, which demonstrates that the investigation results satisfy the DQO data requirements.

\subsubsection{Summary of Analytical Data}

Chemical and radiological results for investigation samples collected at each of the CASs are summarized in Section 2.2.1.1 through Section 2.2.1.7. The preliminary action levels (PALs) for the CAU 224 investigation were determined during the DQO process and are discussed in Section 3.0 of the CAIP (NNSA/NSO, 2004). The FALs used for determining the presence of COCs and for evaluating the need for corrective action are defined in Section 3.1. Details about the methods used during this investigation and a comparison of environmental sample results to the FALs are presented in Appendix A.

\subsubsection{Septic Tank (Buried) (CAS 02-04-01)}

During the investigation of CAS 02-04-01, the only contaminants found above the action levels were several polycyclic aromatic hydrocarbons (PAHs). These results can be found in Table A.3-3 of Appendix A. Field documentation and photographs from the investigation verified that pieces of asphalt are abundantly present within the fill material surrounding the tank and in the soil samples collected. After consulting with the primary decision makers NNSA/NSO and NDEP, it was decided 
that the PAHs are attributed to the presence of asphalt. PAHs are commonly associated with asphalt and are therefore not considered COCs at this site.

\subsubsection{Leachfield (CAS 03-05-01)}

Corrective Action Site 03-05-01 consists of a former leach pit associated with a nearby acetylene generator facility at the Area 3 Subdock. During the CAI hand-augering activities, a whitish-gray substance was found at four locations in the area of the leach pit from approximately $1.5 \mathrm{ft}$ to $6.5 \mathrm{ft}$ bgs. A hard-packed caliche layer caused refusal directly below the foreign material. The material exhibits physical characteristics (color, odor, density) of calcium carbonate, which is a decomposition product of carbide used in acetylene generation. Samples of the foreign material were collected as well as surface samples above it. Total petroleum hydrocarbons-DRO was found at concentrations above PALs in the foreign material, ranging from 330 to 1,100 milligrams per kilogram $(\mathrm{mg} / \mathrm{kg})$. Due to the nature of the caliche layer beneath the former pit, it is believed that this confining layer has bounded the vertical extent of the contamination. The lateral extent was bounded through Decision II sampling around the pit. Also, one surface sample had a slightly elevated plutonium $(\mathrm{Pu})-239$ activity at 8.7 picocuries per gram $(\mathrm{pCi} / \mathrm{g})$, which was also bounded by Decision II step-out sampling.

\subsubsection{Septic Tanks (4)/Discharge Area (CAS 05-04-01)}

Decision I sampling at CAS 05-04-01 consisted of collecting the liquid and sludge from the four tanks and surface soils from the potential discharge area. The tank contents were found to be non-hazardous as no contaminants were present above action levels. During the polychlorinated biphenyl (PCB) analysis of the surface soil samples, the lab tentatively identified pesticides in the samples. A pesticide suite analysis was subsequently added to the analytical suite of all soil samples at the CAS. Chlordane, dichlorodiphenyl-dichloroethylene (DDE) and dichlorodiphenyltrichloroethane (DDT) were detected above the action levels in two of the surface samples. These locations were further sampled at a depth of 2-3 ft bgs, and three new Decision II locations were added in order to delineate the pesticide contamination. 


\subsubsection{Sewage Lagoons (3) (CAS 06-03-01)}

The Decision I sampling at CAS 06-03-01 was performed within the two areas bounded by two sets of concrete monuments identifying the areas as the closed sewage lagoons. Sample locations were excavated to a minimum depth of $8 \mathrm{ft}$ by trenching with a backhoe, with some locations excavated as deep as $12 \mathrm{ft}$ in order to attempt to find any features associated with the former lagoons (e.g., pipes, distribution boxes). No physical evidence of the lagoons was found during the CAI. It is believed that all the features were removed from the ground as part of the closure of the lagoons. None of the samples collected contained any contaminants above the action levels.

\subsubsection{Leachfield (CAS 06-05-01), Decon Pad and Wastewater Catch (CAS 06-17-04), and Decon Pad Discharge Piping (CAS 06-23-01)}

The soil above and below the leachfield and in the area of the associated lagoons (CAS 06-05-01) was collected by hand-sampling and backhoe methods. Three surface samples contain $\mathrm{Pu}-239$ above the FAL and were bounded during Decision II sampling. All soil beneath the decon pad (CAS 06-17-04) was found to be free of COCs, with the exception of an isolated spot of TPH-DRO contamination. The two-chamber sump attached to the decon pad was found to contain elevated levels of radionuclides as well as chemical contaminants above waste disposal criteria (e.g., RCRA). Upon generation of waste, the contents of the sump may need to be managed as mixed waste. Several samples collected around the perimeter of the decon pad, including four samples from one location adjacent to the sump outlet pipe (CAS 06-23-01), were also found to contain elevated TPH-DRO. During the Decision II sampling, the radionuclide contamination was found to spread downgradient toward the area of the former lagoons.

\subsubsection{Sewage Lagoon (CAS 11-04-01)}

All soil, liquid, and sludge samples collected at this CAS were found to contain no COCs.

\subsubsection{Leachfield (CAS 23-05-02)}

The former leachfield is located in an area that is currently an active motor pool parking lot. In order to minimally disturb the lot, the CAI was performed using a Geoprobe ${ }^{\circledR}$ rig. All Decision I locations were sampled to a depth of $8 \mathrm{ft}$ bgs while some locations were drilled to a depth of $12 \mathrm{ft}$ bgs in order 
to attempt to find any features of the former leachfield (piping, leachfield rock). No physical evidence of the leachfield was found during the CAI. It is believed that the leachfield was removed prior to or during the parking lot construction. Total petroleum hydrocarbons-DRO and several PAHs were detected above action levels at CAS 23-05-02. The PAHs can be attributed to the asphalt found either directly above the sample depth of in the fill around the sample, as observed in field documentation. The TPH-DRO hits were delineated by Decision II samples.

\subsubsection{Data Assessment Summary}

The DQA is presented in Appendix B and includes an evaluation of the DQIs to determine the degree of acceptability and usability of the reported data in the decision-making process. The DQO process ensures that the right type, quality, and quantity of data will be available to support the resolution of those decisions at an appropriate level of confidence. Using both the DQO and DQA processes help to ensure that DQO decisions are sound and defensible.

The DQA process as presented in Appendix B is comprised of the following steps:

- Step 1: Review DQOs and Sampling Design.

- Step 2: Conduct a Preliminary Data Review.

- Step 3: Select the Test.

- Step 4: Verify the Assumptions.

- Step 5: Draw Conclusions from the Data.

Sample locations that support the presence and/or extent of contamination at each CAS are shown in Appendix B. Based on the results of the DQA presented in Appendix A, the nature and extent of COCs at CAU 224 have been adequately identified to develop and evaluate corrective action alternatives.

The DQA determined that the data collected met the DQOs and support their intended use in the decision-making process. Additionally, information generated during the investigation support the CSM assumptions.

\subsection{Need for Corrective Action}

Analytes detected during the CAI were evaluated against FALs (defined in Section 3.1) to identify COCs. Contaminants of concern were detected in four of the nine CASs in CAU 224. These CASs 
require corrective action alternatives to be identified and evaluated to determine the appropriate closure strategies. The impacted volume/characteristics are provided in each CAS-specific subsection and the volume calculations for soil to be removed from each area are shown in Appendix C. Site-specific characteristics that might constrain remediation at each of the CASs are underground and/or overhead utilities and facility structures. The corrective action alternatives are identified in Section 3.0 and evaluated for their ability to ensure protection of the public and the environment in accordance with Nevada Administrative Code (NAC) 445A (NAC, 2003), feasibility, and cost effectiveness.

\subsubsection{Septic Tank (Buried) (CAS 02-04-01)}

The PAHs detected above PALs at CAS 02-04-01 were determined to be associated with asphalt in the fill material around the tank; therefore, they are not considered COCs. Because no COCs were detected at this CAS, corrective action is not required at this site. However, contents of the septic tank will be removed and disposed of as sanitary waste and the tank will be closed per Nevada regulation NAC 444.818 (NAC, 2002). Volume estimates for the tank and contents are presented in Appendix C.

\subsubsection{Leachfield (CAS 03-05-01)}

The TPH-DRO concentrations at CAS 03-05-01 do not exceed the FALs defined for TPH-DRO at this site in Section 3.1. No other COCs were identified above FALs. Therefore, no COCs are present at the site, and no corrective action is required.

\subsubsection{Septic Tanks (4)/Discharge Area (CAS 05-04-01)}

The pesticide contamination at this site is limited to the surface soil in the discharge area, at sample locations C03 and C04, with chlordane at 13,000 micrograms per kilogram $(\mu \mathrm{g} / \mathrm{kg})$ at location $\mathrm{C} 03$, and DDE and DDT at $12,000 \mu \mathrm{g} / \mathrm{kg}$ and $11,000 \mu \mathrm{g} / \mathrm{kg}$, respectively at location C04. Step-out samples verified that no pesticides exist above action levels further down the discharge area. The Clean Closure corrective action alternative will consist of removing the contaminated soil. The contents of the septic tank will be removed and disposed of as sanitary waste and the tank will be closed per Nevada regulation NAC 444.818 (NAC, 2002). As a best management practice, the supporting concrete pad, discharge piping, and distribution box will be removed and the piping 
leading to the distribution box will be capped. The volume estimates for soil, concrete, tank, and tank content removal are presented in Appendix C and include a 25 percent swell factor for costing purposes.

\subsubsection{Sewage Lagoons (3) (CAS 06-03-01)}

No COCs are present at this CAS; therefore, no corrective action is needed.

\subsubsection{Leachfield (CAS 06-05-01), Decon Pad and Wastewater Catch (06-17-04), and Decon Pad Discharge Piping (06-23-01)}

The radiological contamination in this area consists of three isolated spots of $\mathrm{Pu}-239$ contamination, as well several locations of TPH-DRO contamination around and underneath the decon pad. The wastewater sump next to the decon pad contains waste that may have to be managed as mixed waste when generated and will need to be removed along with its contents. See Table A.7-24 for a data summary of the sump contents. The soil around the sump also contains radiological and TPH-DRO contamination above the action levels. Clean closure corrective action at the site would consist of removal of the sump and contents, removal of the decon pad and two adjacent concrete pads, as well as soil removal. The volume estimates for soil, concrete and sump content removal are presented in Appendix $\mathrm{C}$ and include a 25 percent swell factor for costing purposes.

\subsubsection{Sewage Lagoon (CAS 11-04-01)}

No COCs were found at this CAS. Therefore, there is no need for corrective action at this site. However, contents of the septic tank will be emptied and disposed of as sanitary waste and the tank will be closed per Nevada regulation NAC 444.818 (NAC, 2002). Also, the piping leading to the tank will be capped and the manholes upstream from the tank will be filled in order to prevent future unauthorized dumping. Volume estimates for the tank and contents are presented in Appendix C.

\subsubsection{Leachfield (CAS 23-05-02)}

The TPH-DRO and PAHs found at this site above PALs are associated with the asphalt parking lot currently covering the site, not the site itself, and are therefore not considered COCs. No corrective action is needed at this CAS. 


\subsection{Summary}

Six of the nine CASs in CAU 224 require further action, four of them requiring corrective actions and two requiring tank closures. Septic tanks and distribution boxes (where applicable) will be removed from CASs 02-04-01, 05-04-01, and 11-04-01 per NAC 444.818 (NAC, 2002). A Clean Closure alternative will include soil removal at CASs 05-04-01, 06-05-01, 06-17-04, and 06-23-01, as well as concrete removal from CASs 05-04-01 and 06-17-04. Clean Closure would also include the removal and disposal of the wastewater sump adjacent to the decon pad at CAS 06-17-04, and of the pad itself. Volume estimates for these actions are presented in Appendix C. 


\subsection{Evaluation of Alternatives}

The purpose of this section is to present the corrective action objectives for CAU 224, describe the general standards and decision factors used to screen the various corrective action alternatives, and develop and evaluate a set of selected corrective action alternatives that will meet the corrective action objectives.

\subsection{Corrective Action Objectives}

The corrective action objectives are to ensure that each release site will not pose an unacceptable risk to human health and the environment and that conditions at each site are in compliance with all applicable laws and regulations. To ensure that these objectives are met, the information identified in the DQOs as necessary to be able to make the DQO decisions was collected and DQO decisions were resolved for each CAS. This process involved the comparison of CAI analytical data to risk-based cleanup goals defined herein as FALs. The FALs for all the constituents detected at CAU 224 CASs are the PALs determined during the DQO process, with the exception of TPH-DRO at CAS 03-05-01, and radiological contaminants at CASs 06-05-01, 06-17-04, and 06-23-01.

For chemical COPCs, PALs are based on EPA Region 9 Industrial Preliminary Remediation Goals (PRGs) (EPA, 2002) and $100 \mathrm{mg} / \mathrm{kg}$ for hydrocarbon-impacted soil (NAC, 2003). For CAS 03-05-01, the FAL for hydrocarbon-impacted soil was determined using the following assessment:

The FFACO Part III, Section III.3 (1996) stipulates conformance with Chapter 445 of the NAC. Section NAC 445A.227 lists requirements for sites with soil contamination. This section stipulates a process to determine the necessary remediation standards (or FALs) based on an evaluation of the risk the site poses to public health and the environment.

Section NAC 445A.22705 states:

1. Except as otherwise provided in NAC 445A.22715, if an owner or operator is required to take corrective action pursuant to NAC 445A.227, the owner or operator may conduct an evaluation of the site, based on the risk it poses to public health and the environment, to determine the necessary remediation standards or to establish that corrective action is not 
necessary. Such an evaluation must be conducted using Method E1739-95, adopted by the American Society for Testing and Materials, as it exists on October 3, 1996, or an equivalent method approved by the Division (i.e. NDEP).

2. The Division shall determine whether an evaluation complies with the requirements of Method E1739-95, or an equivalent method of testing approved by the Division. The Division may reject, require revisions be made to, or withdraw its concurrence with the evaluation at any time after the completion of the evaluation for the following reasons:

- The evaluation does not comply with the applicable requirements for conducting the evaluation;

- Conditions at the site have changed; or

- New information or previously unidentified information which would alter the results of the evaluation becomes available and demonstrates that the release may have a detrimental impact on public health or the environment.

Based on Section NAC 445A.2272, PALs are established by NNSA/NSO and used as action levels for site screening purposes. The PALs are not intended to be used as remediation standards (as defined in Section NAC 445A.22675). Section NAC 445A.22705 requires the use of American Society for Testing and Materials (ASTM) Method E1739-95 to “...conduct an evaluation of the site, based on the risk it poses to public health and the environment, to determine the necessary remediation standards or to establish that corrective action is not necessary." ASTM Method E1739-95 (Section 6.4.3, "Use of Total Petroleum Hydrocarbon Measurements") states: “The TPHs should not be used for risk assessment because the general measure of TPH provides insufficient information about the amounts of individual chemical(s) of concern present" (see also Sections X1.5.4 and X1.42 of the ASTM procedure).

Therefore, the TPH PAL of 100 parts per million (ppm) was used for screening purposes at all CASs and used for FALs at all CASs except CAS 03-05-01. At this CAS, the individual hazardous constituent analytical results (otherwise included in TPH analyses) were compared to corresponding FALs. Tables X1.2 and X1.3 of ASTM Method E1739-95 identify common chemicals of concern for TPH-impacted sites. These TPH chemicals of concern and the corresponding Region 9 PRGs are presented in Table 3-1. These Region 9 PRGs will be used as FALs for the TPH constituents detected in CAS 03-05-01. 
Table 3-1

Total Petroleum Hydrocarbons Constituent Final Action Levels for CAS 03-05-01

\begin{tabular}{|c|c|c|c|}
\hline $\begin{array}{l}\text { Chemical } \\
\text { Abstract } \\
\text { Service ID }\end{array}$ & TPH Constituent & $\begin{array}{c}\mathrm{FAL}^{\mathrm{a}} \\
(\mathrm{mg} / \mathrm{kg})\end{array}$ & Notes \\
\hline $91-57-6$ & 2-methylnaphthalene & 190 & \\
\hline $108-67-8$ & 1,3,5-trimethylbenzene & 70 & \\
\hline $120-12-7$ & anthracene & 100000 & \\
\hline $56-55-3$ & benz(a)anthracene & 2.1 & \\
\hline $71-43-2$ & benzene & 1.4 & \\
\hline $50-32-8$ & benzo(a)pyrene & 0.21 & \\
\hline $205-99-2$ & benzo(b)fluoranthene & 2.1 & \\
\hline $191-24-2$ & benzo(g,h,i)perylene & 29000 & uses pyrene as surrogate $P R G^{b}$ \\
\hline $207-08-9$ & benzo(k)fluoranthene & 21 & \\
\hline $218-01-9$ & chrysene & 210 & \\
\hline $100-41-4$ & ethylbenzene & 400 & \\
\hline $206-44-0$ & fluoranthene & 22000 & \\
\hline $86-73-7$ & fluorene & 26000 & \\
\hline $91-20-3$ & naphthalene & 190 & \\
\hline $85-01-8$ & phenanthrene & 29000 & uses pyrene as surrogate $P R G^{b}$ \\
\hline $129-00-0$ & pyrene & 29000 & \\
\hline $108-88-3$ & toluene & 520 & \\
\hline $1330-20-7$ & xylenes & 420 & \\
\hline
\end{tabular}

${ }^{a}$ FALs equal to Region 9 PRGs

${ }^{b}$ No PRG exists for benzo(g,h,i)perylene and phenanthrene; therefore, pyrene is used as a surrogate based on similar properties.

$\mathrm{FAL}=$ Final action level

PRG $=$ Preliminary Remediation Goal

$\mathrm{TPH}=$ Total petroleum hydrocarbons

The background concentration for arsenic at NTS was used for the arsenic PAL instead of its PRG because natural concentrations at NTS exceed the Region 9 PRG. The background concentration for arsenic is considered to be the mean plus two times the standard deviation for sediment samples collected by the Nevada Bureau of Mines and Geology throughout the Nevada Test and Training Range (formerly the Nellis Air Force Range) (NBMG, 1998; Moore, 1999). 
For radionuclide COPCs the dose-based isotope-specific PALs are based on the National Council on Radiation Protection and Measurement (NCRP) Report No. 129 recommended screening limits for construction, commercial, and industrial land use scenario (NCRP, 1999) based on a 25 millirem (mrem) per year dose and the generic guidelines for residual concentrations of radionuclides in DOE Order 5400.5 (DOE, 1993). The PALs for Ra-226, Ra-228, Th-230, Th-232, and their progeny in secular equilibrium are the generic guidelines for residual concentrations as found in Chapter IV of DOE Order 5400.5, Change 2, "Radiation Protection of the Public and Environment" (DOE, 1993).

The site specific FALs for radionuclides at CASs 06-05-01, 06-17-04 and 06-23-01 were calculated using the RESRAD computer code (version 6.22) and site-specific parameters. The RESRAD analysis was used as a Tier II data evaluation for these CASs after the initial screening criteria (i.e., NCRP PALs) were exceeded in a number of samples. The analysis used a conservative land-use scenario in order to ensure that any future users of the site would not incur an undue exposure and would demonstrate a good practice of the as-low-as-reasonably-achievable (ALARA) principle. The risk based evaluation established the FAL for $\mathrm{Pu}-238$ to be $186 \mathrm{pCi} / \mathrm{g}, \mathrm{Pu}-239$ to be $168 \mathrm{pCi} / \mathrm{g}$, americium (Am)-241 to be $154 \mathrm{pCi} / \mathrm{g}$, and cesium (Cs)-137 to be $36.2 \mathrm{pCi} / \mathrm{g}$. The RESRAD calculations were based on continued industrial use of the site assuming that a worker will be on the site for 250 days per year, 8 hours per day for a duration of 25 years. The method used to calculate the risk-based FALs is provided in Appendix G.

\subsection{Screening Criteria}

The screening criteria used to evaluate and select the preferred corrective action alternatives are identified in the EPA Guidance on RCRA Corrective Action Decision Documents (EPA, 1991) and the Final RCRA Corrective Action Plan (EPA, 1994).

Corrective action alternatives are evaluated based on four general corrective action standards and five remedy selection decision factors. All corrective action alternatives must meet the general standards to be selected for evaluation using the remedy selection decision factors.

The general corrective action standards are as follows:

- Protection of human health and the environment

- Compliance with media cleanup standards 
- Control of the source(s) of the release

- Compliance with applicable federal, state, and local standards for waste management

The remedy selection decision factors are as follows:

- Short-term reliability and effectiveness

- Reduction of toxicity, mobility, and/or volume

- Long-term reliability and effectiveness

- Feasibility

- Cost

\subsubsection{Corrective Action Standards}

The following text describes the corrective action standards used to evaluate the corrective action alternatives.

\section{Protection of Human Health and the Environment}

Protection of human health and the environment is a general mandate of the RCRA statute (EPA, 1994). This mandate requires that the corrective action include any necessary protective measures. These measures may or may not be directly related to media cleanup, source control, or management of wastes. The corrective action alternatives are evaluated for the ability to meet corrective action objectives as defined in Section 3.1.

\section{Compliance With Media Cleanup Standards}

Each corrective action alternative must meet the proposed media cleanup standards as set forth in applicable state and federal regulations, and as specified in the CAIP (NNSA/NSO, 2004). For CAU 224, the media cleanup standards are the FALs defined in Section 3.1.

\section{Control of the Source(s) of the Release}

Another objective of a corrective action remedy is to stop further environmental degradation by controlling or eliminating additional releases that may pose a threat to human health and the environment. Unless source control measures are taken, efforts to clean up releases may be ineffective or, at best, will essentially involve a perpetual cleanup. Therefore, each corrective action alternative must use an effective source control program to ensure the long-term effectiveness and protectiveness of the corrective action. At CAU 224, the sources of the contamination have been 
discontinued, with the exception of the wastewater sump at CAS 06-17-04, which still has the potential for release. To achieve clean closure, the source (i.e. the sump and its contents) will be removed and disposed of.

\section{Compliance With Applicable Federal, State, and Local Standards for Waste Management}

During implementation of any corrective action alternative, all waste management activities must be conducted in accordance with applicable federal and state regulations (e.g., 40 Code of Federal Regulations [CFR] 260-282, "Hazardous Waste Management" [CFR, 2003a]; 40 CFR 761 "Polychlorinated Biphenyls," [CFR, 2003b]; and NAC 444.842 to 98, "Management of Hazardous Waste" [NAC, 2002]).

The requirements for management of the waste, if any, derived from the corrective action will be determined based on applicable federal and state regulations, field observations, process knowledge, waste characterization data, and data collected and analyzed during corrective action implementation. Administrative controls (e.g., decontamination procedures and corrective action strategies) will minimize waste generated during site corrective action activities. Decontamination activities should be performed in accordance with approved procedures and selected according to the COCs present at the site.

\subsubsection{Remedy Selection Decision Factors}

The following text describes the remedy selection decision factors used to evaluate the corrective action alternatives.

\section{Short-Term Reliability and Effectiveness}

Each corrective action alternative must be evaluated with respect to its effects on human health and the environment during implementation of the selected corrective action. The following factors will be addressed for each alternative:

- Protection of the community from potential risks associated with implementation, such as fugitive dusts, transportation of hazardous materials, and explosion.

- Protection of workers during implementation. 
- Environmental impacts that may result from implementation.

- The amount of time until the corrective action objectives are achieved.

\section{Reduction of Toxicity, Mobility, and/or Volume}

Each corrective action alternative must be evaluated for its ability to reduce the toxicity, mobility, and/or volume of the contaminated media. Reduction in toxicity, mobility, and/or volume refers to changes in one or more characteristics of the contaminated media by the use of corrective measures that decrease the inherent threats associated with that media.

\section{Long-Term Reliability and Effectiveness}

Each corrective action alternative must be evaluated in terms of risk remaining at the CAU after the corrective action alternative has been implemented. The primary focus of this evaluation is on the extent and effectiveness of the control that may be required to manage the risk posed by treatment of residuals and/or untreated wastes.

\section{Feasibility}

The feasibility criterion addresses the technical and administrative feasibility of implementing a corrective action alternative and the availability of services and materials needed during implementation. Each corrective action alternative must be evaluated for the following criteria:

- Construction and Operation. Refers to the feasibility of implementing a corrective action alternative given the existing set of waste and site-specific conditions.

- Administrative Feasibility. Refers to the administrative activities needed to implement the corrective action alternative (e.g., permits, use restrictions, public acceptance, rights of way, off-site approval).

- Availability of Services and Materials. Refers to the availability of adequate off-site and on-site treatment, storage capacity, disposal services, necessary technical services and materials, and prospective technologies for each corrective action alternative. 


\section{Cost}

Costs for each alternative are estimated for comparison purposes only. The cost estimate for each corrective action alternative includes both capital, and operation and maintenance costs, as applicable. The following is a brief description of each component:

- Capital Costs. These costs include direct costs that may consist of materials, labor, construction materials, equipment purchase and rental, excavation and backfilling, sampling and analysis, waste disposal, demobilization, and health and safety measures. Indirect costs are separate and not included in the estimates.

- Operation and Maintenance. These costs are separate and include labor, training, sampling and analysis, maintenance materials, utilities, and health and safety measures. These costs are not included in the estimates.

Cost estimates for the corrective action alternatives are provided in Appendix C.

\subsection{Development of Corrective Action Alternatives}

This section identifies and briefly describes the viable corrective action technologies and the corrective action alternatives considered for CAU 224. Based on the review of existing data, future use, and current operations at the NTS, the following alternatives have been developed for consideration at CAU 224:

- Alternative 1 - No Further Action

- Alternative 2 - Clean Closure

- Alternative 3 - Close in Place with Administrative Controls

\subsubsection{Alternative 1 - No Further Action}

Under the No Further Action Alternative, no corrective action activities will be implemented. This alternative is a baseline case with which to compare and assess the other corrective action alternatives and their ability to meet the corrective action standards.

This alternative meets the corrective action objectives for CASs 02-04-01, 03-05-01, 06-03-01, 11-04-01, and 23-05-02 where no COCs were found in any of the samples collected. The septic tanks and a distribution box at CASs 02-04-01 and 11-04-01 will be closed per NAC 444.818 (NAC, 2002). 
At these two CASs, the piping leading to and/or from the tanks will be capped, and any manholes will be filled.

\subsubsection{Alternative 2 - Clean Closure}

For contaminated surface and subsurface soil, Alternative 2 includes excavating and disposing of all impacted soil and debris containing COCs. The Clean Closure alternative for CAS 06-17-04 also includes the removal and disposal of the concrete decon pad, the wastewater sump, and the sump contents. A visual inspection will be conducted to ensure that surface debris has been removed prior to the initiation of the corrective action. Verification soil samples will also be collected and analyzed for the presence of COCs once the known volume of contaminated soil is removed.

Any contaminated material that is removed will be disposed of at an appropriate disposal facility. All excavated areas will be returned to surface conditions compatible with the intended future use of the site. Overburden soil (as feasible), along with additional clean fill, will be used to backfill excavations after removal of the contaminated soil. Clean borrow soil will be removed from a nearby location for placement in depression, as necessary. The following sections provide the area-specific information regarding Alternative 2, Clean Closure. The volume calculations are provided in Appendix $\mathrm{C}$ and include a 25 percent swell factor for costing purposes.

\subsubsection{CAS 05-04-01, Septic Tanks (4)/Discharge Area}

Alternative 2 includes removal and proper disposal of pesticide contaminated soil in the discharge area. Verification samples will be collected from the base of the excavation and analyzed for pesticides to ensure the complete removal of contaminated soil. Contaminated material will be disposed of at an appropriate disposal facility. The excavated area will be backfilled with clean fill and regraded as necessary to return it to conditions compatible with current and potential future on-site activities. The four septic tanks will be removed or closed per NAC 444.818, along with their contents, the discharge piping, the distribution box, and the tank supporting concrete pad (NAC, 2002). The piping leading to the tanks will be capped. 


\subsubsection{CASs 06-05-01, Leachfield, 06-17-04, Decon Pad and Wastewater Catch, and 06-23-01, Decon Pad Discharge Piping}

Alternative 2 includes removal and proper disposal of TPH-DRO and radiologically contaminated soil, the decon pad concrete, and the wastewater sump. The material inside the sump will be disposed of as mixed waste due to the presence of radiological and chemical contamination. Verification samples will be collected and analyzed for the COCs to ensure the complete removal of contaminated soil. Contaminated material will be disposed of at an appropriate disposal facility. The excavated area will be backfilled with clean fill and regraded as necessary to return it to conditions compatible with current and potential future on-site activities.

\subsubsection{Alternative 3 - Close in Place with Administrative Controls}

For contaminated surface and subsurface soil, Alternative 3 includes the administrative activities and costs associated with use restriction for the CASs where contamination is present at levels that exceed the FALs. Administrative controls will restrict inadvertent contact with contaminated media by prohibiting any activity that would cause significant exposure of site occupants to the identified COCs.

\subsection{Evaluation and Comparison of Alternatives}

Each Corrective Action Alternative (CAA) presented in Section 3.3 will be evaluated based on the general corrective action standards and remedy selection decision factors described in Section 3.2. Evaluation and comparison of alternatives for CASs 05-04-01, 06-05-01, 06-17-04, and 06-23-01 will be considered as a group as the contamination characteristics and the potential remediation alternatives at these sites are similar. As discussed in Section 2.3, CAAs will not be evaluated for CASs 02-04-01, 03-05-01, 06-03-01, 11-04-01, and 23-05-02 because no COCs were detected.

\subsubsection{Evaluation of Alternative 1 - No Further Action}

The effectiveness of the No Further Action alternative is evaluated in the following sections. 
- Protection of Human Health and the Environment: Low - Contamination was discovered that exceeded the risk-based action levels.

- Compliance With Media Cleanup Standards: Low - COCs are present above media cleanup standards (final action levels).

- Control of the Source(s) of the Release: Medium - There are no current release sources to control, with the exception of the wastewater sump at CAS 06-17-04, which has the potential to release contamination to the surrounding soil. At CAS 05-04-01, the septic tanks and distribution box represent a potential for future unauthorized dumping, possibly creating a source.

- Compliance With Applicable Federal, State, and Local Standards for Waste Management: High - This alternative will not generate waste.

Remedy Selection Decision Factors

- Short-Term Reliability and Effectiveness: Low - This alternative is not effective at protecting human health and the environment because contamination present at these sites will remain in place.

- Reduction of Toxicity, Mobility, and/or Volume: Medium - This alternative will not reduce toxicity or mobility of the COCs that are present. It will not generate excavation waste volumes.

- Long-Term Reliability and Effectiveness: Low - This alternative is not effective at protecting human health and the environment because contamination present at these sites will remain in place.

- Feasibility: High - This alternative can be readily implemented.

- Cost: High - Scores high because the comparative costs for this alternative are very low.

\subsubsection{Alternative 2 - Clean Closure}

The effectiveness of the Clean Closure alternative is evaluated in the following sections.

\section{Closure Standards}

- Protection of Human Health and the Environment: High - Contamination exceeding the risk-based action levels will be removed. 
- Compliance With Media Cleanup Standards: High - Contamination exceeding the media cleanup standards (FALs) will be removed.

- Control of the Source(s) of the Release: Medium - There are no current release sources to control, with the exception of the sump at CAS 06-17-04, which will be removed under this alternative.

- Compliance With Applicable Federal, State, and Local Standards for Waste Management: Medium - Excavated waste will require characterization.

\section{Remedy Selection Decision Factors}

- Short-Term Reliability and Effectiveness: High - This alternative is reliable and effective at protecting human health and the environment because removal of contaminated media will prevent future exposure of site workers to COCs.

- Reduction of Toxicity, Mobility, and/or Volume: Medium - This alternative will result in a reduction of toxicity and mobility but will generate moderate waste volumes.

- Long-Term Reliability and Effectiveness: High - This alternative is reliable and effective at protecting human health and the environment because removal of contaminated media will prevent future exposure of site workers to COCs.

- Feasibility: High - This alternative can be readily implemented.

- Cost: Medium - The comparative costs for this alternative are relatively high due to significant volumes of soil to be removed.

\subsubsection{Alternative 3 - Close in Place with Administrative Controls}

The effectiveness of Close In Place with Administrative Controls is evaluated in the following sections.

\section{Closure Standards}

- Protection of Human Health and the Environment: High - Contamination exceeding the risk-based action levels will be identified and use restrictions implemented.

- Compliance With Media Cleanup Standards: Medium - Although COCs will not be removed, site workers will not be exposed to COCs above media cleanup standards (FALs). 
- Control of the Source(s) of the Release: Medium - There are no current release sources to control, with the exception of the sump at CAS 06-17-04, which will be removed under this alternative.

- Compliance With Applicable Federal, State, and Local Standards for Waste Management: High - This alternative will not generate waste.

\section{Remedy Selection Decision Factors}

- Short-Term Reliability and Effectiveness: High - This alternative is reliable in the short term before deterioration of site postings and before any changes in the system for tracking use restrictions. It is effective in providing increased protection of human health by preventing contact with COCs.

- Reduction of Toxicity, Mobility, and/or Volume: Medium - This alternative will not reduce toxicity or mobility of the COCs that are present. It will not generate excavation waste volumes.

- Long-Term Reliability and Effectiveness: Medium - This alternative is not as reliable in the long term because site postings and the use restriction system will need ongoing maintenance. It is effective in providing increased protection of human health by preventing contact with COCs.

- Feasibility: Medium - This alternative can be implemented but requires maintenance.

- Cost: Medium - There will be low installation costs but ongoing maintenance costs for this alternative may be cumulatively significant.

Table 3-2 presents a summary of the comparative rating evaluation of closure alternatives for CASs 05-04-01, 06-05-01, 06-17-04, and 06-23-01. The scoring listed in these tables equate to the ratings in the evaluation of corrective action standards and remedy selection decision factors. The high, medium, and low ratings equate to scores of 3,2 , and 1 , respectively. 
Table 3-2

Evaluation of Alternatives for CAS, 05-04-01, 06-05-01, 06-17-04, and 06-23-01

\begin{tabular}{|c|c|c|c|}
\hline \multicolumn{4}{|l|}{ Closure Standards } \\
\hline & CAA 1 & CAA 2 & CAA 3 \\
\hline Protection of Human Health and the Environment & 1 & 3 & 3 \\
\hline Compliance with Media Cleanup Standards & 1 & 3 & 2 \\
\hline Control the Source(s) of the Release & 2 & 2 & 2 \\
\hline Comply with Applicable Federal, State, and Local Standards for Waste Management & 3 & 2 & 3 \\
\hline \multicolumn{4}{|l|}{ Remedy Selection Decision Factors } \\
\hline & CAA 1 & CAA 2 & CAA 3 \\
\hline Short-Term Reliability and Effectiveness & 1 & 3 & 3 \\
\hline Reduction of Toxicity, Mobility, and/or Volume & 2 & 2 & 2 \\
\hline Long-Term Reliability and Effectiveness & 1 & 3 & 2 \\
\hline Feasibility & 3 & 3 & 2 \\
\hline Cost & 3 & 2 & 2 \\
\hline Score & 17 & 23 & 21 \\
\hline
\end{tabular}




\subsection{Recommended Alternatives}

The three corrective action alternatives were evaluated on their technical merits, focusing on performance, reliability, feasibility, and safety. The selected alternatives were judged to meet all requirements for the technical components evaluated. The selected alternatives meet all applicable state and federal regulations for closure of the sites and will minimize potential future exposure pathways to the contaminated media at CAU 224.

Alternative 1, No Further Action, is the preferred corrective action for the following CASs:

- CAS 02-04-01, Septic Tank (Buried), CAS 03-05-01, Leachfield, CAS 06-03-01, Sewage Lagoons (3), CAS 11-04-01, Sewage Lagoon, and CAS 23-05-02, Leachfield. No Further Action is consistent with past practices for CASs that do not contain COCs at concentrations exceeding corresponding FALs. A best management practice will be conducted at CASs 02-04-01 and 11-04-01 to remove septic tanks and a distribution box, cap the tank piping, and fill any manholes in order to prevent unauthorized dumping in the future. The tanks will be closed per NAC 444.818 (NAC, 2002).

Alternative 2, Clean Closure, is the preferred corrective action for the following CASs:

- CAS 05-04-01, Septic Tanks (4)/Discharge Area, CAS 06-05-01, Leachfield, CAS 06-17-04, Decon Pad and Discharge Area, and CAS 06-23-01, Decon Pad Discharge Piping. The highest scoring CAA (as presented in Table 3-2) of Clean Closure is consistent with past practices for CASs that contain COCs at concentrations exceeding corresponding FALs where the removal of contaminated media is feasible and cost-effective. The septic tanks at CAS 05-04-01 will be closed per NAC 444.818 (NAC, 2002).

Alternative 3, Close in Place, was not selected as a preferred corrective action for any CASs. However, physical obstacles such as underground utilities may prevent the implementation of CAA 2, Clean Closure, at CASs 06-05-01, 06-17-04, and 06-23-01, as excavation might not be safely achievable. Therefore Alternative 3 can be implemented in conjunction with Alternative 2 at these sites.

The preferred corrective action alternatives were evaluated on technical merit focusing on performance, reliability, feasibility, and safety. The alternatives were judged to meet all requirements for the technical components evaluated. The alternatives meet all applicable state and federal regulations for closure of the site and will eliminate potential future exposure pathways as a low 
CAU 224 CADD

Section: 4.0

Revision: 0

Date: May 2005

Page 37 of 39

occupancy facility to the contaminated soils at CAU 224. Implementation of corrective actions may potentially present risks to site workers. Therefore, appropriate health and safety procedures will be developed and implemented. 


\subsection{References}

CFR, see Code of Federal Regulations.

Code of Federal Regulations. 2003a. Title 40 CFR Parts 260 - 282, "Hazardous Waste Management." Washington, DC: U.S. Government Printing Office.

Code of Federal Regulations. 2003b. Title 40 CFR 761, "Polychlorinated Biphenyls (PCBs) Manufacturing, Processing, Distribution in Commerce and Prohibitions." Washington, DC: U.S. Government Printing Office.

DOE/NV, see U.S. Department of Energy, Nevada Operations Office.

EPA, see U.S. Environmental Protection Agency.

FFACO, see Federal Facility Agreement and Consent Order.

Federal Facility Agreement and Consent Order. 1996 (as amended). Agreed to by the State of Nevada, the U.S. Department of Energy, and the U.S. Department of Defense.

Moore, J., Science Applications International Corporation. 1999. Memorandum to M. Todd (SAIC) entitled, "Background Concentrations for NTS and TTR Soil Samples," 3 February.

Las Vegas, NV.

NAC, see Nevada Administrative Code.

NBMG, see Nevada Bureau of Mines and Geology.

NCRP, see National Council on Radiation Protection and Measurements.

NNSA/NSO, see U.S. Department of Energy, National Nuclear Security Administration Nevada Site Office.

NNSA/NV, see U.S. Department of Energy, National Nuclear Security Administration Nevada Operations Office.

NRS, see Nevada Revised Statutes.

National Council on Radiation Protection and Measurements. 1999. Recommended Screening Limits for Contaminated Surface Soil and Review of Factors Relevant to Site-Specific Studies, NCRP Report No. 129. Bethesda, MD.

Nevada Administrative Code. 2002. NAC 444, "Sanitation.” Carson City, NV. 
Nevada Administrative Code. 2003. NAC 445A, "Water Controls.” Carson City, NV.

Nevada Bureau of Mines and Geology. 1998. Mineral and Energy Resource Assessment of the Nellis Air Force Range, Open-File Report 98-1. Reno, NV.

U.S. Department of Energy. 1993. DOE Order 5400.5, Change 2. "Radiation Protection of the Public and the Environment." Washington, DC: U.S. Government Printing Office.

U.S. Department of Energy, National Nuclear Security Administration Nevada Operations Office. 2002a. Industrial Sites Quality Assurance Project Plan, Nevada Test Site, Nevada, Rev. 3, DOE/NV--372. Las Vegas, NV.

U.S. Department of Energy, National Nuclear Security Administration Nevada Operations Office. 2002b. Nevada Test Site Waste Acceptance Criteria, Rev. 4, DOE/NV--325. Las Vegas, NV.

U.S. Department of Energy, National Nuclear Security Administration Nevada Site Office. 2004. Corrective Action Investigation Plan for Corrective Action Unit 224: Decon Pad and Septic Systems, Nevada Test Site, Nevada, Rev. 0, DOE/NV--965. Las Vegas, NV.

U.S. Department of Energy, Nevada Operations Office. 1996. Final Environmental Impact Statement for the Nevada Test Site and Off-Site Locations in the State of Nevada, DOE/EIS 02430. Las Vegas, NV.

U.S. Environmental Protection Agency. 1991. Guidance on RCRA Corrective Action Decision Documents, EPA/540/G-91/011. Washington, DC: Office of Research and Development.

U.S. Environmental Protection Agency. 1994. Final RCRA Corrective Action Plan, EPA/520-R-94-004. Washington, DC: Office of Solid Waste and Emergency Response.

U.S. Environmental Protection Agency. 2000. Data Quality Objectives Process for Hazardous Waste Site Investigations, EPA QA/G-4HW, EPA/600/R-00/007. Washington, DC: Office of Environmental Information.

U.S. Environmental Protection Agency. 2002. Region 9 Preliminary Remediation Goals (PRGs). As accessed at www.epa.gov/region09/waste/sfund/prg/index.htm on 8 January 2003. Prepared by S.J. Smucker. San Francisco, CA. 
Appendix A

Corrective Action Investigation Report for CAU 224 


\section{A.1.0 Introduction}

This appendix details corrective action investigation activities and analytical results for CAU 224. Corrective Action Unit 224 is located in Areas 2, 3, 5, 6, 11, and 23 of the NTS (Figure 1-1 of the main document), and is comprised of the nine CASs listed below:

- 02-04-01, Septic Tank (Buried)

- 03-05-01, Leachfield

- 05-04-01, Septic Tanks (4)/Discharge Area

- 06-03-01, Sewage Lagoons, (3)

- 06-05-01, Leachfield

- 06-17-04, Decon Pad and Wastewater Catch

- 06-23-01, Decon Pad Discharge Piping

- 11-04-01, Sewage Lagoon

- 23-05-02, Leachfield

Corrective Action Site 02-04-01 is located in Area 2 of the NTS and consists of one buried septic tank and associated piping, which previously serviced the Area 2 Support Facility.

Corrective Action Site 03-05-01 is located in Area 3 of the NTS, in the Subdock complex. The CAS consists of a former leach pit that has been covered to grade.

Corrective Action Site 05-04-01 is located in Area 5 of the NTS. It consists of four septic tanks, associated piping, a distribution box, and a desert wash area that potentially received overflow from the tanks.

Corrective Action Site 06-03-01 is located in Area 6 of the NTS. It consists of the former Yucca Lake sewage lagoon systems.

Corrective Action Sites 06-05-01, 06-17-04, and 06-23-01 are located on CP-6 Hill in Area 6 of the NTS. These sites consist of a decon pad, leachfield, and associated piping and received wastewater from Buildings CP-2 and CP-6.

Corrective Action Site 11-04-01 is located in Area 11 of the NTS. It consists of an evapotranspiration bed, a two-chamber septic tank, distribution box, former sewage lagoon, and associated piping. The system received waste from the TaDD facility. 
Corrective Action Site 23-05-02 is located in Mercury, in Area 23 of the NTS. The consists of a former leachfield that received waste from former Building 155 and is currently used as a motor pool parking lot.

This CAU was investigated because process knowledge indicated the associated CASs may have been used to store or dispose of material considered to be hazardous or radioactive waste by current standards. The CAI was conducted in accordance with the CAU 224 CAIP as developed under the FFACO (1996).

Additional information regarding the history of each site, planning, and the scope of the investigation is presented in the CAU 224 CAIP (NNSA/NSO, 2004).

\section{A.1.1 Project Objectives}

The primary objective of the investigation was to provide sufficient information and data to develop appropriate corrective action alternatives for each CAS in CAU 224. This objective was achieved by identifying the absence or presence of COPCs, the nature of the COCs (i.e., COPCs at concentrations above FALs), and the vertical and lateral extent of the COCs.

The selection of soil and/or waste characterization sample locations was based on site conditions, and the strategy developed during the DQO process as outlined in the CAU 224 CAIP. The sampling strategy primarily involved collecting soil samples from biased locations and liquid and sludge waste management samples from locations where such material was present.

\section{A.1.2 Content}

This appendix contains information and data in sufficient detail to support the selection of a preferred corrective action alternative in the CADD. The contents of this appendix are as follows:

- Section A.1.0 describes the investigation background, objectives, and content.

- Section A.2.0 provides an investigation overview.

- Section A.3.0 through Section A.9.0 provides CAS-specific information regarding the field activities, sampling methods, and laboratory analytical results from investigation sampling. 
- Section A.10.0 summarizes waste management activities.

- Section A.11.0 discusses the quality assurance (QA) and QC procedures followed and results of the QA/QC activities.

- Section A.12.0 is a summary of the investigation results.

- Section A.13.0 lists the cited references.

The complete field documentation and laboratory data, including field activity daily logs (FADLs), sample collection logs, analysis request/chain-of-custody forms, soil sample descriptions, laboratory certificates of analyses, analytical results, and surveillance results are retained in project files as hard copy files or electronic media. 


\section{A.2.0 Investigation Overview}

Field investigation and sampling activities for the CAU 224 Corrective Action Investigation (CAI) were conducted from August 2004 to January 2005. Table A.2-1 lists the CAI activities that were conducted at each of the CASs.

Table A.2-1

Corrective Action Investigation Activities Conducted at Each Corrective Action Site To Meet Corrective Action Investigation Plan Requirements

\begin{tabular}{|c|c|c|c|c|c|c|c|c|c|}
\hline \multirow[b]{2}{*}{ Corrective Action Investigation Activities } & \multicolumn{9}{|c|}{ Corrective Action Site } \\
\hline & $\begin{array}{l}\text { o } \\
\dot{y} \\
\text { d } \\
\stackrel{1}{\sim}\end{array}$ & 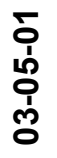 & 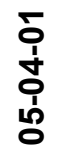 & $\begin{array}{l}\overline{1} \\
\text { ஸ் } \\
\text { ơ }\end{array}$ & 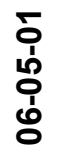 & 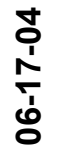 & 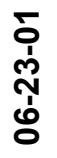 & $\begin{array}{l}5 \\
\stackrel{1}{8} \\
\text { ó } \\
\stackrel{1}{r}\end{array}$ & 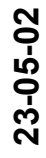 \\
\hline Inspected CAS system components & $\mathrm{X}$ & $\mathrm{X}$ & $\bar{X}$ & $\bar{X}$ & $\bar{X}$ & $\bar{X}$ & $\mathrm{X}$ & $\bar{X}$ & $\bar{X}$ \\
\hline Performed site walkovers & $\mathrm{X}$ & $\mathrm{X}$ & $\mathrm{X}$ & $\mathrm{X}$ & $\mathrm{X}$ & $\mathrm{X}$ & $\mathrm{X}$ & $\bar{x}$ & $\mathrm{X}$ \\
\hline Collected biased soil samples & $\mathrm{X}$ & $\mathrm{X}$ & $\mathrm{X}$ & $\mathrm{X}$ & $\mathrm{X}$ & $\mathrm{X}$ & $\mathrm{X}$ & $\mathrm{X}$ & $\mathrm{X}$ \\
\hline Field-screened samples for alpha and beta/gamma radiation & $\mathrm{X}$ & $\mathrm{X}$ & $\bar{X}$ & $\bar{x}$ & $\mathrm{X}$ & $\mathrm{X}$ & $\mathrm{X}$ & $\bar{x}$ & $\bar{X}$ \\
\hline Field-screened soil samples for volatile organic compounds & $\mathrm{X}$ & $\mathrm{X}$ & $\mathrm{X}$ & $\mathrm{X}$ & $\mathrm{X}$ & $\mathrm{X}$ & $\mathrm{X}$ & $\mathrm{X}$ & $\mathrm{X}$ \\
\hline $\begin{array}{l}\text { Field-screened soil samples for total petroleum hydrocarbons via } \\
\text { gas chromatograph }\end{array}$ & $X$ & $\mathrm{X}$ & $\mathrm{X}$ & $\mathrm{X}$ & $\mathrm{X}$ & $\mathrm{X}$ & $\mathrm{X}$ & $\mathrm{X}$ & $\mathrm{X}$ \\
\hline Submitted select samples for off-site laboratory analysis & $\mathrm{X}$ & $\mathrm{X}$ & $\mathrm{X}$ & $\mathrm{X}$ & $\mathrm{X}$ & $\mathrm{X}$ & $\mathrm{X}$ & $\mathrm{X}$ & $\mathrm{X}$ \\
\hline Conducted waste characterization sampling & $\mathrm{X}$ & & $\mathrm{X}$ & & & & & $\mathrm{X}$ & \\
\hline
\end{tabular}

The investigation and sampling program was managed in accordance with the requirements set forth in the CAU 224 CAIP (NNSA/NSO, 2004). Field activities were performed in accordance with the approved CAU 224 Site-Specific Health and Safety Plan (SNJV, 2004) that is consistent with the DOE Integrated Safety Management System. Samples were collected and documented following approved protocols and procedures indicated in the CAU 224 CAIP. Quality control samples (e.g., field blanks, equipment rinsate blanks, trip blanks, and duplicate samples) were collected as required by the Industrial Sites QAPP (NNSA/NV, 2002) and the CAU 224 CAIP (NNSA/NSO, 2004). During field activities, waste minimization practices were followed according to approved procedures, including segregation of waste by waste stream. 
Weather conditions at the site varied to include sun (moderate to high temperatures), rain, intermittent cloudiness, and light to strong winds. Storms (accompanied by lightning) occasionally delayed site operations.

The CASs were investigated by conducting radiological surface screening and surveys, and sampling potential contaminant sources (e.g. tank liquids), surface, and subsurface soils. Surface soil samples were collected by hand excavation. Subsurface soil samples were collected using hand-augering, backhoe, or drilling operations. Investigation media intervals and soil samples were field screened for VOCs, alpha and beta/gamma radiation, and TPH at specific locations. The results were compared against screening levels to guide in the CAS-specific investigations. Resultant samples were shipped to off-site laboratories to be analyzed for appropriate chemical and radiological parameters. These readings were also used to guide sampling decisions and health and safety controls.

Except as noted in the following CAS-specific sections, CAU 224 sampling locations were generally accessible and sampling activities at planned locations were not restricted by buildings, storage areas, active operations, or aboveground and underground utilities. The required sampling step-out locations were accessible and remained within anticipated spatial boundaries except where otherwise noted.

Section A.2.1 through Section A.2.7 provide the investigation methodology, site geology and hydrology, and laboratory analytical information. Additional activity-specific details for the individual CASs are presented in Section A.3.0 through Section A.4.0.

\section{A.2.1 Preliminary Conceptual Models}

The revised conceptual site model for CAU 224 is consistent with the preliminary CSM provided in the CAU 224 CAIP, with the exception of CAS 06-17-04, Decon Pad and Wastewater Catch. At this CAS, the spatial boundary of the scope of the CAS was expanded from the $45 \mathrm{ft}$ proposed in the CAIP in order to laterally bound the radiological contamination in soils to the south of the site. 


\section{A.2.2 Sample Locations}

Investigation locations selected for sampling were based on interpretation of existing engineering drawings, aerial and land photographs, interviews with former and current site employees, information obtained during site visits, and site conditions as provided in the CAU 224 CAIP. Sampling points for each site were selected based on the approach provided in the CAIP. The planned sample locations are discussed in text and shown on figures in the CAIP. All actual sample locations are depicted on the figures included in Section A.3.0 through Section A.9.0. Some locations were modified slightly from planned positions due to field conditions and observations. In some cases, FSR and/or laboratory analytical results determined the need for step-out sampling locations. Sample locations were staked and labeled appropriately, and the majority were surveyed with a global positioning system (GPS) instrument. The actual locations have been plotted based on the coordinates collected by the GPS instrument. In addition to the sampling locations, the figures in Appendix D of this document show points of interest with their associated GPS coordinates.

No items or materials were removed from any of the CAS area boundaries before or during the CAU $224 \mathrm{CAI}$, with the exception of sampled media.

\section{A.2.3 Investigation Activities}

The investigation activities performed at CAU 224 were based on general field investigation activities discussed in the CAU 224 CAIP (NNSA/NSO, 2004). The technical approach consisted of the activities listed in Table A.2-1. The investigation strategy allowed the nature and extent of contamination associated with each CAS to be established. The following sections describe the specific investigation activities that took place at CAU 224.

\section{A.2.3.1 Site Walkovers}

Site walkovers were performed at all nine CASs within CAU 224. Observations were made to identify biased sampling locations (e.g., soil beneath pipe outlets, low elevation spots). 


\section{A.2.3.2 Field Screening}

Field-screening activities for VOCs, TPH, and alpha and beta/gamma radiation were performed as specified in the CAU 224 CAIP. The FSL for VOC headspace was established at 20 ppm or 2.5 times background, whichever was greater. The TPH FSL was established at 75 ppm. Site-specific FSLs for alpha and beta/gamma radiation were defined as the mean background activity level plus 2 times the standard deviation of readings from 10 background locations selected near each CAS. The radiation FSLs are instrument-specific and were established daily for each instrument and CAS prior to sampling.

All field screening for VOCs was conducted using an FID. Alpha and beta/gamma radiation screening was performed at each CAS using a NE Technologies Electra or E-600 fitted with a DP6 dual-alpha and beta/gamma radiation scintillation probe. Field screening for TPH at CASs 02-04-01, 03-05-01, 05-04-01, 06-03-01, and 23-05-02 was conducted using a SRI Gas Chromatograph. Sludge from the septic tanks at CASs 02-04-01, 05-04-01, and 11-04-01 was screened for fecal coliform.

The CAS-specific sections of this document identify the CASs where field screening was conducted and how the FSLs were used to aid in the selection of sample locations. Field-screening results are recorded on sample collection logs that are retained in project files.

\section{A.2.3.3 Surface and Subsurface Sampling}

Intrusive investigation activities (i.e., surface and subsurface soil sampling) were conducted at all CASs within CAU 224 to support Decision I and Decision II investigation activities. Soil samples were collected using "scoop and trowel" (surface hand-grab sampling), hand-auger, backhoe, and rotosonic drilling methods. Regardless of the sampling method, the sample location was initially field screened for alpha and beta/gamma radiation prior to the start of sampling. Additional screening was conducted during sample collection to both guide the investigation and serve as a health and safety control to protect the sampling team. Labeled sample containers were filled according to the following sequence: total VOCs sample containers were filled with soil directly from the surface location, backhoe bucket or core bag, followed by the collection of soil for VOC field screening and soil for TPH field screening. Additional soil was transferred into an aluminum pan, homogenized, and field screened for alpha and beta/gamma radiation. All remaining sample containers were then 
filled. Excess soil was returned to its original location. No void spaces remained in the boreholes or excavations after backfilling with cuttings.

Surface soil samples were collected from 0.0 to $0.5 \mathrm{ft}$ bgs at biased locations focusing on stained soil, above and underground features (e.g., pipe outlets, buried distribution piping), or areas with elevated radiological measurements. Subsurface soil samples were collected as a continuation at a surface soil sample location. Excavation was required at most CASs in order to uncover buried site features such as tanks and piping, and sample soil adjacent to these features.

\section{A.2.3.3.1 Waste Characterization and Sampling}

Characterization of liquid and sludge from the septic tanks at CASs 02-04-01, 05-04-01, and 11-04-01, as well as concrete from the wastewater trench and sludge from the two-chamber sump at CAS 06-17-04 was conducted in order to support the disposal of these features during anticipated closure activities. This information was used to determine whether the liquid/sludge in question at these CASs could be acting as a source of potential soil contamination, and whether the concrete and tank media would have to be treated as hazardous or radiological waste. Investigation methods included VOC and radiological screening of all tank access points and direct sampling of tank contents. Concrete was sampled using the rotation component of the rotosonic drill rig to break up the surface of the trench. Waste characterization activities were intended to gather adequate information and data about the CAS to support decisions regarding the disposal of materials located within each CAS.

Samples were analyzed in accordance with the procedures specified in the CAU 224 CAIP. The specific analyses for each CAS are listed in CAS-specific sections and the analytical results are compared to the federal limits for hazardous waste, NDEP hydrocarbon action limit, landfill acceptance criteria, and the limits in the NTS performance objective criteria (POC) (BN, 1995). The POC limits have been established for NTS hazardous waste generators to ensure that all hazardous waste being shipped off-site contains no "added radioactivity." 
Specific waste characterization sampling and analysis were conducted on the following potential waste streams:

- Residual liquids in the septic tank at CAS 02-04-01

- Residual liquids and sludge in the septic tanks at CAS 05-04-01

- Residual liquids and sludge in the septic tank at CAS 11-04-01

- Concrete inside the wastewater trench at CAS 06-17-04

- Sludge from both chambers of the sump at CAS 06-17-04

Sample locations were selected so that they were representative of the sampling area and material being sampled. For example, the concrete was sampled inside the trench that carried wastewater from the decon pad to the adjacent sump, representing the concrete most likely to be contaminated.

\section{A.2.3.3.2 Sample Location Documentation}

A GPS instrument was used for determining the sample location coordinates as well as CAS points of interest, such as boundaries. Appendix D presents this data in tabular form.

\section{A.2.4 Laboratory Analytical Information}

Chemical and radiological analyses were performed by Paragon Analytics, Inc., Fort Collins, Colorado. The analytical parameters and laboratory analytical methods used to analyze investigation samples are listed in Table A.2-2. Organic and inorganic analytical results are reported in this appendix if they were detected at or above the minimum reporting levels (MRLs) established in Table 3-2 of the CAU 224 CAIP (NNSA/NSO, 2004). Radionuclide analytical results are reported in this appendix if they are detected at or above minimum detectable concentrations (MDCs).

Validated analytical data for CAU 224 investigation samples have been compiled and evaluated to confirm the presence of contamination and define the extent of contamination, if present. The analytical results for each CAS are presented in Section A.3.0 through Section A.9.0. The analytical results have been compared to MRLs or MDCs, as appropriate, and only those above MRLs or MDCs are included in CAS-specific tables. The complete laboratory data packages are available in the project files.

The analytical parameters are CAS-specific and were selected through the application of site process knowledge according to the EPA's Guidance for the Data Quality Objectives Process (EPA, 1994b). Samples collected during step-out sampling were only analyzed for the COPCs that exceeded FALs 
Table A.2-2

\section{Laboratory Analytical Parameters and Methods, CAU 224 Investigation Samples}

\begin{tabular}{|c|c|}
\hline Analytical Parameter & Analytical Method \\
\hline Total volatile organic compounds & SW-846 8260B ${ }^{a}$ \\
\hline $\begin{array}{l}\text { Total semivolatile organic compounds (including } \\
\text { Hydroquinone at CASs } 11-04-01 \text { and 23-05-02) }\end{array}$ & SW-846 $8270 \mathrm{C}^{\mathrm{a}}$ \\
\hline Total petroleum hydrocarbons - diesel-range organics & SW-846 8015B (modified) ${ }^{a}$ \\
\hline $\begin{array}{l}\text { Total Resource Conservation and Recovery Act (RCRA) } \\
\text { metals }{ }^{\text {b }}\end{array}$ & \multirow{2}{*}{$\begin{array}{l}\text { Water - SW-846 6010B/7470A } \\
\text { Soil - SW-846 6010B/7471A }\end{array}$} \\
\hline $\begin{array}{l}\text { Aluminum, Antimony, Beryllium, Cobalt, Copper, } \\
\text { Manganese, Molybdenum, Nickel, and Zinc }\end{array}$ & \\
\hline Total pesticides, including technical chlordane. & SW-846 8081 \\
\hline Polychlorinated biphenyls & SW-846 8082A \\
\hline Cyanide & SW-846 9010B \\
\hline $\begin{array}{l}\text { Toxicity characteristic leaching procedure (TCLP) volatile } \\
\text { organic compounds }\end{array}$ & SW-846 1311/8260B ${ }^{a}$ \\
\hline TCLP semivolatile organic compounds & SW-846 1311/8270C ${ }^{a}$ \\
\hline TCLP pesticides and herbicides & SW-846 1311/8081 and SW-8461311/ 8151 \\
\hline TCLP RCRA metals ${ }^{b}$ & SW-846 1311/6010B/7470A ${ }^{a}$ \\
\hline Gamma-emitting radionuclides & $\begin{array}{l}\text { Water - EPA } 901.1 \\
\text { Soil - HASL-300 }\end{array}$ \\
\hline Isotopic uranium & $\begin{array}{l}\text { Water - ASTM D3972-02 } \\
\text { Soil - ASTM C1000-02 }\end{array}$ \\
\hline Isotopic plutonium & $\begin{array}{l}\text { Water - ASTM D3865-02 } \\
\text { Soil - ASTM C1001-00 }\end{array}$ \\
\hline Strontium-90 & $\begin{array}{l}\text { Water - ASTM D5811-00 } \\
\quad \text { Soil - HASL-300 }\end{array}$ \\
\hline
\end{tabular}

aU.S. Environmental Protection Agency, Test Methods for Evaluating Solid Waste, Physical/Chemical Methods, 3rd Edition, Parts 1-4, SW-846 CD ROM. Washington, DC. (EPA, 1996)

${ }^{\mathrm{b}}$ Arsenic, barium, cadmium, chromium, lead, mercury, selenium, and silver

in the original samples. Bioassessment samples were not collected because FSRs and observations did not indicate the need.

\section{A.2.5 Comparison to Action Levels}

Chemicals and radionuclides detected in samples at concentrations greater than FALs are identified as COCs. If COCs are present, corrective action must be considered for the CAS. The PALs for the 
CAU 224 investigation were identified and agreed to during the DQO process. The FALs are defined in Section 3.1.

Sample data that are equal to or greater than MRLs are tabulated in the CAS-specific sections that follow. Results that are equal to or greater than FALs (a subset of those that exceed MRLs) are identified by bold text in the corresponding tables and discussed in Section A.3.0 through Section A.9.0.

Nondetected results and results below MRLs have been excluded to minimize the size of this document. However, the unedited dataset for CAU 224 is retained in an electronic format in the project files.

\section{A.2.6 Geology}

Regional native surface soil primarily consists of poorly graded, moderately consolidated, alluvial silty-sand intermixed with poorly sorted cobble- to pea-sized volcanic and sedimentary detritus. Subsurface soils ranged from gravelly sands with little-to-no fines to well-graded sands. The percentage of organic matter is ill-defined with low occurrence on the surface and decreasing with depth beyond the native soil interface.

A general field description for each sample was recorded on sample collection logs (SCLs). A more detailed description of the regional geology for the NTS is provided in the CAU 224 CAIP (NNSA/NSO, 2004).

\section{A.2.7 Hydrology}

Dry washes provide channels that concentrate surface runoff; however, there is no perennial stream flow in the region. Surface topography at the CAU 224 CASs range from flat at CASs 06-03-01 (dry lake bed), 02-04-01, 03-05-01, 11-04-01, and 23-05-01, to hills and ravines at CAS 05-04-01 and hill-top with slopes at CASs 06-05-01, 06-17-04, and 06-23-01.

Due to the depth to groundwater and climatic conditions, groundwater at the NTS in Areas 2, 3, 5, 6, 11, and 23 is not expected to have been impacted by COPCs. Groundwater depths at wells near the CAU 224 CASs range from approximately $700 \mathrm{ft} \mathrm{bgs}$ in Area 5 to 2,053 ft bgs in Area 2. A more 
detailed description of the regional geology for the NTS is provided in the CAU 224 CAIP (NNSA/NSO, 2004).

No saturated zones (e.g., perched water, contaminant saturation) were found anywhere in the subsurface adjacent to or below the CASs, nor were saturated intervals identified during sampling activities.

Potential evapotranspiration at the NTS is significantly greater than precipitation, thus limiting vertical migration of contaminants. The annual average precipitation for this region is only 3 to 6 inches (in.) per year (USGS, 1975). The potential annual evaporation is the dominant factor influencing the movement of water in the upper saturated zone. Therefore, recharge to groundwater from precipitation is not significant at the NTS and does not provide a significant mechanism for migration of contaminants to groundwater. 


\section{A.3.0 CAS 02-04-01, Septic Tank (Buried)}

Corrective Action Site 02-04-01 is a buried septic tank and associated piping that serviced the Area 2 Support Facility. More information regarding the history of the site can be found in the CAIP. The tank is $3 \mathrm{ft} 7 \mathrm{in}$. wide, $9 \mathrm{ft} 5 \mathrm{in}$. long and $2 \mathrm{ft} 7 \mathrm{in}$. deep. There is a 3 - $\mathrm{ft}$ pipe ( 8 in. inner diameter) going from the top of the tank to approximately $1 \mathrm{ft}$ above the surface with a metal lid.

\section{A.3.1 Corrective Action Investigation}

A total of 23 soil characterization samples and 1 liquid waste management sample were collected during investigation activities at CAS 02-04-01. The sample identification numbers (IDs), locations, types, and analyses are listed in Table A.3-1. The sample locations are shown on Figure A.3-1. The specific CAI activities conducted to satisfy the CAIP requirements at this CAS are described in the following sections.

\section{A.3.1.1 Field Screening}

Soil samples were field screened for VOCs, TPH, and alpha and beta/gamma radiation. The FSRs were compared to FSLs to guide subsequent sampling decisions. The VOC headspace, TPH, and alpha and beta/gamma radiation FSLs were not exceeded during sampling activities at this CAS. One sludge sample was collected for fecal coliform field screening, and the results were negative. The laboratory was notified of these results prior to handling the tank liquid sample.

\section{A.3.1.2 Sampling}

Decision I sampling activities included the collection of surface soil samples as well as subsurface soil samples adjacent to the septic tank, and a liquid sample of residual contents within the tank. No obvious release of contaminants was observed. The soil around the tank was excavated using a backhoe, with samples taken at the surface above the tank, the 2-3.5 ft interval near the top of the tank, the 4-6 ft interval near the bottom of the tank, and the 7-8 ft interval below the bottom of the tank. One inlet pipe was located on the east side of the tank, at $30 \mathrm{in}$. bgs. The pipe split into two with a Y-joint $9.5 \mathrm{ft}$ east of the tank. Soil sample location A08 was placed at the pipe inlet point to the tank, and soil sample location A09 was placed at the Y-joint. 
Table A.3-1

Samples Collected at CAS 02-04-01, Septic Tank (Buried) (Page 1 of 2)

\begin{tabular}{|c|c|c|c|c|c|}
\hline $\begin{array}{l}\text { Sample } \\
\text { Location }\end{array}$ & $\begin{array}{l}\text { Sample } \\
\text { Number }\end{array}$ & $\begin{array}{c}\text { Depth } \\
\text { (ft bgs) }\end{array}$ & $\begin{array}{l}\text { Sample } \\
\text { Matrix }\end{array}$ & Purpose & Analyses \\
\hline \multirow{4}{*}{$\mathrm{A} 01$} & $224 \mathrm{~A} 001$ & $0-0.5$ & Soil & SC & Set 2 \\
\hline & $224 \mathrm{~A} 008$ & $2-3$ & Soil & SC & Set 2 \\
\hline & 224A009 & $5-6$ & Soil & SC & Set 1 \\
\hline & $224 \mathrm{~A} 010$ & $7-8$ & Soil & SC & Set 2 \\
\hline \multirow{5}{*}{$\mathrm{A} 02$} & 224A002 & $0-0.5$ & Soil & SC & Set 1 \\
\hline & $224 \mathrm{~A} 011$ & $2-3$ & Soil & SC & Set 2 \\
\hline & 224A012 & $5-6$ & Soil & SC & Set 2 \\
\hline & $224 \mathrm{~A} 013$ & $7-8$ & Soil & SC & Set 1 \\
\hline & 224A014 & $7-8$ & Soil & $\begin{array}{c}\text { Field Duplicate } \\
\text { of \#24A013 }\end{array}$ & Set 1 \\
\hline A03 & $224 \mathrm{~A} 003$ & $0-0.5$ & Soil & $\mathrm{SC}, \mathrm{MS} / \mathrm{MSD}$ & Set 2 \\
\hline A04 & $224 \mathrm{~A} 004$ & $0-0.5$ & Soil & SC & Set 2 \\
\hline A05 & 224A005 & $0-0.5$ & Soil & SC & Set 2 \\
\hline A06 & 224A006 & $0-0.5$ & Soil & SC & Set 1 \\
\hline \multirow{4}{*}{$\mathrm{A} 07$} & 224A007 & $0-0.5$ & Soil & SC & Set 2 \\
\hline & 224A021 & $2-3$ & Soil & SC & Set 2 \\
\hline & $224 \mathrm{~A} 022$ & $4-5$ & Soil & SC & Set 1 \\
\hline & $224 \mathrm{~A} 023$ & $7-8$ & Soil & SC & Set 2 \\
\hline \multirow{3}{*}{ A08 } & $224 \mathrm{~A} 015$ & $2.5-3.5$ & Soil & SC & Set 2 \\
\hline & $224 \mathrm{~A} 016$ & $4-5$ & Soil & SC & Set 2 \\
\hline & $224 \mathrm{~A} 017$ & $7-8$ & Soil & SC & Set 2 \\
\hline \multirow{3}{*}{ A09 } & $224 \mathrm{~A} 018$ & $2.5-3.5$ & Soil & SC & Set 1 \\
\hline & 224A019 & $4-5$ & Soil & SC & Set 2 \\
\hline & $224 \mathrm{~A} 020$ & $7-8$ & Soil & SC & Set 2 \\
\hline NA & $224 \mathrm{~A} 301$ & NA & Water & Trip Blank & Total VOCs \\
\hline NA & 224A302 & NA & Water & Field Blank & Set 1 \\
\hline NA & $224 \mathrm{~A} 303$ & NA & Water & Trip Blank & Total VOCs \\
\hline NA & 224A304 & NA & Water & Trip Blank & Total VOCs \\
\hline NA & 224A304A & NA & Water & Trip Blank & Total VOCs \\
\hline NA & $224 \mathrm{~A} 305$ & NA & Water & Source Blank & Set 1 \\
\hline NA & $224 \mathrm{~A} 306$ & NA & Water & $\begin{array}{c}\text { Equipment } \\
\text { Rinsate Blank }\end{array}$ & Set 1 \\
\hline
\end{tabular}


Table A.3-1

Samples Collected at CAS 02-04-01, Septic Tank (Buried)

(Page 2 of 2)

\begin{tabular}{|c|c|c|c|c|c|}
\hline $\begin{array}{c}\text { Sample } \\
\text { Location }\end{array}$ & $\begin{array}{c}\text { Sample } \\
\text { Number }\end{array}$ & $\begin{array}{c}\text { Depth } \\
\text { (ft bgs) }\end{array}$ & $\begin{array}{c}\text { Sample } \\
\text { Matrix }\end{array}$ & Purpose & Analyses \\
\hline \hline \multirow{2}{*}{ Tank1 } & $224 \mathrm{~A} 501$ & NA & Liquid & WM & Set 1 \\
\cline { 2 - 6 } & $224 \mathrm{~A} 502$ & NA & Sludge & FC & Fecal Coliform \\
\hline
\end{tabular}

Set 1 = Total VOCs, Total SVOCs, Total RCRA Metals, Aluminum, Antimony, Beryllium, Cobalt, Copper, Manganese, Molybdenum, Nickel, Zinc, TPH-DRO, PCBs, Gamma Spectroscopy, Isotopic Plutonium, Isotopic Uranium, and Strontium-90.

Set 2 = Total VOCs, Total SVOCs, Total RCRA Metals, Aluminum, Antimony, Beryllium, Cobalt, Copper, Manganese, Molybdenum, Nickel, Zinc, TPH-DRO, and PCBs.

$\mathrm{ft}$ bgs $=$ Feet below ground surface

$\mathrm{SC}=$ Site characterization

$\mathrm{FC}=$ Fecal Coliform screening only

$\mathrm{WM}=$ Waste Management

MS/MSD = Matrix spike/matrix spike duplicate

NA $=$ Not applicable
$\mathrm{DRO}=$ Diesel-range organics

$\mathrm{PCB}=$ Polychlorinated biphenyl

RCRA = Resource Conservation and Recovery Act

$\mathrm{TPH}=$ Total petroleum hydrocarbons

SVOC = Semivolatile organic compound

$\mathrm{VOC}=$ Volatile organic compound

Samples were collected as outlined in the CAU 224 CAIP (NNSA/NSO, 2004) and submitted for laboratory analysis.

\section{A.3.1.3 Deviations}

There were no significant deviations to the CAIP requirements at this CAS. However, the piping associated with the tank was found to consist of only one pipe on the east side of the tank, as opposed to the three pipes suggested by the CAIP drawings and the utility markings. Two additional sample locations were placed along this pipe in order to satisfy the DQOs. No other deviations were required. The investigation and sampling at CAS 02-04-01 are considered sufficient to meet the DQOs.

\section{A.3.2 Investigation Results}

The following sections provide analytical results from the samples collected to complete investigation activities as outlined in the CAIP. Investigation samples were analyzed for the CAIP-specified COPCs, which included total VOCs, total semivolatile organic compounds (SVOCs), TPH-DRO, total RCRA metals and aluminum, antimony, beryllium, cobalt, copper, manganese, molybdenum, nickel, and zinc (referred to as total metals from this point forward), PCBs, gamma-emitting radionuclides, plutonium isotopes, uranium isotopes, and strontium-90. In 


\begin{tabular}{|c|c|c|c|c|c|c|}
\hline A01 & Depth & BAP & DiBen & & & \\
\hline 224A001 & $0-0.5$ & 340 & 340 & & & \\
\hline 224A008 & $2-3$ & - & - & & & \\
\hline $\mathrm{A} 02$ & Depth & BAP & DiBen & & & \\
\hline 224A011 & $2-3$ & 380 & 350 & & & \\
\hline $224 \mathrm{~A} 012$ & $5-6$ & - & - & & & \\
\hline $\mathrm{A} 03$ & \begin{tabular}{|l} 
Depth \\
\end{tabular} & BAP & DiBen & & & \\
\hline $224 \mathrm{~A} 003$ & $0-0.5$ & 450 & - & & & \\
\hline $\mathrm{A} 04$ & Depth & BAP & DiBen & BAA & BBF & \\
\hline 224A004 & $0-0.5$ & 2100 & 340 & 2500 & 3100 & \\
\hline \begin{tabular}{|l|}
$\mathrm{A} 07$ \\
\end{tabular} & Depth & BAP & DiBen & & & \\
\hline 224A007 & $0-0.5$ & 750 & 340 & & & \\
\hline 224A021 & $2-3$ & - & - & & & \\
\hline \begin{tabular}{|l|}
$\mathrm{A} 09$ \\
\end{tabular} & Depth & BAP & DiBen & BAA & BBF & Indeno \\
\hline \begin{tabular}{|l|}
$224 \mathrm{~A} 018$ \\
\end{tabular} & $2.5-3.5$ & 670 & 140 & 770 & 820 & 520 \\
\hline \begin{tabular}{|l|}
$224 \mathrm{~A} 020$ \\
\end{tabular} & $7.0-8.0$ & 8900 & 2200 & 10000 & 12000 & 4400 \\
\hline
\end{tabular}

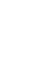

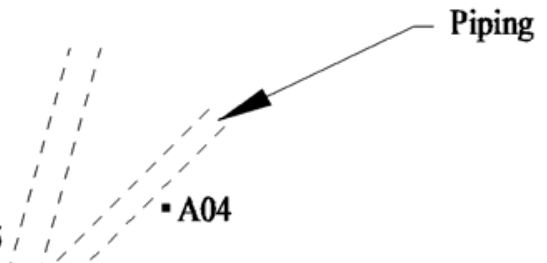

A06 -

A07.<smiles></smiles>

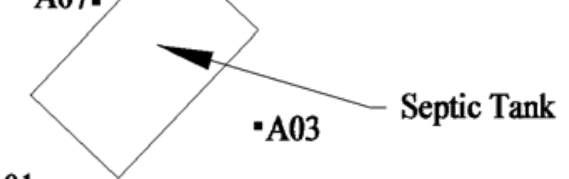

A01- $\quad$ A02

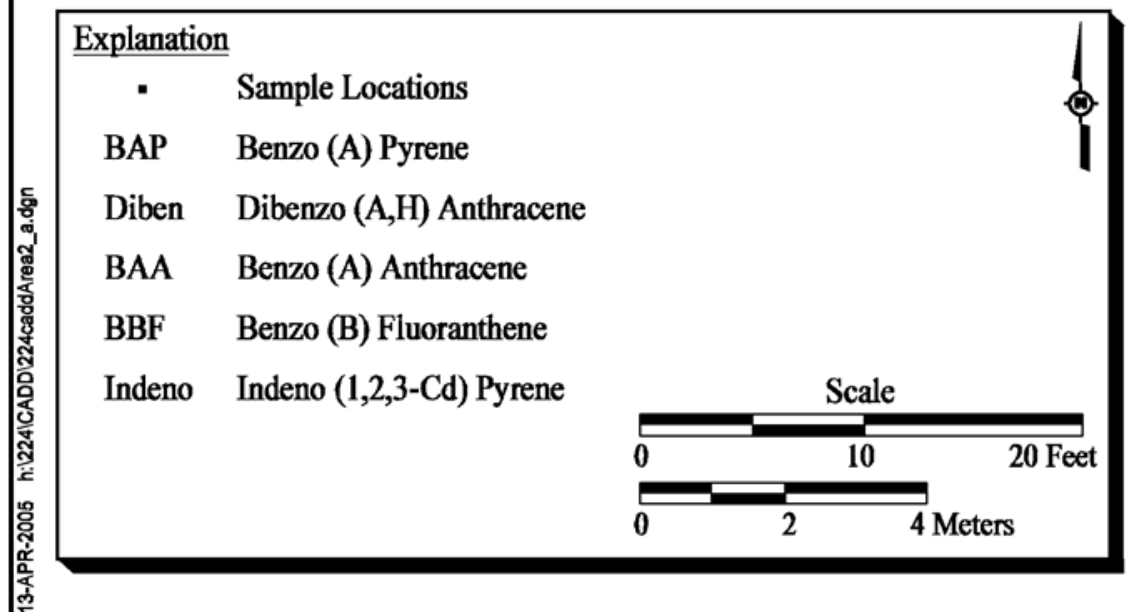

Figure A.3-1

Sample Location Map CAS 02-04-01, Septic Tank (Buried) 
accordance with ROTC CAIP-1, 25 percent of all samples were analyzed for radiological COPCs. The analytical parameters and laboratory methods used to analyze the investigation samples are listed in Table A.2-2. Table A.3-1 lists the sample-specific analytical suite for CAS 02-04-01.

Analytical results from the soil samples with concentrations exceeding MRLs or MDCs are summarized in the following sections. These results are compared to PALs and are a subset of the results that exceed MRLs or MDCs. Results greater than FALs are identified by bold text in the analytical tables. A portion of the analytical results for this CAS was rejected during validation; however, these rejected data did not adversely impact closure decisions as discussed in Appendix B, Section B.1.4.

\section{A.3.2.1 Total Volatile Organic Compounds}

Total VOC analytical results for soil samples collected at CAS 02-04-01 that were detected above MRLs are presented in Table A.3-2. No VOCs were detected in soil samples above FALs.

Table A.3-2

Soil Sample Results for Total VOCs Detected Above Minimum Reporting Limits at CAS 02-04-01, Septic Tank (Buried)

\begin{tabular}{|c|c|c|c|}
\hline \multirow{2}{*}{$\begin{array}{l}\text { Sample } \\
\text { Location }\end{array}$} & \multirow{2}{*}{$\begin{array}{l}\text { Sample } \\
\text { Number }\end{array}$} & \multirow{2}{*}{$\begin{array}{c}\text { Depth } \\
\text { (ft bgs) }\end{array}$} & Contaminants of Potential Concern $(\mu \mathrm{g} / \mathrm{kg})$ \\
\hline & & & Methylene Chloride \\
\hline \multicolumn{3}{|c|}{ Final Action Levels ${ }^{a}$} & 21,000 \\
\hline$\overline{\mathrm{A} 02}$ & $224 \mathrm{A002}$ & $0-0.5$ & $\overline{6.8}$ \\
\hline A04 & $224 \mathrm{~A} 004$ & $0-0.5$ & 6.5 \\
\hline A06 & 224A006 & $0-0.5$ & 7 \\
\hline
\end{tabular}

aBased on U.S. Environmental Protection Agency, Region 9 Preliminary Remediation Goals (PRGs) (EPA, 2002)

$\mathrm{ft}$ bgs $=$ Feet below ground surface $\mu \mathrm{g} / \mathrm{kg}=$ Micrograms per kilogram

\section{A.3.2.2 Total Semivolatile Organic Compounds}

Total SVOC analytical results for soil samples collected at CAS 02-04-01 that were detected above MRLs are presented in Table A.3-3. Several PAHs were detected above FALs; however, these are not considered COCs, as described in Section 2.2.1.1 
Table A.3-3

Soil Sample Results for Total SVOCs Detected Above Minimum Reporting Limits at CAS 02-04-01, Septic Tank (Buried)

\begin{tabular}{|c|c|c|c|c|c|c|c|c|c|c|c|c|c|c|c|c|c|c|c|c|c|c|c|c|}
\hline \multirow[b]{2}{*}{$\begin{array}{l}\text { Sample } \\
\text { Location }\end{array}$} & \multirow[b]{2}{*}{$\begin{array}{l}\text { Sample } \\
\text { Number }\end{array}$} & \multirow[b]{2}{*}{$\begin{array}{l}\text { Depth } \\
\text { (ft bgs) }\end{array}$} & \multicolumn{22}{|c|}{ Contaminants of Potential Concern $(\mu \mathrm{g} / \mathrm{kg})$} \\
\hline & & & 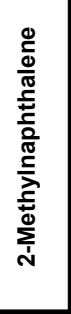 & 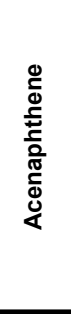 & 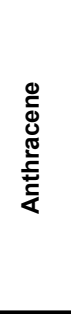 & 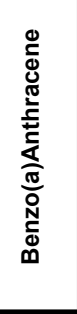 & 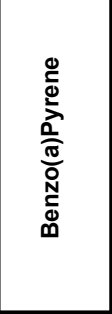 & 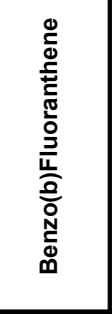 & 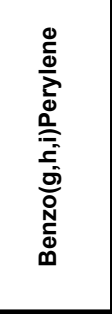 & 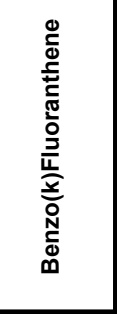 & 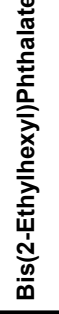 & 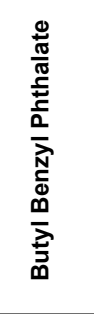 & 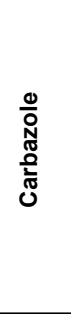 & 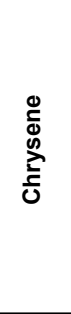 & 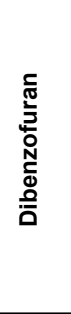 & 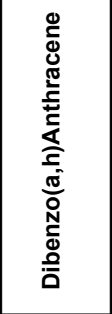 & 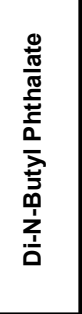 & 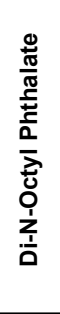 & 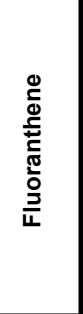 & $\begin{array}{l}\stackrel{0}{0} \\
\stackrel{0}{\frac{0}{0}} \\
\stackrel{ }{\frac{1}{4}}\end{array}$ & 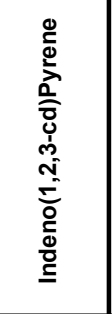 & 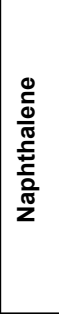 & 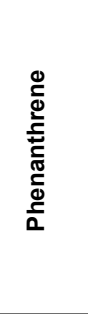 & 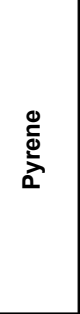 \\
\hline \multicolumn{3}{|c|}{ Final Action Levels ${ }^{a}$} & $\overline{\mathbf{z}}$ & 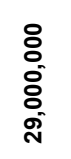 & 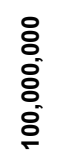 & $\frac{8}{\grave{n}}$ & $\stackrel{ }{N}$ & $\frac{8}{i}$ & $\overline{\mathbf{z}}$ & $\frac{\grave{\Delta}}{i}$ & 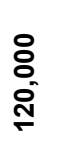 & $\begin{array}{l}8 \\
8 \\
8 \\
8 \\
8 \\
8 \\
8\end{array}$ & $\begin{array}{l}8 \\
8 \\
8 \\
\infty \\
\infty\end{array}$ & $\begin{array}{l}8 \\
\stackrel{0}{0} \\
\stackrel{-}{N}\end{array}$ & $\begin{array}{l}8 \\
\stackrel{\circ}{\circ} \\
\stackrel{-}{\circ} \\
\dot{m}\end{array}$ & 웃 & 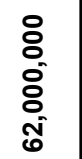 & 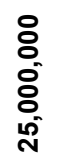 & 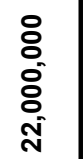 & 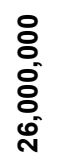 & $\frac{8}{i}$ & $\begin{array}{l}8 \\
8 \\
8 \\
8 \\
\end{array}$ & $\overline{\mathbf{z}}$ & 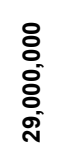 \\
\hline$\overline{\mathrm{A} 01}$ & $224 \mathrm{~A} 001$ & $0-0.5$ & $\overline{---}$ & $\overline{--}$ & $\overline{--}$ & 370 & $340(\mathrm{~J})^{0}$ & $610(\mathrm{~J})^{0}$ & $\overline{---}$ & $\overline{--}$ & $\overline{--}$ & $\overline{--}$ & $\overline{---}$ & 4440 & $\overline{--}$ & $\overline{--}$ & 2,100 & $\overline{--}$ & 1,300 & $\overline{--}$ & $\overline{--}$ & $\overline{---}$ & 1,000 & 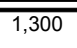 \\
\hline \multirow{2}{*}{ A02 } & 224A002 & $0-0.5$ & -- & -- & -- & -- & -- & -- & -- & -- & -- & -- & -- & -- & -- & -- & 420 & - & -- & -- & -- & -- & - & -- \\
\hline & $224 \mathrm{~A} 011$ & $2-3$ & -- & 420 & 370 & 430 & $380(\mathrm{~J})^{\mathrm{b}}$ & $540(J)^{b}$ & -- & -- & -- & -- & 350 & 460 & - & -- & 3,300 & -- & 1,800 & -- & - & -- & 2,600 & 1,500 \\
\hline A03 & 224A003 & $0-0.5$ & -- & -- & -- & 480 & 450 & 670 & -- & -- & -- & -- & -- & 560 & - & -- & 3,000 & -- & 1,800 & -- & -- & -- & 1,900 & 1,800 \\
\hline A04 & 224A004 & $0-0.5$ & 970 & 940 & 1,200 & 2,500 & $2,100(J)^{b}$ & $3,100(\mathrm{~J})^{b}$ & $1,500(J)^{b}$ & $1,200(\mathrm{~J})^{\mathrm{b}}$ & -- & - & 820 & 2,100 & 940 & - & 6,300 & - & 6,000 & 920 & $1,200(\mathrm{~J})^{b}$ & 380 & 5,700 & 5,800 \\
\hline A05 & 224A005 & $0-0.5$ & -- & -- & -- & -- & -- & -- & -- & -- & -- & -- & -- & -- & -- & -- & 740 & - & 540 & -- & -- & -- & 360 & 470 \\
\hline A06 & 224A006 & $0-0.5$ & -- & -- & -- & -- & -- & 340 & -- & -- & -- & -- & -- & -- & -- & -- & 1,100 & -- & 700 & -- & -- & -- & 480 & 690 \\
\hline \multirow{2}{*}{ A07 } & 224A007 & $0-0.5$ & -- & -- & -- & 760 & $750(\mathrm{~J})^{\mathrm{C}}$ & $1,100(\mathrm{~J})^{\mathrm{C}}$ & $490(\mathrm{~J})^{\mathrm{C}}$ & $500(\mathrm{~J})^{\mathrm{C}}$ & -- & -- & -- & 880 & -- & -- & 3,700 & -- & 2,600 & -- & $440(\mathrm{~J})^{\mathrm{C}}$ & -- & 2,300 & 4,000 \\
\hline & $224 \mathrm{~A} 022$ & $4-5$ & -- & - & -- & - & - & $440(\mathrm{~J})^{b}$ & -- & - & -- & $\overline{--}$ & - & -- & $\overline{--}$ & - & 1,200 & -- & 930 & - & - & -- & 810 & 1,300 \\
\hline \multirow[b]{2}{*}{ A09 } & $224 \mathrm{~A} 018$ & $2.5-3.5$ & -- & -- & -- & 770 & $670(\mathrm{~J})^{b}$ & $820(\mathrm{~J})^{\mathrm{b}}$ & $620(J)^{b}$ & $450(\mathrm{~J})^{\mathrm{b}}$ & 810 & -- & -- & 710 & -- & -- & 2,500 & 710 & 1,800 & -- & $520(\mathrm{~J})^{\mathrm{b}}$ & -- & 1,500 & 3,000 \\
\hline & $224 \mathrm{~A} 020$ & $7-8$ & 1,300 & $\begin{array}{l}4,100 \\
(J)^{\mathrm{e}}\end{array}$ & $\begin{array}{l}5,200 \\
(J)^{\mathrm{e}}\end{array}$ & $\begin{array}{c}10,000 \\
(J)^{e}\end{array}$ & $8,900(J)^{d}$ & $\begin{array}{c}12,000 \\
(J)^{d}\end{array}$ & $5,000(\mathrm{~J})^{f}$ & $4,900(J)^{d}$ & -- & $770(J)^{b}$ & 2,300 & $\begin{array}{l}9,100 \\
(J)^{e}\end{array}$ & 1,900 & $2,200(J)^{c}$ & $\begin{array}{c}24,000 \\
(J)^{e}\end{array}$ & -- & $\begin{array}{c}24,000 \\
(J)^{e}\end{array}$ & $\begin{array}{c}3,500 \\
(J)^{\mathrm{e}}\end{array}$ & $4,400(J)^{f}$ & 570 & $\begin{array}{c}19,000 \\
(J)^{e}\end{array}$ & $\begin{array}{c}33,000 \\
(J)^{e}\end{array}$ \\
\hline
\end{tabular}

${ }_{b}^{a}$ Based on U.S. Environmental Protection Agency, Region 9 Preliminary Remediation Goals (PRGs) (EPA, 2002)

Qualifier added to laboratory data; record accepted. Matrix effects may exist. Internal standard area count outside control limits.

Q Qualifier added to laboratory data; record accepted. Matrix effects may exist. Internal area response show extremely low count.

Qualifier added to laboratory data; record accepted. Surrogates diluted out.

$\mathrm{ft}$ bgs $=$ Feet below ground surface

$\mu \mathrm{g} / \mathrm{kg}=$ Micrograms per kilogram

-- = Not detected above minimum reporting limits. 


\section{A.3.2.3 Total Metals}

Total metals analytical results for soil samples collected at CAS 02-04-01 that were detected above MRLs are presented in Table A.3-4. No metals were detected in soil samples above FALs.

\section{A.3.2.4 Polychlorinated Biphenyls}

Total PCBs analytical results for soil samples collected at CAS 02-04-01 that were detected above MRLs are presented in Table A.3-5. No PCBs were detected in soil samples above FALs.

\section{A.3.2.5 Total Petroleum Hydrocarbons-Diesel-Range Organics}

Total TPH-DRO analytical results for soil samples collected at CAS 02-04-01 that were detected above MRLs are presented in Table A.3-6. No TPH-DRO was detected in soil samples above FALs.

\section{A.3.2.6 Gamma-Emitting Radionuclides}

Gamma-emitting radionuclide analytical results for soil samples collected at CAS 02-04-01 that were detected above MDCs are presented in Table A.3-7. No gamma-emitting radionuclides were detected in soil samples above FALs.

\section{A.3.2.7 Isotopes}

Isotopes analytical results for soil samples collected at CAS 02-04-01 that were detected above MDCs are presented in Table A.3-8. No isotopes were detected in soil samples above FALs.

\section{A.3.2.8 Septic Tank Liquid Results}

Analytical results for the septic tank liquid sample collected at CAS 02-04-01 that were detected above MRLs or MDCs, are presented in Table A.3-9. No COCs were detected in the sample.

\section{A.3.3 Nature and Extent of Contamination}

The only analytes found above FALs at CAS 02-04-01 were several PAHs in a number of subsurface samples. However, these compounds are associated with the asphalt found in the fill around the tank, 
Table A.3-4

Soil Sample Results for Metals Detected Above Minimum Reporting Limits at CAS 02-04-01, Septic Tank (Buried)

(Page 1 of 2)

\begin{tabular}{|c|c|c|c|c|c|c|c|c|c|c|c|c|c|c|}
\hline \multirow[b]{2}{*}{$\begin{array}{l}\text { Sample } \\
\text { Location }\end{array}$} & \multirow[b]{2}{*}{$\begin{array}{l}\text { Sample } \\
\text { Number }\end{array}$} & \multirow[b]{2}{*}{$\begin{array}{c}\text { Depth } \\
\text { (ft bgs) }\end{array}$} & \multicolumn{12}{|c|}{ Contaminants of Potential Concern (mg/kg) } \\
\hline & & & 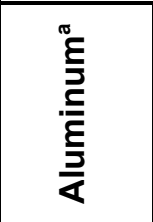 & 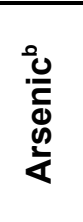 & 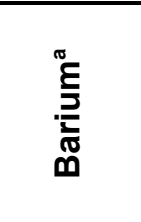 & 占 & 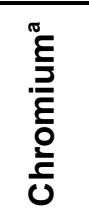 & $\begin{array}{l}\frac{0}{\pi} \\
\frac{\pi}{0} \\
\frac{0}{0} \\
0\end{array}$ & $\begin{array}{l}\frac{0}{0} \\
\frac{0}{0} \\
\frac{0}{0}\end{array}$ & 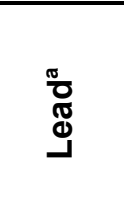 & 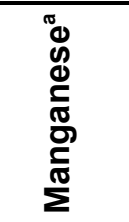 & $\begin{array}{l}\frac{\pi}{0} \\
\frac{x}{0} \\
\frac{x}{Z}\end{array}$ & 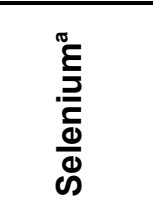 & $\stackrel{\widetilde{N}}{\stackrel{N}{N}}$ \\
\hline \multicolumn{3}{|c|}{ Final Action Levels } & 100,000 & 23 & 67,000 & 1,900 & 450 & 1,900 & 41,000 & 750 & 19,000 & 20,000 & 5,100 & 100,000 \\
\hline \multirow{4}{*}{$\mathrm{A} 01$} & $224 \mathrm{~A} 001$ & $0-0.5$ & $\overline{77,300}$ & $\overline{\overline{4}}$ & 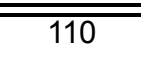 & $\overline{0.65}$ & $\overline{5.8}$ & $\overline{\overline{3.6}}$ & $\overline{12(J)^{c}}$ & $15(J)^{\mathrm{d}}$ & $\overline{310}$ & $\overline{8.4}$ & 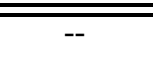 & 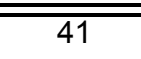 \\
\hline & $224 \mathrm{~A} 008$ & $2-3$ & 8,300 & 4.7 & 140 & 0.87 & 5.1 & 2.9 & $6.8(J)^{c}$ & $7.4(\mathrm{~J})^{\mathrm{d}}$ & 190 & 9.2 & -- & 42 \\
\hline & $224 \mathrm{~A} 009$ & $5-6$ & 6,500 & 4.5 & 140 & 0.67 & 4.2 & 2.7 & $7(J)^{\mathrm{C}}$ & $8.5(J)^{d}$ & 320 & 7.8 & -- & 36 \\
\hline & $224 \mathrm{~A} 010$ & $7-8$ & 5,700 & 3.2 & 93 & 0.57 & 2.8 & 1.9 & $3.7(\mathrm{~J})^{\mathrm{c}}$ & $7.4(\mathrm{~J})^{\mathrm{d}}$ & 220 & 5 & -- & 25 \\
\hline \multirow{5}{*}{$\mathrm{A} 02$} & $224 \mathrm{~A} 002$ & $0-0.5$ & 7,400 & 3.4 & 97 & 0.66 & 4.5 & 2.8 & $7.2(\mathrm{~J})^{\mathrm{c}}$ & $9.5(\mathrm{~J})^{\mathrm{d}}$ & 310 & 5.6 & -- & 31 \\
\hline & $224 \mathrm{~A} 011$ & $2-3$ & 8,200 & 4.6 & 130 & 0.84 & 5.5 & 3.3 & $8.2(\mathrm{~J})^{\mathrm{c}}$ & $13(\mathrm{~J})^{\mathrm{d}}$ & 330 & 7.4 & -- & 34 \\
\hline & $224 \mathrm{~A} 012$ & $5-6$ & 4,800 & 4.7 & 51 & -- & 2.1 & 1.4 & $2.6(\mathrm{~J})^{\mathrm{c}}$ & $5.2(\mathrm{~J})^{\mathrm{d}}$ & 170 & 2.7 & -- & 22 \\
\hline & $224 \mathrm{~A} 013$ & $7-8$ & 6,500 & 3.1 & 79 & 0.63 & 3.2 & 2.2 & $3.5(\mathrm{~J})^{\mathrm{c}}$ & $6.7(\mathrm{~J})^{\mathrm{d}}$ & 260 & 3.8 & -- & 22 \\
\hline & $224 \mathrm{~A} 014$ & $7-8$ & 6,000 & 3.3 & 77 & 0.61 & 3.2 & 2.1 & $3.4(\mathrm{~J})^{\mathrm{c}}$ & $6.3(\mathrm{~J})^{\mathrm{d}}$ & 230 & 3.5 & -- & 20 \\
\hline A03 & $224 \mathrm{~A} 003$ & $0-0.5$ & 7,100 & 3.2 & 94 & 0.63 & 4.6 & 2.6 & $7.5(\mathrm{~J})^{\mathrm{c}}$ & $11(J)^{d}$ & 310 & 5.5 & -- & 31 \\
\hline A04 & $224 \mathrm{~A} 004$ & $0-0.5$ & 8,700 & 3.2 & 110 & 0.8 & 5.7 & 3.4 & $12(\mathrm{~J})^{\mathrm{C}}$ & $15(J)^{d}$ & 310 & 6.2 & -- & 48 \\
\hline A05 & $224 \mathrm{~A} 005$ & $0-0.5$ & 7,400 & 3 & 88 & 0.69 & 4.5 & 2.6 & $6.5(\mathrm{~J})^{\mathrm{c}}$ & $11(J)^{d}$ & 270 & 4.8 & -- & 31 \\
\hline A06 & $224 \mathrm{~A} 006$ & $0-0.5$ & 7,600 & 3.2 & 110 & 0.66 & 4.8 & 3 & $9.8(\mathrm{~J})^{\mathrm{c}}$ & $15(\mathrm{~J})^{\mathrm{d}}$ & 310 & 5.4 & -- & 40 \\
\hline \multirow{4}{*}{$\mathrm{A} 07$} & $224 \mathrm{~A} 007$ & $0-0.5$ & 6,300 & 3 & 100 & 0.56 & 4.6 & 2.7 & $9.5(\mathrm{~J})^{\mathrm{c}}$ & $18(\mathrm{~J})^{\mathrm{d}}$ & 250 & 5 & -- & 38 \\
\hline & $224 \mathrm{~A} 021$ & $2-3$ & 6,900 & 6.1 & 230 & 0.65 & 5.7 & 3.5 & 8.8 & 8.2 & 210 & 14 & -- & 48 \\
\hline & $224 \mathrm{~A} 022$ & $4-5$ & 8,600 & 3.6 & 110 & 0.77 & 5.6 & 2.9 & 7.3 & 9.7 & 320 & 7.4 & $0.66(\mathrm{~J}+)$ & 37 \\
\hline & $224 \mathrm{~A} 023$ & $7-8$ & 10,000 & 3.3 & 110 & 0.93 & 4.4 & 2.5 & 6 & 8.2 & 240 & 4.9 & -- & 29 \\
\hline
\end{tabular}


Table A.3-4

Soil Sample Results for Metals Detected Above Minimum Reporting Limits at CAS 02-04-01, Septic Tank (Buried)

(Page 2 of 2)

\begin{tabular}{|c|c|c|c|c|c|c|c|c|c|c|c|c|c|c|}
\hline \multirow[b]{2}{*}{$\begin{array}{l}\text { Sample } \\
\text { Location }\end{array}$} & \multirow[b]{2}{*}{$\begin{array}{l}\text { Sample } \\
\text { Number }\end{array}$} & \multirow[b]{2}{*}{$\begin{array}{c}\text { Depth } \\
\text { (ft bgs) }\end{array}$} & \multicolumn{12}{|c|}{ Contaminants of Potential Concern (mg/kg) } \\
\hline & & & 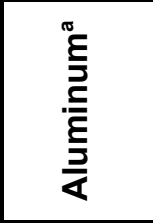 & 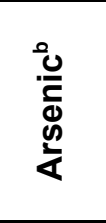 & 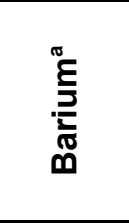 & 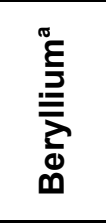 & 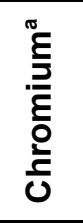 & 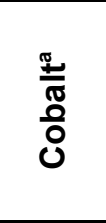 & $\begin{array}{l}\frac{\pi}{0} \\
\frac{0}{0} \\
\frac{0}{0}\end{array}$ & త్ర & 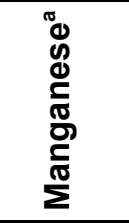 & $\begin{array}{l}\frac{\sigma}{0} \\
\frac{y}{0} \\
\frac{0}{Z}\end{array}$ & 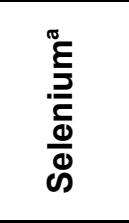 & 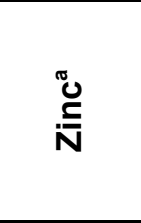 \\
\hline \multicolumn{3}{|c|}{ Final Action Levels } & 100,000 & 23 & 67,000 & 1,900 & 450 & 1,900 & 41,000 & 750 & 19,000 & 20,000 & 5,100 & 100,000 \\
\hline \multirow{3}{*}{ A08 } & $224 \mathrm{~A} 015$ & $2.5-3.5$ & 7,100 & 6.4 & 720 & 0.79 & 8.4 & 4.9 & 19 & 110 & 1,600 & 12 & $1.2(\mathrm{~J}+)$ & 79 \\
\hline & $224 \mathrm{~A} 016$ & $4-5$ & 5,200 & $2(\mathrm{~J}+)$ & 56 & -- & 2 & 2.2 & 4.9 & 7.2 & 290 & 3.8 & -- & 23 \\
\hline & $224 \mathrm{~A} 017$ & $7-8$ & 4,600 & $2(\mathrm{~J}+)$ & 38 & -- & 1.4 & 1.1 & 2 & 3.7 & 190 & -- & -- & 20 \\
\hline \multirow{3}{*}{ A09 } & $224 \mathrm{~A} 018$ & $2.5-3.5$ & 8,100 & 4.4 & 67 & 0.7 & 7.9 & 3.5 & 30 & 22 & 310 & 7.6 & -- & 64 \\
\hline & $224 \mathrm{~A} 019$ & $4-5$ & 7,500 & 2.6 & 60 & 0.66 & 3.4 & 1.9 & 6.6 & 6.1 & 200 & 3.4 & -- & 25 \\
\hline & $224 \mathrm{~A} 020$ & $7-8$ & 7,700 & 2.9 & 87 & 0.68 & 4 & 2.4 & 6.2 & 9.8 & 270 & 4.3 & -- & 32 \\
\hline
\end{tabular}

${ }^{a}$ Based on U.S. Environmental Protection Agency, Region 9 Preliminary Remediation Goals (PRGs) (EPA, 2002)

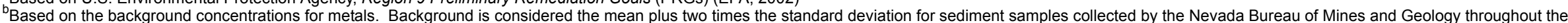
Nevada Test and Training Range (NBMG, 1998; Moore, 1999).

${ }^{c}$ Qualifier added to laboratory data; record accepted. Serial dilution \%D outside control limits. Matrix effects may exist.

${ }^{\mathrm{d}}$ Qualifier added to laboratory data; record accepted. Duplicate precision analysis (relative percent difference) outside control limits.

$\mathrm{ft}$ bgs $=$ Feet below ground surface

$\mathrm{mg} / \mathrm{kg}=$ Milligrams per kilogram

$\mathrm{J}=$ Estimated value.

$\mathrm{J}+=$ The result is an estimated quantity, but the result may be biased high. CRI/CRA \% recovery(ies) criteria was/were not met.

-- = Not detected above minimum reporting limits. 
Table A.3-5

Soil Sample Results for PCBs Detected Above Minimum

Reporting Limits at CAS 02-04-01, Septic Tank (Buried)

\begin{tabular}{||c|c|c|c||}
\hline \multirow{2}{*}{$\begin{array}{c}\text { Sample } \\
\text { Location }\end{array}$} & $\begin{array}{c}\text { Sample } \\
\text { Number }\end{array}$ & \multirow{2}{*}{$\begin{array}{c}\text { Depth } \\
(\mathbf{f t} \text { bgs })\end{array}$} & Contaminants of Potential Concern $(\mu \mathbf{g} / \mathbf{k g})$ \\
\cline { 3 - 4 } & & & Aroclor 1254 \\
\hline \multicolumn{3}{|c|}{ Final Action Levels } & $\mathbf{7 4 0}$ \\
\hline \hline A08 & $224 A 015$ & $2.5-3.5$ & $370(\mathrm{~J})^{\mathrm{b}}$ \\
\hline \multirow{2}{*}{ A09 } & $224 \mathrm{~A} 018$ & $2.5-3.5$ & $650(\mathrm{~J})^{\mathrm{c}}$ \\
\cline { 2 - 5 } & $224 \mathrm{~A} 019$ & $4-5$ & $54(\mathrm{~J})^{\mathrm{b}}$ \\
\hline
\end{tabular}

aBased on U.S. Environmental Protection Agency, Region 9 Preliminary Remediation Goals (PRGs) (EPA, 2002)

${ }^{b}$ Qualifier added to laboratory data; record accepted. \%D between columns $>25$.

${ }^{\mathrm{C}} \mathrm{Qualifier}$ added to laboratory data; record accepted. \%D between columns $>25$. Surrogates diluted out.

$\mathrm{ft}$ bgs $=$ Feet below ground surface

$\mu \mathrm{g} / \mathrm{kg}=$ Micrograms per kilogram

$\mathrm{J}=$ Estimated value.

Table A.3-6

Soil Sample Results for TPH-DRO Detected Above Minimum Reporting Limits at CAS 02-04-01, Septic Tank (Buried)

\begin{tabular}{|c|c|c|c|}
\hline \multirow{2}{*}{$\begin{array}{l}\text { Sample } \\
\text { Location }\end{array}$} & \multirow{2}{*}{$\begin{array}{l}\text { Sample } \\
\text { Number }\end{array}$} & \multirow{2}{*}{$\begin{array}{l}\text { Depth } \\
\text { (ft bgs) }\end{array}$} & Contaminants of Potential Concern (mg/kg) \\
\hline & & & Diesel-Range Organics \\
\hline \multicolumn{3}{|c|}{ Final Action Levels ${ }^{a}$} & 100 \\
\hline A09 & $224 \mathrm{~A} 020$ & $7-8$ & $93(\mathrm{H}, \mathrm{Z})$ \\
\hline
\end{tabular}

aBased on Nevada Administrative Code, "Contamination of Soil: Establishment of Action Levels" (NAC, 2002)

$\mathrm{ft}$ bgs $=$ Feet below ground surface

$\mathrm{mg} / \mathrm{kg}=$ Milligrams per kilogram

$\mathrm{H}=\mathrm{DRO}$ fuel pattern in the heavy end of retention time window.

$Z=A$ significant fraction of the reported result did not resemble the patterns of any of the following petroleum hydrocarbon products: gasoline, JP-4, JP-8, diesel, mineral spirits, motor oil, Stoddard solvent, Bunker C.

not the tank itself, and are, therefore, not considered COCs. No other contaminants were found at the site.

\section{A.3.4 Revised Conceptual Site Model}

No variations in the CSM were identified. 
Table A.3-7

Soil Sample Results for Gamma-Emitting Radionuclides Detected Above Minimum Detectable Concentrations at CAS 02-04-01, Septic Tank (Buried)

\begin{tabular}{|c|c|c|c|c|c|c|c|c|c|c|c|c|c|}
\hline \multirow[b]{2}{*}{$\begin{array}{c}\text { Sample } \\
\text { Location }\end{array}$} & \multirow[b]{2}{*}{$\begin{array}{l}\text { Sample } \\
\text { Number }\end{array}$} & \multirow{3}{*}{$\begin{array}{c}\text { Depth } \\
\text { (ft bgs) }\end{array}$} & \multicolumn{11}{|c|}{ Contaminants of Potential Concern (pCi/g) } \\
\hline & & & \multicolumn{2}{|c|}{ 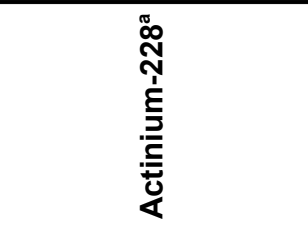 } & \multicolumn{2}{|c|}{ 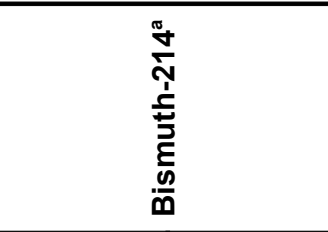 } & \multicolumn{3}{|c|}{ 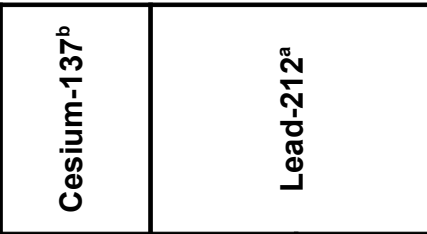 } & \multicolumn{2}{|c|}{ 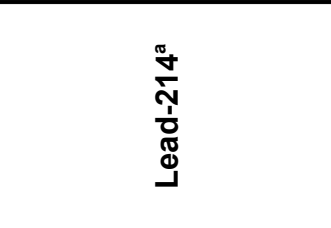 } & \multicolumn{2}{|c|}{ 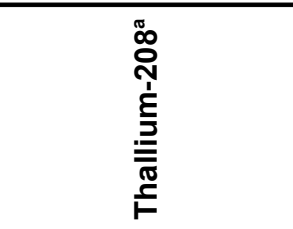 } \\
\hline \multicolumn{2}{|c|}{ Final Action Levels } & & 5 & 15 & 5 & 15 & \multirow{2}{*}{12.2} & 5 & 15 & 5 & 15 & 5 & 15 \\
\hline \multicolumn{3}{|c|}{ Depth bgs (cm) } & $<15$ & $>15$ & $<15$ & $>15$ & & $<15$ & $>15$ & $<15$ & $>15$ & $<15$ & $>15$ \\
\hline$\overline{\mathrm{A} 01}$ & $224 \mathrm{~A} 009$ & $\overline{5-6}$ & $\overline{\mathrm{NA}}$ & $\overline{\overline{1.71(\mathrm{G})}}$ & $\overline{\mathrm{NA}}$ & 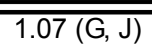 & $\overline{---}$ & $\overline{\mathrm{NA}}$ & $\overline{2.08(J)^{c}}$ & $\overline{\mathrm{NA}}$ & 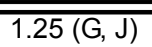 & $\overline{\mathrm{NA}}$ & $\overline{\overline{0.64(G)}}$ \\
\hline \multirow{3}{*}{$\mathrm{A} 02$} & $224 \mathrm{~A} 002$ & $0-0.5$ & $1.86(\mathrm{G})$ & NA & $1(\mathrm{G}, \mathrm{J})$ & $\mathrm{NA}$ & $1.29(\mathrm{G})$ & $1.92(\mathrm{~J})^{\mathrm{c}}$ & NA & $1.35(\mathrm{G}, \mathrm{J})$ & NA & $0.53(\mathrm{G})$ & NA \\
\hline & $224 \mathrm{~A} 013$ & $7-8$ & NA & $2.14(\mathrm{G})$ & $\mathrm{NA}$ & $1.2(\mathrm{G}, \mathrm{J})$ & -- & NA & $2.39(\mathrm{~J})^{\mathrm{c}}$ & $\mathrm{NA}$ & $1.27(\mathrm{G}, \mathrm{J})$ & NA & $0.75(\mathrm{G})$ \\
\hline & $224 \mathrm{~A} 014$ & $7-8$ & $\mathrm{NA}$ & $1.88(\mathrm{G})$ & $\mathrm{NA}$ & $1.1(\mathrm{G}, \mathrm{J})$ & $\overline{--}$ & NA & $2.13(\mathrm{~J})^{\mathrm{c}}$ & $\mathrm{NA}$ & $1.09(\mathrm{G}, \mathrm{J})$ & $\mathrm{NA}$ & $0.72(\mathrm{G})$ \\
\hline A06 & $224 \mathrm{~A} 006$ & $0-0.5$ & $1.97(\mathrm{G})$ & $\mathrm{NA}$ & $1.07(\mathrm{G}, \mathrm{J})$ & $\mathrm{NA}$ & $1.4(\mathrm{G})$ & $2.25(\mathrm{~J})^{\mathrm{c}}$ & $\mathrm{NA}$ & $1.18(\mathrm{G}, \mathrm{J})$ & $\mathrm{NA}$ & $0.58(\mathrm{G})$ & $\mathrm{NA}$ \\
\hline A07 & $224 \mathrm{~A} 022$ & $4-5$ & $\overline{N A}$ & $2.03(\mathrm{G})$ & $\mathrm{NA}$ & $1.21(\mathrm{G}, \mathrm{J})$ & $\overline{--}$ & $\overline{N A}$ & $1.95(\mathrm{~J})^{\mathrm{c}}$ & $\overline{N A}$ & $1.05(\mathrm{G}, \mathrm{J})$ & $\mathrm{NA}$ & $0.62(\mathrm{G})$ \\
\hline A09 & $224 \mathrm{~A} 018$ & $2.5-3.5$ & NA & $1.99(\mathrm{G})$ & $\mathrm{NA}$ & $0.93(G, J)$ & -- & NA & $2.04(\mathrm{~J})^{\mathrm{c}}$ & $\mathrm{NA}$ & $1.04(\mathrm{G}, \mathrm{J})$ & NA & $0.56(\mathrm{G})$ \\
\hline
\end{tabular}

a Taken from the generic guidelines for residual concentrations of actinium-228, bismuth-214, lead-212, lead-214, thallium-208, and thorium-232, as found in Chapter IV of DOE Order 5400.5, Change 2, "Radiation document, $15 \mathrm{~cm}$ is assumed to be equivalent to $0.5 \mathrm{ft}$ ( $6 \mathrm{in}$.); therefore, $5 \mathrm{pCi} / \mathrm{g}$ represents the FALs for these radionuclides in the surface soil ( 0 to $0.5 \mathrm{ft}$ depth)

${ }^{b}$ Taken from the construction, commercial, industrial land use scenario in Table 2.1 of the NCRP Report No. 129, Recommended Screening Limits for Contaminated Surface Soil and Review Factors Relevant to Site-Specific Studies (NCRP, 1999). The values provided in this source document were scaled to a $25-\mathrm{mrem} / \mathrm{yr}$ dose.

QQualifier added to laboratory data; record accepted. Sample does not meet counting geometry requirements.

$\mathrm{ft}$ bgs $=$ Feet below ground surface

$\mathrm{cm}=$ Centimeter

$\mathrm{mrem} / \mathrm{yr}=$ Millirems per year

$\mathrm{pCi} / \mathrm{g}=$ Picocuries per gram

$-=$ Not detected above minimum reporting limits

$>=$ Greater than

$G=$ Sample density differs by more than 15 percent of laboratory control sample density.

$\mathrm{J}=$ Estimated value

$\mathrm{NA}=$ Not applicable 
Table A.3-8

Soil Sample Results for Isotopes Detected Above Minimum Detectable Concentrations at CAS 02-04-01, Septic Tank (Buried)

\begin{tabular}{|c|c|c|c|c|c|c|c|}
\hline \multirow[b]{2}{*}{$\begin{array}{l}\text { Sample } \\
\text { Location }\end{array}$} & \multirow[b]{2}{*}{$\begin{array}{l}\text { Sample } \\
\text { Number }\end{array}$} & \multirow[b]{2}{*}{$\begin{array}{l}\text { Depth } \\
\text { (ft bgs) }\end{array}$} & \multicolumn{5}{|c|}{ Contaminants of Potential Concern $(\mathrm{pCi} / \mathrm{g})$} \\
\hline & & & 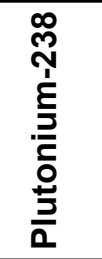 & 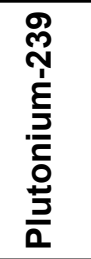 & 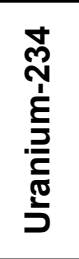 & 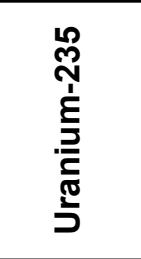 & 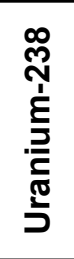 \\
\hline \multicolumn{3}{|c|}{ Final Action Levels ${ }^{a}$} & 13 & 12.7 & 143 & 17.6 & 105 \\
\hline $\mathrm{A} 01$ & 224A009 & $5-6$ & 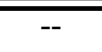 & $\overline{---}$ & 1.04 & $0.05(\mathrm{LT})$ & 1.04 \\
\hline \multirow{3}{*}{$\mathrm{A} 02$} & $224 \mathrm{~A} 002$ & $0-0.5$ & 0.24 & 1.29 & 0.89 & -- & 0.85 \\
\hline & $224 \mathrm{~A} 013$ & $7-8$ & -- & -- & 1.01 & 0.064 & 0.97 \\
\hline & $224 \mathrm{~A} 014$ & $7-8$ & -- & -- & 0.95 & 0.079 & 1 \\
\hline A06 & $224 \mathrm{~A} 006$ & $0-0.5$ & 0.372 & 1.27 & 0.81 & -- & 0.92 \\
\hline A07 & $224 \mathrm{~A} 022$ & $4-5$ & 0.52 & 1.17 & 0.98 & -- & 1.06 \\
\hline A09 & $224 \mathrm{~A} 018$ & $2.5-3.5$ & -- & -- & 1.31 & 0.081 & 1.03 \\
\hline
\end{tabular}

${ }^{a}$ Taken from the construction, commercial, industrial land use scenario in Table 2.1 of the NCRP Report No. 129 Recommended Screening Limits for Contaminated Surface Soil and Review Factors Relevant to Site-Specific Studies (NCRP, 1999). The values provided in this source document were scaled to a $25-\mathrm{mrem} / \mathrm{yr}$ dose.

$\mathrm{ft}$ bgs $=$ Feet below ground surface $\mathrm{pCi} / \mathrm{g}=$ Picocuries per gram

$\mathrm{LT}=$ Result is less than the requested minimum detectable concentration, greater than the sample specific minimum detectable concentration.

-- = Not detected above minimum reporting limits

Table A.3-9

Liquid Sample Results Detected Above Minimum Reporting Limits at CAS 02-04-01, Septic Tank (Buried)

\begin{tabular}{|c|c|c|c|c|c|c|}
\hline \multirow{2}{*}{$\begin{array}{c}\text { Sample } \\
\text { Location }\end{array}$} & \multirow{2}{*}{$\begin{array}{l}\text { Sample } \\
\text { Number }\end{array}$} & \multirow{2}{*}{$\begin{array}{c}\text { Depth } \\
\text { (ft bgs) }\end{array}$} & \multicolumn{4}{|c|}{ Contaminants of Potential Concern (mg/L) } \\
\hline & & & Aluminum & Lead & Manganese & Zinc \\
\hline \multicolumn{3}{|c|}{ Regulatory Limits ${ }^{\mathrm{a}}$} & NI & 5.0 & NI & NI \\
\hline$\overline{~ T a n k 1}$ & $\overline{224 A 501}$ & $\overline{\mathrm{NA}}$ & 1.5 & $\overline{0.0044}$ & 2.6 & 0.027 \\
\hline
\end{tabular}

aBased upon 40 CFR261.24 Table 1-Maximum concentration of contaminants for the toxicity characteristic (CFR, 2004).

$\mathrm{ft}$ bgs $=$ Feet below ground surface $\mathrm{mg} / \mathrm{L}=$ Milligrams per liter $\mathrm{NI}=$ Not identified. 


\section{A.4.0 CAS 03-05-01, Leachfield}

Corrective Action Site 03-05-01 is located at the Subdock Complex in Area 3 of the NTS. The CAS consists of a former leach pit that has since been covered and graded. The pit was associated with acetylene generation activities related to the nearby Bit Sharpening Shop. Investigation activities at CAS 03-05-01 included surface and subsurface sampling at the site of the former pit.

\section{A.4.1 Corrective Action Investigation}

A total of 28 soil characterization samples were collected during investigation activities at CAS 03-05-01. The sample IDs, locations, types, and analyses are listed in Table A.4-1. The sample locations are shown on Figure A.4-1. The specific CAI activities conducted to satisfy the CAIP requirements at this CAS are described in the following sections.

Table A.4-1

Samples Collected at CAS 03-05-01, Leachfield

(Page 1 of 2)

\begin{tabular}{|c|c|c|c|c|c|}
\hline $\begin{array}{c}\text { Sample } \\
\text { Location }\end{array}$ & $\begin{array}{l}\text { Sample } \\
\text { Number }\end{array}$ & $\begin{array}{c}\text { Depth } \\
\text { (ft bgs) }\end{array}$ & $\begin{array}{l}\text { Sample } \\
\text { Matrix }\end{array}$ & Purpose & Analyses \\
\hline \multirow{2}{*}{ B01 } & $\overline{2 \text { 224B001 }}$ & $\overline{00-0.5}$ & 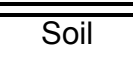 & $\overline{\overline{S C}}$ & $\overline{\overline{\text { Set } 1}}$ \\
\hline & 224B002 & $2-3$ & Soil & SC & Set 1 \\
\hline \multirow{4}{*}{ B02 } & 224B003 & $0-0.5$ & Soil & $\mathrm{SC}$ & Set 1 \\
\hline & 224B004 & $2-3$ & Soil & SC & Set 1 \\
\hline & 224B005 & $2-3$ & Soil & $\begin{array}{c}\text { Field Duplicate } \\
\text { of \#224B004 }\end{array}$ & Set 1 \\
\hline & 224B006 & $5-6$ & Soil & SC & Set 1 \\
\hline \multirow{3}{*}{ B03 } & 224B007 & $0-0.5$ & Soil & SC, MS/MSD & Set 1 \\
\hline & 224B008 & $2-3$ & Soil & $\mathrm{SC}$ & Set 1 \\
\hline & 224B009 & $4-5$ & Soil & SC & Set 1 \\
\hline \multirow{2}{*}{ B04 } & 224B010 & $0-0.5$ & Soil & $\mathrm{SC}$ & Set 2 \\
\hline & 224B018 & $3.5-4$ & Soil & SC & Set 2 \\
\hline \multirow{2}{*}{ B05 } & 224B011 & $0-0.5$ & Soil & SC & Set 2 \\
\hline & 224B019 & $3-4$ & Soil & $\mathrm{SC}$ & Set 2 \\
\hline \multirow{2}{*}{ B06 } & 224B012 & $0-0.5$ & Soil & SC & Set 2 \\
\hline & 224B020 & $2-3$ & Soil & SC & Set 2 \\
\hline
\end{tabular}


Table A.4-1

Samples Collected at CAS 03-05-01, Leachfield

(Page 2 of 2)

\begin{tabular}{|c|c|c|c|c|c|}
\hline $\begin{array}{l}\text { Sample } \\
\text { Location }\end{array}$ & $\begin{array}{l}\text { Sample } \\
\text { Number }\end{array}$ & $\begin{array}{l}\text { Depth } \\
\text { (ft bgs) }\end{array}$ & $\begin{array}{l}\text { Sample } \\
\text { Matrix }\end{array}$ & Purpose & Analyses \\
\hline \multirow{2}{*}{ B07 } & $224 \mathrm{~B} 013$ & $0-0.5$ & Soil & SC & Set 2 \\
\hline & 224B021 & $2-3$ & Soil & SC & Set 2 \\
\hline \multirow{2}{*}{ B08 } & 224B014 & $0-0.5$ & Soil & SC & Set 2 \\
\hline & 224B022 & $3-4$ & Soil & SC & Set 2 \\
\hline \multirow{2}{*}{ B09 } & 224B015 & $0-0.5$ & Soil & SC & Set 2 \\
\hline & 224B023 & $2-3$ & Soil & SC & Set 2 \\
\hline \multirow{2}{*}{ B10 } & 224B016 & $0-0.5$ & Soil & SC & Set 2 \\
\hline & 224B024 & $2.5-3.5$ & Soil & SC & Set 2 \\
\hline \multirow{2}{*}{ B11 } & 224B017 & $0-0.5$ & Soil & SC & Set 2 \\
\hline & 224B025 & $2.5-3.5$ & Soil & SC & Set 2 \\
\hline \multirow{3}{*}{ B12 } & 224B026 & $0-0.5$ & Soil & SC & Set 2 \\
\hline & 224B027 & $0-0.5$ & Soil & $\begin{array}{c}\text { Field Duplicate } \\
\text { of \#224B026 }\end{array}$ & Set 2 \\
\hline & 224B028 & $2-2.5$ & Soil & SC, MS/MSD & Set 2 \\
\hline B13 & 224B029 & $0-0.5$ & Soil & SC & $\begin{array}{l}\text { Gamma Spectroscopy, } \\
\text { Isotopic Plutonium }\end{array}$ \\
\hline B14 & 224B030 & $0-0.5$ & Soil & SC & $\begin{array}{l}\text { Gamma Spectroscopy, } \\
\text { Isotopic Plutonium }\end{array}$ \\
\hline B15 & 224B031 & $0-0.5$ & Soil & SC & $\begin{array}{l}\text { Gamma Spectroscopy, } \\
\text { Isotopic Plutonium }\end{array}$ \\
\hline NA & 224B301 & NA & Water & Trip Blank & Total VOCs \\
\hline NA & 224B302 & NA & Water & Field Blank & Set 1 \\
\hline
\end{tabular}

Set 1 = Total VOCs, Total SVOCs, Total RCRA Metals, Aluminum, Antimony, Beryllium, Cobalt, Copper, Manganese, Molybdenum, Nickel, Zinc, TPH-DRO, PCBs, Gamma Spectroscopy, Isotopic Plutonium, Isotopic Uranium, and Strontium-90.

Set 2 = Total SVOCs, Total RCRA Metals, Aluminum, Antimony, Beryllium, Cobalt, Copper, Manganese, Molybdenum, Nickel, Zinc, TPH-DRO, PCBs, and Isotopic Plutonium

$\mathrm{DRO}=$ Diesel-range organics

$\mathrm{ft}$ bgs $=$ Feet below ground surface

MS/MSD = Matrix spike/matrix spike duplicate

NA = Not applicable

$\mathrm{PCB}=$ Polychlorinated biphenyl

RCRA = Resource Conservation and Recovery Act

$\mathrm{SC}=$ Site characterization

SVOC $=$ Semivolatile organic compound

$\mathrm{TPH}=$ Total petroleum hydrocarbons

VOC $=$ Volatile organic compound 

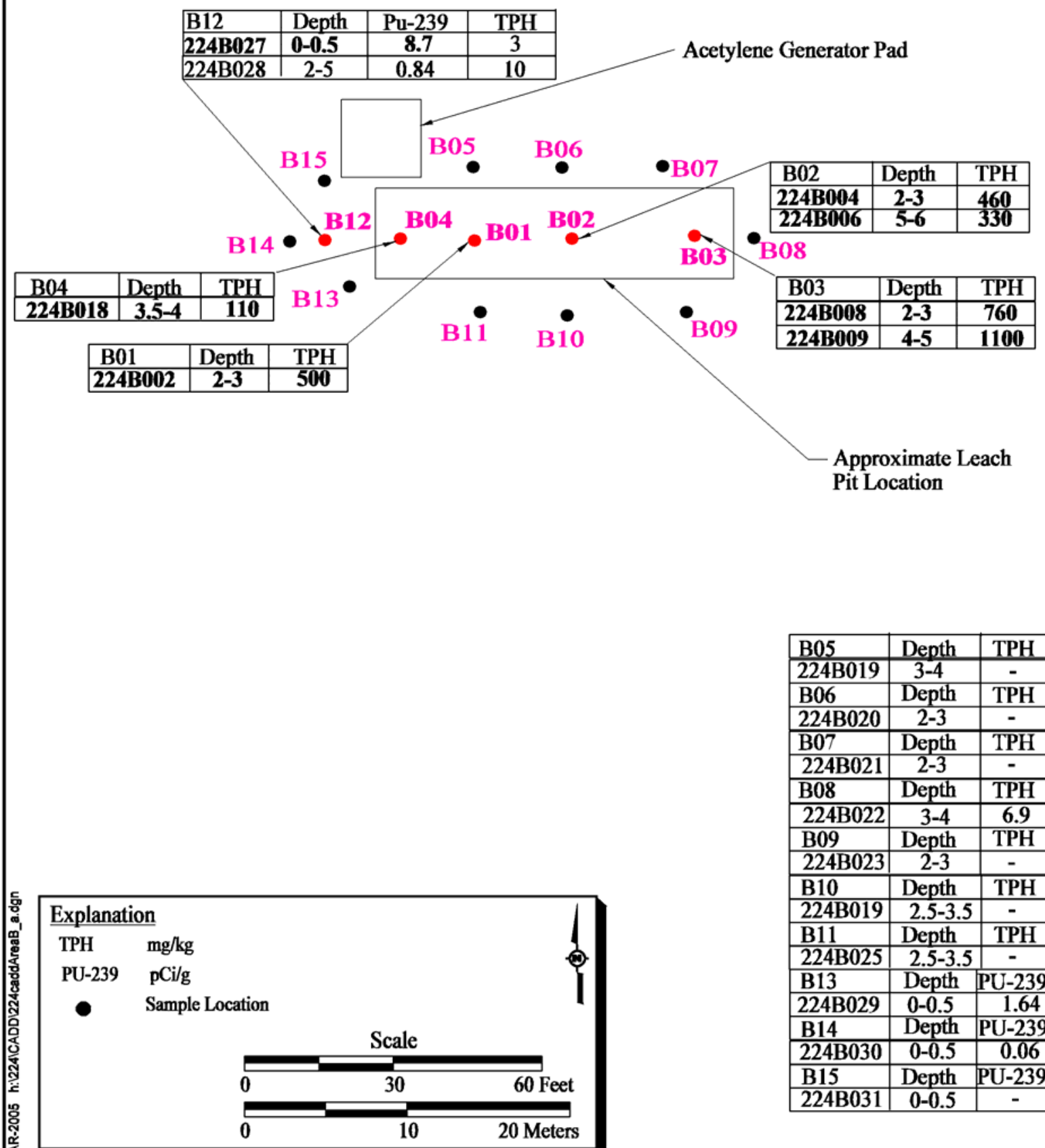

\begin{tabular}{|l|c|c|}
\hline B05 & Depth & TPH \\
\hline 224B019 & $3-4$ & - \\
\hline B06 & Depth & TPH \\
\hline 224B020 & $2-3$ & - \\
\hline B07 & Depth & TPH \\
\hline 224B021 & $2-3$ & - \\
\hline B08 & Depth & TPH \\
\hline 224B022 & $3-4$ & 6.9 \\
\hline B09 & Depth & TPH \\
\hline 224B023 & $2-3$ & - \\
\hline B10 & Depth & TPH \\
\hline 224B019 & $2.5-3.5$ & - \\
\hline B11 & Depth & TPH \\
\hline 224B025 & $2.5-3.5$ & - \\
\hline B13 & Depth & PU-239 \\
\hline 224B029 & $0-0.5$ & 1.64 \\
\hline B14 & Depth & PU-239 \\
\hline 224B030 & $0-0.5$ & 0.06 \\
\hline B15 & Depth & PU-239 \\
\hline 224B031 & $0-0.5$ & - \\
\hline \multicolumn{3}{|c}{} \\
\end{tabular}

Source: SNJV, 2004

Figure A.4-1

Sample Location Map, CAS 03-05-01, Leachfield 


\section{A.4.1.1 Field Screening}

Soil samples were field screened for VOCs, TPH, and alpha and beta/gamma radiation. The FSRs were compared to FSLs to guide subsequent sampling decisions. The VOC headspace, and alpha and beta/gamma radiation FSLs were not exceeded during sampling activities at this CAS. The TPH FSL was exceeded in all the samples collected from a grayish-white substance described in the next section.

\section{A.4.1.2 Sampling}

Initial sample locations were determined by researching several historical aerial photographs. The only feature presently remaining on the site is the former actylene generator concrete pad. Three locations were selected as outlined in the CAIP and the FI. Surface and subsurface samples were collected at each location. The surface sample at each location was a silty sand similar to the native soils in the area. At approximately 1.5 to $2.5 \mathrm{ft}$ bgs at each of the three Decision I locations (B01 through B03), a light-colored grayish-white material was found. The material is fine grained, moist, and has an odor similar to bentonite. Hand-auger refusal was encountered between 5 and $6.5 \mathrm{ft}$ at each location, signifying the bottom of the foreign material and the beginning of a hard native layer.

Decision II samples were collected at eight locations and positioned $15 \mathrm{ft}$ in each direction from the existing Decision I locations. Location B04, $15 \mathrm{ft}$ west of location B01, contained the foreign material at 1 to $3 \mathrm{ft}$ bgs. Another $15 \mathrm{ft}$ step-out, location B15, was placed west of that location and contained no foreign material, nor did any of the other step-out locations (B05 through B11). With the exception of B04, one surface and one subsurface sample were collected at each sample location. The subsurface samples were collected at the refusal depth of 3 to $4 \mathrm{ft} \mathrm{bgs,} \mathrm{indicating} \mathrm{the} \mathrm{native} \mathrm{layer}$ was reached and the foreign substance has been sufficiently delineated. The Decision I and Decision II samples were analyzed for the parameters listed in Table A.4-1. Sample locations are shown on Figure A.4-1.

\section{A.4.1.3 Deviations}

There were no significant deviations to the CAIP requirements at this CAS. Locations were added to the proposed Decision II locations in order to delineate the extent of the foreign material found. The 
investigation and sampling at CAS 03-05-01 are considered sufficient to meet the DQOs, established in the CAIP.

\section{A.4.2 Investigation Results}

The following sections provide descriptions of the CAS-specific activities conducted to complete investigation activities as outlined in the CAIP. Investigation activities included visual inspection of the CAS and collection of Decision I and Decision II samples.

No obvious release of contaminants or biasing factors were observed at the CAS during the visual inspection. Sampling included the collection of Decision I and Decision II samples as outlined in Section A.4.1.2.

Based on the observations made at the site and the analytical results, the contamination present at the site is limited to the foreign material found in the former leach pit.

Decision I samples were analyzed for the CAIP-specified COPCs, which included total VOCs, total SVOCs, TPH-DRO, total metals, PCBs, gamma-emitting radionuclides, plutonium isotopes, uranium isotopes, and strontium-90. Decision II samples were analyzed for SVOCs, total metals, PCBs, TPH-DRO and isotopic plutonium only, guided by the preliminary results from Decision I sampling. The analytical parameters and laboratory methods used to analyze the investigation samples are listed in Table A.2-2. Table A.4-1 lists the sample-specific analytical suite for CAS 03-05-01. A portion of the analytical results for this CAS was rejected during validation; however, these rejected data did not adversely impact closure decisions as discussed in Appendix B, Section B.1.4.

\section{A.4.2.1 Total Volatile Organic Compounds}

Total VOC analytical results for soil samples collected at CAS 03-05-01 that were detected above MRLs are presented in Table A.4-2. No VOCs were detected in soil samples above FALs.

\section{A.4.2.2 Total Semivolatile Organic Compounds}

Total SVOC analytical results for soil samples collected at CAS 03-05-01 that were detected above MRLs are presented in Table A.4-3. No SVOCs were detected in soil samples above FALs. 
Table A.4-2

\section{Soil Sample Results for Total VOCs Detected Above Minimum Reporting Limits at CAS 03-05-01, Leachfield}

\begin{tabular}{|c|c|c|c|c|c||}
\hline \multirow{2}{*}{$\begin{array}{c}\text { Sample } \\
\text { Location }\end{array}$} & \multirow{2}{*}{$\begin{array}{c}\text { Sample } \\
\text { Number }\end{array}$} & \multirow{2}{*}{$\begin{array}{c}\text { Depth } \\
\text { (ft bgs) }\end{array}$} & \multicolumn{3}{|c|}{ Contaminants of Potential Concern $(\mu \mathbf{g} / \mathbf{k g})$} \\
\cline { 4 - 6 } & & & $\mathbf{1 , 2 , 4 - T r i m e t h y l b e n z e n e}$ & Acetone & Naphthalene \\
\hline \multicolumn{3}{|c|}{ Final Action Levels ${ }^{\text {a }}$} & $\mathbf{1 7 0 , 0 0 0}$ & $\mathbf{6 , 0 0 0 , 0 0 0}$ & $\mathbf{1 9 0 , 0 0 0}$ \\
\hline \hline B01 & $224 \mathrm{~B} 002$ & $2-3$ & -- & $67(\mathrm{~J})$ & $15(\mathrm{~J})$ \\
\hline \multirow{3}{*}{ B02 } & $224 \mathrm{~B} 004$ & $2-3$ & $9.9(\mathrm{~J})$ & $88(\mathrm{~J})$ & $32(\mathrm{~J})$ \\
\cline { 2 - 6 } & $224 \mathrm{~B} 005$ & $2-3$ & $8.1(\mathrm{~J})$ & $78(\mathrm{~J})$ & $27(\mathrm{~J})$ \\
\cline { 2 - 6 } & $224 \mathrm{~B} 006$ & $5-6$ & $21(\mathrm{~J})$ & $190(\mathrm{~J})$ & $41(\mathrm{~J})$ \\
\hline \multirow{2}{*}{ B03 } & $224 \mathrm{~B} 008$ & $2-3$ & -- & $190(\mathrm{~J})$ & $13(\mathrm{~J})$ \\
\cline { 2 - 6 } & $224 \mathrm{~B} 009$ & $4-5$ & -- & $29(\mathrm{~J})$ & -- \\
\hline
\end{tabular}

aased on U.S. Environmental Protection Agency, Region 9 Preliminary Remediation Goals (PRGs) (EPA, 2002)

$\mathrm{ft}$ bgs $=$ Feet below ground surface $\mu \mathrm{g} / \mathrm{kg}=$ Micrograms per kilogram

$\mathrm{J}=$ Estimated value. Qualifier added to laboratory data; record accepted. Matrix effects may exist. Surrogate recovery exceeded the lower limits.

Table A.4-3

Soil Sample Results for Total SVOCs Detected Above Minimum Reporting Limits at CAS 03-05-01, Septic Tanks (4)/Discharge Area

\begin{tabular}{|c|c|c|c|}
\hline \multirow{2}{*}{$\begin{array}{c}\text { Sample } \\
\text { Location }\end{array}$} & \multirow{2}{*}{$\begin{array}{l}\text { Sample } \\
\text { Number }\end{array}$} & \multirow{2}{*}{$\begin{array}{c}\text { Depth } \\
\text { (ft bgs) }\end{array}$} & Contaminants of Potential Concern $(\mu \mathrm{g} / \mathrm{kg})$ \\
\hline & & & Pyrene \\
\hline \multicolumn{3}{|c|}{ Final Action Levels ${ }^{a}$} & $29,000,000$ \\
\hline $\mathrm{B} 03$ & 224B009 & $4-5$ & $530(\mathrm{~J})$ \\
\hline
\end{tabular}

${ }^{\mathrm{a} B a s e d}$ on U.S. Environmental Protection Agency, Region 9 Preliminary Remediation Goals (PRGs) (EPA, 2002)

$\mathrm{ft}$ bgs $=$ Feet below ground surface $\mu \mathrm{g} / \mathrm{kg}=$ Micrograms per kilogram

$\mathrm{J}=$ Estimated value. Qualifier added to laboratory data; record accepted. Matrix effects may exist. Internal standard area count outside control limits.

\section{A.4.2.3 Total Metals}

Total metals analytical results for soil samples collected at CAS 03-05-01 are presented in Table A.4-4. No metals were detected above FALs. 
Table A.4-4

Soil Sample Results for Metals Detected Above Minimum Reporting Limits at CAS 03-05-01, Leachfield (Page 1 of 2)

\begin{tabular}{|c|c|c|c|c|c|c|c|c|c|c|c|c|c|c|c|c|c|}
\hline \multirow[b]{2}{*}{$\begin{array}{l}\text { Sample } \\
\text { Location }\end{array}$} & \multirow[b]{2}{*}{$\begin{array}{l}\text { Sample } \\
\text { Number }\end{array}$} & \multirow[b]{2}{*}{$\begin{array}{c}\text { Depth } \\
\text { (ft bgs) }\end{array}$} & \multicolumn{15}{|c|}{ Contaminants of Potential Concern (mg/kg) } \\
\hline & & & 孞 & 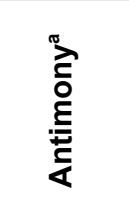 & $\begin{array}{l}\stackrel{0}{0} \\
\frac{0}{2} \\
\stackrel{0}{0} \\
\frac{0}{4}\end{array}$ & 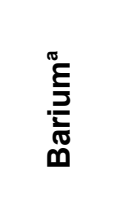 & 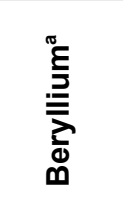 & 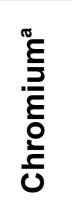 & $\begin{array}{l}\frac{\pi}{ \pm 0} \\
\frac{0}{0} \\
0 \\
0\end{array}$ & $\begin{array}{l}\frac{\pi}{d} \\
\frac{0}{0} \\
\frac{0}{0}\end{array}$ & 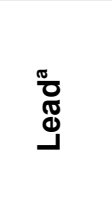 & 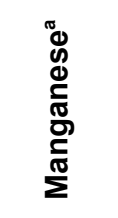 & 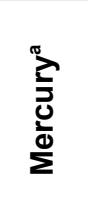 & 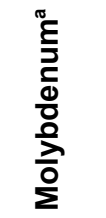 & $\begin{array}{l}\frac{\sigma}{0} \\
\frac{x}{0} \\
\frac{0}{Z}\end{array}$ & "ह & $\stackrel{\widetilde{N}}{\mathrm{~N}}$ \\
\hline \multicolumn{3}{|c|}{ Final Action Levels } & 100,000 & 410 & 23 & 67,000 & 1,900 & 450 & 1,900 & 41,000 & 750 & 19,000 & 310 & 5,100 & 20,000 & 5,100 & 100,000 \\
\hline \multirow{2}{*}{ B01 } & $\overline{2 \text { 224B001 }}$ & $\overline{0-0.5}$ & $\overline{\overline{7,900(\mathrm{~J})^{\mathrm{c}}}}$ & $\overline{---}$ & 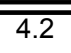 & $\overline{230}$ & 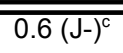 & $\overline{6.1}$ & $\overline{\overline{3.3}}$ & $\overline{9.9}$ & $\overline{16}$ & 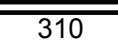 & $\overline{---}$ & $\overline{---}$ & $\overline{14}$ & $\overline{---}$ & 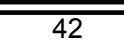 \\
\hline & 224B002 & $2-3$ & $2,100(J)^{c}$ & $\overline{--}$ & $\overline{--}$ & 20 & -- & 3.9 & 2.1 & 3.9 & 4.3 & 15 & -- & -- & 79 & -- & 3.4 \\
\hline \multirow{4}{*}{ B02 } & 224B003 & $0-0.5$ & $7,600(\mathrm{~J})^{\mathrm{c}}$ & $\overline{--}$ & 4.5 & 210 & $0.59(\mathrm{~J})^{\mathrm{c}}$ & 5.8 & 3.4 & 9.7 & 35 & 250 & $\overline{--}$ & $\overline{--}$ & 9.9 & $\overline{--}$ & 60 \\
\hline & 224B004 & $2-3$ & $2,100(\mathrm{~J})^{\mathrm{c}}$ & -- & -- & 24 & -- & 1.6 & -- & 2.7 & -- & 11 & -- & -- & 47 & -- & -- \\
\hline & 224B005 & $2-3$ & $2,000(J)^{c}$ & -- & $\begin{array}{c}1.1 \\
(\mathrm{~J}+)\end{array}$ & 20 & -- & 1.7 & -- & 2.8 & -- & 9.6 & -- & -- & 50 & -- & -- \\
\hline & 224B006 & $5-6$ & $2,900(\mathrm{~J})^{\mathrm{c}}$ & -- & -- & 37 & -- & 2.2 & 1.9 & 5.2 & 6.3 & 47 & -- & -- & 71 & -- & 6.5 \\
\hline \multirow[t]{2}{*}{ B03 } & 224B008 & $2-3$ & $1,900(J)^{c}$ & -- & -- & 27 & -- & -- & -- & 2.3 & 9.1 & 7.6 & -- & -- & 45 & -- & 5.1 \\
\hline & 224B009 & $4-5$ & $7,900(\mathrm{~J})^{\mathrm{c}}$ & -- & 4 & 210 & -- & 5.5 & 2.1 & 6.8 & 17 & 150 & -- & -- & 21 & -- & 32 \\
\hline \multirow{2}{*}{ B04 } & 224B010 & $0-0.5$ & 9,400 & -- & 3.5 & $190(\mathrm{~J})^{\mathrm{d}}$ & 0.7 & 5.2 & 5.2 & 32 & $16(\mathrm{~J})^{d}$ & 390 & -- & 2.8 & 7.5 & 0.8 & $77(\mathrm{~J})^{\mathrm{d}}$ \\
\hline & 224B018 & $3.5-4$ & 12,000 & $\overline{--}$ & 5.3 & $230(\mathrm{~J})^{\mathrm{d}}$ & 1 & 9.2 & 4.7 & 12 & $14(\mathrm{~J})^{\mathrm{d}}$ & 370 & $\overline{--}$ & 1.4 & 16 & 0.64 & $51(\mathrm{~J})^{\mathrm{d}}$ \\
\hline \multirow{2}{*}{ B05 } & 224B011 & $0-0.5$ & 8,400 & -- & 4.7 & $210(\mathrm{~J})^{\mathrm{d}}$ & 0.72 & 6.9 & 3.9 & 13 & $21(\mathrm{~J})^{d}$ & 330 & -- & 1.1 & 10 & -- & $50(\mathrm{~J})^{\mathrm{d}}$ \\
\hline & 224B019 & $3-4$ & 12,000 & -- & 5.9 & $210(\mathrm{~J})^{\mathrm{d}}$ & 1 & 8.4 & 4.6 & 11 & $13(\mathrm{~J})^{\mathrm{d}}$ & 350 & -- & -- & 13 & 0.54 & $50(\mathrm{~J})^{\mathrm{d}}$ \\
\hline \multirow{2}{*}{ B06 } & 224B012 & $0-0.5$ & 8,300 & $\overline{--}$ & 4.9 & $200(\mathrm{~J})^{d}$ & 0.72 & 6.1 & 3.9 & 12 & $18(\mathrm{~J})^{d}$ & 280 & -- & 1 & 11 & 0.6 & $55(\mathrm{~J})^{\mathrm{d}}$ \\
\hline & 224B020 & $2-3$ & 11,000 & -- & 5.3 & $190(\mathrm{~J})^{\mathrm{d}}$ & 1 & 7.9 & 4.2 & 11 & $12(\mathrm{~J})^{\mathrm{d}}$ & 220 & 0.038 & -- & 13 & -- & $45(\mathrm{~J})^{\mathrm{d}}$ \\
\hline \multirow{2}{*}{ B07 } & 224B013 & $0-0.5$ & 8,300 & $0.83(\mathrm{~J})^{\mathrm{e}}$ & 4.8 & $180(\mathrm{~J})^{\mathrm{d}}$ & 0.72 & 6.2 & 3.9 & 12 & $84(J)^{d}$ & 290 & -- & -- & 10 & -- & $49(\mathrm{~J})^{\mathrm{d}}$ \\
\hline & 224B021 & $2-3$ & 7,700 & -- & 4.4 & $200(\mathrm{~J})^{\mathrm{d}}$ & 0.67 & 5.6 & 3.1 & 8.6 & $11(\mathrm{~J})^{\mathrm{d}}$ & 220 & -- & -- & 10 & -- & $38(J)^{d}$ \\
\hline \multirow{2}{*}{ B08 } & 224B014 & $0-0.5$ & 7,600 & $\overline{--}$ & 4.6 & $200(\mathrm{~J})^{\mathrm{d}}$ & 0.66 & 5.6 & 3.6 & 12 & $110(J)^{d}$ & 300 & -- & $\overline{--}$ & 9.3 & 0.85 & $45(\mathrm{~J})^{\mathrm{d}}$ \\
\hline & 224B022 & $3-4$ & 8,100 & -- & 5.9 & $180(\mathrm{~J})^{\mathrm{d}}$ & 0.64 & 4.5 & 2.8 & 6.3 & $8.8(\mathrm{~J})^{\mathrm{d}}$ & 170 & -- & -- & 10 & -- & $26(\mathrm{~J})^{d}$ \\
\hline
\end{tabular}


Table A.4-4

Soil Sample Results for Metals Detected Above Minimum Reporting Limits at CAS 03-05-01, Leachfield

(Page 2 of 2)

\begin{tabular}{|c|c|c|c|c|c|c|c|c|c|c|c|c|c|c|c|c|c|}
\hline \multirow[b]{2}{*}{$\begin{array}{l}\text { Sample } \\
\text { Location }\end{array}$} & \multirow[b]{2}{*}{$\begin{array}{l}\text { Sample } \\
\text { Number }\end{array}$} & \multirow[b]{2}{*}{$\begin{array}{l}\text { Depth } \\
\text { (ft bgs) }\end{array}$} & \multicolumn{15}{|c|}{ Contaminants of Potential Concern (mg/kg) } \\
\hline & & & 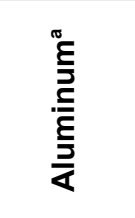 & 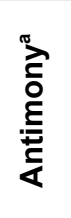 & 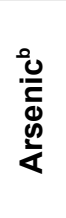 & 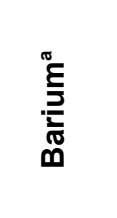 & 吝 & 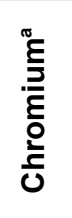 & $\begin{array}{l}\frac{\sqrt[T]{7}}{\pi} \\
\frac{0}{0} \\
0\end{array}$ & $\begin{array}{l}\frac{\pi}{0} \\
\frac{0}{0} \\
0 \\
0\end{array}$ & 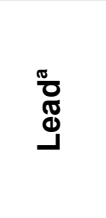 & 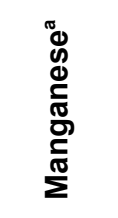 & 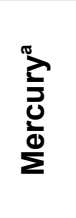 & 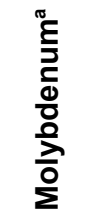 & $\begin{array}{l}\frac{\sigma}{d} \\
\frac{v}{0} \\
\frac{\sigma}{Z}\end{array}$ & $\frac{\stackrel{\pi}{E}}{\frac{\Xi}{5}}$ & 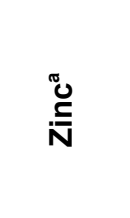 \\
\hline \multicolumn{3}{|c|}{ Final Action Levels } & 100,000 & 410 & 23 & 67,000 & 1,900 & 450 & 1,900 & 41,000 & 750 & 19,000 & 310 & 5,100 & 20,000 & 5,100 & 100,000 \\
\hline \multirow{2}{*}{ B09 } & 224B015 & $0-0.5$ & 7,400 & $\overline{---}$ & $\overline{4.6}$ & $200(J)^{d}$ & $\overline{0.65}$ & 16 & 3.1 & 9.2 & 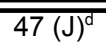 & $\overline{380}$ & $\overline{--}$ & 1.5 & 8.6 & $\overline{---}$ & $43(\mathrm{~J})^{\mathrm{d}}$ \\
\hline & 224B023 & $2-3$ & 12,000 & $\overline{--}$ & 6.1 & $280(\mathrm{~J})^{\mathrm{d}}$ & 1 & 7.4 & 3.6 & 9.2 & $9.9(J)^{d}$ & 210 & -- & -- & 12 & -- & $41(\mathrm{~J})^{\mathrm{d}}$ \\
\hline \multirow{2}{*}{ B10 } & 224B016 & $0-0.5$ & 7,800 & $\overline{--}$ & 4.3 & $190(\mathrm{~J})^{\mathrm{d}}$ & 0.66 & 6 & 3.4 & 10 & $21(\mathrm{~J})^{\mathrm{d}}$ & 240 & $\overline{--}$ & $\overline{--}$ & 9.8 & $\overline{--}$ & $45(J)^{d}$ \\
\hline & 224B024 & $2.5-3.5$ & 12,000 & -- & 5.4 & $260(\mathrm{~J})^{\mathrm{d}}$ & 1.1 & 8.6 & 4.5 & 12 & $13(\mathrm{~J})^{\mathrm{d}}$ & 300 & -- & -- & 15 & 0.67 & $52(\mathrm{~J})^{\mathrm{d}}$ \\
\hline \multirow{2}{*}{ B11 } & 224B017 & $0-0.5$ & 8,000 & - & 4.4 & $190(\mathrm{~J})^{\mathrm{d}}$ & 0.67 & 11 & 3.6 & 11 & $17(\mathrm{~J})^{\mathrm{d}}$ & 270 & - & - & 9.6 & - & $60(J)^{d}$ \\
\hline & 224B025 & $2.5-3.5$ & 7,600 & -- & 4.3 & $230(\mathrm{~J})^{\mathrm{d}}$ & 0.66 & 6.9 & 3.3 & 9.2 & $10(\mathrm{~J})^{\mathrm{d}}$ & 270 & -- & -- & 9.9 & -- & $35(J)^{d}$ \\
\hline \multirow[t]{2}{*}{ B12 } & 224B027 & $0-0.5$ & 9,200 & $\overline{--}$ & 4.9 & $200(\mathrm{~J})^{\mathrm{d}}$ & 0.79 & 7.6 & 4.6 & 12 & $13(\mathrm{~J})^{\mathrm{d}}$ & 320 & -- & $\overline{--}$ & 12 & $\overline{--}$ & $82(\mathrm{~J})^{\mathrm{d}}$ \\
\hline & 224B028 & $2-2.5$ & 9,100 & -- & 4.8 & $190(\mathrm{~J})^{\mathrm{d}}$ & 0.79 & 6.9 & 4.7 & 12 & $13(\mathrm{~J})^{\mathrm{d}}$ & 320 & -- & -- & 11 & -- & $64(\mathrm{~J})^{\mathrm{d}}$ \\
\hline
\end{tabular}

${ }^{\mathrm{a}}$ Based on U.S. Environmental Protection Agency, Region 9 Preliminary Remediation Goals (PRGs) (EPA, 2002)

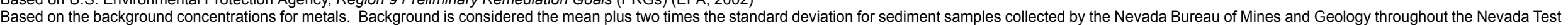
and Training Range (NBMG, 1998; Moore, 1999).

Qualifier added to laboratory data; record accepted. Serial dilution \%D outside control limits. Matrix effects may exist.

Qualifier added to laboratory data; record accepted. Matrix spike recovery outside control limits.

Qualifier added to laboratory data; record accepted. Negative bias found in continuing calibration/method blank. Matrix spike recovery outside control limits.

$\mathrm{ft}$ bgs $=$ Feet below ground surface

$\mathrm{mg} / \mathrm{kg}=$ Milligrams per kilogram

$\mathrm{J}=$ Estimated value.

is an estimated quantity, but the result may be biased high. CRI/CRA \% recovery(ies) criteria was/were not met.

$\mathrm{J}-=$ The result is an estimated quantity, but the result may be biased low.

-- = Not detected above minimum reporting limits. 


\section{A.4.2.4 Polychlorinated Biphenyls}

No PCBs were detected in soil samples above reporting levels.

\section{A.4.2.5 Total Petroleum Hydrocarbons-Diesel-Range Organics}

Total TPH-DRO analytical results for soil samples collected at CAS 03-05-01 that were detected above MRLs are presented in Table A.4-5. Total petroleum hydrocarbons-DRO was found above the NAC threshold of $100 \mathrm{ppm}$ in four soil locations and is confined to the foreign material in the former leach pit. The NAC threshold was not used as the FAL for this CAS. Instead, FALs were established as the EPA Region 9 PRG values corresponding to the hazardous constituents contained in TPH. The results of these TPH hazardous constituents are compared to FALs in the total VOCs and the total SVOCs tables, Table A.4-2 and Table A.4-3, respectively.

Table A.4-5

Soil Sample Results for TPH-DRO Detected Above Minimum Reporting Limits at CAS 03-05-01, Leachfield

\begin{tabular}{|c|c|c|c|}
\hline \multirow{2}{*}{$\begin{array}{c}\text { Sample } \\
\text { Location }\end{array}$} & \multirow{2}{*}{$\begin{array}{l}\text { Sample } \\
\text { Number }\end{array}$} & \multirow{2}{*}{$\begin{array}{l}\text { Depth } \\
\text { (ft bgs) }\end{array}$} & \multirow{2}{*}{$\begin{array}{c}\text { Contaminants of Potential Concern }(\mathrm{mg} / \mathrm{kg}) \\
\text { Diesel-Range Organics }\end{array}$} \\
\hline & & & \\
\hline$\overline{\mathrm{B} 01}$ & 224B002 & $2-3$ & 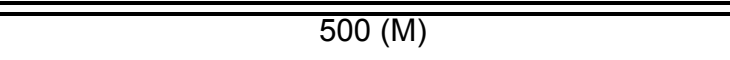 \\
\hline \multirow{3}{*}{ B02 } & 224B004 & $2-3$ & $460(\mathrm{M})$ \\
\hline & 224B005 & $2-3$ & $410(\mathrm{M})$ \\
\hline & 224B006 & $5-6$ & $330(\mathrm{M})$ \\
\hline \multirow{2}{*}{ B03 } & 224B008 & $2-3$ & $760(\mathrm{M})$ \\
\hline & 224B009 & $4-5$ & $1,100(\mathrm{M})$ \\
\hline \multirow{2}{*}{ B04 } & 224B010 & $0-0.5$ & $80(\mathrm{M})$ \\
\hline & 224B018 & $3.5-4$ & $110(\mathrm{M})$ \\
\hline B05 & 224B011 & $0-0.5$ & $61(\mathrm{M})$ \\
\hline B11 & 224B017 & $0-0.5$ & $31(\mathrm{M})$ \\
\hline
\end{tabular}

aBased on Nevada Administrative Code, "Contamination of Soil: Establishment of Action Levels" (NAC, 2002)

$\mathrm{ft}$ bgs $=$ Feet below ground surface

$\mathrm{mg} / \mathrm{kg}=$ Milligrams per

$\mathrm{M}=\mathrm{A}$ pattern resembling motor oil was detected. 


\section{A.4.2.6 Gamma-Emitting Radionuclides}

Gamma-emitting radionuclide analytical results for soil samples collected at CAS 03-05-01 that were detected above MDCs are presented in Table A.4-6.

\section{A.4.2.6.1 Isotopes}

Isotope analytical results for soil samples collected at CAS 03-05-01 that were detected above MDCs are presented in Table A.4-7. Sample 224B027 contained Pu-239 activity at $8.7 \mathrm{pCi} / \mathrm{g}$ and was bounded laterally and vertically by additional step-out sampling. However, this isolated occurrence is below the FAL of $12.7 \mathrm{pCi} / \mathrm{g}$. Therefore, $\mathrm{Pu}-239$ is not considered a $\mathrm{COC}$ at this site.

\section{A.4.3 Nature and Extent of Contamination}

No COCs were identified at CAS 03-05-01.

\section{A.4.4 Revised Conceptual Site Model}

No variations in the CSM were identified. 
Table A.4-6

Soil Sample Results for Gamma-Emitting Radionuclides

Detected Above Minimum Detectable Concentrations at CAS 03-05-01, Leachfield

\begin{tabular}{|c|c|c|c|c|c|c|c|c|c|c|c|c|c|}
\hline \multirow[b]{2}{*}{$\begin{array}{l}\text { Sample } \\
\text { Location }\end{array}$} & \multirow[b]{2}{*}{$\begin{array}{l}\text { Sample } \\
\text { Number }\end{array}$} & \multirow{3}{*}{$\begin{array}{l}\text { Depth } \\
\text { (ft bgs) }\end{array}$} & \multicolumn{11}{|c|}{ Contaminants of Potential Concern (pCi/g) } \\
\hline & & & \multicolumn{2}{|c|}{ 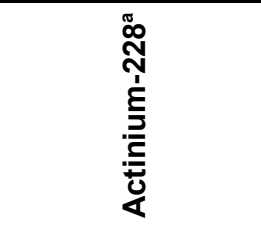 } & \multicolumn{2}{|c|}{ 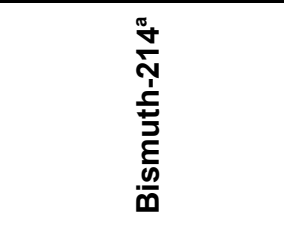 } & \multicolumn{3}{|l|}{ 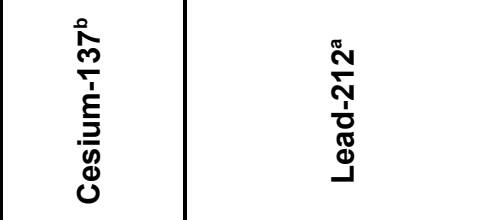 } & \multicolumn{2}{|c|}{ 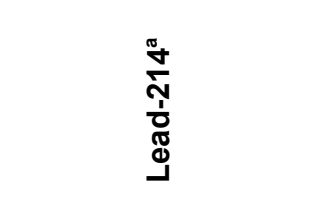 } & \multicolumn{2}{|c|}{ 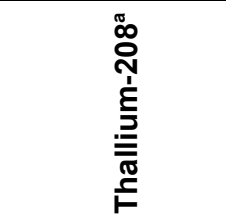 } \\
\hline \multirow{2}{*}{\multicolumn{3}{|c|}{ Final Action Levels }} & 5 & 15 & 5 & 15 & \multirow{2}{*}{12.2} & 5 & 15 & 5 & 15 & 5 & 15 \\
\hline & & & $<15$ & $>15$ & $<15$ & $>15$ & & $<15$ & $>15$ & $<15$ & $>15$ & $<15$ & $>15$ \\
\hline B01 & 224B001 & $0-0.5$ & 1.36 & NA & $\overline{--}$ & $\overline{N A}$ & 1.54 & 1.26 & NA & $0.83(\mathrm{~J})$ & NA & 0.35 & $\overline{\mathrm{NA}}$ \\
\hline B02 & 224B003 & $0-0.5$ & - & NA & $0.86(\mathrm{~J})$ & NA & 0.67 & 1.37 & NA & $0.99(\mathrm{~J})$ & NA & 0.33 & NA \\
\hline \multirow{2}{*}{ B03 } & 224B007 & $0-0.5$ & -- & NA & $0.77(\mathrm{~J})$ & NA & 0.5 & 1.28 & NA & $1.08(\mathrm{~J})$ & NA & -- & NA \\
\hline & 224B009 & $4-5$ & NA & -- & NA & -- & -- & NA & $1.05(J)^{c}$ & NA & $1.25(\mathrm{G}, \mathrm{J})$ & NA & -- \\
\hline B13 & 224B029 & $0-0.5$ & $1.23(\mathrm{G})$ & NA & $0.89(\mathrm{G}, \mathrm{J})$ & NA & $0.47(\mathrm{G}, \mathrm{LT})$ & $1.66(J)^{c}$ & NA & $1.11(\mathrm{G}, \mathrm{J})$ & NA & $0.46(\mathrm{G})$ & NA \\
\hline B14 & 224B030 & $0-0.5$ & $1.66(\mathrm{G})$ & NA & $0.86(\mathrm{G}, \mathrm{J})$ & NA & -- & $1.71(\mathrm{~J})^{\mathrm{c}}$ & NA & $1.19(\mathrm{G}, \mathrm{J})$ & NA & $0.54(\mathrm{G})$ & NA \\
\hline B15 & 224B031 & $0-0.5$ & $1.17(\mathrm{G})$ & NA & $0.77(\mathrm{G}, \mathrm{J})$ & NA & -- & $1.4(\mathrm{~J})^{\mathrm{c}}$ & NA & $1.08(\mathrm{G}, \mathrm{J})$ & NA & $0.47(\mathrm{G})$ & NA \\
\hline
\end{tabular}

${ }^{2}$ Taken from the generic guidelines for residual concentrations of actinium-228, bismuth-214, lead-212, lead-214, thallium-208, and thorium-232, as found in Chapter IV of DOE Order 5400.5, Change 2, "Radiation Protection of the Pulc document, $15 \mathrm{~cm}$ is assumed to be equivalent to $0.5 \mathrm{ft}$ ( 6 in.); therefore, $5 \mathrm{pCi} / \mathrm{g}$ represents the FALs for these radionuclides in the surface soil ( 0 to $0.5 \mathrm{ft}$ depth).

${ }^{\mathrm{b}}$ Taken from the construction, commercial, industrial land use scenario in Table 2.1 of the NCRP Report No. 129, Recommended Screening Limits for Contaminated Surface Soil and Review Factors Relevant to Site-Specific Studies (NCRP, 1999). The values provided in this source document were scaled to a $25-\mathrm{mrem} / \mathrm{yr}$ dose.

QQualifier added to laboratory data; record accepted. Sample does not meet counting geometry requirements.

$\mathrm{ft}$ bgs $=$ Feet below ground surface

$\mathrm{cm}=$ Centimeter

$\mathrm{mrem} / \mathrm{yr}=$ Millirems per year

$\mathrm{pCi} / \mathrm{g}=$ Picocuries per gram

$=$ Not detected above minimum reporting limits

$>=$ Greater than

$G=$ Sample density differs by more than 15 percent of laboratory control sample density.

$\mathrm{J}=$ Estimated value.

$\mathrm{NA}=$ Not applicable 


\section{Table A.4-7 \\ Soil Sample Results for Isotopes Detected \\ Above Minimum Detectable Concentrations at CAS 03-05-01, Leachfield}

\begin{tabular}{|c|c|c|c|c|c|c|c|}
\hline \multirow{2}{*}{$\begin{array}{l}\text { Sample } \\
\text { Location }\end{array}$} & \multirow{2}{*}{$\begin{array}{l}\text { Sample } \\
\text { Number }\end{array}$} & \multirow{2}{*}{$\begin{array}{l}\text { Depth } \\
\text { (ft bgs) }\end{array}$} & \multicolumn{5}{|c|}{ Contaminants of Potential Concern (pCi/g) } \\
\hline & & & Plutonium-238 & Plutonium-239 & Uranium-234 & Uranium-235 & Uranium-238 \\
\hline \multicolumn{3}{|c|}{ Final Action Levels ${ }^{a}$} & 13 & 12.7 & 143 & 17.6 & 105 \\
\hline \multirow{2}{*}{ B01 } & 224B001 & $0-0.5$ & 0.96 & 6.9 & 0.85 & 0.062 & $\overline{0.79}$ \\
\hline & 224B002 & $2-3$ & -- & -- & 0.47 & -- & 0.409 \\
\hline \multirow{4}{*}{ B02 } & 224B003 & $0-0.5$ & -- & 0.311 & 0.88 & 0.067 & 0.85 \\
\hline & 224B004 & $2-3$ & -- & -- & 0.419 & -- & 0.348 \\
\hline & 224B005 & $2-3$ & -- & -- & 0.423 & -- & 0.334 \\
\hline & 224B006 & $5-6$ & -- & 0.345 & 0.446 & -- & 0.401 \\
\hline \multirow{3}{*}{ B03 } & 224B007 & $0-0.5$ & 0.71 & 2.78 & 1.09 & -- & 1.08 \\
\hline & 224B008 & $2-3$ & -- & -- & 0.258 & -- & 0.254 \\
\hline & 224B009 & $4-5$ & -- & 0.226 & 0.91 & $0.04(\mathrm{LT})$ & 0.85 \\
\hline B04 & 224B010 & $0-0.5$ & -- & 0.174 & -- & -- & -- \\
\hline B05 & 224B011 & $0-0.5$ & 0.04 (LT) & 0.136 & $\overline{--}$ & - & $\overline{--}$ \\
\hline B06 & 224B012 & $0-05$ & 0.148 & 0.8 & -- & -- & -- \\
\hline B07 & 224B013 & $0-0.5$ & -- & 0.105 & -- & -- & -- \\
\hline B08 & 224B014 & $0-0.5$ & -- & 1.89 & -- & -- & -- \\
\hline B09 & 224B015 & $0-0.5$ & 0.82 & 4.75 & -- & - & -- \\
\hline B10 & 224B016 & $0-0.5$ & 0.81 & 3.76 & -- & -- & -- \\
\hline B11 & 224B017 & $0-0.5$ & 0.72 & 2.51 & -- & -- & -- \\
\hline \multirow{3}{*}{ B12 } & 224B026 & $0-0.5$ & -- & 0.43 & -- & -- & -- \\
\hline & 224B027 & $0-0.5$ & 1.35 & 8.7 & -- & -- & -- \\
\hline & 224B028 & $2-2.5$ & 0.228 & 0.84 & -- & -- & -- \\
\hline B13 & 224B029 & $0-0.5$ & 0.243 & 1.64 & -- & -- & -- \\
\hline B14 & 224B030 & $0-0.5$ & -- & 0.058 & -- & -- & -- \\
\hline
\end{tabular}

\footnotetext{
${ }^{a}$ Taken from the construction, commercial, industrial land use scenario in Table 2.1 of the NCRP Report No. 129 Recommended Screening Limits for Contaminated Surface Soil and Review Factors Relevant to Site-Specific Studies (NCRP, 1999). The values provided in this source document were scaled to a 25-mrem/yr dose.
}

$\mathrm{ft}$ bgs $=$ Feet below ground surface $\mathrm{mrem} / \mathrm{yr}=$ Millirems per year $\mathrm{pCi} / \mathrm{g}=$ Picocuries per gram

$\mathrm{LT}=$ Result is less than the requested minimum detectable concentration, greater than the sample specific minimum detectable concentration.

-- = Not detected above minimum reporting limits 


\section{A.5.0 CAS 05-04-01, Septic Tanks (4)/Discharge Area}

Corrective Action Site 05-04-01 is located in Area 5 of the NTS. The CAS consists of four septic tanks and associated piping, and a potential overflow area. The septic system serviced the former Area 5 Trailer Park. Investigation activities at CAS 05-04-01 included collection of liquid and sludge samples from the four tanks, surface soil samples in the discharge area, as well as a visual inspection of piping and manholes upstream from the tanks.

\section{A.5.1 Corrective Action Investigation}

A total of 4 liquid and 4 sludge waste management samples and 12 soil characterization samples were collected during investigation activities at CAS 05-04-01. The IDs, locations, types, and analyses are listed in Table A.5-1. The sample locations are shown on Figure A.5-1. The specific CAI activities conducted to satisfy the CAIP requirements at this CAS are described in the following sections.

\section{A.5.1.1 Field Screening}

Soil samples were field screened for VOCs, TPH, and alpha and beta/gamma radiation. The FSRs were compared to FSLs to guide subsequent sampling decisions. The VOC headspace, TPH, and alpha and beta/gamma radiation FSLs were not exceeded during sampling activities at this CAS. Fecal coliform screening was also performed on contents of all four tanks. All results were negative with the exception of sample 224C506 (Tank 2), which contained two positive readings out of five tubes tested, resulting in 51,000 colonies per 100 milliliters. The laboratory was notified of these results prior to handling the liquid and sludge samples.

\section{A.5.1.2 Sampling}

The DQO requirements set forth in the CAIP called for tank samples to confirm the results of a 1995 characterization of the tank contents (NNSA/NSO, 2004). Liquid and sludge samples were collected from each of the four tanks in the system. All manholes and the distribution box were free of liquid or sludge, and were not sampled. Surface soil samples were taken at four locations in the discharge area, near each of the four overflow pipes. The samples were analyzed for the parameters listed in Table A.5-1. Sample locations are shown on Figure A.5-1. 
Table A.5-1

Samples Collected at CAS 05-04-01, Septic Tanks (4)/Discharge Area

\begin{tabular}{|c|c|c|c|c|c|}
\hline $\begin{array}{l}\text { Sample } \\
\text { Location }\end{array}$ & $\begin{array}{l}\text { Sample } \\
\text { Number }\end{array}$ & $\begin{array}{l}\text { Depth } \\
\text { (ft bgs) }\end{array}$ & $\begin{array}{l}\text { Sample } \\
\text { Matrix }\end{array}$ & Purpose & Analyses \\
\hline $\mathrm{C} 01$ & $224 \mathrm{C} 001$ & $0-0.5$ & Soil & $\mathrm{SC}$ & Set 3, Pesticide \\
\hline $\mathrm{C} 02$ & $224 \mathrm{C} 002$ & $0-0.5$ & Soil & SC & Set 1, Pesticide \\
\hline \multirow{2}{*}{$\mathrm{C03}$} & $224 \mathrm{C} 003$ & $0-0.5$ & Soil & SC, MS/MSD & Set 3, Pesticide \\
\hline & $224 \mathrm{C} 011$ & $1.5-2.5$ & Soil & SC & Pesticide \\
\hline \multirow{2}{*}{$\mathrm{CO4}$} & $224 \mathrm{C} 004$ & $0-0.5$ & Soil & SC & Set 3, Pesticide \\
\hline & $224 \mathrm{C} 012$ & $1.5-2.5$ & Soil & SC & Pesticide \\
\hline $\mathrm{C} 05$ & $224 \mathrm{C} 005$ & $0-0.5$ & Soil & SC & Set 3, Pesticide \\
\hline \multirow[b]{2}{*}{ C06 } & $224 \mathrm{C} 006$ & $0-0.5$ & Soil & SC & Set 1, Pesticide \\
\hline & $224 \mathrm{C} 007$ & $0-0.5$ & Soil & $\begin{array}{c}\text { Field Duplicate } \\
\text { of \#224C006 }\end{array}$ & Set 1, Pesticide \\
\hline $\mathrm{C} 07$ & $224 \mathrm{C} 008$ & $0-0.5$ & Soil & SC & Pesticide \\
\hline $\mathrm{C08}$ & $224 C 009$ & $0-0.5$ & Soil & SC & Pesticide \\
\hline $\mathrm{C09}$ & $224 \mathrm{C} 010$ & $0-0.5$ & Soil & SC & Pesticide \\
\hline NA & $224 C 301$ & NA & Water & Trip Blank & Total VOCs \\
\hline NA & $224 C 302$ & NA & Water & Field blank & Set 1 \\
\hline \multirow{2}{*}{ Tank 1} & $224 C 501$ & NA & Liquid & WM & Set 1 \\
\hline & $224 C 505$ & NA & Sludge & WM & Set 2 \\
\hline \multirow{2}{*}{ Tank 2} & $224 \mathrm{C} 502$ & NA & Liquid & WM & Set 1 \\
\hline & $224 \mathrm{C} 506$ & NA & Sludge & WM & Set 2 \\
\hline \multirow{2}{*}{ Tank 3} & $224 \mathrm{C} 503$ & NA & Liquid & WM & Set 1 \\
\hline & $224 \mathrm{C} 507$ & NA & Sludge & WM & Set 2 \\
\hline \multirow{2}{*}{ Tank 4} & $224 \mathrm{C} 504$ & NA & Liquid & WM & Set 1 \\
\hline & $224 C 508$ & NA & Sludge & WM & Set 2 \\
\hline
\end{tabular}

Set 1 = Total VOCs, Total SVOCs, Total RCRA Metals, Aluminum, Antimony, Beryllium, Cobalt, Copper, Manganese, Molybdenum, Nickel, Zinc, TPH-DRO, PCBs, Gamma Spectroscopy, Isotopic Plutonium, Isotopic Uranium, and Strontium-90.

Set 2 = TCLP VOCs, TCLP SVOCs, TCLP RCRA Metals.

Set 3 = Total VOCs, Total SVOCs, Total RCRA Metals, Aluminum, Antimony, Beryllium, Cobalt, Copper, Manganese, Molybdenum, Nickel, Zinc, TPH-DRO, and PCBs.

DRO = Diesel-range organics

$\mathrm{ft}$ bgs $=$ Feet below ground surface

$\mathrm{PCB}=$ Polychlorinated biphenyl

RCRA = Resource Conservation and Recovery Act

$\mathrm{SC}=$ Site characterization

SVOC $=$ Semivolatile organic compound
TCLP = Toxicity characteristic leaching procedure

$\mathrm{TPH}=$ Total petroleum hydrocarbons

WM = Waste Management

MS/MSD = Matrix spike/matrix spike duplicate

$\mathrm{NA}=$ Not applicable

VOC $=$ Volatile organic compound 


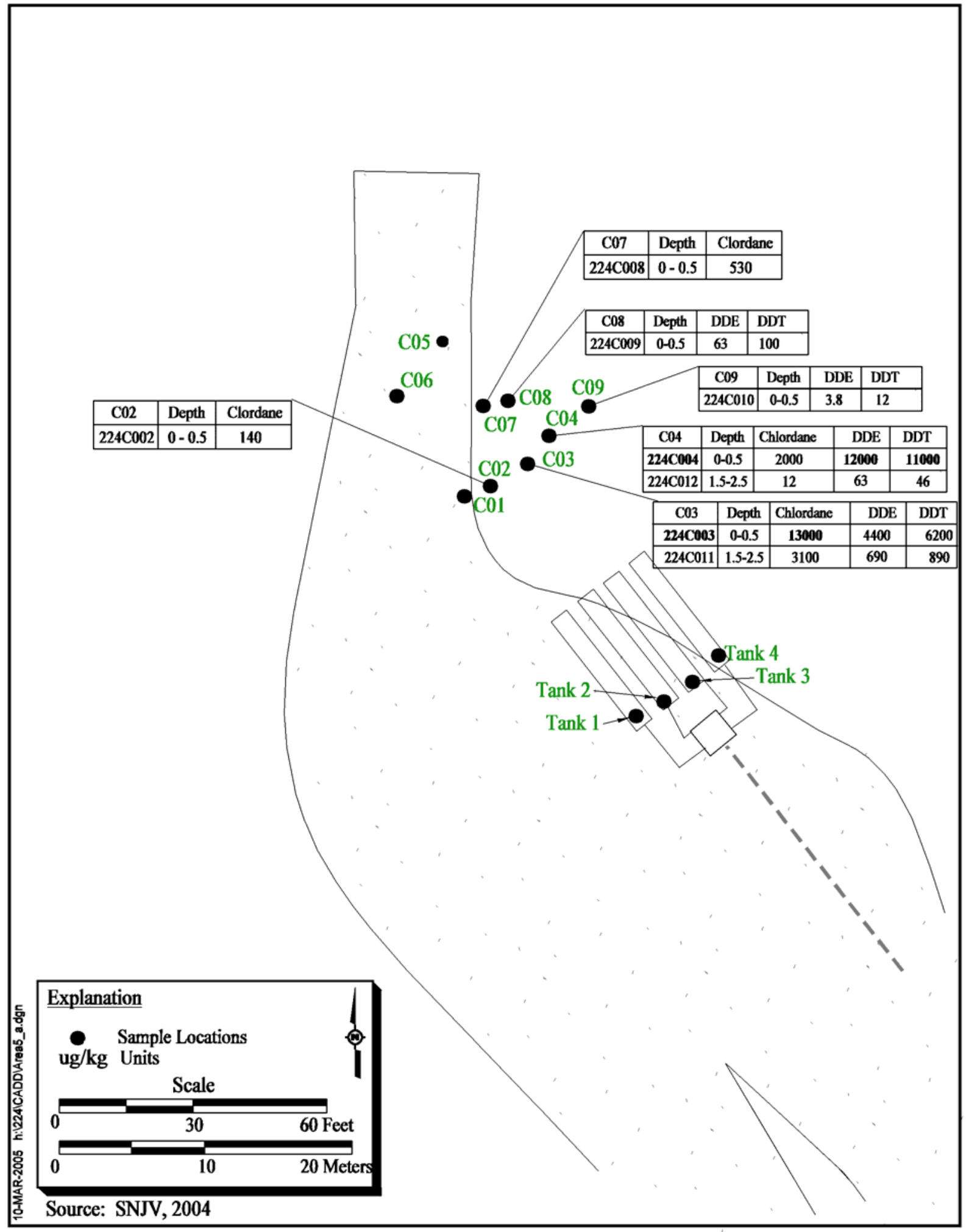

Figure A.5-1

Sample Location Map, CAS 05-04-01, Septic Tanks (4)/Discharge Area 


\section{A.5.1.3 Deviations}

There were no significant deviations to the CAIP requirements at this CAS. The locations of the surface samples in the discharge area were moved slightly from the drawings in the CAIP in order to achieve a more representative characterization of the area.

\section{A.5.2 Investigation Results}

The following sections provide descriptions of the CAS-specific activities conducted to complete investigation activities as outlined in the CAIP. Investigation activities included visual inspection of the CAS and collection of Decision I and Decision II samples.

No obvious release of contaminants was observed at the CAS during the visual inspection. Sampling included the collection of liquid, sludge, and soil samples as outlined in Section A.5.1.2.

Tank liquid and surface soil samples were analyzed for the CAIP-specified COPCs, which included total VOCs, total SVOCs, TPH-DRO, total metals, PCBs, gamma-emitting radionuclides, plutonium isotopes, uranium isotopes, and strontium-90. In accordance with the ROTC CAIP-1, 25 percent of all samples were analyzed for radiological COPCs. Sludge samples were analyzed for TCLP VOCs, TCLP SVOCs, and TCLP RCRA metals. The analytical parameters and laboratory methods used to analyze the investigation samples are listed in Table A.2-2. Table A.5-1 lists the sample-specific analytical suite for CAS 05-04-01.

\section{A.5.2.1 Total Volatile Organic Compounds}

No VOCs were detected in soil samples above reporting limits.

\section{A.5.2.2 Total Semivolatile Organic Compounds}

No SVOCs were detected in soil samples above reporting limits. 


\section{A.5.2.3 Total Metals}

Total metals (including RCRA metals plus aluminum, antimony, beryllium, cobalt, copper, manganese, molybdenum, nickel, and zinc) analytical results for soil samples collected at CAS 05-04-01 are presented in Table A.5-2. No metals were detected above FALs.

\section{A.5.2.4 Polychlorinated Biphenyls}

No PCBs were detected in soil samples above reporting limits.

\section{A.5.2.5 Total Petroleum Hydrocarbon-Diesel-Range Organics}

No TPH-DRO was detected in soil samples above reporting limits.

\section{A.5.2.6 Gamma-Emitting Radionuclides}

Gamma-emitting radionuclide analytical results for soil samples collected at CAS 05-04-01 that were detected above MDCs are presented in Table A.5-3. No gamma-emitting radionuclides were detected above FALs.

\section{A.5.2.7 Isotopes}

Isotope analytical results for soil samples collected at CAS 05-04-01 detected above MDCs are presented in Table A.5-4. No isotopes were detected above FALs.

\section{A.5.2.8 Pesticides}

Pesticide analysis was added to the analytical suite at CAS 05-04-01, due to a number of tentatively identified pesticide compounds during the PCB analysis. Pesticide analytical results for soil samples collected at CAS 05-04-01 detected above MDCs are presented in Table A.5-4. Two soil samples contained pesticides above the FALs. 
Table A.5-2

Soil Sample Results for Metals Detected Above Minimum Reporting Limits at CAS 05-04-01, Septic Tanks (4)/Discharge Area

\begin{tabular}{|c|c|c|c|c|c|c|c|c|c|c|c|c|c|}
\hline \multirow[b]{2}{*}{$\begin{array}{l}\text { Sample } \\
\text { Location }\end{array}$} & \multirow[b]{2}{*}{$\begin{array}{l}\text { Sample } \\
\text { Number }\end{array}$} & \multirow[b]{2}{*}{$\begin{array}{l}\text { Depth } \\
\text { (ft bgs) }\end{array}$} & \multicolumn{11}{|c|}{ Contaminants of Potential Concern $(\mathrm{mg} / \mathrm{kg})$} \\
\hline & & & 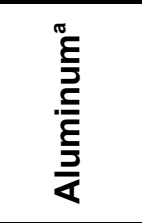 & $\begin{array}{l}\stackrel{0}{0} \\
\frac{0}{c} \\
\frac{0}{0} \\
\frac{0}{4}\end{array}$ & 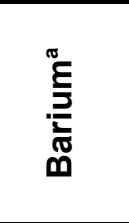 & 疍 & 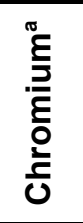 & $\begin{array}{l}\frac{\pi}{\pi} \\
\frac{0}{0} \\
0 \\
0\end{array}$ & $\begin{array}{l}\frac{\pi}{2} \\
\frac{0}{0} \\
\frac{0}{0} \\
0\end{array}$ & ర్ల & 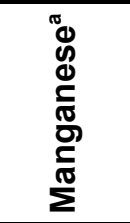 & $\begin{array}{l}\frac{\sigma}{\Phi} \\
\frac{v}{0} \\
\frac{v}{Z}\end{array}$ & $\stackrel{0}{\stackrel{0}{c}}$ \\
\hline \multicolumn{3}{|c|}{ Final Action Levels } & 100,000 & 23 & 67,000 & 1,900 & 450 & 1,900 & 41,000 & 750 & 19,000 & 20,000 & 100,000 \\
\hline$\overline{\mathrm{C} 01}$ & $224 \mathrm{C} 001$ & $0-0.5$ & 9,700 & 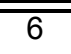 & $\overline{110}$ & $0.55(\mathrm{~J})$ & $\overline{77.6}$ & 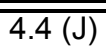 & 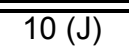 & 8.9 & $3 \overline{310(\mathrm{~J})}$ & $\overline{77.7}$ & $32(\mathrm{~J})$ \\
\hline $\mathrm{C02}$ & $224 \mathrm{C} 002$ & $0-0.5$ & 7,500 & 4.4 & 76 & -- & 5.6 & $3.1(\mathrm{~J})$ & $7.6(\mathrm{~J})$ & 5.5 & $170(\mathrm{~J})$ & 5.8 & $21(\mathrm{~J})$ \\
\hline $\mathrm{C03}$ & $224 \mathrm{C003}$ & $0-0.5$ & 8,200 & 4 & 87 & -- & 6.3 & $3.3(\mathrm{~J})$ & $9.6(\mathrm{~J})$ & 8.2 & $220(\mathrm{~J})$ & 6.3 & $29(\mathrm{~J})$ \\
\hline $\mathrm{CO4}$ & $224 \mathrm{C} 004$ & $0-0.5$ & 8,700 & 4.5 & 93 & -- & 6.7 & $3.5(\mathrm{~J})$ & $9.4(\mathrm{~J})$ & 9.3 & $230(\mathrm{~J})$ & 6.7 & $27(\mathrm{~J})$ \\
\hline $\mathrm{C05}$ & $224 \mathrm{C005}$ & $0-0.5$ & 9,200 & 4.4 & 120 & $0.51(\mathrm{~J})$ & 7.3 & $3.9(\mathrm{~J})$ & $9.6(\mathrm{~J})$ & 7.4 & $290(\mathrm{~J})$ & 7.3 & $30(\mathrm{~J})$ \\
\hline \multirow{2}{*}{$\mathrm{C} 06$} & $224 \mathrm{C} 006$ & $0-0.5$ & 10,000 & 4.6 & 120 & $0.54(\mathrm{~J})$ & 7.9 & $4.1(\mathrm{~J})$ & $9.9(\mathrm{~J})$ & 9.3 & $350(\mathrm{~J})$ & 7.8 & $34(\mathrm{~J})$ \\
\hline & $224 \mathrm{C} 007$ & $0-0.5$ & 9,700 & 4.2 & 120 & $0.53(\mathrm{~J})$ & 7.7 & $4(\mathrm{~J})$ & $9.4(\mathrm{~J})$ & 8.7 & $340(\mathrm{~J})$ & 7.5 & $33(\mathrm{~J})$ \\
\hline
\end{tabular}

${ }^{\mathrm{a}}$ Based on U.S. Environmental Protection Agency, Region 9 Preliminary Remediation Goals (PRGs) (EPA, 2002)

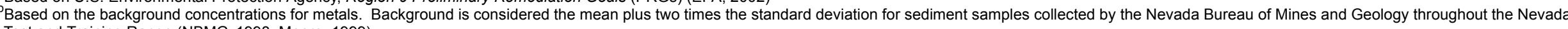
Test and Training Range (NBMG, 1998; Moore, 1999).

$\mathrm{ft}$ bgs $=$ Feet below ground surface

$\mathrm{mg} / \mathrm{kg}=$ Milligrams per kilogram

orting limits.

Qualfier added to laboratory data; record accepted. Serial dilution \%D outside control limits.

Matrix effects may exist. 
Table A.5-3

\section{Soil Sample Results for Gamma-Emitting Radionuclides Detected Above Minimum Detectable Concentrations at CAS 05-04-01, Septic Tanks (4)/Discharge Area}

\begin{tabular}{||c|c|c|c|c|c|c|c||}
\hline \multirow{2}{*}{$\begin{array}{c}\text { Sample } \\
\text { Location }\end{array}$} & \multirow{2}{*}{$\begin{array}{c}\text { Sample } \\
\text { Number }\end{array}$} & $\begin{array}{c}\text { Depth } \\
\text { (ft bgs) }\end{array}$ & \multicolumn{5}{|c|}{ Contaminants of Potential Concern (pCi/g) } \\
\cline { 3 - 8 } & & Actinium-228 & Bismuth-214 & Lead-212 & Lead-214 & Thallium-208 \\
\hline \multicolumn{3}{|c|}{ Final Action Levels ${ }^{\mathrm{a}}$} & $\mathbf{5}$ & $\mathbf{5}$ & $\mathbf{5}$ & $\mathbf{5}$ & $\mathbf{5}$ \\
\hline \multicolumn{3}{|c|}{ Depth bgs (cm) } & $<15$ & $<15$ & $<15$ & $<15$ & $<15$ \\
\hline \hline C02 & $224 C 002$ & $0-0.5$ & -- & $0.52(\mathrm{G}, \mathrm{J})$ & $0.43(\mathrm{~J})^{\mathrm{b}}$ & $0.73(\mathrm{G}, \mathrm{J})$ & $0.192(\mathrm{G})$ \\
\hline \multirow{2}{*}{$\mathrm{C} 06$} & $224 \mathrm{C} 006$ & $0-0.5$ & $0.96(\mathrm{G})$ & $0.98(\mathrm{G}, \mathrm{J})$ & $1.12(\mathrm{~J})^{\mathrm{b}}$ & $0.82(\mathrm{G}, \mathrm{J})$ & $0.31(\mathrm{G})$ \\
\cline { 2 - 8 } & $224 \mathrm{C} 007$ & $0-0.5$ & $1.05(\mathrm{G})$ & $0.78(\mathrm{G}, \mathrm{J})$ & $1.12(\mathrm{~J})^{\mathrm{b}}$ & $0.97(\mathrm{G}, \mathrm{J})$ & $0.223(\mathrm{G})$ \\
\hline
\end{tabular}

atTaken from the generic guidelines for residual concentrations of actinium-228, bismuth-214, lead-212, lead-214, thallium-208, and thorium-232, as found in Chapter IV of DOE Order 5400.5, Change 2, "Radiation Protection of the Public and Environment." (DOE, 1993). The FALs for these isotopes is specified as $5 \mathrm{pCi} / \mathrm{g}$ averaged over the first $15 \mathrm{~cm}$ of soil and $15 \mathrm{pCi} / \mathrm{g}$ for deeper soils (DOE, 1993). For purposes of this document, $15 \mathrm{~cm}$ is assumed to be equivalent to $0.5 \mathrm{ft}$ ( $6 \mathrm{in}$.); therefore, $5 \mathrm{pCi} / \mathrm{g}$ represents the FALs for these radionuclides in the surface soil ( 0 to $0.5 \mathrm{ft}$ depth).

${ }^{b}$ Qualifier added to laboratory data; record accepted. Sample does not meet counting geometry requirements.

$\mathrm{ft}$ bgs $=$ Feet below ground surface

$\mathrm{cm}=$ Centimeter

$\mathrm{pCi} / \mathrm{g}=$ Picocuries per gram

$--=$ Not detected above minimum reporting limits

$<=$ Less than

$\mathrm{G}=$ Sample density differs by more than 15 percent of laboratory control sample density.

$\mathrm{J}=$ Estimated value.

Table A.5-4

Soil Sample Results for Isotopes Detected Above Minimum Detectable Concentrations at CAS 05-04-01, Septic Tanks (4)/Discharge Area

\begin{tabular}{||c|c|c|c|c|c|c||}
\hline \multirow{2}{*}{$\begin{array}{c}\text { Sample } \\
\text { Location }\end{array}$} & \multirow{2}{*}{$\begin{array}{c}\text { Sample } \\
\text { Number }\end{array}$} & \multirow{2}{*}{$\begin{array}{c}\text { Depth } \\
\text { (ft bgs) }\end{array}$} & \multicolumn{4}{|c|}{ Contaminants of Potential Concern (pCi/g) } \\
\cline { 5 - 7 } & & & Plutonium-238 & Plutonium-239 & Uranium-234 & Uranium-238 \\
\hline \multicolumn{3}{|c|}{ Final Action Levels ${ }^{\mathrm{a}}$} & $\mathbf{1 3}$ & $\mathbf{1 2 . 7}$ & $\mathbf{1 4 3}$ & $\mathbf{1 0 5}$ \\
\hline \hline $\mathrm{C} 02$ & $224 \mathrm{C} 002$ & $0-0.5$ & -- & 0.265 & 0.413 & 0.39 \\
\hline \multirow{2}{*}{$\mathrm{C} 06$} & $224 \mathrm{C} 006$ & $0-0.5$ & 0.056 & 3.32 & 0.55 & 0.49 \\
\cline { 2 - 7 } & $224 \mathrm{C} 007$ & $0-0.5$ & -- & 0.129 & 0.62 & 0.53 \\
\hline
\end{tabular}

aTaken from the construction, commercial, industrial land use scenario in Table 2.1 of the NCRP Report No. 129 Recommended Screening Limits for Contaminated Surface Soil and Review Factors Relevant to Site-Specific Studies (NCRP, 1999). The values provided in this source document were scaled to a 25 -mrem/yr dose.

$\mathrm{ft}$ bgs $=$ Feet below ground surface

$\mathrm{mrem} / \mathrm{yr}=$ Millirems per year

$\mathrm{pCi} / \mathrm{g}=$ Picocuries per gram

$--=$ Not detected above minimum reporting limits 
Table A.5-5

Soil Sample Results for Pesticides Detected Above Minimum Reporting Limits at CAS 05-04-01, Septic Tanks (4)/Discharge Area

\begin{tabular}{|c|c|c|c|c|c|c|c|c|c|c|}
\hline \multirow{2}{*}{$\begin{array}{c}\text { Sample } \\
\text { Location }\end{array}$} & \multirow{2}{*}{$\begin{array}{l}\text { Sample } \\
\text { Number }\end{array}$} & \multirow{2}{*}{$\begin{array}{c}\text { Depth } \\
\text { (ft bgs) }\end{array}$} & \multicolumn{8}{|c|}{ Contaminants of Potential Concern $(\mu \mathrm{g} / \mathrm{kg})$} \\
\hline & & & 4,4'-DDD & 4,4'-DDE & 4,4'-DDT & Alpha-Chlordane & Chlordane & Dieldrin & Endosulfan II & Gamma-Chlordane \\
\hline \multicolumn{3}{|c|}{ Final Action Levels ${ }^{a}$} & 10,000 & 7,000 & 7,000 & NI & 6,500 & 110 & $3,700,000$ & NI \\
\hline 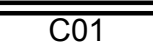 & $224 \mathrm{C001}$ & $0-0.5$ & $180(\mathrm{~J})^{\mathrm{b}}$ & 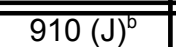 & 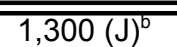 & $\overline{---}$ & 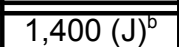 & $\overline{---}$ & $\overline{---}$ & $\overline{---}$ \\
\hline $\mathrm{CO2}$ & $224 \mathrm{C002}$ & $0-0.5$ & $78(J)^{b}$ & $310(J)^{b}$ & $470(\mathrm{~J})^{\mathrm{b}}$ & -- & 140 & -- & -- & -- \\
\hline \multirow{2}{*}{$\mathrm{C03}$} & $224 \mathrm{C} 003$ & $0-0.5$ & $1,200(\mathrm{~J})^{\mathrm{b}}$ & $4,400(J)^{b}$ & $6,200(J)^{b}$ & $2,000(J)^{c}$ & $13,000(J)^{b}$ & -- & - & $2,600(J)^{b}$ \\
\hline & $224 \mathrm{C} 011$ & $1.5-2.5$ & $350(\mathrm{~J})^{\mathrm{c}}$ & $690(J)^{b}$ & $890(J)^{b}$ & $430(J)^{b}$ & $3,100(J)^{b}$ & -- & -- & $460(\mathrm{~J})^{\mathrm{b}}$ \\
\hline \multirow{2}{*}{$\mathrm{CO} 4$} & $224 \mathrm{C004}$ & $0-0.5$ & -- & $12,000(\mathrm{~J})^{\mathrm{b}}$ & $11,000(\mathrm{~J})^{\mathrm{b}}$ & $\overline{--}$ & $2,000(J)^{b}$ & $\overline{--}$ & -- & -- \\
\hline & $224 \mathrm{C} 012$ & $1.5-2.5$ & 12 & 63 & 46 & -- & -- & -- & -- & -- \\
\hline $\mathrm{C05}$ & $224 \mathrm{C} 005$ & $0-0.5$ & -- & $540(\mathrm{~J})^{\mathrm{b}}$ & $500(J)^{b}$ & -- & 290 & -- & -- & -- \\
\hline \multirow{2}{*}{$\mathrm{C} 06$} & $224 \mathrm{C} 006$ & $0-0.5$ & -- & 6.7 & $6.3(J)^{d}$ & -- & -- & -- & -- & -- \\
\hline & $224 \mathrm{C} 007$ & $0-0.5$ & -- & 6.4 & $5.8(J)^{d}$ & -- & -- & -- & -- & -- \\
\hline $\mathrm{C07}$ & $224 \mathrm{C} 008$ & $0-0.5$ & $29(J)^{\mathrm{e}}$ & $190(\mathrm{~J})^{\mathrm{b}}$ & $88(J)^{c}$ & $74(\mathrm{~J})^{\mathrm{b}}$ & 530 & $4.4(J)^{\mathrm{C}}$ & $2.5(\mathrm{~J})^{\mathrm{C}}$ & $86(J)^{b}$ \\
\hline $\mathrm{C08}$ & $224 C 009$ & $0-0.5$ & $20(J)^{f}$ & $63(J)^{f}$ & $100(\mathrm{~J})^{f}$ & -- & $55(J)^{c}$ & -- & -- & -- \\
\hline C09 & $224 \mathrm{C} 010$ & $0-0.5$ & 2.4 & 3.8 & $12(\mathrm{~J})^{\mathrm{c}}$ & -- & -- & -- & -- & -- \\
\hline
\end{tabular}

${ }^{a}$ Based on U.S. Environmental Protection Agency, Region 9 Preliminary Remediation Goals (PRGs) (EPA, 2002)

Qualifier added to laboratory data; record accepted. Surrogates diluted out.

${ }^{c} Q u a l i f i e r$ added to laboratory data; record accepted. \%D between columns $>25$. Surrogates diluted out.

${ }^{d}$ Qualifier added to laboratory data; record accepted. \%D between columns $>25$.

${ }^{\text {Q }}$ Qualifier added to laboratory data; record accepted. Value exceeded linear/calibration range of instrument. The reported value is from the dilution run. \%D between columns $>25$.

Qualifier added to laboratory data; record accepted. Surrogate recovery exceeded the upper limits.

DDD = Dichlorodiphenyl-dichloroethane

$\mathrm{DDE}=$ Dichlorodiphenyl-dichloroethylen

DDT = Dichlorodiphenyl-trichloroethane

$\mathrm{ft}$ bgs $=$ Feet below ground surface

$\mu \mathrm{g} / \mathrm{kg}=$ Micrograms per kilogram

$\mathrm{J}=$ Estimated value.

Not detect above minimum reporting limits.

$\mathrm{NI}=$ Not identified 


\section{A.5.2.9 Liquid and Sludge Sample Results}

Analytical results for the septic tank liquid samples collected at CAS 05-04-01 that were detected above MRLs or MDCs are presented in Table A.5-6. No analytes were detected in the sludge samples above MRLs. No COCs were detected in the samples.

Table A.5-6

Liquid Sample Results Detected Above Minimum Reporting Limits at CAS 05-04-01, Septic Tanks (4)/Discharge Area

\begin{tabular}{|c|c|c|c|c|}
\hline Sample Number & Sample Matrix & Parameter & Result & Units \\
\hline \hline $224 \mathrm{C502}$ & Liquid & Aluminum & 0.22 & $\mathrm{mg} / \mathrm{L}$ \\
\hline $224 \mathrm{C502}$ & Liquid & Manganese & 0.2 & $\mathrm{mg} / \mathrm{L}$ \\
\hline $224 \mathrm{C503}$ & Liquid & Aluminum & 0.53 & $\mathrm{mg} / \mathrm{L}$ \\
\hline $224 \mathrm{C501}$ & Liquid & Uranium-234 & 0.152 & $\mathrm{pCi} / \mathrm{L}$ \\
\hline $224 \mathrm{C502}$ & Liquid & Uranium-234 & 0.127 & $\mathrm{pCi} / \mathrm{L}$ \\
\hline $224 \mathrm{C502}$ & Liquid & Uranium-238 & 0.103 & $\mathrm{pCi} / \mathrm{L}$ \\
\hline $224 \mathrm{C503}$ & Liquid & Uranium-234 & 0.37 & $\mathrm{pCi} / \mathrm{L}$ \\
\hline $224 \mathrm{C503}$ & Liquid & Uranium-238 & 0.166 & $\mathrm{pCi} / \mathrm{L}$ \\
\hline
\end{tabular}

$\mathrm{mg} / \mathrm{L}=$ Milligrams per liter $\mathrm{pCi} / \mathrm{L}=$ Picocuries per liter

\section{A.5.3 Nature and Extent of Contamination}

Two surface samples were found to contain pesticides above the FALs. The extent of the pesticide contamination was bounded during Decision II sampling. See Figure B.1-1 for a graphical representation of the extent of contamination at CAS 05-04-01.

\section{A.5.4 Revised Conceptual Site Model}

No variations in the CSM were identified. 


\section{A.6.0 CAS 06-03-01, Sewage Lagoons (3)}

Corrective Action Site 06-03-01 is located on the Yucca Lake bed in Area 6 of the NTS. The CAS consists of the former Yucca Lake sewage lagoon systems, including Sewage Lagoons I and II and the Domestic Lagoons. Investigation activities at CAS 06-03-01 included collection of surface and subsurface soil samples.

\section{A.6.1 Corrective Action Investigation}

A total of 50 soil characterization samples were collected during investigation activities at CAS 06-03-01. The sample IDs, locations, types, and analyses are listed in Table A.6-1. The sample locations are shown on Figure A.6-1. The specific CAI activities conducted to satisfy the CAIP requirements at this CAS are described in the following sections.

\section{A.6.1.1 Field Screening}

Soil samples were field screened for VOCs, TPH, and alpha and beta/gamma radiation. The FSRs were compared to FSLs to guide subsequent sampling decisions. The VOC headspace, TPH, and alpha and beta/gamma radiation FSLs were not exceeded during sampling activities at this CAS.

\section{A.6.1.2 Sampling}

Surface and subsurface samples were collected at locations specified in the CAIP. The locations were chosen to be associated with features (i.e., piping, distribution box) of the former lagoons, according to engineering drawings. However, during the investigation none of the features were found to still exist, leading to the assumption that the features were removed when the lagoons were closed. Samples were collected at the surface and at two subsurface intervals at each location. The samples were analyzed for the parameters listed in Table A.6-1. Sample locations are shown on Figure A.6-1.

\section{A.6.1.3 Deviations}

There were no significant deviations to the CAIP requirements at this CAS. No samples were associated with piping, manholes, or distribution boxes, because none of these features could be located. The manhole indicated in Figure A.6-1 was found to be part of the active Yucca Lake 
Table A.6-1

Samples Collected at CAS 06-03-01, Sewage Lagoons (3) (Page 1 of 2)

\begin{tabular}{|c|c|c|c|c|c|}
\hline $\begin{array}{l}\text { Sample } \\
\text { Location }\end{array}$ & $\begin{array}{l}\text { Sample } \\
\text { Number }\end{array}$ & $\begin{array}{c}\text { Depth } \\
\text { (ft bgs) }\end{array}$ & $\begin{array}{l}\text { Sample } \\
\text { Matrix }\end{array}$ & Purpose & Analyses \\
\hline \multirow{3}{*}{ D01 } & $224 \mathrm{D001}$ & $0-0.5$ & Soil & SC & Set 2 \\
\hline & $224 \mathrm{D} 007$ & $2-3$ & Soil & SC & Set 2 \\
\hline & $224 D 008$ & $4-5$ & Soil & SC & Set 1 \\
\hline \multirow{2}{*}{ D02 } & $224 \mathrm{D} 002$ & $0-0.5$ & Soil & SC & Set 1 \\
\hline & 224D017 & $4-5$ & Soil & SC & Set 1 \\
\hline \multirow{2}{*}{ D03 } & $224 D 003$ & $0-0.5$ & Soil & $\mathrm{SC}, \mathrm{MS} / \mathrm{MSD}$ & Set 1 \\
\hline & $224 D 015$ & $4-5$ & Soil & SC & Set 2 \\
\hline \multirow{3}{*}{ D04 } & $224 D 004$ & $0-0.5$ & Soil & SC & Set 2 \\
\hline & 224D005 & $0-0.5$ & Soil & $\begin{array}{c}\text { Field Duplicate } \\
\text { of \#224D004 }\end{array}$ & Set 2 \\
\hline & $224 D 013$ & $4-5$ & Soil & SC & Set 2 \\
\hline \multirow{3}{*}{ D05 } & $224 D 006$ & $0-0.5$ & Soil & SC & Set 2 \\
\hline & 224D009 & $2-3$ & Soil & SC & Set 2 \\
\hline & $224 \mathrm{D} 011$ & $5-6$ & Soil & SC & Set 1 \\
\hline \multirow{2}{*}{ D06 } & 224D019 & $0-0.5$ & Soil & SC & Set 1 \\
\hline & 224D020 & $4-5$ & Soil & SC & Set 2 \\
\hline \multirow{3}{*}{ D07 } & $224 D 022$ & $0-0.5$ & Soil & SC & Set 2 , Herbicide \\
\hline & 224D023 & $0-0.5$ & Soil & $\begin{array}{c}\text { Field Duplicate } \\
\text { of \#224D022 }\end{array}$ & Set 2 \\
\hline & $224 D 024$ & $3-4$ & Soil & $\mathrm{SC}, \mathrm{MS} / \mathrm{MSD}$ & Set 1 \\
\hline \multirow{2}{*}{ D08 } & $224 D 026$ & $0-0.5$ & Soil & SC & Set 2 \\
\hline & 224D034 & $4-5$ & Soil & SC & Set 2 \\
\hline \multirow{2}{*}{ D09 } & $224 \mathrm{D} 027$ & $0-0.5$ & Soil & SC & Set 2 \\
\hline & $224 D 036$ & $4-5$ & Soil & SC & Set 1 \\
\hline \multirow{2}{*}{ D10 } & 224D028 & $0-0.5$ & Soil & SC & Set 2 \\
\hline & 224D038 & $4-5$ & Soil & SC & Set 2 \\
\hline \multirow{2}{*}{ D11 } & $224 \mathrm{D} 029$ & $0-0.5$ & Soil & SC & Set 2 \\
\hline & $224 D 040$ & $4-5$ & Soil & SC, MS/MSD & Set 1 \\
\hline \multirow{2}{*}{ D12 } & 224D030 & $0-0.5$ & Soil & SC & Set 2 \\
\hline & $224 \mathrm{D} 042$ & $4-5$ & Soil & SC & Set 2 \\
\hline
\end{tabular}


Table A.6-1

Samples Collected at CAS 06-03-01, Sewage Lagoons (3) (Page 2 of 2)

\begin{tabular}{|c|c|c|c|c|c|}
\hline $\begin{array}{l}\text { Sample } \\
\text { Location }\end{array}$ & $\begin{array}{l}\text { Sample } \\
\text { Number }\end{array}$ & $\begin{array}{l}\text { Depth } \\
\text { (ft bgs) }\end{array}$ & $\begin{array}{l}\text { Sample } \\
\text { Matrix }\end{array}$ & Purpose & Analyses \\
\hline \multirow{3}{*}{ D13 } & $224 \mathrm{D031}$ & $0-0.5$ & Soil & SC & Set 1 \\
\hline & $224 \mathrm{D} 044$ & $4-5$ & Soil & SC & Set 1 \\
\hline & 224D045 & $4-5$ & Soil & $\begin{array}{c}\text { Field Duplicate } \\
\text { of \#224D022 }\end{array}$ & Set 1 \\
\hline \multirow{2}{*}{ D14 } & $224 \mathrm{D} 032$ & $0-0.5$ & Soil & $\mathrm{SC}$ & Set 2 \\
\hline & $224 \mathrm{D} 047$ & $4-5$ & Soil & $\mathrm{SC}$ & Set 2 \\
\hline \multirow{2}{*}{ D15 } & $224 \mathrm{D} 033$ & $0-0.5$ & Soil & SC & Set 2 \\
\hline & $224 \mathrm{D} 049$ & $4-5$ & Soil & SC & Set 1 \\
\hline NA & $224 \mathrm{D} 301$ & NA & Water & Trip Blank & Total VOCs \\
\hline NA & $224 \mathrm{D} 302$ & NA & Water & Field Blank & Set 1 \\
\hline NA & $224 \mathrm{D} 303$ & NA & Water & Trip Blank & Total VOCs \\
\hline NA & $224 \mathrm{D} 304$ & NA & Water & Trip Blank & Total VOCs \\
\hline NA & $224 \mathrm{D} 305$ & NA & Water & Trip Blank & Total VOCs \\
\hline NA & $224 \mathrm{D} 306$ & NA & Water & Trip Blank & Total VOCs \\
\hline NA & $224 \mathrm{D} 307$ & NA & Water & Trip Blank & Total VOCs \\
\hline
\end{tabular}

Note: Certain sample numbers are missing. This is due to the fact that the samples they represented were not shipped to the laboratory according to the specifications in the CAIP.

Set 1 = Total VOCs, Total SVOCs, Total RCRA Metals, Aluminum, Antimony, Beryllium, Cobalt, Copper, Manganese, Molybdenum, Nickel, Zinc, TPH-DRO, PCBs, Cyanide, Gamma Spectroscopy, Isotopic Plutonium, Isotopic Uranium, and Strontium-90.

Set 2 = Total VOCs, Total SVOCs, Total RCRA Metals, Aluminum, Antimony, Beryllium, Cobalt, Copper, Manganese, Molybdenum, Nickel, Zinc, TPH-DRO, PCBs and Cyanide.

CAIP = Corrective action investigation plan

$\mathrm{DRO}=$ Diesel-range organics

$\mathrm{ft}$ bgs $=$ Feet below ground surface

PCB = Polychlorinated biphenyl

RCRA = Resource Conservation and Recovery Act

$\mathrm{SC}=$ Site characterization
$\mathrm{SC}=$ Site characterization

SVOC = Semivolatile organic compound

$\mathrm{TPH}=$ Total petroleum hydrocarbons

WM $=$ Waste Management

MS/MSD = Matrix spike/matrix spike duplicate

$\mathrm{NA}=$ Not applicable

VOC $=$ Volatile organic compound 


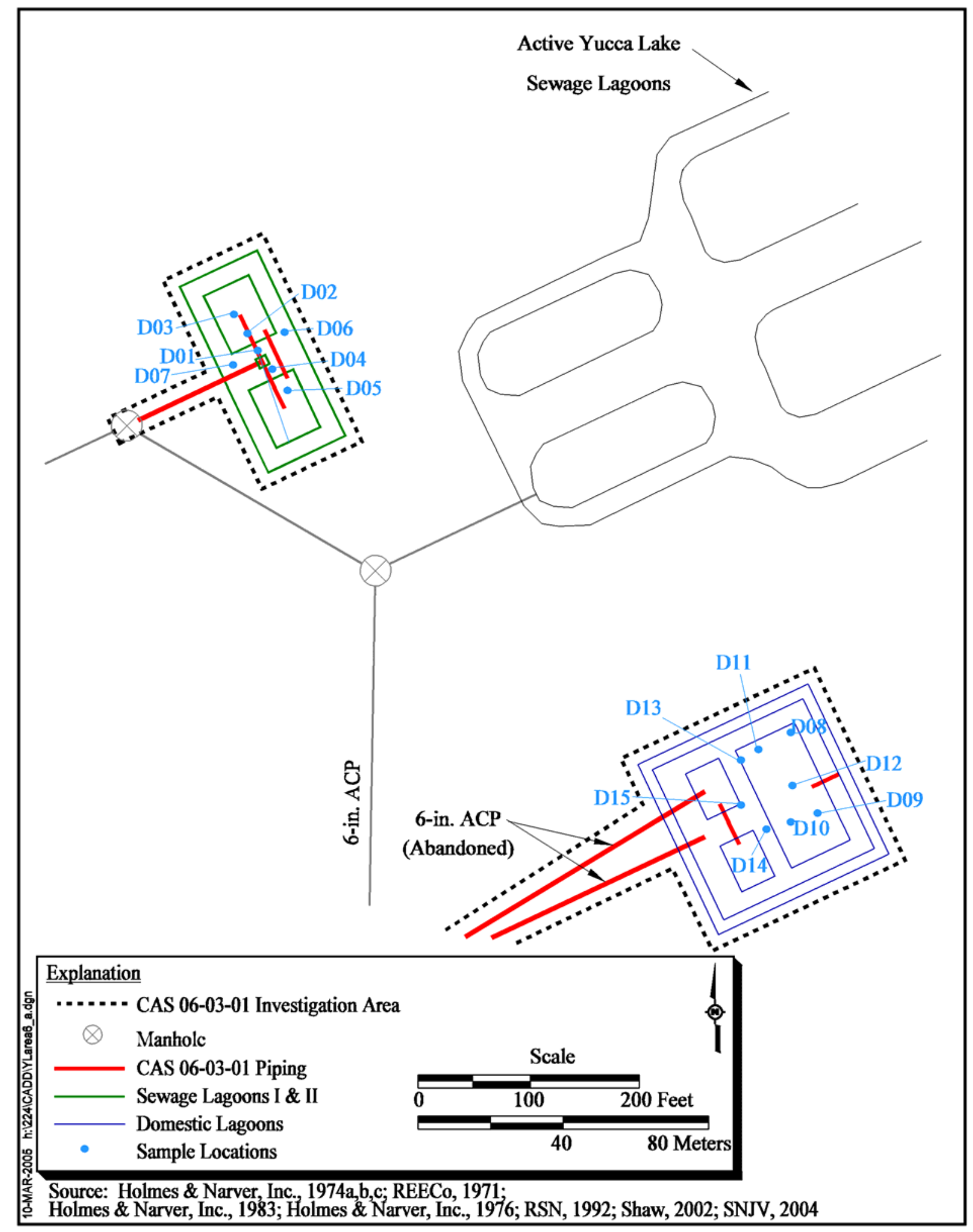

Figure A.6-1

Sample Location Map, CAS 06-03-01, Sewage Lagoons (3) 
Sewage Lagoon System. Therefore, it was inaccessible and it was not involved in the CAI. The investigation and sampling at CAS 06-03-01 are considered sufficient to meet the DQOs.

\section{A.6.2 Investigation Results}

The following sections provide descriptions of the CAS-specific activities conducted to complete investigation activities as outlined in the CAIP. Investigation activities included visual inspection of the CAS and collection of surface and subsurface soil samples, as outlined in Section A.6.1.2.

Surface and subsurface soil samples were analyzed for the CAIP-specified COPCs, which included total VOCs, total SVOCs, TPH-DRO, total metals, PCBs, cyanide, gamma-emitting radionuclides, plutonium isotopes, uranium isotopes, and strontium-90. In accordance with the ROTC CAIP-1, 25 percent of all samples were analyzed for radiological COPCs. The analytical parameters and laboratory methods used to analyze the investigation samples are listed in Table A.2-2. Table A.6-1 lists the sample-specific analytical suite for CAS 06-03-01. A portion of the analytical results for this CAS was rejected during validation; however, these rejected data did not adversely impact closure decisions as discussed in Appendix B, Section B.1.4.

\section{A.6.2.1 Total Volatile Organic Compounds}

Total VOC analytical results for soil samples collected at CAS 06-03-01 that were detected above MRLs are presented in Table A.6-2. No VOCs were detected in soil samples above FALs.

Table A.6-2

Soil Sample Results for Total VOCs Detected Above Minimum Reporting Limits at CAS 06-03-01, Sewage Lagoons (3)

\begin{tabular}{|c|c|c|c|}
\hline \multirow{2}{*}{$\begin{array}{l}\text { Sample } \\
\text { Location }\end{array}$} & \multirow{2}{*}{$\begin{array}{l}\text { Sample } \\
\text { Number }\end{array}$} & \multirow{2}{*}{$\begin{array}{c}\text { Depth } \\
\text { (ft bgs) }\end{array}$} & \multirow{2}{*}{$\begin{array}{c}\text { Contaminants of Potential Concern }(\mu \mathrm{g} / \mathrm{kg}) \\
\text { Methylene Chloride }\end{array}$} \\
\hline & & & \\
\hline \multicolumn{3}{|c|}{ Final Action Levels ${ }^{a}$} & 21,000 \\
\hline \multirow{2}{*}{ D04 } & 224D004 & $0-0.5$ & $\overline{\overline{6.4}}$ \\
\hline & $224 \mathrm{D} 005$ & $0-0.5$ & 11 \\
\hline D05 & 224D006 & $0-0.5$ & 11 \\
\hline
\end{tabular}

${ }^{a}$ Based on U.S. Environmental Protection Agency, Region 9 Preliminary Remediation Goals (PRGs) (EPA, 2002)

$\mathrm{ft}$ bgs $=$ Feet below ground surface $\mu \mathrm{g} / \mathrm{kg}=$ Micrograms per kilogram 


\section{A.6.2.2 Total Semivolatile Organic Compounds}

No SVOCs were detected in soil samples above reporting limits.

\section{A.6.2.3 Total Metals}

Total metals analytical results for soil samples collected at CAS 06-03-01 are presented in Table A.6-3. No metals were detected above FALs.

\section{A.6.2.4 Polychlorinated Biphenyls}

No PCBs were detected in soil samples above reporting limits.

\section{A.6.2.5 Total Petroleum Hydrocarbons-Diesel-Range Organics}

No TPH-DRO was detected in soil samples above reporting limits.

\section{A.6.2.6 Gamma-Emitting Radionuclides}

Gamma-emitting radionuclide analytical results for soil samples collected at CAS 06-03-01 that were detected above MDCs are presented in Table A.6-4.

\section{A.6.2.7 Isotopes}

Isotope analytical results for soil samples collected at CAS 06-03-01 that were detected above MDCs are presented in Table A.6-5.

\section{A.6.2.8 Cyanide}

Cyanide analytical results for soil samples collected at CAS 06-03-01 that were detected above MRLs are presented in Table A.6-6.

\section{A.6.2.9 Herbicides}

Herbicide analysis was added to the analytical suite for sample 224D022 at the recommendation of the laboratory. No herbicides were detected in soil samples above reporting limits. 
Table A.6-3

Soil Sample Results for Metals Detected Above Minimum Reporting Limits at CAS 06-03-01, Sewage Lagoons (3) (Page 1 of 2)

\begin{tabular}{|c|c|c|c|c|c|c|c|c|c|c|c|c|c|c|}
\hline \multirow[b]{2}{*}{$\begin{array}{l}\text { Sample } \\
\text { Location }\end{array}$} & \multirow[b]{2}{*}{$\begin{array}{l}\text { Sample } \\
\text { Number }\end{array}$} & \multirow[b]{2}{*}{$\begin{array}{c}\text { Depth } \\
\text { (ft bgs) }\end{array}$} & \multicolumn{12}{|c|}{ Contaminants of Potential Concern $(\mathrm{mg} / \mathrm{kg})$} \\
\hline & & & 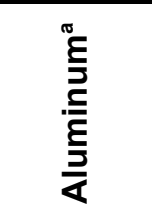 & 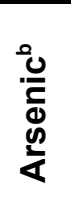 & 占 & & 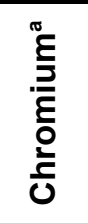 & $\begin{array}{l}\frac{\pi}{\frac{\pi}{\pi}} \\
\frac{0}{0} \\
0 \\
0\end{array}$ & $\begin{array}{l}\frac{0}{0} \\
\frac{0}{0} \\
\frac{0}{0}\end{array}$ & స్రం & 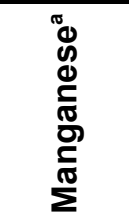 & $\begin{array}{l}\frac{\pi}{0} \\
\frac{x}{0} \\
\frac{0}{Z}\end{array}$ & 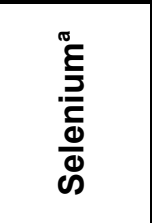 & $\stackrel{0}{\underline{N}}$ \\
\hline \multicolumn{3}{|c|}{ Final Action Levels } & 100,000 & 23 & 67,000 & 1,900 & 450 & 1,900 & 41,000 & 750 & 19,000 & 20,000 & 5,100 & 100,000 \\
\hline \multirow{3}{*}{ D01 } & 224D001 & $0-0.5$ & 25,000 & $\overline{99.1}$ & $\overline{2220}$ & 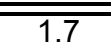 & $\overline{\overline{16}}$ & 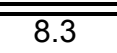 & $\overline{23}$ & $\overline{\overline{19}}$ & $\overline{570}$ & $\overline{21(\mathrm{~J})^{\mathrm{c}}}$ & $\overline{1.1(\mathrm{~J}+)}$ & $\overline{90(J)^{c}}$ \\
\hline & 224D007 & $2-3$ & 24,000 & 8.6 & 220 & 1.7 & 15 & 6.9 & 21 & 16 & 460 & 19 & -- & 82 \\
\hline & 224D008 & $4-5$ & 22,000 & 8.4 & 200 & 1.5 & 14 & 6.9 & 18 & 16 & 490 & 17 & $0.62(\mathrm{~J}+)$ & 72 \\
\hline \multirow{2}{*}{ D02 } & 224D002 & $0-0.5$ & 23,000 & 8 & 200 & 1.5 & 14 & 7 & 20 & 17 & 500 & $18(\mathrm{~J})^{\mathrm{C}}$ & $1.2(\mathrm{~J}+)$ & $80(\mathrm{~J})^{\mathrm{c}}$ \\
\hline & 224D017 & $4-5$ & 20,000 & 9 & 200 & 1.6 & 13 & 6.8 & 20 & 16 & 450 & $18(\mathrm{~J})^{\mathrm{C}}$ & -- & 77 \\
\hline \multirow{2}{*}{ D03 } & 224D003 & $0-0.5$ & 20,000 & 7.7 & 190 & 1.3 & 12 & 6.3 & 17 & 15 & 430 & $15(\mathrm{~J})^{\mathrm{c}}$ & -- & $64(J)^{c}$ \\
\hline & 224D015 & $4-5$ & 20,000 & 8.5 & 210 & 1.7 & 13 & 6.8 & 20 & 16 & 460 & $18(\mathrm{~J})^{\mathrm{c}}$ & -- & 77 \\
\hline \multirow[t]{2}{*}{ D04 } & $224 \mathrm{D} 005$ & $0-0.5$ & 21,000 & 7.9 & 220 & 1.5 & 13 & 6.4 & 19 & 15 & 430 & $17(\mathrm{~J})^{\mathrm{C}}$ & $0.65(\mathrm{~J}+)$ & $74(J)^{c}$ \\
\hline & $224 \mathrm{D} 013$ & $4-5$ & 21,000 & 8.8 & 240 & 1.7 & 14 & 7.1 & 21 & 17 & 480 & $19(\mathrm{~J})^{\mathrm{c}}$ & -- & 81 \\
\hline \multirow{3}{*}{ D05 } & 224D006 & $0-0.5$ & 24,000 & 8.4 & 210 & 1.7 & 15 & 7.1 & 21 & 17 & 480 & $20(\mathrm{~J})^{\mathrm{C}}$ & -- & $84(J)^{c}$ \\
\hline & 224D009 & $2-3$ & 22,000 & 9.5 & 220 & 1.9 & 15 & 7.7 & 24 & 18 & 520 & $21(\mathrm{~J})^{\mathrm{C}}$ & -- & 91 \\
\hline & 224D011 & $5-6$ & 18,000 & 7.9 & 190 & 1.4 & 12 & 6.2 & 17 & 14 & 410 & $16(\mathrm{~J})^{\mathrm{C}}$ & -- & 65 \\
\hline \multirow{2}{*}{ D06 } & 224D019 & $0-0.5$ & 20,000 & 8.7 & 220 & 1.7 & 14 & 7.2 & 21 & 17 & 500 & $19(\mathrm{~J})^{\mathrm{C}}$ & -- & 81 \\
\hline & $224 \mathrm{D} 020$ & $4-5$ & 19,000 & 8.2 & 190 & 1.6 & 13 & 6.5 & 19 & 15 & 430 & $17(\mathrm{~J})^{\mathrm{C}}$ & -- & 73 \\
\hline \multirow{3}{*}{ D07 } & 224D022 & $0-0.5$ & 21,000 & 8.3 & 240 & 1.7 & 15 & 7.2 & 22 & 17 & 480 & $20(J)^{c}$ & -- & 85 \\
\hline & $224 \mathrm{D} 023$ & $0-0.5$ & 21,000 & 8.4 & 240 & 1.7 & 15 & 7.2 & 22 & 17 & 490 & $20(\mathrm{~J})^{\mathrm{C}}$ & -- & 86 \\
\hline & 224D024 & $3-4$ & 14,000 & 7.6 & 150 & 1 & 9.1 & 5.3 & 12 & 13 & 400 & $11(\mathrm{~J})^{\mathrm{C}}$ & -- & 49 \\
\hline \multirow{2}{*}{ D08 } & 224D026 & $0-0.5$ & 22,000 & 9.7 & 220 & 1.8 & 15 & 7.5 & 23 & 18 & 510 & 21 & -- & $85(J)^{c}$ \\
\hline & 224D034 & $4-5$ & 19,000 & 8.4 & 220 & 1.6 & 13 & 6.9 & 21 & 16 & 480 & 19 & -- & $77(\mathrm{~J})^{\mathrm{c}}$ \\
\hline \multirow{2}{*}{ D09 } & 224D027 & $0-0.5$ & 24,000 & 9.6 & 220 & 1.9 & 16 & 8.6 & 25 & 19 & 600 & 27 & $0.93(\mathrm{~J}+)$ & $96(\mathrm{~J})^{\mathrm{C}}$ \\
\hline & $224 \mathrm{D} 036$ & $4-5$ & 21,000 & 9 & 210 & 1.7 & 15 & 7.3 & 22 & 17 & 500 & 20 & -- & $84(\mathrm{~J})^{\mathrm{c}}$ \\
\hline
\end{tabular}


Table A.6-3

Soil Sample Results for Metals Detected Above Minimum Reporting Limits at CAS 06-03-01, Sewage Lagoons (3) (Page 2 of 2)

\begin{tabular}{|c|c|c|c|c|c|c|c|c|c|c|c|c|c|c|}
\hline \multirow[b]{2}{*}{$\begin{array}{l}\text { Sample } \\
\text { Location }\end{array}$} & \multirow[b]{2}{*}{$\begin{array}{l}\text { Sample } \\
\text { Number }\end{array}$} & \multirow[b]{2}{*}{$\begin{array}{l}\text { Depth } \\
\text { (ft bgs) }\end{array}$} & \multicolumn{12}{|c|}{ Contaminants of Potential Concern (mg/kg) } \\
\hline & & & 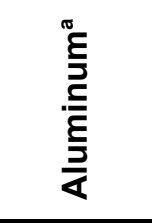 & 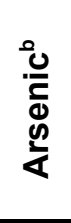 & 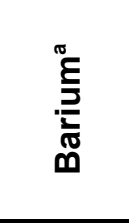 & 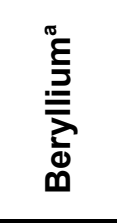 & 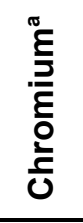 & $\begin{array}{l}\frac{\pi}{\pi} \\
\frac{0}{0} \\
0 \\
0\end{array}$ & $\begin{array}{l}\frac{0}{0} \\
\frac{0}{0} \\
\frac{0}{0} \\
0\end{array}$ & 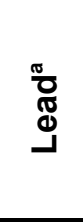 & 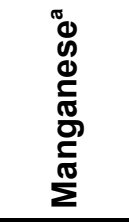 & $\begin{array}{l}\frac{\sigma}{\Phi} \\
\frac{x}{0} \\
\frac{1}{Z}\end{array}$ & 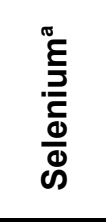 & $\stackrel{0}{\underline{N}}$ \\
\hline \multicolumn{3}{|c|}{ Final Action Levels } & 100,000 & 23 & 67,000 & 1,900 & 450 & 1,900 & 41,000 & 750 & 19,000 & 20,000 & 5,100 & 100,000 \\
\hline \multirow{2}{*}{ D10 } & 224D028 & $0-0.5$ & 20,000 & 9.4 & 220 & 1.7 & $\overline{14}$ & 7 & 21 & $\overline{16}$ & 480 & 19 & -- & $79(\mathrm{~J})^{\mathrm{C}}$ \\
\hline & 224D038 & $4-5$ & 22,000 & 9.9 & 220 & 1.8 & 15 & 7.7 & 24 & 18 & 520 & 21 & -- & $86(J)^{c}$ \\
\hline \multirow{2}{*}{ D11 } & 224D029 & $0-0.5$ & 20,000 & 9.2 & 210 & 1.7 & 14 & 7.2 & 22 & 17 & 500 & 20 & -- & $81(\mathrm{~J})^{\mathrm{C}}$ \\
\hline & 224D040 & $4-5$ & 21,000 & 8.9 & 210 & 1.7 & 14 & 7.1 & 21 & 16 & 480 & 20 & -- & $80(\mathrm{~J})^{\mathrm{c}}$ \\
\hline \multirow{2}{*}{ D12 } & $224 \mathrm{D} 030$ & $0-0.5$ & 21,000 & 9.5 & 210 & 1.8 & 15 & 7.4 & 23 & 17 & 500 & 21 & -- & $87(\mathrm{~J})^{\mathrm{C}}$ \\
\hline & $224 \mathrm{D} 042$ & $4-5$ & 21,000 & 9.6 & 230 & 1.7 & 14 & 7.3 & 22 & 17 & 510 & 20 & -- & $82(\mathrm{~J})^{\mathrm{C}}$ \\
\hline \multirow{2}{*}{ D13 } & $224 \mathrm{D} 031$ & $0-0.5$ & 21,000 & 8.9 & 210 & 1.7 & 14 & 7 & 21 & 17 & 490 & 19 & -- & $80(\mathrm{~J})^{\mathrm{C}}$ \\
\hline & 224D045 & $4-5$ & 19,000 & 8.6 & 230 & 1.6 & 13 & 6.5 & 20 & 15 & 430 & 18 & -- & $73(\mathrm{~J})^{\mathrm{C}}$ \\
\hline \multirow{2}{*}{ D14 } & 224D032 & $0-0.5$ & 22,000 & 9.7 & 230 & 1.8 & 15 & 7.6 & 23 & 18 & 520 & 21 & -- & $87(\mathrm{~J})^{\mathrm{C}}$ \\
\hline & $224 \mathrm{D} 047$ & $4-5$ & 18,000 & 8.7 & 200 & 1.5 & 12 & 6.6 & 19 & 15 & 470 & 17 & -- & $72(\mathrm{~J})^{\mathrm{C}}$ \\
\hline \multirow{2}{*}{ D15 } & $224 \mathrm{D} 033$ & $0-0.5$ & 22,000 & 9.7 & 220 & 1.8 & 15 & 7.7 & 23 & 18 & 540 & 21 & -- & $86(J)^{c}$ \\
\hline & 224D049 & $4-5$ & 17,000 & 7.8 & 200 & 1.3 & 11 & 6.2 & 17 & 15 & 440 & 16 & -- & $65(\mathrm{~J})^{\mathrm{C}}$ \\
\hline
\end{tabular}

${ }^{a}$ Based on U.S. Environmental Protection Agency, Region 9 Preliminary Remediation Goals (PRGs) (EPA, 2002)

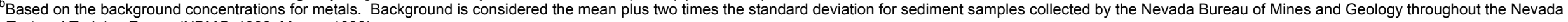

'Test and Training Range (NBMG, 1998; Moore, 1999).
Q

$\mathrm{ft}$ bgs $=$ Feet below ground surface

$\mathrm{mg} / \mathrm{kg}=$ Milligrams per kilogram

$\mathrm{J}=$ Estimated value.

$--=$ Not detected above minimum reporting limits. 
Table A.6-4

Soil Sample Results for Gamma-Emitting Radionuclides Detected Above Minimum Detectable Concentrations at CAS 06-03-01, Sewage Lagoons (3)

\begin{tabular}{|c|c|c|c|c|c|c|c|c|c|c|c|c|c|c|}
\hline \multirow[b]{2}{*}{$\begin{array}{l}\text { Sample } \\
\text { Location }\end{array}$} & \multirow[b]{2}{*}{$\begin{array}{c}\text { Sample } \\
\text { Number }\end{array}$} & \multirow{3}{*}{$\begin{array}{l}\text { Depth } \\
\text { (ft bgs) }\end{array}$} & \multicolumn{12}{|c|}{ Contaminants of Potential $(\mathrm{pCi} / \mathrm{g})$} \\
\hline & & & \multicolumn{2}{|c|}{ 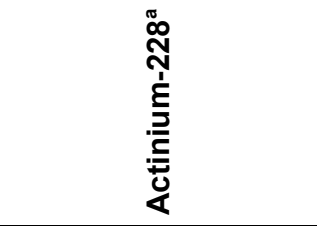 } & \multicolumn{2}{|c|}{ 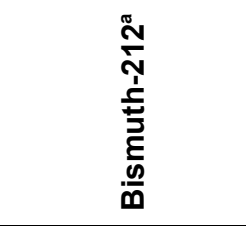 } & \multicolumn{2}{|c|}{ 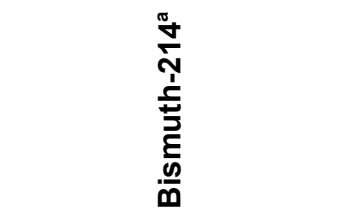 } & \multicolumn{2}{|c|}{ 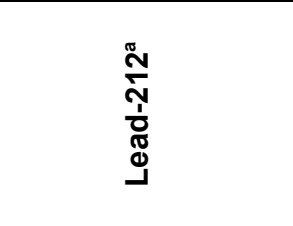 } & \multicolumn{2}{|c|}{ 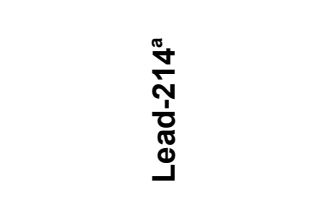 } & \multicolumn{2}{|c|}{ 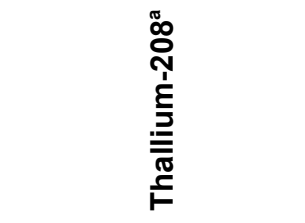 } \\
\hline \multicolumn{2}{|c|}{ Final Action Levels ${ }^{a}$} & & 5 & 15 & 5 & 15 & 5 & 15 & 5 & 15 & 5 & 15 & 5 & 15 \\
\hline \multicolumn{3}{|c|}{ Depth bgs (cm) } & $<15$ & $>15$ & $<15$ & $>15$ & $<15$ & $>15$ & $<15$ & $>15$ & $<15$ & $>15$ & $<15$ & $>15$ \\
\hline D01 & 224D008 & $4-5$ & NA & $2.05(\mathrm{G})$ & NA & -- & NA & $1.25(\mathrm{G}, \mathrm{J})$ & NA & $2.15(\mathrm{~J})^{b}$ & NA & $1.25(\mathrm{G}, \mathrm{J})$ & NA & $0.49(\mathrm{G})$ \\
\hline D02 & 224D017 & $4-5$ & NA & $1.61(\mathrm{G})$ & NA & -- & NA & $1.34(\mathrm{G}, \mathrm{J})$ & NA & $2.47(\mathrm{~J})^{\mathrm{b}}$ & NA & $1.46(\mathrm{G}, \mathrm{J})$ & NA & $0.89(\mathrm{G})$ \\
\hline D03 & 224D003 & $0-0.5$ & $2.16(\mathrm{G})$ & NA & $1.97(\mathrm{G})$ & NA & $0.96(\mathrm{G}, \mathrm{J})$ & NA & $1.81(\mathrm{G})$ & NA & $1.16(\mathrm{G}, \mathrm{J})$ & NA & $0.57(\mathrm{G})$ & NA \\
\hline D05 & 224D011 & $5-6$ & NA & $1.93(\mathrm{G})$ & NA & -- & NA & $1.18(\mathrm{G}, \mathrm{J})$ & NA & $2.86(\mathrm{~J})^{\mathrm{b}}$ & NA & $1.16(\mathrm{G}, \mathrm{J})$ & NA & $0.62(\mathrm{G})$ \\
\hline D06 & 224D019 & $0-0.5$ & $1.94(\mathrm{G})$ & NA & -- & NA & $1.47(\mathrm{G}, \mathrm{J})$ & NA & $2.43(\mathrm{~J})^{\mathrm{b}}$ & NA & $1.4(\mathrm{G}, \mathrm{J})$ & NA & $0.83(\mathrm{G})$ & NA \\
\hline D07 & 224D024 & $3-4$ & NA & $1.78(\mathrm{G})$ & NA & -- & NA & $0.94(\mathrm{G}, \mathrm{J})$ & NA & $1.84(\mathrm{~J})^{\mathrm{b}}$ & NA & $1.12(\mathrm{G}, \mathrm{J})$ & NA & $0.53(\mathrm{G})$ \\
\hline D09 & 224D036 & $4-5$ & NA & $1.88(\mathrm{G})$ & NA & -- & NA & $1.3(\mathrm{G}, \mathrm{J})$ & NA & $2.41(\mathrm{~J})^{\mathrm{b}}$ & NA & $1.44(\mathrm{G}, \mathrm{J})$ & NA & $0.61(\mathrm{G})$ \\
\hline D11 & 224D040 & $4-5$ & NA & $1.97(\mathrm{G})$ & NA & -- & NA & $1.19(\mathrm{G}, \mathrm{J})$ & NA & $2.8(\mathrm{~J})^{\mathrm{b}}$ & NA & $1.62(\mathrm{G}, \mathrm{J})$ & NA & $0.73(\mathrm{G})$ \\
\hline \multirow{3}{*}{ D13 } & 224D031 & $0-0.5$ & $2.18(\mathrm{G})$ & NA & -- & NA & $1.37(\mathrm{G}, \mathrm{J})$ & NA & $2.32(\mathrm{~J})^{\mathrm{b}}$ & NA & $1.52(\mathrm{G}, \mathrm{J})$ & NA & $0.62(\mathrm{G})$ & NA \\
\hline & 224D044 & $4-5$ & NA & $2(\mathrm{G})$ & NA & -- & NA & $1.2(\mathrm{G}, \mathrm{J})$ & NA & $2.39(\mathrm{~J})^{\mathrm{b}}$ & NA & $1.57(\mathrm{G}, \mathrm{J})$ & NA & $0.71(\mathrm{G})$ \\
\hline & 224D045 & $4-5$ & NA & $2.08(\mathrm{G})$ & NA & -- & NA & $1.14(\mathrm{G}, \mathrm{J})$ & NA & $2.21(\mathrm{~J})^{\mathrm{b}}$ & NA & $1.48(\mathrm{G}, \mathrm{J})$ & NA & $0.58(\mathrm{G})$ \\
\hline D15 & 224D049 & $4-5$ & NA & $2.01(\mathrm{G})$ & NA & -- & NA & $1.3(\mathrm{G}, \mathrm{J})$ & NA & $2.09(\mathrm{~J})^{\mathrm{b}}$ & NA & $1.42(\mathrm{G}, \mathrm{J})$ & NA & $0.7(\mathrm{G})$ \\
\hline
\end{tabular}

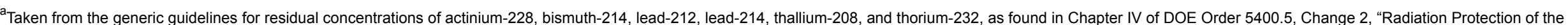

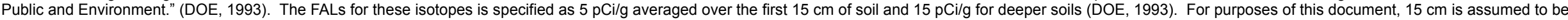
equivalent to $0.5 \mathrm{ft}$ ( $6 \mathrm{in}$.); therefore, $5 \mathrm{pCi} / \mathrm{g}$ represents the $\mathrm{FALs}$ for these radionuclides in the surface soil ( 0 to $0.5 \mathrm{ft}$ depth).

Qualifier added to laboratory data; record accepted. Sample does not meet counting geometry requirements.

ft bgs = Feet below ground surface $\mathrm{cm}=$ Centimeter

$\mathrm{Ci} / \mathrm{g}=$ Picocuries per gram

= Not detected above minimum reporting limits
$>=$ Greater than

$<=$ Less than

$\mathrm{G}=$ Sample density differs by more than 15 percent of laboratory control sample density. $\mathrm{NA}=$ Not applicable 


\section{Table A.6-5 \\ Soil Sample Results for Isotopes Detected Above \\ Minimum Detectable Concentrations at CAS 06-03-01, Sewage Lagoons (3)}

\begin{tabular}{|c|c|c|c|c|c|}
\hline \multirow{2}{*}{$\begin{array}{l}\text { Sample } \\
\text { Location }\end{array}$} & \multirow{2}{*}{$\begin{array}{l}\text { Sample } \\
\text { Number }\end{array}$} & \multirow{2}{*}{$\begin{array}{c}\text { Depth } \\
\text { (ft bgs) }\end{array}$} & \multicolumn{3}{|c|}{ Contaminants of Potential Concern (pCi/g) } \\
\hline & & & Uranium-234 & Uranium-235 & Uranium-238 \\
\hline \multicolumn{3}{|c|}{ Final Action Levels ${ }^{a}$} & 143 & 17.6 & 105 \\
\hline$\overline{\mathrm{D} 01}$ & $\overline{2224 \mathrm{D008}}$ & 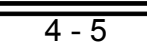 & $\overline{\overline{0.96}}$ & $\overline{---}$ & 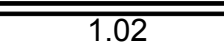 \\
\hline D02 & $224 \mathrm{D} 017$ & $4-5$ & 1.01 & -- & 1.08 \\
\hline D03 & $224 \mathrm{D} 003$ & $0-0.5$ & 0.88 & $\overline{--}$ & 0.93 \\
\hline D05 & $224 \mathrm{D} 011$ & $5-6$ & 1.05 & $\overline{--}$ & 0.94 \\
\hline D06 & $224 D 019$ & $0-0.5$ & 0.98 & -- & 0.99 \\
\hline D07 & $224 \mathrm{D} 024$ & $3-4$ & 0.75 & $\overline{--}$ & 0.72 \\
\hline D09 & $224 \mathrm{D} 036$ & $4-5$ & 0.94 & -- & 1 \\
\hline D11 & 224D040 & $4-5$ & 1.07 & -- & 1.04 \\
\hline \multirow{3}{*}{ D13 } & $224 \mathrm{D} 031$ & $0-0.5$ & 1.06 & -- & 1.08 \\
\hline & $224 \mathrm{D} 044$ & $4-5$ & 0.91 & -- & 0.91 \\
\hline & $224 \mathrm{D} 045$ & $4-5$ & 1.06 & 0.09 & 1.13 \\
\hline D15 & 224D049 & $4-5$ & 0.88 & -- & 0.91 \\
\hline
\end{tabular}

aTaken from the construction, commercial, industrial land use scenario in Table 2.1 of the NCRP Report No. 129 Recommended Screening Limits for Contaminated Surface Soil and Review Factors Relevant to Site-Specific Studies (NCRP, 1999). The values provided in this source document were scaled to a $25-\mathrm{mrem} / \mathrm{yr}$ dose.

$\mathrm{ft}$ bgs $=$ Feet below ground surface

$\mathrm{mrem} / \mathrm{yr}$ Millirems per year

$\mathrm{pCi} / \mathrm{g}=$ Picocuries per gram

$--=$ Not detected above minimum reporting limits

Table A.6-6

Soil Sample Results for Cyanide Detected Above

Minimum Reporting Limits at CAS 06-03-01, Sewage Lagoons (3)

\begin{tabular}{|c|c|c|c|}
\hline \multirow{2}{*}{$\begin{array}{l}\text { Sample } \\
\text { Location }\end{array}$} & \multirow{2}{*}{$\begin{array}{l}\text { Sample } \\
\text { Number }\end{array}$} & \multirow{2}{*}{$\begin{array}{c}\text { Depth } \\
\text { (ft bgs) }\end{array}$} & Contaminants of Potential Concern (mg/kg) \\
\hline & & & Cyanide \\
\hline \multicolumn{3}{|c|}{ Final Action Levels ${ }^{a}$} & 12,000 \\
\hline \multirow{2}{*}{ D04 } & 224D004 & $0-0.5$ & $\overline{0.67}$ \\
\hline & 224D005 & $0-0.5$ & 0.61 \\
\hline D10 & 224D028 & $0-0.5$ & 1.3 \\
\hline
\end{tabular}

${ }^{a}$ Based on U.S. Environmental Protection Agency, Region 9 Preliminary Remediation Goals (PRGs) (EPA, 2002) 


\section{A.6.3 Nature and Extent of Contamination}

No contamination was identified at the CAS.

\section{A.6.4 Revised Conceptual Site Model}

No variations in the CSM were identified. 


\section{A.7.0 CASs 06-05-01, Leachfield, 06-17-04, Decon Pad and Wastewater Catch, and 06-23-01, Decon Pad Discharge Piping}

Corrective Action Sites 06-05-01, 06-17-04, and 06-23-01 comprise a system that was used for decontamination of radiologically contaminated equipment in Area 6 of the NTS. CAS 06-05-01 consists of a leachfield and associated sewage lagoons; CAS 06-17-04 is a decon pad used for decontamination of equipment and the associated wastewater catch; CAS 06-23-01 consists of piping connecting the other two CASs to each other and to former nearby buildings. Investigation activities at these CASs included collection of surface and subsurface soil samples, as well as one waste management concrete sample from the wastewater trench at CAS 06-17-04.

\section{A.7.1 Corrective Action Investigation}

A total of 95 soil characterization samples were collected at CAS 06-05-01, 43 at CAS 06-17-04 (including two sludge samples from the sump attached to the decon pad), and 6 at CAS 06-23-01. The sample IDs, locations, types, and analyses for each of the three CASs are listed in Table A.7-1 through Table A.7-3. The sample locations are shown on Figure A.7-1. The specific CAI activities conducted to satisfy the CAIP requirements at this CAS are described in the following sections.

\section{A.7.1.1 Field Screening}

Soil samples were field screened for VOCs, TPH (at some locations only), and alpha and beta/gamma radiation. The FSRs were compared to FSLs to guide subsequent sampling decisions. The VOC headspace and TPH FSLs were not exceeded during sampling activities at this CAS. Alpha and beta/gamma FSLs were exceeded in several samples.

\section{A.7.1.2 Sampling}

Surface and subsurface samples were collected at locations specified in the CAIP. The locations were chosen to be associated with features (i.e. piping, trench) of the sites, according to engineering drawings and visual inspections. Samples were collected at the surface (or directly beneath the concrete at CAS 06-17-04), and at two or three subsurface intervals at each location. Surface samples 
Table A.7-1

Samples Collected at CASs 06-05-01, Leachfield (Page 1 of 4 )

\begin{tabular}{|c|c|c|c|c|c|}
\hline $\begin{array}{c}\text { Sample } \\
\text { Location }\end{array}$ & $\begin{array}{l}\text { Sample } \\
\text { Number }\end{array}$ & $\begin{array}{c}\text { Depth } \\
\text { (ft bgs) }\end{array}$ & $\begin{array}{c}\text { Sample } \\
\text { Matrix }\end{array}$ & Purpose & Analyses \\
\hline \multirow{4}{*}{ E01 } & 224E001 & $0-0.5$ & Soil & $\overline{S C, M S / M S D}$ & Set 1 \\
\hline & 224E002 & $3-4$ & Soil & SC & Set 1 \\
\hline & 224E003 & $3-4$ & Soil & $\begin{array}{c}\text { Field Duplicate } \\
\text { of \#224E002 }\end{array}$ & Set 1 \\
\hline & 224E004 & $5-6$ & Soil & $\overline{S C}$ & Set 1 \\
\hline \multirow{3}{*}{ E02 } & 224E005 & $0-0.5$ & Soil & SC & Set 1 \\
\hline & 224E007 & $4-5$ & Soil & SC & Set 1 \\
\hline & 224E009 & $7-8$ & Soil & $\mathrm{SC}$ & Set 1 \\
\hline \multirow{3}{*}{ E03 } & 224E006 & $0-0.5$ & Soil & SC & Set 1 \\
\hline & 224E013 & $4-5$ & Soil & SC & Set 1 \\
\hline & 224E016 & $7-8$ & Soil & SC & Set 1 \\
\hline \multirow{4}{*}{ E04 } & 224E008 & $0-0.5$ & Soil & $\mathrm{SC}$ & Set 1 \\
\hline & 224E052 & $3.5-4.5$ & Soil & $\mathrm{SC}$ & Set 1 \\
\hline & 224E053 & $7-8$ & Soil & SC & Set 1 \\
\hline & 224E054 & $9-10$ & Soil & SC & Set 1 \\
\hline \multirow{5}{*}{ E05 } & 224E010 & $0-0.5$ & Soil & SC & Set 1 \\
\hline & 224E020 & $4-5$ & Soil & SC & Set 1 \\
\hline & 224E021 & $4-5$ & Soil & $\begin{array}{l}\text { Field Duplicate } \\
\text { of \#224E020 }\end{array}$ & Set 1 \\
\hline & 224E022 & $7-8$ & Soil & $\mathrm{SC}, \mathrm{MS} / \mathrm{MSD}$ & Set 1 \\
\hline & 224E023 & $9-10$ & Soil & SC & Set 1 \\
\hline \multirow{4}{*}{ E06 } & 224E011 & $0-0.5$ & Soil & SC & Set 1 \\
\hline & 224E055 & $3.5-4.5$ & Soil & $\mathrm{SC}$ & Set 1 \\
\hline & 224E056 & $7-8$ & Soil & SC & Set 1 \\
\hline & 224E057 & $9-10$ & Soil & SC & Set 1 \\
\hline \multirow{3}{*}{ E07 } & 224E012 & $0-0.5$ & Soil & SC & Set 1 \\
\hline & 224E037 & $5-6$ & Soil & SC & Set 1 \\
\hline & 224E038 & $9-10$ & Soil & $\mathrm{SC}$ & Set 1 \\
\hline \multirow{4}{*}{ E08 } & 224E014 & $0-0.5$ & Soil & SC & Set 1 \\
\hline & 224E025 & $2-3$ & Soil & SC & Set 1 \\
\hline & 224E026 & $7-8$ & Soil & SC & Set 1 \\
\hline & 224E027 & $9-10$ & Soil & $\overline{S C}$ & Set 1 \\
\hline \multirow{3}{*}{ E09 } & 224E015 & $0-0.5$ & Soil & SC & Set 1 \\
\hline & 224E035 & $6-7$ & Soil & $\overline{S C}$ & Set 1 \\
\hline & 224E036 & $9-10$ & Soil & SC & Set 1 \\
\hline \multirow{3}{*}{ E10 } & 224E017 & $0-0.5$ & Soil & SC & Set 1 \\
\hline & 224E033 & $5-6$ & Soil & $\mathrm{SC}$ & Set 1 \\
\hline & 224E034 & $8-9$ & Soil & SC & Set 1 \\
\hline
\end{tabular}


Table A.7-1

Samples Collected at CASs 06-05-01, Leachfield (Page 2 of 4 )

\begin{tabular}{|c|c|c|c|c|c|}
\hline $\begin{array}{c}\text { Sample } \\
\text { Location }\end{array}$ & $\begin{array}{l}\text { Sample } \\
\text { Number }\end{array}$ & $\begin{array}{c}\text { Depth } \\
\text { (ft bgs) }\end{array}$ & $\begin{array}{l}\text { Sample } \\
\text { Matrix }\end{array}$ & Purpose & Analyses \\
\hline \multirow{4}{*}{ E11 } & $\overline{2 \text { 224E018 }}$ & $0-0.5$ & $\overline{\text { Soil }}$ & $\overline{\mathrm{SC}}$ & $\overline{\text { Set } 1}$ \\
\hline & 224E028 & $2-3$ & Soil & $\mathrm{SC}$ & Set 1 \\
\hline & 224E029 & $5-6$ & Soil & SC & Set 1 \\
\hline & 224E030 & $7-8$ & Soil & SC & Set 1 \\
\hline \multirow{3}{*}{ E12 } & 224E019 & $0-0.5$ & Soil & $\mathrm{SC}$ & Set 1 \\
\hline & 224E031 & $4-5$ & Soil & SC & Set 1 \\
\hline & 224E032 & $7-8$ & Soil & SC & Set 1 \\
\hline E13 & 224E024 & $7-8$ & Soil & SC & Set 1 \\
\hline \multirow{5}{*}{ E14 } & 224E039 & $0-0.5$ & Soil & $\mathrm{SC}$ & Set 1 \\
\hline & 224E040 & $2-3$ & Soil & SC, MS/MSD & Set 1 \\
\hline & 224E041 & $6-7$ & Soil & $\mathrm{SC}$ & Set 1 \\
\hline & 224E042 & $6-7$ & Soil & $\begin{array}{c}\text { Field Duplicate } \\
\text { of \#224E041 }\end{array}$ & Set 1 \\
\hline & 224E043 & $9-10$ & Soil & $\mathrm{SC}$ & Set 1 \\
\hline \multirow{4}{*}{ E15 } & 224E044 & $0-0.5$ & Soil & $\mathrm{SC}$ & Set 1 \\
\hline & 224E045 & $2-3$ & Soil & $\mathrm{SC}$ & Set 1 \\
\hline & 224E046 & $6-7$ & Soil & $\overline{S C}$ & Set 1 \\
\hline & 224E047 & $9-10$ & Soil & SC & Set 1 \\
\hline \multirow{4}{*}{ E16 } & 224E048 & $0-0.5$ & Soil & $\overline{S C}$ & Set 1 \\
\hline & 224E049 & $2-3$ & Soil & SC & Set 1 \\
\hline & 224E050 & $7-8$ & Soil & $\mathrm{SC}$ & Set 1 \\
\hline & 224E051 & $10-11$ & Soil & SC & Set 1 \\
\hline \multirow{3}{*}{ E17 } & 224E058 & $0-0.5$ & Soil & $\mathrm{SC}$ & Set 1 \\
\hline & 224E068 & $4-5$ & Soil & $\overline{S C}$ & Set 1 \\
\hline & 224E069 & $6.5-7.5$ & Soil & $\overline{S C}$ & Set 1 \\
\hline \multirow{3}{*}{ E18 } & 224E059 & $0-0.5$ & Soil & $\overline{S C}$ & Set 1 \\
\hline & 224E070 & $4-5$ & Soil & SC & Set 1 \\
\hline & 224E071 & $7-8$ & Soil & $\overline{S C}$ & Set 1 \\
\hline \multirow{3}{*}{ E19 } & 224E060 & $0-0.5$ & Soil & SC & Set 1 \\
\hline & 224E072 & $4-5$ & Soil & $\mathrm{SC}$ & Set 1 \\
\hline & 224E073 & $7-8$ & Soil & $\mathrm{SC}$ & Set 1 \\
\hline \multirow{4}{*}{ E20 } & 224E061 & $0-0.5$ & Soil & $\mathrm{SC}$ & Set 1 \\
\hline & 224E063 & $4-5$ & Soil & $\mathrm{SC}$ & Set 1 \\
\hline & 224E066 & $6.5-7.5$ & Soil & $\mathrm{SC}$ & Set 1 \\
\hline & 224E067 & $6.5-7.5$ & Soil & $\begin{array}{c}\text { Field Duplicate } \\
\text { of \#224E066 }\end{array}$ & Set 1 \\
\hline
\end{tabular}


Table A.7-1

Samples Collected at CASs 06-05-01, Leachfield (Page 3 of 4 )

\begin{tabular}{|c|c|c|c|c|c|}
\hline $\begin{array}{c}\text { Sample } \\
\text { Location }\end{array}$ & $\begin{array}{l}\text { Sample } \\
\text { Number }\end{array}$ & $\begin{array}{l}\text { Depth } \\
\text { (ft bgs) }\end{array}$ & $\begin{array}{l}\text { Sample } \\
\text { Matrix }\end{array}$ & Purpose & Analyses \\
\hline \multirow{3}{*}{ E21 } & 224E062 & $0-0.5$ & 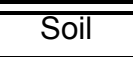 & $\overline{\overline{S C}}$ & $\overline{\text { Set } 1}$ \\
\hline & 224E074 & $4-5$ & Soil & SC & Set 1 \\
\hline & 224E075 & $7-8$ & Soil & $\mathrm{SC}$ & Set 1 \\
\hline \multirow{3}{*}{ E22 } & 224E064 & $0-0.5$ & Soil & $\mathrm{SC}$ & Set 1 \\
\hline & 224E076 & $4-5$ & Soil & SC & Set 1 \\
\hline & 224E077 & $7-8$ & Soil & SC & Set 1 \\
\hline \multirow{3}{*}{ E23 } & 224E065 & $0-0.5$ & Soil & SC, MS/MSD & Set 1 \\
\hline & 224E078 & $4-5$ & Soil & $\mathrm{SC}$ & Set 1 \\
\hline & 224E079 & $7-8$ & Soil & SC & Set 1 \\
\hline \multirow{3}{*}{ E24 } & 224E080 & $0-1$ & Soil & SC & Set 1 \\
\hline & 224E082 & $4-5$ & Soil & $\mathrm{SC}$ & Set 1 \\
\hline & 224E083 & $7-8$ & Soil & $\overline{S C}$ & Set 1 \\
\hline \multirow{4}{*}{ E25 } & 224E081 & $0-1$ & Soil & $\overline{S C}$ & Set 1 \\
\hline & 224E084 & $4-5$ & Soil & $\overline{S C}$ & Set 1 \\
\hline & 224E085 & $4-5$ & Soil & $\begin{array}{l}\text { Field Duplicate } \\
\text { of \#224E084 }\end{array}$ & Set 1 \\
\hline & 224E086 & $7-8$ & Soil & SC, MS/MSD & Set 1 \\
\hline E26 & 224E087 & $0-1$ & Soil & $\mathrm{SC}$ & Set 1 \\
\hline E27 & 224E090 & $0-1$ & Soil & $\overline{S C}$ & Set 1 \\
\hline E28 & 224E093 & $0-1$ & Soil & SC & Set 1 \\
\hline E29 & 224E096 & $0-0.5$ & Soil & SC & $\begin{array}{l}\text { Gamma Spectroscopy, } \\
\text { Isotopic Plutonium }\end{array}$ \\
\hline E30 & 224E097 & $0-0.5$ & Soil & SC & $\begin{array}{l}\text { Gamma Spectroscopy, } \\
\text { Isotopic Plutonium }\end{array}$ \\
\hline E31 & 224E098 & $0-0.5$ & Soil & SC & $\begin{array}{l}\text { Gamma Spectroscopy, } \\
\text { Isotopic Plutonium }\end{array}$ \\
\hline E32 & 224E099 & $0-0.5$ & Soil & SC & $\begin{array}{l}\text { Gamma Spectroscopy, } \\
\text { Isotopic Plutonium }\end{array}$ \\
\hline E33 & 224E100 & $0-0.5$ & Soil & SC & $\begin{array}{l}\text { Gamma Spectroscopy, } \\
\text { Isotopic Plutonium }\end{array}$ \\
\hline E34 & 224E101 & $0-0.5$ & Soil & SC & $\begin{array}{l}\text { Gamma Spectroscopy, } \\
\text { Isotopic Plutonium }\end{array}$ \\
\hline E35 & 224E102 & $0-0.5$ & Soil & SC & $\begin{array}{l}\text { Gamma Spectroscopy, } \\
\text { Isotopic Plutonium }\end{array}$ \\
\hline \multirow{3}{*}{ E36 } & 224E103 & $0-0.5$ & Soil & SC & $\begin{array}{l}\text { Gamma Spectroscopy, } \\
\text { Isotopic Plutonium }\end{array}$ \\
\hline & 224E104 & $2-2.5$ & Soil & SC & $\begin{array}{l}\text { Gamma Spectroscopy } \\
\text { Isotopic Plutonium }\end{array}$ \\
\hline & 224E108 & $3-3.5$ & Soil & SC & $\begin{array}{l}\text { Gamma Spectroscopy, } \\
\text { Isotopic Plutonium }\end{array}$ \\
\hline
\end{tabular}


Table A.7-1

Samples Collected at CASs 06-05-01, Leachfield

(Page 4 of 4 )

\begin{tabular}{|c|c|c|c|c|c|}
\hline $\begin{array}{c}\text { Sample } \\
\text { Location }\end{array}$ & $\begin{array}{l}\text { Sample } \\
\text { Number }\end{array}$ & $\begin{array}{l}\text { Depth } \\
\text { (ft bgs) }\end{array}$ & $\begin{array}{l}\text { Sample } \\
\text { Matrix }\end{array}$ & Purpose & Analyses \\
\hline \multirow{2}{*}{ E37 } & 224E105 & $0-0.5$ & Soil & SC & $\begin{array}{l}\text { Gamma Spectroscopy } \\
\text { Isotopic Plutonium }\end{array}$ \\
\hline & 224E109 & $1-1.5$ & Soil & SC & $\begin{array}{l}\text { Gamma Spectroscopy, } \\
\text { Isotopic Plutonium }\end{array}$ \\
\hline \multirow{2}{*}{ E38 } & 224E106 & $0-0.5$ & Soil & SC & $\begin{array}{l}\text { Gamma Spectroscopy } \\
\text { Isotopic Plutonium }\end{array}$ \\
\hline & 224E110 & $1-1.5$ & Soil & SC & $\begin{array}{l}\text { Gamma Spectroscopy, } \\
\text { Isotopic Plutonium }\end{array}$ \\
\hline \multirow{2}{*}{ E39 } & 224E107 & $0-0.5$ & Soil & SC & $\begin{array}{l}\text { Gamma Spectroscopy } \\
\text { Isotopic Plutonium }\end{array}$ \\
\hline & 224E111 & $0.5-1$ & Soil & SC & $\begin{array}{l}\text { Gamma Spectroscopy, } \\
\text { Isotopic Plutonium }\end{array}$ \\
\hline $\mathrm{E} 40$ & 224E112 & $0-0.5$ & Soil & SC & $\begin{array}{l}\text { Gamma Spectroscopy, } \\
\text { Isotopic Plutonium }\end{array}$ \\
\hline NA & 224E301 & $\overline{N A}$ & Water & Trip Blank & Total VOCs \\
\hline NA & 224E302 & NA & Water & Trip Blank & Total VOCs \\
\hline NA & 224E303 & NA & Water & Trip Blank & Total VOCs \\
\hline NA & 224E304 & NA & Water & Trip Blank & Total VOCs \\
\hline$\overline{N A}$ & 224E305 & $\overline{N A}$ & Water & Trip Blank & Total VOCs \\
\hline NA & 224E306 & NA & Water & Field Blank & Set 1 \\
\hline NA & 224E307 & NA & Water & Trip Blank & Total VOCs \\
\hline $\mathrm{NA}$ & 224E308 & $\mathrm{NA}$ & Water & Trip Blank & Total VOCs \\
\hline NA & 224E309 & NA & Water & Trip Blank & Total VOCs \\
\hline NA & 224E310 & $\overline{N A}$ & Water & Trip Blank & Total VOCs \\
\hline$\overline{N A}$ & 224E311 & $\overline{N A}$ & Water & Trip Blank & Total VOCs \\
\hline NA & 224E312 & NA & Water & Trip Blank & Total VOCs \\
\hline NA & 224E313 & NA & Water & Trip Blank & Total VOCs \\
\hline NA & 224E501 & NA & Liquid & WM & $\begin{array}{c}\text { Gross Alpha/Beta, } \\
\text { Tritium }\end{array}$ \\
\hline
\end{tabular}

Note: Certain sample numbers are missing. This is due to the fact that the samples they represented were not shipped to the laboratory according to the specifications in the CAIP.

Set 1 = Total VOCs, Total SVOCs, Total RCRA Metals, Aluminum, Antimony, Beryllium, Cobalt, Copper, Manganese, Molybdenum, Nickel, Zinc, TPH-DRO, PCBs, Gamma Spectroscopy, Isotopic Plutonium, Isotopic Uranium, and Strontium-90.

CAIP = Corrective Action Investigation Plan

DRO = Diesel-range organics

$\mathrm{ft}$ bgs $=$ Feet below ground surface

$\mathrm{PCB}=$ Polychlorinated biphenyl

RCRA = Resource Conservation and Recovery Act

$\mathrm{SC}=$ Site characterization
SVOC = Semivolatile organic compound

$\mathrm{TPH}=$ Total petroleum hydrocarbons

$\mathrm{WM}=$ Waste Management

MS/MSD = Matrix spike/matrix spike duplicate

$\mathrm{NA}=$ Not applicable

$\mathrm{VOC}=$ Volatile organic compound 
Table A.7-2

Samples Collected at CAS 06-17-04, Decon Pad and Wastewater Catch (Page 1 of 6 )

\begin{tabular}{|c|c|c|c|c|c|}
\hline $\begin{array}{c}\text { Sample } \\
\text { Location }\end{array}$ & $\begin{array}{l}\text { Sample } \\
\text { Number }\end{array}$ & $\begin{array}{l}\text { Depth } \\
\text { (ft bgs) }\end{array}$ & $\begin{array}{l}\text { Sample } \\
\text { Matrix }\end{array}$ & Purpose & Analyses \\
\hline \multirow{4}{*}{ F01 } & 224F001 & $1-2$ & $\overline{\text { Soil }}$ & $\overline{\overline{S C}}$ & $\overline{\text { Set } 1}$ \\
\hline & 224F002 & $1-2$ & Soil & $\begin{array}{c}\text { Field Duplicate } \\
\text { of \#224F001 }\end{array}$ & Set 1 \\
\hline & 224F003 & $4-5$ & Soil & $\mathrm{SC}, \mathrm{MS} / \mathrm{MSD}$ & Set 1 \\
\hline & 224F004 & $7-8$ & Soil & SC & Set 1 \\
\hline \multirow{3}{*}{ F02 } & 224F005 & $1-2$ & Soil & SC & Set 1 \\
\hline & $224 \mathrm{~F} 006$ & $4-5$ & Soil & SC & Set 1 \\
\hline & 224F007 & $7-8$ & Soil & SC & Set 1 \\
\hline \multirow{3}{*}{ F03 } & 224F008 & $1-2$ & Soil & SC & Set 1 \\
\hline & 224F009 & $4-5$ & Soil & SC & Set 1 \\
\hline & $224 \mathrm{~F} 010$ & $7-8$ & Soil & $\overline{S C}$ & Set 1 \\
\hline \multirow{3}{*}{ F04 } & $224 \mathrm{~F} 011$ & $1-2$ & Soil & SC & Set 1 \\
\hline & $224 \mathrm{~F} 012$ & $4-5$ & Soil & SC & Set 1 \\
\hline & $224 \mathrm{~F} 013$ & $7-8$ & Soil & SC & Set 1 \\
\hline \multirow{3}{*}{ F05 } & $224 \mathrm{~F} 014$ & $1-2$ & Soil & SC & Set 1 \\
\hline & $224 \mathrm{~F} 015$ & $4-5$ & Soil & SC & Set 1 \\
\hline & 224F016 & $7-8$ & Soil & SC & Set 1 \\
\hline \multirow{3}{*}{ F06 } & $224 \mathrm{~F} 017$ & $1-2$ & Soil & $\mathrm{SC}$ & Set 1 \\
\hline & $224 \mathrm{~F} 018$ & $4-5$ & Soil & SC & Set 1 \\
\hline & 224F019 & $7-8$ & Soil & SC & Set 1 \\
\hline \multirow{3}{*}{ F07 } & $224 \mathrm{~F} 020$ & $1-2$ & Soil & SC & Set 1 \\
\hline & $224 \mathrm{~F} 021$ & $4-5$ & Soil & SC & Set 1 \\
\hline & $224 \mathrm{~F} 022$ & $7-8$ & Soil & $\mathrm{SC}, \mathrm{MS} / \mathrm{MSD}$ & Set 1 \\
\hline \multirow{4}{*}{ F08 } & $224 \mathrm{~F} 023$ & $0.2-1$ & Soil & SC & Set 1 \\
\hline & $224 \mathrm{~F} 024$ & $4-5$ & Soil & SC & Set 1 \\
\hline & $224 \mathrm{~F} 025$ & $7-8$ & Soil & SC & Set 1 \\
\hline & 224F026 & $7-8$ & Soil & $\begin{array}{c}\text { Field Duplicate } \\
\text { of \#224F025 }\end{array}$ & Set 1 \\
\hline \multirow{3}{*}{ F09 } & 224F027 & $0.2-1$ & Soil & SC & Set 1 \\
\hline & 224F028 & $4-5$ & Soil & SC & Set 1 \\
\hline & 224F029 & $7-8$ & Soil & SC & Set 1 \\
\hline \multirow{4}{*}{ F10 } & 224F030 & $0-1$ & Soil & SC & Set 1 \\
\hline & 224F031 & $4-5$ & Soil & SC & Set 1 \\
\hline & 224F032 & $7-8$ & Soil & SC & Set 1 \\
\hline & 224F081 & $0-0.5$ & Soil & SC, MS/MSD & Chlordane \\
\hline
\end{tabular}


Table A.7-2

Samples Collected at CAS 06-17-04, Decon Pad and Wastewater Catch (Page 2 of 6 )

\begin{tabular}{|c|c|c|c|c|c|}
\hline $\begin{array}{l}\text { Sample } \\
\text { Location }\end{array}$ & $\begin{array}{l}\text { Sample } \\
\text { Number }\end{array}$ & $\begin{array}{l}\text { Depth } \\
\text { (ft bgs) }\end{array}$ & $\begin{array}{l}\text { Sample } \\
\text { Matrix }\end{array}$ & Purpose & Analyses \\
\hline \multirow{5}{*}{ F11 } & 224F033 & $0-1$ & Soil & SC & Set 1 \\
\hline & 224F034 & $4-5$ & Soil & SC & Set 1 \\
\hline & 224F035 & $7-8$ & Soil & $\mathrm{SC}$ & Set 1 \\
\hline & 224F082 & $0-0.5$ & Soil & $\mathrm{SC}$ & Chlordane \\
\hline & 224F083 & $0-0.5$ & Soil & $\begin{array}{l}\text { Field Duplicate } \\
\text { of \#224F082 }\end{array}$ & Chlordane \\
\hline \multirow{4}{*}{$\mathrm{F} 12$} & $224 \mathrm{~F} 036$ & $0-1$ & Soil & $\mathrm{SC}$ & Set 1 \\
\hline & 224F037 & $4-5$ & Soil & SC & Set 1 \\
\hline & 224F038 & $7-8$ & Soil & $\mathrm{SC}$ & Set 1 \\
\hline & 224F084 & $0-0.5$ & Soil & SC & Chlordane \\
\hline \multirow{4}{*}{ F13 } & 224F039 & $0-1$ & Soil & SC & Set 1 \\
\hline & 224F040 & $4-5$ & Soil & SC & Set 1 \\
\hline & 224F041 & $7-8$ & Soil & SC, MS/MSD & Set 1 \\
\hline & 224F501 & $0-0.3$ & Concrete & WM & Set 1 \\
\hline F14 & 224F042 & $3-4$ & Sludge & SC & Set 1 , Set 2 \\
\hline F15 & 224F043 & $3-4$ & Sludge & SC & Set 1 , Set 2 \\
\hline F16 & 224F044 & $1-2$ & Soil & SC & TPH-DRO \\
\hline F17 & 224F045 & $1-2$ & Soil & SC & TPH-DRO \\
\hline \multirow[b]{2}{*}{ F18 } & 224F046 & $1-2$ & Soil & SC & TPH-DRO \\
\hline & 224F047 & $1-2$ & Soil & $\begin{array}{l}\text { Field Duplicate } \\
\text { of \#224F046 }\end{array}$ & TPH-DRO \\
\hline F19 & 224F048 & $1-3$ & Soil & SC, MS/MSD & TPH-DRO \\
\hline $\mathrm{F} 20$ & 224F049 & $1-2$ & Soil & SC & TPH-DRO \\
\hline F21 & 224F050 & $1-3$ & Soil & SC & $\begin{array}{l}\text { TPH-DRO, Isotopic Plutonium, } \\
\text { Gamma Spectroscopy }\end{array}$ \\
\hline F22 & 224F051 & $1-3$ & Soil & SC & $\begin{array}{l}\text { TPH-DRO, Isotopic Plutonium, } \\
\text { Gamma Spectroscopy }\end{array}$ \\
\hline F23 & 224F052 & $1-3$ & Soil & SC & $\begin{array}{l}\text { TPH-DRO, Isotopic Plutonium, } \\
\text { Gamma Spectroscopy }\end{array}$ \\
\hline \multirow{3}{*}{ F24 } & 224F053 & $0-2$ & Soil & SC & $\begin{array}{l}\text { TPH-DRO, Isotopic Plutonium, } \\
\text { Gamma Spectroscopy }\end{array}$ \\
\hline & 224F054 & $3-5$ & Soil & SC & $\begin{array}{l}\text { TPH-DRO, Isotopic Plutonium, } \\
\text { Gamma Spectroscopy }\end{array}$ \\
\hline & 224F055 & $6-7$ & Soil & SC & $\begin{array}{l}\text { TPH-DRO, Isotopic Plutonium, } \\
\text { Gamma Spectroscopy }\end{array}$ \\
\hline
\end{tabular}


Table A.7-2

Samples Collected at CAS 06-17-04, Decon Pad and Wastewater Catch (Page 3 of 6 )

\begin{tabular}{|c|c|c|c|c|c|}
\hline $\begin{array}{l}\text { Sample } \\
\text { Location }\end{array}$ & $\begin{array}{l}\text { Sample } \\
\text { Number }\end{array}$ & $\begin{array}{l}\text { Depth } \\
\text { (ft bgs) }\end{array}$ & $\begin{array}{c}\text { Sample } \\
\text { Matrix }\end{array}$ & Purpose & Analyses \\
\hline \multirow{2}{*}{ F25 } & 224F056 & $0-3$ & Soil & SC & $\begin{array}{c}\text { TPH-DRO, Isotopic Plutonium, } \\
\text { Gamma Spectroscopy }\end{array}$ \\
\hline & 224F057 & $5-7$ & Soil & SC & $\begin{array}{l}\text { TPH-DRO, Isotopic Plutonium, } \\
\text { Gamma Spectroscopy }\end{array}$ \\
\hline \multirow{2}{*}{ F26 } & 224F058 & $0-4$ & Soil & SC & $\begin{array}{l}\text { TPH-DRO, Isotopic Plutonium, } \\
\text { Gamma Spectroscopy }\end{array}$ \\
\hline & 224F059 & $4-7$ & Soil & SC & $\begin{array}{l}\text { TPH-DRO, Isotopic Plutonium, } \\
\text { Gamma Spectroscopy }\end{array}$ \\
\hline \multirow{2}{*}{ F27 } & 224F060 & $0-2$ & Soil & SC & $\begin{array}{l}\text { TPH-DRO, Isotopic Plutonium, } \\
\text { Gamma Spectroscopy }\end{array}$ \\
\hline & 224F061 & $2-4$ & Soil & SC & $\begin{array}{l}\text { TPH-DRO, Isotopic Plutonium, } \\
\text { Gamma Spectroscopy }\end{array}$ \\
\hline \multirow{3}{*}{ F28 } & 224F062 & $0-0.5$ & Soil & SC & $\begin{array}{l}\text { TPH-DRO, Isotopic Plutonium, } \\
\text { Gamma Spectroscopy }\end{array}$ \\
\hline & 224F063 & $0-0.5$ & Soil & $\begin{array}{l}\text { Field Duplicate } \\
\text { of \#224F062 }\end{array}$ & $\begin{array}{l}\text { TPH-DRO, Isotopic Plutonium, } \\
\text { Gamma Spectroscopy }\end{array}$ \\
\hline & 224F064 & $3-4$ & Soil & SC & $\begin{array}{l}\text { TPH-DRO, Isotopic Plutonium, } \\
\text { Gamma Spectroscopy }\end{array}$ \\
\hline \multirow{2}{*}{ F29 } & 224F065 & $0-0.5$ & Soil & SC, MS/MSD & $\begin{array}{l}\text { TPH-DRO, Isotopic Plutonium, } \\
\text { Gamma Spectroscopy }\end{array}$ \\
\hline & 224F066 & $3-4$ & Soil & SC & $\begin{array}{l}\text { TPH-DRO, Isotopic Plutonium, } \\
\text { Gamma Spectroscopy }\end{array}$ \\
\hline \multirow{2}{*}{ F30 } & 224F067 & $0-0.5$ & Soil & SC & $\begin{array}{l}\text { TPH-DRO, Isotopic Plutonium, } \\
\text { Gamma Spectroscopy }\end{array}$ \\
\hline & 224F068 & $3-4$ & Soil & SC & $\begin{array}{l}\text { TPH-DRO, Isotopic Plutonium, } \\
\text { Gamma Spectroscopy }\end{array}$ \\
\hline F31 & 224F069 & $0-0.5$ & Soil & SC & Set 1 \\
\hline F32 & $224 \mathrm{~F} 070$ & $0-0.5$ & Soil & SC & Set 1 \\
\hline F33 & 224F071 & $0-0.5$ & Soil & SC & $\begin{array}{l}\text { TPH-DRO, Isotopic Plutonium, } \\
\text { Gamma Spectroscopy }\end{array}$ \\
\hline F34 & 224F072 & $0-0.5$ & Soil & SC & $\begin{array}{l}\text { TPH-DRO, Isotopic Plutonium, } \\
\text { Gamma Spectroscopy }\end{array}$ \\
\hline F35 & 224F073 & $0-0.5$ & Soil & SC & $\begin{array}{l}\text { TPH-DRO, Isotopic Plutonium, } \\
\text { Gamma Spectroscopy }\end{array}$ \\
\hline F36 & 224F074 & $0-0.5$ & Soil & SC & $\begin{array}{l}\text { Gamma Spectroscopy, } \\
\text { Isotopic Plutonium }\end{array}$ \\
\hline F37 & 224F075 & $0-0.5$ & Soil & SC & $\begin{array}{l}\text { Gamma Spectroscopy, } \\
\text { Isotopic Plutonium }\end{array}$ \\
\hline F38 & 224F076 & $0-0.5$ & Soil & SC & $\begin{array}{l}\text { Gamma Spectroscopy, } \\
\text { Isotopic Plutonium }\end{array}$ \\
\hline
\end{tabular}


Table A.7-2

Samples Collected at CAS 06-17-04, Decon Pad and Wastewater Catch (Page 4 of 6 )

\begin{tabular}{|c|c|c|c|c|c|}
\hline $\begin{array}{l}\text { Sample } \\
\text { Location }\end{array}$ & $\begin{array}{l}\text { Sample } \\
\text { Number }\end{array}$ & $\begin{array}{l}\text { Depth } \\
\text { (ft bgs) }\end{array}$ & $\begin{array}{l}\text { Sample } \\
\text { Matrix }\end{array}$ & Purpose & Analyses \\
\hline \multirow{2}{*}{ F39 } & 224F077 & $0-0.5$ & Soil & SC & $\begin{array}{l}\text { Gamma Spectroscopy, } \\
\text { Isotopic Plutonium }\end{array}$ \\
\hline & 224F104 & $1-1.5$ & Soil & SC & $\begin{array}{l}\text { Gamma Spectroscopy, } \\
\text { Isotopic Plutonium }\end{array}$ \\
\hline \multirow{2}{*}{$\mathrm{F} 40$} & 224F078 & $0-0.5$ & Soil & SC & $\begin{array}{l}\text { Gamma Spectroscopy, } \\
\text { Isotopic Plutonium }\end{array}$ \\
\hline & 224F105 & $0.5-1$ & Soil & SC & $\begin{array}{l}\text { Gamma Spectroscopy, } \\
\text { Isotopic Plutonium }\end{array}$ \\
\hline F41 & 224F079 & $0-0.5$ & Soil & SC & $\begin{array}{c}\text { TPH-DRO, Isotopic Plutonium, } \\
\text { Gamma Spectroscopy }\end{array}$ \\
\hline \multirow{2}{*}{ F42 } & 224F080 & $0-0.5$ & Soil & SC & $\begin{array}{c}\text { TPH-DRO, Isotopic Plutonium, } \\
\text { Gamma Spectroscopy }\end{array}$ \\
\hline & 224F090 & $1-1.5$ & Soil & $\mathrm{SC}$ & Isotopic Plutonium \\
\hline \multirow{2}{*}{$\mathrm{F} 43$} & 224F091 & $0-0.5$ & Soil & SC & Gamma Spectroscopy \\
\hline & 224F093 & $1.5-2$ & Soil & SC & Gamma Spectroscopy \\
\hline \multirow{2}{*}{ F44 } & 224F092 & $0-0.5$ & Soil & SC & Gamma Spectroscopy \\
\hline & 224F094 & $1-1.5$ & Soil & SC & Gamma Spectroscopy \\
\hline \multirow{2}{*}{ F45 } & 224F095 & $0-0.5$ & Soil & SC & $\begin{array}{l}\text { Gamma Spectroscopy, } \\
\text { Isotopic Plutonium }\end{array}$ \\
\hline & 224F096 & $1-1.5$ & Soil & SC & $\begin{array}{l}\text { Gamma Spectroscopy, } \\
\text { Isotopic Plutonium }\end{array}$ \\
\hline \multirow{3}{*}{ F46 } & 224F097 & $0.5-1$ & Soil & SC & $\begin{array}{l}\text { Gamma Spectroscopy, } \\
\text { Isotopic Plutonium }\end{array}$ \\
\hline & $224 \mathrm{~F} 098$ & $1-1.5$ & Soil & SC & $\begin{array}{l}\text { Gamma Spectroscopy, } \\
\text { Isotopic Plutonium }\end{array}$ \\
\hline & 224F099 & $1-1.5$ & Soil & $\begin{array}{l}\text { Field Duplicate } \\
\text { of \#224F098 }\end{array}$ & $\begin{array}{l}\text { Gamma Spectroscopy, } \\
\text { Isotopic Plutonium }\end{array}$ \\
\hline \multirow{2}{*}{ F47 } & $224 \mathrm{~F} 100$ & $0-0.5$ & Soil & $S C, Q C$ & $\begin{array}{l}\text { Gamma Spectroscopy, } \\
\text { Isotopic Plutonium }\end{array}$ \\
\hline & 224F101 & $0.5-1.5$ & Soil & SC & $\begin{array}{l}\text { Gamma Spectroscopy, } \\
\text { Isotopic Plutonium }\end{array}$ \\
\hline \multirow{2}{*}{ F48 } & 224F102 & $0-0.5$ & Soil & SC & $\begin{array}{l}\text { Gamma Spectroscopy, } \\
\text { Isotopic Plutonium }\end{array}$ \\
\hline & 224F103 & $0.5-1$ & Soil & SC & $\begin{array}{l}\text { Gamma Spectroscopy, } \\
\text { Isotopic Plutonium }\end{array}$ \\
\hline \multirow{2}{*}{ F49 } & 224F085 & $0-0.5$ & Soil & SC & $\begin{array}{l}\text { Gamma Spectroscopy, } \\
\text { Isotopic Plutonium }\end{array}$ \\
\hline & $224 \mathrm{~F} 086$ & $1-1.5$ & Soil & SC & $\begin{array}{l}\text { Gamma Spectroscopy, } \\
\text { Isotopic Plutonium }\end{array}$ \\
\hline
\end{tabular}


Table A.7-2

Samples Collected at CAS 06-17-04, Decon Pad and Wastewater Catch (Page 5 of 6 )

\begin{tabular}{|c|c|c|c|c|c|}
\hline $\begin{array}{l}\text { Sample } \\
\text { Location }\end{array}$ & $\begin{array}{l}\text { Sample } \\
\text { Number }\end{array}$ & $\begin{array}{l}\text { Depth } \\
\text { (ft bgs) }\end{array}$ & $\begin{array}{l}\text { Sample } \\
\text { Matrix }\end{array}$ & Purpose & Analyses \\
\hline \multirow{2}{*}{ F50 } & 224F087 & $0-0.5$ & Soil & SC & $\begin{array}{l}\text { Gamma Spectroscopy, } \\
\text { Isotopic Plutonium }\end{array}$ \\
\hline & 224F088 & $1-1.5$ & Soil & SC & $\begin{array}{l}\text { Gamma Spectroscopy, } \\
\text { Isotopic Plutonium }\end{array}$ \\
\hline \multirow{2}{*}{ F51 } & 224F089 & $0-0.5$ & Soil & SC & $\begin{array}{l}\text { Gamma Spectroscopy, } \\
\text { Isotopic Plutonium }\end{array}$ \\
\hline & 224F106 & $1-1.5$ & Soil & SC & $\begin{array}{l}\text { Gamma Spectroscopy, } \\
\text { Isotopic Plutonium }\end{array}$ \\
\hline F52 & 224F107 & $0-0.5$ & Soil & SC & $\begin{array}{l}\text { Gamma Spectroscopy, } \\
\text { Isotopic Plutonium }\end{array}$ \\
\hline F53 & 224F108 & $0-0.5$ & Soil & SC & $\begin{array}{l}\text { Gamma Spectroscopy, } \\
\text { Isotopic Plutonium }\end{array}$ \\
\hline \multirow{2}{*}{ F54 } & 224F109 & $0-0.5$ & Soil & SC & $\begin{array}{l}\text { Gamma Spectroscopy, } \\
\text { Isotopic Plutonium }\end{array}$ \\
\hline & $224 \mathrm{~F} 110$ & $0.5-1.5$ & Soil & SC & $\begin{array}{l}\text { Gamma Spectroscopy, } \\
\text { Isotopic Plutonium }\end{array}$ \\
\hline F55 & 224F111 & $0-0.5$ & Soil & SC & $\begin{array}{l}\text { Gamma Spectroscopy, } \\
\text { Isotopic Plutonium }\end{array}$ \\
\hline F56 & 224F112 & $0-0.5$ & Soil & SC & $\begin{array}{l}\text { Gamma Spectroscopy, } \\
\text { Isotopic Plutonium }\end{array}$ \\
\hline \multirow{2}{*}{ F57 } & $224 \mathrm{~F} 113$ & $0-0.5$ & Soil & SC & $\begin{array}{l}\text { Gamma Spectroscopy, } \\
\text { Isotopic Plutonium }\end{array}$ \\
\hline & $224 \mathrm{~F} 114$ & $0.5-1.5$ & Soil & SC & $\begin{array}{l}\text { Gamma Spectroscopy, } \\
\text { Isotopic Plutonium }\end{array}$ \\
\hline F58 & $224 \mathrm{~F} 115$ & $0-0.5$ & Soil & SC & $\begin{array}{l}\text { Gamma Spectroscopy, } \\
\text { Isotopic Plutonium }\end{array}$ \\
\hline F59 & 224F116 & $0-0.5$ & Soil & SC & $\begin{array}{l}\text { Gamma Spectroscopy, } \\
\text { Isotopic Plutonium }\end{array}$ \\
\hline F60 & $224 \mathrm{~F} 117$ & $0-0.5$ & Soil & SC & $\begin{array}{l}\text { Gamma Spectroscopy, } \\
\text { Isotopic Plutonium }\end{array}$ \\
\hline F61 & 224F118 & $0-0.5$ & Soil & SC & $\begin{array}{l}\text { Gamma Spectroscopy, } \\
\text { Isotopic Plutonium }\end{array}$ \\
\hline F62 & 224F119 & $0-0.5$ & Soil & SC & $\begin{array}{l}\text { Gamma Spectroscopy, } \\
\text { Isotopic Plutonium }\end{array}$ \\
\hline \multirow{3}{*}{ F63 } & $224 \mathrm{~F} 120$ & $0-0.5$ & Soil & SC & $\begin{array}{l}\text { Gamma Spectroscopy, } \\
\text { Isotopic Plutonium }\end{array}$ \\
\hline & 224F121 & $0-0.5$ & Soil & $\begin{array}{l}\text { Field Duplicate } \\
\text { of \#224F120 }\end{array}$ & $\begin{array}{l}\text { Gamma Spectroscopy, } \\
\text { Isotopic Plutonium }\end{array}$ \\
\hline & 224F122 & $1-2$ & Soil & $S C, Q C$ & $\begin{array}{l}\text { Gamma Spectroscopy, } \\
\text { Isotopic Plutonium }\end{array}$ \\
\hline
\end{tabular}


Table A.7-2

Samples Collected at CAS 06-17-04, Decon Pad and Wastewater Catch (Page 6 of 6 )

\begin{tabular}{|c|c|c|c|c|c|}
\hline $\begin{array}{l}\text { Sample } \\
\text { Location }\end{array}$ & $\begin{array}{l}\text { Sample } \\
\text { Number }\end{array}$ & $\begin{array}{c}\text { Depth } \\
\text { (ft bgs) }\end{array}$ & $\begin{array}{l}\text { Sample } \\
\text { Matrix }\end{array}$ & Purpose & Analyses \\
\hline F64 & 224F123 & $0-0.5$ & Soil & SC & $\begin{array}{l}\text { Gamma Spectroscopy, } \\
\text { Isotopic Plutonium }\end{array}$ \\
\hline F65 & 224F124 & $0-0.5$ & Soil & SC & $\begin{array}{l}\text { Gamma Spectroscopy, } \\
\text { Isotopic Plutonium }\end{array}$ \\
\hline \multirow{2}{*}{ F66 } & 224F125 & $0-0.5$ & Soil & $\mathrm{SC}$ & $\begin{array}{l}\text { Gamma Spectroscopy, } \\
\text { Isotopic Plutonium }\end{array}$ \\
\hline & $224 \mathrm{~F} 126$ & $1.5-2.5$ & Soil & SC & $\begin{array}{l}\text { Gamma Spectroscopy, } \\
\text { Isotopic Plutonium }\end{array}$ \\
\hline F67 & $224 \mathrm{~F} 127$ & $0-0.5$ & Soil & SC & $\begin{array}{l}\text { Gamma Spectroscopy, } \\
\text { Isotopic Plutonium }\end{array}$ \\
\hline F68 & 224F128 & $0-0.5$ & Soil & SC & $\begin{array}{l}\text { Gamma Spectroscopy, } \\
\text { Isotopic Plutonium }\end{array}$ \\
\hline F69 & $224 \mathrm{~F} 129$ & $0-0.5$ & Soil & SC & $\begin{array}{l}\text { Gamma Spectroscopy, } \\
\text { Isotopic Plutonium }\end{array}$ \\
\hline NA & $224 \mathrm{~F} 301$ & NA & Water & Trip Blank & Total VOCs \\
\hline NA & $224 \mathrm{~F} 302$ & NA & Water & Trip Blank & Total VOCs \\
\hline NA & $224 \mathrm{~F} 303$ & NA & Water & $\begin{array}{c}\text { Equipment } \\
\text { Rinsate Blank }\end{array}$ & Set 1 \\
\hline NA & $224 \mathrm{~F} 304$ & NA & Water & Trip Blank & Total VOCs \\
\hline NA & $224 \mathrm{~F} 305$ & NA & Water & Trip Blank & Total VOCs \\
\hline NA & $224 \mathrm{~F} 306$ & NA & Water & Trip Blank & Total VOCs \\
\hline NA & 224F502 & NA & Liquid & WM & $\begin{array}{c}\text { Gross Alpha/Beta, } \\
\text { Tritium }\end{array}$ \\
\hline
\end{tabular}

Set 1 = Total VOCs, Total SVOCs, Total RCRA Metals, Aluminum, Antimony, Beryllium, Cobalt, Copper, Manganese, Molybdenum, Nickel, Zinc, TPH-DRO, PCBs, Gamma Spectroscopy, Isotopic Plutonium, Isotopic Uranium, and Strontium-90. Set 2 = TCLP VOCs, TCLP SVOCs, and TCLP RCRA Metals.

DRO = Diesel-range organics

$\mathrm{ft}$ bgs $=$ Feet below ground surface

MS/MSD = Matrix spike/matrix spike duplicate

NA $=$ Not applicable

$\mathrm{PCB}=$ Polychlorinated biphenyl

RCRA = Resource Conservation and Recovery Act

$\mathrm{SC}=$ Site characterization

SVOC = Semivolatile organic compound

TCLP $=$ Toxicity characteristic leaching procedure

$\mathrm{TPH}=$ Total petroleum hydrocarbons

$\mathrm{WM}=$ Waste Management

$\mathrm{VOC}=$ Volatile organic compound 
Table A.7-3

Samples Collected at CAS 06-23-01, Decon Pad Discharge Piping

\begin{tabular}{||c|c|c|c|c|c||}
\hline $\begin{array}{c}\text { Sample } \\
\text { Location }\end{array}$ & $\begin{array}{c}\text { Sample } \\
\text { Number }\end{array}$ & $\begin{array}{c}\text { Depth } \\
\text { (ft bgs) }\end{array}$ & $\begin{array}{c}\text { Sample } \\
\text { Matrix }\end{array}$ & Purpose & Analyses \\
\hline \hline \multirow{4}{*}{ G01 } & $224 G 001$ & $0-1$ & Soil & SC & Set 1 \\
\cline { 2 - 6 } & $224 G 002$ & $0-1$ & Soil & $\begin{array}{c}\text { Field Duplicate } \\
\text { of \#224G001 }\end{array}$ & Set 1 \\
\cline { 2 - 6 } & $224 G 003$ & $2-3$ & Soil & SC, MS/MSD & Set 1 \\
\cline { 2 - 6 } & $224 G 004$ & $3-4$ & Soil & SC & Set 1 \\
\hline \multirow{3}{*}{ G02 } & $224 G 005$ & $0-1$ & Soil & SC & Set 1 \\
\cline { 2 - 7 } & $224 G 007$ & $0-0.5$ & Soil & SC & Chlordane \\
\hline \multirow{2}{*}{ G03 } & $224 G 006$ & $0-1$ & Soil & SC & Set 1 \\
\cline { 2 - 7 } & $224 G 008$ & $0-0.5$ & Soil & SC & Chlordane \\
\hline
\end{tabular}

Set 1 = Total VOCs, Total SVOCs, Total RCRA Metals, Aluminum, Antimony, Beryllium, Cobalt, Copper, Manganese, Molybdenum, Nickel, Zinc, TPH-DRO, PCBs, Gamma Spectroscopy, Isotopic Plutonium, Isotopic Uranium, and Strontium-90.

$\mathrm{DRO}=$ Diesel-range organics $\mathrm{ft}$ bgs $=$ Feet below ground surface MS/MSD = Matrix spike/matrix spike duplicate NA $=$ Not applicable

PCB $=$ Polychlorinated biphenyl
RCRA = Resource Conservation and Recovery Act $\mathrm{SC}=$ Site characterization SVOC $=$ Semivolatile organic compound $\mathrm{TPH}=$ Total petroleum hydrocarbons

$\mathrm{VOC}=$ Volatile organic compound

were collected using disposable scoops and pans. Subsurface samples at CAS 06-05-01 were collected using a backhoe, while a rotosonic drill rig was used at CAS 06-17-04, and a hand-auger was used at CAS 06-23-01. The samples were analyzed for the parameters listed in Table A.7-1 through Table A.7-3. Sample locations are shown on Figure A.7-1.

\section{A.7.1.3 Deviations}

There were no significant deviations to the CAIP requirements at these CASs. Some locations had to be added to CAS 06-05-01, Leachfield, in order to delineate the extent of the leachfield. The investigation and sampling at CASs 06-05-01, 06-17-04, and 06-23-01 are considered sufficient to meet the DQOs.

\section{A.7.2 Investigation Results}

The following sections provide descriptions of the CAS-specific activities conducted to complete investigation activities as outlined in the CAIP. Investigation activities included visual inspection of 


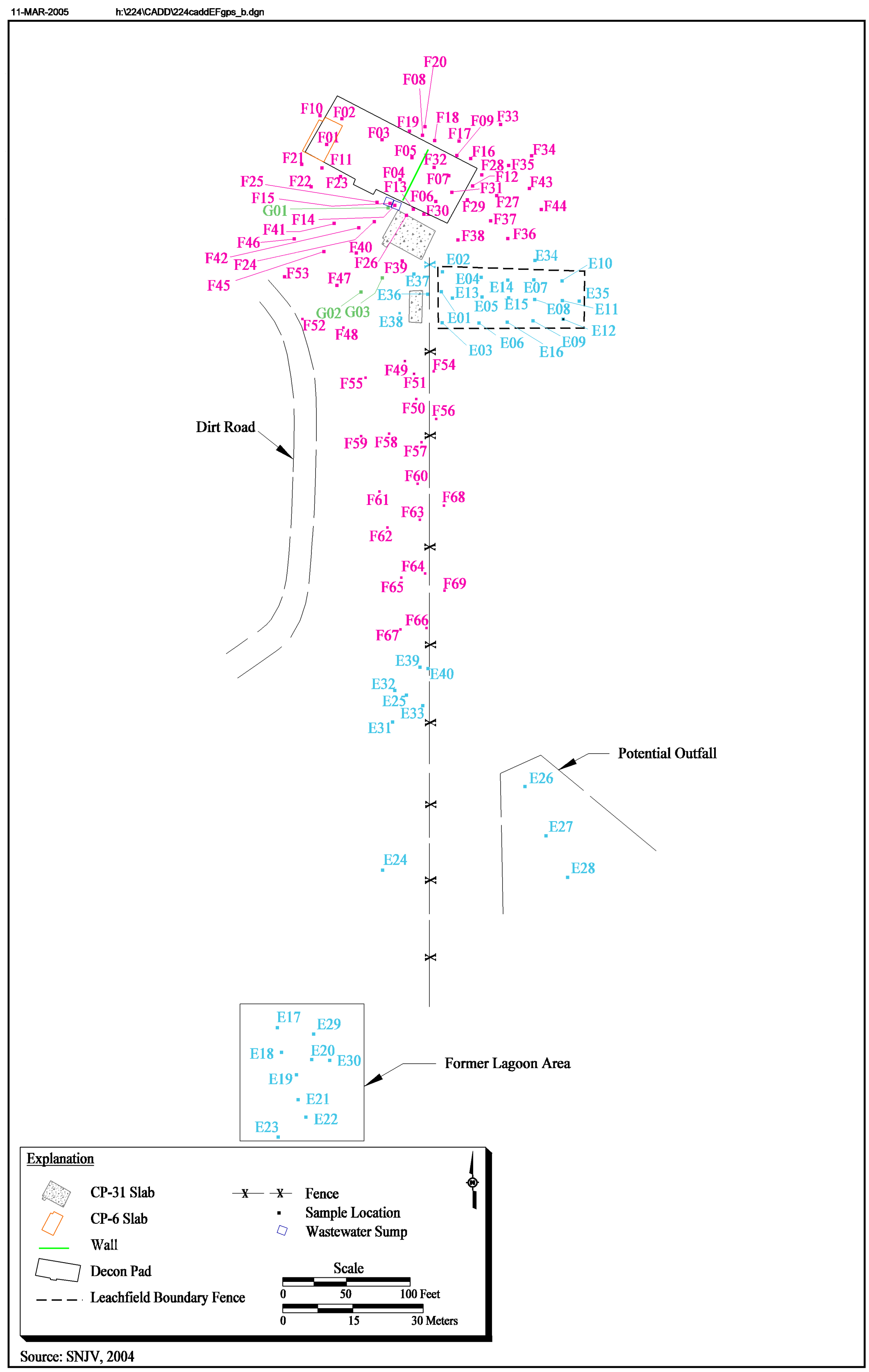

Figure A.7-1

Sample Location Map, CAS 06-05-01 (E), CAS 06-17-04 (F), and CAS 06-23-01 (G) 
the CASs and collection of surface and subsurface soil samples, as well as concrete and sludge samples, as outlined in Section A.6.1.2.

Surface and subsurface soil samples, sludge samples, and the concrete sample were analyzed for the CAIP-specified COPCs, which included total VOCs, total SVOCs, TPH-DRO, total metals, PCBs, gamma-emitting radionuclides, plutonium isotopes, uranium isotopes, and strontium-90. The two sludge samples were also analyzed for TCLP VOCs, TCLP SVOCs, and TCLP RCRA metals. The analytical parameters and laboratory methods used to analyze the investigation samples are listed in Table A.2-2. Table A.7-1 through Table A.7-3 list the sample-specific analytical suite for the three CASs. A portion of the analytical results for these CASs was rejected during validation; however, these rejected data did not adversely impact closure decisions as discussed in Appendix B, Section B.1.4.

\section{A.7.2.1 Total Volatile Organic Compounds}

Total VOC analytical results for soil samples collected at CAS 06-05-01 that were detected above MRLs are presented in Table A.7-4. No VOCs were detected above reporting limits in soil samples at CASs 06-17-04 and 06-23-01. No VOCs were detected in soil samples above FALs.

Table A.7-4

Soil Sample Results for Total VOCs Detected Above Minimum Reporting Limits at CAS 06-05-01, Leachfield

\begin{tabular}{|c|c|c|c|}
\hline \multirow{2}{*}{$\begin{array}{l}\text { Sample } \\
\text { Location }\end{array}$} & \multirow{2}{*}{$\begin{array}{c}\text { Sample } \\
\text { Number }\end{array}$} & \multirow{2}{*}{$\begin{array}{l}\text { Depth } \\
\text { (ft bgs) }\end{array}$} & Contaminants of Potential Concern $(\mu \mathrm{g} / \mathrm{kg})$ \\
\hline & & & Methylene Chloride \\
\hline \multicolumn{3}{|c|}{ Final Action Levels ${ }^{a}$} & 21,000 \\
\hline$\overline{\mathrm{E} 18}$ & 224E071 & $77-8$ & $\overline{11(\mathrm{~J})}$ \\
\hline
\end{tabular}

aased on U.S. Environmental Protection Agency, Region 9 Preliminary Remediation Goals (PRGs) (EPA, 2002)

$\mathrm{ft}$ bgs $=$ Feet below ground surface $\mu \mathrm{g} / \mathrm{kg}=$ Micrograms per kilogram

$\mathrm{J}=$ Estimated value. Qualifier added to laboratory data; record accepted. Value was $<10 \mathrm{x}$ the contamination in the calibration/method blank. 


\section{A.7.2.2 Total Semivolatile Organic Compounds}

Total SVOC analytical results for soil samples collected at CASs 06-05-01, 06-17-04, and 06-23-01 that were detected above MRLs are presented in Table A.7-5 through Table A.7-7. No SVOCs were detected in soil samples above FALs.

Table A.7-5

Soil Sample Results for Total SVOCs Detected

Above Minimum Reporting Limits at CAS 06-05-01, Leachfield

\begin{tabular}{|c|c|c|c|c|c|c|c|c|}
\hline \multirow[b]{2}{*}{$\begin{array}{c}\text { Sample } \\
\text { Location }\end{array}$} & \multirow[b]{2}{*}{$\begin{array}{l}\text { Sample } \\
\text { Number }\end{array}$} & \multirow[b]{2}{*}{$\begin{array}{c}\text { Depth } \\
\text { (ft bgs) }\end{array}$} & \multicolumn{6}{|c|}{ Contaminants of Potential Concern $(\mu \mathrm{g} / \mathrm{kg})$} \\
\hline & & & 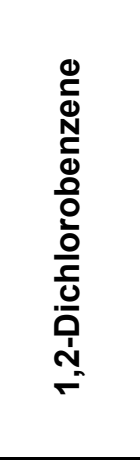 & 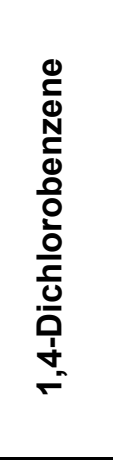 & 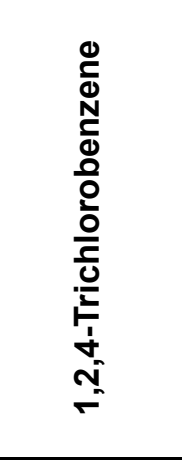 & 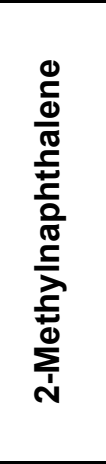 & 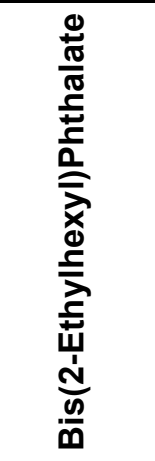 & 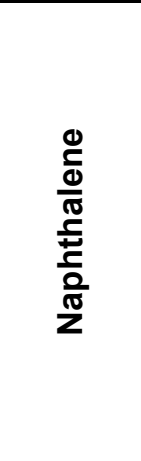 \\
\hline \multicolumn{3}{|c|}{ Final Action Levels ${ }^{a}$} & 370,000 & 7,900 & $3,100,000$ & NI & 120,000 & 190,000 \\
\hline \multirow{2}{*}{ E04 } & 224E008 & $0-0.5$ & 2,200 & 380 & 1,200 & 1,300 & $\overline{---}$ & $\overline{980}$ \\
\hline & 224E052 & $3.5-4.5$ & 400 & -- & -- & -- & 390 & -- \\
\hline
\end{tabular}

aased on U.S. Environmental Protection Agency, Region 9 Preliminary Remediation Goals (PRGs) (EPA, 2002)

$\mathrm{ft}$ bgs $=$ Feet below ground surface $\mu \mathrm{g} / \mathrm{kg}=$ Micrograms per kilogram

$--=$ Not detected above minimum reporting limits

Table A.7-6

Soil Sample Results for Total SVOCs Detected Above Minimum Reporting Limits at CAS 06-17-04, Decon Pad and Wastewater Catch

\begin{tabular}{|c|c|c|c|c|}
\hline \multirow{2}{*}{$\begin{array}{l}\text { Sample } \\
\text { Location }\end{array}$} & \multirow{2}{*}{$\begin{array}{l}\text { Sample } \\
\text { Number }\end{array}$} & \multirow{2}{*}{$\begin{array}{l}\text { Depth } \\
\text { (ft bgs) }\end{array}$} & \multicolumn{2}{|c|}{ Contaminants of Potential Concern $(\mu \mathrm{g} / \mathrm{kg})$} \\
\hline & & & Bis(2-Ethylhexyl)Phthalate & Butyl Benzyl Phthalate \\
\hline \multicolumn{3}{|c|}{ Final Action Levels ${ }^{a}$} & 120,000 & $100,000,000$ \\
\hline F10 & $224 \mathrm{~F} 030$ & $0-1$ & $\overline{---}$ & 440 \\
\hline F32 & $224 \mathrm{~F} 070$ & $0-0.5$ & 780 & -- \\
\hline
\end{tabular}

äBased on U.S. Environmental Protection Agency, Region 9 Preliminary Remediation Goals (PRGs) (EPA, 2002)

$\mathrm{ft}$ bgs = Feet below ground surface

$\mu \mathrm{g} / \mathrm{kg}=$ Micrograms per kilogram 
Table A.7-7

Soil Sample Results for Total SVOCs Detected

Above Minimum Reporting Limits at CAS 06-23-01, Decon Pad Discharge Piping

\begin{tabular}{|c|c|c|c|}
\hline \multirow{2}{*}{$\begin{array}{l}\text { Sample } \\
\text { Location }\end{array}$} & \multirow{2}{*}{$\begin{array}{l}\text { Sample } \\
\text { Number }\end{array}$} & \multirow{2}{*}{$\begin{array}{l}\text { Depths } \\
\text { (ft bgs) }\end{array}$} & Contaminants of Potential Concern $(\mu \mathrm{g} / \mathrm{kg})$ \\
\hline & & & Bis(2-Ethylhexyl)Phthalate \\
\hline \multicolumn{3}{|c|}{ Final Action Levels ${ }^{a}$} & 120,000 \\
\hline \multirow{4}{*}{ G01 } & $224 \mathrm{G} 001$ & $0-1$ & $\overline{5,200(\mathrm{~J})}$ \\
\hline & $224 \mathrm{G} 002$ & $0-1$ & $7,700(\mathrm{~J})$ \\
\hline & $224 G 003$ & $2-3$ & $7,900(\mathrm{~J})$ \\
\hline & 224G004 & $3-4$ & 1,100 \\
\hline
\end{tabular}

a'Based on U.S. Environmental Protection Agency, Region 9 Preliminary Remediation Goals (PRGs) (EPA, 2002)

$\mathrm{ft}$ bgs $=$ Feet below ground surface

$\mu \mathrm{g} / \mathrm{kg}=$ Micrograms per kilogram

$\mathrm{J}=$ Estimated value. Qualifier added to laboratory data; record accepted. Matrix effects may exist. Internal standard area count outside control limits.

Surrogate recovery exceeded the upper limits.

\section{A.7.2.3 Total Metals}

Total metals analytical results for soil samples collected at CASs 06-05-01, 06-17-04, and 06-23-01 are presented in Table A.7-8 through Table A.7-10. No metals were detected in soil samples above FALs.

\section{A.7.2.4 Polychlorinated Biphenyls}

Total PCB analytical results for soil samples collected at CASs 06-05-01, 06-17-04, and 06-23-01 that were detected above MRLs are presented in Table A.7-11 through Table A.7-13. No PCBs were detected in soil samples above FALs.

\section{A.7.2.5 Total Petroleum Hydrocarbons-Diesel-Range Organics}

Total TPH-DRO analytical results for soil samples collected at CASs 06-05-01, 06-17-04, and 06-23-01 that were detected above MRLs are presented in Table A.7-14 through Table A.7-16. Total petroleum hydrocarbons-DRO was detected in several soil samples above FALs. 
Table A.7-8

Soil Sample Results for Metals Detected Above Minimum Reporting Limits at CAS 06-05-01, Leachfield (Page 1 of 6$)$

\begin{tabular}{|c|c|c|c|c|c|c|c|c|c|c|c|c|c|c|c|c|c|}
\hline \multirow[b]{2}{*}{$\begin{array}{l}\text { Sample } \\
\text { Location }\end{array}$} & \multirow[b]{2}{*}{$\begin{array}{l}\text { Sample } \\
\text { Number }\end{array}$} & \multirow[b]{2}{*}{$\begin{array}{c}\text { Depth } \\
\text { (ft bgs) }\end{array}$} & \multicolumn{15}{|c|}{ Contaminants of Potential Concern $(\mathrm{mg} / \mathrm{kg})$} \\
\hline & & & 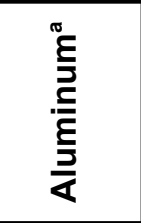 & 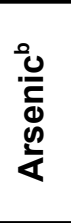 & 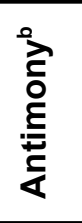 & 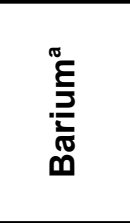 & 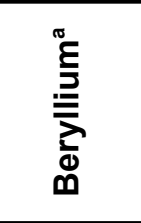 & 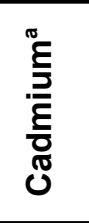 & 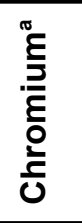 & $\begin{array}{l}\frac{\pi}{\pi} \\
\frac{0}{\pi} \\
0 \\
0\end{array}$ & $\begin{array}{l}\frac{0}{0} \\
\frac{0}{0} \\
\frac{0}{0}\end{array}$ & 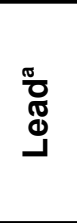 & 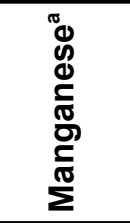 & $\begin{array}{l}\stackrel{0}{Z} \\
\frac{0}{0} \\
\frac{0}{d} \\
\sum\end{array}$ & $\begin{array}{l}\frac{\sigma}{0} \\
\frac{x}{0} \\
\frac{\underline{z}}{Z}\end{array}$ & 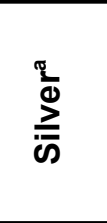 & $\stackrel{0}{\text { Ñ }}$ \\
\hline \multicolumn{3}{|c|}{ Final Action Levels } & 100,000 & 23 & 410 & 67,000 & 1,900 & 450 & 450 & 1,900 & 41,000 & 750 & 19,000 & 310 & 20,000 & 5,100 & 100,000 \\
\hline \multirow{4}{*}{ E01 } & 224E001 & $0-0.5$ & 6,500 & 5.6 & $\overline{--}$ & 98 & $0.52(\mathrm{~J})^{\mathrm{c}}$ & $\overline{---}$ & 6.1 & 3.2 & 11 & 38 & $220(\mathrm{~J})^{\mathrm{d}}$ & $\overline{--}$ & 6.1 & $\overline{--}$ & 87 \\
\hline & 224E002 & $3-4$ & 7,500 & 6.2 & -- & 91 & $0.57(\mathrm{~J})^{\mathrm{c}}$ & -- & 5 & 3.2 & 6.8 & 8.9 & $200(J)^{d}$ & -- & 5.8 & -- & 20 \\
\hline & 224E003 & $3-4$ & 7,400 & 6 & -- & 87 & $0.56(\mathrm{~J})^{\mathrm{c}}$ & -- & 4.8 & 3.2 & 6.9 & 8.5 & $200(\mathrm{~J})^{\mathrm{d}}$ & -- & 5.7 & -- & 20 \\
\hline & 224E004 & $5-6$ & 5,600 & 6 & -- & 77 & -- & -- & 4.2 & 2.8 & 5.4 & 7 & $210(\mathrm{~J})^{\mathrm{d}}$ & -- & 5.1 & -- & 18 \\
\hline \multirow{3}{*}{ E02 } & 224E005 & $0-0.5$ & 5,800 & 6.1 & -- & 77 & -- & 0.65 & 6.3 & 3.4 & 20 & 25 & 200 & -- & 11 & -- & 1,300 \\
\hline & 224E007 & $4-5$ & 7,400 & 6 & -- & 83 & 0.56 & -- & 4.6 & 3.1 & 7 & 7.3 & 180 & -- & 5.5 & -- & 19 \\
\hline & 224E009 & $7-8$ & 7,600 & 5.3 & -- & 75 & 0.58 & -- & 4.2 & 3.1 & 6.8 & 6.8 & 170 & -- & 5.3 & -- & 18 \\
\hline \multirow{3}{*}{ E03 } & 224E006 & $0-0.5$ & 7,800 & 6 & -- & 94 & 0.6 & -- & 6.5 & 3.6 & 9.5 & 23 & 210 & -- & 6.4 & -- & 58 \\
\hline & 224E013 & $4-5$ & 8,400 & 7 & $\overline{--}$ & 96 & 0.62 & $\overline{--}$ & 5.7 & 3.8 & 7.6 & 9.9 & 220 & $\overline{--}$ & 6.5 & -- & 24 \\
\hline & 224E016 & $7-8$ & 8,000 & 6.2 & $\overline{--}$ & 81 & 0.61 & -- & 4.8 & 3.4 & 7.6 & 7.9 & 170 & -- & 6.3 & -- & 21 \\
\hline \multirow{4}{*}{ E04 } & 224E008 & $0-0.5$ & 6,100 & 5.8 & -- & 91 & -- & $\overline{--}$ & 5.6 & 2.9 & 9 & 40 & 210 & -- & 5.5 & -- & 89 \\
\hline & 224E052 & $3.5-4.5$ & 7,300 & 6.2 & $\overline{--}$ & $89(\mathrm{~J})^{\mathrm{C}}$ & $0.53(\mathrm{~J})^{\mathrm{c}}$ & -- & 5 & $3.5(\mathrm{~J})^{\mathrm{c}}$ & 7.2 & 7.5 & $210(\mathrm{~J})^{\mathrm{c}}$ & $\overline{--}$ & 6 & $\overline{--}$ & $21(\mathrm{~J})^{\mathrm{C}}$ \\
\hline & 224E053 & $7-8$ & 7,900 & 5.4 & -- & $89(\mathrm{~J})^{\mathrm{C}}$ & $0.57(\mathrm{~J})^{\mathrm{c}}$ & -- & 5.4 & $2.5(\mathrm{~J})^{\mathrm{C}}$ & 8.5 & 17 & $170(\mathrm{~J})^{\mathrm{C}}$ & -- & 5.8 & -- & $24(J)^{c}$ \\
\hline & 224E054 & $9-10$ & 6,200 & 6.4 & -- & $61(\mathrm{~J})^{\mathrm{c}}$ & -- & -- & 3.7 & $2.2(\mathrm{~J})^{\mathrm{c}}$ & 6.6 & 8.3 & $130(\mathrm{~J})^{\mathrm{c}}$ & -- & 5 & -- & $16(\mathrm{~J})^{\mathrm{c}}$ \\
\hline
\end{tabular}


Table A.7-8

Soil Sample Results for Metals Detected Above Minimum Reporting Limits at CAS 06-05-01, Leachfield (Page 2 of 6 )

\begin{tabular}{|c|c|c|c|c|c|c|c|c|c|c|c|c|c|c|c|c|c|}
\hline \multirow[b]{2}{*}{$\begin{array}{l}\text { Sample } \\
\text { Location }\end{array}$} & \multirow[b]{2}{*}{$\begin{array}{l}\text { Sample } \\
\text { Number }\end{array}$} & \multirow[b]{2}{*}{$\begin{array}{c}\text { Depth } \\
\text { (ft bgs) }\end{array}$} & \multicolumn{15}{|c|}{ Contaminants of Potential Concern $(\mathrm{mg} / \mathrm{kg})$} \\
\hline & & & 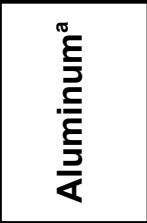 & 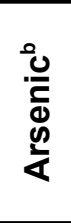 & 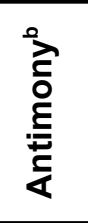 & 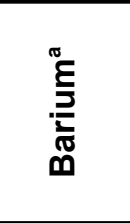 & 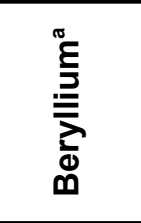 & 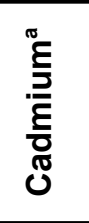 & 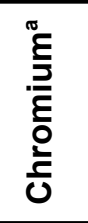 & $\begin{array}{l}\frac{\pi}{\pi} \\
\frac{0}{\pi} \\
0 \\
0\end{array}$ & $\begin{array}{l}\frac{0}{0} \\
\frac{0}{0} \\
\frac{0}{0}\end{array}$ & ণ్ర & 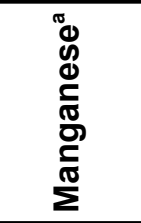 & $\begin{array}{l}\stackrel{0}{Z} \\
\frac{0}{0} \\
\frac{0}{d} \\
\sum\end{array}$ & $\begin{array}{l}\frac{\sigma}{0} \\
\frac{x}{0} \\
\frac{\underline{z}}{Z}\end{array}$ & 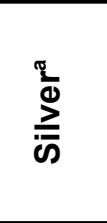 & $\stackrel{0}{\text { Ñ }}$ \\
\hline \multicolumn{3}{|c|}{ Final Action Levels } & 100,000 & 23 & 410 & 67,000 & 1,900 & 450 & 450 & 1,900 & 41,000 & 750 & 19,000 & 310 & 20,000 & 5,100 & 100,000 \\
\hline \multirow{5}{*}{ E05 } & 224E010 & $0-0.5$ & 6,200 & $\overline{5.6}$ & $\overline{---}$ & 95 & $\overline{--}$ & $\overline{--}$ & 6.2 & 2.9 & 9.3 & $\overline{41}$ & 210 & $\overline{---}$ & 5.8 & $\overline{--}$ & $\overline{58}$ \\
\hline & 224E020 & $4-5$ & 7,300 & 5.9 & $\overline{--}$ & 89 & $0.56(\mathrm{~J})^{\mathrm{c}}$ & $\overline{--}$ & 5.1 & 3.4 & 7 & 8.9 & $220(\mathrm{~J})^{\mathrm{d}}$ & $\overline{--}$ & 6 & $\overline{--}$ & 22 \\
\hline & 224E021 & $4-5$ & 7,600 & 6.3 & -- & 95 & $0.58(\mathrm{~J})^{\mathrm{c}}$ & -- & 5.3 & 3.4 & 7.2 & 9.2 & $230(\mathrm{~J})^{\mathrm{d}}$ & -- & 6 & -- & 22 \\
\hline & 224E022 & $7-8$ & 9,000 & 6.4 & -- & 84 & $0.68(J)^{c}$ & -- & 4.8 & 2.8 & 7.7 & 12 & $190(\mathrm{~J})^{\mathrm{d}}$ & -- & 5.8 & -- & 22 \\
\hline & 224E023 & $9-10$ & 7,500 & 6.3 & -- & 78 & $0.59(\mathrm{~J})^{\mathrm{c}}$ & -- & 4.2 & 2.9 & 6.7 & 9.6 & $180(\mathrm{~J})^{\mathrm{d}}$ & -- & 5.4 & -- & 19 \\
\hline \multirow{4}{*}{ E06 } & 224E011 & $0-0.5$ & 6,400 & 5.7 & -- & 82 & -- & -- & 5 & 2.9 & 7.5 & 43 & 200 & -- & 5.3 & -- & 35 \\
\hline & 224E055 & $3.5-4.5$ & 7,200 & 6.6 & -- & $91(\mathrm{~J})^{\mathrm{C}}$ & $0.53(\mathrm{~J})^{\mathrm{c}}$ & $\begin{array}{l}- \\
\end{array}$ & 5.1 & $3.2(\mathrm{~J})^{\mathrm{c}}$ & 7.2 & 8.8 & $210(\mathrm{~J})^{\mathrm{C}}$ & -- & 6 & -- & $22(\mathrm{~J})^{\mathrm{C}}$ \\
\hline & 224E056 & $7-8$ & 6,900 & 5.9 & -- & $89(\mathrm{~J})^{\mathrm{C}}$ & $0.53(\mathrm{~J})^{\mathrm{c}}$ & -- & 5 & $3.2(\mathrm{~J})^{\mathrm{C}}$ & 7 & 7.9 & $200(J)^{C}$ & $\overline{--}$ & 5.9 & $\overline{--}$ & $20(J)^{c}$ \\
\hline & 224E057 & $9-10$ & 6,800 & 5.4 & $\overline{--}$ & $73(\mathrm{~J})^{\mathrm{c}}$ & $\overline{--}$ & $\overline{--}$ & 4.1 & $2.3(\mathrm{~J})^{\mathrm{c}}$ & 6 & 8.6 & $150(\mathrm{~J})^{\mathrm{C}}$ & $\overline{--}$ & 4.8 & $\overline{--}$ & $18(\mathrm{~J})^{\mathrm{c}}$ \\
\hline \multirow{3}{*}{ E07 } & 224E012 & $0-0.5$ & 6,000 & 5.7 & $\overline{--}$ & 90 & -- & -- & 5.4 & 2.8 & 8.9 & 100 & 200 & -- & 5.3 & $\overline{--}$ & 42 \\
\hline & 224E037 & $5-6$ & 8,500 & 5.7 & -- & $90(\mathrm{~J})^{\mathrm{C}}$ & $0.62(\mathrm{~J})^{\mathrm{c}}$ & -- & 5.1 & 3 & 6.9 & 11 & $230(\mathrm{~J})^{\mathrm{C}}$ & -- & 5.9 & -- & 23 \\
\hline & 224E038 & $9-10$ & 9,500 & 5.1 & $\overline{--}$ & $72(\mathrm{~J})^{\mathrm{C}}$ & $0.69(\mathrm{~J})^{\mathrm{c}}$ & $\overline{--}$ & 3.7 & 3.8 & 6.3 & 8 & $150(J)^{c}$ & -- & 4.9 & -- & 18 \\
\hline \multirow{4}{*}{ E08 } & 224E014 & $0-0.5$ & 6,100 & 5.6 & -- & 85 & -- & $\overline{--}$ & 4.9 & 2.8 & 9 & 31 & 200 & -- & 5.3 & $\overline{--}$ & 29 \\
\hline & 224E025 & $2-3$ & 6,900 & 8 & -- & 85 & $0.55(\mathrm{~J})^{\mathrm{c}}$ & -- & 4.8 & 3.1 & 6.4 & 7.9 & $200(J)^{d}$ & $\overline{--}$ & 5.8 & -- & 21 \\
\hline & 224E026 & $7-8$ & 5,100 & 4.4 & -- & 55 & -- & -- & 2.7 & 1.9 & $\overline{4}$ & 7.4 & $130(\mathrm{~J})^{\mathrm{d}}$ & -- & 3.6 & -- & 14 \\
\hline & 224E027 & $9-10$ & 7,400 & 5.5 & -- & 76 & $0.57(\mathrm{~J})^{\mathrm{c}}$ & -- & 3.6 & 2.4 & 5.7 & 7.9 & $160(\mathrm{~J})^{\mathrm{d}}$ & -- & 4.6 & -- & 17 \\
\hline
\end{tabular}


Table A.7-8

Soil Sample Results for Metals Detected Above Minimum Reporting Limits at CAS 06-05-01, Leachfield (Page 3 of 6 )

\begin{tabular}{|c|c|c|c|c|c|c|c|c|c|c|c|c|c|c|c|c|c|}
\hline \multirow[b]{2}{*}{$\begin{array}{l}\text { Sample } \\
\text { Location }\end{array}$} & \multirow[b]{2}{*}{$\begin{array}{l}\text { Sample } \\
\text { Number }\end{array}$} & \multirow[b]{2}{*}{$\begin{array}{c}\text { Depth } \\
\text { (ft bgs) }\end{array}$} & \multicolumn{15}{|c|}{ Contaminants of Potential Concern $(\mathrm{mg} / \mathrm{kg})$} \\
\hline & & & 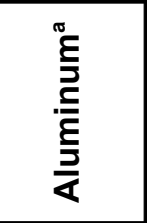 & 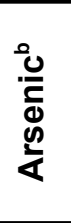 & 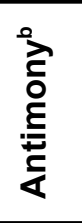 & 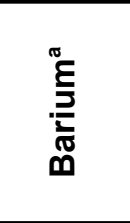 & 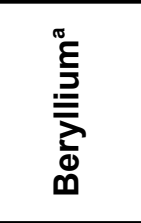 & 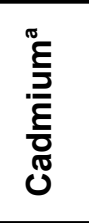 & 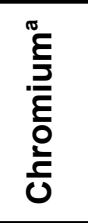 & $\begin{array}{l}\frac{\pi}{\pi} \\
\frac{0}{\pi} \\
0 \\
0\end{array}$ & $\begin{array}{l}\frac{0}{0} \\
\frac{0}{0} \\
\frac{0}{0}\end{array}$ & 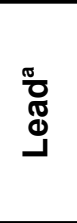 & 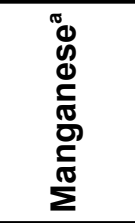 & $\begin{array}{l}\stackrel{0}{Z} \\
\frac{0}{0} \\
\frac{0}{d} \\
\sum\end{array}$ & $\begin{array}{l}\frac{\sigma}{0} \\
\frac{x}{0} \\
\frac{y}{Z}\end{array}$ & 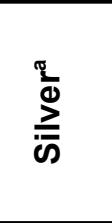 & $\stackrel{0}{\text { Ñ }}$ \\
\hline \multicolumn{3}{|c|}{ Final Action Levels } & 100,000 & 23 & 410 & 67,000 & 1,900 & 450 & 450 & 1,900 & 41,000 & 750 & 19,000 & 310 & 20,000 & 5,100 & 100,000 \\
\hline \multirow{3}{*}{ E09 } & 224E015 & $0-0.5$ & 6,300 & 5.9 & $\overline{---}$ & 89 & $\overline{--}$ & $\overline{--}$ & 5.2 & 3.1 & 7.5 & 40 & 240 & $\overline{--}$ & 5.6 & $\overline{--}$ & 45 \\
\hline & 224E035 & $6-7$ & 6,100 & 4.6 & -- & $75(\mathrm{~J})^{\mathrm{C}}$ & -- & -- & 3.4 & 1.8 & 5.2 & 10 & $140(\mathrm{~J})^{\mathrm{c}}$ & -- & 3.9 & 2.5 & 17 \\
\hline & 224E036 & $9-10$ & 6,500 & 4.9 & -- & $74(\mathrm{~J})^{\mathrm{C}}$ & $0.53(\mathrm{~J})^{\mathrm{c}}$ & -- & 4 & 1.8 & 5 & 10 & $150(\mathrm{~J})^{\mathrm{C}}$ & -- & 4.2 & -- & 17 \\
\hline \multirow{3}{*}{ E10 } & 224E017 & $0-0.5$ & 6,300 & 6.2 & -- & 91 & -- & -- & 4.7 & 2.9 & 6.7 & 31 & 230 & -- & 5.3 & -- & 44 \\
\hline & 224E033 & $5-6$ & 7,500 & 5.4 & -- & $78(\mathrm{~J})^{\mathrm{C}}$ & $0.55(\mathrm{~J})^{\mathrm{c}}$ & -- & 3.6 & 2.6 & 4.8 & 7.3 & $150(\mathrm{~J})^{\mathrm{C}}$ & -- & 4.4 & -- & 18 \\
\hline & 224E034 & $8-9$ & 6,900 & 5.3 & -- & $63(\mathrm{~J})^{\mathrm{c}}$ & $0.54(J)^{c}$ & -- & 3 & 2.2 & 4.5 & 5.8 & $140(\mathrm{~J})^{\mathrm{C}}$ & -- & 4.2 & -- & 15 \\
\hline \multirow{4}{*}{ E11 } & 224E018 & $0-0.5$ & 6,700 & 6.9 & -- & 98 & 0.53 & $\begin{array}{l}- \\
\end{array}$ & 6.4 & 3.1 & 12 & 73 & 210 & -- & 5.8 & -- & 39 \\
\hline & 224E028 & $2-3$ & 5,800 & 5.9 & -- & 82 & $\overline{--}$ & $\overline{--}$ & 4.4 & 2.8 & 6.3 & 15 & $200(J)^{d}$ & -- & 5.3 & $\overline{--}$ & 24 \\
\hline & 224E029 & $5-6$ & 8,100 & 7.4 & -- & 90 & $0.61(\mathrm{~J})^{\mathrm{c}}$ & -- & 4.9 & 3.3 & 6.9 & 10 & $210(\mathrm{~J})^{\mathrm{d}}$ & -- & 5.8 & -- & 22 \\
\hline & 224E030 & $7-8$ & 6,100 & 4.9 & $\overline{--}$ & 68 & -- & -- & 2.7 & 2 & 4.2 & 6 & $140(\mathrm{~J})^{\mathrm{d}}$ & -- & 3.7 & -- & 15 \\
\hline \multirow{3}{*}{ E12 } & 224E019 & $0-0.5$ & 5,700 & 6.2 & -- & 77 & -- & -- & 4.2 & 2.7 & 5.9 & 24 & 190 & -- & 4.9 & -- & 22 \\
\hline & 224E031 & $4-5$ & 6,600 & 5.5 & $\overline{--}$ & $77(\mathrm{~J})^{\mathrm{C}}$ & $\overline{--}$ & $\overline{--}$ & 3.3 & 2.4 & 4.6 & 8.6 & $180(\mathrm{~J})^{\mathrm{c}}$ & $\overline{--}$ & 4.4 & -- & 15 \\
\hline & 224E032 & $7-8$ & 6,400 & 5.4 & -- & $69(\mathrm{~J})^{\mathrm{C}}$ & $\overline{--}$ & -- & 3.4 & 2.5 & 4.6 & 7.3 & $150(\mathrm{~J})^{\mathrm{C}}$ & $\overline{--}$ & 4.4 & -- & 15 \\
\hline E13 & 224E024 & $7-8$ & 7,400 & 6.2 & -- & 89 & $0.58(\mathrm{~J})^{\mathrm{c}}$ & -- & 5.3 & 3.8 & 7.1 & 10 & $220(\mathrm{~J})^{\mathrm{d}}$ & -- & 6.6 & -- & 22 \\
\hline
\end{tabular}


Table A.7-8

Soil Sample Results for Metals Detected Above Minimum Reporting Limits at CAS 06-05-01, Leachfield (Page 4 of 6 )

\begin{tabular}{|c|c|c|c|c|c|c|c|c|c|c|c|c|c|c|c|c|c|}
\hline \multirow[b]{2}{*}{$\begin{array}{l}\text { Sample } \\
\text { Location }\end{array}$} & \multirow[b]{2}{*}{$\begin{array}{l}\text { Sample } \\
\text { Number }\end{array}$} & \multirow[b]{2}{*}{$\begin{array}{c}\text { Depth } \\
\text { (ft bgs) }\end{array}$} & \multicolumn{15}{|c|}{ Contaminants of Potential Concern $(\mathrm{mg} / \mathrm{kg})$} \\
\hline & & & 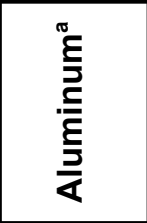 & 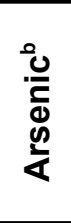 & 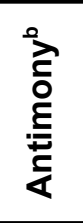 & 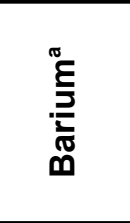 & 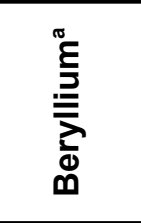 & 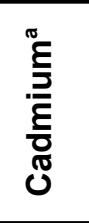 & 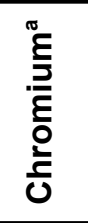 & $\begin{array}{l}\frac{\pi}{\pi} \\
\frac{0}{\pi} \\
0 \\
0\end{array}$ & $\begin{array}{l}\frac{0}{0} \\
\frac{0}{0} \\
\frac{0}{0}\end{array}$ & శ్ర & 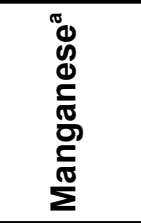 & 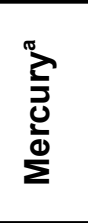 & $\begin{array}{l}\frac{\sigma}{0} \\
\frac{x}{0} \\
\frac{\underline{z}}{Z}\end{array}$ & 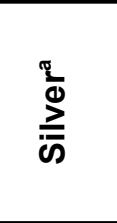 & ָ̃ \\
\hline \multicolumn{3}{|c|}{ Final Action Levels } & 100,000 & 23 & 410 & 67,000 & 1,900 & 450 & 450 & 1,900 & 41,000 & 750 & 19,000 & 310 & 20,000 & 5,100 & 100,000 \\
\hline \multirow{5}{*}{ E14 } & 224E039 & $\overline{0-0.5}$ & $\overline{\overline{5,900}}$ & $\overline{6}$ & $\overline{---}$ & $\overline{\overline{78(J)^{c}}}$ & $\overline{---}$ & $\overline{---}$ & $\overline{4.3}$ & $\overline{2.9}$ & $\overline{\overline{6.1}}$ & $7 \overline{7.1}$ & $\overline{200(J)^{c}}$ & $\overline{--}$ & $\overline{5.1}$ & $\overline{---}$ & $\overline{\overline{19}}$ \\
\hline & 224E040 & $2-3$ & 7,200 & 6.5 & $\overline{--}$ & $93(\mathrm{~J})^{\mathrm{c}}$ & $0.57(\mathrm{~J})^{\mathrm{c}}$ & $\overline{--}$ & 5.3 & $3.5(\mathrm{~J})^{\mathrm{c}}$ & 7.1 & 8.7 & $250(\mathrm{~J})^{\mathrm{c}}$ & $\overline{--}$ & 6.4 & $\overline{--}$ & $25(J)^{c}$ \\
\hline & 224E041 & $6-7$ & 6,600 & 6.5 & -- & $88(\mathrm{~J})^{\mathrm{C}}$ & $0.53(\mathrm{~J})^{\mathrm{c}}$ & -- & 4 & $2.5(\mathrm{~J})^{\mathrm{c}}$ & 5.9 & 7.3 & $170(\mathrm{~J})^{\mathrm{C}}$ & -- & 5 & -- & $19(\mathrm{~J})^{\mathrm{C}}$ \\
\hline & 224E042 & $6-7$ & 6,600 & 5.8 & -- & $80(\mathrm{~J})^{\mathrm{C}}$ & $0.53(\mathrm{~J})^{\mathrm{c}}$ & -- & 4 & $2.4(\mathrm{~J})^{\mathrm{c}}$ & 6 & 6.7 & $180(\mathrm{~J})^{\mathrm{C}}$ & -- & 4.6 & -- & $18(\mathrm{~J})^{\mathrm{C}}$ \\
\hline & 224E043 & $9-10$ & 6,500 & 7.2 & -- & $78(\mathrm{~J})^{\mathrm{C}}$ & -- & -- & 3 & $2.5(\mathrm{~J})^{\mathrm{c}}$ & 5.3 & 7.4 & $140(\mathrm{~J})^{\mathrm{c}}$ & -- & 3.7 & -- & $15(\mathrm{~J})^{\mathrm{c}}$ \\
\hline \multirow{4}{*}{ E15 } & 224E044 & $0-0.5$ & 5,300 & 5.2 & -- & $73(\mathrm{~J})^{\mathrm{C}}$ & -- & -- & 4.2 & $2.7(\mathrm{~J})^{\mathrm{C}}$ & 7 & 11 & $170(\mathrm{~J})^{\mathrm{c}}$ & -- & 4.9 & -- & $22(\mathrm{~J})^{\mathrm{c}}$ \\
\hline & 224E045 & $2-3$ & 6,100 & 5.4 & -- & $75(\mathrm{~J})^{\mathrm{C}}$ & -- & -- & 4.2 & $2.7(\mathrm{~J})^{\mathrm{c}}$ & 6.2 & 6.1 & $180(\mathrm{~J})^{\mathrm{C}}$ & -- & 4.8 & -- & $18(\mathrm{~J})^{\mathrm{c}}$ \\
\hline & 224E046 & $6-7$ & 7,400 & 5.6 & -- & $100(\mathrm{~J})^{\mathrm{c}}$ & $0.54(J)^{c}$ & -- & 6.2 & $2.8(\mathrm{~J})^{\mathrm{C}}$ & 10 & 32 & $300(\mathrm{~J})^{\mathrm{c}}$ & -- & 6 & 2.3 & $30(\mathrm{~J})^{\mathrm{c}}$ \\
\hline & 224E047 & $9-10$ & 7,400 & 4.7 & $\overline{--}$ & $73(\mathrm{~J})^{\mathrm{c}}$ & $0.53(\mathrm{~J})^{\mathrm{c}}$ & $\overline{--}$ & 3.5 & $2.3(\mathrm{~J})^{\mathrm{c}}$ & 5.1 & 6.3 & $150(\mathrm{~J})^{\mathrm{c}}$ & $\overline{--}$ & 4.2 & $\overline{--}$ & $17(\mathrm{~J})^{\mathrm{c}}$ \\
\hline \multirow{4}{*}{ E16 } & 224E048 & $0-0.5$ & 5,700 & 5.6 & $\overline{--}$ & $83(\mathrm{~J})^{\mathrm{C}}$ & -- & -- & 4.5 & $3.5(\mathrm{~J})^{\mathrm{c}}$ & 6.9 & 13 & $210(J)^{c}$ & -- & 5.1 & -- & $22(J)^{c}$ \\
\hline & 224E049 & $2-3$ & 8,000 & 6 & -- & $94(J)^{c}$ & $0.59(\mathrm{~J})^{\mathrm{c}}$ & -- & 5.9 & $3.6(\mathrm{~J})^{\mathrm{c}}$ & 7.9 & 8.4 & $250(\mathrm{~J})^{\mathrm{c}}$ & -- & 6.7 & -- & $24(\mathrm{~J})^{\mathrm{C}}$ \\
\hline & 224E050 & $7-8$ & 7,200 & 4.5 & $\overline{--}$ & $71(\mathrm{~J})^{\mathrm{C}}$ & $0.54(\mathrm{~J})^{\mathrm{c}}$ & $\overline{--}$ & 3.8 & $1.9(\mathrm{~J})^{\mathrm{c}}$ & 5.5 & 7.5 & $120(\mathrm{~J})^{\mathrm{c}}$ & $\overline{--}$ & 4.3 & -- & $17(\mathrm{~J})^{\mathrm{C}}$ \\
\hline & 224E051 & $10-11$ & 6,600 & 5.5 & -- & $75(\mathrm{~J})^{\mathrm{C}}$ & $\overline{--}$ & $\overline{--}$ & 3.9 & $2.3(\mathrm{~J})^{\mathrm{C}}$ & 6.5 & 12 & $160(\mathrm{~J})^{\mathrm{c}}$ & -- & 4.9 & -- & $19(\mathrm{~J})^{\mathrm{c}}$ \\
\hline \multirow{3}{*}{ E17 } & 224E058 & $0-0.5$ & 9,400 & 6.6 & -- & $120(\mathrm{~J})^{\mathrm{C}}$ & $0.7(J)^{c}$ & -- & 7.7 & $5.1(\mathrm{~J})^{\mathrm{c}}$ & 10 & 23 & $300(J)^{c}$ & 0.15 & 8.9 & -- & $36(J)^{c}$ \\
\hline & 224E068 & $4-5$ & 7,300 & 7.4 & -- & $91(\mathrm{~J})^{\mathrm{C}}$ & $0.56(\mathrm{~J})^{\mathrm{c}}$ & -- & 5.9 & $3.4(\mathrm{~J})^{\mathrm{c}}$ & 8 & 20 & $240(J)^{c}$ & -- & 6.6 & -- & $27(J)^{c}$ \\
\hline & 224E069 & $6.5-7.5$ & 6,700 & 6.3 & -- & $83(\mathrm{~J})^{\mathrm{C}}$ & $0.52(\mathrm{~J})^{\mathrm{c}}$ & -- & 7.4 & $2.9(\mathrm{~J})^{\mathrm{c}}$ & 12 & 41 & $220(J)^{c}$ & -- & 6 & -- & $35(\mathrm{~J})^{\mathrm{c}}$ \\
\hline
\end{tabular}


Table A.7-8

Soil Sample Results for Metals Detected Above Minimum Reporting Limits at CAS 06-05-01, Leachfield (Page 5 of 6 )

\begin{tabular}{|c|c|c|c|c|c|c|c|c|c|c|c|c|c|c|c|c|c|}
\hline \multirow[b]{2}{*}{$\begin{array}{l}\text { Sample } \\
\text { Location }\end{array}$} & \multirow[b]{2}{*}{$\begin{array}{l}\text { Sample } \\
\text { Number }\end{array}$} & \multirow[b]{2}{*}{$\begin{array}{c}\text { Depth } \\
\text { (ft bgs) }\end{array}$} & \multicolumn{15}{|c|}{ Contaminants of Potential Concern $(\mathrm{mg} / \mathrm{kg})$} \\
\hline & & & 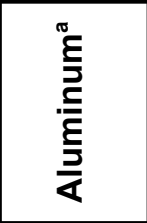 & 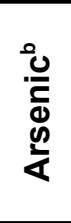 & 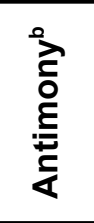 & 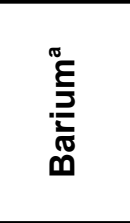 & 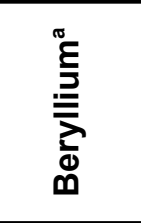 & 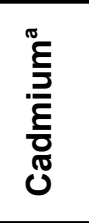 & 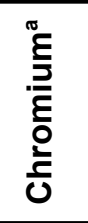 & $\begin{array}{l}\frac{\pi}{\pi} \\
\frac{0}{\pi} \\
0 \\
0\end{array}$ & $\begin{array}{l}\frac{0}{0} \\
\frac{0}{0} \\
\frac{0}{0}\end{array}$ & శ్ర & 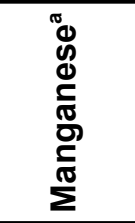 & $\begin{array}{l}\stackrel{0}{Z} \\
\frac{0}{0} \\
\frac{0}{d} \\
\sum\end{array}$ & $\begin{array}{l}\frac{\sigma}{0} \\
\frac{x}{0} \\
\frac{\underline{z}}{Z}\end{array}$ & 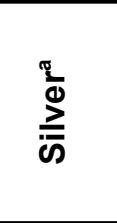 & 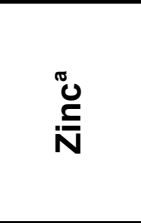 \\
\hline \multicolumn{3}{|c|}{ Final Action Levels } & 100,000 & 23 & 410 & 67,000 & 1,900 & 450 & 450 & 1,900 & 41,000 & 750 & 19,000 & 310 & 20,000 & 5,100 & 100,000 \\
\hline \multirow{3}{*}{ E18 } & $224 \mathrm{E} 059$ & $\overline{0-0.5}$ & $\overline{7,200}$ & 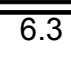 & $\overline{---}$ & $88(\mathrm{~J})^{\mathrm{C}}$ & $0.55(\mathrm{~J})^{\mathrm{c}}$ & $\overline{---}$ & $\overline{5.8}$ & $3.3(\mathrm{~J})^{\mathrm{c}}$ & 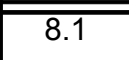 & 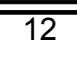 & $230(\mathrm{~J})^{\mathrm{C}}$ & $\overline{--}$ & 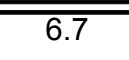 & $\overline{---}$ & $\overline{27(J)^{c}}$ \\
\hline & 224E070 & $4-5$ & 9,800 & 6.1 & -- & $120(\mathrm{~J})^{\mathrm{C}}$ & $0.69(\mathrm{~J})^{\mathrm{c}}$ & -- & 7.2 & $4.2(\mathrm{~J})^{\mathrm{C}}$ & 9 & 11 & $300(\mathrm{~J})^{\mathrm{c}}$ & -- & 8.2 & -- & $32(\mathrm{~J})^{\mathrm{c}}$ \\
\hline & 224E071 & $7-8$ & 7,000 & 7.3 & -- & $94(\mathrm{~J})^{\mathrm{C}}$ & $0.54(\mathrm{~J})^{\mathrm{c}}$ & -- & 6.2 & $3.1(\mathrm{~J})^{\mathrm{c}}$ & 13 & 23 & $240(\mathrm{~J})^{\mathrm{C}}$ & -- & 6.5 & -- & $30(\mathrm{~J})^{\mathrm{c}}$ \\
\hline \multirow{3}{*}{ E19 } & 224E060 & $0-0.5$ & 9,200 & 6.3 & -- & $110(\mathrm{~J})^{\mathrm{c}}$ & $0.66(J)^{c}$ & -- & 7.4 & $4.4(J)^{\mathrm{C}}$ & 10 & 21 & $300(\mathrm{~J})^{\mathrm{c}}$ & -- & 8 & -- & $36(J)^{c}$ \\
\hline & 224E072 & $4-5$ & 7,300 & 6.6 & -- & $150(\mathrm{~J})^{\mathrm{c}}$ & $0.57(\mathrm{~J})^{\mathrm{c}}$ & 0.66 & 11 & $3.2(\mathrm{~J})^{\mathrm{c}}$ & 29 & 84 & $240(\mathrm{~J})^{\mathrm{C}}$ & -- & 6.7 & -- & $56(J)^{c}$ \\
\hline & 224E073 & $7-8$ & 7,700 & 6.7 & -- & $98(\mathrm{~J})^{\mathrm{C}}$ & $0.58(\mathrm{~J})^{\mathrm{c}}$ & -- & 6.3 & $3.2(\mathrm{~J})^{\mathrm{c}}$ & 9.2 & 27 & $230(\mathrm{~J})^{\mathrm{c}}$ & -- & 6.8 & -- & $30(\mathrm{~J})^{\mathrm{c}}$ \\
\hline \multirow{4}{*}{ E20 } & 224E061 & $0-0.5$ & 8,900 & 5.6 & -- & $97(\mathrm{~J})^{\mathrm{C}}$ & $0.63(\mathrm{~J})^{\mathrm{c}}$ & 0.77 & 14 & $3.5(\mathrm{~J})^{\mathrm{c}}$ & 18 & 62 & $220(\mathrm{~J})^{\mathrm{C}}$ & -- & 7.5 & -- & $60(\mathrm{~J})^{\mathrm{c}}$ \\
\hline & 224E063 & $4-5$ & 4,400 & 5.9 & -- & $59(\mathrm{~J})^{\mathrm{C}}$ & $\overline{--}$ & $\overline{--}$ & 3.8 & $1.8(\mathrm{~J})^{\mathrm{C}}$ & 4.6 & 7.6 & $180(\mathrm{~J})^{\mathrm{C}}$ & -- & 4.3 & $\overline{--}$ & $16(J)^{c}$ \\
\hline & 224E066 & $6.5-7.5$ & 4,400 & 5.6 & $\overline{--}$ & $58(\mathrm{~J})^{\mathrm{C}}$ & $\overline{--}$ & $\overline{--}$ & 4.2 & $2(\mathrm{~J})^{\mathrm{c}}$ & 5.4 & 9.1 & $160(\mathrm{~J})^{\mathrm{c}}$ & $\overline{--}$ & 4.1 & $\overline{--}$ & $17(\mathrm{~J})^{\mathrm{c}}$ \\
\hline & 224E067 & $6.5-7.5$ & 4,500 & 6.6 & $\overline{--}$ & $59(\mathrm{~J})^{\mathrm{C}}$ & -- & -- & 4.4 & $2(J)^{c}$ & 5.6 & 9.7 & $160(\mathrm{~J})^{\mathrm{C}}$ & -- & 4.3 & -- & $18(\mathrm{~J})^{\mathrm{c}}$ \\
\hline \multirow{3}{*}{ E21 } & 224E062 & $0-0.5$ & 7,900 & 6.2 & $2(J)^{e}$ & $98(J)^{c}$ & $0.59(\mathrm{~J})^{\mathrm{c}}$ & -- & 6.4 & $3.7(\mathrm{~J})^{\mathrm{C}}$ & 8.8 & 15 & $270(\mathrm{~J})^{\mathrm{C}}$ & -- & 7.3 & -- & $29(\mathrm{~J})^{\mathrm{C}}$ \\
\hline & 224E074 & $4-5$ & 7,300 & 6.8 & -- & $96(\mathrm{~J})^{\mathrm{C}}$ & $0.55(\mathrm{~J})^{\mathrm{c}}$ & -- & 7.3 & $3.3(\mathrm{~J})^{\mathrm{c}}$ & 13 & 31 & $230(\mathrm{~J})^{\mathrm{C}}$ & -- & 6.6 & -- & $33(\mathrm{~J})^{\mathrm{c}}$ \\
\hline & 224E075 & $7-8$ & 7,100 & 6.4 & -- & $86(\mathrm{~J})^{\mathrm{C}}$ & $0.53(\mathrm{~J})^{\mathrm{c}}$ & -- & 5.8 & $2.9(\mathrm{~J})^{\mathrm{C}}$ & 8.5 & 17 & $210(\mathrm{~J})^{\mathrm{C}}$ & -- & 6.1 & $\overline{--}$ & $27(\mathrm{~J})^{\mathrm{C}}$ \\
\hline \multirow{3}{*}{ E22 } & 224E064 & $0-0.5$ & 9,700 & 6 & -- & $110(\mathrm{~J})^{\mathrm{c}}$ & $0.7(J)^{c}$ & -- & 7.7 & $4.1(\mathrm{~J})^{\mathrm{c}}$ & 10 & 18 & $300(J)^{c}$ & $\overline{--}$ & 8.2 & $\overline{--}$ & $35(J)^{c}$ \\
\hline & 224E076 & $4-5$ & 7,000 & 7.6 & $\overline{--}$ & 87 & $\overline{--}$ & $\overline{--}$ & 4.3 & 2.5 & 6.9 & 8.6 & 200 & $\overline{--}$ & 5.4 & $\overline{--}$ & 19 \\
\hline & 224E077 & $7-8$ & 5,900 & 6.6 & -- & 72 & -- & -- & 3.8 & 2 & 5.9 & 7.6 & 180 & -- & 4.7 & -- & 16 \\
\hline
\end{tabular}


Table A.7-8

Soil Sample Results for Metals Detected Above Minimum Reporting Limits at CAS 06-05-01, Leachfield (Page 6 of 6 )

\begin{tabular}{|c|c|c|c|c|c|c|c|c|c|c|c|c|c|c|c|c|c|}
\hline \multirow[b]{2}{*}{$\begin{array}{l}\text { Sample } \\
\text { Location }\end{array}$} & \multirow[b]{2}{*}{$\begin{array}{l}\text { Sample } \\
\text { Number }\end{array}$} & \multirow[b]{2}{*}{$\begin{array}{c}\text { Depth } \\
\text { (ft bgs) }\end{array}$} & \multicolumn{15}{|c|}{ Contaminants of Potential Concern $(\mathrm{mg} / \mathrm{kg})$} \\
\hline & & & 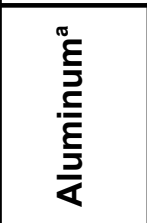 & 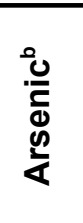 & 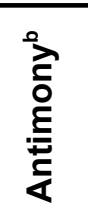 & 톨 & 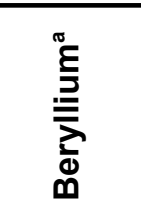 & 立 & 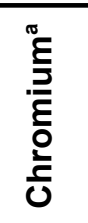 & $\begin{array}{l}\frac{\mathfrak{T}}{\pi} \\
\frac{0}{0} \\
0 \\
ن\end{array}$ & $\begin{array}{l}\frac{\pi}{0} \\
\frac{0}{0} \\
0 \\
0\end{array}$ & ֻٓ & 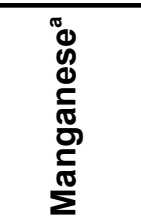 & 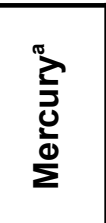 & $\begin{array}{l}\frac{\sigma}{\Phi} \\
\frac{x}{0} \\
\frac{\underline{y}}{Z}\end{array}$ & 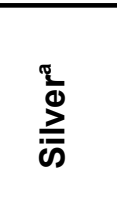 & $\stackrel{0}{\frac{0}{N}}$ \\
\hline \multicolumn{3}{|c|}{ Final Action Levels } & 100,000 & 23 & 410 & 67,000 & 1,900 & 450 & 450 & 1,900 & 41,000 & 750 & 19,000 & 310 & 20,000 & 5,100 & 100,000 \\
\hline \multirow{3}{*}{ E23 } & $224 \mathrm{E} 065$ & $\overline{0-0.5}$ & $\overline{99,300}$ & 6.8 & $\overline{---}$ & $1110(\mathrm{~J})^{\mathrm{C}}$ & $0.68(\mathrm{~J})^{\mathrm{c}}$ & 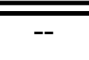 & $\overline{7}$ & $4.3(\mathrm{~J})^{\mathrm{C}}$ & $\overline{9.1}$ & 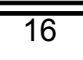 & $310(\mathrm{~J})^{\mathrm{c}}$ & $\overline{---}$ & $\overline{8.5}$ & $\overline{---}$ & $\overline{\overline{33(J)^{c}}}$ \\
\hline & 224E078 & $4-5$ & 4,800 & 6.5 & -- & 61 & -- & -- & 3.3 & 1.9 & 4.6 & 6.8 & 170 & -- & 4.2 & -- & 14 \\
\hline & 224E079 & $7-8$ & 5,600 & 6.9 & -- & 72 & -- & -- & 4 & 2.2 & 6.1 & 11 & 180 & -- & 4.7 & -- & 18 \\
\hline \multirow{3}{*}{ E24 } & 224E080 & $0-1$ & 9,100 & 6.4 & -- & 120 & 0.62 & -- & 7.2 & 3.8 & 10 & 16 & 280 & 0.044 & 7.8 & -- & 32 \\
\hline & 224E082 & $4-5$ & 4,900 & 6.6 & -- & 67 & -- & -- & 3.3 & 2.3 & 5.3 & 8.9 & 160 & -- & 4.3 & -- & 15 \\
\hline & 224E083 & $7-8$ & 4,500 & 6.8 & -- & 63 & -- & -- & 3.1 & 2.6 & 4.4 & 8.5 & 190 & -- & 4.2 & -- & 15 \\
\hline \multirow{4}{*}{ E25 } & 224E081 & $0-1$ & 8,900 & 5.8 & -- & 130 & 0.63 & -- & 6.7 & 3.8 & 17 & 22 & 260 & 0.1 & 8.4 & -- & 34 \\
\hline & 224E084 & $4-5$ & 4,000 & 7.8 & -- & $55(J)^{c}$ & -- & -- & 3 & 2.3 & 5.3 & 10 & $170(\mathrm{~J})^{\mathrm{c}}$ & -- & 4.1 & -- & $16(\mathrm{~J})^{\mathrm{c}}$ \\
\hline & 224E085 & $4-5$ & 3,800 & 6.6 & -- & $51(\mathrm{~J})^{\mathrm{C}}$ & -- & -- & 2.9 & 2 & 5.3 & 9.9 & $160(\mathrm{~J})^{\mathrm{c}}$ & -- & 3.8 & -- & $17(\mathrm{~J})^{\mathrm{c}}$ \\
\hline & 224E086 & $7-8$ & 5,900 & 5.2 & -- & $77(\mathrm{~J})^{\mathrm{C}}$ & -- & -- & 3.9 & 2.7 & 5.7 & 8.8 & $210(J)^{c}$ & -- & 5 & -- & $18(\mathrm{~J})^{\mathrm{c}}$ \\
\hline E26 & 224E087 & $0-1$ & 8,000 & 6.6 & -- & $100(\mathrm{~J})^{\mathrm{c}}$ & $0.58(\mathrm{~J})^{\mathrm{c}}$ & -- & 6.1 & 3.7 & 8.4 & 15 & $290(\mathrm{~J})^{\mathrm{c}}$ & 0.038 & 7.4 & -- & $30(\mathrm{~J})^{\mathrm{c}}$ \\
\hline E27 & 224E090 & $0-1$ & 5,300 & 6 & $\overline{--}$ & $82(\mathrm{~J})^{\mathrm{c}}$ & $\overline{--}$ & $\overline{--}$ & 3.7 & 2.7 & 6.2 & 8.5 & $160(\mathrm{~J})^{\mathrm{c}}$ & 0.036 & 5.1 & $\overline{--}$ & $16(\mathrm{~J})^{\mathrm{C}}$ \\
\hline E28 & 224E093 & $0-1$ & 4,700 & 6.1 & -- & $58(\mathrm{~J})^{\mathrm{c}}$ & -- & -- & 3.2 & 2.4 & 5.2 & 6.8 & $150(\mathrm{~J})^{\mathrm{c}}$ & -- & 4.4 & -- & $15(\mathrm{~J})^{\mathrm{C}}$ \\
\hline
\end{tabular}

${ }^{a}$ Based on U.S. Environmental Protection Agency, Region 9 Preliminary Remediation Goals (PRGs) (EPA, 2002)

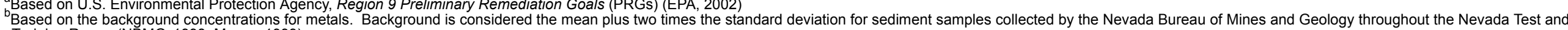
c

${ }^{\mathrm{d}}$ Qualifier added to laboratory data; record accepted. Matrix spike recovery outside control limits. Serial dilution \%D outside control limits. Matrix effects may exist. ${ }^{\mathrm{e}} \mathrm{Qualifier}$ added to laboratory data; record accepted. Matrix spike recovery outside control limits.

$\mathrm{ft}$ bgs = Feet below ground surface

$\mathrm{mg} / \mathrm{kg}=$ Milligrams per kilogram

$J=$ Estimated value. 
Table A.7-9

Soil Sample Results for Metals Detected Above Minimum Reporting Limits at CAS 06-17-04, Decon Pad and Wastewater Catch (Page 1 of 3)

\begin{tabular}{|c|c|c|c|c|c|c|c|c|c|c|c|c|c|c|c|c|}
\hline \multirow[b]{2}{*}{$\begin{array}{l}\text { Sample } \\
\text { Location }\end{array}$} & \multirow[b]{2}{*}{$\begin{array}{l}\text { Sample } \\
\text { Number }\end{array}$} & \multirow[b]{2}{*}{$\begin{array}{c}\text { Depth } \\
\text { (ft bgs) }\end{array}$} & \multicolumn{14}{|c|}{ Contaminants of Potential Concern $(\mathrm{mg} / \mathrm{kg})$} \\
\hline & & & & 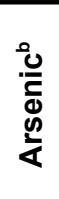 & 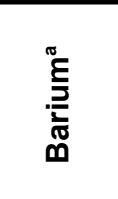 & 疍 & 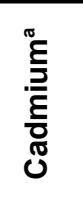 & 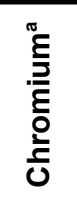 & 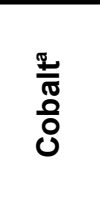 & $\begin{array}{l}\frac{0}{0} \\
\frac{0}{0} \\
0 \\
ن\end{array}$ & 总 & 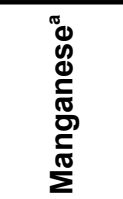 & $\begin{array}{l}\stackrel{0}{Z} \\
\frac{0}{0} \\
\frac{0}{0} \\
\sum\end{array}$ & 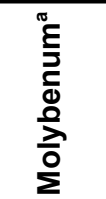 & $\begin{array}{l}\frac{\sigma}{\Phi} \\
\frac{\partial}{0} \\
\frac{0}{Z}\end{array}$ & $\stackrel{0}{\stackrel{0}{N}}$ \\
\hline \multicolumn{3}{|c|}{ Final Action Levels } & 100,000 & 23 & 67,000 & 1,900 & 450 & 450 & 1,900 & 41,000 & 750 & 19,000 & 310 & 5,100 & 20,000 & 100,000 \\
\hline \multirow{4}{*}{ F01 } & $\overline{224 \mathrm{~F} 001}$ & $\overline{11-2}$ & $\overline{\overline{4,700}}$ & $\overline{5.9}$ & $\overline{67}$ & $\overline{---}$ & $\overline{---}$ & 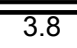 & $\overline{2.5}$ & 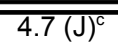 & 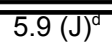 & $\overline{180(\mathrm{~J})^{\mathrm{e}}}$ & $\overline{---}$ & $\overline{---}$ & $\overline{4.5}$ & $\overline{15(J)^{c}}$ \\
\hline & $224 \mathrm{~F} 002$ & $1-2$ & 4,600 & 5.8 & 64 & -- & -- & 3.6 & 2.3 & $4.4(\mathrm{~J})^{\mathrm{c}}$ & $6(J)^{d}$ & $160(\mathrm{~J})^{\mathrm{e}}$ & $\overline{--}$ & -- & 4.3 & $15(\mathrm{~J})^{\mathrm{c}}$ \\
\hline & $224 \mathrm{~F} 003$ & $4-5$ & 3,100 & 5.4 & 42 & -- & -- & 2.5 & 2.2 & $3.4(\mathrm{~J})^{\mathrm{c}}$ & $5.2(\mathrm{~J})^{\mathrm{d}}$ & $150(\mathrm{~J})^{\mathrm{e}}$ & $\overline{--}$ & -- & 3.5 & $11(\mathrm{~J})^{\mathrm{c}}$ \\
\hline & 224F004 & $7-8$ & 4,100 & 5.9 & 47 & -- & -- & 2.5 & 1.9 & $3.9(\mathrm{~J})^{\mathrm{c}}$ & $4.7(J)^{d}$ & $120(\mathrm{~J})^{\mathrm{e}}$ & 0.083 & -- & 3.3 & $11(\mathrm{~J})^{\mathrm{c}}$ \\
\hline \multirow{3}{*}{ F02 } & $224 \mathrm{~F} 005$ & $1-2$ & 3,000 & 5.4 & 40 & -- & -- & 2.7 & 1.8 & $3(J)^{c}$ & $4.7(\mathrm{~J})^{\mathrm{d}}$ & $130(\mathrm{~J})^{\mathrm{e}}$ & -- & -- & 3.3 & $11(\mathrm{~J})^{\mathrm{c}}$ \\
\hline & $224 \mathrm{~F} 006$ & $4-5$ & 1,700 & 5.2 & 28 & -- & -- & 1.9 & 1.2 & $1.9(\mathrm{~J})^{\mathrm{c}}$ & $3.4(J)^{d}$ & $110(\mathrm{~J})^{\mathrm{e}}$ & -- & -- & 2.2 & $7.2(\mathrm{~J})^{\mathrm{c}}$ \\
\hline & 224F007 & $7-8$ & 4,000 & 5 & 47 & -- & -- & 3.4 & 1.9 & $4.3(\mathrm{~J})^{\mathrm{c}}$ & $4.2(J)^{d}$ & $120(\mathrm{~J})^{\mathrm{e}}$ & $\overline{--}$ & -- & 3.5 & $13(\mathrm{~J})^{\mathrm{c}}$ \\
\hline \multirow{3}{*}{ F03 } & $224 \mathrm{~F} 008$ & $1-2$ & 6,100 & 5.6 & 76 & -- & $\overline{--}$ & 4.5 & 2.9 & $5.6(\mathrm{~J})^{\mathrm{c}}$ & $6.3(J)^{d}$ & $150(\mathrm{~J})^{\mathrm{e}}$ & $\overline{--}$ & -- & 5.3 & $18(\mathrm{~J})^{\mathrm{c}}$ \\
\hline & 224F009 & $4-5$ & 3,700 & 6 & 45 & -- & -- & 2.8 & 2.2 & $3.3(\mathrm{~J})^{\mathrm{c}}$ & $5.9(\mathrm{~J})^{\mathrm{d}}$ & $150(\mathrm{~J})^{\mathrm{e}}$ & -- & -- & 3.8 & $12(\mathrm{~J})^{\mathrm{c}}$ \\
\hline & $224 \mathrm{~F} 010$ & $7-8$ & 2,500 & 4.2 & 34 & -- & -- & 1.9 & 1.5 & $2.6(J)^{c}$ & 4.9 & $110(\mathrm{~J})^{\mathrm{c}}$ & $\overline{--}$ & -- & 2.3 & $9.7(\mathrm{~J})^{\mathrm{c}}$ \\
\hline \multirow{3}{*}{ F04 } & 224F011 & $1-2$ & 4,900 & 4.9 & 71 & -- & -- & 3.8 & 2.4 & $4.9(\mathrm{~J})^{\mathrm{c}}$ & 6.5 & $180(\mathrm{~J})^{\mathrm{c}}$ & -- & -- & 4.3 & $16(\mathrm{~J})^{c}$ \\
\hline & $224 \mathrm{~F} 012$ & $4-5$ & 5,600 & 4 & 59 & -- & -- & 2.6 & 1.7 & $3.5(\mathrm{~J})^{\mathrm{c}}$ & 4.6 & $120(\mathrm{~J})^{\mathrm{c}}$ & -- & -- & 3.1 & $12(\mathrm{~J})^{c}$ \\
\hline & $224 \mathrm{~F} 013$ & $7-8$ & 4,300 & 3.4 & 44 & $\overline{--}$ & $\overline{--}$ & 2.1 & 1.7 & $3.1(\mathrm{~J})^{\mathrm{c}}$ & 4 & $120(\mathrm{~J})^{\mathrm{c}}$ & $\overline{--}$ & -- & 2.8 & $10(\mathrm{~J})^{\mathrm{c}}$ \\
\hline \multirow{3}{*}{ F05 } & $224 \mathrm{~F} 014$ & $1-2$ & 5,100 & 4.8 & 67 & -- & -- & 4 & 2.4 & $5.4(\mathrm{~J})^{\mathrm{c}}$ & 5.2 & $190(\mathrm{~J})^{\mathrm{c}}$ & $\overline{--}$ & -- & 4.4 & $17(\mathrm{~J})^{\mathrm{C}}$ \\
\hline & $224 \mathrm{~F} 015$ & $4-5$ & 3,100 & 3.7 & 39 & -- & -- & 1.7 & 1.1 & $2.5(\mathrm{~J})^{\mathrm{c}}$ & 3.5 & $100(\mathrm{~J})^{\mathrm{C}}$ & -- & -- & $2.1(\mathrm{~B})$ & $8.4(\mathrm{~J})^{\mathrm{c}}$ \\
\hline & $224 \mathrm{~F} 016$ & $7-8$ & 4,100 & 5.1 & 44 & -- & -- & 2.2 & 1.6 & $2.9(\mathrm{~J})^{\mathrm{c}}$ & 4.4 & $110(\mathrm{~J})^{\mathrm{c}}$ & $\overline{--}$ & -- & 2.8 & $9.5(\mathrm{~J})^{\mathrm{c}}$ \\
\hline \multirow{3}{*}{ F06 } & $224 \mathrm{~F} 017$ & $1-2$ & 8,100 & 6.5 & 100 & $0.66(\mathrm{~J})^{\mathrm{c}}$ & -- & 6.5 & 3.9 & $8(J)^{c}$ & 8.7 & $240(\mathrm{~J})^{\mathrm{c}}$ & $\overline{--}$ & -- & 7.1 & $26(J)^{c}$ \\
\hline & $224 \mathrm{~F} 018$ & $4-5$ & 9,100 & 5.8 & 110 & $0.71(\mathrm{~J})^{\mathrm{C}}$ & $\overline{--}$ & 6.9 & 4 & $8.4(\mathrm{~J})^{\mathrm{c}}$ & 8.4 & $260(\mathrm{~J})^{\mathrm{c}}$ & $\overline{--}$ & $\overline{--}$ & 7.5 & $28(J)^{c}$ \\
\hline & 224F019 & $7-8$ & 4,400 & 5.1 & 59 & -- & -- & 3.4 & 2.3 & $4.4(\mathrm{~J})^{\mathrm{c}}$ & 5.6 & $190(\mathrm{~J})^{\mathrm{c}}$ & -- & -- & 4.1 & $16(\mathrm{~J})^{\mathrm{c}}$ \\
\hline \multirow{3}{*}{$\mathrm{F} 07$} & $224 \mathrm{~F} 020$ & $1-2$ & 4,800 & 5.9 & 68 & -- & -- & 4 & 2.6 & $5(J)^{c}$ & 6.1 & $190(\mathrm{~J})^{\mathrm{c}}$ & -- & -- & 4.7 & $18(\mathrm{~J})^{c}$ \\
\hline & $224 \mathrm{~F} 021$ & $4-5$ & 5,300 & 5.7 & 71 & -- & -- & 4.2 & 2.7 & $5.4(\mathrm{~J})^{\mathrm{c}}$ & 6.9 & $190(\mathrm{~J})^{\mathrm{c}}$ & $\overline{--}$ & -- & 4.6 & $19(\mathrm{~J})^{\mathrm{c}}$ \\
\hline & $224 \mathrm{~F} 022$ & $7-8$ & 4,400 & 3.8 & 58 & -- & -- & 2.5 & 1.6 & $3.2(\mathrm{~J})^{\mathrm{c}}$ & 3.8 & $140(\mathrm{~J})^{\mathrm{c}}$ & -- & -- & 2.9 & $13(\mathrm{~J})^{\mathrm{c}}$ \\
\hline
\end{tabular}


Table A.7-9

Soil Sample Results for Metals Detected Above Minimum Reporting Limits at CAS 06-17-04, Decon Pad and Wastewater Catch (Page 2 of 3 )

\begin{tabular}{|c|c|c|c|c|c|c|c|c|c|c|c|c|c|c|c|c|}
\hline \multirow[b]{2}{*}{$\begin{array}{l}\text { Sample } \\
\text { Location }\end{array}$} & \multirow[b]{2}{*}{$\begin{array}{l}\text { Sample } \\
\text { Number }\end{array}$} & \multirow[b]{2}{*}{$\begin{array}{c}\text { Depth } \\
\text { (ft bgs) }\end{array}$} & \multicolumn{14}{|c|}{ Contaminants of Potential Concern (mg/kg) } \\
\hline & & & & 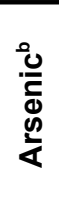 & 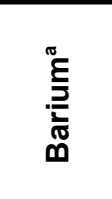 & 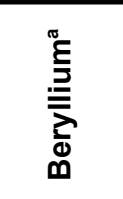 & 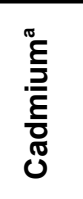 & 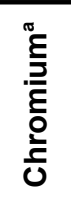 & $\begin{array}{l}\frac{\text { N }}{\pi} \\
\text { 융 } \\
0\end{array}$ & $\begin{array}{l}\frac{\pi}{\delta} \\
\frac{0}{0} \\
\frac{0}{0}\end{array}$ & 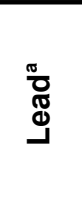 & 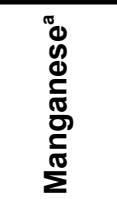 & $\begin{array}{l}\stackrel{0}{2} \\
\frac{0}{0} \\
\frac{0}{2} \\
\Sigma\end{array}$ & 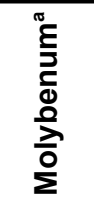 & $\begin{array}{l}\frac{\sigma}{d} \\
\frac{v}{0} \\
\frac{0}{Z}\end{array}$ & $\stackrel{\text { Ũ }}{\stackrel{N}{N}}$ \\
\hline \multicolumn{3}{|c|}{ Final Action Levels } & 100,000 & 23 & 67,000 & 1,900 & 450 & 450 & 1,900 & 41,000 & 750 & 19,000 & 310 & 5,100 & 20,000 & 100,000 \\
\hline \multirow{4}{*}{ F08 } & $\overline{2224 \mathrm{~F} 023}$ & $\overline{0.2-1}$ & $\overline{4,200}$ & $\overline{\overline{7.1}}$ & $\overline{\overline{62}}$ & $\overline{---}$ & $\overline{---}$ & $\overline{\overline{4}}$ & $\overline{2.1}$ & $\overline{\overline{5.8(J)^{\mathrm{c}}}}$ & $\overline{244}$ & 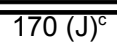 & $\overline{---}$ & $\overline{---}$ & $\overline{4.6}$ & $24(\mathrm{~J})^{\mathrm{c}}$ \\
\hline & $224 \mathrm{~F} 024$ & $4-5$ & 4,400 & 4.6 & 57 & -- & -- & 3.5 & 2.1 & $4.9(\mathrm{~J})^{\mathrm{c}}$ & 5 & $140(\mathrm{~J})^{\mathrm{c}}$ & $0.05(J)^{f}$ & -- & 3.7 & $14(\mathrm{~J})^{\mathrm{c}}$ \\
\hline & $224 \mathrm{~F} 025$ & $7-8$ & 3,100 & 4.5 & 39 & -- & -- & 1.8 & 1.3 & $2.6(\mathrm{~J})^{\mathrm{c}}$ & 3.7 & $110(\mathrm{~J})^{\mathrm{c}}$ & -- & -- & 2.2 & $8.6(\mathrm{~J})^{\mathrm{c}}$ \\
\hline & $224 \mathrm{~F} 026$ & $7-8$ & 3,100 & 3.9 & 39 & -- & -- & 1.8 & 1.2 & $2.4(\mathrm{~J})^{\mathrm{c}}$ & 3.2 & 120 & $\overline{--}$ & -- & $2.1(\mathrm{~J})^{\mathrm{c}}$ & $8.9(\mathrm{~J})^{\mathrm{c}}$ \\
\hline \multirow{3}{*}{ F09 } & 224F027 & $0.2-1$ & 9,400 & 6.2 & 110 & $0.73(\mathrm{~J})^{\mathrm{c}}$ & -- & 7.9 & 4.2 & $9.5(\mathrm{~J})^{\mathrm{c}}$ & 10 & $290(\mathrm{~J})^{\mathrm{C}}$ & $0.18(J)^{f}$ & -- & 8.6 & $32(\mathrm{~J})^{c}$ \\
\hline & $224 \mathrm{~F} 028$ & $4-5$ & 6,700 & 5.4 & 86 & $0.54(\mathrm{~J})^{\mathrm{c}}$ & -- & 4.6 & 3 & $6.6(J)^{c}$ & 6 & $190(\mathrm{~J})^{\mathrm{c}}$ & -- & -- & 5.1 & $20(\mathrm{~J})^{c}$ \\
\hline & $224 \mathrm{~F} 029$ & $7-8$ & 2,800 & 4.4 & 31 & $\overline{--}$ & $\overline{--}$ & 1.9 & 1.1 & $2.4(\mathrm{~J})^{\mathrm{c}}$ & 3.1 & $98(J)^{c}$ & -- & $\overline{--}$ & -- & $8(\mathrm{~J})^{\mathrm{c}}$ \\
\hline \multirow{3}{*}{ F10 } & $224 \mathrm{~F} 030$ & $0-1$ & 4,300 & 5.1 & 64 & -- & $\overline{--}$ & 3.8 & 2 & $6.3(\mathrm{~J})^{\mathrm{c}}$ & 12 & $150(\mathrm{~J})^{\mathrm{e}}$ & $\overline{--}$ & -- & 4 & $26(\mathrm{~J})^{\mathrm{e}}$ \\
\hline & 224F031 & $4-5$ & 2,900 & 5.3 & 44 & -- & -- & 2.7 & 1.6 & $2.9(\mathrm{~J})^{\mathrm{c}}$ & -- & $130(\mathrm{~J})^{\mathrm{e}}$ & -- & -- & 3.2 & $9.6(\mathrm{~J})^{\mathrm{e}}$ \\
\hline & $224 \mathrm{~F} 032$ & $7-8$ & 3,300 & 4.8 & 44 & -- & -- & 3 & 1.6 & $3.6(J)^{c}$ & 6 & $130(\mathrm{~J})^{\mathrm{e}}$ & $\overline{--}$ & -- & 3.2 & $12(\mathrm{~J})^{\mathrm{e}}$ \\
\hline \multirow{3}{*}{ F11 } & $224 \mathrm{~F} 033$ & $0-1$ & 5,500 & 6.6 & 92 & -- & 1.8 & 13 & 2.3 & $26(\mathrm{~J})^{\mathrm{c}}$ & -- & $180(\mathrm{~J})^{\mathrm{e}}$ & -- & -- & 4.8 & $66(\mathrm{~J})^{\mathrm{e}}$ \\
\hline & 224F034 & $4-5$ & 2,900 & 4.7 & 41 & -- & -- & 2.7 & 1.5 & $3.5(J)^{c}$ & 49 & $87(\mathrm{~J})^{\mathrm{e}}$ & $\begin{array}{c}0.045 \\
(J)^{g}\end{array}$ & -- & 2.4 & $7.2(\mathrm{~J})^{\mathrm{e}}$ \\
\hline & $224 \mathrm{~F} 035$ & $7-8$ & 9,600 & 6.4 & 65 & -- & -- & 5.1 & 2.8 & $6.4(\mathrm{~J})^{\mathrm{c}}$ & 7.1 & $160(\mathrm{~J})^{\mathrm{e}}$ & $0.11(\mathrm{~J})^{g}$ & -- & 5.7 & $18(\mathrm{~J})^{\mathrm{e}}$ \\
\hline \multirow{3}{*}{ F12 } & 224F036 & $0-1$ & 8,700 & 5.5 & 130 & -- & -- & 8.6 & 4 & $19(\mathrm{~J})^{\mathrm{c}}$ & 59 & $270(J)^{\mathrm{e}}$ & $\begin{array}{c}0.056 \\
(J)^{g}\end{array}$ & -- & 8.4 & $140(\mathrm{~J})^{\mathrm{e}}$ \\
\hline & 224F037 & $4-5$ & 5,300 & 6 & 75 & -- & -- & 5.4 & 2.8 & $6.6(J)^{c}$ & 18 & $210(\mathrm{~J})^{\mathrm{e}}$ & $\overline{--}$ & -- & 5.3 & $28(\mathrm{~J})^{\mathrm{e}}$ \\
\hline & $224 \mathrm{~F} 038$ & $7-8$ & 6,300 & 5.1 & 72 & -- & -- & 3.5 & 2.3 & $5.3(\mathrm{~J})^{\mathrm{c}}$ & 6.9 & $160(\mathrm{~J})^{\mathrm{e}}$ & $\overline{--}$ & -- & 4.4 & $15(\mathrm{~J})^{\mathrm{e}}$ \\
\hline \multirow{3}{*}{ F13 } & $224 \mathrm{~F} 039$ & $0-1$ & 5,300 & 5.1 & 110 & $\overline{--}$ & $\overline{--}$ & 4.9 & 2.5 & $14(\mathrm{~J})^{\mathrm{c}}$ & 41 & $200(J)^{e}$ & -- & $\overline{--}$ & 4.9 & $29(\mathrm{~J})^{\mathrm{e}}$ \\
\hline & $224 \mathrm{~F} 040$ & $4-5$ & 4,600 & 5.2 & 59 & -- & -- & 3.2 & 2.2 & $4.3(\mathrm{~J})^{\mathrm{c}}$ & -- & $160(\mathrm{~J})^{\mathrm{e}}$ & -- & -- & 3.8 & $14(\mathrm{~J})^{\mathrm{e}}$ \\
\hline & $224 \mathrm{~F} 041$ & $7-8$ & 3,500 & 5.5 & 59 & -- & $\overline{--}$ & 2.7 & 1.9 & $3.4(\mathrm{~J})^{\mathrm{c}}$ & -- & $150(\mathrm{~J})^{\mathrm{e}}$ & -- & -- & 3.4 & $13(\mathrm{~J})^{\mathrm{e}}$ \\
\hline
\end{tabular}


Table A.7-9

Soil Sample Results for Metals Detected Above Minimum Reporting Limits at CAS 06-17-04, Decon Pad and Wastewater Catch (Page 3 of 3 )

\begin{tabular}{|c|c|c|c|c|c|c|c|c|c|c|c|c|c|c|c|c|}
\hline \multirow[b]{2}{*}{$\begin{array}{l}\text { Sample } \\
\text { Location }\end{array}$} & \multirow[b]{2}{*}{$\begin{array}{l}\text { Sample } \\
\text { Number }\end{array}$} & \multirow[b]{2}{*}{$\begin{array}{c}\text { Depth } \\
\text { (ft bgs) }\end{array}$} & \multicolumn{14}{|c|}{ Contaminants of Potential Concern (mg/kg) } \\
\hline & & & 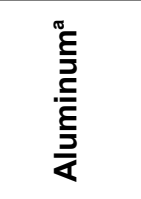 & 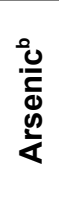 & 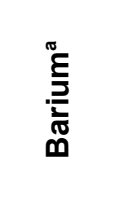 & 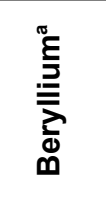 & 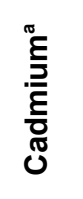 & 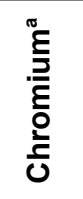 & $\begin{array}{l}\frac{\pi}{\pi} \\
\frac{0}{0} \\
0 \\
0\end{array}$ & $\begin{array}{l}\frac{\pi}{0} \\
\frac{0}{0} \\
0 \\
0\end{array}$ & ర్ల & 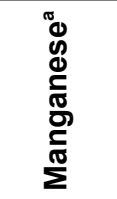 & 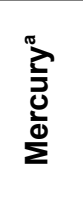 & 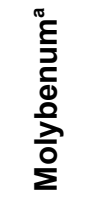 & $\begin{array}{l}\frac{\sigma}{d} \\
\frac{v}{0} \\
\frac{\sigma}{Z}\end{array}$ & 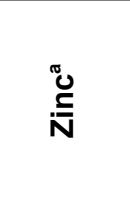 \\
\hline \multicolumn{3}{|c|}{ Final Action Levels } & 100,000 & 23 & 67,000 & 1,900 & 450 & 450 & 1,900 & 41,000 & 750 & 19,000 & 310 & 5,100 & 20,000 & 100,000 \\
\hline$\overline{\text { F31 }}$ & 224F069 & $\overline{0-0.5}$ & $\overline{6,000}$ & 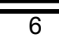 & $\overline{220}$ & $\overline{---}$ & $\overline{\overline{6.8}}$ & $\overline{21}$ & $\overline{\overline{4}}$ & $\overline{65}$ & $\overline{440}$ & 240 & $\overline{0.071}$ & $\overline{\overline{3.4}}$ & $\overline{16}$ & 370 \\
\hline F32 & 224F070 & $0-0.5$ & 4,900 & 5.1 & 140 & -- & 2.9 & 19 & 2.8 & 76 & 230 & 190 & 0.063 & 2.3 & 11 & 160 \\
\hline
\end{tabular}

${ }^{a}$ Based on U.S. Environmental Protection Agency, Region 9 Preliminary Remediation Goals (PRGs) (EPA, 2002)

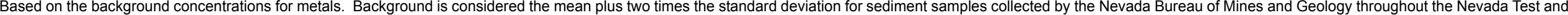
Training Range (NBMG, 1998; Moore, 1999).

Qualifier added to laboratory data; record accepted. Serial dilution \%D outside control limits. Matrix effects may exist.

Qualifier added to laboratory data; record accepted. Matrix spike recovery outside control limits.

Qualifier added to laboratory data; record accepted. Matrix spike recovery outside control limits. Serial dilution \%D outside control limits. Matrix effects may exist.

Qualifier added to laboratory data; record accepted. Exceeded holding time. Matrix spike recovery outside control limits.

Qualifier added to laboratory data; record accepted. Exceeded holding time.

$\mathrm{ft}$ bgs $=$ Feet below ground surface

$\mathrm{mg} / \mathrm{kg}=$ Milligrams per kilogram

$B=$ Value less than the contract required detection limit, but greater than or equal to the instrument detection limit.

$\mathrm{J}=$ Estimated value

$--=$ Not detected above minimum reporting limits. 
Table A.7-10

Soil Sample Results for Metals Detected Above Minimum Reporting Limits at CAS 06-23-01, Decon Pad Discharge Piping

\begin{tabular}{|c|c|c|c|c|c|c|c|c|c|c|c|c|c|c|c|c|}
\hline \multirow[b]{2}{*}{$\begin{array}{l}\text { Sample } \\
\text { Location }\end{array}$} & \multirow[b]{2}{*}{$\begin{array}{l}\text { Sample } \\
\text { Number }\end{array}$} & \multirow[b]{2}{*}{$\begin{array}{l}\text { Depths } \\
\text { (ft bgs) }\end{array}$} & \multicolumn{14}{|c|}{ Contaminants of Potential Concern (mg/kg) } \\
\hline & & & 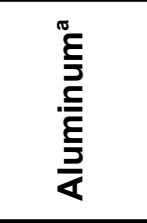 & 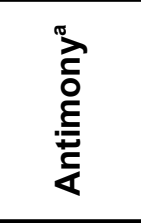 & 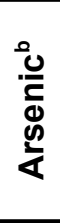 & 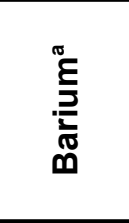 & 疍 & 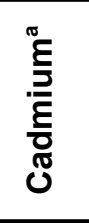 & 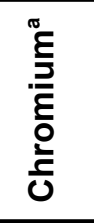 & $\begin{array}{l}\frac{0}{\pi} \\
\frac{0}{0} \\
0 \\
0\end{array}$ & $\begin{array}{l}\frac{0}{0} \\
\frac{0}{0} \\
\frac{0}{0}\end{array}$ & 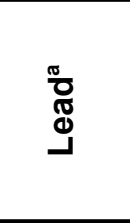 & 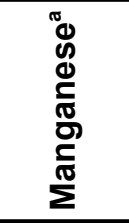 & $\begin{array}{l}\stackrel{0}{2} \\
\frac{2}{2} \\
\frac{0}{0} \\
\sum\end{array}$ & $\begin{array}{l}\frac{\sigma}{0} \\
\frac{x}{0} \\
\frac{x}{Z}\end{array}$ & $\stackrel{U}{\underline{N}}$ \\
\hline \multicolumn{3}{|c|}{ Final Action Levels } & 100,000 & 410 & 23 & 67,000 & 1,900 & 450 & 450 & 1,900 & 41,000 & 750 & 19,000 & 310 & 20,000 & 100,000 \\
\hline \multirow{4}{*}{ G01 } & $224 \mathrm{G} 001$ & $\overline{0-1}$ & 5,600 & $0.47(\mathrm{~J})^{\mathrm{c}}$ & 4.7 & 120 & $0.52(\mathrm{~J})^{\mathrm{d}}$ & 0.63 & $\overline{8.5}$ & $\overline{3}$ & $25(J)^{d}$ & $100(\mathrm{~J})^{\mathrm{c}}$ & $220(\mathrm{~J})^{\mathrm{e}}$ & $\overline{0.65}$ & $\overline{7}$ & $63(\mathrm{~J})^{\mathrm{d}}$ \\
\hline & $224 G 002$ & $0-1$ & 6,300 & $0.41(\mathrm{~J})^{\mathrm{c}}$ & 5.6 & 200 & $0.55(J)^{d}$ & 0.61 & 10 & 3.6 & $32(J)^{d}$ & $130(J)^{c}$ & $230(\mathrm{~J})^{\mathrm{e}}$ & 0.71 & 8.4 & $72(J)^{d}$ \\
\hline & $224 \mathrm{G} 003$ & $2-3$ & 5,500 & $1.2(\mathrm{~J})^{\mathrm{c}}$ & 4.7 & 150 & -- & 0.65 & 8.8 & 2.9 & $24(J)^{d}$ & $100(J)^{c}$ & $210(\mathrm{~J})^{\mathrm{e}}$ & 0.6 & 6.5 & $58(J)^{d}$ \\
\hline & $224 \mathrm{G} 004$ & $3-4$ & 5,700 & $0.59(\mathrm{~J})^{\mathrm{c}}$ & 5.6 & 160 & $0.59(\mathrm{~J})^{\mathrm{d}}$ & -- & 5.5 & 2.6 & $10(\mathrm{~J})^{\mathrm{d}}$ & $25(\mathrm{~J})^{\mathrm{c}}$ & $250(\mathrm{~J})^{\mathrm{e}}$ & 0.15 & 9.4 & $42(J)^{d}$ \\
\hline G02 & $224 G 005$ & $0-1$ & 3,900 & $0.61(\mathrm{~J})^{\mathrm{c}}$ & 6.7 & 68 & -- & -- & 3.5 & 2.3 & $6.6(J)^{d}$ & $19(\mathrm{~J})^{\mathrm{e}}$ & $150(\mathrm{~J})^{\mathrm{e}}$ & 0.042 & 4.1 & $17(\mathrm{~J})^{\mathrm{d}}$ \\
\hline G03 & $224 G 006$ & $0-1$ & 4,800 & $0.36(\mathrm{~J})^{\mathrm{c}}$ & 6.9 & 72 & -- & -- & 5.2 & 2.4 & $6.8(J)^{d}$ & $14(J)^{c}$ & $140(\mathrm{~J})^{\mathrm{e}}$ & 0.04 & 4.9 & $17(\mathrm{~J})^{\mathrm{d}}$ \\
\hline
\end{tabular}

${ }^{\mathrm{a} B}$ Based on U.S. Environmental Protection Agency, Region 9 Preliminary Remediation Goals (PRGs) (EPA, 2002)

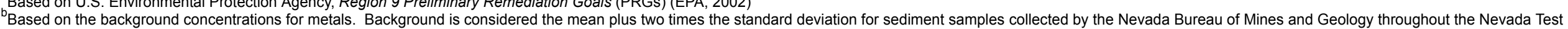
and Training Range (NBMG, 1998; Moore, 1999).

Qualifier added to laboratory data; record accepted. Matrix spike recovery outside control limits.

QQualifier added to laboratory data; record accepted. Matrix spike recovery outside control limits. Serial dilution \% $\%$ outside control limits. Matrix effects may exist.

$\mathrm{ft}$ bgs $=$ Feet below ground surface

$\mathrm{mg} / \mathrm{kg}=$ Milligrams per kilogram

- = Not detected above minimum reporting limits. 
Table A.7-11

\section{Soil Sample Results for PCBs Detected Above Minimum Reporting Limits at CAS 06-05-01, Leachfield}

\begin{tabular}{|c|c|c|c|}
\hline \multirow{2}{*}{$\begin{array}{l}\text { Sample } \\
\text { Location }\end{array}$} & \multirow{2}{*}{$\begin{array}{l}\text { Sample } \\
\text { Number }\end{array}$} & \multirow{2}{*}{$\begin{array}{l}\text { Depth } \\
\text { (ft bgs) }\end{array}$} & \multirow{2}{*}{$\begin{array}{c}\text { Contaminants of Potential Concern }(\mu \mathrm{g} / \mathrm{kg}) \\
\text { Aroclor } 1260\end{array}$} \\
\hline & & & \\
\hline \multicolumn{3}{|c|}{ Final Action Levels ${ }^{a}$} & 740 \\
\hline$\overline{\overline{E 04}}$ & 224E008 & $0-0.5$ & $\overline{72(\mathrm{~J})}$ \\
\hline E05 & 224E010 & $0-0.5$ & 53 \\
\hline E07 & 224E012 & $0-0.5$ & 35 \\
\hline E17 & 224E069 & $6.5-7.5$ & $69(\mathrm{~J})$ \\
\hline E19 & 224E072 & $4-5$ & 94 \\
\hline E20 & 224E061 & $0-0.5$ & 130 \\
\hline $\mathrm{E} 21$ & 224E074 & $4-5$ & 41 \\
\hline
\end{tabular}

${ }^{\mathrm{a} B a s e d}$ on U.S. Environmental Protection Agency, Region 9 Preliminary Remediation Goals (PRGs) (EPA, 2002)

$\mathrm{ft}$ bgs $=$ Feet below ground surface $\mu \mathrm{g} / \mathrm{kg}=$ Micrograms per kilogram

$\mathrm{J}=$ Estimated value. Qualifier added to laboratory data; record accepted. \%D between columns $>25$.

Table A.7-12

Soil Sample Results for PCBs Detected Above Minimum Reporting Limits at CAS 06-17-04, Decon Pad and Wastewater Catch

\begin{tabular}{|c|c|c|c|}
\hline \multirow{2}{*}{$\begin{array}{l}\text { Sample } \\
\text { Location }\end{array}$} & \multirow{2}{*}{$\begin{array}{l}\text { Sample } \\
\text { Number }\end{array}$} & \multirow{2}{*}{$\begin{array}{l}\text { Depth } \\
\text { (ft bgs) }\end{array}$} & Contaminants of Potential Concern $(\mu \mathrm{g} / \mathrm{kg})$ \\
\hline & & & Aroclor 1260 \\
\hline \multicolumn{3}{|c|}{ Final Action Levels ${ }^{a}$} & 740 \\
\hline F08 & 224F023 & $0.2-1$ & 69 \\
\hline F11 & 224F033 & $0-1$ & $79(\mathrm{~J})^{\mathrm{b}}$ \\
\hline F12 & 224F036 & $0-1$ & 47 \\
\hline F31 & 224F069RR1 & $0-0.5$ & $730(\mathrm{~J})^{\mathrm{c}}$ \\
\hline F32 & $224 \mathrm{~F} 070$ & $0-0.5$ & $120(\mathrm{~J})^{\mathrm{b}}$ \\
\hline
\end{tabular}

${ }^{a}$ Based on U.S. Environmental Protection Agency, Region 9 Preliminary Remediation Goals (PRGs) (EPA, 2002)

${ }^{b}$ Qualifier added to laboratory data; record accepted. \%D between columns $>25$.

${ }^{\circ} Q$ ualifier added to laboratory data; record accepted. Matrix spike recovery grossly outside control limits. Matrix effects may exist.

$\% \mathrm{D}$ between columns $>25$. Surrogates diluted out.

$\mathrm{ft}$ bgs $=$ Feet below ground surface $\mu \mathrm{g} / \mathrm{kg}=$ Micrograms per kilogram $\mathrm{J}=$ Estimated value. 
Table A.7-13

Soil Sample Results for PCBs Detected Above Minimum Reporting Limits at CAS 06-23-01, Decon Pad Discharge Piping

\begin{tabular}{|c|c|c|c|}
\hline \multirow{2}{*}{$\begin{array}{l}\text { Sample } \\
\text { Location }\end{array}$} & \multirow{2}{*}{$\begin{array}{l}\text { Sample } \\
\text { Number }\end{array}$} & \multirow{2}{*}{$\begin{array}{l}\text { Depths } \\
\text { (ft bgs) }\end{array}$} & Contaminants of Potential Concern $(\mu \mathrm{g} / \mathrm{kg})$ \\
\hline & & & Aroclor 1254 \\
\hline \multicolumn{3}{|c|}{ Final Action Levels ${ }^{a}$} & 740 \\
\hline$\overline{\mathrm{G} 01}$ & $224 \mathrm{G} 002$ & $\overline{0-1}$ & $\overline{700(\mathrm{~J})}$ \\
\hline
\end{tabular}

${ }^{a}$ Based on U.S. Environmental Protection Agency, Region 9 Preliminary Remediation Goals (PRGs) (EPA, 2002)

$\mathrm{ft}$ bgs $=$ Feet below ground surface

$\mu \mathrm{g} / \mathrm{kg}=$ Micrograms per kilogram

$\mathrm{J}=$ Estimated value. Qualifier added to laboratory data; record accepted. Matrix effects may exist. Surrogates diluted out.

Table A.7-14

Soil Sample Results for TPH-DRO Detected Above Minimum Reporting Limits at CAS 06-05-01, Leachfield

\begin{tabular}{|c|c|c|c|}
\hline \multirow{2}{*}{$\begin{array}{l}\text { Sample } \\
\text { Location }\end{array}$} & \multirow{2}{*}{$\begin{array}{l}\text { Sample } \\
\text { Number }\end{array}$} & \multirow{2}{*}{$\begin{array}{l}\text { Depth } \\
\text { (ft bgs) }\end{array}$} & \multirow{2}{*}{$\begin{array}{c}\text { Contaminants of Potential Concern }(\mathrm{mg} / \mathrm{kg}) \\
\text { Diesel-Range Organics }\end{array}$} \\
\hline & & & \\
\hline \multicolumn{3}{|c|}{ Final Action Levels ${ }^{a}$} & 100 \\
\hline$\overline{\overline{E 04}}$ & 224E008 & $0-0.5$ & $\overline{\overline{31(\mathrm{M})}}$ \\
\hline E05 & 224E010 & $0-0.5$ & $51(\mathrm{M})$ \\
\hline E07 & 224E012 & $0-0.5$ & $90(\mathrm{M})$ \\
\hline E08 & 224E014 & $0-0.5$ & $34(\mathrm{M})$ \\
\hline E09 & 224E015 & $0-0.5$ & $40(M)$ \\
\hline E11 & 224E018 & $0-0.5$ & $78(\mathrm{M})$ \\
\hline E12 & 224E019 & $0-0.5$ & $25(\mathrm{M})$ \\
\hline E15 & 224E046 & $6-7$ & $32(\mathrm{M})$ \\
\hline E17 & 224E069 & $6.5-7.5$ & $26(\mathrm{H}, \mathrm{M})$ \\
\hline E19 & 224E072 & $4-5$ & $60(\mathrm{H}, \mathrm{M})$ \\
\hline E20 & 224E061 & $0-0.5$ & $34(\mathrm{H}, \mathrm{M})$ \\
\hline
\end{tabular}

a'Based on Nevada Administrative Code, "Contamination of Soil: Establishment of Action Levels" (NAC, 2002)

$\mathrm{ft}$ bgs $=$ Feet below ground surface

$\mathrm{mg} / \mathrm{kg}=$ Milligrams per kilogram

$\mathrm{H}=\mathrm{DRO}$ fuel pattern in the heavy end of retention time window.

$\mathrm{M}=\mathrm{A}$ pattern resembling motor oil was detected. 
Table A.7-15

Soil Sample Results for TPH-DRO Detected Above Minimum Reporting Limits at CAS 06-17-04, Decon Pad and Wastewater Catch

\begin{tabular}{|c|c|c|c|}
\hline \multirow{2}{*}{$\begin{array}{l}\text { Sample } \\
\text { Location }\end{array}$} & \multirow{2}{*}{$\begin{array}{l}\text { Sample } \\
\text { Number }\end{array}$} & \multirow{2}{*}{$\begin{array}{l}\text { Depth } \\
\text { (ft bgs) }\end{array}$} & Contaminants of Potential Concern $(\mathrm{mg} / \mathrm{kg})$ \\
\hline & & & Diesel-Range Organics \\
\hline \multicolumn{3}{|c|}{ Final Action Levels $^{a}$} & 100 \\
\hline F04 & $224 \mathrm{F011}$ & $1-2$ & $280(\mathrm{H})$ \\
\hline F08 & $224 \mathrm{~F} 023$ & $0.2-1$ & $190(\mathrm{M})$ \\
\hline F09 & $224 \mathrm{~F} 027$ & $0.2-1$ & $210(\mathrm{H})$ \\
\hline F10 & $224 \mathrm{~F} 030$ & $0-1$ & $47(\mathrm{~J})$ \\
\hline F11 & $224 \mathrm{~F} 033$ & $0-1$ & $380(\mathrm{H})$ \\
\hline F19 & $224 \mathrm{~F} 048$ & $1-3$ & $34(\mathrm{M})$ \\
\hline F20 & $224 \mathrm{~F} 049$ & $1-2$ & $33(\mathrm{M})$ \\
\hline F21 & $224 \mathrm{~F} 050$ & $1-3$ & $33(\mathrm{D})$ \\
\hline F24 & 224F054 & $3-5$ & $31(\mathrm{M})$ \\
\hline $\mathrm{F} 25$ & $224 \mathrm{~F} 056$ & $0-3$ & $110(\mathrm{M})$ \\
\hline F26 & 224F058 & $0-4$ & $36(\mathrm{M})$ \\
\hline \multirow{2}{*}{ F28 } & $224 \mathrm{~F} 062$ & $0-0.5$ & $160(\mathrm{M}, \mathrm{Z})$ \\
\hline & $224 \mathrm{~F} 063$ & $0-0.5$ & $170(\mathrm{M}, \mathrm{Z})$ \\
\hline F29 & 224F065 & $0-0.5$ & $32(\mathrm{M})$ \\
\hline F31 & 224F069 & $0-0.5$ & $190(\mathrm{M})$ \\
\hline F32 & $224 \mathrm{~F} 070$ & $0-0.5$ & $88(\mathrm{M})$ \\
\hline
\end{tabular}

aBased on Nevada Administrative Code, "Contamination of Soil: Establishment of Action Levels" (NAC, 2002)

$\mathrm{ft}$ bgs $=$ Feet below ground surface $\mathrm{mg} / \mathrm{kg}=$ Milligrams per kilogram

$\mathrm{D}=\mathrm{A}$ pattern resembling diesel was detected.

$\mathrm{H}=\mathrm{DRO}$ fuel pattern in the heavy end of retention time window.

$\mathrm{J}=$ Estimated value. Qualifier added to laboratory data; record accepted. Matrix effects may exist. Surrogate recovery exceeded the upper limits.

$M=A$ pattern resembling motor oil was detected.

$Z=A$ significant fraction of the reported result did not resemble the patterns of any of the following petroleum hydrocarbon products: gasoline, JP-4, JP-8, diesel, mineral spirits, motor oil, Stoddard solvent, Bunker C.

\section{A.7.2.6 Gamma-Emitting Radionuclides}

Gamma-emitting radionuclide analytical results for soil samples collected at CASs 06-05-01, 06-17-04, and 06-23-01 that were detected above MDCs are presented in Table A.7-17 through Table A.7-19. No gamma-emitting radionuclides were found above FALs. 
Table A.7-16

Soil Sample Results for TPH-DRO Detected Above Minimum Reporting Limits at CAS 06-23-01, Decon Pad Discharge Piping

\begin{tabular}{|c|c|c|c|}
\hline \multirow{2}{*}{$\begin{array}{l}\text { Sample } \\
\text { Location }\end{array}$} & \multirow{2}{*}{$\begin{array}{l}\text { Sample } \\
\text { Number }\end{array}$} & \multirow{2}{*}{$\begin{array}{l}\text { Depths } \\
\text { (ft bgs) }\end{array}$} & \multirow{2}{*}{$\begin{array}{c}\text { Contaminants of Potential Concern }(\mathrm{mg} / \mathrm{kg}) \\
\text { Diesel-Range Organics }\end{array}$} \\
\hline & & & \\
\hline \multicolumn{3}{|c|}{ Final Action Levels ${ }^{a}$} & 100 \\
\hline \multirow{4}{*}{ G01 } & $224 \mathrm{G} 001$ & $0-1$ & $270(H, Z)$ \\
\hline & $224 \mathrm{G} 002$ & $0-1$ & $290(H, Z)$ \\
\hline & $224 G 003$ & $2-3$ & $200(H, Z)$ \\
\hline & $224 G 004$ & $3-4$ & 65 \\
\hline
\end{tabular}

aBased on Nevada Administrative Code, "Contamination of Soil: Establishment of Action Levels" (NAC, 2002)

$\mathrm{ft}$ bgs $=$ Feet below ground surface

$\mathrm{mg} / \mathrm{kg}=$ Milligrams per kilogram

$\mathrm{H}=\mathrm{DRO}$ fuel pattern in the heavy end of retention time window.

$Z$ = A significant fraction of the reported result did not resemble the patterns of any of the following petroleum hydrocarbon products: gasoline, JP-4, JP-8, diesel, mineral spirits, motor oil, Stoddard solvent, Bunker C.

\section{A.7.2.7 Isotopes}

Isotope analytical results for soil samples collected at CASs 06-05-01, 06-17-04, and 06-23-01 that were detected above MDCs are presented in Table A.7-20 through Table A.7-22. Plutonium-239 was found above the FAL in three surface samples.

\section{A.7.2.8 Pesticides}

Pesticide analysis was added to the analytical suite at CASs 06-17-04 and 06-23-01 at the request of the Stoller-Navarro Joint Venture Analytical Services Department, due to tentative identification of the compound in the SVOC analysis. Pesticide analytical results for soil samples collected at CAS 06-23-01 that were detected above MRLs are presented in Table A.7-23. No pesticides were detected above MRLs at CAS 06-17-04.

\section{A.7.2.9 Sludge and Concrete Results}

The analytes detected above MRLs and MDCs for the two sludge and one concrete sample are presented in Table A.7-24. Several chemical and radiological analytes are present above waste disposal criteria (e.g., RCRA). Therefore, the sludge may need to be managed as mixed waste, when generated. 
Table A.7-17

Soil Sample Results for Gamma-Emitting Radionuclides Detected Above Minimum Detectable Concentrations at CAS 06-05-01, Leachfield (Page 1 of 8 )

\begin{tabular}{|c|c|c|c|c|c|c|c|c|c|c|c|c|c|c|}
\hline \multirow[b]{2}{*}{$\begin{array}{l}\text { Sample } \\
\text { Location }\end{array}$} & \multirow[b]{2}{*}{$\begin{array}{l}\text { Sample } \\
\text { Number }\end{array}$} & \multirow{3}{*}{$\begin{array}{c}\text { Depth } \\
\text { (ft bgs) }\end{array}$} & \multicolumn{12}{|c|}{ Contaminants of Potential Concern (pCi/g) } \\
\hline & & & \multicolumn{2}{|c|}{ 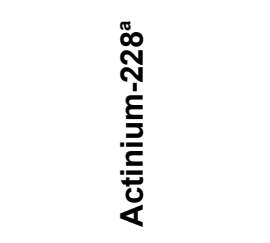 } & \multirow{3}{*}{ 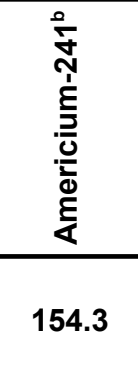 } & \multicolumn{2}{|c|}{ 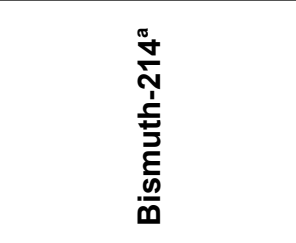 } & 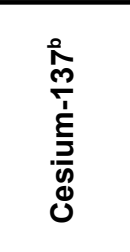 & \multicolumn{2}{|c|}{ 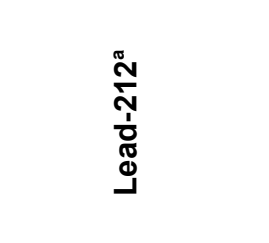 } & \multicolumn{2}{|c|}{ 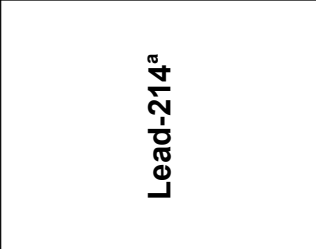 } & \multicolumn{2}{|c|}{ 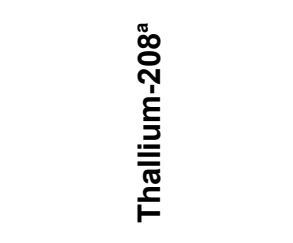 } \\
\hline \multirow{2}{*}{\multicolumn{3}{|c|}{ Final Action Levels }} & 5 & 15 & & 5 & 15 & & 5 & 15 & 5 & 15 & 5 & 15 \\
\hline & & & $<15$ & $>15$ & & $<15$ & $>15$ & & $<15$ & $>15$ & $<15$ & $>15$ & $<15$ & $>15$ \\
\hline \multirow{4}{*}{ E01 } & 224E001 & $0-0.5$ & $0.84(\mathrm{G})$ & $\overline{\mathrm{NA}}$ & $29.1(\mathrm{~J})^{\mathrm{C}}$ & $0.71(\mathrm{G}, \mathrm{J})$ & $\overline{\mathrm{NA}}$ & $0.99(\mathrm{G})$ & $1.06(\mathrm{~J})^{\mathrm{d}}$ & $\overline{\mathrm{NA}}$ & $0.73(\mathrm{G}, \mathrm{J})$ & $\overline{\mathrm{NA}}$ & $0.278(G)$ & $\overline{\mathrm{NA}}$ \\
\hline & 224E002 & $3-4$ & NA & $\overline{--}$ & $0.049(\mathrm{~J})^{\mathrm{c}}$ & NA & $0.66(\mathrm{~J})$ & $\overline{--}$ & $\mathrm{NA}$ & 0.83 & $\mathrm{NA}$ & $0.6(\mathrm{~J})$ & $\mathrm{NA}$ & 0.196 \\
\hline & 224E003 & $3-4$ & NA & 0.82 & $0(\mathrm{~J})^{\mathrm{c}}$ & NA & $0.59(\mathrm{~J})$ & $\overline{--}$ & $\mathrm{NA}$ & 0.6 & $\mathrm{NA}$ & $0.48(\mathrm{~J})$ & $\overline{N A}$ & 0.186 \\
\hline & 224E004 & $5-6$ & NA & 0.62 & $0.5(J)^{c}$ & $\overline{N A}$ & $0.45(\mathrm{~J})$ & $\overline{--}$ & $\mathrm{NA}$ & 0.75 & NA & $0.59(\mathrm{~J})$ & NA & $\overline{--}$ \\
\hline \multirow{3}{*}{ E02 } & 224E005 & $0-0.5$ & $0.71(\mathrm{G})$ & NA & -- & $0.5(\mathrm{G}, \mathrm{J})$ & NA & -- & $0.6(J)^{d}$ & NA & $0.55(\mathrm{G}, \mathrm{J})$ & $\mathrm{NA}$ & -- & NA \\
\hline & 224E007 & $4-5$ & NA & $\overline{--}$ & -- & NA & -- & -- & NA & 0.78 & NA & $0.46(\mathrm{~J})$ & NA & -- \\
\hline & 224E009 & $7-8$ & NA & $\overline{--}$ & -- & NA & $0.45(\mathrm{~J})$ & $\overline{--}$ & $\mathrm{NA}$ & 0.73 & $\mathrm{NA}$ & $0.52(\mathrm{~J})$ & NA & -- \\
\hline \multirow{3}{*}{ E03 } & 224E006 & $0-0.5$ & $0.77(\mathrm{G})$ & NA & -- & $0.71(\mathrm{G}, \mathrm{J})$ & NA & $\begin{array}{l}0.337 \\
(L T, G)\end{array}$ & $0.88(J)^{d}$ & NA & $0.66(\mathrm{G}, \mathrm{J})$ & NA & $0.269(\mathrm{G})$ & NA \\
\hline & 224E013 & $4-5$ & $\mathrm{NA}$ & 0.89 & -- & NA & $0.42(\mathrm{~J})$ & -- & $\mathrm{NA}$ & 0.8 & $\mathrm{NA}$ & $0.75(\mathrm{~J})$ & $\mathrm{NA}$ & 0.354 \\
\hline & 224E016 & $7-8$ & NA & -- & -- & NA & $0.6(\mathrm{~J})$ & -- & NA & 0.85 & $\mathrm{NA}$ & $0.64(\mathrm{~J})$ & $\mathrm{NA}$ & 0.225 \\
\hline \multirow{4}{*}{ E04 } & 224E008 & $0-0.5$ & $\overline{--}$ & NA & -- & $0.57(\mathrm{~J})$ & $\mathrm{NA}$ & 0.78 & 0.59 & $\mathrm{NA}$ & $0.69(\mathrm{~J})$ & $\mathrm{NA}$ & 0.205 & $\mathrm{NA}$ \\
\hline & 224E052 & $3.5-4.5$ & NA & 0.81 & $\overline{--}$ & NA & $0.52(\mathrm{~J})$ & $\overline{--}$ & $\mathrm{NA}$ & 0.72 & $\overline{N A}$ & $0.65(\mathrm{~J})$ & $\overline{N A}$ & 0.257 \\
\hline & 224E053 & $7-8$ & $\overline{N A}$ & $0.87(\mathrm{G})$ & $\overline{--}$ & $\overline{N A}$ & $0.66(\mathrm{G}, \mathrm{J})$ & $\overline{--}$ & $\mathrm{NA}$ & $0.69(\mathrm{~J})^{\mathrm{d}}$ & $\overline{N A}$ & $0.65(\mathrm{G}, \mathrm{J})$ & $\overline{N A}$ & $0.232(\mathrm{G})$ \\
\hline & 224E054 & $9-10$ & $\mathrm{NA}$ & $\overline{--}$ & $\overline{--}$ & $\mathrm{NA}$ & $0.64(\mathrm{G}, \mathrm{J})$ & $\overline{--}$ & $\overline{N A}$ & $0.66(\mathrm{~J})^{\mathrm{d}}$ & $\overline{N A}$ & $0.47(\mathrm{G}, \mathrm{J})$ & $\mathrm{NA}$ & $0.222(\mathrm{G})$ \\
\hline
\end{tabular}


Table A.7-17

Soil Sample Results for Gamma-Emitting Radionuclides Detected Above Minimum Detectable Concentrations at CAS 06-05-01, Leachfield (Page 2 of 8)

\begin{tabular}{|c|c|c|c|c|c|c|c|c|c|c|c|c|c|c|}
\hline \multirow[b]{2}{*}{$\begin{array}{l}\text { Sample } \\
\text { Location }\end{array}$} & \multirow[b]{2}{*}{$\begin{array}{l}\text { Sample } \\
\text { Number }\end{array}$} & \multirow{3}{*}{$\begin{array}{c}\text { Depth } \\
\text { (ft bgs) }\end{array}$} & \multicolumn{12}{|c|}{ Contaminants of Potential Concern (pCi/g) } \\
\hline & & & \multicolumn{2}{|c|}{ 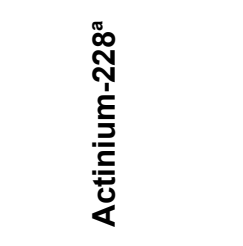 } & \multirow{3}{*}{ 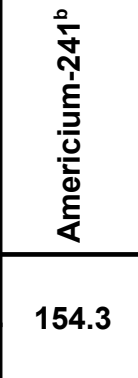 } & \multicolumn{2}{|c|}{ 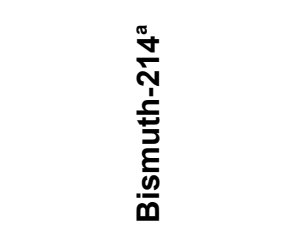 } & \multicolumn{3}{|c|}{ 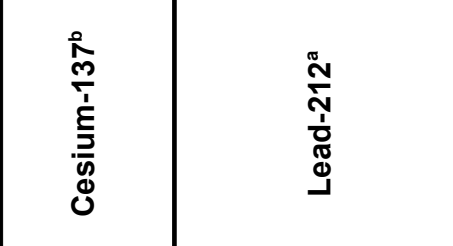 } & \multicolumn{2}{|c|}{ 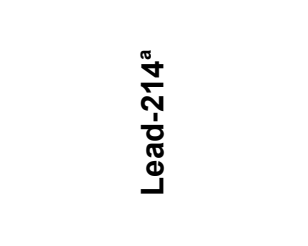 } & \multicolumn{2}{|c|}{ 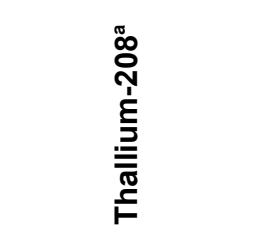 } \\
\hline \multicolumn{2}{|c|}{ Final Action Levels } & & 5 & 15 & & 5 & 15 & & 5 & 15 & 5 & 15 & 5 & 15 \\
\hline \multicolumn{3}{|c|}{ Depth bgs (cm) } & $<15$ & $>15$ & & $<15$ & $>15$ & & $<15$ & $>15$ & $<15$ & $>15$ & $<15$ & $>15$ \\
\hline \multirow{5}{*}{ E05 } & $224 \mathrm{E} 010$ & $0-0.5$ & $\overline{---}$ & $\overline{\mathrm{NA}}$ & $\overline{---}$ & $0.73(\mathrm{G}, \mathrm{J})$ & $\overline{\mathrm{NA}}$ & $6.87(\mathrm{G})$ & $0.81(J)^{d}$ & $\overline{\mathrm{NA}}$ & $0.71(\mathrm{G}, \mathrm{J})$ & $\overline{\mathrm{NA}}$ & $\overline{---}$ & $\overline{\mathrm{NA}}$ \\
\hline & 224E020 & $4-5$ & NA & 0.67 & $-0.29(\mathrm{~J})^{\mathrm{c}}$ & NA & $0.43(\mathrm{~J})$ & -- & $\mathrm{NA}$ & 0.82 & $\overline{N A}$ & $0.59(\mathrm{~J})$ & $\overline{N A}$ & 0.207 \\
\hline & 224E021 & $4-5$ & NA & 0.69 & $0.03(\mathrm{~J})^{\mathrm{c}}$ & NA & $0.51(\mathrm{~J})$ & -- & NA & 0.77 & NA & $0.63(\mathrm{~J})$ & NA & 0.225 \\
\hline & 224E022 & $7-8$ & NA & -- & $-0.02(\mathrm{~J})^{\mathrm{c}}$ & NA & $0.35(\mathrm{~J})$ & -- & $\mathrm{NA}$ & 0.66 & $\mathrm{NA}$ & $0.59(\mathrm{~J})$ & NA & 0.262 \\
\hline & 224E023 & $9-10$ & NA & - & $0.02(J)^{c}$ & NA & - & - & NA & 0.4 & NA & $0.47(\mathrm{~J})$ & NA & 0.195 \\
\hline \multirow{4}{*}{ E06 } & 224E011 & $0-0.5$ & 0.55 & NA & -- & $0.44(\mathrm{~J})$ & NA & $0.193(\mathrm{LT})$ & 0.74 & NA & $0.58(\mathrm{~J})$ & NA & 0.202 & $\mathrm{NA}$ \\
\hline & 224E055 & $3.5-4.5$ & NA & -- & -- & NA & $0.54(\mathrm{~J})$ & -- & NA & 0.55 & $\mathrm{NA}$ & $0.55(\mathrm{~J})$ & NA & 0.222 \\
\hline & 224E056 & $7-8$ & NA & 0.7 & -- & NA & $0.45(\mathrm{~J})$ & -- & $\mathrm{NA}$ & 0.78 & $\mathrm{NA}$ & $0.73(\mathrm{~J})$ & NA & 0.325 \\
\hline & 224E057 & $9-10$ & $\mathrm{NA}$ & 0.9 & $\overline{--}$ & $\mathrm{NA}$ & $0.68(J)$ & $\overline{--}$ & $\overline{N A}$ & 0.93 & $\overline{N A}$ & $0.58(\mathrm{~J})$ & $\overline{N A}$ & 0.36 \\
\hline \multirow{3}{*}{ E07 } & 224E012 & $0-0.5$ & 0.59 & $\overline{N A}$ & -- & $0.58(\mathrm{~J})$ & $\mathrm{NA}$ & 0.72 & 0.59 & $\overline{N A}$ & $0.51(\mathrm{~J})$ & $\mathrm{NA}$ & 0.179 & $\overline{N A}$ \\
\hline & 224E037 & $5-6$ & NA & $1.09(\mathrm{G})$ & -- & $\overline{N A}$ & $0.78(\mathrm{G}, \mathrm{J})$ & -- & $\mathrm{NA}$ & $0.98(\mathrm{~J})^{\mathrm{d}}$ & $\mathrm{NA}$ & $0.75(\mathrm{G}, \mathrm{J})$ & $\mathrm{NA}$ & $0.32(\mathrm{G})$ \\
\hline & 224E038 & $9-10$ & NA & $0.96(\mathrm{G})$ & -- & NA & $0.63(\mathrm{G}, \mathrm{J})$ & -- & $\mathrm{NA}$ & $1.15(\mathrm{~J})^{\mathrm{d}}$ & $\mathrm{NA}$ & $0.67(\mathrm{G}, \mathrm{J})$ & $\mathrm{NA}$ & $0.299(\mathrm{G})$ \\
\hline \multirow{4}{*}{ E08 } & 224E014 & $0-0.5$ & 0.8 & NA & 6.28 & $0.68(\mathrm{~J})$ & NA & $0.42(\mathrm{LT})$ & 0.79 & NA & $0.55(\mathrm{~J})$ & NA & -- & NA \\
\hline & 224E025 & $2-3$ & NA & 0.82 & $0.47(\mathrm{~J})^{\mathrm{c}}$ & NA & $0.74(\mathrm{~J})$ & -- & NA & 0.81 & NA & $0.49(\mathrm{~J})$ & NA & 0.237 \\
\hline & 224E026 & $7-8$ & NA & 0.65 & $0.006(\mathrm{~J})^{\mathrm{c}}$ & NA & $0.48(\mathrm{~J})$ & -- & $\mathrm{NA}$ & 0.73 & NA & $0.56(\mathrm{~J})$ & NA & 0.33 \\
\hline & 224E027 & $9-10$ & NA & 0.79 & $-0.5(\mathrm{~J})^{\mathrm{c}}$ & NA & $0.6(\mathrm{~J})$ & -- & $\mathrm{NA}$ & 0.87 & NA & $0.78(\mathrm{~J})$ & NA & 0.293 \\
\hline
\end{tabular}


Table A.7-17

Soil Sample Results for Gamma-Emitting Radionuclides Detected Above Minimum Detectable Concentrations at CAS 06-05-01, Leachfield (Page 3 of 8 )

\begin{tabular}{|c|c|c|c|c|c|c|c|c|c|c|c|c|c|c|}
\hline \multirow[b]{2}{*}{$\begin{array}{l}\text { Sample } \\
\text { Location }\end{array}$} & \multirow[b]{2}{*}{$\begin{array}{l}\text { Sample } \\
\text { Number }\end{array}$} & \multirow{3}{*}{$\begin{array}{c}\text { Depth } \\
\text { (ft bgs) }\end{array}$} & \multicolumn{12}{|c|}{ Contaminants of Potential Concern (pCi/g) } \\
\hline & & & \multicolumn{2}{|c|}{ 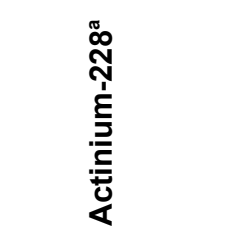 } & 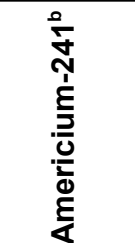 & \multicolumn{2}{|c|}{ 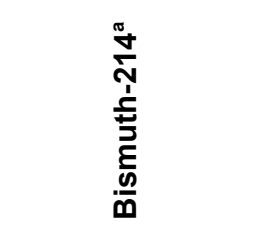 } & 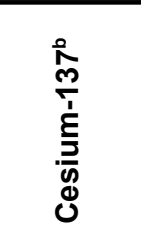 & \multicolumn{2}{|c|}{ 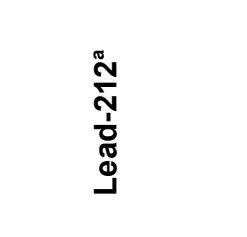 } & \multicolumn{2}{|c|}{ 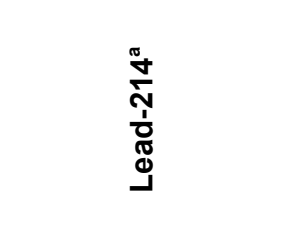 } & \multicolumn{2}{|c|}{ 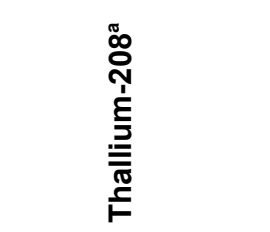 } \\
\hline \multicolumn{2}{|c|}{ Final Action Levels } & & 5 & 15 & \multirow{2}{*}{154.3} & 5 & 15 & \multirow{2}{*}{36.22} & 5 & 15 & 5 & 15 & 5 & 15 \\
\hline \multicolumn{3}{|c|}{ Depth bgs (cm) } & $<15$ & $>15$ & & $<15$ & $>15$ & & $<15$ & $>15$ & $<15$ & $>15$ & $<15$ & $>15$ \\
\hline \multirow{3}{*}{ E09 } & $224 \mathrm{E} 015$ & $\overline{0-0.5}$ & $\overline{0.76}$ & $\overline{\mathrm{NA}}$ & 2.66 & $\overline{0.61(\mathrm{~J})}$ & $\overline{\mathrm{NA}}$ & $\overline{0.43(\mathrm{LT})}$ & 0.93 & $\overline{\mathrm{NA}}$ & 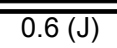 & $\overline{\mathrm{NA}}$ & $\overline{0.246}$ & $\overline{\mathrm{NA}}$ \\
\hline & 224E035 & $6-7$ & NA & -- & -- & NA & $0.58(\mathrm{~J})$ & -- & NA & 0.74 & NA & $0.63(\mathrm{~J})$ & NA & 0.25 \\
\hline & 224E036 & $9-10$ & NA & 0.88 & -- & NA & $0.75(\mathrm{~J})$ & -- & NA & 1.2 & NA & $0.82(\mathrm{~J})$ & NA & 0.354 \\
\hline \multirow{3}{*}{ E10 } & 224E017 & $0-0.5$ & 0.87 & NA & -- & $0.62(\mathrm{~J})$ & NA & -- & 0.77 & $\mathrm{NA}$ & $0.63(\mathrm{~J})$ & NA & 0.236 & NA \\
\hline & 224E033 & $5-6$ & NA & $0.83(\mathrm{G})$ & -- & NA & $0.64(\mathrm{G}, \mathrm{J})$ & -- & NA & $1.1(\mathrm{~J})^{\mathrm{d}}$ & NA & $0.66(\mathrm{G}, \mathrm{J})$ & NA & $0.24(\mathrm{G})$ \\
\hline & 224E034 & $8-9$ & NA & $1.02(\mathrm{G})$ & -- & NA & $0.7(\mathrm{G}, \mathrm{J})$ & $\overline{--}$ & NA & $1.06(J)^{d}$ & NA & $0.69(\mathrm{G}, \mathrm{J})$ & NA & $0.315(\mathrm{G})$ \\
\hline \multirow{4}{*}{ E11 } & 224E018 & $0-0.5$ & 0.78 & NA & -- & $0.62(\mathrm{~J})$ & NA & $0.36(\mathrm{LT})$ & 0.7 & NA & $0.61(\mathrm{~J})$ & NA & 0.194 & NA \\
\hline & 224E028 & $2-3$ & NA & 0.63 & $0.09(\mathrm{~J})^{\mathrm{c}}$ & NA & $0.49(\mathrm{~J})$ & -- & NA & 0.8 & NA & $0.63(\mathrm{~J})$ & $\mathrm{NA}$ & 0.23 \\
\hline & 224E029 & $5-6$ & NA & 0.87 & $0.022(\mathrm{~J})^{\mathrm{c}}$ & NA & $0.56(\mathrm{~J})$ & -- & NA & 0.85 & $\mathrm{NA}$ & $0.53(\mathrm{~J})$ & $\mathrm{NA}$ & 0.31 \\
\hline & 224E030 & $7-8$ & NA & $0.91(\mathrm{G})$ & $0.02(\mathrm{~J})^{\mathrm{c}}$ & NA & $0.68(\mathrm{G}, \mathrm{J})$ & -- & NA & $1.05(\mathrm{~J})^{\mathrm{d}}$ & NA & $0.81(\mathrm{G}, \mathrm{J})$ & $\mathrm{NA}$ & $0.26(\mathrm{G})$ \\
\hline \multirow{3}{*}{ E12 } & 224E019 & $0-0.5$ & 0.63 & NA & -- & -- & NA & -- & 0.47 & NA & $0.52(\mathrm{~J})$ & NA & 0.246 & NA \\
\hline & 224E031 & $4-5$ & NA & $0.9(\mathrm{G})$ & -- & NA & $0.56(\mathrm{G}, \mathrm{J})$ & - & NA & $1.08(\mathrm{~J})^{\mathrm{d}}$ & NA & $0.6(\mathrm{G}, \mathrm{J})$ & NA & $0.336(\mathrm{G})$ \\
\hline & 224E032 & $7-8$ & NA & $1.11(\mathrm{G})$ & -- & NA & $0.66(\mathrm{G}, \mathrm{J})$ & -- & NA & $1.32(J)^{d}$ & NA & $0.79(\mathrm{G}, \mathrm{J})$ & NA & $0.37(\mathrm{G})$ \\
\hline E13 & 224E024 & $7-8$ & NA & 0.8 & $-0.04(J)^{c}$ & NA & $0.52(\mathrm{~J})$ & -- & NA & 0.82 & NA & $0.61(\mathrm{~J})$ & NA & 0.269 \\
\hline
\end{tabular}


Table A.7-17

Soil Sample Results for Gamma-Emitting Radionuclides Detected Above Minimum Detectable Concentrations at CAS 06-05-01, Leachfield (Page 4 of 8 )

\begin{tabular}{|c|c|c|c|c|c|c|c|c|c|c|c|c|c|c|}
\hline \multirow[b]{2}{*}{$\begin{array}{c}\text { Sample } \\
\text { Location }\end{array}$} & \multirow[b]{2}{*}{$\begin{array}{l}\text { Sample } \\
\text { Number }\end{array}$} & \multirow{3}{*}{$\begin{array}{c}\text { Depth } \\
\text { (ft bgs) }\end{array}$} & \multicolumn{12}{|c|}{ Contaminants of Potential Concern (pCi/g) } \\
\hline & & & \multicolumn{2}{|c|}{ 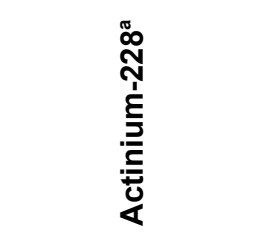 } & \multirow{3}{*}{ 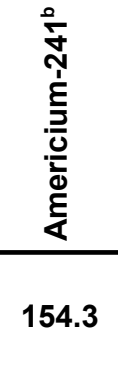 } & \multicolumn{2}{|c|}{ 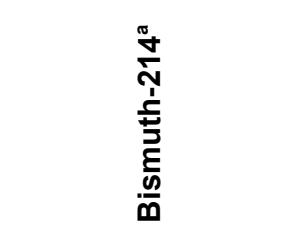 } & 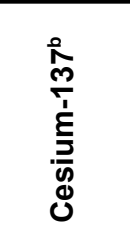 & \multicolumn{2}{|c|}{ 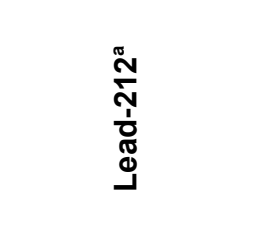 } & \multicolumn{2}{|c|}{ 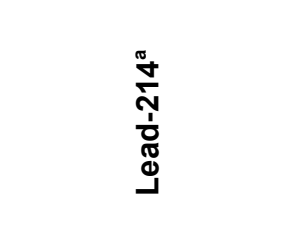 } & \multicolumn{2}{|c|}{ 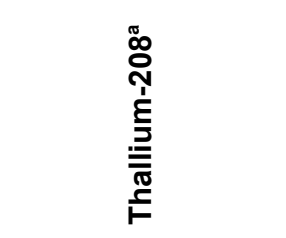 } \\
\hline \multicolumn{2}{|c|}{ Final Action Levels } & & 5 & 15 & & 5 & 15 & & 5 & 15 & 5 & 15 & 5 & 15 \\
\hline \multicolumn{3}{|c|}{ Depth bgs (cm) } & $<15$ & $>15$ & & $<15$ & $>15$ & & $<15$ & $>15$ & $<15$ & $>15$ & $<15$ & $>15$ \\
\hline \multirow{5}{*}{ E14 } & 224E039 & $0-0.5$ & $\overline{---}$ & $\overline{\mathrm{NA}}$ & $\overline{---}$ & $0.46(\mathrm{~J})$ & $\overline{\mathrm{NA}}$ & $\overline{---}$ & 0.98 & $\overline{\mathrm{NA}}$ & $0.56(\mathrm{~J})$ & $\overline{\mathrm{NA}}$ & 0.213 & $\overline{\mathrm{NA}}$ \\
\hline & 224E040 & $2-3$ & NA & -- & -- & $\overline{N A}$ & $0.62(\mathrm{G}, \mathrm{J})$ & -- & $\overline{N A}$ & $0.86(\mathrm{~J})^{d}$ & $\overline{N A}$ & $0.64(\mathrm{G}, \mathrm{J})$ & $\overline{N A}$ & $0.249(\mathrm{G})$ \\
\hline & 224E041 & $6-7$ & NA & 0.6 & -- & NA & $0.46(\mathrm{~J})$ & -- & NA & 0.72 & NA & $0.4(\mathrm{~J})$ & NA & 0.243 \\
\hline & 224E042 & $6-7$ & NA & 0.78 & -- & NA & $0.69(\mathrm{~J})$ & -- & $\mathrm{NA}$ & 0.77 & NA & $0.61(\mathrm{~J})$ & NA & 0.227 \\
\hline & 224E043 & $9-10$ & NA & $0.81(\mathrm{G})$ & $\overline{--}$ & NA & $0.6(\mathrm{G}, \mathrm{J})$ & - & NA & $0.87(\mathrm{~J})^{\mathrm{d}}$ & NA & $0.67(\mathrm{G}, \mathrm{J})$ & NA & - \\
\hline \multirow{4}{*}{ E15 } & 224E044 & $0-0.5$ & -- & NA & $0.32(\mathrm{~J})^{\mathrm{d}}$ & $0.58(\mathrm{G}, \mathrm{J})$ & NA & -- & $0.79(\mathrm{~J})^{\mathrm{d}}$ & NA & $0.52(\mathrm{G}, \mathrm{J})$ & NA & -- & $\mathrm{NA}$ \\
\hline & 224E045 & $2-3$ & NA & -- & -- & NA & $0.42(\mathrm{~J})$ & -- & NA & 0.73 & $\mathrm{NA}$ & $0.61(\mathrm{~J})$ & NA & 0.246 \\
\hline & 224E046 & $6-7$ & NA & 0.79 & -- & NA & $0.53(\mathrm{~J})$ & -- & NA & 0.68 & NA & $0.63(\mathrm{~J})$ & NA & 0.205 \\
\hline & 224E047 & $9-10$ & $\mathrm{NA}$ & $0.94(G)$ & $\overline{--}$ & $\mathrm{NA}$ & $0.6(\mathrm{G}, \mathrm{J})$ & -- & NA & $1.1(\mathrm{~J})^{\mathrm{d}}$ & $\mathrm{NA}$ & $0.82(\mathrm{G}, \mathrm{J})$ & NA & $0.312(\mathrm{G})$ \\
\hline \multirow{4}{*}{ E16 } & 224E048 & $0-0.5$ & 0.65 & NA & -- & $0.57(\mathrm{~J})$ & $\overline{N A}$ & -- & 0.67 & $\mathrm{NA}$ & $0.58(\mathrm{~J})$ & $\overline{N A}$ & 0.24 & $\overline{N A}$ \\
\hline & 224E049 & $2-3$ & NA & 0.78 & -- & NA & $0.66(\mathrm{~J})$ & -- & NA & 0.92 & NA & $0.68(\mathrm{~J})$ & NA & 0.322 \\
\hline & 224E050 & $7-8$ & NA & $0.83(\mathrm{G})$ & -- & NA & $0.61(\mathrm{G}, \mathrm{J})$ & -- & $\mathrm{NA}$ & $1.37(\mathrm{~J})^{\mathrm{d}}$ & $\mathrm{NA}$ & $0.77(\mathrm{G}, \mathrm{J})$ & $\mathrm{NA}$ & $0.289(\mathrm{G})$ \\
\hline & 224E051 & $10-11$ & NA & -- & -- & NA & $0.55(\mathrm{~J})$ & -- & $\mathrm{NA}$ & 0.77 & $\mathrm{NA}$ & $0.63(\mathrm{~J})$ & NA & -- \\
\hline \multirow{3}{*}{ E17 } & 224E058 & $0-0.5$ & $0.92(\mathrm{G})$ & NA & -- & $0.64(\mathrm{G}, \mathrm{J})$ & NA & $1.21(\mathrm{G})$ & $1.18(\mathrm{~J})^{\mathrm{d}}$ & NA & $0.87(\mathrm{G}, \mathrm{J})$ & NA & $0.39(\mathrm{G})$ & NA \\
\hline & 224E068 & $4-5$ & NA & $0.74(\mathrm{G})$ & -- & NA & $0.79(\mathrm{G}, \mathrm{J})$ & $0.97(\mathrm{G})$ & $\mathrm{NA}$ & $1.04(\mathrm{~J})^{\mathrm{d}}$ & $\mathrm{NA}$ & $0.8(G, J)$ & NA & $0.34(\mathrm{G})$ \\
\hline & 224E069 & $6.5-7.5$ & NA & 0.67 & -- & NA & $0.63(\mathrm{~J})$ & 3.32 & NA & 0.71 & NA & $0.73(\mathrm{~J})$ & NA & 0.26 \\
\hline
\end{tabular}


Table A.7-17

Soil Sample Results for Gamma-Emitting Radionuclides Detected Above Minimum Detectable Concentrations at CAS 06-05-01, Leachfield (Page 5 of 8)

\begin{tabular}{|c|c|c|c|c|c|c|c|c|c|c|c|c|c|c|}
\hline \multirow[b]{2}{*}{$\begin{array}{l}\text { Sample } \\
\text { Location }\end{array}$} & \multirow[b]{2}{*}{$\begin{array}{l}\text { Sample } \\
\text { Number }\end{array}$} & \multirow{3}{*}{$\begin{array}{c}\text { Depth } \\
\text { (ft bgs) }\end{array}$} & \multicolumn{12}{|c|}{ Contaminants of Potential Concern (pCi/g) } \\
\hline & & & \multicolumn{2}{|c|}{ 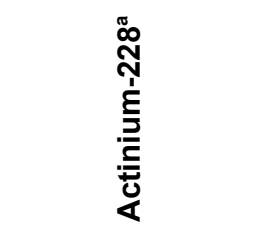 } & \multirow{3}{*}{ 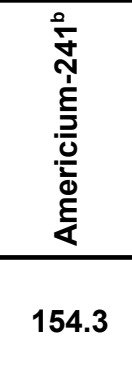 } & \multicolumn{2}{|c|}{ 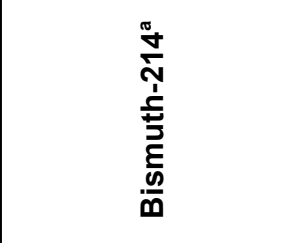 } & 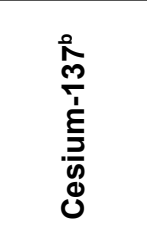 & \multicolumn{2}{|c|}{ 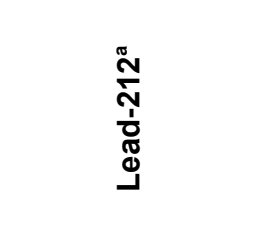 } & \multicolumn{2}{|c|}{ 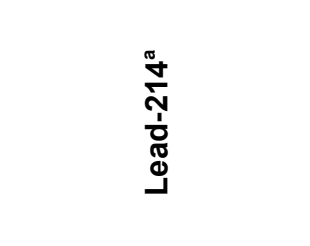 } & \multicolumn{2}{|c|}{ 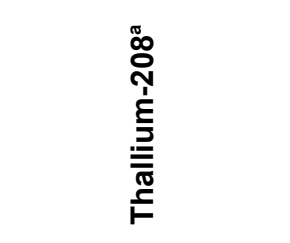 } \\
\hline \multicolumn{2}{|c|}{ Final Action Levels } & & 5 & 15 & & 5 & 15 & & 5 & 15 & 5 & 15 & 5 & 15 \\
\hline \multicolumn{3}{|c|}{ Depth bgs (cm) } & $<15$ & $>15$ & & $<15$ & $>15$ & & $<15$ & $>15$ & $<15$ & $>15$ & $<15$ & $>15$ \\
\hline \multirow{3}{*}{ E18 } & 224E059 & $0-0.5$ & $\overline{0.73}$ & $\overline{\mathrm{NA}}$ & $\overline{---}$ & $0.62(\mathrm{~J})$ & $\overline{\mathrm{NA}}$ & $0.292(\mathrm{LT})$ & 0.69 & $\overline{\mathrm{NA}}$ & $0.7(\mathrm{~J})$ & $\overline{\mathrm{NA}}$ & 0.3 & $\overline{\mathrm{NA}}$ \\
\hline & 224E070 & $4-5$ & NA & 1.12 & -- & NA & $0.53(\mathrm{~J})$ & -- & NA & 1.17 & $\mathrm{NA}$ & $0.92(\mathrm{~J})$ & NA & 0.309 \\
\hline & 224E071 & $7-8$ & NA & 0.99 & -- & NA & $0.53(\mathrm{~J})$ & 1.53 & NA & 0.75 & NA & $0.6(\mathrm{~J})$ & NA & 0.212 \\
\hline \multirow{3}{*}{ E19 } & 224E060 & $0-0.5$ & $1.13(\mathrm{G})$ & NA & $0.4(\mathrm{~J})^{\mathrm{d}}$ & $0.92(\mathrm{G}, \mathrm{J})$ & NA & $1.25(\mathrm{G})$ & $1.02(\mathrm{~J})^{\mathrm{d}}$ & NA & $1(\mathrm{G}, \mathrm{J})$ & $\mathrm{NA}$ & $0.24(\mathrm{G})$ & NA \\
\hline & 224E072 & $4-5$ & NA & -- & -- & NA & $0.6(\mathrm{~J})$ & 6.15 & NA & 0.8 & NA & $0.56(\mathrm{~J})$ & NA & 0.24 \\
\hline & 224E073 & $7-8$ & NA & 0.86 & -- & NA & $0.6(\mathrm{~J})$ & 1.64 & NA & 1.06 & NA & $0.72(\mathrm{~J})$ & NA & 0.257 \\
\hline \multirow{4}{*}{ E20 } & 224E061 & $0-0.5$ & -- & NA & -- & $0.6(\mathrm{G}, \mathrm{J})$ & NA & $10(\mathrm{G})$ & $0.97(\mathrm{~J})^{\mathrm{d}}$ & $\mathrm{NA}$ & $0.69(\mathrm{G}, \mathrm{J})$ & $\mathrm{NA}$ & $0.31(\mathrm{G})$ & $\mathrm{NA}$ \\
\hline & 224E063 & $4-5$ & NA & -- & -- & NA & -- & $0.251(\mathrm{LT})$ & NA & 0.55 & NA & $0.49(\mathrm{~J})$ & NA & 0.208 \\
\hline & 224E066 & $6.5-7.5$ & $\mathrm{NA}$ & 0.73 & $\overline{--}$ & $\mathrm{NA}$ & $0.4(\mathrm{~J})$ & 0.85 & $\mathrm{NA}$ & 0.54 & $\mathrm{NA}$ & $0.41(\mathrm{~J})$ & $\overline{N A}$ & $\overline{--}$ \\
\hline & 224E067 & $6.5-7.5$ & NA & 0.4 & -- & NA & $0.4(\mathrm{~J})$ & 0.74 & NA & 0.478 & NA & $0.393(\mathrm{~J})$ & NA & 0.116 \\
\hline \multirow{3}{*}{ E21 } & 224E062 & $0-0.5$ & -- & NA & -- & $0.65(\mathrm{~J})$ & NA & $0.42(\mathrm{LT})$ & 0.93 & NA & $0.6(\mathrm{~J})$ & NA & 0.204 & NA \\
\hline & 224E074 & $4-5$ & NA & 0.61 & -- & NA & $0.57(\mathrm{~J})$ & 1.99 & NA & 0.92 & NA & $0.72(\mathrm{~J})$ & NA & 0.234 \\
\hline & 224E075 & $7-8$ & NA & $0.85(\mathrm{G})$ & -- & NA & $0.52(\mathrm{G}, \mathrm{J})$ & $1.4(\mathrm{G})$ & $\mathrm{NA}$ & $0.8(\mathrm{~J})^{\mathrm{d}}$ & $\mathrm{NA}$ & $0.65(\mathrm{G}, \mathrm{J})$ & $\mathrm{NA}$ & $0.269(\mathrm{G})$ \\
\hline \multirow{3}{*}{ E22 } & 224E064 & $0-0.5$ & $1.1(\mathrm{G})$ & NA & $0.204(J)^{d}$ & $0.79(\mathrm{G}, \mathrm{J})$ & NA & $0.87(\mathrm{G})$ & $1.03(\mathrm{~J})^{\mathrm{d}}$ & $\mathrm{NA}$ & $0.73(\mathrm{G}, \mathrm{J})$ & NA & $0.3(\mathrm{G})$ & NA \\
\hline & 224E076 & $4-5$ & NA & 0.77 & -- & NA & -- & -- & $\mathrm{NA}$ & 0.75 & NA & $0.48(\mathrm{~J})$ & NA & 0.252 \\
\hline & 224E077 & $7-8$ & NA & -- & -- & NA & $0.49(\mathrm{~J})$ & -- & NA & 0.42 & NA & $0.62(\mathrm{~J})$ & NA & 0.251 \\
\hline
\end{tabular}


Table A.7-17

Soil Sample Results for Gamma-Emitting Radionuclides Detected Above Minimum Detectable Concentrations at CAS 06-05-01, Leachfield (Page 6 of 8)

\begin{tabular}{|c|c|c|c|c|c|c|c|c|c|c|c|c|c|c|}
\hline \multirow[b]{2}{*}{$\begin{array}{c}\text { Sample } \\
\text { Location }\end{array}$} & \multirow[b]{2}{*}{$\begin{array}{l}\text { Sample } \\
\text { Number }\end{array}$} & \multirow{3}{*}{$\begin{array}{c}\text { Depth } \\
\text { (ft bgs) }\end{array}$} & \multicolumn{12}{|c|}{ Contaminants of Potential Concern (pCi/g) } \\
\hline & & & \multicolumn{2}{|c|}{ 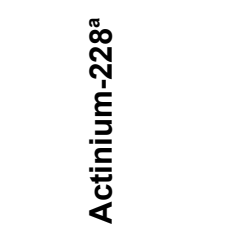 } & \multirow{3}{*}{ 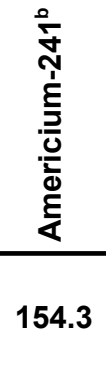 } & \multicolumn{2}{|c|}{ 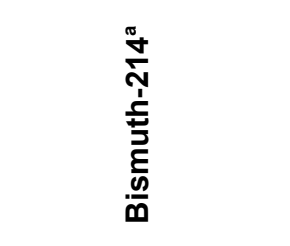 } & 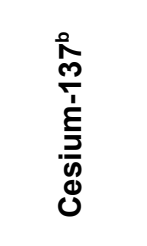 & \multicolumn{2}{|c|}{ 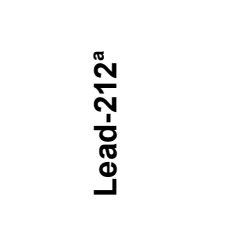 } & \multicolumn{2}{|c|}{ 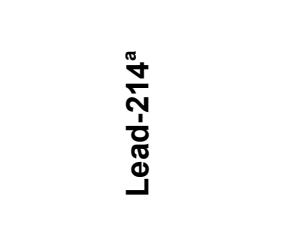 } & \multicolumn{2}{|c|}{ 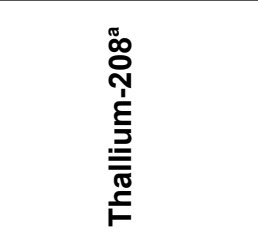 } \\
\hline \multicolumn{2}{|c|}{ Final Action Levels } & & 5 & 15 & & 5 & 15 & & 5 & 15 & 5 & 15 & 5 & 15 \\
\hline \multicolumn{3}{|c|}{ Depth bgs (cm) } & $<15$ & $>15$ & & $<15$ & $>15$ & & $<15$ & $>15$ & $<15$ & $>15$ & $<15$ & $>15$ \\
\hline \multirow{3}{*}{ E23 } & 224E065 & $0-0.5$ & 1.09 & $\overline{\mathrm{NA}}$ & $\overline{---}$ & $0.61(\mathrm{~J})$ & $\overline{\mathrm{NA}}$ & $0.34(\mathrm{LT})$ & 1.27 & $\overline{N A}$ & $0.75(\mathrm{~J})$ & NA & 0.319 & $\overline{N A}$ \\
\hline & 224E078 & $4-5$ & NA & 0.49 & -- & NA & $0.39(\mathrm{~J})$ & -- & NA & 0.41 & NA & $0.46(\mathrm{~J})$ & NA & -- \\
\hline & 224E079 & $7-8$ & NA & 0.54 & - & NA & $0.49(\mathrm{~J})$ & $0.346(\mathrm{LT})$ & NA & 0.64 & NA & $0.443(\mathrm{~J})$ & NA & 0.171 \\
\hline \multirow{3}{*}{ E24 } & 224E080 & $0-1$ & NA & $1.2(\mathrm{G})$ & -- & NA & $0.84(\mathrm{G}, \mathrm{J})$ & $1.57(\mathrm{G})$ & NA & $1.46(\mathrm{~J})^{\mathrm{d}}$ & NA & $0.85(\mathrm{G}, \mathrm{J})$ & NA & $0.35(\mathrm{G})$ \\
\hline & 224E082 & $4-5$ & NA & -- & -- & NA & -- & -- & NA & 0.62 & NA & $0.53(\mathrm{~J})$ & NA & -- \\
\hline & 224E083 & $7-8$ & NA & -- & -- & NA & $0.63(\mathrm{~J})$ & -- & NA & 0.49 & NA & $0.4(\mathrm{~J})$ & NA & 0.17 \\
\hline \multirow{4}{*}{ E25 } & 224E081 & $0-1$ & NA & $0.97(\mathrm{G})$ & -- & NA & $0.83(\mathrm{G}, \mathrm{J})$ & $2.1(\mathrm{G})$ & NA & $0.99(\mathrm{~J})^{\mathrm{d}}$ & NA & $0.64(\mathrm{G}, \mathrm{J})$ & NA & $0.34(\mathrm{G})$ \\
\hline & 224E084 & $4-5$ & NA & -- & -- & NA & $0.46(\mathrm{~J})$ & $0.323(\mathrm{LT})$ & NA & 0.32 & NA & $0.48(\mathrm{~J})$ & NA & -- \\
\hline & 224E085 & $4-5$ & NA & 0.44 & -- & NA & $0.319(\mathrm{~J})$ & $0.27(\mathrm{LT})$ & NA & 0.476 & NA & $0.395(\mathrm{~J})$ & $\mathrm{NA}$ & 0.155 \\
\hline & 224E086 & $7-8$ & NA & -- & $\overline{--}$ & NA & $0.55(\mathrm{~J})$ & $\overline{--}$ & NA & 0.68 & NA & $0.56(\mathrm{~J})$ & $\overline{N A}$ & 0.204 \\
\hline E26 & 224E087 & $0-1$ & NA & 0.87 & -- & NA & $0.69(\mathrm{~J})$ & $0.238(\mathrm{LT})$ & NA & 0.95 & NA & $0.73(\mathrm{~J})$ & NA & 0.267 \\
\hline E27 & 224E090 & $0-1$ & NA & 0.52 & -- & NA & $0.44(\mathrm{~J})$ & -- & NA & 0.66 & NA & $0.48(\mathrm{~J})$ & NA & 0.17 \\
\hline E28 & 224E093 & $0-1$ & NA & -- & -- & NA & $0.48(\mathrm{~J})$ & -- & NA & 0.52 & NA & $0.53(\mathrm{~J})$ & NA & 0.158 \\
\hline
\end{tabular}


Table A.7-17

Soil Sample Results for Gamma-Emitting Radionuclides Detected Above Minimum Detectable Concentrations at CAS 06-05-01, Leachfield (Page 7 of 8 )

\begin{tabular}{|c|c|c|c|c|c|c|c|c|c|c|c|c|c|c|}
\hline \multirow[b]{2}{*}{$\begin{array}{c}\text { Sample } \\
\text { Location }\end{array}$} & \multirow[b]{2}{*}{$\begin{array}{l}\text { Sample } \\
\text { Number }\end{array}$} & \multirow{3}{*}{$\begin{array}{c}\text { Depth } \\
\text { (ft bgs) }\end{array}$} & \multicolumn{12}{|c|}{ Contaminants of Potential Concern (pCi/g) } \\
\hline & & & \multicolumn{2}{|c|}{ 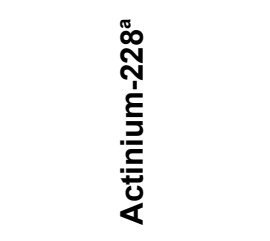 } & \multirow{3}{*}{ 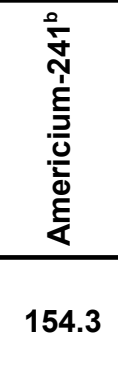 } & \multicolumn{2}{|c|}{ 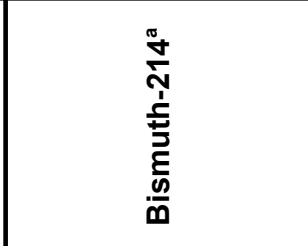 } & \multicolumn{3}{|c|}{ 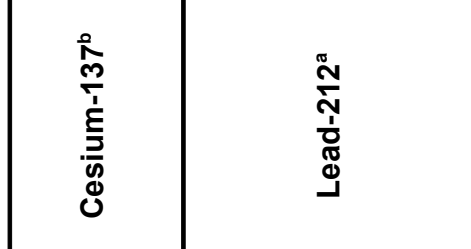 } & \multicolumn{2}{|c|}{ 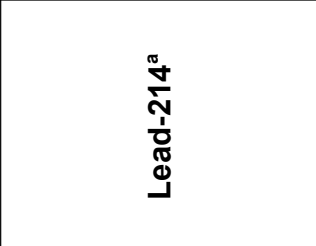 } & \multicolumn{2}{|c|}{ 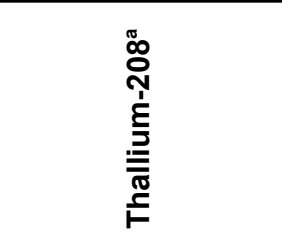 } \\
\hline \multicolumn{2}{|c|}{ Final Action Levels } & & 5 & 15 & & 5 & 15 & & 5 & 15 & 5 & 15 & 5 & 15 \\
\hline \multicolumn{3}{|c|}{ Depth bgs (cm) } & $<15$ & $>15$ & & $<15$ & $>15$ & & $<15$ & $>15$ & $<15$ & $>15$ & $<15$ & $>15$ \\
\hline$\overline{E 29}$ & 224E096 & $0-0.5$ & $1.19(\mathrm{G})$ & $\overline{\mathrm{NA}}$ & $\overline{--}$ & $0.82(\mathrm{G}, \mathrm{J})$ & $\overline{\mathrm{NA}}$ & $0.67(\mathrm{G})$ & $1.16(\mathrm{~J})^{\mathrm{d}}$ & $\overline{\mathrm{NA}}$ & $\overline{0.91(G, J)}$ & $\overline{\mathrm{NA}}$ & $0.35(\mathrm{G})$ & $\overline{\mathrm{NA}}$ \\
\hline E30 & 224E097 & $0-0.5$ & $1.32(\mathrm{G})$ & NA & $\overline{--}$ & $0.96(\mathrm{G}, \mathrm{J})$ & $\mathrm{NA}$ & $\overline{--}$ & $1.5(\mathrm{~J})^{\mathrm{d}}$ & $\mathrm{NA}$ & $1.03(\mathrm{G}, \mathrm{J})$ & $\mathrm{NA}$ & $0.42(\mathrm{G})$ & $\mathrm{NA}$ \\
\hline E31 & 224E098 & $0-0.5$ & $0.93(\mathrm{G})$ & NA & -- & $0.91(\mathrm{G}, \mathrm{J})$ & $\mathrm{NA}$ & $1.18(\mathrm{G})$ & $1.25(\mathrm{~J})^{\mathrm{d}}$ & $\mathrm{NA}$ & $0.79(\mathrm{G}, \mathrm{J})$ & $\mathrm{NA}$ & $0.36(\mathrm{G})$ & $\mathrm{NA}$ \\
\hline E32 & 224E099 & $0-0.5$ & $1.06(\mathrm{G})$ & NA & $\overline{--}$ & $0.75(\mathrm{G}, \mathrm{J})$ & $\mathrm{NA}$ & $1.24(\mathrm{G})$ & $1.02(\mathrm{~J})^{\mathrm{d}}$ & $\mathrm{NA}$ & $0.64(\mathrm{G}, \mathrm{J})$ & $\mathrm{NA}$ & $\overline{--}$ & $\overline{N A}$ \\
\hline E33 & 224E100 & $0-0.5$ & $1.54(\mathrm{G})$ & NA & -- & $0.94(\mathrm{G}, \mathrm{J})$ & $\mathrm{NA}$ & -- & $1.8(\mathrm{~J})^{\mathrm{d}}$ & $\mathrm{NA}$ & $1.1(\mathrm{G}, \mathrm{J})$ & $\mathrm{NA}$ & 0.53 & $\mathrm{NA}$ \\
\hline E34 & 224E101 & $0-0.5$ & -- & NA & $1.01(\mathrm{~J})^{\mathrm{d}}$ & $0.45(\mathrm{G}, \mathrm{J})$ & NA & $1.06(\mathrm{G})$ & $0.63(\mathrm{~J})^{\mathrm{d}}$ & NA & $0.43(\mathrm{G}, \mathrm{J})$ & NA & 0.247 & NA \\
\hline E35 & 224E102 & $0-0.5$ & $0.61(\mathrm{G})$ & NA & $1.02(\mathrm{~J})^{\mathrm{d}}$ & $0.61(\mathrm{G}, \mathrm{J})$ & $\mathrm{NA}$ & $1.03(\mathrm{G})$ & $0.82(\mathrm{~J})^{\mathrm{d}}$ & $\mathrm{NA}$ & $0.63(\mathrm{G}, \mathrm{J})$ & $\mathrm{NA}$ & 0.225 & NA \\
\hline \multirow{3}{*}{ E36 } & 224E103 & $0-0.5$ & -- & NA & $5.3(\mathrm{~J})^{\mathrm{d}}$ & -- & NA & $1.31(\mathrm{G})$ & $0.91(\mathrm{~J})^{\mathrm{d}}$ & NA & $0.75(\mathrm{G}, \mathrm{J})$ & NA & 0.28 & NA \\
\hline & 224E104 & $2-2.5$ & NA & - & $5(\mathrm{G})$ & NA & - & $0.77(\mathrm{G})$ & NA & $0.77(\mathrm{~J})^{\mathrm{d}}$ & $\mathrm{NA}$ & $0.66(G, J)$ & NA & -- \\
\hline & 224E108 & $3-3.5$ & NA & $0.81(G)$ & $2.03(\mathrm{~J})^{\mathrm{d}}$ & NA & $0.62(\mathrm{G}, \mathrm{J})$ & $0.46(\mathrm{G}, \mathrm{LT})$ & NA & $0.8(\mathrm{~J})$ & $\mathrm{NA}$ & $0.73(\mathrm{G}, \mathrm{J})$ & NA & $0.23(\mathrm{G})$ \\
\hline \multirow{2}{*}{ E37 } & 224E105 & $0-0.5$ & -- & NA & 17.4 & -- & NA & 5.25 & 0.58 & $\mathrm{NA}$ & $0.7(\mathrm{~J})$ & $\mathrm{NA}$ & -- & $\mathrm{NA}$ \\
\hline & 224E109 & $1-1.5$ & NA & $0.78(\mathrm{G})$ & $3(\mathrm{~J})$ & $\mathrm{NA}$ & $0.6(\mathrm{G}, \mathrm{J})$ & $1.38(\mathrm{G})$ & $\mathrm{NA}$ & $0.84(\mathrm{~J})^{\mathrm{d}}$ & $\mathrm{NA}$ & $0.69(\mathrm{G}, \mathrm{J})$ & NA & $0.33(\mathrm{G})$ \\
\hline \multirow{2}{*}{ E38 } & 224E106 & $0-0.5$ & -- & NA & 13.7 & -- & $\mathrm{NA}$ & 1.94 & 0.5 & $\mathrm{NA}$ & -- & $\mathrm{NA}$ & -- & $\mathrm{NA}$ \\
\hline & 224E110 & $1-1.5$ & NA & -- & $4.08(J)^{d}$ & NA & $0.58(\mathrm{G}, \mathrm{J})$ & $0.41(\mathrm{G}, \mathrm{LT})$ & $\mathrm{NA}$ & $0.43(\mathrm{~J})^{\mathrm{d}}$ & $\mathrm{NA}$ & $0.36(G, J)$ & $\mathrm{NA}$ & -- \\
\hline \multirow{2}{*}{ E39 } & 224E107 & $0-0.5$ & -- & NA & 5.61 & -- & $\mathrm{NA}$ & 7.57 & 0.97 & $\mathrm{NA}$ & $0.87(\mathrm{~J})$ & $\mathrm{NA}$ & -- & $\mathrm{NA}$ \\
\hline & $224 \mathrm{E} 111$ & $0.5-1.0$ & NA & $0.91(\mathrm{G})$ & $4.22(\mathrm{~J})$ & NA & $0.78(\mathrm{G}, \mathrm{J})$ & $5.97(\mathrm{G})$ & $\mathrm{NA}$ & $1.34(\mathrm{~J})^{\mathrm{d}}$ & $\mathrm{NA}$ & $0.71(\mathrm{G}, \mathrm{J})$ & $\mathrm{NA}$ & $0.33(\mathrm{G})$ \\
\hline
\end{tabular}


Table A.7-17

Soil Sample Results for Gamma-Emitting Radionuclides Detected Above Minimum Detectable Concentrations at CAS 06-05-01, Leachfield

(Page 8 of 8 )

\begin{tabular}{|c|c|c|c|c|c|c|c|c|c|c|c|c|c|c|}
\hline \multirow[b]{2}{*}{$\begin{array}{c}\text { Sample } \\
\text { Location }\end{array}$} & \multirow[b]{2}{*}{$\begin{array}{l}\text { Sample } \\
\text { Number }\end{array}$} & \multirow[b]{2}{*}{$\begin{array}{c}\text { Depth } \\
\text { (ft bgs) }\end{array}$} & \multicolumn{12}{|c|}{ Contaminants of Potential Concern (pCi/g) } \\
\hline & & & \multicolumn{2}{|c|}{ 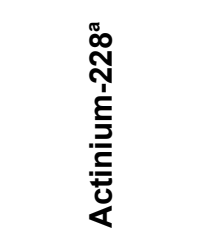 } & 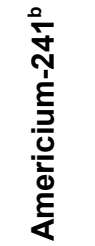 & \multicolumn{2}{|c|}{ 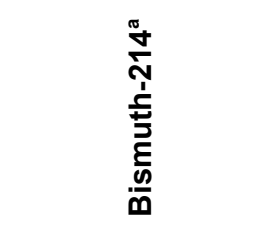 } & 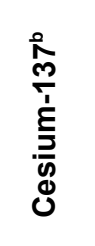 & \multicolumn{2}{|c|}{ 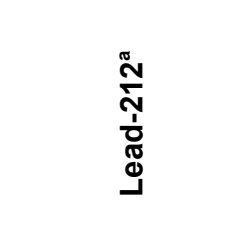 } & \multicolumn{2}{|c|}{ 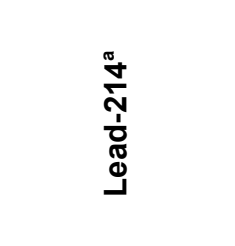 } & \multicolumn{2}{|c|}{ 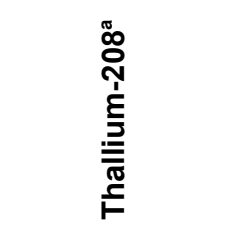 } \\
\hline \multicolumn{3}{|c|}{ Final Action Levels } & 5 & 15 & \multirow{2}{*}{154.3} & 5 & 15 & \multirow{2}{*}{36.22} & 5 & 15 & 5 & 15 & 5 & 15 \\
\hline \multicolumn{3}{|c|}{ Depth bgs (cm) } & $<15$ & $>15$ & & $<15$ & $>15$ & & $<15$ & $>15$ & $<15$ & $>15$ & $<15$ & $>15$ \\
\hline$\overline{\mathrm{E} 40}$ & $224 \mathrm{E} 112$ & $0-0.5$ & $\overline{---}$ & NA & $\overline{---}$ & $0.74(\mathrm{G}, \mathrm{J})$ & $\overline{\mathrm{NA}}$ & $\overline{---}$ & $1.09(\mathrm{~J})^{\mathrm{c}}$ & NA & $\overline{---}$ & NA & $\overline{---}$ & $\overline{\mathrm{NA}}$ \\
\hline
\end{tabular}

${ }^{a}$ Taken from the generic guidelines for residual concentrations of actinium-228, bismuth-214, lead-212, lead-214, thallium-208, and thorium-232, as found in Chapter IV of DOE Order 5400.5,

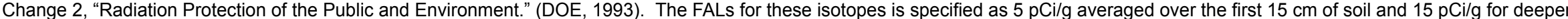

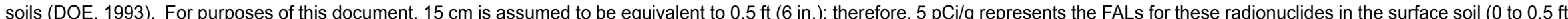
depth)

${ }^{\mathrm{b}} \mathrm{Am}-241$ and Cs-137 FALs determined by RESRAD evaluation.

${ }^{C}$ Qualifier added to laboratory data; record accepted. Duplicate normalized difference outside control limits.

${ }^{\mathrm{d}}$ Qualifier added to laboratory data; record accepted. Sample does not meet counting geometry requirements.

$\mathrm{ft}$ bgs $=$ Feet below ground surface

$\mathrm{cm}=$ Centimeter

$\mathrm{pCi} / \mathrm{g}=$ Picocuries per gram

RESRAD = Residual radiation

$--=$ Not detected above minimum reporting limits

$>=$ Greater than

$<=$ Less than

$\mathrm{G}=$ Sample density differs by more than 15 percent of laboratory control sample density.

$\mathrm{J}=$ Estimated value

$\mathrm{LT}=$ Result is less than the requested minimum detectable concentration, greater than the sample specific minimum detectable concentration

NA = Not applicable 
Table A.7-18

Soil Sample Results for Gamma-Emitting Radionuclides Detected Above Minimum Detectable Concentrations at CAS 06-17-04, Decon Pad and Wastewater Catch (Page 1 of 7 )

\begin{tabular}{|c|c|c|c|c|c|c|c|c|c|c|c|c|c|c|c|c|}
\hline \multirow[b]{2}{*}{$\begin{array}{c}\text { Sample } \\
\text { Location }\end{array}$} & \multirow[b]{2}{*}{$\begin{array}{l}\text { Sample } \\
\text { Number }\end{array}$} & \multirow{3}{*}{$\begin{array}{l}\text { Depth } \\
\text { (ft bgs) }\end{array}$} & \multicolumn{14}{|c|}{ Contaminants of Potential Concern (pCi/g) } \\
\hline & & & \multicolumn{2}{|c|}{ 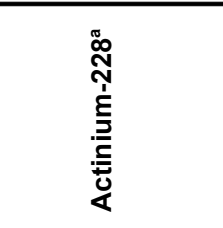 } & \multirow{3}{*}{ 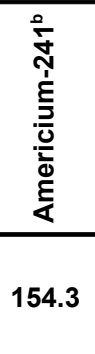 } & \multicolumn{2}{|c|}{ 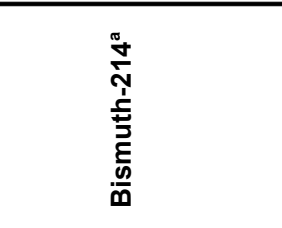 } & \multirow{3}{*}{ 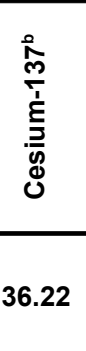 } & \multirow{3}{*}{$\begin{array}{l}\stackrel{0}{0} \\
\frac{1}{\pi} \\
\frac{0}{0} \\
0\end{array}$} & \multirow{3}{*}{ 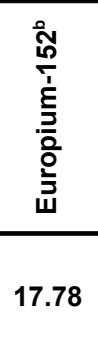 } & \multicolumn{2}{|c|}{ 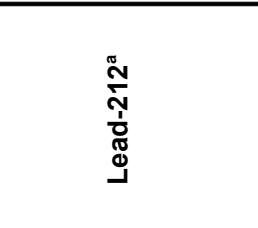 } & \multicolumn{2}{|c|}{ 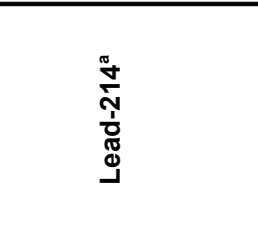 } & \multicolumn{2}{|c|}{ 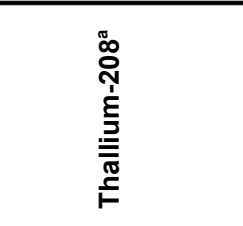 } \\
\hline \multicolumn{2}{|c|}{ Final Action Levels } & & 5 & 15 & & 5 & 15 & & & & 5 & 15 & 5 & 15 & 5 & 15 \\
\hline \multicolumn{3}{|c|}{ Depth bgs $(\mathrm{cm})$} & $<15$ & $>15$ & & $<15$ & $>15$ & & & & $<15$ & $>15$ & $<15$ & $>15$ & $<15$ & $>15$ \\
\hline \multirow{4}{*}{ F01 } & $224 \mathrm{~F} 001$ & $1-2$ & NA & $0.66(\mathrm{G})$ & $\overline{---}$ & $\overline{\mathrm{NA}}$ & $0.45(\mathrm{G}, \mathrm{J})$ & $\overline{---}$ & $\overline{---}$ & $\overline{---}$ & $\mathrm{NA}$ & $0.7(\mathrm{~J})^{\mathrm{c}}$ & $\overline{N A}$ & $0.48(G, J)$ & NA & $\overline{0.26(\mathrm{G})}$ \\
\hline & $224 \mathrm{~F} 002$ & $1-2$ & NA & -- & -- & NA & $0.61(\mathrm{G}, \mathrm{J})$ & -- & -- & - & NA & $0.75(J)^{c}$ & NA & $0.47(\mathrm{G}, \mathrm{J})$ & NA & $0.162(G)$ \\
\hline & $224 \mathrm{~F} 003$ & $4-5$ & NA & -- & -- & NA & $0.59(\mathrm{G}, \mathrm{J})$ & -- & -- & - & NA & $0.47(J)^{c}$ & NA & $0.48(\mathrm{G}, \mathrm{J})$ & NA & -- \\
\hline & 224F004 & $7-8$ & NA & -- & -- & NA & $0.43(\mathrm{G}, \mathrm{J})$ & -- & -- & -- & NA & $0.65(J)^{c}$ & NA & $0.5(G, J)$ & NA & $0.226(\mathrm{G})$ \\
\hline \multirow{3}{*}{ F02 } & 224F005 & $1-2$ & NA & -- & -- & NA & -- & -- & -- & -- & NA & 0.36 & NA & $0.35(\mathrm{~J})$ & $\mathrm{NA}$ & -- \\
\hline & 224F006 & $4-5$ & NA & -- & -- & NA & $0.3(\mathrm{~J})$ & -- & -- & -- & $N A$ & -- & NA & -- & NA & -- \\
\hline & $224 \mathrm{~F} 007$ & $7-8$ & NA & -- & -- & NA & -- & -- & -- & - & NA & $0.41(J)^{c}$ & NA & $0.37(\mathrm{G}, \mathrm{J})$ & NA & -- \\
\hline \multirow{3}{*}{ F03 } & 224F008 & $1-2$ & NA & $\begin{array}{c}0.95(\mathrm{G} \\
\mathrm{TI})\end{array}$ & -- & NA & $0.53(\mathrm{G}, \mathrm{J})$ & -- & -- & -- & NA & $0.76(\mathrm{~J})^{\mathrm{c}}$ & NA & $0.57(\mathrm{G}, \mathrm{J})$ & NA & $0.21(G)$ \\
\hline & 224F009 & $4-5$ & NA & - & -- & NA & $0.38(\mathrm{~J})$ & -- & -- & -- & NA & 0.27 & NA & $0.3(\mathrm{~J})$ & NA & -- \\
\hline & $224 \mathrm{~F} 010$ & $7-8$ & NA & -- & -- & NA & $0.43(\mathrm{~J})$ & -- & -- & - & NA & 0.51 & NA & $0.55(\mathrm{~J})$ & NA & 0.195 \\
\hline \multirow{3}{*}{ F04 } & 224F011 & $1-2$ & NA & $0.7(\mathrm{G})$ & -- & NA & $0.64(\mathrm{G}, \mathrm{J})$ & -- & -- & -- & NA & $0.73(\mathrm{~J})^{\mathrm{c}}$ & NA & $0.58(\mathrm{G}, \mathrm{J})$ & NA & $0.2(\mathrm{G})$ \\
\hline & $224 \mathrm{~F} 012$ & $4-5$ & NA & -- & -- & NA & $0.57(\mathrm{G}, \mathrm{J})$ & -- & -- & -- & NA & $0.54(J)^{c}$ & NA & $0.45(\mathrm{G}, \mathrm{J})$ & NA & $0.204(\mathrm{G})$ \\
\hline & $224 \mathrm{~F} 013$ & $7-8$ & NA & $0.85(\mathrm{G})$ & -- & NA & $0.63(\mathrm{G}, \mathrm{J})$ & -- & -- & -- & $N A$ & $0.98(J)^{c}$ & NA & $0.56(\mathrm{G}, \mathrm{J})$ & NA & $0.255(\mathrm{G})$ \\
\hline \multirow{3}{*}{ F05 } & $224 \mathrm{~F} 014$ & $1-2$ & NA & $0.66(\mathrm{G})$ & -- & NA & $0.58(\mathrm{G}, \mathrm{J})$ & -- & -- & - & $N A$ & $0.82(\mathrm{~J})^{\mathrm{c}}$ & NA & $0.63(\mathrm{G}, \mathrm{J})$ & NA & $0.205(G)$ \\
\hline & $224 \mathrm{~F} 015$ & $4-5$ & NA & -- & -- & NA & -- & -- & -- & -- & NA & 0.55 & $\mathrm{NA}$ & $0.47(\mathrm{~J})$ & $\mathrm{NA}$ & 0.22 \\
\hline & $224 \mathrm{~F} 016$ & $7-8$ & NA & $0.74(\mathrm{G})$ & -- & NA & $0.47(\mathrm{G}, \mathrm{J})$ & -- & - & - & NA & $0.82(\mathrm{~J})^{\mathrm{c}}$ & NA & $0.64(\mathrm{G}, \mathrm{J})$ & NA & $0.206(\mathrm{G})$ \\
\hline \multirow{3}{*}{ F06 } & 224F017 & $1-2$ & NA & -- & -- & NA & $0.99(\mathrm{G}, \mathrm{J})$ & -- & -- & -- & NA & $1.24(\mathrm{~J})^{\mathrm{c}}$ & NA & $0.96(G, J)$ & NA & $0.33(\mathrm{G})$ \\
\hline & $224 \mathrm{~F} 018$ & $4-5$ & NA & $1.03(\mathrm{G})$ & -- & NA & $0.93(\mathrm{G}, \mathrm{J})$ & -- & -- & -- & NA & $0.96(\mathrm{~J})^{\mathrm{c}}$ & NA & $0.87(\mathrm{G}, \mathrm{J})$ & $\mathrm{NA}$ & $0.32(\mathrm{G})$ \\
\hline & 224F019 & $7-8$ & NA & $0.6(\mathrm{G})$ & -- & NA & $0.44(\mathrm{G}, \mathrm{J})$ & -- & -- & -- & $N A$ & $0.76(J)^{\circ}$ & NA & $0.6(G, J)$ & NA & $0.247(G)$ \\
\hline
\end{tabular}


Table A.7-18

Soil Sample Results for Gamma-Emitting Radionuclides Detected Above Minimum Detectable Concentrations at CAS 06-17-04, Decon Pad and Wastewater Catch (Page 2 of 7 )

\begin{tabular}{|c|c|c|c|c|c|c|c|c|c|c|c|c|c|c|c|c|}
\hline \multirow[b]{2}{*}{$\begin{array}{c}\text { Sample } \\
\text { Location }\end{array}$} & \multirow[b]{2}{*}{$\begin{array}{c}\text { Sample } \\
\text { Number }\end{array}$} & \multirow{3}{*}{$\begin{array}{c}\text { Depth } \\
\text { (ft bgs) }\end{array}$} & \multicolumn{14}{|c|}{ Contaminants of Potential Concern (pCi/g) } \\
\hline & & & \multicolumn{2}{|c|}{ 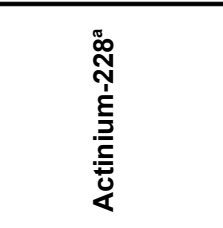 } & \multirow{3}{*}{ 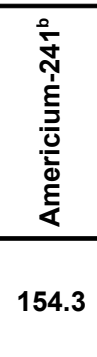 } & \multicolumn{2}{|c|}{ 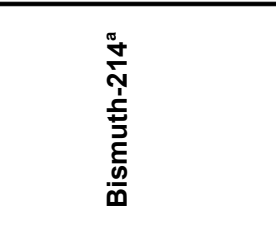 } & \multirow{3}{*}{ 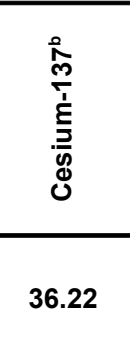 } & \multirow{3}{*}{$\begin{array}{l}\stackrel{0}{0} \\
\frac{1}{\pi} \\
\frac{0}{0} \\
0 \\
0\end{array}$} & \multirow{3}{*}{ 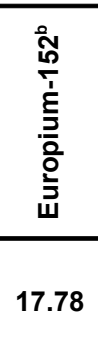 } & \multicolumn{2}{|c|}{ 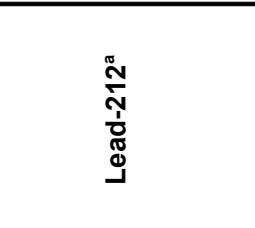 } & \multicolumn{2}{|c|}{ 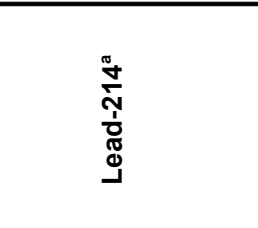 } & \multicolumn{2}{|c|}{ 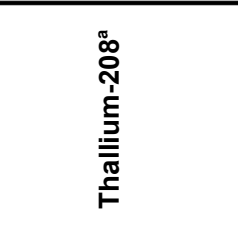 } \\
\hline \multicolumn{2}{|c|}{ Final Action Levels } & & 5 & 15 & & 5 & 15 & & & & 5 & 15 & 5 & 15 & 5 & 15 \\
\hline \multicolumn{3}{|c|}{ Depth bgs $(\mathrm{cm})$} & $<15$ & $>15$ & & $<15$ & $>15$ & & & & $<15$ & $>15$ & $<15$ & $>15$ & $<15$ & $>15$ \\
\hline \multirow{3}{*}{ F07 } & $224 \mathrm{~F} 020$ & $1-2$ & $\overline{\mathrm{NA}}$ & $\overline{0.89}$ & $\overline{---}$ & $\overline{\mathrm{NA}}$ & 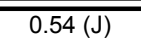 & $\overline{---}$ & $\overline{---}$ & $\overline{---}$ & NA & $\overline{0.74}$ & NA & $0.6(\mathrm{~J})$ & $\mathrm{NA}$ & $\overline{---}$ \\
\hline & 224F021 & $4-5$ & NA & $0.57(\mathrm{G})$ & -- & NA & $0.52(\mathrm{G}, \mathrm{J})$ & -- & -- & -- & NA & $0.75(J)^{c}$ & NA & $0.53(\mathrm{G}, \mathrm{J})$ & NA & $0.219(\mathrm{G})$ \\
\hline & 224F022 & $7-8$ & NA & 0.89 & -- & NA & $0.64(J)$ & -- & -- & -- & NA & 0.94 & NA & $0.6(\mathrm{~J})$ & NA & 0.371 \\
\hline \multirow{4}{*}{ F08 } & 224F023 & $0.2-1$ & NA & 0.73 & -- & NA & $0.54(\mathrm{~J})$ & $0.224(\mathrm{LT})$ & -- & -- & NA & 0.81 & NA & $0.61(\mathrm{~J})$ & $N A$ & 0.222 \\
\hline & 224F024 & $4-5$ & NA & -- & -- & NA & -- & -- & -- & -- & NA & $0.53(\mathrm{~J})^{\mathrm{c}}$ & NA & $0.58(\mathrm{G}, \mathrm{J})$ & NA & -- \\
\hline & 224F025 & $7-8$ & NA & 0.51 & -- & NA & $0.36(\mathrm{~J})$ & -- & -- & -- & NA & 0.59 & NA & $0.412(\mathrm{~J})$ & NA & 0.184 \\
\hline & 224F026 & $7-8$ & NA & -- & -- & NA & -- & -- & -- & -- & NA & 0.7 & NA & $0.46(J)$ & NA & -- \\
\hline \multirow{3}{*}{ F09 } & 224F027 & $0.2-1$ & $\mathrm{NA}$ & $1.35(\mathrm{G})$ & -- & NA & $0.92(\mathrm{G}, \mathrm{J})$ & -- & -- & -- & NA & $1.32(\mathrm{~J})^{\mathrm{c}}$ & NA & $0.92(\mathrm{G}, \mathrm{J})$ & NA & $0.43(\mathrm{G})$ \\
\hline & 224F028 & $4-5$ & NA & $0.83(\mathrm{G})$ & -- & NA & $0.64(\mathrm{G}, \mathrm{J})$ & -- & -- & -- & NA & $1.02(\mathrm{~J})^{\mathrm{c}}$ & NA & $0.65(\mathrm{G}, \mathrm{J})$ & NA & $0.274(G)$ \\
\hline & 224F029 & $7-8$ & $N A$ & -- & -- & NA & $0.4(\mathrm{~J})$ & -- & -- & -- & NA & 0.53 & NA & $0.4(\mathrm{~J})$ & NA & 0.178 \\
\hline \multirow{3}{*}{ F10 } & 224F030 & $0-1$ & NA & -- & -- & NA & $0.54(\mathrm{~J})$ & -- & -- & -- & NA & 0.55 & NA & $0.46(J)$ & NA & 0.184 \\
\hline & 224F031 & $4-5$ & NA & 0.32 & -- & NA & $0.31(\mathrm{~J})$ & -- & -- & -- & NA & 0.339 & NA & $0.277(\mathrm{~J})$ & $N A$ & 0.096 \\
\hline & 224F032 & $7-8$ & NA & -- & -- & NA & -- & -- & -- & -- & NA & 0.43 & NA & $0.4(\mathrm{~J})$ & NA & -- \\
\hline \multirow{3}{*}{ F11 } & 224F033 & $0-1$ & $\mathrm{NA}$ & 0.62 & 7.01 & NA & $0.42(\mathrm{~J})$ & 0.521 & -- & -- & NA & 0.77 & NA & $0.51(\mathrm{~J})$ & NA & 0.221 \\
\hline & 224F034 & $4-5$ & NA & $0.56(\mathrm{TI})$ & -- & NA & $0.38(\mathrm{~J})$ & - & -- & -- & NA & 0.36 & NA & $0.39(\mathrm{~J})$ & NA & -- \\
\hline & 224F035 & $7-8$ & NA & $1(\mathrm{G})$ & -- & NA & $0.53(\mathrm{G}, \mathrm{J})$ & -- & -- & -- & NA & $0.86(J)^{c}$ & NA & $0.56(\mathrm{G}, \mathrm{J})$ & NA & $0.29(\mathrm{G})$ \\
\hline \multirow{3}{*}{ F12 } & 224F036 & $0-1$ & NA & 0.88 & 0.75 & NA & $0.75(\mathrm{~J})$ & 1.7 & -- & -- & NA & 1.18 & NA & $0.78(\mathrm{~J})$ & $N A$ & 0.45 \\
\hline & $224 \mathrm{~F} 037$ & $4-5$ & NA & -- & -- & NA & $0.46(J)$ & 0.53 & - & - & $\mathrm{NA}$ & 0.73 & $\mathrm{NA}$ & $0.62(\mathrm{~J})$ & NA & 0.246 \\
\hline & 224F038 & $7-8$ & NA & 0.71 & -- & NA & $0.53(\mathrm{~J})$ & -- & -- & -- & NA & 0.81 & NA & $0.57(\mathrm{~J})$ & NA & 0.238 \\
\hline
\end{tabular}


Table A.7-18

Soil Sample Results for Gamma-Emitting Radionuclides Detected Above Minimum Detectable Concentrations at CAS 06-17-04, Decon Pad and Wastewater Catch (Page 3 of 7 )

\begin{tabular}{|c|c|c|c|c|c|c|c|c|c|c|c|c|c|c|c|c|}
\hline \multirow[b]{2}{*}{$\begin{array}{c}\text { Sample } \\
\text { Location }\end{array}$} & \multirow[b]{2}{*}{$\begin{array}{l}\text { Sample } \\
\text { Number }\end{array}$} & \multirow{3}{*}{$\begin{array}{c}\text { Depth } \\
\text { (ft bgs) }\end{array}$} & \multicolumn{14}{|c|}{ Contaminants of Potential Concern (pCi/g) } \\
\hline & & & \multicolumn{2}{|c|}{ 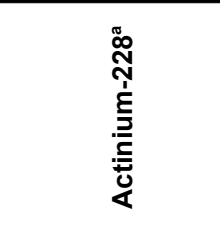 } & 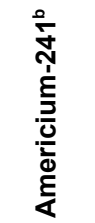 & \multicolumn{2}{|c|}{ 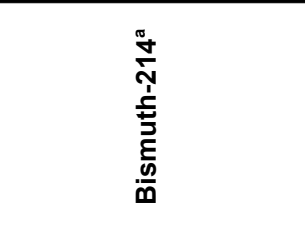 } & \multirow{3}{*}{ 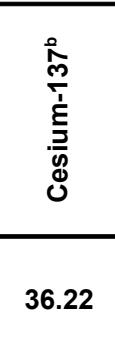 } & \multirow{3}{*}{$\begin{array}{l}\stackrel{0}{0} \\
\frac{1}{\pi} \\
\frac{0}{0} \\
0\end{array}$} & \multirow{3}{*}{ 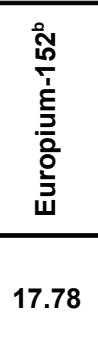 } & \multicolumn{2}{|c|}{ 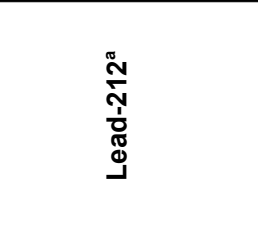 } & \multicolumn{2}{|c|}{ 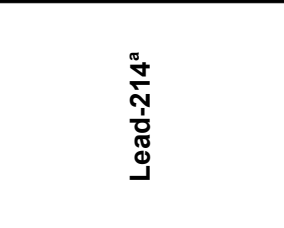 } & \multicolumn{2}{|c|}{ 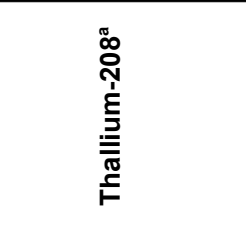 } \\
\hline \multicolumn{2}{|c|}{ Final Action Levels } & & 5 & 15 & \multirow{2}{*}{154.3} & 5 & 15 & & & & 5 & 15 & 5 & 15 & 5 & 15 \\
\hline \multicolumn{3}{|c|}{ Depth bgs (cm) } & $<15$ & $>15$ & & $<15$ & $>15$ & & & & $<15$ & $>15$ & $<15$ & $>15$ & $<15$ & $>15$ \\
\hline \multirow{3}{*}{ F13 } & $224 \mathrm{~F} 039$ & $0-1$ & $\overline{N A}$ & $\overline{---}$ & $\overline{---}$ & $\overline{N A}$ & $0.53(\mathrm{G}, \mathrm{J})$ & $\overline{---}$ & $\overline{--}$ & $\overline{---}$ & $\overline{\mathrm{NA}}$ & $\overline{0.74(\mathrm{~J})^{\mathrm{c}}}$ & $\overline{N A}$ & $0.57(G, J)$ & $\mathrm{NA}$ & $0.196(\mathrm{G})$ \\
\hline & 224F040 & $4-5$ & NA & -- & -- & NA & -- & -- & -- & -- & NA & $0.73(\mathrm{~J})^{\mathrm{c}}$ & NA & $0.56(G, J)$ & NA & $0.27(\mathrm{G})$ \\
\hline & 224F041 & $7-8$ & NA & - & -- & NA & $0.34(\mathrm{~J})$ & -- & - & -- & NA & 0.44 & NA & $0.37(\mathrm{~J})$ & NA & 0.147 \\
\hline F21 & 224F050 & $1-3$ & $\mathrm{NA}$ & -- & - & NA & -- & -- & -- & -- & NA & 0.5 & NA & -- & NA & -- \\
\hline F22 & 224F051 & $1-3$ & $\mathrm{NA}$ & -- & - & NA & -- & -- & -- & -- & NA & 0.57 & NA & - & $N A$ & -- \\
\hline F23 & 224F052 & $1-3$ & NA & -- & -- & NA & -- & -- & -- & -- & NA & 0.65 & NA & $0.73(\mathrm{~J})$ & NA & -- \\
\hline F24 & 224F053 & $0-2$ & $\mathrm{NA}$ & -- & -- & NA & -- & $0.55(\mathrm{G})$ & -- & -- & NA & -- & NA & -- & NA & -- \\
\hline \multirow{2}{*}{ F25 } & 224F056 & $0-3$ & NA & -- & -- & NA & -- & $1.69(\mathrm{G})$ & -- & -- & NA & $1(\mathrm{~J})^{\mathrm{c}}$ & NA & $0.74(\mathrm{G}, \mathrm{J})$ & NA & -- \\
\hline & 224F057 & $5-7$ & NA & - & -- & NA & -- & $0.54(\mathrm{G})$ & -- & -- & NA & $0.77(J)^{c}$ & NA & $0.71(\mathrm{G}, \mathrm{J})$ & NA & -- \\
\hline \multirow{2}{*}{ F26 } & 224F058 & $0-4$ & NA & -- & -- & NA & -- & $1.9(\mathrm{G})$ & -- & -- & NA & $1.01(\mathrm{~J})^{\mathrm{c}}$ & NA & $0.85(\mathrm{G}, \mathrm{J})$ & NA & -- \\
\hline & 224F059 & $4-7$ & NA & $\overline{--}$ & -- & NA & $\overline{--}$ & -- & $\overline{--}$ & -- & NA & 0.58 & NA & -- & $\mathrm{NA}$ & $\overline{--}$ \\
\hline \multirow{2}{*}{ F27 } & 224F060 & $0-2$ & NA & - & -- & NA & -- & -- & -- & -- & NA & 0.52 & NA & $0.54(\mathrm{~J})$ & $N A$ & -- \\
\hline & 224F061 & $2-4$ & NA & -- & -- & NA & -- & -- & -- & -- & NA & $1.12(\mathrm{~J})^{\mathrm{c}}$ & NA & NA & NA & $0.43(\mathrm{G})$ \\
\hline \multirow{3}{*}{ F28 } & 224F062 & $0-0.5$ & $1.34(\mathrm{TI})$ & NA & -- & -- & NA & 3.31 & -- & -- & 1.05 & NA & $0.65(\mathrm{~J})$ & NA & -- & NA \\
\hline & 224F063 & $0-0.5$ & 1.38 & NA & 5.9 & $1.14(\mathrm{~J})$ & NA & 5.41 & - & -- & 1.29 & NA & $0.92(\mathrm{~J})$ & NA & 0.4 & NA \\
\hline & 224F064 & $3-4$ & NA & -- & - & NA & 0.99 & -- & -- & -- & NA & 0.68 & NA & - & NA & -- \\
\hline \multirow{2}{*}{ F29 } & 224F065 & $0-0.5$ & - & NA & -- & $0.99(\mathrm{~J})$ & NA & 8.5 & - & - & 1 & NA & $0.98(\mathrm{~J})$ & NA & -- & NA \\
\hline & 224F066 & $3-4$ & NA & -- & -- & -- & NA & -- & -- & -- & NA & $1.1(\mathrm{~J})^{\mathrm{c}}$ & NA & $0.93(\mathrm{G}, \mathrm{J})$ & NA & -- \\
\hline
\end{tabular}


Table A.7-18

Soil Sample Results for Gamma-Emitting Radionuclides Detected Above Minimum Detectable Concentrations at CAS 06-17-04, Decon Pad and Wastewater Catch (Page 4 of 7 )

\begin{tabular}{|c|c|c|c|c|c|c|c|c|c|c|c|c|c|c|c|c|}
\hline \multirow[b]{2}{*}{$\begin{array}{l}\text { Sample } \\
\text { Location }\end{array}$} & \multirow[b]{2}{*}{$\begin{array}{l}\text { Sample } \\
\text { Number }\end{array}$} & \multirow{3}{*}{$\begin{array}{l}\text { Depth } \\
\text { (ft bgs) }\end{array}$} & \multicolumn{14}{|c|}{ Contaminants of Potential Concern (pCi/g) } \\
\hline & & & \multicolumn{2}{|c|}{ 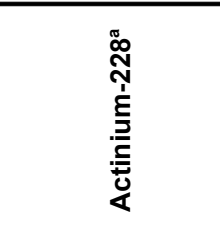 } & \multirow{3}{*}{ 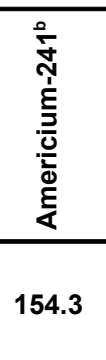 } & \multicolumn{2}{|c|}{ 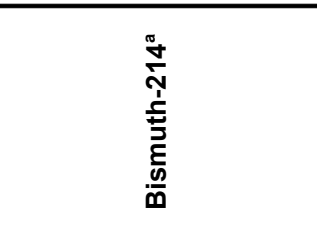 } & \multirow{3}{*}{ 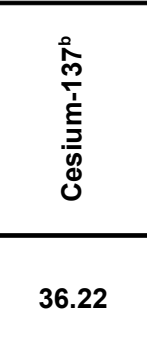 } & \multirow{3}{*}{$\begin{array}{l}\stackrel{0}{0} \\
\frac{1}{\pi} \\
\frac{0}{0} \\
0\end{array}$} & \multirow{3}{*}{ 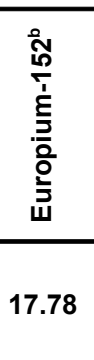 } & \multicolumn{2}{|c|}{ 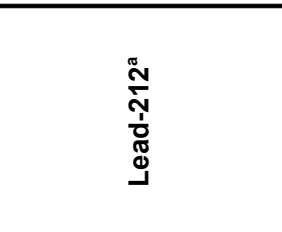 } & \multicolumn{2}{|c|}{ 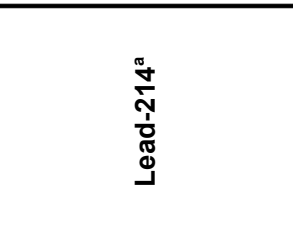 } & \multicolumn{2}{|c|}{ 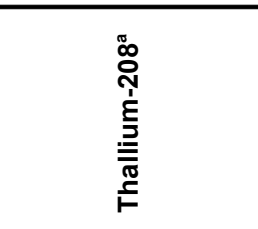 } \\
\hline \multicolumn{2}{|c|}{ Final Action Levels } & & 5 & 15 & & 5 & 15 & & & & 5 & 15 & 5 & 15 & 5 & 15 \\
\hline \multicolumn{3}{|c|}{ Depth bgs (cm) } & $<15$ & $>15$ & & $<15$ & $>15$ & & & & $<15$ & $>15$ & $<15$ & $>15$ & $<15$ & $>15$ \\
\hline \multirow{2}{*}{$\mathrm{F} 30$} & $224 \mathrm{~F} 067$ & $\overline{0-0.5}$ & $\overline{---}$ & $\overline{\mathrm{NA}}$ & $\overline{--}$ & $\overline{---}$ & $\overline{\mathrm{NA}}$ & $\overline{1.43(\mathrm{G})}$ & $\overline{---}$ & $\overline{--}$ & $0.91(\mathrm{~J})^{\mathrm{c}}$ & $\overline{\mathrm{NA}}$ & $0.89(\mathrm{G}, \mathrm{J})$ & $\overline{\mathrm{NA}}$ & $\overline{--}$ & $\overline{\mathrm{NA}}$ \\
\hline & $224 \mathrm{~F} 068$ & $3-4$ & NA & -- & -- & NA & -- & -- & -- & -- & NA & $0.89(\mathrm{~J})^{\mathrm{c}}$ & NA & $1.02(\mathrm{G}, \mathrm{J})$ & NA & -- \\
\hline F31 & 224F069 & $0-0.5$ & $1.12(\mathrm{G})$ & NA & $\overline{--}$ & $0.76(G, J)$ & NA & $4.43(\mathrm{G})$ & $\overline{--}$ & $\overline{--}$ & $1.24(\mathrm{~J})^{\mathrm{c}}$ & $\overline{N A}$ & $\overline{1(G, J)}$ & $\overline{N A}$ & $0.34(\mathrm{G})$ & $\overline{N A}$ \\
\hline F32 & $224 \mathrm{~F} 070$ & $0-0.5$ & $1(\mathrm{G})$ & NA & $0.77(\mathrm{~J})$ & $0.89(\mathrm{G}, \mathrm{J})$ & NA & $1.52(\mathrm{G})$ & $\overline{--}$ & $\overline{--}$ & $1.23(\mathrm{~J})^{\mathrm{c}}$ & NA & $0.86(\mathrm{G}, \mathrm{J})$ & NA & $0.38(\mathrm{G})$ & NA \\
\hline F33 & 224F071 & $00-0.5$ & 1.08 & NA & -- & $0.68(\mathrm{~J})$ & NA & -- & -- & -- & 1.15 & NA & $0.78(\mathrm{~J})$ & NA & 0.34 & NA \\
\hline F34 & 224F072 & $00-0.5$ & $\overline{--}$ & $\overline{N A}$ & $\overline{--}$ & $\overline{--}$ & NA & $\overline{--}$ & $\overline{--}$ & $\overline{--}$ & 0.69 & $\overline{N A}$ & $0.62(\mathrm{~J})$ & $\overline{N A}$ & $\overline{--}$ & $\overline{N A}$ \\
\hline F35 & $224 \mathrm{~F} 073$ & $00-0.5$ & $\overline{--}$ & NA & 20.1 & - & NA & 1.37 & -- & -- & 0.79 & NA & $\overline{--}$ & NA & -- & NA \\
\hline F36 & $224 \mathrm{~F} 074$ & $00-0.5$ & -- & NA & -- & -- & NA & -- & $0.138(\mathrm{G}, \mathrm{TI})$ & -- & -- & NA & -- & NA & -- & NA \\
\hline F37 & $224 \mathrm{~F} 075$ & $00-0.5$ & $\overline{--}$ & $\overline{N A}$ & $\overline{--}$ & $\overline{--}$ & NA & 1.04 & $\overline{--}$ & $\overline{--}$ & 0.67 & $\overline{N A}$ & $\overline{--}$ & $\overline{N A}$ & $\overline{--}$ & $\overline{N A}$ \\
\hline F38 & $224 \mathrm{~F} 076$ & $00-0.5$ & -- & NA & -- & -- & NA & -- & -- & -- & $0.76(\mathrm{~J})^{\mathrm{c}}$ & NA & -- & NA & -- & NA \\
\hline \multirow{2}{*}{ F39 } & 224F077 & $00-0.5$ & -- & NA & 19 & -- & NA & 4.23 & - & - & 0.95 & NA & $0.85(\mathrm{~J})$ & NA & 0.27 & NA \\
\hline & 224F104 & $1-1.5$ & NA & -- & $20.6(\mathrm{~J})$ & NA & $0.6(\mathrm{G}, \mathrm{J})$ & $3.15(\mathrm{G})$ & -- & $\overline{--}$ & NA & $1.12(\mathrm{~J})^{\mathrm{c}}$ & NA & $0.64(G, J)$ & NA & $0.4(\mathrm{G})$ \\
\hline \multirow{2}{*}{$\mathrm{F} 40$} & 224F078 & $00-0.5$ & -- & NA & 27.5 & -- & NA & 2.01 & -- & -- & 0.64 & NA & -- & NA & -- & NA \\
\hline & 224F105 & $0.5-1$ & NA & $\overline{--}$ & $4.1(\mathrm{~J})$ & NA & $0.33(\mathrm{G}, \mathrm{J})$ & -- & - & - & NA & $0.44(\mathrm{~J})^{\mathrm{c}}$ & NA & $0.35(\mathrm{G}, \mathrm{J})$ & NA & $0.169(\mathrm{G})$ \\
\hline F41 & 224F079 & $00-0.5$ & -- & NA & -- & -- & NA & 1.5 & - & - & 0.53 & NA & $0.76(\mathrm{~J})$ & NA & -- & NA \\
\hline F42 & $224 \mathrm{~F} 080$ & $00-0.5$ & -- & NA & $3.8(\mathrm{G})$ & - & NA & $0.98(\mathrm{G})$ & - & - & $0.77(\mathrm{~J})^{\mathrm{c}}$ & NA & -- & NA & $\overline{--}$ & NA \\
\hline \multirow{2}{*}{ F43 } & 224F091 & $0-0.5$ & $0.75(\mathrm{G})$ & NA & $0.43(\mathrm{~J})$ & $0.52(\mathrm{G}, \mathrm{J})$ & NA & $0.27(\mathrm{G}, \mathrm{LT})$ & - & - & $1.04(J)^{\mathrm{c}}$ & NA & $0.65(\mathrm{G}, \mathrm{J})$ & NA & $0.27(\mathrm{G})$ & NA \\
\hline & 224F093 & $1.5-2$ & NA & 1.26 & -- & NA & -- & -- & -- & -- & NA & 1.24 & NA & $0.77(\mathrm{~J})$ & NA & 0.34 \\
\hline
\end{tabular}


Table A.7-18

Soil Sample Results for Gamma-Emitting Radionuclides Detected Above Minimum Detectable Concentrations at CAS 06-17-04, Decon Pad and Wastewater Catch (Page 5 of 7 )

\begin{tabular}{|c|c|c|c|c|c|c|c|c|c|c|c|c|c|c|c|c|}
\hline \multirow[b]{2}{*}{$\begin{array}{c}\text { Sample } \\
\text { Location }\end{array}$} & \multirow[b]{2}{*}{$\begin{array}{l}\text { Sample } \\
\text { Number }\end{array}$} & \multirow{3}{*}{$\begin{array}{c}\text { Depth } \\
\text { (ft bgs) }\end{array}$} & \multicolumn{14}{|c|}{ Contaminants of Potential Concern (pCi/g) } \\
\hline & & & \multicolumn{2}{|c|}{ 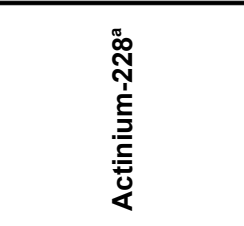 } & \multirow{3}{*}{ 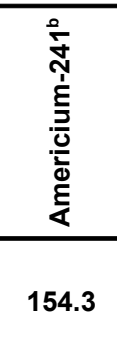 } & \multicolumn{2}{|c|}{ 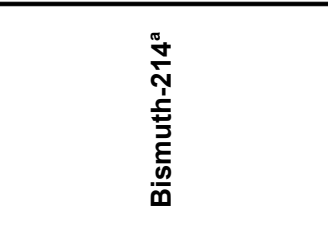 } & \multirow{3}{*}{ 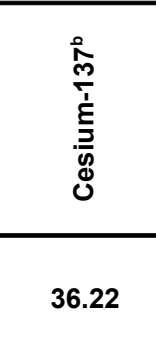 } & \multirow{3}{*}{$\begin{array}{l}\stackrel{0}{0} \\
\frac{1}{\pi} \\
\frac{0}{0} \\
0\end{array}$} & \multirow{3}{*}{ 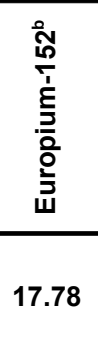 } & \multicolumn{2}{|c|}{ 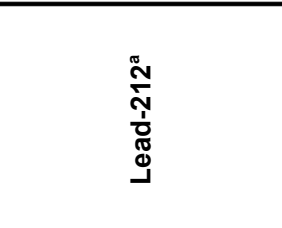 } & \multicolumn{2}{|c|}{ 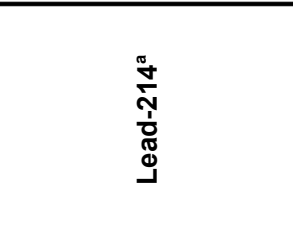 } & \multicolumn{2}{|c|}{ 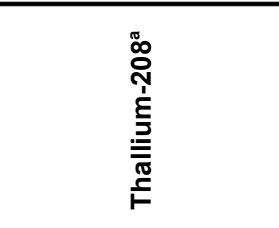 } \\
\hline \multicolumn{2}{|c|}{ Final Action Levels } & & 5 & 15 & & 5 & 15 & & & & 5 & 15 & 5 & 15 & 5 & 15 \\
\hline \multicolumn{3}{|c|}{ Depth bgs $(\mathrm{cm})$} & $<15$ & $>15$ & & $<15$ & $>15$ & & & & $<15$ & $>15$ & $<15$ & $>15$ & $<15$ & $>15$ \\
\hline \multirow{2}{*}{ F44 } & $224 \mathrm{~F} 092$ & $0-0.5$ & $\overline{---}$ & $\overline{\mathrm{NA}}$ & $\overline{---}$ & $\overline{---}$ & $\overline{\mathrm{NA}}$ & $\overline{---}$ & $\overline{---}$ & $\overline{---}$ & $0.72(\mathrm{~J})^{\mathrm{c}}$ & NA & $0.76(\mathrm{G}, \mathrm{J})$ & $\overline{N A}$ & $\overline{---}$ & NA \\
\hline & 224F094 & $1-1.5$ & NA & -- & -- & NA & -- & $0.33(\mathrm{LT})$ & -- & -- & NA & 0.66 & NA & -- & NA & -- \\
\hline \multirow{2}{*}{ F45 } & 224F095 & $0-0.5$ & $0.81(\mathrm{G})$ & NA & -- & $0.38(\mathrm{G}, \mathrm{J})$ & NA & $0.43(\mathrm{G}, \mathrm{LT})$ & -- & -- & $0.76(\mathrm{~J})^{\mathrm{c}}$ & NA & $0.63(\mathrm{G}, \mathrm{J})$ & NA & $0.28(\mathrm{G})$ & NA \\
\hline & 224F096 & $1-1.5$ & NA & $0.91(\mathrm{G})$ & $0.34(\mathrm{~J})$ & NA & $0.52(\mathrm{G}, \mathrm{J})$ & $0.28(\mathrm{G}, \mathrm{LT})$ & -- & -- & NA & $0.8(\mathrm{~J})^{\mathrm{c}}$ & NA & $0.5(G, J)$ & NA & $0.31(\mathrm{G})$ \\
\hline \multirow{3}{*}{ F46 } & 224F097 & $0.5-1$ & NA & $0.94(G)$ & $1.11(\mathrm{~J})$ & NA & $0.69(\mathrm{G}, \mathrm{J})$ & $0.188(\mathrm{G}, \mathrm{LT})$ & -- & -- & NA & $1.08(\mathrm{~J})^{\mathrm{c}}$ & NA & $0.63(\mathrm{G}, \mathrm{J})$ & NA & $0.272(\mathrm{G})$ \\
\hline & 224F098 & $1-1.5$ & NA & $0.56(\mathrm{G})$ & -- & NA & $0.292(\mathrm{G}, \mathrm{J})$ & -- & -- & -- & NA & $0.48(J)^{c}$ & NA & $0.419(\mathrm{G}, \mathrm{J})$ & NA & $0.123(\mathrm{G})$ \\
\hline & 224F099 & $1-1.5$ & NA & -- & -- & NA & $0.49(\mathrm{G}, \mathrm{J})$ & -- & -- & -- & NA & $0.46(J)^{c}$ & NA & $0.42(\mathrm{G}, \mathrm{J})$ & NA & $0.22(\mathrm{G})$ \\
\hline \multirow{2}{*}{ F47 } & 224F100 & $0-0.5$ & $0.77(\mathrm{G})$ & NA & -- & $0.56(\mathrm{G}, \mathrm{J})$ & NA & $0.228(G, L T)$ & -- & -- & $0.59(\mathrm{~J})^{\mathrm{c}}$ & NA & $0.58(\mathrm{G}, \mathrm{J})$ & NA & $0.236(\mathrm{G})$ & NA \\
\hline & 224F101 & $0.5-1.5$ & NA & -- & -- & NA & $0.43(\mathrm{G}, \mathrm{J})$ & -- & -- & -- & NA & $0.73(\mathrm{~J})^{\mathrm{c}}$ & NA & $0.56(\mathrm{G}, \mathrm{J})$ & NA & $0.203(\mathrm{G})$ \\
\hline \multirow{2}{*}{ F48 } & 224F102 & $0-0.5$ & 0.53 & NA & -- & $0.52(\mathrm{~J})$ & NA & 0.207 (LT) & -- & -- & 0.71 & NA & $0.35(\mathrm{~J})$ & NA & 0.158 & NA \\
\hline & 224F103 & $0.5-1$ & NA & $0.61(\mathrm{G})$ & -- & NA & $0.46(\mathrm{G}, \mathrm{J})$ & -- & -- & -- & NA & $0.5(\mathrm{~J})^{\mathrm{c}}$ & NA & $0.46(\mathrm{G}, \mathrm{J})$ & NA & $0.174(\mathrm{G})$ \\
\hline \multirow{2}{*}{ F49 } & 224F085 & $0-0.5$ & -- & NA & $22.1(\mathrm{~J})^{\mathrm{c}}$ & $0.53(\mathrm{G}, \mathrm{J})$ & NA & $1.17(\mathrm{G})$ & -- & -- & $0.74(\mathrm{~J})^{\mathrm{d}}$ & NA & $0.53(\mathrm{G}, \mathrm{J})$ & NA & $0.24(\mathrm{G})$ & NA \\
\hline & 224F086 & $1-1.5$ & NA & $0.55(\mathrm{G})$ & $3.32(\mathrm{~J})^{\mathrm{c}}$ & NA & -- & -- & - & -- & NA & $0.65(\mathrm{~J})^{\mathrm{d}}$ & NA & $0.44(\mathrm{G}, \mathrm{J})$ & NA & $0.149(\mathrm{G})$ \\
\hline \multirow{2}{*}{ F50 } & 224F087 & $0-0.5$ & $0.81(\mathrm{G})$ & NA & $26.2(\mathrm{~J})$ & $0.61(\mathrm{G}, \mathrm{J})$ & NA & $1.51(\mathrm{G})$ & -- & -- & $0.83(\mathrm{~J})^{\mathrm{d}}$ & NA & $0.61(\mathrm{G}, \mathrm{J})$ & NA & -- & NA \\
\hline & 224F088 & $1-1.5$ & NA & $0.77(\mathrm{G})$ & -- & NA & $0.67(\mathrm{G}, \mathrm{J})$ & -- & -- & -- & NA & $0.81(\mathrm{~J})^{\mathrm{d}}$ & NA & $0.65(\mathrm{G}, \mathrm{J})$ & NA & $0.27(\mathrm{G})$ \\
\hline \multirow{2}{*}{ F51 } & 224F089 & $0-0.5$ & $1.23(\mathrm{G})$ & NA & -- & $0.8(\mathrm{G}, \mathrm{J})$ & NA & - & -- & -- & $1.34(\mathrm{~J})^{\mathrm{d}}$ & NA & $0.88(G, J)$ & NA & $0.32(\mathrm{G})$ & NA \\
\hline & 224F106 & $1-1.5$ & NA & $0.88(G)$ & -- & NA & $0.68(\mathrm{G}, \mathrm{J})$ & -- & -- & -- & NA & $0.98(J)^{d}$ & NA & $0.67(\mathrm{G}, \mathrm{J})$ & NA & $0.3(\mathrm{G})$ \\
\hline F52 & 224F107 & $0-0.5$ & $0.88(\mathrm{G})$ & NA & -- & $0.76(\mathrm{G}, \mathrm{J})$ & NA & $0.358(\mathrm{G}, \mathrm{LT})$ & -- & -- & $1.12(\mathrm{~J})^{\mathrm{d}}$ & NA & $0.79(\mathrm{G}, \mathrm{J})$ & NA & $0.299(\mathrm{G})$ & NA \\
\hline F53 & 224F108 & $1-1.5$ & NA & -- & -- & NA & $0.54(\mathrm{G}, \mathrm{J})$ & -- & -- & -- & NA & $0.72(\mathrm{~J})^{\mathrm{d}}$ & NA & $0.52(\mathrm{G}, \mathrm{J})$ & NA & -- \\
\hline
\end{tabular}


Table A.7-18

Soil Sample Results for Gamma-Emitting Radionuclides Detected Above Minimum Detectable Concentrations at CAS 06-17-04, Decon Pad and Wastewater Catch (Page 6 of 7 )

\begin{tabular}{|c|c|c|c|c|c|c|c|c|c|c|c|c|c|c|c|c|}
\hline \multirow[b]{2}{*}{$\begin{array}{c}\text { Sample } \\
\text { Location }\end{array}$} & \multirow[b]{2}{*}{$\begin{array}{l}\text { Sample } \\
\text { Number }\end{array}$} & \multirow{3}{*}{$\begin{array}{l}\text { Depth } \\
\text { (ft bgs) }\end{array}$} & \multicolumn{14}{|c|}{ Contaminants of Potential Concern (pCi/g) } \\
\hline & & & \multicolumn{2}{|c|}{ 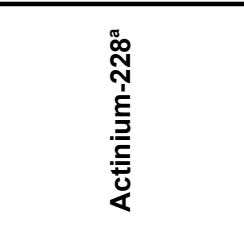 } & \multirow{3}{*}{ 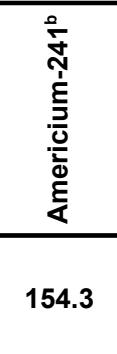 } & \multicolumn{2}{|c|}{ 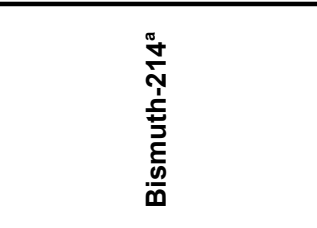 } & \multirow{3}{*}{ 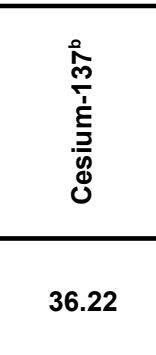 } & \multirow{3}{*}{$\begin{array}{l}\stackrel{0}{0} \\
\frac{1}{\pi} \\
\frac{0}{0} \\
0 \\
0\end{array}$} & \multirow{3}{*}{ 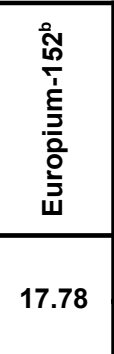 } & \multicolumn{2}{|c|}{ 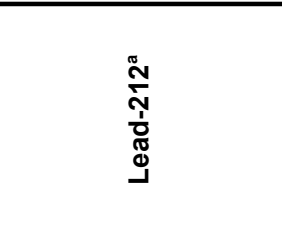 } & \multicolumn{2}{|c|}{ 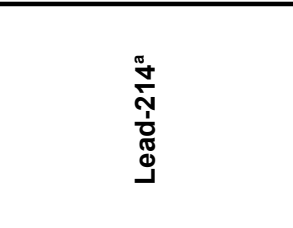 } & \multicolumn{2}{|c|}{ 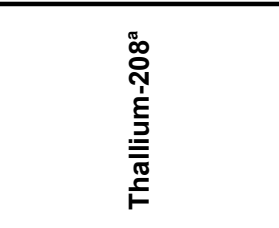 } \\
\hline \multicolumn{2}{|c|}{ Final Action Levels } & & 5 & 15 & & 5 & 15 & & & & 5 & 15 & 5 & 15 & 5 & 15 \\
\hline \multicolumn{3}{|c|}{ Depth bgs (cm) } & $<15$ & $>15$ & & $<15$ & $>15$ & & & & $<15$ & $>15$ & $<15$ & $>15$ & $<15$ & $>15$ \\
\hline \multirow{2}{*}{ F54 } & $224 \mathrm{~F} 109$ & $0-0.5$ & $\overline{---}$ & $\overline{N A}$ & $\overline{---}$ & $\overline{---}$ & $\overline{N A}$ & $0.35(\mathrm{G}, \mathrm{LT})$ & $\overline{---}$ & $\overline{---}$ & $0.77(\mathrm{~J})^{\mathrm{c}}$ & $\overline{N A}$ & $0.69(\mathrm{G}, \mathrm{J})$ & $\overline{N A}$ & $0.25(\mathrm{G})$ & $\overline{\overline{N A}}$ \\
\hline & $224 \mathrm{~F} 110$ & $0.5-1.5$ & NA & $0.82(\mathrm{G})$ & -- & NA & $0.67(\mathrm{G}, \mathrm{J})$ & -- & -- & - & NA & $0.95(J)^{c}$ & NA & $0.7(\mathrm{G}, \mathrm{J})$ & NA & $0.251(\mathrm{G})$ \\
\hline F55 & 224F111 & $0-0.5$ & $0.92(G)$ & NA & -- & $0.48(\mathrm{G}, \mathrm{J})$ & NA & $1.31(\mathrm{G})$ & -- & - & $0.63(J)^{c}$ & NA & $0.59(\mathrm{G}, \mathrm{J})$ & NA & -- & NA \\
\hline F56 & $224 \mathrm{~F} 112$ & $0-0.5$ & $1.35(\mathrm{G})$ & NA & -- & $0.99(\mathrm{G}, \mathrm{J})$ & NA & -- & -- & -- & $1.31(\mathrm{~J})^{\mathrm{c}}$ & NA & $0.98(\mathrm{G}, \mathrm{J})$ & NA & $0.52(\mathrm{G})$ & NA \\
\hline \multirow{2}{*}{ F57 } & $224 \mathrm{~F} 113$ & $0-0.5$ & -- & NA & $4.41(\mathrm{~J})^{\mathrm{c}}$ & $0.71(\mathrm{G}, \mathrm{J})$ & NA & $1.07(\mathrm{G})$ & -- & -- & $0.87(\mathrm{~J})^{\mathrm{c}}$ & NA & $0.65(\mathrm{G}, \mathrm{J})$ & NA & -- & NA \\
\hline & $224 \mathrm{~F} 114$ & $0.5-1.5$ & NA & $0.87(G)$ & -- & NA & $0.57(\mathrm{G}, \mathrm{J})$ & -- & -- & -- & NA & $1.21(\mathrm{~J})^{\mathrm{c}}$ & NA & $0.64(\mathrm{G}, \mathrm{J})$ & NA & $0.36(\mathrm{G})$ \\
\hline F58 & $224 \mathrm{~F} 115$ & $0-0.5$ & $0.78(\mathrm{G})$ & NA & -- & $0.55(\mathrm{G}, \mathrm{J})$ & NA & $0.225(\mathrm{G}, \mathrm{LT})$ & -- & -- & $0.91(\mathrm{~J})^{\mathrm{c}}$ & NA & $0.62(G, J)$ & NA & $0.249(\mathrm{G})$ & NA \\
\hline F59 & $224 \mathrm{~F} 116$ & $0-0.5$ & $1.19(\mathrm{G})$ & NA & $1.04(J)^{c}$ & $1.2(\mathrm{G}, \mathrm{J})$ & NA & $0.96(G)$ & -- & $1.03(\mathrm{~J})^{\mathrm{c}}$ & $1.52(\mathrm{~J})^{\mathrm{c}}$ & NA & $1.1(\mathrm{G}, \mathrm{J})$ & NA & $0.47(\mathrm{G})$ & NA \\
\hline F60 & $224 \mathrm{~F} 117$ & $0-0.5$ & $0.82(G)$ & NA & -- & $0.48(\mathrm{G}, \mathrm{J})$ & NA & $0.69(\mathrm{G})$ & -- & -- & $0.79(\mathrm{~J})^{\mathrm{C}}$ & NA & $0.54(\mathrm{G}, \mathrm{J})$ & NA & -- & NA \\
\hline F61 & $224 \mathrm{~F} 118$ & $0-0.5$ & $1.02(G)$ & NA & $1.09(\mathrm{~J})^{\mathrm{c}}$ & $0.92(\mathrm{G}, \mathrm{J})$ & NA & $1.75(\mathrm{G})$ & -- & $0.98(J)^{c}$ & $1.39(\mathrm{~J})^{\mathrm{c}}$ & NA & $1.04(\mathrm{G}, \mathrm{J})$ & NA & $0.354(\mathrm{G})$ & NA \\
\hline F62 & $224 \mathrm{~F} 119$ & $0-0.5$ & -- & NA & -- & -- & NA & -- & -- & -- & $0.66(J)^{c}$ & NA & $0.67(\mathrm{G}, \mathrm{J})$ & NA & $0.25(\mathrm{G})$ & NA \\
\hline \multirow{3}{*}{ F63 } & $224 \mathrm{~F} 120$ & $0-0.5$ & $1.2(\mathrm{G})$ & NA & $3.4(J)^{c}$ & $0.81(\mathrm{G}, \mathrm{J})$ & NA & $4.73(\mathrm{G})$ & -- & $1.14(\mathrm{~J})^{\mathrm{c}}$ & $1.73(\mathrm{~J})^{\mathrm{C}}$ & NA & $0.92(\mathrm{G}, \mathrm{J})$ & NA & $0.38(G)$ & NA \\
\hline & $224 \mathrm{~F} 121$ & $0-0.5$ & $1.36(\mathrm{G})$ & NA & -- & $0.97(\mathrm{G}, \mathrm{J})$ & NA & $2.89(\mathrm{G})$ & -- & $1.06(\mathrm{~J})^{\mathrm{c}}$ & $1.44(J)^{c}$ & NA & $0.95(\mathrm{G}, \mathrm{J})$ & NA & $0.48(\mathrm{G})$ & NA \\
\hline & 224F122 & $1-2$ & NA & $0.9(\mathrm{G})$ & $3.88(J)^{c}$ & NA & $0.78(\mathrm{G}, \mathrm{J})$ & $8.01(\mathrm{G})$ & -- & - & NA & $1.01(\mathrm{~J})^{\mathrm{c}}$ & NA & $0.75(\mathrm{G}, \mathrm{J})$ & NA & $0.421(\mathrm{G})$ \\
\hline F64 & $224 \mathrm{~F} 123$ & $0-0.5$ & -- & NA & $29.6(J)^{c}$ & $0.67(\mathrm{G}, \mathrm{J})$ & NA & $25.3(\mathrm{G})$ & -- & - & $0.92(J)^{c}$ & NA & $0.88(\mathrm{G}, \mathrm{J})$ & NA & $0.42(\mathrm{G})$ & NA \\
\hline F65 & $224 \mathrm{~F} 124$ & $0-0.5$ & $0.98(G)$ & NA & -- & $0.68(\mathrm{G}, \mathrm{J})$ & NA & $1.64(\mathrm{G})$ & -- & -- & $0.86(\mathrm{~J})^{\mathrm{c}}$ & NA & $0.7(\mathrm{G}, \mathrm{J})$ & NA & $0.33(\mathrm{G})$ & NA \\
\hline \multirow{2}{*}{ F66 } & $224 \mathrm{~F} 125$ & $0-0.5$ & $1.44(\mathrm{G})$ & NA & $10.1(\mathrm{~J})^{\mathrm{c}}$ & $0.99(\mathrm{G}, \mathrm{J})$ & NA & $8.1(\mathrm{G})$ & -- & $1.37(\mathrm{~J})^{\mathrm{c}}$ & $1.62(J)^{\mathrm{C}}$ & NA & $1.03(\mathrm{G}, \mathrm{J})$ & NA & $0.47(\mathrm{G})$ & NA \\
\hline & $224 \mathrm{~F} 126$ & $1.5-2.5$ & NA & $0.83(\mathrm{G})$ & -- & NA & $0.74(\mathrm{G}, \mathrm{J})$ & $1.12(\mathrm{G})$ & -- & -- & NA & $0.76(\mathrm{~J})^{\mathrm{c}}$ & NA & $0.52(\mathrm{G}, \mathrm{J})$ & NA & $0.28(\mathrm{G})$ \\
\hline F67 & $224 \mathrm{~F} 127$ & $0-0.5$ & $1.19(\mathrm{G})$ & NA & -- & $0.63(\mathrm{G}, \mathrm{J})$ & NA & $2.03(\mathrm{G})$ & -- & -- & $1.02(J)^{c}$ & NA & $0.74(\mathrm{G}, \mathrm{J})$ & NA & $0.346(\mathrm{G})$ & NA \\
\hline
\end{tabular}


Table A.7-18

Soil Sample Results for Gamma-Emitting Radionuclides Detected Above Minimum Detectable Concentrations at CAS 06-17-04, Decon Pad and Wastewater Catch (Page 7 of 7 )

\begin{tabular}{|c|c|c|c|c|c|c|c|c|c|c|c|c|c|c|c|c|}
\hline \multirow[b]{2}{*}{$\begin{array}{l}\text { Sample } \\
\text { Location }\end{array}$} & \multirow[b]{2}{*}{$\begin{array}{l}\text { Sample } \\
\text { Number }\end{array}$} & \multirow[b]{2}{*}{$\begin{array}{c}\text { Depth } \\
\text { (ft bgs) }\end{array}$} & \multicolumn{14}{|c|}{ Contaminants of Potential Concern $(\mathrm{pCi} / \mathrm{g})$} \\
\hline & & & \multicolumn{2}{|c|}{ 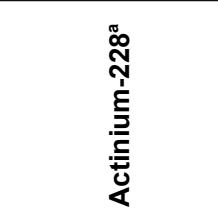 } & 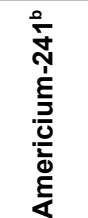 & \multicolumn{2}{|c|}{ 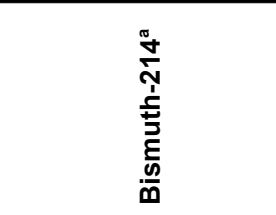 } & \multirow{3}{*}{ 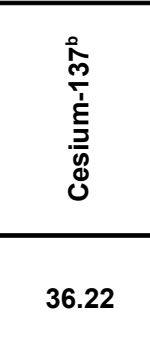 } & \multirow{3}{*}{$\begin{array}{l}\stackrel{0}{0} \\
\frac{d}{0} \\
\frac{1}{0} \\
0 \\
0\end{array}$} & \multirow{3}{*}{ 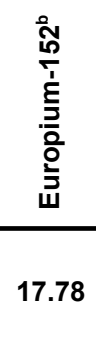 } & \multicolumn{2}{|c|}{ 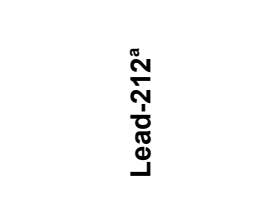 } & \multicolumn{2}{|c|}{ 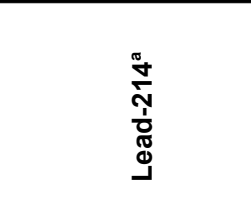 } & \multicolumn{2}{|c|}{ 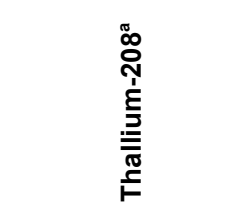 } \\
\hline \multicolumn{3}{|c|}{ Final Action Levels } & 5 & 15 & & 5 & 15 & & & & 5 & 15 & 5 & 15 & 5 & 15 \\
\hline \multicolumn{3}{|c|}{ Depth bgs (cm) } & $<15$ & $>15$ & & $<15$ & $>15$ & & & & $<15$ & $>15$ & $<15$ & $>15$ & $<15$ & $>15$ \\
\hline F68 & $224 \mathrm{~F} 128$ & $\overline{00-0.5}$ & $\overline{--}$ & NA & $\overline{--}$ & $\overline{0.44(\mathrm{G}, \mathrm{J})}$ & NA & $\overline{0.39(\mathrm{G}, \mathrm{LT})}$ & $\overline{--}$ & $\overline{--}$ & $0.71(\mathrm{~J})^{\mathrm{c}}$ & $\mathrm{NA}$ & $0.54(\mathrm{G}, \mathrm{J})$ & NA & $\overline{--}$ & NA \\
\hline F69 & $224 \mathrm{~F} 129$ & $0-0.5$ & $0.66(\mathrm{G})$ & NA & -- & $0.58(\mathrm{G}, \mathrm{J})$ & NA & -- & $\overline{--}$ & $\overline{--}$ & $0.86(\mathrm{~J})^{\mathrm{c}}$ & $\mathrm{NA}$ & $0.65(\mathrm{G}, \mathrm{J})$ & NA & $0.33(\mathrm{G})$ & NA \\
\hline
\end{tabular}

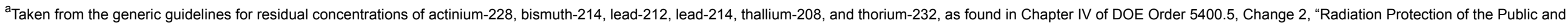

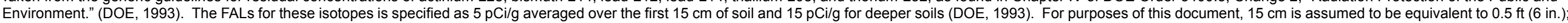
therefore, $5 \mathrm{pCi} / \mathrm{g}$ represents the FALs for these radionuclides in the surface soil (0 to $0.5 \mathrm{ft}$ depth)

Am-241, Eu-152, and Cs-137 FALs determined by RESRAD evaluation.

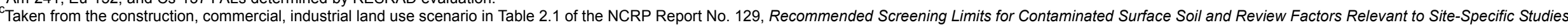
(NCRP, 1999). The values provided in this source document were scaled to a $25-\mathrm{mrem} / \mathrm{yr}$ dose.

Qualifier added to laboratory data, record accepted. Sample does not meet counting geometry requirements.

Qualifier added to laboratory data; record accepted. Duplicate precision analysis (relative percent difference) outside control limits.

$\mathrm{ft}$ bgs $=$ Feet below ground surface

$\mathrm{cm}=$ Centimeter

$\mathrm{mrem} / \mathrm{yr}=$ Millirems per year

pCi/g = Picocuries per gram

RESRAD = Residual radiation

$-=$ Not detected above minimum reporting limits

$=$ Greater than

$G=$ Sample density differs by more than 15 percent of laboratory control sample density.

= Estimated value.

$\mathrm{LT}=$ Result is less than the requested minimum detectable concentration, greater than the sample specific minimum detectable concentration.

$\mathrm{TI}=$ Tentatively identified 
Table A.7-19

Soil Sample Results for Gamma-Emitting Radionuclides Detected Above Minimum Detectable Concentrations at CAS 06-23-01, Decon Pad Discharge Piping

\begin{tabular}{|c|c|c|c|c|c|c|c|c|c|}
\hline \multirow{2}{*}{$\begin{array}{l}\text { Sample } \\
\text { Location }\end{array}$} & \multirow{2}{*}{$\begin{array}{l}\text { Sample } \\
\text { Number }\end{array}$} & \multirow{2}{*}{$\begin{array}{l}\text { Depth } \\
\text { (ft bgs) }\end{array}$} & \multicolumn{7}{|c|}{ Contaminants of Potential Concern $(\mathrm{pCi} / \mathrm{g})$} \\
\hline & & & Actinium-228 ${ }^{a}$ & Americium-241 ${ }^{\mathrm{b}}$ & Bismuth-214 ${ }^{a}$ & Cesium-137 ${ }^{\mathrm{b}}$ & Lead-212 ${ }^{\mathrm{a}}$ & Lead-214 ${ }^{a}$ & Thallium-208 \\
\hline \multicolumn{3}{|c|}{ Final Action Levels } & 15 & \multirow{2}{*}{154.3} & 15 & \multirow{2}{*}{36.22} & 15 & 15 & 15 \\
\hline \multicolumn{3}{|c|}{ Depth bgs (cm) } & $>15$ & & $>15$ & & $>15$ & $>15$ & $>15$ \\
\hline \multirow{4}{*}{ G01 } & 224G001 & $0-1$ & 1.03 & $2.88(J)^{c}$ & $0.81(\mathrm{~J})$ & 2.15 & 0.85 & $0.78(\mathrm{~J})$ & 0.32 \\
\hline & $224 G 002$ & $0-1$ & 0.86 & -- & $0.57(\mathrm{~J})$ & 3.03 & 1 & $0.86(\mathrm{~J})$ & -- \\
\hline & $224 G 003$ & $2-3$ & 0.87 & $4.12(\mathrm{~J})^{\mathrm{c}}$ & $0.58(\mathrm{~J})$ & 2.36 & 0.86 & $0.78(\mathrm{~J})$ & 0.286 \\
\hline & $224 G 004$ & $3-4$ & $1.14(\mathrm{G})$ & $1.36(\mathrm{G})$ & $0.75(\mathrm{G}, \mathrm{J})$ & $0.264(\mathrm{G}, \mathrm{LT})$ & $1.23(\mathrm{~J})^{\mathrm{C}}$ & $0.97(\mathrm{G}, \mathrm{J})$ & $0.39(\mathrm{G})$ \\
\hline G02 & $224 G 005$ & $0-1$ & -- & -- & $0.39(\mathrm{~J})$ & $0.36(\mathrm{LT})$ & 0.44 & $0.46(\mathrm{~J})$ & -- \\
\hline G03 & $224 G 006$ & $0-1$ & 0.58 & 0.74 & $0.44(\mathrm{~J})$ & $0.211(\mathrm{LT})$ & 0.59 & $0.42(\mathrm{~J})$ & 0.172 \\
\hline
\end{tabular}

${ }^{a}$ Taken from the generic guidelines for residual concentrations of actinium-228, bismuth-214, lead-212, lead-214, thallium-208, and thorium-232, as found in Chapter IV of DOE Order 5400.5, Change 2, "Radiation Protection of the Public and Environment." (DOE, 1993). The FALs for these isotopes is specified as $5 \mathrm{pCi} / \mathrm{g}$ averaged over the first $15 \mathrm{~cm}$ of soil and $15 \mathrm{pCi} / \mathrm{g}$ for deeper soils (DOE, 1993). For purposes of this document, $15 \mathrm{~cm}$ is assumed to be equivalent to $0.5 \mathrm{ft}$ ( $6 \mathrm{in}$.); therefore, $5 \mathrm{pCi} / \mathrm{g}$ represents the FALs for these radionuclides in the surface soil ( 0 to $0.5 \mathrm{ft}$ depth).

'Qualifier added to laboratory data; record accepted. Sample does not meet counting geometry requirements.

$\mathrm{ft}$ bgs $=$ Feet below ground surface

$\mathrm{cm}=$ Centimeter

$\mathrm{pCi} / \mathrm{g}=$ Picocuries per gram

RESRAD = Residual radiation

$--=$ Not detected above minimum reporting limits

$>=$ Greater than

$G=$ Sample density differs by more than 15 percent of laboratory control sample density.

$\mathrm{J}=$ Estimated value.

$\mathrm{LT}=$ Result is less than the requested minimum detectable concentration, greater than the sample specific minimum detectable concentration. 
Table A.7-20

Soil Sample Results for Isotopes Detected Above Minimum Detectable Concentrations at CAS 06-05-01, Leachfield (Page 1 of 4 )

\begin{tabular}{|c|c|c|c|c|c|c|c|c|}
\hline \multirow[b]{2}{*}{$\begin{array}{l}\text { Sample } \\
\text { Location }\end{array}$} & \multirow[b]{2}{*}{$\begin{array}{l}\text { Sample } \\
\text { Number }\end{array}$} & \multirow[b]{2}{*}{$\begin{array}{c}\text { Depth } \\
\text { (ft bgs) }\end{array}$} & \multicolumn{6}{|c|}{ Contaminants of Potential Concern (pCi/g) } \\
\hline & & & 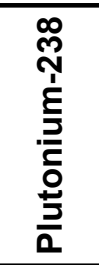 & 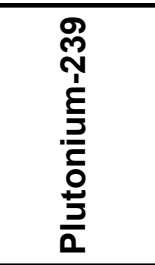 & 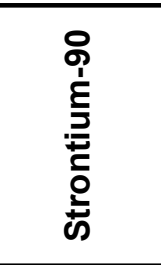 & 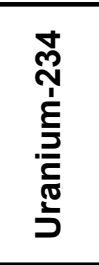 & 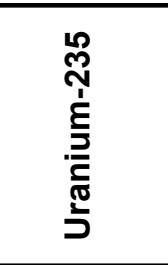 & 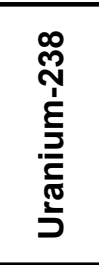 \\
\hline \multicolumn{3}{|c|}{ Final Action Levels ${ }^{a}$} & 186 & 168.1 & 2692 & 1052 & 138 & 471.6 \\
\hline \multirow{4}{*}{ E01 } & 224E001 & $0-0.5$ & $\overline{--}$ & $\overline{0.44}$ & $\overline{0.84}$ & $\overline{0.75}$ & $\overline{0.089}$ & $\overline{0.66}$ \\
\hline & 224E002 & $3-4$ & 0.119 & 6.3 & $\overline{--}$ & 0.57 & $\overline{--}$ & 0.54 \\
\hline & 224E003 & $3-4$ & -- & 0.45 & $\overline{--}$ & 0.62 & $\overline{--}$ & 0.55 \\
\hline & 224E004 & $5-6$ & -- & 0.129 & $\overline{--}$ & 0.54 & 0.054 & 0.48 \\
\hline \multirow{3}{*}{ E02 } & 224E005 & $0-0.5$ & $\overline{--}$ & $\overline{1.79}$ & $\overline{--}$ & 0.6 & $\overline{--}$ & 0.59 \\
\hline & 224E007 & $4-5$ & -- & -- & -- & 0.54 & -- & 0.62 \\
\hline & 224E009 & $7-8$ & -- & $\overline{--}$ & -- & 0.59 & -- & 0.61 \\
\hline \multirow{3}{*}{ E03 } & 224E006 & $0-0.5$ & -- & 1.31 & -- & 0.65 & 0.054 & 0.59 \\
\hline & 224E013 & $4-5$ & -- & -- & -- & 0.68 & -- & 0.68 \\
\hline & 224E016 & $7-8$ & -- & $\overline{--}$ & -- & 0.54 & $0.031(\mathrm{LT})$ & 0.57 \\
\hline \multirow{4}{*}{ E04 } & 224E008 & $0-0.5$ & 0.106 & 2.63 & -- & 0.59 & $\overline{--}$ & 0.6 \\
\hline & 224E052 & $3.5-4.5$ & -- & -- & $-0.075(J)^{b}$ & 0.58 & -- & 0.55 \\
\hline & 224E053 & $7-8$ & -- & 0.058 & $0.02(\mathrm{~J})^{\mathrm{b}}$ & 0.54 & $\overline{--}$ & 0.48 \\
\hline & 224E054 & $9-10$ & -- & 0.086 & $0.13(\mathrm{~J})^{\mathrm{b}}$ & 0.47 & -- & 0.54 \\
\hline \multirow{5}{*}{ E05 } & 224E010 & $0-0.5$ & 0.095 & 4.41 & 32.5 & 0.73 & $\overline{--}$ & 0.67 \\
\hline & 224E020 & $4-5$ & -- & 0.114 & $\overline{--}$ & 0.68 & $\overline{--}$ & 0.5 \\
\hline & 224E021 & $4-5$ & -- & 0.159 & -- & 0.54 & -- & 0.57 \\
\hline & 224E022 & $7-8$ & -- & $\overline{--}$ & $\overline{--}$ & 0.58 & $\overline{--}$ & 0.57 \\
\hline & 224E023 & $9-10$ & -- & 0.061 & -- & 0.62 & $\overline{--}$ & 0.65 \\
\hline \multirow{4}{*}{ E06 } & 224E011 & $0-0.5$ & 0.067 & 3.78 & $0.19(\mathrm{~J})^{\mathrm{c}}$ & 0.56 & -- & 0.53 \\
\hline & 224E055 & $3.5-4.5$ & $\overline{--}$ & 0.074 & $0.07(\mathrm{~J})^{\mathrm{b}}$ & 0.45 & -- & 0.446 \\
\hline & 224E056 & $7-8$ & -- & -- & $0.03(\mathrm{~J})^{\mathrm{b}}$ & 0.56 & -- & 0.62 \\
\hline & 224E057 & $9-10$ & -- & -- & $0.07(\mathrm{~J})^{\mathrm{b}}$ & 0.67 & $\overline{--}$ & 0.68 \\
\hline \multirow{3}{*}{ E07 } & 224E012 & $0-0.5$ & 0.143 & 10.7 & -- & 0.62 & -- & 0.49 \\
\hline & 224E037 & $5-6$ & -- & -- & -- & 0.78 & -- & 0.77 \\
\hline & 224E038 & $9-10$ & -- & -- & -- & 0.56 & $0.036(\mathrm{LT})$ & 0.54 \\
\hline \multirow{4}{*}{ E08 } & 224E014 & $0-0.5$ & 0.069 & 5.61 & -- & 0.53 & -- & 0.61 \\
\hline & 224E025 & $2-3$ & $\overline{--}$ & -- & -- & 0.5 & 0.064 & 0.62 \\
\hline & 224E026 & $7-8$ & $\overline{--}$ & $\overline{--}$ & $\overline{--}$ & 0.68 & 0.05 & 0.75 \\
\hline & 224E027 & $9-10$ & $\overline{--}$ & $0.038(\mathrm{LT})$ & $\overline{--}$ & 0.8 & $\overline{--}$ & 0.91 \\
\hline
\end{tabular}


Table A.7-20

Soil Sample Results for Isotopes Detected Above Minimum Detectable Concentrations at CAS 06-05-01, Leachfield (Page 2 of 4 )

\begin{tabular}{|c|c|c|c|c|c|c|c|c|}
\hline \multirow[b]{2}{*}{$\begin{array}{l}\text { Sample } \\
\text { Location }\end{array}$} & \multirow[b]{2}{*}{$\begin{array}{l}\text { Sample } \\
\text { Number }\end{array}$} & \multirow[b]{2}{*}{$\begin{array}{l}\text { Depth } \\
\text { (ft bgs) }\end{array}$} & \multicolumn{6}{|c|}{ Contaminants of Potential Concern (pCi/g) } \\
\hline & & & 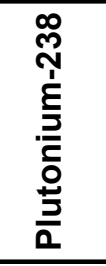 & 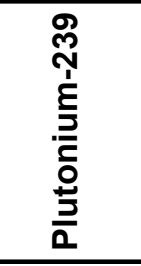 & 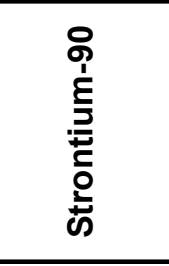 & 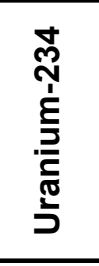 & 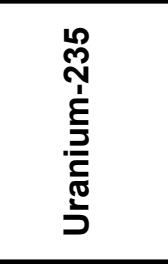 & 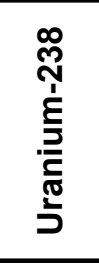 \\
\hline \multicolumn{3}{|c|}{ Final Action Levels ${ }^{a}$} & 186 & 168.1 & 2692 & 1052 & 138 & 471.6 \\
\hline \multirow{3}{*}{ E09 } & 224E015 & $0-0.5$ & 0.306 & $\overline{5.69}$ & $\overline{---}$ & 0.53 & $\overline{---}$ & $\overline{0.54}$ \\
\hline & 224E035 & $6-7$ & -- & -- & -- & 0.62 & -- & 0.73 \\
\hline & 224E036 & $9-10$ & -- & -- & -- & 0.7 & $0.031(\mathrm{LT})$ & 0.72 \\
\hline \multirow{3}{*}{ E10 } & 224E017 & $0-0.5$ & -- & 1.05 & -- & 0.61 & -- & 0.59 \\
\hline & 224E033 & $5-6$ & -- & -- & -- & 0.7 & -- & 0.73 \\
\hline & 224E034 & $8-9$ & -- & -- & -- & 0.75 & 0.072 & 0.64 \\
\hline \multirow{4}{*}{ E11 } & 224E018 & $0-0.5$ & 0.415 & 33.5 & -- & 0.6 & -- & 0.46 \\
\hline & 224E028 & $2-3$ & 0.068 & 0.83 & -- & 0.76 & 0.079 & 0.55 \\
\hline & 224E029 & $5-6$ & -- & -- & -- & 0.79 & 0.083 & 0.78 \\
\hline & 224E030 & $7-8$ & -- & -- & -- & 0.8 & 0.122 & 0.8 \\
\hline \multirow{3}{*}{ E12 } & 224E019 & $0-0.5$ & - & 0.55 & -- & 0.52 & -- & 0.54 \\
\hline & 224E031 & $4-5$ & -- & -- & -- & 0.57 & -- & 0.64 \\
\hline & 224E032 & $7-8$ & -- & -- & -- & 0.72 & -- & 0.75 \\
\hline E13 & 224E024 & $7-8$ & -- & 0.294 & -- & 0.64 & -- & 0.62 \\
\hline \multirow{5}{*}{ E14 } & 224E039 & $0-0.5$ & - & 0.055 & -- & 0.5 & -- & 0.57 \\
\hline & 224E040 & $2-3$ & -- & -- & $0.06(J)^{b}$ & 0.55 & -- & 0.56 \\
\hline & 224E041 & $6-7$ & -- & -- & $-0.041(\mathrm{~J})^{\mathrm{b}}$ & 0.59 & -- & 0.57 \\
\hline & 224E042 & $6-7$ & -- & -- & $0.05(\mathrm{~J})^{\mathrm{b}}$ & 0.59 & -- & 0.51 \\
\hline & 224E043 & $9-10$ & -- & -- & $-0.045(J)^{b}$ & 0.66 & -- & 0.59 \\
\hline \multirow{4}{*}{ E15 } & 224E044 & $0-0.5$ & -- & 1.37 & $0.02(J)^{b}$ & 0.65 & $0.032(\mathrm{LT})$ & 0.52 \\
\hline & 224E045 & $2-3$ & -- & -- & $-0.01(\mathrm{~J})^{\mathrm{b}}$ & 0.64 & $0.04(\mathrm{LT})$ & 0.47 \\
\hline & 224E046 & $6-7$ & -- & 0.135 & $0.15(\mathrm{~J})^{\mathrm{b}}$ & 0.53 & -- & 0.55 \\
\hline & 224E047 & $9-10$ & -- & -- & $0.04(J)^{b}$ & 0.65 & -- & 0.72 \\
\hline \multirow{4}{*}{ E16 } & 224E048 & $0-0.5$ & -- & 0.46 & $0.02(\mathrm{~J})^{\mathrm{b}}$ & 0.5 & -- & 0.49 \\
\hline & 224E049 & $2-3$ & -- & -- & $-0.089(\mathrm{~J})^{\mathrm{b}}$ & 0.5 & -- & 0.53 \\
\hline & 224E050 & $7-8$ & -- & -- & $-0.057(J)^{b}$ & 0.87 & -- & 0.81 \\
\hline & 224E051 & $10-11$ & -- & -- & $0.34(J)^{b}$ & 0.415 & -- & 0.46 \\
\hline \multirow{3}{*}{ E17 } & 224E058 & $0-0.5$ & -- & $1.92(\mathrm{~J})^{\mathrm{d}}$ & -- & 0.74 & $0.038(\mathrm{LT})$ & 0.78 \\
\hline & 224E068 & $4-5$ & -- & $0.7(\mathrm{~J})^{\mathrm{d}}$ & -- & 0.75 & -- & 0.65 \\
\hline & 224E069 & $6.5-7.5$ & 0.075 & $1.7(J)^{d}$ & $\overline{--}$ & 0.93 & 0.051 & 0.58 \\
\hline
\end{tabular}


Table A.7-20

Soil Sample Results for Isotopes Detected Above Minimum Detectable Concentrations at CAS 06-05-01, Leachfield (Page 3 of 4 )

\begin{tabular}{|c|c|c|c|c|c|c|c|c|}
\hline \multirow[b]{2}{*}{$\begin{array}{l}\text { Sample } \\
\text { Location }\end{array}$} & \multirow[b]{2}{*}{$\begin{array}{l}\text { Sample } \\
\text { Number }\end{array}$} & \multirow[b]{2}{*}{$\begin{array}{c}\text { Depth } \\
\text { (ft bgs) }\end{array}$} & \multicolumn{6}{|c|}{ Contaminants of Potential Concern (pCi/g) } \\
\hline & & & 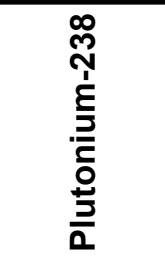 & 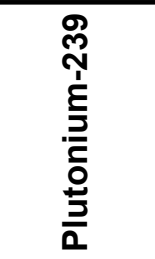 & 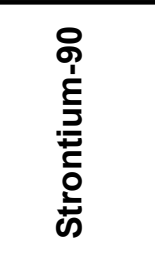 & 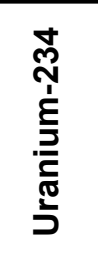 & 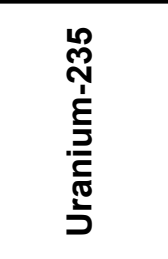 & 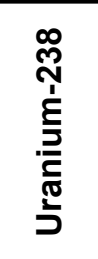 \\
\hline \multicolumn{3}{|c|}{ Final Action Levels ${ }^{a}$} & 186 & 168.1 & 2692 & 1052 & 138 & 471.6 \\
\hline \multirow{3}{*}{ E18 } & 224E059 & $\overline{0-0.5}$ & $\overline{--}$ & $0.56(\mathrm{~J})^{\mathrm{d}}$ & $\overline{---}$ & $\overline{0.61}$ & $\overline{--}$ & 0.57 \\
\hline & 224E070 & $4-5$ & -- & $0.101(\mathrm{~J})^{\mathrm{d}}$ & -- & 0.68 & -- & 0.67 \\
\hline & 224E071 & $7-8$ & -- & $1.23(J)^{d}$ & $0.42(\mathrm{LT})$ & 0.73 & -- & 0.63 \\
\hline \multirow{3}{*}{ E19 } & 224E060 & $0-0.5$ & 0.059 & $3.09(J)^{d}$ & $\overline{--}$ & 0.69 & $\overline{--}$ & 0.69 \\
\hline & 224E072 & $4-5$ & 0.216 & $2.54(\mathrm{~J})^{\mathrm{d}}$ & $0.56(\mathrm{Y} 1)$ & 1.08 & 0.043 (LT) & 0.81 \\
\hline & 224E073 & $7-8$ & $\overline{--}$ & $0.132(\mathrm{~J})^{\mathrm{d}}$ & -- & 0.75 & -- & 0.65 \\
\hline \multirow{4}{*}{ E20 } & 224E061 & $0-0.5$ & -- & $3.5(\mathrm{~J})^{\mathrm{d}}$ & $0.58(\mathrm{Y} 1)$ & 1.07 & - & 0.7 \\
\hline & 224E063 & $4-5$ & $\overline{--}$ & $0.05(\mathrm{~J})^{\mathrm{d}}$ & -- & 0.67 & -- & 0.65 \\
\hline & 224E066 & $6.5-7.5$ & $\overline{--}$ & $0.173(\mathrm{~J})^{\mathrm{d}}$ & $\overline{--}$ & 0.6 & 0.042 (LT) & 0.53 \\
\hline & 224E067 & $6.5-7.5$ & -- & $0.142(\mathrm{~J})^{d}$ & -- & 0.59 & -- & 0.49 \\
\hline \multirow{3}{*}{ E21 } & 224E062 & $0-0.5$ & -- & $0.46(\mathrm{~J})^{\mathrm{d}}$ & -- & 0.58 & - & 0.62 \\
\hline & 224E074 & $4-5$ & -- & $0.84(J)^{d}$ & -- & 0.9 & 0.042 (LT) & 0.64 \\
\hline & 224E075 & $7-8$ & -- & $0.34(\mathrm{~J})^{\mathrm{d}}$ & $\overline{--}$ & 0.85 & $\overline{--}$ & 0.67 \\
\hline \multirow{3}{*}{ E22 } & 224E064 & $0-0.5$ & -- & $0.73(\mathrm{~J})^{\mathrm{d}}$ & -- & 0.64 & -- & 0.71 \\
\hline & 224E076 & $4-5$ & $\overline{--}$ & $\overline{--}$ & $\overline{--}$ & 0.67 & 0.071 & 0.56 \\
\hline & 224E077 & $7-8$ & -- & -- & $\overline{--}$ & 0.66 & -- & 0.66 \\
\hline \multirow{3}{*}{ E23 } & 224E065 & $0-0.5$ & -- & $0.59(J)^{d}$ & -- & 0.64 & 0.066 & 0.65 \\
\hline & 224E078 & $4-5$ & -- & -- & $\overline{--}$ & 0.6 & 0.036 (LT) & 0.47 \\
\hline & 224E079 & $7-8$ & -- & 0.097 & -- & 0.65 & $0.039(\mathrm{LT})$ & 0.56 \\
\hline \multirow{3}{*}{ E24 } & 224E080 & $0-1$ & $0.04(\mathrm{LT})$ & $\overline{1.8}$ & $\overline{--}$ & 0.93 & -- & 0.78 \\
\hline & 224E082 & $4-5$ & -- & 0.186 & $\overline{--}$ & 0.6 & $0.048(\mathrm{LT})$ & 0.61 \\
\hline & 224E083 & $7-8$ & -- & 0.206 & -- & 0.51 & -- & 0.52 \\
\hline \multirow{4}{*}{ E25 } & 224E081 & $0-1$ & 0.124 & 8.2 & -- & 0.87 & 0.047 (LT) & 0.72 \\
\hline & 224E084 & $4-5$ & $\overline{--}$ & 0.298 & -- & 0.62 & -- & 0.52 \\
\hline & 224E085 & $4-5$ & $\overline{--}$ & 0.29 & -- & 0.71 & -- & 0.52 \\
\hline & 224E086 & $7-8$ & -- & 0.171 & -- & 0.55 & -- & 0.64 \\
\hline $\bar{E} 26$ & 224E087 & $0-1$ & $0.033(\mathrm{LT})$ & 1.21 & -- & 0.63 & -- & 0.63 \\
\hline $\bar{E} 27$ & 224E090 & $0-1$ & $\overline{--}$ & -- & -- & 0.435 & -- & 0.47 \\
\hline E28 & 224E093 & $0-1$ & $\overline{--}$ & $\overline{--}$ & $\overline{--}$ & 0.52 & 0.037 (LT) & 0.52 \\
\hline E29 & 224E096 & $0-0.5$ & -- & 1.12 & -- & -- & -- & -- \\
\hline
\end{tabular}




\section{Table A.7-20 \\ Soil Sample Results for Isotopes Detected Above Minimum Detectable Concentrations at CAS 06-05-01, Leachfield}

(Page 4 of 4 )

\begin{tabular}{|c|c|c|c|c|c|c|c|c|}
\hline \multirow[b]{2}{*}{$\begin{array}{l}\text { Sample } \\
\text { Location }\end{array}$} & \multirow[b]{2}{*}{$\begin{array}{l}\text { Sample } \\
\text { Number }\end{array}$} & \multirow[b]{2}{*}{$\begin{array}{l}\text { Depth } \\
\text { (ft bgs) }\end{array}$} & \multicolumn{6}{|c|}{ Contaminants of Potential Concern $(\mathrm{pCi} / \mathrm{g})$} \\
\hline & & & 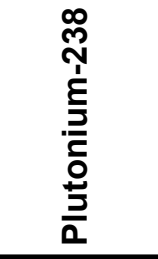 & 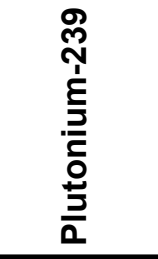 & 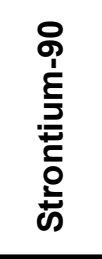 & 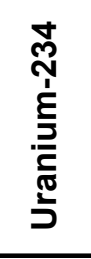 & 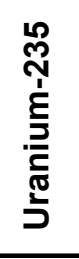 & 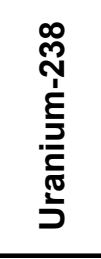 \\
\hline \multicolumn{3}{|c|}{ Final Action Levels ${ }^{a}$} & 186 & 168.1 & 2692 & 1052 & 138 & 471.6 \\
\hline E30 & 224E097 & $0-0.5$ & -- & 0.346 & -- & $\overline{--}$ & -- & -- \\
\hline E31 & 224E098 & $0-0.5$ & -- & 2.31 & -- & -- & -- & -- \\
\hline E32 & 224E099 & $0-0.5$ & -- & 1.87 & -- & -- & -- & -- \\
\hline E33 & 224E100 & $0-0.5$ & -- & 0.108 & -- & -- & -- & -- \\
\hline E34 & 224E101 & $0-0.5$ & 0.082 & 4.51 & -- & -- & -- & -- \\
\hline E35 & 224E102 & $0-0.5$ & 0.095 & 4.07 & -- & -- & -- & -- \\
\hline \multirow{3}{*}{ E36 } & 224E103 & $0-0.5$ & 0.42 & 34.9 & -- & -- & -- & -- \\
\hline & 224E104 & $2-2.5$ & 0.32 (M3) & $21.3(\mathrm{~J})^{\mathrm{d}}$ & -- & -- & -- & -- \\
\hline & 224E108 & $3-3.5$ & 0.187 & 11.3 & -- & -- & -- & -- \\
\hline \multirow{2}{*}{ E37 } & 224E105 & $0-0.5$ & $3.9(\mathrm{~J})^{\mathrm{d}}$ & $218(\mathrm{~J})^{\mathrm{d}}$ & -- & -- & -- & -- \\
\hline & 224E109 & $1-1.5$ & 0.34 & 17.5 & -- & -- & -- & -- \\
\hline \multirow{2}{*}{ E38 } & 224E106 & $0-0.5$ & $1.75(\mathrm{~J})^{\mathrm{d}}$ & $100(J)^{d}$ & -- & -- & -- & -- \\
\hline & 224E110 & $1-1.5$ & 0.299 & 19.9 & -- & -- & -- & -- \\
\hline \multirow{2}{*}{ E39 } & 224E107 & $0-0.5$ & $0.97(\mathrm{Y} 1)$ & $104(\mathrm{~J})^{\mathrm{d}}$ & -- & -- & -- & -- \\
\hline & 224E111 & $0.5-1.0$ & $0.24(\mathrm{~J})^{\mathrm{d}}$ & $15.5(\mathrm{~J})^{\mathrm{d}}$ & -- & -- & -- & -- \\
\hline E40 & 224E112 & $0-0.5$ & -- & $0.296(J)^{d}$ & -- & -- & -- & -- \\
\hline
\end{tabular}

${ }^{a}$ FALs determined by RESRAD evaluation.

${ }^{\mathrm{b}}$ Qualifier added to laboratory data; record accepted. Sample does not meet counting geometry requirements.

${ }^{\mathrm{C}} \mathrm{Qualifier}$ added to laboratory data; record accepted. Chemical yield above control limits.

${ }^{\mathrm{d}}$ Qualifier added to laboratory data; record accepted. Duplicate precision analysis (relative percent difference) outside control limits.

$\mathrm{ft}$ bgs $=$ Feet below ground surface

$\mathrm{pCi} / \mathrm{g}=$ Picocuries per gram

RESRAD $=$ Residual radiation

$\mathrm{J}=$ Estimated value.

$\mathrm{LT}=$ Result is less than the requested minimum detectable concentration, greater than the sample specific minimum detectable concentration.

M3 = The requested minimum detectable concentration was not met, but the reported activity is greater than the reported minimum detectable concentration.

$\mathrm{Y} 1=$ Chemical yield is in control at $100-110$ percent. Quantitative yield is assumed.

-- = Not detected above minimum reporting limits 
Table A.7-21

Soil Sample Results for Isotopes Detected Above Minimum Detectable Concentrations at CAS 06-17-04, Decon Pad and Wastewater Catch (Page 1 of 4 )

\begin{tabular}{|c|c|c|c|c|c|c|c|c|}
\hline \multirow[b]{2}{*}{$\begin{array}{c}\text { Sample } \\
\text { Location }\end{array}$} & \multirow[b]{2}{*}{$\begin{array}{l}\text { Sample } \\
\text { Number }\end{array}$} & \multirow[b]{2}{*}{$\begin{array}{c}\text { Depth } \\
\text { (ft bgs) }\end{array}$} & \multicolumn{6}{|c|}{ Contaminants of Potential Concern (pCi/g) } \\
\hline & & & 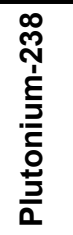 & 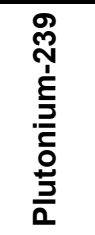 & 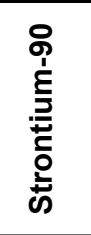 & 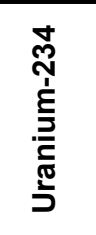 & 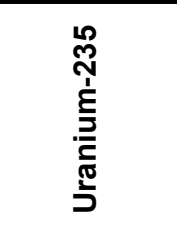 & 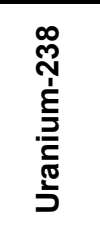 \\
\hline \multicolumn{3}{|c|}{ Final Action Levels ${ }^{a}$} & 186 & 168.1 & 2692 & 1052 & 138 & 471.6 \\
\hline \multirow{4}{*}{$\mathrm{F} 01$} & $224 \mathrm{~F} 001$ & $1-2$ & $-\overline{-}$ & - & - & 0.57 & -- & 0.56 \\
\hline & $224 \mathrm{~F} 002$ & $1-2$ & -- & -- & - & 0.5 & -- & 0.59 \\
\hline & $224 \mathrm{~F} 003$ & $4-5$ & - & - & - & 0.53 & - & 0.54 \\
\hline & 224F004 & $7-8$ & - & - & - & 0.78 & 0.067 & 0.68 \\
\hline \multirow{3}{*}{ F02 } & $224 \mathrm{~F} 005$ & $1-2$ & - & - & - & 0.54 & -- & 0.54 \\
\hline & 224F006 & $4-5$ & - & - & - & 0.39 & - & 0.43 \\
\hline & 224F007 & $7-8$ & - & - & -- & 0.392 & -- & 0.416 \\
\hline \multirow{3}{*}{ F03 } & 224F008 & $1-2$ & -- & -- & - & 0.52 & -- & 0.48 \\
\hline & 224F009 & $4-5$ & - & -- & - & 0.47 & - & 0.47 \\
\hline & $224 \mathrm{~F} 010$ & $7-8$ & - & - & - & 0.56 & - & 0.65 \\
\hline \multirow{3}{*}{ F04 } & 224F011 & $1-2$ & - & - & - & 0.67 & - & 0.58 \\
\hline & 224F012 & $4-5$ & - & - & -- & 0.49 & $0.038(\mathrm{LT}))$ & 0.47 \\
\hline & $224 \mathrm{~F} 013$ & $7-8$ & - & - & - & 0.54 & - & 0.58 \\
\hline \multirow{3}{*}{ F05 } & $224 F 014$ & $1-2$ & -- & - & -- & 0.53 & -- & 0.57 \\
\hline & $224 \mathrm{~F} 015$ & $4-5$ & -- & -- & -- & 0.56 & -- & 0.46 \\
\hline & $224 \mathrm{~F} 016$ & $7-8$ & -- & -- & -- & 0.56 & 0.037 (LT) & 0.62 \\
\hline \multirow{3}{*}{ F06 } & $224 \mathrm{~F} 017$ & $1-2$ & - & - & - & 0.55 & - & 0.66 \\
\hline & 224F018 & $4-5$ & - & - & - & 0.53 & 0.043 (LT) & 0.62 \\
\hline & 224F019 & $7-8$ & - & - & - & 0.52 & - & 0.55 \\
\hline \multirow{3}{*}{ F07 } & $224 \mathrm{~F} 020$ & $1-2$ & - & - & - & 0.52 & - & 0.5 \\
\hline & $224 F 021$ & $4-5$ & - & - & -- & 0.53 & - & 0.49 \\
\hline & 224F022 & $7-8$ & - & - & -- & 0.7 & $0.033(\mathrm{LT})$ & 0.74 \\
\hline \multirow{4}{*}{ F08 } & 224F023 & $0.2-1$ & - & 0.322 & -- & 0.65 & 0.055 & 0.62 \\
\hline & 224F024 & $4-5$ & - & $\overline{--}$ & - & 0.46 & 0.034 (LT) & 0.5 \\
\hline & $224 \mathrm{~F} 025$ & $7-8$ & - & - & - & 0.56 & 0.037 (LT) & 0.51 \\
\hline & 224F026 & $7-8$ & - & - & - & 0.43 & - & 0.62 \\
\hline \multirow{3}{*}{ F09 } & $224 \mathrm{~F} 027$ & $0.2-1$ & - & - & -- & 0.55 & - & 0.65 \\
\hline & 224F028 & $4-5$ & - & -- & -- & 0.59 & 0.052 & 0.62 \\
\hline & 224F029 & $7-8$ & - & - & - & 0.365 & - & 0.417 \\
\hline
\end{tabular}


Table A.7-21

Soil Sample Results for Isotopes Detected Above Minimum Detectable Concentrations at CAS 06-17-04, Decon Pad and Wastewater Catch (Page 2 of 4 )

\begin{tabular}{|c|c|c|c|c|c|c|c|c|}
\hline \multirow[b]{2}{*}{$\begin{array}{c}\text { Sample } \\
\text { Location }\end{array}$} & \multirow[b]{2}{*}{$\begin{array}{l}\text { Sample } \\
\text { Number }\end{array}$} & \multirow[b]{2}{*}{$\begin{array}{c}\text { Depth } \\
\text { (ft bgs) }\end{array}$} & \multicolumn{6}{|c|}{ Contaminants of Potential Concern (pCi/g) } \\
\hline & & & 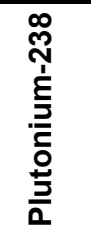 & 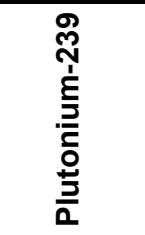 & 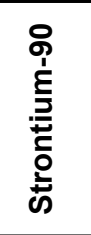 & 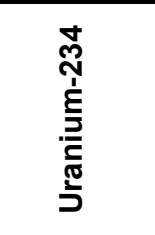 & 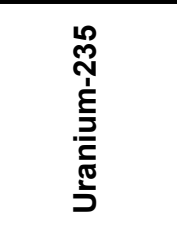 & 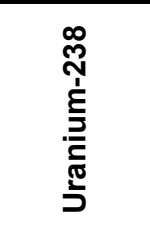 \\
\hline \multicolumn{3}{|c|}{ Final Action Levels ${ }^{a}$} & 186 & 168.1 & 2692 & 1052 & 138 & 471.6 \\
\hline \multirow{3}{*}{ F10 } & $224 \mathrm{~F} 030$ & $0-1$ & $\overline{---}$ & - & - & 0.388 & -- & 0.49 \\
\hline & $224 \mathrm{~F} 031$ & $4-5$ & -- & -- & -- & 0.401 & -- & 0.414 \\
\hline & $224 \mathrm{~F} 032$ & $7-8$ & - & - & - & 0.48 & 0.033 (LT) & 0.4 \\
\hline \multirow{3}{*}{ F11 } & 224F033 & $0-1$ & 0.78 & 52.4 & - & 0.88 (M3) & - & 0.63 (M3) \\
\hline & 224F034 & $4-5$ & -- & 0.243 & -- & 0.365 & -- & 0.381 \\
\hline & 224F035 & $7-8$ & - & - & - & 0.48 & - & 0.55 \\
\hline \multirow{3}{*}{ F12 } & $224 \mathrm{~F} 036$ & $0-1$ & 0.226 & 10.5 & -- & 0.6 & $0.038(\mathrm{LT}))$ & 0.56 \\
\hline & 224F037 & $4-5$ & 0.147 & 10.4 & - & 0.57 & - & 0.52 \\
\hline & $224 \mathrm{~F} 038$ & $7-8$ & -- & -- & -- & 0.6 & -- & 0.49 \\
\hline \multirow{3}{*}{ F13 } & 224F039 & $0-1$ & - & 0.42 & - & 0.48 & $\overline{--}$ & 0.53 \\
\hline & $224 \mathrm{~F} 040$ & $4-5$ & - & - & - & 0.52 & - & 0.53 \\
\hline & $224 \mathrm{~F} 041$ & $7-8$ & -- & - & -- & 0.402 & - & 0.439 \\
\hline F21 & 224F050 & $1-3$ & - & 0.257 & -- & - & - & - \\
\hline F23 & 224F052 & $1-3$ & - & $1.73(\mathrm{~J})$ & -- & -- & - & - \\
\hline \multirow{3}{*}{ F24 } & 224F053 & $0-2$ & 0.72 & 19.8 (J) & -- & -- & -- & -- \\
\hline & 224F054 & $3-5$ & -- & $0.75(\mathrm{~J})$ & -- & -- & -- & -- \\
\hline & 224F055 & $6-7$ & - & $0.6(\mathrm{~J})$ & - & - & $\overline{--}$ & - \\
\hline \multirow{2}{*}{ F25 } & 224F056 & $0-3$ & 0.119 & $5.1(\mathrm{~J})$ & - & - & - & - \\
\hline & 224F057 & $5-7$ & - & 0.117 (J) & -- & - & - & -- \\
\hline \multirow{2}{*}{ F26 } & 224F058 & $0-4$ & 0.099 & $4.56(\mathrm{~J})$ & -- & - & - & - \\
\hline & 224F059 & $4-7$ & -- & 0.78 (J) & -- & -- & -- & -- \\
\hline F27 & 224F061 & $2-4$ & -- & 0.116 (J) & -- & -- & -- & -- \\
\hline \multirow{3}{*}{ F28 } & 224F062 & $0-0.5$ & 10 & $201(\mathrm{~J})$ & -- & -- & -- & -- \\
\hline & 224F063 & $0-0.5$ & 0.54 & $11.8(\mathrm{~J})$ & - & - & -- & - \\
\hline & 224F064 & $3-4$ & - & $0.181(\mathrm{~J})$ & - & - & - & - \\
\hline \multirow{2}{*}{ F29 } & 224F065 & $0-0.5$ & 0.56 & $8.6(\mathrm{~J})$ & -- & -- & -- & -- \\
\hline & 224F066 & $3-4$ & - & $0.36(\mathrm{~J})$ & -- & -- & - & - \\
\hline \multirow{2}{*}{$\mathrm{F} 30$} & 224F067 & $0-0.5$ & 0.56 & $9.4(\mathrm{~J})$ & -- & -- & -- & - \\
\hline & 224F068 & $3-4$ & -- & $2.63(\mathrm{~J})$ & - & -- & -- & - \\
\hline
\end{tabular}


Table A.7-21

Soil Sample Results for Isotopes Detected Above Minimum Detectable Concentrations at CAS 06-17-04, Decon Pad and Wastewater Catch (Page 3 of 4 )

\begin{tabular}{|c|c|c|c|c|c|c|c|c|}
\hline \multirow[b]{2}{*}{$\begin{array}{l}\text { Sample } \\
\text { Location }\end{array}$} & \multirow[b]{2}{*}{$\begin{array}{l}\text { Sample } \\
\text { Number }\end{array}$} & \multirow[b]{2}{*}{$\begin{array}{c}\text { Depth } \\
\text { (ft bgs) }\end{array}$} & \multicolumn{6}{|c|}{ Contaminants of Potential Concern (pCi/g) } \\
\hline & & & 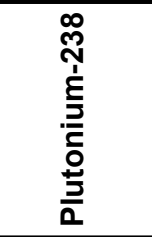 & 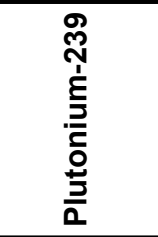 & 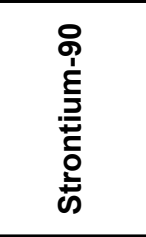 & 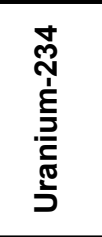 & 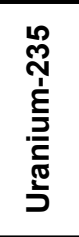 & 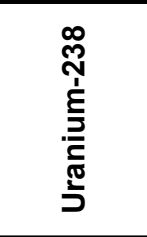 \\
\hline \multicolumn{3}{|c|}{ Final Action Levels ${ }^{a}$} & 186 & 168.1 & 2692 & 1052 & 138 & 471.6 \\
\hline$\overline{\text { F31 }}$ & $224 \mathrm{~F} 069$ & $0-0.5$ & $\overline{0.34}$ & $\overline{3.29(\mathrm{~J})}$ & $\overline{0.37(\mathrm{LT})}$ & 0.88 & $\overline{---}$ & $0.85(\mathrm{M} 3)$ \\
\hline F32 & $224 \mathrm{~F} 070$ & $0-0.5$ & -- & $0.62(\mathrm{~J})$ & - & 0.82 & -- & 0.83 \\
\hline F33 & $224 \mathrm{~F} 071$ & $0-0.5$ & -- & $0.129(\mathrm{~J})$ & -- & -- & -- & -- \\
\hline F34 & $224 \mathrm{~F} 072$ & $0-0.5$ & -- & $0.074(\mathrm{~J})$ & -- & -- & -- & -- \\
\hline F35 & $224 \mathrm{~F} 073$ & $0-0.5$ & 0.108 & $2.41(\mathrm{~J})$ & -- & -- & -- & -- \\
\hline F36 & $224 \mathrm{~F} 074$ & $0-0.5$ & -- & $0.42(\mathrm{~J})$ & -- & -- & -- & -- \\
\hline F37 & $224 \mathrm{~F} 075$ & $0-0.5$ & 0.05 & $4.6(\mathrm{~J})$ & -- & -- & -- & -- \\
\hline F38 & $224 \mathrm{~F} 076$ & $0-0.5$ & -- & $0.343(\mathrm{~J})$ & -- & -- & -- & -- \\
\hline \multirow{2}{*}{ F39 } & $224 \mathrm{~F} 077$ & $0-0.5$ & 1.55 & $89(\mathrm{~J})$ & $\overline{--}$ & -- & -- & -- \\
\hline & 224F104 & $1-1.5$ & 1.12 & 80 & -- & -- & -- & -- \\
\hline \multirow{2}{*}{$\mathrm{F} 40$} & $224 \mathrm{~F} 078$ & $0-0.5$ & 0.68 & $39(\mathrm{~J})$ & $\overline{--}$ & $\overline{--}$ & $\overline{--}$ & $\overline{--}$ \\
\hline & $224 \mathrm{~F} 105$ & $0.5-1$ & 0.053 & 3.13 & -- & -- & -- & -- \\
\hline F41 & 224F079 & $0-0.5$ & -- & $1.26(\mathrm{~J})$ & -- & -- & -- & -- \\
\hline F42 & 224F080 & $0-0.5$ & 0.297 & $10.2(\mathrm{~J})$ & -- & -- & -- & -- \\
\hline \multirow{2}{*}{$\mathrm{F} 45$} & 224F095 & $0-0.5$ & $\overline{--}$ & 1.15 & $\overline{--}$ & -- & -- & -- \\
\hline & $224 \mathrm{~F} 096$ & $1-1.5$ & -- & 1.1 & -- & -- & -- & -- \\
\hline F46 & 224F097 & $0.5-1$ & - & 1.05 & - & -- & - & - \\
\hline \multirow{2}{*}{$\mathrm{F} 47$} & $224 \mathrm{~F} 100$ & $0-0.5$ & $\overline{--}$ & 1.4 & $\overline{--}$ & $\overline{--}$ & $\overline{--}$ & $\overline{--}$ \\
\hline & 224F101 & $0.5-1.5$ & -- & 0.239 & -- & -- & -- & - \\
\hline \multirow{2}{*}{$\mathrm{F} 48$} & $224 \mathrm{~F} 102$ & $0-0.5$ & -- & 0.129 & -- & -- & -- & -- \\
\hline & $224 \mathrm{~F} 103$ & $0.5-1$ & -- & 0.261 & -- & -- & -- & -- \\
\hline \multirow{2}{*}{ F49 } & $224 \mathrm{~F} 085$ & $0-0.5$ & $2.92(\mathrm{~J})$ & $170(\mathrm{~J})$ & $\overline{--}$ & - & -- & -- \\
\hline & $224 \mathrm{~F} 086$ & $1-1.5$ & $0.084(\mathrm{~J})$ & $6.5(\mathrm{~J})$ & -- & -- & -- & -- \\
\hline \multirow{2}{*}{$\mathrm{F} 50$} & $224 \mathrm{~F} 087$ & $0-0.5$ & $1.03(\mathrm{~J})$ & $71(\mathrm{~J})$ & -- & -- & -- & $\overline{--}$ \\
\hline & $224 \mathrm{~F} 088$ & $1-1.5$ & -- & $1.4(\mathrm{~J})$ & -- & -- & - & - \\
\hline \multirow{2}{*}{ F51 } & $224 \mathrm{~F} 089$ & $0-0.5$ & -- & $1.71(\mathrm{~J})$ & - & -- & -- & -- \\
\hline & $224 \mathrm{~F} 106$ & $1-1.5$ & -- & $0.38(\mathrm{~J})$ & -- & -- & -- & -- \\
\hline F52 & $224 \mathrm{~F} 107$ & $0-0.5$ & -- & $0.269(\mathrm{~J})$ & -- & -- & -- & -- \\
\hline F54 & 224F109 & $0-0.5$ & 0.122 & 6.5 & -- & -- & -- & -- \\
\hline
\end{tabular}


Table A.7-21

Soil Sample Results for Isotopes Detected Above Minimum Detectable Concentrations at CAS 06-17-04, Decon Pad and Wastewater Catch (Page 4 of 4 )

\begin{tabular}{|c|c|c|c|c|c|c|c|c|}
\hline \multirow[b]{2}{*}{$\begin{array}{c}\text { Sample } \\
\text { Location }\end{array}$} & \multirow[b]{2}{*}{$\begin{array}{l}\text { Sample } \\
\text { Number }\end{array}$} & \multirow[b]{2}{*}{$\begin{array}{c}\text { Depth } \\
\text { (ft bgs) }\end{array}$} & \multicolumn{6}{|c|}{ Contaminants of Potential Concern (pCi/g) } \\
\hline & & & 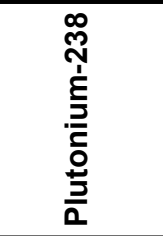 & 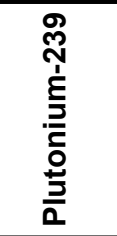 & 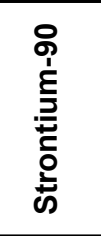 & 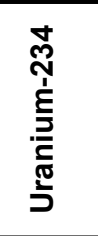 & 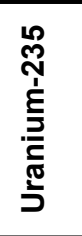 & 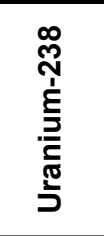 \\
\hline \multicolumn{3}{|c|}{ Final Action Levels ${ }^{a}$} & 186 & 168.1 & 2692 & 1052 & 138 & 471.6 \\
\hline $\mathrm{F} 55$ & $224 \mathrm{~F} 111$ & $0-0.5$ & 0.42 & 22.3 & -- & -- & -- & - \\
\hline \multirow{2}{*}{ F57 } & $224 \mathrm{~F} 113$ & $0-0.5$ & 0.11 & 6.8 & -- & -- & -- & -- \\
\hline & $224 \mathrm{~F} 114$ & $0.5-1.5$ & -- & 0.35 & -- & -- & -- & -- \\
\hline F58 & $224 F 115$ & $0-0.5$ & -- & 0.85 & -- & -- & -- & -- \\
\hline $\mathrm{F} 59$ & $224 \mathrm{~F} 116$ & $0-0.5$ & 0.307 & 1.89 & -- & -- & -- & -- \\
\hline $\mathrm{F} 60$ & $224 \mathrm{~F} 117$ & $0-0.5$ & 0.28 & 11.3 & -- & -- & -- & - \\
\hline F61 & 224F118 & $0-0.5$ & 0.68 & 4.14 & -- & -- & -- & -- \\
\hline F62 & $224 F 119$ & $0-0.5$ & -- & 0.068 & -- & -- & -- & -- \\
\hline \multirow{3}{*}{ F63 } & $224 F 120$ & $0-0.5$ & 0.47 & 3.17 & -- & -- & -- & -- \\
\hline & 224F121 & $0-0.5$ & 0.78 & 7.1 & -- & -- & $\overline{--}$ & - \\
\hline & 224F122 & $1-2$ & 0.187 & 10.5 & -- & -- & -- & -- \\
\hline F64 & $224 \mathrm{~F} 123$ & $0-0.5$ & 3.1 (M3) & $144(\mathrm{~J})$ & -- & -- & -- & -- \\
\hline F65 & $224 F 124$ & $0-0.5$ & 0.117 & 4.58 & -- & -- & - & - \\
\hline \multirow{2}{*}{ F66 } & $224 \mathrm{~F} 125$ & $0-0.5$ & 0.74 & 41.1 & - & - & - & - \\
\hline & 224F126 & $1.5-2.5$ & 0.104 & 5.9 & -- & -- & -- & -- \\
\hline F67 & $224 F 127$ & $0-0.5$ & $0.045(\mathrm{LT})$ & 2.41 & -- & -- & -- & -- \\
\hline F68 & 224F128 & $0-0.5$ & 0.145 & 1.11 & -- & -- & -- & -- \\
\hline F69 & 224F129 & $0-0.5$ & -- & 0.4 & -- & -- & -- & -- \\
\hline
\end{tabular}

${ }^{a}$ FALs determined by RESRAD evaluation.

$\mathrm{ft}$ bgs = Feet below ground surface

$\mathrm{pCi} / \mathrm{g}=$ Picocuries per gram

RESRAD $=$ Residual radiation

$\mathrm{J}=$ Estimated value. Qualifier added to laboratory data; record accepted. Duplicate precision analysis (relative percent difference) outside control limits.

$\mathrm{LT}=$ Result is less than the requested minimum detectable concentration, greater than the sample specific minimum detectable concentration.

M3 = The requested minimum detectable concentration was not met, but the reported activity is greater than the reported minimum detectable concentration.

-- = Not detected above minimum reporting limits 
Table A.7-22

Soil Sample Results for Isotopes Detected Above

Minimum Detectable Concentrations at CAS 06-23-01, Decon Pad Discharge Piping

\begin{tabular}{|c|c|c|c|c|c|c|c|c|}
\hline \multirow[b]{2}{*}{$\begin{array}{l}\text { Sample } \\
\text { Location }\end{array}$} & \multirow[b]{2}{*}{$\begin{array}{l}\text { Sample } \\
\text { Number }\end{array}$} & \multirow[b]{2}{*}{$\begin{array}{l}\text { Depths } \\
\text { (ft bgs) }\end{array}$} & \multicolumn{6}{|c|}{ Contaminants of Potential Concern (pCi/g) } \\
\hline & & & 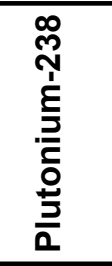 & 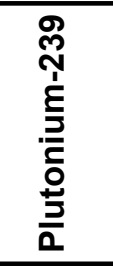 & 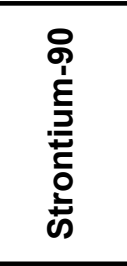 & 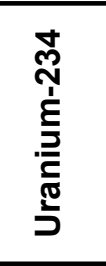 & 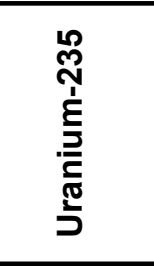 & 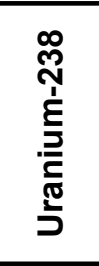 \\
\hline \multicolumn{3}{|c|}{ Final Action Levels $^{a}$} & 186 & 168.1 & 2692 & 1052 & 138 & 471.6 \\
\hline \multirow{4}{*}{ G01 } & $\overline{224 G 001}$ & $\overline{0-1}$ & 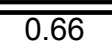 & 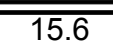 & $\overline{---}$ & $\overline{0.7}$ & $\overline{0.051}$ & $\overline{0.81}$ \\
\hline & $224 G 002$ & $0-1$ & 0.308 & 10.4 & $0.56(J)$ & 0.78 & $0.05(\mathrm{LT})$ & 0.63 \\
\hline & $224 G 003$ & $2-3$ & 0.49 & 31.3 & $0.64(\mathrm{~J})$ & 0.78 & $\overline{--}$ & 0.68 \\
\hline & $224 \mathrm{G} 004$ & $3-4$ & 0.264 & 17.7 & -- & 0.96 & 0.068 & 0.94 \\
\hline G02 & $224 \mathrm{G} 005$ & $0-1$ & 0.086 & 5.43 & -- & 0.421 & -- & 0.47 \\
\hline G03 & $224 G 006$ & $0-1$ & 0.117 & 6.4 & -- & 0.52 & -- & 0.397 \\
\hline
\end{tabular}

${ }^{a}$ FALs determined by RESRAD evaluation.

$\mathrm{ft}$ bgs $=$ Feet below ground surface

$\mathrm{pCi} / \mathrm{g}=$ Picocuries per gram

RESRAD = Residual radiation

$\mathrm{J}=$ Estimated value

$\mathrm{LT}=$ Result is less than the requested minimum detectable concentration, greater than the sample specific minimum detectable concentration.

$--=$ Not detected above minimum reporting limits

Table A.7-23

Soil Sample Results for Pesticides Detected Above Minimum Reporting Limits at CAS 06-23-01, Decon Pad Discharge Piping

\begin{tabular}{|c|c|c|c|c|c|c|c|c|}
\hline \multirow{2}{*}{$\begin{array}{l}\text { Sample } \\
\text { Location }\end{array}$} & \multirow{2}{*}{$\begin{array}{l}\text { Sample } \\
\text { Number }\end{array}$} & \multirow{2}{*}{$\begin{array}{l}\text { Depths } \\
\text { (ft bgs) }\end{array}$} & \multicolumn{6}{|c|}{ Contaminants of Potential Concern $(\mu \mathrm{g} / \mathrm{kg})$} \\
\hline & & & 4,4'-DDD & 4,4'-DDT & Alpha-Chlordane & Chlordane & Dieldrin & Gamma-Chlordane \\
\hline \multicolumn{3}{|c|}{ Final Action Levels ${ }^{a}$} & 10,000 & 7,000 & NI & 6,500 & 110 & NI \\
\hline$\overline{\mathrm{G} 02}$ & $224 G 007$ & $0-0.5$ & $\overline{--}$ & $2.3(\mathrm{~J})$ & $\overline{--}$ & $\overline{--}$ & -- & $1.8(\mathrm{~J})$ \\
\hline G03 & $224 G 008$ & $0-0.5$ & $3.6(\mathrm{~J})$ & 20 & $4.9(\mathrm{~J})$ & $36(\mathrm{~J})$ & $2.8(\mathrm{~J})$ & $5.2(\mathrm{~J})$ \\
\hline
\end{tabular}

\footnotetext{
${ }^{\mathrm{a}}$ Based on U.S. Environmental Protection Agency, Region 9 Preliminary Remediation Goals (PRGs) (EPA, 2002)
}

DDD = Dichlorodiphenyl - dichlorethane

DDT = Dichlorodiphenyl - trichloroethane

$\mathrm{ft}$ bgs = Feet below ground surface

$\mu \mathrm{g} / \mathrm{kg}=$ Micrograms per kilogram

$\mathrm{J}=$ Estimated value. Qualifier added to laboratory data; record accepted. \%D between columns $>25$ 
Table A.7-24

\section{Sludge and Concrete Samples Detected Above MRLs or MDCs at CAS 06-17-04, Decon Pad and Wastewater Catch}

(Page 1 of 4 )

\begin{tabular}{|c|c|c|c|c|}
\hline $\begin{array}{l}\text { Sample } \\
\text { Number }\end{array}$ & $\begin{array}{l}\text { Sample } \\
\text { Matrix }\end{array}$ & Parameter & Result & Units \\
\hline 224F042 & Sludge & Aroclor 1254 & $1,600(\mathrm{~J})^{\mathrm{a}}$ & $\mu \mathrm{g} / \mathrm{kg}$ \\
\hline $224 \mathrm{~F} 042$ & Sludge & Aroclor 1260 & $2,000(J)^{b}$ & $\mu \mathrm{g} / \mathrm{kg}$ \\
\hline 224F042 & Sludge & Diesel-Range Organics & $23,000(\mathrm{~J})^{\mathrm{C}}$ & $\mathrm{mg} / \mathrm{kg}$ \\
\hline $224 \mathrm{~F} 042$ & Sludge & 3+4-Methylphenol & 0.15 & $\mathrm{mg} / \mathrm{L}$ \\
\hline $224 \mathrm{~F} 042$ & Sludge & 2-Methylnaphthalene & $29,000(\mathrm{~J})^{\mathrm{d}}$ & $\mu \mathrm{g} / \mathrm{kg}$ \\
\hline $224 \mathrm{~F} 042$ & Sludge & Di-N-Butyl Phthalate & $11,000(\mathrm{~J})^{\mathrm{d}}$ & $\mu \mathrm{g} / \mathrm{kg}$ \\
\hline $224 \mathrm{~F} 042$ & Sludge & Phenanthrene & $5,200(J)^{d}$ & $\mu \mathrm{g} / \mathrm{kg}$ \\
\hline $224 \mathrm{~F} 042$ & Sludge & 4-Methylphenol & 4,600 & $\mu \mathrm{g} / \mathrm{kg}$ \\
\hline 224F042 & Sludge & N-Nitrosodiphenylamine & $15,000(\mathrm{~J})^{\mathrm{d}}$ & $\mu \mathrm{g} / \mathrm{kg}$ \\
\hline 224F042RR1 & Sludge & Chlorobenzene & 4.5 & $\mathrm{mg} / \mathrm{L}$ \\
\hline 224F042RR1 & Sludge & Trichloroethene & 12 & $\overline{\mathrm{mg} / \mathrm{L}}$ \\
\hline 224F042RR1 & Sludge & Tetrachloroethene & 1.2 & $\mathrm{mg} / \mathrm{L}$ \\
\hline 224F042RR1 & Sludge & Naphthalene & $30,000(\mathrm{~J})^{\mathrm{c}}$ & $\mu \mathrm{g} / \mathrm{kg}$ \\
\hline 224F042RR1 & Sludge & Bis(2-Ethylhexyl)Phthalate & $280,000(\mathrm{~J})^{\mathrm{c}}$ & $\mu \mathrm{g} / \mathrm{kg}$ \\
\hline 224F042 & Sludge & Cobalt-60 & $0.333(\mathrm{G})$ & $\mathrm{pCi} / \mathrm{g}$ \\
\hline $224 \mathrm{~F} 042$ & Sludge & Actinium-228 & $1.54(\mathrm{G})$ & $\mathrm{pCi} / \mathrm{g}$ \\
\hline $224 \mathrm{~F} 042$ & Sludge & Americium-241 & $153(\mathrm{~J})^{\mathrm{e}}$ & $\mathrm{pCi} / \mathrm{g}$ \\
\hline $224 \mathrm{~F} 042$ & Sludge & Bismuth-214 & $1.21(\mathrm{G}, \mathrm{J})$ & $\mathrm{pCi} / \mathrm{g}$ \\
\hline $224 \mathrm{~F} 042$ & Sludge & Cesium-137 & $67.2(\mathrm{G})$ & $\mathrm{pCi} / \mathrm{g}$ \\
\hline $224 \mathrm{~F} 042$ & Sludge & Europium-152 & $1.36(\mathrm{G})$ & $\mathrm{pCi} / \mathrm{g}$ \\
\hline $224 \mathrm{~F} 042$ & Sludge & Lead-212 & $1.67(\mathrm{~J})^{\mathrm{e}}$ & $\mathrm{pCi} / \mathrm{g}$ \\
\hline $224 \mathrm{~F} 042$ & Sludge & Lead-214 & $0.93(\mathrm{G}, \mathrm{J})$ & $\mathrm{pCi} / \mathrm{g}$ \\
\hline $224 \mathrm{~F} 042$ & Sludge & Thorium-234 & $7(\mathrm{~J})^{\mathrm{e}}$ & $\mathrm{pCi} / \mathrm{g}$ \\
\hline $224 \mathrm{~F} 042$ & Sludge & Thallium-208 & $0.53(\mathrm{G})$ & $\mathrm{pCi} / \mathrm{g}$ \\
\hline $224 \mathrm{~F} 042$ & Sludge & Uranium-234 & 7.5 & $\mathrm{pCi} / \mathrm{g}$ \\
\hline $224 \mathrm{~F} 042$ & Sludge & Uranium-235 & 0.47 & $\mathrm{pCi} / \mathrm{g}$ \\
\hline $224 \mathrm{~F} 042$ & Sludge & Uranium-238 & 8 & $\mathrm{pCi} / \mathrm{g}$ \\
\hline $224 \mathrm{~F} 042$ & Sludge & Plutonium-238 & $23.7(J)^{f}$ & $\mathrm{pCi} / \mathrm{g}$ \\
\hline $224 \mathrm{~F} 042$ & Sludge & Plutonium-239 & $940(\mathrm{~J})^{f}$ & $\mathrm{pCi} / \mathrm{g}$ \\
\hline $224 \mathrm{~F} 042$ & Sludge & Zinc & $930(\mathrm{~J})^{\mathrm{g}}$ & $\mathrm{mg} / \mathrm{kg}$ \\
\hline $224 \mathrm{~F} 042$ & Sludge & Lead & 19,000 & $\mathrm{mg} / \mathrm{kg}$ \\
\hline 224F042 & Sludge & Barium & 1,100 & $\mathrm{mg} / \mathrm{kg}$ \\
\hline
\end{tabular}


Table A.7-24

\section{Sludge and Concrete Samples Detected Above MRLs or MDCs at CAS 06-17-04, Decon Pad and Wastewater Catch}

(Page 2 of 4 )

\begin{tabular}{|c|c|c|c|c|}
\hline $\begin{array}{l}\text { Sample } \\
\text { Number }\end{array}$ & $\begin{array}{c}\text { Sample } \\
\text { Matrix }\end{array}$ & Parameter & Result & Units \\
\hline 224F042 & Sludge & $\overline{\text { Cobalt }}$ & 7.3 & $\mathrm{mg} / \mathrm{kg}$ \\
\hline $224 \mathrm{~F} 042$ & Sludge & Copper & $200(\mathrm{~J})^{\mathrm{h}}$ & $\mathrm{mg} / \mathrm{kg}$ \\
\hline $224 \mathrm{~F} 042$ & Sludge & Nickel & 25 & $\mathrm{mg} / \mathrm{kg}$ \\
\hline $224 \mathrm{~F} 042$ & Sludge & Arsenic & 22 & $\mathrm{mg} / \mathrm{kg}$ \\
\hline $224 \mathrm{~F} 042$ & Sludge & Cadmium & 9.3 & $\mathrm{mg} / \mathrm{kg}$ \\
\hline $224 \mathrm{~F} 042$ & Sludge & Aluminum & 11,000 & $\mathrm{mg} / \mathrm{kg}$ \\
\hline $224 \mathrm{~F} 042$ & Sludge & Chromium & 130 & $\mathrm{mg} / \mathrm{kg}$ \\
\hline 224F042 & Sludge & Selenium & 3 & $\mathrm{mg} / \mathrm{kg}$ \\
\hline 224F042 & Sludge & Manganese & $550(\mathrm{~J})^{9}$ & $\mathrm{mg} / \mathrm{kg}$ \\
\hline 224F042 & Sludge & Molybdenum & 51 & $\mathrm{mg} / \mathrm{kg}$ \\
\hline 224F042 & Sludge & Lead & 19,000 & $\mathrm{mg} / \mathrm{L}$ \\
\hline $224 \mathrm{~F} 042$ & Sludge & Mercury & 0.15 & $\mathrm{mg} / \mathrm{kg}$ \\
\hline $224 \mathrm{~F} 043$ & Sludge & Aroclor 1254 & $5,200(\mathrm{~J})^{\mathrm{C}}$ & $\mu \mathrm{g} / \mathrm{kg}$ \\
\hline $224 \mathrm{~F} 043$ & Sludge & Diesel-Range Organics & $41,000(\mathrm{~J})^{\mathrm{C}}$ & $\mathrm{mg} / \mathrm{kg}$ \\
\hline $224 \mathrm{~F} 043$ & Sludge & 3+4-Methylphenol & 0.19 & $\mathrm{mg} / \mathrm{L}$ \\
\hline $224 \mathrm{~F} 043$ & Sludge & Bis(2-Ethylhexyl)Phthalate & $250,000(\mathrm{~J})^{\mathrm{c}}$ & $\mu \mathrm{g} / \mathrm{kg}$ \\
\hline $224 \mathrm{~F} 043$ & Sludge & Naphthalene & $72,000(\mathrm{~J})^{\mathrm{c}}$ & $\mu \mathrm{g} / \mathrm{kg}$ \\
\hline $224 \mathrm{~F} 043$ & Sludge & 2-Methylnaphthalene & $66,000(\mathrm{~J})^{\mathrm{c}}$ & $\mu \mathrm{g} / \mathrm{kg}$ \\
\hline $224 \mathrm{~F} 043$ & Sludge & Di-N-Butyl Phthalate & $30,000(\mathrm{~J})^{\mathrm{c}}$ & $\mu \mathrm{g} / \mathrm{kg}$ \\
\hline 224F043RR1 & Sludge & Trichloroethene & 4.9 & $\mathrm{mg} / \mathrm{L}$ \\
\hline 224F043RR1 & Sludge & Tetrachloroethene & 0.61 & $\mathrm{mg} / \mathrm{L}$ \\
\hline 224F043RR1 & Sludge & Phenanthrene & 13,000 & $\mu \mathrm{g} / \mathrm{kg}$ \\
\hline 224F043RR1 & Sludge & 2,4-Dimethylphenol & 3,800 & $\mu \mathrm{g} / \mathrm{kg}$ \\
\hline 224F043RR1 & Sludge & N-Nitrosodiphenylamine & 12,000 & $\mu \mathrm{g} / \mathrm{kg}$ \\
\hline 224F043RR2 & Sludge & Chlorobenzene & 5.2 & $\mathrm{mg} / \mathrm{L}$ \\
\hline 224F043RR2 & Sludge & Trichloroethene & 5 & $\mathrm{mg} / \mathrm{L}$ \\
\hline 224F043RR2 & Sludge & Tetrachloroethene & 0.62 & $\mathrm{mg} / \mathrm{L}$ \\
\hline $224 \mathrm{~F} 043$ & Sludge & Cobalt-60 & $0.4(\mathrm{G}, \mathrm{TI})$ & $\mathrm{pCi} / \mathrm{g}$ \\
\hline $224 \mathrm{~F} 043$ & Sludge & Americium-241 & $67.2(\mathrm{~J})^{\mathrm{e}}$ & $\mathrm{pCi} / \mathrm{g}$ \\
\hline $224 \mathrm{~F} 043$ & Sludge & Cesium-137 & $322(\mathrm{G}, \mathrm{M} 3)$ & $\mathrm{pCi} / \mathrm{g}$ \\
\hline $224 \mathrm{~F} 043$ & Sludge & Lead-212 & $2.8(\mathrm{G})$ & $\mathrm{pCi} / \mathrm{g}$ \\
\hline $224 \mathrm{~F} 043$ & Sludge & Uranium-234 & 7.9 & $\mathrm{pCi} / \mathrm{g}$ \\
\hline
\end{tabular}


Table A.7-24

\section{Sludge and Concrete Samples Detected Above MRLs or MDCs at CAS 06-17-04, Decon Pad and Wastewater Catch}

(Page 3 of 4 )

\begin{tabular}{|c|c|c|c|c|}
\hline $\begin{array}{l}\text { Sample } \\
\text { Number }\end{array}$ & $\begin{array}{l}\text { Sample } \\
\text { Matrix }\end{array}$ & Parameter & Result & Units \\
\hline 224F043 & Sludge & Uranium-235 & 0.423 & $\overline{\mathrm{pCi} / \mathrm{g}}$ \\
\hline $224 \mathrm{~F} 043$ & Sludge & Uranium-238 & 11.6 & $\mathrm{pCi} / \mathrm{g}$ \\
\hline $224 \mathrm{~F} 043$ & Sludge & Plutonium-238 & $4.4(J)^{f}$ & $\mathrm{pCi} / \mathrm{g}$ \\
\hline $224 \mathrm{~F} 043$ & Sludge & Plutonium-239 & $108(J)^{f}$ & $\mathrm{pCi} / \mathrm{g}$ \\
\hline $224 \mathrm{~F} 043$ & Sludge & Selenium & 2 & $\mathrm{mg} / \mathrm{kg}$ \\
\hline $224 \mathrm{~F} 043$ & Sludge & Manganese & $460(\mathrm{~J})^{\mathrm{g}}$ & $\mathrm{mg} / \mathrm{kg}$ \\
\hline $224 \mathrm{~F} 043$ & Sludge & Molybdenum & 55 & $\mathrm{mg} / \mathrm{kg}$ \\
\hline $224 \mathrm{~F} 043$ & Sludge & Cobalt & 6 & $\mathrm{mg} / \mathrm{kg}$ \\
\hline $224 \mathrm{~F} 043$ & Sludge & Nickel & 25 & $\mathrm{mg} / \mathrm{kg}$ \\
\hline $224 \mathrm{~F} 043$ & Sludge & Lead & 10,000 & $\mathrm{mg} / \mathrm{kg}$ \\
\hline $224 \mathrm{~F} 043$ & Sludge & Zinc & $790(\mathrm{~J})^{9}$ & $\mathrm{mg} / \mathrm{kg}$ \\
\hline $224 \mathrm{~F} 043$ & Sludge & Barium & 640 & $\mathrm{mg} / \mathrm{kg}$ \\
\hline $224 \mathrm{~F} 043$ & Sludge & Copper & $280(\mathrm{~J})^{\mathrm{h}}$ & $\mathrm{mg} / \mathrm{kg}$ \\
\hline $224 \mathrm{~F} 043$ & Sludge & Arsenic & 18 & $\mathrm{mg} / \mathrm{kg}$ \\
\hline $224 \mathrm{~F} 043$ & Sludge & Cadmium & 11 & $\mathrm{mg} / \mathrm{kg}$ \\
\hline $224 \mathrm{~F} 043$ & Sludge & Aluminum & 9,500 & $\mathrm{mg} / \mathrm{kg}$ \\
\hline $224 \mathrm{~F} 043$ & Sludge & Chromium & 77 & $\mathrm{mg} / \mathrm{kg}$ \\
\hline $224 \mathrm{~F} 043$ & Sludge & Lead & 10,000 & $\mathrm{mg} / \mathrm{L}$ \\
\hline $224 \mathrm{~F} 043$ & Sludge & Mercury & 0.18 & $\mathrm{mg} / \mathrm{kg}$ \\
\hline $224 \mathrm{~F} 501$ & Concrete & Diesel-Range Organics & $150(\mathrm{H})$ & $\mathrm{mg} / \mathrm{kg}$ \\
\hline $224 \mathrm{~F} 501$ & Concrete & Di-N-Butyl Phthalate & 1,600 & $\mu \mathrm{g} / \mathrm{kg}$ \\
\hline $224 \mathrm{~F} 501$ & Concrete & Bismuth-214 & $0.4(G, J)$ & $\mathrm{pCi} / \mathrm{g}$ \\
\hline $224 \mathrm{~F} 501$ & Concrete & Cesium-137 & $1.77(\mathrm{G})$ & $\mathrm{pCi} / \mathrm{g}$ \\
\hline $224 \mathrm{~F} 501$ & Concrete & Lead-212 & $0.44(\mathrm{~J})^{\mathrm{e}}$ & $\mathrm{pCi} / \mathrm{g}$ \\
\hline $224 \mathrm{~F} 501$ & Concrete & Lead-214 & $0.52(\mathrm{G}, \mathrm{J})$ & $\mathrm{pCi} / \mathrm{g}$ \\
\hline $224 \mathrm{~F} 501$ & Concrete & Plutonium-239 & 0.58 & $\mathrm{pCi} / \mathrm{g}$ \\
\hline $224 \mathrm{~F} 501$ & Concrete & Uranium-234 & 0.91 & $\mathrm{pCi} / \mathrm{g}$ \\
\hline $224 \mathrm{~F} 501$ & Concrete & Uranium-235 & 0.063 & $\mathrm{pCi} / \mathrm{g}$ \\
\hline $224 \mathrm{~F} 501$ & Concrete & Uranium-238 & 0.85 & $\mathrm{pCi} / \mathrm{g}$ \\
\hline
\end{tabular}


Table A.7-24

\section{Sludge and Concrete Samples Detected Above MRLs or MDCs at CAS 06-17-04, Decon Pad and Wastewater Catch}

(Page 4 of 4 )

\begin{tabular}{|c|c|c|c|c|}
\hline $\begin{array}{l}\text { Sample } \\
\text { Number }\end{array}$ & $\begin{array}{l}\text { Sample } \\
\text { Matrix }\end{array}$ & Parameter & Result & Units \\
\hline 224F501 & Concrete & Manganese & $\overline{170(\mathrm{~J})^{9}}$ & $\mathrm{mg} / \mathrm{kg}$ \\
\hline 224F501 & Concrete & Lead & 870 & $\mathrm{mg} / \mathrm{kg}$ \\
\hline 224F501 & Concrete & Zinc & $42(\mathrm{~J})^{\mathrm{g}}$ & $\mathrm{mg} / \mathrm{kg}$ \\
\hline $224 \mathrm{~F} 501$ & Concrete & Barium & 69 & $\mathrm{mg} / \mathrm{kg}$ \\
\hline 224F501 & Concrete & Cobalt & 2.9 & $\mathrm{mg} / \mathrm{kg}$ \\
\hline $224 \mathrm{~F} 501$ & Concrete & Copper & $12(\mathrm{~J})^{\mathrm{h}}$ & $\mathrm{mg} / \mathrm{kg}$ \\
\hline 224F501 & Concrete & Nickel & 9.4 & $\mathrm{mg} / \mathrm{kg}$ \\
\hline 224F501 & Concrete & Arsenic & 5.2 & $\mathrm{mg} / \mathrm{kg}$ \\
\hline 224F501 & Concrete & Aluminum & 5,000 & $\mathrm{mg} / \mathrm{kg}$ \\
\hline 224F501 & Concrete & Chromium & 19 & $\mathrm{mg} / \mathrm{kg}$ \\
\hline $224 \mathrm{~F} 501$ & Concrete & Molybdenum & 3.1 & $\mathrm{mg} / \mathrm{kg}$ \\
\hline
\end{tabular}

${ }^{a}$ Qualifier added to laboratory data; record accepted. Matrix effects may exist. Surrogate recovery exceeded the lower limits. ${ }^{b}$ Qualifier added to laboratory data; record accepted. Matrix effects may exist. \%D between columns $>25$. Surrogate recovery exceeded the lower limits.

${ }^{\circ} Q u a l i f i e r$ added to laboratory data; record accepted. Surrogates diluted out.

${ }^{d}$ Qualifier added to laboratory data; record accepted. Matrix effects may exist. Surrogate recovery exceeded upper limits. ${ }^{e} Q u a l i f i e r$ added to laboratory data; record accepted. Samples does not meet counting geometry requirements. ${ }^{f}$ Qualifier added to laboratory data; record accepted. Duplicate precision analysis (relative percent difference) outside control limits.

${ }^{g}$ Qualifier added to laboratory data; record accepted. Matrix spike recovery outside control limits. Serial dilution \%D outside control limits. Matrix effects may exist.

${ }^{\mathrm{h}}$ Qualifier added to laboratory data; record accepted. Serial dilution \%D outside control limits. Matrix effects may exist.

$\mathrm{mg} / \mathrm{kg}=$ Milligrams per kilogram

$\mathrm{mg} / \mathrm{L}=$ Milligrams per liter

$\mathrm{pCi} / \mathrm{g}=$ Picocuries per gram

$\mu \mathrm{g} / \mathrm{kg}=$ Micrograms per kilogram

$\mathrm{G}=$ Sample density differs by more than 15 percent of laboratory control sample density.

$\mathrm{H}=\mathrm{DRO}$ fuel pattern in the heavy end of retention time window.

$\mathrm{J}=$ Estimated value.

M3 = The requested minimum detectable concentration was not met, but the reported activity is greater than the reported minimum detectable concentration.

$\mathrm{TI}=$ Tentatively identified. 


\section{A.7.3 Nature and Extent of Contamination}

Total petroleum hydrocarbons-DRO as well as radiological contamination was found at these CASs.

The extent of the contamination was bound during Decision II sampling. See Figure B.1-2 and

Figure B.1-3 for a graphical representation of the extent of contamination at CASs 06-05-01, 06-17-04, and 06-23-01.

\section{A.7.4 Revised Conceptual Site Model}

No variations in the CSM were identified. 


\section{A.8.0 CAS 11-04-01, Sewage Lagoon}

Corrective Action Site 11-04-01 is located adjacent to the TaDD facility in Area 11 of the NTS. The CAS consists of a former sewage lagoon, an evapotranspiration bed (shallow leachfield), a two-chamber septic tank, and associated piping. Investigation activities at CAS 11-04-01 included collection of liquid and sludge samples from the tanks, as well as surface and subsurface soil samples.

\section{A.8.1 Corrective Action Investigation}

A total of 75 soil characterization samples, 3 tank liquid samples (from the two sections of the tank and the distribution box) and 1 sludge sample (Tank 1 was the only one that contained sludge) were collected during investigation activities at CAS 11-04-01. The sample IDs, locations, types, and analyses are listed in Table A.8-1. The sample locations are shown on Figure A.8-1

The specific CAI activities conducted to satisfy the CAIP requirements at this CAS are described in the following sections.

\section{A.8.1.1 Field Screening}

Soil samples were field screened for VOCs and alpha and beta/gamma radiation. Total petroleum hydrocarbons screening was not done at this CAS because TPH is not a major concern at the site. The FSRs were compared to FSLs to guide subsequent sampling decisions. The VOC headspace and alpha and beta/gamma radiation FSLs were not exceeded during sampling activities at this CAS.

\section{A.8.1.2 Sampling}

Surface and subsurface samples were collected at locations specified in the CAIP. The locations were chosen to be associated with features (i.e. piping, septic tank, etc.) of the site, according to engineering drawings and field observations. In the case of the former sewage lagoon, a geophysics survey was used to generate GPS locations for the three sample locations, due to the fact that no visible features could be found. Samples were collected at the surface and at two or more subsurface intervals at each location, via hand-sampling and backhoe methods. The samples were analyzed for the parameters listed in Table A.8-1. Sample locations are shown on Figure A.8-1. 
Table A.8-1

Samples Collected at CAS 11-04-01, Sewage Lagoon (Page 1 of 3 )

\begin{tabular}{|c|c|c|c|c|c|}
\hline $\begin{array}{c}\text { Sample } \\
\text { Location }\end{array}$ & $\begin{array}{l}\text { Sample } \\
\text { Number }\end{array}$ & $\begin{array}{c}\text { Depth } \\
\text { (ft bgs) }\end{array}$ & $\begin{array}{l}\text { Sample } \\
\text { Matrix }\end{array}$ & Purpose & Analyses \\
\hline \multirow{4}{*}{$\mathrm{H} 01$} & $224 \mathrm{H} 001$ & $0-1$ & Soil & $\overline{S C}$ & $\overline{\text { Set } 1}$ \\
\hline & $224 \mathrm{H} 002$ & $0-1$ & Soil & $\begin{array}{c}\text { Field Duplicate } \\
\text { of \#224H001 }\end{array}$ & Set 1 \\
\hline & $224 \mathrm{H} 003$ & $4-5$ & Soil & SC, MS/MSD & Set 1 \\
\hline & $224 \mathrm{H} 004$ & $7-8$ & Soil & $\mathrm{SC}$ & Set 3 \\
\hline \multirow{2}{*}{$\mathrm{H} 02$} & $224 \mathrm{H} 005$ & $0-1$ & Soil & SC & Set 3 \\
\hline & $224 \mathrm{H} 006$ & $4-5$ & Soil & SC & Set 1 \\
\hline \multirow{3}{*}{$\mathrm{H} 03$} & $224 \mathrm{H} 007$ & $0-0.5$ & Soil & SC & Set 3 \\
\hline & $224 \mathrm{H} 008$ & $7-8$ & Soil & SC & Set 3 \\
\hline & $224 \mathrm{H} 009$ & $9-10$ & Soil & SC & Set 3 \\
\hline \multirow{4}{*}{$\mathrm{H} 04$} & $224 \mathrm{H} 010$ & $0-1$ & Soil & SC & Set 1 \\
\hline & $224 \mathrm{H} 011$ & $1.5-2.5$ & Soil & SC & Set 3 \\
\hline & $224 \mathrm{H} 012$ & $3-4$ & Soil & SC & Set 3 \\
\hline & $224 \mathrm{H} 013$ & $6-7$ & Soil & SC & Set 3 \\
\hline \multirow{4}{*}{$\mathrm{H} 05$} & $224 \mathrm{H} 014$ & $0-1$ & Soil & SC & Set 1 \\
\hline & $224 \mathrm{H} 016$ & $2-2.5$ & Soil & SC & Set 3 \\
\hline & $224 \mathrm{H} 017$ & $7-8$ & Soil & SC & Set 3 \\
\hline & $224 \mathrm{H} 019$ & $10-11$ & Soil & SC & Set 3 \\
\hline \multirow{4}{*}{$\mathrm{H} 06$} & $224 \mathrm{H} 015$ & $0-1$ & Soil & SC & Set 3 \\
\hline & $224 \mathrm{H} 021$ & $3-4$ & Soil & SC & Set 3 \\
\hline & $224 \mathrm{H} 018$ & $7-8$ & Soil & SC & Set 1 \\
\hline & $224 \mathrm{H} 020$ & $10-11$ & Soil & SC & Set 3 \\
\hline \multirow{4}{*}{$\mathrm{H} 07$} & $224 \mathrm{H} 022$ & $0-1$ & Soil & SC & Set 1 \\
\hline & $224 \mathrm{H} 023$ & $1.5-2$ & Soil & SC & Set 3 \\
\hline & $224 \mathrm{H} 024$ & $3.5-4.5$ & Soil & SC & Set 3 \\
\hline & $224 \mathrm{H} 025$ & $3.5-4.5$ & Soil & $\begin{array}{c}\text { Field Duplicate } \\
\text { of \#224H024 }\end{array}$ & Set 3 \\
\hline \multirow{3}{*}{$\mathrm{H} 08$} & $224 \mathrm{H} 026$ & $0-0.5$ & Soil & SC, MS/MSD & Set 1 \\
\hline & $224 \mathrm{H} 027$ & $2-2.5$ & Soil & SC & Set 3 \\
\hline & $224 \mathrm{H} 028$ & $3.5-4.5$ & Soil & $\mathrm{SC}$ & Set 3 \\
\hline \multirow{3}{*}{ H09 } & $224 \mathrm{H} 029$ & $0-0.5$ & Soil & SC & Set 3 \\
\hline & $224 \mathrm{H} 030$ & $2-2.5$ & Soil & SC & Set 1 \\
\hline & $224 \mathrm{H} 031$ & $4-5$ & Soil & SC & Set 3 \\
\hline
\end{tabular}


Table A.8-1

Samples Collected at CAS 11-04-01, Sewage Lagoon (Page 2 of 3 )

\begin{tabular}{|c|c|c|c|c|c|}
\hline $\begin{array}{l}\text { Sample } \\
\text { Location }\end{array}$ & $\begin{array}{l}\text { Sample } \\
\text { Number }\end{array}$ & $\begin{array}{l}\text { Depth } \\
\text { (ft bgs) }\end{array}$ & $\begin{array}{l}\text { Sample } \\
\text { Matrix }\end{array}$ & Purpose & Analyses \\
\hline \multirow{3}{*}{$\mathrm{H} 10$} & $224 \mathrm{H} 032$ & $0-0.5$ & Soil & $\overline{\mathrm{SC}}$ & $\overline{\text { Set } 3}$ \\
\hline & $224 \mathrm{H} 033$ & $2.5-3$ & Soil & $\mathrm{SC}$ & Set 3 \\
\hline & $224 \mathrm{H} 034$ & $4-5$ & Soil & SC & Set 1 \\
\hline \multirow{3}{*}{$\mathrm{H} 11$} & $224 \mathrm{H} 035$ & $0-0.5$ & Soil & SC & Set 3 \\
\hline & $224 \mathrm{H} 036$ & $2-2.5$ & Soil & $\mathrm{SC}$ & Set 3 \\
\hline & $224 \mathrm{H} 037$ & $3.5-4.5$ & Soil & SC & Set 3 \\
\hline \multirow{3}{*}{$\mathrm{H} 12$} & $224 \mathrm{H} 038$ & $0-0.5$ & Soil & SC & Set 1 \\
\hline & $224 \mathrm{H} 039$ & $2-2.5$ & Soil & SC & Set 3 \\
\hline & $224 \mathrm{H} 040$ & $3.5-4.5$ & Soil & $\mathrm{SC}$ & Set 3 \\
\hline \multirow{4}{*}{$\mathrm{H} 13$} & $224 \mathrm{H} 041$ & $0-0.5$ & Soil & SC & Set 3 \\
\hline & $224 \mathrm{H} 042$ & $2-2.5$ & Soil & SC & Set 1 \\
\hline & $224 \mathrm{H} 043$ & $2-2.5$ & Soil & $\begin{array}{c}\text { Field Duplicate } \\
\text { of \#224H042 }\end{array}$ & Set 1 \\
\hline & $224 \mathrm{H} 044$ & $3.5-4.5$ & Soil & SC & Set 3 \\
\hline \multirow{3}{*}{$\mathrm{H} 14$} & $224 \mathrm{H} 045$ & $0-0.5$ & Soil & SC, MS/MSD & Set 3 \\
\hline & $224 \mathrm{H} 046$ & $2-2.5$ & Soil & SC & Set 3 \\
\hline & $224 \mathrm{H} 047$ & $3-4$ & Soil & SC & Set 3 \\
\hline \multirow{3}{*}{$\mathrm{H} 15$} & $224 \mathrm{H} 048$ & $0-0.5$ & Soil & SC & Set 1 \\
\hline & $224 \mathrm{H} 049$ & $2-2.5$ & Soil & SC & Set 3 \\
\hline & $224 \mathrm{H} 050$ & $3.5-4.5$ & Soil & SC & Set 3 \\
\hline \multirow{3}{*}{$\mathrm{H} 16$} & $224 \mathrm{H} 051$ & $0-0.5$ & Soil & SC & Set 3 \\
\hline & $224 \mathrm{H} 052$ & $1.5-2.0$ & Soil & SC & Set 1 \\
\hline & $224 \mathrm{H} 053$ & $3.5-4.5$ & Soil & $\mathrm{SC}$ & Set 3 \\
\hline \multirow{3}{*}{$\mathrm{H} 17$} & $224 \mathrm{H} 054$ & $0-0.5$ & Soil & SC & Set 3 \\
\hline & $224 \mathrm{H} 055$ & $2-2.5$ & Soil & SC & Set 3 \\
\hline & $224 \mathrm{H} 056$ & $3.5-4.5$ & Soil & SC & Set 1 \\
\hline \multirow{3}{*}{$\mathrm{H} 18$} & $224 \mathrm{H} 057$ & $0-0.5$ & Soil & SC & Set 3 \\
\hline & $224 \mathrm{H} 058$ & $2-2.5$ & Soil & SC & Set 3 \\
\hline & $224 \mathrm{H} 059$ & $3.5-4.5$ & Soil & SC & Set 3 \\
\hline \multirow{3}{*}{$\mathrm{H} 19$} & $224 \mathrm{H} 060$ & $0-0.5$ & Soil & $\mathrm{SC}$ & Set 1 \\
\hline & $224 \mathrm{H} 061$ & $2-2.5$ & Soil & SC & Set 3 \\
\hline & $224 \mathrm{H} 062$ & $3.5-4.5$ & Soil & SC & Set 3 \\
\hline
\end{tabular}


Table A.8-1

Samples Collected at CAS 11-04-01, Sewage Lagoon (Page 3 of 3 )

\begin{tabular}{|c|c|c|c|c|c|}
\hline $\begin{array}{l}\text { Sample } \\
\text { Location }\end{array}$ & $\begin{array}{l}\text { Sample } \\
\text { Number }\end{array}$ & $\begin{array}{l}\text { Depth } \\
\text { (ft bgs) }\end{array}$ & $\begin{array}{l}\text { Sample } \\
\text { Matrix }\end{array}$ & Purpose & Analyses \\
\hline \multirow{4}{*}{$\mathrm{H} 20$} & $224 \mathrm{H} 063$ & $0-0.5$ & Soil & SC & Set 1 \\
\hline & $224 \mathrm{H} 064$ & $0-0.5$ & Soil & $\begin{array}{c}\text { Field Duplicate } \\
\text { of \#224H063 }\end{array}$ & Set 1 \\
\hline & $224 \mathrm{H} 065$ & $2-2.5$ & Soil & SC, MS/MSD & Set 3 \\
\hline & $224 \mathrm{H} 066$ & $3.5-4.5$ & Soil & SC & Set 3 \\
\hline \multirow{3}{*}{$\mathrm{H} 21$} & $224 \mathrm{H} 067$ & $0-0.5$ & Soil & SC & Set 3 \\
\hline & $224 \mathrm{H} 068$ & $2-2.5$ & Soil & SC & Set 1 \\
\hline & $224 \mathrm{H} 069$ & $3.5-4.5$ & Soil & SC & Set 3 \\
\hline \multirow{3}{*}{$\mathrm{H} 22$} & $224 \mathrm{H} 070$ & $0-0.5$ & Soil & SC & Set 3 \\
\hline & $224 \mathrm{H} 073$ & $4-5$ & Soil & SC & Set 3 \\
\hline & $224 \mathrm{H} 075$ & $6-7$ & Soil & SC & Set 3 \\
\hline \multirow{3}{*}{$\mathrm{H} 23$} & $224 \mathrm{H} 071$ & $0-0.5$ & Soil & SC & Set 3 \\
\hline & $224 \mathrm{H} 072$ & $4-5$ & Soil & SC & Set 3 \\
\hline & $224 \mathrm{H} 074$ & $6-7$ & Soil & SC & Set 3 \\
\hline NA & $224 \mathrm{H} 301$ & NA & Water & Trip Blank & Total VOCs \\
\hline NA & $224 \mathrm{H} 302$ & NA & Water & Trip Blank & Total VOCs \\
\hline NA & $224 \mathrm{H} 303$ & NA & Water & Trip Blank & Total VOCs \\
\hline NA & $224 \mathrm{H} 304$ & NA & Water & Trip Blank & Total VOCs \\
\hline NA & $224 \mathrm{H} 305$ & NA & Water & Trip Blank & Total VOCs \\
\hline NA & $224 \mathrm{H} 306$ & NA & Water & Trip Blank & Total VOCs \\
\hline NA & $224 \mathrm{H} 307$ & NA & Water & Field Blank & Set 1 \\
\hline \multirow{2}{*}{ Tank 1} & $224 \mathrm{H} 501$ & NA & Liquid & WM & Set 1, Pesticide, Herbicide \\
\hline & $224 \mathrm{H} 504$ & NA & Sludge & WM & Set 2 \\
\hline Tank 2 & $224 \mathrm{H} 502$ & NA & Liquid & WM & Set 1, Pesticide, Herbicide \\
\hline Tank 3 & $224 \mathrm{H} 503$ & NA & Liquid & WM & Set 1, Pesticide, Herbicide \\
\hline
\end{tabular}

Set 1 = Total VOCs, Total SVOCs plus Hydroquinone, Total RCRA Metals, Aluminum, Antimony, Beryllium, Cobalt, Copper, Manganese, Molybdenum, Nickel, Zinc, TPH-DRO, PCBs, Gamma Spectroscopy, Isotopic Plutonium, Isotopic Uranium, and Strontium-90.

Set 2 = TCLP VOCs, TCLP SVOCs, and TCLP RCRA Metals

Set 3 = Total VOCs, Total SVOCs plus Hydroquinone, Total RCRA Metals, Aluminum, Antimony, Beryllium, Cobalt, Copper, Manganese, Molybdenum, Nickel, Zinc, TPH-DRO, and PCBs.

$\mathrm{DRO}=$ Diesel-range organics

$\mathrm{ft}$ bgs $=$ Feet below ground surface

MS/MSD = Matrix spike/matrix spike duplicate

NA $=$ Not applicable

$\mathrm{PCB}=$ Polychlorinated biphenyl

RCRA = Resource Conservation and Recovery Act
$\mathrm{SC}=$ Site characterization

SVOC = Semivolatile organic compound

$\mathrm{TPH}=$ Total petroleum hydrocarbons

$\mathrm{WM}=$ Waste Management

VOC $=$ Volatile organic compound 


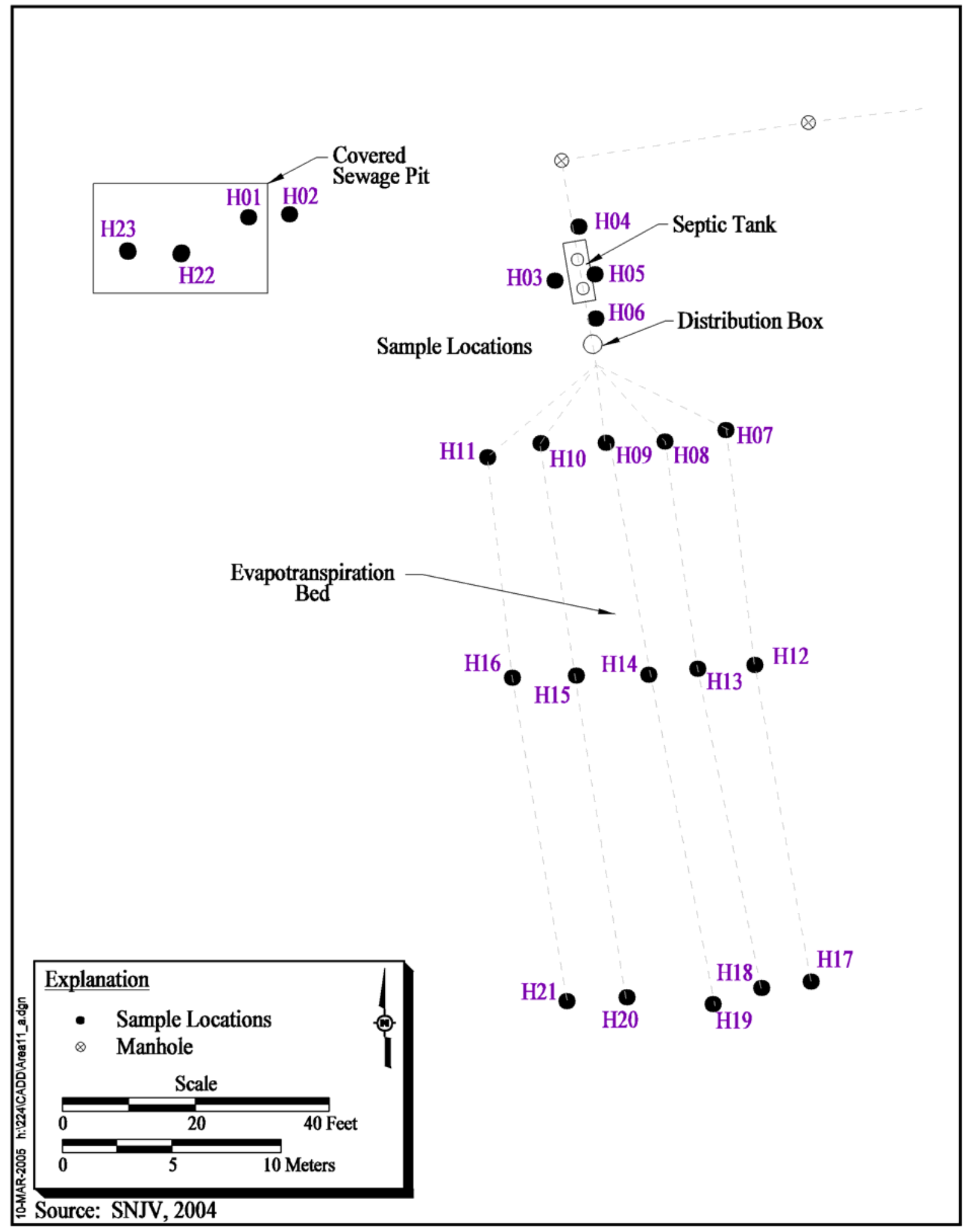

Figure A.8-1

Sample Location Map, CAS 11-04-01, Sewage Lagoon 


\section{A.8.1.3 Deviations}

There were no significant deviations to the CAIP requirements at this CAS. No samples were taken in any of the manholes, because none contained liquid or sludge. The investigation and sampling at CAS 11-04-01 are considered sufficient to meet the DQOs.

\section{A.8.2 Investigation Results}

The following sections provide descriptions of the CAS-specific activities conducted to complete investigation activities as outlined in the CAIP. Investigation activities included visual inspection of the CAS and collection of surface and subsurface soil samples, as outlined in Section A.8.1.2.

Surface and subsurface soil samples were analyzed for the CAIP-specified COPCs, which included total VOCs, total SVOCs, TPH-DRO, total metals, PCBs, cyanide, gamma-emitting radionuclides, plutonium isotopes, uranium isotopes, and strontium-90. In accordance with the ROTC CAIP-1, 25 percent of all samples were analyzed for radiological COPCs. The analytical parameters and laboratory methods used to analyze the investigation samples are listed in Table A.2-2. Table A.8-1 lists the sample-specific analytical suite for CAS 11-04-01.

\section{A.8.2.1 Total Volatile Organic Compounds}

No VOCs were detected in soil samples above the reporting limits.

\section{A.8.2.2 Total Semivolatile Organic Compounds}

No SVOCs were detected in soil samples above the reporting limits.

\section{A.8.2.3 Total Metals}

Total metals analytical results for soil samples collected at CAS 11-04-01 are presented in Table A.8-2. No metals were detected above FALs.

\section{A.8.2.4 Polychlorinated Biphenyls}

No PCBs were detected in soil samples above the reporting limits. 
Table A.8-2

Soil Sample Results for Metals Detected Above Minimum Reporting Limits at CAS 11-04-01, Sewage Lagoon (Page 1 of 5 )

\begin{tabular}{|c|c|c|c|c|c|c|c|c|c|c|c|c|c|c|c|c|}
\hline \multirow[b]{2}{*}{$\begin{array}{l}\text { Sample } \\
\text { Location }\end{array}$} & \multirow[b]{2}{*}{$\begin{array}{l}\text { Sample } \\
\text { Number }\end{array}$} & \multirow[b]{2}{*}{$\begin{array}{l}\text { Depth } \\
\text { (ft bgs) }\end{array}$} & \multicolumn{14}{|c|}{ Contaminants of Potential Concern (mg/kg) } \\
\hline & & & 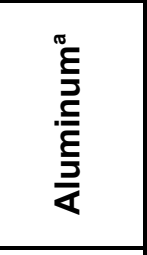 & 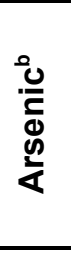 & 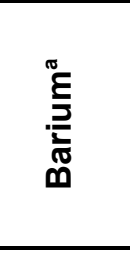 & 点 & 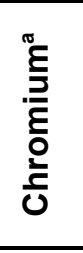 & $\begin{array}{l}\frac{\pi}{\pi} \\
\frac{0}{\pi} \\
0 \\
0\end{array}$ & $\begin{array}{l}\frac{\pi}{0} \\
\frac{0}{0} \\
\frac{0}{0} \\
ن\end{array}$ & 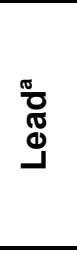 & 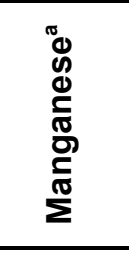 & $\begin{array}{l}\stackrel{\pi}{2} \\
\frac{0}{0} \\
\sum\end{array}$ & 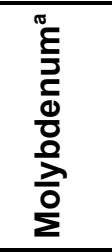 & $\begin{array}{l}\frac{\sigma}{0} \\
\frac{x}{0} \\
\frac{.0}{Z}\end{array}$ & 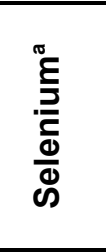 & $\stackrel{0}{\stackrel{0}{c}}$ \\
\hline \multirow{4}{*}{ H01 } & $224 \mathrm{H} 001$ & $0-1$ & $\overline{9,800}$ & 4.8 & 110 & $\overline{0.74}$ & 7.3 & $\overline{4}$ & $7.9(\mathrm{~J})^{\mathrm{C}}$ & $\overline{8}$ & 290 & $-\overline{--}$ & $-\overline{--}$ & $\overline{77.6}$ & $-\overline{-}$ & 32 \\
\hline & $224 \mathrm{H} 002$ & $0-1$ & 9,100 & 4.6 & 110 & 0.71 & 7 & 3.9 & $7.9(J)^{c}$ & 9.4 & 290 & -- & -- & 7.4 & -- & 30 \\
\hline & $224 \mathrm{H} 003$ & $4-5$ & 11,000 & 5.3 & 100 & 0.82 & 7.2 & 4.3 & $8.2(\mathrm{~J})^{\mathrm{c}}$ & 8.1 & 270 & -- & -- & 7.7 & -- & 31 \\
\hline & $224 \mathrm{H} 004$ & $7-8$ & 8,700 & 5.6 & 110 & 0.65 & 5.7 & 3.6 & $6.5(J)^{c}$ & 7 & 210 & -- & -- & 6.5 & -- & 25 \\
\hline H02 & $224 \mathrm{H} 005$ & $0-1$ & 5,900 & 2.7 & 80 & -- & 6.6 & 3 & $4.1(J)^{c}$ & 9.4 & 190 & -- & -- & 4.7 & -- & 26 \\
\hline \multirow{3}{*}{$\mathrm{H} 03$} & $224 \mathrm{H} 007$ & $0-0.5$ & 9,700 & 4.6 & 110 & 0.75 & 6.7 & 4.1 & $7.7(\mathrm{~J})^{\mathrm{c}}$ & 8.4 & 280 & -- & -- & 7.3 & -- & 30 \\
\hline & $224 \mathrm{H} 008$ & $7-8$ & 6,100 & 5.5 & 130 & 0.59 & 6.4 & 3 & $7.8(J)^{\mathrm{c}}$ & 7 & 190 & -- & -- & 11 & -- & 36 \\
\hline & $224 \mathrm{H} 009$ & $9-10$ & 9,300 & 5.5 & 100 & 0.8 & 6.6 & 4.1 & $6.5(J)^{c}$ & 8.1 & 240 & -- & -- & 7.5 & -- & 29 \\
\hline \multirow{4}{*}{ H04 } & $224 \mathrm{H} 010$ & $0-1$ & 9,900 & 4.9 & 110 & 0.74 & 7 & 3.8 & 7.4 & 8.4 & 280 & -- & -- & 7.5 & -- & 28 \\
\hline & $224 \mathrm{H} 011$ & $1.5-2.5$ & 5,800 & 6.2 & 260 & 0.64 & 5.7 & 3.6 & 7.9 & 22 & 500 & -- & 1.7 & 13 & -- & 45 \\
\hline & $224 \mathrm{H} 012$ & $3-4$ & 11,000 & 5.6 & 120 & 0.87 & 7.4 & 3.9 & 9.6 & 8.5 & 240 & 0.07 & -- & 11 & -- & 36 \\
\hline & $224 \mathrm{H} 013$ & $6-7$ & 8,800 & 4.8 & 110 & 0.72 & 5.9 & 3.6 & 6.6 & 7.4 & 220 & -- & -- & 6.8 & -- & 24 \\
\hline \multirow{4}{*}{ H05 } & $224 \mathrm{H} 014$ & $0-1$ & 10,000 & 4.5 & 120 & 0.73 & 7.8 & 4.5 & 8.9 & 10 & 380 & -- & -- & 8.3 & -- & 38 \\
\hline & $224 \mathrm{H} 016$ & $2-2.5$ & 6,500 & 5.8 & 190 & 0.67 & 6.3 & 3.5 & 9.1 & 11 & 410 & -- & 1.2 & 12 & -- & 39 \\
\hline & $224 \mathrm{H} 017$ & $7-8$ & 5,900 & 6 & 140 & 0.6 & 5.4 & 2.8 & 7.8 & 6.6 & 190 & -- & -- & 12 & 0.71 & 36 \\
\hline & $224 \mathrm{H} 019$ & $10-11$ & 9,700 & 5.8 & 140 & 0.75 & 6.7 & 3.8 & 7.5 & 7.4 & 210 & $\begin{array}{l}- \\
\end{array}$ & -- & 9.2 & -- & 30 \\
\hline
\end{tabular}


Table A.8-2

Soil Sample Results for Metals Detected Above Minimum Reporting Limits at CAS 11-04-01, Sewage Lagoon (Page 2 of 5 )

\begin{tabular}{|c|c|c|c|c|c|c|c|c|c|c|c|c|c|c|c|c|}
\hline \multirow[b]{2}{*}{$\begin{array}{l}\text { Sample } \\
\text { Location }\end{array}$} & \multirow[b]{2}{*}{$\begin{array}{l}\text { Sample } \\
\text { Number }\end{array}$} & \multirow[b]{2}{*}{$\begin{array}{l}\text { Depth } \\
\text { (ft bgs) }\end{array}$} & \multicolumn{14}{|c|}{ Contaminants of Potential Concern (mg/kg) } \\
\hline & & & 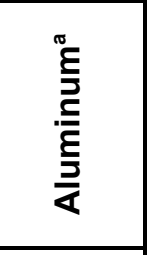 & 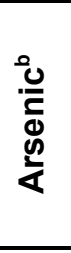 & 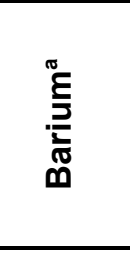 & 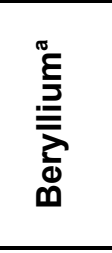 & 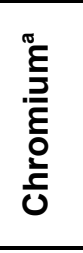 & $\begin{array}{l}\frac{\sigma}{\frac{\pi}{\pi}} \\
\frac{0}{0} \\
0 \\
ن\end{array}$ & $\begin{array}{l}\frac{\pi}{0} \\
\frac{0}{0} \\
\frac{0}{0} \\
ن\end{array}$ & ర్ల & 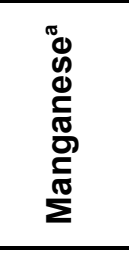 & 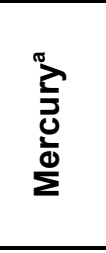 & 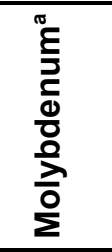 & $\begin{array}{l}\frac{\sigma}{0} \\
\frac{v}{0} \\
\frac{.0}{z}\end{array}$ & 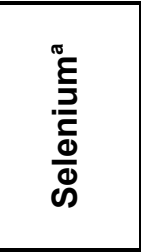 & $\stackrel{\text { U. }}{\stackrel{0}{\mathbf{N}}}$ \\
\hline \multirow{4}{*}{ H06 } & $224 \mathrm{H} 015$ & $0-1$ & 10,000 & 4.8 & 130 & 0.72 & 8.1 & 4.6 & 9.5 & 9.8 & 410 & $\overline{---}$ & -- & 8.6 & -- & $\overline{37}$ \\
\hline & $224 \mathrm{H} 021$ & $3-4$ & 6,000 & 5.7 & 180 & 0.61 & 5.5 & 3.3 & 8 & 7.2 & 170 & 0.076 & -- & 12 & -- & 36 \\
\hline & $224 \mathrm{H} 018$ & $7-8$ & 8,600 & 5.1 & 99 & 0.7 & 6.2 & 3.9 & 6.5 & 7.6 & 270 & -- & -- & 7.3 & -- & 26 \\
\hline & $224 \mathrm{H} 020$ & $10-11$ & 10,000 & 6 & 130 & 0.74 & 6.7 & 4.1 & 7.1 & 7.5 & 240 & -- & -- & 7.7 & -- & 26 \\
\hline \multirow{3}{*}{$\mathrm{H} 07$} & $224 \mathrm{H} 022$ & $0-1$ & 8,300 & 4.2 & 120 & 0.66 & 5.9 & 3.6 & 7 & 8 & 290 & -- & -- & 7.4 & -- & 29 \\
\hline & $224 \mathrm{H} 024$ & $3.5-4.5$ & 9,000 & 5.5 & 130 & 0.82 & 6.5 & 4.1 & 8.4 & 8 & 250 & -- & -- & 8.1 & -- & 34 \\
\hline & $224 \mathrm{H} 025$ & $3.5-4.5$ & 9,000 & 5.4 & 140 & 0.83 & 6.6 & 4.1 & 8.7 & 9.2 & 270 & -- & -- & 9 & $0.57(\mathrm{~J}+)$ & 35 \\
\hline \multirow{3}{*}{$\mathrm{H} 08$} & $224 \mathrm{H} 026$ & $0-0.5$ & 8,500 & 5.1 & 100 & 0.77 & 6.5 & $4.1(\mathrm{~J})^{\mathrm{c}}$ & 7.1 & 8.1 & 260 & -- & -- & 7.2 & -- & 31 \\
\hline & $224 \mathrm{H} 027$ & $2-2.5$ & 4,600 & 5.7 & 160 & 0.59 & 5.2 & 3.3 & 8.5 & 13 & 240 & -- & -- & 11 & 1.2 & 40 \\
\hline & $224 \mathrm{H} 028$ & $3.5-4.5$ & 11,000 & 6.4 & 120 & 1 & 7.4 & 3.9 & 9.1 & 8.9 & 210 & 0.046 & -- & 9.6 & $0.9(\mathrm{~J}+)$ & 36 \\
\hline \multirow{3}{*}{ H09 } & $224 \mathrm{H} 029$ & $0-0.5$ & 9,200 & 5.2 & 110 & 0.79 & 6.6 & 4.2 & 8.5 & 8.3 & 250 & -- & -- & 7.5 & -- & 30 \\
\hline & $224 \mathrm{H} 030$ & $2-2.5$ & 5,000 & 5.6 & 160 & 0.62 & 5.6 & 3.3 & 7.9 & 8.8 & 300 & -- & -- & 11 & $1(\mathrm{~J}+)$ & 43 \\
\hline & $224 \mathrm{H} 031$ & $4-5$ & 14,000 & 6.4 & 110 & 1.3 & 9.1 & 5 & 10 & 10 & 260 & -- & -- & 11 & -- & 38 \\
\hline \multirow{3}{*}{$\mathrm{H} 10$} & $224 \mathrm{H} 032$ & $0-0.5$ & 9,000 & 5.1 & 100 & 0.76 & 6.8 & 4 & 7.9 & 7.8 & 250 & -- & -- & 7.5 & -- & 30 \\
\hline & $224 \mathrm{H} 033$ & $2.5-3$ & 4,700 & 5.4 & 250 & 0.58 & 5.3 & 3.5 & 9.2 & 15 & 690 & -- & 4.3 & 11 & -- & 40 \\
\hline & $224 \mathrm{H} 034$ & $4-5$ & 6,700 & 5.4 & 130 & 0.69 & 5.8 & 3.6 & 8.4 & 7.9 & 210 & -- & -- & 10 & -- & 35 \\
\hline
\end{tabular}


Table A.8-2

Soil Sample Results for Metals Detected Above Minimum Reporting Limits at CAS 11-04-01, Sewage Lagoon

(Page 3 of 5 )

\begin{tabular}{|c|c|c|c|c|c|c|c|c|c|c|c|c|c|c|c|c|}
\hline \multirow[b]{2}{*}{$\begin{array}{l}\text { Sample } \\
\text { Location }\end{array}$} & \multirow[b]{2}{*}{$\begin{array}{l}\text { Sample } \\
\text { Number }\end{array}$} & \multirow[b]{2}{*}{$\begin{array}{l}\text { Depth } \\
\text { (ft bgs) }\end{array}$} & \multicolumn{14}{|c|}{ Contaminants of Potential Concern (mg/kg) } \\
\hline & & & 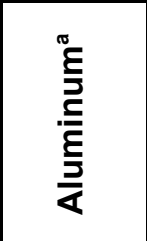 & 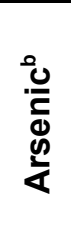 & 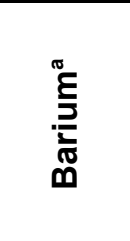 & 吝 & 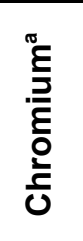 & $\begin{array}{l}\frac{\pi}{\pi} \\
\frac{\pi}{0} \\
0 \\
0\end{array}$ & $\begin{array}{l}\frac{\pi}{0} \\
\frac{0}{0} \\
\frac{0}{0}\end{array}$ & ֻٓ & 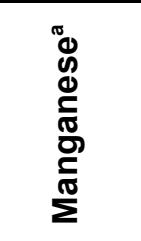 & 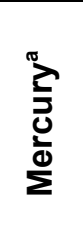 & 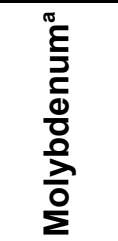 & $\begin{array}{l}\frac{\pi}{\Phi} \\
\frac{x}{0} \\
\frac{0}{Z}\end{array}$ & 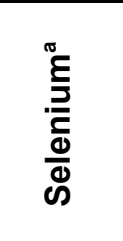 & 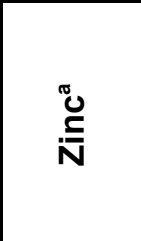 \\
\hline \multicolumn{3}{|c|}{ Final Action Levels } & 100,000 & 23 & 67,000 & 1,900 & 450 & 1,900 & 41,000 & 750 & 19,000 & 310 & 5,100 & 20,000 & 5,100 & 100,000 \\
\hline \multirow{3}{*}{$\mathrm{H} 11$} & $224 \mathrm{H} 035$ & $\overline{0-0.5}$ & 8,900 & $\overline{5.4}$ & $\overline{1110}$ & $\overline{0.76}$ & $\overline{76.6}$ & 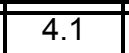 & 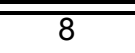 & $\overline{7.7}$ & 250 & $\overline{---}$ & $\overline{---}$ & $\overline{7.4}$ & $\overline{---}$ & $\overline{29}$ \\
\hline & $224 \mathrm{H} 036$ & $2-2.5$ & 4,600 & 6.1 & 160 & 0.54 & 4.5 & 3.7 & 8.7 & 6.1 & 200 & -- & -- & 13 & -- & 36 \\
\hline & $224 \mathrm{H} 037$ & $3.5-4.5$ & 8,700 & 5.1 & 99 & 0.76 & 6 & 3.8 & 7.7 & 7.9 & 190 & -- & -- & 6.9 & -- & 27 \\
\hline \multirow{3}{*}{$\mathrm{H} 12$} & $224 \mathrm{H} 038$ & $0-0.5$ & 8,600 & 4.8 & 110 & 0.73 & 6.5 & 3.9 & 8.2 & 7.8 & 260 & -- & -- & 7.6 & -- & 31 \\
\hline & $224 \mathrm{H} 039$ & $2-2.5$ & 5,100 & 6.6 & 190 & 0.64 & 5.9 & 4.6 & 9.8 & 17 & 470 & -- & 1.6 & 17 & -- & 50 \\
\hline & $224 \mathrm{H} 040$ & $3.5-4.5$ & 9,300 & 5.6 & 130 & 0.84 & 6.9 & 4.6 & 8.1 & 8.4 & 250 & -- & -- & 8 & -- & 31 \\
\hline \multirow{4}{*}{$\mathrm{H} 13$} & $224 \mathrm{H} 041$ & $0-0.5$ & 8,800 & 5.3 & 130 & 0.77 & 7.1 & 4.1 & 8.7 & 9.8 & 260 & -- & -- & 9 & -- & 33 \\
\hline & $224 \mathrm{H} 042$ & $2-2.5$ & 4,400 & 5.2 & 310 & 0.58 & 4.3 & $2.3(\mathrm{~J})^{\mathrm{c}}$ & 7 & 170 & 3000 & - & -- & 10 & $1.1(\mathrm{~J}-)$ & 34 \\
\hline & $224 \mathrm{H} 043$ & $2-2.5$ & 4,900 & 11 & 200 & 0.66 & 5.5 & 3.1 & $7.8(J)^{\mathrm{c}}$ & 27 & 460 & - & 3 & 13 & 2.8 & 46 \\
\hline & $224 \mathrm{H} 044$ & $3.5-4.5$ & 11,000 & 6.6 & 140 & 1.2 & 7.8 & $4.7(\mathrm{~J})^{\mathrm{c}}$ & 7.7 & 10 & 230 & -- & -- & 9.2 & -- & 34 \\
\hline \multirow{3}{*}{$\mathrm{H} 14$} & $224 \mathrm{H} 045$ & $0-0.5$ & 8,600 & 5 & 110 & 0.77 & 6.6 & $4(J)^{\mathrm{C}}$ & 7.1 & 8.4 & 270 & -- & -- & 7.2 & -- & 30 \\
\hline & $224 \mathrm{H} 046$ & $2-2.5$ & 4,400 & 5.1 & 140 & 0.56 & 5 & $2.6(\mathrm{~J})^{\mathrm{c}}$ & 8 & 7.7 & 170 & -- & 1.2 & 11 & -- & 40 \\
\hline & $224 \mathrm{H} 047$ & $3-4$ & 8,000 & 5.1 & 110 & 0.74 & 6.3 & $4(J)^{c}$ & 6.6 & 7.8 & 210 & -- & -- & 7.5 & -- & 87 \\
\hline \multirow{3}{*}{$\mathrm{H} 15$} & $224 \mathrm{H} 048$ & $0-0.5$ & 8,400 & 4.5 & 100 & 0.74 & 6.5 & $3.8(\mathrm{~J})^{\mathrm{c}}$ & 6.8 & 7.9 & 260 & -- & -- & 7 & -- & 29 \\
\hline & $224 \mathrm{H} 049$ & $2-2.5$ & 4,300 & 6.2 & 130 & 0.63 & 5.5 & $3.6(\mathrm{~J})^{\mathrm{c}}$ & 8.1 & 8.6 & 230 & -- & 1.5 & 13 & -- & 48 \\
\hline & $224 \mathrm{H} 050$ & $3.5-4.5$ & 8,200 & 4.8 & 110 & 0.86 & 6.7 & $4.1(\mathrm{~J})$ & 6.4 & 8.3 & 210 & -- & -- & 8.1 & -- & 30 \\
\hline
\end{tabular}


Table A.8-2

Soil Sample Results for Metals Detected Above Minimum Reporting Limits at CAS 11-04-01, Sewage Lagoon (Page 4 of 5)

\begin{tabular}{|c|c|c|c|c|c|c|c|c|c|c|c|c|c|c|c|c|}
\hline \multirow[b]{2}{*}{$\begin{array}{l}\text { Sample } \\
\text { Location }\end{array}$} & \multirow[b]{2}{*}{$\begin{array}{l}\text { Sample } \\
\text { Number }\end{array}$} & \multirow[b]{2}{*}{$\begin{array}{l}\text { Depth } \\
\text { (ft bgs) }\end{array}$} & \multicolumn{14}{|c|}{ Contaminants of Potential Concern (mg/kg) } \\
\hline & & & 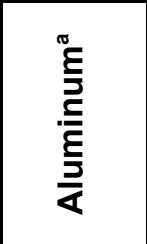 & 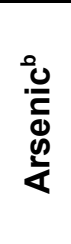 & 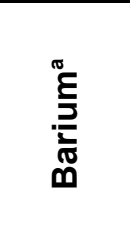 & 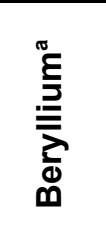 & 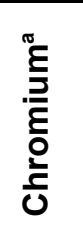 & $\begin{array}{l}\frac{10}{\pi} \\
\frac{0}{0} \\
0 \\
0\end{array}$ & $\begin{array}{l}\frac{\pi}{0} \\
\frac{0}{0} \\
\frac{0}{0}\end{array}$ & ర్ల & 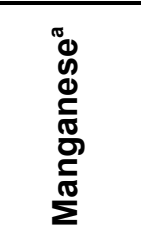 & 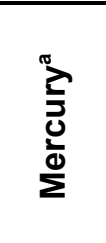 & 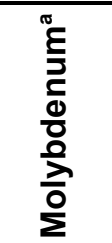 & $\begin{array}{l}\frac{\sigma}{\Phi} \\
\frac{x}{0} \\
\frac{0}{Z}\end{array}$ & 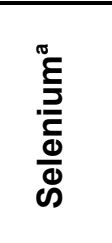 & $\stackrel{\widetilde{N}}{\underline{N}}$ \\
\hline \multicolumn{3}{|c|}{ Final Action Levels } & 100,000 & 23 & 67,000 & 1,900 & 450 & 1,900 & 41,000 & 750 & 19,000 & 310 & 5,100 & 20,000 & 5,100 & 100,000 \\
\hline \multirow{3}{*}{$\mathrm{H} 16$} & $224 \mathrm{H} 051$ & $\overline{0-0.5}$ & $\overline{99,300}$ & 4.9 & 110 & $\overline{0.83}$ & $\overline{77}$ & $4.3(\mathrm{~J})^{\mathrm{c}}$ & $\overline{7.3}$ & 8.5 & 270 & -- & -- & $\overline{7.7}$ & -- & 32 \\
\hline & $224 \mathrm{H} 052$ & $1.5-2$ & 4,600 & 5.4 & 150 & 0.61 & 6.1 & $3.4(\mathrm{~J})^{\mathrm{c}}$ & 7.3 & 15 & 330 & -- & 1.4 & 12 & -- & 43 \\
\hline & $224 \mathrm{H} 053$ & $3.5-4.5$ & 8,300 & 5.2 & 150 & 0.75 & 6.3 & $3.6(\mathrm{~J})^{\mathrm{c}}$ & 7 & 7.6 & 220 & -- & -- & 8 & -- & 33 \\
\hline \multirow{3}{*}{$\mathrm{H} 17$} & $224 \mathrm{H} 054$ & $0-0.5$ & 8,600 & 4.7 & 110 & 0.78 & 6.6 & $4.2(\mathrm{~J})^{\mathrm{c}}$ & 7.2 & 8.6 & 280 & -- & -- & 7.5 & -- & 30 \\
\hline & $224 \mathrm{H} 055$ & $2-2.5$ & 5,000 & 5.5 & 160 & 0.63 & 5.7 & $3.2(\mathrm{~J})^{\mathrm{c}}$ & 7.5 & 7.6 & 300 & -- & 1.2 & 11 & -- & 41 \\
\hline & $224 \mathrm{H} 056$ & $3.5-4.5$ & 8,100 & 5.2 & 130 & 0.82 & 6.7 & $4.4(\mathrm{~J})^{\mathrm{c}}$ & 7.1 & 8.4 & 270 & -- & -- & 8.6 & -- & 31 \\
\hline \multirow{3}{*}{$\mathrm{H} 18$} & $224 \mathrm{H} 057$ & $0-0.5$ & 9,700 & 4.9 & 120 & 0.77 & 6.9 & 3.9 & 7.7 & 8.3 & $270(\mathrm{~J})^{\mathrm{d}}$ & -- & -- & 7.8 & -- & $29(\mathrm{~J})^{\mathrm{e}}$ \\
\hline & $224 \mathrm{H} 058$ & $2-2.5$ & 5,300 & 5.3 & 170 & 0.61 & 5.2 & 3.3 & 8.2 & 8.3 & $310(\mathrm{~J})^{\mathrm{d}}$ & -- & -- & 12 & -- & $36(\mathrm{~J})^{\mathrm{e}}$ \\
\hline & $224 \mathrm{H} 059$ & $3.5-4.5$ & 11,000 & 5.8 & 130 & 0.85 & 7.2 & 3.9 & 8 & 8.6 & $220(\mathrm{~J})^{\mathrm{d}}$ & 0.04 & -- & 7.9 & -- & $28(\mathrm{~J})^{\mathrm{e}}$ \\
\hline \multirow{3}{*}{$\mathrm{H} 19$} & $224 \mathrm{H} 060$ & $0-0.5$ & 10,000 & 4.9 & 110 & 0.79 & 7.3 & 4.1 & 7.8 & 8.5 & $270(\mathrm{~J})^{\mathrm{d}}$ & -- & -- & 7.7 & -- & $29(\mathrm{~J})^{\mathrm{e}}$ \\
\hline & $224 \mathrm{H} 061$ & $2-2.5$ & 5,200 & 5.4 & 130 & 0.6 & 6.7 & 3.1 & 8.2 & 8 & $190(\mathrm{~J})^{\mathrm{d}}$ & -- & 1.3 & 15 & 0.74 & $46(\mathrm{~J})^{\mathrm{e}}$ \\
\hline & $224 \mathrm{H} 062$ & $3.5-4.5$ & 11,000 & 4.9 & 110 & 0.96 & 7.7 & 4.6 & 7.6 & 9.5 & $230(\mathrm{~J})^{\mathrm{d}}$ & -- & -- & 8.5 & -- & $30(\mathrm{~J})^{\mathrm{e}}$ \\
\hline \multirow{4}{*}{$\mathrm{H} 20$} & $224 \mathrm{H} 063$ & $0-0.5$ & 10,000 & 4.8 & 110 & 0.79 & 6.8 & 4 & 7.5 & 8.3 & $250(\mathrm{~J})^{\mathrm{d}}$ & -- & -- & 7.7 & -- & $28(J)^{e}$ \\
\hline & $224 \mathrm{H} 064$ & $0-0.5$ & 11,000 & 5 & 120 & 0.81 & 7.1 & 4 & 7.9 & 8.3 & $250(\mathrm{~J})^{\mathrm{d}}$ & -- & -- & 7.9 & -- & $29(\mathrm{~J})^{\mathrm{e}}$ \\
\hline & $224 \mathrm{H} 065$ & $2-2.5$ & 6,100 & 6 & 170 & 0.63 & 5.6 & 2.7 & 7.9 & 7.6 & $200(J)^{d}$ & 0.047 & 1.1 & 12 & -- & $42(J)^{\mathrm{e}}$ \\
\hline & $224 \mathrm{H} 066$ & $3.5-4.5$ & 11,000 & 4.8 & 130 & 1 & 7.3 & 4.2 & 7.1 & 9.7 & $220(\mathrm{~J})^{\mathrm{d}}$ & -- & -- & 7.7 & -- & $28(\mathrm{~J})^{\mathrm{e}}$ \\
\hline
\end{tabular}


Table A.8-2

Soil Sample Results for Metals Detected Above Minimum Reporting Limits at CAS 11-04-01, Sewage Lagoon

(Page 5 of 5 )

\begin{tabular}{|c|c|c|c|c|c|c|c|c|c|c|c|c|c|c|c|c|}
\hline \multirow[b]{2}{*}{$\begin{array}{l}\text { Sample } \\
\text { Location }\end{array}$} & \multirow[b]{2}{*}{$\begin{array}{l}\text { Sample } \\
\text { Number }\end{array}$} & \multirow[b]{2}{*}{$\begin{array}{l}\text { Depth } \\
\text { (ft bgs) }\end{array}$} & \multicolumn{14}{|c|}{ Contaminants of Potential Concern (mg/kg) } \\
\hline & & & 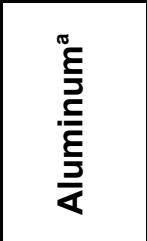 & 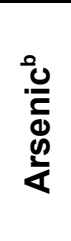 & 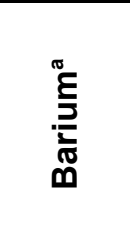 & 吝 & 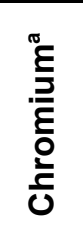 & $\begin{array}{l}\frac{\pi}{\pi} \\
\frac{\pi}{0} \\
0 \\
0\end{array}$ & $\begin{array}{l}\frac{\pi}{0} \\
\frac{0}{0} \\
\frac{0}{0}\end{array}$ & ర్ల & 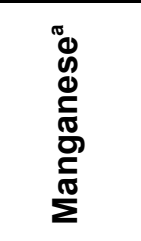 & 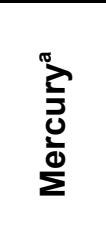 & 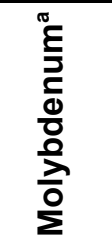 & $\begin{array}{l}\frac{\sigma}{\Phi} \\
\frac{x}{0} \\
\frac{0}{Z}\end{array}$ & 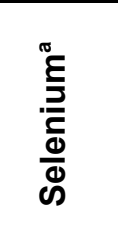 & 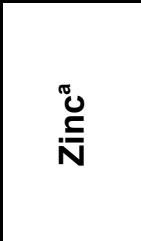 \\
\hline \multicolumn{3}{|c|}{ Final Action Levels } & 100,000 & 23 & 67,000 & 1,900 & 450 & 1,900 & 41,000 & 750 & 19,000 & 310 & 5,100 & 20,000 & 5,100 & 100,000 \\
\hline \multirow{3}{*}{$\mathrm{H} 21$} & $224 \mathrm{H} 067$ & $\overline{0-0.5}$ & 8,900 & $\overline{5.8}$ & $\overline{250}$ & $\overline{0.75}$ & $\overline{6.8}$ & 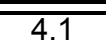 & $\overline{99.1}$ & $\overline{277}$ & $760(\mathrm{~J})^{\mathrm{d}}$ & $\overline{---}$ & $\overline{---}$ & $\overline{10}$ & $\overline{---}$ & $\overline{\overline{36(\mathrm{~J})^{\mathrm{e}}}}$ \\
\hline & $224 \mathrm{H} 068$ & $2-2.5$ & 5,200 & 5.4 & 140 & 0.58 & 5.7 & 3 & 7.6 & 8.3 & $250(\mathrm{~J})^{\mathrm{d}}$ & -- & 1.3 & 12 & 0.75 & $39(\mathrm{~J})^{\mathrm{e}}$ \\
\hline & $224 \mathrm{H} 069$ & $3.5-4.5$ & 12,000 & 5.1 & 120 & 0.92 & 7.7 & 4.4 & 8.6 & 9.2 & $250(\mathrm{~J})^{\mathrm{d}}$ & -- & -- & 8.7 & -- & $31(\mathrm{~J})^{\mathrm{e}}$ \\
\hline \multirow{3}{*}{$\mathrm{H} 22$} & $224 \mathrm{H} 070$ & $0-0.5$ & 10,000 & 5 & 140 & 0.77 & 7.1 & 4 & 8.1 & 8.4 & $280(\mathrm{~J})^{\mathrm{d}}$ & 0.044 & -- & 8 & -- & $31(\mathrm{~J})^{\mathrm{e}}$ \\
\hline & $224 \mathrm{H} 073$ & $4-5$ & 10,000 & 5.3 & 120 & 0.75 & 6.5 & 3.7 & 7.3 & 7.3 & $240(\mathrm{~J})^{\mathrm{d}}$ & -- & -- & 7.6 & -- & $27(\mathrm{~J})^{\mathrm{e}}$ \\
\hline & $224 \mathrm{H} 075$ & $6-7$ & 12,000 & 6.7 & 160 & 0.99 & 7.8 & 4.3 & 7.7 & 9 & $260(\mathrm{~J})^{\mathrm{d}}$ & - & -- & 8.8 & -- & $31(\mathrm{~J})^{\mathrm{e}}$ \\
\hline \multirow{3}{*}{$\mathrm{H} 23$} & $224 \mathrm{H} 071$ & $0-0.5$ & 9,600 & 4.5 & 120 & 0.73 & 7 & 4 & 8.1 & 9.3 & $300(\mathrm{~J})^{\mathrm{d}}$ & -- & -- & 7.7 & -- & $31(\mathrm{~J})^{\mathrm{e}}$ \\
\hline & $224 \mathrm{H} 072$ & $4-5$ & 11,000 & 4.9 & 130 & 0.84 & 7.1 & 4 & 8 & 8.2 & $240(J)^{d}$ & -- & -- & 8.1 & -- & $29(\mathrm{~J})^{\mathrm{e}}$ \\
\hline & $224 \mathrm{H} 074$ & $6-7$ & 12,000 & 7.3 & 210 & 0.89 & 6.9 & 3.8 & 7.3 & 8 & $220(\mathrm{~J})^{\mathrm{d}}$ & 0.049 & -- & 7.8 & -- & $27(\mathrm{~J})^{\mathrm{e}}$ \\
\hline
\end{tabular}

${ }^{\mathrm{a}}$ Based on U.S. Environmental Protection Agency, Region 9 Preliminary Remediation Goals (PRGs) (EPA, 2002)

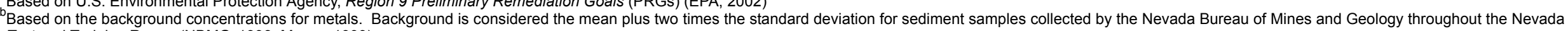
Test and Training Range (NBMG, 1998; Moore, 1999).

Qualifier added to laboratory data; record accepted. Serial dilution \%D outside control limits. Matrix effects may exist.

Qualifier added to laboratory data; record accepted. CRI/CRA \% recovery(ies) criteria was/were not met.

$\mathrm{ft}$ bgs $=$ Feet below ground surface

$\mathrm{mg} / \mathrm{kg}=$ Milligrams per

$+=$ The result is an estimated quantity, but the result may be biased high. CRDL check sample recovery was outside control limits.

$\mathrm{J}=$ The result is an estimated quantity, but the result may be biased low. Negative bias found in continuing calibration/method blank.

$=$ Not detected above minimum reporting limits. 


\section{A.8.2.5 Total Petroleum Hydrocarbons-Diesel-Range Organics}

Total TPH-DRO analytical results for soil samples collected at CAS 11-04-01 that were detected above MRLs are presented in Table A.8-3. No TPH-DRO was detected in soil samples above FALs.

Table A.8-3

Soil Sample Results for TPH-DRO Detected Above Minimum Reporting Limits at CAS 11-04-01, Sewage Lagoon

\begin{tabular}{|c|c|c|c|}
\hline \multirow{2}{*}{$\begin{array}{l}\text { Sample } \\
\text { Location }\end{array}$} & \multirow{2}{*}{$\begin{array}{l}\text { Sample } \\
\text { Number }\end{array}$} & \multirow{2}{*}{$\begin{array}{l}\text { Depth } \\
\text { (ft bgs) }\end{array}$} & Contaminants of Potential Concern (mg/kg) \\
\hline & & & Diesel-Range Organics \\
\hline \multicolumn{3}{|c|}{ Final Action Levels ${ }^{a}$} & 100 \\
\hline$\overline{\mathrm{H} 08}$ & $224 \mathrm{H} 027$ & $2-2.5$ & $33(\mathrm{H})$ \\
\hline
\end{tabular}

aBased on Nevada Administrative Code, "Contamination of Soil: Establishment of Action Levels" (NAC, 2002)

$\mathrm{ft}$ bgs $=$ Feet below ground surface $\mathrm{mg} / \mathrm{kg}=$ Milligrams per kilogram

$\mathrm{H}=\mathrm{DRO}$ fuel pattern in the heavy end of retention time window.

\section{A.8.2.6 Gamma-Emitting Radionuclides}

Gamma-emitting radionuclide analytical results for soil samples collected at CAS 11-04-01 that were detected above MDCs are presented in Table A.8-4.

\section{A.8.2.7 Isotopes}

Isotope analytical results for soil samples collected at CAS 11-04-01 that were detected above MDCs are presented in Table A.8-5.

\section{A.8.2.8 Liquid and Sludge Sample Results}

Analytical results for the septic tank liquid samples collected at CAS 11-04-01 that were detected above MRLs or MDCs are presented in Table A.8-6. No analytes were detected in the sludge sample above MRLs.

\section{A.8.3 Nature and Extent of Contamination}

No contamination was found at this site. 
Table A.8-4

Soil Sample Results for Gamma-Emitting Radionuclides Detected Above Minimum Detectable Concentrations at CAS 11-04-01, Sewage Lagoon (Page 1 of 2)

\begin{tabular}{|c|c|c|c|c|c|c|c|c|c|c|c|c|c|c|c|}
\hline \multirow[b]{2}{*}{$\begin{array}{c}\text { Sample } \\
\text { Location }\end{array}$} & \multirow[b]{2}{*}{$\begin{array}{l}\text { Sample } \\
\text { Number }\end{array}$} & \multirow{3}{*}{$\begin{array}{c}\text { Depth } \\
\text { (ft bgs) }\end{array}$} & \multicolumn{13}{|c|}{ Contaminants of Potential Concern (pCi/g) } \\
\hline & & & \multicolumn{2}{|c|}{ 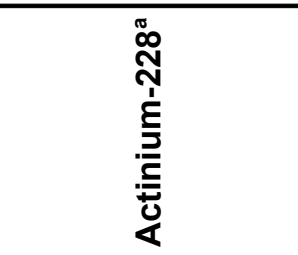 } & \multicolumn{2}{|c|}{ 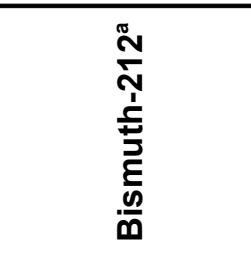 } & \multicolumn{2}{|c|}{ 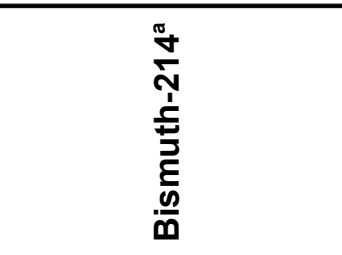 } & \multicolumn{2}{|c|}{ 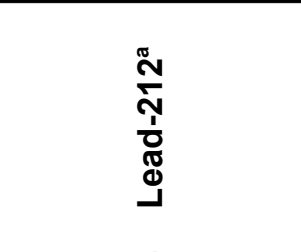 } & \multicolumn{2}{|c|}{ 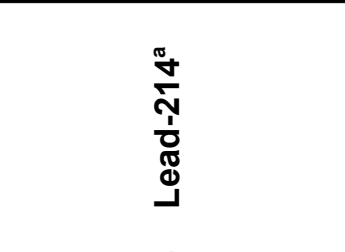 } & \multicolumn{2}{|c|}{ 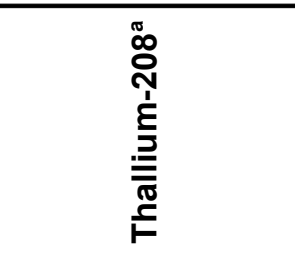 } & \multirow{3}{*}{ 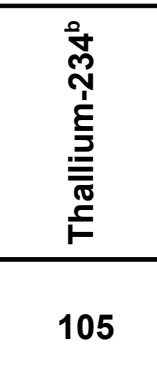 } \\
\hline \multicolumn{2}{|c|}{ Final Action Levels } & & 5 & 15 & 5 & 15 & 5 & 15 & 5 & 15 & 5 & 15 & 5 & 15 & \\
\hline \multicolumn{3}{|c|}{ Depth bgs (cm) } & $<15$ & $>15$ & $<15$ & $>15$ & $<15$ & $>15$ & $<15$ & $>15$ & $<15$ & $>15$ & $<15$ & $>15$ & \\
\hline \multirow{3}{*}{ H01 } & $\overline{2224 \mathrm{H} 001}$ & $\overline{0-1}$ & $\overline{\overline{\mathrm{NA}}}$ & 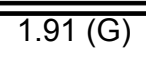 & $\overline{\overline{\mathrm{NA}}}$ & 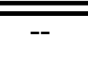 & $\overline{\mathrm{NA}}$ & $1.14(\mathrm{G}, \mathrm{J})$ & $\overline{\overline{N A}}$ & $\overline{2.37(\mathrm{~J})^{\mathrm{c}}}$ & $\overline{\mathrm{NA}}$ & $1.39(\mathrm{G}, \mathrm{J})$ & $\overline{\mathrm{NA}}$ & $\overline{0.59(\mathrm{G})}$ & 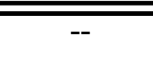 \\
\hline & $224 \mathrm{H} 002$ & $0-1$ & $\overline{N A}$ & $1.88(\mathrm{G})$ & $\overline{N A}$ & -- & NA & $1.54(\mathrm{G}, \mathrm{J})$ & $\overline{N A}$ & $1.75(\mathrm{~J})^{\mathrm{c}}$ & $\overline{N A}$ & $1.43(\mathrm{G}, \mathrm{J})$ & NA & $0.5(\mathrm{G})$ & -- \\
\hline & $224 \mathrm{H} 003$ & $4-5$ & $\overline{\mathrm{NA}}$ & $1.73(\mathrm{G})$ & $\overline{\mathrm{NA}}$ & -- & NA & $1.18(\mathrm{G}, \mathrm{J})$ & $\overline{\mathrm{NA}}$ & $2.21(\mathrm{~J})^{\mathrm{c}}$ & $\overline{\mathrm{NA}}$ & $1.32(\mathrm{G}, \mathrm{J})$ & $\overline{N A}$ & $0.64(\mathrm{G})$ & -- \\
\hline $\mathrm{H} 02$ & $224 \mathrm{H} 006$ & $4-5$ & $\overline{N A}$ & $1.8(\mathrm{G})$ & $\overline{N A}$ & -- & $\overline{N A}$ & $1.32(\mathrm{G}, \mathrm{J})$ & $\overline{\mathrm{NA}}$ & $2.35(\mathrm{~J})^{\mathrm{c}}$ & $\overline{N A}$ & $1.51(\mathrm{G}, \mathrm{J})$ & $\overline{N A}$ & $0.54(\mathrm{G})$ & -- \\
\hline $\mathrm{H} 04$ & $224 \mathrm{H} 010$ & $0-1$ & $\overline{\mathrm{NA}}$ & $1.56(\mathrm{G})$ & $\overline{\mathrm{NA}}$ & -- & NA & $1.21(\mathrm{G}, \mathrm{J})$ & $\overline{\mathrm{NA}}$ & $2.27(\mathrm{~J})^{\mathrm{c}}$ & $\overline{\mathrm{NA}}$ & $1.5(\mathrm{G}, \mathrm{J})$ & $\overline{\mathrm{NA}}$ & $0.55(\mathrm{G})$ & $\overline{--}$ \\
\hline $\mathrm{H} 05$ & $224 \mathrm{H} 014$ & $0-1$ & $\overline{\mathrm{NA}}$ & $2.04(\mathrm{G})$ & $\overline{\mathrm{NA}}$ & $\overline{--}$ & $\overline{N A}$ & $1.41(\mathrm{G}, \mathrm{J})$ & $\overline{N A}$ & $2.1(\mathrm{~J})^{\mathrm{c}}$ & $\overline{N A}$ & $1.56(\mathrm{G}, \mathrm{J})$ & NA & $0.6(\mathrm{G})$ & -- \\
\hline $\mathrm{H} 06$ & $224 \mathrm{H} 018$ & $7-8$ & $\overline{N A}$ & $1.96(\mathrm{G})$ & $\overline{N A}$ & -- & NA & $1.06(\mathrm{G}, \mathrm{J})$ & $\overline{N A}$ & $2.25(\mathrm{~J})^{\mathrm{c}}$ & $\overline{N A}$ & $1.5(\mathrm{G}, \mathrm{J})$ & $\overline{N A}$ & $0.53(\mathrm{G})$ & -- \\
\hline $\mathrm{H} 08$ & $224 \mathrm{H} 026$ & $0-0.5$ & $1.65(\mathrm{G})$ & NA & $1.88(\mathrm{G})$ & $\mathrm{NA}$ & $1.32(\mathrm{G}, \mathrm{J})$ & $\overline{N A}$ & $2.03(\mathrm{~J})^{\mathrm{c}}$ & NA & $1.46(\mathrm{G}, \mathrm{J})$ & $\overline{N A}$ & $0.58(\mathrm{G})$ & $\mathrm{NA}$ & $4.2(\mathrm{G}, \mathrm{TI})$ \\
\hline $\mathrm{H} 09$ & $224 \mathrm{H} 030$ & $2-2.5$ & $\overline{\mathrm{NA}}$ & $1.21(\mathrm{G})$ & $\overline{N A}$ & $\overline{--}$ & $\overline{N A}$ & $0.95(\mathrm{G}, \mathrm{J})$ & $\overline{\mathrm{NA}}$ & $1.49(\mathrm{~J})^{\mathrm{c}}$ & $\overline{\mathrm{NA}}$ & $1.13(\mathrm{G}, \mathrm{J})$ & $\overline{N A}$ & $0.433(\mathrm{G})$ & $\overline{--}$ \\
\hline $\mathrm{H} 10$ & $224 \mathrm{H} 034$ & $4-5$ & $\overline{N A}$ & $1.63(\mathrm{G})$ & $\overline{N A}$ & -- & NA & $1.11(\mathrm{G}, \mathrm{J})$ & $\overline{N A}$ & $1.83(\mathrm{~J})^{\mathrm{c}}$ & $\overline{N A}$ & $1.25(\mathrm{G}, \mathrm{J})$ & NA & $0.5(\mathrm{G})$ & $\overline{--}$ \\
\hline $\mathrm{H} 12$ & $224 \mathrm{H} 038$ & $0-0.5$ & $1.85(\mathrm{G})$ & NA & $2.4(\mathrm{G})$ & NA & $1.2(\mathrm{G}, \mathrm{J})$ & $\overline{N A}$ & $2.04(\mathrm{~J})^{\mathrm{c}}$ & NA & $1.34(\mathrm{G}, \mathrm{J})$ & $\overline{N A}$ & $0.56(\mathrm{G})$ & $\overline{N A}$ & -- \\
\hline \multirow{2}{*}{$\mathrm{H} 13$} & $224 \mathrm{H} 042$ & $2-2.5$ & $\overline{N A}$ & $1.37(\mathrm{G})$ & $\overline{N A}$ & - & NA & $1.16(\mathrm{G}, \mathrm{J})$ & $\overline{N A}$ & $1.37(\mathrm{~J})^{\mathrm{c}}$ & $\overline{N A}$ & $0.91(\mathrm{G}, \mathrm{J})$ & $\overline{N A}$ & $0.59(\mathrm{G})$ & $\overline{--}$ \\
\hline & $224 \mathrm{H} 043$ & $2-2.5$ & $\mathrm{NA}$ & $1.25(\mathrm{G})$ & $\mathrm{NA}$ & -- & NA & $0.84(\mathrm{G}, \mathrm{J})$ & $\mathrm{NA}$ & $1.49(\mathrm{~J})^{\mathrm{c}}$ & $\mathrm{NA}$ & $1.07(\mathrm{G}, \mathrm{J})$ & $\mathrm{NA}$ & $0.45(\mathrm{G})$ & -- \\
\hline $\mathrm{H} 15$ & $224 \mathrm{H} 048$ & $0-0.5$ & $1.48(\mathrm{G})$ & $\overline{\mathrm{NA}}$ & -- & $\mathrm{NA}$ & $1.48(\mathrm{G}, \mathrm{J})$ & $\overline{N A}$ & $2.28(\mathrm{~J})^{\mathrm{c}}$ & $\overline{N A}$ & $1.38(\mathrm{G}, \mathrm{J})$ & $\overline{N A}$ & $0.54(\mathrm{G})$ & $\overline{N A}$ & -- \\
\hline $\mathrm{H} 16$ & $224 \mathrm{H} 052$ & $1.5-2$ & $\overline{\mathrm{NA}}$ & $1.36(\mathrm{G})$ & $\overline{\mathrm{NA}}$ & -- & NA & $0.93(G, J)$ & NA & $1.22(\mathrm{~J})^{\mathrm{c}}$ & $\overline{\mathrm{NA}}$ & $0.99(\mathrm{G}, \mathrm{J})$ & NA & $0.43(\mathrm{G})$ & $\overline{--}$ \\
\hline
\end{tabular}


Table A.8-4

Soil Sample Results for Gamma-Emitting Radionuclides Detected Above Minimum Detectable Concentrations at CAS 11-04-01, Sewage Lagoon

(Page 2 of 2)

\begin{tabular}{|c|c|c|c|c|c|c|c|c|c|c|c|c|c|c|c|}
\hline \multirow[b]{2}{*}{$\begin{array}{l}\text { Sample } \\
\text { Location }\end{array}$} & \multirow[b]{2}{*}{$\begin{array}{l}\text { Sample } \\
\text { Number }\end{array}$} & \multirow{3}{*}{$\begin{array}{c}\text { Depth } \\
\text { (ft bgs) }\end{array}$} & \multicolumn{13}{|c|}{ Contaminants of Potential Concern (pCi/g) } \\
\hline & & & \multicolumn{2}{|c|}{ 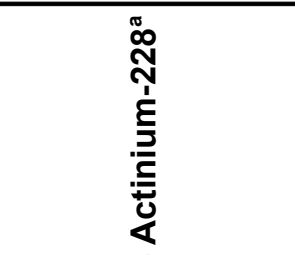 } & \multicolumn{2}{|c|}{ 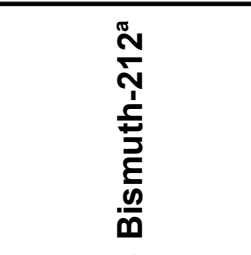 } & \multicolumn{2}{|c|}{ 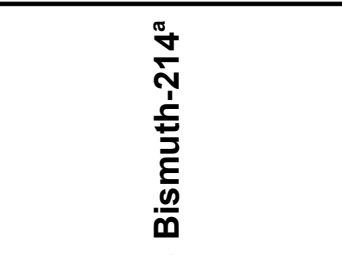 } & \multicolumn{2}{|c|}{ 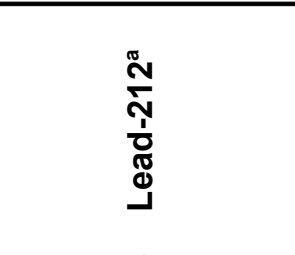 } & \multicolumn{2}{|c|}{ 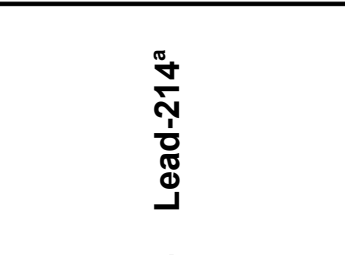 } & \multicolumn{2}{|c|}{ 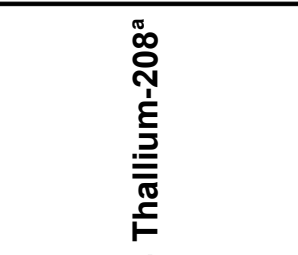 } & \multirow{3}{*}{ 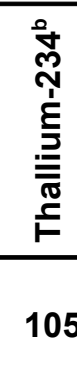 } \\
\hline \multicolumn{2}{|c|}{ Final Action Levels } & & 5 & 15 & 5 & 15 & 5 & 15 & 5 & 15 & 5 & 15 & 5 & 15 & \\
\hline \multicolumn{3}{|c|}{ Depth bgs (cm) } & $<15$ & $>15$ & $<15$ & $>15$ & $<15$ & $>15$ & $<15$ & $>15$ & $<15$ & $>15$ & $<15$ & $>15$ & \\
\hline$\overline{\mathrm{H} 17}$ & $224 \mathrm{H} 056$ & $3.5-4.5$ & $\overline{\mathrm{NA}}$ & $1.7(\mathrm{G})$ & $\overline{\mathrm{NA}}$ & $\overline{---}$ & $\overline{\mathrm{NA}}$ & $1.08(\mathrm{G}, \mathrm{J})$ & $\overline{\mathrm{NA}}$ & $1.59(\mathrm{~J})^{\mathrm{c}}$ & $\overline{\mathrm{NA}}$ & $1.33(\mathrm{G}, \mathrm{J})$ & $\overline{\mathrm{NA}}$ & $0.5(\mathrm{G})$ & 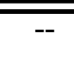 \\
\hline $\mathrm{H} 19$ & $224 \mathrm{H} 060$ & $0-0.5$ & $1.91(\mathrm{G})$ & NA & $2.26(\mathrm{G})$ & $\mathrm{NA}$ & $1.32(\mathrm{G}, \mathrm{J})$ & NA & $2.29(\mathrm{~J})^{\mathrm{c}}$ & NA & $1.29(\mathrm{G}, \mathrm{J})$ & NA & $0.61(\mathrm{G})$ & NA & -- \\
\hline \multirow{2}{*}{$\mathrm{H} 2 \mathrm{O}$} & $224 \mathrm{H} 063$ & $0-0.5$ & $1.7(\mathrm{G})$ & NA & $\overline{--}$ & NA & $1.29(\mathrm{G}, \mathrm{J})$ & NA & $1.8(\mathrm{~J})^{\mathrm{c}}$ & NA & $1.3(\mathrm{G}, \mathrm{J})$ & NA & $0.66(\mathrm{G})$ & $\overline{N A}$ & $\overline{--}$ \\
\hline & $224 \mathrm{H} 064$ & $0-0.5$ & $1.66(\mathrm{G})$ & NA & $\overline{--}$ & NA & $1.12(\mathrm{G}, \mathrm{J})$ & NA & $1.59(\mathrm{~J})^{\mathrm{c}}$ & NA & $1.47(\mathrm{G}, \mathrm{J})$ & $\mathrm{NA}$ & $0.64(\mathrm{G})$ & NA & -- \\
\hline $\mathrm{H} 21$ & $224 \mathrm{H} 068$ & $2-2.5$ & $\overline{N A}$ & $1.27(\mathrm{G})$ & $\overline{N A}$ & -- & NA & $1(G, J)$ & NA & $1.69(\mathrm{~J})^{\mathrm{c}}$ & $\overline{N A}$ & $0.94(G, J)$ & $\mathrm{NA}$ & $0.48(\mathrm{G})$ & -- \\
\hline $\mathrm{H} 23$ & $224 \mathrm{H} 072$ & $4-5$ & $\overline{\mathrm{NA}}$ & $1.56(\mathrm{G})$ & $\overline{\mathrm{NA}}$ & $\overline{--}$ & NA & $1.03(\mathrm{G}, \mathrm{J})$ & $\overline{N A}$ & $2.12(\mathrm{~J})^{\mathrm{C}}$ & $\overline{N A}$ & $1.19(\mathrm{G}, \mathrm{J})$ & $\mathrm{NA}$ & $0.5(\mathrm{G})$ & -- \\
\hline
\end{tabular}

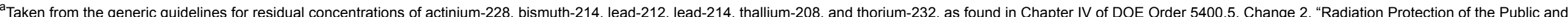

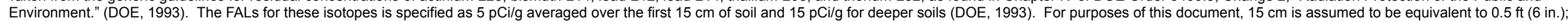
therefore, $5 \mathrm{pCi} / \mathrm{g}$ represents the FALs for these radionuclides in the surface soil ( 0 to $0.5 \mathrm{ft}$ depth).

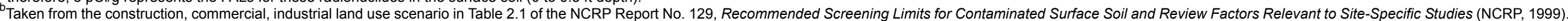
The values provided in this source document were scaled to a $25-\mathrm{mrem} / \mathrm{yr}$ dose.

${ }^{c}$ Qualifier added to laboratory data; record accepted. Sample does not meet counting geometry requirements.

$\mathrm{ft}$ bgs $=$ Feet below ground surface

$\mathrm{mrem} / \mathrm{yr}=$ Millirems per year

$\mathrm{pCi} / \mathrm{g}=$ Picocuries per gram

$=$ Not detected above minimum reporting limits

$>=$ Greater than

$G=$ Sample density differs by more than 15 percent of laboratory control sample density.

$\mathrm{J}=$ Estimated value

$\mathrm{TI}=$ Tentatively identified 


\section{Table A.8-5 \\ Soil Sample Results for Isotopes Detected Above \\ Minimum Detectable Concentrations at CAS 11-04-01, Sewage Lagoon}

\begin{tabular}{|c|c|c|c|c|c|c|}
\hline \multirow{2}{*}{$\begin{array}{c}\text { Sample } \\
\text { Location }\end{array}$} & \multirow{2}{*}{$\begin{array}{l}\text { Sample } \\
\text { Number }\end{array}$} & \multirow{2}{*}{$\begin{array}{l}\text { Depth } \\
\text { (ft bgs) }\end{array}$} & \multicolumn{4}{|c|}{ Contaminants of Potential Concern (pCi/g) } \\
\hline & & & Plutonium-239 & Uranium-234 & Uranium-235 & Uranium-238 \\
\hline \multicolumn{3}{|c|}{ Final Action Levels ${ }^{a}$} & 12.7 & 143 & 17.6 & 105 \\
\hline \multirow{3}{*}{ H01 } & $2224 \mathrm{H} 001$ & $\overline{0-1}$ & $\overline{---}$ & $\overline{1.08}$ & 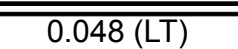 & $\overline{1.2}$ \\
\hline & $224 \mathrm{H} 002$ & $0-1$ & $0.042(\mathrm{LT})$ & 1.25 & 0.075 & 1.23 \\
\hline & $224 \mathrm{H} 003$ & $4-5$ & $\overline{--}$ & 1.36 & 0.083 & 1.23 \\
\hline $\mathrm{H} 02$ & $224 \mathrm{H} 006$ & $4-5$ & $\overline{--}$ & 1.48 & 0.082 & 1.19 \\
\hline $\mathrm{H} 04$ & $224 \mathrm{H} 010$ & $0-1$ & -- & 1.41 & 0.06 & 1.2 \\
\hline $\mathrm{H} 05$ & $224 \mathrm{H} 014$ & $0-1$ & 0.105 & 1.05 & -- & 1.01 \\
\hline $\mathrm{H} 06$ & $224 \mathrm{H} 018$ & $7-8$ & $\overline{--}$ & 1.45 & $\overline{--}$ & 1.25 \\
\hline $\mathrm{H} 07$ & $224 \mathrm{H} 022$ & $0-1$ & -- & 1.09 & 0.08 & 1 \\
\hline $\mathrm{H} 08$ & $224 \mathrm{H} 026$ & $0-0.5$ & -- & 1.26 & $0.038(\mathrm{LT})$ & 1.13 \\
\hline $\mathrm{H} 09$ & $224 \mathrm{H} 030$ & $2-2.5$ & -- & 1.1 & 0.053 & 1.29 \\
\hline $\mathrm{H} 10$ & $224 \mathrm{H} 034$ & $4-5$ & -- & 1 & 0.049 (LT) & 0.95 \\
\hline $\mathrm{H} 12$ & $224 \mathrm{H} 038$ & $0-0.5$ & -- & 1.12 & 0.056 & 1.03 \\
\hline \multirow{2}{*}{$\mathrm{H} 13$} & $224 \mathrm{H} 042$ & $2-2.5$ & -- & 1.12 & 0.058 & 1.09 \\
\hline & $224 \mathrm{H} 043$ & $2-2.5$ & $\overline{--}$ & 1.08 & 0.077 & 1.11 \\
\hline $\mathrm{H} 15$ & $224 \mathrm{H} 048$ & $0-0.5$ & -- & 0.99 & $0.05(\mathrm{LT})$ & 1.18 \\
\hline $\mathrm{H} 16$ & $224 \mathrm{H} 052$ & $1.5-2$ & -- & 1.03 & 0.051 & 1.02 \\
\hline $\mathrm{H} 17$ & $224 \mathrm{H} 056$ & $3.5-4.5$ & -- & 1.06 & 0.051 & 0.99 \\
\hline $\mathrm{H} 19$ & $224 \mathrm{H} 060$ & $0-0.5$ & -- & 1.12 & 0.087 & 1.05 \\
\hline \multirow{2}{*}{$\mathrm{H} 2 \mathrm{O}$} & $224 \mathrm{H} 063$ & $0-0.5$ & $\overline{--}$ & 1.06 & 0.061 & 1.01 \\
\hline & $224 \mathrm{H} 064$ & $0-0.5$ & 0.154 & 1.15 & 0.06 & 1.04 \\
\hline $\mathrm{H} 21$ & $224 \mathrm{H} 068$ & $2-2.5$ & -- & 0.95 & 0.051 & 1.04 \\
\hline $\mathrm{H} 23$ & $224 \mathrm{H} 072$ & $4-5$ & -- & 1.28 & 0.083 & 1.12 \\
\hline
\end{tabular}

${ }^{a}$ Taken from the construction, commercial, industrial land use scenario in Table 2.1 of the NCRP Report No. 129 Recommended Screening Limits for Contaminated Surface Soil and Review Factors Relevant to Site-Specific Studies (NCRP, 1999). The values provided in this source document were scaled to a 25 -mrem/yr dose.

$\mathrm{ft}$ bgs $=$ Feet below ground surface

$\mathrm{mrem} / \mathrm{yr}=$ Millirems per year

$\mathrm{pCi} / \mathrm{g}=$ Picocuries per gram

$\mathrm{LT}=$ Result is less than the requested minimum detectable concentration, greater than the sample specific minimum detectable

concentration.

-- = Not detected above minimum reporting limits 
Table A.8-6

Liquid Sample Results Detected

Above MRLs or MDCs at CAS 11-04-01, Sewage Lagoon

\begin{tabular}{||c|c|c|c|c|}
\hline Sample Number & Sample Matrix & Parameter & Result & Units \\
\hline \hline $224 \mathrm{H} 501$ & Liquid & Copper & 0.041 & $\mathrm{mg} / \mathrm{L}$ \\
\hline $224 \mathrm{H} 502$ & Liquid & Copper & 0.047 & $\mathrm{mg} / \mathrm{L}$ \\
\hline $224 \mathrm{H} 503$ & Liquid & Copper & 0.087 & $\mathrm{mg} / \mathrm{L}$ \\
\hline $224 \mathrm{H} 503$ & Liquid & Nickel & 0.026 & $\mathrm{mg} / \mathrm{L}$ \\
\hline $224 \mathrm{H} 503$ & Liquid & Aluminium & 0.27 & $\mathrm{mg} / \mathrm{L}$ \\
\hline $224 \mathrm{H} 501$ & Liquid & Uranium-234 & 1.53 & $\mathrm{pCi} / \mathrm{L}$ \\
\hline $224 \mathrm{H} 501$ & Liquid & Uranium-238 & 0.52 & $\mathrm{pCi} / \mathrm{L}$ \\
\hline $224 \mathrm{H} 502$ & Liquid & Uranium-234 & 1.81 & $\mathrm{pCi} / \mathrm{L}$ \\
\hline $224 \mathrm{H} 502$ & Liquid & Uranium-238 & 0.41 & $\mathrm{pCi} / \mathrm{L}$ \\
\hline $224 \mathrm{H} 503$ & Liquid & Uranium-234 & 2.86 & $\mathrm{pCi} / \mathrm{L}$ \\
\hline $224 \mathrm{H} 503$ & Liquid & Uranium-238 & 0.85 & $\mathrm{pCi} / \mathrm{L}$ \\
\hline
\end{tabular}

$\mathrm{mg} / \mathrm{L}=$ Milligrams per liter

$\mathrm{pCi} / \mathrm{L}=$ Picocuries per liter

\section{A.8.4 Revised Conceptual Site Model}

No variations in the CSM were identified. 


\section{A.9.0 CAS 23-05-02, Leachfield}

Corrective Action Site 23-05-02 is located adjacent to Building 753 in Mercury in Area 23 of the NTS. The CAS consists of a leachfield that serviced former Building 155. Investigation activities at CAS 23-05-02 included collection of surface and subsurface soil samples.

\section{A.9.1 Corrective Action Investigation}

A total of 41 soil characterization samples were collected during investigation activities at CAS 23-05-02. The sample IDs, locations, types, and analyses are listed in Table A.9-1. The sample locations are shown on Figure A.9-1. The specific CAI activities conducted to satisfy the CAIP requirements at this CAS are described in the following sections.

\section{A.9.1.1 Field Screening}

Soil samples were field screened for VOCs, TPH-DRO, and alpha and beta/gamma radiation. The FSRs were compared to FSLs to guide subsequent sampling decisions. The VOC headspace and alpha and beta/gamma radiation FSLs were not exceeded during sampling activities at this CAS. Total petroleum hydrocarbons-DRO FSL was exceeded in sample 224J008.

\section{A.9.1.2 Sampling}

Surface and subsurface samples were collected at locations specified in the CAIP. The locations were chosen to be associated with features (i.e., piping, drain) of the site, according to engineering drawings and field observations. Samples were collected at the surface (or directly below the asphalt), and at two or more subsurface intervals at each location, via the Geoprobe ${ }^{\circledR}$ sampling method. The samples were analyzed for the parameters listed in Table A.9-1. Sample locations are shown on Figure A.9-1.

\section{A.9.1.3 Deviations}

There were no significant deviations to the CAIP requirements at this CAS. The investigation and sampling at CAS 23-05-02 are considered sufficient to meet the DQOs. 


\section{A.9.2 Investigation Results}

The following sections provide descriptions of the CAS-specific activities conducted to complete investigation activities as outlined in the CAIP. Investigation activities included visual inspection of the CAS and collection of surface and subsurface soil samples, as outlined in Section A.9.1.2.

Surface and subsurface soil samples were analyzed for the CAIP-specified COPCs, which included total VOCs, total SVOCs, TPH-DRO, total metals, PCBs, cyanide, gamma-emitting radionuclides, plutonium isotopes, uranium isotopes, and strontium-90. The analytical parameters and laboratory methods used to analyze the investigation samples are listed in Table A.2-2. Table A.9-1 lists the sample-specific analytical suite for CAS 23-05-02. A portion of the analytical results for this CAS was rejected during validation; however, these rejected data did not adversely impact closure decisions as discussed in Appendix B, Section B.1.4.

\section{A.9.2.1 Total Volatile Organic Compounds}

Total VOC analytical results for soil samples collected at CAS 23-05-02 that were detected above MRLs are presented in Table A.9-2. No VOCs were detected in soil samples above FALs.

\section{A.9.2.2 Total Semivolatile Organic Compounds}

Total SVOC analytical results for soil samples collected at CAS 23-05-02 that were detected above MRLs are presented in Table A.9-3. Several PAHs were detected in soil samples above FALs. However, these samples were collected directly beneath the asphalt parking lot, or at intervals at which asphalt was present in the fill material. Therefore, PAHs at these locations are attributable to the asphalt, not the CAS, and are not COCs.

\section{A.9.2.3 Total Metals}

Total metals (including RCRA metals plus aluminum, antimony, beryllium, cobalt, copper, manganese, molybdenum, nickel, and zinc) analytical results for soil samples collected at CAS 23-05-02 are presented in Table A.9-4. No metals were detected above FALs. 


\section{A.9.2.4 Polychlorinated Biphenyls}

Total PCB analytical results for soil samples collected at CAS 23-05-02 that were detected above MRLs are presented in Table A.9-5. No PCBs were detected in soil samples above FALs.

\section{A.9.2.5 Total Petroleum Hydrocarbons-Diesel-Range Organics}

Total TPH-DRO analytical results for soil samples collected at CAS 23-05-02 that were detected above MRLs are presented in Table A.9-6. Total petroleum hydrocarbons-DRO was detected in several soil samples above FALs. However, these results can be attributed to the active parking lot that currently exists at the site, not the former leachfield, and the TPH-DRO is therefore not a COC.

\section{A.9.2.6 Gamma-Emitting Radionuclides}

Gamma-emitting radionuclide analytical results for soil samples collected at CAS 23-05-02 that were detected above MDCs are presented in Table A.9-7.

\section{A.9.2.7 Isotopes}

Isotope analytical results for soil samples collected at CAS 23-05-02 that were detected above MDCs are presented in Table A.9-8.

\section{A.9.3 Nature and Extent of Contamination}

Total petroleum hydrocarbons-DRO and PAH contamination was found at the site. However, the contamination is confined to the soil directly below the active asphalt parking lot and is, therefore, not attributable to the site.

\section{A.9.4 Revised Conceptual Site Model}

No variations in the CSM were identified. 
Table A.9-1

Samples Collected at CAS 23-05-02, Leachfield

(Page 1 of 2)

\begin{tabular}{|c|c|c|c|c|c|}
\hline $\begin{array}{l}\text { Sample } \\
\text { Location }\end{array}$ & $\begin{array}{l}\text { Sample } \\
\text { Number }\end{array}$ & $\begin{array}{c}\text { Depth } \\
\text { (ft bgs) }\end{array}$ & $\begin{array}{l}\text { Sample } \\
\text { Matrix }\end{array}$ & Purpose & Analyses \\
\hline \multirow{4}{*}{ J01 } & 224J001 & $0-2$ & Soil & $\begin{array}{c}\text { SC, } \\
\text { MS/MSD } \\
\text { (metals only) }\end{array}$ & Set 1 \\
\hline & 224J002 & $0-2$ & Soil & $\begin{array}{c}\text { Field Duplicate } \\
\text { of \#224H001 }\end{array}$ & Set 1 \\
\hline & 224J003 & $4-5$ & Soil & $\begin{array}{c}\mathrm{SC} \\
\mathrm{MS} / \mathrm{MSD} \\
\text { (except metals) }\end{array}$ & Set 1 \\
\hline & 224J004 & $7-8$ & Soil & SC & Set 1 \\
\hline \multirow{3}{*}{ J02 } & 224J005 & $0.5-2$ & Soil & SC & Set 1 \\
\hline & 224J006 & $4-5$ & Soil & SC & Set 1 \\
\hline & 224J007 & $7-8$ & Soil & SC & Set 1 \\
\hline \multirow{3}{*}{ J03 } & 224J008 & $0.5-2$ & Soil & SC & Set 1 \\
\hline & 224J009 & $4-5$ & Soil & SC & Set 1 \\
\hline & 224J010 & $7-8$ & Soil & SC & Set 1 \\
\hline \multirow{3}{*}{ J05 } & 224J014 & $0.5-2$ & Soil & SC & Set 1 \\
\hline & 224J015 & $4-5$ & Soil & SC & Set 1 \\
\hline & 224J016 & $7-8$ & Soil & SC & Set 1 \\
\hline \multirow{3}{*}{ J06 } & 224J017 & $0.5-2$ & Soil & SC & Set 1 \\
\hline & 224J018 & $4-5$ & Soil & SC & Set 1 \\
\hline & 224J019 & $7-8$ & Soil & SC & Set 1 \\
\hline \multirow{3}{*}{ J08 } & 224J023 & $0.5-2$ & Soil & SC & Set 1 \\
\hline & 224J024 & $4-5$ & Soil & SC & Set 1 \\
\hline & 224J025 & $7-8$ & Soil & SC & Set 1 \\
\hline \multirow{4}{*}{ J09 } & 224J026 & $0.5-2$ & Soil & SC & Set 1 \\
\hline & 224J027 & $0.5-2$ & Soil & $\begin{array}{c}\text { Field Duplicate } \\
\text { of \#224J026 }\end{array}$ & Set 1 \\
\hline & 224J028 & $4-5$ & Soil & $\mathrm{SC}, \mathrm{MS} / \mathrm{MSD}$ & Set 1 \\
\hline & 224J029 & $7-8$ & Soil & SC & Set 1 \\
\hline \multirow{3}{*}{$\mathrm{J} 10$} & 224J030 & $0.5-2$ & Soil & SC & Set 1 \\
\hline & 224J031 & $4-5$ & Soil & SC & Set 1 \\
\hline & 224J032 & $7-8$ & Soil & SC & Set 1 \\
\hline \multirow{3}{*}{ J11 } & 224J033 & $0.5-2$ & Soil & SC & Set 1 \\
\hline & 224J034 & $4-5$ & Soil & SC & Set 1 \\
\hline & 224J035 & $7-8$ & Soil & SC & Set 1 \\
\hline
\end{tabular}


Table A.9-1

Samples Collected at CAS 23-05-02, Leachfield

(Page 2 of 2)

\begin{tabular}{|c|c|c|c|c|c|}
\hline $\begin{array}{c}\text { Sample } \\
\text { Location }\end{array}$ & $\begin{array}{l}\text { Sample } \\
\text { Number }\end{array}$ & $\begin{array}{c}\text { Depth } \\
\text { (ft bgs) }\end{array}$ & $\begin{array}{l}\text { Sample } \\
\text { Matrix }\end{array}$ & Purpose & Analyses \\
\hline \multirow{3}{*}{ J12 } & 2 224J036 & $0.5-2$ & $\overline{\text { Soil }}$ & $\overline{S C}$ & $\overline{\text { Set } 1}$ \\
\hline & 224J037 & $4-5$ & Soil & SC & Set 1 \\
\hline & 224J038 & $7-8$ & Soil & $\mathrm{SC}$ & Set 1 \\
\hline $\mathrm{J} 13$ & 224J044 & $0.5-1.5$ & Soil & SC & TPH-DRO \\
\hline \multirow{2}{*}{ J14 } & 224J039 & $0.5-1.5$ & Soil & $\mathrm{SC}$ & TPH-DRO \\
\hline & 224J040 & $4.5-5.5$ & Soil & SC & TPH-DRO \\
\hline \multirow{2}{*}{ J15 } & 224J041 & $0.5-1.5$ & Soil & SC & TPH-DRO \\
\hline & 224J042 & $4.5-5.5$ & Soil & SC & TPH-DRO \\
\hline J16 & 224J043 & $0.5-1.5$ & Soil & SC & TPH-DRO \\
\hline \multirow[b]{2}{*}{ J17 } & 224J045 & $0.5-1.5$ & Soil & SC & TPH-DRO \\
\hline & 224J046 & $0.5-1.5$ & Soil & $\begin{array}{l}\text { Field Duplicate } \\
\text { of \#224J045 }\end{array}$ & TPH-DRO \\
\hline J18 & 224J047 & $0.5-1.5$ & Soil & $\mathrm{SC}, \mathrm{MS} / \mathrm{MSD}$ & TPH-DRO \\
\hline NA & $224 J 301$ & NA & Water & Trip Blank & Total VOCs \\
\hline NA & $224 J 302$ & NA & Water & Trip Blank & Total VOCs \\
\hline NA & $224 \mathrm{~J} 303$ & NA & Water & Trip Blank & Total VOCs \\
\hline NA & $224 J 304$ & NA & Water & Field Blank & Set 1 \\
\hline NA & 224J305 & NA & Water & $\begin{array}{c}\text { Equipment } \\
\text { Rinsate Blank }\end{array}$ & Set 1 \\
\hline NA & $224 J 306$ & NA & Water & Trip Blank & Total VOCs \\
\hline NA & 224J501 & NA & Liquid & WM & $\begin{array}{c}\text { Gross Alpha/Beta, } \\
\text { Tritium }\end{array}$ \\
\hline
\end{tabular}

Note: Certain sample numbers are missing. This is due to the fact that the samples they represented were not shipped to the laboratory according to the specifications in the CAIP and FI.

Set 1 = Total VOCs, Total SVOCs plus Hydroquinone, Total RCRA Metals, Aluminum, Antimony, Beryllium, Cobalt, Copper, Manganese, Molybdenum, Nickel, Zinc, TPH-DRO, PCBs, Cyanide, Gamma Spectroscopy, Isotopic Plutonium, Isotopic Uranium, and Strontium-90.

$\mathrm{DRO}=$ Diesel-range organics $\mathrm{ft}$ bgs $=$ Feet below ground surface MS/MSD = Matrix spike/matrix spike duplicate NA = Not applicable

$\mathrm{PCB}=$ Polychlorinated biphenyl
RCRA = Resource Conservation and Recovery Act

$\mathrm{SC}=$ Site characterization

SVOC = Semivolatile organic compound

$\mathrm{TPH}=$ Total petroleum hydrocarbons

$\mathrm{WM}=$ Waste Management

VOC $=$ Volatile organic compound 


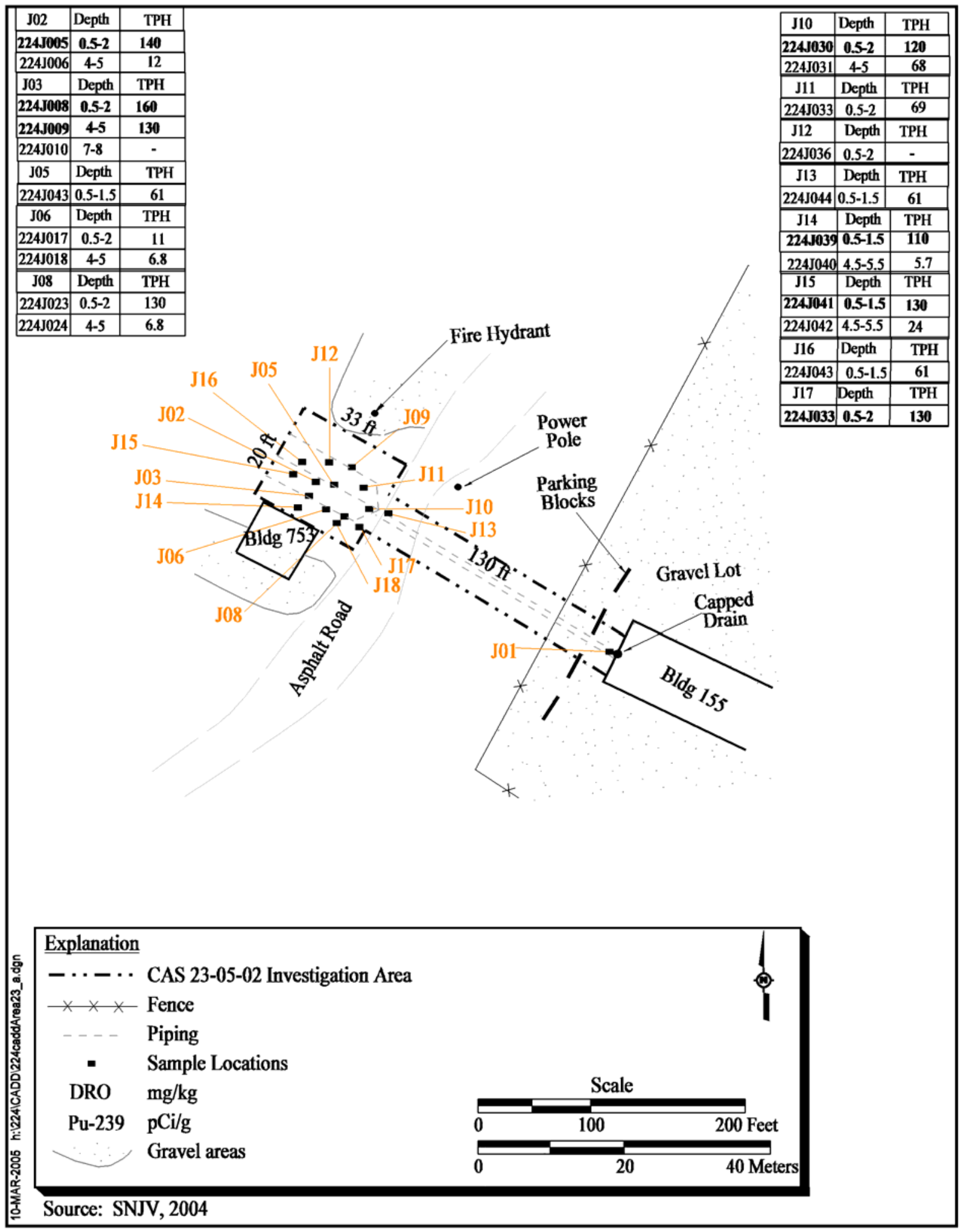

Figure A.9-1

Sample Location Map, CAS 23-05-02, Leachfield 
Table A.9-2

Soil Sample Results for Total VOCs Detected Above
Minimum Reporting Limits at CAS 23-05-02, Leachfield

\begin{tabular}{|c|c|c|c|c|c|c|}
\hline \multirow{2}{*}{$\begin{array}{l}\text { Sample } \\
\text { Location }\end{array}$} & \multirow{2}{*}{$\begin{array}{l}\text { Sample } \\
\text { Number }\end{array}$} & \multirow{2}{*}{$\begin{array}{c}\text { Depth } \\
\text { (ft bgs) }\end{array}$} & \multicolumn{4}{|c|}{ Contaminants of Potential Concern $(\mu \mathrm{g} / \mathrm{kg})$} \\
\hline & & & Methylene Chloride & $m+p-X y l e n e$ & Naphthalene & Toluene \\
\hline \multicolumn{3}{|c|}{ Final Action Levels ${ }^{a}$} & 21,000 & 420,000 & 190,000 & 520,000 \\
\hline \multirow{2}{*}{ J01 } & 224J003 & $4-5$ & 8.7 & -- & -- & -- \\
\hline & 224J004 & $7-8$ & 8.9 & -- & -- & -- \\
\hline \multirow{2}{*}{$\mathrm{J} 02$} & 224J005 & $0.5-2$ & 5.7 & -- & -- & -- \\
\hline & 224J007 & $7-8$ & 6.8 & -- & -- & -- \\
\hline \multirow{3}{*}{$\mathrm{J} 03$} & 224J008 & $0.5-2$ & 7.6 & -- & -- & -- \\
\hline & 224J009 & $4-5$ & -- & -- & 28 & -- \\
\hline & 224J010 & $7-8$ & 7.6 & -- & -- & -- \\
\hline \multirow{2}{*}{ J05 } & 224J014 & $0.5-2$ & 11 & -- & -- & -- \\
\hline & 224J015 & $4-5$ & 11 & -- & -- & -- \\
\hline \multirow{2}{*}{$\mathrm{J} 10$} & 224J030 & $0.5-2$ & -- & -- & 37 & 8 \\
\hline & 224J031 & $4-5$ & -- & 6.2 & 96 & 24 \\
\hline
\end{tabular}

${ }^{a}$ Based on U.S. Environmental Protection Agency, Region 9 Preliminary Remediation Goals (PRGs) (EPA, 2002)

$\mathrm{ft}$ bgs $=$ Feet below ground surface $\mu \mathrm{g} / \mathrm{kg}=$ Micrograms per kilogram

-- = Not detected above minimum reporting limits. 
Table A.9-3

Soil Sample Results for Total SVOCs Detected Above Minimum Reporting Limits at CAS 23-05-02, Leachfield

\begin{tabular}{|c|c|c|c|c|c|c|c|c|c|c|c|c|c|c|c|c|c|c|c|c|c|}
\hline \multirow[b]{2}{*}{$\begin{array}{c}\text { Sample } \\
\text { Location }\end{array}$} & \multirow[b]{2}{*}{$\begin{array}{l}\text { Sample } \\
\text { Number }\end{array}$} & \multirow[b]{2}{*}{$\begin{array}{c}\text { Depth } \\
\text { (ft bgs) }\end{array}$} & \multicolumn{19}{|c|}{ Contaminants of Potential Concern $(\mu \mathrm{g} / \mathrm{kg})$} \\
\hline & & & 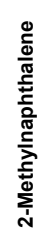 & 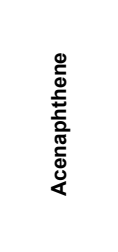 & 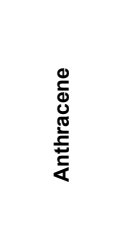 & 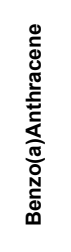 & 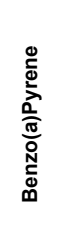 & 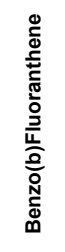 & 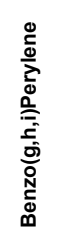 & 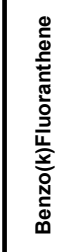 & 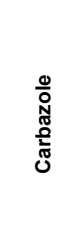 & 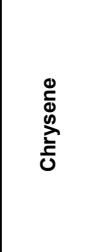 & 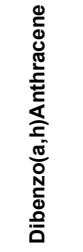 & 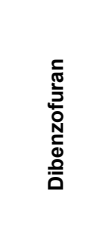 & 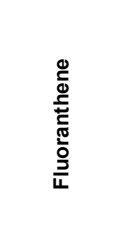 & 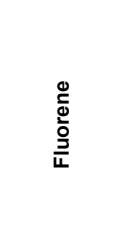 & 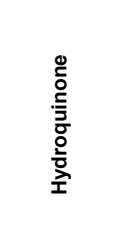 & 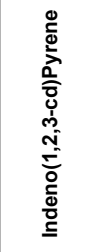 & 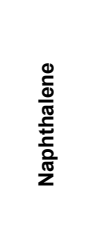 & 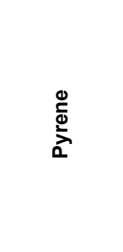 & 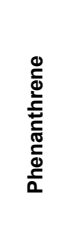 \\
\hline \multicolumn{3}{|c|}{ Final Action Levels } & NI & $29,000,000$ & $100,000,00$ & 2,100 & 210 & 2,100 & $\mathbf{N I}$ & 21,000 & 86,000 & 210,000 & 210 & $3,100,000$ & $22,000,000$ & $26,000,000$ & $25,000,000$ & 2,100 & 190,000 & $29,000,000$ & NI \\
\hline J03 & 224J009RR1RX1 & $4-5$ & -- & $3,700(J)^{b}$ & $8,000(J)^{b}$ & $\begin{array}{c}18,000 \\
(J)^{\circ}\end{array}$ & $\begin{array}{c}\begin{array}{c}16,000 \\
(J)^{b}\end{array} \\
\text { s. }\end{array}$ & $\begin{array}{c}14,000 \\
(J)^{\circ}\end{array}$ & $\begin{array}{c}16,000 \\
(J)^{d}\end{array}$ & $\begin{array}{c}, 6000 \\
(J)^{e}\end{array}$ & -- & $19,000(J)^{b}$ & -- & $1,400(J)^{\prime}$ & $30,000(J)^{b}$ & $2,400(\mathrm{~J})^{t}$ & - & $14,000(J)^{d}$ & $1,100(J)$ & $41,000(J)^{\prime}$ & $\begin{array}{c}24,000 \\
(J)^{b}\end{array}$ \\
\hline J06 & 224J018RX1 & $4-5$ & -- & -- & -- & -- & - & - & -- & - & -- & - & -- & - & -- & - & -- & -- & - & $470(J)^{\prime}$ & - \\
\hline \multirow{2}{*}{ J08 } & $224 J 023$ & $0.5-2$ & -- & - & - & -- & - & - & -- & - & -- & -- & -- & -- & -- & -- & $3,600(J)^{9}$ & -- & -- & -- & - \\
\hline & $224 \mathrm{~J} 025$ & $7-8$ & -- & -- & -- & -- & - & - & - & - & -- & -- & -- & -- & -- & -- & $730(\mathrm{~J})^{9}$ & -- & -- & -- & - \\
\hline \multirow{2}{*}{$J 10$} & $224 J 030$ & $0.5-2$ & -- & -- & 850 & $\begin{array}{c}2,600 \\
(J)^{n}\end{array}$ & $\begin{array}{c}2,300 \\
(J)^{n}\end{array}$ & $\begin{array}{c}2,300 \\
(J)^{n}\end{array}$ & $\begin{array}{c}2,700 \\
(J)^{n}\end{array}$ & $810(J)^{h}$ & - & $2,800(\mathrm{~J})^{h}$ & $860(J)^{h}$ & - & 3,300 & -- & - & $2,200(J)^{n}$ & - & $12,000(J)^{h}$ & 2,700 \\
\hline & $224 J 031$ & $4-5$ & 480 & 1,900 & 3,500 & $\begin{array}{c}6,600 \\
(J)^{\prime}\end{array}$ & $\begin{array}{c}6,200 \\
(\mathrm{~J})^{\prime}\end{array}$ & $\begin{array}{l}6,200 \\
(\mathrm{~J})\end{array}$ & $\begin{array}{c}7,400 \\
(J)^{k}\end{array}$ & $\begin{array}{l}1,900 \\
(J)^{n}\end{array}$ & 970 & $7,200(\mathrm{~J})^{\prime}$ & $\begin{array}{l}1,900 \\
(J)^{n}\end{array}$ & 960 & $11,000(J)^{\prime}$ & 1,600 & - & $5,500(J)^{\prime}$ & 700 & $34,000(J)^{\prime}$ & $\begin{array}{c}12,000 \\
(\mathcal{J})^{m}\end{array}$ \\
\hline
\end{tabular}

${ }^{a}$ Based on U.S. Environmental Protection Agency, Region 9 Preliminary Remediation Goals (PRGs) (EPA, 2002)

Qualifier added to laboratory data; record accepted. Exceeded holding time. Matrix effects may exist. Surrogates diluted out. Internal area response show extremely low count.

Qualifier added to laboratory data; record accepted. Exceeded holding time. Calibration verification did not meet criteria or was not performed. Surrogates diluted out

Qualifier added to laboratory data; record accepted. Exceeded holding time. Matrix effects may exist. Internal standard area count outside control limits.

Qualifier added to laboratory data, record accepled. Exceeded holding time.

hQualifier added to laboratory data, record accepted. Surrogater

Qualifier added to laboratory data; record accepted. Matrix effects may exist. Surrogates dillted out. Internal standard area count outside control limits.

Qualifier added to laboratory data; record accepted. Value exceeded linear/calibration range of instrument. Matrix effects may exist. The reported value is from the dilution run. Internal standard area count outside control limits.

Qualifier added to laboratory data; record accepted. Serial dilution \%D outside control limits. Matrix effects may exist. The reported value is from the dilution run. Internal standard area count outside control limits

Qualifier added to laboratory data; record accepted. Surrogates diluted out.

$\mathrm{ft}$ bgs $=$ Feet below ground surface

.

$\mathrm{J}=$ Estimated value

$\mathrm{NI}=$ Not identified 
Table A.9-4

Soil Sample Results for Metals Detected Above Minimum Reporting Limits at CAS 23-05-02, Leachfield (Page 1 of 2)

\begin{tabular}{|c|c|c|c|c|c|c|c|c|c|c|c|c|c|c|c|c|}
\hline \multirow[b]{2}{*}{$\begin{array}{c}\text { Sample } \\
\text { Location }\end{array}$} & \multirow[b]{2}{*}{$\begin{array}{l}\text { Sample } \\
\text { Number }\end{array}$} & \multirow[b]{2}{*}{$\begin{array}{l}\text { Depth } \\
\text { (ft bgs) }\end{array}$} & \multicolumn{14}{|c|}{ Contaminants of Potential Concern $(\mathrm{mg} / \mathrm{kg})$} \\
\hline & & & 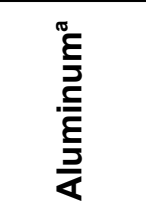 & 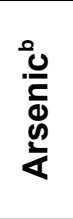 & 㺼 & 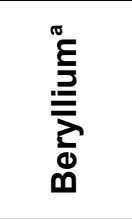 & 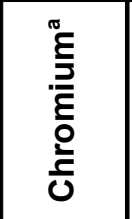 & $\begin{array}{l}\frac{\pi}{\pi} \\
\frac{1}{0} \\
0\end{array}$ & $\begin{array}{l}\frac{\pi}{d} \\
\frac{0}{0} \\
\frac{0}{0}\end{array}$ & 总 & 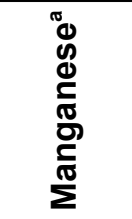 & $\begin{array}{l}\frac{\pi}{J} \\
\frac{0}{0} \\
\sum \\
\Sigma\end{array}$ & $\begin{array}{l}\frac{\sigma}{d} \\
\frac{d}{d} \\
\frac{u}{z}\end{array}$ & 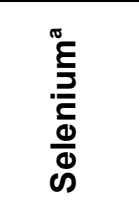 & $\frac{\stackrel{s}{\bar{d}}}{\bar{c}}$ & 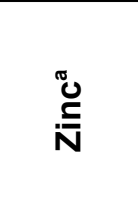 \\
\hline \multicolumn{3}{|c|}{ Final Action Levels } & 100,000 & 23 & 67,000 & 1,900 & 450 & 1,900 & 41,000 & 750 & 19,000 & 310 & 20,000 & 5,100 & 5,100 & 100,000 \\
\hline \multirow{4}{*}{ J01 } & 2 224J001 & $0-2$ & $\overline{\overline{5,400}}$ & 4.2 & $83(\mathrm{~J})^{\mathrm{c}}$ & $\overline{---}$ & $4.2(\mathrm{~J})^{\mathrm{c}}$ & 2.3 & $\overline{6.9}$ & $26(\mathrm{~J})^{\mathrm{d}}$ & 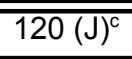 & 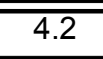 & 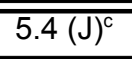 & $\overline{---}$ & $\overline{-\overline{--}}$ & $20(\mathrm{~J})^{\mathrm{c}}$ \\
\hline & 224J002 & $0-2$ & 5,500 & 5.3 & $75(J)^{c}$ & -- & $3.8(\mathrm{~J})^{\mathrm{c}}$ & 2.4 & 6.8 & $12(\mathrm{~J})^{\mathrm{d}}$ & $110(J)^{c}$ & 1.5 & $4.7(\mathrm{~J})^{\mathrm{C}}$ & -- & -- & $16(\mathrm{~J})^{\mathrm{c}}$ \\
\hline & 224J003 & $4-5$ & 3,200 & 3 & $43(\mathrm{~J})^{c}$ & -- & $2.4(\mathrm{~J})^{\mathrm{c}}$ & 1.3 & 3.5 & $5.2(\mathrm{~J})^{\mathrm{d}}$ & $85(\mathrm{~J})^{\mathrm{C}}$ & 0.36 & $2.8(\mathrm{~J})^{\mathrm{c}}$ & -- & -- & $9.4(\mathrm{~J})^{\mathrm{c}}$ \\
\hline & 224J004 & $7-8$ & 4,100 & 5 & $53(\mathrm{~J})^{c}$ & $\overline{--}$ & $2.6(\mathrm{~J})^{\mathrm{c}}$ & 1.8 & 4.7 & $2.6(J)^{d}$ & $87(\mathrm{~J})^{\mathrm{c}}$ & $\overline{--}$ & $3.2(\mathrm{~J})^{\mathrm{c}}$ & $\overline{--}$ & $\overline{--}$ & $9.4(J)^{c}$ \\
\hline \multirow{3}{*}{ J02 } & 224J005 & $0.5-2$ & 6,100 & 3.8 & $76(\mathrm{~J})^{c}$ & $\overline{--}$ & $4.7(\mathrm{~J})^{\mathrm{c}}$ & 2.6 & 6.2 & $6.2(J)^{d}$ & $170(\mathrm{~J})^{\mathrm{c}}$ & $\overline{--}$ & $5.4(\mathrm{~J})^{\mathrm{c}}$ & $\overline{--}$ & $\overline{--}$ & $18(\mathrm{~J})^{\mathrm{c}}$ \\
\hline & 224J006 & $4-5$ & 2,900 & 2.8 & $43(\mathrm{~J})^{c}$ & -- & $2.4(\mathrm{~J})^{\mathrm{c}}$ & 1.1 & 14 & $2.7(\mathrm{~J})^{\mathrm{d}}$ & $81(\mathrm{~J})^{\mathrm{c}}$ & 0.11 & $3.2(\mathrm{~J})^{\mathrm{c}}$ & -- & 12 & $16(\mathrm{~J})^{\mathrm{c}}$ \\
\hline & 224J007 & $7-8$ & 3,800 & 3.6 & $42(\mathrm{~J})^{c}$ & $\overline{--}$ & $2.5(\mathrm{~J})^{\mathrm{c}}$ & 1.4 & 4.6 & $3.5(J)^{d}$ & $73(\mathrm{~J})^{c}$ & 0.17 & $3.1(\mathrm{~J})^{\mathrm{c}}$ & -- & 2.4 & $9.4(\mathrm{~J})^{\mathrm{C}}$ \\
\hline \multirow{3}{*}{ J03 } & 224J008 & $0.5-2$ & 5,000 & 4.2 & $67(\mathrm{~J})^{\mathrm{c}}$ & $\overline{--}$ & $3.7(\mathrm{~J})^{\mathrm{c}}$ & 2.2 & 5.5 & $6.3(\mathrm{~J})^{\mathrm{d}}$ & $130(\mathrm{~J})^{\mathrm{c}}$ & -- & $4.5(\mathrm{~J})^{\mathrm{c}}$ & $\overline{--}$ & -- & $16(\mathrm{~J})^{\mathrm{c}}$ \\
\hline & 224J009 & $4-5$ & 7,500 & 4.2 & $51(\mathrm{~J})^{\mathrm{c}}$ & $0.78(\mathrm{~J})^{\mathrm{c}}$ & $23(\mathrm{~J})^{\mathrm{c}}$ & 2.5 & 15 & $66(\mathrm{~J})^{\mathrm{d}}$ & $140(\mathrm{~J})^{\mathrm{c}}$ & 41 & $5.8(\mathrm{~J})^{\mathrm{c}}$ & $\overline{--}$ & 280 & $97(\mathrm{~J})^{\mathrm{c}}$ \\
\hline & 224J010 & $7-8$ & 6,000 & 3.8 & $70(\mathrm{~J})^{c}$ & -- & $4.2(\mathrm{~J})^{\mathrm{c}}$ & 1.6 & 6.5 & $4.2(\mathrm{~J})^{\mathrm{d}}$ & $120(\mathrm{~J})^{\mathrm{c}}$ & 0.23 & $4.3(\mathrm{~J})^{\mathrm{c}}$ & -- & 7.3 & $14(\mathrm{~J})^{\mathrm{c}}$ \\
\hline \multirow{3}{*}{ J05 } & 224J014 & $0.5-2$ & 4,600 & 4.5 & $58(\mathrm{~J})^{c}$ & -- & $3.5(\mathrm{~J})^{\mathrm{c}}$ & 1.9 & 4.8 & $3.5(\mathrm{~J})^{\mathrm{d}}$ & $100(\mathrm{~J})^{\mathrm{c}}$ & 0.057 & $3.8(\mathrm{~J})^{\mathrm{c}}$ & -- & -- & $12(\mathrm{~J})^{\mathrm{c}}$ \\
\hline & 224J015 & $4-5$ & 5,300 & 4.2 & $55(\mathrm{~J})^{\mathrm{c}}$ & $\overline{--}$ & $4.4(\mathrm{~J})^{\mathrm{c}}$ & 2.1 & 5 & $3.7(\mathrm{~J})^{\mathrm{d}}$ & $110(\mathrm{~J})^{\mathrm{c}}$ & 0.18 & $4.2(\mathrm{~J})^{\mathrm{c}}$ & $\overline{--}$ & $\overline{--}$ & $13(\mathrm{~J})^{\mathrm{c}}$ \\
\hline & 224J016 & $7-8$ & 6,600 & 5.8 & $77(\mathrm{~J})^{\mathrm{C}}$ & -- & $4.2(\mathrm{~J})^{\mathrm{c}}$ & 2.7 & 6.7 & $3.5(\mathrm{~J})^{\mathrm{d}}$ & $110(J)^{c}$ & 0.066 & $4.7(\mathrm{~J})^{\mathrm{C}}$ & -- & -- & $14(\mathrm{~J})^{\mathrm{c}}$ \\
\hline \multirow{3}{*}{ J06 } & 224J017 & $0.5-2$ & 5,500 & 4.4 & $73(\mathrm{~J})^{\mathrm{c}}$ & -- & $4.1(\mathrm{~J})^{\mathrm{c}}$ & 2.3 & $5.7(\mathrm{~J})^{\mathrm{c}}$ & 4.9 & $140(\mathrm{~J})^{\mathrm{c}}$ & 0.46 & $4.5(\mathrm{~J})^{\mathrm{c}}$ & -- & 2.2 & $15(\mathrm{~J})^{\mathrm{c}}$ \\
\hline & 224J018 & $4-5$ & 4,900 & 4.2 & $53(\mathrm{~J})^{\mathrm{c}}$ & $\overline{--}$ & $25(\mathrm{~J})^{\mathrm{c}}$ & 1.9 & $18(\mathrm{~J})^{\mathrm{c}}$ & 77 & $110(\mathrm{~J})^{\mathrm{c}}$ & 45 & $4.8(\mathrm{~J})^{\mathrm{c}}$ & $0.55(\mathrm{~J}-)$ & 360 & $140(\mathrm{~J})^{\mathrm{c}}$ \\
\hline & 224J019 & $7-8$ & 3,400 & 4.5 & $54(\mathrm{~J})^{\mathrm{c}}$ & -- & $2.2(\mathrm{~J})^{\mathrm{c}}$ & $\overline{--}$ & $4.8(\mathrm{~J})^{\mathrm{c}}$ & 3 & $58(\mathrm{~J})^{\mathrm{C}}$ & 0.77 & -- & -- & 5 & $8.1(\mathrm{~J})^{\mathrm{c}}$ \\
\hline \multirow{3}{*}{ J08 } & 224J023 & $0.5-2$ & 5,800 & 4.1 & $72(\mathrm{~J})^{\mathrm{c}}$ & $\overline{--}$ & $4.3(\mathrm{~J})^{\mathrm{c}}$ & 2.4 & $5.4(\mathrm{~J})^{\mathrm{c}}$ & 3.9 & $130(\mathrm{~J})^{\mathrm{c}}$ & $\overline{--}$ & $5(J)^{c}$ & $\overline{--}$ & -- & $14(\mathrm{~J})^{\mathrm{C}}$ \\
\hline & 224J024 & $4-5$ & 3,200 & 2.9 & $48(J)^{c}$ & -- & $4.4(\mathrm{~J})^{\mathrm{c}}$ & 1.2 & $6.3(\mathrm{~J})^{\mathrm{c}}$ & 9.2 & $81(\mathrm{~J})^{\mathrm{C}}$ & 2.5 & $2.8(\mathrm{~J})^{\mathrm{e}}$ & -- & 31 & $14(\mathrm{~J})^{\mathrm{c}}$ \\
\hline & 224J025 & $7-8$ & 4,300 & 3.9 & $60(\mathrm{~J})^{\mathrm{c}}$ & -- & $2.7(\mathrm{~J})^{\mathrm{c}}$ & 1.5 & $5.7(\mathrm{~J})^{\mathrm{c}}$ & 2.7 & $60(\mathrm{~J})^{c}$ & 0.49 & -- & -- & 2.4 & $9(\mathrm{~J})^{\mathrm{c}}$ \\
\hline
\end{tabular}


Table A.9-4

Soil Sample Results for Metals Detected Above Minimum Reporting Limits at CAS 23-05-02, Leachfield

(Page 2 of 2)

\begin{tabular}{|c|c|c|c|c|c|c|c|c|c|c|c|c|c|c|c|c|}
\hline \multirow[b]{2}{*}{$\begin{array}{c}\text { Sample } \\
\text { Location }\end{array}$} & \multirow[b]{2}{*}{$\begin{array}{c}\text { Sample } \\
\text { Number }\end{array}$} & \multirow[b]{2}{*}{$\begin{array}{c}\text { Depth } \\
\text { (ft bgs) }\end{array}$} & \multicolumn{14}{|c|}{ Contaminants of Potential Concern $(\mathrm{mg} / \mathrm{kg})$} \\
\hline & & & 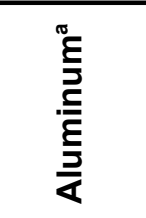 & 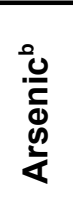 & 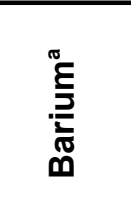 & 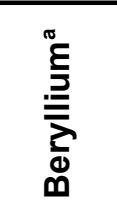 & 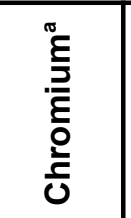 & $\begin{array}{l}\frac{\pi}{\pi} \\
\frac{0}{0} \\
ن\end{array}$ & $\begin{array}{l}\frac{\pi}{\delta} \\
\frac{0}{0} \\
0 \\
0\end{array}$ & 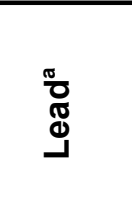 & 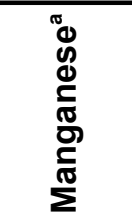 & 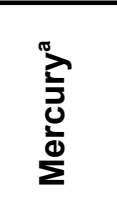 & $\begin{array}{l}\frac{\sigma}{0} \\
\frac{\bar{v}}{0} \\
\frac{0}{z}\end{array}$ & 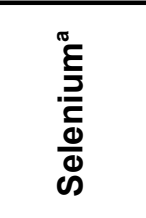 & $\sum_{\bar{c}}^{\frac{\pi}{\omega}}$ & 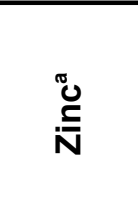 \\
\hline \multicolumn{3}{|c|}{ Final Action Levels } & 100,000 & 23 & 67,000 & 1,900 & 450 & 1,900 & 41,000 & 750 & 19,000 & 310 & 20,000 & 5,100 & 5,100 & 100,000 \\
\hline \multirow{4}{*}{ J09 } & $\overline{\overline{224 J 026}}$ & $0.5-2$ & 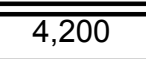 & 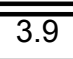 & 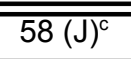 & $\overline{---}$ & $3.4(\mathrm{~J})^{\mathrm{c}}$ & $\overline{\overline{1.7}}$ & 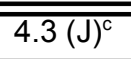 & $\overline{\overline{3.7}}$ & 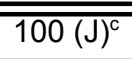 & 0.053 & $\overline{-\overline{--}}$ & 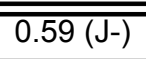 & $\overline{---}$ & 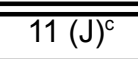 \\
\hline & 224J027 & $0.5-2$ & 4,400 & 4.1 & $58(\mathrm{~J})^{\mathrm{c}}$ & $\overline{--}$ & $3.4(\mathrm{~J})^{\mathrm{c}}$ & $\overline{1.7}$ & $4.6(\mathrm{~J})^{\mathrm{c}}$ & 3 & $100(\mathrm{~J})^{\mathrm{c}}$ & 0.088 & $\overline{--}$ & -- & -- & $11(\mathrm{~J})^{\mathrm{c}}$ \\
\hline & 224J028 & $4-5$ & 4,400 & 4.7 & $52(\mathrm{~J})^{\mathrm{c}}$ & -- & $3.3(\mathrm{~J})^{\mathrm{c}}$ & 1.9 & $5.1(\mathrm{~J})^{\mathrm{c}}$ & 1.6 & $76(\mathrm{~J})^{\mathrm{C}}$ & 0.11 & -- & -- & -- & $9.7(\mathrm{~J})^{\mathrm{c}}$ \\
\hline & 224J029 & $7-8$ & 4,300 & 5 & $63(\mathrm{~J})^{\mathrm{c}}$ & $\overline{--}$ & $2.9(\mathrm{~J})^{\mathrm{c}}$ & 1.9 & $6(J)^{c}$ & 2.5 & $64(\mathrm{~J})^{\mathrm{c}}$ & 0.043 & $\overline{--}$ & -- & $\overline{--}$ & $8.9(\mathrm{~J})^{\mathrm{c}}$ \\
\hline \multirow{3}{*}{$\mathrm{J} 10$} & $224 \mathrm{~J} 030$ & $0.5-2$ & 4,500 & 5.3 & 71 & $\overline{--}$ & 5.3 & 1.9 & 6.7 & 13 & 130 & 5.2 & 4.8 & -- & 34 & 21 \\
\hline & 224J031 & $4-5$ & 2,600 & 3 & 33 & -- & 4.6 & -- & 4.5 & 8.9 & 69 & 5.4 & 2.5 & -- & 53 & 14 \\
\hline & 224J032 & $7-8$ & 3,500 & 3.7 & 55 & $\overline{--}$ & 2.3 & 1.3 & 4.6 & 2.3 & 60 & 0.68 & 2.9 & -- & 4.4 & 6.6 \\
\hline \multirow{3}{*}{ J11 } & 224J033 & $0.5-2$ & 5,600 & 4.1 & 74 & $\overline{--}$ & 4.2 & 2.2 & 5.5 & 4.4 & 130 & -- & 4.8 & -- & -- & 12 \\
\hline & 224J034 & $4-5$ & 2,600 & 2.7 & 41 & -- & 2.5 & -- & 3.3 & -- & 59 & 0.061 & 2.5 & -- & 3.2 & 5.6 \\
\hline & 224J035 & $7-8$ & 3,600 & 5.9 & 58 & -- & 2.2 & 1.3 & 3.8 & -- & 61 & -- & 2.8 & -- & 6.4 & 7 \\
\hline \multirow{3}{*}{ J12 } & 224J036 & $0.5-2$ & 3,600 & 3.9 & 51 & -- & 2.7 & 1.4 & 3.6 & $1.8(\mathrm{~J}-)$ & 95 & -- & 3.2 & -- & -- & 8.4 \\
\hline & 224J037 & $4-5$ & 3,000 & 3.1 & 43 & -- & 2.9 & 1.2 & 3.6 & $2.9(\mathrm{~J}-)$ & 78 & 0.37 & 2.8 & -- & 4.1 & 8.1 \\
\hline & 224J038 & $7-8$ & 2,600 & 4.2 & 38 & -- & 1.9 & 1.1 & 2.8 & -- & 60 & 0.039 & 2.2 & -- & -- & 5.8 \\
\hline
\end{tabular}

${ }^{a}$ Based on U.S. Environmental Protection Agency, Region 9 Preliminary Remediation Goals (PRGs) (EPA, 2002)

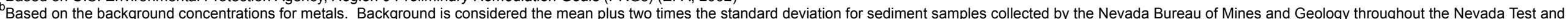
Training Range (NBMG, 1998; Moore, 1999).

${ }^{c}$ Qualifier added to laboratory data; record accepted. Serial dilution \%D outside control limits. Matrix effects may exist.

dualifier added to laboratory data; record accepted. Matrix spike reDevery outside control limits. Duplicate precision analysis (relative percent difference) outside control limits.

${ }^{e}$ Qualifier added to laboratory data; record accepted. Serial dilution \%D outside control limits. Value $<5$ times contamination in continuing calibration/method blank. Matrix effects may exist.

$\mathrm{ft}$ bgs $=$ Feet below ground surface

$\mathrm{mg} / \mathrm{kg}=$ Milligrams per kilogram

$J=$ Estimated value.

$\mathrm{J}-=$ The result is an estimated quantity, but the result may be biased low. Negative bias found in continuing calibration/method blank.

-- = Not detected above minimum reporting limits. 
Table A.9-5

Soil Sample Results for PCBs Detected Above Minimum Reporting Limits at CAS 23-05-02, Leachfield

\begin{tabular}{||c|c|c|c||}
\hline \multirow{2}{*}{$\begin{array}{c}\text { Sample } \\
\text { Location }\end{array}$} & $\begin{array}{c}\text { Sample } \\
\text { Number }\end{array}$ & $\begin{array}{c}\text { Depth } \\
(\mathrm{ft} \text { bgs) }\end{array}$ & Contaminants of Potential Concern $(\mu \mathrm{g} / \mathrm{kg})$ \\
\cline { 3 - 4 } & & & Aroclor 1260 \\
\hline \multicolumn{3}{|c||}{ Final Action Levels } & $\mathbf{7 4 0}$ \\
\hline \hline \multirow{2}{*}{$\mathrm{J} 10$} & $224 \mathrm{~J} 030$ & $0.5-2$ & $44(\mathrm{~J})$ \\
\cline { 2 - 4 } & $224 \mathrm{~J} 031$ & $4-5$ & $41(\mathrm{~J})$ \\
\hline
\end{tabular}

aBased on U.S. Environmental Protection Agency, Region 9 Preliminary Remediation Goals (PRGs) (EPA, 2002)

$\mathrm{ft}$ bgs = Feet below ground surface

$\mu \mathrm{g} / \mathrm{kg}=$ Micrograms per kilogram

$\mathrm{J}=$ Estimated value. Qualifier added to laboratory data; record accepted. \%D between columns $>25$.

Table A.9-6

Soil Sample Results for TPH-DRO Detected Above Minimum Reporting Limits at CAS 23-05-02, Leachfield (Page 1 of 2)

\begin{tabular}{|c|c|c|c|}
\hline \multirow{2}{*}{$\begin{array}{l}\text { Sample } \\
\text { Location }\end{array}$} & \multirow{2}{*}{$\begin{array}{l}\text { Sample } \\
\text { Number }\end{array}$} & \multirow{2}{*}{$\begin{array}{l}\text { Depth } \\
\text { (ft bgs) }\end{array}$} & Contaminants of Potential Concern (mg/kg) \\
\hline & & & Diesel-Range Organics \\
\hline \multicolumn{3}{|c|}{ Final Action Levels ${ }^{a}$} & 100 \\
\hline \multirow{3}{*}{ J01 } & 224J001 & $0-2$ & $85(\mathrm{M})$ \\
\hline & 224J002 & $0-2$ & $46(\mathrm{M})$ \\
\hline & 224J003 & $4-5$ & $38(\mathrm{M})$ \\
\hline $\mathrm{J} 02$ & 224J005 & $0.5-2$ & $140(\mathrm{M})$ \\
\hline \multirow{2}{*}{$\mathrm{J} 03$} & 224J008 & $0.5-2$ & $160(\mathrm{M})$ \\
\hline & 224J009 & $4-5$ & $130(\mathrm{H}, \mathrm{M}, \mathrm{Z})$ \\
\hline \multirow{2}{*}{$\mathrm{J} 05$} & 224J014 & $0.5-2$ & $27(\mathrm{M})$ \\
\hline & 224J015 & $4-5$ & $77(\mathrm{M})$ \\
\hline \multirow{2}{*}{$\mathrm{J} 08$} & 224J023 & $0.5-2$ & $130(\mathrm{M})$ \\
\hline & 224J024 & $4-5$ & $30(\mathrm{M})$ \\
\hline J09 & 224J026 & $0.5-2$ & $26(\mathrm{M})$ \\
\hline \multirow{2}{*}{ J10 } & 224J030 & $0.5-2$ & $120(\mathrm{M}, \mathrm{Z})$ \\
\hline & 224J031 & $4-5$ & $68(\mathrm{M}, \mathrm{Z})$ \\
\hline J11 & 224J033 & $0.5-2$ & $69(\mathrm{M})$ \\
\hline J12 & 224J037 & $4-5$ & $65(\mathrm{M})$ \\
\hline $\mathrm{J} 14$ & 224J039 & $0.5-1.5$ & $110(\mathrm{M})$ \\
\hline J15 & 224J041 & $0.5-1.5$ & $130(\mathrm{M})$ \\
\hline
\end{tabular}


Table A.9-6

Soil Sample Results for TPH-DRO Detected Above
Minimum Reporting Limits at CAS 23-05-02, Leachfield (Page 2 of 2)

\begin{tabular}{||c|c|c|c||}
\hline \multirow{2}{*}{$\begin{array}{c}\text { Sample } \\
\text { Location }\end{array}$} & $\begin{array}{c}\text { Sample } \\
\text { Number }\end{array}$ & \multirow{2}{*}{$\begin{array}{c}\text { Depth } \\
\text { (ft bgs) }\end{array}$} & Contaminants of Potential Concern (mg/kg) \\
\cline { 3 - 4 } & & & Diesel-Range Organics \\
\hline \multicolumn{3}{|c|}{ Final Action Levels ${ }^{\mathrm{a}}$} & $\mathbf{1 0 0}$ \\
\hline \hline $\mathrm{J} 16$ & $224 \mathrm{~J} 043$ & $0.5-1.5$ & $61(\mathrm{M})$ \\
\hline \multirow{2}{*}{$\mathrm{J} 17$} & $224 \mathrm{~J} 045$ & $0.5-1.5$ & $49(\mathrm{M})$ \\
\cline { 2 - 4 } & $224 \mathrm{~J} 046$ & $0.5-1.5$ & $53(\mathrm{M})$ \\
\hline $\mathrm{J} 18$ & $224 \mathrm{~J} 047$ & $0.5-1.5$ & $25(\mathrm{M})$ \\
\hline
\end{tabular}

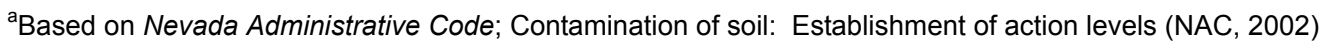

$\mathrm{ft}$ bgs $=$ Feet below ground surface

$\mathrm{mg} / \mathrm{kg}=$ Milligrams per kilogram

$\mathrm{H}=\mathrm{DRO}$ fuel pattern in the heavy end of retention time window.

$M=A$ pattern resembling motor oil was detected.

$Z=A$ significant fraction of the reported result did not resemble the patterns of any of the following petroleum hydrocarbon products: gasoline, JP-4, JP-8, diesel, mineral spirits, motor oil, Stoddard solvent, Bunker C.

Table A.9-7

Soil Sample Results for Gamma-Emitting Radionuclides Detected Above Minimum Detectable Concentrations at CAS 23-05-02, Leachfield (Page 1 of 2)

\begin{tabular}{|c|c|c|c|c|c|c|c|c|}
\hline \multirow{2}{*}{$\begin{array}{c}\text { Sample } \\
\text { Location }\end{array}$} & \multirow{2}{*}{$\begin{array}{l}\text { Sample } \\
\text { Number }\end{array}$} & \multirow{2}{*}{$\begin{array}{c}\text { Depth } \\
\text { (ft bgs) }\end{array}$} & \multicolumn{6}{|c|}{ Contaminants of Potential Concern (pCi/g) } \\
\hline & & & Aluminum-26 & Bismuth-214a & Cesium-137 & Lead-212 & Lead-214 ${ }^{\mathrm{a}}$ & Thallium-208 \\
\hline \multicolumn{3}{|c|}{ Depth bgs (cm) } & 2.3 & $>15$ & 12.2 & $>15$ & $>15$ & $>15$ \\
\hline \multirow{2}{*}{ J02 } & 224J006 & $4-5$ & -- & $0.313(\mathrm{~J})$ & -- & 0.223 & $0.442(\mathrm{~J})$ & -- \\
\hline & 224J007 & $7-8$ & -- & $0.47(\mathrm{G}, \mathrm{J})$ & -- & -- & $0.67(\mathrm{G}, \mathrm{J})$ & -- \\
\hline J03 & 224J008 & $0.5-2$ & -- & -- & -- & 0.54 & $0.55(\mathrm{~J})$ & -- \\
\hline \multirow[t]{2}{*}{ J05 } & 224J015 & $4-5$ & $\overline{--}$ & $0.49(\mathrm{~J})$ & -- & 0.26 & $0.46(\mathrm{~J})$ & -- \\
\hline & 224J016 & $7-8$ & -- & $0.63(\mathrm{G}, \mathrm{J})$ & -- & $0.47(\mathrm{~J})^{\mathrm{c}}$ & $0.59(\mathrm{G}, \mathrm{J})$ & -- \\
\hline
\end{tabular}




\section{Table A.9-7 \\ Soil Sample Results for Gamma-Emitting Radionuclides Detected Above Minimum Detectable Concentrations at CAS 23-05-02, Leachfield}

(Page 2 of 2)

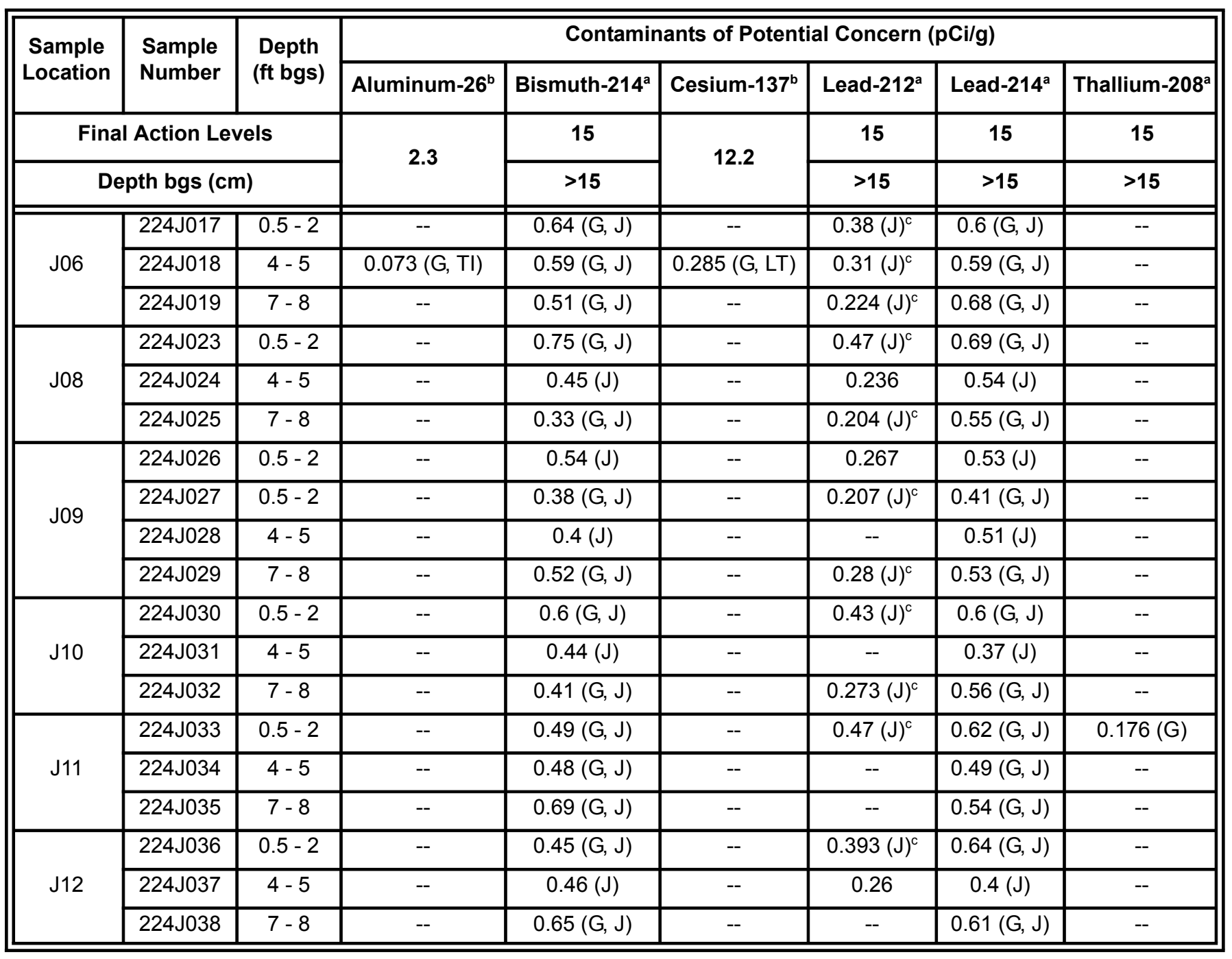

ataken from the generic guidelines for residual concentrations of aluminum-26, bismuth-214, lead-212, lead-214, thallium-208, and thorium-232, as found in Chapter IV of DOE Order 5400.5, Change 2, "Radiation Protection of the Public and Environment." (DOE, 1993). The FALs for these isotopes is specified as 5 $\mathrm{pCi} / \mathrm{g}$ averaged over the first $15 \mathrm{~cm}$ of soil and $15 \mathrm{pCi} / \mathrm{g}$ for deeper soils (DOE, 1993). For purposes of this document, $15 \mathrm{~cm}$ is assumed to be equivalent to $0.5 \mathrm{ft}$ (6 in.); therefore, $5 \mathrm{pCi} / \mathrm{g}$ represents the FALs for these radionuclides in the surface soil ( 0 to $0.5 \mathrm{ft}$ depth).

b Taken from the construction, commercial, industrial land use scenario in Table 2.1 of the NCRP Report No. 129, Recommended Screening Limits for

Contaminated Surface Soil and Review Factors Relevant to Site-Specific Studies (NCRP, 1999). The values provided in this source document were scaled to a 25-mrem/yr dose.

${ }^{\mathrm{c}}$ Qualifier added to laboratory data; record accepted. Sample does not meet counting geometry requirements.

$\mathrm{ft}$ bgs $=$ Feet below ground surface

$\mathrm{cm}=$ Centimeter

mrem = Millirems per year

$\mathrm{pCi} / \mathrm{g}=$ Picocuries per gram

-- = Not detected above minimum reporting limits

$>=$ Greater than

$\mathrm{G}=$ Sample density differs by more than 15 percent of laboratory control sample density.

$\mathrm{J}=$ Estimated value.

$\mathrm{LT}=$ Result is less than the requested minimum detectable concentration, greater than the sample specific minimum detectable

concentration.

$\mathrm{TI}=$ Tentatively identified 


\section{Table A.9-8 \\ Soil Sample Results for Isotopes Detected Above Minimum Detectable Concentrations at CAS 23-05-02, Leachfield} (Page 1 of 2)

\begin{tabular}{|c|c|c|c|c|c|c|}
\hline \multirow{2}{*}{$\begin{array}{l}\text { Sample } \\
\text { Location }\end{array}$} & \multirow{2}{*}{$\begin{array}{l}\text { Sample } \\
\text { Number }\end{array}$} & \multirow{2}{*}{$\begin{array}{c}\text { Depth } \\
\text { (ft bgs) }\end{array}$} & \multicolumn{4}{|c|}{ Contaminants of Potential Concern $(\mathrm{pCi} / \mathrm{g})$} \\
\hline & & & Plutonium-239 & Uranium-234 & Uranium-235 & Uranium-238 \\
\hline \multicolumn{3}{|c|}{ Final Action Levels ${ }^{a}$} & 12.7 & 143 & 17.6 & 105 \\
\hline \multirow{4}{*}{ J01 } & $224 \mathrm{~J} 001$ & $\overline{0-2}$ & 0.052 & $\overline{0.53}$ & 0.058 & $\overline{0.5}$ \\
\hline & 224J002 & $0-2$ & $\begin{array}{l}- \\
-\end{array}$ & 0.55 & -- & 0.5 \\
\hline & 224J003 & $4-5$ & $\overline{--}$ & 0.47 & $\overline{--}$ & 0.51 \\
\hline & 224J004 & $7-8$ & -- & 0.76 & -- & 0.59 \\
\hline \multirow{3}{*}{$\mathrm{J} 02$} & 224J005 & $0.5-2$ & $\begin{array}{l}- \\
-\end{array}$ & 0.61 & -- & 0.54 \\
\hline & 224J006 & $4-5$ & -- & 0.51 & -- & 0.46 \\
\hline & 224J007 & $7-8$ & $\overline{--}$ & 0.53 & $\overline{--}$ & 0.53 \\
\hline \multirow{3}{*}{$\mathrm{J} 03$} & 224J008 & $0.5-2$ & -- & 0.57 & -- & 0.48 \\
\hline & 224J009 & $4-5$ & 0.5 & 0.7 & $\overline{--}$ & 0.58 \\
\hline & 224J010 & $7-8$ & -- & 0.65 & 0.045 (LT) & 0.53 \\
\hline \multirow{3}{*}{$\mathrm{J} 05$} & 224J014 & $0.5-2$ & $\overline{--}$ & 0.52 & $\overline{--}$ & 0.53 \\
\hline & 224J015 & $4-5$ & -- & 0.56 & $0.043(\mathrm{LT})$ & 0.58 \\
\hline & 224J016 & $7-8$ & -- & 0.77 & $\overline{--}$ & 0.64 \\
\hline \multirow{3}{*}{$\mathrm{J} 06$} & 224J017 & $0.5-2$ & -- & 0.57 & -- & 0.58 \\
\hline & 224J018 & $4-5$ & 0.43 & 0.58 & $\overline{--}$ & 0.54 \\
\hline & 224J019 & $7-8$ & -- & $0.76(\mathrm{M} 3)$ & -- & 0.57 \\
\hline \multirow{3}{*}{$\mathrm{J} 08$} & 224J023 & $0.5-2$ & $\overline{--}$ & 0.51 & $\overline{--}$ & 0.45 \\
\hline & 224J024 & $4-5$ & 0.077 & 0.61 & $\overline{--}$ & 0.57 \\
\hline & 224J025 & $7-8$ & -- & 0.57 & -- & 0.41 \\
\hline \multirow{4}{*}{ J09 } & 224J026 & $0.5-2$ & -- & 0.7 & -- & 0.71 \\
\hline & $224 \mathrm{~J} 027$ & $0.5-2$ & -- & 0.6 & -- & 0.52 \\
\hline & 224J028 & $4-5$ & -- & 0.49 & -- & 0.404 \\
\hline & 224J029 & $7-8$ & $\overline{--}$ & 0.56 & $\overline{--}$ & 0.46 \\
\hline \multirow{3}{*}{ J10 } & 224J030 & $0.5-2$ & $0.171(\mathrm{~J})$ & 0.51 & -- & 0.53 \\
\hline & 224J031 & $4-5$ & $0.117(\mathrm{~J})$ & 0.44 & $0.043(\mathrm{LT})$ & 0.45 \\
\hline & $224 \mathrm{~J} 032$ & $7-8$ & -0.001 & 0.46 & $0.041(\mathrm{LT})$ & 0.406 \\
\hline \multirow{3}{*}{$\mathrm{J} 11$} & $224 \mathrm{~J} 033$ & $0.5-2$ & $0.004(\mathrm{~J})$ & 0.51 & -- & 0.47 \\
\hline & 224J034 & $4-5$ & $0.007(\mathrm{~J})$ & 0.56 & $0.041(\mathrm{LT})$ & 0.47 \\
\hline & 224J035 & $7-8$ & $0.016(\mathrm{~J})$ & 0.75 & $\overline{--}$ & 0.58 \\
\hline
\end{tabular}




\section{Table A.9-8 \\ Soil Sample Results for Isotopes Detected Above Minimum Detectable Concentrations at CAS 23-05-02, Leachfield} (Page 2 of 2)

\begin{tabular}{||c|c|c|c|c|c|c||}
\hline \multirow{2}{*}{$\begin{array}{c}\text { Sample } \\
\text { Location }\end{array}$} & \multirow{2}{*}{$\begin{array}{c}\text { Sample } \\
\text { Number }\end{array}$} & \multirow{2}{*}{$\begin{array}{c}\text { Depth } \\
\text { (ft bgs) }\end{array}$} & \multicolumn{3}{|c|}{ Contaminants of Potential Concern (pCi/g) } \\
\cline { 4 - 7 } & & & Plutonium-239 & Uranium-234 & Uranium-235 & Uranium-238 \\
\hline \multicolumn{3}{|c|}{ Final Action Levels ${ }^{\mathrm{a}}$} & $\mathbf{1 2 . 7}$ & $\mathbf{1 4 3}$ & $\mathbf{1 7 . 6}$ & $\mathbf{1 0 5}$ \\
\hline \hline \multirow{3}{*}{$\mathrm{J} 12$} & 224J036 & $0.5-2$ & $0.009(\mathrm{~J})$ & 0.54 & -- & 0.47 \\
\cline { 2 - 7 } & 224J037 & $4-5$ & $0.013(\mathrm{~J})$ & 0.61 & -- & 0.5 \\
\cline { 2 - 7 } & 224J038 & $7-8$ & $0.004(\mathrm{~J})$ & 0.77 & -- & 0.61 \\
\hline
\end{tabular}

aTaken from the construction, commercial, industrial land use scenario in Table 2.1 of the NCRP Report No. 129 Recommended Screening Limits for Contaminated Surface Soil and Review Factors Relevant to Site-Specific Studies (NCRP, 1999). The values provided in this source document were scaled to a 25-mrem/yr dose.

$\mathrm{ft}$ bgs $=$ Feet below ground surface

$\mathrm{mrem} / \mathrm{yr}=$ Millirems per year

$\mathrm{pCi} / \mathrm{g}=$ Picocuries per gram

$\mathrm{J}=$ Estimated value. Duplicate precision analysis (relative percent difference) outside control limits.

$\mathrm{LT}=$ Result is less than the requested minimum detectable concentration, greater than the sample specific minimum detectable concentration.

M3 = The requested minimum detectable concentration was not met, but the reported activity is greater than the reported minimum detectable concentration.

-- = Not detected above minimum reporting limits 


\section{A.10.0 Waste Management}

Investigation-derived waste was generated during the field investigation activities of CAU 224. The waste streams generated include decontamination rinse water, disposable personal protective equipment (PPE), disposable sampling equipment, and miscellaneous waste removed as best management practice during the investigation activities. Investigation-derived waste was segregated to the greatest extent possible and waste minimization techniques were integrated into the field activities to reduce the amount of waste generated. Controls were in place to minimize the use of hazardous materials and the unnecessary generation of hazardous and/or mixed waste.

Decontamination activities were planned and executed to minimize the volume of rinsate generated.

The amount, type, and source of waste placed into each drum was recorded in waste management logbooks. Potentially hazardous waste generated during the investigation was placed in containers and labeled as "Hazardous Waste - Pending Analysis." Six Hazardous Waste Accumulation Areas (HWAAs) and two Satellite Accumulation Areas were established to manage hazardous and potentially hazardous waste generated during the CAI.

\section{A.10.1 Characterization}

Analytical results from associated field characterization samples were reviewed to ensure compliance with federal regulations, state regulations, DOE directives/policies, guidance, and waste disposal criteria.

\section{A.10.2 Waste Streams}

Investigation-derived waste generated during the investigation was segregated into the following waste streams:

- Personal Protective Equipment and disposable sampling equipment

- Decontamination rinsate

- Debris including, but not limited to: plastic sheeting, glass/plastic sample jars, PPE, soil, sampling scoops, aluminum foil, and bowls 


\section{A.10.3 Investigation-Derived Waste Generated}

A total of 13 drums of IDW were generated during the investigation:

- All the drums are characterized as non-hazardous waste. Currently, the drums are being reviewed to determine whether any will require disposal as radioactive waste exceeding landfill criteria.

Office waste and lunch trash was disposed of throughout the project at the NTS sanitary landfill. Sanitary industrial waste was inspected and disposed of in the NTS industrial waste landfill. Additional waste (e.g., decontamination pad liners) may be generated during completion of waste management activities and closure of the HWAAs.

\section{A.10.4 Waste Characterization Samples}

Waste characterization samples were collected from the septic tanks at CASs 02-04-01, 05-04-01, and 11-04-01, and from drummed waste, as necessary, to facilitate full characterization of the waste for disposal. Two samples were collected from the wastewater sump at CAS 06-17-04. Although these samples are classified as site characterization samples, the results will also be used for waste characterization. Results of waste characterization samples are not presented in this document except as necessary to support site characterization decisions. Complete results for all samples are maintained in project files. The following sections describe the waste characterization samples collected during the investigation of CAU 224.

\section{A.10.4.1 CAS 02-04-01, Septic Tank (Buried)}

One liquid sample was collected from the septic tank. The amount of sludge at the bottom of the tank was minimal (less than 2 in.); therefore, no sludge sample was collected. The analytical results indicated that the liquid in the tank is not a RCRA hazardous waste does not contain radioactive material above the NTS landfill acceptance criteria.

\section{A.10.4.2 CAS 05-04-01, Septic Tanks (4)/Discharge Area}

One liquid and one sludge sample was collected from each of the four septic tanks in this CAS. The analytical results indicated that the liquid and sludge in the tanks are not RCRA hazardous waste and do not contain radioactive material above the NTS landfill acceptance criteria. 


\section{A.10.4.3 CAS 06-17-04, Decon Pad and Wastewater Catch}

One sludge sample was collected from each chamber of the wastewater sump attached to the decon pad. Both samples (224F042 and 224F043) contained radiological and chemical contamination above waste disposal criteria. Therefore the sludge may need to be disposed of as mixed waste.

\section{A.10.4.4 CAS 11-04-01, Sewage Lagoon}

Three liquid samples were collected at the CAS, one from each chamber of the septic tank, and one from the distribution box. The upstream tank chamber was the only one containing a significant amount of sludge; one sludge sample was collected there. Analytical results indicated the liquid and the sludge are not RCRA hazardous waste and do not contain radioactive material above the NTS landfill acceptance criteria. 


\section{A.11.0 Quality Assurance}

This section contains a summary of QA/QC measures implemented during the sampling and analysis activities conducted in support of the CAU 224 CAI. The following sections discuss the data validation process, QC samples, and nonconformances. A detailed evaluation of the DQIs is presented in Appendix B.

Laboratory analyses were conducted for samples used in the decision-making process to provide a quantitative measurement of any COPCs present. Rigorous QA/QC was implemented for all laboratory samples including documentation, verification and validation of analytical results, and affirmation of DQI requirements related to laboratory analysis. Detailed information regarding the QA program is contained in the Industrial Sites QAPP (NNSA/NV, 2002).

\section{A.11.1 Data Validation}

Data validation was performed in accordance with the Industrial Sites QAPP (NNSA/NV, 2002) and approved protocols and procedures. All laboratory data from samples collected and analyzed for CAU 224 were evaluated for data quality according to the EPA Functional Guidelines (EPA, 1994a and 1999). These guidelines are implemented in a tiered process and are presented in Section A.11.1.1, Section A.11.1.2, and Section A.11.1.3. Data were reviewed to ensure that samples were appropriately processed and analyzed, and the results were evaluated using validation criteria. Documentation of the data qualifications resulting from these reviews is retained in project files as a hard copy and electronic media.

One hundred percent of the data analyzed as part of this investigation were subjected to Tier I and Tier II evaluations. A Tier III evaluation was performed on approximately five percent of the data analyzed.

\section{A.11.1.1 Tier I Evaluation}

Tier I evaluation for chemical and radiochemical analysis examines, but is not limited to:

- Sample count/type consistent with chain of custody.

- Analysis count/type consistent with chain of custody. 
- Correct sample matrix.

- Significant problems stated in cover letter or case narrative.

- Completeness of certificates of analysis.

- Completeness of Contract Laboratory Program (CLP) or CLP-like packages.

- Completeness of signatures, dates, and times on chain of custody.

- Condition-upon-receipt variance form included.

- Requested analyses performed on all samples.

- Date received/analyzed given for each sample.

- Correct concentration units indicated.

- Electronic data transfer supplied.

- Results reported for field and laboratory QC samples.

- Whether or not the deliverable met the overall objectives of the project.

\section{A.11.1.2 Tier II Evaluation}

Tier II evaluation for chemical and radiochemical analysis examines, but is not limited to:

\section{Chemical:}

- Correct detection limits achieved.

- Sample date, preparation date, and analysis date for each sample.

- Holding time criteria met.

- Quality control batch association for each sample.

- Cooler temperature upon receipt.

- Sample $\mathrm{pH}$ for aqueous samples, as required.

- Detection limits properly adjusted for dilution, as required.

- Blank contamination evaluated and applied to sample results/qualifiers.

- Matrix spike (MS)/matrix spike duplicate (MSD) percent recoveries (\%R) and relative percent differences (RPDs) evaluated and qualifiers applied to laboratory results, as necessary.

- Field duplicate RPDs evaluated using professional judgment and qualifiers applied to laboratory results, as necessary.

- Laboratory duplicate RPDs evaluated and qualifiers applied to laboratory results, as necessary.

- Surrogate $\% \mathrm{R}$ evaluated and qualifiers applied to laboratory results, as necessary.

- Laboratory control sample \%R evaluated and qualifiers applied to laboratory results, as necessary. 
- Initial and continuing calibration evaluated and qualifiers applied to laboratory results, as necessary.

- Internal standard evaluation.

- Mass spectrometer tuning criteria.

- Organic compound quantitation.

- Inductively coupled plasma interference check sample evaluation.

- Graphite furnace atomic absorption quality control.

- Inductively coupled plasma serial dilution effects.

- Recalculation of 10 percent of laboratory results from raw data.

\section{Radioanalytical:}

- Correct detection limits achieved.

- Blank contamination evaluated and, if significant, qualifiers are applied to sample results.

- Certificate of Analysis consistent with data package documentation.

- Quality control sample results (duplicates, laboratory control samples, laboratory blanks) evaluated and used to determine laboratory result qualifiers.

- Sample results, uncertainty, and minimum detectable concentration evaluated.

- Detector system calibrated with National Institute for Standards and Technology (NIST)traceable sources.

- Calibration sources preparation was documented, demonstrating proper preparation and appropriateness for sample matrix, emission energies, and concentrations.

- Detector system response to daily or weekly background and calibration checks for peak energy, peak centroid, peak full-width half-maximum, and peak efficiency, depending on the detection system.

- Tracers NIST-traceable, appropriate for the analysis performed, and recoveries that met QC requirements.

- Documentation of all QC sample preparation complete and properly performed.

- Spectra lines, photon emissions, particle energies, peak areas, and background peak areas support the identified radionuclide and its concentration.

\section{A.11.1.3 Tier III}

The Tier III review is an independent examination of the Tier II evaluation. The Tier III review duplicates the Tier II review for a limited number of samples (typically 5 percent) by an independent agency and includes the following additional evaluations: 


\section{Chemical:}

- Recalculation of all laboratory results from raw data.

\section{Radioanalytical:}

- QC sample results (e.g., calibration source concentration, \%R, and RPD) verified.

- Radionuclides and their concentration validated as appropriate considering their decay schemes, half-lives, and process knowledge and history of the facility and site.

- Each identified line in spectra verified against emission libraries and calibration results.

- Independent identification of spectra lines, area under the peaks, and quantification of radionuclide concentration in a random number of sample results.

A Tier III review of 5 percent of the sample analytical data was performed by TechLaw, Inc., of Lakewood, Colorado. Tier II and Tier III results were compared and where differences were noted, data were reviewed and changes made accordingly.

\section{A.11.2 Field Quality Control Samples}

Field quality control samples consisted of 39 trip blanks, 3 equipment rinsate blanks, 7 field blanks, 1 source blank, $27 \mathrm{MS} / \mathrm{MSDs}$, and 27 field duplicates collected and submitted for analysis by the laboratory analytical methods shown in Table A.2-2. The QC samples were assigned individual sample numbers and sent to the laboratory "blind." Additional samples were selected by the laboratory to be analyzed as laboratory duplicates.

\section{A.11.2.1 Field Quality Control Samples}

Review of the field blank analytical data for soil sampling indicates that there was no cross-contamination due to transportation practices or the ambient conditions, and that decontamination was adequate.

During the sampling events, 27 field duplicates were sent as blind samples to the laboratory to be analyzed for the investigation parameters listed in Table A.2-2. For these samples, the duplicate results precision (i.e., RPDs between the environmental sample results and their corresponding field duplicate sample results) were evaluated to the guidance set forth in the EPA Functional Guidelines (EPA, 1994a). 


\section{A.11.2.2 Laboratory Quality Control Samples}

Analyses of method QC blanks were performed on each sample delivery group (SDG) for inorganics. Analyses for surrogate spikes and preparation blanks (PBs) were performed on each SDG for organics only. Initial and continuing calibration and laboratory control samples (LCSs) were performed for each SDG by Paragon Analytical, Inc. The results of these analyses were used to qualify associated environmental sample results according to the EPA Functional Guidelines (EPA, 1994a and 1999). Documentation of data qualifications resulting from the application of these guidelines is retained in project files as both hard copy and electronic media.

The laboratory included a PB, LCS, and a laboratory duplicate sample with each batch of field samples analyzed for radionuclides.

\section{A.11.3 Field Nonconformances}

There were no field nonconformances identified for the corrective action investigation.

\section{A.11.4 Laboratory Nonconformances}

Laboratory nonconformances are generally due to inconsistencies in the analytical instrumentation operation, sample preparations, extractions, missed holding times, and fluctuations in internal standard and calibration results. Thirty one nonconformances were issued by the laboratory that may or may not have resulted in qualifying data. These laboratory nonconformances have been accounted for during the data qualification process. 


\section{A.12.0 Summary}

Analytes detected in soil samples during the CAI were evaluated against FALs to determine the nature and extent of COCs for CAU 224. Assessment of the data generated from investigation activities indicates the FALs were exceeded in soil samples at several CAU 224 CASs. The following summarizes the results for each CAS.

\section{CAS 02-04-01, Septic Tank (Buried)}

Based on observations made and analytical results of samples collected at CAS 02-04-01, the septic tank and associated piping that make up CAS 02-04-01 are not contributing contamination to the surrounding soil that is not already present in the fill around the tank. That is, COPCs present at the site are due to asphalt in the fill around the tank.

\section{CAS 03-05-01, Leachfield}

Based on observations made and analytical results of samples collected at CAS 03-05-01, the Pu-239 contamination at the site is not a result of activities at the site. No other contaminants are present at CAS 03-05-01.

\section{CAS 05-04-01, Septic Tanks (4)/Discharge Area}

Based on observations made and analytical results of samples collected at CAS 05-04-01, pesticide contamination exists in the outfall/discharge area north of the tanks.

\section{CAS 06-03-01, Sewage Lagoons}

Based on observations made and analytical results of samples collected at CAS 06-03-01, no contamination is present at CAS 06-03-01.

\section{CASs 06-05-01, Leachfield, 06-17-04, Decon Pad and Discharge Area, and 06-23-01, Decon Pad} Discharge Piping

Based on observations made and analytical results of samples collected at these three CASs, radiological and TPH-DRO contamination is present in the soil at the sites, and waste that may need to be managed as mixed waste when generated is present in the wastewater sump. 


\section{CAS 11-04-01, Sewage Lagoon}

Based on observations made and analytical results of samples collected at CAS 11-04-01, no contamination is present at CAS 11-04-01.

\section{CAS 23-05-02, Leachfield}

Based on observations made and analytical results of samples collected at CAS 23-05-02, the former leachfield is not contributing contamination to the surrounding soil that is not already present. That is, COPCs present at the site are due to asphalt in the covering parking lot above the former leachfield. 


\section{A.13.0 References}

BN, see Bechtel Nevada.

Bechtel Nevada. 1995. Nevada Test Site Performance Objective for Certification of Nonradioactive Hazardous Waste, Rev. 0, G-E11/96.01. Las Vegas, NV.

CFR, see Code of Federal Regulations.

Code of Federal Regulations. 2004. Title 40 CFR, "Protection of Environment," Parts 260-282,

"Hazardous Waste Management." Washington, DC: U.S. Government Printing Office.

DOE, see U.S. Department of Energy.

DOE/NV, see U.S. Department of Energy, Nevada Operations Office.

EPA, see U.S. Environmental Protection Agency.

FFACO, see Federal Facility Agreement and Consent Order.

Federal Facility Agreement and Consent Order. 1996 (as amended). Agreed to by the State of Nevada, the U.S. Department of Energy, and the U.S. Department of Defense.

H\&N, see Holmes \& Narver, Inc.

Holmes \& Narver, Inc. 1974a. Engineering drawing 006-082-C7 entitled, "Sewer \& Steam Cleaning Effluent Sys., Yucca Lake Plan \& Profile,” 18 December. Mercury, NV: Archives and Records Center.

Holmes \& Narver, Inc. 1974b. Engineering drawing 006-082-C8 entitled, "Sewer \& Steam Cleaning Effluent Sys., Yucca Lake Plan \& Profile,” 18 December. Mercury, NV: Archives and Records Center.

Holmes \& Narver, Inc. 1974c. Engineering drawing 006-082-C6 entitled, "Sewer \& Steam Cleaning Effluent Sys., Yucca Lake Plot Plan \& Details,” 18 December. Mercury, NV: Archives and Records Center.

Holmes \& Narver, Inc. 1976. Engineering drawing JS-006-082-C9 entitled, "Nevada Test Site Area 6, 6" Sanitary Sewer Line Yucca Lake Plan Profile," Sheet 1 of 2, 22 April. Mercury, NV: Archives and Records Center.

Holmes \& Narver, Inc. 1983. Engineering drawing JS-006-624-C2 entitled, "Nevada Test Site Area 6 Heavy Duty Drilling Repair Facility, Bldg. 6-624 Plot Plan and Detail," Sheet 2 of 25, 22 April. Mercury, NV: Archives and Records Center. 
Moore, J., Science Applications International Corporation. 1999. Memorandum to M. Todd (SAIC), "Background Concentrations for NTS and TTR Soil Samples," 3 February. Las Vegas, NV.

NAC, see Nevada Administrative Code.

NBMG, see Nevada Bureau of Mines and Geology.

NCRP, see National Council on Radiation Protection and Measurements.

NDEP, see Nevada Division of Environmental Protection.

NNSA/NV, see U.S. Department of Energy, National Nuclear Security Administration Nevada Operations Office.

NNSA/NSO, see U.S. Department of Energy, National Nuclear Security Administration Nevada Site Office.

National Council on Radiation Protection and Measurements. 1999. Recommended Screening Limits for Contaminated Surface Soil and Review of Factors Relevant to Site-Specific Studies, Report No. 129. Bethesda, MD.

Nevada Administrative Code. 2002. NAC 445A.2272, "Contamination of Soil: Establishment of Action Levels." Carson City, NV.

Nevada Bureau of Mines and Geology. 1998. Mineral and Energy Resource Assessment of the Nellis Air Force Range, Open-File Report 98-1. Reno, NV.

REECo, see Reynolds Electrical \& Engineering Co., Inc.

RSN, see Raytheon Services Nevada.

Raytheon Services Nevada. 1992. Engineering Drawing JS-006-002-C7 entitled "Area 6 Yucca Lake Facilities Map,” 29 May. Mercury, NV: Archives and Records Center.

Reynolds Electrical \& Engineering Co., Inc. 1971. Engineering Drawing 6-CH-C1 entitled "Nevada Test Site - Area 6 Craft Change House Architectural Plan - Detail,” 21 April. Mercury, NV: Archives and Records Center.

Shaw, see Shaw Environmental, Inc.

SNJV, see Stoller-Navarro Joint Venture.

Shaw Environmental, Inc. 2002. Site sketch from the CAU 224 Project Files. Las Vegas, NV. 
Stoller-Navarro Joint Venture. 2004. Site-Specific Health and Safety Plan for CAU 224, Decon Pad and Septic Systems, Nevada Test Site, Nevada. Las Vegas, NV.

USGS, see U.S. Geological Survey.

U.S. Department of Energy. 1993. DOE Order 5400.5, Change 2, "Radiation Protection of the Public and the Environment." Washington, DC.

U.S. Department of Energy, National Nuclear Security Administration Nevada Operations Office. 2002. Industrial Sites Quality Assurance Project Plan, Nevada Test Site, Nevada, Rev. 3, DOE/NV--372. Las Vegas, NV.

U.S. Department of Energy, National Nuclear Security Administration Nevada Site Office. 2004. Corrective Action Investigation Plan for Corrective Action Unit 224: Decon Pad and Septic Systems, Nevada Test Site, Nevada, DOE/NV--965, Rev. 0. Las Vegas, NV.

U.S. Environmental Protection Agency. 1994a. Contract Laboratory Program National Functional Guidelines for Inorganic Data Review, EPA/540/R-94/013. Washington, DC.

U.S. Environmental Protection Agency. 1994b. Guidance for the Data Quality Objectives Process, EPA QA/G-4. Washington, DC.

U.S. Environmental Protection Agency. 1996. Test Methods for Evaluating Solid Waste, Physical/Chemical Methods, SW-846, 3rd Edition, CD-ROM PB97-501928GEI. Washington, DC.

U.S. Environmental Protection Agency. 1999. Contract Laboratory Program National Functional Guidelines for Organic Data Review, EPA 540/R-99/008. Washington, DC.

U.S. Environmental Protection Agency. 2002 (as revised). Region 9 Preliminary Remediation Goals (PRGs). As accessed at www.epa.gov/region09/waste/sfund/prg/htm on 8 January 2004.

U.S. Geological Survey. 1975. Hydrogeologic and Hydrochemical Framework, South Central Great Basin Nevada-California, with Special Reference to the Nevada Test Site, U.S. Geological Survey Professional Paper 712-C. Prepared by I.J. Winograd and W. Thordarson. Denver, CO. 
Appendix B

Data Assessment 


\section{B.1.0 Data Assessment}

The DQA process is the scientific evaluation of the actual investigation results to determine whether the DQO criteria established in the CAU 224 CAIP were met and whether DQO decisions can be resolved at the desired level of confidence. The DQO process ensures that the right type, quality, and quantity of data will be available to support the resolution of those decisions at an appropriate level of confidence. Using both the DQO and DQA processes help to ensure that DQO decisions are sound and defensible.

The DQA involves five steps that begin with a review of the DQOs and end with an answer to the DQO decisions. The five steps are briefly summarized as follows:

Step 1: Review DQOs and Sampling Design - Review the DQO process to provide context for analyzing the data. State the primary statistical hypotheses; confirm the limits on decision errors for committing false rejection (Type I) or false acceptance (Type II) decision errors; and review any special features, potential problems, or any deviations to the sampling design.

Step 2: Conduct a Preliminary Data Review - A preliminary data review should be performed by reviewing QA reports and inspecting the data both numerically and graphically, validating and verifying the data to ensure that the measurement systems performed in accordance with the criteria specified, and using the validated data set to determine whether the quality of the data is satisfactory.

Step 3: Select the Test - Select the test based on the population of interest, population parameter, and the hypotheses. Identify the key underlying assumptions that could cause a change in one of the DQO decisions.

Step 4: Verify the Assumptions - Perform tests of assumptions. If data are missing or are censored, determine the impact on DQO decision error.

Step 5: Draw Conclusions from the Data - Perform the calculations required for the test. 


\section{B.1.1 Review DQOs and Sampling Design}

This section contains a review of the DQO process presented in Appendix A of the CAU 224 CAIP (NNSA/NSO, 2004). The DQO decisions are presented with the DQO provisions to limit false rejection (false negative) or false acceptance (false positive) decision errors. Special features, potential problems, or any deviations to the sampling design are also presented.

\section{B.1.1.1 Decision I}

Decision I - "Is a COC present in environmental media within the CAS at a concentration that could pose an unacceptable risk to human health and the environment?"

\section{Decision Rules:}

- If the population parameter of any COPC in a target population exceeds the PAL for that $\mathrm{COPC}$, then that COPC is identified as a COC, and Decision II samples will be collected and the extent determined.

- If COPC concentrations are less than the corresponding PAL, then the decision will be no further action.

Population Parameter: The maximum observed sample result

\section{B.1.1.1 DQO Provisions To Limit False Negative Decision Error}

A false rejection decision error (where consequences are more severe) was controlled by meeting the following criteria:

1. Having a high degree of confidence that locations selected will identify COCs if present anywhere within the CAS.

2. Having a high degree of confidence that analyses conducted will be sufficient to detect any COCs present in the samples.

3. Having a high degree of confidence that the data set is of sufficient quality and completeness. 


\section{Criteria 1:}

The following criteria (stipulated in the CAU 224 DQOs [NNSA/NSO, 2004]) were used in selecting sample locations.

1. Selection of sampling locations associated with field-screening results was accomplished by analyzing samples for TPH-DRO using a gas chromatograph, VOCs using an FID, alpha- and beta/gamma-emitting radionuclides using a hand-held NE Technology Electra, and gamma-emitting radionuclides using gamma spectroscopy.

2. Selection of sampling locations associated with piping was accomplished by performing visual inspections of the pipes, pipe joints and breaches in pipes, as well as video mole surveys.

3. Selection of sampling locations based on specific site features (e.g., leachfield piping, distribution boxes) was accomplished by reviewing "as-built" engineering drawings, aerial photographs, and/or geophysical survey results.

4. Selection of sampling locations associated with outfalls was accomplished by identifying the following two areas:

- A: At the discharge point of the outfall

- B: Downgradient from the discharge

5. Selection of sampling locations associated with professional judgment based on acceptable knowledge was accomplished by:

- Source and location of release

- Chemical nature and fate properties

- Physical transport pathways and properties

- Transport drivers

6. Selection of liquid and sludge sampling locations was accomplished by visual observation of the tanks, distribution boxes, and/or sumps.

Decision I sample locations are presented in relation to this criteria in Table B.1-1. 
Table B.1-1

Decision I Sample Location Justification

\begin{tabular}{|c|c|c|c|}
\hline CAS & $\begin{array}{l}\text { Sample } \\
\text { Location }\end{array}$ & Justification & Notes \\
\hline \multirow{3}{*}{ 02-04-01 } & Tank1 & 6 & Liquid sampled from septic tank \\
\hline & A01 through A07 & 3,5 & $\begin{array}{l}\text { Sample locations based on engineering drawings of the septic } \\
\text { systems and professional judgement }\end{array}$ \\
\hline & $\mathrm{A} 08$ and $\mathrm{A} 09$ & 2 & Sampled beneath excavated pipe tie-ins with possible breaches \\
\hline 03-05-01 & B01 through B03 & 3 & \\
\hline \multirow{3}{*}{ 05-04-01 } & C01 through C04 & $4 \mathrm{~A}$ & Sampled beneath overflow pipe in wash \\
\hline & C05 and C06 & 4B & Sampled downgradient in wash/outfall area \\
\hline & $\begin{array}{l}\text { Tank1 through } \\
\text { Tank } 4\end{array}$ & 6 & Septic Tanks 1 through 4 \\
\hline 06-03-01 & D01 through D15 & 3 & \\
\hline \multirow{3}{*}{ 06-05-01 } & E01 through E12 & 3 & \\
\hline & E13 through E16 & 2,3 & Sample locations associated with excavated leachfield pipe \\
\hline & E17 through E28 & 3 & \\
\hline \multirow{2}{*}{$06-17-04$} & F01 through F13 & 3,5 & Samples locations placed on and around the decon pad \\
\hline & $\mathrm{F} 14$ and $\mathrm{F} 15$ & 1,6 & Sample taken from bottom of wastewater sump \\
\hline \multirow{3}{*}{ CAS 06-23-01 } & G01 & 2 & Sample taken beneath the wastewater sump outlet pipe \\
\hline & G02 & 2,3 & \\
\hline & G03 & 3 & \\
\hline \multirow{9}{*}{ CAS 11-04-01 } & $\mathrm{H} 01$ and $\mathrm{H} 02$ & 3 & Sample locations associated with former sewage pit \\
\hline & $\mathrm{H} 03$ & 3 & \\
\hline & $\mathrm{H} 04$ & 2 & Samples collected above and below the inlet pipe to the tank \\
\hline & $\mathrm{H} 05$ & 3 & \\
\hline & $\mathrm{H} 06$ & 2 & Samples collected above and below the outlet pipe to the tank \\
\hline & $\mathrm{H} 07$ through $\mathrm{H} 21$ & 2 & Locations $\mathrm{H} 07$ through $\mathrm{H} 21$ are associated with leachfield pipe \\
\hline & $\mathrm{H} 22$ and $\mathrm{H} 23$ & 3 & Sample locations associated with former sewage pit \\
\hline & $\begin{array}{l}\text { Tank1 and } \\
\text { Tank2 }\end{array}$ & 7 & Septic tank compartments 1 and 2 \\
\hline & Tank3 & 7 & Distribution box \\
\hline \multirow{4}{*}{ CAS 23-05-02 } & $\mathrm{J} 01$ & 2,3 & Sample taken near plugged drain of leachfield inlet pipe \\
\hline & J02 and J03 & 3 & \\
\hline & J05 and J06 & 3 & \\
\hline & J08 through J12 & 3 & \\
\hline
\end{tabular}




\section{Criteria 2:}

All samples were analyzed using the analytical methods listed in Table A.1-6 of the CAIP and for the chemical and radiological parameters listed in the table. Table B.1-2 provides a reconciliation of samples analyzed to the planned analytical program. All analyses that were required by the CAIP were collected and analyzed.

Table B.1-2

CAU 224 Analytical Program

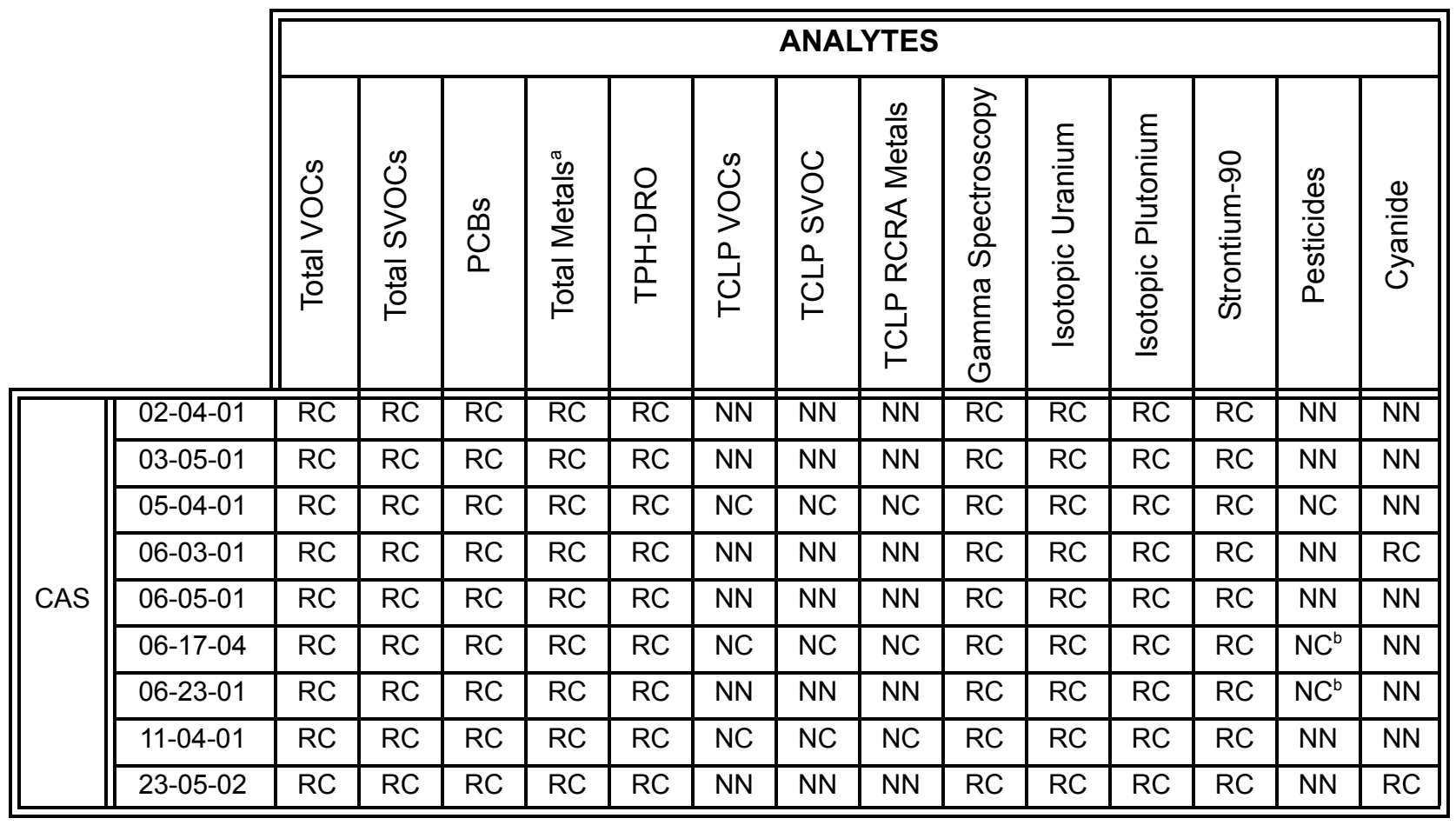

${ }^{\mathrm{a}}$ Total Metals = RCRA Metals plus Aluminum, Antimony, Beryllium, Cobalt, Copper, Manganese, Molybdenum, Nickel and Zinc ${ }^{\mathrm{b}}$ Technical Chlordane Only

$\mathrm{RC}=$ Required , Collected

$\mathrm{NC}=$ Not Required, Collected

$\mathrm{NN}=$ Not Required, Not Collected

$\mathrm{RN}=$ Required, Not Collected

$\mathrm{DRO}=$ Diesel-range organics

$\mathrm{PCB}=$ Polychlorinated biphenyl

RCRA = Resource Conservation and Recovery Act

SVOC = Semivolatile organic compound

TCLP = Toxicity characterization leaching procedure

$\mathrm{TPH}=$ Total petroleum hydrocarbons

VOC $=$ Volatile organic compound 
Sample results were assessed against the DQI of sensitivity as defined in the Industrial Sites QAPP (NNSA/NV, 2002). The sensitivity goal defined in the CAIP is that analytical detection limits will be less than the corresponding action level. This goal was not achieved for the analytical results listed in Table B.1-3 and Table B.1-4. Results not meeting the sensitivity goal will not be used in making DQO decisions and will therefore be considered as rejected data. The impact on DQO decisions is addressed in the assessment of completeness.

Table B.1-3

Chemical Analytes Failing Sensitivity Criteria

(Page 1 of 3)

\begin{tabular}{|c|c|c|c|c|c|}
\hline $\begin{array}{l}\text { Sample } \\
\text { Number }\end{array}$ & Parameter & Result & Units & $\begin{array}{l}\text { Detect } \\
\text { Limit }\end{array}$ & $\begin{array}{c}\text { Industrial } 2002 \\
\text { PRG }\end{array}$ \\
\hline$\overline{\overline{\text { 224A020RR1 }}}$ & "N-Nitrosodimethylamine & $\overline{3,500}$ & $\overline{\mu \mathrm{gg} / \mathrm{kg}}$ & 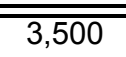 & $\overline{\overline{34}}$ \\
\hline 224B011RR1 & N-Nitrosodimethylamine & 170 & $\mu \mathrm{g} / \mathrm{kg}$ & 170 & 34 \\
\hline 224C003RR1 & Toxaphene & 34,000 & $\mu \mathrm{g} / \mathrm{kg}$ & 34,000 & 1,600 \\
\hline 224C004RR1 & Toxaphene & 85,000 & $\mu \mathrm{g} / \mathrm{kg}$ & 85,000 & 1,600 \\
\hline 224F023RR1 & Benzo(a)Pyrene & 4,200 & $\mu \mathrm{g} / \mathrm{kg}$ & 4,200 & 210 \\
\hline 224F023RR1 & Dibenzo(a,h)Anthracene & 4,200 & $\mu \mathrm{g} / \mathrm{kg}$ & 4,200 & 210 \\
\hline 224F023RR1 & N-Nitrosodimethylamine & 690 & $\mu \mathrm{g} / \mathrm{kg}$ & 690 & 34 \\
\hline 224F023RR1 & N-Nitroso-Di-N-Propylamine & 4,800 & $\mu \mathrm{g} / \mathrm{kg}$ & 4,800 & 250 \\
\hline 224F027RR1 & Benzo(a)Pyrene & 4,200 & $\mu \mathrm{g} / \mathrm{kg}$ & 4,200 & 210 \\
\hline 224F027RR1 & Dibenzo(a,h)Anthracene & 4,200 & $\mu \mathrm{g} / \mathrm{kg}$ & 4,200 & 210 \\
\hline 224F027RR1 & N-Nitrosodimethylamine & 710 & $\mu \mathrm{g} / \mathrm{kg}$ & 710 & 34 \\
\hline 224F027RR1 & N-Nitroso-Di-N-Propylamine & 4,900 & $\mu \mathrm{g} / \mathrm{kg}$ & 4,900 & 250 \\
\hline 224F042 & 1,2-Dibromoethane & 470 & $\mu \mathrm{g} / \mathrm{kg}$ & 470 & 28 \\
\hline 224F042 & 1,2,3-Trichloropropane & 470 & $\mu \mathrm{g} / \mathrm{kg}$ & 470 & 11 \\
\hline $224 \mathrm{~F} 042$ & Dibenzo(a,h)Anthracene & 2,300 & $\mu \mathrm{g} / \mathrm{kg}$ & 2,300 & 210 \\
\hline $224 \mathrm{~F} 042$ & N-Nitrosodimethylamine & 380 & $\mu \mathrm{g} / \mathrm{kg}$ & 380 & 34 \\
\hline 224F042RR1 & 1,4-DichloroBenzene & 47,000 & $\mu \mathrm{g} / \mathrm{kg}$ & 47,000 & 7,900 \\
\hline 224F042RR1 & 1,2-DiChloroethane & 47,000 & $\mu \mathrm{g} / \mathrm{kg}$ & 47,000 & 600 \\
\hline 224F042RR1 & DibromoChloromethane & 47,000 & $\mu \mathrm{g} / \mathrm{kg}$ & 47,000 & 2,600 \\
\hline 224F042RR1 & Carbon Tetrachloride & 47,000 & $\mu \mathrm{g} / \mathrm{kg}$ & 47,000 & 550 \\
\hline 224F042RR1 & Chloroform & 47,000 & $\mu \mathrm{g} / \mathrm{kg}$ & 47,000 & 12,000 \\
\hline 224F042RR1 & Benzene & 47,000 & $\mu \mathrm{g} / \mathrm{kg}$ & 47,000 & 1,300 \\
\hline 224F042RR1 & Chloromethane & 47,000 & $\mu \mathrm{g} / \mathrm{kg}$ & 47,000 & 2,600 \\
\hline 224F042RR1 & Chloroethane & 47,000 & $\mu \mathrm{g} / \mathrm{kg}$ & 47,000 & 6,500 \\
\hline 224F042RR1 & Chloroethane & 47,000 & $\mu \mathrm{g} / \mathrm{kg}$ & 47,000 & 6,500 \\
\hline 224F042RR1 & Vinyl Chloride & 47,000 & $\mu \mathrm{g} / \mathrm{kg}$ & 47,000 & 750 \\
\hline 224F042RR1 & Bromodichloromethane & 47,000 & $\mu \mathrm{g} / \mathrm{kg}$ & 47,000 & 1,800 \\
\hline 224F042RR1 & 1,2-Dichloropropane & 47,000 & $\mu \mathrm{g} / \mathrm{kg}$ & 47,000 & 740 \\
\hline
\end{tabular}




\section{Table B.1-3 \\ Chemical Analytes Failing Sensitivity Criteria}

(Page 2 of 3 )

\begin{tabular}{|c|c|c|c|c|c|}
\hline $\begin{array}{l}\text { Sample } \\
\text { Number }\end{array}$ & Parameter & Result & Units & $\begin{array}{l}\text { Detect } \\
\text { Limit }\end{array}$ & $\begin{array}{c}\text { Industrial } 2002 \\
\text { PRG }\end{array}$ \\
\hline 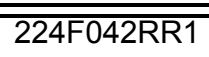 & $\overline{\text { 1,1,2-Trichloroethane }}$ & 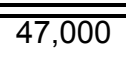 & $\overline{\mu g} / \mathrm{kg}$ & 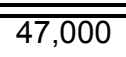 & $\overline{1,600}$ \\
\hline 224F042RR1 & 1,1,2,2-Tetrachloroethane & 47,000 & $\mu \mathrm{g} / \mathrm{kg}$ & 47,000 & 930 \\
\hline 224F042RR1 & Hexachlorobutadiene & 47,000 & $\mu \mathrm{g} / \mathrm{kg}$ & 47,000 & 22,000 \\
\hline 224F042RR1 & 1,2-Dibromo-3-Chloropropane & 95,000 & $\mu \mathrm{g} / \mathrm{kg}$ & 95,000 & 2,000 \\
\hline 224F042RR1 & 1,2-Dibromo-3-Chloropropane & 95,000 & $\mu \mathrm{g} / \mathrm{kg}$ & 95,000 & 2,000 \\
\hline 224F042RR1 & Bis(2-Chloroethyl)Ether & 38,000 & $\mu \mathrm{g} / \mathrm{kg}$ & 38,000 & 550 \\
\hline 224F042RR1 & Hexachlorobenzene & 38,000 & $\mu \mathrm{g} / \mathrm{kg}$ & 38,000 & 1,100 \\
\hline 224F042RR1 & Indeno(1,2,3-cd)Pyrene & 38,000 & $\mu \mathrm{g} / \mathrm{kg}$ & 38,000 & 2,100 \\
\hline 224F042RR1 & Benzo(a)Pyrene & 23,000 & $\mu \mathrm{g} / \mathrm{kg}$ & 23,000 & 210 \\
\hline 224F042RR1 & N-Nitroso-Di-N-Propylamine & 26,000 & $\mu \mathrm{g} / \mathrm{kg}$ & 26,000 & 250 \\
\hline 224F042RR1 & Pentachlorophenol & 75,000 & $\mu \mathrm{g} / \mathrm{kg}$ & 75,000 & 9,000 \\
\hline 224F042RR1 & 2-Nitroaniline & 75,000 & $\mu \mathrm{g} / \mathrm{kg}$ & 75,000 & 18,000 \\
\hline $224 \mathrm{~F} 043$ & Aroclor 1221 & 6,800 & $\mu \mathrm{g} / \mathrm{kg}$ & 6,800 & 740 \\
\hline $224 \mathrm{~F} 043$ & 1,2-Dibromoethane & 440 & $\mu \mathrm{g} / \mathrm{kg}$ & 440 & 28 \\
\hline $224 \mathrm{~F} 043$ & 1,2,3-Trichloropropane & 440 & $\mu \mathrm{g} / \mathrm{kg}$ & 440 & 11 \\
\hline $224 \mathrm{~F} 043$ & Bis(2-Chloroethyl)Ether & 35,000 & $\mu \mathrm{g} / \mathrm{kg}$ & 35,000 & 550 \\
\hline $224 \mathrm{~F} 043$ & Hexachlorobenzene & 35,000 & $\mu \mathrm{g} / \mathrm{kg}$ & 35,000 & 1100 \\
\hline $224 \mathrm{~F} 043$ & Indeno(1,2,3-cd)Pyrene & 35,000 & $\mu \mathrm{g} / \mathrm{kg}$ & 35,000 & 2100 \\
\hline $224 \mathrm{~F} 043$ & Benzo(a)Pyrene & 21,000 & $\mu \mathrm{g} / \mathrm{kg}$ & 21,000 & 210 \\
\hline $224 \mathrm{~F} 043$ & Dibenzo(a,h)Anthracene & 21,000 & $\mu \mathrm{g} / \mathrm{kg}$ & 21,000 & 210 \\
\hline $224 \mathrm{~F} 043$ & N-Nitrosodimethylamine & 3,500 & $\mu \mathrm{g} / \mathrm{kg}$ & 3,500 & 34 \\
\hline $224 \mathrm{~F} 043$ & N-Nitroso-Di-N-Propylamine & 24,000 & $\mu \mathrm{g} / \mathrm{kg}$ & 24,000 & 250 \\
\hline $224 \mathrm{~F} 043$ & Pentachlorophenol & 69,000 & $\mu \mathrm{g} / \mathrm{kg}$ & 69,000 & 9,000 \\
\hline $224 \mathrm{~F} 043$ & 2-Nitroaniline & 69,000 & $\mu \mathrm{g} / \mathrm{kg}$ & 69,000 & 18,000 \\
\hline 224F043RR1 & 1,4-DICHLOROBenzene & 44,000 & $\mu \mathrm{g} / \mathrm{kg}$ & 44,000 & 7900 \\
\hline 224F043RR1 & 1,2-DiChloroethane & 44,000 & $\mu \mathrm{g} / \mathrm{kg}$ & 44,000 & 600 \\
\hline 224F043RR1 & DibromoChloromethane & 44,000 & $\mu \mathrm{g} / \mathrm{kg}$ & 44,000 & 2,600 \\
\hline 224F043RR1 & Carbon Tetrachloride & 44,000 & $\mu \mathrm{g} / \mathrm{kg}$ & 44,000 & 550 \\
\hline 224F043RR1 & Chloroform & 44,000 & $\mu \mathrm{g} / \mathrm{kg}$ & 44,000 & 12,000 \\
\hline 224F043RR1 & Chloroform & 44,000 & $\mu \mathrm{g} / \mathrm{kg}$ & 44,000 & 12,000 \\
\hline 224F043RR1 & Benzene & 44,000 & $\mu \mathrm{g} / \mathrm{kg}$ & 44,000 & 1,300 \\
\hline 224F043RR1 & Chloromethane & 44,000 & $\mu \mathrm{g} / \mathrm{kg}$ & 44,000 & 2,600 \\
\hline 224F043RR1 & Chloroethane & 44,000 & $\mu \mathrm{g} / \mathrm{kg}$ & 44,000 & 6,500 \\
\hline 224F043RR1 & Chloroethane & 44,000 & $\mu \mathrm{g} / \mathrm{kg}$ & 44,000 & 6,500 \\
\hline 224F043RR1 & Vinyl Chloride & 44,000 & $\mu \mathrm{g} / \mathrm{kg}$ & 44,000 & 750 \\
\hline 224F043RR1 & Bromodichloromethane & 44,000 & $\mu \mathrm{g} / \mathrm{kg}$ & 44,000 & 1,800 \\
\hline
\end{tabular}




\section{Table B.1-3 \\ Chemical Analytes Failing Sensitivity Criteria}

(Page 3 of 3)

\begin{tabular}{|c|c|c|c|c|c|}
\hline $\begin{array}{l}\text { Sample } \\
\text { Number }\end{array}$ & Parameter & Result & Units & $\begin{array}{l}\text { Detect } \\
\text { Limit }\end{array}$ & $\begin{array}{c}\text { Industrial } 2002 \\
\text { PRG }\end{array}$ \\
\hline 224F043RR1 & 1,2-Dichloropropane & 44,000 & $\mu \mathrm{g} / \mathrm{kg}$ & 44,000 & 740 \\
\hline 224F043RR1 & 1,1,2-TRIChloroethane & 44,000 & $\mu \mathrm{g} / \mathrm{kg}$ & 44,000 & 1,600 \\
\hline 224F043RR1 & 1,1,2,2-TETRAChloroethane & 44,000 & $\mu \mathrm{g} / \mathrm{kg}$ & 44,000 & 930 \\
\hline 224F043RR1 & 1,2-Dibromo-3-Chloropropane & 87,000 & $\mu \mathrm{g} / \mathrm{kg}$ & 87,000 & 2,000 \\
\hline 224F043RR1 & 1,2-Dibromo-3-Chloropropane & 87,000 & $\mu \mathrm{g} / \mathrm{kg}$ & 87,000 & 2,000 \\
\hline 224F043RR1 & N-Nitrosodimethylamine & 350 & $\mu \mathrm{g} / \mathrm{kg}$ & 350 & 34 \\
\hline 224G003RR1 & N-Nitrosodimethylamine & 2,100 & $\mu \mathrm{g} / \mathrm{kg}$ & 2,100 & 34 \\
\hline 224J001 & N-Nitrosodimethylamine & 180 & $\mu \mathrm{g} / \mathrm{kg}$ & 180 & 34 \\
\hline 224J001RR1 & Benzo(a)Pyrene & 5,300 & $\mu \mathrm{g} / \mathrm{kg}$ & 5,300 & 210 \\
\hline 224J001RR1 & Dibenzo(a,h)Anthracene & 5,300 & $\mu \mathrm{g} / \mathrm{kg}$ & 5,300 & 210 \\
\hline 224J001RR1 & N-Nitroso-Di-N-Propylamine & 6,200 & $\mu \mathrm{g} / \mathrm{kg}$ & 6,200 & 250 \\
\hline 224J001RR1 & Pentachlorophenol & 18,000 & $\mu \mathrm{g} / \mathrm{kg}$ & 18,000 & 9,000 \\
\hline 224J002 & N-Nitrosodimethylamine & 180 & $\mu \mathrm{g} / \mathrm{kg}$ & 180 & 34 \\
\hline 224J002RR1 & Dibenzo(a,h)Anthracene & 2,100 & $\mu \mathrm{g} / \mathrm{kg}$ & 2,100 & 210 \\
\hline 224J003 & N-Nitrosodimethylamine & 170 & $\mu \mathrm{g} / \mathrm{kg}$ & 170 & 34 \\
\hline 224J003RR1 & Dibenzo(a,h)Anthracene & 2,100 & $\mu \mathrm{g} / \mathrm{kg}$ & 2,100 & 210 \\
\hline 224J005 & N-Nitrosodimethylamine & 170 & $\mu \mathrm{g} / \mathrm{kg}$ & 170 & 34 \\
\hline 224J005RR1 & Dibenzo(a,h)Anthracene & 3,100 & $\mu \mathrm{g} / \mathrm{kg}$ & 3,100 & 210 \\
\hline 224J008 & N-Nitrosodimethylamine & 180 & $\mu \mathrm{g} / \mathrm{kg}$ & 180 & 34 \\
\hline 224J008RR1 & Benzo(a)Pyrene & 4,200 & $\mu \mathrm{g} / \mathrm{kg}$ & 4,200 & 210 \\
\hline 224J008RR1 & Dibenzo(a,h)Anthracene & 4,200 & $\mu \mathrm{g} / \mathrm{kg}$ & 4,200 & 210 \\
\hline 224J008RR1 & N-Nitroso-Di-N-Propylamine & 4,900 & $\mu \mathrm{g} / \mathrm{kg}$ & 4,900 & 250 \\
\hline 224J009 & N-Nitrosodimethylamine & 180 & $\mu \mathrm{g} / \mathrm{kg}$ & 180 & 34 \\
\hline 224J009RR1RX1 & Dibenzo(a,h)Anthracene & 4,400 & $\mu \mathrm{g} / \mathrm{kg}$ & 4,400 & 210 \\
\hline 224J009RR1RX1 & N-Nitroso-Di-N-Propylamine & 5,100 & $\mu \mathrm{g} / \mathrm{kg}$ & 5,100 & 250 \\
\hline 224J009RR2RX1 & Bis(2-Chloroethyl)Ether & 11,000 & $\mu \mathrm{g} / \mathrm{kg}$ & 11,000 & 550 \\
\hline 224J009RR2RX1 & Pentachlorophenol & 22,000 & $\mu \mathrm{g} / \mathrm{kg}$ & 22,000 & 9,000 \\
\hline 224J015 & N-Nitrosodimethylamine & 180 & $\mu \mathrm{g} / \mathrm{kg}$ & 180 & 34 \\
\hline $224 \mathrm{~J} 023$ & N-Nitrosodimethylamine & 180 & $\mu \mathrm{g} / \mathrm{kg}$ & 180 & 34 \\
\hline 224J023RR1 & Dibenzo(a,h)Anthracene & 2,100 & $\mu \mathrm{g} / \mathrm{kg}$ & 2,100 & 210 \\
\hline 224J030RR1 & N-Nitrosodimethylamine & 170 & $\mu \mathrm{g} / \mathrm{kg}$ & 170 & 34 \\
\hline 224J031RR1 & Dibenzo(a,h)Anthracene & 3,100 & $\mu \mathrm{g} / \mathrm{kg}$ & 3,100 & 210 \\
\hline 224J031RR1 & N-Nitrosodimethylamine & 520 & $\mu \mathrm{g} / \mathrm{kg}$ & 520 & 34 \\
\hline
\end{tabular}

$\mathrm{mg} / \mathrm{L}=$ Milligrams per liter

$\mu \mathrm{g} / \mathrm{kg}=$ Micrograms per kilogram 
Table B.1-4

Radiological Analytes Failing Sensitivity Criteria

\begin{tabular}{|c|c|c|c|c|}
\hline Sample Number & Parameter & Result & Detection Limit & PAL \\
\hline \hline 224B005 & Europium-154 & -0.8 & 3.3 & 3.24 \\
\hline
\end{tabular}

\section{Criteria 3:}

To satisfy the third criterion, the entire data set as well as individual sample results were assessed against the DQIs of precision, accuracy, comparability, completeness, and representativeness, as defined in the Industrial Sites QAPP (NNSA/NV, 2002). The DQI goals were presented in Table 6-1 of the CAIP. As presented in Table B.1-5 through Table B.1-17, these goals were met for each the DQIs.

\section{Precision}

Precision is a measurement of agreement among a replicate set of measurements of the same property under similar conditions and is measured through the use of MS/MSDs, laboratory duplicates, LCS/laboratory control sample duplicates, and field duplicate samples. Precision for chemical analyses is expressed as the RPD between duplicate measurements. Table B.1-5 provides the chemical precision analysis results.

To evaluate precision for radiological analyses, the duplicate precision is evaluated using the RPD or normalized difference. The RPD is applicable when both the sample and its duplicate have concentrations of the target radionuclide exceeding five times their minimum detectable concentration. This excludes many measurements because the samples contain non-detectable or low levels of the target radionuclide. In situations where the RPD does not apply, duplicate results are evaluated using the normalized difference. Table B.1-6 provides the radiological precision analysis results.

\section{Accuracy}

For the purpose of determining data accuracy of sample analyses, all water and soil samples, including field QC samples (i.e., trip blanks, equipment rinsate samples, field blanks) were evaluated and incorporated into the accuracy calculation. As shown in Table B.1-7 and Table B.1-8, all 
Table B.1-5

Chemical Precision Measurements

\begin{tabular}{|c|c|c|c|c|c|c|c|c|c|}
\hline \multirow{2}{*}{ Measurements } & \multicolumn{6}{|c|}{ ORGANICS } & \multicolumn{3}{|c|}{ INORGANICS } \\
\hline & VOCs & SVOCs & TPH-DRO & PCBs & Pesticides & Herbicides & Cyanide & Metals* & Mercury \\
\hline \multicolumn{10}{|c|}{ Matrix Spike Duplicate (MSD) Precision } \\
\hline MSD & 125 & 273 & 30 & 42 & 12 & 0 & 8 & 352 & 25 \\
\hline $\begin{array}{l}\text { Relative Percent } \\
\text { Differences (RPDs) } \\
\text { Within Criteria }\end{array}$ & 125 & 273 & 30 & 42 & 12 & 0 & 8 & 352 & 25 \\
\hline MSD Percent Precision & 100 & 100 & 100 & 100 & 100 & NA & 100 & 100 & 100 \\
\hline \multicolumn{10}{|c|}{ Laboratory Control Sample Duplicate (LCSD) Precision } \\
\hline LCSD & 220 & 417 & 34 & 68 & 24 & 6 & 6 & 488 & 34 \\
\hline RPDs Within Criteria & 220 & 417 & 34 & 68 & 24 & 6 & 6 & 488 & 34 \\
\hline $\begin{array}{l}\text { LCSD Percent } \\
\text { Precision }\end{array}$ & 100 & 100 & 100 & 100 & 100 & 100 & 100 & 100 & 100 \\
\hline \multicolumn{10}{|c|}{ Field Sample Duplicate (FD) Precision } \\
\hline FD & 1,380 & 1,638 & 24 & 147 & 44 & 10 & 5 & 336 & 21 \\
\hline RPDs Within Criteria & 1,376 & 1,634 & 20 & 146 & 43 & 10 & 5 & 321 & 20 \\
\hline FD Percent Precision & 99.7 & 99.8 & 83.3 & 99.3 & 97.7 & 100 & 100 & 95.5 & 95.2 \\
\hline \multicolumn{10}{|c|}{ Laboratory Sample Duplicate (Lab-Dup) Precision } \\
\hline Lab-Dup & NA & NA & NA & NA & NA & NA & 0 & 352 & 25 \\
\hline RPDs Within Criteria & NA & NA & NA & NA & NA & NA & 0 & 352 & 25 \\
\hline $\begin{array}{l}\text { Lab-Dup Percent } \\
\text { Precision }\end{array}$ & NA & NA & NA & NA & NA & NA & NA & 100 & 100 \\
\hline
\end{tabular}

*Measurements include Aluminum, Antimony, Arsenic, Barium, Beryllium, Chromium, Cobalt, Coppery, Lead, Manganese, Molybdenum, Nickel, Selenium, Silver, Zinc

NA $=$ Not applicable

$\mathrm{DRO}=$ Diesel-range organics

$\mathrm{PCB}=$ Polychlorinated biphenyls

SVOC $=$ Semivolatile organic compound

$\mathrm{TPH}=$ Total petroleum hydrocarbons

VOCs $=$ Volatile organic compound 
Table B.1-6

Radiological Precision

\begin{tabular}{|c|c|c|c|c|c|c|}
\hline Measurements & $\begin{array}{c}\text { Gamma } \\
\text { Spectroscopy }\end{array}$ & $\begin{array}{l}\text { Isotopic } \\
\text { Uranium }\end{array}$ & $\begin{array}{l}\text { Isotopic } \\
\text { Plutonium }\end{array}$ & Strontium-90 & Tritium & $\begin{array}{c}\text { Gross } \\
\text { Alpha/Beta }\end{array}$ \\
\hline \multicolumn{7}{|c|}{ Field Sample Duplicate (FD) Precision } \\
\hline $\begin{array}{l}\text { Relative Percent } \\
\text { Difference (RPD) }\end{array}$ & 31 & 34 & 11 & 0 & 0 & 0 \\
\hline RPDs Within Criteria & 29 & 34 & 3 & 0 & 0 & 0 \\
\hline Percent Precision & 93.5 & 100 & 27.3 & NA & NA & NA \\
\hline $\begin{array}{l}\text { Normalized Difference } \\
\text { (ND) }\end{array}$ & 409 & 17 & 31 & 17 & 0 & 0 \\
\hline NDs Within Criteria & 409 & 17 & 29 & 17 & 0 & 0 \\
\hline Percent Precision & 100 & 100 & 93.5 & 100 & NA & NA \\
\hline \multicolumn{7}{|c|}{ Laboratory Sample Duplicate (Lab-Dup) Precision } \\
\hline RPD & 70 & 65 & 39 & 8 & 0 & 0 \\
\hline RPDs Within Criteria & 70 & 65 & 28 & 8 & 0 & 0 \\
\hline Percent Precision & 100 & 100 & 71.8 & 100 & NA & NA \\
\hline ND & 1,076 & 47 & 69 & 33 & 1 & 2 \\
\hline NDs Within Criteria & 1,074 & 47 & 69 & 32 & 1 & 2 \\
\hline Percent Precision & 99.8 & 100 & 100 & 97.0 & 100 & 100 \\
\hline
\end{tabular}

NA = Not applicable

accuracy rates are at or above 75 percent. Therefore, the data set is determined to be acceptable for the DQI of accuracy.

Table B.1-7 provides the chemical accuracy analysis results. Table B.1-8 provides the radiological accuracy analysis results.

\section{Representativeness}

The DQO process as identified in Appendix A.1 of the CAIP was used to address sampling and analytical requirements for CAU 224. During this process, appropriate locations were selected that enabled the samples collected to be representative of the population parameters identified in the DQO (the most likely locations to contain contamination and locations that bound COCs). The sampling 
Table B.1-7

Chemical Accuracy Measurements

\begin{tabular}{|c|c|c|c|c|c|c|c|c|c|}
\hline \multirow{2}{*}{ Measurements } & \multicolumn{6}{|c|}{ ORGANICS } & \multicolumn{3}{|c|}{ INORGANICS } \\
\hline & VOCs & SVOCs & $\begin{array}{l}\text { TPH- } \\
\text { DRO }\end{array}$ & PCBs & Pesticides & Herbicides & Cyanide & Metals* & Mercury \\
\hline \multicolumn{10}{|c|}{ Matrix Spike (MS) Accuracy } \\
\hline MS & 250 & 546 & 60 & 84 & 24 & 0 & 16 & 704 & 6 \\
\hline MS Within Criteria & 250 & 546 & 60 & 82 & 18 & 0 & 16 & 650 & 5 \\
\hline MS Percent Accuracy & 100 & 100 & 100 & 97.6 & 75.0 & NA & 100 & 92.3 & 83.3 \\
\hline \multicolumn{10}{|c|}{ Laboratory Control Sample (LCS) Accuracy } \\
\hline LCS & 440 & 845 & 68 & 136 & 48 & 12 & 12 & 976 & 68 \\
\hline LCS Within Criteria & 440 & 845 & 68 & 136 & 48 & 12 & 12 & 976 & 68 \\
\hline LCS Percent Accuracy & 100 & 100 & 100 & 100 & 100 & 100 & 100 & 100 & 100 \\
\hline \multicolumn{10}{|c|}{ Surrogate Accuracy } \\
\hline Analyzed & 26,345 & 28,745 & 400 & 2,527 & 484 & 50 & NA & NA & NA \\
\hline $\begin{array}{l}\text { Not Affected by } \\
\text { Out-of-Control Surrogates }\end{array}$ & 25,891 & 27,377 & 397 & 2,422 & 324 & 50 & NA & NA & NA \\
\hline $\begin{array}{l}\text { Surrogate Percent } \\
\text { Accuracy }\end{array}$ & 98.3 & 95.2 & 99.3 & 95.8 & 66.9 & 100 & NA & NA & NA \\
\hline
\end{tabular}

*Measurements include Aluminum, Antimony, Arsenic, Barium, Beryllium, Chromium, Cobalt, Coppery, Lead, Manganese, Molybdenum, Nickel, Selenium, Silver, Zinc

NA $=$ Not applicable

$\mathrm{DRO}=$ Diesel-range organics

$\mathrm{PCB}=$ Polychlorinated biphenyls

SVOC = Semivolatile organic compound

$\mathrm{TPH}=$ Total petroleum hydrocarbons

VOCs $=$ Volatile organic compound

locations identified in Table B.1-1 and the Criteria 1 discussion meet this criteria. Therefore, the analytical data acquired during the CAU $224 \mathrm{CAI}$ are considered representative of the population parameters.

\section{Comparability}

Field sampling, as described in the CAU 224 CAIP (NNSA/NSO, 2004), was performed and documented in accordance with approved procedures that are comparable to standard industry practices. Approved analytical methods and procedures per DOE were used to analyze, report, and validate the data. These are comparable to other methods used not only in industry and government practices, but most importantly are comparable to other investigations conducted for the NTS. 
Table B.1-8

Radiological Accuracy Measurements for CAU 224

\begin{tabular}{||l|c|c|c|c|c|c||}
\hline \multicolumn{1}{|c|}{ Measurements } & $\begin{array}{c}\text { Gamma } \\
\text { Spectroscopy }\end{array}$ & $\begin{array}{c}\text { Isotopic } \\
\text { Uranium }\end{array}$ & $\begin{array}{c}\text { Isotopic } \\
\text { Plutonium }\end{array}$ & Strontium-90 & Tritium & $\begin{array}{c}\text { Gross } \\
\text { Alpha/Beta }\end{array}$ \\
\hline \hline \multicolumn{7}{|c||}{ Matrix Spike Sample (MS) Accuracy } \\
\hline \hline Measurements & 0 & 0 & 0 & 0 & 1 & 2 \\
\hline $\begin{array}{l}\text { Percent RECs Within } \\
\text { Criteria }\end{array}$ & 0 & 0 & 0 & 0 & 1 & 1 \\
\hline Percent Accuracy & NA & NA & NA & NA & 100 & 50 \\
\hline \hline & Laboratory Control Sample (LCS) Accuracy & & 1 \\
\hline \hline Measurements & 123 & 60 & 42 & 42 & 1 & 2 \\
\hline $\begin{array}{l}\text { Percent RECs Within } \\
\text { Criteria }\end{array}$ & 123 & 60 & 42 & 42 & 1 & 2 \\
\hline Percent Accuracy & 100 & 100 & 100 & 100 & 100 & 100 \\
\hline
\end{tabular}

Therefore, data sets within this project are considered comparable to other data sets generated using these same standardized DOE procedures, thereby meeting DQO requirements.

Also, standard, approved field and analytical methods ensured that data were appropriate for comparison to the investigation action levels specified in the CAIP.

\section{Completeness}

The CAU 224 CAIP defines acceptable criteria for completeness to be 80 percent of CAS-specific possible analytes identified in the CAIP having valid results and 90 percent of critical analytes (including Decision II samples) having valid results. Also, the data must be of sufficient high-quality to be able to make the DQO decisions.

Rejected data were not used in the resolution of DQO decisions and are not counted towards meeting the completeness goals. Table B.1-9 through Table B.1-15 provide the rejected data for CAU 224 CASs. Table B.1-3 and Table B.1-4 provide the rejected data failing the criteria for sensitivity. The rejected data were not needed to define the presence or extent of COC contamination at any CAS within CAU 224. As shown in Table B.1-16 and Table B.1-17, the criteria for completeness was met. 
Table B.1-9

CAU 224 Rejected Data for CAS 02-04-01, Septic Tank (Buried)

\begin{tabular}{|l|c|c|c|c|c|c|}
\hline $\begin{array}{l}\text { Sample } \\
\text { Number }\end{array}$ & $\begin{array}{c}\text { Laboratory } \\
\text { Method }\end{array}$ & Parameter & Matrix & Qualifier & Reason 1 & Reason 2 \\
\hline \hline $224 \mathrm{~A} 007$ & SW8270 & Dibenzo(a,h)Anthracene & Soil & R & 153 & 41 \\
\hline
\end{tabular}

Reason Codes:

$41=$ Matrix effects may exist.

$153=$ Internal area response show extremely low count.

Table B.1-10

CAU 224 Rejected Data for CAS 03-05-01, Leachfield

(Page 1 of 3 )

\begin{tabular}{|c|c|c|c|c|c|c|}
\hline $\begin{array}{l}\text { Sample } \\
\text { Number }\end{array}$ & $\begin{array}{c}\text { Laboratory } \\
\text { Method }\end{array}$ & Parameter & Matrix & Qualifier & Reason 1 & Reason 2 \\
\hline$\overline{224 \mathrm{~B} 002}$ & SW8270 & $\overline{\text { Phenol }}$ & $\overline{\text { Soil }}$ & $\overline{\overline{\mathrm{R}}}$ & $\overline{411}$ & $\overline{127}$ \\
\hline 224B002 & SW8270 & 2-Chlorophenol & Soil & $\mathrm{R}$ & 41 & 127 \\
\hline 224B002 & SW8270 & 4,6-Dinitro-2-Methylphenol & Soil & $\mathrm{R}$ & 41 & 127 \\
\hline 224B002 & SW8270 & Pentachlorophenol & Soil & $\mathrm{R}$ & 41 & 127 \\
\hline 224B002 & SW8270 & 2,4-Dichlorophenol & Soil & $\mathrm{R}$ & 41 & 127 \\
\hline 224B002 & SW8270 & 2,4-Dimethylphenol & Soil & $\mathrm{R}$ & 41 & 127 \\
\hline 224B002 & SW8270 & Benzoic Acid & Soil & $\mathrm{R}$ & 41 & 127 \\
\hline 224B002 & SW8270 & 2-Nitrophenol & Soil & $\mathrm{R}$ & 41 & 127 \\
\hline 224B002 & SW8270 & 4-Nitrophenol & Soil & $\mathrm{R}$ & 41 & 127 \\
\hline 224B002 & SW8270 & 2-Methylphenol & Soil & $\mathrm{R}$ & 41 & 127 \\
\hline 224B002 & SW8270 & 2,4-Dinitrophenol & Soil & $\mathrm{R}$ & 41 & 127 \\
\hline 224B002 & SW8270 & 4-Methylphenol & Soil & $\mathrm{R}$ & 41 & 127 \\
\hline 224B002 & SW8270 & 4-Chloro-3-Methylphenol & Soil & $\mathrm{R}$ & 41 & 127 \\
\hline 224B002 & SW8270 & 2,3,4,6-Tetrachlorophenol & Soil & $\mathrm{R}$ & 41 & 127 \\
\hline 224B002 & SW8270 & 2,4,5-Trichlorophenol & Soil & $\mathrm{R}$ & 41 & 127 \\
\hline 224B002 & SW8270 & 2,4,6-Trichlorophenol & Soil & $\mathrm{R}$ & 41 & 127 \\
\hline 224B005 & SW8270 & Benzoic Acid & Soil & $\mathrm{R}$ & 41 & 127 \\
\hline 224B005 & SW8270 & 2-Nitrophenol & Soil & $\mathrm{R}$ & 41 & 127 \\
\hline 224B005 & SW8270 & Pentachlorophenol & Soil & $\mathrm{R}$ & 41 & 127 \\
\hline 224B005 & SW8270 & 2,4-Dichlorophenol & Soil & $\mathrm{R}$ & 41 & 127 \\
\hline 224B005 & SW8270 & 2,4-Dimethylphenol & Soil & $\mathrm{R}$ & 41 & 127 \\
\hline 224B005 & SW8270 & 2,4,5-Trichlorophenol & Soil & $\mathrm{R}$ & 41 & 127 \\
\hline $224 \mathrm{~B} 005$ & SW8270 & 2,3,4,6-Tetrachlorophenol & Soil & $\mathrm{R}$ & 41 & 127 \\
\hline $224 \mathrm{~B} 005$ & SW8270 & Phenol & Soil & $\mathrm{R}$ & 41 & 127 \\
\hline
\end{tabular}


Table B.1-10

CAU 224 Rejected Data for CAS 03-05-01, Leachfield (Page 2 of 3 )

\begin{tabular}{|c|c|c|c|c|c|c|}
\hline $\begin{array}{l}\text { Sample } \\
\text { Number }\end{array}$ & $\begin{array}{c}\text { Laboratory } \\
\text { Method }\end{array}$ & Parameter & Matrix & Qualifier & Reason 1 & Reason 2 \\
\hline$\overline{2224 \mathrm{~B} 005}$ & $\overline{\text { SW8270 }}$ & 2,4-Dinitrophenol & $\overline{\overline{\text { Soil }}}$ & $\overline{\overline{\mathrm{R}}}$ & $\overline{\overline{41}}$ & $\overline{127}$ \\
\hline 224B005 & SW8270 & 2,4,6-Trichlorophenol & Soil & $\bar{R}$ & 41 & 127 \\
\hline 224B005 & SW8270 & 4-Chloro-3-Methylphenol & Soil & $\bar{R}$ & 41 & 127 \\
\hline 224B005 & SW8270 & 4,6-Dinitro-2-Methylphenol & Soil & $\mathrm{R}$ & 41 & 127 \\
\hline 224B005 & SW8270 & 4-Nitrophenol & Soil & $\mathrm{R}$ & 41 & 127 \\
\hline 224B005 & SW8270 & 2-Chlorophenol & Soil & $\bar{R}$ & 41 & 127 \\
\hline 224B005 & SW8270 & 2-Methylphenol & Soil & $\mathrm{R}$ & 41 & 127 \\
\hline 224B005 & SW8270 & 4-Methylphenol & Soil & $\mathrm{R}$ & 41 & 127 \\
\hline 224B008 & SW8270 & 2,4,5-Trichlorophenol & Soil & $\mathrm{R}$ & 41 & 127 \\
\hline 224B008 & SW8270 & 2,4,6-Trichlorophenol & Soil & $\mathrm{R}$ & 41 & 127 \\
\hline 224B008 & SW8270 & Dibenzo(a,h)Anthracene & Soil & $\mathrm{R}$ & 41 & 153 \\
\hline 224B008 & SW8270 & Indeno(1,2,3-cd)Pyrene & Soil & $\mathrm{R}$ & 41 & 153 \\
\hline 224B008 & SW8270 & 4-Chloro-3-Methylphenol & Soil & $\bar{R}$ & 41 & 127 \\
\hline 224B008 & SW8270 & 2,3,4,6-Tetrachlorophenol & Soil & $R$ & 41 & 127 \\
\hline 224B008 & SW8270 & 4,6-Dinitro-2-Methylphenol & Soil & $R$ & 41 & 127 \\
\hline 224B008 & SW8270 & 2,4-Dinitrophenol & Soil & $\bar{R}$ & 41 & 127 \\
\hline 224B008 & SW8270 & 4-Nitrophenol & Soil & $R$ & 41 & 127 \\
\hline 224B008 & SW8270 & Phenol & Soil & $\mathrm{R}$ & 41 & 127 \\
\hline 224B008 & SW8270 & Benzoic Acid & Soil & $\bar{R}$ & 41 & 127 \\
\hline 224B008 & SW8270 & 2-Nitrophenol & Soil & $\bar{R}$ & 41 & 127 \\
\hline 224B008 & SW8270 & 2-Chlorophenol & Soil & $\bar{R}$ & 41 & 127 \\
\hline 224B008 & SW8270 & 2-Methylphenol & Soil & $R$ & 41 & 127 \\
\hline 224B008 & SW8270 & 4-Methylphenol & Soil & $\bar{R}$ & 41 & 127 \\
\hline 224B008 & SW8270 & Benzo(a)Pyrene & Soil & $R$ & 41 & 153 \\
\hline 224B008 & SW8270 & Pentachlorophenol & Soil & $R$ & 41 & 127 \\
\hline 224B008 & SW8270 & 2,4-Dichlorophenol & Soil & $\bar{R}$ & 41 & 127 \\
\hline 224B008 & SW8270 & 2,4-Dimethylphenol & Soil & $\mathrm{R}$ & 41 & 127 \\
\hline 224B008 & SW8270 & Benzo(b)Fluoranthene & Soil & $\bar{R}$ & 41 & 153 \\
\hline 224B008 & SW8270 & Benzo(g,h,i)Perlyene & Soil & $\mathrm{R}$ & 41 & 153 \\
\hline 224B008 & SW8270 & Benzo(k)Fluoranthene & Soil & $\mathrm{R}$ & 41 & 153 \\
\hline 224B009 & SW8270 & Benzo(g,h,i)Perlyene & Soil & $\bar{R}$ & $\overline{41}$ & 153 \\
\hline 224B009 & SW8270 & Benzo(k)Fluoranthene & Soil & $R$ & 41 & 153 \\
\hline 224B009 & SW8270 & Dibenzo(a,h)Anthracene & Soil & $\mathrm{R}$ & 41 & 153 \\
\hline
\end{tabular}


Table B.1-10

CAU 224 Rejected Data for CAS 03-05-01, Leachfield

(Page 3 of 3 )

\begin{tabular}{||c|c|c|c|c|c|c||}
\hline $\begin{array}{c}\text { Sample } \\
\text { Number }\end{array}$ & $\begin{array}{c}\text { Laboratory } \\
\text { Method }\end{array}$ & Parameter & Matrix & Qualifier & Reason 1 & Reason 2 \\
\hline \hline 224B009 & SW8270 & Indeno(1,2,3-cd)Pyrene & Soil & $\mathrm{R}$ & 41 & 153 \\
\hline $224 \mathrm{~B} 011$ & SW8270 & Benzo(a)Pyrene & Soil & $\mathrm{R}$ & 153 & 41 \\
\hline 224B011 & SW8270 & Benzo(b)Fluoranthene & Soil & $\mathrm{R}$ & 153 & 41 \\
\hline 224B011 & SW8270 & Benzo(g,h,i)Perlyene & Soil & $\mathrm{R}$ & 153 & 41 \\
\hline 224B011 & SW8270 & Benzo(k)Fluoranthene & Soil & $\mathrm{R}$ & 153 & 41 \\
\hline 224B011 & SW8270 & Dibenzo(a,h)Anthracene & Soil & $\mathrm{R}$ & 153 & 41 \\
\hline 224B011 & SW8270 & Indeno(1,2,3-cd)Pyrene & Soil & $\mathrm{R}$ & 153 & 41 \\
\hline
\end{tabular}

Reason Codes:

41 = Matrix effects may exist.

127 = Surrogate recovery $<10$ percent.

153 = Internal area response show extremely low count.

Table B.1-11

CAU 224 Rejected Data for CAS 06-05-01, Leachfield

\begin{tabular}{||c|c|c|c|c|c|c||}
\hline $\begin{array}{c}\text { Sample } \\
\text { Number }\end{array}$ & Matrix & $\begin{array}{c}\text { Laboratory } \\
\text { Method }\end{array}$ & Parameter & Qualifier & Reason 1 & Reason 2 \\
\hline \hline 224E018 & Soil & SW8270 & Dibenzo(a,h)Anthracene & R & 153 & 41 \\
\hline 224E018 & Soil & SW8270 & Indeno(1,2,3-cd)Pyrene & R & 153 & 41 \\
\hline 224E018 & Soil & SW8270 & Benzo(g,h,i)Perylene & R & 153 & 41 \\
\hline
\end{tabular}

Reason Codes:

41 = Matrix effects may exist.

$153=$ Internal area response show extremely low count.

The analyte hydroquinone was listed as a critical analyte at CASs 11-04-01 and 23-05-02 because of its possible use as a reducing agent in photo-finishing processes. The analytical results for hydroquinone were rejected in a majority of cases due to the compound being readily oxidized during the extraction and analysis procedures. However, the valid hydroquinone results indicate absence of the compound. At CAS 11-04-01, hydroquinone was not detected in the three liquid samples collected from the septic tanks and distribution box, which are considered the sources at the CAS. Hydroquinone was also not detected in nine soil samples from the leachfield at CAS 11-04-01 above 
Table B.1-12

CAU 224 Rejected Data for CAS 06-17-04, Decon Pad and Wastewater Catch (Page 1 of 2)

\begin{tabular}{|c|c|c|c|c|c|c|c|}
\hline $\begin{array}{l}\text { Sample } \\
\text { Number }\end{array}$ & $\begin{array}{l}\text { Laboratory } \\
\text { Method }\end{array}$ & Parameter & Matrix & Qualifier & Reason 1 & Reason 2 & Reason 3 \\
\hline $224 \mathrm{~F} 023$ & SW8270 & Benzo(a)Anthracene & $\overline{\text { Soil }}$ & $\overline{\mathrm{R}}$ & $\overline{153}$ & $\overline{41}$ & \\
\hline $224 \mathrm{~F} 023$ & SW8270 & Benzo(b)Fluoranthene & Soil & $\mathrm{R}$ & 153 & 41 & \\
\hline $224 \mathrm{~F} 023$ & SW8270 & Benzo(g,h,i)Perylene & Soil & $\mathrm{R}$ & 153 & 41 & 117 \\
\hline $224 \mathrm{~F} 023$ & SW8270 & Benzo(k)Fluoranthene & Soil & $\mathrm{R}$ & 153 & 41 & \\
\hline $224 \mathrm{~F} 023$ & SW8270 & Di-N-Octyl Phthalate & Soil & $\mathrm{R}$ & 153 & 41 & \\
\hline $224 \mathrm{~F} 023$ & SW8270 & 3,3'-Dichlorobenzidine & Soil & $\mathrm{R}$ & 153 & 41 & \\
\hline $224 \mathrm{~F} 023$ & SW8270 & Butyl Benzyl Phthalate & Soil & $\mathrm{R}$ & 153 & 41 & \\
\hline $224 \mathrm{~F} 023$ & SW8270 & Indeno(1,2,3-cd)Pyrene & Soil & $\mathrm{R}$ & 153 & 41 & 117 \\
\hline $224 \mathrm{~F} 023$ & SW8270 & Pyrene & Soil & $\mathrm{R}$ & 153 & 41 & \\
\hline 224F023 & SW8270 & Chrysene & Soil & $\mathrm{R}$ & 153 & 41 & \\
\hline $224 \mathrm{~F} 023$ & SW8270 & Bis(2-Ethylhexyl)Phthalate & Soil & $\mathrm{R}$ & 153 & 41 & \\
\hline 224F027 & SW8270 & Indeno(1,2,3-cd)Pyrene & Soil & $\mathrm{R}$ & 153 & 41 & 117 \\
\hline $224 \mathrm{~F} 027$ & SW8270 & Bis(2-Ethylhexyl)Phthalate & Soil & $\mathrm{R}$ & 153 & 41 & \\
\hline $224 \mathrm{~F} 027$ & SW8270 & Benzo(a)Anthracene & Soil & $\mathrm{R}$ & 153 & 41 & \\
\hline $224 \mathrm{~F} 027$ & SW8270 & Pyrene & Soil & $\mathrm{R}$ & 153 & 41 & \\
\hline $224 \mathrm{~F} 027$ & SW8270 & Chrysene & Soil & $\mathrm{R}$ & 153 & 41 & \\
\hline $224 \mathrm{~F} 027$ & SW8270 & Benzo(b)Fluoranthene & Soil & $\mathrm{R}$ & 153 & 41 & \\
\hline $224 \mathrm{~F} 027$ & SW8270 & Benzo(g,h,i)Perylene & Soil & $\mathrm{R}$ & 153 & 41 & 117 \\
\hline $224 \mathrm{~F} 027$ & SW8270 & Benzo(k)Fluoranthene & Soil & $\mathrm{R}$ & 153 & 41 & \\
\hline $224 \mathrm{~F} 027$ & SW8270 & Di-N-Octyl Phthalate & Soil & $\mathrm{R}$ & 153 & 41 & \\
\hline $224 \mathrm{~F} 027$ & SW8270 & 3,3'-Dichlorobenzidine & Soil & $\mathrm{R}$ & 153 & 41 & \\
\hline $224 \mathrm{~F} 027$ & SW8270 & Butyl Benzyl Phthalate & Soil & $\mathrm{R}$ & 153 & 41 & \\
\hline $224 \mathrm{~F} 042$ & SW8270 & Pyrene & Sludge & $\mathrm{R}$ & 41 & 153 & \\
\hline $224 \mathrm{~F} 042$ & SW8270 & Chrysene & Sludge & $\mathrm{R}$ & 41 & 153 & \\
\hline $224 \mathrm{~F} 042$ & SW8270 & Benzo(a)Anthracene & Sludge & $\mathrm{R}$ & 41 & 153 & \\
\hline $224 \mathrm{~F} 042$ & SW8270 & Benzo(b)Fluoranthene & Sludge & $\mathrm{R}$ & 41 & 153 & \\
\hline $224 \mathrm{~F} 042$ & SW8270 & Benzo(k)Fluoranthene & Sludge & $\mathrm{R}$ & 41 & 153 & \\
\hline $224 \mathrm{~F} 042$ & SW8270 & Di-N-Octyl Phthalate & Sludge & $\mathrm{R}$ & 41 & 153 & \\
\hline 224F042 & SW8270 & 3,3'-Dichlorobenzidine & Sludge & $\mathrm{R}$ & 41 & 153 & \\
\hline $224 \mathrm{~F} 042$ & SW8270 & Butyl Benzyl Phthalate & Sludge & $\mathrm{R}$ & 41 & 153 & \\
\hline 224F043RR1 & SW8270 & Benzo(k)Fluoranthene & Sludge & $\mathrm{R}$ & 41 & 153 & \\
\hline 224F043RR1 & SW8270 & Benzo(a)Pyrene & Sludge & $\mathrm{R}$ & 41 & 153 & \\
\hline 224F043RR1 & SW8270 & Benzo(a)Anthracene & Sludge & $\mathrm{R}$ & 41 & 153 & \\
\hline 224F043RR1 & SW8270 & Benzo(b)Fluoranthene & Sludge & $\mathrm{R}$ & 41 & 153 & \\
\hline 224F043RR1 & SW8270 & Di-N-Octyl Phthalate & Sludge & $\mathrm{R}$ & 41 & 153 & \\
\hline 224F043RR1 & SW8270 & 3,3'-Dichlorobenzidine & Sludge & $\mathrm{R}$ & 41 & 153 & \\
\hline
\end{tabular}


Table B.1-12

CAU 224 Rejected Data for CAS 06-17-04, Decon Pad and Wastewater Catch (Page 2 of 2)

\begin{tabular}{|c|c|c|c|c|c|c|c|}
\hline $\begin{array}{l}\text { Sample } \\
\text { Number }\end{array}$ & $\begin{array}{l}\text { Laboratory } \\
\text { Method }\end{array}$ & Parameter & Matrix & Qualifier & Reason 1 & Reason 2 & Reason 3 \\
\hline 224F043RR1 & SW8270 & Pyrene & $\overline{\text { Sludge }}$ & $\overline{\overline{\mathrm{R}}}$ & $\overline{41}$ & $\overline{153}$ & \\
\hline $224 \mathrm{~F} 501$ & SW8270 & Benzoic Acid & Concrete & $\mathrm{R}$ & 41 & 127 & \\
\hline $224 \mathrm{~F} 501$ & SW8270 & 2-Nitrophenol & Concrete & $\mathrm{R}$ & 41 & 127 & \\
\hline $224 \mathrm{~F} 501$ & SW8270 & 4-Nitrophenol & Concrete & $\mathrm{R}$ & 41 & 127 & \\
\hline $224 \mathrm{~F} 501$ & SW8270 & 2-Chlorophenol & Concrete & $\mathrm{R}$ & 41 & 127 & \\
\hline 224F501 & SW8270 & 2-Methylphenol & Concrete & $\mathrm{R}$ & 41 & 127 & \\
\hline 224F501 & SW8270 & 2,4-Dinitrophenol & Concrete & $\mathrm{R}$ & 41 & 127 & \\
\hline $224 \mathrm{~F} 501$ & SW8270 & Pentachlorophenol & Concrete & $\mathrm{R}$ & 41 & 127 & \\
\hline $224 \mathrm{~F} 501$ & SW8270 & 2,4-Dichlorophenol & Concrete & $\mathrm{R}$ & 41 & 127 & \\
\hline $224 \mathrm{~F} 501$ & SW8270 & 2,4-Dimethylphenol & Concrete & $\mathrm{R}$ & 41 & 127 & \\
\hline $224 \mathrm{~F} 501$ & SW8270 & 2,4,5-Trichlorophenol & Concrete & $\mathrm{R}$ & 41 & 127 & \\
\hline $224 \mathrm{~F} 501$ & SW8270 & 2,4,6-Trichlorophenol & Concrete & $\mathrm{R}$ & 41 & 127 & \\
\hline $224 \mathrm{~F} 501$ & SW8270 & 4-Chloro-3-Methylphenol & Concrete & $\mathrm{R}$ & 41 & 127 & \\
\hline $224 \mathrm{~F} 501$ & SW8270 & 2,3,4,6-Tetrachlorophenol & Concrete & $\mathrm{R}$ & 41 & 127 & \\
\hline $224 \mathrm{~F} 501$ & SW8270 & 4,6-Dinitro-2-Methylphenol & Concrete & $\mathrm{R}$ & 41 & 127 & \\
\hline
\end{tabular}

Reason Codes:

1 = Exceeded holding time.

21 = Matrix spike recovery grossly outside control limits.

41 = Matrix effects may exist.

$117=$ Calibration verification did not meet criteria or was not performed.

127 = Surrogates recovery $<10$ percent.

153 = Internal area response show extremely low count.

the minimum detection limit, which varied from 0.69 to $0.73 \mathrm{ppm}$, well below the PAL of $25,000 \mathrm{ppm}$.

At CAS 23-05-02, hydroquinone analysis was successful for four soil samples in the area of the former leachfield. Two of these samples estimated (lab qualified as "estimated") the presence of hydroquinone at values of $0.73 \mathrm{ppm}$ and $3.6 \mathrm{ppm}$. In both CASs the distribution of the successful hydroquinone results indicates an absence above minimum detectable limits. Although the 90 percent completeness criterion was not met for hydroquinone, sufficient data were available to determine that hydroquinone is not a $\mathrm{COC}$ at these CASs. Therefore, the completeness criterion of sufficient data of acceptable quality to make DQO decisions was met. 
Table B.1-13

CAU 224 Rejected Data for CAS 06-23-01, Decon Pad Discharge Piping

\begin{tabular}{|c|c|c|c|c|c|c|}
\hline $\begin{array}{l}\text { Sample } \\
\text { Number }\end{array}$ & $\begin{array}{l}\text { Laboratory } \\
\text { Method }\end{array}$ & Parameter & Matrix & Qualifier & Reason 1 & Reason 2 \\
\hline $224 G 001$ & SW8270 & 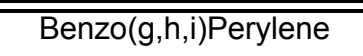 & $\overline{\text { Soil }}$ & $\overline{\overline{\mathrm{R}}}$ & 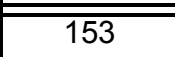 & $\overline{\overline{41}}$ \\
\hline $224 G 001$ & SW8270 & Benzo(k)Fluoranthene & Soil & $\bar{R}$ & 153 & 41 \\
\hline $224 G 001$ & SW8270 & Dibenzo(a,h)Anthracene & Soil & $R$ & 153 & 41 \\
\hline $224 G 001$ & SW8270 & Indeno(1,2,3-cd)Pyrene & Soil & $\mathrm{R}$ & 153 & 41 \\
\hline $224 G 001$ & SW8270 & Benzo(b)Fluoranthene & Soil & $\bar{R}$ & 153 & 41 \\
\hline $224 G 001$ & SW8270 & Benzo(a)Pyrene & Soil & $R$ & 153 & 41 \\
\hline $224 G 003$ & SW8270 & Pentachlorophenol & Soil & $\bar{R}$ & 42 & 41 \\
\hline $224 G 003$ & SW8270 & 4-Nitrophenol & Soil & $\bar{R}$ & 42 & 41 \\
\hline
\end{tabular}

Reason Codes:

41 = Matrix effects may exist.

42 = Matrix spike recovery was zero percent.

$153=$ Internal area response show extremely low count.

Table B.1-14

CAU 224 Rejected Data for CAS 11-04-01, Sewage Lagoons (Page 1 of 3 )

\begin{tabular}{|c|c|c|c|c|c|c|c|}
\hline $\begin{array}{l}\text { Sample } \\
\text { Number }\end{array}$ & $\begin{array}{l}\text { Laboratory } \\
\text { Method }\end{array}$ & Parameter & Matrix & Qualifier & Reason 1 & Reason 2 & Reason 3 \\
\hline $224 \mathrm{H} 001$ & SW8270 & Hydroquinone & Soil & $\overline{\mathrm{R}}$ & 127 & $\overline{41}$ & \\
\hline $224 \mathrm{H} 002$ & SW8270 & Hydroquinone & Soil & $\mathrm{R}$ & 127 & 41 & \\
\hline $224 \mathrm{H} 003$ & SW8270 & Hydroquinone & Soil & $\mathrm{R}$ & 127 & 41 & 21 \\
\hline $224 \mathrm{H} 004$ & SW8270 & Hydroquinone & Soil & $\mathrm{R}$ & 127 & 41 & \\
\hline $224 \mathrm{H} 005$ & SW8270 & Hydroquinone & Soil & $\mathrm{R}$ & 127 & 41 & \\
\hline $224 \mathrm{H} 006$ & SW8270 & Hydroquinone & Soil & $\mathrm{R}$ & 127 & 41 & \\
\hline $224 \mathrm{H} 007$ & SW8270 & Hydroquinone & Soil & $\mathrm{R}$ & 127 & 41 & \\
\hline $224 \mathrm{H} 008$ & SW8270 & Hydroquinone & Soil & $\mathrm{R}$ & 127 & 41 & \\
\hline $224 \mathrm{H} 009$ & SW8270 & Hydroquinone & Soil & $\mathrm{R}$ & 127 & 41 & \\
\hline $224 \mathrm{H} 010$ & SW8270 & Hydroquinone & Soil & $R$ & 127 & 41 & \\
\hline $224 \mathrm{H} 011$ & SW8270 & Hydroquinone & Soil & $\mathrm{R}$ & 127 & 41 & \\
\hline $224 \mathrm{H} 012$ & SW8270 & Hydroquinone & Soil & $\mathrm{R}$ & 127 & 41 & \\
\hline $224 \mathrm{H} 013$ & SW8270 & Hydroquinone & Soil & $\mathrm{R}$ & 127 & 41 & \\
\hline $224 \mathrm{H} 014$ & SW8270 & Hydroquinone & Soil & $\mathrm{R}$ & 127 & 41 & \\
\hline $224 \mathrm{H} 015$ & SW8270 & Hydroquinone & Soil & $\mathrm{R}$ & 127 & 41 & \\
\hline $224 \mathrm{H} 016$ & SW8270 & Hydroquinone & Soil & $\mathrm{R}$ & 127 & 41 & \\
\hline $224 \mathrm{H} 018$ & SW8270 & Hydroquinone & Soil & $\mathrm{R}$ & 127 & 41 & \\
\hline
\end{tabular}


Table B.1-14

CAU 224 Rejected Data for CAS 11-04-01, Sewage Lagoons (Page 2 of 3 )

\begin{tabular}{|c|c|c|c|c|c|c|c|}
\hline $\begin{array}{l}\text { Sample } \\
\text { Number }\end{array}$ & $\begin{array}{c}\text { Laboratory } \\
\text { Method }\end{array}$ & Parameter & Matrix & Qualifier & Reason 1 & Reason 2 & Reason 3 \\
\hline$\overline{\overline{224 \mathrm{H} 019}}$ & $\overline{\overline{S W} 8270}$ & "Hydroquinone & $\overline{\overline{\text { Soil }}}$ & $\overline{\overline{\mathrm{R}}}$ & $\overline{\overline{127}}$ & $\overline{\overline{41}}$ & \\
\hline $224 \mathrm{H} 020$ & SW8270 & Hydroquinone & Soil & $\mathrm{R}$ & 127 & 41 & \\
\hline $224 \mathrm{H} 021$ & SW8270 & Hydroquinone & Soil & $\bar{R}$ & 127 & 41 & \\
\hline $224 \mathrm{H} 022$ & SW8270 & Hydroquinone & Soil & $\bar{R}$ & 127 & 41 & \\
\hline $224 \mathrm{H} 023$ & SW8270 & Hydroquinone & Soil & $R$ & 127 & 41 & \\
\hline $224 \mathrm{H} 024$ & SW8270 & Hydroquinone & Soil & $\bar{R}$ & 127 & & \\
\hline $224 \mathrm{H} 025$ & SW8270 & Hydroquinone & Soil & $\mathrm{R}$ & 127 & & \\
\hline $224 \mathrm{H} 026$ & SW8270 & Hydroquinone & Soil & $\mathrm{R}$ & 21 & 127 & 41 \\
\hline $224 \mathrm{H} 028$ & SW8270 & Hydroquinone & Soil & $\mathrm{R}$ & 127 & & \\
\hline $224 \mathrm{H} 029$ & SW8270 & Hydroquinone & Soil & $\mathrm{R}$ & 127 & & \\
\hline $224 \mathrm{H} 030$ & SW8270 & Hydroquinone & Soil & $\bar{R}$ & 143 & 21 & \\
\hline $224 \mathrm{H} 031$ & SW8270 & Hydroquinone & Soil & $\bar{R}$ & 127 & & \\
\hline $224 \mathrm{H} 032$ & SW8270 & Hydroquinone & Soil & $\bar{R}$ & 127 & & \\
\hline $224 \mathrm{H} 034$ & SW8270 & Hydroquinone & Soil & $R$ & 127 & & \\
\hline $224 \mathrm{H} 035$ & SW8270 & Hydroquinone & Soil & $\mathrm{R}$ & 127 & & \\
\hline $224 \mathrm{H} 037$ & SW8270 & Hydroquinone & Soil & $\bar{R}$ & 127 & & \\
\hline $224 \mathrm{H} 038$ & SW8270 & Hydroquinone & Soil & $R$ & 127 & & \\
\hline $224 \mathrm{H} 039$ & SW8270 & Hydroquinone & Soil & $\bar{R}$ & 127 & & \\
\hline $224 \mathrm{H} 040$ & SW8270 & Hydroquinone & Soil & $\bar{R}$ & 127 & & \\
\hline $224 \mathrm{H} 041$ & SW8270 & Hydroquinone & Soil & $\bar{R}$ & 127 & & \\
\hline $224 \mathrm{H} 044$ & SW8270 & Hydroquinone & Soil & $\bar{R}$ & 127 & & \\
\hline $224 \mathrm{H} 045$ & SW8270 & Hydroquinone & Soil & $R$ & 127 & & \\
\hline $224 \mathrm{H} 047$ & SW8270 & Hydroquinone & Soil & $\mathrm{R}$ & 127 & & \\
\hline $224 \mathrm{H} 048$ & SW8270 & Hydroquinone & Soil & $\mathrm{R}$ & 127 & & \\
\hline $224 \mathrm{H} 050$ & SW8270 & Hydroquinone & Soil & $R$ & 127 & & \\
\hline $224 \mathrm{H} 051$ & SW8270 & Hydroquinone & Soil & $\bar{R}$ & 127 & & \\
\hline $224 \mathrm{H} 053$ & SW8270 & Hydroquinone & Soil & $\bar{R}$ & 127 & & \\
\hline $224 \mathrm{H} 054$ & SW8270 & Hydroquinone & Soil & $\bar{R}$ & 127 & & \\
\hline $224 \mathrm{H} 055$ & SW8270 & Hydroquinone & Soil & $R$ & 127 & & \\
\hline $224 \mathrm{H} 056$ & SW8270 & Hydroquinone & Soil & $R$ & 127 & & \\
\hline $224 \mathrm{H} 057$ & SW8270 & Hydroquinone & Soil & $\bar{R}$ & 127 & & \\
\hline $224 \mathrm{H} 058$ & SW8270 & Hydroquinone & Soil & $\mathrm{R}$ & 127 & & \\
\hline $224 \mathrm{H} 059$ & SW8270 & Hydroquinone & Soil & $\mathrm{R}$ & 127 & & \\
\hline
\end{tabular}


Table B.1-14

CAU 224 Rejected Data for CAS 11-04-01, Sewage Lagoons (Page 3 of 3 )

\begin{tabular}{|c|c|c|c|c|c|c|c|}
\hline $\begin{array}{l}\text { Sample } \\
\text { Number }\end{array}$ & $\begin{array}{c}\text { Laboratory } \\
\text { Method }\end{array}$ & Parameter & Matrix & Qualifier & Reason 1 & Reason 2 & Reason 3 \\
\hline$\overline{224 \mathrm{H} 060}$ & $\overline{\overline{S W} 8270}$ & Hydroquinone & $\overline{\text { Soil }}$ & $\overline{\overline{\mathrm{R}}}$ & $\overline{\overline{127}}$ & & \\
\hline $224 \mathrm{H} 061$ & SW8270 & Hydroquinone & Soil & $\mathrm{R}$ & 127 & & \\
\hline $224 \mathrm{H} 062$ & SW8270 & Hydroquinone & Soil & $\mathrm{R}$ & 127 & & \\
\hline $224 \mathrm{H} 063$ & SW8270 & Hydroquinone & Soil & $\mathrm{R}$ & 127 & & \\
\hline $224 \mathrm{H} 064$ & SW8270 & Hydroquinone & Soil & $\mathrm{R}$ & 127 & & \\
\hline $224 \mathrm{H} 065$ & SW8270 & Hydroquinone & Soil & $\mathrm{R}$ & 15 & 127 & \\
\hline $224 \mathrm{H} 066$ & SW8270 & Hydroquinone & Soil & $\mathrm{R}$ & 127 & & \\
\hline $224 \mathrm{H} 067$ & SW8270 & Hydroquinone & Soil & $\mathrm{R}$ & 127 & & \\
\hline $224 \mathrm{H} 068$ & SW8270 & Hydroquinone & Soil & $\mathrm{R}$ & 127 & & \\
\hline $224 \mathrm{H} 069$ & SW8270 & Hydroquinone & Soil & $\mathrm{R}$ & 127 & & \\
\hline $224 \mathrm{H} 070$ & SW8270 & Hydroquinone & Soil & $\mathrm{R}$ & 127 & & \\
\hline $224 \mathrm{H} 071$ & SW8270 & Hydroquinone & Soil & $\mathrm{R}$ & 127 & & \\
\hline $224 \mathrm{H} 072$ & SW8270 & Hydroquinone & Soil & $\mathrm{R}$ & 127 & & \\
\hline $224 \mathrm{H} 073$ & SW8270 & Hydroquinone & Soil & $\mathrm{R}$ & 127 & & \\
\hline $224 \mathrm{H} 074$ & SW8270 & Hydroquinone & Soil & $\mathrm{R}$ & 127 & & \\
\hline $224 \mathrm{H} 075$ & SW8270 & Hydroquinone & Soil & $\mathrm{R}$ & 127 & & \\
\hline
\end{tabular}

Reason Codes:

15 = Matrix spike recovery outside control limits.

21 = Matrix spike recovery grossly outside control limits.

41 = Matrix effects may exist.

127 = Surrogate recovery $<10$ percent.

\section{B.1.1.1.2 DQO Provisions To Limit False Positive Decision Error}

The false acceptance decision error was controlled by assessing the potential for false positive analytical results. Quality assurance/QC samples such as field blanks, trip blanks, laboratory control samples, and method blanks were used to determine whether a false positive analytical result may have occurred. Of $104 \mathrm{QA} / \mathrm{QC}$ samples submitted, no false positive analytical results were detected.

Proper decontamination of sampling equipment and the use of certified clean sampling equipment and containers also minimized the potential for cross contamination that could lead to a false positive analytical result. 
Table B.1-15

CAU 224 Rejected Data at CAS 23-05-02, Leachfield

(Page 1 of 3 )

\begin{tabular}{|c|c|c|c|c|c|c|c|c|}
\hline $\begin{array}{l}\text { Sample } \\
\text { Number }\end{array}$ & $\begin{array}{l}\text { Laboratory } \\
\text { Method }\end{array}$ & Parameter & Matrix & Qualifier & Reason 1 & Reason 2 & Reason 3 & Reason 4 \\
\hline 224J001 & SW8270 & Benzo(a)Anthracene & Soil & $\overline{\mathrm{R}}$ & 153 & $\overline{41}$ & & \\
\hline 224J001 & SW8270 & Di-N-Octyl Phthalate & Soil & $R$ & 153 & 41 & & \\
\hline 224J001 & SW8270 & 3,3'-Dichlorobenzidine & Soil & $R$ & 153 & 41 & & \\
\hline 224J001 & SW8270 & Butyl Benzyl Phthalate & Soil & $\bar{R}$ & 153 & 41 & & \\
\hline 224J001 & SW8270 & Pyrene & Soil & $R$ & 153 & 41 & & \\
\hline 224J001 & SW8270 & Chrysene & Soil & $R$ & 153 & 41 & & \\
\hline 224J002 & SW8270 & Hydroquinone & Soil & $R$ & 127 & 41 & & \\
\hline 224J003 & SW8270 & 4-Nitrophenol & Soil & $R$ & 21 & 41 & & \\
\hline 224J003 & SW8270 & Pentachlorophenol & Soil & $\bar{R}$ & 21 & 41 & & \\
\hline 224J003 & SW8270 & Hydroquinone & Soil & $R$ & 127 & 41 & 21 & \\
\hline 224J004 & SW8270 & Hydroquinone & Soil & $R$ & 127 & 41 & & \\
\hline 224J005 & SW8270 & Benzo(a)Anthracene & Soil & $R$ & 153 & 41 & & \\
\hline 224J005 & SW8270 & Di-N-Octyl Phthalate & Soil & $R$ & 153 & 41 & & \\
\hline 224J005 & SW8270 & 3,3'-Dichlorobenzidine & Soil & $R$ & 153 & 41 & & \\
\hline 224J005 & SW8270 & Bis(2-Ethylhexyl)Phthalate & Soil & $R$ & 153 & 41 & & \\
\hline 224J005 & SW8270 & Butyl Benzyl Phthalate & Soil & $\bar{R}$ & 153 & 41 & & \\
\hline 224J005 & SW8270 & Pyrene & Soil & $R$ & 153 & 41 & & \\
\hline 224J005 & SW8270 & Chrysene & Soil & $\bar{R}$ & 153 & 41 & & \\
\hline 224J005 & SW8270 & Hydroquinone & Soil & $\bar{R}$ & 127 & & & \\
\hline 224J006 & SW8270 & Hydroquinone & Soil & $R$ & 127 & 41 & & \\
\hline 224J007 & SW8270 & Hydroquinone & Soil & $\bar{R}$ & 127 & 41 & & \\
\hline 224J008 & SW8270 & Pyrene & Soil & $\bar{R}$ & 153 & 41 & & \\
\hline 224J008 & SW8270 & Benzo(a)Anthracene & Soil & $\bar{R}$ & 153 & 41 & & \\
\hline 224J008 & SW8270 & 3,3'-Dichlorobenzidine & Soil & $\bar{R}$ & 153 & 41 & & \\
\hline 224J008 & SW8270 & Chrysene & Soil & $\bar{R}$ & 153 & 41 & & \\
\hline 224J008 & SW8270 & Carbazole & Soil & $R$ & 153 & 41 & & \\
\hline 224J008 & SW8270 & Bis(2-Ethylhexyl)Phthalate & Soil & $R$ & 153 & 41 & & \\
\hline 224J008 & SW8270 & Di-N-Octyl Phthalate & Soil & $R$ & 153 & 41 & & \\
\hline 224J008 & SW8270 & Hydroquinone & Soil & $R$ & 127 & & & \\
\hline 224J009 & SW8270 & Hydroquinone & Soil & $\bar{R}$ & 127 & & & \\
\hline 224J010 & SW8270 & Hydroquinone & Soil & $R$ & 127 & 41 & & \\
\hline 224J014 & SW8270 & Hydroquinone & Soil & $\bar{R}$ & 127 & 41 & & \\
\hline 224J015 & SW8270 & Hydroquinone & Soil & $R$ & 127 & & & \\
\hline 224J016 & SW8270 & Hydroquinone & Soil & $\bar{R}$ & 127 & 41 & & \\
\hline 224J017 & SW8270 & Hydroquinone & Soil & $\bar{R}$ & 127 & & & \\
\hline 224J018 & SW8270 & Hydroquinone & Soil & $\bar{R}$ & 127 & & & \\
\hline 224J019 & SW8270 & Hydroquinone & Soil & $\mathrm{R}$ & 127 & & & \\
\hline $224 \mathrm{~J} 023$ & SW8270 & Dibenzo(a,h)Anthracene & Soil & $\bar{R}$ & 153 & 41 & & \\
\hline
\end{tabular}


Table B.1-15

CAU 224 Rejected Data at CAS 23-05-02, Leachfield

(Page 2 of 3 )

\begin{tabular}{|c|c|c|c|c|c|c|c|c|}
\hline $\begin{array}{l}\text { Sample } \\
\text { Number }\end{array}$ & $\begin{array}{l}\text { Laboratory } \\
\text { Method }\end{array}$ & Parameter & Matrix & Qualifier & Reason 1 & Reason 2 & Reason 3 & Reason 4 \\
\hline $224 \mathrm{~J} 023$ & SW8270 & Benzo(b)Fluoranthene & Soil & $\bar{R}$ & 153 & 41 & & \\
\hline 224J023 & SW8270 & Indeno(1,2,3-cd)Pyrene & Soil & $\mathrm{R}$ & 153 & 41 & & \\
\hline 224J023 & SW8270 & Bis(2-Ethylhexyl)Phthalate & Soil & $\mathrm{R}$ & 153 & 41 & & \\
\hline 224J023 & SW8270 & Pyrene & Soil & $\mathrm{R}$ & 153 & 41 & & \\
\hline 224J023 & SW8270 & Chrysene & Soil & $\mathrm{R}$ & 153 & 41 & & \\
\hline 224J023 & SW8270 & Benzo(g,h,i)Perylene & Soil & $\mathrm{R}$ & 153 & 41 & & \\
\hline 224J023 & SW8270 & Benzo(k)Fluoranthene & Soil & $\mathrm{R}$ & 153 & 41 & & \\
\hline 224J023 & SW8270 & Di-N-Octyl Phthalate & Soil & $\mathrm{R}$ & 153 & 41 & & \\
\hline 224J023 & SW8270 & 3,3'-Dichlorobenzidine & Soil & $\mathrm{R}$ & 153 & 41 & & \\
\hline 224J023 & SW8270 & Butyl Benzyl Phthalate & Soil & $\mathrm{R}$ & 153 & 41 & & \\
\hline 224J023 & SW8270 & Benzo(a)Pyrene & Soil & $\mathrm{R}$ & 153 & 41 & & \\
\hline 224J023 & SW8270 & Benzo(a)Anthracene & Soil & $\mathrm{R}$ & 153 & 41 & & \\
\hline 224J024 & SW8270 & Benzo(b)Fluoranthene & Soil & $\mathrm{R}$ & 153 & 41 & & \\
\hline 224J024 & SW8270 & Benzo(g,h,i)Perylene & Soil & $R$ & 153 & 41 & & \\
\hline 224J024 & SW8270 & Benzo(k)Fluoranthene & Soil & $\mathrm{R}$ & 153 & 41 & & \\
\hline 224J024 & SW8270 & Dibenzo(a,h)Anthracene & Soil & $\mathrm{R}$ & 153 & 41 & & \\
\hline 224J024 & SW8270 & Indeno(1,2,3-cd)Pyrene & Soil & $R$ & 153 & 41 & & \\
\hline 224J024 & SW8270 & Benzo(a)Pyrene & Soil & $\mathrm{R}$ & 153 & 41 & & \\
\hline 224J024 & SW8270 & Hydroquinone & Soil & $R$ & 127 & & & \\
\hline 224J026 & SW8270 & Hydroquinone & Soil & $\mathrm{R}$ & 127 & & & \\
\hline $224 \mathrm{~J} 027$ & SW8270 & Hydroquinone & Soil & $\mathrm{R}$ & 127 & & & \\
\hline 224J028 & SW8270 & Hydroquinone & Soil & $\mathrm{R}$ & 127 & 42 & 41 & \\
\hline 224J029 & SW8270 & Hydroquinone & Soil & $\mathrm{R}$ & 127 & & & \\
\hline 224J030 & SW8270 & Bis(2-Ethylhexyl)Phthalate & Soil & $\mathrm{R}$ & 153 & 41 & & \\
\hline 224J030 & SW8270 & Hydroquinone & Soil & $\mathrm{R}$ & 127 & & & \\
\hline $224 \mathrm{~J} 030$ & SW8270 & Di-N-Octyl Phthalate & Soil & $R$ & 153 & 41 & & \\
\hline 224J030 & SW8270 & 3,3'-Dichlorobenzidine & Soil & $\mathrm{R}$ & 153 & 41 & & \\
\hline $224 \mathrm{~J} 030$ & SW8270 & Butyl Benzyl Phthalate & Soil & $R$ & 153 & 41 & & \\
\hline 224J031 & SW8270 & Di-N-Octyl Phthalate & Soil & $R$ & 153 & 41 & & \\
\hline 224J031 & SW8270 & 3,3'-Dichlorobenzidine & Soil & $\mathrm{R}$ & 153 & 41 & & \\
\hline 224J031 & SW8270 & Butyl Benzyl Phthalate & Soil & $R$ & 153 & 41 & & \\
\hline 224J031 & SW8270 & Bis(2-Ethylhexyl)Phthalate & Soil & $R$ & 153 & 41 & & \\
\hline 224J031 & SW8270 & Hydroquinone & Soil & $\mathrm{R}$ & 127 & & & \\
\hline 224J032 & SW8270 & Hydroquinone & Soil & $\mathrm{R}$ & 127 & & & \\
\hline 224J034 & SW8270 & Hydroquinone & Soil & $\mathrm{R}$ & 127 & & & \\
\hline 224J035 & SW8270 & Hydroquinone & Soil & $\mathrm{R}$ & 127 & & & \\
\hline 224J036 & SW8270 & Hydroquinone & Soil & $R$ & 127 & & & \\
\hline 224J037 & SW8270 & Hydroquinone & Soil & $\mathrm{R}$ & 127 & & & \\
\hline
\end{tabular}


Table B.1-15

CAU 224 Rejected Data at CAS 23-05-02, Leachfield

(Page 3 of 3 )

\begin{tabular}{|c|c|c|c|c|c|c|c|c|}
\hline $\begin{array}{l}\text { Sample } \\
\text { Number }\end{array}$ & $\begin{array}{l}\text { Laboratory } \\
\text { Method }\end{array}$ & Parameter & Matrix & Qualifier & Reason 1 & Reason 2 & Reason 3 & Reason 4 \\
\hline 224J038 & SW8270 & Hydroquinone & Soil & $\bar{R}$ & 127 & & & \\
\hline
\end{tabular}

Reason Codes:

$21=$ Matrix spike recovery grossly outside control limits.

41 = Matrix effects may exist.

42 = Matrix spike effects may exist.

117 = Calibration verification did not meet criteria or was not performed.

126 = Surrogates diluted out.

127 = Surrogates recovery $<10$ percent.

134 = Internal standard area count outside control limits.

$153=$ Internal area response show extremely low count.

Table B.1-16

Chemical Completeness Measurements

\begin{tabular}{|c|c|c|c|c|c|c|c|c|c|}
\hline \multirow{2}{*}{$\begin{array}{c}\text { Completeness } \\
\text { Parameters }\end{array}$} & \multicolumn{6}{|c|}{ ORGANICS } & \multicolumn{3}{|c|}{ INORGANICS } \\
\hline & VOCs & SVOCs & $\begin{array}{l}\text { TPH- } \\
\text { DRO }\end{array}$ & PCBs & Pesticides & Herbicides & Cyanide & Metals* & Mercury \\
\hline \multicolumn{10}{|c|}{ Sample Analysis Completeness } \\
\hline Samples Submitted & 380 & 360 & 399 & 360 & 21 & 5 & 70 & 360 & 360 \\
\hline Samples Analyzed & 380 & 360 & 399 & 360 & 21 & 5 & 70 & 360 & 360 \\
\hline Percent Completeness & 100 & 100 & 100 & 100 & 100 & 100 & 100 & 100 & 100 \\
\hline \multicolumn{10}{|c|}{ Measurement Usability Completeness } \\
\hline Measurements ** & 26,345 & 28,745 & 400 & 2,527 & 484 & 50 & 70 & 5,760 & 360 \\
\hline Rejected - Field & 0 & 0 & 0 & 0 & 0 & 0 & 0 & 0 & 0 \\
\hline Rejected - Lab/Matrix & 0 & 1863 & 1 & 0 & 0 & 0 & 0 & 0 & 0 \\
\hline Percent Completeness & 100 & 93.5 & 99.8 & 100 & 100 & 100 & 100 & 100 & 100 \\
\hline
\end{tabular}

*Measurements include Aluminum, Antimony, Arsenic, Barium, Beryllium, Chromium, Cobalt, Coppery, Lead, Manganese,

Molybdenum, Nickel, Selenium, Silver, Zinc

**Measurements include re-analyses

DRO = Diesel-range organics

$\mathrm{PCB}=$ Polychlorinated biphenyl

RCRA = Resource Conservation and Recovery Act

SVOC = Semivolatile organic compound

TCLP = Toxicity characterization leaching procedure

$\mathrm{TPH}=$ Total petroleum hydrocarbons

VOC $=$ Volatile organic compound 
Table B.1-17

Radiological Completeness Measurements for CAU 224

\begin{tabular}{|c|c|c|c|c|c|c|}
\hline $\begin{array}{c}\text { Completeness } \\
\text { Parameters }\end{array}$ & $\begin{array}{c}\text { Gamma } \\
\text { Spectroscopy }\end{array}$ & $\begin{array}{l}\text { Isotopic } \\
\text { Uranium }\end{array}$ & $\begin{array}{l}\text { Isotopic } \\
\text { Plutonium }\end{array}$ & Strontium-90 & Tritium & $\begin{array}{c}\text { Gross } \\
\text { Alpha/Beta }\end{array}$ \\
\hline \multicolumn{7}{|c|}{ Sample Analysis Completeness } \\
\hline Samples submitted & 338 & 245 & 354 & 245 & 3 & 3 \\
\hline Samples analyzed & 338 & 245 & 354 & 245 & 3 & 3 \\
\hline Percent Completeness & 100 & 100 & 100 & 100 & 100 & 100 \\
\hline \multicolumn{7}{|c|}{ Measurement Usability Completeness } \\
\hline Total Measurements & 7,436 & 735 & 708 & 245 & 3 & 6 \\
\hline Rejected - Field & 0 & 0 & 0 & 0 & 0 & 0 \\
\hline Rejected - Lab/Matrix & 0 & 0 & 0 & 0 & 0 & 0 \\
\hline Percent Completeness & 100 & 100 & 100 & 100 & 100 & 100 \\
\hline
\end{tabular}

\section{B.1.1.2 Decision II}

Decision II - "If a COC is present, is sufficient information available to evaluate appropriate corrective action alternatives?"

\section{Decision Rules:}

- If the observed concentration of any COC in a Decision II sample exceeds the PALs, then additional samples will be collected to complete the determination of the extent.

- If observed COC concentrations in a sample from all bounding directions are less than the PALs, then the decision will be that the extent of contamination has been defined in the lateral and/or vertical direction.

\section{B.1.1.2.1 DQO Provisions To Limit False Negative Decision Error}

A false rejection decision error (where consequences are more severe) is controlled by meeting the following criteria:

1. Having a high degree of confidence that the sample locations selected will identify the extent of the COCs. 
2. Having a high degree of confidence that analyses conducted will be sufficient to detect any COCs present in the samples.

3. Having a high degree of confidence that the data set is of sufficient quality and completeness.

\section{Criteria 1:}

The following criteria (stipulated in the CAU 224 DQOs [NNSA/NV, 2004]) were used in selecting Decision II sample locations.

- Selection of sampling locations associated with vertical extent was accomplished by the depth sequences used during the Decision I sampling for each CAS.

- Selection of sampling locations associated with lateral extent was accomplished by measuring approximately $15 \mathrm{ft}$ from the Decision I sample location in a triangular pattern surrounding the Decision I location. Where locations were not achievable at $15 \mathrm{ft}$ laterally, the distances were recorded on the sample collection log and in the Site Supervisor's Daily Log. At CAS 06-17-04, surface radiological contamination was found to be relatively widespread; therefore, Decision II samples were placed at greater intervals.

In general, soil sample results demonstrated that the vertical and lateral extent of COCs were defined. The extent sample locations and concentrations for the contaminants driving the extent of contamination are shown in Figure B.1-1 through Figure B.1-3.

\section{Criteria 2:}

All samples were analyzed for the COCs present at the corresponding CAS.

- CAS 03-05-01- TPH-DRO, Pu-239

- CAS 05-04-01- Pesticides (DDE, DDT, chlordane)

- CAS 06-05-01- Am-241, Cs-137, Pu-239

- CAS 06-17-04- TPH-DRO, Pu-238, Pu-239, Cs-137, Am-241

- CAS 23-05-02- TPH-DRO

The second criterion for extent (sensitivity) was accomplished for all analyses as demonstrated in Table B.1-3 and Table B.1-4.

\section{Criteria 3:}

To satisfy the third criterion for extent, the entire data set, as well as individual sample results, were assessed against the DQIs of precision, accuracy, comparability, completeness, and 


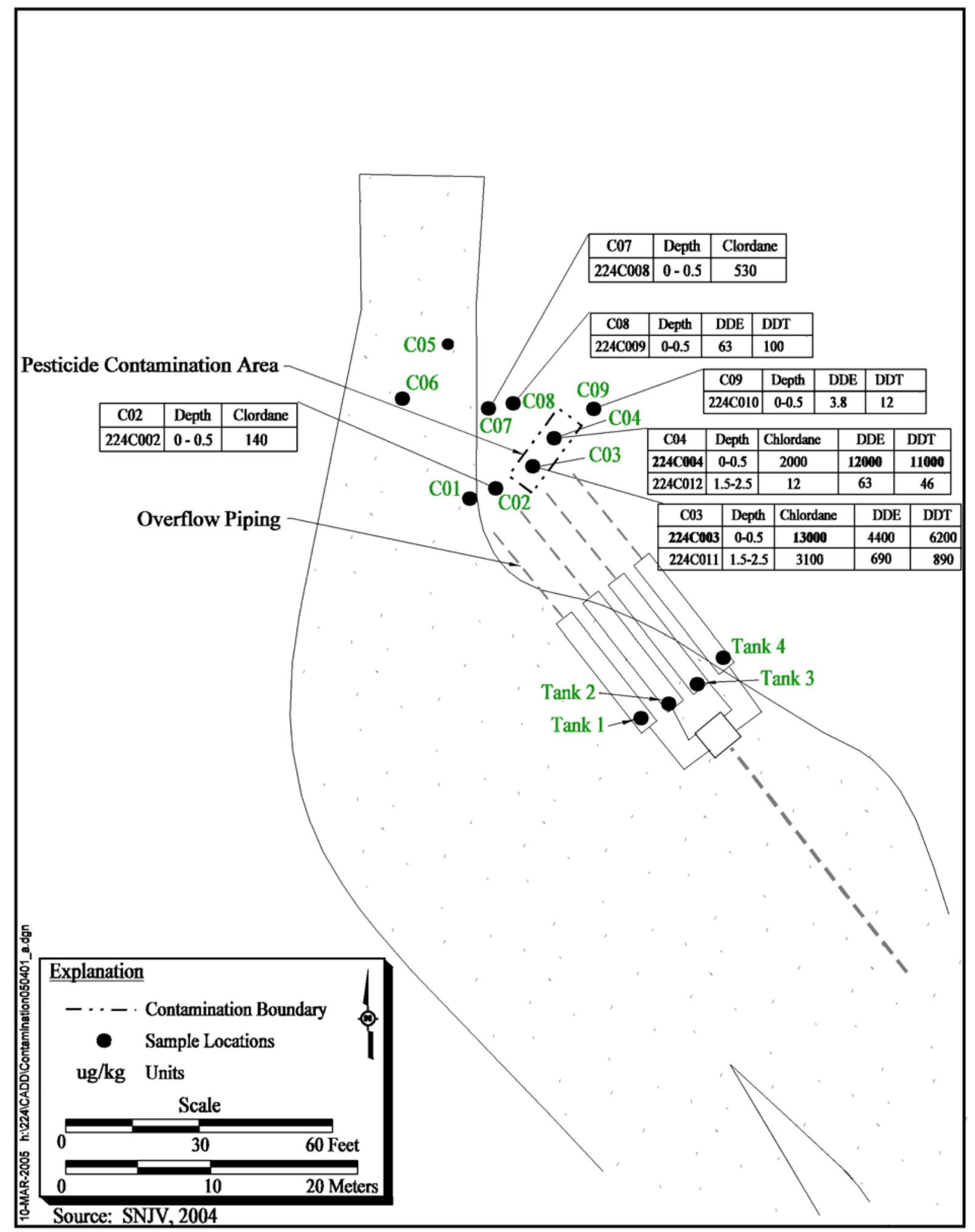

Figure B.1-1

Extent of Contamination, CAS 05-04-01, Septic Tanks (4)/Discharge Area 


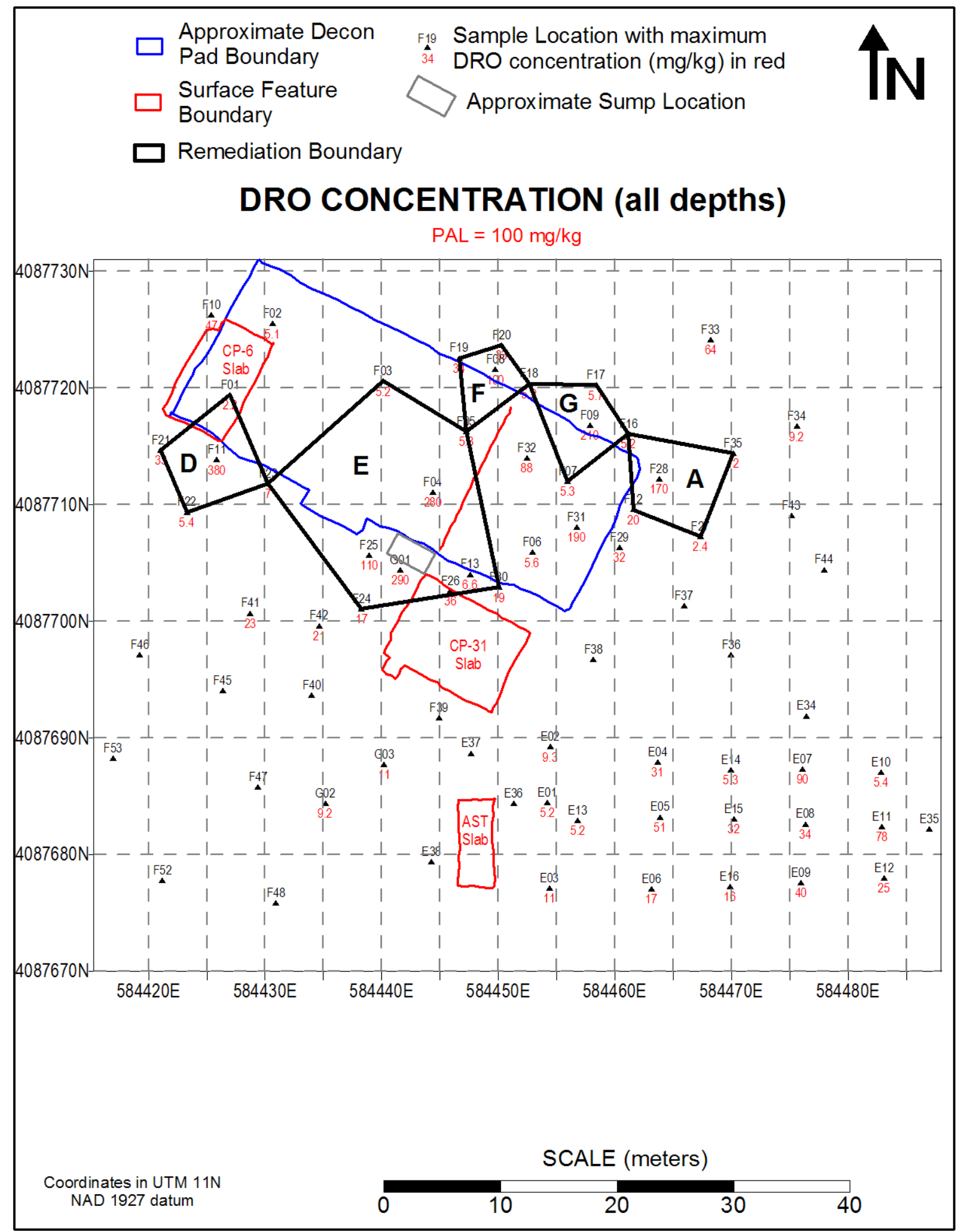

Figure B.1-2

Extent of TPH-DRO Contamination, CASs 06-05-01, Leachfield, 06-17-04, Decon Pad and Wastewater Catch, and 06-23-01, Decon Pad Discharge Piping 


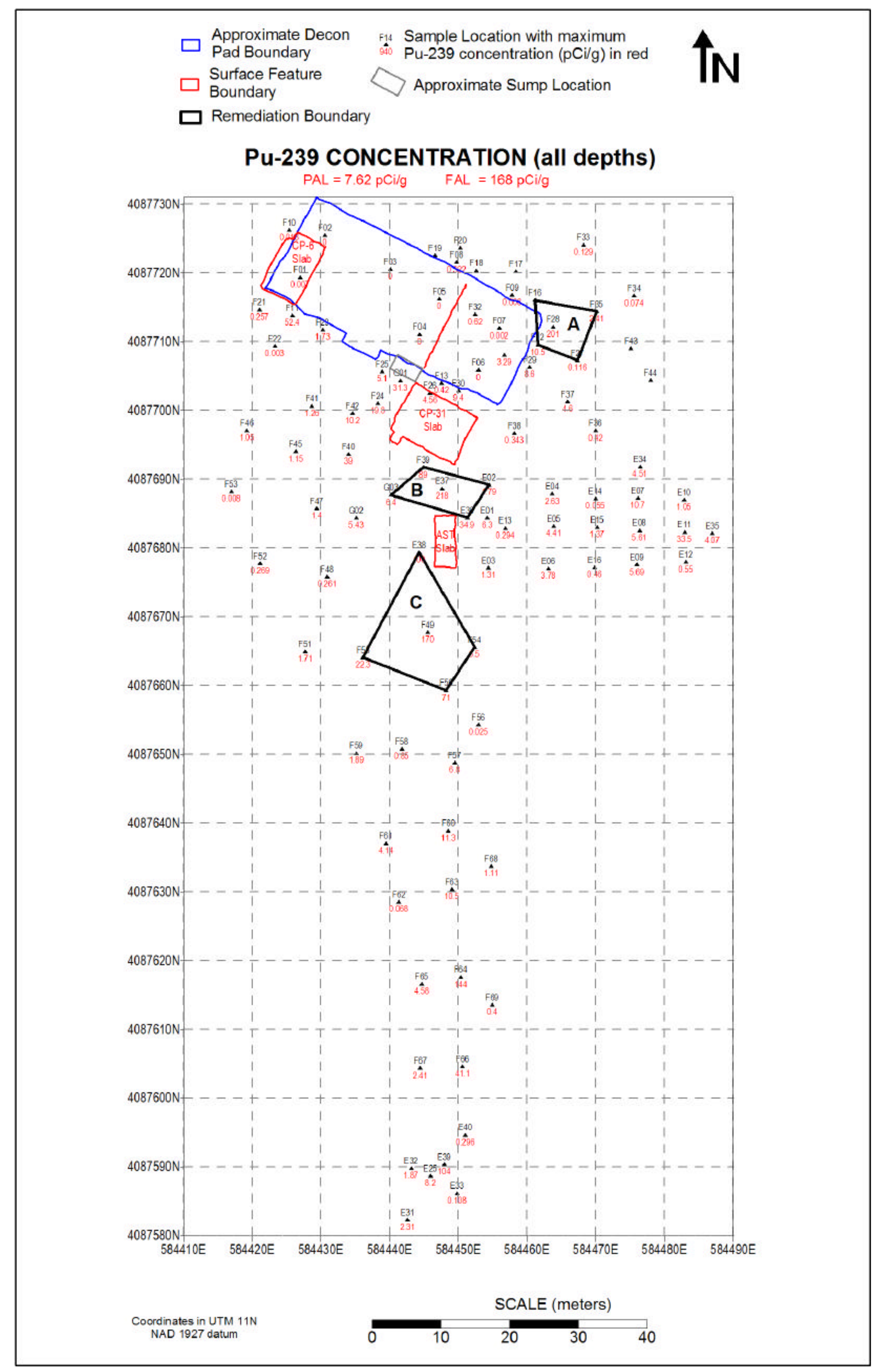

Figure B.1-3

Extent of Pu-239 Contamination, CASs 06-05-01, Leachfield, 06-17-04,

Decon Pad and Wastewater Catch, and 06-23-01, Decon Pad Discharge Piping 
representativeness, as defined in the Industrial Sites QAPP (NNSA/NV, 2002). The DQI discussion is presented under Criteria 3 for Decision I.

\section{B.1.1.2.2 DQO Provisions To Limit False Positive Decision Error}

The false acceptance decision error was controlled by assessing the potential for false positive analytical results. Quality assurance/QC samples such as field blanks, trip blanks, laboratory control samples, and method blanks were used to determine whether a false positive analytical result may have occurred. Of $42 \mathrm{QA} / \mathrm{QC}$ samples submitted, no false positive analytical results were detected.

Proper decontamination of sampling equipment and the use of certified clean sampling equipment and containers also minimized the potential for cross contamination that could lead to a false positive analytical result.

\section{B.1.2 Conduct a Preliminary Data Review}

A preliminary data review was conducted by reviewing QA reports and inspecting the data. The contract analytical laboratories generate a QA report when data quality does not meet contractual requirements. All data received from the analytical laboratories met contractual requirements and a QA report was not generated. Data were validated and verified to ensure that the measurement systems performed in accordance with the criteria specified and that the validated data set quality is satisfactory.

\section{B.1.3 Select the Test and Identify Key Assumptions}

The test for making DQO Decision I was the comparison of the maximum analyte result from each CAS to the corresponding PAL. The test for making DQO Decision II was the comparison of all COC analyte results from each bounding sample to the corresponding PALs.

The key assumptions that could impact a DQO decision are listed in Table B.1-18.

\section{B.1.4 Verify the Assumptions}

The results of the investigation support the key assumptions identified in the CAU 224 DQOs and Table B.1-18 except as listed below: 


\section{Table B.1-18}

Key Assumptions

\begin{tabular}{|c|c|}
\hline Exposure Scenario & $\begin{array}{l}\text { Site workers are only exposed to contaminants of concern (COCs) through oral } \\
\text { ingestion, inhalation, external exposure to radiation, or dermal contact (by absorption) } \\
\text { of COCs absorbed onto the soils. } \\
\text { Exposure to contamination is limited to industrial site workers, } \\
\text { construction/remediation workers, and military personnel conducting training. } \\
\text { The investigation results did not reveal any potential exposures other than those } \\
\text { identified in the Conceptual Site Models (CSMs). }\end{array}$ \\
\hline Affected Media & $\begin{array}{l}\text { Surface soil, shallow subsurface soil, and potentially perched (shallow) groundwater. } \\
\text { Deep groundwater contamination is not a concern. } \\
\text { Contaminants migrating to regional aquifers are not considered. } \\
\text { The investigation results did not reveal any affected media other than those identified } \\
\text { in the CSMs. }\end{array}$ \\
\hline $\begin{array}{l}\text { Location of } \\
\text { Contamination/Release Points }\end{array}$ & $\begin{array}{l}\text { The area of contamination is contiguous. } \\
\text { The extent of COC concentration decreases away from the area of contamination. } \\
\text { The investigation results did not reveal any locations of contamination or release } \\
\text { points other than those identified in the CSMs. }\end{array}$ \\
\hline Transport Mechanisms & $\begin{array}{l}\text { Surface transport may occur as a result of a spill or storm water runoff. } \\
\text { Surface transport beyond shallow substrate is not a concern. } \\
\text { The investigation results did not reveal any transport mechanisms other than those } \\
\text { identified in the CSMs. }\end{array}$ \\
\hline Preferential Pathways & $\begin{array}{l}\text { None. } \\
\text { The investigation results did not reveal any preferential pathways other than those } \\
\text { identified in the CSMs. }\end{array}$ \\
\hline $\begin{array}{l}\text { Lateral and Vertical Extent of } \\
\text { Contamination }\end{array}$ & $\begin{array}{l}\text { Subsurface contamination, if present, is contiguous and decreases with distance and } \\
\text { depth from the source. } \\
\text { Surface contamination may occur laterally as a result of a spill or storm water runoff. } \\
\text { The investigation results did not reveal any lateral and vertical extent of contamination } \\
\text { other than those identified in the CSMs. }\end{array}$ \\
\hline Groundwater impacts & $\begin{array}{l}\text { None. } \\
\text { The investigation results did not reveal groundwater impacts other than those } \\
\text { identified in the CSMs. }\end{array}$ \\
\hline Future Land Use & $\begin{array}{l}\text { Nonresidential. } \\
\text { The investigation results did not reveal any future land uses other than those identified } \\
\text { in the CSMs. }\end{array}$ \\
\hline
\end{tabular}

- Exception: The lateral extent of the contamination did not decrease with distance from the source for CAS 06-17-04. Downgradient from the decon pad, radiological contamination was identified at one location above the FALs. The downgradient contamination identified for CAS 06-17-04 has been defined laterally.

- Impact: No impact to the CSM. 
All data collected during the CAI supported CSMs with the exceptions noted in this section. These exceptions did not invalidate the CSMs presented in the CAIP, nor did they necessitate revisions to the CSMs.

\section{B.1.5 Results}

This section resolves the two DQO decisions for each of the CAU 224 CASs.

\section{B.1.5.1 Decision I}

\section{Decision Rule:}

If the concentration of any COPC in a target population exceeds the FAL for that COPC during the initial investigation, then that COPC is identified as a COC and Decision II sampling will be conducted.

\section{Result:}

In the following CASs, COCs exceeded FALs. Decision II was then resolved for these COCs in each CAS.

- CAS 05-04-01 - Pesticides (DDE, DDT, chlordane)

- CAS 06-05-01 - Pu-239

- CAS 06-17-04 - TPH-DRO, Pu-239

- CAS 06-23-01 - TPH-DRO

\section{Decision Rule:}

If all COPC concentrations are less than the corresponding FALs, then the decision will be no further action.

\section{Result:}

No COCs were identified in samples collected from CAS 02-04-01, 03-05-01, 06-03-01, 11-04-01, and 23-05-02. At CASs 02-04-01, and 23-05-02, COPCs were encountered above PALs (PAHs at CAS 02-04-01, and TPH-DRO and PAHs at CAS 23-05-02). However these contaminants are not associated with the CASs and are not considered COCs. Total petroleum hydrocarbons-DRO were encountered above PALs in CAS 03-05-01. However, a component breakdown of hazardous 
constituents of TPH established new FALs for the site as described in Section 3.1. Corrective Action Site 03-05-01 also contained one sample with a $\mathrm{Pu}-239$ activity of $8.7 \mathrm{pCi} / \mathrm{g}$; however, the activity is below the FAL of $12.7 \mathrm{pCi} / \mathrm{g}$. Therefore, Pu-239 is not considered a COC at this CAS. No further action was identified as the recommended corrective action alternative for these CASs.

\section{B.1.5.2 Decision II}

\section{Decision Rule:}

If the observed concentration of any COC in a Decision II sample exceeds the PALs, then additional samples will be collected to complete the determination of the extent.

\section{Result:}

Samples to define extent were collected from CASs 03-05-01, 05-04-01, 06-05-01, 06-17-04, and 23-05-02. Samples collected at CAS 23-05-02 were analyzed for TPH-DRO in order to confirm that the diesel contamination found in Decision I samples was not associated with the former leachfield. The fact that contamination continued to be found most prevalently directly below the asphalt parking lot lead to the decision that the TPH-DRO contamination is not related to the CAS. Samples were also collected at CAS 03-05-01 to delineate the extent of contamination above PALs; however, the initial Decision I samples are all below FALs. Due to the proximity of CAS 06-23-01 to CASs 06-17-04 and 06-05-01, the DRO contamination at location G01 (CAS 06-23-01) was bounded by samples from CASs 06-17-04 and 06-05-01.

\section{Decision Rule:}

If all observed COC population parameters are less than the PALs, then the decision will be that the extent of contamination has been defined in the lateral and/or vertical direction.

\section{Result:}

The vertical and lateral extent of contamination at CASs 05-04-01, 06-05-01, 06-17-04, and 06-23-01 were defined. The extent of COC plumes are displayed in Figure B.1-1, Figure B.1-2, and Figure B.1-3. 


\section{B.2.0 References}

NNSA/NSO, see U.S. Department of Energy, National Nuclear Security Administration Nevada Site Office.

NNSA/NV, see U.S. Department of Energy, National Nuclear Security Administration Nevada Operations Office.

SNJV, see Stoller-Navarro Joint Venture.

Stoller-Navarro Joint Venture. 2004. Site-Specific Health and Safety Plan for CAU 224, Decon Pad and Septic Systems, Nevada Test Site, Nevada. Las Vegas, NV.

U.S. Department of Energy, National Nuclear Security Administration Nevada Operations Office. 2002. Industrial Sites Quality Assurance Project Plan, Nevada Test Site, Nevada, Rev. 3, DOE/NV--372. Las Vegas, NV.

U.S. Department of Energy, National Nuclear Security Administration Nevada Site Office. 2004. Corrective Action Investigation Plan for Corrective Action Unit 224: Decon Pad and Septic Systems, Nevada Test Site, Nevada, Rev. 0, DOE/NV--965. Las Vegas, NV. 


\section{Appendix C}

\section{Cost Estimates for CAU 224}

(14 Pages) 


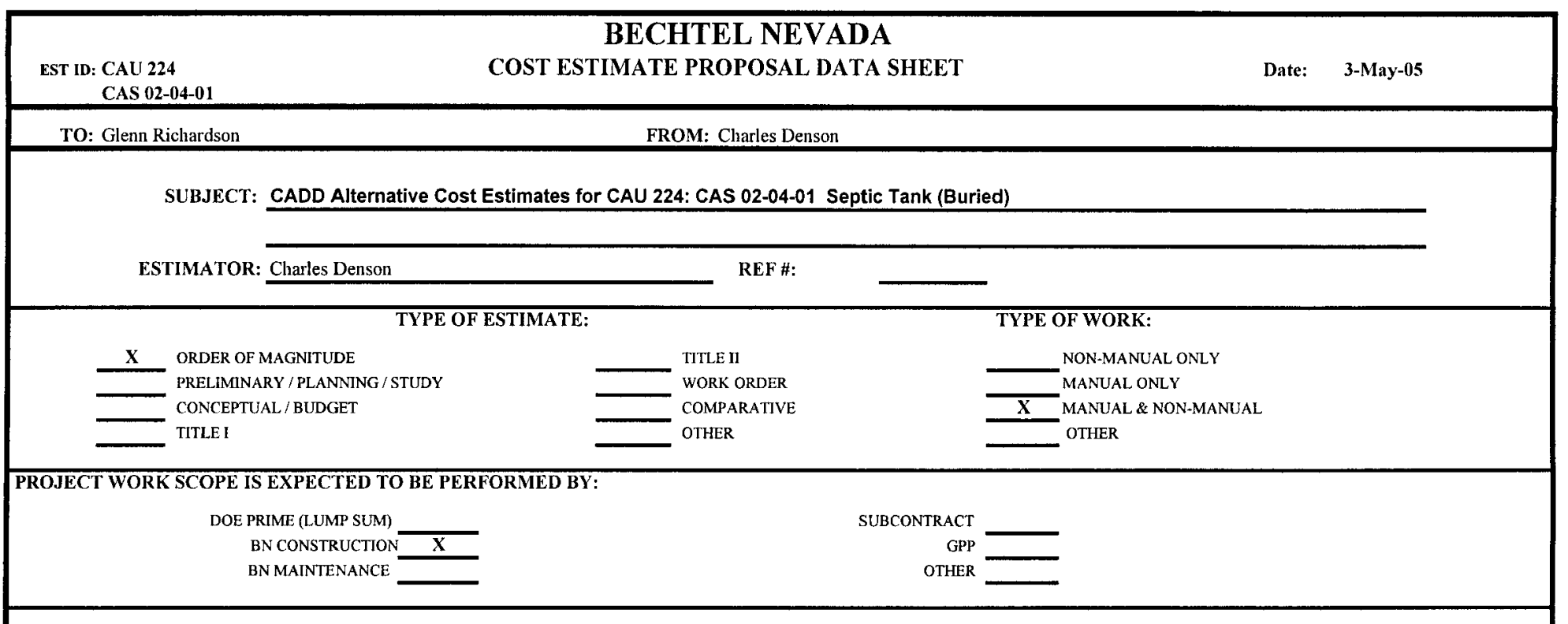

\section{STATEMENT OF WORK}

This estimate has been prepared to provide remedial alternative costs for the closure of Corrective Action Site (CAS) 02-04-01, which is included within Corrective Action Unit (CAU) 224. CAU 224 CAS 02-04-01 is an environmental restoration site listed in the Federal Facility Agreement and Consent Order (FFACO). CAS 02-04-01 is specifically described within the FFACO as Septic Tank (Buried), located in Area 2 RSM $2 \mathrm{~L} 13$. Two alternatives have been evaluated for closure of the CAS: I. No Further Action and III. Closure in Place with Administrative Controls. This estimate will be used to identify the most cost effective alternative for closure of the site while remaining protective of human health and the environment. The total estimated costs are intended for comparative analysis of remedial fieldwork cost only. Cost for project management, plan preparation, project support, and/or other activities are not included herein.

\section{SCOPE:}

Provide site closure using one of the following alternatives:

I) NO FURTHER ACTION

III) CLOSURE IN PLACE WITH ADMINISTRATIVE CONTROLS

\section{$\underline{B A S I S:}$}

The characterization contractor recently completed field investigations of the Buried Septic Tank and associated piping located in the Area 2 Support Facility area. The septic tank is estimated to be approximately $9.5 \mathrm{ft}$ by $3.7 \mathrm{ft}$. by $2.7 \mathrm{ft}$. The types and amounts of materials requiring remedial action are as follows: 126 gallons of tank liquid with 1 inch of sludge in the septic tank, the septic tank structure, and the associated subsurface piping. Site closure estimates for each alternative were priced using standard construction references such as RS Means, Richardson's, and the BN estimating database.

\section{ALTERNATIVE SPECIFIC BASIS OF ESTIMATE/ASSUMPTIONS}

Alternative I: No Further Action with Best Management Practices

- Excavate to expose the septic tank or manhole access

- Add bacteria disinfectant to septic tank contents

- Solidify septic tank contents with clean backfill material

- Remove and load septic tank and contents into end dump for Area 6 hydrocarbon landfill disposal

- Field Screening and verification sampling to analyze for TPH

- Grout associated subsurface piping

- Backfill to the approximate existing grade

Alternative III: Closure in Place with Administrative Controls

- Excavate to Expose Septic Tank or Manhole Access

- Add Grout Mixture inside of Septic Tank

- Install Administrative Controls (identification/warning signs) 


\section{ASSUMPTIONS:}

- No corrective actions are required for the surrounding areas outside the CAS boundary.

- All COCs at the site have been identified during the site investigation and analytical data accurately represent site conditions and waste characteristics.

- Assumes the type of septic tank requiring remedial action is concrete.

- Assumes associated subsurface piping will not require removal and will be grouted in place as a closure action

- Assumes there is no evidence of an associated leachfield or lagoon based on site reconnaissance that requires a corrective action.

- Assumes no radioactive constituents nor pesticide contamination are present in the septic tank.

- Equipment will remain operational to support the planned/scheduled completion of each CADD alternative.

- Waste volumes are based on field measurements collected during the corrective action investigation.

- Work to be performed by BN during a "normal" workday (no provision for overtime has been provided). Shifts are based on 10-hour days / 4-days per week

- This estimate does not include the efficiencies which may be realized if work for similar activities at similar sites can be completed concurrently.

- Dimensions, volumes, measurements, and analytical data provided by the characterization contractor accurately represent site conditions and waste

characteristics.

- This estimate does not include costs for preparation of required project plans, permits, reports, site preparations, or project management.

\section{ESCALATION:}

No escalation factors have been applied. All costs are in FY05 Rev. 1 dollars.

\section{CONTINGENCY:}

Contingency costs are not included in this estimate.

\section{RATES:}

Rates are based on FY05 final rates (Rev. 1) effective 10/20/05 and were applied using the BN FY05 cost model.

\section{COST ALTERNATIVES SUMMARY:}

Alternative I: No Further Action
a. Excavate to Expose Septic Tank or Manhole Access
b. Add Disinfectant and Solidify Septic Tank Contents
c. Remove and Load Septic Tank and Contents into End Dump
d. Field Screening and Verification Sampling
e. Grout Associated Subsurface Piping
f. Backfill to Approx. Existing Grade
g. Waste Management (TPH and Sanitary)

Alternative III: Closure in Place with Administrative Controls
a. Excavate to Expose Septic Tank or Manhole Access
b. Add Grout Mixture inside of Septic Tank
c. Install Administrative Controls
d. Waste Management (Sanitary)

\section{REVIEW/CONCURRENCE:}
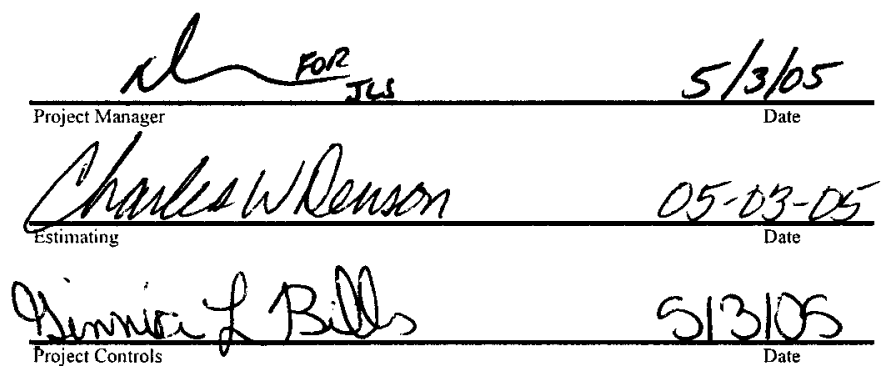


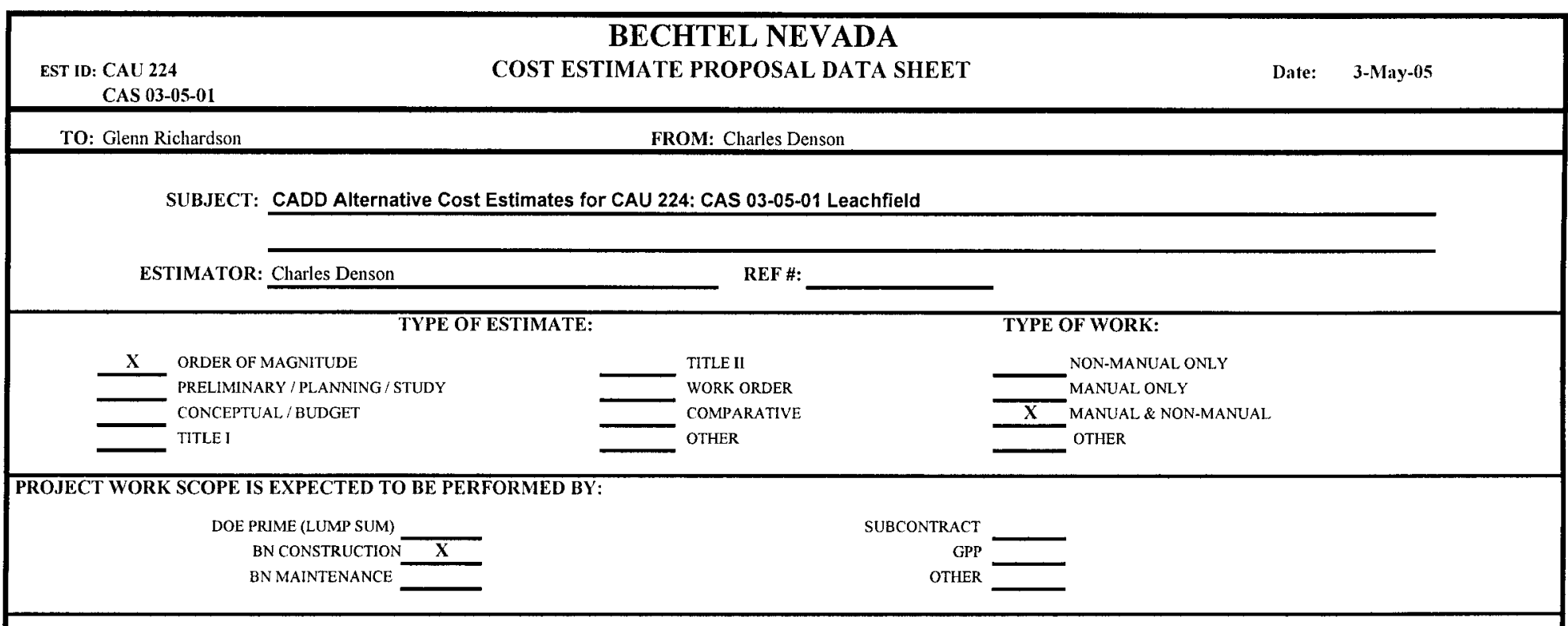

\section{STATEMENT OF WORK}

This estimate has been prepared to provide remedial alternative costs for the closure of Corrective Action Site (CAS) 03-05-01, which is included within Corrective Action Unit (CAU) 224. CAU 224 CAS 03-05-01 is an environmental restoration site listed in the Federal Facility Agreement and Consent Order (FFACO). CAS 03-05-01 is specifically described within the FFACO as Leachfield, located in Area 3 Subdock/Steampit. Three alternatives have been evaluated for closure of the CAS: I. No Further Action; II. Clean Closure; and III. Closure in Place with Administrative Controls. This estimate will be used to identify the most cost effective alternative for closure of the site while remaining protective of human health and the environment. The total estimated costs are intended for comparative analysis of remedial fieldwork cost only. Cost for project management, plan preparation, project support, and/or other activities are not included herein.

\section{SCOPE:}

Provide site closure using one of the following alternatives:

I) NO FURTHER ACTION

II) CLEAN CLOSURE

III) CLOSURE IN PLACE WITH ADMINISTRATIVE CONTROLS

\section{BASIS:}

The characterization contractor recently completed field investigations of the Leach Pit area that indicates the following: no contaminants of concern exceed the final action levels. Therefore, no further action is the recommended alternative. Site closure estimates for each alternative were priced using standard construction references such as RS Means, Richardson's, and the BN estimating database. There is no estimate required for evaluation of the No Further Action alternative since no cost is incurred.

\section{ALTERNATIVE SPECIFIC BASIS OF ESTIMATE/ASSUMPTIONS}

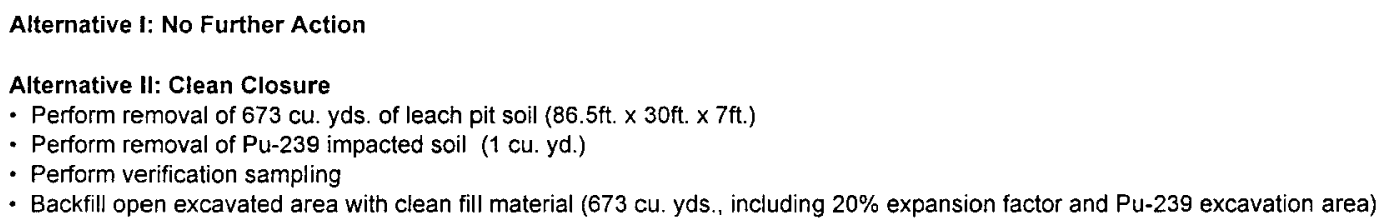

Alternative III: Closure in Place with Administrative Controls

- Perform removal of Pu-239 impacted soil near the Leach Pit

- Install Wire Fencing

- Implement Administrative Controls (identification/warning signs) 


\section{ASSUMPTIONS:}

- No corrective actions are required for the surrounding areas outside the CAS boundary

- All COCs at the site have been identified during the site investigation and analytical data accurately represent site conditions and waste characteristics.

- The nature of contamination is limited to the leach pit area.

- Assumes the estimated dimensions of the leach pit are $86.5 \mathrm{ft}$. by $30 \mathrm{ft}$. by $7 \mathrm{ft}$

- Assumes subsurface piping associated with the leach pit will not require removal and will be grouted in place as a closure action.

- Assumes no radioactive constituents nor pesticide contamination is present in the leach pit area.

- Equipment will remain operational to support the planned/scheduled completion of each CADD alternative.

- Waste volumes are based on field measurements collected during the corrective action investigation.

- Work to be performed by $\mathrm{BN}$ during a "normal" workday (no provision for overtime has been provided). Shifts are based on 10-hour days / 4-days per week.

- This estimate does not include the efficiencies which may be realized if work for similar activities at similar sites can be completed concurrently.

- Dimensions, volumes, measurements, and analytical data provided by the characterization contractor accurately represent site conditions and waste characteristics.

- This estimate does not include costs for preparation of required project plans, permits, reports, site preparations, or project management.

\section{ESCALATION:}

No escalation factors have been applied. All costs are in FY05 Rev. 1 dollars.

\section{CONTINGENCY:}

Contingency costs are not included in this estimate.

\section{RATES:}

Rates are based on FY05 final rates (Rev. 1) effective 10/20/05 and were applied using the BN FY05 cost model.

\section{COSTALTERNATIVES SUMMARY:}

Alternative I: No Further Action

a. Perfonn removal of $673 \mathrm{cu}$. yds. of leach pit soil $(86.5 \mathrm{ft} . \times 30 \mathrm{ft} . \times 7 \mathrm{ft}$.)

b. Perform removal of Pu-239 impacted soil (1 cu. yd.)

c. Perforn verification sampling

d. Backfill open excavated area with clean fill material ( $673 \mathrm{cu}$. yds., including $20 \%$ expansion factor)

e. Waste Management (Sanitary)

Alternative III: Closure in Place with Administrative Controls

a. Perform removal of Pu-239 impacted soil near the Leach Pit (1 cu. yd.)

b. Install Wire Fencing

c. Implement Administrative Controls (identification / warning signs)

d. Waste Management (Sanitary)

\section{REVIEW/CONCURRENCE:}
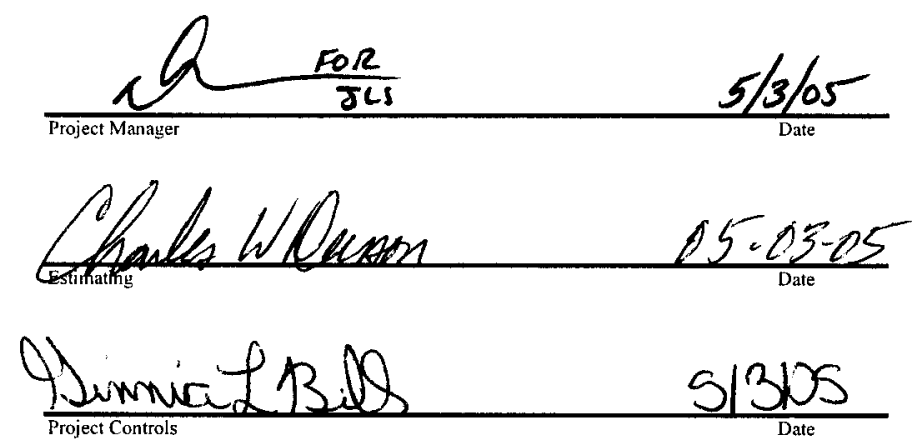


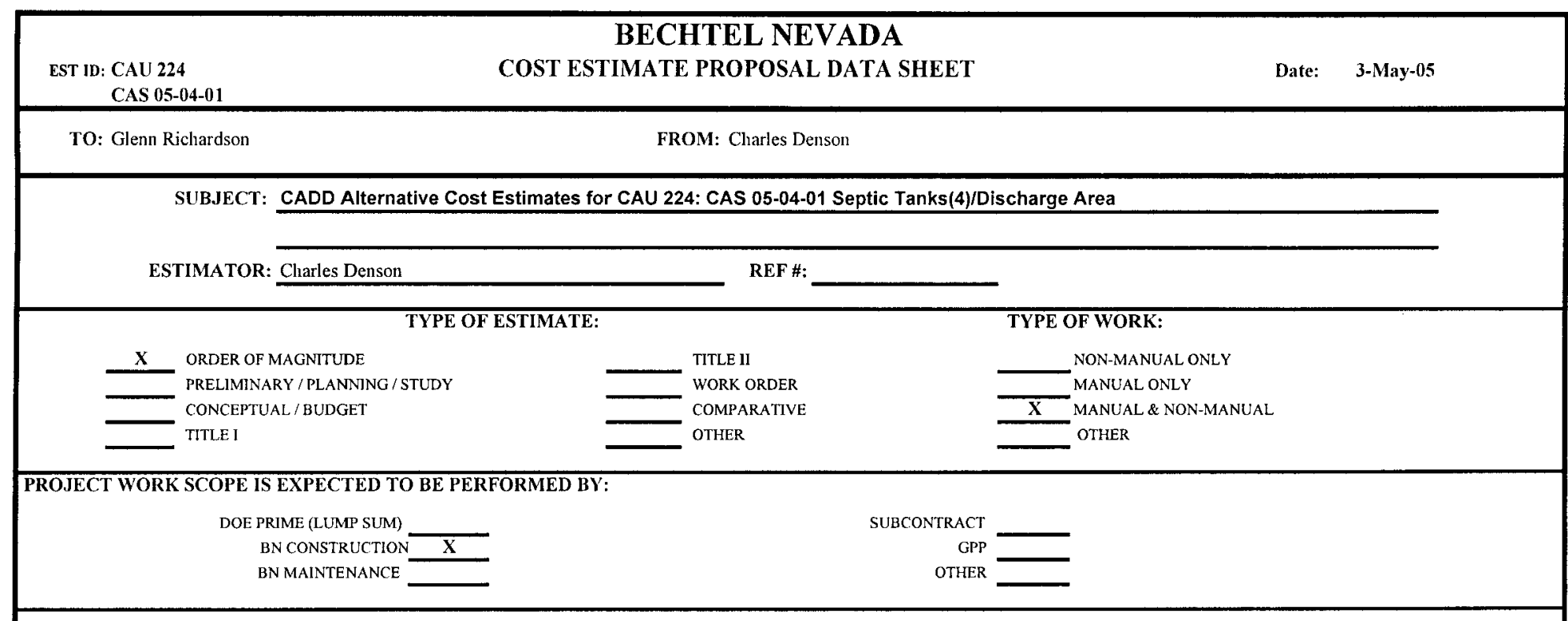

\section{STATEMENT OF WORK}

This estimate has been prepared to provide remedial alternative costs for the closure of Corrective Action Site (CAS) 05-04-01, which is included within Corrective Action Unit (CAU) 224. CAU 224 CAS 05-04-01 is an environmental restoration site listed in the Federal Facility Agreement and Consent Order (FFACO). CAS 05-04-01 is specifically described within the FFACO as Septic Tanks(4)/Discharge Area, located in Area 5 Trailer Park. Three alternatives have been evaluated for closure of the CAS: I. No Further Action; II. Clean Closure; and III. Closure in Place with Administrative Controls. This estimate will be used to identify the most cost effective alternative for closure of the site while remaining protective of human health and the environment. The total estimated costs are intended for comparative analysis of remedial fieldwork cost only. Cost for project management, plan preparation, project support, and/or other activities are not included herein.

\section{SCOPE:}

Provide site closure using one of the following alternatives:

I) NO FURTHER ACTION

II) CLEAN CLOSURE

III) CLOSURE IN PLACE WITH ADMINISTRATIVE CONTROLS

\section{BASIS:}

The characterization contractor recently completed field investigations of four (4) 7,500 gallon septic tanks, a distribution box, associated discharge piping, and a desert wash area located northwest of a former Area 5 Trailer Park. Approximately 15,000 gallons of tank liquid, 2 cu. yds. of tank sludge, approximately 20 $\mathrm{cu}$. yds. of concrete, and $2 \mathrm{cu}$. yds. of pesticide contaminated soil require a remedial action. Site closure estimates for each alternative were priced using standard construction references such as RS Means, Richardson's, and the BN estimating database. There is no estimate required for evaluation of the No Further Action alternative since no cost is incurred

\section{ALTERNATIVE SPECIFIC BASIS OF ESTIMATE/ASSUMPTIONS}

Alternative I: No Further Action

Alternative II: Clean Closure

- Excavate to expose the septic tanks (4) or manhole access

- Add bacteria disinfectant to all septic tank (4) contents

- Solidify septic tank contents with clean backfill material

- Remove and load septic tanks (4) and contents into end dump for Area 6 hydrocarbon landfill disposal

- Field Screening and verification sampling to analyze for TPH

- Grout associated subsurface piping

- Backfill to the approximate natural existing grade

Alternative III: Closure in Place with Administrative Controls

- Excavate to expose Septic Tanks (4) or manhole access

- Add grout mixture inside of Septic Tanks (4)

- Install Administrative Controls (identification/warning signs) 


\section{ASSUMPTIONS:}

- No corrective actions are required for the surrounding areas outside the CAS boundary.

- All COCs at the site have been identified during the site investigation and analytical data accurately represent site conditions and waste characteristics.

- The nature of contamination is limited to TPH (DRO and GRO)

- Assumes the type of septic tank requiring remedial action is concrete

- Assumes the distribution box will be disposed of as a component of the septic tank system.

- Assumes a chlorine disinfectant will be used to neutralize the fecal coliform bacteria

- Assumes associated subsurface piping will not require removal and will be grouted in place as a closure action

- Assumes there is no evidence of an associated leachfield or lagoon based on site reconnaissance that requires a corrective action.

- Assumes no radioactive constituents nor pesticide contamination are present in the septic tank.

- Equipment will remain operational to support the planned/scheduled completion of each CADD alternative.

- Waste volumes are based on field measurements collected during the corrective action investigation

- Work to be performed by BN during a "normal" workday (no provision for overtime has been provided). Shifts are based on 10-hour days / 4-days per week.

- This estimate does not include the efficiencies which may be realized if work for similar activities at similar sites can be completed concurrently.

- Dimensions, volumes, measurements, and analytical data provided by the characterization contractor accurately represent site conditions and waste characteristics.

- This estimate does not include costs for preparation of required project plans, permits, reports, site preparations, or project management.

\section{ESCALATION:}

No escalation factors have been applied. All costs are in FY05 Rev. 1 dollars.

\section{CONTINGENCY:}

Contingency costs are not included in this estimate.

\section{RATES:}

Rates are based on FY05 final rates (Rev. 1) effective 10/20/05 and were applied using the BN FY05 cost model.

\section{COST ALTERNATIVES SUMMARY:}

Alternative I: No Further Action

Alternative II: Clean Closure

a. Excavate to Expose Septic Tanks (4) or Manhole Access

b. Add Disinfectant and Solidify Septic Tank Contents

c. Remove and Load Septic Tanks (4) and Contents into End Dump

d. Field Screening and Verification Sampling

e. Grout Associated Subsurface Piping

f. Backfill to Approx. Natural Existing Grade

g. Waste Management (TPH and Sanitary)

Alternative III: Closure in Place with Administrative Controls

a. Excavate to Expose Top of Septic Tanks (4) or Manhole Access

b. Add Grout Mixture inside of Septic Tanks (4)

c. Install Administrative Controls

d. Waste Management (Sanitary)

\section{REVIEW / CONCURRENCE:}
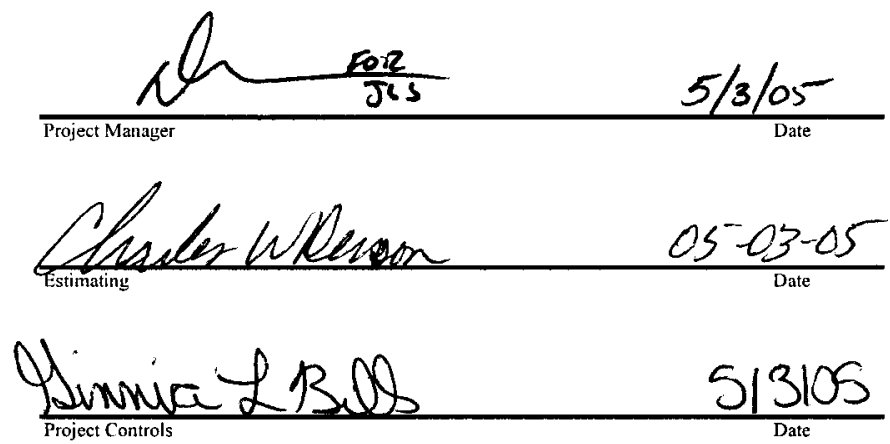


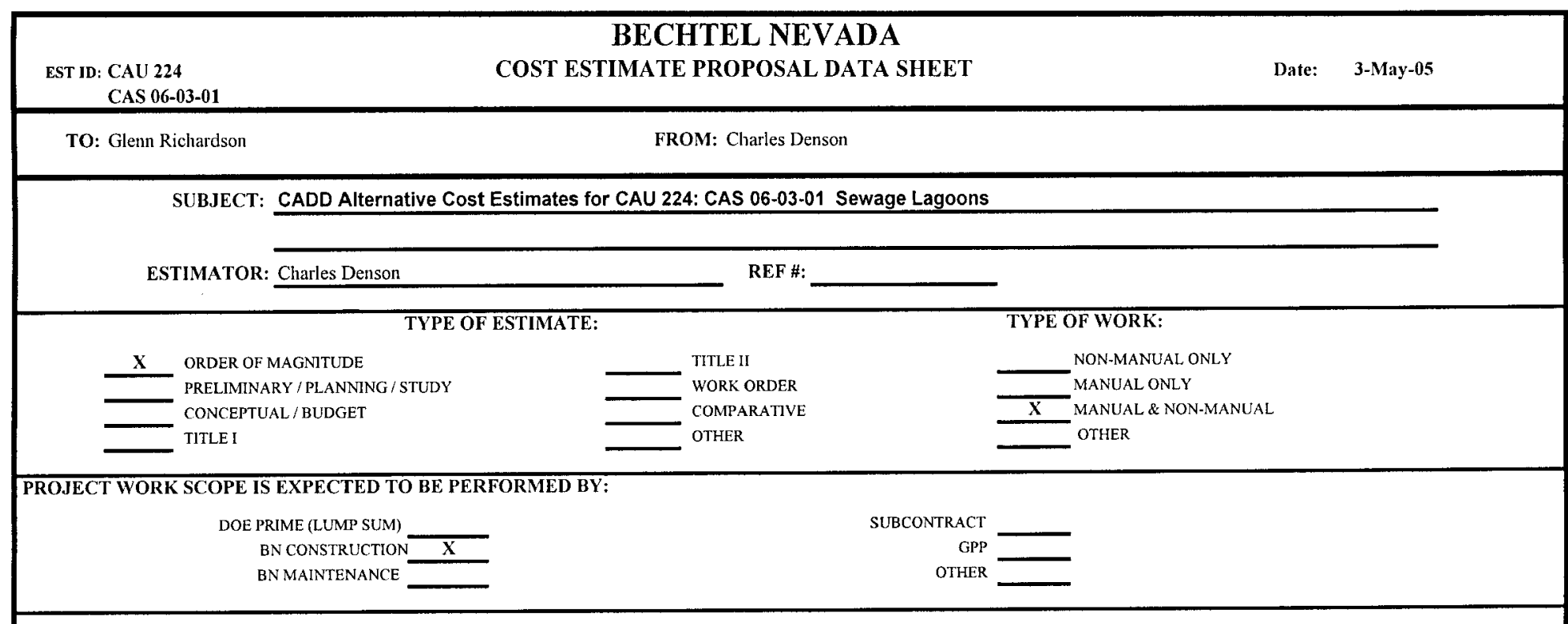

\section{STATEMENT OF WORK}

This estimate has been prepared to provide remedial alternative costs for the closure of Corrective Action Site (CAS) 06-03-01, which is included within Corrective Action Unit (CAU) 224. CAU 224 CAS 06-03-01 is an environmental restoration site listed in the Federal Facility Agreement and Consent Order (FFACO). CAS 06-03-01 is specifically described within the FFACO as Sewage Lagoons, located in Yucca Lake.

\section{SCOPE:}

Provide site closure using one of the following alternatives:

I) NO FURTHER ACTION

\section{BASIS:}

No constituents of concern were identified that exceeded the preliminary action level (PAL).

\section{ALTERNATIVE SPECIFIC BASIS OF ESTIMATE/ASSUMPTIONS}

\section{Alternative I: No Further Action}

No further action was the preferred alternative

\section{ASSUMPTIONS:}

- No actions and no associated costs. No administrative controls are implied.

\section{ESCALATION:}

No escalation factors have been applied. All costs are in FY05 Rev. 1 dollars.

\section{CONTINGENCY:}

Contingency costs are not included in this estimate.

\section{RATES:}

Rates are based on FY05 final rates (Rev. 1) effective 10/20/05 and were applied using the BN FY05 cost model. 


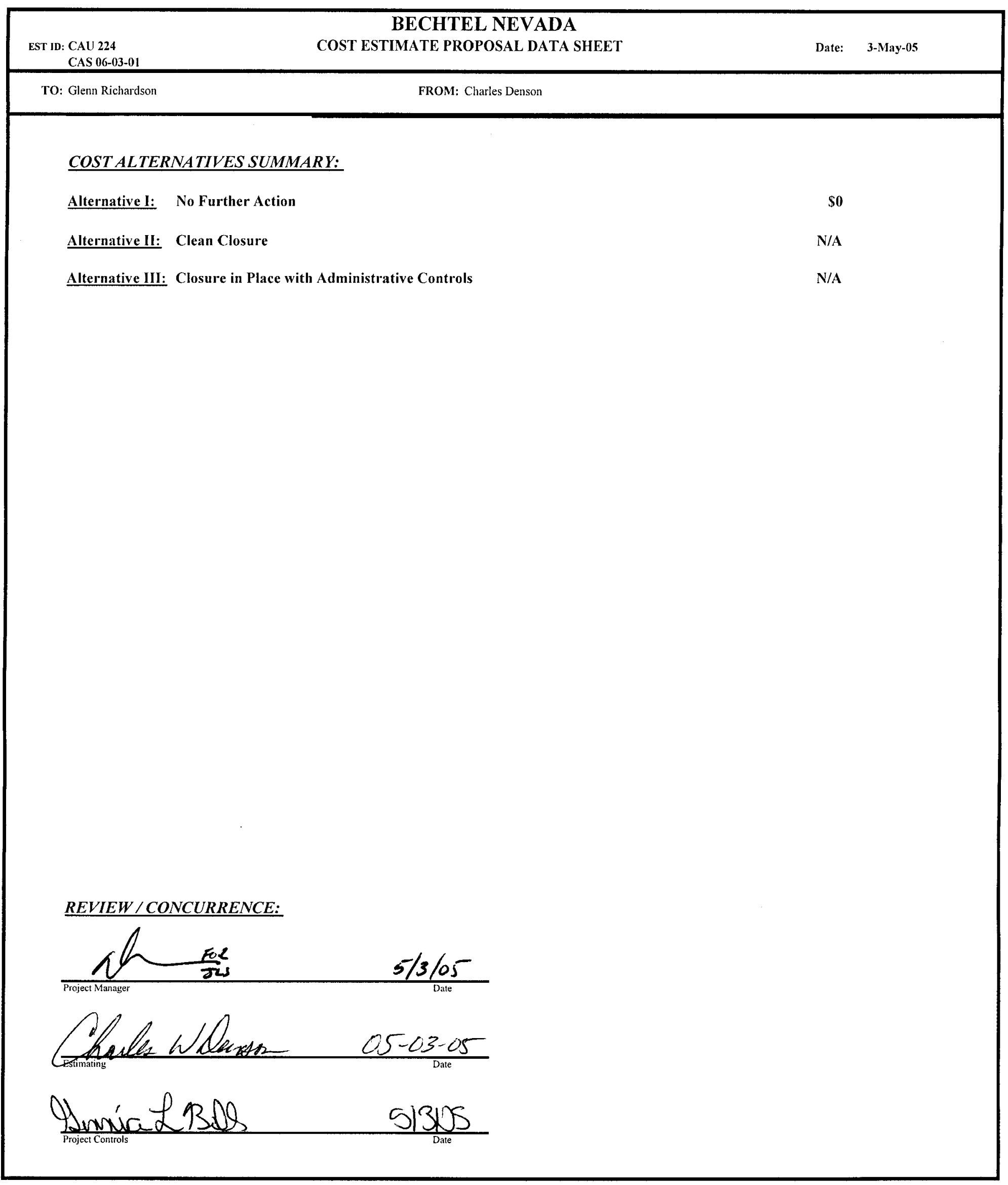

Page 2 of 2 


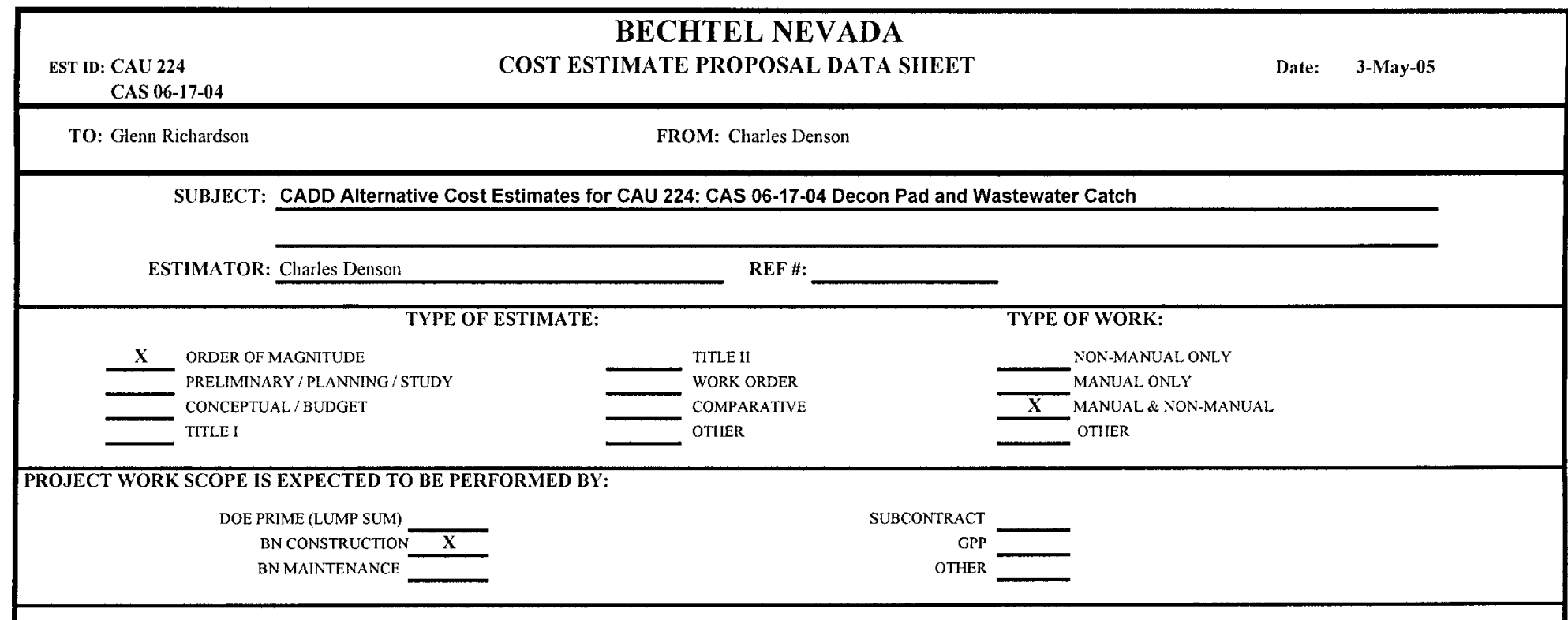

\section{STATEMENT OF WORK}

This estimate has been prepared to provide remedial alternative costs for the closure of Corrective Action Site (CAS) 06-17-04, 06-05-01, \& 06-23-01, which is included within Corrective Action Unit (CAU) 224. CAU 224 CAS 06-17-04, 06-05-01, \& 06-23-01, are environmental restoration sites listed in the Federal Facility Agreement and Consent Order (FFACO). These CASs are specifically described within the FFACO as Decon Pad and Wastewater Catch, Leachfield, and Decon Pad Discharge Piping, located in CP-6 Decon Pad Area. Three alternatives have been evaluated for closure of the CAS: I. No Further Action; II. Clean Closure; and III. Closure in Place with Administrative Controls. This estimate will be used to identify the most cost effective alternative for closure of the site while remaining protective of human health and the environment. The total estimated costs are intended for comparative analysis of remedial fieldwork cost only. Cost for project management, plan preparation, project support, and/or other activities are not included herein

\section{SCOPE:}

Provide site closure using one of the following alternatives:

I) NO FURTHER ACTION

II) CLEAN CLOSURE

III) CLOSURE IN PLACE WITH ADMINISTRATIVE CONTROLS

\section{BASIS:}

The characterization contractor recently completed field investigations of the Area 6 Compound area that includes a leachfield, decon pad, and a sump. Site closure estimates for each alternative were priced using standard construction references such as RS Means, Richardson's, and the BN estimating database. There is no estimate required for evaluation of the No Further Action alternative since no cost is incurred.

\section{ALTERNATIVE SPECIFIC BASIS OF ESTIMATE/ASSUMPTIONS}

\section{Alternative I: No Further Action}

Alternative II: Clean Closure

- Removal of $5 \mathrm{cu}$. yds. of Sludge and $4 \mathrm{cu}$. yds. of Concrete Volume in the Sump

- Perform Removal of the Decon Pad Concrete (Approx. $151 \mathrm{cu}$. yds.)

- Perform removal of TPH and Pu-239 contaminated soil from the surrounding pad area (392 cu. yds.)

- Perform verification sampling. (TPH-DRO)

- Backfill open excavated area with clean fill material (including 20\% expansion factor)

Alternative III: Closure in Place with Administrative Controls

- Install Wire Fencing

- Implement Administrative Controls (identification/warning signs) 


\section{ASSUMPTIONS:}

- No corrective actions are required for the surrounding areas outside the CAS boundary

- All COCs at the site have been identified during the site investigation and analytical data accurately represent site conditions and waste characteristics.

- Assumes associated piping will be cut and grouted in place.

- The nature of contamination is limited to TPH (DRO) and PU-239 in the surrounding soil on the south and east sides of the decon pad.

- Assumes a radiological waste profile currently exists and is available.

- TPH DRO contamination decreases with depth.

- Equipment will remain operational to support the planned/scheduled completion of each CADD alternative.

- Waste volumes are based on field measurements collected during the corrective action investigation.

- Work to be performed by BN during a "normal" workday (no provision for overtime has been provided). Shifts are based on 10-hour days / 4-days per week.

- This estimate does not include the efficiencies which may be realized if work for similar activities at similar sites can be completed concurrently.

- Dimensions, volumes, measurements, and analytical data provided by the characterization contractor accurately represent site conditions and waste characteristics.

- This estimate does not include costs for preparation of required project plans, permits, reports, site preparations, or project management.

\section{ESCALATION:}

No escalation factors have been applied. All costs are in FY05 Rev. 1 dollars.

\section{CONTINGENCY:}

Contingency costs are not included in this estimate.

\section{RATES:}

Rates are based on FY05 final rates (Rev. 1) effective 10/20/05 and were applied using the BN FY05 cost model.

\section{COST ALTERNATIVES SUMMARY:}

Alternative I: No Further Action

a. Removal of sludge and concrete in the Sump Area

b. Perform Removal of Decon Pad Concrete (Approx. 151 cu. yds.).

c. Perform removal of TPH and Pu-239 Contaminated Soil

d. Perform Verification Sampling

e. Backfill Open Excavated Area with Clean Fill Material

f. Waste Management (TPH and Sanitary)

Alternative III: Closure in Place with Administrative Controls a. Install Wire Fencing

b. Implement Administrative Controls (identification / warning signs)

\section{REVIEW / CONCURRENCE:}
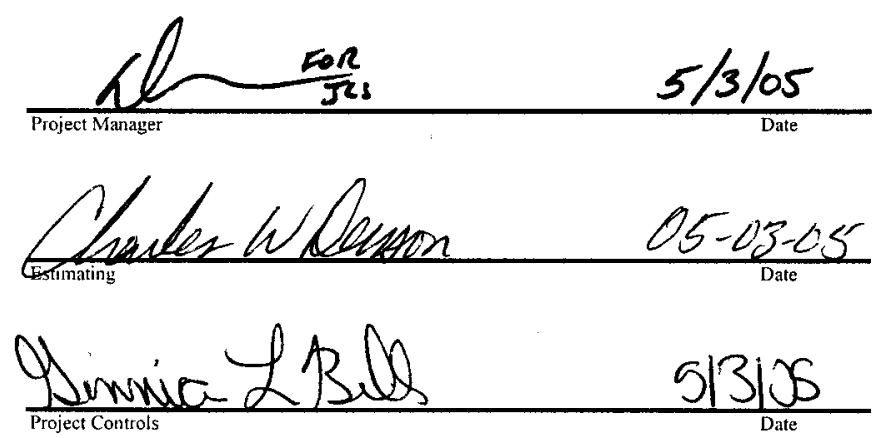


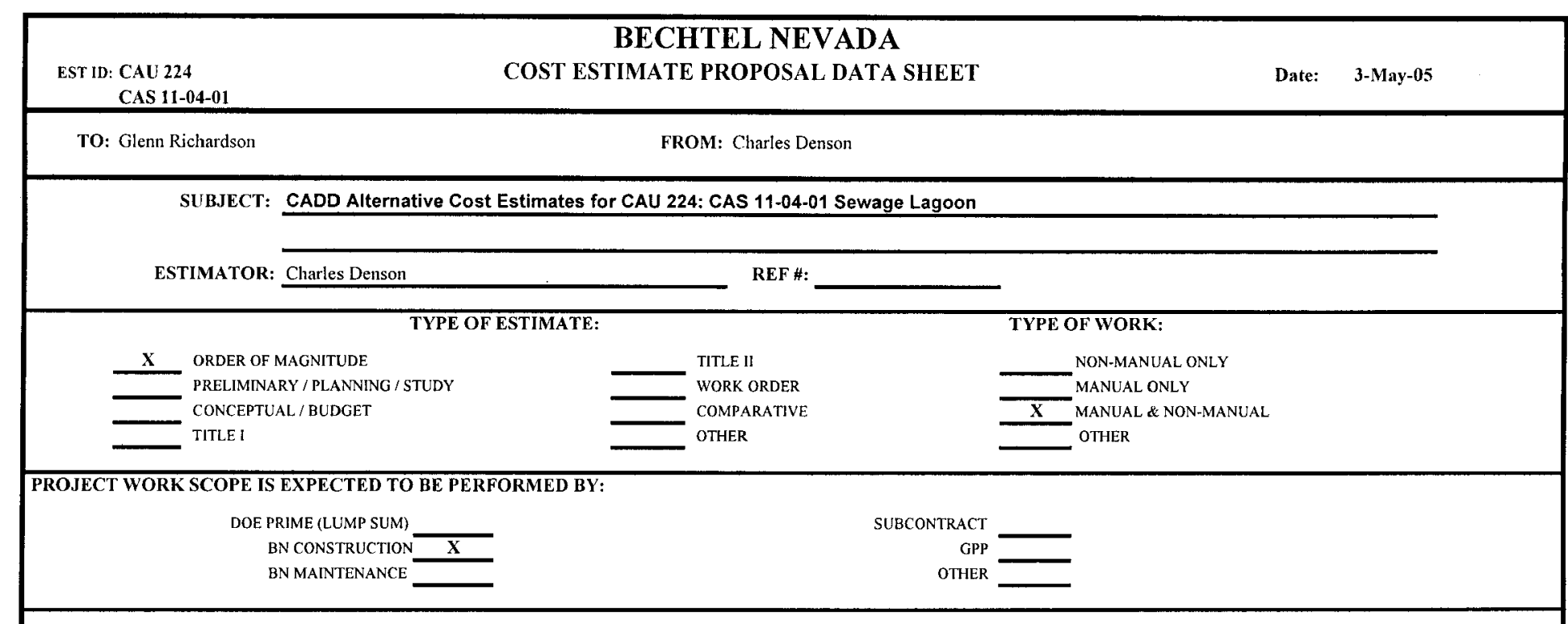

\section{STATEMENT OF WORK}

This estimate has been prepared to provide remedial alternative costs for the closure of Corrective Action Site (CAS) 11-04-01, which is included within Corrective Action Unit (CAU) 224. CAU 224 CAS 11-04-01 is an environmental restoration site listed in the Federal Facility Agreement and Consent Order (FFACO). CAS 11-04-01 is specifically described within the FFACO as Sewage Lagoon, located in LLNL Tech Facility. Two alternatives have been evaluated for closure of the CAS: 1. No Further Action and III. Closure in Place with Administrative Controls. This estimate will be used to identify the most cost effective alternative for closure of the site while remaining protective of human health and the environment. The total estimated costs are intended for comparative analysis of remedial fieldwork cost only. Cost for project management, plan preparation, project support, and/or other activities are not included herein.

\section{SCOPE:}

Provide site closure using one of the following alternatives:

I) NO FURTHER ACTION

III) CLOSURE IN PLACE WITH ADMINISTRATIVE CONTROLS

\section{BASIS:}

The characterization contractor recently completed field investigations of the septic tank in Area 11. Remedial action is associated with two chambers in septic tank, leachfield, subsurface piping, and a former lagoon. Site closure estimates for each alternative were priced using standard construction references such as RS Means, Richardson's, and the BN estimating database. There is no estimate required for evaluation of the No Further Action alternative since no cost is incurred.

\section{ALTERNATIVE SPECIFIC BASIS OF ESTIMATE/ASSUMPTIONS}

Alternative I: No Further Action

- Excavate to expose the septic tank or manhole access

- Add bacteria disinfectant to septic tank contents

- Solidify septic tank contents with clean backfill material

- Remove and load septic tank and contents into end dump for Area 6 hydrocarbon landfill disposal

- Field Screening and verification sampling to analyze for TPH

- Grout associated subsurface piping

- Backfill to the approximate existing grade

Alternative III: Closure in Place with Administrative Controls

- Excavate to Expose Septic Tank or Manhole Access

- Add Grout Mixture inside of Septic Tank

- Install Administrative Controls (identification/warning signs) 


\section{ASSUMPTIONS:}

- No corrective actions are required for the surrounding areas outside the CAS boundary.

- All COCs at the site have been identified during the site investigation and analytical data accurately represent site conditions and waste characteristics.

- Assumes the type of septic tank requiring remedial action is concrete.

- Assumes associated subsurface piping will not require removal and will be grouted in place as a closure action.

- Assumes no radioactive constituents nor pesticide contamination are present in the septic tank.

- TPH DRO contamination is not present above PALs.

- Equipment will remain operational to support the planned/scheduled completion of each CADD alternative.

- Waste volumes are based on field measurements collected during the corrective action investigation.

- Work to be performed by BN during a "normal" workday (no provision for overtime has been provided). Shifts are based on 10-hour days / 4-days per week.

- This estimate does not include the efficiencies which may be realized if work for similar activities at similar sites can be completed concurrently.

- Dimensions, volumes, measurements, and analytical data provided by the characterization contractor accurately represent site conditions and waste characteristics.

- This estimate does not include costs for preparation of required project plans, permits, reports, site preparations, or project management.

\section{ESCALATION:}

No escalation factors have been applied. All costs are in FY05 Rev. 1 dollars.

\section{CONTINGENCY:}

Contingency costs are not included in this estimate.

\section{RATES:}

Rates are based on FY05 final rates (Rev. 1) effective 10/20/05 and were applied using the BN FY05 cost model.

\section{COST ALTERNATIVES SUMMARY:}

Alternative I: No Further Action

a. Excavate to Expose Septic Tank or Manhole Access

b. Add Disinfectant and Solidify Septic Tank Contents

c. Remove and Load Septic Tank and Contents into End Dump

d. Field Screening and Verification Sampling

e. Grout Associated Subsurface Piping

f. Backfill to Approx. Natural Existing Grade

g. Waste Management (TPH and Sanitary)

Alternative III: Closure in Place with Administrative Controls

a. Excavate to Expose Septic Tank or Manhole Access

b. Add Grout Mixture inside of Septic Tank

c. Install Administrative Controls

d. Waste Management (Sanitary)

\section{REVIEW/CONCURRENCE:}
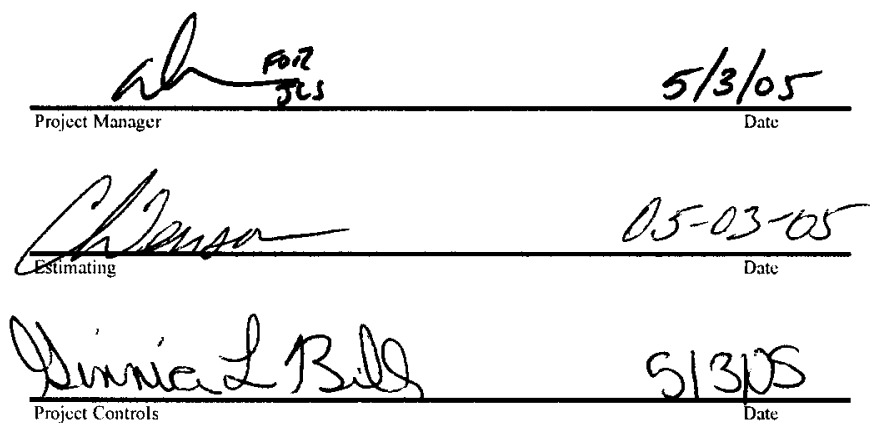


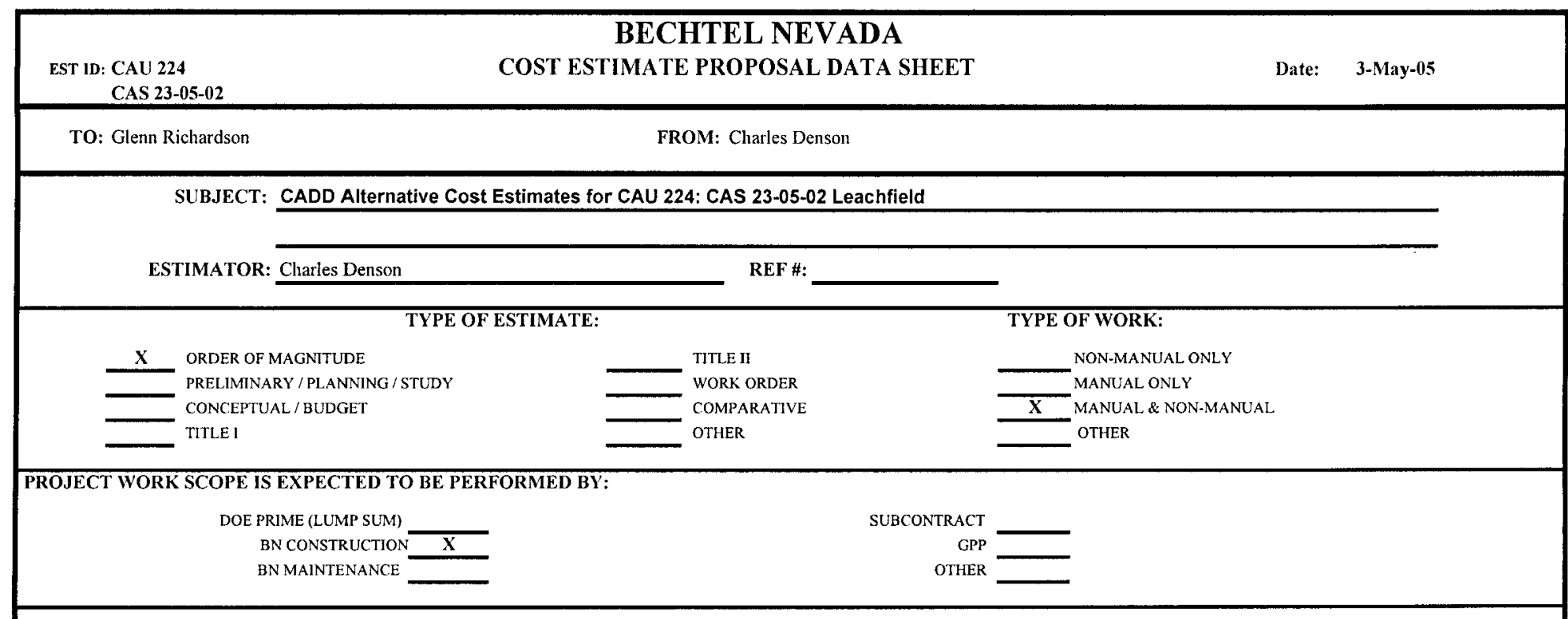

\section{STATEMENT OF WORK}

This estimate has been prepared to provide remedial alternative costs for the closure of Corrective Action Site (CAS) 23-05-02, which is included within Corrective Action Unit (CAU) 224. CAU 224 CAS 23-05-02 is an environmental restoration site listed in the Federal Facility Agreement and Consent Order (FFACO). CAS 23-05-02 is specifically described within the FFACO as Leachfield, located in Building 155.

\section{SCOPE:}

Provide site closure using one of the following alternatives:

I) NO FURTHER ACTION

\section{$\underline{B A S I S:}$}

No constituents of concern were identified that exceeded the preliminary action level (PAL).

\section{ALTERNATIVE SPECIFIC BASIS OF ESTIMATE/ASSUMPTIONS}

Alternative I: No Further Action

No further action was the preferred alternative

\section{ASSUMPTIONS:}

- No actions and no associated costs. No administrative controls are implied.

\section{ESCALATION:}

No escalation factors have been applied. All costs are in FY05 Rev. 1 dollars

\section{CONTINGENCY:}

Contingency costs are not included in this estimate.

\section{RATES:}

Rates are based on FY05 final rates (Rev. 1) effective 10/20/05 and were applied using the BN FY05 cost model. 
BECHTEL NEVADA

COST ESTIMATE PROPOSAL DATA SHEET

FROM: Charles Denson

TO: Glenn Richardson

Alternative I: No Further Action

N/A

Alternative II: Clean Closure

N/A

Alternative III: Closure in Place with Administrative Controls

Date: $\quad 3-$ May-05

\section{COST ALTERNATIVES SUMMARY:}

REVIEW/CONCURRENCE:
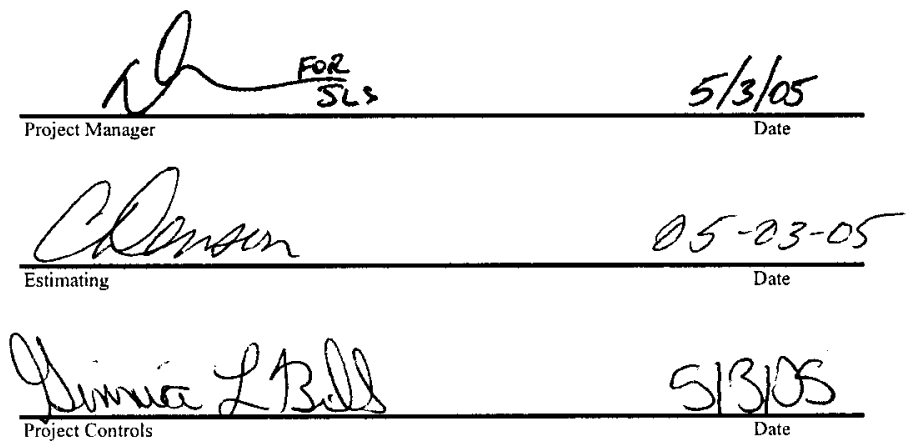


\section{Appendix D}

Sample Location Coordinates for CAU 224 


\section{D.1.0 Sample Location Coordinates}

\section{D.1.1 CAS 02-04-01, Septic Tank (Buried)}

Sample location coordinates were collected during the CAI using a Trimble GPS, Model TSCI.

These coordinates identify the field-sampling locations (e.g., latitude, longitude) at CAU 224, CAS 02-04-01.

Sample locations and pertinent locations of interest at 02-04-01 are shown on Figure A.3-1. The corresponding coordinates for CAS 02-04-01 sample locations are listed in Table D.1-1.

Table D.1-1

Sample Location Coordinates and Locations of Interest for CAS 02-04-01

\begin{tabular}{|c|c|c|c|c|c|c|c||}
\hline Latitude & Longitude & Northing $^{\mathbf{a}}$ & Easting $^{\mathbf{a}}$ & $\begin{array}{c}\text { HAE } \\
\text { (meters) }\end{array}$ & Location & $\begin{array}{c}\text { Vertical } \\
\text { Precision }\end{array}$ & $\begin{array}{c}\text { Horizontal } \\
\text { Precision }\end{array}$ \\
\hline \hline 37.16260665 & -116.0971866 & 4113089.025 & 580160.270 & NA & A01 & NA & NA \\
\hline 37.16260603 & -116.0971648 & 4113088.974 & 580162.209 & NA & A02 & NA & NA \\
\hline 37.16261389 & -116.0971529 & 4113089.856 & 580163.258 & NA & A03 & NA & NA \\
\hline 37.16265508 & -116.0971252 & 4113094.448 & 580165.668 & NA & A04 & NA & NA \\
\hline 37.16264847 & -116.0971565 & 4113093.689 & 580162.900 & NA & A05 & NA & NA \\
\hline 37.16264453 & -116.0971631 & 4113093.247 & 580162.319 & NA & A06 & NA & NA \\
\hline 37.16262959 & -116.0971780 & 4113091.576 & 580161.015 & NA & A07 & NA & NA \\
\hline 37.16263093 & -116.0971541 & 4113091.746 & 580163.135 & NA & A08 & NA & NA \\
\hline 37.16264464 & -116.0971400 & 4113093.279 & 580164.372 & NA & A09 & NA & NA \\
\hline
\end{tabular}

aUniversal Transverse Mercator (UTM) Zone 11, North American Datum (NAD) 1927 (U.S. Western)

$\mathrm{HAE}=$ Height above ellipsoid NA = Not applicable

\section{D.1.2 CAS 03-05-01, Leachfield}

Sample location coordinates were collected during the CAI using a Trimble GPS, Model TSCI.

These coordinates identify the field-sampling locations (e.g., latitude, longitude) at CAU 224, CAS 03-05-01. 
Sample locations and pertinent locations of interest at CAS 03-05-01 are shown on Figure A.4-1. The corresponding coordinates for CAS 03-05-01 sample locations are listed in Table D.1-2.

Table D.1-2

Sample Location Coordinates and Locations of Interest for CAS 03-05-01

\begin{tabular}{|c|c|c|c|c|c|c|c||}
\hline Latitude & Longitude & Northing $^{\text {a }}$ & Easting $^{\text {a }}$ & $\begin{array}{c}\text { HAE } \\
\text { (meters) }\end{array}$ & Location & $\begin{array}{c}\text { Vertical } \\
\text { Precision }\end{array}$ & $\begin{array}{c}\text { Horizontal } \\
\text { Precision }\end{array}$ \\
\hline \hline 37.04890445 & -116.0499677 & 4100516.216 & 584479.099 & NA & B01 & NA & NA \\
\hline 37.04890503 & -116.0499002 & 4100516.340 & 584485.103 & NA & B02 & NA & NA \\
\hline 37.04890603 & -116.0498155 & 4100516.527 & 584492.631 & NA & B03 & NA & NA \\
\hline 37.04890595 & -116.0500188 & 4100516.337 & 584474.557 & NA & B04 & NA & NA \\
\hline 37.04894528 & -116.0499680 & 4100520.746 & 584479.034 & NA & B05 & NA & NA \\
\hline 37.04894467 & -116.0499066 & 4100520.732 & 584484.488 & NA & B06 & NA & NA \\
\hline 37.04894472 & -116.0498372 & 4100520.800 & 584490.667 & NA & B07 & NA & NA \\
\hline 37.04890427 & -116.0497744 & 4100516.368 & 584496.295 & NA & B08 & NA & NA \\
\hline 37.04886360 & -116.0498218 & 4100511.814 & 584492.126 & NA & B09 & NA & NA \\
\hline 37.04886237 & -116.0499042 & 4100511.605 & 584484.797 & NA & B10 & NA & NA \\
\hline 37.04886476 & -116.0499641 & 4100511.816 & 584479.464 & NA & B11 & NA & NA \\
\hline 37.04890569 & -116.0500711 & 4100516.261 & 584469.903 & NA & B12 & NA & NA \\
\hline 37.04887953 & -116.0500544 & 4100513.374 & 584471.420 & NA & B13 & NA & NA \\
\hline 37.04890491 & -116.0500952 & 4100516.153 & 584467.764 & NA & B14 & NA & NA \\
\hline 37.04893843 & -116.0500709 & 4100519.894 & 584469.892 & NA & B15 & NA & NA \\
\hline
\end{tabular}

aUniversal Transverse Mercator (UTM) Zone 11, North American Datum (NAD) 1927 (U.S. Western)

HAE = Height above ellipsoid

NA $=$ Not applicable

\section{D.1.3 CAS 05-04-01, Septic Tanks (4)/Discharge Area}

Sample location coordinates were collected during the CAI using a Trimble GPS, Model TSCI.

These coordinates identify the field-sampling locations (e.g., latitude, longitude) at CAU 224, CAS 05-04-01.

Sample locations and pertinent locations of interest at CAS 05-04-01 are shown on Figure A.5-1. The corresponding coordinates for CAS 05-04-01 sample locations are listed in Table D.1-3. 
Table D.1-3

Sample Location Coordinates and Locations of Interest for CAS 05-04-01

\begin{tabular}{|c|c|c|c|c|c|c|c|}
\hline Latitude & Longitude & Northing $^{a}$ & Easting $^{a}$ & $\begin{array}{c}\text { HAE } \\
\text { (meters) }\end{array}$ & Location & $\begin{array}{c}\text { Vertical } \\
\text { Precision }\end{array}$ & $\begin{array}{l}\text { Horizontal } \\
\text { Precision }\end{array}$ \\
\hline 36.71192010 & -115.9747710 & 4063202.849 & 591567.452 & NA & C_Tank_1 & NA & $\mathrm{NA}$ \\
\hline 36.71192870 & -115.9747498 & 4063203.823 & 591569.337 & NA & C_Tank_2 & NA & NA \\
\hline 36.71194077 & -115.9747277 & 4063205.183 & 591571.293 & NA & C_Tank_3 & NA & NA \\
\hline 36.71195693 & -115.9747075 & 4063206.995 & 591573.075 & NA & C_Tank_4 & NA & NA \\
\hline 36.71205660 & -115.9749008 & 4063217.867 & 591555.690 & NA & $\mathrm{C01}$ & NA & NA \\
\hline 36.71206287 & -115.9748808 & 4063218.581 & 591557.476 & NA & $\mathrm{C02}$ & NA & NA \\
\hline 36.71207642 & -115.9748521 & 4063220.112 & 591560.017 & NA & $\mathrm{C03}$ & NA & NA \\
\hline 36.71209341 & -115.9748356 & 4063222.013 & 591561.477 & NA & $\mathrm{C04}$ & NA & NA \\
\hline 36.71215248 & -115.9749160 & 4063228.488 & 591554.224 & NA & $\mathrm{C05}$ & NA & NA \\
\hline 36.71211890 & -115.9749515 & 4063224.729 & 591551.093 & NA & $\mathrm{C06}$ & NA & NA \\
\hline 36.71211239 & -115.9748855 & 4063224.070 & 591556.997 & NA & $\mathrm{C07}$ & NA & NA \\
\hline 36.71211537 & -115.9748667 & 4063224.419 & 591558.670 & NA & $\mathrm{C08}$ & NA & NA \\
\hline 36.71211129 & -115.9748053 & 4063224.025 & 591564.156 & NA & $\mathrm{C09}$ & NA & NA \\
\hline
\end{tabular}

aUniversal Transverse Mercator (UTM) Zone 11, North American Datum (NAD) 1927 (U.S. Western)

HAE $=$ Height above ellipsoid

NA = Not applicable

\section{D.1.4 CAS 06-03-01, Sewage Lagoons (3)}

Sample location coordinates were collected during the CAI using a Trimble GPS, Model TSCI.

These coordinates identify the field-sampling locations (e.g., latitude, longitude) at CAU 224, CAS 06-03-01.

Sample locations and pertinent locations of interest at CAS 06-03-01 are shown on Figure A.6-1. The corresponding coordinates for CAS 06-03-01 sample locations are listed in Table D.1-4

\section{D.1.5 CAS 06-05-01, Leachfield}

Sample location coordinates were collected during the CAI using a Trimble GPS, Model TSCI. These coordinates identify the field-sampling locations (e.g., latitude, longitude) at CAU 224, CAS 06-05-01. 
Table D.1-4

Sample Location Coordinates and Locations of Interest for CAS 06-03-01

\begin{tabular}{||c|c|c|c|c|c|c|c||}
\hline Latitude & Longitude & Northing $^{\text {a }}$ & Easting $^{\text {a }}$ & $\begin{array}{c}\text { HAE } \\
\text { (meters) }\end{array}$ & Location & $\begin{array}{c}\text { Vertical } \\
\text { Precision }\end{array}$ & $\begin{array}{c}\text { Horizontal } \\
\text { Precision }\end{array}$ \\
\hline \hline 36.94259221 & -116.0391053 & 4088732.183 & 585564.068 & NA & D01 & NA & NA \\
\hline 36.94263552 & -116.0391393 & 4088736.957 & 585560.997 & NA & D02 & NA & NA \\
\hline 36.94268289 & -116.0391778 & 4088742.176 & 585557.517 & NA & D03 & NA & NA \\
\hline 36.94254645 & -116.0390628 & 4088727.145 & 585567.907 & NA & D04 & NA & NA \\
\hline 36.94249336 & -116.0390171 & 4088721.296 & 585572.035 & NA & D05 & NA & NA \\
\hline 36.94263625 & -116.0390218 & 4088737.143 & 585571.455 & NA & D06 & NA & NA \\
\hline 36.94255738 & -116.0391829 & 4088728.250 & 585557.196 & NA & D07 & NA & NA \\
\hline 36.94162989 & -116.0374746 & 4088626.894 & 585710.364 & NA & D08 & NA & NA \\
\hline 36.94143269 & -116.0373904 & 4088605.093 & 585718.081 & NA & D09 & NA & NA \\
\hline 36.94141098 & -116.0374817 & 4088602.603 & 585709.969 & NA & D10 & NA & NA \\
\hline 36.94158852 & -116.0375771 & 4088622.213 & 585701.282 & NA & D11 & NA & NA \\
\hline 36.94150208 & -116.0374728 & 4088612.716 & 585710.665 & NA & D12 & NA & NA \\
\hline 36.94156474 & -116.0376355 & 4088619.522 & 585696.103 & NA & D13 & NA & NA \\
\hline 36.94139376 & -116.0375544 & 4088600.627 & 585703.517 & NA & D14 & NA & NA \\
\hline 36.94145309 & -116.0376362 & 4088607.135 & 585696.166 & NA & D15 & NA & NA \\
\hline
\end{tabular}

aUniversal Transverse Mercator (UTM) Zone 11, North American Datum (NAD) 1927 (U.S. Western)

HAE = Height above ellipsoid

NA = Not applicable

Sample locations and pertinent locations of interest at CAS 06-05-01 are shown on Figure A.7-1. The corresponding coordinates for CAS 06-05-01 sample locations are listed in Table D.1-5.

\section{D.1.6 CAS 06-17-04, Decon Pad and Wastewater Catch}

Sample location coordinates were collected during the CAI using a Trimble GPS, Model TSCI.

These coordinates identify the field-sampling locations (e.g., latitude, longitude) at CAU 224, CAS 06-17-04.

Sample locations and pertinent locations of interest at CAS 06-17-04 are shown on Figure A.7-1. The corresponding coordinates for CAS 06-17-04 sample locations are listed in Table D.1-6. 
Table D.1-5

Sample Location Coordinates and Locations of Interest for CAS 06-05-01

(Page 1 of 2)

\begin{tabular}{|c|c|c|c|c|c|c|c|}
\hline Latitude & Longitude & Northing $^{a}$ & Easting $^{a}$ & $\begin{array}{c}\text { HAE } \\
\text { (meters) }\end{array}$ & Location & $\begin{array}{c}\text { Vertical } \\
\text { Precision }\end{array}$ & $\begin{array}{l}\text { Horizontal } \\
\text { Precision }\end{array}$ \\
\hline (36.93324850 & -116.0516845 & 4087684.432 & 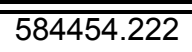 & $\overline{\overline{N A}}$ & $\overline{\mathrm{E} 01}$ & $\overline{\overline{N A}}$ & $\overline{\overline{\mathrm{NA}}}$ \\
\hline 36.93329160 & -116.0516813 & 4087689.216 & 584454.455 & NA & E02 & NA & NA \\
\hline 36.93318259 & -116.0516835 & 4087677.122 & 584454.386 & NA & E03 & NA & NA \\
\hline 36.93327887 & -116.0515779 & 4087687.896 & 584463.678 & NA & E04 & NA & NA \\
\hline 36.93323651 & -116.0515763 & 4087683.198 & 584463.875 & NA & E05 & NA & NA \\
\hline 36.93318100 & -116.0515853 & 4087677.032 & 584463.133 & NA & E06 & NA & NA \\
\hline 36.93327244 & -116.0514383 & 4087687.306 & 584476.119 & NA & E07 & NA & NA \\
\hline 36.93322998 & -116.0514363 & 4087682.597 & 584476.347 & NA & E08 & NA & NA \\
\hline 36.93318458 & -116.0514410 & 4087677.557 & 584475.975 & NA & E09 & NA & NA \\
\hline 36.93326957 & -116.0513629 & 4087687.055 & 584482.836 & NA & E10 & NA & NA \\
\hline 36.93322732 & -116.0513627 & 4087682.368 & 584482.908 & NA & E11 & NA & NA \\
\hline 36.93318766 & -116.0513610 & 4087677.969 & 584483.097 & NA & E12 & NA & NA \\
\hline 36.93323463 & -116.0516554 & 4087682.919 & 584456.830 & NA & E13 & NA & NA \\
\hline 36.93327226 & -116.0515077 & 4087687.224 & 584469.939 & NA & E14 & NA & NA \\
\hline 36.93323440 & -116.0515054 & 4087683.027 & 584470.190 & NA & E15 & NA & NA \\
\hline 36.93318219 & -116.0515099 & 4087677.231 & 584469.846 & NA & E16 & NA & NA \\
\hline 36.93167713 & -116.0521411 & 4087509.710 & 584415.294 & NA & E17 & NA & NA \\
\hline 36.93162457 & -116.0521308 & 4087503.888 & 584416.269 & NA & E18 & NA & NA \\
\hline 36.93157596 & -116.0520914 & 4087498.531 & 584419.828 & NA & E19 & NA & NA \\
\hline 36.93160829 & -116.0520505 & 4087502.154 & 584423.438 & NA & E20 & NA & NA \\
\hline 36.93152287 & -116.0520878 & 4087492.645 & 584420.210 & NA & E21 & NA & NA \\
\hline 36.93148505 & -116.0520678 & 4087488.467 & 584422.027 & NA & E22 & NA & NA \\
\hline 36.93144340 & -116.0521422 & 4087483.781 & 584415.454 & NA & E23 & NA & NA \\
\hline 36.93201185 & -116.0518566 & 4087547.093 & 584440.259 & NA & E24 & NA & NA \\
\hline 36.93238625 & -116.0517883 & 4087588.687 & 584445.929 & NA & E25 & NA & NA \\
\hline 36.93218793 & -116.0514747 & 4087566.965 & 584474.075 & NA & E26 & NA & NA \\
\hline 36.93208221 & -116.0514202 & 4087555.285 & 584479.047 & NA & E27 & NA & NA \\
\hline 36.93199296 & -116.0513638 & 4087545.434 & 584484.166 & NA & E28 & NA & NA \\
\hline 36.93166280 & -116.0520449 & 4087508.206 & 584423.873 & NA & E29 & NA & NA \\
\hline 36.93160638 & -116.0520025 & 4087501.984 & 584427.709 & NA & E30 & NA & NA \\
\hline 36.93232888 & -116.0518264 & 4087582.290 & 584442.599 & NA & E31 & NA & NA \\
\hline 36.93239667 & -116.0518197 & 4087589.816 & 584443.122 & NA & E32 & NA & NA \\
\hline 36.93236308 & -116.0517449 & 4087586.155 & 584449.815 & NA & E33 & NA & NA \\
\hline
\end{tabular}


Table D.1-5

Sample Location Coordinates and Locations of Interest for CAS 06-05-01

(Page 2 of 2)

\begin{tabular}{||c|c|c|c|c|c|c|c||}
\hline Latitude & Longitude & Northing $^{\mathrm{a}}$ & Easting $^{\mathrm{a}}$ & $\begin{array}{c}\text { HAE } \\
\text { (meters) }\end{array}$ & Location & $\begin{array}{c}\text { Vertical } \\
\text { Precision }\end{array}$ & $\begin{array}{c}\text { Horizontal } \\
\text { Precision }\end{array}$ \\
\hline \hline 36.93331352 & -116.0514344 & 4087691.867 & 584476.428 & NA & E34 & NA & NA \\
\hline 36.93322531 & -116.0513172 & 4087682.185 & 584486.959 & NA & E35 & NA & NA \\
\hline 36.93324373 & -116.0517212 & 4087683.870 & 584450.957 & NA & E36 & NA & NA \\
\hline 36.93328732 & -116.0517577 & 4087688.673 & 584447.664 & NA & E37 & NA & NA \\
\hline 36.93320390 & -116.0517967 & 4087679.385 & 584444.279 & NA & E38 & NA & NA \\
\hline 36.93240131 & -116.0517654 & 4087590.378 & 5844247.948 & NA & E39 & NA & NA \\
\hline 36.93243943 & -116.0517305 & 4087594.638 & 584451.018 & NA & E40 & NA & NA \\
\hline
\end{tabular}

aUniversal Transverse Mercator (UTM) Zone 11, North American Datum (NAD) 1927 (U.S. Western)

$\mathrm{HAE}=$ Height above ellipsoid

$\mathrm{NA}=$ Not applicable

Table D.1-6

Sample Location Coordinates and Locations of Interest for CAS 06-17-04

(Page 1 of 3 )

\begin{tabular}{||c|c|c|c|c|c|c|c||}
\hline Latitude & Longitude & Northing $^{\text {a }}$ & Easting $^{\text {a }}$ & $\begin{array}{c}\text { HAE } \\
\text { (meters) }\end{array}$ & Location & $\begin{array}{c}\text { Vertical } \\
\text { Precision }\end{array}$ & $\begin{array}{c}\text { Horizontal } \\
\text { Precision }\end{array}$ \\
\hline \hline 36.93356615 & -116.0519864 & 4087719.403 & 584426.981 & NA & F01 & NA & NA \\
\hline 36.93362105 & -116.0519451 & 4087725.530 & 584430.605 & NA & F02 & NA & NA \\
\hline 36.93357560 & -116.0518388 & 4087720.582 & 584440.117 & NA & F03 & NA & NA \\
\hline 36.93348966 & -116.0517921 & 4087711.089 & 584444.374 & NA & F04 & NA & NA \\
\hline 36.93353606 & -116.0517593 & 4087716.265 & 584447.241 & NA & F05 & NA & NA \\
\hline 36.93344213 & -116.0516970 & 4087705.901 & 584452.899 & NA & F06 & NA & NA \\
\hline 36.93349669 & -116.0516619 & 4087711.985 & 584455.960 & NA & F07 & NA & NA \\
\hline 36.93358371 & -116.0517306 & 4087721.577 & 584449.743 & NA & F08 & NA & NA \\
\hline 36.93353999 & -116.0516403 & 4087716.807 & 584457.840 & NA & F09 & NA & NA \\
\hline 36.93362794 & -116.0520036 & 4087726.242 & 584425.386 & NA & F10 & NA & NA \\
\hline 36.93351619 & -116.0519999 & 4087713.849 & 584425.841 & NA & F11 & NA & NA \\
\hline 36.93347442 & -116.0515990 & 4087709.570 & 584461.586 & NA & F12 & NA & NA \\
\hline 36.93342569 & -116.0517571 & 4087704.024 & 584447.556 & NA & F13 & NA & NA \\
\hline 36.93343469 & -116.0518068 & 4087704.979 & 584443.121 & NA & F14 & NA & NA \\
\hline 36.93343901 & -116.0518188 & 4087705.448 & 584442.052 & NA & F15 & NA & NA \\
\hline 36.93353292 & -116.0516031 & 4087716.056 & 584461.157 & NA & F16 & NA & NA \\
\hline 36.93357092 & -116.0516337 & 4087720.244 & 584458.386 & NA & F17 & NA & NA \\
\hline 36.93357251 & -116.0516983 & 4087720.363 & 584452.631 & NA & F18 & NA & NA \\
\hline
\end{tabular}




\section{Table D.1-6 \\ Sample Location Coordinates and Locations of Interest for CAS 06-17-04}

(Page 2 of 3 )

\begin{tabular}{|c|c|c|c|c|c|c|c|}
\hline Latitude & Longitude & Northing $^{a}$ & Easting $^{a}$ & $\begin{array}{c}\text { HAE } \\
\text { (meters) }\end{array}$ & Location & $\begin{array}{c}\text { Vertical } \\
\text { Precision }\end{array}$ & $\begin{array}{c}\text { Horizontal } \\
\text { Precision }\end{array}$ \\
\hline 36.93359292 & -116.0517656 & 4087722.569 & 584446.620 & $\overline{\mathrm{NA}}$ & $\overline{F 19}$ & $\overline{\mathrm{NA}}$ & $\overline{\mathrm{NA}}$ \\
\hline 36.93360249 & -116.0517244 & 4087723.666 & 584450.280 & NA & F20 & NA & NA \\
\hline 36.93352418 & -116.0520535 & 4087714.687 & 584421.052 & NA & F21 & NA & NA \\
\hline 36.93347595 & -116.0520292 & 4087709.359 & 584423.273 & NA & $\mathrm{F} 22$ & NA & $\mathrm{NA}$ \\
\hline 36.93349721 & -116.0519505 & 4087711.787 & 584430.254 & NA & F23 & NA & NA \\
\hline 36.93339984 & -116.0518618 & 4087701.064 & 584438.268 & NA & F24 & NA & NA \\
\hline 36.93344138 & -116.0518539 & 4087705.679 & 584438.920 & NA & F25 & NA & NA \\
\hline 36.93341289 & -116.0517762 & 4087702.587 & 584445.877 & $\mathrm{NA}$ & F26 & $\mathrm{NA}$ & $\mathrm{NA}$ \\
\hline 36.93345325 & -116.0515347 & 4087707.279 & 584467.337 & $\mathrm{NA}$ & F27 & $\mathrm{NA}$ & NA \\
\hline 36.93349802 & -116.0515740 & 4087712.210 & 584463.786 & NA & F28 & NA & NA \\
\hline 36.93344524 & -116.0516126 & 4087706.320 & 584460.409 & $\mathrm{NA}$ & F29 & $\mathrm{NA}$ & $\mathrm{NA}$ \\
\hline 36.93341527 & -116.0517295 & 4087702.892 & 584450.028 & NA & F30 & NA & NA \\
\hline 36.93346143 & -116.0516539 & 4087708.081 & 584456.710 & NA & F31 & $\mathrm{NA}$ & NA \\
\hline 36.93351503 & -116.0517009 & 4087713.985 & 584452.463 & NA & F32 & $\mathrm{NA}$ & NA \\
\hline 36.93360514 & -116.0515228 & 4087724.139 & 584468.231 & NA & F33 & NA & NA \\
\hline 36.93353753 & -116.0514409 & 4087716.711 & 584475.595 & $\mathrm{NA}$ & F34 & $\mathrm{NA}$ & $\mathrm{NA}$ \\
\hline 36.93351730 & -116.0515024 & 4087714.412 & 584470.145 & NA & F35 & NA & NA \\
\hline 36.93336129 & -116.0515062 & 4087697.102 & 584469.976 & NA & F36 & NA & $\overline{N A}$ \\
\hline 36.93339945 & -116.0515513 & 4087701.296 & 584465.918 & NA & F37 & NA & NA \\
\hline 36.93335889 & -116.0516389 & 4087696.718 & 584458.157 & $\mathrm{NA}$ & F38 & $\mathrm{NA}$ & $\mathrm{NA}$ \\
\hline 36.93331513 & -116.0517881 & 4087691.732 & 584444.920 & $\mathrm{NA}$ & F39 & $\mathrm{NA}$ & $\mathrm{NA}$ \\
\hline 36.93333348 & -116.0519105 & 4087693.660 & 584434.001 & $\mathrm{NA}$ & $\mathrm{F} 40$ & NA & NA \\
\hline 36.93339726 & -116.0511969 & 4087700.683 & 584428.717 & $\mathrm{NA}$ & $\mathrm{F} 41$ & $\mathrm{NA}$ & $\mathrm{NA}$ \\
\hline 36.93338710 & -116.0519031 & 4087699.614 & 584434.604 & $\mathrm{NA}$ & $\mathrm{F} 42$ & $\mathrm{NA}$ & NA \\
\hline 36.93346843 & -116.051447 & 4087709.04 & 584475.132 & NA & F43 & NA & NA \\
\hline 36.93342658 & -116.0514161 & 4087704.425 & 584477.933 & NA & F44 & NA & NA \\
\hline 36.93333779 & -116.0519965 & 4087694.061 & 584426.333 & $\mathrm{NA}$ & $\mathrm{F} 45$ & $\mathrm{NA}$ & $\mathrm{NA}$ \\
\hline 36.93336591 & -116.0520757 & 4087697.111 & 584419.25 & NA & $\mathrm{F} 46$ & NA & $\mathrm{NA}$ \\
\hline 36.93326262 & -116.0519631 & 4087685.751 & 584429.392 & $\mathrm{NA}$ & F47 & $\mathrm{NA}$ & $\mathrm{NA}$ \\
\hline 36.9331731 & -116.0519471 & 4087675.835 & 584430.917 & NA & F48 & NA & NA \\
\hline 36.93309927 & -116.0517839 & 4087667.789 & 584445.534 & $\mathrm{NA}$ & F49 & $\mathrm{NA}$ & $\mathrm{NA}$ \\
\hline 36.93302264 & -116.0517545 & 4087659.315 & 584448.238 & $\mathrm{NA}$ & F50 & NA & $\mathrm{NA}$ \\
\hline 36.9330752 & -116.0519842 & 4087664.942 & 584427.718 & NA & F51 & NA & NA \\
\hline
\end{tabular}




\section{Table D.1-6 \\ Sample Location Coordinates and Locations of Interest for CAS 06-17-04}

(Page 3 of 3 )

\begin{tabular}{|c|c|c|c|c|c|c|c|}
\hline Latitude & Longitude & Northing $^{a}$ & Easting $^{a}$ & $\begin{array}{c}\text { HAE } \\
\text { (meters) }\end{array}$ & Location & $\begin{array}{c}\text { Vertical } \\
\text { Precision }\end{array}$ & $\begin{array}{l}\text { Horizontal } \\
\text { Precision }\end{array}$ \\
\hline 36.93319148 & -116.0520564 & 4087677.777 & 584421.163 & $\overline{\mathrm{NA}}$ & F52 & $\overline{\mathrm{NA}}$ & $\overline{\mathrm{NA}}$ \\
\hline 36.93328621 & -116.0521024 & 4087688.245 & 584416.96 & NA & F53 & NA & NA \\
\hline 36.933078935 & -116.051707842 & 4087665.601 & 584452.33 & NA & F54 & NA & NA \\
\hline 36.933066712 & -116.051889609 & 4087664.084 & 584436.155 & NA & F55 & NA & NA \\
\hline 36.932976934 & -116.051702191 & 4087654.291 & 584452.946 & NA & F56 & NA & NA \\
\hline 36.93292765 & -116.05174192 & 4087648.788 & 584449.462 & $\mathrm{NA}$ & F57 & NA & NA \\
\hline 36.932946534 & -116.051828102 & 4087650.807 & 584441.765 & NA & F58 & NA & NA \\
\hline 36.932941625 & -116.05190253 & 4087650.196 & 584435.142 & NA & F59 & NA & NA \\
\hline 36.932838623 & -116.051753251 & 4087638.902 & 584448.551 & NA & F60 & NA & NA \\
\hline 36.932822779 & -116.051855478 & 4087637.054 & 584439.464 & NA & F61 & NA & NA \\
\hline 36.932745819 & -116.05183532 & 4087628.534 & 584441.344 & NA & F62 & $\overline{N A}$ & NA \\
\hline 36.932761529 & -116.051748638 & 4087630.354 & 584449.047 & NA & F63 & NA & NA \\
\hline 36.932646401 & -116.051735503 & 4087617.594 & 584450.343 & NA & F64 & NA & NA \\
\hline 36.932637867 & -116.051799311 & 4087616.591 & 584444.67 & $\overline{N A}$ & F65 & $\overline{N A}$ & $\overline{N A}$ \\
\hline 36.932529487 & -116.051733749 & 4087604.626 & 584450.629 & NA & F66 & NA & NA \\
\hline 36.932527438 & -116.051803026 & 4087604.337 & 584444.461 & $\overline{N A}$ & F67 & $\overline{N A}$ & $\overline{N A}$ \\
\hline 36.932791306 & -116.051683771 & 4087633.715 & 584454.791 & NA & F68 & NA & NA \\
\hline 36.932609543 & -116.051684375 & 4087613.551 & 584454.938 & NA & F69 & $\overline{N A}$ & NA \\
\hline
\end{tabular}

aUniversal Transverse Mercator (UTM) Zone 11, North American Datum (NAD) 1927 (U.S. Western)

HAE = Height above ellipsoid

NA $=$ Not applicable

\section{D.1.7 CAS 06-23-01, Decon Pad Discharge Piping}

Sample location coordinates were collected during the corrective action investigation using a Trimble GPS, Model TSCI. These coordinates identify the field sampling locations (e.g., latitude, longitude) at CAU 224, CAS 06-23-01.

Sample locations and pertinent locations of interest at CAS 06-23-01 are shown on Figure A.7-1. The corresponding coordinates for CAS 06-23-01 sample locations are listed in Table D.1-7. 
Table D.1-7

Sample Location Coordinates and Locations of Interest for CAS 06-23-01

\begin{tabular}{|c|c|c|c|c|c|c|c|}
\hline Latitude & Longitude & Northing & Easting $^{\mathrm{a}}$ & $\begin{array}{c}\text { HAE } \\
\text { (meters) }\end{array}$ & Location & $\begin{array}{c}\text { Vertical } \\
\text { Precision }\end{array}$ & $\begin{array}{c}\text { Horizontal } \\
\text { Precision }\end{array}$ \\
\hline \hline 36.93342938 & -116.0518241 & 4087704.374 & 584441.592 & NA & G01 & NA & NA \\
\hline 36.93324978 & -116.0518988 & 4087684.385 & 584435.133 & NA & G02 & NA & NA \\
\hline 36.93327950 & -116.0518415 & 4087687.732 & 584440.204 & NA & G03 & NA & NA \\
\hline
\end{tabular}

aUniversal Transverse Mercator (UTM) Zone 11, North American Datum (NAD) 1927 (U.S. Western)

HAE = Height above ellipsoid

NA $=$ Not applicable

\section{D.1.8 CAS 11-04-01, Sewage Lagoon}

Sample location coordinates were collected during the CAI using a Trimble GPS, Model TSCI.

These coordinates identify the field-sampling locations (e.g., latitude, longitude) at CAU 224,

CAS 11-04-01.

Sample locations and pertinent locations of interest at CAS 11-04-01 are shown on Figure A.8-1. The corresponding coordinates for CAS 11-04-01 sample locations are listed in Table D.1-8.

Table D.1-8

Sample Location Coordinates and Locations of Interest for CAS 11-04-01 (Page 1 of 2)

\begin{tabular}{||c|c|c|c|c|c|c|c||}
\hline Latitude & Longitude & Northing $^{\text {a }}$ & Easting $^{\text {a }}$ & $\begin{array}{c}\text { HAE } \\
\text { (meters) }\end{array}$ & Location & $\begin{array}{c}\text { Vertical } \\
\text { Precision }\end{array}$ & $\begin{array}{c}\text { Horizontal } \\
\text { Precision }\end{array}$ \\
\hline \hline 36.94677188 & -115.9681678 & 4089261.880 & 591875.953 & NA & H01 & NA & NA \\
\hline 36.94677284 & -115.9681470 & 4089262.007 & 591877.802 & NA & H02 & NA & NA \\
\hline 36.94674433 & -115.9680113 & 4089258.975 & 591889.919 & NA & H03 & NA & NA \\
\hline 36.94676671 & -115.9679987 & 4089261.470 & 591891.013 & NA & H04 & NA & NA \\
\hline 36.94674696 & -115.9679908 & 4089259.287 & 591891.745 & NA & H05 & NA & NA \\
\hline 36.94672866 & -115.9679907 & 4089257.257 & 591891.777 & NA & H06 & NA & NA \\
\hline 36.94668215 & -115.9679249 & 4089252.161 & 591897.691 & NA & H07 & NA & NA \\
\hline 36.94667749 & -115.9679558 & 4089251.615 & 591894.946 & NA & H08 & NA & NA \\
\hline 36.94667751 & -115.9679860 & 4089251.587 & 591892.256 & NA & H09 & NA & NA \\
\hline 36.94667754 & -115.9680195 & 4089251.558 & 591889.273 & NA & H10 & NA & NA \\
\hline 36.94667177 & -115.9680469 & 4089250.892 & 591886.840 & NA & H11 & NA & NA \\
\hline 36.94658499 & -115.9679110 & 4089241.396 & 591899.046 & NA & H12 & NA & NA \\
\hline 36.94658386 & -115.9679404 & 4089241.242 & 591896.425 & NA & H13 & NA & NA \\
\hline
\end{tabular}


Table D.1-8

Sample Location Coordinates and Locations of Interest for CAS 11-04-01 (Page 2 of 2)

\begin{tabular}{||c|c|c|c|c|c|c|c||}
\hline Latitude & Longitude & Northing $^{\text {a }}$ & Easting $^{\text {a }}$ & $\begin{array}{c}\text { HAE } \\
\text { (meters) }\end{array}$ & Location & $\begin{array}{c}\text { Vertical } \\
\text { Precision }\end{array}$ & $\begin{array}{c}\text { Horizontal } \\
\text { Precision }\end{array}$ \\
\hline \hline 36.94658142 & -115.9679655 & 4089240.947 & 591894.199 & NA & H14 & NA & NA \\
\hline 36.94658144 & -115.9680027 & 4089240.913 & 591890.881 & NA & H15 & NA & NA \\
\hline 36.94658086 & -115.9680355 & 4089240.818 & 591887.961 & NA & H16 & NA & NA \\
\hline 36.94645447 & -115.9678838 & 4089226.943 & 591901.622 & NA & H17 & NA & NA \\
\hline 36.94645168 & -115.9679094 & 4089226.609 & 591899.352 & NA & H18 & NA & NA \\
\hline 36.94644562 & -115.9679346 & 4089225.913 & 591897.108 & NA & H19 & NA & NA \\
\hline 36.94644851 & -115.9679784 & 4089226.191 & 591893.204 & NA & H20 & NA & NA \\
\hline 36.94644752 & -115.9680089 & 4089226.052 & 591890.489 & NA & H21 & NA & NA \\
\hline 36.94675641 & -115.9682018 & 4089260.132 & 591872.944 & NA & H22 & NA & NA \\
\hline 36.94675815 & -115.9682295 & 4089260.298 & 591870.474 & NA & H23 & NA & NA \\
\hline
\end{tabular}

aUniversal Transverse Mercator (UTM) Zone 11, North American Datum (NAD) 1927 (U.S. Western)

$\mathrm{HAE}=$ Height above ellipsoid

NA = Not applicable

\section{D.1.9 CAS 23-05-02, Leachfield}

Sample location coordinates were collected during the CAI using a Trimble GPS, Model TSCI.

These coordinates identify the field-sampling locations (e.g., latitude, longitude) at CAU 224, CAS 23-05-02.

Sample locations and pertinent locations of interest at CAS 23-05-02 are shown on Figure A.9-1. The corresponding coordinates for CAS 23-05-02 sample locations are listed in Table D.1-9. 
Table D.1-9

Sample Location Coordinates and Locations of Interest for CAS 23-05-02

\begin{tabular}{|c|c|c|c|c|c|c|c|}
\hline Latitude & Longitude & Northing $^{a}$ & Easting $^{a}$ & $\begin{array}{c}\text { HAE } \\
\text { (meters) }\end{array}$ & Location & $\begin{array}{c}\text { Vertical } \\
\text { Precision }\end{array}$ & $\begin{array}{l}\text { Horizontal } \\
\text { Precision }\end{array}$ \\
\hline 36.66115992 & -115.9991991 & 4057548.979 & 589444.381 & NA & J01 & NA & NA \\
\hline 36.66137367 & -115.9996449 & 4057572.274 & 589404.282 & NA & J02 & NA & NA \\
\hline 36.66135626 & -115.9996561 & 4057570.333 & 589403.304 & NA & J03 & NA & NA \\
\hline 36.66137026 & -115.9996169 & 4057571.922 & 589406.794 & NA & J05 & NA & NA \\
\hline 36.66133941 & -115.9996294 & 4057568.488 & 589405.710 & NA & J06 & NA & NA \\
\hline 36.66133044 & -115.9996019 & 4057567.519 & 589408.180 & NA & J08 & NA & NA \\
\hline 36.66139110 & -115.9995898 & 4057574.260 & 589409.193 & NA & J09 & NA & NA \\
\hline 36.66133920 & -115.9995636 & 4057568.527 & 589411.588 & NA & $\mathrm{J} 10$ & NA & NA \\
\hline 36.66136616 & -115.9995721 & 4057571.509 & 589410.801 & NA & J11 & NA & NA \\
\hline 36.66139707 & -115.9996240 & 4057574.889 & 589406.128 & NA & $\mathrm{J} 12$ & NA & NA \\
\hline 36.66133336 & -115.9995347 & 4057567.906 & 589414.179 & NA & J13 & NA & NA \\
\hline 36.66134294 & -115.9996735 & 4057568.840 & 589401.763 & NA & J14 & NA & NA \\
\hline 36.66138332 & -115.9996796 & 4057573.313 & 589401.175 & NA & J15 & NA & NA \\
\hline 36.66139804 & -115.9996660 & 4057574.958 & 589402.369 & NA & J16 & NA & NA \\
\hline 36.66131725 & -115.9995791 & 4057566.078 & 589410.233 & NA & J17 & NA & NA \\
\hline 36.66132254 & -115.9996147 & 4057566.631 & 589407.045 & NA & J18 & NA & NA \\
\hline
\end{tabular}

aUniversal Transverse Mercator (UTM) Zone 11, North American Datum (NAD) 1927 (U.S. Western)

$\mathrm{HAE}=$ Height above ellipsoid

NA $=$ Not applicable 
Appendix E

Evaluation of Risk 


\section{E.1.0 Evaluation of Risk}

A detailed assessment of risk for no further action and evaluated alternatives was not performed because COCs exceeding FALs are not present or will not be left in place without appropriate controls. 


\section{Appendix F}

\section{Project Organization for CAU 224}




\section{F.1.0 Project Organization}

The NNSA/NSO Project Manager is Janet Appenzeller-Wing and her telephone number is (702) 295-0461.

The identification of the project Health and Safety Officer and the Quality Assurance Officers can be found in the appropriate plan. However, personnel are subject to change and it is suggested that the appropriate U.S. Department of Energy Project Manager be contacted for further information. The Task Manager is identified in the FFACO Biweekly Activity Report. 


\section{Appendix G}

Derivation of Residual Radioactive Material Guidelines for Radionuclides in Soil at CAU 224 


\section{Introduction}

Residual radioactive material guidelines for fission products, activation products, uranium isotopes, and plutonium isotopes were derived for Corrective Action Sites (CASs) 06-05-01, 06-17-01, and 06-23-01 within Corrective Action Unit (CAU) 224, Decon Pad and Septic Systems, located in Area 6 of the Nevada Test Site (NTS). These sites were contaminated with fission products, activation products, uranium isotopes, and plutonium isotopes from decontamination activities conducted at the Area 6 decontamination pads and catch basin. Fission product, activation products, uranium isotopes, and plutonium isotope guidelines for single radionuclides were derived on the basis of the requirement that the total effective dose equivalent (TEDE) to a hypothetical individual working within the identified area of contamination within the boundary of these CASs at CAU 224 should not exceed an annual dose limitation of 25 millirem per year (mrem/yr).

The radiation dose (TEDE) is defined as the sum of the effective dose equivalent (EDE) from external radiation and the committed effective dose equivalent (CEDE) from internal radiation. A dose equivalent is the radiation dose absorbed by a tissue multiplied by a dimensionless quality factor that represents the biological effectiveness of the radiation type (i.e., alpha particles, photons) for causing stochastic biological effects such as cancer or heredity effects. The EDE is the weighted sum of the dose equivalents to different organs and tissues, where the weighing factor is the ratio of the stochastic risk for an individual tissue to the total stochastic risk for all tissue following whole body irradiation. The CEDE is the dose equivalent to organs and tissues that will be received by an individual from an intake of radioactive material during the 50 -year period following the intake.

The Residual Radiation (RESRAD) computer code (version 6.21) was developed by Argonne National Laboratory for the U.S. Department of Energy (DOE). The RESRAD implements the dose assessment methodology described in DOE Order 5400.5, "Radiation Protection of the Public and the Environment" (DOE, 1990) and is used to implement residual radioactive material guidelines contained in the Order. One scenario (scenario A) was considered for this site due to its proximity to the Area 6 Control Point and potential for future long-term use (NDEP, 2004). The two approved scenarios vary regarding the future land use at the site and indicate that a $25 \mathrm{mrem} / \mathrm{yr}$ dose constraint would not be exceeded within 1,000 years for the single radionuclide 
guidelines for each scenario. The scenarios were chosen based on the consideration of all significant exposures to the critical population group via the following pathways:

- Direct exposure to external radiation from the contaminated soil

- Internal dose from inhalation of airborne radionuclides

- Internal dose from ingestion of contaminated soil

Fission product, activation product, uranium isotope, and plutonium isotope radionuclide guidelines have been derived on a site-specific basis due to the fact that no generic fission product, activation product, uranium isotope, or plutonium isotope radionuclide guidelines exits for the NTS. The derived guidelines represent the residual concentrations of amercium-241, cesium-137, europium-152, plutonium-238, plutonium-239, uranium-234, uranium-235, and uranium-238. The radionuclides are assumed to be in a homogeneously contaminated volume at a concentration that must not be exceeded if the dose criterion is not to be exceeded. The fission product, activation product, uranium isotopes, and plutonium isotopes were chosen from detected sample results associated with the characterization of the CAU 224 CASs. The highest concentration (maximum result) was used for each detected radionuclide for the hot-spot analysis, and the average concentration plus the distribution dependent 95 percent upper confidence level was used for each radionuclide for the dose calculation. Table 1 lists the input concentrations used in the calculations. The U. S. Environmental Protection Agency (EPA) software application, ProUCL Version 3.0, was used to calculate the appropriate 95 percent upper confidence level based on the distribution of the unknown mean.

Of the following two potential exposure scenarios, only scenario A was considered for the CASs within CAU 224 because of the proximity to the Area 6 Control Point and the potential for future long-term use. For all scenarios it is assumed that at some time within 1,000 years the site will be released for use without radiological restrictions following remediation. 


\section{Table 1 \\ Input Concentrations}

\begin{tabular}{||c|c|c|c||}
\hline \multicolumn{2}{|c|}{ Dose Calculation } & \multicolumn{2}{c|}{ Hot-Spot Calculation } \\
\hline Radionuclide & $\begin{array}{c}\text { Average Activity plus } \\
\text { 95\% UCL } \\
\text { Concentration (pCi/g) }\end{array}$ & Radionuclide & $\begin{array}{c}\text { Maximum Activity } \\
\text { Concentration (pCi/g) }\end{array}$ \\
\hline Americium-241 & 20.49 & Americium-241 & 29.60 \\
\hline Cesium-137 & 4.76 & Cesium-137 & 10 \\
\hline Europium-152 & 2.46 & Europium-152 & $1.37^{*}$ \\
\hline Plutonium-238 & 1.99 & Plutonium-238 & 10 \\
\hline Plutonium-239 & 39.25 & Plutonium-239 & 218 \\
\hline Strontium-90 & 21.27 & Strontium-90 & 32.50 \\
\hline Uranium-234 & 1.26 & Uranium-234 & $1.08^{*}$ \\
\hline Uranium-235 & 0.11 & Uranium-235 & 0.12 \\
\hline Uranium-238 & 1.21 & Uranium-238 & $0.94^{*}$ \\
\hline \hline
\end{tabular}

${ }^{*}$ For Eu-152, U-234 and U-234 the average plus the 95 percent UCL values were used in the hot-spot calculation.

$\mathrm{UCL}=$ Upper Confidence Level

$\mathrm{pCi} / \mathrm{g}=$ Picocuries per gram

Scenario A assumes continued industrial use of the site. This scenario addresses long-term exposure to industrial workers exposed daily to residual levels of radionuclides in soil during an average work day outdoors on site (EPA, 1991). This scenario assumes that a worker will be on the site for 250-days per year, 8-hours per day for a duration of 25 years. The indoor time fraction is zero, since there are no buildings on the site nor are any buildings anticipated to be built since the site lies within a wash. The outdoor time fraction is determined by assuming that workers will be on-site and outdoors for 250 days/year x $8 \mathrm{hrs} /$ day $=2,000 \mathrm{hrs} / \mathrm{year}$. The outdoor time fraction is then given by $2,000 / 8,760$ (number of work hours in a year) $=0.228$. The worker exposures are limited to working hours and do not include contributions from ingestion of drinking water, plant foods, meat, or fish taken from the immediate area.

Scenario B assumes a low-occupancy activity at the site. This scenario addresses long-term exposure to workers exposed to residual levels of radionuclides in soil during an average work day outdoors on site. This scenario assumes that a worker will be on the site for a total of 335-hours per year for a duration of 25 years. The indoor time is zero. The outdoor time fraction is determined by $335 / 8,760=0.038$. The worker exposures are limited to working hours and do not include contributions from ingestion of drinking water, plant foods, meat, or fish taken from the immediate area. 
The pathways considered for Scenarios A and B are summarized in Table 2.

\section{Table 2}

Summary of Pathways Considered for

Scenarios A and B

\begin{tabular}{||l|c|c||}
\hline \multicolumn{1}{|c|}{ Pathway } & Scenario A & Scenario B \\
\hline \hline External exposure & Yes & Yes \\
\hline Particulate inhalation & Yes & Yes \\
\hline Radon inhalation & No & No \\
\hline Ingestion of soil & Yes & Yes \\
\hline $\begin{array}{l}\text { Ingestion of produce from on-site } \\
\text { garden }\end{array}$ & No & No \\
\hline $\begin{array}{l}\text { Ingestion of meat from on-site } \\
\text { livestock }\end{array}$ & No & No \\
\hline $\begin{array}{l}\text { ingestion of milk from on-site } \\
\text { livestock }\end{array}$ & No & No \\
\hline ingestion of fish from on-site pond & No & No \\
\hline ingestion of water from on-site well & No & No \\
\hline
\end{tabular}

The RESRAD computer code (version 6.21) was used to calculate the dose/source concentration ratios $\mathrm{DSR}_{\mathrm{ip}}(\mathrm{t})$ for all identified radionuclides (i) and pathways $(\mathrm{p})$ at time $(\mathrm{t})$ after remediation. The period considered in this analysis was 1,000 years. Radioactive decay and ingrowth were considered in deriving the dose/source concentration ratios. The various parameters used in the RESRAD code for this analysis are listed in Attachment A. The calculated maximum dose contributions for all considered pathways are presented in Table 3 and the dose/source concentration ratios summed over all pathways are presented in Table 4 for scenario A. The maximum dose contributions and total dose/source concentration ratios for scenario A have been predicted to occur at time zero. The dose contributions are based on scenario A with a mean plus 95 percent UCL radionuclide activity concentration and a 1,800 square meter $\left(\mathrm{m}^{2}\right)$ contamination area. 
Uncertainty in the derivation of dose contributions and dose/source concentration ratios comes from the distribution of possible input parameter values, as well as uncertainty in the conceptual model used to represent the site. For Scenario A the external exposure and soil ingestion pathways contribute 80 percent of the dose. Therefore, uncertainties in occupancy factors, mass loading, inhalation rate, and wind speed have the greatest significance on the model predictions. A sensitivity analysis was performed on these parameters in Scenario A. The sensitivity analysis was performed by multiplying or dividing the mean parameter value by a factor of 2 . Figures 1 through 5 graphically represent the effects on the summed dose contributions for all radionuclides and all pathways for each above mentioned parameter. 
Table 3

Maximum Dose Contributions for Scenario A (occurs at $t=0$ )

\begin{tabular}{|c|c|c|c|c|c|c|c|c|}
\hline \multirow[b]{3}{*}{ Radionuclide } & \multicolumn{8}{|c|}{$\begin{array}{l}\text { Maximum Dose Contributions } \\
\text { (mrem/yr) }\end{array}$} \\
\hline & \multicolumn{2}{|c|}{ Ground } & \multicolumn{2}{|c|}{ Inhalation } & \multicolumn{2}{|c|}{ Soil } & \multicolumn{2}{|c|}{ Total } \\
\hline & mrem/yr & Fraction & mrem/yr & Fraction & mrem/yr & Fraction & mrem/yr & Fraction \\
\hline Americium-241 & 1.894E-01 & 0.0115 & $1.096 \mathrm{E}+00$ & 0.0665 & $2.035 E+00$ & 0.1235 & $3.320 \mathrm{E}+00$ & 0.2015 \\
\hline Cesium-137 & $3.279 E+00$ & 0.1990 & 1.813E-05 & 0.0000 & 6.437E-03 & 0.0004 & $3.285 E+00$ & 0.1994 \\
\hline Europium-152 & $3.457 E+00$ & 0.2098 & 6.399E-05 & 0.0000 & 4.250E-04 & 0.0000 & 3.457E+00 & 0.2098 \\
\hline Plutonium-238 & 6.661E-05 & 0.0000 & 9.385E-02 & 0.0057 & 1.753E-01 & 0.0105 & 2.692E-01 & 0.0162 \\
\hline Plutonium-239 & $2.465 \mathrm{E}-03$ & 0.0001 & $2.034 \mathrm{E}+00$ & 0.1234 & $3.801 \mathrm{E}+00$ & 0.2307 & $5.837 \mathrm{E}+00$ & 0.3542 \\
\hline Strontium-90 & 1.063E-01 & 0.0064 & 3.322E-03 & 0.0002 & 8.788E-02 & 0.0053 & 1.975E-01 & 0.0119 \\
\hline Uranium-234 & 1.081E-04 & 0.0000 & 2.008E-02 & 0.0012 & $9.749 \mathrm{E}-03$ & 0.0006 & 2.993E-02 & 0.0018 \\
\hline Uranium-235 & 1.749E-02 & 0.0011 & 1.633E-03 & 0.0001 & 8.033E-04 & 0.0000 & 1.992E-02 & 0.0012 \\
\hline Uranium-238 & 3.801E-02 & 0.0023 & 1.723E-02 & 0.0010 & 8.899E-03 & 0.0005 & 6.413E-02 & 0.0038 \\
\hline Total & $7.091 \mathrm{E}+00$ & 0.4302 & $3.266 \mathrm{E}+00$ & 0.1982 & $6.124 \mathrm{E}+00$ & 0.3716 & $1.648 \mathrm{E}+01$ & 1.0000 \\
\hline
\end{tabular}

$\mathrm{mrem} / \mathrm{yr}=$ Milligrams per year 


\section{Table 4}

\section{Maximum Dose/Source Concentration Ratios Summed Over All Pathways for Scenarios A}

\begin{tabular}{|c|c|}
\hline & $\begin{array}{l}\text { Summed Dose/Source Concentration Ratios at Time } \mathrm{t}=0.0 \text { Years } \\
\qquad(\mathrm{mrem} / \mathrm{yr}) /(\mathrm{pCi} / \mathrm{g})\end{array}$ \\
\hline & Scenario A \\
\hline Radionuclide & $(\mathrm{mrem} / \mathrm{yr}) /(\mathrm{pCi} / \mathrm{g})$ \\
\hline Americium-241 & $1.621 \mathrm{E}-01$ \\
\hline Cesium-137 & 6.903E-01 \\
\hline Europium-152 & $1.406 \mathrm{E}+00$ \\
\hline Plutonium-238 & $1.344 \mathrm{E}-01$ \\
\hline Plutonium-239 & 1.487E-01 \\
\hline Strontium-90 & $9.285 \mathrm{E}-03$ \\
\hline Uranium-234 & 2.376E-02 \\
\hline Uranium-235 & $1.812 \mathrm{E}-01$ \\
\hline Uranium-238 & 5.301E-02 \\
\hline
\end{tabular}

Mrem/yr = Milligrams per year

$\mathrm{pCi} / \mathrm{g}=$ Picocuries per gram

RESRAD default parameters were used if no site-specific data were available. The default values are based on national averages or reasonable maximum values. The contaminated zone thickness of 1.524 meters $(\mathrm{m})$ (5 feet [ft]) with no cover was used to derive the dose contribution and is based on the results of the soil sampling. The calculated dose contributions and dose/source concentration ratios are conservative since in reality contamination is rarely distributed uniformly, but rather occurs in localized areas.

The residual radioactive material guideline represents the concentration of residual radioactive material that can remain in place and still allow use of that area without radiological restrictions. The radioactive material guideline, $\mathrm{G}$, can be calculated given a dose limit of $\mathrm{H}_{\mathrm{EL}}$ for an individual as follows:$$
\mathrm{G}=\mathrm{H}_{\mathrm{EL}} / \mathrm{DSR},
$$

where DSR is the total dose/source concentration ratio listed in Table 4. The dose limit $\mathrm{H}_{\mathrm{EL}}$, used to derive the residual radioactive material guideline is $25 \mathrm{mrem} / \mathrm{yr}$ for Scenario A. The 
calculated residual radioactive material guidelines for individual radionuclides are presented in Table 5.

Table 5

Residual Radioactive Material Guidelines for Scenario A

\begin{tabular}{|c|c|}
\cline { 2 - 2 } \multicolumn{1}{c|}{} & Guideline $(\mathrm{pCi} / \mathrm{g})$, by Scenario \\
\hline Radionuclide & Scenario A \\
\hline Americium-241 & $1.543 \mathrm{E}+02$ \\
\hline Cesium-137 & $3.622 \mathrm{E}+01$ \\
\hline Europium-152 & $1.778 \mathrm{E}+01$ \\
\hline Plutonium-238 & $1.86 \mathrm{E}+02$ \\
\hline Plutonium-239 & $1.681 \mathrm{E}+02$ \\
\hline Strontium-90 & $2.692 \mathrm{E}+03$ \\
\hline Uranium-234 & $1.052 \mathrm{E}+03$ \\
\hline Uranium-235 & $1.380 \mathrm{E}+02$ \\
\hline Uranium-238 & $4.716 \mathrm{E}+02$ \\
\hline
\end{tabular}

$\mathrm{pCi} / \mathrm{g}=$ Picocuries per gram

The derived guidelines presented in Table 5 apply to a large, homogeneously contaminated area. For a small, isolated area of contamination (i.e., hot spot), the allowable concentration that can remain on-site may be higher due to the averaging process than what is allowed by the homogeneous area guideline. To evaluate the potential for greater exposure to individuals using a site contaminated with hot spots versus a site that is homogeneously contaminated, the following hot spot criterion must be applied. The hot spot soil guideline $\mathrm{G}_{\mathrm{i}}$, can be calculated with RESRAD by using the area of the hot spot or can be conservatively estimated on a single radionuclide bases for field applications by using the following;

$$
\mathrm{G}_{\mathrm{i}}=\mathrm{G}_{\mathrm{i}}\left(\mathrm{t}_{\mathrm{m}}\right) \times(100 / \mathrm{A})^{1 / 2}
$$

where:

$\mathrm{G}_{\mathrm{i}}\left(\mathrm{t}_{\mathrm{m}}\right)=$ the single radionuclide soil guideline in a homogeneously contaminated zone A $\quad=$ area of hot spot $\left(\mathrm{m}^{2}\right)$.

The RESRAD code was used to calculate hot spot guideline values for Scenario A and B for the following hot spot areas; $100 \mathrm{~m}^{2}, 10 \mathrm{~m}^{2}$, and $1 \mathrm{~m}^{2}$. The initial radionuclide concentrations used were the maximum results and would represent the worst-case radionuclide ratio. The results for these calculations are presented in Table 6. 


\section{Table 6. \\ Calculated Hot Spot Guidelines for Scenario A}

\begin{tabular}{|l|c|c|c|}
\cline { 2 - 4 } \multicolumn{1}{c|}{} & \multicolumn{3}{c|}{ Guideline (pCi/g), by Size of Hot Spot } \\
\cline { 2 - 4 } \multicolumn{1}{c|}{} & \multicolumn{3}{c||}{ Scenario A } \\
\hline Radionuclide & $100 \mathbf{~ m}^{2}$ & $10 \mathbf{~ m}^{\mathbf{2}}$ & $1 \mathbf{~ m}^{\mathbf{2}}$ \\
\hline Americium- & $4.346 \mathrm{E}+02$ & $6.805 \mathrm{E}+02$ & $9.952 \mathrm{E}+02$ \\
\hline Cesium-137 & $4.138 \mathrm{E}+01$ & $7.011 \mathrm{E}+01$ & $3.188 \mathrm{E}+02$ \\
\hline Europium-152 & $2.038 \mathrm{E}+01$ & $3.469 \mathrm{E}+01$ & $1.584 \mathrm{E}+02$ \\
\hline Plutonium-238 & $5.759 \mathrm{E}+02$ & $8.965 \mathrm{E}+02$ & $1.188 \mathrm{E}+03$ \\
\hline Plutonium-239 & $5.228 \mathrm{E}+02$ & $8.153 \mathrm{E}+02$ & $1.081 \mathrm{E}+03$ \\
\hline Strontium-90 & $5.116 \mathrm{E}+03$ & $9.291 \mathrm{E}+03$ & $3.943 \mathrm{E}+04$ \\
\hline Uranium-234 & $1.991 \mathrm{E}+03$ & $2.705 \mathrm{E}+03$ & $3.522 \mathrm{E}+03$ \\
\hline Uranium-235 & $1.624 \mathrm{E}+02$ & $2.666 \mathrm{E}+02$ & $9.704 \mathrm{E}+02$ \\
\hline Uranium-238 & $6.453 \mathrm{E}+02$ & $1.020 \mathrm{E}+03$ & $2.513 \mathrm{E}+03$ \\
\hline
\end{tabular}

$\mathrm{pCi} / \mathrm{g}=$ Picocuries per gram
$\mathrm{m}^{2} \stackrel{\text { Square meters }}{=}$

The hot-spot criteria can be used as long as the average radionuclide concentration for any $100 \mathrm{~m}^{2}$ does not exceed the homogeneous residual radioactive material guidelines. Therefore, it is recommended for the chosen scenario that any encountered plutonium-239 hot spot be remediated to the Table 5 guideline level of 168 picocuries per gram (pCi/g) for ALARA purposes.

The following graphs identify the dose contribution and excess cancer risk for each radionuclide summed across all pathways for Scenario A. The EPA generally sets site-specific remediation levels for carcinogens at a level that represents an excess upper bound lifetime cancer risk to an individual of between $1 \times 10^{-4}$ to $1 \times 10^{-6}$. Furthermore, additional clarification is provided in a memorandum from S. D. Luftig and L. Weinstock to addressees titled, "Establishment of Cleanup Levels for CERCLA Sites with Radioactive Contamination” (1997). The subject memo provides the following clarification, "If a dose assessment is conducted at a site then 15 millirem per year (mrem/yr) effective dose equivalent (EDE) should generally be the maximum does limit for humans. This level equates to approximately $3 \times 10^{-4}$ increased lifetime risk and is consistent 
with levels generally considered protective in other governmental actions, particularly regulations and guidance developed by EPA in other radiation control programs." Additionally, it states, "Cleanup should generally achieve a level of risk within $10^{-4}$ to $10^{-6}$ carcinogenic risk range based on reasonable maximum exposure for an individual." The cleanup levels to be specified include exposures from all potential pathways, and through all media (e.g., soil, groundwater, surface sediment, air, structures, biota). As noted in previous policy, the "Upper boundary of risk range is not a discrete line at $1 \times 10^{-4}$, although EPA generally uses $1 \times 10^{-4}$ in making risk management decisions. A specific risk estimate around $10^{-4}$ may be considered acceptable if justified based on site-specific conditions" (OSWER 9355.0-30, April 22, 1991).

Figures 6 and 7, represent the total dose rate per year and total excess cancer risk summed across all pathways for an individual under Scenario A. At time zero, the total dose rate per year to an individual on-site is $16.48 \mathrm{mrem} / \mathrm{year}$ and the total excess cancer risk to the same individual is $1.07 \times 10^{-4}$. Therefore, no remediation is warranted under Scenario A, since the total dose rate and total excess cancer risk to an individual on-site is well within the prescribed EPA guidance. However, the site contains discrete areas of elevated radiological contamination (plutonium-239 hot spots), as a best management practice of ALARA these discrete areas of elevated radiological contamination should be remediated to the appropriate guideline levels in Table 5. 


\section{DOSE: All Nuclides Summed, All Pathways Summed With SA on Inhalation rate}

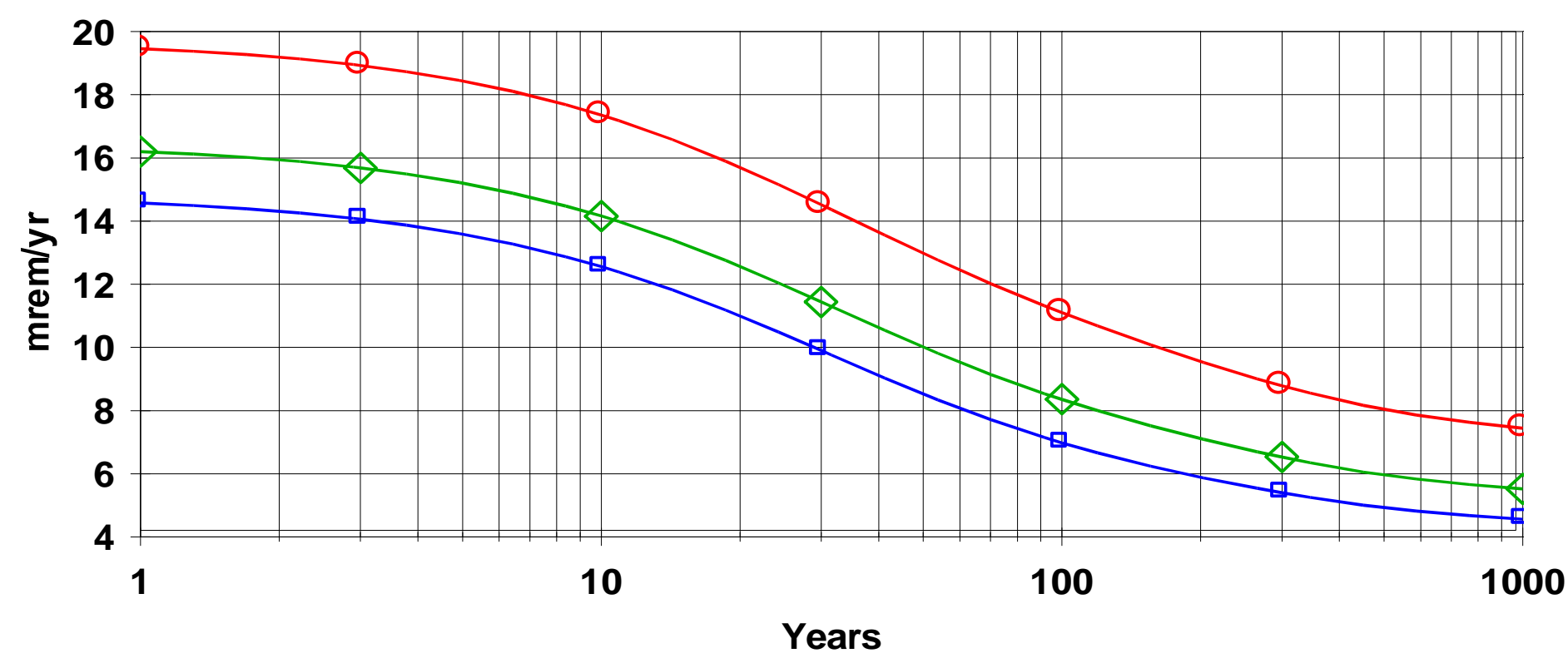

$\checkmark$ Upper: $16800-$ Mid: $8400 \quad \square$ Lower: 4200

AU 224250 day-yr average sensiivity.RAD 02/23/2005 11:19 Includes All P athway

Figure 1

Scenario A: Sensitivity Analysis Inhalation Rate Parameter 
DOSE: All Nuclides Summed, All Pathways Summed With SA on Mass loading for inhalation

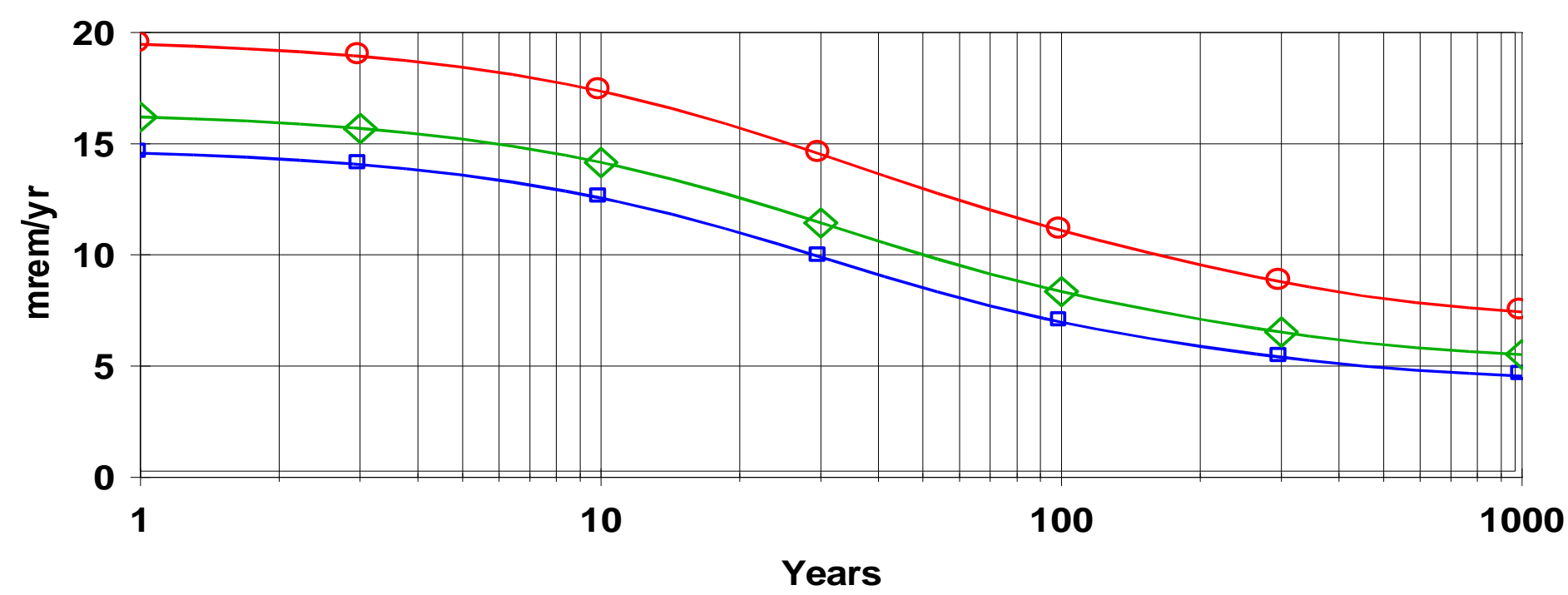

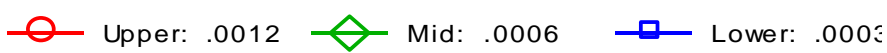

AU 224250 day-yr average sensiivity.RAD 02/23/2005 11:19 Includes All Pathway

Figure 2

Scenario A: Sensitivity Analysis Mass Loading Parameter 
DOSE: Am-241, All Pathways Summed With SA on Soil ingestion

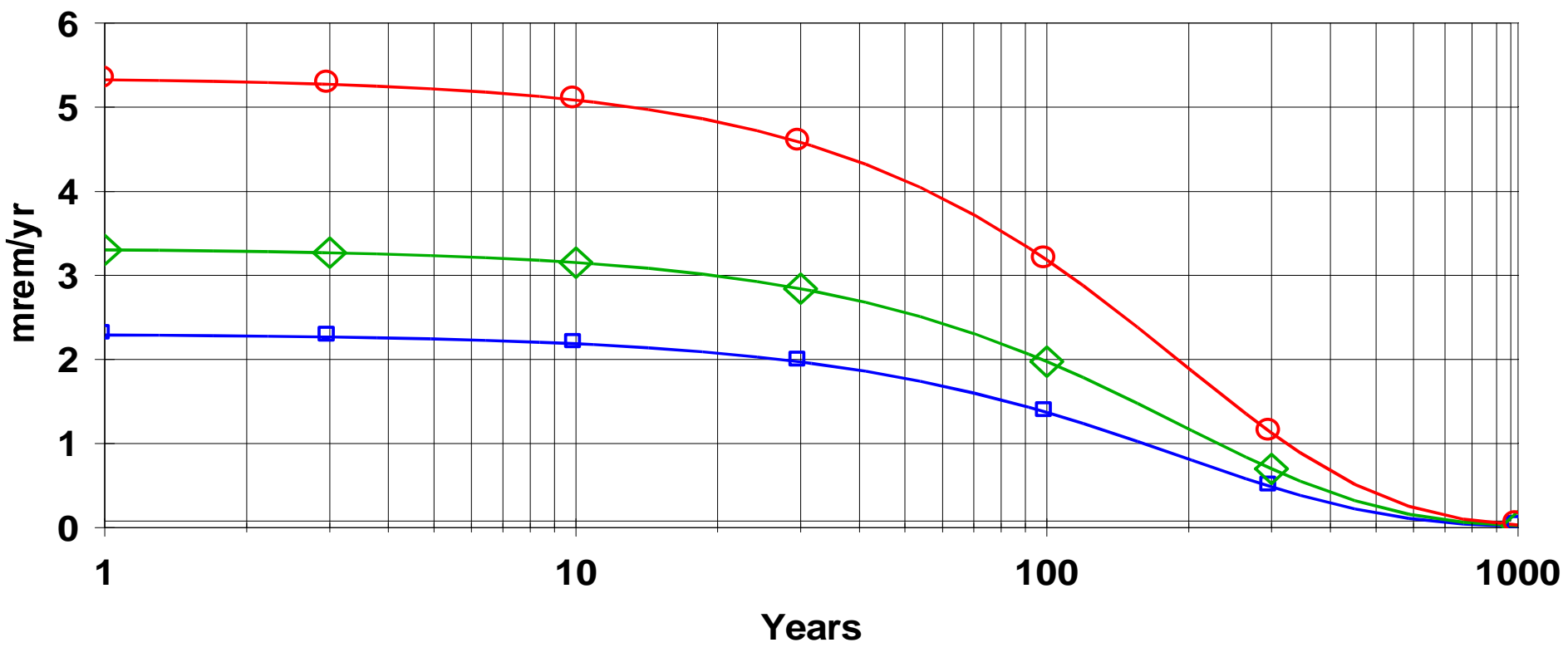

$\curvearrowright$ Upper: $240 \multimap$ Mid: $120 \rightarrow-$ Lower: 60

AU 224250 day-yr average sensiivity.RAD 02/23/2005 11:19 Includes All P athway

Figure 3

Scenario A: Sensitivity Analysis Soil Ingestion Parameter 
DOSE: Am-241, All Pathways Summed With SA on Average annual wind speed

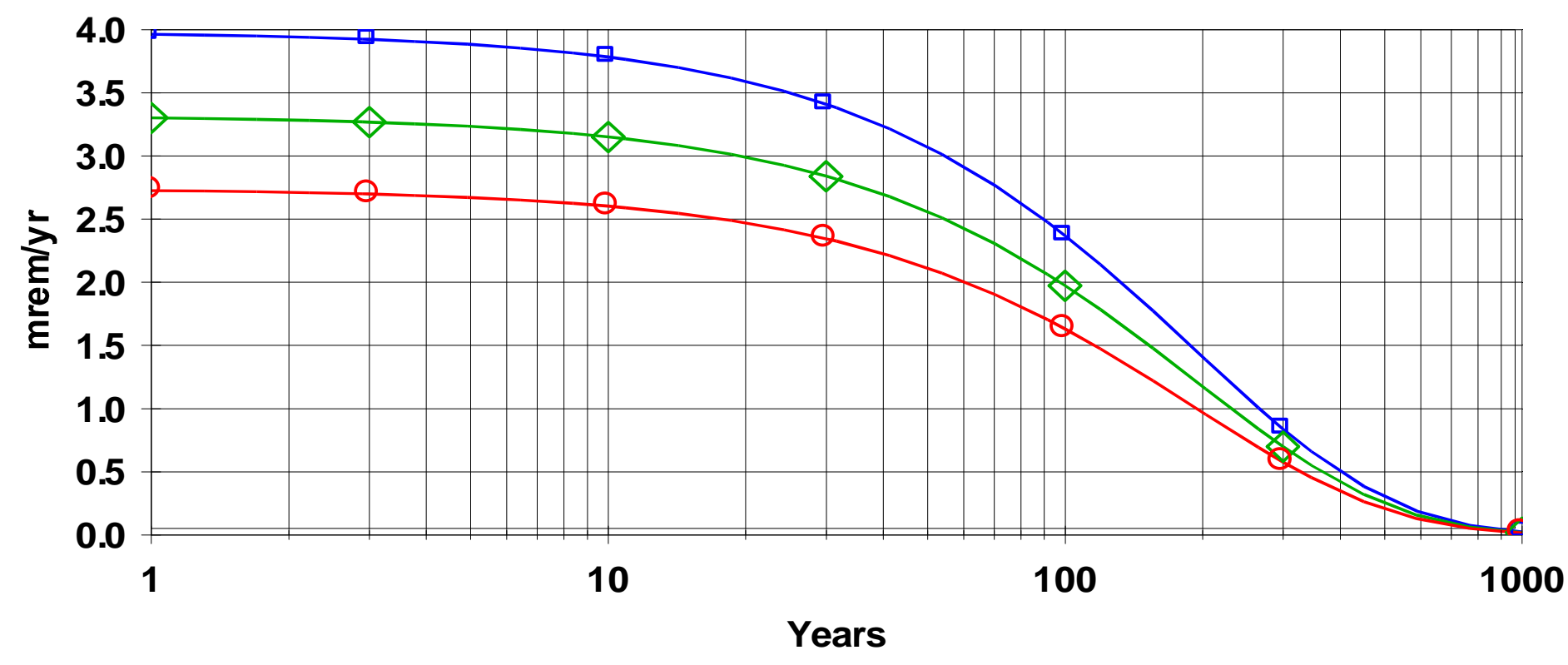

- Upper: $6.8 \curvearrowright$ Mid: $3.4 \longrightarrow$ Lower: 1.7

AU 224250 day-yr average sensiivity.RAD 02/23/2005 11:19 Includes All Pathway

Figure 4

Scenario A: Sensitivity Analysis Wind Speed Parameter 
DOSE: Am-241, All Pathways Summed With SA on Outdoor Time fraction

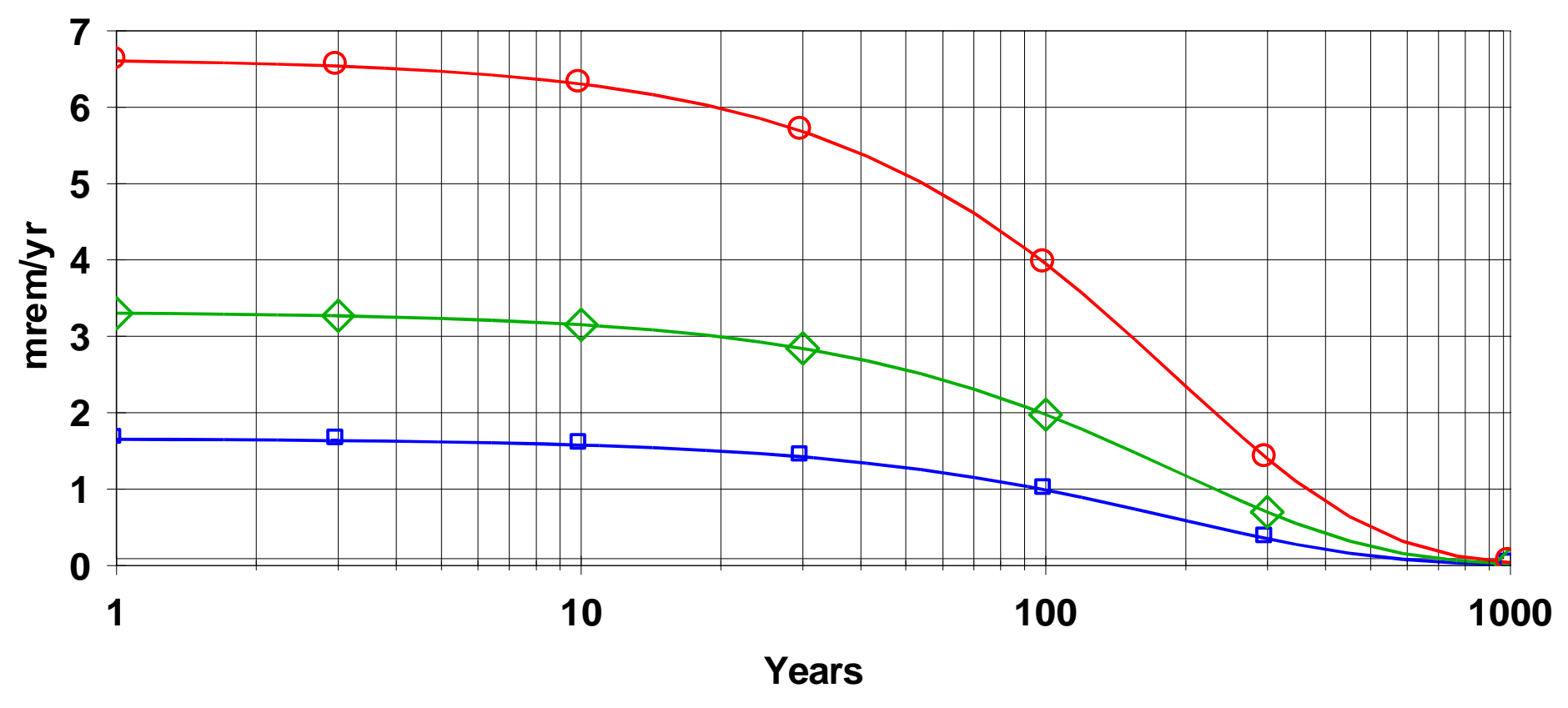

$\bigcirc$ Upper: $.456 \curvearrowright$ Mid: $.228 \multimap-$ Lower: .114

AU 224250 day-yr average sensiivity.RAD 02/23/2005 11:19 Includes All Pathway

Figure 5

Scenario A: Sensitivity Analysis Outdoor Time Fraction Parameter 
DOSE: All Nuclides Summed, All Pathways Summed
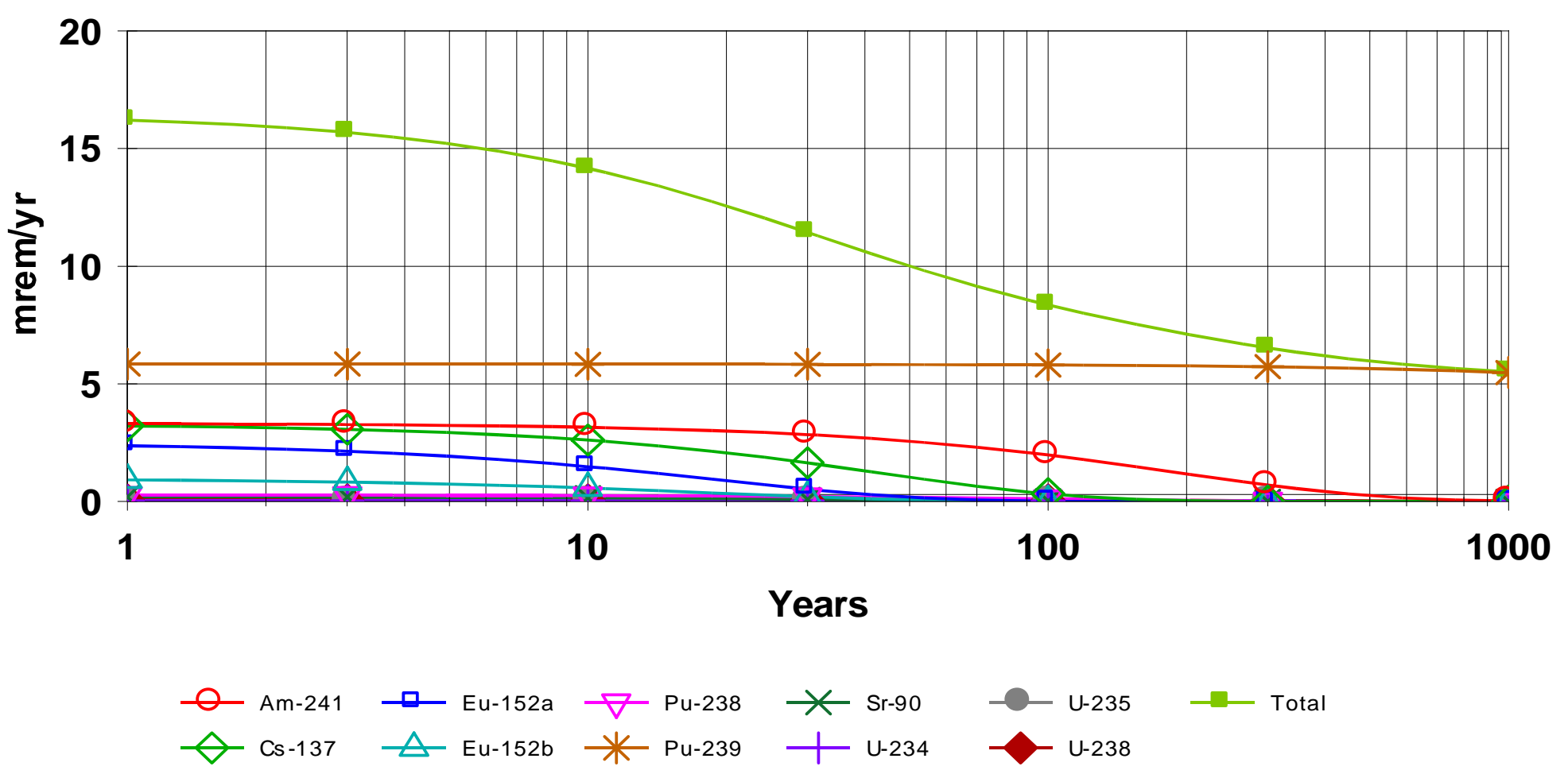

;AU 224250 day-yr average.RAD 02/22/2005 16:32 Includes All Pathway؛

Figure 6

Scenario A: Dose Rate Per Year Summed Across all Pathways 
EXCESS CANCER RISK: All Nuclides Summed, All Pathways Summed

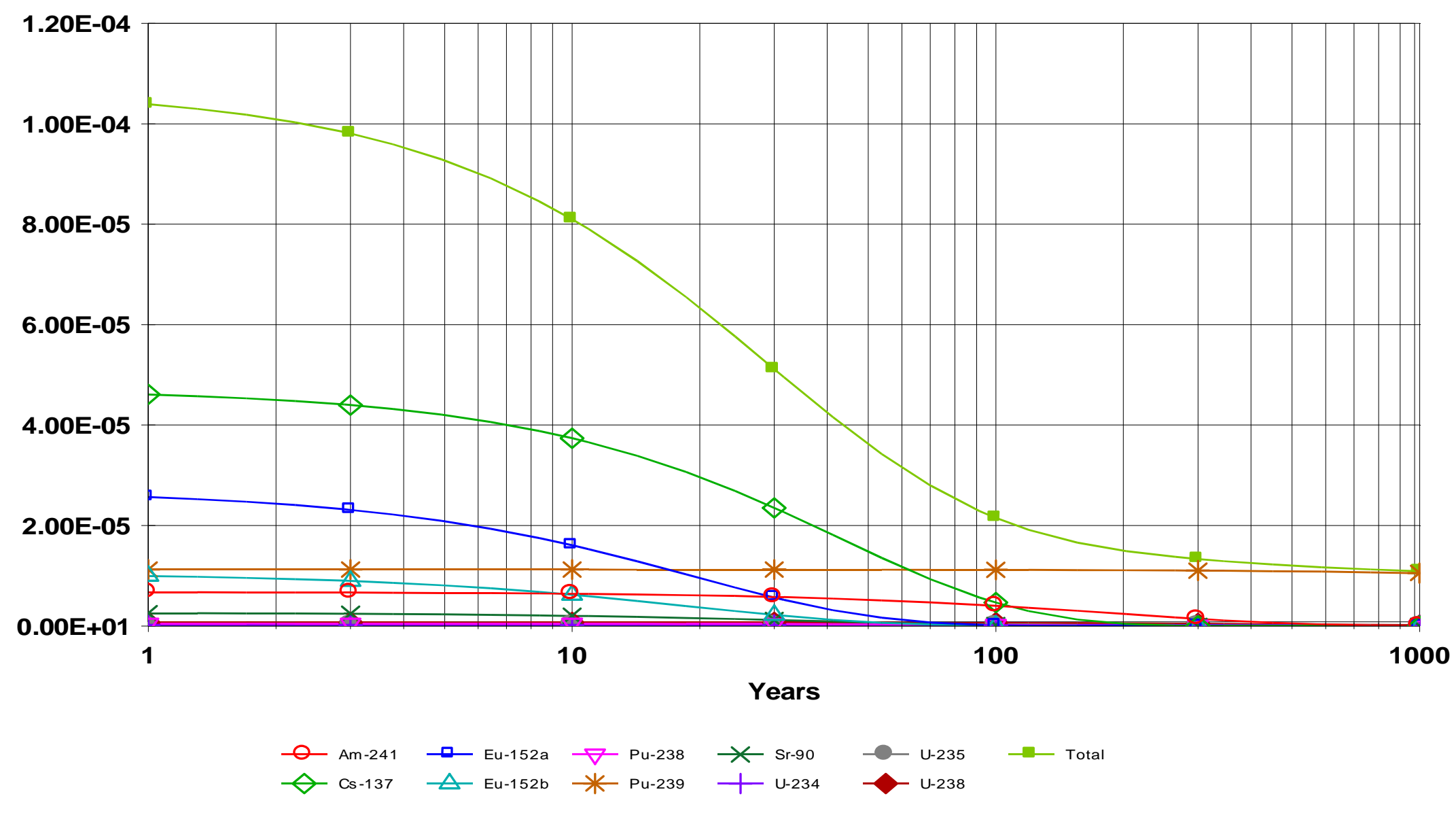

:AU 224250 day-yr average.RAD 02/22/2005 16:38 Includes All Pathways

\section{Figure 7}

Scenario A: Excess Cancer Risk Per Year Summed Across all Pathways 


\section{References:}

Air Resources Laboratory/Special Operations and Research Division. 2005. Web site for atmospheric research. As accessed on 2/10/2005 at http://www.sord.nv.doe.gov/arlsord-1.htm.

Luftig, S.D., and L. Weinstock, U.S. Environmental Protection Agency. 1997. Memorandum to addresses entitled, "Establishment of Cleanup Levels for CERCLA Sites with Radioactive Contamination," 22 August, OSWER 9200.4-18. Washington, DC.

Murphy, T., Nevada Division of Environmental Protection. 2004. Letter to R. Bangerter (NNSA/NSO), entitled, “Guidance for Calculating Industrial Sites Project Remediation Goals for Radionuclides in Soil Using the Residual Radiation (RESRAD) Computer Code," 19 November. Las Vegas, NV.

U. S. Department of Energy 1990. DOE Order 5400.5, "Radiation Protection of the Public and the Environment." Washington, DC.

U. S. Department of Energy, National Nuclear Security Administration Nevada Operations Office. 2001. Corrective Action Decision Document for Corrective Action Unit 262: Area 25 Septic Systems and Underground Discharge Point, Nevada Test Site, Nevada, DOE/NVB744Rev 1. Las Vegas, NV.

U.S. Environmental Protection Agency 1991. Human Health Evaluation Manual Supplemental Guidance: "Standard Default Exposure Factor," OSWER 9285.6-03. Washington, DC.

U. S. Nuclear Regulatory Commission. 1997. Radiation Dose Limits for Individual Members of the Public, 10 CFR Part 20 Subpart E. Washington, DC.

Yu C., A.J. Zielen, J.-J. Cheng, D.J. LePoire, E. Gnanapragasam, S. Kamboj, J. Arnish, A.Wallo III, W.A. Williams, and H. Peterson. 2001. User ¥Manual for RESRAD Version 6, ANL/EAD-4. Argonne, IL: Argonne National Laboratory, Environmental Assessment Division.

Yu C., C. Loureiro, C.C. Cheng, L.G. Jones, Y.Y. Wang, Y.P. Chia, and E. Faillace. 1993. Manual for Data Collection Handbook to Support Modeling the Impacts of Radioactive Material in Soil, ANL/EAIS-8. Chicago, IL: Argonne National Laboratory, Environmental Assessment Division. 


\section{Attachment A
Scenarios and Parameters Used for Analysis of The CAU 224 CASs}

The following exposure scenario was analyzed for the CAU 224 CASs, in Area 6 of the NTS:

C Scenario A assumes continued industrial use of the site. This scenario addresses long-term exposure to industrial workers exposed daily to residual levels of radionuclides in soil during an average work day outdoors onsite (EPA, 1991). This scenario assumes that a worker will be on the site for 250-days per year, 8 -hours per day for a duration of 25 years. The indoor time fraction is zero, since there are no buildings on the site nor are any buildings anticipated to be built since the site lies within a wash. The outdoor time fraction is determined by assuming that workers will be on-site and outdoors for 250 days/year $\times 1 \mathrm{hrs} /$ day $=2,000 \mathrm{hrs} /$ year. The outdoor time fraction is then given by $2,000 / 8,760$ (number of work hours in a year) $=0.228$. The worker exposures are limited to working hours and do not include contributions from ingestion of drinking water, plant foods, meat or fish taken from the immediate area.

The parametric values used in the RESRAD code for the analysis of the CAU 529 site are listed in Table A.1. Some parameters are site specific, while other values are default RESRAD values. The dose conversion factors used for inhalation and ingestion were the default FGR 13 morbidity values and correspond to the guidance and recommendations per the memorandum from A. Lawrence Office of Environmental Policy \& Guidance to Distribution titled, "Radiation Risk Estimation from Total Effective Dose Equivalents (TEDEs)” (2002). 
Table A.1 RESRAD Parameters Used for $25 \mathrm{mrem} / \mathrm{yr}$ Analysis of the CAU 224 Area 6 CASs (Page 1 of 6)

\begin{tabular}{|c|c|c|c|c|c|}
\hline Parameter & Units & Scenario A & Hot Spot & Defaults & Reference/Rationale \\
\hline \multicolumn{6}{|l|}{ R011 Contaminated Zone } \\
\hline Area of Contaminated Zone & $\mathrm{m}^{2}$ & $1.800 \mathrm{E}+03$ & $100,10,1$ & $1.000 \mathrm{E}+04$ & Area of contamination and hot spot area \\
\hline Thickness of Contaminated Zone & $\mathrm{m}$ & $1.52 \mathrm{E}+00$ & $1.52 \mathrm{E}+00$ & $2.000 \mathrm{E}+00$ & Based on sampling data \\
\hline Length Parallel to Aquifer Flow & $\mathrm{m}$ & not used & not used & $1.000 \mathrm{E}+02$ & \\
\hline Radiation Dose Limit & $\mathrm{mrem} / \mathrm{yr}$ & 25 & 25 & $2.5 \mathrm{E}+001$ & \\
\hline Elapsed Time Since Placement of Material & $\mathrm{yr}$ & 0.0 & 0.0 & 0.0 & \\
\hline \multicolumn{6}{|l|}{ R012 Initial Principal Radionuclide } \\
\hline Americium-241 (soil) & $\mathrm{pCi} / \mathrm{g}$ & 20.49 & 29.60 & 0.0 & CAU 224 soil sampling results \\
\hline Cesium-137 (soil) & $\mathrm{pCi} / \mathrm{g}$ & 4.76 & 10.00 & 0.0 & CAU 224 soil sampling results \\
\hline Europium-152 (soil) & $\mathrm{pCi} / \mathrm{g}$ & 2.46 & 2.46 & 0.0 & CAU 224 soil sampling results \\
\hline Plutonium-238 (soil) & $\mathrm{pCi} / \mathrm{g}$ & 1.99 & 10.00 & 0.0 & CAU 224 soil sampling results \\
\hline Plutonium-239 (soil) & $\mathrm{pCi} / \mathrm{g}$ & 39.25 & 218 & 0.0 & CAU 224 soil sampling results \\
\hline Strontium-90 (soil) & $\mathrm{pCi} / \mathrm{g}$ & 21.27 & 32.50 & 0.0 & CAU 224 soil sampling results \\
\hline Uranium-234 (soil) & $\mathrm{pCi} / \mathrm{g}$ & 1.26 & 1.26 & 0.0 & CAU 224 soil sampling results \\
\hline Uranium-235 (soil) & $\mathrm{pCi} / \mathrm{g}$ & 0.11 & 0.12 & 0.0 & CAU 224 soil sampling results \\
\hline Uranium-238 (soil) & $\mathrm{pCi} / \mathrm{g}$ & 1.21 & 1.21 & 0.0 & CAU 224 soil sampling results \\
\hline
\end{tabular}


Table A.1 RESRAD Parameters Used for 25 mrem/yr Analysis of the CAU 224 Area 6 CASs (Page 2 of 6).

\begin{tabular}{|c|c|c|c|c|c|}
\hline Parameter & Units & Scenario A & Hot Spot & Defaults & Reference/Rationale \\
\hline \multicolumn{6}{|c|}{ R013 Cover and Contaminated Zone Hydrological Data } \\
\hline Cover Depth & $\mathrm{m}$ & 0.0 & 0.0 & 0.0 & No Cover Assumed \\
\hline Density of Cover Material & $\mathrm{g} / \mathrm{cm}^{3}$ & not used & not used & 1.5 & No Cover Assumed \\
\hline Cover Depth Erosion Rate & $\mathrm{m} / \mathrm{yr}$ & not used & not used & $1.000 \mathrm{E}-03$ & No Cover Assumed \\
\hline Density of Contaminated Zone & $\mathrm{g} / \mathrm{cm}^{3}$ & 1.5 & 1.6 & 1.5 & RESRAD Default \\
\hline Contaminated Zone Erosion Rate & $\mathrm{m} / \mathrm{yr}$ & $1.000 \mathrm{E}-03$ & $1.000 \mathrm{E}-03$ & $1.000 \mathrm{E}-03$ & RESRAD Default \\
\hline Contaminated Zone Total Porosity & - & $4.000 \mathrm{E}-01$ & $4.000 \mathrm{E}-01$ & $4.000 \mathrm{E}-01$ & RESRAD Default \\
\hline Contaminated Zone Field Capacity & - & $2.000 \mathrm{E}-01$ & $2.000 \mathrm{E}-01$ & $2.000 \mathrm{E}-01$ & RESRAD Default \\
\hline Contaminated Zone Hydraulic Conductivity & $\mathrm{m} / \mathrm{yr}$ & $1.000 \mathrm{E}+01$ & $1.000 \mathrm{E}+01$ & $1.000 \mathrm{E}+01$ & RESRAD Default \\
\hline Contaminated Zone b Parameter & - & $5.300 \mathrm{E}+00$ & $5.300 \mathrm{E}+00$ & $5.300 \mathrm{E}+00$ & RESRAD Default \\
\hline Average Annual Wind Speed & $\mathrm{m} / \mathrm{sec}$ & 3.40 & 3.40 & $2.000 \mathrm{E}+00$ & Data from Air Resource Laboratory \\
\hline Humidity in Air & $\mathrm{g} / \mathrm{m}^{3}$ & not used & not used & $8.000 \mathrm{E}+00$ & \\
\hline Evapotranspiration Coefficient & - & $5.000 \mathrm{E}-01$ & $5.000 \mathrm{E}-01$ & $5.000 \mathrm{E}-01$ & Calculated based on site parameters \\
\hline Precipitation & $\mathrm{m} / \mathrm{yr}$ & $1.650 \mathrm{E}-01$ & $1.650 \mathrm{E}-01$ & $1.000 \mathrm{E}+00$ & Data from Air Resources Laboratory \\
\hline Irrigation & $\mathrm{m} / \mathrm{yr}$ & $2.000 \mathrm{E}-01$ & $2.000 \mathrm{E}-01$ & $2.000 \mathrm{E}-01$ & \\
\hline Irrigation Mode & - & overhead & overhead & overhead & \\
\hline Runoff Coefficient & - & $2.000 \mathrm{E}-01$ & $2.000 \mathrm{E}-01$ & $2.000 \mathrm{E}-01$ & \\
\hline Watershed Area for Nearby Stream or Pond & $\mathrm{m}^{2}$ & not used & not used & $1.000 \mathrm{E}+06$ & \\
\hline Accuracy for Water/Soil Computations & - & not used & not used & $1.000 \mathrm{E}-03$ & \\
\hline
\end{tabular}


Table A.1 RESRAD Parameters Used for 25 mrem/yr Analysis of the CAU 224 Area 6 CASs (Page 3 of 6).

\begin{tabular}{|c|c|c|c|c|c|}
\hline Parameter & Units & Scenario A & Hot Spot & Defaults & Reference/Rationale \\
\hline \multicolumn{6}{|l|}{ R014 Saturated Zone Hydrological Data } \\
\hline Density of Saturated Zone & $\mathrm{g} / \mathrm{cm}^{3}$ & not used & not used & $1.500 \mathrm{E}+00$ & \\
\hline Saturated Zone Total Porosity & - & not used & not used & $4.000 \mathrm{E}-01$ & \\
\hline Saturated Zone Effective Porosity & - & not used & not used & $2.000 \mathrm{E}-01$ & \\
\hline Saturated Zone Field Capacity & - & not used & not used & $2.000 \mathrm{E}-01$ & \\
\hline Saturated Zone Hydraulic Conductivity & $\mathrm{m} / \mathrm{yr}$ & not used & not used & $1.000 \mathrm{E}+02$ & \\
\hline Saturated Zone Hydraulic Gradient & - & not used & not used & $2.000 \mathrm{E}-02$ & \\
\hline Saturated Zone b Parameter & - & not used & not used & $5.300 \mathrm{E}+00$ & \\
\hline Water Table Drop Rate & $\mathrm{m} / \mathrm{yr}$ & not used & not used & $1.000 \mathrm{E}-03$ & \\
\hline Well Pump Intake Depth & $\mathrm{m}$ & not used & not used & $1.000 \mathrm{E}+01$ & \\
\hline Model: Nondispersion or Mass-Balance & - & not used & not used & ND & \\
\hline Well Pumping Rate & $\mathrm{m}^{3} / \mathrm{yr}$ & not used & not used & $2.500 \mathrm{E}+02$ & \\
\hline \multicolumn{6}{|c|}{ R015 Uncontaminated and Unsaturated Strata Hydrological Data } \\
\hline Number of Unsaturated Zone Strata & - & not used & not used & 1 & \\
\hline Thickness & $\mathrm{m}$ & not used & not used & $4.000 \mathrm{E}+00$ & \\
\hline Soil Density & $\mathrm{g} / \mathrm{cm}^{3}$ & not used & not used & $1.500 \mathrm{E}+00$ & \\
\hline Total Porosity & - & not used & not used & $4.000 \mathrm{E}-01$ & \\
\hline Effective Porosity & - & not used & not used & $2.000 \mathrm{E}-01$ & \\
\hline Field Capacity & - & not used & not used & $2.000 \mathrm{E}-01$ & \\
\hline Soil-specific b Parameter & - & not used & not used & $5.300 \mathrm{E}+00$ & \\
\hline Hydraulic Conductivity & $\mathrm{m} / \mathrm{yr}$ & not used & not used & $1.000 \mathrm{E}+01$ & \\
\hline
\end{tabular}


Table A.1 RESRAD Parameters Used for 25 mrem/yr Analysis of the CAU 224 Area 6 CASs (Page 4 of 6).

\begin{tabular}{|c|c|c|c|c|c|}
\hline Parameter & Units & Scenario A & Hot Spot & Defaults & Reference/Rationale \\
\hline \multicolumn{6}{|l|}{ R016 Distribution Coefficients and Leach Rates } \\
\hline Contaminated Zone $\mathrm{K}_{\mathrm{d}}$ (all Zones) & $\mathrm{cm}^{3} / \mathrm{g}$ & & & & RESRAD Defaults \\
\hline Saturated Leach Rate & /yr & 0.0 & 0.0 & 0.0 & \\
\hline Solubility Constant & - & 0.0 & 0.0 & 0.0 & \\
\hline \multicolumn{6}{|l|}{ R017 Inhalation and External Gamma } \\
\hline Inhalation Rate & $\mathrm{m}^{3} / \mathrm{yr}$ & $8.400 \mathrm{E}+03$ & $8.400 \mathrm{E}+03$ & $8.400 \mathrm{E}+03$ & RESRAD Default \\
\hline Mass Loading for Inhalation & $\mathrm{g} / \mathrm{m}^{3}$ & $6.00 \mathrm{E}-04$ & $6.00 \mathrm{E}-04$ & $1 \mathrm{E}-04$ & $\begin{array}{l}\text { The estimated mass loading for construction } \\
\text { activities (Yu, et. al., 1993) }\end{array}$ \\
\hline Exposure Duration & $\mathrm{yr}$ & 25 & 25 & 30 & Standard for Industrial/Commercial Scenario \\
\hline Shielding Factor Inhalation & - & 1 & 1 & 0.4 & Assumes no indoor time fraction \\
\hline Shielding Factor External Gamma & - & 1 & 1 & 0.7 & Assumes no indoor time fraction \\
\hline Fraction of Time Spent Indoors & - & 0.0 & 0.0 & 0.5 & Assumes no indoor time fraction \\
\hline Fraction of Time Spent Outdoors & - & 0.228 & 0.228 & 0.25 & Scenario A time fraction \\
\hline Shape Factor & - & 1.0 & 1.0 & 1.0 & RESRAD Default \\
\hline \multicolumn{6}{|c|}{ R018 Ingestion Pathway Data, Dietary Parameters } \\
\hline Fruits, Vegetables, and Grain Consumption & $\mathrm{kg} / \mathrm{yr}$ & not used & not used & $1.600 \mathrm{E}+02$ & \\
\hline Leafy Vegetable Consumption & $\mathrm{kg} / \mathrm{yr}$ & not used & not used & $1.400 \mathrm{E}+01$ & \\
\hline Milk Consumption & $\mathrm{L} / \mathrm{yr}$ & not used & not used & $9.200 \mathrm{E}+01$ & \\
\hline Meat and Poultry Consumption & $\mathrm{kg} / \mathrm{yr}$ & not used & not used & $6.300 \mathrm{E}+01$ & \\
\hline
\end{tabular}


Table A.1 RESRAD Parameters Used for 15 mrem/yr Analysis of the CAU 529 Wash Site (Page 5 of 6)

\begin{tabular}{|c|c|c|c|c|c|}
\hline Parameter & Units & Scenario A & Hot Spot & Defaults & Reference/Rationale \\
\hline Fish Consumption & $\mathrm{kg} / \mathrm{yr}$ & not used & not used & $5.400 \mathrm{E}+00$ & \\
\hline Other Seafood Consumption & $\mathrm{kg} / \mathrm{yr}$ & not used & not used & $9.000 \mathrm{E}-01$ & \\
\hline Soil Ingestion Rate & $\mathrm{g} / \mathrm{yr}$ & $1.200 \mathrm{E}+02$ & $1.200 \mathrm{E}+02$ & 36.5 & 480 mg/day (EPA, 1991) \\
\hline Drinking Water Intake & $\mathrm{L} / \mathrm{yr}$ & not used & not used & $5.100 \mathrm{E}+02$ & \\
\hline Drinking Water Contaminated Fraction & - & not used & not used & $1.000 \mathrm{E}+00$ & \\
\hline Household Water Contaminated Fraction & - & not used & not used & $1.000 \mathrm{E}+00$ & \\
\hline Livestock Water Contaminated Fraction & - & not used & not used & $1.000 \mathrm{E}+00$ & \\
\hline Irrigation Water Contaminated Fraction & - & not used & not used & $1.000 \mathrm{E}+00$ & \\
\hline Aquatic Food Contaminated Fraction & - & not used & not used & $5.000 \mathrm{E}-01$ & \\
\hline Plant Food Contaminated Fraction & - & not used & not used & -1 & \\
\hline Meat Contaminated Fraction & - & not used & not used & -1 & \\
\hline Milk Contaminated Fraction & - & not used & not used & -1 & \\
\hline \multicolumn{6}{|l|}{ R019 Ingestion Pathway Data, Nondietary } \\
\hline Livestock Fodder Intake for Meat & $\mathrm{kg} /$ day & not used & not used & $6.800 \mathrm{E}+01$ & \\
\hline Livestock Fodder Intake for Milk & $\mathrm{kg} /$ day & not used & not used & $5.500 \mathrm{E}+01$ & \\
\hline Livestock Water Intake for Meat & L/day & not used & not used & $5.000 \mathrm{E}+01$ & \\
\hline Livestock Water Intake for Milk & L/day & not used & not used & $1.600 \mathrm{E}+02$ & \\
\hline Livestock Soil Intake & $\mathrm{kg} /$ day & not used & not used & $5.000 \mathrm{E}-01$ & \\
\hline Mass Loading for Foliar Deposition & $\mathrm{g} / \mathrm{m}^{3}$ & not used & not used & $1.000 \mathrm{E}-04$ & \\
\hline Depth of Soil Mixing layer & $\mathrm{m}$ & not used & not used & $1.500 \mathrm{E}-01$ & \\
\hline Depth of Roots & $\mathrm{m}$ & not used & not used & $9.000 \mathrm{E}-01$ & \\
\hline
\end{tabular}


Table A.1 RESRAD Parameters Used for 15 mrem/yr Analysis of the CAU 529 Wash Site (Page 6 of 6)

\begin{tabular}{lcccc}
\hline \multicolumn{1}{c}{ Parameter } & Units & Scenario A & Hot Spot & Defaults \\
\hline Drinking Water Fraction from Groundwater & - & not used & not used & $1.000 \mathrm{E}+00$ \\
Household Water Fraction from Groundwater & - & not used & not used & $1.000 \mathrm{E}+00$ \\
Livestock Water Fraction from Groundwater & - & not used & not used & $1.000 \mathrm{E}+00$ \\
Irrigation Fraction from Groundwater & - & not used & not used & $1.000 \mathrm{E}+00$ \\
R021 Radon & & & & \\
Radon Parameters Not Used & & & \\
\hline
\end{tabular}




\section{Exhibit 1}

\section{RESRAD Scenario A Average Dose}

(27 Pages) 
1RESRAD, Version $6.21 \quad T^{1 / 2}$ Limit $=0.5$ year

Summary : CAU 224250 day-yr average worker scenario
File : CAU 224250 day-yr average. RAD

$$
\text { Table of Contents }
$$

Part I: Mixture Sums and Single Radionuclide Guidelines

Dose Conversion Eactor (and Relat

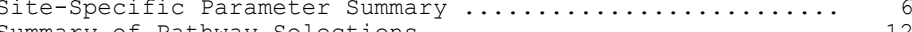
Contaminated Zone and Total Dose Summary $\ldots \ldots \ldots \ldots \ldots \ldots, \ldots \ldots \ldots,{ }_{13}$ Cotal Dose Components Time $=0.000 \mathrm{E}+00$

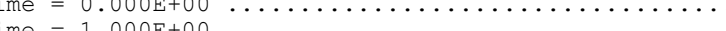

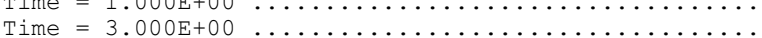

Time $=1.000 \mathrm{E}+01$

Time $=3.000 \mathrm{E}+01$

Time $=3.000 \mathrm{E}+02$

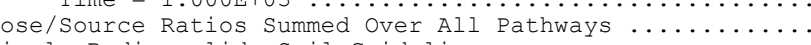

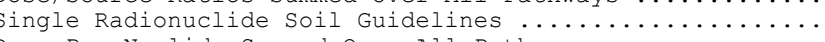

Soil Concentration Per nuclide ............ 
1RESRAD, Version $6.21 \quad T^{1 / 2}$ Limit $=0.5$ year

02/22/2005 17:24 Page

$\begin{array}{llll}\text { Summary } & \text { : CAU } 224 & 250 & \text { day-yr average wor } \\ \text { File } & \text { CAU } 224 & 250 & \text { day-yr average. RAD }\end{array}$

Dose Conversion Factor (and Related) Parameter Summary File: FGR 13 Morbidity

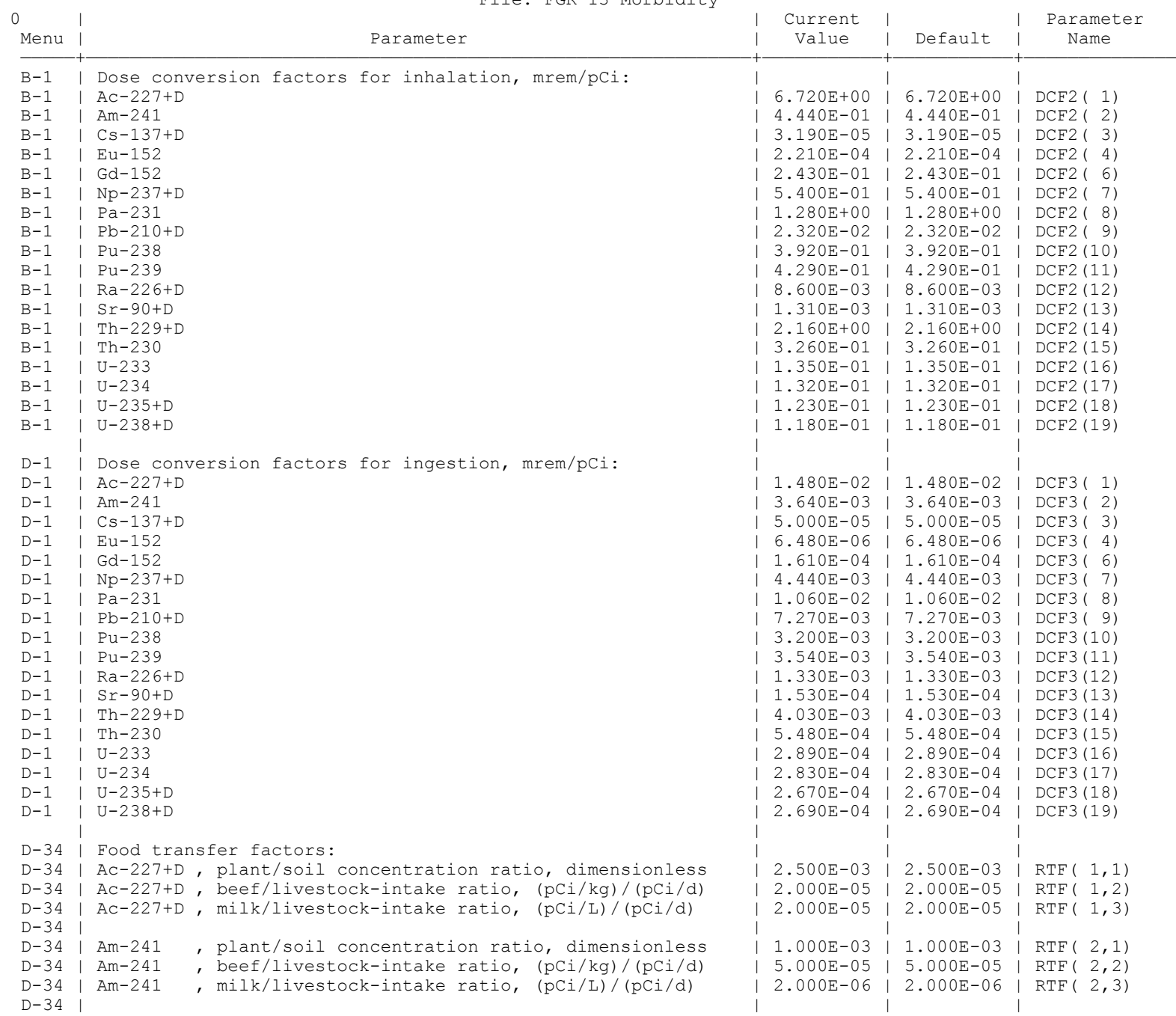


1RESRAD, Version $6.21 \quad$ T3/2 Limit $=0.5$ year

02/22/2005 17:24 Page 3

$\begin{array}{llll}\text { Summary : CAU } & 224 & 250 & \text { day-yr average worker } \\ \text { File : CAU } 224 & 250 \text { day-yr average. RAD }\end{array}$

Dose Conversion Factor (and Related) Parameter Summary (continued) File: FGR 13 Morbidity

| Current | Default | Paran |

Menu

\begin{tabular}{|c|c|c|}
\hline $4.000 \mathrm{E}-02$ & I $4.000 \mathrm{E}-02$ & | $\operatorname{RTF}(3,1)$ \\
\hline $3.000 \mathrm{E}-02$ & $3.000 \mathrm{E}-02$ & I $\operatorname{RTF}(3,2)$ \\
\hline $8.000 \mathrm{E}-03$ & $8.000 \mathrm{E}-03$ & $\operatorname{RTE}(3,3)$ \\
\hline $2.500 \mathrm{E}-03$ & $2.500 \mathrm{E}-03$ & $\operatorname{RTF}(4,1)$ \\
\hline $2.000 \mathrm{E}-03$ & $2.000 \mathrm{E}-03$ & I $\operatorname{RTE}(4,2)$ \\
\hline $2.000 \mathrm{E}-05$ & $2.000 \mathrm{E}-05$ & $\operatorname{RTE}(4,3)$ \\
\hline $2.500 \mathrm{E}-03$ & $2.500 \mathrm{E}-03$ & $\operatorname{RTE}(6,1)$ \\
\hline $2.000 \mathrm{E}-03$ & $2.000 \mathrm{E}-03$ & $\operatorname{RTE}(6,2)$ \\
\hline $2.000 \mathrm{E}-05$ & $2.000 \mathrm{E}-05$ & $\operatorname{RTE}(6,3)$ \\
\hline $2.000 \mathrm{E}-02$ & $2.000 \mathrm{E}-02$ & $\operatorname{RTE}(7,1)$ \\
\hline $1.000 \mathrm{E}-03$ & $1.000 \mathrm{E}-03$ & $\operatorname{RTE}(7,2)$ \\
\hline $5.000 \mathrm{E}-06$ & $5.000 \mathrm{E}-06$ & $\operatorname{RTE}(7,3)$ \\
\hline $1.000 \mathrm{E}-02$ & $1.000 \mathrm{E}-02$ & $\operatorname{RTF}(8,1)$ \\
\hline $5.000 \mathrm{E}-03$ & $5.000 \mathrm{E}-03$ & $\operatorname{RTE}(8,2)$ \\
\hline $5.000 \mathrm{E}-06$ & $5.000 \mathrm{E}-06$ & $\operatorname{RTE}(8,3)$ \\
\hline & I & \\
\hline $\begin{array}{l}1.000 \mathrm{E}-02 \\
8.000 \mathrm{E}-04\end{array}$ & $\begin{array}{l}1.000 \mathrm{E}-02 \\
8.000 \mathrm{E}-04\end{array}$ & $\begin{array}{l}\mid \operatorname{RTF}(9,1) \\
\operatorname{RTF}(9,2)\end{array}$ \\
\hline $3.000 \mathrm{E}-04$ & $3.000 \mathrm{E}-04$ & $\operatorname{RTE}(9,3)$ \\
\hline $1.000 \mathrm{E}-03$ & $1.000 \mathrm{E}-03$ & $\operatorname{RTF}(10,1)$ \\
\hline $1.000 \mathrm{E}-04$ & $1.000 \mathrm{E}-04$ & ITTE $(10,2)$ \\
\hline $1.000 \mathrm{E}-06$ & $1.000 \mathrm{E}-06$ & I $\operatorname{RTF}(10,3)$ \\
\hline $1.000 \mathrm{E}-03$ & $1.000=-03$ & RTF \\
\hline $1.000 \mathrm{E}-04$ & $1.000 \mathrm{E}-04$ & $\operatorname{RTF}(11,2)$ \\
\hline $1.000 \mathrm{E}-06$ & $1.000 \mathrm{E}-06$ & $\operatorname{RTE}(11,3)$ \\
\hline $4.000 \mathrm{E}-02$ & $4.000 \mathrm{E}-02$ & $\operatorname{RTF}(12,1)$ \\
\hline $1.000 \mathrm{E}-03$ & $1.000 \mathrm{E}-03$ & I $\operatorname{RTF}(12,2)$ \\
\hline $1.000 \mathrm{E}-03$ & $1.000 \mathrm{E}-03$ & $\operatorname{RTE}(12,3)$ \\
\hline $3.000 \mathrm{E}-01$ & $3.000 \mathrm{E}-01$ & $\operatorname{RTF}(13,1)$ \\
\hline $8.000 \mathrm{E}-03$ & $8.000 \mathrm{E}-03$ & I $\operatorname{RTF}(13,2)$ \\
\hline $2.000 \mathrm{E}-03$ & $12.000 \mathrm{E}-03$ & $\operatorname{RTF}(13,3)$ \\
\hline & & $\operatorname{RTF}(14,1)$ \\
\hline $1.000 \mathrm{E}-04$ & $1.000 \mathrm{E}-04$ & $\operatorname{RTF}(14,2)$ \\
\hline $5.000 \mathrm{E}-06$ & $5.000 \mathrm{E}-06$ & $\operatorname{RTF}(14,3)$ \\
\hline & & \\
\hline $\begin{array}{l}1.000 \mathrm{E}-03 \\
1.000 \mathrm{E}-04\end{array}$ & $1.000 \mathrm{E}-03$ & $\begin{array}{l}\mid \operatorname{RTF}(15,1) \\
\operatorname{RTF}(15,2)\end{array}$ \\
\hline $5.000 \mathrm{E}-06$ & $5.000 \mathrm{E}-06$ & $\operatorname{RTF}(15,3)$ \\
\hline
\end{tabular}

Diant/soil concentration ratio, dimensionless D-34 I Cs-137+D, plant/soil concentration ratio, (pin/kg)/(pCi/d)

D-34 Eu-152, plant/soil concentration ratio, dimensionless D -34 Eu-152, beef/livestock-intake ratio, (pCi/kg)/(pCi/d) D-34

D-34 Gd-152, plant/soil concentration ratio, dimensionless D-34 Gd-152, beef/livestock-intake ratio, (pCi/kg)/(pCi/d) D-34 Gd-152, milk/livestock-intake ratio, (pCi/L)/(pCi/d) D-34 Np-237+D', beef/livestock-intake ratio, (pCi/kg)/(pCi/d) -34 Np-237+D, milk/livestock-intake ratio, (pCi/L)/(pCi/d) D -34

Da-231, plant/soil concentration ratio, dimensionless $\mathrm{D}-34$ Pa-231, beef/livestock-intake ratio, $(\mathrm{pCi} / \mathrm{kg}) /(\mathrm{pCi} / \mathrm{d})$ D-34 $\mathrm{Pa}-231, \mathrm{milk} /$ livestock-intake ratio, (PCi/L)/(PCi/d) D-34

D-34 Pb-210+D, plant/soil concentration ratio, dimensionless D-34 Pb-210+D, beef/livestock-intake ratio, (pCi/kg)/(pCi/d) D-34 Pb-210+D, milk/livestock-intake ratio, (pCi/L)/(pCi/d)

D-34 Pu-238, plant/soil concentration ratio, dimensionless D-34 Pu-238, beef/livestock-intake ratio, $(\mathrm{pCi} / \mathrm{kg}) /(\mathrm{pCi} / \mathrm{d})$ D-34 | Pu-238, milk/livestock-intake ratio, (pCi/L)/(pCi/d) D -34

D Pu-239, plant/soil concentration ratio, dimensionless , beef/livestock-intake ratio, (pCi/ $\mathrm{kg}) /(\mathrm{pCi} / \mathrm{d})$

D-34 Ra-226+D, plant/soil concentration ratio, dimensionless D-34 Ra-226+D, beef/livestock-intake ratio, (pCi/kg)/(pCi/d) D-34 Ra-226+D , milk/livestock-intake ratio, (pCi/L)/(pCi/d)

D-34 Sr-90+D, plant/soil concentration ratio, dimensionless D-34 Sr-90+D, beef/livestock-intake ratio, (pCi/kg)/(pCi/d) D-34 Sr-90+D , milk/livestock-intake ratio, (pCi/L)/(pCi/d)

D-34 Th-229+D, plant/soil concentration ratio, dimensionless (D) $229+\mathrm{D}$, beef/livestock-intake ratio, (pCi/kg)/(pCi/d) , milk/livestock-intake ratio, (pCi/L)/(pCi/d)

D-34 Th-230, plant/soil concentration ratio, dimensionless D-34 Th-230, beef/livestock-intake ratio, $(\mathrm{pCi} / \mathrm{kg}) /(\mathrm{pCi} / \mathrm{d})$ $\mathrm{D}-34$ Th-230, milk/livestock-intake ratio, (pCi/L)/(pCi/d) 
Dose Conversion Factor (and Related) Parameter Summary (continued)

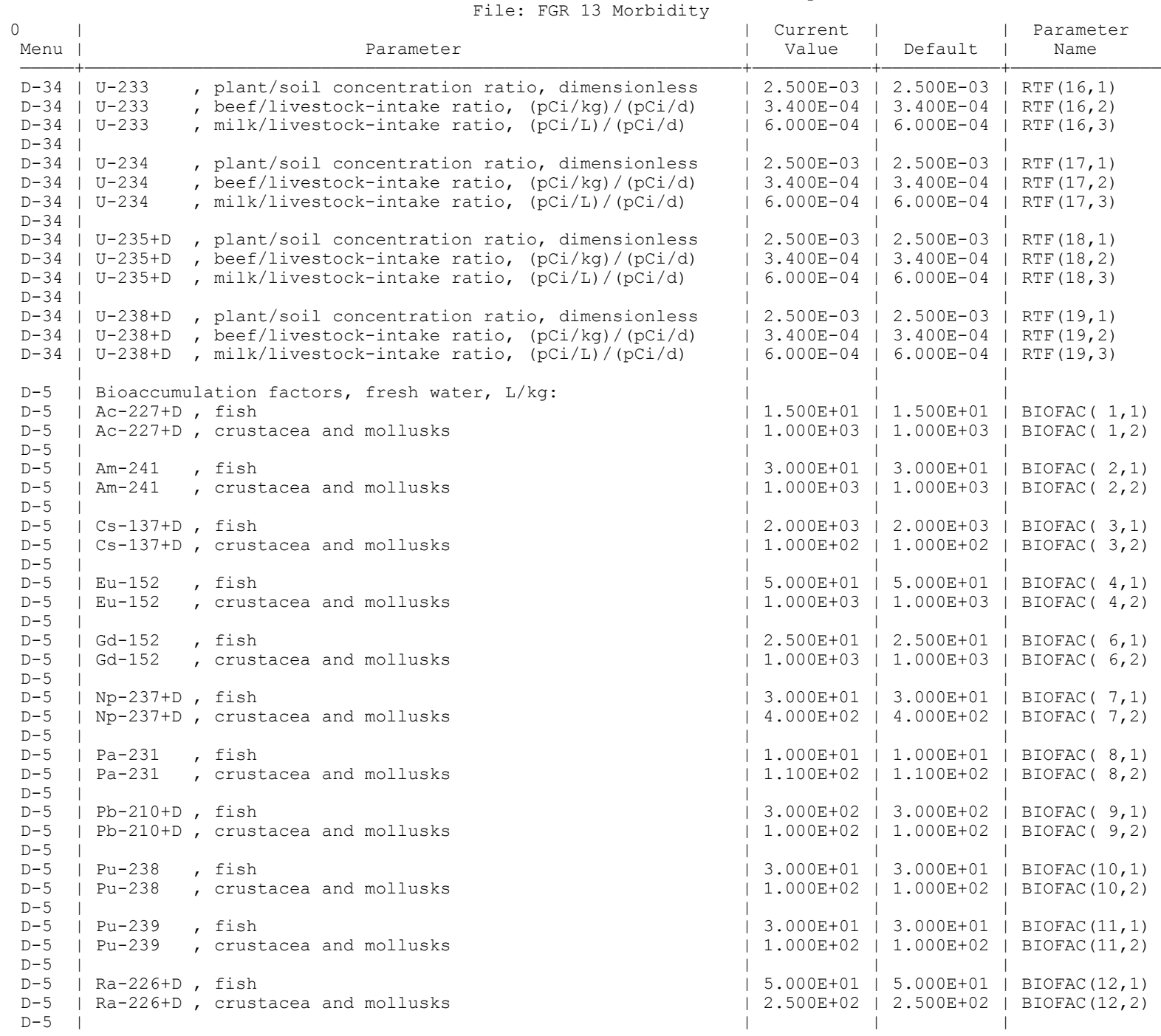


1RESRAD, Version $6.21 \quad T \frac{1}{2}$ Limit $=0.5$ year

Summary : CAU 224250 day-yr average worker scenario
File

02/22/2005 17:24 Page

Dose Conversion Factor (and Related) Parameter Summary (continued)

\begin{tabular}{|c|c|c|c|c|c|}
\hline Menu & । & Parameter & $\begin{array}{l}\text { Current } \\
\text { Value }\end{array}$ & I Default & $\begin{array}{c}\text { Parameter } \\
\text { Name }\end{array}$ \\
\hline D-5 & $S r-90+D$ & , fish & $6.000 \mathrm{E}+01$ & $6.000 \mathrm{E}+01$ & $\operatorname{BIOFAC}(13,1)$ \\
\hline $\begin{array}{l}D-5 \\
D-5\end{array}$ & $\mathrm{Sr}-90+\mathrm{D}$ & crustacea and mollusks & $1.000 \mathrm{E}+02$ & $1.000 \mathrm{E}+02$ & $\operatorname{BIOFAC}(13,2)$ \\
\hline$D-5$ & Th-229+D & , fish & $1.000 \mathrm{E}+02$ & $1.000 \mathrm{E}+02$ & $\operatorname{BIOFAC}(14,1)$ \\
\hline $\begin{array}{l}D-5 \\
D-5\end{array}$ & Th-229+D & , crustacea and mollusks & $5.000 \mathrm{E}+02$ & $5.000 \mathrm{E}+02$ & $\operatorname{BIOFAC}(14,2)$ \\
\hline$D-5$ & Th-230 & , fish & $1.000 \mathrm{E}+02$ & $1.000 \mathrm{E}+02$ & $\operatorname{BIOFAC}(15,1)$ \\
\hline $\begin{array}{l}D-5 \\
D-5\end{array}$ & $\operatorname{Th}-230$ & , crustacea and mollusks & $5.000 \mathrm{E}+02$ & $5.000 \mathrm{E}+02$ & $\operatorname{BIOFAC}(15,2)$ \\
\hline$D-5$ & U-233 & , fish & $1.000 \mathrm{E}+01$ & $1.000 \mathrm{E}+01$ & $\operatorname{BIOFAC}(16,1)$ \\
\hline $\begin{array}{l}D-5 \\
D-5\end{array}$ & $\mathrm{U}-233$ & crustacea and mollusks & $6.000 \mathrm{E}+01$ & $6.000 \mathrm{E}+01$ & $\operatorname{BIOFAC}(16,2)$ \\
\hline D-5 & U-234 & , fish & $1.000 \mathrm{E}+01$ & $1.000 \mathrm{E}+01$ & $\operatorname{BIOFAC}(17,1)$ \\
\hline $\begin{array}{l}D-5 \\
D-5\end{array}$ & $\mathrm{U}-234$ & crustacea and mollusks & $6.000 \mathrm{E}+01$ & $6.000 \mathrm{E}+01$ & $\operatorname{BIOFAC}(17,2)$ \\
\hline$D-5$ & $\mathrm{U}-235+\mathrm{D}$ & , fish & $1.000 \mathrm{E}+01$ & $1.000 \mathrm{E}+01$ & $\operatorname{BIOFAC}(18,1)$ \\
\hline $\begin{array}{l}D-5 \\
D-5\end{array}$ & $U-235+D$ & , crustacea and mollusks & $6.000 \mathrm{E}+01$ & $6.000 \mathrm{E}+01$ & $\operatorname{BIOFAC}(18,2)$ \\
\hline D-5 & $\mathrm{U}-238+\mathrm{D}$ & , fish & $1.000 \mathrm{E}+01$ & $1.000 \mathrm{E}+01$ & $\operatorname{BIOFAC}(19$, \\
\hline$D-5$ & $\mathrm{U}-238+\mathrm{D}$ & , crustacea and mollusks & $6.000 \mathrm{E}+01$ & $6.000 \mathrm{E}+01$ & $\operatorname{BIOFAC}(19,2)$ \\
\hline
\end{tabular}


1RESRAD, Version $6.21 \quad T \frac{1}{2}$ Limit $=0.5$ year

02/22/2005 17:24 Page Summary : CAU 224250 day-yr average work
File : CAU 224250 day-yr average. RAD

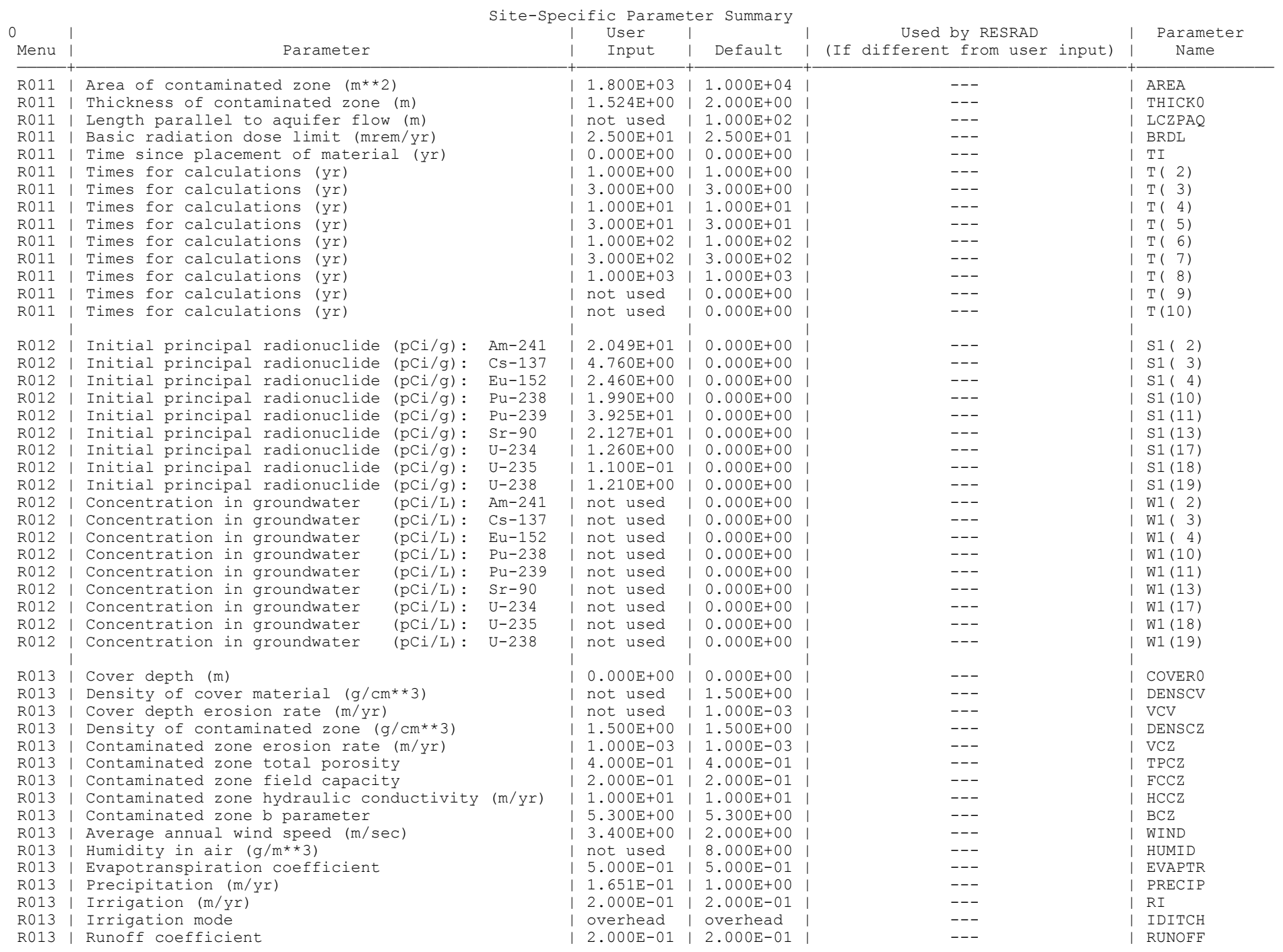


1RESRAD, Version $6.21 \quad$ T⿱13/2 Limit $=0.5$ year Summary : CAU 224250 day-yr average worker scenario

\section{Site-Specific Parameter summary (continued)}

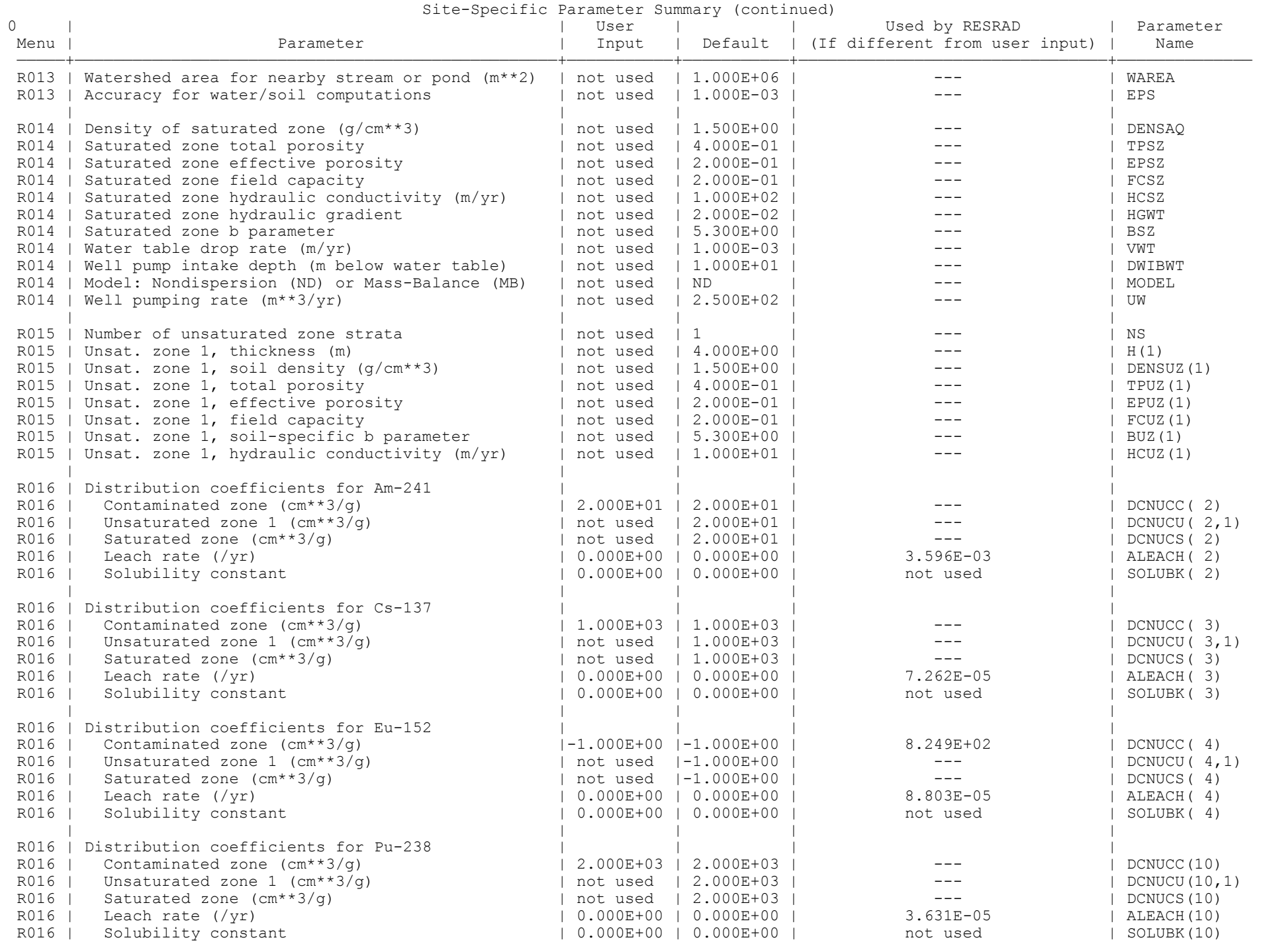


1RESRAD, Version $6.21 \quad T \frac{1}{2}$ Limit $=0.5$ year

02/22/2005 17:24 Page

$\begin{array}{lll}\text { Summary : CAU } 224 & 250 \text { day-yr average work } \\ \text { File } & \text { CAU } 224250 \text { day-yr average. RAD }\end{array}$

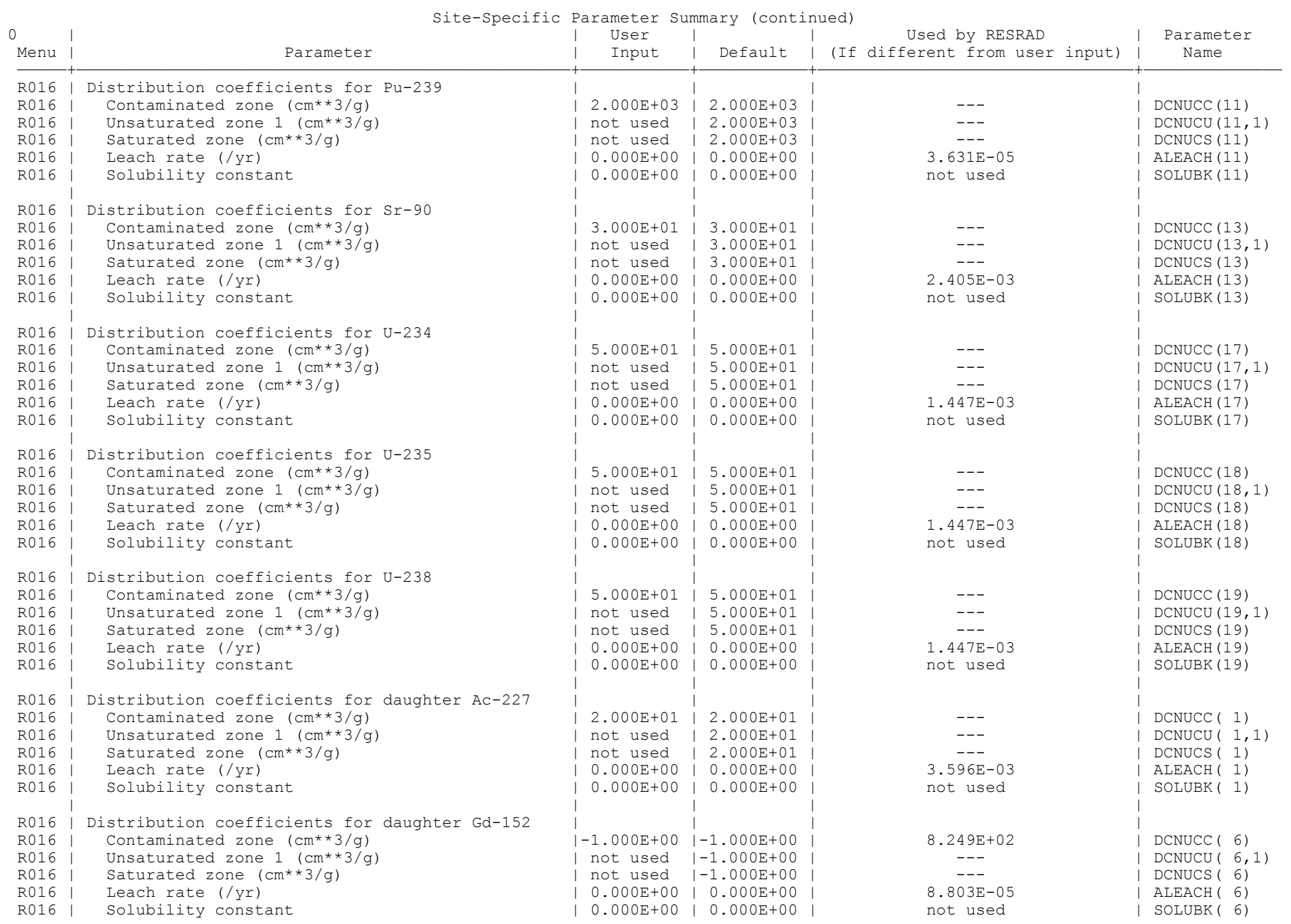




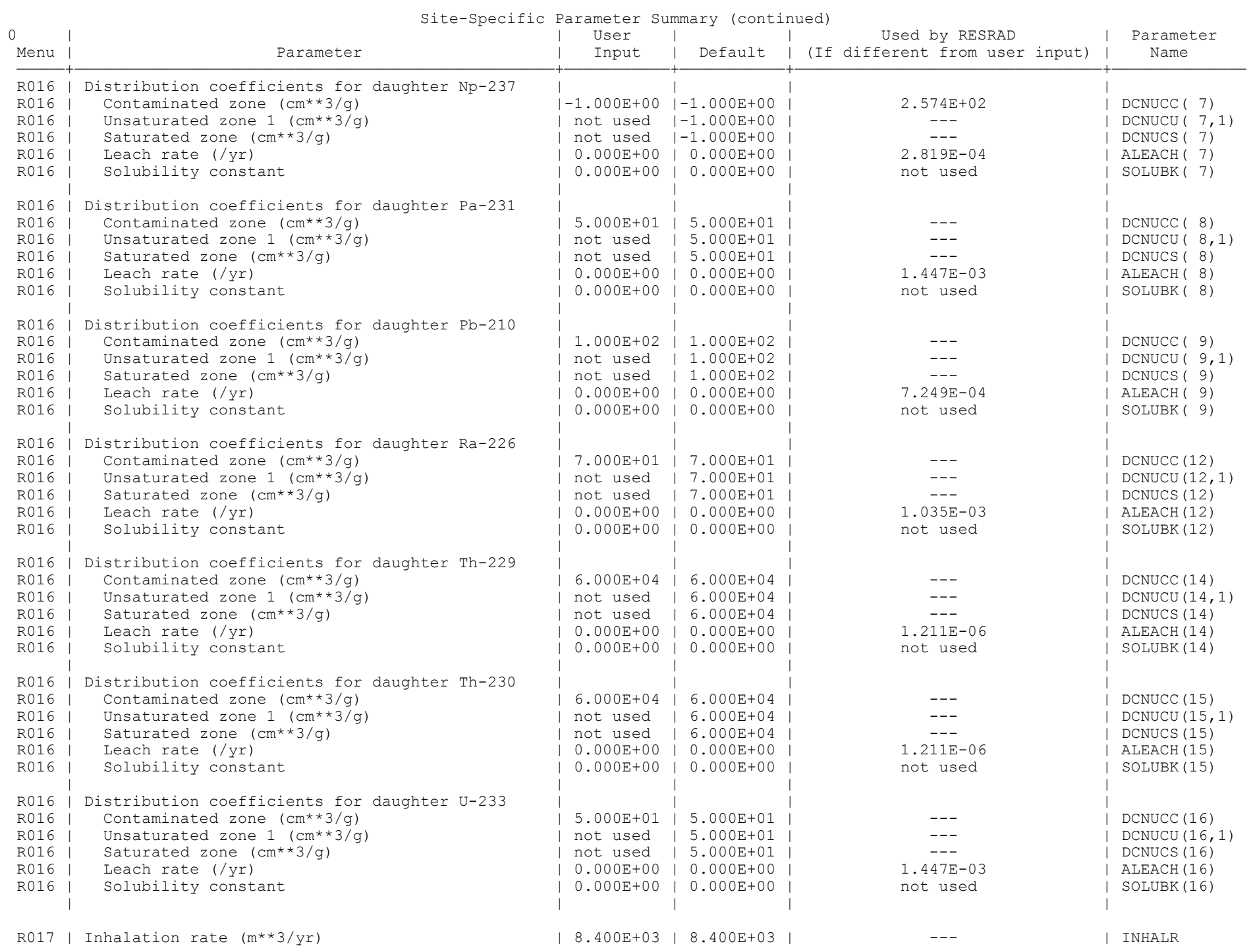


1RESRAD, Version $6.21 \quad T^{1 / 2}$ Limit $=0.5$ year Summary : CAU 224250 day-yr average worker scenario

File CAU 224-250 day-yr average.RAD

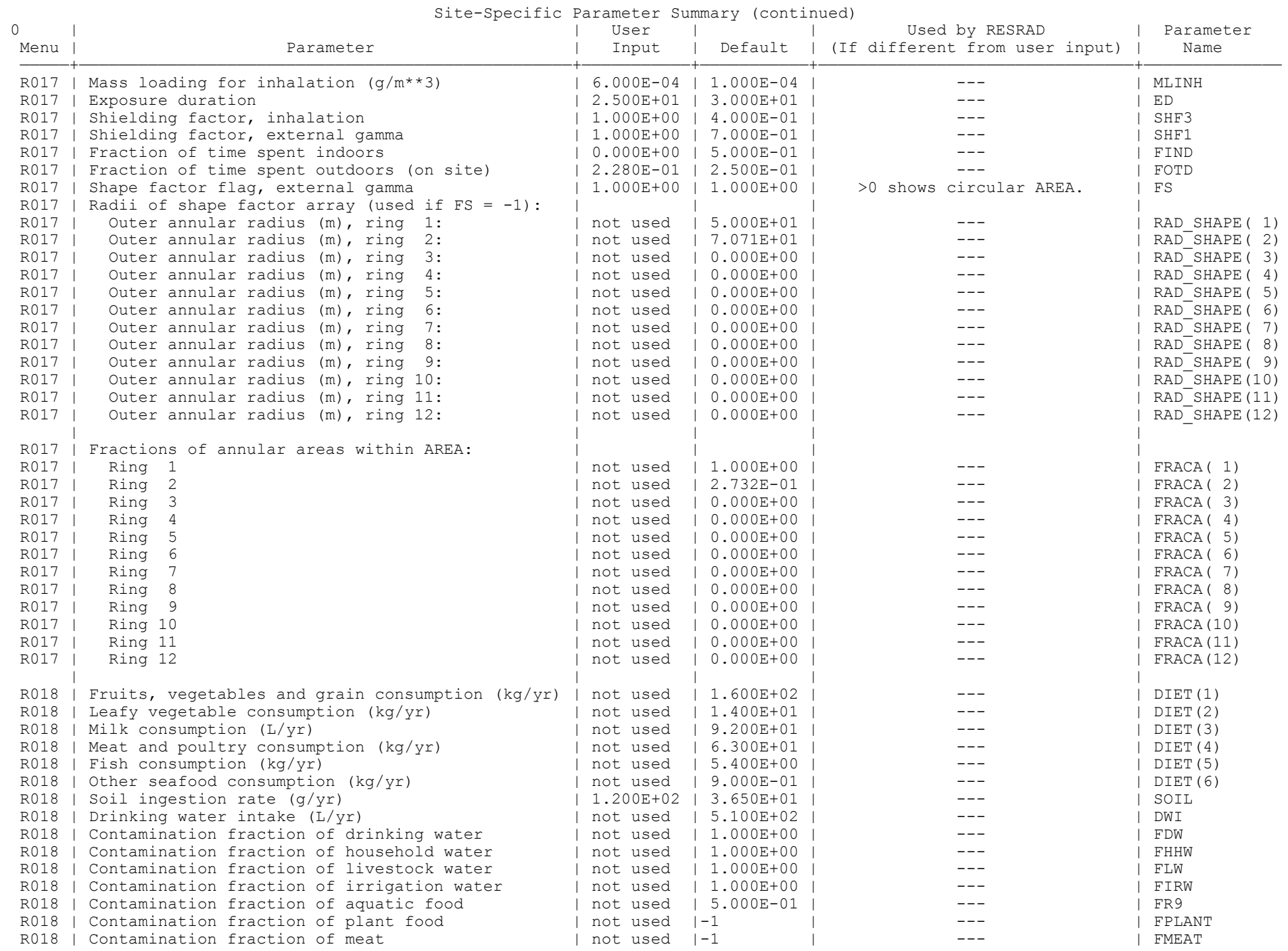


1RESRAD, Version $6.21 \quad T^{1 / 2}$ Limit $=0.5$ year Summary : CAU 224250 day-yr average worker scenario

Site-Specific Parameter Summary (continued)

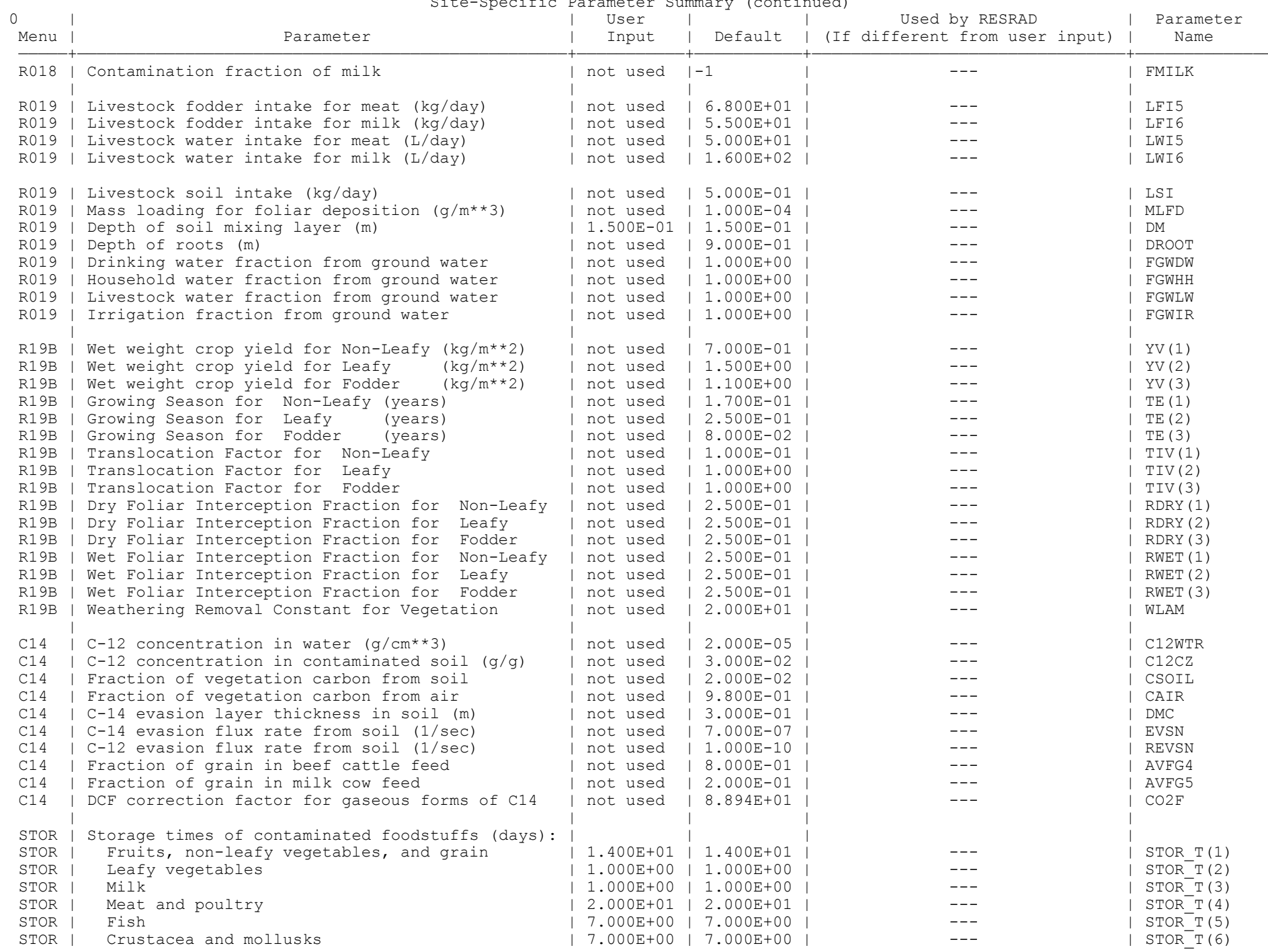


1RESRAD, Version $6.21 \quad T^{\frac{1}{2}}$ Limit $=0.5$ year

02/22/2005 17:24 Page 12 Summary : CAU 224250 day-yr average worker
File : CAU 224250 day-yr average. RAD

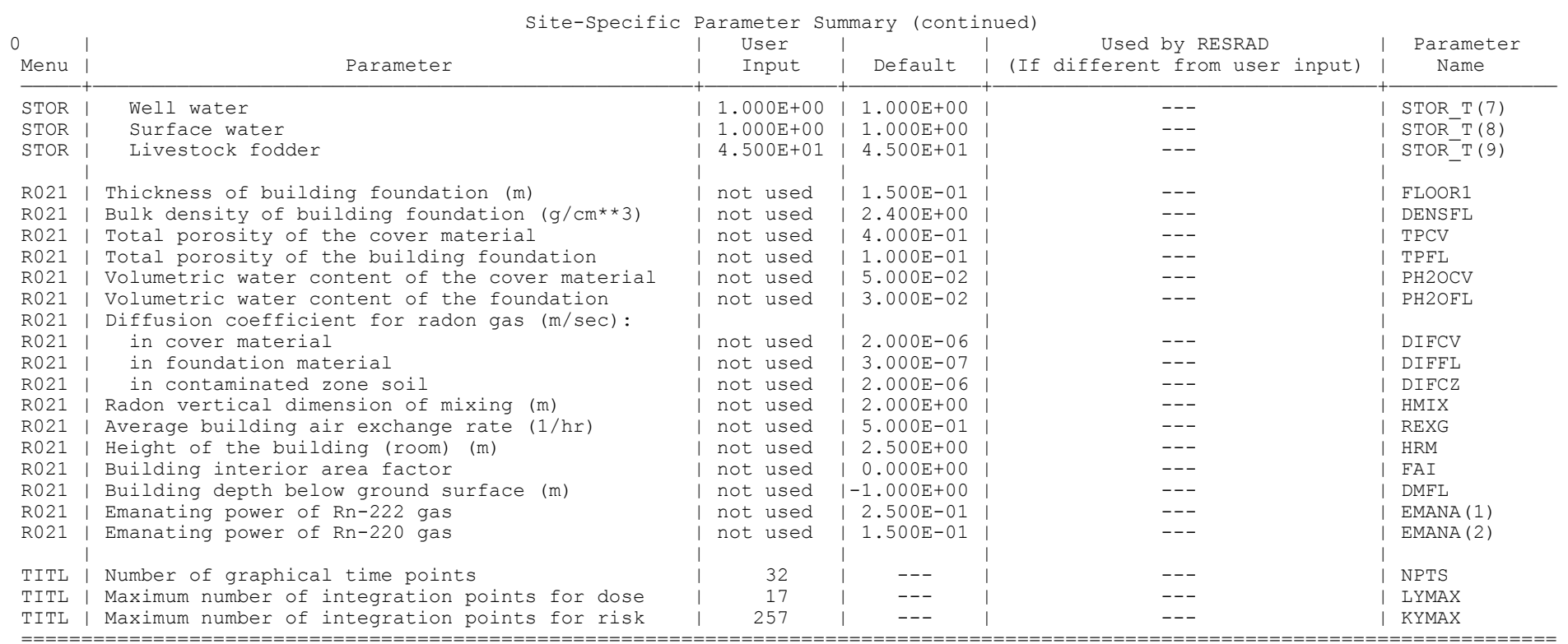

Summary of Pathway Selections

\begin{tabular}{|c|c|}
\hline Pathway & User Selectior \\
\hline 1 -- external gamma & active \\
\hline 2 -- inhalation (w/o radon) & active \\
\hline 3 -- plant ingestion & suppressed \\
\hline 4 -- meat ingestion & suppressed \\
\hline 5 -- milk ingestion & suppressed \\
\hline 6 -- aquatic foods & suppressed \\
\hline 7 -- drinking water & suppressed \\
\hline 8 -- soil ingestion & active \\
\hline 9 -- radon & suppressed \\
\hline Find peak pathway doses & active \\
\hline
\end{tabular}


1RESRAD, Version $6.21 \quad$ Th/2 Limit $=0.5$ year

Summary : CAU 224250 day-yr average worker scenario
File : CAU 224250 day-yr average. RAD

02/22/2005 17:24 Page 13

Contaminated Zone Dimensions

Area: 1800.00 square meters

Thickness: $\quad 1.52$ meters

\begin{tabular}{cc} 
Initial Soil Concentrations, $\mathrm{pCi} / \mathrm{g}$ \\
\hline $\mathrm{Am}-241$ & $2.049 \mathrm{E}+01$ \\
$\mathrm{Cs}-137$ & $4.760 \mathrm{E}+00$ \\
$\mathrm{Eu}-152$ & $2.460 \mathrm{E}+00$ \\
$\mathrm{Pu}-238$ & $1.990 \mathrm{E}+00$ \\
$\mathrm{Pu}-239$ & $3.925 \mathrm{E}+01$ \\
$\mathrm{Sr}-90$ & $2.127 \mathrm{E}+01$ \\
$\mathrm{U}-234$ & $1.260 \mathrm{E}+00$ \\
$\mathrm{U}-235$ & $1.100 \mathrm{E}-01$ \\
$\mathrm{U}-238$ & $1.210 \mathrm{E}+00$
\end{tabular}

Total Dose TDOSE (t), mrem/yr

Basic Radiation Dose Limit $=2.500 \mathrm{E}+01 \mathrm{mrem} / \mathrm{yr}$

Total Mixture Sum $M(t)=$ Fraction of Basic Dose Limit Received at Time (t)

$t$ (years): $\begin{array}{llllllll}0.000 \mathrm{E}+00 & 1.000 \mathrm{E}+00 & 3.000 \mathrm{E}+00 & 1.000 \mathrm{E}+01 & 3.000 \mathrm{E}+01 & 1.000 \mathrm{E}+02 & 3.000 \mathrm{E}+02 & 1.000 \mathrm{E}+03\end{array}$

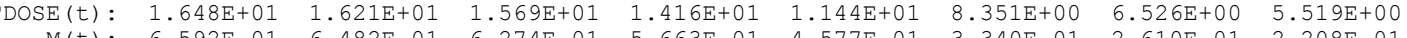

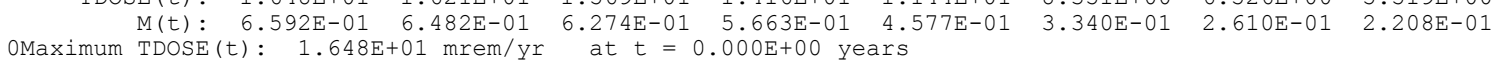


$\mathrm{T} \frac{1}{2}$ Limit $=0.5$ year

02/22/2005 17:24 Page 14

224250 day-yr average worker scenario

Total Dose Contributions TDOSE (i,p,t) for Individual Radionuclides (i) and Pathways (p)

As mrem/yr and Fraction of Total Dose At $t=0.000 \mathrm{E}+00$ years

\begin{tabular}{|c|c|c|c|c|c|c|c|c|c|c|c|c|c|c|}
\hline \multirow{2}{*}{$\begin{array}{l}\text { Radio- } \\
\text { Nuclide }\end{array}$} & \multicolumn{2}{|c|}{ Ground } & \multicolumn{2}{|c|}{ Inhalation } & \multicolumn{2}{|c|}{$\begin{array}{l}r \text { Independent } \\
\text { Radon }\end{array}$} & \multicolumn{2}{|c|}{$\begin{array}{l}\text { (Inhalation } \\
\text { Plant }\end{array}$} & \multicolumn{2}{|c|}{$\begin{array}{c}\text { xcludes radon) } \\
\text { Meat }\end{array}$} & \multicolumn{2}{|c|}{ Milk } & \multicolumn{2}{|l|}{ Soil } \\
\hline & $\mathrm{mrem} / \mathrm{yr}$ & fract. & mrem/yr & fract. & $\mathrm{mrem} / \mathrm{yr}$ & fract. & $\mathrm{mrem} / \mathrm{yr}$ & fract. & mrem/yr & fract. & $\mathrm{mrem} / \mathrm{yr}$ & fract. & mrem/yr & frac \\
\hline & & & & & & & & & & & & & & \\
\hline & & & & & & & & & & & & & & \\
\hline & & & & & & & & & & & & & & \\
\hline & & & & & & & & & & & & & & \\
\hline & & & & & & & & & & & & & & \\
\hline & & & & & & & & & & & & & & \\
\hline & & & & & & & & & & & & & & \\
\hline & & & & & & & & & & & & & & \\
\hline (0) & & & & & & & & & & & & & & \\
\hline Jat & $91 E+00$ & 0.4302 & $266 \mathrm{E}+00$ & 1982 & $000 \mathrm{E}+00$ & 0.0000 & $0.000 \mathrm{E}+00$ & 0000 & $.000 \mathrm{E}+00$ & 0.0000 & $.000 \mathrm{E}+00$ & 0.0000 & $6.124 \mathrm{E}+00$ & 0.3716 \\
\hline
\end{tabular}

Total Dose Contributions TDOSE $(i, p, t)$ for Individual Radionuclides (i) and Pathways (p) As mrem/yr and Fraction of Total Dose At $t=0.000 \mathrm{E}+00$ years

Fish

Radon

Plant

Meat

Milk

mrem/yr fract.

$0.000 \mathrm{E}+00 \quad 0.0000$ $0.000 \mathrm{E}+00 \quad 0.0000$ $0.000 \mathrm{E}+00 \quad 0.0000$ $0.000 \mathrm{E}+000.000$ $0.000 \mathrm{E}+00 \quad 0.0000$ $0.000 \mathrm{E}+000.0000$

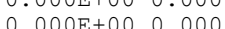
$0.000 \mathrm{E}+00 \quad 0.0000$ $\begin{array}{ll}1.993 \mathrm{E}-02 & 0.0012 \\ 6.414 \mathrm{E}-02 & 0.0039\end{array}$ $\begin{array}{llll}0.000 \mathrm{E}+00 & 0.0000 & 1.648 \mathrm{E}+01 & 1.0000\end{array}$
All Pathways* mrem/yr fract. $3.286 \mathrm{E}+00 \quad 0.1994$ $\begin{array}{lll}3.458 \mathrm{E}+00 & 0.2098 \\ 2.675 \mathrm{E}-01 & 0.0162\end{array}$ $\begin{array}{lll}2.675 \mathrm{E}-01 & 0.0162 \\ 5.838 \mathrm{E}+00 & 0.3542\end{array}$ $\begin{array}{lll}1.975 \mathrm{E}-01 & 0.0120\end{array}$ $.993 \mathrm{E}-02 \quad 0.0018$ $3.321 \mathrm{E}+00 \quad 0.2015$

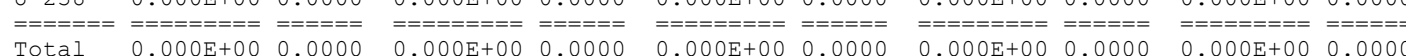

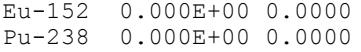

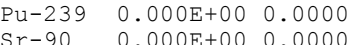

$\begin{array}{lll}-234 & 0.000 \mathrm{E}+00 & 0.0000\end{array}$

* Sum of all water independent and dependent pathways. 
1RESRAD, Version $6.21 \quad$ T3/2 Limit $=0.5$ year

02/22/2005 17:24 Page 15

$\begin{array}{lll}\text { Summary : CAU } 224 & 250 \text { day-yr average worker } \\ \text { File : CAU } 224250 \text { day-yr average. RAD }\end{array}$

Total Dose Contributions TDOSE (i,p,t) for Individual Radionuclides (i) and Pathways (p)

As mrem/yr and Fraction of Total Dose At $t=1.000 \mathrm{E}+00$ years

0

\begin{tabular}{|c|c|c|c|c|c|c|c|c|c|c|c|c|c|c|}
\hline \multirow{2}{*}{$\begin{array}{l}\text { Radio- } \\
\text { Nuclide }\end{array}$} & \multicolumn{2}{|c|}{ Ground } & \multicolumn{2}{|c|}{ Inhalation } & \multicolumn{2}{|c|}{$\begin{array}{l}\text { Radon } \\
\text { Radent }\end{array}$} & \multicolumn{2}{|c|}{$\begin{array}{l}\text { Mnala } \\
\text { Plant }\end{array}$} & \multicolumn{2}{|c|}{$\begin{array}{l}\text { radon) } \\
\text { Meat }\end{array}$} & \multicolumn{2}{|c|}{ Milk } & \multicolumn{2}{|c|}{ Soil } \\
\hline & em/yr & ict & mrem/yr & ract & mrem/yr & fract & $\mathrm{mrem} / \mathrm{yr}$ & frac & mrem/yr & frac & mren & fract & mrem, & frac \\
\hline & & & & & & & & & & & & & & \\
\hline & & & & & & & & & & & & & & \\
\hline & & & & & & & & & & & & & & \\
\hline & & & & & & & & & & & & & & \\
\hline & & & & & & & & & & & & & & \\
\hline & & & & & & & & & & & & & & \\
\hline & & & & & & & & & & & & & & \\
\hline & & & & & & & & & & & & & & \\
\hline 250 & & 023 & -02 & & & & & & & & & & & \\
\hline & .00 & 218 & $259 \mathrm{E}+00$ & 11 & +00 & 000 & $E+00$ & 000 & $0 \mathrm{E}+00$ & 000 & $0 \mathrm{E}+00$ & 000 & $10 \mathrm{E}+00$ & 0.3770 \\
\hline
\end{tabular}

Total Dose Contributions TDOSE (i,p,t) for Individual Radionuclides (i) and Pathways (p) As mrem/yr and Fraction of Total Dose At $t=1.000 \mathrm{E}+00$ years Radio- $\frac{\text { Water }}{\text { mrem/yr fract }}$ Fish Radon Plant

Meat

mrem/yr fract.

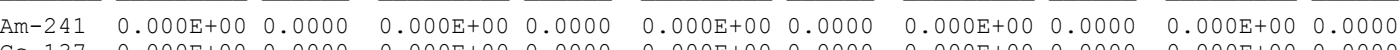
Cs-137 $0.000 \mathrm{E}+00 \quad 0.0000$

$\begin{array}{lll}\mathrm{Eu}-152 & 0.000 \mathrm{E}+00 & 0.0000 \\ \mathrm{Pu}-238 & 0.0000 \mathrm{n}+00 & 0.0000\end{array}$

$\begin{array}{lll}\mathrm{Pu}-238 & 0.000 \mathrm{E}+00 & 0.0000 \\ \mathrm{Pu}-239 & 0.000 \mathrm{E}+00 & 0.0000 \\ \mathrm{Sr}-90 & 0.000 \mathrm{C}+00 & 0.0000\end{array}$

$\begin{array}{lll}\mathrm{Su}-230 & 0.000 \mathrm{E}+00 \quad 0.0000\end{array}$

$\mathrm{U}-234 \quad 0.000 \mathrm{E}+00 \quad 0.0000$

$\mathrm{U}-235 \quad 0.000 \mathrm{E}+00 \quad 0.0000$

$\mathrm{U}-238 \quad 0.000 \mathrm{E}+00 \quad 0.0000$

$===========$

$0.000 \mathrm{E}+00 \quad 0.0000$
0 $\begin{array}{ll}0.000 \mathrm{E}+00 & 0.0000 \\ 0.000 \mathrm{E}+00 & 0.0000\end{array}$ $\begin{array}{lll}0.000 \mathrm{E}+00 & 0.0000 \\ 0.000 \mathrm{E}+00 & 0.0000 \\ 0.000 \mathrm{E}+00 & 0.0000\end{array}$ $\begin{array}{ll}0.000 \mathrm{E}+00 & 0.0000 \\ 0.000 \mathrm{E}+00 & 0.0000 \\ 0.0000 \mathrm{E}+00 & 0.0000\end{array}$ $\begin{array}{lll}0.000 \mathrm{E}+00 & 0.0000 \\ 0.000 \mathrm{E}+00 & 0.0000\end{array}$ $\begin{array}{llll}0.000 \mathrm{E}+00 & 0.0000 & 0.000 \mathrm{E}+00 & 0.0000\end{array}$ $\begin{array}{llll}0.000 \mathrm{E}+00 & 0.0000 & 0.000 \mathrm{E}+00 & 0.0000 \\ 0.000 \mathrm{E}+00 & 0.0000 & 0.000 \mathrm{E}+00 & 0.0000\end{array}$ $\begin{array}{llll}0.000 \mathrm{E}+00 & 0.0000 & 0.000 \mathrm{E}+00 & 0.0000 \\ 0.000 \mathrm{E}+00 & 0.0000 & 0.000 \mathrm{E}+00 & 0.0000 \\ 0.0000 \mathrm{E}+00 & 0.0000 & 0.000 \mathrm{E}+00 & 0.0000\end{array}$ $0.000 \mathrm{E}+00 \quad 0.0000$ $0.000 \mathrm{E}+00 \quad 0.0000$ Milk mrem/yr fract. $0.000 \mathrm{E}+00 \quad 0.0000$ $0.000 \mathrm{E}+00 \quad 0.0000$ $0.000 \mathrm{E}+00 \quad 0.0000$ $0.000 \mathrm{E}+00 \quad 0.000$ $\begin{array}{lll}0.000 \mathrm{E}+00 & 0.0000 \\ 0.000 \mathrm{E}+00 & 0.0000\end{array}$

$0.000 \mathrm{E}+00 \quad 0.0000$ $\begin{array}{llllll}0.000 \mathrm{E}+00 & 0.0000 & 0.000 \mathrm{E}+00 & 0.0000 & 0.000 \mathrm{E}+00 & 0.0000\end{array}$ $0.000 \mathrm{E}+00$

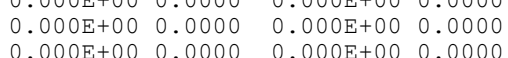
$0.000 \mathrm{E}+00 \quad 0.0000$ $.000 \mathrm{E}+00 \quad 0.0000$ $0.000 \mathrm{E}+00 \quad 0.0000$ $\begin{array}{ll}6.405 \mathrm{E}-02 & 0.0040\end{array}$ $\begin{array}{llll}0.000 \mathrm{E}+00 & 0.0000 & 1.621 \mathrm{E}+01 & 1.0000\end{array}$

$0 *$ Sum of all water independent and dependent pathways. 
$\mathrm{T} 1 / 2$ Limit $=0.5$ year

250 day-yr average worker scenario

02/22/2005 17:24 Page 16

File : CAU 224250 day-yr average.RAD

Total Dose Contributions TDOSE (i,p,t) for Individual Radionuclides (i) and Pathways (p)

As mrem/yr and Fraction of Total Dose At $t=3.000 \mathrm{E}+00$ years

0

\begin{tabular}{|c|c|c|c|c|c|c|c|c|c|c|c|c|c|c|}
\hline \multirow{2}{*}{$\begin{array}{l}\text { Radio- } \\
\text { Nuclide }\end{array}$} & \multicolumn{2}{|c|}{ Ground } & \multicolumn{2}{|c|}{ Inhalation } & \multicolumn{2}{|l|}{ Rac } & \multicolumn{2}{|c|}{$\begin{array}{l}\text { Inhalat } \\
\text { Plant }\end{array}$} & \multicolumn{2}{|c|}{$\begin{array}{l}\text { S radon) } \\
\text { Meat }\end{array}$} & \multicolumn{2}{|c|}{ Milk } & \multicolumn{2}{|c|}{ Soil } \\
\hline & $\mathrm{mrem} / \mathrm{yr}$ & fract. & mrem/yr & fract. & $\mathrm{mrem} / \mathrm{yr}$ & fract. & mrem/yr & fract. & mrem/yr & fract. & mrem/yr & fract. & mrem/yr & fract. \\
\hline 1 & 1 & 0 & 0 & 38 & 0 & 0.0000 & 0 & 0 & 0 & 0 & 00 & 0 & 0 & 0 \\
\hline & $E+00$ & & $591 \mathrm{E}-05$ & 000 & 0 & 0 & & & & & $E+00$ & & -03 & \\
\hline & & 8 & $473 \mathrm{E}-05$ & 0.000 & 00 & 0 & & & & & & & & \\
\hline & $6.504 \mathrm{E}-05$ & 0.0000 & $.164 \mathrm{E}-02$ & 0.0058 & $.000 \mathrm{E}+00$ & 0000 & $\mathrm{E}+00$ & 0 & $E+00$ & 0 & $0 \mathrm{E}+00$ & 0 & 01 & 108 \\
\hline Pu-239 & $2.464 \mathrm{E}-03$ & 0.0002 & $.033 \mathrm{E}+00$ & 0.1296 & $0.000 \mathrm{E}+00$ & 0.0000 & $0.000 \mathrm{E}+00$ & 0.0000 & $0.000 \mathrm{E}+00$ & 0.0000 & $0.000 \mathrm{E}+00$ & 0.0000 & $3.801 \mathrm{E}+00$ & 0.2423 \\
\hline $\mathrm{Sr}-$ & $9.826 \mathrm{E}-02$ & 0.0063 & $.071 \mathrm{E}-03$ & 0.0002 & 0.00 & 0. & 0.00 & 0. & 0.0 & 0. & $10 E+00$ & 0 & -02 & 0 \\
\hline & $1.077 \mathrm{E}-04$ & 0.0000 & $.999 \mathrm{E}-02$ & 0.0013 & $0.000 \mathrm{E}+00$ & 0.0000 & $0.000 \mathrm{E}$ & 0.0 & 0.00 & 0.0 & $0.000 \mathrm{E}+00$ & 0.0 & -03 & 0.0 \\
\hline & & 0.0011 & $.628 \mathrm{E}-03$ & 0.0001 & $.000 \mathrm{E}-$ & 0.0000 & $0.000 \mathrm{E}$ & 0.0 & & & $0.000 \mathrm{E}+00$ & 0.0 & & 0.0 \\
\hline U-238 & $3.784 \mathrm{E}-02$ & 0.0024 & $1.716 \mathrm{E}-02$ & 0.0011 & $0.000 \mathrm{E}+00$ & 0.0000 & $0.000 \mathrm{E}+00$ & 0.0000 & $0.000 \mathrm{E}+00$ & 0.0000 & $0.000 \mathrm{E}+00$ & 0.0000 & $8.861 \mathrm{E}-03$ & 0.0006 \\
\hline$====$ & $====$ & $====$ & & & & & & & & & & & & \\
\hline Total & $6.359 \mathrm{E}+00$ & 0.4054 & $3.246 \mathrm{E}+00$ & 0.2069 & $0.000 \mathrm{E}+00$ & 0.0000 & $0.000 \mathrm{E}+00$ & 0.0000 & $0.000 \mathrm{E}+00$ & 0.0000 & $0.000 \mathrm{E}+00$ & 0.0000 & $6.081 \mathrm{E}+00$ & 0.3877 \\
\hline
\end{tabular}

Total Dose Contributions TDOSE (i,p,t) for Individual Radionuclides (i) and Pathways (p) As mrem/yr and Fraction of Total Dose At $t=3.000 \mathrm{E}+00$ years

0 Radio-
Nuclide Fish Radon Plant $\frac{\text { Milk }}{\text { mrem/yr fract. }}$

\section{$\frac{\mathrm{Am}-241}{0.000 \mathrm{E}+00} \frac{\text { ract. }}{0.0000}$}

mrem/yr fract. mrem/yr fract.

mrem/yr fract.

mrem/yr fract. All Pathways* $\begin{array}{lll}\mathrm{Cs}-137 & 0.000 \mathrm{E}+00 & 0.0000 \\ \mathrm{Eu}-152 & 0.000 \mathrm{E}+00 & 0.0000\end{array}$ $0.000 \mathrm{E}+00 \quad 0.0000$ $\begin{array}{lllll}0.000 \mathrm{E}+00 & 0.0000 & 0.000 \mathrm{E}+00 & 0.0000\end{array}$ $\begin{array}{llll}0.000 \mathrm{E}+00 & 0.0000 & 0.000 \mathrm{E}+00 & 0.0000 \\ 0.000 \mathrm{E}+00 & 0.0000 & 0.000 \mathrm{c}+00 & 0.0000\end{array}$ $\begin{array}{lllllllll}0.0000 & 0.000 \mathrm{E}+00 & 0.0000 & 0.000 \mathrm{E}+00 & 0.0000 & 0.000 \mathrm{E}+00 & 0.0000 & 0.000 \mathrm{E}+00 & 0.0000 \\ 0.000 \mathrm{E}+00 & 0.0000 & 0.000 \mathrm{E}+00 & 0.0000\end{array}$ $\begin{array}{lllllllllll}\mathrm{Pu}-238 & 0.000 \mathrm{E}+00 & 0.0000 & 0.000 \mathrm{E}+00 & 0.0000 & 0.000 \mathrm{E}+00 & 0.0000 & 0.000 \mathrm{E}+00 & 0.0000 & 0.000 \mathrm{E}+00 & 0.0000\end{array}$ $\begin{array}{lll}\text { Pu-239 } & 0.000 \mathrm{E}+00 \quad 0.0000\end{array}$ $\begin{array}{lll}\mathrm{Sr}-90 & 0.000 \mathrm{E}+00 & 0.0000\end{array}$ $\begin{array}{llll}0.000 \mathrm{E}+00 & 0.0000 & 0.000 \mathrm{E}+00 & 0.000\end{array}$ $0.000 \mathrm{E}+00 \quad 0.0000$ $0.000 \mathrm{E}+00 \quad 0.0000$ $\begin{array}{lll}\mathrm{U}-234 & 0.000 \mathrm{E}+00 & 0.0000 \\ \mathrm{U}-235 & 0.000 \mathrm{E}+00 & 0.0000\end{array}$

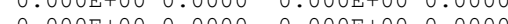

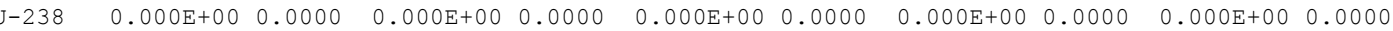

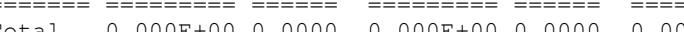
$0.000 \mathrm{E}+000.0000$ $0.000 \mathrm{E}+00 \quad 0.000$ $0.000 \mathrm{E}+00 \quad 0.0000$ $0.000 \mathrm{E}+00 \quad 0.0000$ $0.000 \mathrm{E}+00 \quad 0.0000$

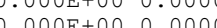
$0.000 \mathrm{E}+00 \quad 0.0000$ $0.000 \mathrm{E}+00 \quad 0.0000$ $0 *$ Sum of all water independent and dependent pathways. 
$\mathrm{T} 1 / 2$ Limit $=0.5$ year

250 day-yr average worker scenario

02/22/2005 17:24 Page 17

File : CAU 224250 day-yr average.RAD

Total Dose Contributions TDOSE (i,p,t) for Individual Radionuclides (i) and Pathways (p)

As mrem/yr and Fraction of Total Dose At $t=1.000 \mathrm{E}+01$ years

0

\begin{tabular}{|c|c|c|c|c|c|c|c|c|c|c|c|c|c|c|}
\hline \multirow{2}{*}{$\begin{array}{l}\text { Radio- } \\
\text { Nuclide }\end{array}$} & \multicolumn{2}{|c|}{ Ground } & \multicolumn{2}{|c|}{ Inhalation } & \multicolumn{2}{|c|}{$\begin{array}{l}r \text { Independent } \\
\text { Radon }\end{array}$} & \multicolumn{2}{|c|}{$\begin{array}{l}\text { (Inhalation } \\
\text { Plant }\end{array}$} & \multicolumn{2}{|c|}{$\begin{array}{c}\text { xcludes radon) } \\
\text { Meat }\end{array}$} & \multicolumn{2}{|c|}{ Milk } & \multicolumn{2}{|l|}{ Soil } \\
\hline & $\mathrm{mrem} / \mathrm{yr}$ & fract. & mrem/yr & fract. & $\mathrm{mrem} / \mathrm{yr}$ & fract. & $\mathrm{mrem} / \mathrm{yr}$ & fract. & mrem/yr & fract. & $\mathrm{mrem} / \mathrm{yr}$ & fract. & $\mathrm{mrem} / \mathrm{yr}$ & frac \\
\hline & & & & & & & & & & & & & & \\
\hline & & & & & & & & & & & & & & \\
\hline & & & & & & & & & & & & & & \\
\hline & & & & & & & & & & & & & & \\
\hline & & & & & & & & & & & & & & \\
\hline & & & & & & & & & & & & & & \\
\hline & & & & & & & & & & & & & & \\
\hline & & & & & & & & & & & & & & \\
\hline the & & & & & & & & & & & & & & \\
\hline Jat & $74 \mathrm{E}+00$ & 3513 & $201 E+00$ & 2261 & $000 \mathrm{E}+00$ & 0.0000 & $0.000 \mathrm{E}+00$ & 0.0000 & $.000 \mathrm{E}+00$ & 0.0000 & $0.000 \mathrm{E}+00$ & 0.0000 & $5.984 \mathrm{E}+00$ & 0.4226 \\
\hline
\end{tabular}

Total Dose Contributions TDOSE (i,p,t) for Individual Radionuclides (i) and Pathways (p) As mrem/yr and Fraction of Total Dose At $t=1.000 \mathrm{E}+01$ years

0 Radio- $\frac{\text { Water }}{\text { Nuclide }}$ Fish Radon Plant mrem/yr fract. mrem/yr fract. mrem/yr fract. $0.000 \mathrm{E}+00 \quad 0.0000$ $0.000 \mathrm{E}+00 \quad 0.0000$ $\begin{array}{llllllllllll}\mathrm{Am}-241 & 0.000 \mathrm{E}+00 & 0.0000 & 0.000\end{array}$ $\begin{array}{lll}C s-137 & 0.000 E+00 & 0.0000 \\ \text { Eu-152 } & 0.000 E+00 & 0.0000\end{array}$ $\begin{array}{lll}\mathrm{Eu}-152 & 0.000 \mathrm{E}+00 & 0.0000 \\ \mathrm{Pu}-238 & 0.000 \mathrm{E}+00 & 0.0000\end{array}$ $\begin{array}{lll}\mathrm{Pu}-239 & 0.000 \mathrm{E}+00 & 0.0000\end{array}$ $\begin{array}{lll}\mathrm{Sr}-90 & 0.000 \mathrm{E}+00 & 0.0000 \\ \mathrm{U}-234 & 0.000 \mathrm{E}+00 & 0.0000\end{array}$ $\begin{array}{lll}\mathrm{U}-234 & 0.000 \mathrm{E}+00 & 0.0000 \\ \mathrm{U}-235 & 0.000 \mathrm{E}+00 & 0.0000\end{array}$ $0.000 \mathrm{E}+00 \quad 0.0000$ $\begin{array}{lll}0.000 \mathrm{E}+00 & 0.0000 \\ 0.000 & 0 & 0 \\ 0.000 & 0.0000\end{array}$ $\begin{array}{llll}0.000 \mathrm{E}+00 & 0.0000 & 0.000 \mathrm{E}+00 & 0.0000 \\ 0.000 \mathrm{E}+00 & 0.0000 & 0.000 \mathrm{E}+00 & 0.0000\end{array}$ $\begin{array}{lllll}0.000 \mathrm{E}+00 & 0.0000 & 0.000 \mathrm{E}+00 & 0.0000\end{array}$ $0.000 \mathrm{E}+00 \quad 0.0000$ $\begin{array}{llll}0.000 \mathrm{E}+00 & 0.0000 & 0.000 \mathrm{E}+00 & 0.0000\end{array}$

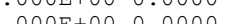
$\begin{array}{lll}0.000 \mathrm{E}+00 & 0.0000 \\ 0.000 \mathrm{E}+00 & 0.0000\end{array}$ $0.000 \mathrm{E}+00 \quad 0.0000$ $\mathrm{J}-238 \quad 0.000 \mathrm{E}+00 \quad 0.0000$ $0.000 \mathrm{E}+00 \quad 0.0000$ $0.000 \mathrm{E}+00 \quad 0.0000$

$0.000 \mathrm{E}+000.0000$ 0.000E+00 $0.00000 .000 \mathrm{E}+000.0000$ $0.000 \mathrm{E}+00 \quad 0.0000$

$0 *$ Sum of all water independent and dependent pathways.
Meat

Milk

mrem/yr fract. mrem/yr fract. $\begin{array}{lll}3.152 \mathrm{E}+00 & 0.2227\end{array}$ $2.606 \mathrm{E}+000.1841$ $2.054 \mathrm{E}+00 \quad 0.1451$ $\begin{array}{ll}2.471 \mathrm{E}-01 & 0.0174 \\ 5.834 \mathrm{E}+00 & 0.4121\end{array}$ $1.520 \mathrm{E}-010.0107$ $\begin{array}{lll}1.520 \mathrm{E}-01 & 0.0107 \\ 2.951 \mathrm{E}-02 & 0.0021\end{array}$ $1.966 \mathrm{E}-02 \quad 0.0014$ $\begin{array}{ll}6.322 \mathrm{E}-02 & 0.0045\end{array}$ $1.416 \mathrm{E}+01$ 1. 0000 
$\mathrm{T} 1 / 2$ Limit $=0.5$ year

250 day-yr average worker scenario

Total Dose Contributions TDOSE (i,p,t) for Individual Radionuclides (i) and Pathways (p)

As mrem/yr and Fraction of Total Dose At $t=3.000 \mathrm{E}+01$ years

\begin{tabular}{|c|c|c|c|c|c|c|c|c|c|c|c|c|c|c|}
\hline \multirow{2}{*}{$\begin{array}{l}\text { Radio- } \\
\text { Nuclide }\end{array}$} & \multicolumn{2}{|c|}{ Ground } & \multicolumn{2}{|c|}{ Inhalation $^{\mathrm{W}}$} & \multicolumn{2}{|c|}{$\begin{array}{l}r \text { Independent } \\
\text { Radon }\end{array}$} & \multicolumn{2}{|c|}{$\begin{array}{l}\text { (Inhalation } \\
\text { Plant }\end{array}$} & \multicolumn{2}{|c|}{$\begin{array}{c}\text { xcludes radon) } \\
\text { Meat }\end{array}$} & \multicolumn{2}{|c|}{ Milk } & \multicolumn{2}{|c|}{ Soil } \\
\hline & $\mathrm{mrem} / \mathrm{yr}$ & fract. & mrem/yr & fract. & $\mathrm{mrem} / \mathrm{yr}$ & fract. & $\mathrm{mrem} / \mathrm{yr}$ & fract. & mrem/yr & fract. & $\mathrm{mrem} / \mathrm{yr}$ & fract. & $\mathrm{mrem} / \mathrm{yr}$ & frac \\
\hline & & & & & & & & & & & & & & \\
\hline & & & & & & & & & & & & & & \\
\hline & & & & & & & & & & & & & & \\
\hline & & & & & & & & & & & & & & \\
\hline & & & & & & & & & & & & & & \\
\hline & & & & & & & & & & & & & & \\
\hline & & & & & & & & & & & & & & \\
\hline 2 & & & & & & & & & & & & & & \\
\hline 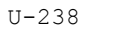 & & & & & & & & & & & & & & \\
\hline old1 & $2.627 \mathrm{E}+00$ & 0.2296 & $.080 \mathrm{E}+00$ & 0.2692 & $000 E+00$ & 0.0000 & $0.000 \mathrm{E}+00$ & 0.0000 & $0.000 \mathrm{E}+00$ & 0.0000 & $0.000 \mathrm{E}+00$ & 0.0000 & $5.734 \mathrm{E}+00$ & 0.5012 \\
\hline
\end{tabular}

Total Dose Contributions TDOSE (i,p,t) for Individual Radionuclides (i) and Pathways (p) As mrem/yr and Fraction of Total Dose At $t=3.000 \mathrm{E}+01$ years

0 Radio- $\frac{\text { Water }}{\text { Nuclide }}$ Fish Radon Plant

Meat mrem/yr fract. mrem/yr fract. mrem/yr fract. $0.000 \mathrm{E}+00 \quad 0.0000$ $0.000 \mathrm{E}+00 \quad 0.0000$ $\begin{array}{lll}A m-241 & 0.000 E+00 \quad 0.0000\end{array}$ $\begin{array}{lll}C s-137 & 0.000 E+00 & 0.0000 \\ \text { Eu-152 } & 0.000 E+00 & 0.0000 \\ P u-238 & 0.000 E+00 & 0.0000\end{array}$ $\begin{array}{lll}\mathrm{Eu}-152 & 0.000 \mathrm{E}+00 & 0.0000 \\ \mathrm{Pu}-238 & 0.000 \mathrm{E}+00 & 0.0000\end{array}$ $\begin{array}{lll}\mathrm{Pu}-239 & 0.000 \mathrm{E}+00 & 0.0000\end{array}$ $\begin{array}{lll}\mathrm{Sr}-90 & 0.000 \mathrm{E}+00 & 0.0000 \\ \mathrm{U}-234 & 0.000 \mathrm{E}+00 & 0.0000\end{array}$ $\begin{array}{lll}\mathrm{U}-234 & 0.000 \mathrm{E}+00 & 0.0000 \\ \mathrm{U}-235 & 0.000 \mathrm{E}+00 & 0.0000\end{array}$ $0.000 \mathrm{E}+00 \quad 0.0000$ $0.000 \mathrm{E}+00 \quad 0.0000$ $\begin{array}{llll}0.000 \mathrm{E}+00 & 0.0000 & 0.000 \mathrm{E}+00 & 0.0000\end{array}$ $\begin{array}{lllll}0.000 \mathrm{E}+00 & 0.0000 & 0.000 \mathrm{E}+00 & 0.0000\end{array}$ $0.000 \mathrm{E}+00 \quad 0.0000$ $\begin{array}{llll}0.000 \mathrm{E}+00 & 0.0000 & 0.000 \mathrm{E}+00 \quad 0.0000\end{array}$

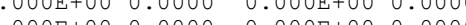
$0.000 \mathrm{E}+00 \quad 0.0000$ $\begin{array}{ll}0.000 \mathrm{E}+00 & 0.0000 \\ 0.000 \mathrm{E}+00 & 0.0000 \\ 0.000 \mathrm{E}+00 & 0.0000\end{array}$ $\begin{array}{lll}-238 & 0.000 \mathrm{E}+00 & 0.0000\end{array}$ $\begin{array}{llll}0.0000 & 0.000 \mathrm{E}+00 & 0.0000\end{array}$ $\begin{array}{ll}0.000 \mathrm{E}+00 & 0.0000\end{array}$ Total $0.000 \mathrm{E}+00 \quad 0.0000$ $0.000 \mathrm{E}+000.0000$ 0.000E+00 0.0000 $0.000 \mathrm{E}+00 \quad 0.0000$ mrem/yr fract. o*Sum of all water independent and dependent pathways. $\operatorname{Milk}$

\begin{tabular}{|c|c|c|c|}
\hline \multicolumn{2}{|c|}{ Milk } & \multicolumn{2}{|c|}{ All Pathways* } \\
\hline$m / y r$ & fract. & $\mathrm{mrem} / \mathrm{yr}$ & fract. \\
\hline 0 & 0.00 & $2.841 E+00$ & 0.2483 \\
\hline & & & \\
\hline $.000 \mathrm{E}+00$ & 0.0000 & $7.248 \mathrm{E}-01$ & 0.0633 \\
\hline $.000 \mathrm{E}+00$ & 0.0000 & $2.108 \mathrm{E}-01$ & 0.0184 \\
\hline $.000 \mathrm{E}+00$ & 0.0000 & $5.826 \mathrm{E}+00$ & 0.5092 \\
\hline $.000 \mathrm{E}+00$ & 0.0000 & $8.997 \mathrm{E}-02$ & 0.0079 \\
\hline $0 E+00$ & 0.0000 & $2.868 \mathrm{E}-02$ & 0.0025 \\
\hline $.000 \mathrm{E}+00$ & 0.0000 & $1.915 \mathrm{E}-02$ & 0.0017 \\
\hline $.000 \mathrm{E}+00$ & 0.0000 & $6.142 \mathrm{E}-02$ & 0.0054 \\
\hline & & & \\
\hline
\end{tabular}


$\mathrm{T} \frac{1}{2}$ Limit $=0.5$ year

02/22/2005 17:24 Page 19

worker scenario

File : CAU 224250 day-yr average.RAD

Total Dose Contributions TDOSE (i,p,t) for Individual Radionuclides (i) and Pathways (p)

As mrem/yr and Fraction of Total Dose At $t=1.000 \mathrm{E}+02$ years

0

\begin{tabular}{|c|c|c|c|c|c|c|c|c|c|c|c|c|c|c|}
\hline \multirow{2}{*}{$\begin{array}{l}\text { Radio- } \\
\text { Nuclide }\end{array}$} & \multicolumn{2}{|c|}{ Ground } & \multicolumn{2}{|c|}{ Inhalation } & \multicolumn{2}{|c|}{ Radon } & \multicolumn{2}{|c|}{ Plant } & \multicolumn{2}{|c|}{$\begin{array}{l}\text { Sadon) } \\
\text { Meat }\end{array}$} & \multicolumn{2}{|c|}{ Milk } & \multicolumn{2}{|l|}{ Soil } \\
\hline & $r$ & $\mathrm{f}$ & mrem/yr & fract. & mrem/yr & fract. & $\mathrm{m}$ & $\mathrm{f}$ & $\mathrm{m}$ & ra & rem/yr & $\mathrm{f}$ & $r$ & rac \\
\hline & & & & & & & & & & & & & & \\
\hline & & & & & & & & & & & & & & \\
\hline & & & & & & & & & & & & & & \\
\hline & & & & & & & & & & & & & & \\
\hline & & & & & & & & & & & & & & \\
\hline & & & & & & & & & & & & & & \\
\hline & & & & & & & & & & & & & & \\
\hline & & & & & & & & & & & & & & \\
\hline 20 & $3.289 \mathrm{E}-02$ & 0.003 & $1.492 \mathrm{E}-02$ & 0.0018 & $0.000 \mathrm{E}+00$ & 0.0000 & $0.000 \mathrm{E}+00$ & 0.0000 & $0.000 \mathrm{E}+00$ & 0.0000 & $0.000 \mathrm{E}+00$ & 0.0000 & 03 & 0.0009 \\
\hline & $130 \mathrm{E}-01$ & 0.0614 & $.749 \mathrm{E}+00$ & 0.3292 & $.000 \mathrm{E}+00$ & 0.0000 & $0.000 \mathrm{E}+00$ & 0.0000 & $0.000 \mathrm{E}+00$ & 0.0000 & $0.000 \mathrm{E}+00$ & 0.0000 & $5.089 \mathrm{E}+00$ & 0.6094 \\
\hline
\end{tabular}
Tota

Total Dose Contributions TDOSE (i,p,t) for Individual Radionuclides (i) and Pathways (p) As mrem/yr and Fraction of Total Dose At $t=1.000 \mathrm{E}+02$ years Radio- $\frac{\text { Water }}{\text { Nuclide }}$ Fish Radon Plant

Meat

mrem/yr fract. mrem/yr fract. $\begin{array}{lllllllllll}\mathrm{Am}-241 & 0.000 \mathrm{E}+00 & 0.0000 & 0.000 \mathrm{E}+00 & 0.0000 & 0.000 \mathrm{E}+00 & 0.0000 & 0.000 \mathrm{E}+00 & 0.0000 & 0.000 \mathrm{E}+00 & 0.0000 \\ \mathrm{Cs}-137 & 0.000 \mathrm{E}+00 & 0.0000 & 0.000 \mathrm{E}+00 & 0.0000 & 0.000 \mathrm{E}+00 & 0.0000 & 0.000 \mathrm{E}+00 & 0.0000 & 0.000 \mathrm{E}+00 & 0.0000 \\ \mathrm{E} 13-152 & 0.000 \mathrm{E}+00 & 0.0000 & 0.000 \mathrm{E}+00 & 0.0000 & 0.000 \mathrm{E}+00 & 0.0000 & 0.000 \mathrm{E}+00 & 0.0000 & 0.000 \mathrm{E}+00 & 0.0000\end{array}$ $\begin{array}{lllllllllll}\mathrm{Am}-241 & 0.000 \mathrm{E}+00 & 0.0000 & 0.000 \mathrm{E}+00 & 0.0000 & 0.000 \mathrm{E}+00 & 0.0000 & 0.000 \mathrm{E}+00 & 0.0000 & 0.000 \mathrm{E}+00 & 0.0000 \\ \mathrm{Cs}-137 & 0.000 \mathrm{E}+00 & 0.0000 & 0.000 \mathrm{E}+00 & 0.0000 & 0.000 \mathrm{E}+00 & 0.0000 & 0.000 \mathrm{E}+00 & 0.0000 & 0.000 \mathrm{E}+00 & 0.0000 \\ \mathrm{Eu}-152 & 0.000 \mathrm{E}+00 & 0.0000 & 0.000 \mathrm{E}+00 & 0.0000 & 0.000 \mathrm{E}+00 & 0.0000 & 0.000 \mathrm{E}+00 & 0.0000 & 0.000 \mathrm{E}+00 & 0.0000 \\ \mathrm{Pu}-238 & 0.000 \mathrm{E}+00 & 0.0000 & 0.000 \mathrm{E}+00 & 0.0000 & 0.000 \mathrm{E}+00 & 0.0000 & 0.000 \mathrm{E}+00 & 0.0000 & 0.000 \mathrm{E}+00 & 0.0000\end{array}$ $\begin{array}{lllllllllll}\mathrm{Pu}-238 & 0.000 \mathrm{E}+00 & 0.0000 & 0.000 \mathrm{E}+00 & 0.0000 & 0.000 \mathrm{E}+00 & 0.0000 & 0.000 \mathrm{E}+00 & 0.0000 & 0.000 \mathrm{E}+00 & 0.0000\end{array}$ Pu-239 $0.000 \mathrm{E}+00 \quad 0.0000$ $\begin{array}{lll}\mathrm{Sr}-90 & 0.000 \mathrm{E}+00 \quad 0.0000\end{array}$

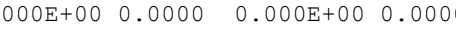

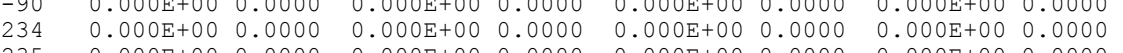
$\mathrm{U}-2380.000 \mathrm{E}+00$ 0.0000 $0.000 \mathrm{E}+000.0000$ - $0.000 \mathrm{E}+000.0000$

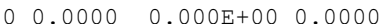

$\begin{array}{lllllllllllllllll}\text { Thtal } & 0.000 \mathrm{E}+00 & 0.0000 & 0.000 \mathrm{E}+00 & 0.0000 & 0.000 \mathrm{E}+00 & 0.0000 & 0.000 \mathrm{E}+00 & 0.0000 & 0.000 \mathrm{E}+00 & 0.0000\end{array}$ $0 *$ Sum of all water independent and dependent pathways.
Milk

mrem/yr fract.

$0.000 \mathrm{E}+00 \quad 0.0000$ $0.000 \mathrm{E}+00 \quad 0.000$ $0.000 \mathrm{E}+00 \quad 0.0000$ $0.000 \mathrm{E}+00 \quad 0.0000$ $0.000 \mathrm{E}+00 \quad 0.0000$ $0.000 \mathrm{E}+00 \quad 0.0000$ $0.000 \mathrm{E}+00 \quad 0.000$ $0.000 \mathrm{E}+00 \quad 0.0000$ $0.000 \mathrm{E}+00 \quad 0.0000$ All Pathways* $.974 E+00 \quad 0.2364$ $\begin{array}{lll}.236 \mathrm{E}-01 & 0.0388 \\ 1.891 \mathrm{E}-02 & 0.0023\end{array}$ $1.891 \mathrm{E}-02 \quad 0.0023$ $\begin{array}{ll}1.210 \mathrm{E}-01 & 0.0145 \\ 5.800 \mathrm{E}+00 & 0.6945\end{array}$ $\begin{array}{lll}1.756 \mathrm{E}-02 & 0.0021\end{array}$ $8.351 \mathrm{E}+001.0000$ 
$\mathrm{T} \frac{1}{2}$ Limit $=0.5$ year

02/22/2005 17:24 Page 20

2240 day-y average worker scenario

Total Dose Contributions TDOSE (i,p,t) for Individual Radionuclides (i) and Pathways (p) As mrem/yr and Fraction of Total Dose At $t=3.000 \mathrm{E}+02$ years

\begin{tabular}{|c|c|c|c|c|c|c|c|c|c|c|c|c|c|c|}
\hline \multirow{2}{*}{$\begin{array}{l}\text { Radio- } \\
\text { Nuclide }\end{array}$} & \multicolumn{2}{|c|}{ Ground } & \multicolumn{2}{|c|}{ Inhalation } & \multicolumn{2}{|c|}{$\begin{array}{l}r \text { Independent } \\
\text { Radon }\end{array}$} & \multicolumn{2}{|c|}{$\begin{array}{l}\text { (Inhalation } \\
\text { Plant }\end{array}$} & \multicolumn{2}{|c|}{$\begin{array}{c}\text { xcludes radon) } \\
\text { Meat }\end{array}$} & \multicolumn{2}{|c|}{ Milk } & \multicolumn{2}{|l|}{ Soil } \\
\hline & $\mathrm{rem} / \mathrm{yr}$ & fract. & mrem/yr & fract. & $\mathrm{mrem} / \mathrm{yr}$ & fract. & $\mathrm{mrem} / \mathrm{yr}$ & fract. & mrem/yr & fract. & $\mathrm{mrem} / \mathrm{yr}$ & fract. & $\mathrm{mrem} / \mathrm{yr}$ & frac \\
\hline & & & & & & & & & & & & & & \\
\hline & & & & & & & & & & & & & & \\
\hline & & & & & & & & & & & & & & \\
\hline & & & & & & & & & & & & & & \\
\hline & & & & & & & & & & & & & & \\
\hline & & & & & & & & & & & & & & \\
\hline & & & & & & & & & & & & & & \\
\hline & & & & & & & & & & & & & & \\
\hline 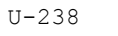 & & & & & & & & & & & & & & \\
\hline old1 & $220 \mathrm{E}-02$ & 0.0126 & $.259 \mathrm{E}+00$ & 0.3462 & $000 E+00$ & 0.0000 & $0.000 \mathrm{E}+00$ & 0.0000 & $0.000 \mathrm{E}+00$ & 0.0000 & $0.000 \mathrm{E}+00$ & 0.0000 & $4.185 \mathrm{E}+00$ & 0.6412 \\
\hline
\end{tabular}

Total Dose Contributions TDOSE (i,p,t) for Individual Radionuclides (i) and Pathways (p) As mrem/yr and Fraction of Total Dose At $t=3.000 \mathrm{E}+02$ years

Radon

Plant

Radio-
Nuclide mrem/yr fract.

mrem/yr fract.

mrem/yr fract.

$\mathrm{mrem} / \mathrm{yr}$ fract.

mrem/yr fract.

Milk

All Pathways*

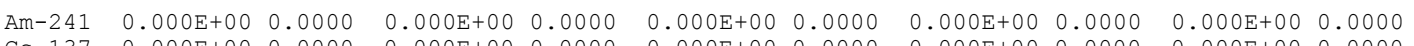

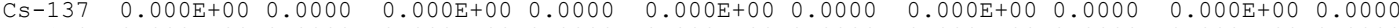

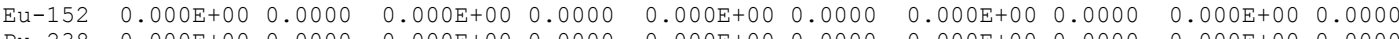

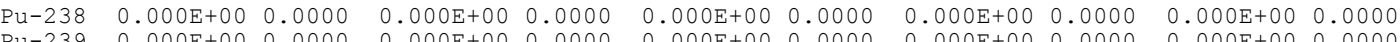

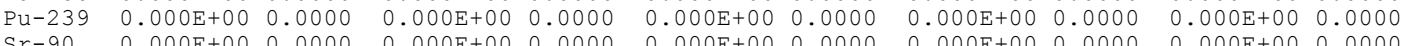

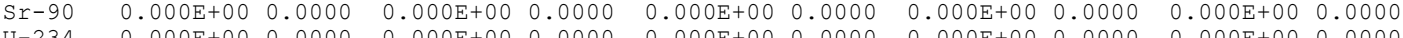

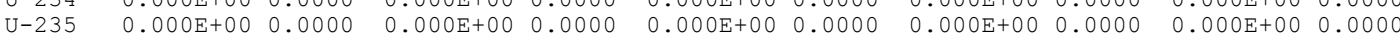

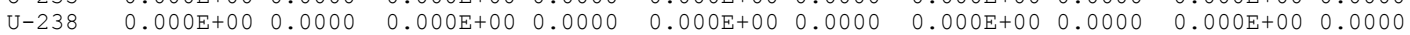

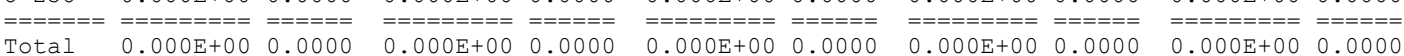
$0 *$ Sum of all water independent and dependent pathways.

mrem/yr fract.

$6.982 \mathrm{E}-01 \quad 0.1070$ $3.140 \mathrm{E}-03 \quad 0.0005$ $.994 \mathrm{E}-020.0031$ $4.157 \mathrm{E}-02 \quad 0.0064$ $6.526 \mathrm{E}+00 \quad 1.0000$ 
$\mathrm{T} 1 / 2$ Limit $=0.5$ year

2240 day-yr average worker scenario

Total Dose Contributions TDOSE (i,p,t) for Individual Radionuclides (i) and Pathways (p)

As mrem/yr and Fraction of Total Dose At $t=1.000 \mathrm{E}+03$ years

\begin{tabular}{|c|c|c|c|c|c|c|c|c|c|c|c|c|c|c|}
\hline \multirow{2}{*}{$\begin{array}{l}\text { Radio- } \\
\text { Nuclide }\end{array}$} & \multicolumn{2}{|c|}{ Ground } & \multicolumn{2}{|c|}{ Inhalation } & \multicolumn{2}{|l|}{ Ra } & \multicolumn{2}{|c|}{$\begin{array}{l}\text { Inhalat } \\
\text { Plant }\end{array}$} & \multicolumn{2}{|c|}{$\begin{array}{l}\text { S radon) } \\
\text { Meat }\end{array}$} & \multicolumn{2}{|c|}{ Milk } & \multicolumn{2}{|c|}{ Soil } \\
\hline & $\mathrm{mrem} / \mathrm{yr}$ & fract. & mrem/yr & fract. & $\mathrm{mrem} / \mathrm{yr}$ & fract. & mrem/yr & fract. & mrem/yr & fract. & mrem/yr & fract. & mrem/yr & fract. \\
\hline 1 & 1 & 0 & 3 & 1 & 0 & 0.0000 & 0 & 0 & 0 & 0 & 00 & 0 & 22 & 1 \\
\hline & $6 E-10$ & & -15 & 000 & 00 & 0 & & & & & $E+00$ & & -13 & \\
\hline & & c & -15 & 0.000 & 00 & 0 & & & & & & & & \\
\hline & $1.134 \mathrm{E}-06$ & 0.0000 & $692 \mathrm{E}-05$ & 0.0000 & $000 \mathrm{E}+00$ & 0000 & $\mathrm{E}+00$ & 0 & $\mathrm{E}+00$ & 0.0 & $0.000 \mathrm{E}+00$ & 0 & -05 & 000 \\
\hline Pu-239 & $2.313 \mathrm{E}-03$ & 0.0004 & $.906 \mathrm{E}+00$ & 0.3453 & $0.000 \mathrm{E}+00$ & 0.0000 & $0.000 \mathrm{E}+00$ & 0.0000 & $0.000 \mathrm{E}+00$ & 0.0000 & $0.000 \mathrm{E}+00$ & 0.0000 & $3.562 \mathrm{E}+00$ & 0.6453 \\
\hline & $4.409 \mathrm{E}-13$ & 0.0000 & $378 E-14$ & 0.0000 & 0.000 & 0.0 & 0.00 & 0. & 00 & 0. & $10 E+00$ & 0 & $45 E-13$ & 100 \\
\hline 234 & $2.304 \mathrm{E}-03$ & 0.0004 & $.948 \mathrm{E}-03$ & 0.0009 & $0.000 \mathrm{E}+00$ & 0.0000 & $0.000 \mathrm{E}+00$ & 0. & 0.00 & 0.0 & $0.000 \mathrm{E}+00$ & & -03 & 0.0005 \\
\hline & $4.343 E-03$ & 0.0008 & $.679 \mathrm{E}-04$ & 0.0002 & $0.000 \mathrm{E}$ & 0.0000 & 0.000 & 0.0 & & & & & & \\
\hline U-238 & $8.939 \mathrm{E}-03$ & 0.0016 & $4.068 \mathrm{E}-03$ & 0.0007 & $0.000 \mathrm{E}+00$ & 0.0000 & $0.000 \mathrm{E}+00$ & 0.0000 & $0.000 \mathrm{E}+00$ & 0.0000 & $0.000 \mathrm{E}+00$ & 0.0 & $2.100 \mathrm{E}-03$ & 0.0004 \\
\hline$====$ & & $==$ & & & & & & & & & & & & \\
\hline Total & $1.918 \mathrm{E}-02$ & 0.0035 & $1.922 \mathrm{E}+00$ & 0.3482 & $0.000 \mathrm{E}+00$ & 0.0000 & $0.000 \mathrm{E}+00$ & 0.0000 & $0.000 \mathrm{E}+00$ & 0.0000 & $0.000 \mathrm{E}+00$ & 0.0000 & $3.578 \mathrm{E}+00$ & 0.6484 \\
\hline
\end{tabular}

Total Dose Contributions TDOSE (i,p,t) for Individual Radionuclides (i) and Pathways (p) As mrem/yr and Fraction of Total Dose At $t=1.000 \mathrm{E}+03$ years

0 Radio- $\frac{\text { Water }}{\text { Nuclide }}$ Fish Radon Plant

Meat mrem/yr fract. mrem/yr fract. mrem/yr fract. $0.000 \mathrm{E}+00 \quad 0.0000$ $0.000 \mathrm{E}+00 \quad 0.0000$ $\begin{array}{lll}A m-241 & 0.000 E+00 \quad 0.0000\end{array}$ $\begin{array}{lll}C s-137 & 0.000 E+00 & 0.0000 \\ \text { Eu-152 } & 0.000 E+00 & 0.0000\end{array}$ $\begin{array}{lll}\mathrm{Eu}-152 & 0.000 \mathrm{E}+00 & 0.0000 \\ \mathrm{Pu}-238 & 0.000 \mathrm{E}+00 & 0.0000\end{array}$ $\begin{array}{lll}\mathrm{Pu}-239 & 0.000 \mathrm{E}+00 & 0.000\end{array}$ $\begin{array}{lll}\mathrm{Sr}-90 & 0.000 \mathrm{E}+00 & 0.0000 \\ \mathrm{U}-234 & 0.000 \mathrm{E}+00 & 0.0000\end{array}$ $\begin{array}{lll}\mathrm{U}-234 & 0.000 \mathrm{E}+00 & 0.0000 \\ \mathrm{U}-235 & 0.000 \mathrm{E}+00 & 0.0000\end{array}$ $0.000 \mathrm{E}+00 \quad 0.0000$ $0.000 \mathrm{E}+00 \quad 0.0000$ $0.000 \mathrm{E}+000.0000$ $0.000 \mathrm{E}+000.0000$ $\begin{array}{lll}.0000 & 0.000 \mathrm{E}+00 & 0.0000 \\ 0.0000 & 0.000 \mathrm{E}+00 & 0.0000\end{array}$ $0.000 \mathrm{E}+00 \quad 0.0000$ $\mathrm{J}-238 \quad 0.000 \mathrm{E}+00 \quad 0.0000$

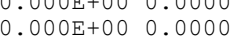
$0.000 \mathrm{E}+000.0000$ $0.0 \mathrm{E}+00 \quad 0.0000$

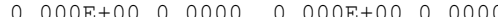
$0.000 \mathrm{E}+00 \quad 0.0000$ mrem/yr fract. $0 *$ Sum of all water independent and dependent pathways. Milk

\begin{tabular}{|c|c|c|c|}
\hline \multicolumn{2}{|c|}{ Milk } & \\
\hline $\mathrm{m} / \mathrm{yr}$ & fract. & mrem/yr & fract. \\
\hline & 0.0 & $1.874 \mathrm{E}-02$ & 0.0034 \\
\hline & & & \\
\hline ) & 0.0000 & $2.620 \mathrm{E}-15$ & 0.0000 \\
\hline $000 \mathrm{E}+00$ & 0.0000 & $1.018 \mathrm{E}-04$ & 0.0000 \\
\hline+00 & 0.0 & 5.4 & 910 \\
\hline$E+00$ & 0.0000 & $8.192 \mathrm{E}-13$ & 0.0000 \\
\hline & 0.0000 & $9.849 \mathrm{E}-03$ & 0.0018 \\
\hline $.000 \mathrm{E}+00$ & 0.0000 & $5.756 \mathrm{E}-03$ & 0.0010 \\
\hline $.000 \mathrm{E}+00$ & 0.0000 & $1.511 \mathrm{E}-02$ & 0.0027 \\
\hline & & & \\
\hline
\end{tabular}


1RESRAD, Version 6.21

$\mathrm{T}^{1} \frac{1}{2}$ Limit $=0.5$ year

Summary : CAU 224250 day-yr average worker

Dose/Source Ratios Summed Over All Pathways

Parent and Progeny Principal Radionuclide Contributions Indicated

OParent Product Branch

(i) (j) Fraction* $t=0.000 \mathrm{E}+00 \quad 1.000 \mathrm{E}+00 \quad 3.000 \mathrm{E}+00 \quad 1.000 \mathrm{E}+01 \quad 3.000 \mathrm{E}+01 \quad 1.000 \mathrm{E}+02 \quad 3.000 \mathrm{E}+02 \quad 1.000 \mathrm{E}+03$

$\overline{\mathrm{Am}-241} \overline{\mathrm{Am}-241} \overline{1.000 \mathrm{E}+00} \overline{1.621 \mathrm{E}-01} \overline{1.612 \mathrm{E}-01} \overline{1.596 \mathrm{E}-01} \overline{1.539 \mathrm{E}-01} \overline{1.387 \mathrm{E}-01} \overline{9.635 \mathrm{E}-02} \overline{3.406 \mathrm{E}-02} \overline{8.941 \mathrm{E}-04}$ $\begin{array}{lllllllllll}\mathrm{Am}-241 & \mathrm{~Np}-237 & 1.000 \mathrm{E}+00 & 6.717 \mathrm{E}-08 & 2.010 \mathrm{E}-07 & 4.666 \mathrm{E}-07 & 1.373 \mathrm{E}-06 & 3.779 \mathrm{E}-06 & 1.037 \mathrm{E}-05 & 1.941 \mathrm{E}-05 & 2.048 \mathrm{E}-05\end{array}$ $\begin{array}{lllllllllll}\mathrm{Am}-241 & \mathrm{U}-233 & 1.000 \mathrm{E}+00 & 5.776 \mathrm{E}-15 & 4.035 \mathrm{E}-14 & 2.123 \mathrm{E}-13 & 1.869 \mathrm{E}-12 & 1.506 \mathrm{E}-11 & 1.397 \mathrm{E}-10 & 8.204 \mathrm{E}-10 & 2.709 \mathrm{E}-09 \\ \mathrm{Am}-241 & \mathrm{Th}-229 & 1.000 \mathrm{E}+00 & 3.915 \mathrm{E}-18 & 5.864 \mathrm{E}-17 & 6.819 \mathrm{E}-16 & 1.786 \mathrm{E}-14 & 4.220 \mathrm{E}-13 & 1.340 \mathrm{E}-11 & 2.601 \mathrm{E}-10 & 3.792 \mathrm{E}-09\end{array}$

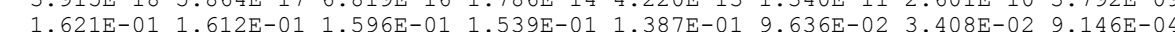
$\begin{array}{lllllllllll}\mathrm{Am}-241 & \text { SDSR (j) } & 1.000 \mathrm{E}+00 & 6.923 \mathrm{E}-01 & 6.745 \mathrm{E}-01 & 6.439 \mathrm{E}-01 & 5.475 \mathrm{E}-01 & 3.444 \mathrm{E}-01 & 6.799 \mathrm{E}-02 & 6.596 \mathrm{E}-04 & 5.928 \mathrm{E}-11\end{array}$ 0Eu-152 Eu-152 7.208E-01 $\quad \begin{array}{llllllll}1.013 \mathrm{E}+00 & 9.618 \mathrm{E}-01 & 8.666 \mathrm{E}-01 & 6.019 \mathrm{E}-01 & 2.124 \mathrm{E}-01 & 5.541 \mathrm{E}-03 & 1.657 \mathrm{E}-07 & 2.421 \mathrm{E}-23\end{array}$

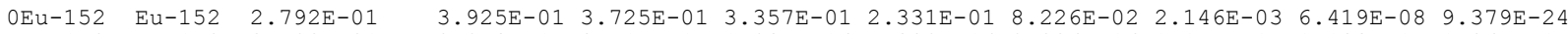

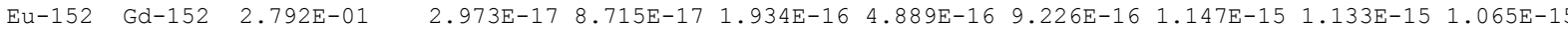
Pu-238 Pu-238 $1.000 \mathrm{E}+00$ $\mathrm{Pu}-238 \mathrm{U}-234 \quad 1.000 \mathrm{E}+00$ $\mathrm{Pu}-238 \mathrm{Th}-230 \quad 1.000 \mathrm{E}+00$ $\begin{array}{lll}\mathrm{Pu}-238 & \mathrm{Ra}-226 & 1.000 \mathrm{E}+00\end{array}$ Pu-238 $\mathrm{Pu}-238 \mathrm{SDSR}(j)$

$\mathrm{Pu}-239 \quad 1.000 \mathrm{E}+00$ $\mathrm{U}-2351.000 \mathrm{E}+00$ $\begin{array}{lll}\mathrm{Pu}-239 & \mathrm{~Pa}-231 & 1.000 \mathrm{E}+00\end{array}$ $\mathrm{Pu}-239 \quad \mathrm{Ac}-227 \quad 1.000 \mathrm{E}+00$ $\mathrm{Pu}-239$ SDSR $(j)$ $\mathrm{OU}-234 \mathrm{U}-234000 \mathrm{E}+00$ U-234 Th-230 $1.000 \mathrm{E}+00$ $\begin{array}{lll}\mathrm{U}-234 & \mathrm{R} a-226 & 1.000 \mathrm{E}+00\end{array}$ $\mathrm{U}-234$ Po-210 1.000E+0 $\mathrm{U}-234 \quad \operatorname{SDSR}(j)$

$\begin{array}{lll}0-235 & 1.000 \mathrm{E}+00\end{array}$ $\mathrm{U}-235$ AC-227 $1.000 \mathrm{E}+00$ UU-238 $\mathrm{UDSR}(j)$ $\mathrm{U}-238 \mathrm{U}-234-1.000 \mathrm{E}+00$ $\begin{array}{lll}\mathrm{U}-238 & \mathrm{Th}-230 & 1.000 \mathrm{E}+00\end{array}$ $\mathrm{U}-238 \mathrm{Ra}-226 \quad 1.000 \mathrm{E}+00$ $\mathrm{U}-238 \quad \mathrm{~Pb}-210 \quad 1.000 \mathrm{E}+00$ $\mathrm{U}-238$

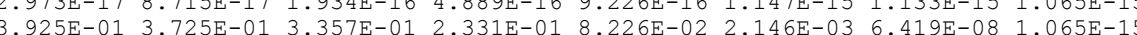

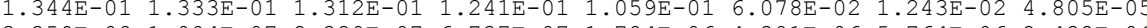

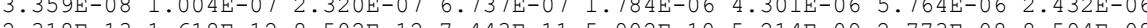

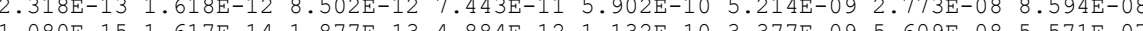

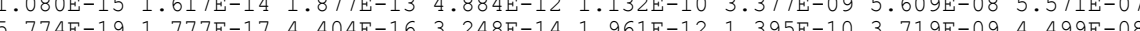

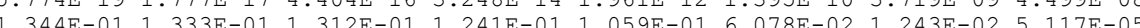
$\begin{array}{llllllll}1.487 \mathrm{E}-01 & 1.487 \mathrm{E}-01 & 1.487 \mathrm{E}-01 & 1.486 \mathrm{E}-01 & 1.484 \mathrm{E}-01 & 1.478 \mathrm{E}-01 & 1.459 \mathrm{E}-01 & 1.394 \mathrm{E}-01\end{array}$ $\begin{array}{llllllll}8.923 \mathrm{E}-11 & 2.675 \mathrm{E}-10 & 6.233 \mathrm{E}-10 & 1.860 \mathrm{E}-09 & 5.322 \mathrm{E}-09 & 1.664 \mathrm{E}-08 & 4.306 \mathrm{E}-08 & 9.068 \mathrm{E}-08\end{array}$ $\begin{array}{llllllll}1.682 \mathrm{E}-15 & 1.176 \mathrm{E}-14 & 6.206 \mathrm{E}-14 & 5.513 \mathrm{E}-13 & 4.558 \mathrm{E}-12 & 4.618 \mathrm{E}-11 & 3.400 \mathrm{E}-10 & 1.980 \mathrm{E}-09\end{array}$ $\begin{array}{llllllll}4.484 \mathrm{E}-17 & 6.671 \mathrm{E}-16 & 7.642 \mathrm{E}-15 & 1.900 \mathrm{E}-13 & 3.902 \mathrm{E}-12 & 8.405 \mathrm{E}-11 & 8.743 \mathrm{E}-10 & 5.808 \mathrm{E}-09\end{array}$

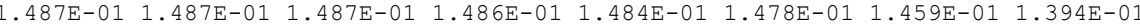

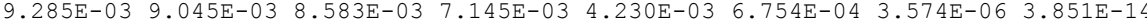

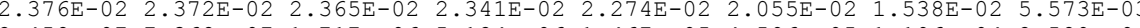

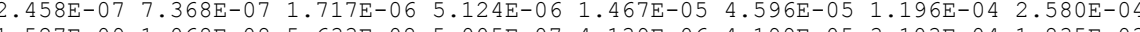
$\begin{array}{llllllll}1.527 \mathrm{E}-09 & 1.068 \mathrm{E}-08 & 5.633 \mathrm{E}-08 & 5.005 \mathrm{E}-07 & 4.139 \mathrm{E}-06 & 4.199 \mathrm{E}-05 & 3.103 \mathrm{E}-04 & 1.835 \mathrm{E}-03\end{array}$

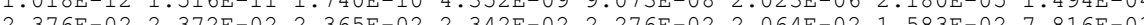

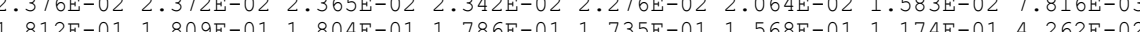

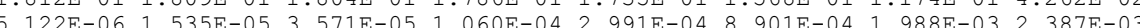
$\begin{array}{llllllll}1.818 \mathrm{E}-07 & 1.258 \mathrm{E}-06 & 6.491 \mathrm{E}-06 & 5.328 \mathrm{E}-05 & 3.568 \mathrm{E}-04 & 2.016 \mathrm{E}-03 & 5.669 \mathrm{E}-03 & 7.321 \mathrm{E}-03\end{array}$ $\begin{array}{lllllllll}1.812 \mathrm{E}-01 & 1.809 \mathrm{E}-01 & 1.804 \mathrm{E}-01 & 1.787 \mathrm{E}-01 & 1.741 \mathrm{E}-01 & 1.597 \mathrm{E}-01 & 1.250 \mathrm{E}-01 & 5.233 \mathrm{E}-02\end{array}$

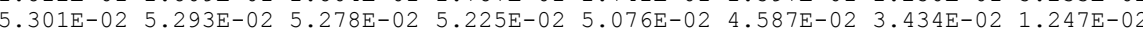

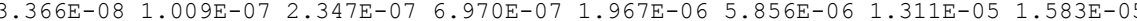

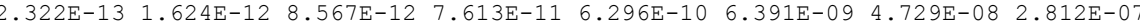

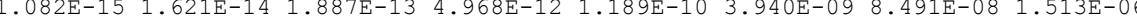

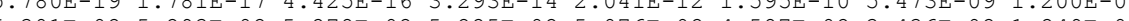

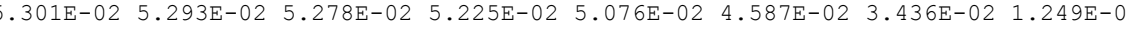

*Branch Fraction is the cumulative factor for the $j$ 't principal radionuclide daughter: $\operatorname{CUMBRF}(j)=\operatorname{BRF}(1) * \operatorname{BRF}(2) * \ldots \operatorname{BRF}(j)$. \$ is used to indicate sumation; the Greek sigma is not included in this font. 
1RESRAD, Version $6.21 \quad$ T3/2 Limit $=0.5$ year

02/22/2005 17:24 Page 23

Summary : CAU 224250 day-yr average worker scenario
File : CAU 224250 day-yr average. RAD

Single Radionuclide Soil Guidelines $\mathrm{G}(\mathrm{i}, \mathrm{t})$ in $\mathrm{pCi} / \mathrm{g}$ Basic Radiation Dose Limit $=2.500 \mathrm{E}+01 \mathrm{mrem} / \mathrm{yr}$

ONuclide

\begin{tabular}{|c|c|c|c|c|c|c|c|c|c|}
\hline $\begin{array}{l}\mathrm{C} \perp 1 \\
\text { (i) }\end{array}$ & $t=$ & $0.000 \mathrm{E}+00$ & $1.000 \mathrm{E}+00$ & $3.000 \mathrm{E}+00$ & $1.000 \mathrm{E}+01$ & $3.000 \mathrm{E}+01$ & $1.000 \mathrm{E}+02$ & $3.000 \mathrm{E}+02$ & $1.000 \mathrm{E}+03$ \\
\hline & & $\mathrm{E}+02$ & $1 E+02$ & $567 \mathrm{E}+02$ & $5 \mathrm{E}$ & 1.8 & $2.594 \mathrm{E}+02$ & $7.337 \mathrm{E}+02$ & $2.734 \mathrm{E}+04$ \\
\hline & & & & & & & & & \\
\hline 52 & & +01 & $\mathrm{E}+01$ & 1 & 2 & 1 & 3. & 8 & $* 1$. \\
\hline & & $0 \mathrm{E}+02$ & $5 E+02$ & $5 E+02$ & $14 \mathrm{E}+02$ & 2 & 22 & $E+03$ & $86 \mathrm{E}+05$ \\
\hline & & & & & & & & 1. & $1.794 \mathrm{E}+02$ \\
\hline 0 & & $2.692 \mathrm{E}+03$ & $2.764 \mathrm{E}+03$ & $2.913 \mathrm{E}$ & $3.499 \mathrm{E}$ & $5.910 \mathrm{E}$ & $3.701 \mathrm{E}$ & $6.995 \mathrm{E}+06$ & $* 1.365 \mathrm{E}+14$ \\
\hline$U-234$ & & $1.052 \mathrm{E}+03$ & $1.054 \mathrm{E}+$ & $1.057 \mathrm{E}$ & $1.068 \mathrm{E}+$ & 1.09 & $1.211 \mathrm{E}$ & $1.579 \mathrm{E}$ & $3.198 \mathrm{E}+03$ \\
\hline $\mathrm{U}-235$ & & $1.380 \mathrm{E}+02$ & $1.382 \mathrm{E}+02$ & $1.386 \mathrm{E}+02$ & $1.399 \mathrm{E}+02$ & $1.436 \mathrm{E}+02$ & $1.566 \mathrm{E}+02$ & $2.000 \mathrm{E}+02$ & $4.777 \mathrm{E}+02$ \\
\hline U-238 & & $4.716 \mathrm{E}+02$ & $4.723 \mathrm{E}+02$ & $737 E+02$ & $5 \mathrm{E}+02$ & $5 \mathrm{E}+02$ & $5.450 \mathrm{E}+02$ & $7.277 \mathrm{E}+02$ & $2.002 E+03$ \\
\hline
\end{tabular}

At specific activity limit

Summed Dose/Source Ratios DSR $(i, t)$ in (mrem/yr)/(pCi/g)

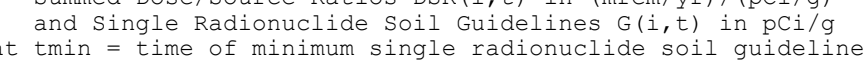
$\begin{aligned} \text { at } \operatorname{tmin} & =\text { time of minimum single radionuclide soil guid } \\ \text { and at } \operatorname{tmax} & =\text { time of maximum total dose }=0.000 \mathrm{E}+00 \text { years }\end{aligned}$

\begin{tabular}{|c|c|c|c|c|c|c|}
\hline $\begin{array}{l}\text { Nuclide } \\
\text { (i) }\end{array}$ & $\begin{array}{l}\text { Initial } \\
(\mathrm{pCi} / \mathrm{g})\end{array}$ & $\begin{array}{c}\text { tmin } \\
\text { (years) }\end{array}$ & $\operatorname{DSR}(i, \operatorname{tmin})$ & $\begin{array}{l}\mathrm{G}(\mathrm{i}, \mathrm{tmin}) \\
(\mathrm{pCi} / \mathrm{g})\end{array}$ & $\operatorname{DSR}(i, \operatorname{tmax})$ & $\begin{array}{c}G(i, \operatorname{tmax}) \\
(\mathrm{pC} i / \mathrm{g})\end{array}$ \\
\hline & $2.049 \mathrm{E}+01$ & $0.000 \mathrm{E}+$ & $1.621 \mathrm{E}-01$ & $1.543 \mathrm{E}+02$ & $1.621 \mathrm{E}-01$ & $1.543 \mathrm{E}+02$ \\
\hline & & & & & & \\
\hline Eu-152 & $2.460 \mathrm{E}+00$ & $.000 \mathrm{E}+00$ & +00 & $1.778 \mathrm{E}+01$ & $1.406 \mathrm{E}+00$ & $1.778 \mathrm{E}+01$ \\
\hline & 1.99 & .000 & & & & $\mathrm{E}+02$ \\
\hline Pu-239 & 3.925 & 0.00 & 1.48 & 1.68 & 1.4 & $1.681 \mathrm{~F}$ \\
\hline Sr-90 & $2.127 \mathrm{E}+01$ & $0.000 \mathrm{E}$ & $9.285 \mathrm{E}$ & $2.692 \mathrm{E}+03$ & $9.285 \mathrm{E}-03$ & $2.692 \mathrm{E}+03$ \\
\hline $\mathrm{U}-234$ & $1.260 \mathrm{E}+00$ & 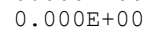 & $2.376 \mathrm{E}-02$ & $1.052 \mathrm{E}+03$ & $2.376 \mathrm{E}-02$ & $1.052 \mathrm{E}+03$ \\
\hline $\mathrm{U}-235$ & $1.100 \mathrm{E}-01$ & $0.000 \mathrm{E}+00$ & -01 & 1.38 & $1.812 \mathrm{E}-01$ & $1.380 \mathrm{E}+02$ \\
\hline $\mathrm{U}-238$ & $1.210 \mathrm{E}+00$ & $00 \mathrm{E}+00$ & $5.301 \mathrm{E}-02$ & $4.716 \mathrm{E}+02$ & $5.301 \mathrm{E}-02$ & $4.716 \mathrm{E}+02$ \\
\hline
\end{tabular}


1RESRAD, Version $6.21 \quad T^{1 / 2}$ Limit $=0.5$ year

$\begin{array}{lll}\text { Summary : CAU } 224 & 250 \text { day-yr average worker } \\ \text { File : CAU } 224250 \text { day-yr average. RAD }\end{array}$

Individual Nuclide Dose Summed Over All Pathways

Parent Nuclide and Branch Fraction Indicated

ONuclide Parent BRF(i)

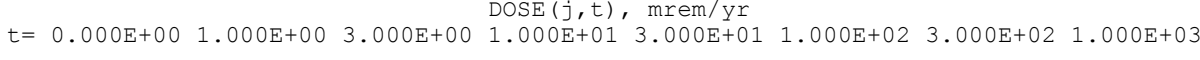

$\overline{\mathrm{Am}-241} \overline{\mathrm{Am}-241} \overline{1.000 \mathrm{E}+00}$ ONp-237 Am-241 $1.000 \mathrm{E}+00$ OU $-233, \mathrm{Am}-241 \quad 1.000 \mathrm{E}+00$

$\mathrm{OCs}-137$ Cs $-137 \quad 1.000 \mathrm{E}+00$

OEu-152 Eu-152 $7.208 \mathrm{E}-01$

Eu-152 Eu-152 2.792E-01

$\mathrm{Eu}-152$ \$DOSE (j)

Eu-152 2.792E-01

$0 \mathrm{Pu}-238 \quad \mathrm{Pu}-238 \quad 1.000 \mathrm{E}+00$

OU-234 $\quad \mathrm{Pu}-238 \quad 1.000 \mathrm{E}+00$

$\mathrm{U}-234-\mathrm{U}-234-1.000 \mathrm{E}+00$

$\mathrm{U}-234-\mathrm{U}-238 \quad 1.000 \mathrm{E}+0$

$\mathrm{U}-234$ SDOSE (j)

Th-230 U U-234 $1.000 \mathrm{E}+00$

Th-230 U -234 1.000 E+00

Th-230 SDOSE (j)

$\begin{array}{ll}\mathrm{Th}-230 & \text { SDOSE (j) } \\ \mathrm{OR} a-226 & \mathrm{Pu}-238 \\ \mathrm{Ra} & 1.000 \mathrm{E}+00\end{array}$

$\mathrm{Ra}-226 \mathrm{U}-234 \quad 1.000 \mathrm{E}+00$

$\mathrm{Ra}-226 \mathrm{U}-238 \quad 1.000 \mathrm{E}+00$

$\mathrm{Ra}-226$ \$DOSE $(j)$

$\mathrm{Pbb}-210 \quad \mathrm{Pu}-238 \quad 1.000 \mathrm{E}+00$

$\mathrm{Pb}-210 \quad \mathrm{U}-234 \quad 1.000 \mathrm{E}+00$

$\mathrm{Pb}-210 \quad \mathrm{U}-238 \quad 1.000 \mathrm{E}+00$

$\mathrm{Pb}-210$ \$DOSE $(\mathrm{j})$

OPu-239 $\mathrm{Pu}-239 \quad 1.000 \mathrm{E}+00$

$\begin{array}{lll}\mathrm{U}-235 & \mathrm{Pu}-239 & 1.000 \mathrm{E}+0\end{array}$

$\mathrm{U}-235$ SDOSE (j)

$\begin{array}{lll}\mathrm{Pa}-231 & \mathrm{Pu}-239 & 1.000 \mathrm{E}+00\end{array}$

$\mathrm{Pa}-231 \mathrm{U}-235$ 1.000E+00

Pa-231 \$DOSE (j)

$\begin{array}{llllllll}3.321 \mathrm{E}+00 & 3.303 \mathrm{E}+00 & 3.269 \mathrm{E}+00 & 3.152 \mathrm{E}+00 & 2.841 \mathrm{E}+00 & 1.974 \mathrm{E}+00 & 6.978 \mathrm{E}-01 & 1.832 \mathrm{E}-02\end{array}$

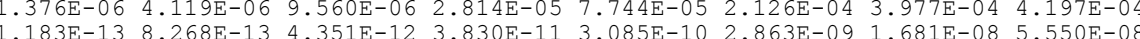

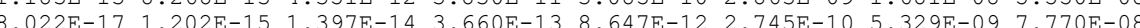
$\begin{array}{llllllll}3.286 \mathrm{E}+00 & 3.210 \mathrm{E}+00 & 3.065 \mathrm{E}+00 & 2.606 \mathrm{E}+00 & 1.639 \mathrm{E}+00 & 3.236 \mathrm{E}-01 & 3.140 \mathrm{E}-03 & 2.822 \mathrm{E}-10\end{array}$ $\begin{array}{llllllll}2.493 \mathrm{E}+00 & 2.366 \mathrm{E}+00 & 2.132 \mathrm{E}+00 & 1.481 \mathrm{E}+00 & 5.224 \mathrm{E}-01 & 1.363 \mathrm{E}-02 & 4.077 \mathrm{E}-07 & 5.956 \mathrm{E}-23\end{array}$ $\begin{array}{llllllll}9.655 \mathrm{E}-01 & 9.165 \mathrm{E}-01 & 8.258 \mathrm{E}-01 & 5.735 \mathrm{E}-01 & 2.024 \mathrm{E}-01 & 5.280 \mathrm{E}-03 & 1.579 \mathrm{E}-07 & 2.307 \mathrm{E}-23\end{array}$

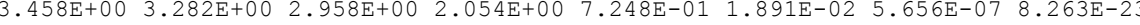
$\begin{array}{llllllll}7.312 \mathrm{E}-17 & 2.144 \mathrm{E}-16 & 4.757 \mathrm{E}-16 & 1.203 \mathrm{E}-15 & 2.270 \mathrm{E}-15 & 2.821 \mathrm{E}-15 & 2.787 \mathrm{E}-15 & 2.620 \mathrm{E}-15\end{array}$

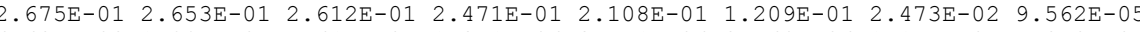

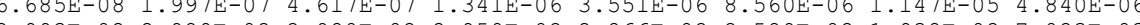

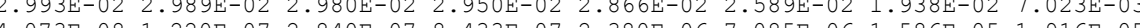

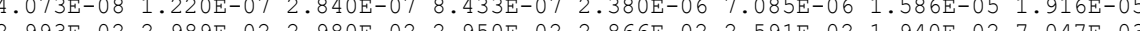

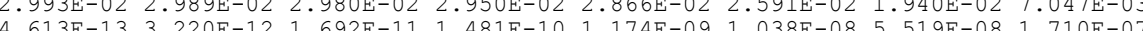

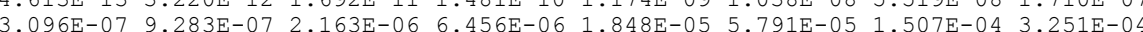

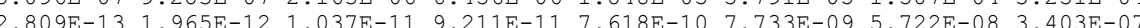

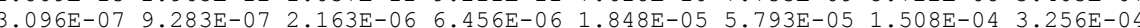
$\begin{array}{llllllll}2.150 \mathrm{E}-15 & 3.218 \mathrm{E}-14 & 3.734 \mathrm{E}-13 & 9.719 \mathrm{E}-12 & 2.254 \mathrm{E}-10 & 6.721 \mathrm{E}-09 & 1.116 \mathrm{E}-07 & 1.109 \mathrm{E}-06\end{array}$

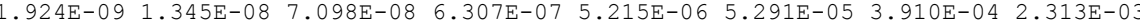

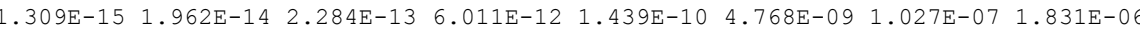

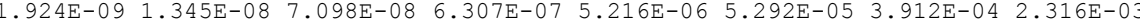

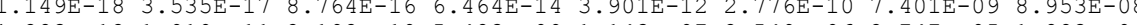

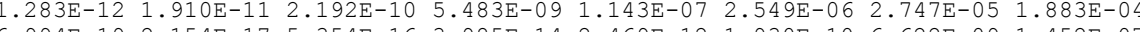

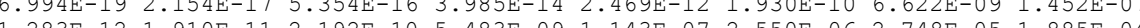

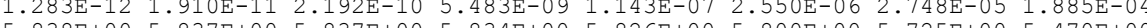

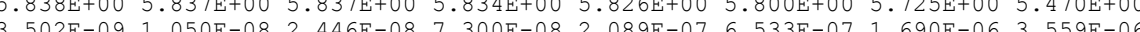

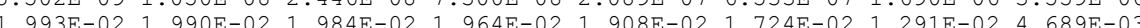

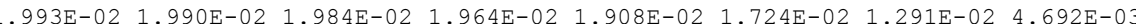
$\begin{array}{llllllll}6.601 \mathrm{E}-14 & 4.617 \mathrm{E}-13 & 2.436 \mathrm{E}-12 & 2.164 \mathrm{E}-11 & 1.789 \mathrm{E}-10 & 1.813 \mathrm{E}-09 & 1.334 \mathrm{E}-08 & 7.773 \mathrm{E}-08\end{array}$ $\begin{array}{llllllll}5.634 \mathrm{E}-07 & 1.688 \mathrm{E}-06 & 3.928 \mathrm{E}-0 & 1.166 \mathrm{E}-0 & 3.291 \mathrm{E}-05 & 9.791 \mathrm{E}-05 & 2.187 \mathrm{E}-04 & 2.625 \mathrm{E}-04\end{array}$

5.634E-07 1.688E-06 $3.928 \mathrm{E}-06 \quad 1.166 \mathrm{E}-05 \quad 3.291 \mathrm{E}-05 \quad 9.791 \mathrm{E}-05 \quad 2.187 \mathrm{E}-04 \quad 2.626 \mathrm{E}-04$ 
Individual Nuclide Dose Summed Over All Pathways

Parent Nuclide and Branch Fraction Indicated

Nuclide Parent BRF(i)

(j) (i)

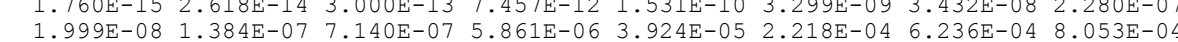

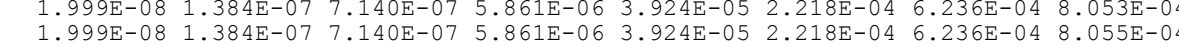

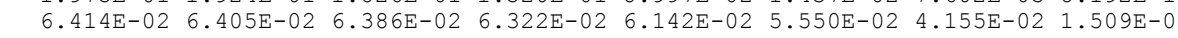
BRF(i) is the branch fraction of the parent nuclide.

$\$$ is used to indicate summation; the Greek sigma is not included in this font. 
1RESRAD, Version 6.21

$T^{1 / 2}$ Limit $=0.5$ year

02/22/2005 17:24 Page 26

Summary : CAU 224250 day-yr average worker scenario

File : CAU 224250 day-yr average.RAD

Individual Nuclide Soil Concentration

Parent Nuclide and Branch Fraction Indicated

ONuclide Parent BRF(i)

(j) (i)

$\overline{\mathrm{Am}-241} \overline{\mathrm{Am}-241} \overline{1.000 \mathrm{E}+00}$ $\begin{array}{lll}0 \mathrm{~Np}-237 & \mathrm{Am}-241 & 1.000 \mathrm{E}+00\end{array}$

$0 \mathrm{O}-233 \mathrm{Am}-241 \mathrm{1} 1.000 \mathrm{E}+00$

$\mathrm{OCs}-137$ Cs $-137 \quad 1.000 \mathrm{E}+00$

OEu-152 Eu-152 $7.208 \mathrm{E}-01$

Eu-152 Eu-152 2.792E-01

$\mathrm{Eu}-152$ \$S $(j):$

$\begin{array}{lll}\text { OGd-152 } & \mathrm{Eu}-152 & 2.792 \mathrm{E}-01 \\ \mathrm{OPu}-238 & \mathrm{Pu}-238 & 1.000 \mathrm{E}+00\end{array}$

OU-234 Pu-238 $1.000 \mathrm{E}+00$

$\mathrm{U}-234 \quad \mathrm{U}-234 \quad 1.000 \mathrm{E}+00$

$\begin{array}{lll}\mathrm{U}-234 & \mathrm{U}-238 & 1.000 \mathrm{E}+00 \\ \mathrm{U}-234 & \$ \mathrm{~S}(\mathrm{j}): & \end{array}$

$\begin{array}{ccc}U-234 & \$ S(j): & \\ 0 T h-230 & P u-238 & 1.000 E+00\end{array}$

$\begin{array}{ccc}0 \mathrm{Th}-230 & \mathrm{Pu}-238 & 1.000 \mathrm{E}+00 \\ \mathrm{Th}-230 & \mathrm{U}-234 & 1.000 \mathrm{E}+00\end{array}$

$\begin{array}{lll}\text { Th }-230 & \mathrm{U}-234 & 1.000 \mathrm{E}+00 \\ \mathrm{Th}-230 & \mathrm{U}-238 & 1.000 \mathrm{E}+00\end{array}$

$\begin{array}{lll}\text { Th }-230 & \$ S(j): & \\ 0 R a-226 & \text { Pu-238 } & 1.000 E+00\end{array}$

ORa 226 PU $1.000 \mathrm{E}+00$

$\mathrm{Ra}-226 \mathrm{U}-238 \quad 1.000 \mathrm{E}+00$

$\begin{array}{lll}\mathrm{Ra}-226 & \$ S(j): & 1.000 \mathrm{E}+00 \\ 0 \mathrm{~Pb}-210 & \mathrm{Pu}-238 & 1.000 \mathrm{E}+00\end{array}$

$\begin{array}{ccc}0 \mathrm{~Pb}-210 & \mathrm{Pu}-238 & 1.000 \mathrm{E}+00 \\ \mathrm{~Pb}-210 & \mathrm{U}-234 & 1.000 \mathrm{E}+00\end{array}$

$\begin{array}{lll}\mathrm{Pb}-210 & \mathrm{U}-234 & 1.000 \mathrm{E}+00 \\ \mathrm{~Pb}-210 & \mathrm{U}-238 & 1.000 \mathrm{E}+00\end{array}$

$\begin{array}{lll}\mathrm{Pb}-210 & \mathrm{U}-238 & 1.000 \mathrm{E}+00 \\ \mathrm{~Pb}-210 & \$ \mathrm{~S}(\mathrm{j}): & \\ 0 \mathrm{~Pb}-239 & \mathrm{Pu}-239 & 1.000 \mathrm{E}+00\end{array}$

$\begin{array}{lll}0 \mathrm{Pu}-239 & \mathrm{Pu}-239 & 1.000 \mathrm{E}+00\end{array}$

OU-235 Pu-239 $1.000 \mathrm{E}+00$

$\mathrm{U}-235$ \$S $(j):$

$\begin{array}{lll}\mathrm{OPa}-231 & \mathrm{Pu}-239 & 1.000 \mathrm{E}+00 \\ \mathrm{~Pa}-231 & \mathrm{U}-235 & 1.000 \mathrm{E}+00\end{array}$

$\mathrm{Pa}-231 \$ S(j)$

$\begin{array}{lllllll}0.000 \mathrm{E}+00 & 1.000 \mathrm{E}+00 & 3.000 \mathrm{E}+00 & \begin{array}{rlll}\mathrm{S}(j, \mathrm{j}), & \mathrm{pCi} / \mathrm{g}\end{array} & & \\ 1.000 \mathrm{E}+01 & 3.000 \mathrm{E}+01 & 1.000 \mathrm{E}+02 & 3.000 \mathrm{E}+02 & 1.000 \mathrm{E}+03\end{array}$

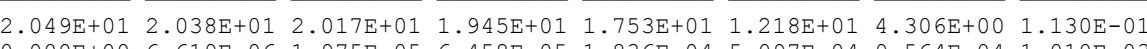

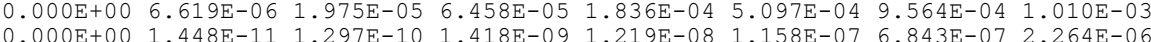

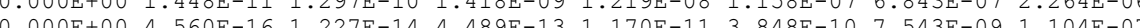

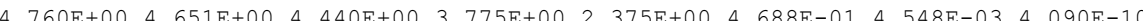
$\begin{array}{llllllll}1.773 \mathrm{E}+00 & 1.683 \mathrm{E}+00 & 1.417 \mathrm{E}+00 & 1.7753 \mathrm{E}+00 & 3.716 \mathrm{E}-01 & 9.697 \mathrm{E}-03 & 2.900 \mathrm{E}-07 & 4.242 \mathrm{E}-23\end{array}$ $\begin{array}{lllllllll}6.868 \mathrm{E}-01 & 6.520 \mathrm{E}-01 & 5.875 \mathrm{E}-01 & 4.080 \mathrm{E}-01 & 1.440 \mathrm{E}-01 & 3.756 \mathrm{E}-03 & 1.123 \mathrm{E}-07 & 1.643 \mathrm{E}-23\end{array}$ $2.460 \mathrm{E}+00 \quad 2.335 \mathrm{E}+00 \quad 2.104 \mathrm{E}+00 \quad 1.461 \mathrm{E}+00 \quad 5.156 \mathrm{E}-01 \quad 1.345 \mathrm{E}-02 \quad 4.023 \mathrm{E}-07 \quad 5.886 \mathrm{E}-23$ $\begin{array}{llllllll}0.000 \mathrm{E}+00 & 4.295 \mathrm{E}-15 & 1.224 \mathrm{E}-14 & 3.434 \mathrm{E}-14 & 6.678 \mathrm{E}-14 & 8.357 \mathrm{E}-14 & 8.256 \mathrm{E}-14 & 7.763 \mathrm{E}-14\end{array}$

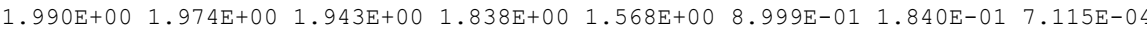

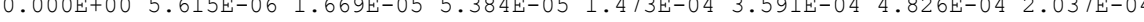

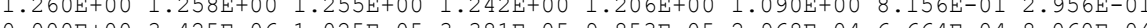

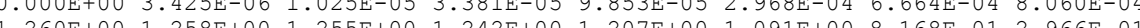

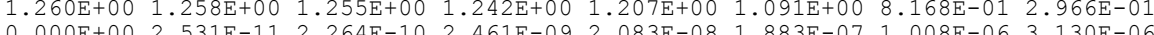

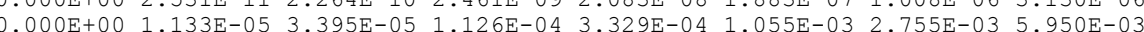
$0.000 \mathrm{E}+00.1 .542 \mathrm{E}-11 \quad 1.386 \mathrm{E}-10 \quad 1.529 \mathrm{E}-09 \quad 1.350 \mathrm{E}-08 \quad 1.402 \mathrm{E}-07 \quad 1.045 \mathrm{E}-06 \quad 6.226 \mathrm{E}-03$

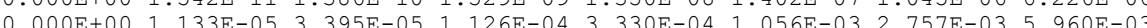

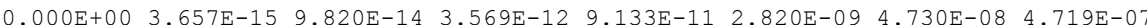

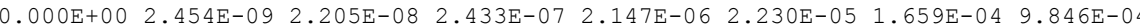

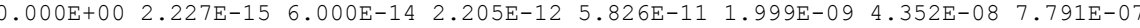

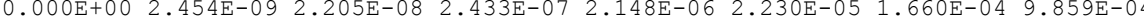

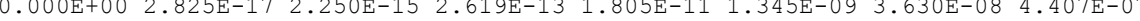

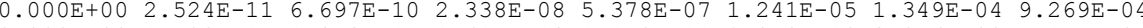

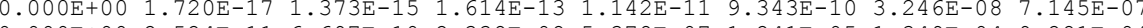

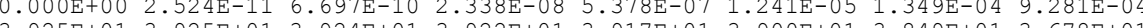

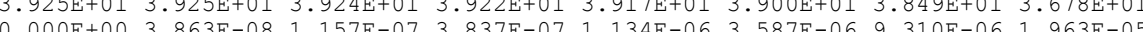
$\begin{array}{llllll}0 & \end{array}$

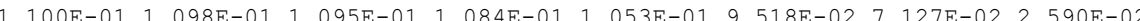

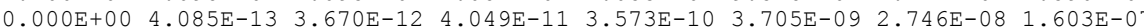

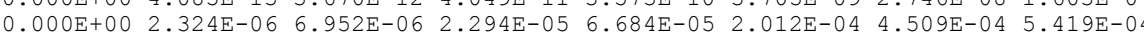

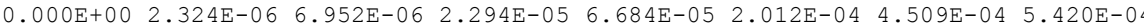


1RESRAD, Version $6.21 \quad T^{\frac{1}{2}}$ Limit $=0.5$ year $\begin{array}{ll}\text { Summary : CAU } 224 & 250 \text { day-yr average worker scenario } \\ \text { File } & \text { CAU } 224250 \text { day-yr average. RAD }\end{array}$

$02 / 22 / 2005 \quad 17: 24 \quad$ Page 27

Individual Nuclide Soil Concentration

Parent Nuclide and Branch Fraction Indicated

ONuclide Parent BRF(i)

(j) (i)

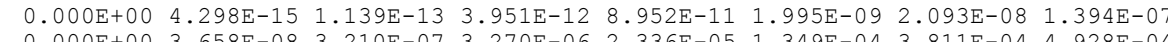

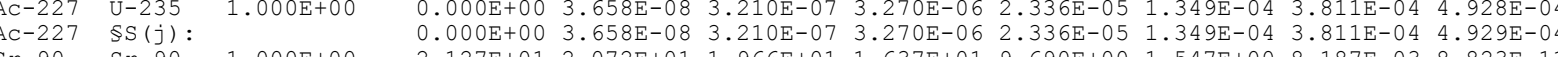
$\begin{array}{llllllll}2.127 \mathrm{E}+01 & 2.072 \mathrm{E}+01 & 1.966 \mathrm{E}+01 & 1.637 \mathrm{E}+01 & 9.690 \mathrm{E}+00 & 1.547 \mathrm{E}+00 & 8.187 \mathrm{E}-03 & 8.823 \mathrm{E}-11\end{array}$

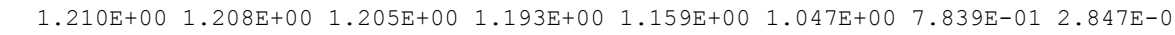
$B R F(i)$ is the branch fraction of the parent nuclide.

$\$$ is used to indicate summation; the Greek sigma is not included in this font. ORESCALC.EXE execution time $=2.28$ seconds 


\section{Exhibit 2}

\section{RESRAD Scenario A Hot Spot $100 \mathrm{~m}^{2}$}

(27 Pages) 
1RESRAD, Version 6.21 TT1/2 Limit $=0.5$ year $02 / 22 / 2005 \quad 17: 38$ Page 1 Summary : CAU 224250 day/yr worker scenario $100 \mathrm{~m}$ hotspot

Table of Contents

Part I: Mixture Sums and Single Radionuclide Guidelines

Dose Conversion Eactor (and Relate

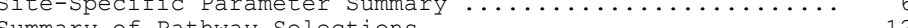
Contaminated Zone and Total Dose Summary $\ldots \ldots \ldots \ldots \ldots \ldots,{ }_{1}$ Total Dose Components Time $=0.000 \mathrm{E}+00$

.

Time $=1.000 \mathrm{E}+01$

Time $=3.000 \mathrm{E}+02$

Time $=1.000 \mathrm{E}+03$

Dose/Source Ratios Summed Over All Pathways $\ldots \ldots \ldots \ldots \ldots$ Single Radionuclide soil Guidelines...

Soil Concentration Per Muclide ........... 
1RESRAD, Version $6.21 \quad$ T1/2 Limit $=0.5$ year

Summary : CAU 224250 day/yr worker scenario $100 \mathrm{~m}$ hotspot

02/22/2005 17:38 Page 2

Dose Conversion Factor (and Related) Parameter Summary

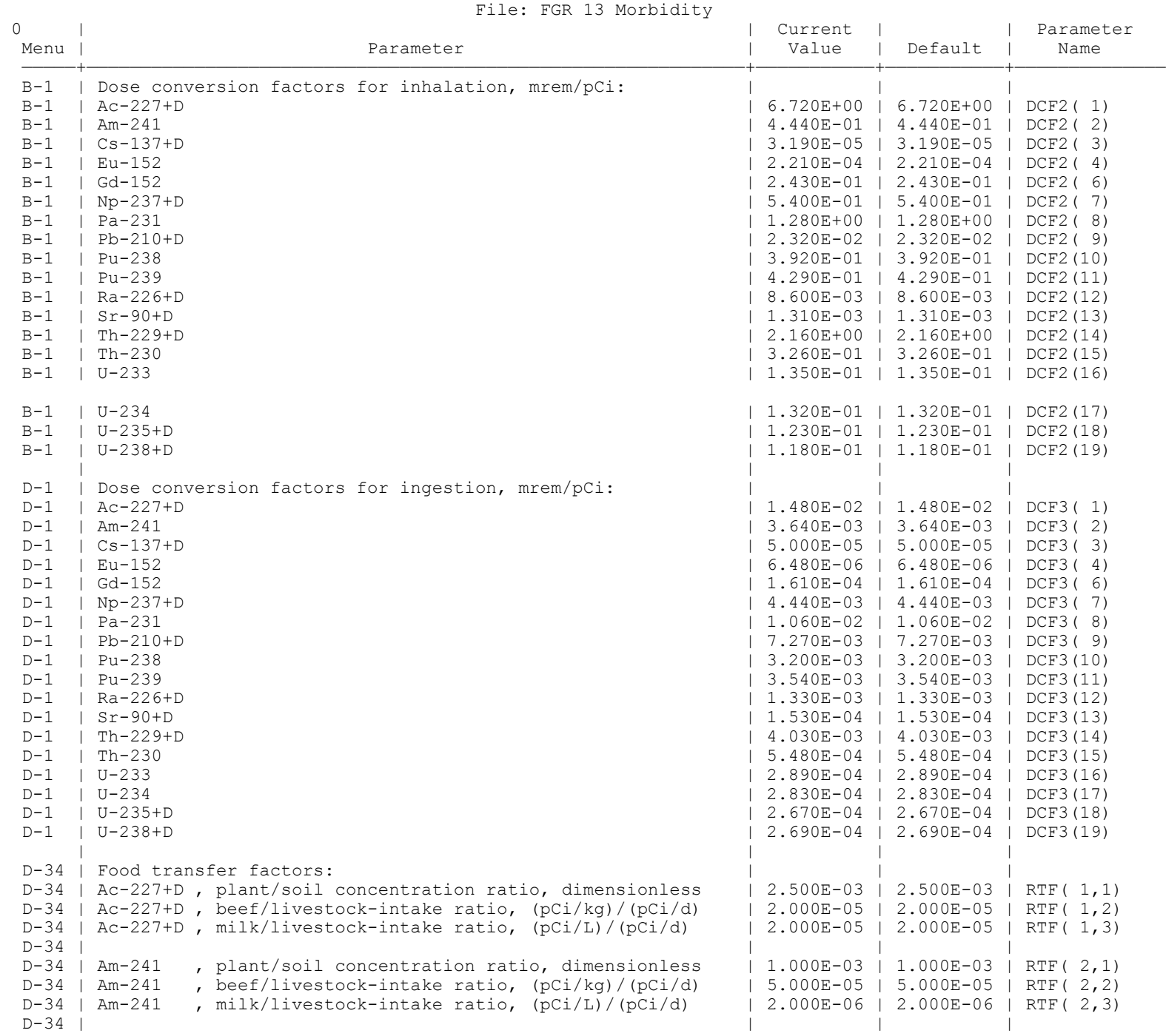


1RESRAD, Version $6.21 \quad T^{\frac{1}{2}}$ Limit $=0.5$ year $\quad 02 / 22 / 2005 \quad 17: 38$ Page 3

Summary : CAU 224250 day/yr worker scenario $100 \mathrm{~m}$ hotspot

Dose Conversion Factor (and Related) Parameter Summary (continued) File: FGR 13 Morbidity

Menu Paramete

\begin{tabular}{|c|c|c|}
\hline $\begin{array}{l}\text { Current } \\
\text { Value }\end{array}$ & Default & $\begin{array}{l}\text { Parameter } \\
\text { Name }\end{array}$ \\
\hline $4.000 \mathrm{E}-02$ & $14.000 \mathrm{E}-02$ & ( $\operatorname{RTF}(3,1)$ \\
\hline $3.000 \mathrm{E}-02$ & $3.000 \mathrm{E}-02$ & $\operatorname{RTE}(3,2)$ \\
\hline $8.000 \mathrm{E}-03$ & $8.000 \mathrm{E}-03$ & $\operatorname{RTF}(3,3)$ \\
\hline $2.500 \mathrm{E}-03$ & $2.500 \mathrm{E}-03$ & $\operatorname{RTF}(4,1)$ \\
\hline $2.000 \mathrm{E}-03$ & $2.000 \mathrm{E}-03$ & $\operatorname{RTF}(4,2)$ \\
\hline $2.000 \mathrm{E}-05$ & $2.000 \mathrm{E}-05$ & $\operatorname{RTE}(4,3)$ \\
\hline $2.500 \mathrm{E}-03$ & $2.500 \mathrm{E}-03$ & $\operatorname{RTF}(6,1)$ \\
\hline $2.000 \mathrm{E}-03$ & $2.000 \mathrm{E}-03$ & $\operatorname{RTF}(6,2)$ \\
\hline $2.000 \mathrm{E}-05$ & $2.000 \mathrm{E}-05$ & $\operatorname{RTF}(6,3)$ \\
\hline $2.000 \mathrm{E}-02$ & $2.000 \mathrm{E}-02$ & $\operatorname{RTF}(7,1)$ \\
\hline $1.000 \mathrm{E}-03$ & $1.000 \mathrm{E}-03$ & $\operatorname{RTF}(7,2)$ \\
\hline $5.000 \mathrm{E}-06$ & $5.000 \mathrm{E}-06$ & $\operatorname{RTF}(7,3)$ \\
\hline $1.000 \mathrm{E}-02$ & $1.000 \mathrm{E}-02$ & $\operatorname{RTF}(8,1)$ \\
\hline $5.000 \mathrm{E}-03$ & $5.000 \mathrm{E}-03$ & $\operatorname{RTF}(8,2)$ \\
\hline $5.000 \mathrm{E}-06$ & $5.000 \mathrm{E}-06$ & $\operatorname{RTF}(8,3)$ \\
\hline & 1 & । \\
\hline $1.000 \mathrm{E}-02$ & $1.000 \mathrm{E}-02$ & $\operatorname{RTF}(9,1)$ \\
\hline $8.000 \mathrm{E}-04$ & $8.000 \mathrm{E}-04$ & $\operatorname{RTF}(9,2)$ \\
\hline $3.000 \mathrm{E}-04$ & $3.000 \mathrm{E}-04$ & $\operatorname{RTE}(9,3)$ \\
\hline $1.000 \mathrm{E}-03$ & | & | \\
\hline $\begin{array}{l}1.000 \mathrm{E}-03 \\
1.000 \mathrm{E}-04\end{array}$ & $\begin{array}{r}1.000 \mathrm{E}-03 \\
1.000 \mathrm{E}-04\end{array}$ & $\begin{array}{l}\operatorname{RTH}(10,1) \\
\operatorname{RTF}(10,2)\end{array}$ \\
\hline $1.000 \mathrm{E}-06$ & $1.000 \mathrm{E}-06$ & $\operatorname{RTF}(10,3)$ \\
\hline $1.000 \mathrm{E}-03$ & $1.000 \mathrm{E}-03$ & $\operatorname{RTF}(11,1)$ \\
\hline $1.000 \mathrm{E}-04$ & $1.000 \mathrm{E}-04$ & $\operatorname{RTF}(11,2)$ \\
\hline $1.000 \mathrm{E}-06$ & $1.000 \mathrm{E}-06$ & $\operatorname{RTF}(11,3)$ \\
\hline & & \\
\hline & $4.000 \mathrm{E}-02$ & I RTF (12, \\
\hline $1.000 \mathrm{E}-0$ & $1.000 \mathrm{E}-03$ & RTF (12, \\
\hline $1.000 \mathrm{E}-03$ & $1.000 \mathrm{E}-03$ & $\operatorname{RTE}(12,3)$ \\
\hline $3.000 \mathrm{E}-01$ & $3.000 \mathrm{E}-01$ & $\operatorname{RTF}(13$, \\
\hline $8.000 \mathrm{E}-03$ & $8.000 \mathrm{E}-03$ & $\operatorname{RTF}(13,2)$ \\
\hline $2.000 \mathrm{E}-03$ & $2.000 \mathrm{E}-03$ & $\operatorname{RTE}(13,3)$ \\
\hline $1.000 \mathrm{E}-03$ & $1.000 \mathrm{E}-03$ & $\operatorname{RTF}(14$, \\
\hline $1.000 \mathrm{E}-04$ & $1.000 \mathrm{E}-04$ & $\operatorname{RTF}(14,2)$ \\
\hline $5.000 \mathrm{E}-06$ & $5.000 \mathrm{E}-06$ & $\operatorname{RTE}(14,3)$ \\
\hline & $1.000 E-03$ & RTF $(15,1)$ \\
\hline $\begin{array}{l}1.000 \mathrm{E}-03 \\
1.000 \mathrm{E}-04\end{array}$ & $1.000 \mathrm{E}-03$ & 苜TF $(15,1)$ \\
\hline $.000 \mathrm{E}-06$ & $5.000 \mathrm{E}-06$ & RTE $(15,3)$ \\
\hline & | & \\
\hline
\end{tabular}

D-34 | Cs-137+D, plant/soil concentration ratio, dimensionless D-34 Cs-137+D, beef/livestock-intake ratio, $(\mathrm{pCi} / \mathrm{kg}) /(\mathrm{pCi} / \mathrm{d})$ (pCi/L)/ ( $\mathrm{pCi} / \mathrm{d})$

D-34 Eu-152, plant/soil concentration ratio, dimensionless D-34 | Eu-152, beef/livestock-intake ratio, (pCi/kg)/(pCi/d) D- -34

D-34 Gd-152, plant/soil concentration ratio, dimensionless D-34 Gd-152, beef/livestock-intake ratio, $(\mathrm{pCi} / \mathrm{kg}) /(\mathrm{pCi} / \mathrm{d})$ D-34 | Gd-152, milk/livestock-intake ratio, (pCi/L)/(pCi/d) D- 34

D-34 Np-237+D, plant/soil concentration ratio, dimensionless D-34 Np-237+D, beef/livestock-intake ratio, (pCi/kg)/(pCi/d) D-34 Np-237+D, milk/livestock-intake ratio, (pCi/L)/(pCi/d) D -34

D-34 $\mathrm{Pa}-231$, plant/soil concentration ratio, dimensionless D-34 $\mathrm{Pa}-231$, beef/livestock-intake ratio, (pCi/ $\mathrm{kg}) /(\mathrm{pCi} / \mathrm{d})$

D-34 Pb-210+D, plant/soil concentration ratio, dimensionless D-34 Pb-210tD, beef/livestock-intake ratio, $(\mathrm{pCi} / \mathrm{kg}) /(\mathrm{pCl} / \mathrm{d})$ D-34 Pb-210+D, milk/livestock-intake ratio, (pCi/L)/(pCi/d) $D-34$

D-34 $\mathrm{Pu}-238$, plant/soil concentration ratio, dimensionless D-34 Pu-238, beef/livestock-intake ratio, (pCi/kg)/(pCi/d) D-34 -34 (1

Pu-239, plant/soil concentration ratio, dimensionless D-34 $\mid \mathrm{Pu}-239$, beef/livestock-intake ratio, $(\mathrm{pCi} / \mathrm{kg}) /(\mathrm{pCi} / \mathrm{d})$ D -34

D-34 Ra-226+D, plant/soil concentration ratio, dimensionless D-34 Ra-226+D, beef/livestock-intake ratio, (pCi/kg)/(pCi/d) D-34 | Ra-226+D, milk/livestock-intake ratio, (pCi/L)/(pCi/d) $\mathrm{D}-34$

D-34 Sr-90+D, plant/soil concentration ratio, dimensionless

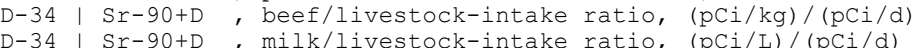
D -34

4 Th-229+D, plant/soil concentration ratio, dimensionless $\mathrm{D}-34$ Th-229+D , beef/livestock-intake ratio, (pCi/kg)/(pCi/d) D-34 Th-229+D, milk/livestock-intake ratio, (pCi/L)/(pCi/d) D-34

D-34 Th-230, plant/soil concentration ratio, dimensionless D-34 Th-230, beef/livestock-intake ratio, (pCi/kg)/(pCi/d) D-34 | Th-230, milk/livestock-intake ratio, (pCi/L)/(pCi/d) 
1RESRAD, Version 6.21 T3/2 Limit $=0.5$ year $02 / 22 / 2005 \quad 17: 38$ Page 4 Summary : CAU 224250 day/yr worker scenario $100 \mathrm{~m}$ hotspot

Dose Conversion Factor (and Related) Parameter Summary (continued)

\begin{tabular}{|c|c|c|c|c|c|}
\hline Menu & & Parameter & $\begin{array}{l}\text { Current } \\
\text { Value }\end{array}$ & Default & $\begin{array}{l}\text { Parameter } \\
\text { Name }\end{array}$ \\
\hline D-34 & $\mathrm{U}-233$ & , plant/soil concentration ratio, dimensionless & $12.500 \mathrm{E}-03$ & $2.500 \mathrm{E}-03$ & $\operatorname{RTF}(16,1)$ \\
\hline$D-34$ & & ef/livestock-intake ratio, (pCi/k & $3.400 \mathrm{E}-04$ & $3.400 \mathrm{E}-04$ & $\operatorname{RTF}(16,2)$ \\
\hline $\begin{array}{l}D-34 \\
D-34\end{array}$ & $\mathrm{U}-233$ & milk/livestock-intake ratio, (pCi/L)/(pCi & $6.000 \mathrm{E}-04$ & $6.000 \mathrm{E}-04$ & $\operatorname{RTE}(16,3)$ \\
\hline$D-34$ & $\mathrm{U}-234$ & , plant/soil concentration ratio, dimensionless & $2.500 \mathrm{E}-03$ & $2.500 \mathrm{E}-03$ & $\operatorname{RTF}(17,1)$ \\
\hline$D-34$ & $\mathrm{U}-234$ & , beef/livestock-intake ratio, $(\mathrm{pCi} / \mathrm{kg}) /(\mathrm{pCi} / \mathrm{d})$ & $3.400 \mathrm{E}-04$ & $3.400 \mathrm{E}-04$ & $\operatorname{RTE}(17,2)$ \\
\hline $\begin{array}{l}D-34 \\
D-34\end{array}$ & $\mathrm{U}-234$ & milk/livestock-intake ratio, $(\mathrm{pCi} / \mathrm{L}) /(\mathrm{pCi} / \mathrm{d})$ & $6.000 \mathrm{E}-04$ & $6.000 \mathrm{E}-04$ & $\operatorname{RTF}(17,3)$ \\
\hline$D-34$ & $\mathrm{U}-235+\mathrm{D}$ & , plant/soil concentration ratio, dimensionless & $2.500 \mathrm{E}-03$ & $2.500 \mathrm{E}-03$ & $\operatorname{RTF}(18,1)$ \\
\hline$D-34$ & $\mathrm{U}-235+\mathrm{D}$ & , beef/livestock-intake ratio, (pCi/kg)/(pC & $3.400 \mathrm{E}-04$ & $3.400 \mathrm{E}-04$ & $\operatorname{RTF}(18,2)$ \\
\hline $\begin{array}{l}D-34 \\
D-34\end{array}$ & U-235+D & , milk/livestock-intake ratio, (pCi/L)/(pCi/ & $6.000 \mathrm{E}-04$ & $6.000 \mathrm{E}-04$ & $\operatorname{RTE}(18,3)$ \\
\hline$D-34$ & U-238+D & , plant/soil concentration ratio, dimensionless & $2.500 \mathrm{E}-03$ & $2.500 \mathrm{E}-03$ & $\operatorname{RTF}(19,1)$ \\
\hline D-34 & $\mathrm{U}-238+\mathrm{D}$ & , beef/livestock-intake ratio, (pCi/k & $3.400 \mathrm{E}-04$ & $3.400 \mathrm{E}-04$ & $\operatorname{RTE}(19,2)$ \\
\hline$D-34$ & $\mathrm{U}-238+\mathrm{D}$ & milk/livestock-intake ratio, $(\mathrm{pCi} / \mathrm{L}) /(\mathrm{pCi} / \mathrm{d})$ & $6.000 \mathrm{E}-04$ & $6.000 \mathrm{E}-04$ & $\operatorname{RTE}(19,3)$ \\
\hline D-5 & Bioaccum & ulation factors, fresh water, L/kg: & & & \\
\hline D-5 & Ac-227+D & , fish & $1.500 \mathrm{E}+01$ & $1.500 \mathrm{E}+01$ & $\operatorname{BIOFAC}(1,1)$ \\
\hline $\begin{array}{l}D-5 \\
D-5\end{array}$ & $A C-227+D$ & , crustacea and mollusks & $1.000 \mathrm{E}+03$ & $1.000 \mathrm{E}+03$ & $\operatorname{BIOFAC}(1,2)$ \\
\hline D-5 & $\mathrm{Am}-241$ & , fish & $3.000 \mathrm{E}+01$ & $3.000 \mathrm{E}+01$ & $\operatorname{BIOFAC}(2,1)$ \\
\hline $\begin{array}{l}D-5 \\
D-5\end{array}$ & $\mathrm{Am}-241$ & crustacea and mollusks & $1.000 \mathrm{E}+03$ & $1.000 \mathrm{E}+03$ & $\operatorname{BIOFAC}(2,2)$ \\
\hline$D-5$ & Cs $-137+D$ & , fish & $2.000 \mathrm{E}+03$ & $2.000 \mathrm{E}+03$ & $\operatorname{BIOFAC}(3,1)$ \\
\hline $\begin{array}{l}D-5 \\
D-5\end{array}$ & $\mathrm{Cs}-137+\mathrm{D}$ & , crustacea and mollusks & $1.000 \mathrm{E}+02$ & $1.000 \mathrm{E}+02$ & $\operatorname{BIOFAC}(3,2)$ \\
\hline$D-5$ & Eu-152 & , fish & $5.000 \mathrm{E}+01$ & $5.000 \mathrm{E}+01$ & $\operatorname{BIOFAC}(4,1)$ \\
\hline $\begin{array}{l}D-5 \\
D-5\end{array}$ & Eu-152 & , crustacea and mollusks & $1.000 \mathrm{E}+03$ & $1.000 \mathrm{E}+03$ & $\operatorname{BIOFAC}(4,2)$ \\
\hline D-5 & Gd-152 & , fish & $2.500 \mathrm{E}+01$ & $2.500 \mathrm{E}+01$ & $\operatorname{BIOFAC}(6,1)$ \\
\hline$D-5$ & $\mathrm{Gd}-152$ & , crustacea and mollusks & $1.000 \mathrm{E}+03$ & $1.000 \mathrm{E}+03$ & $\operatorname{BIOFAC}(6,2)$ \\
\hline D-5 & & & & & \\
\hline D-5 & I $N p-237+D$ & , fish & 3.00 & 3.00 & $\operatorname{BIOFAC}(7,1)$ \\
\hline $\begin{array}{l}D-5 \\
D-5\end{array}$ & $\mathrm{~Np}-237+\mathrm{D}$ & , crustacea and mollusks & $4.000 \mathrm{E}+02$ & $4.000 \mathrm{E}+02$ & $\operatorname{BIOFAC}(7,2)$ \\
\hline D-5 & $\mathrm{Pa}-231$ & , fist & 1.0 & $0 E+01$ & $\operatorname{BIOFAC}(8,1)$ \\
\hline $\begin{array}{l}D-5 \\
D-5\end{array}$ & $\mathrm{~Pa}-231$ & crustacea and mollusks & $1.100 \mathrm{E}+02$ & $1.100 \mathrm{E}+02$ & $\operatorname{BIOFAC}(8,2)$ \\
\hline & $\mathrm{Pb}-210+\mathrm{D}$ & , fish & $3.000 \mathrm{E}+02$ & $3.000 \mathrm{E}+02$ & BIOFAC ( \\
\hline $\begin{array}{l}D-5 \\
D-5\end{array}$ & $\mathrm{~Pb}-210+\mathrm{D}$ & , crustacea and mollusks & $1.000 \mathrm{E}+02$ & $1.000 \mathrm{E}+02$ & $\operatorname{BIOFAC}(9,2)$ \\
\hline $\begin{array}{l}D-5 \\
D-5\end{array}$ & $\mathrm{Pu}-238$ & , fi & 3.0 & & $\mathrm{AC}(1$ \\
\hline $\begin{array}{l}D-5 \\
D-5\end{array}$ & $\mathrm{Pu}-238$ & , crustacea and mollusks & $1.000 \mathrm{E}+02$ & $1.000 \mathrm{E}+02$ & $\operatorname{BIOFAC}(10,2)$ \\
\hline D-5 & & & & & \\
\hline D-5 & $\mathrm{Pu}-239$ & , fis & 13.0 & 3. & $\operatorname{BIOFAC}(11,1)$ \\
\hline $\begin{array}{l}\text { D-5 } \\
\text { D-5 }\end{array}$ & Pu-239 & , crustacea and mollusks & $1.000 \mathrm{E}+02$ & $1.000 \mathrm{E}+02$ & $\operatorname{BIOFAC}(11,2)$ \\
\hline D-5 & $\mathrm{Ra}-226+\mathrm{D}$ & , fish & 5.0 & $\mathrm{DE}+01$ & $\operatorname{BIOFAC}(12,1)$ \\
\hline D-5 & $\mathrm{Ra}-226+\mathrm{D}$ & , crustacea and mollusks & $2.500 \mathrm{E}+02$ & $2.500 \mathrm{E}+02$ & $\operatorname{BIOFAC}(12,2)$ \\
\hline
\end{tabular}


Summary : CAU 224250 day/yr worker scenario 100m hotspot

File : cau224 $250100 \mathrm{~m}$ parcel. RAD

Dose Conversion Factor (and Related) Parameter Summary (continued)

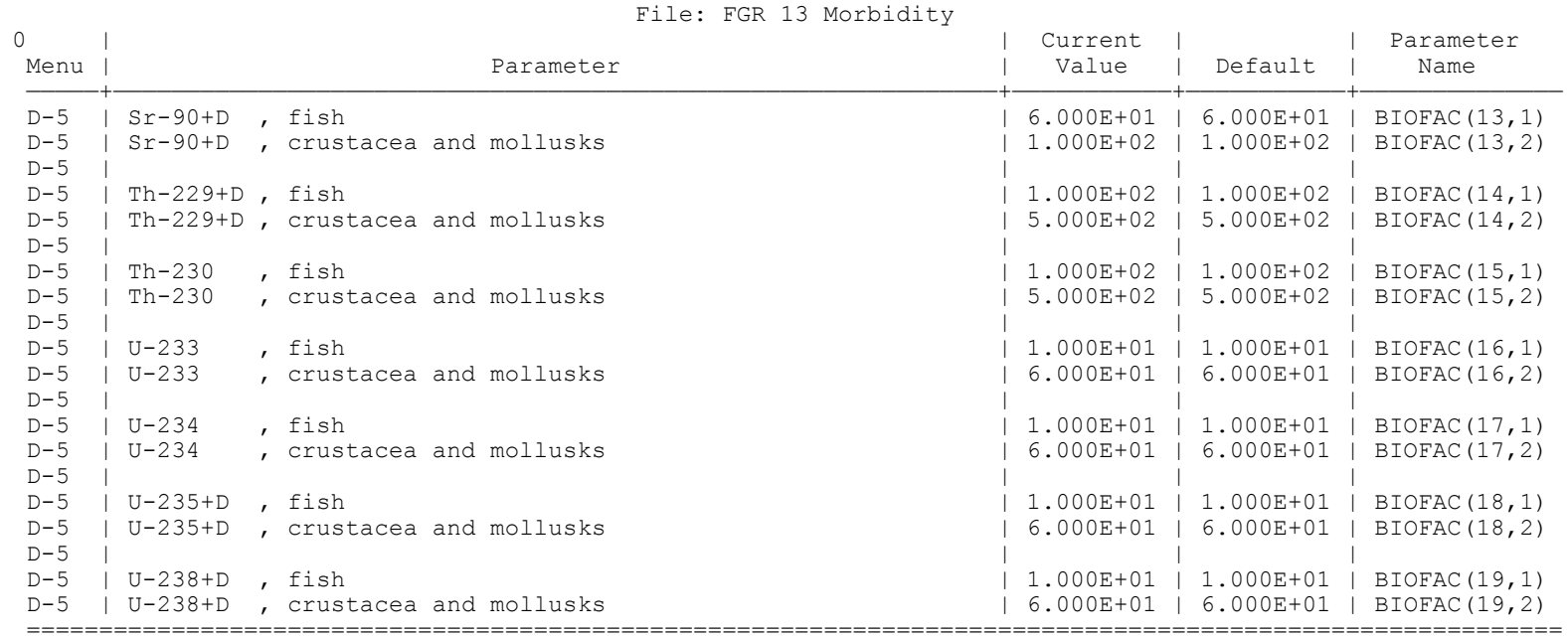




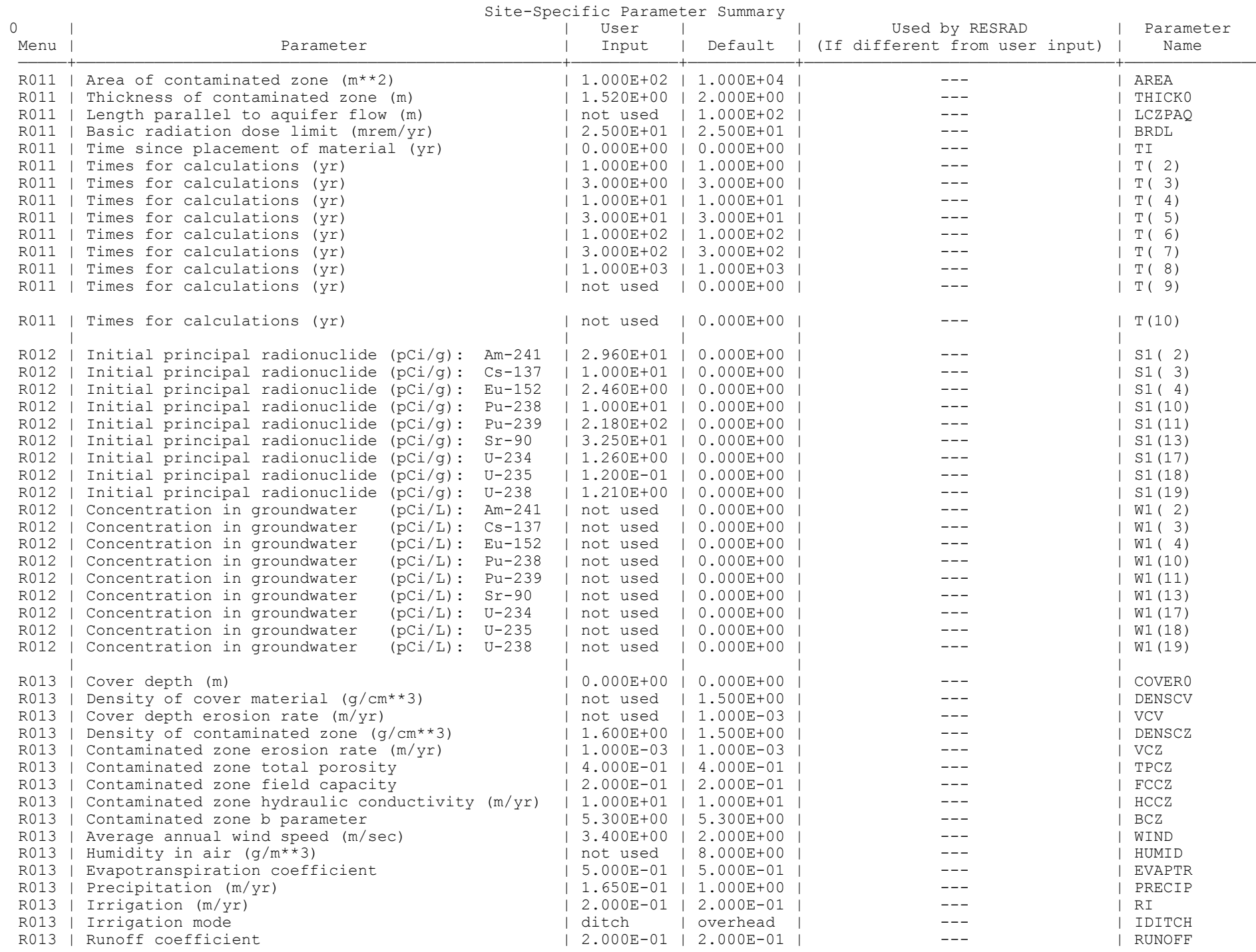


1RESRAD, Version $6.21 \quad T^{3} / 2$ Limit $=0.5$ year Summary : CAU 224250 day/yr worker scenario $100 \mathrm{~m}$ hotspot

Site-Specific Parameter Summary (continued)

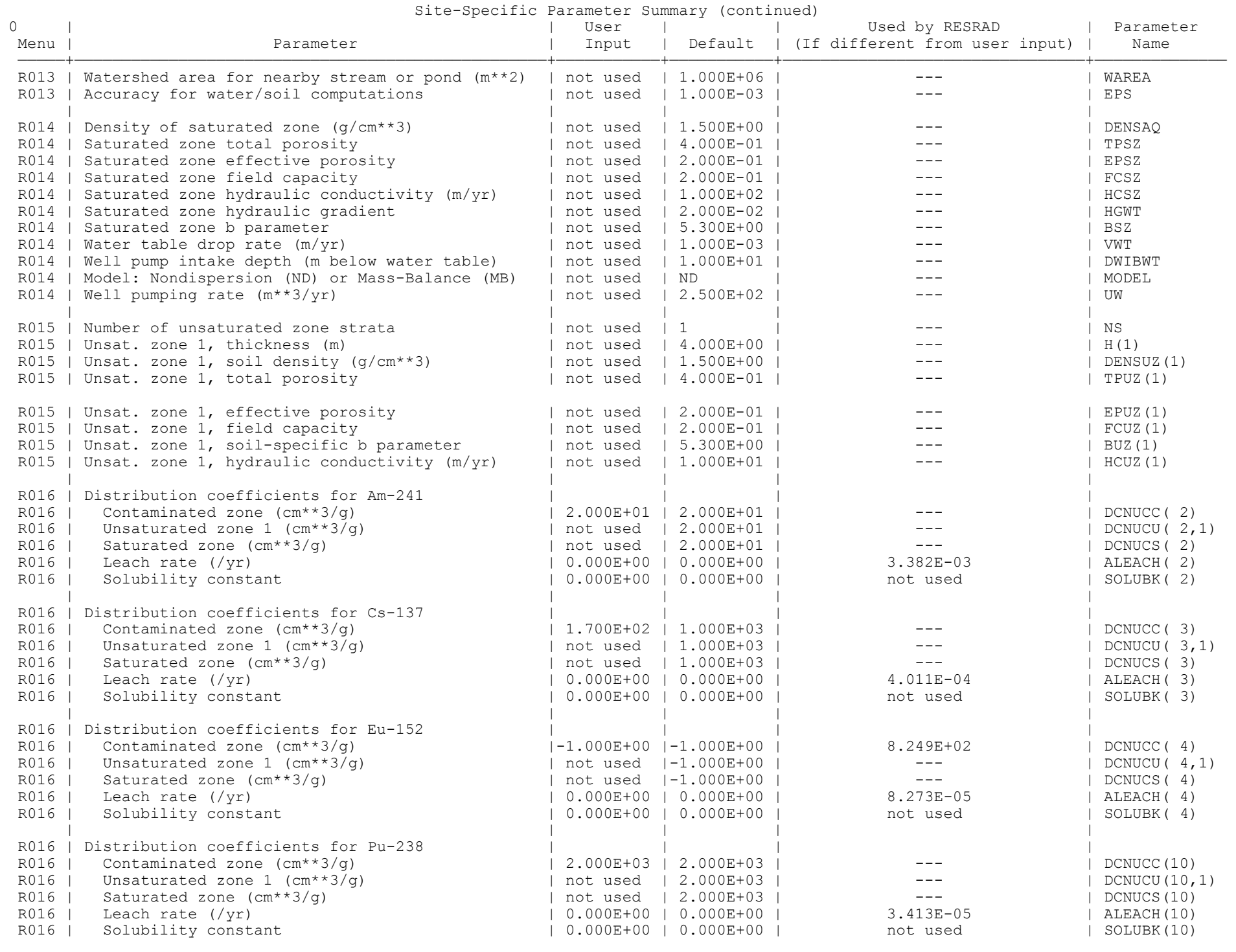


1RESRAD, Version $6.21 \quad T 3 / 2$ Limit $=0.5$ year Summary : CAU 224250 day/yr worker scenario $100 \mathrm{~m}$ hotspot

Site-Specific Parameter Summary (continued)

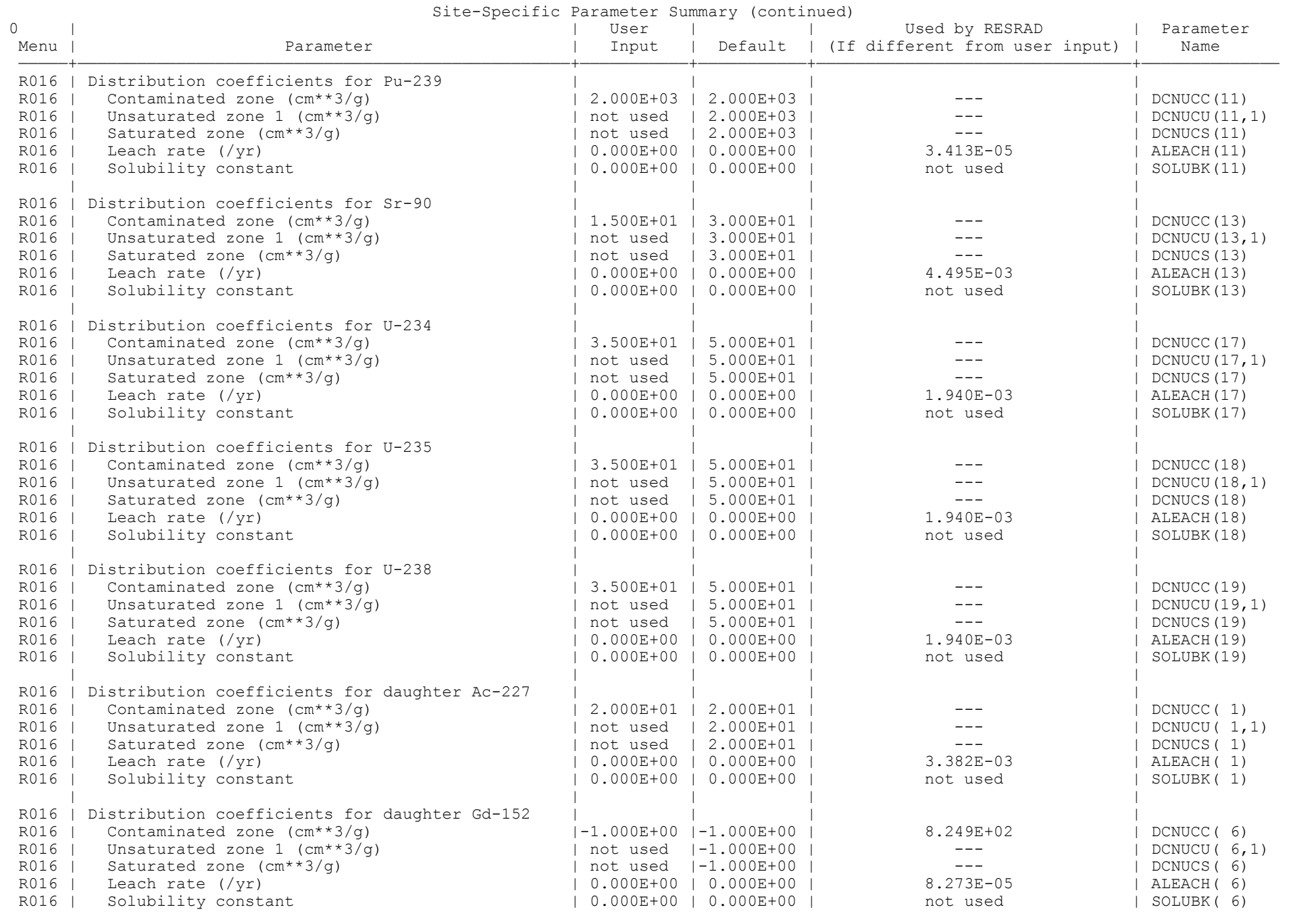


1RESRAD, Version $6.21 \quad T^{3} / 2$ Limit $=0.5$ year

Summary : CAU 224250 day/yr worker scenario $100 \mathrm{~m}$ hotspot

Site-Specific Parameter Summary (continued)

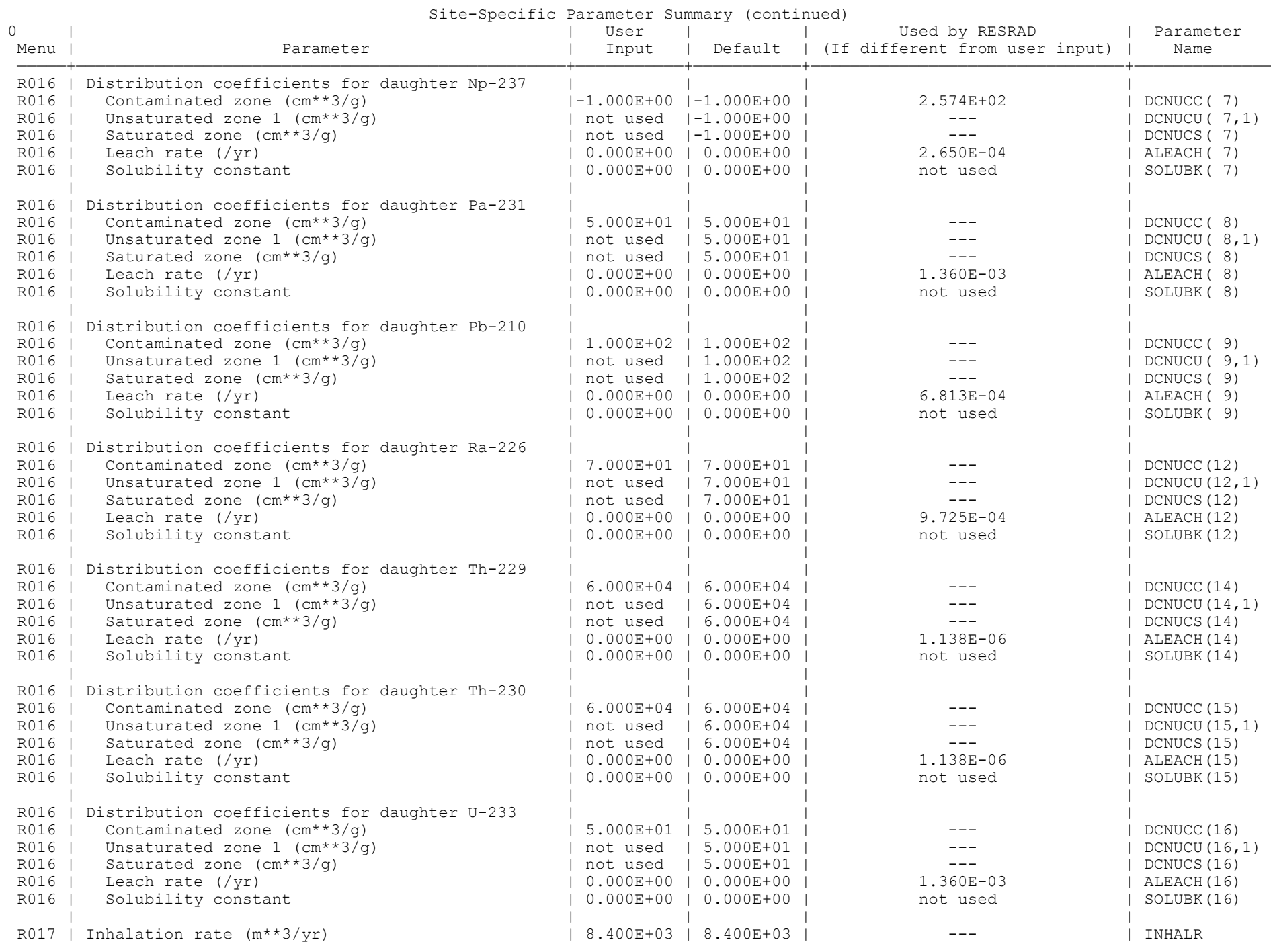


1RESRAD, Version $6.21 \quad T^{3} / 2$ Limit $=0.5$ year Summary : CAU 224250 day/yr worker scenario $100 \mathrm{~m}$ hotspot

Site-Specific Parameter Summary (continued)

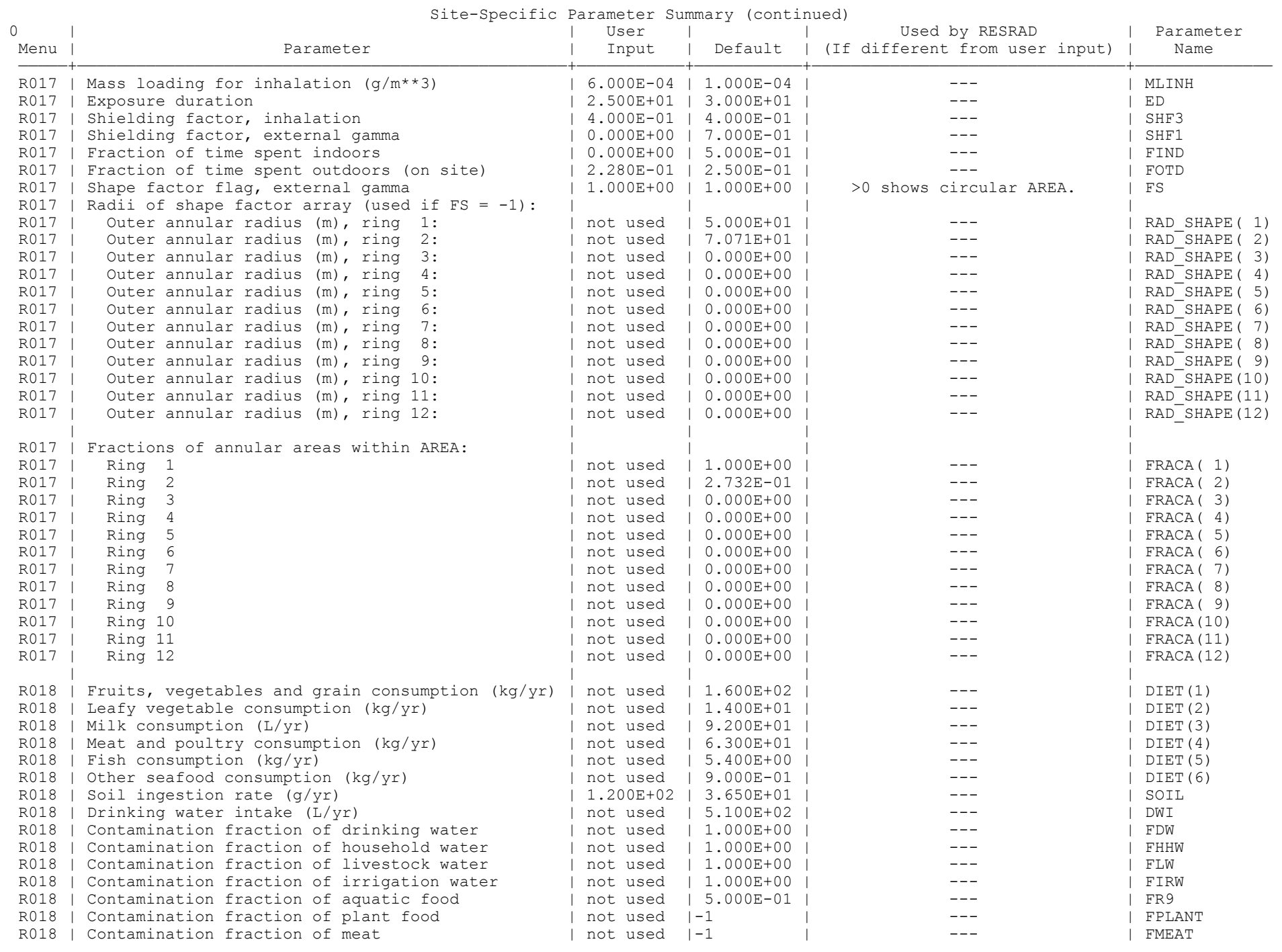




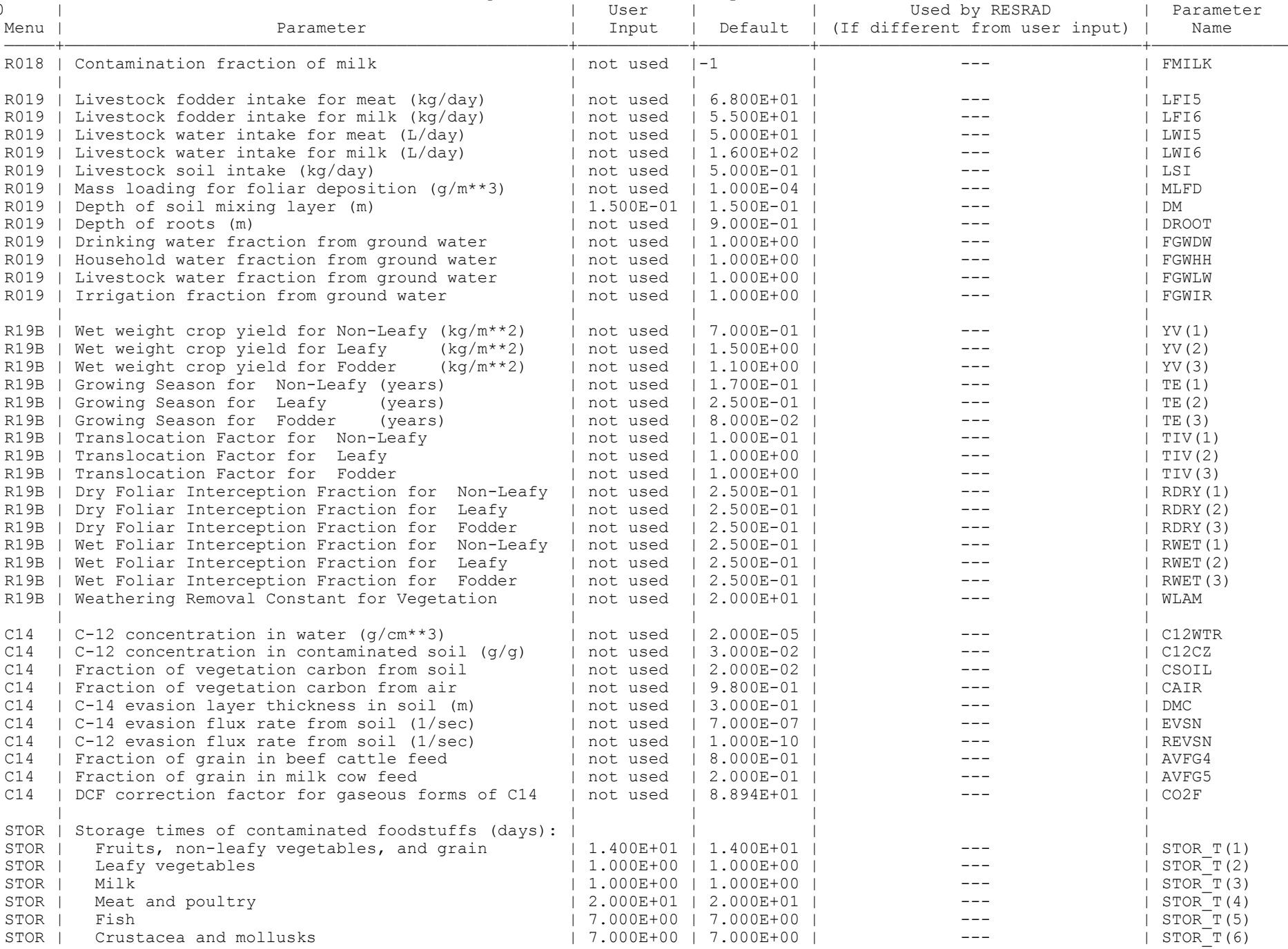


1RESRAD, Version $6.21 \quad \mathrm{~T} \frac{1}{2}$ Limit $=0.5$ year

Summary : CAU 224250 day/yr worker scenario $100 \mathrm{~m}$ hotspot

02/22/2005 17:38 Page 12

Site-Specific Parameter Summary (continued)

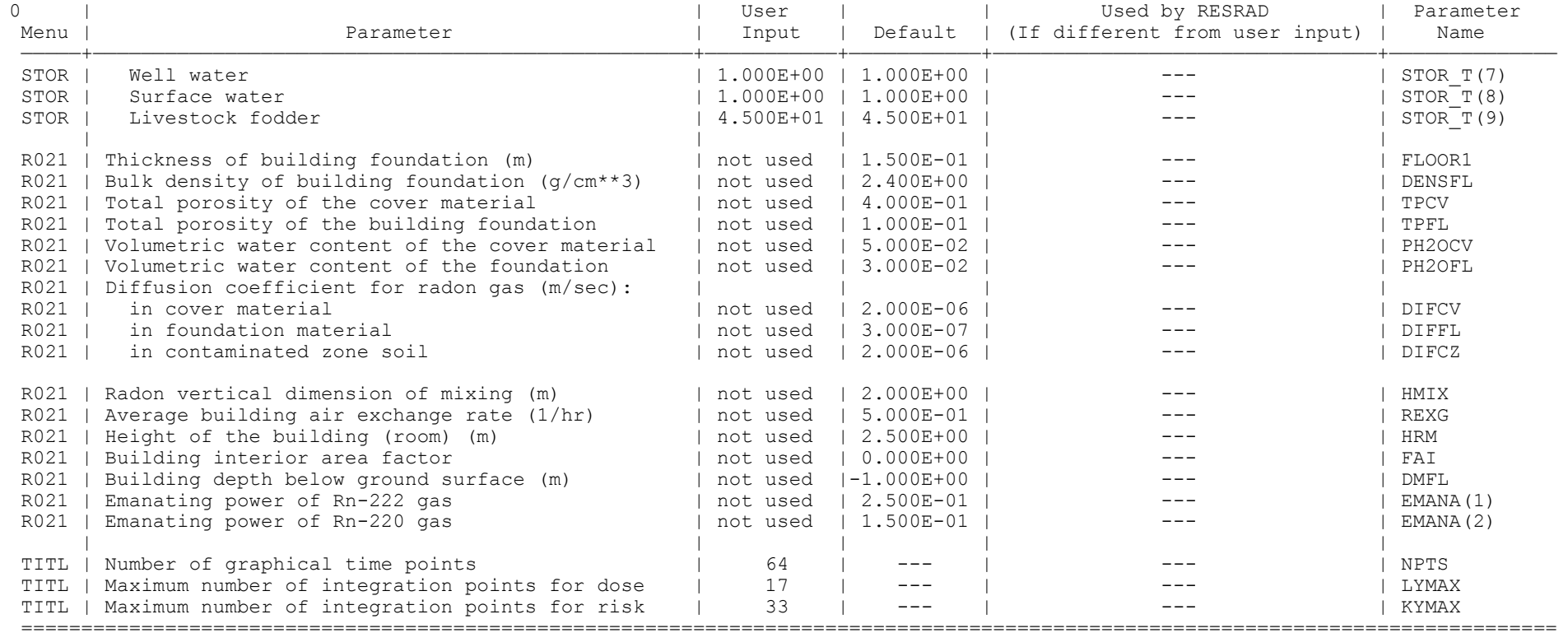

Summary of Pathway Selections

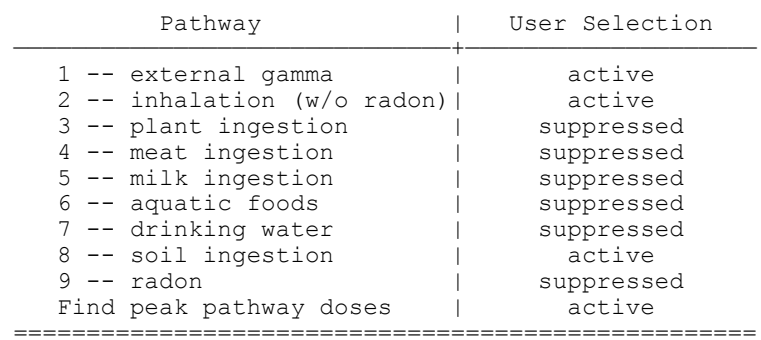


1RESRAD, Version $6.21 \quad T^{3 / 2}$ Limit $=0.5$ year $02 / 22 / 2005 \quad 17: 38$ Page 13 Summary : CAU 224250 day/yr worker scenario $100 \mathrm{~m}$ hotspot

Contaminated Zone Dimensions

Area: 100.00 square meters

Thickness:

over Depth:

1.52 meters

\begin{tabular}{cr} 
Initial Soil & \\
\hline $\mathrm{Am}-241$ & $2.960 \mathrm{E}+01$ \\
$\mathrm{Cs}-137$ & $1.000 \mathrm{E}+01$ \\
$\mathrm{Eu}-152$ & $2.460 \mathrm{E}+00$ \\
$\mathrm{Pu}-238$ & $1.000 \mathrm{E}+01$ \\
$\mathrm{Pu}-239$ & $2.180 \mathrm{E}+02$ \\
$\mathrm{Sr}-90$ & $3.250 \mathrm{E}+01$ \\
$\mathrm{U}-234$ & $1.260 \mathrm{E}+00$ \\
$\mathrm{U}-235$ & $1.200 \mathrm{E}-01$ \\
$\mathrm{U}-238$ & $1.210 \mathrm{E}+00$
\end{tabular}

Total Dose TDOSE(t), mrem/yr

Basic Radiation Dose Limit $=2.500 \mathrm{E}+01 \mathrm{mrem} / \mathrm{yr}$

Total Mixture Sum $M(t)=$ Fraction of Basic Dose Limit Received at Time (t)

$t$ (years): $\begin{array}{lllllllll}0.000 \mathrm{E}+00 & 1.000 \mathrm{E}+00 & 3.000 \mathrm{E}+00 & 1.000 \mathrm{E}+01 & 3.000 \mathrm{E}+01 & 1.000 \mathrm{E}+02 & 3.000 \mathrm{E}+02 & 1.000 \mathrm{E}+03\end{array}$

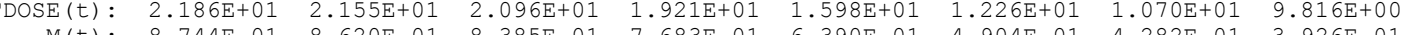
$\begin{array}{llllllllll}M(t): & 8.744 \mathrm{E}-01 & 8.620 \mathrm{E}-01 & 8.385 \mathrm{E}-01 & 7.683 \mathrm{E}-01 & 6.390 \mathrm{E}-01 & 4.904 \mathrm{E}-01 & 4.282 \mathrm{E}-01 & 3.926 \mathrm{E}-01\end{array}$ 
$T^{1 / 2}$ Limit $=0.5$ year

02/22/2005 17:38 Page 14

: CAU $224250 \mathrm{day} / \mathrm{yr}$ worker scenario $100 \mathrm{~m}$ hotspot
: cau224 $250100 \mathrm{~m}$ parcel. RAD

Total Dose Contributions TDOSE (i,p,t) for Individual Radionuclides (i) and Pathways (p) As mrem/yr and Fraction of Total Dose At $t=0.000 \mathrm{E}+00$ years

\begin{tabular}{|c|c|c|c|c|c|c|c|c|c|c|c|c|c|c|}
\hline \multirow{2}{*}{$\begin{array}{l}\text { Radio- } \\
\text { Nuclide }\end{array}$} & \multicolumn{2}{|c|}{ Ground } & \multicolumn{2}{|c|}{ Inhalation } & \multicolumn{2}{|c|}{$\begin{array}{l}\text { Radont } \\
\text { Radont }\end{array}$} & \multicolumn{2}{|c|}{$\begin{array}{l}\text { Plant } \\
\text { Plant }\end{array}$} & \multicolumn{2}{|c|}{$\begin{array}{l}\text { Sadon) } \\
\text { Meat }\end{array}$} & \multicolumn{2}{|c|}{ Milk } & \multicolumn{2}{|l|}{ Soil } \\
\hline & $\mathrm{m} / \mathrm{yr}$ & ict & mrem/yr & ract & mrem/yr & fract & mrem/yr & frac & mrem/yr & frac & mren & fract & mrem, & frac \\
\hline & & & & & & & & & & & & & & \\
\hline & & & & & & & & & & & & & & \\
\hline & & & & & & & & & & & & & & \\
\hline & & & & & & & & & & & & & & \\
\hline & & & & & & & & & & & & & & \\
\hline & & & & & & & & & & & & & & \\
\hline & & & & & & & & & & & & & & \\
\hline & & & & & & & & & & & & & & \\
\hline 250 & 2 & 15 & -02 & & & & & & & & & & & \\
\hline & 100 & 349 & +00 & 503 & +00 & 000 & $E+00$ & 000 & $0 \mathrm{E}+00$ & 000 & $0 E+00$ & 000 & $509 \mathrm{E}+00$ & 0.1148 \\
\hline
\end{tabular}

Total Dose Contributions TDOSE (i,p,t) for Individual Radionuclides (i) and Pathways (p) As mrem/yr and Fraction of Total Dose At $t=0.000 \mathrm{E}+00$ years

0 $\begin{array}{ll}\text { Radio- } & \text { Water } \\ \text { Nuclide } & \text { mrem/yr fract. }\end{array}$ Fish Radon Plant

Meat mrem/yr fract. mrem/yr fract. $0.000 \mathrm{E}+00 \quad 0.0000$ $0.000 \mathrm{E}+00 \quad 0.0000$ $\begin{array}{lll}\mathrm{Am}-241 & 0.000 \mathrm{E}+00 & 0.0000\end{array}$ $\begin{array}{lll}\mathrm{Cs}-137 & 0.000 \mathrm{E}+00 & 0.0000 \\ \mathrm{Eu}-152 & 0.000 \mathrm{E}+00 & 0.0000\end{array}$ $\begin{array}{lll}\mathrm{Pu}-238 & 0.000 \mathrm{E}+00 & 0.0000 \\ \mathrm{Pu}-239 & 0.000 \mathrm{E}+00 & 0.0000\end{array}$ $\begin{array}{lll}\mathrm{Sr}-90 & 0.000 \mathrm{E}+00 & 0.0000\end{array}$ $\mathrm{J}-234 \quad 0.000 \mathrm{E}+00 \quad 0.0000$ $.000 \mathrm{E}+00 \quad 0.0000$ $0.000 \mathrm{E}+00 \quad 0.0000$

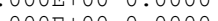
$0.000 \mathrm{E}+00 \quad 0.000$ $\mathrm{U}-238 \quad 0.000 \mathrm{E}+00 \quad 0.0000$ $0.000 \mathrm{E}+00.0000 \quad 0.000 \mathrm{E}+00 \quad 0.0000$ $\begin{array}{ll}0.000 \mathrm{E}+00 & 0.000 \\ ========= & ===== \\ 0.000 \mathrm{E}+00 & 0.0000\end{array}$ mrem/yr fract. mrem/yr fract. $\frac{\text { Milk }}{\text { mrem/yr fract. }}$ $\begin{array}{lllll}0.000 E+00 & 0.0000 & 0.000 \mathrm{E}+00 & 0.0000\end{array}$ $0.000 \mathrm{E}+00 \quad 0.0000$ $\begin{array}{lllll}0.000 \mathrm{E}+00 & 0.0000 & 0.000 \mathrm{E}+00 & 0.0000\end{array}$ $\begin{array}{llllll}0.000 \mathrm{E}+00 & 0.0000 & 0.000 \mathrm{E}+00 & 0.0000\end{array}$ $\begin{array}{llll}0.000 \mathrm{E}+00 & 0.0000 & 0.000 \mathrm{E}+00 & 0.0000\end{array}$ $\begin{array}{llll}0.000 \mathrm{E}+00 & 0.0000 & 0.000 \mathrm{E}+00 & 0.0000\end{array}$ $0.000 \mathrm{E}+000.0000$

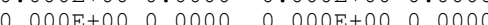
$0.000 \mathrm{E}+00 \quad 0.0000$

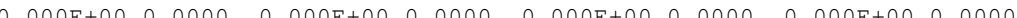
$0.000 \mathrm{E}+00 \quad 0.0000$ All Pathways* mrem/yr fract. $0.000 \mathrm{E}+000.0000$ $0.000 \mathrm{E}+00 \quad 0.000$ $0.000 \mathrm{E}+00 \quad 0.0000$ $0.000 \mathrm{E}+00 \quad 0.0000$ $0.000 \mathrm{E}+00 \quad 0.0000$ $\begin{array}{ll}0.000 \mathrm{E}+00 & 0.0000 \\ 0.0000 & 0\end{array}$ $0.0005+000.0000$ $0.000 \mathrm{E}+000.0000$ $=======\quad====+2$ $\begin{array}{lll}1.703 E+00 & 0.0779\end{array}$ $6.041 \mathrm{E}+00 \quad 0.2764$ $3.018 \mathrm{E}+000.1380$ $1.588 \mathrm{E}-010.0073$ $\begin{array}{ll}.587 \mathrm{E}-02 & 0.0008\end{array}$ $\begin{array}{ll}1.688 \mathrm{E}-02 & 0.0021\end{array}$ $2.186 \mathrm{E}+01 \quad 1.0000$

$0 *$ Sum of all water independent and dependent pathways. 
$T^{1 / 2}$ Limit $=0.5$ year

02/22/2005 17:38 Page 15

224250 day/yr worker scenario $100 \mathrm{~m}$ hotspot

Total Dose Contributions TDOSE(i,p,t) for Individual Radionuclides (i) and Pathways ( $p$ )

As mrem/yr and Fraction of Total Dose At $t=1.000 \mathrm{E}+00$ years

0

\begin{tabular}{|c|c|c|c|c|c|c|c|c|c|c|c|c|c|c|}
\hline \multirow{2}{*}{$\begin{array}{l}\text { Radio- } \\
\text { Nuclide }\end{array}$} & \multicolumn{2}{|c|}{ Ground } & \multicolumn{2}{|c|}{ Inhalation } & \multicolumn{2}{|l|}{ Rado } & \multicolumn{2}{|c|}{ Plant } & \multicolumn{2}{|l|}{ Meat } & \multicolumn{2}{|c|}{ Milk } & \multicolumn{2}{|c|}{ Soil } \\
\hline & mrem/yr & fract. & mrem/yr & fract. & mrem/yr & fract. & mrem/yr & fract. & mrem/yr & fract. & mrem/yr & fract. & mrem/yr & fract \\
\hline & & & & & & & & & & & & & 1 & \\
\hline & & & & & & & & & & & & & & \\
\hline & & & & & & & & & & & & & & \\
\hline & & 0 & & & & & & & & & & & & \\
\hline 39 & 1 & 0 & & 0.3 & 0 & 0 & 0 & 0. & & & & & & \\
\hline & & & & & & & & & & & & & & \\
\hline & & & & & & & & & & & & & & \\
\hline & & & & & & & & & & & & & & \\
\hline U -23 & & & & & & & 0 & & & & & & & \\
\hline & 100 & 273 & $35 E+00$ & 564 & $10 E+00$ & 0000 & $0 \mathrm{E}+00$ & 000 & $000 \mathrm{E}+00$ & 0000 & $.000 \mathrm{E}+00$ & 0.0000 & $.507 \mathrm{E}+00$ & 0.1163 \\
\hline
\end{tabular}

Total Dose Contributions TDOSE (i,p,t) for Individual Radionuclides (i) and Pathways (p) As mrem/yr and Fraction of Total Dose At $t=1.000 \mathrm{E}+00$ years

0 Radio- $\frac{\text { Water }}{\text { Nuclide }}$ Fish Radon Plant mrem/yr fract. mrem/yr fract. mrem/yr fract. $0.000 \mathrm{E}+00 \quad 0.0000$ $0.000 \mathrm{E}+000.0000$ $\begin{array}{lll}A m-241 & 0.000 E+00 \quad 0.0000\end{array}$ $\begin{array}{lll}C s-137 & 0.000 E+00 & 0.0000 \\ \text { Eu-152 } & 0.000 E+00 & 0.0000 \\ P u-238 & 0.000 E+00 & 0.0000\end{array}$ $\begin{array}{lll}\mathrm{Eu}-152 & 0.000 \mathrm{E}+00 & 0.0000 \\ \mathrm{Pu}-238 & 0.000 \mathrm{E}+00 & 0.0000\end{array}$ $\begin{array}{lll}\mathrm{Pu}-239 & 0.000 \mathrm{E}+00 & 0.000\end{array}$ $\begin{array}{lll}\mathrm{Sr}-90 & 0.000 \mathrm{E}+00 & 0.0000 \\ \mathrm{U}-234 & 0.000 \mathrm{E}+00 & 0.0000\end{array}$ $\begin{array}{lll}\mathrm{U}-234 & 0.000 \mathrm{E}+00 & 0.0000 \\ \mathrm{U}-235 & 0.000 \mathrm{E}+00 & 0.0000\end{array}$ $0.000 \mathrm{E}+00 \quad 0.0000$ $0.000 \mathrm{E}+00 \quad 0.0000$ $\begin{array}{llll}0.000 \mathrm{E}+00 & 0.0000 & 0.000 \mathrm{E}+00 & 0.0000\end{array}$ $\begin{array}{llllll}0.000 \mathrm{E}+00 & 0.0000 & 0.000 \mathrm{E}+00 & 0.0000\end{array}$ $0.000 \mathrm{E}+00 \quad 0.0000$ $\begin{array}{llll}0.000 \mathrm{E}+00 & 0.0000 & 0.000 \mathrm{E}+00 \quad 0.000\end{array}$

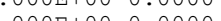
$\begin{array}{lll}0.000 \mathrm{E}+00 & 0.0000 \\ 0.000 \mathrm{E}+00 & 0.0000\end{array}$ $0.000 \mathrm{E}+00 \quad 0.0000$ $\mathrm{U}-238 \quad 0.000 \mathrm{E}+00 \quad 0.0000$ $0.000 \mathrm{E}+00 \quad 0.0000$ $0.000 \mathrm{E}+00 \quad 0.0000$

$0.000 \mathrm{E}+000.00000 .000 \mathrm{E}+000.0000-0.000 \mathrm{E}+000.0000$ $0.000 \mathrm{E}+00 \quad 0.0000$ 0 * Sum of all water independent and dependent pathways.
Meat

Milk

mrem/yr fract. mrem/yr fract. $0.000 \mathrm{E}+00 \quad 0.0000$ $0.000 \mathrm{E}+000.0000$ $0.000 \mathrm{E}+000.0000$ $0.000 \mathrm{E}+00 \quad 0.0000$ $0.000 \mathrm{E}+00 \quad 0.000$ $0.000 \mathrm{E}+00 \quad 0.0000$ $0.000 \mathrm{E}+000.0000$ $0.000 \mathrm{t}+00.0000$ 0.0000 $+00 \quad 0.0000$ $\begin{array}{llll}0.000 \mathrm{E}+00 & 0.0000 & 0.000 \mathrm{E}+00 & 0.000\end{array}$ All Pathways* mrem/yr fract. $\begin{array}{lll}1.694 \mathrm{E}+00 & 0.0786\end{array}$ $5.901 \mathrm{E}+00 \quad 0.2738$ $2.864 \mathrm{E}+00 \quad 0.1329$ $.843 E-02 \quad 0.0009$ $\begin{array}{lll}1.879 \mathrm{E}-02 & 0.00022\end{array}$ $2.155 \mathrm{E}+01 \quad 1.0000$ 
$T^{1 / 2}$ Limit $=0.5$ year

02/22/2005 17:38 Page 16

224250 day/yr worker scenario $100 \mathrm{~m}$ hotspot

Total Dose Contributions TDOSE(i,p,t) for Individual Radionuclides (i) and Pathways ( $p$ )

As mrem/yr and Fraction of Total Dose At $t=3.000 \mathrm{E}+00$ years

0

\begin{tabular}{|c|c|c|c|c|c|c|c|c|c|c|c|c|c|c|}
\hline \multirow{2}{*}{$\begin{array}{l}\text { Radio- } \\
\text { Nuclide }\end{array}$} & \multicolumn{2}{|c|}{ Ground } & \multicolumn{2}{|c|}{ Inhalation } & \multicolumn{2}{|c|}{$\begin{array}{l}\text { Radont } \\
\text { Radont }\end{array}$} & \multicolumn{2}{|c|}{$\begin{array}{l}\text { nhalat } \\
\text { Plant }\end{array}$} & \multicolumn{2}{|c|}{$\begin{array}{l}\text { Sadon) } \\
\text { Meat }\end{array}$} & \multicolumn{2}{|c|}{ Milk } & \multicolumn{2}{|l|}{ Soil } \\
\hline & $\mathrm{m} / \mathrm{yr}$ & ict & mrem/yr & ract & mrem/yr & fract & mrem/yr & frac & mrem/yr & frac & mren & frac & mrem, & frac \\
\hline & & & & & & & & & & & & & & \\
\hline & & & & & & & & & & & & & & \\
\hline & & & & & & & & & & & & & & \\
\hline & & & & & & & & & & & & & & \\
\hline & & & & & & & & & & & & & & \\
\hline & & & & & & & & & & & & & & \\
\hline & & & & & & & & & & & & & & \\
\hline & & & & & & & & & & & & & & \\
\hline 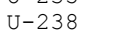 & 2 & 10 & -02 & & & & & & & & & & & \\
\hline & .00 & 124 & +00 & 683 & +00 & 000 & $0 E+00$ & 000 & $0 \mathrm{E}+00$ & 000 & $0 E+00$ & 000 & $501 \mathrm{E}+00$ & 0.1193 \\
\hline
\end{tabular}

Total Dose Contributions TDOSE (i,p,t) for Individual Radionuclides (i) and Pathways (p) As mrem/yr and Fraction of Total Dose At $t=3.000 \mathrm{E}+00$ years Radio- $\frac{\text { Water }}{\text { Nuclide }}$ Fish Radon Plant

Meat mrem/yr fract. mrem/yr fract. $0.000 \mathrm{E}+00 \quad 0.0000$ $0.000 \mathrm{E}+00 \quad 0.0000$ $\begin{array}{lll}\mathrm{Am}-241 & 0.000 \mathrm{E}+00 & 0.0000\end{array}$ $\begin{array}{lll}\mathrm{Cs}-137 & 0.000 \mathrm{E}+00 & 0.0000 \\ \mathrm{Eu}-152 & 0.000 \mathrm{E}+00 & 0.0000 \\ \mathrm{Pu}-238 & 0.000 \mathrm{O}+00 & 0.0000\end{array}$ $\begin{array}{lll}\mathrm{Eu}-152 & 0.000 \mathrm{E}+00 & 0.0000 \\ \mathrm{Pu}-238 & 0.000 \mathrm{E}+00 & 0.0000\end{array}$ $\begin{array}{lll}\mathrm{Pu}-239 & 0.000 \mathrm{E}+00 & 0.000\end{array}$ $\begin{array}{lll}\mathrm{Sr}-90 & 0.000 \mathrm{E}+00 & 0.0000 \\ \mathrm{U}-234 & 0.000 \mathrm{E}+00 & 0.0000\end{array}$ $\begin{array}{lll}\mathrm{U}-235 & 0.000 \mathrm{E}+00 & 0.0000\end{array}$ $0.000 \mathrm{E}+00 \quad 0.0000$ $0.000 \mathrm{E}+00 \quad 0.0000$ $.000 \mathrm{E}+00 \quad 0.0000$ $\begin{array}{llll}0.000 \mathrm{E}+00 & 0.0000 & 0.000 \mathrm{E}+00 & 0.000\end{array}$ $0.000 \mathrm{E}+00 \quad 0.0000$ $0.000 \mathrm{E}+00 \quad 0.0000 \quad 0.000 \mathrm{E}+00 \quad 0.000$ $0.000 \mathrm{E}+00 \quad 0.0000$ $0.000 \mathrm{E}+00 \quad 0.0000$

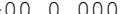

$\begin{array}{lllllllll}\text { Total } & 0.000 \mathrm{E}+00 & 0.0000 & 0.000 \mathrm{E}+00 & 0.0000 & 0.000 \mathrm{E}+00 & 0.0000\end{array}$ mrem/yr fract. mrem/yr fract. Milk $\begin{array}{llll}0.000 \mathrm{E}+00 & 0.0000 & 0.000 \mathrm{E}+00 & 0.0000\end{array}$ $\begin{array}{llll}0.000 \mathrm{E}+00 & 0.0000 & 0.000 \mathrm{E}+00 & 0.0000\end{array}$ $\begin{array}{llll}0.000 \mathrm{E}+00 & 0.0000 & 0.000 \mathrm{E}+00 & 0.0000\end{array}$ $\begin{array}{llll}0.000 E+00 & 0.0000 & 0.000 E+00 & 0.0000\end{array}$ $\begin{array}{llllllllllll}0.000 \mathrm{E}+00 & 0.0000 & 0.000 \mathrm{E}+00 & 0.0000\end{array}$

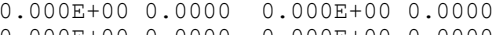

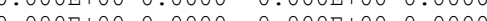
$\begin{array}{llll}0.000 \mathrm{E}+00 & 0.0000 & 0.000 \mathrm{E}+00 & 0.0000\end{array}$ $\begin{array}{ll}=.000 \mathrm{E}+00 & 0.0000\end{array}$ $0.000 \mathrm{E}+00 \quad 0.0000$ $0.000 \mathrm{E}+00 \quad 0.0000$
All Pathways* mrem/yr fract. $1.677 \mathrm{E}+00 \quad 0.0800$ $\begin{array}{ll}5.630 \mathrm{E}+00 & 0.2686\end{array}$ $2.581 \mathrm{E}+000.1231$ $\begin{array}{lll}4.239 \mathrm{E}-01 & 0.0202 \\ 1.042 \mathrm{E}+01 & 0.4972\end{array}$ $\begin{array}{lll}1.836 \mathrm{E}-02 & 0.0009\end{array}$ $2.096 \mathrm{E}+01 \quad 1.0000$

$0 *$ Sum of all water independent and dependent pathways. 
$T^{1 / 2}$ Limit $=0.5$ year

02/22/2005 17:38 Page 17

22425 day/yr worker scenario $100 \mathrm{~m}$ hotspot

Total Dose Contributions TDOSE(i,p,t) for Individual Radionuclides (i) and Pathways ( $p$ )

As mrem/yr and Fraction of Total Dose At $t=1.000 \mathrm{E}+01$ years

0

\begin{tabular}{|c|c|c|c|c|c|c|c|c|c|c|c|c|c|c|}
\hline \multirow{2}{*}{$\begin{array}{l}\text { Radio- } \\
\text { Nuclide }\end{array}$} & \multicolumn{2}{|c|}{ Ground } & \multicolumn{2}{|c|}{ Inhalation } & \multicolumn{2}{|l|}{ Rado } & \multicolumn{2}{|c|}{ Plant } & \multicolumn{2}{|l|}{ Meat } & \multicolumn{2}{|c|}{ Milk } & \multicolumn{2}{|l|}{ Soil } \\
\hline & & fract. & yr & fract. & mrem/ & frac & HILE & frac & $\mathrm{mrem} / \mathrm{yr}$ & fra & 1ent/ & fract. & $\mathrm{mrem} / \mathrm{yr}$ & ract. \\
\hline-247 & & & & & & & & & & & & & & \\
\hline & & & & & & & & & & & & & & \\
\hline & & & & & & & & & & & & & & \\
\hline & & & & & & & & & & & & & & \\
\hline & & & & & & & & & & & & & & \\
\hline & 1 & & & & & 0 & & & & & & & & \\
\hline & & & & & & & & & & & & & & \\
\hline & & & & & & & & & & & & & & \\
\hline 23 & $3.269 \mathrm{E}-02$ & 0.0017 & $1.242 \mathrm{E}-02$ & 0.0006 & $0.000 \mathrm{E}+00$ & 0.0000 & $0.000 \mathrm{E}+00$ & 0.0 & $0 \mathrm{E}+00$ & 0.0 & $0 \mathrm{E}+00$ & 0. & $6 \mathrm{E}-04$ & 0.0000 \\
\hline ta & $369 \mathrm{E}+00$ & 3628 & $\begin{array}{l}====== \\
754 \mathrm{E}+00\end{array}$ & 079 & $000 \mathrm{E}+00$ & 0000 & $\begin{array}{l}======== \\
0.000 \mathrm{E}+00\end{array}$ & 0.0000 & $000 \mathrm{E}+00$ & .0000 & $\begin{array}{l}====-= \\
0 \mathrm{E}+00\end{array}$ & 0000 & $E+00$ & 293 \\
\hline
\end{tabular}

Total Dose Contributions TDOSE (i,p,t) for Individual Radionuclides (i) and Pathways (p) As mrem/yr and Fraction of Total Dose At $t=1.000 \mathrm{E}+01$ years Radio- $\frac{\text { Water }}{\text { Nuclide }}$ Fish Radon Plant

Meat

mrem/yr fract. mrem/yr fract. $0.000 \mathrm{E}+00 \quad 0.0000$ $\begin{array}{ll}0.000 \mathrm{E}+00 & 0.0000 \\ 0.000 \mathrm{E}+00 & 0.0000\end{array}$ $\begin{array}{lll}A m-241 & 0.000 E+00 & 0.0000\end{array}$ $\begin{array}{lll}\mathrm{Cs}-137 & 0.000 \mathrm{E}+00 & 0.0000 \\ \mathrm{Eu}-152 & 0.000 \mathrm{E}+00 & 0.0000 \\ \mathrm{Pu}-238 & 0.000 \mathrm{O}+00 & 0.0000\end{array}$ $\begin{array}{lll}\mathrm{Pu}-238 & 0.000 \mathrm{E}+00 & 0.0000\end{array}$ $\begin{array}{lll}\mathrm{Pu}-239 & 0.000 \mathrm{E}+00 & 0.000\end{array}$ $\begin{array}{lll}\mathrm{Sr}-90 & 0.000 \mathrm{E}+00 & 0.0000 \\ \mathrm{U}-234 & 0.000 \mathrm{E}+00 & 0.0000\end{array}$ $\begin{array}{lll}\mathrm{U}-235 & 0.000 \mathrm{E}+00 & 0.0000\end{array}$ mrem/yr fract. $0.000 \mathrm{E}+00 \quad 0.0000$ $0.000 \mathrm{E}+000.0000$

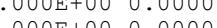

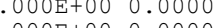
$\begin{array}{llll}0.000 \mathrm{E}+00 & 0.0000 & 0.000 \mathrm{E}+00 & 0.000\end{array}$ $0.000 \mathrm{E}+00 \quad 0.0000$ $0.000 \mathrm{E}+00 \quad 0.0000$ $0.000 \mathrm{E}+00 \quad 0.0000$

$\begin{array}{llllllll}\text { Total } & 0.000 \mathrm{E}+00 & 0.0000 & 0.000 \mathrm{E}+00 & 0.0000 & 0.000 \mathrm{E}+00 & 0.0000\end{array}$ mrem/yr fract. $0.000 \mathrm{E}+000.0000$ $\begin{array}{lllll}0.000 \mathrm{E}+00 & 0.0000 & 0.000 \mathrm{E}+00 & 0.0000\end{array}$ $\begin{array}{llll}0.000 \mathrm{E}+00 & 0.0000 & 0.000 \mathrm{E}+00 & 0.0000\end{array}$ $\begin{array}{llll}0.000 \mathrm{E}+00 & 0.0000 & 0.000 \mathrm{E}+00 & 0.0000\end{array}$ $0.000 \mathrm{E}+00 \quad 0.0000$ $\begin{array}{llll}0.000 \mathrm{E}+00 & 0.0000 & 0.000 \mathrm{E}+00 & 0.0000\end{array}$ $\begin{array}{llll}0.000 \mathrm{E}+00 & 0.0000 & 0.000 \mathrm{E}+00 & 0.00000\end{array}$ $\begin{array}{llll}0.000 \mathrm{E}+00 & 0.0000 & 0.000 \mathrm{E}+00 & 0.0000\end{array}$ $\begin{array}{ll}0.000 \mathrm{E}+00 & 0.0000\end{array}$ $0 *$ Sum of all water independent and dependent pathways.
Milk

mrem/yr fract. $0.000 \mathrm{E}+00 \quad 0.0000$ $0.000 \mathrm{E}+00 \quad 0.0000$ $0.000 \mathrm{E}+00 \quad 0.000$ $0.000 \mathrm{E}+00 \quad 0.0000$ $0.000 \mathrm{E}+00 \quad 0.0000$ $0.000 \mathrm{E}+00 \quad 0.0000$ $0.000 \mathrm{E}+00$ 0.0000 $0.000 \mathrm{E}+00 \quad 0.0000$
All Pathways* mrem/yr fract. $\begin{array}{lll}1.620 \mathrm{E}+00 & 0.0843\end{array}$ $4.776 \mathrm{E}+00 \quad 0.2486$ $1.793 \mathrm{E}+000.0933$ $\begin{array}{ll}1.552 \mathrm{E}-02 & 0.0008\end{array}$ $\begin{array}{ll}4.598 \mathrm{E}-02 & 0.0024\end{array}$ $1.921 \mathrm{E}+011.0000$ 
$T^{1 / 2}$ Limit $=0.5$ year

02/22/2005 17:38 Page 18

224250 day/yr worker scenario $100 \mathrm{~m}$ hotspot

Total Dose Contributions TDOSE(i,p,t) for Individual Radionuclides (i) and Pathways ( $p$ )

As mrem/yr and Fraction of Total Dose At $t=3.000 \mathrm{E}+01$ years

0

\begin{tabular}{|c|c|c|c|c|c|c|c|c|c|c|c|c|c|c|}
\hline \multirow{2}{*}{$\begin{array}{l}\text { Radio- } \\
\text { Nuclide }\end{array}$} & \multicolumn{2}{|c|}{ Ground } & \multicolumn{2}{|c|}{ Inhalation } & \multicolumn{2}{|c|}{$\begin{array}{l}\text { Radon } \\
\text { Radent }\end{array}$} & \multicolumn{2}{|c|}{$\begin{array}{l}\text { Mnala } \\
\text { Plant }\end{array}$} & \multicolumn{2}{|c|}{$\begin{array}{l}\text { radon) } \\
\text { Meat }\end{array}$} & \multicolumn{2}{|c|}{ Milk } & \multicolumn{2}{|l|}{ Soil } \\
\hline & $\mathrm{em} / \mathrm{yr}$ & ct & mrem/yr & ract & mrem/yr & fract & mrem/yr & frac & mrem/yr & frac & mren & fract & mrem, & frac \\
\hline & & & & & & & & & & & & & & \\
\hline & & & & & & & & & & & & & & \\
\hline & & & & & & & & & & & & & & \\
\hline & & & & & & & & & & & & & & \\
\hline & & & & & & & & & & & & & & \\
\hline & & & & & & & & & & & & & & \\
\hline & & & & & & & & & & & & & & \\
\hline & & & & & & & & & & & & & & \\
\hline 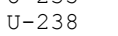 & 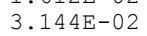 & 20 & -02 & 07 & & & & & & & & & & \\
\hline & 100 & 471 & +00 & 03 & +00 & 000 & $E+00$ & 000 & $0 \mathrm{E}+00$ & 000 & $0 \mathrm{E}+00$ & 000 & $38 \mathrm{E}+00$ & 0.1526 \\
\hline
\end{tabular}

Total Dose Contributions TDOSE (i,p,t) for Individual Radionuclides (i) and Pathways (p) As mrem/yr and Fraction of Total Dose At $t=3.000 \mathrm{E}+01$ years Radio- $\frac{\text { Water }}{\text { Nuclide }}$ Fish Radon Plant

mrem/yr fract.

$\begin{array}{lll}\mathrm{Am}-241 & 0.000 \mathrm{E}+00 & 0.0000\end{array}$ $\begin{array}{lll}\mathrm{Cs}-137 & 0.000 \mathrm{E}+00 & 0.0000 \\ \mathrm{Eu}-152 & 0.000 \mathrm{E}+00 & 0.0000\end{array}$ $\begin{array}{lll}\mathrm{Eu}-152 & 0.000 \mathrm{E}+00 & 0.0000 \\ \mathrm{Pu}-238 & 0.000 \mathrm{E}+00 & 0.0000\end{array}$ $\begin{array}{lll}\mathrm{Pu}-239 & 0.000 \mathrm{E}+00 & 0.000\end{array}$ $\begin{array}{lll}\mathrm{Sr}-90 & 0.000 \mathrm{E}+00 & 0.0000 \\ \mathrm{U}-234 & 0.000 \mathrm{E}+00 & 0.0000\end{array}$

$\mathrm{J}-235 \quad 0.000 \mathrm{E}+00 \quad 0.0000$

mrem/yr fract. mrem/yr fract. mrem/yr fract. $\begin{array}{llll}0.000 \mathrm{E}+00 & 0.0000 & 0.000 \mathrm{E}+00 & 0.0000 \\ 0.000 \mathrm{E}+00 & 0.0000 & 0.000 \mathrm{E}+00 & 0.0000 \\ 0.000 \mathrm{E}+00 & 0.0000 & 0.000 \mathrm{E}+00 & 0.0000\end{array}$ $\begin{array}{llll}0.000 \mathrm{E}+00 & 0.0000 & 0.000 \mathrm{E}+00 & 0.0000\end{array}$ $.000 \mathrm{E}+00 \quad 0.0000$ $.000 \mathrm{E}+00 \quad 0.0000$

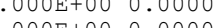

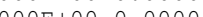
$0.000 \mathrm{E}+00 \quad 0.0000 \quad 0.000 \mathrm{E}+00 \quad 0.0000$ $\begin{array}{llll}0.000 \mathrm{E}+00 & 0.0000 & 0.000 \mathrm{E}+00 & 0.0000 \\ 0.000 \mathrm{E}+00 & 0.0000 & 0.000 \mathrm{E}+00 & 0.0000\end{array}$ $0.000 \mathrm{E}+00 \quad 0.0000$ $\mathrm{U}-238 \quad 0.000 \mathrm{E}+00 \quad 0.0000$ (1.000 $\begin{array}{lllllllll}0.000 \mathrm{E}+00 & 0.0000 & 0.000 \mathrm{E}+00 & 0.0000 & 0.000 \mathrm{E}+00 & 0.0000 & 0.000 \mathrm{E}+00 & 0.0000\end{array}$ $0.0005+000.0000$ 0.000E+00 0.0000 0.0005+00 0.0000 $0.000 \mathrm{E}+00 \quad 0.0000$ $0.0005+000.0$

Milk mrem/yr fract. $0.000 \mathrm{E}+00 \quad 0.0000$ $\begin{array}{lll}0.000 \mathrm{E}+00 & 0.0000\end{array}$ $0.000 \mathrm{E}+00 \quad 0.0000$ $0.000 \mathrm{E}+00 \quad 0.0000$ $0.000 \mathrm{E}+00 \quad 0.0000$ $0.000 \mathrm{E}+00 \quad 0.0000$ $0.000 \mathrm{E}+00 \quad 0.0000$ $0.000 \mathrm{E}+00.0000===$ $0.000 \mathrm{E}+00 \quad 0.0000$
All Pathways* mrem/yr fract. $1.466 \mathrm{E}+00 \quad 0.0918$ $2.984 \mathrm{E}+00 \quad 0.1868$ $\begin{array}{lll}6.325 \mathrm{E}-01 & 0.0396\end{array}$ $\begin{array}{ll}3.422 \mathrm{E}-01 & 0.0214\end{array}$ $\begin{array}{lll}.795 \mathrm{E}-02 & 0.0043\end{array}$ $\begin{array}{ll}1.746 \mathrm{E}-02 & 0.0011\end{array}$ $============$

$0 *$ Sum of all water independent and dependent pathways. 
$T^{1 / 2}$ Limit $=0.5$ year

02/22/2005 17:38 Page 19

$224250 \mathrm{day} / \mathrm{yr}$ worker scenario $100 \mathrm{~m}$ hotspot

Total Dose Contributions TDOSE (i,p,t) for Individual Radionuclides (i) and Pathways ( $p$ )

As mrem/yr and Fraction of Total Dose At $t=1.000 \mathrm{E}+02$ years Water Independent
Radon Plant

Milk

\begin{tabular}{|c|c|c|c|c|c|c|c|c|c|c|c|c|c|c|}
\hline \multirow{2}{*}{$\begin{array}{l}\text { Radio- } \\
\text { Nuclide }\end{array}$} & \multicolumn{2}{|c|}{ Ground } & \multicolumn{2}{|c|}{ Inhalation } & \multicolumn{2}{|c|}{ Radon } & \multicolumn{2}{|c|}{ Plant } & \multicolumn{2}{|c|}{ Meat } & \multicolumn{2}{|c|}{ Milk } & \multicolumn{2}{|l|}{ Soil } \\
\hline & + & frac & . & fract. & mrem/yr & fra & . & trues & the & trace. & $\mathrm{rem} / \mathrm{yr}$ & fract. & mrem/yr & fract. \\
\hline & & & & & & & & & & & & & & \\
\hline & & & & & & & & & & & & & & \\
\hline & & & & & & & & & & & & & & \\
\hline & & & & & & & & & & & & & & \\
\hline & & & & & & & & & & & & & & \\
\hline & & & & & & & & & & & & & & \\
\hline & & & & & & & & & & & & & & \\
\hline & & & & & & & & & & & & & & \\
\hline-238 & $2.745 \mathrm{E}-02$ & 0.0022 & $1.043 \mathrm{E}-02$ & 0.0009 & $0.000 \mathrm{E}+00$ & 0.0000 & $0.000 \mathrm{E}+00$ & 0.0000 & $0.000 \mathrm{E}+00$ & 0.0000 & $0.000 \mathrm{E}+00$ & 0.0000 & $7.330 \mathrm{E}-04$ & 0.0001 \\
\hline & $37 \mathrm{E}-01$ & 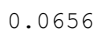 & 0 & & 0 & & +00 & ( & +00 & 0.0000 & $000 \mathrm{E}+00$ & & $.319 \mathrm{E}+00$ & \\
\hline
\end{tabular}
0

Total Dose Contributions TDOSE (i,p,t) for Individual Radionuclides (i) and Pathways (p) As mrem/yr and Fraction of Total Dose At $t=1.000 \mathrm{E}+02$ years Water Fish Radon Plater Dependent Pathways Water Fish 1 Radon Miant Meat $\quad$ Milk Nuclide mrem/yr fract. mrem/yr fract. mrem/yr fract. mrem/yr fract. mrem/yr fract. $\mathrm{mrem} / \mathrm{yr}$ fract. $\mathrm{mrem} / \mathrm{yr}$ fract.

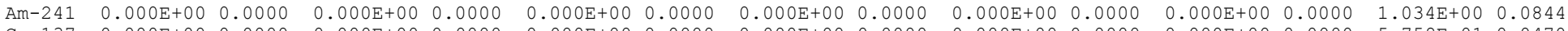

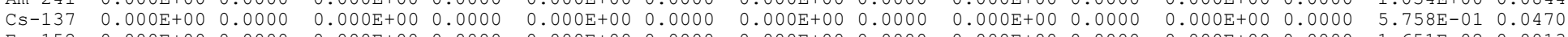

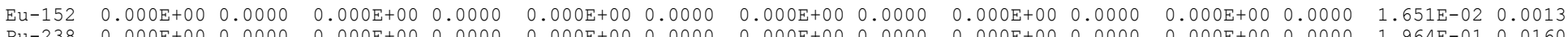

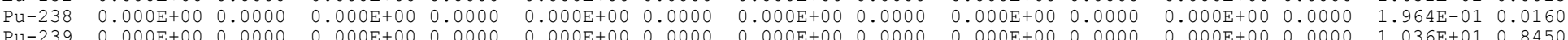

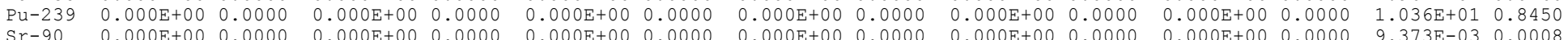

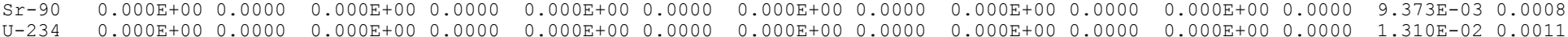
$\begin{array}{llllllllllllllll}\mathrm{U}-235 & 0.000 \mathrm{E}+00 & 0.0000 & 0.000 \mathrm{E}+00 & 0.0000 & 0.000 \mathrm{E}+00 & 0.0000 & 0.000 \mathrm{E}+00 & 0.0000 & 0.000 \mathrm{E}+00 & 0.0000 & 0.000 \mathrm{E}+00 & 0.0000 & 1.540 \mathrm{E}-02 & 0.0013\end{array}$ $\begin{array}{lllllllllllllll}\mathrm{U}-238 & 0.000 \mathrm{E}+00 & 0.0000 & 0.000 \mathrm{E}+00 & 0.0000 & 0.000 \mathrm{E}+00 & 0.0000 & 0.000 \mathrm{E}+00 & 0.0000 & 0.000 \mathrm{E}+00 & 0.0000 & 0.000 \mathrm{E}+00 & 0.0000 & 3.862 \mathrm{E}-02 & 0.0032\end{array}$

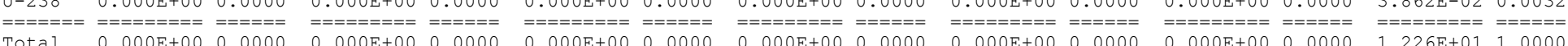
$0 *$ Sum of all water independent and dependent pathways. 
$T^{1 / 2}$ Limit $=0.5$ year

02/22/2005 17:38 Page 20

2240 day/yr worker scenario $100 \mathrm{~m}$ hotspot

Total Dose Contributions TDOSE (i,p,t) for Individual Radionuclides (i) and Pathways (p) As mrem/yr and Fraction of Total Dose At $t=3.000 \mathrm{E}+02$ years

\begin{tabular}{|c|c|c|c|c|c|c|c|c|c|c|c|c|c|c|}
\hline \multirow{2}{*}{$\begin{array}{l}\text { Radio- } \\
\text { Nuclide }\end{array}$} & \multicolumn{2}{|c|}{ Ground } & \multicolumn{2}{|c|}{ Inhalation } & \multicolumn{2}{|c|}{$\begin{array}{l}\text { Radont } \\
\text { Radont }\end{array}$} & \multicolumn{2}{|c|}{$\begin{array}{l}\text { nhalat } \\
\text { Plant }\end{array}$} & \multicolumn{2}{|c|}{$\begin{array}{l}\text { Sadon) } \\
\text { Meat }\end{array}$} & \multicolumn{2}{|c|}{ Milk } & \multicolumn{2}{|l|}{ Soil } \\
\hline & $\mathrm{em} / \mathrm{y}$ & ct & mrem/yr & ract & mrem/yr & fract & mrem/yr & frac & mrem/yr & frac & mren & frac & mrem, & frac \\
\hline & & & & & & & & & & & & & & \\
\hline & & & & & & & & & & & & & & \\
\hline & & & & & & & & & & & & & & \\
\hline & & & & & & & & & & & & & & \\
\hline & & & & & & & & & & & & & & \\
\hline & & & & & & & & & & & & & & \\
\hline & & & & & & & & & & & & & & \\
\hline & & & & & & & & & & & & & & \\
\hline 250 & 1 & 017 & 3 & & & & & & & & & & & \\
\hline & -01 & 095 & +00 & 399 & +00 & 000 & $0 E+00$ & 000 & $0 \mathrm{E}+00$ & 000 & $0 \mathrm{E}+00$ & b0o & $.147 \mathrm{E}+00$ & 2006 \\
\hline
\end{tabular}

Total Dose Contributions TDOSE (i,p,t) for Individual Radionuclides (i) and Pathways (p) As mrem/yr and Fraction of Total Dose At $t=3.000 \mathrm{E}+02$ years

0 $\begin{array}{ll}\text { Radio- } & \text { Water } \\ \text { Nuclide } & \text { mrem/yr fract. }\end{array}$ Fish Radon Plant

Meat

mrem/yr fract. $\begin{array}{lll}\mathrm{Am}-241 & 0.000 \mathrm{E}+00 & 0.0000\end{array}$ $\begin{array}{lll}\mathrm{Cs}-137 & 0.000 \mathrm{E}+00 & 0.0000\end{array}$ $\begin{array}{lll}\mathrm{Eu}-152 & 0.000 \mathrm{E}+00 & 0.0000 \\ \mathrm{Pu}-238 & 0.000 \mathrm{E}+00 & 0.0000 \\ \mathrm{Pu}-239 & 0.000 \mathrm{O}+00 & 0.0000\end{array}$ $\begin{array}{lll}\mathrm{Pu}-239 & 0.000 \mathrm{E}+00 & 0.0000\end{array}$ $\begin{array}{lll}\mathrm{Sr}-90 & 0.000 \mathrm{E}+00 \quad 0.000\end{array}$ $\begin{array}{lll}\mathrm{U}-234 & 0.000 \mathrm{E}+00 & 0.0000 \\ \mathrm{U}-235 & 0.000 \mathrm{E}+00 & 0.0000\end{array}$ $\begin{array}{lll}-238 & 0.000 \mathrm{E}+00 & 0.0000\end{array}$ mrem/yr fract. mrem/yr fract. mrem/yr fract. $0.000 \mathrm{E}+00 \quad 0.0000$ $\begin{array}{llll}0.000 \mathrm{E}+00 & 0.0000 & 0.000 \mathrm{E}+00 & 0.0000 \\ 0.000 \mathrm{E}+00 & 0.0000 & 0.000 \mathrm{E}+00 & 0.0000 \\ 0.000 \mathrm{E}+00 & 0.0000 & 0.000 \mathrm{E}+00 & 0.0000\end{array}$ $\begin{array}{lllll}0.000 \mathrm{E}+00 & 0.0000 & 0.000 \mathrm{E}+00 & 0.0000\end{array}$ $\begin{array}{lll}.000 \mathrm{E}+00 & 0.0000 \\ 0.000 & 0.0000\end{array}$ $0.000 \mathrm{E}+00 \mathrm{0} 0.0000$ $\begin{array}{ll}0.000 \mathrm{E}+00 & 0.00000 \\ 0.000 & 0\end{array}$ $0.000 \mathrm{E}+00 \quad 0.0000$ $\begin{array}{ll}0.000 \mathrm{E}+00 & 0.0000 \\ 0.000 \mathrm{E}+00 & 0.0000 \\ 0.000 \mathrm{E}+00 & 0.0000\end{array}$ $\begin{array}{ll}.000 \mathrm{E}+00 & 0.0000 \\ .000 \mathrm{E}+00 & 0.0000 \\ 0.0000 \mathrm{E}+00 & 0.0000\end{array}$ $\begin{array}{ll}0.000 \mathrm{E}+00 & 0.0000 \\ 0.000 \mathrm{E}+00 & 0.0000\end{array}$ $\begin{array}{lll}0.000 \mathrm{E}+00 & 0.0000 \\ 0.000 \mathrm{C}+00 & 0.0000\end{array}$ $\begin{array}{lll}0.000 \mathrm{E}+00 & 0.0000 \\ 0.000 \mathrm{E}+00 & 0.0000 & 0\end{array}$ $0.000 \mathrm{E}+0.0000$ $0.000 \mathrm{E}+00 \quad 0.0000$ $0.000 \mathrm{E}+00 \quad 0.0000$ $0.000 \mathrm{E}+00 \quad 0.0000$ -10
0 $0.000 \mathrm{E}+000.0000$ $0.000 \mathrm{E}+00 \quad 0.0000$
Milk mrem/yr fract. $0.000 \mathrm{E}+00 \quad 0.0000$ $0.000 \mathrm{E}+00 \quad 0.000$ $0.000 \mathrm{E}+00 \quad 0.0000$ $0.000 \mathrm{E}+00 \quad 0.0000$ $0.000 \mathrm{E}+00 \quad 0.0000$ $0.000 \mathrm{E}+00 \quad 0.0000$ $0.000 \mathrm{E}+00 \quad 0.0000$ $\begin{array}{ll}========= & ===== \\ 0.000 \mathrm{E}+00 & 0.0000\end{array}$
All Pathways* $\begin{array}{ll}3.820 \mathrm{E}-01 & 0.0357\end{array}$ $\begin{array}{lll}5.231 \mathrm{E}-03 & 0.0005\end{array}$ $.943 \mathrm{E}-07 \quad 0.0000$
$. .020 \mathrm{E}-02$ $\begin{array}{ll}1.080 \mathrm{E}-02 & 0.0010\end{array}$ .0024 $\begin{array}{ll}1.070 \mathrm{E}+01 & 1.0000\end{array}$

$0 *$ Sum of all water independent and dependent pathways. 
Total Dose Contributions TDOSE (i,p,t) for Individual Radionuclides (i) and Pathways (p) As mrem/yr and Fraction of Total Dose At $t=1.000 \mathrm{E}+03$ years

\begin{tabular}{|c|c|c|c|c|c|c|c|c|c|c|c|c|c|c|}
\hline \multirow{2}{*}{$\begin{array}{l}\text { Radio- } \\
\text { Nuclide }\end{array}$} & \multicolumn{2}{|c|}{ Ground } & \multicolumn{2}{|c|}{ Inhalation } & \multicolumn{2}{|c|}{$\begin{array}{r}\text { Independent } \\
\text { Radon }\end{array}$} & \multicolumn{2}{|c|}{$\begin{array}{l}\text { (Inhalation } \\
\text { Plant }\end{array}$} & \multicolumn{2}{|c|}{$\begin{array}{c}\text { excludes radon) } \\
\text { Meat }\end{array}$} & \multicolumn{2}{|c|}{ Milk } & \multicolumn{2}{|l|}{ Soil } \\
\hline & $\mathrm{mrem} / \mathrm{yr}$ & fract. & mrem/yr & fract. & mrem/yr & fract. & mrem/yr & fract. & mrem/yr & fract. & mrem/yr & fract. & mrem/yr & fract. \\
\hline & & 0 & $8.031 \mathrm{E}-03$ & 0.0 & $0.000 \mathrm{E}+00$ & 0.0000 & $0.000 \mathrm{E}+00$ & 0.0 & $0.000 \mathrm{E}+00$ & 0.0 & $0.000 \mathrm{E}+00$ & 0.0 & $2.029 \mathrm{E}-03$ & 0 \\
\hline & & 0.0 & 15 & 0.0 & 0 & 0.0 & $0.000 \mathrm{E}+00$ & 0.0 & 0.0 & 0.0 & $E+00$ & & -14 & \\
\hline $1-152$ & $7.252 \mathrm{E}-23$ & 0.0000 & $1.683 \mathrm{E}-15$ & 0.0000 & $0.000 \mathrm{E}+00$ & 0.0000 & $0.000 \mathrm{E}+00$ & 0.0000 & $0.000 \mathrm{E}+00$ & 0.0000 & $0.000 \mathrm{E}+00$ & 0.0 & $3.438 \mathrm{E}-17$ & \\
\hline Pu-238 & $4.469 \mathrm{E}-06$ & 0.0 & $1.325 \mathrm{E}-04$ & & $0 \mathrm{E}+00$ & 0.0000 & $0.000 \mathrm{E}+00$ & 0.0000 & $0.000 \mathrm{E}+00$ & & $0 \mathrm{E}+00$ & & -05 & 0 \\
\hline $\mathrm{Pu}-2$ & 1.166 & 0.0 & 7.7 & & $.000 \mathrm{E}+00$ & 0.00 & $0.000 \mathrm{E}+00$ & 0.0 & & 0 & 00 & & 00 & 0 \\
\hline & $7.270 \mathrm{~F}$ & 0.00 & $1.912 \mathrm{E}-15$ & 0 . & $E+00$ & 0.00 & & 0.0 & & & & & & \\
\hline$U-23$ & $1.782 \mathrm{E}-03$ & 0.0002 & $2.260 \mathrm{E}-03$ & 0.0002 & $0.000 \mathrm{E}+00$ & 0.00 & $0.000 \mathrm{E}+00$ & 0.0 & $0.000 \mathrm{E}+00$ & 0.0 & $0.000 \mathrm{E}+00$ & 0.0 & -04 & 0. \\
\hline $\mathrm{U}-23$ & -03 & 0.0 & 5.117 & & & & $E+00$ & 0.0 & & & & & & \\
\hline $\mathrm{U}$ & $89 \mathrm{E}-03$ & 0. & $1.826 \mathrm{E}-03$ & 0.0002 & $E+00$ & 0. & $E+00$ & 0.0 & $0.000 \mathrm{E}+00$ & 0.0 & $0 \mathrm{E}+00$ & 0 . & BE-04 & 0 \\
\hline tal & $2.287 \mathrm{E}-02$ & 0.0023 & $7.808 \mathrm{E}+00$ & 0.7954 & $0.000 \mathrm{E}+00$ & 0.0000 & $\begin{array}{l}======== \\
0.000 \mathrm{E}+00\end{array}$ & $\begin{array}{l}===== \\
0.0000\end{array}$ & $\begin{array}{l}======== \\
0.000 \mathrm{E}+00\end{array}$ & $\begin{array}{l}===== \\
0.0000\end{array}$ & $0.000 \mathrm{E}+00$ & 0.0000 & $1.985 \mathrm{E}+00$ & 0.20 \\
\hline
\end{tabular}
$2.287 \mathrm{E}-02 \quad 0.0023$

Total Dose Contributions TDOSE(i,p,t) for Individual Radionuclides (i) and Pathways (p) As mrem/yr and Fraction of Total Dose At $t=1.000 \mathrm{E}+03$ years

0 As mrem/yr and
Water Dependent Pathways Fish

Radon Plant

Meat

Milk

Radio-
Nuclide $\overline{m r e m / y r}$ fract.

mrem/yr fract.

mrem/yr fract.

mrem/yr fract.

mrem/yr fract. $\begin{array}{lll}\mathrm{Am}-241 & 0.000 \mathrm{E}+00 & 0.0000 \\ \mathrm{Cs}-137 & 0.000 \mathrm{E}+00 & 0.0000\end{array}$

$0.000 \mathrm{E}+00 \quad 0.0000$ $0.000 \mathrm{E}+00 \quad 0.0000$ $\begin{array}{lll}\mathrm{Eu}-152 & 0.000 \mathrm{E}+00 & 0.0000 \\ \mathrm{Pu}-238 & 0.000 \mathrm{E}+00 & 0.0000\end{array}$ $\mathrm{Pu}-2390.000 \mathrm{E}+00 \quad 0.0000$ $0.000 \mathrm{E}+00 \quad 0.0000$ $\begin{array}{lll}0.000 \mathrm{E}+00 & 0.0000 \\ 0.000 \mathrm{e} & 0.0000\end{array}$ $0.000 \mathrm{E}+00 \quad 0.0000$ $\begin{array}{lllll}0.000 \mathrm{E}+00 & 0.0000 & 0.000 \mathrm{E}+00 & 0.0000\end{array}$ $\begin{array}{llllllllll}0.000 \mathrm{E}+00 & 0.0000 & 0.000 \mathrm{E}+00 & 0.00000\end{array}$ $\begin{array}{lllll}0.000 \mathrm{E}+00 & 0.0000 & 0.000 \mathrm{E}+00 & 0.0000\end{array}$ $\begin{array}{lll}\mathrm{Sr}-90 & 0.000 \mathrm{E}+00 & 0.0000\end{array}$

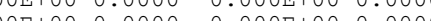
$.000 \mathrm{E}+00 \quad 0.0000 \quad 0.000 \mathrm{E}+00 \quad 0.0000$
$. .000 \mathrm{E}+00 \quad 0.0000 \quad 0.000 \mathrm{E}+000.0000$

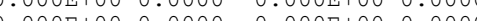

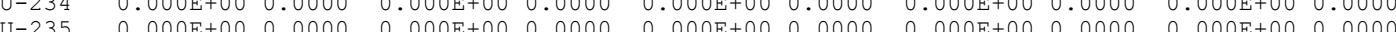
$\mathrm{U}-238$ $============$ $0.0 \mathrm{E}+00 \quad 0.0000$ 
Dose/Source Ratios Summed Over All Pathways

Parent and Progeny Principal Radionuclide Contributions Indicated

OParent Product Branch $\operatorname{DSR}(j, \mathrm{t}) \quad(\mathrm{mrem} / \mathrm{yr}) /(\mathrm{pCi} / \mathrm{g})$

\begin{tabular}{|c|c|c|c|c|c|c|c|c|c|c|c|}
\hline (i) & $(j)$ & Fraction* & $t=$ & $0.000 \mathrm{E}+00$ & $1.000 \mathrm{E}+00$ & $3.000 \mathrm{E}+00$ & $1.000 \mathrm{E}+01$ & $3.000 \mathrm{E}+01$ & +02 & +02 & $1.000 \mathrm{E}+03$ \\
\hline 241 & & $E+00$ & & & & 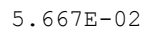 & 5.473 & $4.953 \mathrm{E}-02$ & 3.49 & $E-02$ & $3.933 \mathrm{E}-04$ \\
\hline$A m-241$ & Np-237 & $1.000 \mathrm{E}+00$ & & $4.252 \mathrm{E}-08$ & $1.273 \mathrm{E}-07$ & $2.954 \mathrm{E}-07$ & $8.703 E-07$ & $2.401 \mathrm{E}-06$ & $6.638 \mathrm{E}-06$ & $1.263 \mathrm{E}-05$ & $1.372 \mathrm{E}-05$ \\
\hline$A m-241$ & $\mathrm{U}-233$ & $1.000 \mathrm{E}+00$ & & $3.073 \mathrm{E}-15$ & $2.147 \mathrm{E}-14$ & $1.130 \mathrm{E}-13$ & $9.956 \mathrm{E}-13$ & $8.036 \mathrm{E}-12$ & $7.511 \mathrm{E}-11$ & $4.490 \mathrm{E}-10$ & $1.552 \mathrm{E}-09$ \\
\hline$A m-241$ & Th-229 & & & $2.767 \mathrm{E}-18$ & $4.144 \mathrm{E}-17$ & $4.819 \mathrm{E}-16$ & $1.263 \mathrm{E}-14$ & $.989 \mathrm{E}-13$ & $9.541 \mathrm{E}-12$ & $.877 \mathrm{E}-10$ & $2.831 \mathrm{E}-09$ \\
\hline$A m-241$ & $\operatorname{SDSR}(j)$ & & & $5.752 \mathrm{E}-02$ & $5.724 \mathrm{E}-02$ & $667 \mathrm{E}-02$ & $5.473 \mathrm{E}-02$ & & & & \\
\hline $0 \mathrm{Cs}-137$ & $\mathrm{Cs}-137$ & $1.000 \mathrm{E}+00$ & & $6.041 \mathrm{E}-01$ & $5.901 \mathrm{E}-01$ & $5.630 \mathrm{E}-01$ & $4.776 \mathrm{E}-01$ & $2.984 \mathrm{E}-01$ & $5.758 \mathrm{E}-02$ & & $3.736 \mathrm{E}-11$ \\
\hline $0 \mathrm{Eu}-152$ & $\mathrm{Eu}-152$ & $7.208 \mathrm{E}-01$ & & $8.842 \mathrm{E}-01$ & $8.393 \mathrm{E}-01$ & $7.563 \mathrm{E}-01$ & $5.252 \mathrm{E}-01$ & $1.853 \mathrm{E}-01$ & $4.838 \mathrm{E}-03$ & $1.448 \mathrm{E}-07$ & $2.125 \mathrm{E}-23$ \\
\hline $0 \mathrm{Eu}-152$ & $\mathrm{Eu}-152$ & $2.792 \mathrm{E}-01$ & & $3.425 \mathrm{E}-01$ & $3.251 \mathrm{E}-01$ & $2.929 \mathrm{E}-01$ & $2.034 \mathrm{E}-01$ & $7.179 \mathrm{E}-02$ & $1.874 \mathrm{E}-03$ & $5.610 \mathrm{E}-08$ & $8.231 \mathrm{E}-24$ \\
\hline $\mathrm{Eu}-152$ & $\mathrm{Gd}-152$ & $2.792 \mathrm{E}-01$ & & $1.938 \mathrm{E}-17$ & $5.683 \mathrm{E}-17$ & $1261 \mathrm{~F}-16$ & $3.188 \mathrm{E}-16$ & $6.017 \mathrm{E}-16$ & $7.482 \mathrm{E}-16$ & & $6.982 \mathrm{E}-16$ \\
\hline $\mathrm{Eu}-152$ & $\operatorname{SDSR}(j)$ & & & & & & & & & & \\
\hline $0 \mathrm{Pu}-238$ & $\mathrm{Pu}-238$ & مOF- & & $4.341 \mathrm{E}-02$ & $4.307 \mathrm{E}-02$ & $4.239 \mathrm{E}-02$ & $4.010 \mathrm{E}-02$ & $3.422 \mathrm{E}-02$ & $1.963 \mathrm{E}$ & 03 & 1 \\
\hline $\mathrm{Pu}-238$ & $U-234$ & $1.000 \mathrm{E}+00$ & & $1.776 \mathrm{E}-08$ & $5.304 \mathrm{E}-08$ & $1.226 \mathrm{E}-07$ & $3.553 \mathrm{E}-07$ & $9.361 \mathrm{E}-07$ & $2.213 \mathrm{E}-06$ & $2.769 \mathrm{E}-06$ & $8.493 \mathrm{E}-07$ \\
\hline $\mathrm{Pu}-238$ & Th-230 & $1.000 \mathrm{E}+00$ & & $1.301 \mathrm{E}-13$ & $9.082 \mathrm{E}-13$ & $4.770 \mathrm{E}-12$ & $4.171 \mathrm{E}-11$ & $3.297 \mathrm{E}-10$ & $2.878 \mathrm{E}-09$ & $1.477 \mathrm{E}-08$ & $4.092 \mathrm{E}-08$ \\
\hline & -226 & & & & & & & & & & \\
\hline $\mathrm{Pu}-238$ & $\mathrm{~Pb}-210$ & $1.000 \mathrm{E}+00$ & & 6.57 & 2.022 & 5.012 & $3.695 \mathrm{E}-15$ & $2.226 \mathrm{E}-13$ & 1.57 & & -09 \\
\hline $\mathrm{Pu}-238$ & $\operatorname{SDSR}(j)$ & & & $4.341 \mathrm{E}-02$ & $4.307 \mathrm{E}-02$ & $4.239 \mathrm{E}-02$ & $4.010 \mathrm{E}-02$ & $3.422 \mathrm{E}-02$ & $1.964 \mathrm{E}-02$ & 4.0201 & $1.688 \mathrm{E}-05$ \\
\hline $0 \mathrm{Pu}-239$ & $\mathrm{Pu}-239$ & $1.000 \mathrm{E}+00$ & & $4.782 \mathrm{E}-02$ & $4.782 \mathrm{E}-02$ & $4.781 \mathrm{E}-02$ & $4.779 \mathrm{E}-02$ & $4.773 \mathrm{E}$ & 4.752 & 2 & 4 \\
\hline $\mathrm{Pu}-239$ & $\mathrm{U}-235$ & & & & & & 1.5 & & & & \\
\hline 9 & $\mathrm{P}$ & 1.0 & & & 4.3 & & 2.02 & & & & \\
\hline $\mathrm{Pu}-239$ & Ac- 227 & $1.000 \mathrm{E}+00$ & & $2.763 \mathrm{E}-17$ & $4.111 \mathrm{E}-16$ & $4.710 \mathrm{E}-15$ & $1.170 \mathrm{E}-13$ & $2.401 \mathrm{E}-12$ & $5.140 \mathrm{E}-11$ & $5.227 \mathrm{E}-10$ & $3.230 \mathrm{E}-09$ \\
\hline $\mathrm{Pu}-239$ & $\operatorname{SDSR}(j)$ & & & $4.782 \mathrm{E}-02$ & $4.782 \mathrm{E}-02$ & $4.781 \mathrm{E}-02$ & $4.779 \mathrm{E}-02$ & $4.773 \mathrm{E}-02$ & $4.752 \mathrm{E}-02$ & $4.693 \mathrm{E}-02$ & $4.490 \mathrm{E}-02$ \\
\hline $0 \mathrm{Sr}-90$ & $\mathrm{Sr}-90$ & $1.000 \mathrm{E}+00$ & & 4.88 & 4.750 & 4.48 & 3.68 & 2.091 & 2.884 & 1 & 2.50 \\
\hline & & & & 1.2 & & & & & & & \\
\hline $\mathrm{U}-234$ & 230 & & & 1.3 & 4.13 & 0 & 2. & & & & \\
\hline $\mathrm{U}-234$ & $\mathrm{Ra}-226$ & $1.000 \mathrm{E}+00$ & & $1.315 \mathrm{E}-09$ & $9.195 \mathrm{E}-09$ & $4.850 \mathrm{E}-08$ & $4.305 \mathrm{E}-07$ & 3.550 & 3.566 & 04 & $1.405 \mathrm{E}-03$ \\
\hline $\mathrm{U}-234$ & $\mathrm{~Pb}-210$ & $1.000 \mathrm{E}+00$ & & 1.15 & 1.726 & 1.980 & 4.948 & 1.030 & 2.2781 & 6 & $17 \mathrm{E}-05$ \\
\hline $\mathrm{U}-234$ & $\operatorname{SDSR}(j)$ & & & $1.256 \mathrm{E}-02$ & $1.253 \mathrm{E}-02$ & $1.248 \mathrm{E}-02$ & $1.232 \mathrm{E}-02$ & $1.186 \mathrm{E}-02$ & $1.040 \mathrm{E}-02$ & 7.3321 & $3.340 \mathrm{E}-03$ \\
\hline $0-234$ & (1) & & & 1.539 & & & 1.510 & & 1.049 & 8.60 & $\begin{array}{l}.044 \\
2.21\end{array}$ \\
\hline 35 & $\mathrm{~Pa}-231$ & & & 1.88 & 5.6441 & 05 & 3.89 & 04 & 3.209 & 6.891 & $7.260 \mathrm{E}-04$ \\
\hline 35 & Ac -22 & 1 & & 1.12 & 7.756 & 4 & 3.28 & 2.193 & & & 3 \\
\hline$U-235$ & $\operatorname{SDSR}(j)$ & & & $1.539 \mathrm{E}-01$ & $1.536 \mathrm{E}-01$ & $1.530 \mathrm{E}$ & $1.510 \mathrm{E}-01$ & $1.455 \mathrm{E}-01$ & $1.283 \mathrm{E}-01$ & 102E & $2.662 \mathrm{E}$ \\
\hline OU -238 & $\mathrm{U}-238$ & 0 & & $3.874 \mathrm{E}-02$ & $3.867 \mathrm{E}-02$ & & & & $3.191 \mathrm{E}-02$ & & $66 \mathrm{E}$ \\
\hline 2 200 & & & & & & & & & & & \\
\hline $\mathrm{U}-238$ & Th & 1.00 & & 1. & 9.11 & 4.80 & 4.25 & 3.50 & 3.47 & 2. & $5 E-07$ \\
\hline U. & $\mathrm{Ra}$ & 1 & & $9.317 \mathrm{E}-$ & $1.396 \mathrm{E}$ & $1.625 \mathrm{E}-13$ & $4.269 \mathrm{E}-12$ & $1.017 \mathrm{E}-10$ & $3.317 \mathrm{E}-09$ & & 1 \\
\hline $\mathrm{U}-2$ & $\mathrm{~Pb}-210$ & $1.000 \mathrm{E}+00$ & & $6.578 \mathrm{E}-20$ & $2.026 \mathrm{E}-18$ & $5.034 \mathrm{E}-17$ & $3.742 \mathrm{E}-15$ & $2.310 \mathrm{E}-13$ & $1.781 \mathrm{E}-11$ & $5.861 \mathrm{E}-10$ & $1.119 \mathrm{E}$ \\
\hline $\mathrm{U}-238$ & $\operatorname{SDSR}(j)$ & & & $3.874 \mathrm{E}-02$ & $3.867 \mathrm{E}-02$ & $3.852 \mathrm{E}-02$ & $3.800 \mathrm{E}-02$ & $3.655 \mathrm{E}-02$ & $3.191 \mathrm{E}-02$ & $2.166 \mathrm{E}-02$ & $5.573 \mathrm{E}-03$ \\
\hline
\end{tabular}

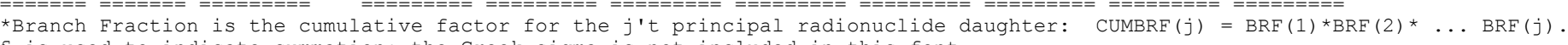
$\$$ is used to indicate summation; the Greek sigma is not included in this font. 
1RESRAD, Version $6.21 \quad$ T3/ Limit $=0.5$ year

02/22/2005 17:38 Page 23

RESRAD, Version 6.210 d T//2 Limit $=0.5$ year
Summary : CAU 224250 day/yr worker scenario $100 \mathrm{~m}$ hotspot
File : cau224 $250100 \mathrm{~m}$ parcel. RAD

Single Radionuclide Soil Guidelines $\mathrm{G}(\mathrm{i}, \mathrm{t})$ in $\mathrm{pCi} / \mathrm{g}$

ONuclide

Basic Radiation Dose Limit $=2.500 \mathrm{E}+01 \mathrm{mrem} / \mathrm{yr}$

\begin{tabular}{|c|c|c|c|c|c|c|c|c|c|}
\hline $\begin{array}{l}\text { (i) } \\
\text { (i) }\end{array}$ & $t=$ & $0.000 \mathrm{E}+00$ & $1.000 \mathrm{E}+00$ & $3.000 \mathrm{E}+00$ & $1.000 \mathrm{E}+01$ & $3.000 \mathrm{E}+01$ & $1.000 \mathrm{E}+02$ & $3.000 \mathrm{E}+02$ & $1.000 \mathrm{E}+03$ \\
\hline 241 & & $4.346 \mathrm{E}+02$ & $4.368 \mathrm{E}+02$ & $4.411 E+02$ & $4.568 \mathrm{E}+02$ & $5.047 \mathrm{E}+02$ & $7.153 E+02$ & $1.937 \mathrm{E}+03$ & $6.142 \mathrm{E}+04$ \\
\hline & & $4.138 \mathrm{E}+01$ & $4.237 \mathrm{E}+01$ & $41 \mathrm{E}+01$ & $235 E+01$ & $8.377 \mathrm{E}+01$ & $4.342 \mathrm{E}+02$ & $779 E+04$ & $91 \mathrm{E}+11$ \\
\hline Eu-152 & & $2.038 \mathrm{E}+01$ & $2.147 \mathrm{E}+01$ & $2.383 E+01$ & $3.431 \mathrm{E}+01$ & $9.723 \mathrm{E}+01$ & $3.725 \mathrm{E}+03$ & $1.244 \mathrm{E}+08$ & $* 1.765 \mathrm{E}+14$ \\
\hline $\mathrm{Pu}-238$ & & $5.759 \mathrm{E}+02$ & $5.805 \mathrm{E}+02$ & $5.898 \mathrm{E}+02$ & $6.234 \mathrm{E}+02$ & $7.306 \mathrm{E}+02$ & $1.273 \mathrm{E}+03$ & $6.220 \mathrm{E}+03$ & $1.481 \mathrm{E}+06$ \\
\hline $\mathrm{Pu}-239$ & & $5.228 \mathrm{E}+02$ & $5.228 \mathrm{E}+02$ & $5.229 \mathrm{E}+02$ & $5.231 \mathrm{E}+02$ & $5.238 \mathrm{E}+02$ & $5.261 \mathrm{E}+02$ & $5.327 \mathrm{E}+02$ & $5.567 \mathrm{E}+02$ \\
\hline $\mathrm{Sr}-90$ & & $5.116 \mathrm{E}+03$ & $5.263 E+03$ & $5.570 \mathrm{E}+03$ & $6.790 \mathrm{E}+03$ & $1.196 \mathrm{E}+04$ & $8.668 \mathrm{E}+04$ & $2.488 \mathrm{E}+07$ & $* 1.365 \mathrm{E}+14$ \\
\hline $\mathrm{U}-234$ & & $1.991 \mathrm{E}+03$ & $1.995 \mathrm{E}+03$ & $2.002 \mathrm{E}+03$ & $2.029 \mathrm{E}+03$ & $2.108 \mathrm{E}+03$ & $2.404 \mathrm{E}+03$ & $3.410 \mathrm{E}+03$ & $7.485 \mathrm{E}+03$ \\
\hline $\mathrm{U}-235$ & & $1.624 \mathrm{E}+02$ & $1.627 \mathrm{E}+02$ & $1.634 \mathrm{E}+02$ & $1.655 \mathrm{E}+02$ & $1.718 \mathrm{E}+02$ & $1.948 \mathrm{E}+02$ & $2.777 \mathrm{E}+02$ & $9.392 \mathrm{E}+02$ \\
\hline $\mathrm{U}-238$ & & $6.453 \mathrm{E}+02$ & $6.465 \mathrm{E}+02$ & $6.490 \mathrm{E}+02$ & $6.579 \mathrm{E}+02$ & $6.839 \mathrm{E}+02$ & $7.833 \mathrm{E}+02$ & $1.154 \mathrm{E}+03$ & $4.486 \mathrm{E}+03$ \\
\hline$======$ & & $===-===-=$ & $=========$ & $=======-=$ & $=========$ & $=========$ & $=========$ & $=========$ & $========$ \\
\hline
\end{tabular}

Summed Dose/Source Ratios DSR(i,t) in (mrem/yr)/(pCi/g)

and Single Radionuclide Soil Guidelines $G(i, t)$ in $\mathrm{pCi} / \mathrm{g}$
tmin = time of minimum single radionuclide soil guideline $\begin{aligned} \text { at } \operatorname{tmin} & =\text { time of minimum single radionuclide soil gui } \\ \text { and at } \operatorname{tmax} & =\text { time of maximum total dose }=0.000 \mathrm{E}+00 \text { years }\end{aligned}$

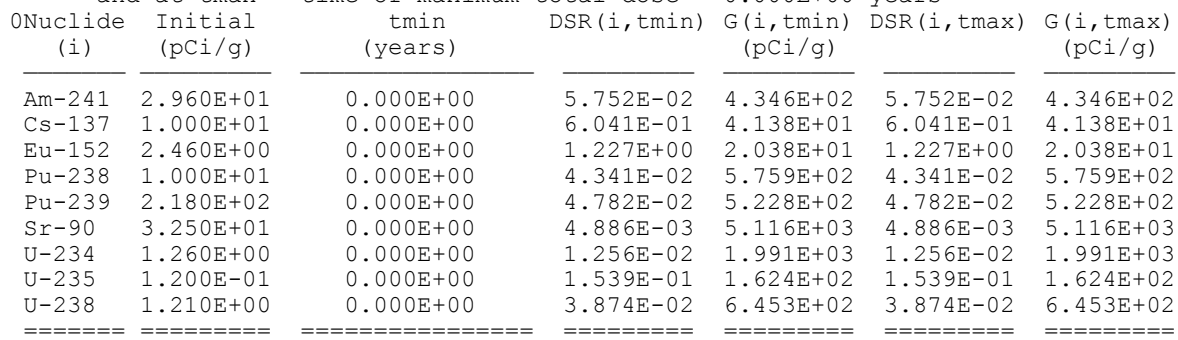


1RESRAD, Version $6.21 \quad \mathrm{~T} \frac{1}{2}$ Limit $=0.5$ year

Pl/ Limit $=0.5$ year 02/22/2005 17:38 Page 24

Summary : CAU 224250 day/yr worker scenario $100 \mathrm{~m}$ hotspot

File : cau224 $250100 \mathrm{~m}$ parcel. RAD

Individual Nuclide Dose Summed Over All Pathways

Parent Nuclide and Branch Fraction Indicated

ONuclide Parent BRF(i)

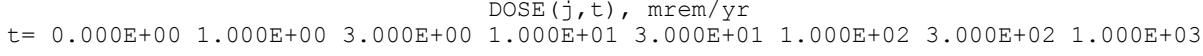

$\overline{\mathrm{Am}-241} \overline{\mathrm{Am}-241} \overline{1.000 \mathrm{E}+00}$ ONp-237 Am-241 $1.000 \mathrm{E}+00$ $00-233$ Am-241 $1.000 \mathrm{E}+00$

$\mathrm{OCs}-137$ Cs $-137 \quad 1.000 \mathrm{E}+00$

OEu-152 Eu-152 $7.208 \mathrm{E}-01$

Eu-152 Eu-152 2.792E-01

$\mathrm{Eu}-152$ \$DOSE (j)

$\begin{array}{ll}\mathrm{Eu}-152 & 2.792 \mathrm{E}-01 \\ \mathrm{Eu}-238 & 1.000 \mathrm{t}+00\end{array}$

$0 \mathrm{Pu}-238 \quad \mathrm{Pu}-238 \quad 1.000 \mathrm{E}+00$

OU-234 $\quad \mathrm{Pu}-238 \quad 1.000 \mathrm{E}+00$

$\mathrm{U}-234 \quad \mathrm{U}-234 \quad 1.000 \mathrm{E}+00$

$\mathrm{U}-234-\mathrm{U}-238 \quad 1.000 \mathrm{E}+0$

$\mathrm{U}-234$ SDOSE (j)

Th-230 U -234 - $1.000 \mathrm{E}+00$

Th-230 U $-234 \quad 1.000 \mathrm{E}+00$

Th-230 SDOSE (j)

$\begin{array}{ll}\mathrm{Th}-230 & \text { SDOSE (j) } \\ \mathrm{OR} a-226 & \mathrm{Pu}-238 \\ \mathrm{Ra} & 1.000 \mathrm{E}+00\end{array}$

Ra-226 U-234 $1.000 \mathrm{E}+00$

$\mathrm{Ra}-226 \mathrm{U}-238 \quad 1.000 \mathrm{E}+00$

$\mathrm{Ra}-226$ \$DOSE (j)

$\mathrm{Pb}-210 \quad \mathrm{Pu}-238 \quad 1.000 \mathrm{E}+00$

$\mathrm{Pb}-210 \mathrm{U}-234 \quad 1.000 \mathrm{E}+00$

$\mathrm{Pb}-210 \quad \mathrm{U}-238 \quad 1.000 \mathrm{E}+00$

$\mathrm{Pb}-210$ \$DOSE $(j)$

OPu-239 $\mathrm{Pu}-239 \quad 1.000 \mathrm{E}+00$

$\begin{array}{lll}0 \mathrm{U}-235 & \mathrm{Pu}-239 & 1.000 \mathrm{E}+0\end{array}$

$\mathrm{U}-235$ SDOSE (j)

$\begin{array}{lll}\mathrm{PPa}-231 & \mathrm{Pu}-239 & 1.000 \mathrm{E}+00\end{array}$

$\mathrm{Pa}-231 \mathrm{U}-235 \quad 1.000 \mathrm{E}+00$

$\mathrm{Pa}-231$ \$DOSE $(j)$

$\begin{array}{llllllll}1.703 \mathrm{E}+00 & 1.694 \mathrm{E}+00 & 1.677 \mathrm{E}+00 & 1.620 \mathrm{E}+00 & 1.466 \mathrm{E}+00 & 1.034 \mathrm{E}+00 & 3.816 \mathrm{E}-01 & 1.164 \mathrm{E}-02\end{array}$ $\begin{array}{llllllll}1.259 \mathrm{E}-06 & 3.767 \mathrm{E}-06 & 8.745 \mathrm{E}-06 & 2.576 \mathrm{E}-05 & 7.106 \mathrm{E}-05 & 1.965 \mathrm{E}-04 & 3.739 \mathrm{E}-04 & 4.061 \mathrm{E}-04\end{array}$

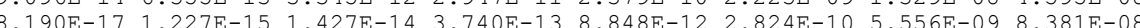
$\begin{array}{llllllll}6.041 \mathrm{E}+00 & 5.901 \mathrm{E}+00 & 5.630 \mathrm{E}+00 & 4.776 \mathrm{E}+00 & 2.984 \mathrm{E}+00 & 5.758 \mathrm{E}-01 & 5.231 \mathrm{E}-03 & 3.736 \mathrm{E}-10\end{array}$ $\begin{array}{llllllll}2.175 \mathrm{E}+00 & 2.065 \mathrm{E}+00 & 1.860 \mathrm{E}+00 & 1.292 \mathrm{E}+00 & 4.559 \mathrm{E}-01 & 1.190 \mathrm{E}-02 & 3.563 \mathrm{E}-07 & 5.227 \mathrm{E}-23\end{array}$

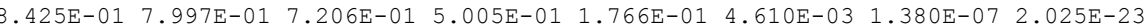

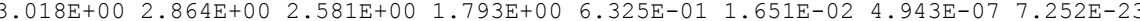

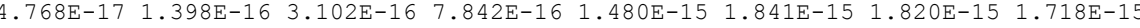

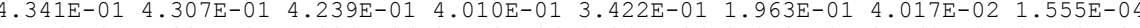

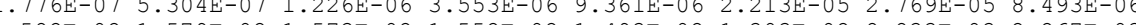

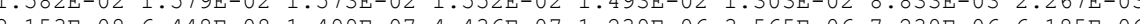

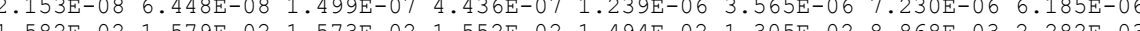

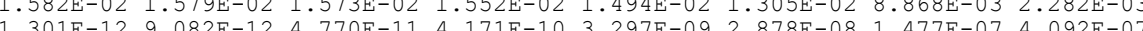
$\begin{array}{llll}1.738 \mathrm{E}-07 & 5.210 \mathrm{E}-07 & 1.213 \mathrm{E}-06 & 3\end{array}$ $1.577 \mathrm{E}-13 \quad 1.103 \mathrm{E}-12 \quad 5.813 \mathrm{E}-12 \quad 5.153 \mathrm{E}-11 \quad 4.234 \mathrm{E}-10 \quad 4.202 \mathrm{E}-09 \quad 2.923 \mathrm{E}-08 \quad 1.446 \mathrm{E}-07$

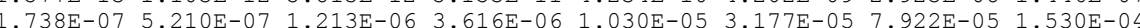
$\begin{array}{llllllll}9.306 \mathrm{E}-15 & 1.393 \mathrm{E}-13 & 1.616 \mathrm{E}-12 & 4.202 \mathrm{E}-11 & 9.723 \mathrm{E}-10 & 2.877 \mathrm{E}-08 & 4.672 \mathrm{E}-07 & 4.308 \mathrm{E}-06\end{array}$

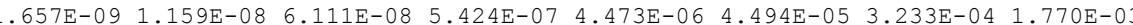

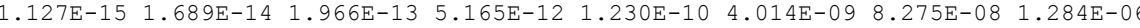

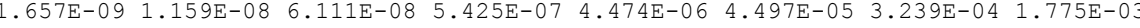

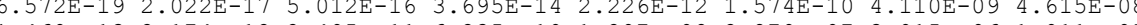

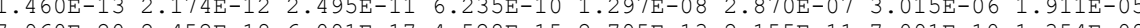
$\begin{array}{llllllll}7.960 \mathrm{E}-20 & 2.452 \mathrm{E}-18 & 6.091 \mathrm{E}-17 & 4.528 \mathrm{E}-15 & 2.795 \mathrm{E}-13 & 2.155 \mathrm{E}-11 & 7.091 \mathrm{E}-10 & 1.354 \mathrm{E}-08\end{array}$ $1.460 \mathrm{E}-132.174 \mathrm{E}-122.495 \mathrm{E}-112.235 \mathrm{E}-10 \mathrm{1} 1.298 \mathrm{E}-082.872 \mathrm{E}-073.020 \mathrm{E}-061.917 \mathrm{E}-05$

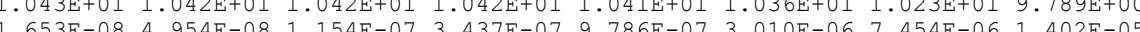
$1.847 \mathrm{E}-02 \quad 1.843 \mathrm{E}-02 \quad 1.836 \mathrm{E}-02 \quad 1.811 \mathrm{E}-02 \quad 1.743 \mathrm{E}-02 \quad 1.521 \mathrm{E}-02 \quad 1.032 \mathrm{E}-02 \quad 2.654 \mathrm{E}-03$ $\begin{array}{lllllllll}1.847 \mathrm{E}-02 & 1.843 \mathrm{E}-02 & 1.836 \mathrm{E}-02 & 1.811 \mathrm{E}-02 & 1.743 \mathrm{E}-02 & 1.5215 \mathrm{E}-02 & 1.032 \mathrm{E}-02 & 2.65 \mathrm{E}-03 \\ 1.349 \mathrm{E}-13 & 9.433 \mathrm{E}-13 & 4.975 \mathrm{E}-12 & 4.416 \mathrm{E}-11 & 3.641 \mathrm{E}-10 & 3.655 \mathrm{E}-02 & 1.033 \mathrm{E}-02 & 2.668 \mathrm{E}-03\end{array}$ $\begin{array}{lllllllll}1.349 \mathrm{E}-13 & 9.433 \mathrm{E}-13 & 4.975 \mathrm{E}-12 & 4.416 \mathrm{E}-11 & 3.641 \mathrm{E}-10 & 3.655 \mathrm{E}-0 & 2.633 \mathrm{E}-02 & 2.668 \mathrm{E}-03\end{array}$ $\begin{array}{llllllll}2.261 \mathrm{E}-07 & 6.773 \mathrm{E}-07 & 1.575 \mathrm{E}-06 & 4.671 \mathrm{E}-06 & 1.313 \mathrm{E}-05 & 3.851 \mathrm{E}-05 & 8.269 \mathrm{E}-05 & 8.712 \mathrm{E}-05\end{array}$ $2.261 \mathrm{E}-07 \quad 6.773 \mathrm{E}-07 \quad 1.575 \mathrm{E}-06 \quad 4.671 \mathrm{E}-06 \quad 1.313 \mathrm{E}-05 \quad 3.851 \mathrm{E}-05 \quad 8.272 \mathrm{E}-05 \quad 8.726 \mathrm{E}-05$ 
1RESRAD, Version $6.21 \quad T^{\frac{1}{2} / 2}$ Limit $=0.5$ year $\quad 02 / 22 / 2005 \quad 17: 38$ Page 25

Summary : CAU 224250 day/yr worker scenario $100 \mathrm{~m}$ hotspot
File : cau224 $250100 \mathrm{~m}$ parcel. RAD

Individual Nuclide Dose Summed Over All Pathways

Parent Nuclide and Branch Fraction Indicated

ONuclide Parent BRF(i)

$\frac{\text { (j) }}{\mathrm{AC}-227} \frac{\text { (i) }}{\mathrm{Pu}-239} \frac{}{1.000 \mathrm{E}+00}$

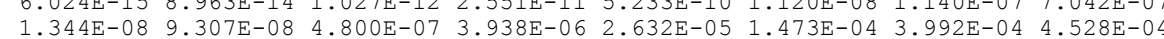

AC-227 \$DOSE $(j)$

(1.000

$\begin{array}{llllllll}1.344 \mathrm{E}-08 & 9.307 \mathrm{E}-08 & 4.800 \mathrm{E}-07 & 3.938 \mathrm{E}-06 & 2.632 \mathrm{E}-05 & 1.473 \mathrm{E}-04 & 3.992 \mathrm{E}-04 & 4.528 \mathrm{E}-04 \\ 1.344 \mathrm{E}-08 & 9.307 \mathrm{E}-08 & 4.800 \mathrm{E}-07 & 3.938 \mathrm{E}-06 & 2.632 \mathrm{E}-05 & 1.473 \mathrm{E}-04 & 3.994 \mathrm{E}-04 & 4.535 \mathrm{E}-04\end{array}$

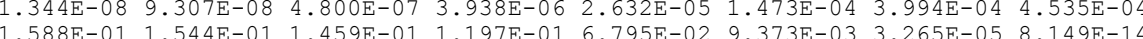

$\begin{array}{llllllll}4.688 \mathrm{E}-02 & 4.679 \mathrm{E}-02 & 4.661 \mathrm{E}-02 & 4.598 \mathrm{E}-02 & 4.423 \mathrm{E}-02 & 3.861 \mathrm{E}-02 & 2.620 \mathrm{E}-02 & 6.735 \mathrm{E}-03\end{array}$

BRF(i) is the branch fraction of the parent nuclide.

$\$$ is used to indicate summation; the Greek sigma is not included in this font. 
1RESRAD, Version $6.21 \quad \mathrm{~T}^{1} / 2$ Limit $=0.5$ year

T2/2 Limit $=0.5$ year $\quad 02 / 22 / 2005 \quad 17: 38$ Page 26

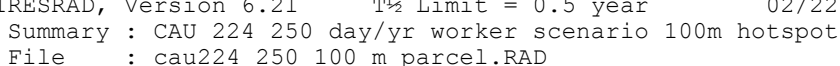

Individual Nuclide Soil Concentration

Parent Nuclide and Branch Fraction Indicated

ONuclide Parent BRF(i)

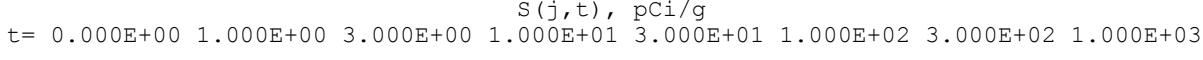

$\overline{\mathrm{Am}-241} \overline{\mathrm{Am}-241} \overline{1.000 \mathrm{E}+00}$ ONp-237 Am-241 $1.000 \mathrm{E}+00$ OU -233 Am-241 $1.000 \mathrm{E}+00$

$\mathrm{OCs}-137 \mathrm{Cs}-137 \quad 1.000 \mathrm{E}+00$

OEu-152 Eu-152 $7.208 \mathrm{E}-01$

Eu-152 Eu-152 $2.792 \mathrm{E}-01$

$\mathrm{Eu}-152$ \$S $(j):$

$\begin{array}{lll}\mathrm{OGd}-152 & \mathrm{Eu}-152 & 2.792 \mathrm{E}-01 \\ \mathrm{OPu}-238 & \mathrm{Pu}-238 & 1.000 \mathrm{E}+00\end{array}$

OU-234 $\quad \mathrm{Pu}-238 \quad 1.000 \mathrm{E}+00$

$\mathrm{U}-234 \quad \mathrm{U}-234 \quad 1.000 \mathrm{E}+00$

$\mathrm{U}-234 \quad \mathrm{U}-238 \quad 1.000 \mathrm{E}+00$

$\begin{array}{ccc}\mathrm{U}-234 & \$ S(j): & \\ 0 \mathrm{Th}-230 & \mathrm{Pu}-238 & 1.000 \mathrm{E}+00\end{array}$

Th-230 Pu-238 $1.000 \mathrm{E}+00$

Th-230 $1.000 \mathrm{E}+00$

Th-230 \$S(j):

ORa-226 Pu-238 1.000E+00

$\begin{array}{lll}\mathrm{Ra}-226 & \mathrm{U}-234 & 1.000 \mathrm{E}+00 \\ \mathrm{Ra}-226 & \mathrm{U}-238 & 1.000 \mathrm{E}+00\end{array}$

$\begin{array}{lll}\mathrm{Ra}-226 & \$ \mathrm{~S}(\mathrm{j}): & \\ & & \\ \mathrm{Rb}-210 & \mathrm{Pu}-238 & 1.000 \mathrm{E}+0\end{array}$

$\begin{array}{lll}0 \mathrm{~Pb}-210 & \mathrm{Pu}-238 & 1.000 \mathrm{E}+00 \\ \mathrm{~Pb}-210 & \mathrm{U}-234 & 1.000 \mathrm{E}+00\end{array}$

$\begin{array}{lll}\mathrm{Pb}-210 & \mathrm{U}-234 & 1.000 \mathrm{E}+00 \\ \mathrm{~Pb}-210 & \mathrm{U}-238 & 1.000 \mathrm{E}+00\end{array}$

$\begin{array}{lll}\mathrm{Pb}-210 & \mathrm{U}-238 & 1.000 \mathrm{E}+00 \\ \mathrm{~Pb}-210 & \$ \mathrm{~S}(\mathrm{j}): & \\ 0 & & \end{array}$

OPu-239 Pu-239 $1.000 \mathrm{E}+00$

OU-235 Pu-239 $1.000 \mathrm{E}+00$

$\mathrm{U}-235$ \$S $(j):$

$\begin{array}{ccc}0 \mathrm{~Pa}-231 & \mathrm{Pu}-239 & 1.000 \mathrm{E}+0 \\ \mathrm{~Pa}-231 & \mathrm{U}-235 & 1.000 \mathrm{E}+0\end{array}$

$\mathrm{Pa}-231 \$ S(j)$

$\begin{array}{llllllllll}2.960 \mathrm{E}+01 & 2.945 \mathrm{E}+01 & 2.916 \mathrm{E}+01 & 2.816 \mathrm{E}+01 & 2.549 \mathrm{E}+01 & 1.798 \mathrm{E}+01 & 6.634 \mathrm{E}+00 & 2.024 \mathrm{E}-01\end{array}$

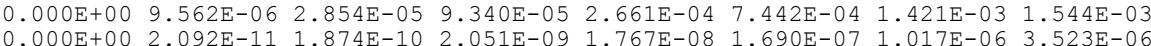

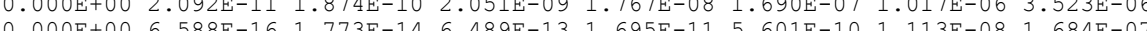
$1.000 \mathrm{E}+01 \quad 9.768 \mathrm{E}+00 \quad 9.319 \mathrm{E}+00 \quad 7.905 \mathrm{E}+00 \quad 4.940 \mathrm{E}+00 \quad 9.531 \mathrm{E}-01 \quad 8.659 \mathrm{E}-03 \quad 6.187 \mathrm{E}-10$ $\begin{array}{llllllll}1.773 \mathrm{E}+00 & 1.683 \mathrm{E}+00 & 1.517 \mathrm{E}+00 & 1.053 \mathrm{E}+00 & 3.717 \mathrm{E}-01 & 9.702 \mathrm{E}-03 & 2.905 \mathrm{E}-07 & 4.265 \mathrm{E}-23\end{array}$ $\begin{array}{llllllll}6.868 \mathrm{E}-01 & 6.520 \mathrm{E}-01 & 5.875 \mathrm{E}-01 & 4.080 \mathrm{E}-01 & 1.440 \mathrm{E}-01 & 3.758 \mathrm{E}-03 & 1.125 \mathrm{E}-07 & 1.652 \mathrm{E}-23\end{array}$

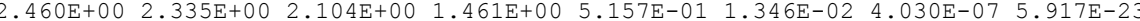

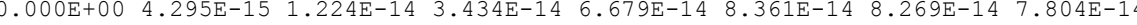

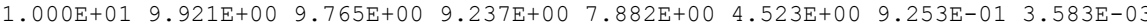

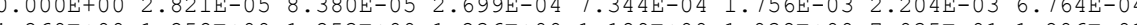

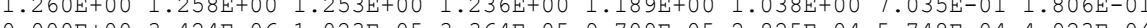

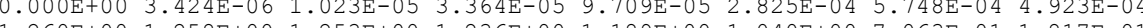

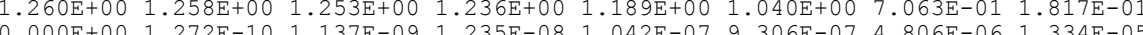
$0.000 \mathrm{E}+001.133 \mathrm{E}-05 \quad 3.393 \mathrm{E}-05 \quad 1.123 \mathrm{E}-04 \quad 3.305 \mathrm{E}-04 \quad 1.030 \mathrm{E}-032.574 \mathrm{E}-034.369 \mathrm{E}-03$

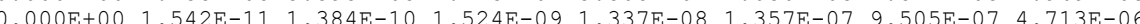
$\begin{array}{llllllll}0.000 \mathrm{E}+00 & 1.133 \mathrm{E}-05 & 3.393 \mathrm{E}-05 & 1.123 \mathrm{E}-04 & 3.306 \mathrm{E}-04 & 1.031 \mathrm{E}-03 & 2.580 \mathrm{E}-03 & 4.987 \mathrm{E}-03\end{array}$

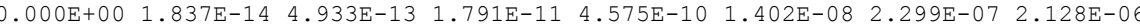

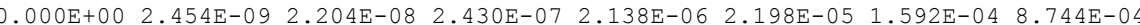

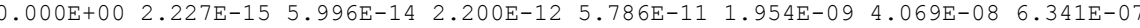

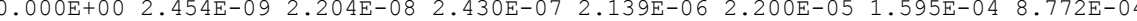

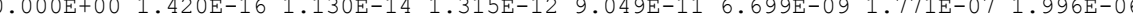

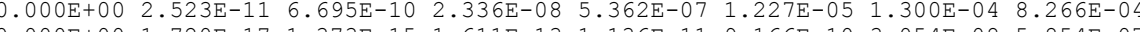

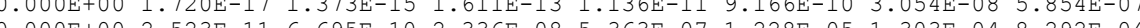

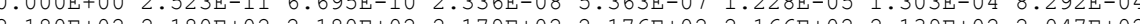

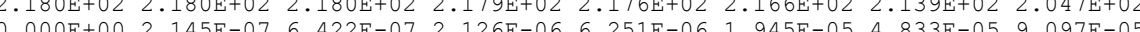

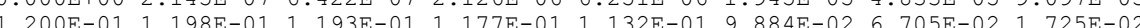

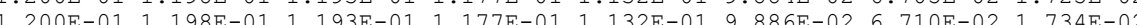

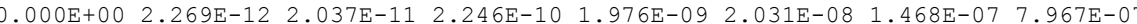
$\begin{array}{llllllll}0.000 \mathrm{E}+00 & 2.535 \mathrm{E}-06 & 7.579 \mathrm{E}-06 & 2.497 \mathrm{E}-05 & 7.247 \mathrm{E}-05 & 2.151 \mathrm{E}-04 & 4.634 \mathrm{E}-04 & 4.888 \mathrm{E}-04\end{array}$ $0.000 \mathrm{E}+00 \quad 2.535 \mathrm{E}-06 \quad 7.579 \mathrm{E}-06 \quad 2.497 \mathrm{E}-05 \quad 7.247 \mathrm{E}-05 \quad 2.151 \mathrm{E}-04 \quad 4.635 \mathrm{E}-04 \quad 4.896 \mathrm{E}-04$ 
1RESRAD, Version $6.21 \quad T^{3 / 2}$ Limit $=0.5$ year $02 / 22 / 2005 \quad 17: 38$ Page 27 Summary : CAU 224250 day/yr worker scenario $100 \mathrm{~m}$ hotspot

Individual Nuclide Soil Concentration

Parent Nuclide and Branch Fraction Indicated

ONuclide Parent BRF(i)

$\frac{(j)}{\text { (i) }} \frac{}{1.000 \mathrm{E}+00}$

Ac-227 Pu-239 $1.000 \mathrm{E}+00 \quad 0.000 \mathrm{E}+00 \frac{2.387 \mathrm{E}-14}{6.32}$

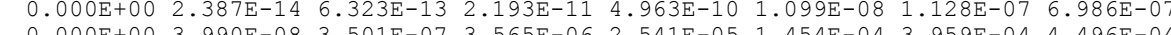
$\begin{array}{llllllllll}A c-227 & \$ S(j): & 0.000 \mathrm{E}+00 & 3.990 \mathrm{E}-08 & 3.501 \mathrm{E}-07 & 3.565 \mathrm{E}-06 & 2.541 \mathrm{E}-05 & 1.454 \mathrm{E}-04 & 3.960 \mathrm{E}-04 & 4.503 \mathrm{E}-04\end{array}$

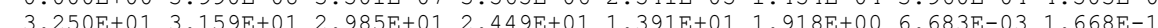

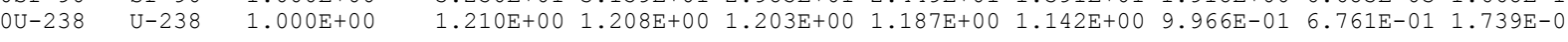
RF(i) is the branch fraction of the parent nuclide.

$\$$ is used to indicate summation; the Greek sigma is not included in this font. 


\section{Exhibit 3}

\section{RESRAD Scenario A Hot Spot $10 \mathrm{~m}^{2}$}

(27 Pages) 
1RESRAD, Version 6.21 TT1/2 Limit $=0.5$ year $02 / 22 / 2005 \quad 17: 45$ Page 1 Summary : CAU 224250 day/yr worker scenario $10 \mathrm{~m}$ hotspot

Table of Contents

Part I: Mixture Sums and Single Radionuclide Guidelines

Dose Conversion Eactor (and Relate

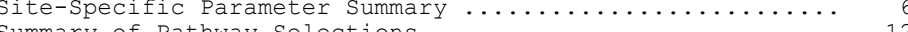
Contaminated Zone and Total Dose Summary $\ldots \ldots \ldots \ldots \ldots \ldots \ldots,{ }_{1}$ Total Dose Components Time $=0.000 \mathrm{E}+00$

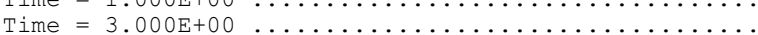

Time $=1.000 \mathrm{E}+01$

Time $=1.000 \mathrm{E}+02 \ldots$

Time $=3.000 \mathrm{E}+02$

Iime $=1.000 \mathrm{E}+03$

Dose/Source Ratios Summed Over All Pathways $\ldots \ldots \ldots \ldots \ldots$ Single Radionuclide Soll Guldelines... 
1RESRAD, Version $6.21 \quad T^{1 / 2}$ Limit $=0.5$ year

Summary : CAU 224250 day/yr worker scenario $10 \mathrm{~m}$ hotspot

02/22/2005 17:45 Page

Dose Conversion Factor (and Related) Parameter Summary

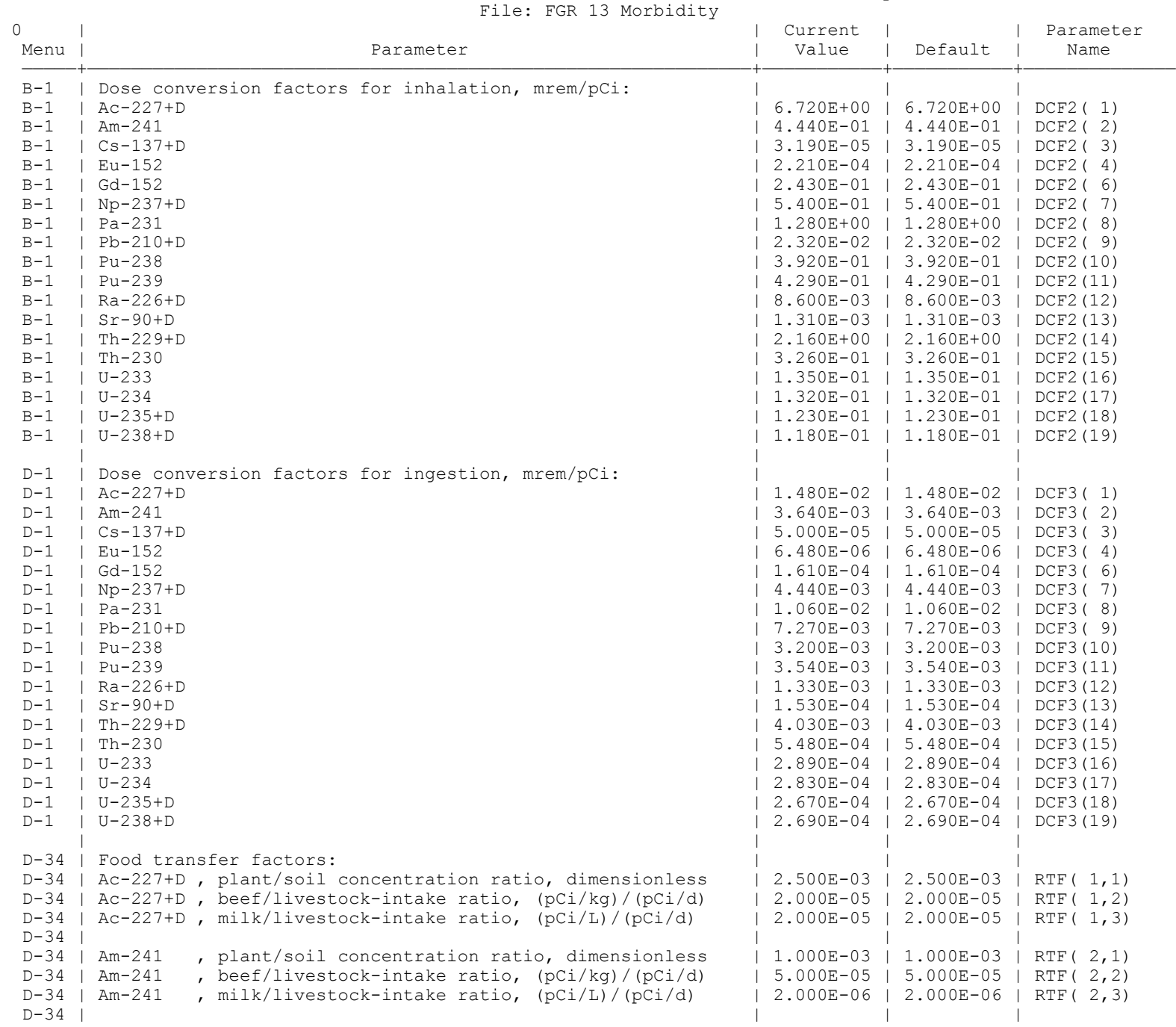


1RESRAD, Version $6.21 \quad T^{\frac{1}{2} / 2}$ Limit $=0.5$ year $\quad 02 / 22 / 2005 \quad 17: 45$ Page 3

Summary : CAU 224250 day/yr worker scenario $10 \mathrm{~m}$ hotspot

Dose Conversion Factor (and Related) Parameter Summary (continued) File: FGR 13 Morbidit

Menu Paramete

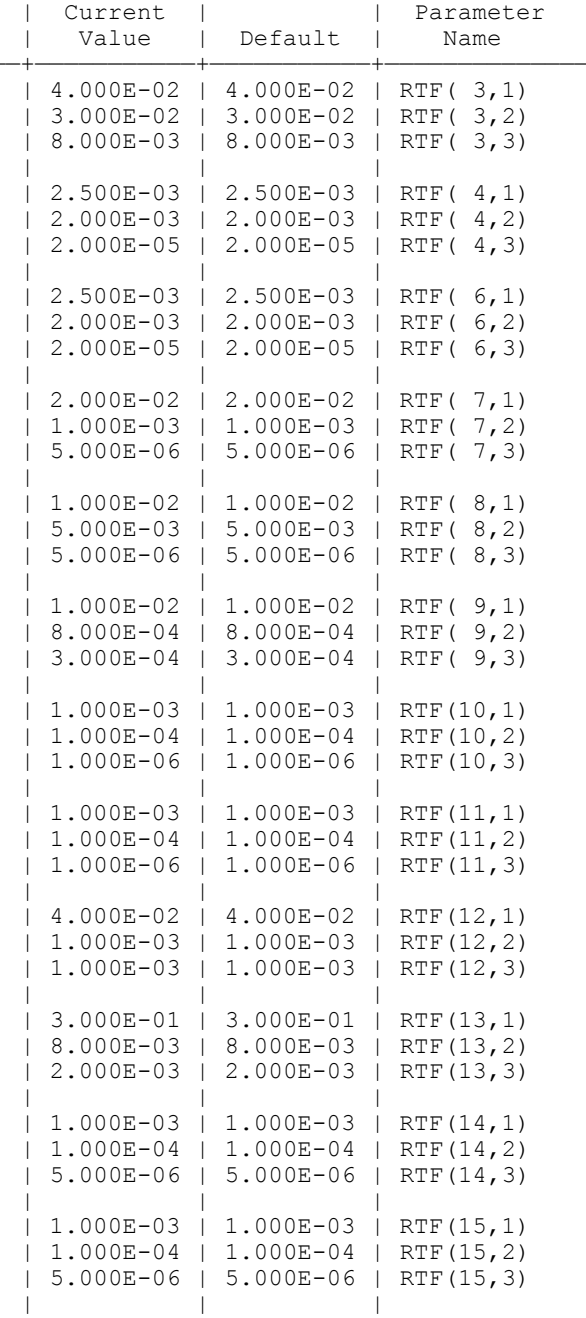

D-34 | Cs-137+D, plant/soil concentration ratio, dimensionless D-34 Cs-137+D, beef/livestock-intake ratio, $(\mathrm{pCi} / \mathrm{kg}) /(\mathrm{pCi} / \mathrm{d})$ $(\mathrm{pCi} / \mathrm{L}) /(\mathrm{pCi} / \mathrm{d})$

D-34 Eu-152, plant/soil concentration ratio, dimensionless D-34 Eu-152, beef/livestock-intake ratio, (pCi/kg)/(pCi/d) D -34

D-34 Gd-152, plant/soil concentration ratio, dimensionless D-34 Gd-152, beef/livestock-intake ratio, $(\mathrm{pCi} / \mathrm{kg}) /(\mathrm{pCi} / \mathrm{d})$ D-34 | Gd-152, milk/livestock-intake ratio, (pCi/L)/(pCi/d) D- 34

D-34 Np-237+D, plant/soil concentration ratio, dimensionless D-34 Np-237+D, beef/livestock-intake ratio, (pCi/kg)/(pCi/d) -34 Np-237+D, milk/livestock-intake ratio, (pCi/L)/(pCi/d) D -34

D-34 $\mid \mathrm{Pa}-231$, plant/soil concentration ratio, dimensionless $\mathrm{D}-34 \mid \mathrm{Pa}-231$, beef/livestock-intake ratio, $(\mathrm{pCi} / \mathrm{kg}) /(\mathrm{pCi} / \mathrm{d})$ D-34 | Pa-231, milk/livestock-intake ratio, (PCi/L)/(pCi/d) D-34

D-34 $\mathrm{Pb}-210+\mathrm{D}$, plant/soil concentration ratio, dimensionless D-34 Pb-210+D, beef/livestock-intake ratio, (pCi/kg)/(pCi/d) $\mathrm{D}-34 \mathrm{~Pb}-210+\mathrm{D}, \mathrm{milk} /$ livestock-intake ratio, (pCi/L)/(pCi/d)

D-34 Pu-238, plant/soil concentration ratio, dimensionless D-34 $\mid \mathrm{Pu}-238$ D-34 Pu-238, milk/livestock-intake ratio, ( $\mathrm{pCi} / \mathrm{L}) /(\mathrm{pCi} / \mathrm{d})$ D -34

D , beef/livestock-intake ratio, (pCi/kg)/(pCi/d) ivestock-intake ratio,

D-34 Ra-226+D, plant/soil concentration ratio, dimensionless D-34 Ra-226+D, beef/livestock-intake ratio, (pCi/kg)/(pCi/d) D-34 Ra-226+D, milk/livestock-intake ratio, (pCi/L)/(pCi/d) D-34 I

D-34 Sr-90+D, plant/soil concentration ratio, dimensionless D-34 Sr-90+D, beef/livestock-intake ratio, $(\mathrm{pCi} / \mathrm{kg}) /(\mathrm{pCi} / \mathrm{d})$ D-34 Sr-90+D , milk/livestock-intake ratio, (pCi/L)/(pCi/d)

D-34 Th-229+D, plant/soil concentration ratio, dimensionless (Th-229+D, beef/livestock-intake ratio, (pCi/kg)/(pCi/d) ( Th-229+D, milk/livestock-intake ratio, (pCi/L)/(pCi/d) D-34

D-34 Th-230, plant/soil concentration ratio, dimensionless D-34 Th-230, beef/livestock-intake ratio, $(\mathrm{pCi} / \mathrm{kg}) /(\mathrm{pCi} / \mathrm{d})$ D-34 
1RESRAD, Version 6.21 T1/2 Limit $=0.5$ year $02 / 22 / 2005 \quad 17: 45$ Page 4 Summary : CAU 224250 day/yr worker scenario $10 \mathrm{~m}$ hotspot

Dose Conversion Factor (and Related) Parameter Summary (continued)

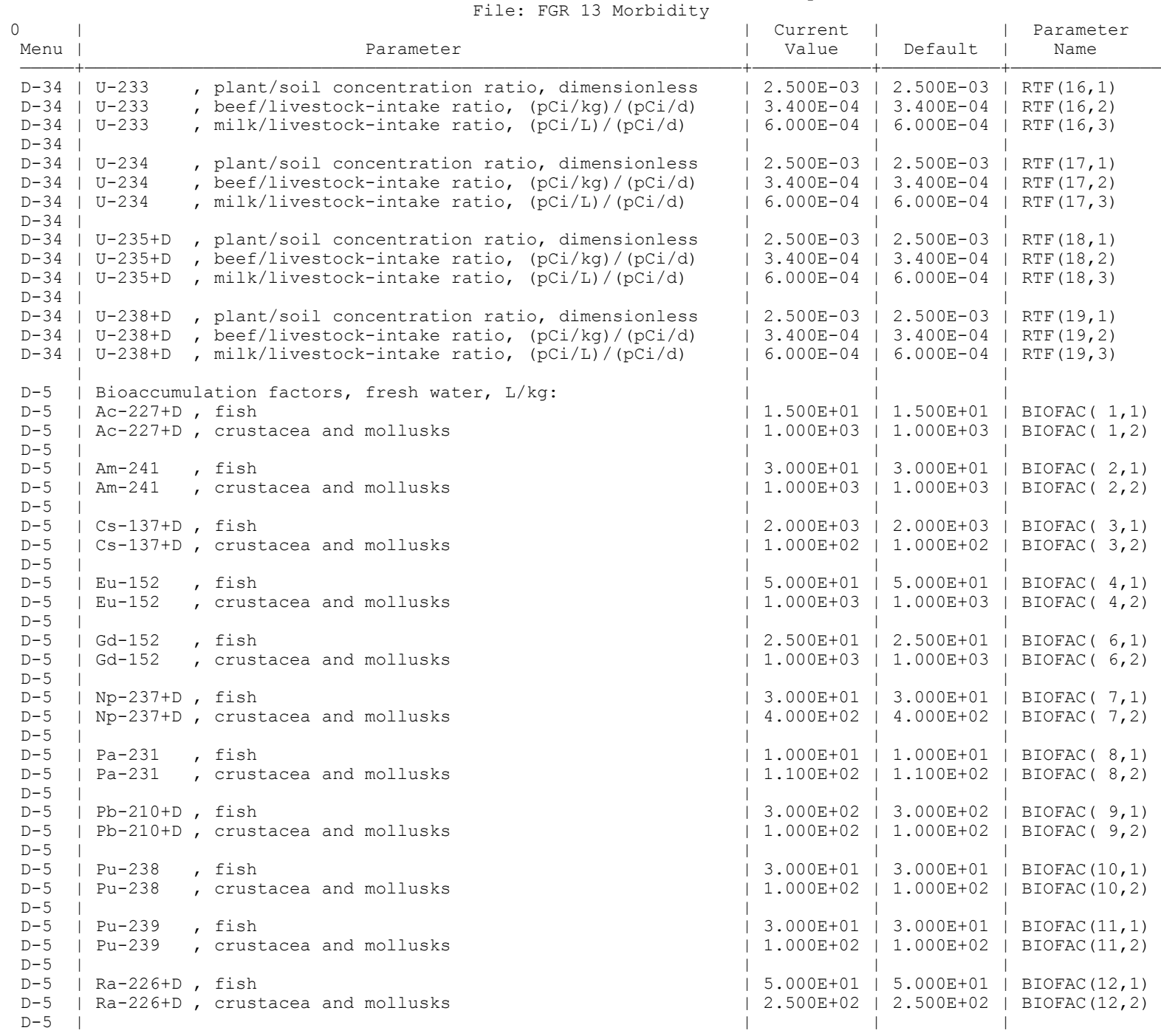


1RESRAD, Version 6.21 T13/2 Limit $=0.5$ year $\quad 02 / 22 / 2005 \quad 17: 45$ Page 5 Summary : CAU 224250 day/yr worker scenario $10 \mathrm{~m}$ hotspot

Dose Conversion Factor (and Related) Parameter Summary (continued)

\begin{tabular}{|c|c|c|c|c|c|}
\hline Menu & ! & Parameter & $\begin{array}{c}\text { Current } \\
\text { Value }\end{array}$ & Default & $\begin{array}{l}\text { Parameter } \\
\text { Name }\end{array}$ \\
\hline D-5 & Sr-90+D & fish & $6.000 \mathrm{E}+01$ & $6.000 \mathrm{E}+01$ & $\operatorname{BIOFAC}(13,1)$ \\
\hline$D-5$ & Sr $-90+D$ & crustacea and mollusks & $1.000 \mathrm{E}+02$ & $1.000 \mathrm{E}+02$ & $\operatorname{BIOFAC}(13,2)$ \\
\hline $\begin{array}{l}D-5 \\
D-5\end{array}$ & $T h-229+D$ & fish & $1.000 \mathrm{E}+02$ & $1.000 \mathrm{E}+02$ & $\operatorname{BIOFAC}(14,1)$ \\
\hline $\begin{array}{l}D-5 \\
D-5\end{array}$ & Th-229+D & , crustacea and mollusks & $5.000 \mathrm{E}+02$ & $5.000 \mathrm{E}+02$ & $\operatorname{BIOFAC}(14,2)$ \\
\hline$D-5$ & Th-230 & , fish & $1.000 \mathrm{E}+02$ & $1.000 \mathrm{E}+02$ & $\operatorname{BIOFAC}(15,1)$ \\
\hline $\begin{array}{l}D-5 \\
D-5\end{array}$ & Th-230 & , crustacea and mollusks & $5.000 \mathrm{E}+02$ & $5.000 \mathrm{E}+02$ & $\operatorname{BIOFAC}(15,2)$ \\
\hline D-5 & U-233 & , fish & $1.000 \mathrm{E}+01$ & $1.000 \mathrm{E}+01$ & $\operatorname{BIOFAC}(16,1)$ \\
\hline $\begin{array}{l}D-5 \\
D-5\end{array}$ & $\mathrm{U}-233$ & crustacea and mollusks & $6.000 \mathrm{E}+01$ & $6.000 \mathrm{E}+01$ & $\operatorname{BIOFAC}(16,2)$ \\
\hline$D-5$ & U-234 & , fish & $1.000 \mathrm{E}+01$ & $1.000 \mathrm{E}+01$ & $\operatorname{BIOFAC}(17,1)$ \\
\hline $\begin{array}{l}D-5 \\
D-5\end{array}$ & $\mathrm{U}-234$ & , crustacea and mollusks & $6.000 \mathrm{E}+01$ & $6.000 \mathrm{E}+01$ & $\operatorname{BIOFAC}(17,2)$ \\
\hline$D-5$ & $\mathrm{U}-235+\mathrm{D}$ & , fish & $1.000 \mathrm{E}+01$ & $1.000 \mathrm{E}+01$ & $\operatorname{BIOFAC}(18,1)$ \\
\hline $\begin{array}{l}D-5 \\
D-5\end{array}$ & $\mathrm{U}-235+\mathrm{D}$ & , crustacea and mollusks & $6.000 \mathrm{E}+01$ & $6.000 \mathrm{E}+01$ & $\operatorname{BIOFAC}(18,2)$ \\
\hline D-5 & $\mathrm{U}-238+\mathrm{D}$ & , fish & $1.000 \mathrm{E}+01$ & $1.000 \mathrm{E}+01$ & $\operatorname{BIOFAC}(19,1)$ \\
\hline$D-5$ & $U-238+D$ & crustacea and mollusks & $6.000 \mathrm{E}+01$ & $6.000 \mathrm{E}+01$ & BTOFAC $(19,2), 2$ \\
\hline
\end{tabular}




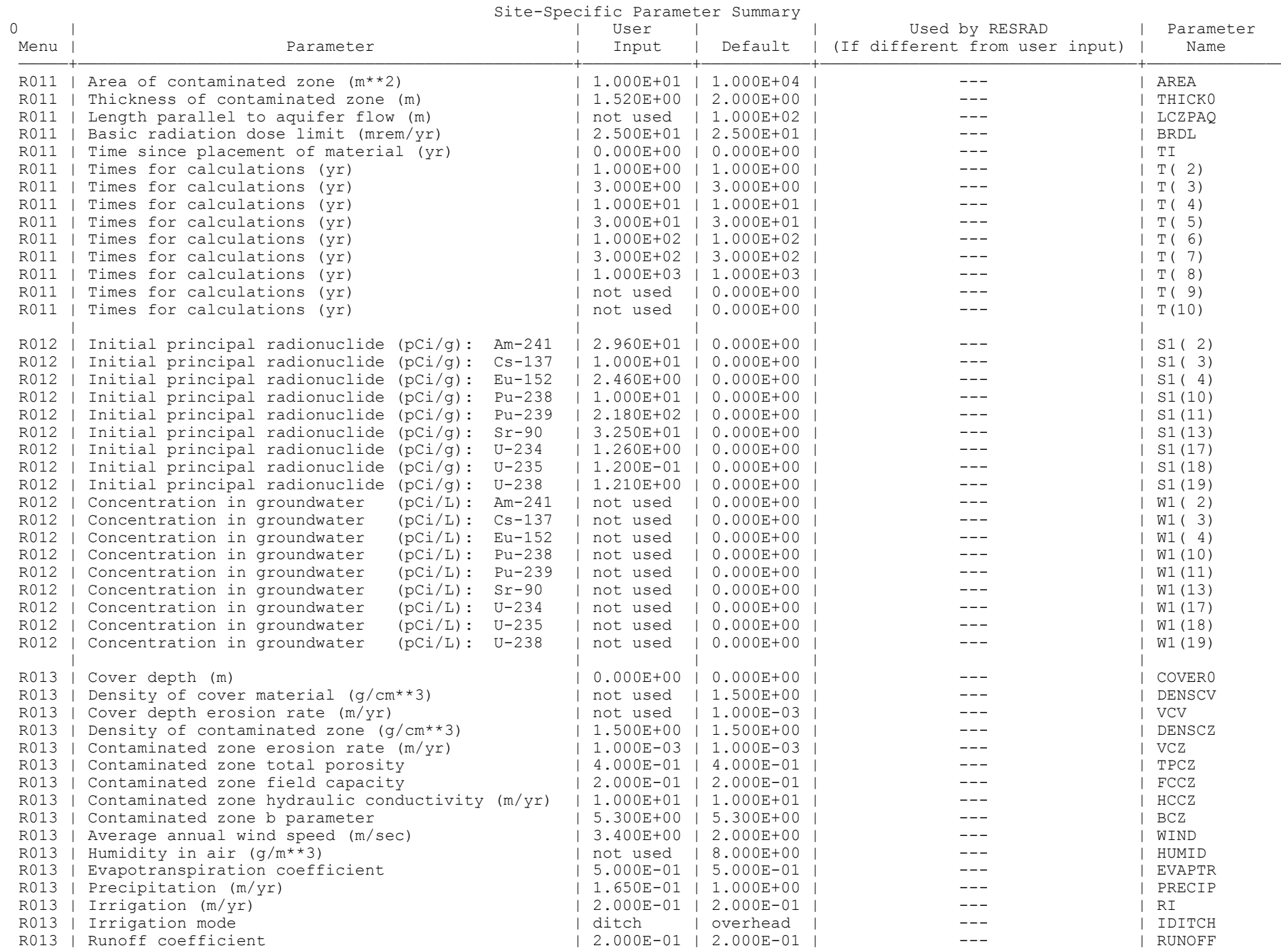


1RESRAD, Version $6.21 \quad T^{3} / 2$ Limit $=0.5$ year

Summary : CAU 224250 day/yr worker scenario $10 \mathrm{~m}$ hotspot

Site-Specific Parameter summary (continued)

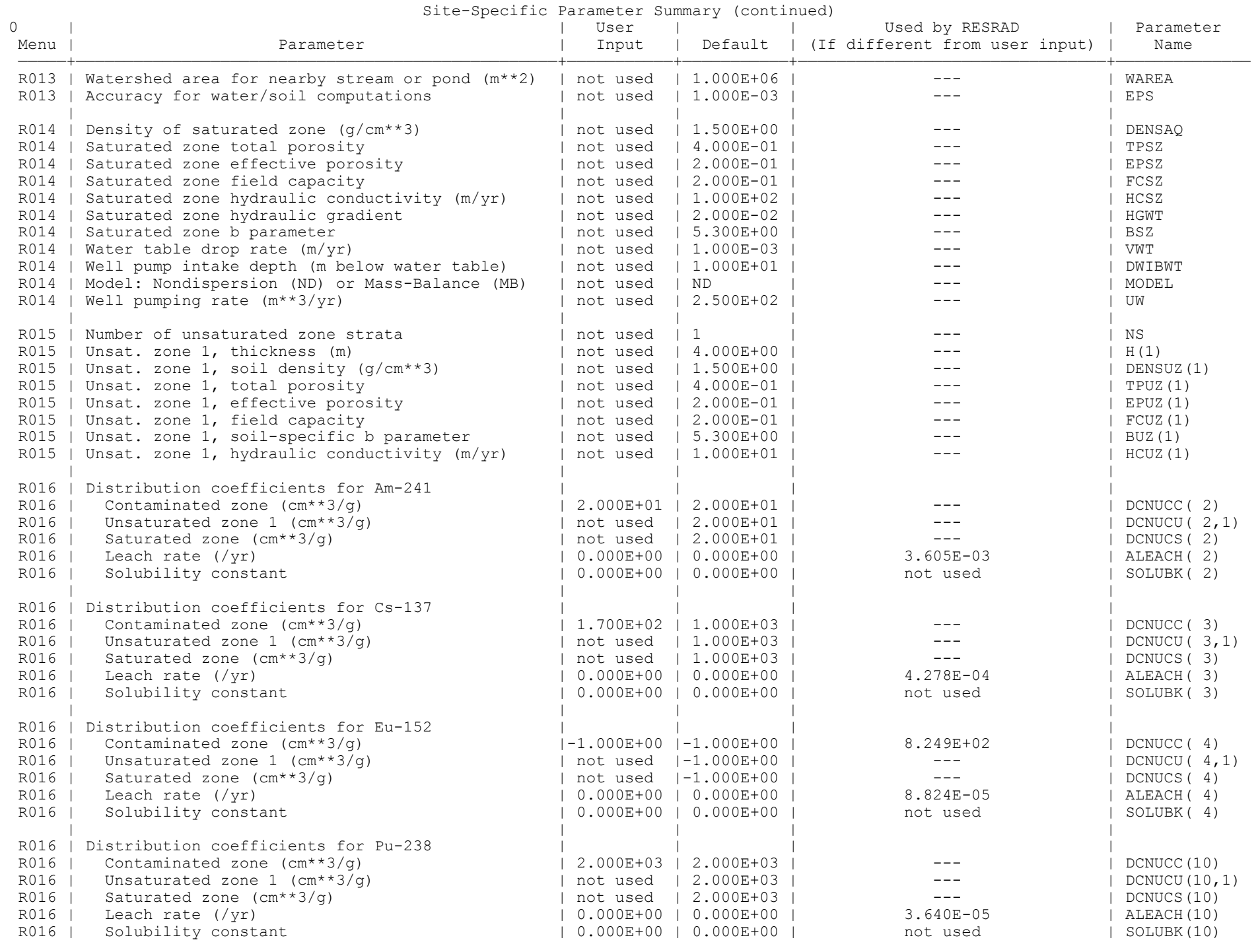


1RESRAD, Version $6.21 \quad T^{3 / 2}$ Limit $=0.5$ year

Summary : CAU 224250 day/yr worker scenario $10 \mathrm{~m}$ hotspot

Site-Specific Parameter Summary (continued)

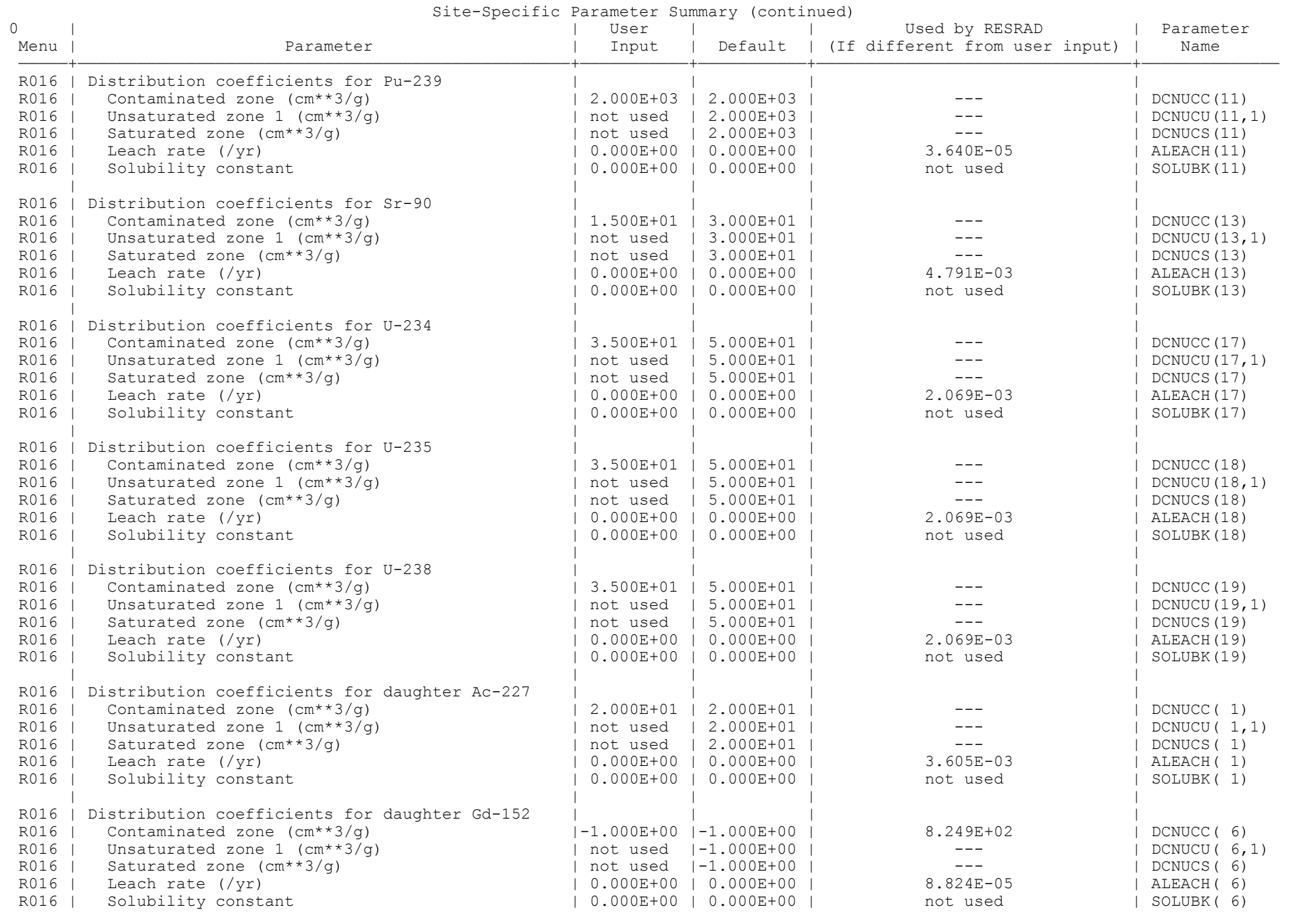


1RESRAD, Version $6.21 \quad T^{1 / 2}$ Limit $=0.5$ year

Summary : CAU 224250 day/yr worker scenario $10 \mathrm{~m}$ hotspot

Site-Specific Parameter Summary (continued)

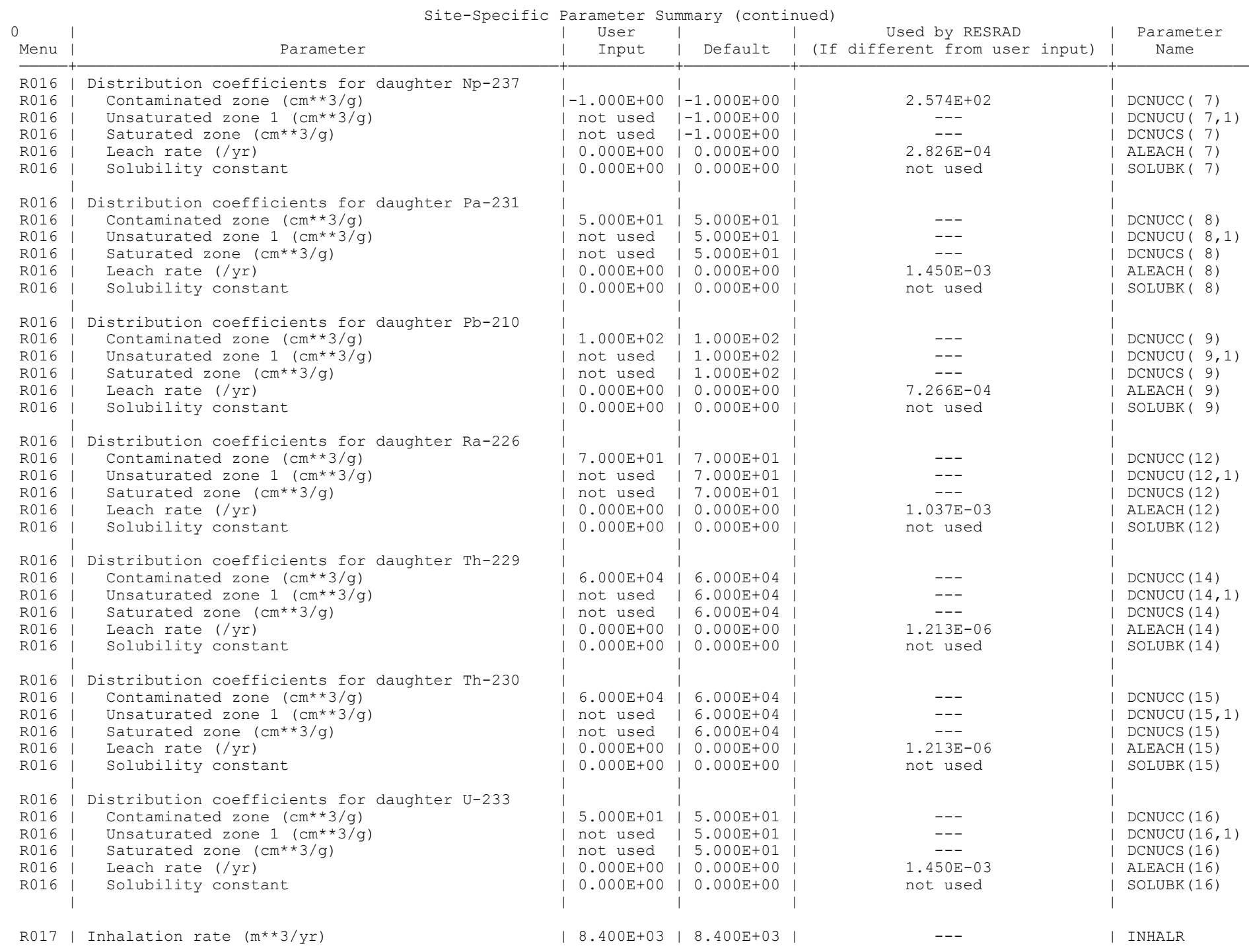




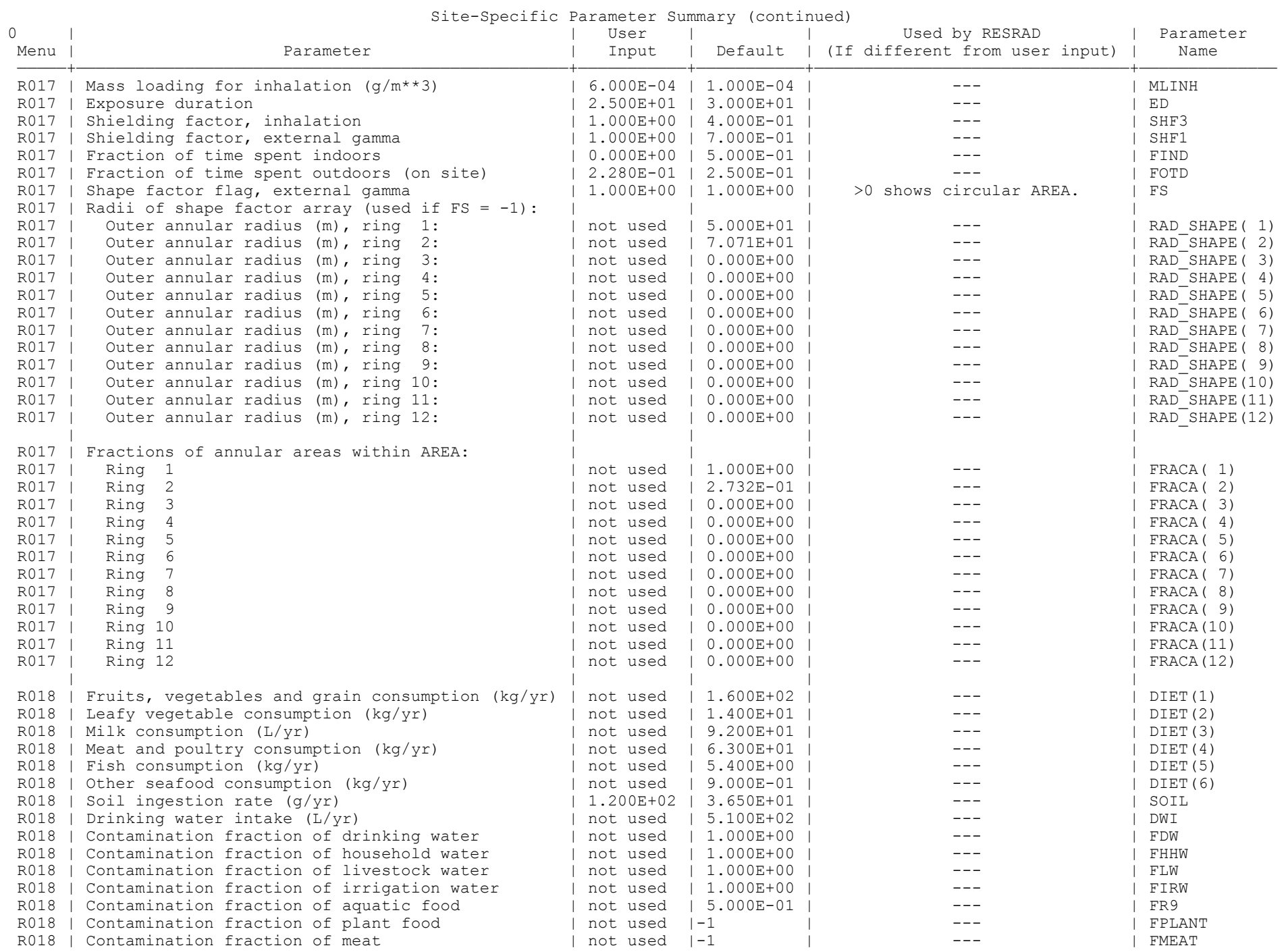


Site-Specific Parameter summary (continued)

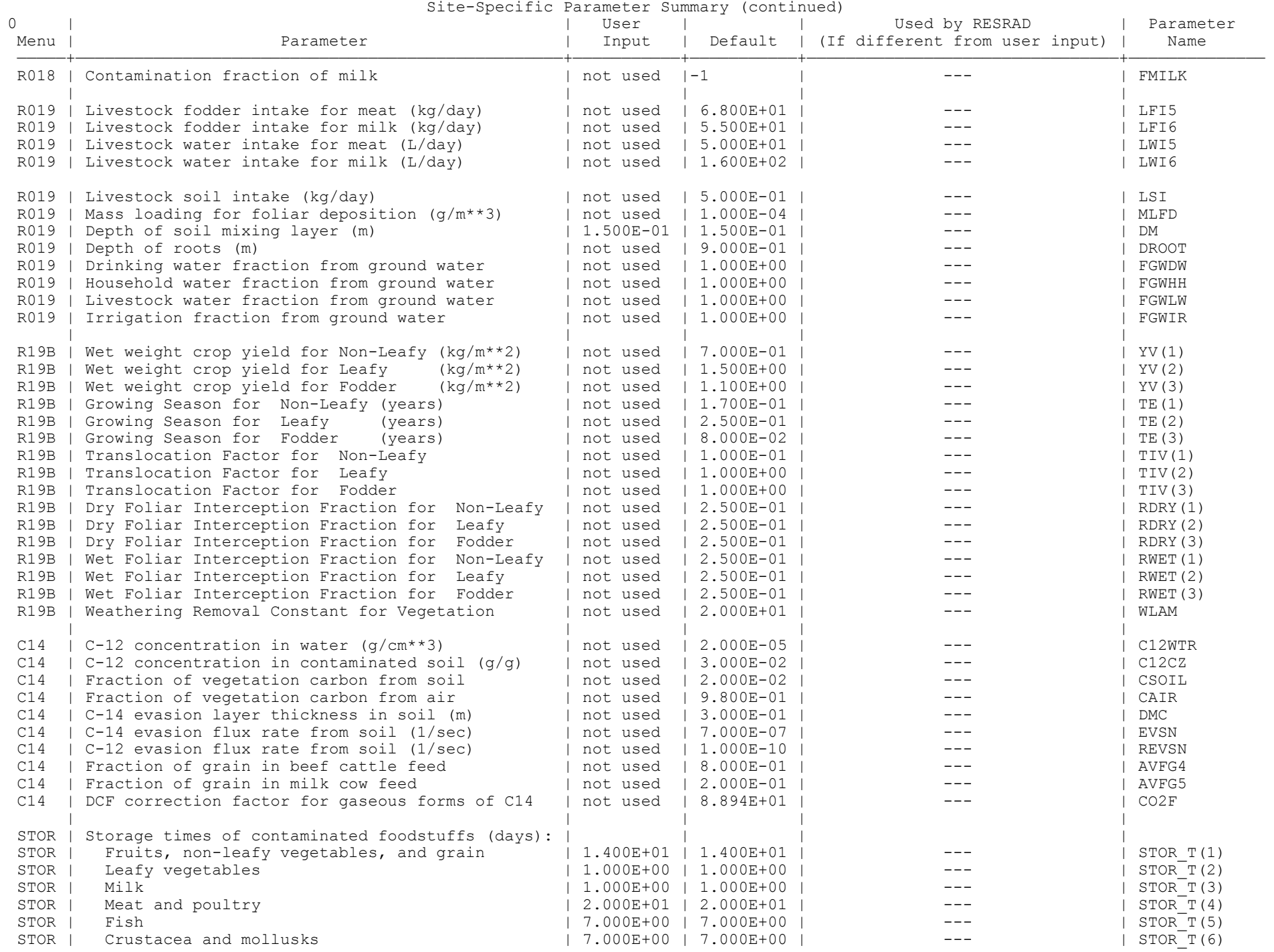


1RESRAD, Version $6.21 \quad T^{1 / 2}$ Limit $=0.5$ year Summary : CAU 224250 day/yr worker scenario $10 \mathrm{~m}$ hotspot

\section{Site-Specific Parameter Summary (continued)}

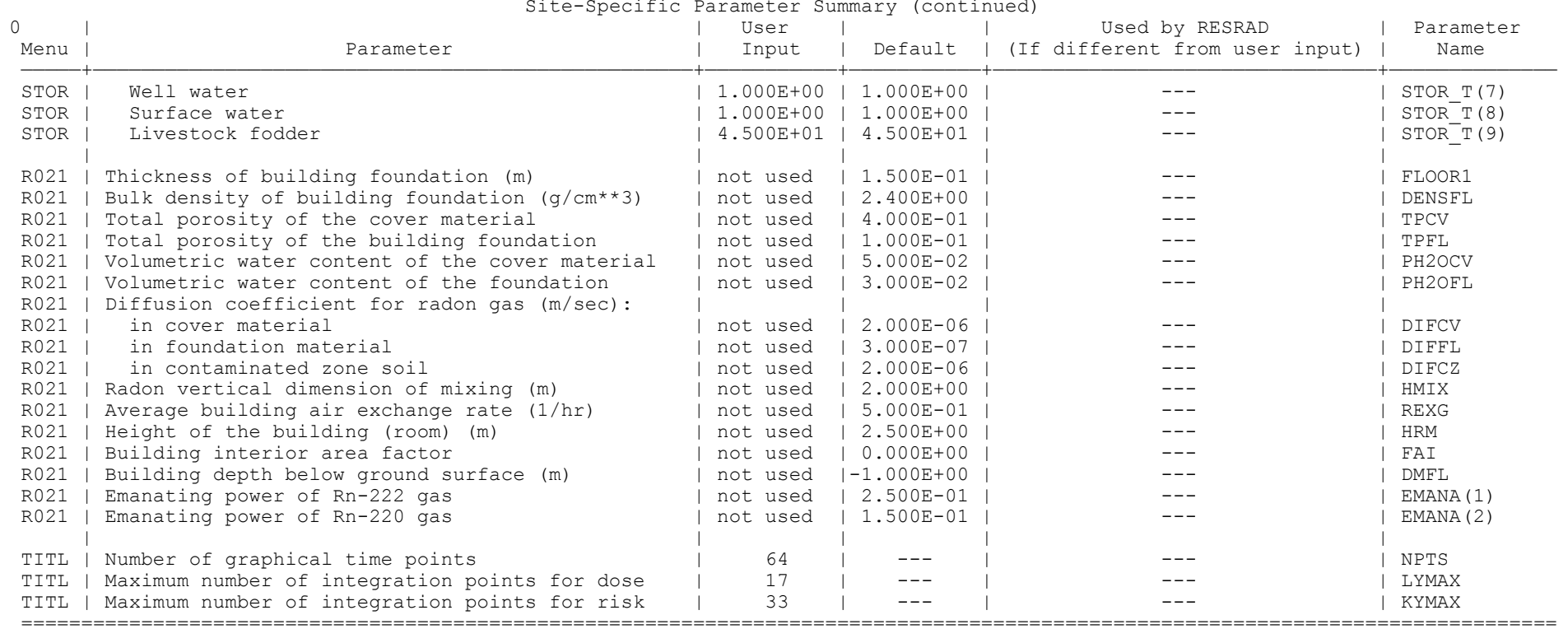

Summary of Pathway Selections

\begin{tabular}{c|c} 
Pathway & User Selection \\
\hline $1--$ external gamma & active \\
$2--$ inhalation (w/o radon) & active \\
$3--$ plant ingestion & suppressed \\
$4--$ meat ingestion & suppressed \\
$5--$ milk ingestion & suppressed \\
6 -- aquatic foods & suppressed \\
$7--$ drinking water & active \\
$8--$ soil ingestion & suppressed \\
9-- radon & active \\
Find peak pathway doses & $\mid$
\end{tabular}


1RESRAD, Version $6.21 \quad T^{3 / 2}$ Limit $=0.5$ year $\quad 02 / 22 / 2005 \quad 17: 45$ Page 13 Summary : CAU 224250 day/yr worker scenario $10 \mathrm{~m}$ hotspot

Contaminated Zone Dimensions

Area: $\quad 10.00$ square meters

over Depth:

1.52 meters

Total Dose TDOSE(t), mrem/y

\begin{tabular}{cc} 
Initial Soil Concentrations, $\mathrm{pCi} / \mathrm{g}$ \\
\hline $\mathrm{Am}-241$ & $2.960 \mathrm{E}+01$ \\
$\mathrm{Cs}-137$ & $1.000 \mathrm{E}+01$ \\
$\mathrm{Eu}-152$ & $2.460 \mathrm{E}+00$ \\
$\mathrm{Pu}-238$ & $1.000 \mathrm{E}+01$ \\
$\mathrm{Pu}-239$ & $2.180 \mathrm{E}+02$ \\
$\mathrm{Sr}-90$ & $3.250 \mathrm{E}+01$ \\
$\mathrm{U}-234$ & $1.260 \mathrm{E}+00$ \\
$\mathrm{U}-235$ & $1.200 \mathrm{E}-01$ \\
$\mathrm{U}-238$ & $1.210 \mathrm{E}+00$
\end{tabular}

Basic Radiation Dose Limit $=2.500 \mathrm{E}+01 \mathrm{mrem} / \mathrm{yr}$

Total Mixture Sum $M(t)=$ Fraction of Basic Dose Limit Received at Time (t)

$t$ (years): $\begin{array}{llllllll}0.000 \mathrm{E}+00 & 1.000 \mathrm{E}+00 & 3.000 \mathrm{E}+00 & 1.000 \mathrm{E}+01 & 3.000 \mathrm{E}+01 & 1.000 \mathrm{E}+02 & 3.000 \mathrm{E}+02 & 1.000 \mathrm{E}+03\end{array}$ $\begin{array}{ccccccccc}\text { DOSE }(t): & 1.353 \mathrm{E}+01 & 1.335 \mathrm{E}+01 & 1.300 \mathrm{E}+01 & 1.196 \mathrm{E}+01 & 1.004 \mathrm{E}+01 & 7.810 \mathrm{E}+00 & 6.841 \mathrm{E}+00 & 6.277 \mathrm{E}+00 \\ M(t): & 5.412 \mathrm{E}-01 & 5.338 \mathrm{E}-01 & 5.199 \mathrm{E}-01 & 4.783 \mathrm{E}-01 & 4.016 \mathrm{E}-01 & 3.124 \mathrm{E}-01 & 2.736 \mathrm{E}-01 & 2.511 \mathrm{E}-01\end{array}$ 
Total Dose Contributions TDOSE (i,p,t) for Individual Radionuclides (i) and Pathways (p) As mrem/yr and Fraction of Total Dose At $t=0.000 \mathrm{E}+00$ years Water Independent
Radon Plant

Milk

\begin{tabular}{|c|c|c|c|c|c|}
\hline \multicolumn{2}{|c|}{ Meat } & \multicolumn{2}{|c|}{ Milk } & \multicolumn{2}{|c|}{ Soil } \\
\hline mrem/y & fract & mrem/yr & fract. & $\mathrm{mrem} / \mathrm{yr}$ & fract. \\
\hline & & & & & \\
\hline & & & & & \\
\hline & & & & & \\
\hline & & & & & \\
\hline & 0 & & & & \\
\hline & 0.0 & & & & \\
\hline+00 & 0.0000 & 0 & 0.0 & 9.7 . & 0 \\
\hline & 0.0 & & & & \\
\hline & 0.00 & $0.000 \mathrm{E}+00$ & 0.00 & 05 & \\
\hline & & & $======$ & $=========$ & 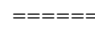 \\
\hline 0 & 0000 & $000 E+00$ & 0000 & $.509 \mathrm{E}-01$ & 01 \\
\hline
\end{tabular}

mrem/yr fract. mrem/yr fract. $9.063 \mathrm{E}-010.0670$ Nuclide mrem/yr fract. Am-241 $1.518 \mathrm{E}-01 \quad 0.0112$

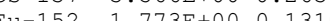
$\mathrm{Pu}-238 \quad 2.095 \mathrm{E}-04 \quad 0.0000$ Pu-239 $7.751 \mathrm{E}-03 \quad 0.0006$ $\begin{array}{lll}\mathrm{Sr}-90 & 8.321 \mathrm{E}-02 & 0.0062 \\ \mathrm{U}-234 & 6.102 \mathrm{E}-05 & 0.0000\end{array}$ $\begin{array}{lll}\mathrm{U}-235 & 1.022 \mathrm{E}-02 & 0.0008\end{array}$ $\mathrm{U}-238 \quad 1.971 \mathrm{E}-02 \quad 0.0015$ Total $\quad 5.611 \mathrm{E}+00 \quad 0.4148$

Total Dose Contributions TDOSE (i,p,t) for Individual Radionuclides (i) and Pathways (p) As mrem/yr and Fraction of Total Dose At $t=0.000 \mathrm{E}+00$ years

Fish

Radon Plant

Meat

Milk

mrem/yr fract. $\begin{array}{lll}0.000 \mathrm{E}+00 & 0.0000 \\ 0.000 \mathrm{E}+00 & 0.0000 \\ 0.000 \mathrm{E}+00 & 0.0000 & 1\end{array}$ $0.000 \mathrm{E}+00 \quad 0.0000$ $0.000 \mathrm{E}+00 \quad 0.0000$ $0.000 \mathrm{E}+00 \quad 0.000$ $0.000 \mathrm{E}+00 \quad 0.0000$ $\begin{array}{ll}0.000 \mathrm{E}+00 & 0.0000 \\ 0.000 \mathrm{E}+00 & 0.0000\end{array}$ $0.000 \mathrm{E}+00.0000$ $0.000 \mathrm{E}+00 \quad 0.0000$ $0.000 \mathrm{E}+00 \quad 0.0000$ $\begin{array}{lllllll}.000 \mathrm{E}+00 & 0.0000 & 0.000 \mathrm{E}+00 & 0.0000 & 0.000 \mathrm{E}+00 & 0.0000 & 0.000 \mathrm{E}+00\end{array}$
All Pathways* mrem/yr fract. $1.087 \mathrm{E}+000.0804$ $3.566 \mathrm{E}+00 \quad 0.2636$ $\begin{array}{lll}1.773 \mathrm{E}+00 & 0.1310\end{array}$ $\begin{array}{lll}2.789 \mathrm{E}-01 & 0.0206 \\ 6.684 \mathrm{E}+00 & 0.4941\end{array}$

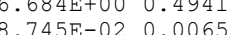
$\begin{array}{ll}02 & 0.0009\end{array}$ $2.966 \mathrm{E}-02 \quad 0.0022$ $1.353 \mathrm{E}+01 \quad 1.0000$

$\begin{array}{lll}\mathrm{U}-235 & 0.000 \mathrm{E}+00 & 0.0000 \\ \mathrm{U}-238 & 0.000 \mathrm{E}+00 & 0.0000\end{array}$ $\begin{array}{lllllllll} & \end{array}$

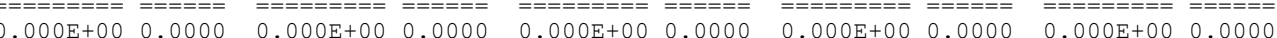
0 *Sum of all water independent and dependent pathways. 
Total Dose Contributions TDOSE(i,p,t) for Individual Radionuclides (i) and Pathways ( $p$ ) As mrem/yr and Fraction of Total Dose At $t=1.000 \mathrm{E}+00$ years Water Independent
Radon Plant

$\operatorname{Milk}$

\begin{tabular}{|c|c|c|c|c|c|c|c|c|c|c|c|}
\hline $\mathrm{em} / \mathrm{yr}$ & fract. & mrem/yr & fract. & mrem/yr & fract. & mrem/yr & fract. & mrem/yr & fract. & $\mathrm{mrem} / \mathrm{yr}$ & fract. \\
\hline & & 0 & 0 & 00 & 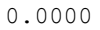 & 00 & 0 & 00 & 0 & 02 & 0 \\
\hline & & & & & & & & & & & \\
\hline & & & & & & & & & & & \\
\hline & & +00 & 0.0000 & 00 & 0.0 & 0. & 0. & & & & \\
\hline & 0.4 & +00 & 0.0 & & 0 . & & & & & 1 & 58 \\
\hline & & & & & & & & & & & \\
\hline & & & & & & & & & & & \\
\hline .01 & 0.0 & +00 & 0.0000 & 00 & 0 & 0. & 0. & 0 & & 6 & 0 \\
\hline 84 & & 100 & & & & & & & & & \\
\hline$D E+00$ & 0.5740 & $0.000 \mathrm{E}+00$ & 0.0000 & $0.000 \mathrm{E}+00$ & 0.0000 & $0.000 \mathrm{E}+00$ & 0.0000 & $0.000 \mathrm{E}+00$ & 0.0000 & $2.507 \mathrm{E}-01$ & 0.0188 \\
\hline
\end{tabular}
Total Dose Contributions TDOSE (i,p,t) for Individual Radionuclides (i) and Pathways (p)
As mrem/yr and Fraction of Total Dose At $t=1.000 \mathrm{E}+00$ years Total Dose Contributions TDOSE (i,p,t) for Individual Radionuclides (i) and
As mrem/yr and Fraction of Total Dose At $t=1.000 \mathrm{E}+00$ years

Radio-
Nuclide
mrem/yr fract. $\overline{\mathrm{Am}-241} \overline{1.510 \mathrm{E}-01} \overline{0.0113}$ $\mathrm{Eu}-152 \quad 1.683 \mathrm{E}+00 \quad 0.1261$ $\mathrm{Pu}-238 \quad 2.078 \mathrm{E}-04 \quad 0.0000$ Pu-239 7.750E-03 0.0006 $\begin{array}{lll}\mathrm{Sr}-90 & 8.086 \mathrm{E}-02 & 0.0061 \\ \mathrm{U}-234 & 6.091 \mathrm{E}-05 & 0.0000\end{array}$ $\mathrm{U}-235 \quad 1.020 \mathrm{E}-02 \quad 0.0008$ $\mathrm{U}-238 \quad \begin{array}{lll}1.967 \mathrm{E}-02 & 0.0015\end{array}$ Total $5.435 \mathrm{E}+00 \quad 0.4073$ Radio- $\frac{\text { Water }}{\text { Nuclide }}$ Fish Radon Plant mrem/yr fract. $\frac{\text { Nuclide }}{\mathrm{Am}-241} \frac{\text { mrem/yr }}{0.000 \mathrm{E}+00} \frac{\text { fract. }}{0.0000}$ mrem/yr fract. mrem/yr fract. mrem/yr fract. $\begin{array}{ccc}C s-137 & 0.000 E+00 & 0.0000 \\ \mathrm{E}-152 & 0.000 \mathrm{E}+00 & 0.0000\end{array}$ $\begin{array}{lll}\mathrm{Eu}-152 & 0.000 \mathrm{E}+00 & 0.0000 \\ \mathrm{Pu}-238 & 0.000 \mathrm{E}+00 & 0.0000\end{array}$ Pu-239 $0.000 \mathrm{E}+00 \quad 0.0000$ $\begin{array}{lll}\mathrm{Sr}-90 & 0.000 \mathrm{E}+00 \quad 0.000\end{array}$ $0.000 \mathrm{E}+00 \quad 0.0000$ $\begin{array}{lllll}0.000 \mathrm{E}+00 & 0.0000 & 0.000 \mathrm{E}+00 & 0.0000\end{array}$ $\begin{array}{llll}0.000 \mathrm{E}+00 & 0.0000 & 0.000 \mathrm{E}+00 & 0.000\end{array}$ $\begin{array}{llll}0.000 \mathrm{E}+00 & 0.0000 & 0.000 \mathrm{E}+00 & 0.0000 \\ 0.000 \mathrm{E}+00 & 0.0000 & 0.000 \mathrm{E}+00 & 0.0000\end{array}$ $0.000 \mathrm{E}+000.0000$ $\begin{array}{llll}0.000 \mathrm{E}+00 & 0.0000 & 0.000 \mathrm{E}+00 & 0.0000 \\ 0.000 \mathrm{E}+00 & 0.0000 & 0.000 \mathrm{E}+00 & 0.0000 \\ 0\end{array}$ $\begin{array}{llll}0.000 \mathrm{E}+00 & 0.0000 \quad 0.000 \mathrm{E}+00 & 0.0000\end{array}$

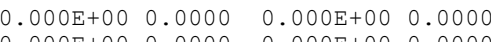
$\begin{array}{llllllll}\mathrm{U}-234 & 0.000 \mathrm{E}+00 & 0.0000 & 0.000 \mathrm{E}+00 & 0.0000 & 0.000 \mathrm{E}+00 & 0.0000 & 0.000 \mathrm{E}\end{array}$ $\begin{array}{lllllllllll}\mathrm{U}-238 & 0.000 \mathrm{E}+00 & 0.0000 & 0.000 \mathrm{E}+00 & 0.0000 & 0.000 \mathrm{E}+00 & 0.0000 & 0.000 \mathrm{E}+00 & 0.0000 & 0.000 \mathrm{E}+00 & 0.0000\end{array}$

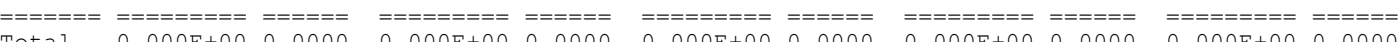
$0 *$ Sum of all water independent and dependent pathways.
Milk

$\mathrm{mrem} / \mathrm{yr}$ fract. $0.000 \mathrm{E}+00 \quad 0.0000$ $0.000 \mathrm{E}+00 \quad 0.0000$ $\begin{array}{ll}0.000 \mathrm{E}+00 & 0.0000 \\ 0.000 \mathrm{E}+00 & 0.0000 \\ 0.000 \mathrm{E}+00 & 0.0000\end{array}$ $\begin{array}{ll}0.000 \mathrm{E}+00 & 0.0000 \\ 0.000 \mathrm{E}+00 & 0.0000 \\ 0.000 & 0.000\end{array}$ $0.000 \mathrm{E}+00 \quad 0.0000$ $0.000 \mathrm{E}+00 \quad 0.0000$ $\begin{array}{llll}0.000 \mathrm{E}+00 & 0.0000 & 1.123 \mathrm{E}-02 & 0.0008 \\ 0.000 \mathrm{E}+00 & 0.0000 & 2.960 \mathrm{E}-02 & 0.0022\end{array}$ $\begin{array}{llll}======== \pm & ===== \pm & ======== \pm & ===== \pm \\ 0.000 \mathrm{E}+00 & 0.0000 & 1.335 \mathrm{E}+01 & 1.0000\end{array}$
All Pathways* mrem/yr fract. $.082 \mathrm{E}+00 \quad 0.0811$ $\begin{array}{lll}3.483 \mathrm{E}+00 & 0.2610\end{array}$ $1.683 \mathrm{E}+00 \quad 0.1261$ $\begin{array}{lll}2.767 \mathrm{E}-01 & 0.0207 \\ 6.684 \mathrm{E}+00 & 0.5008\end{array}$ $\begin{array}{ll}-02 & 0.0064\end{array}$ 
Total Dose Contributions TDOSE (i,p,t) for Individual Radionuclides (i) and Pathways ( $p$ ) As mrem/yr and Fraction of Total Dose At $t=3.000 \mathrm{E}+00$ years

\begin{tabular}{|c|c|c|c|c|c|c|c|c|c|c|c|c|c|c|}
\hline \multirow{2}{*}{$\begin{array}{l}\text { Radio- } \\
\text { Nuclide }\end{array}$} & \multicolumn{2}{|c|}{ Ground } & \multicolumn{2}{|c|}{ Inhalation } & \multicolumn{2}{|l|}{ Rac } & \multicolumn{2}{|c|}{$\begin{array}{l}\text { Inhalat } \\
\text { Plant }\end{array}$} & \multicolumn{2}{|c|}{$\begin{array}{l}\text { S radon) } \\
\text { Meat }\end{array}$} & \multicolumn{2}{|c|}{ Milk } & \multicolumn{2}{|c|}{ Soil } \\
\hline & $\mathrm{mrem} / \mathrm{yr}$ & fract. & mrem/yr & fract. & $\mathrm{mrem} / \mathrm{yr}$ & fract. & mrem/yr & fract. & mrem/yr & fract. & mrem/yr & fract. & mrem/yr & fract. \\
\hline 1 & 1 & 0 & 1 & 6 & 0 & 0.0000 & 0 & 0 & 0 & 0 & 00 & 0 & 22 & 2 \\
\hline & 3 & & 05 & 000 & 0 & 0 & & & & & $E+00$ & & 04 & \\
\hline & & 11 & 13. & 0.000 & 0 & .0000 & & & & & & & & \\
\hline & $2.046 \mathrm{E}-04$ & 0.0000 & $636 \mathrm{E}-01$ & 0.0203 & $0.000 \mathrm{E}+00$ & 0000 & $\mathrm{E}+00$ & 0 & $E+00$ & 0 & $0 \mathrm{E}+00$ & 0 & $E-03$ & 007 \\
\hline Pu-239 & $7.749 \mathrm{E}-03$ & 0.0006 & $6.464 \mathrm{E}+00$ & 0.4973 & $0.000 \mathrm{E}+00$ & 0.0000 & $0.000 \mathrm{E}+00$ & 0.0000 & $0.000 \mathrm{E}+00$ & 0.0000 & $0.000 \mathrm{E}+00$ & 0.0000 & $2.111 \mathrm{E}-01$ & 0.0162 \\
\hline & $7.637 \mathrm{E}-02$ & 0.0059 & $.663 \mathrm{E}-03$ & 0.0002 & 0.000 & 0.0 & 0.00 & 0. & 0.0 & 0. & $10 E+00$ & 0 & 03 & 0 \\
\hline 234 & $6.069 \mathrm{E}-05$ & 0.0000 & $.142 \mathrm{E}-02$ & 0.0009 & $0.000 \mathrm{E}+00$ & 0.0000 & $0.000 \mathrm{E}+00$ & 0.0 & 0.00 & 0.0 & $0.000 \mathrm{E}+00$ & 0.0 & 9.68 & 0.0000 \\
\hline & $1.016 \mathrm{E}-02$ & 0.0008 & $.014 \mathrm{E}-03$ & 0.0001 & $0.000 \mathrm{E}+00$ & 0.0000 & $0.000 \mathrm{E}$ & 0.0 & & & $0.000 \mathrm{E}+00$ & 0.0 & & 0.0 \\
\hline U-238 & $1.959 \mathrm{E}-02$ & 0.0015 & $9.800 \mathrm{E}-03$ & 0.0008 & $0.000 \mathrm{E}+00$ & 0.0000 & $0.000 \mathrm{E}+00$ & 0.0000 & $0.000 \mathrm{E}+00$ & 0.0000 & $0.000 \mathrm{E}+00$ & 0.0000 & $8.841 \mathrm{E}-05$ & 0.0000 \\
\hline$====$ & $====$ & $==$ & & & & & & & & & & & & \\
\hline Total & $5.102 \mathrm{E}+00$ & 0.3926 & $7.645 \mathrm{E}+00$ & 0.5882 & $0.000 \mathrm{E}+00$ & 0.0000 & $0.000 \mathrm{E}+00$ & 0.0000 & $0.000 \mathrm{E}+00$ & 0.0000 & $0.000 \mathrm{E}+00$ & 0.0000 & $2.501 \mathrm{E}-01$ & 0.0192 \\
\hline
\end{tabular}

Total Dose Contributions TDOSE (i,p,t) for Individual Radionuclides (i) and Pathways (p) As mrem/yr and Fraction of Total Dose At $t=3.000 \mathrm{E}+00$ years Radio- $\frac{\text { Water }}{\text { Nuclide }}$ Fish Radon Plant

Meat

mrem/yr fract. Am-241 $0.000 \mathrm{E}+00 \quad 0.0000$ Cs-137 $0.000 \mathrm{E}+00 \quad 0.0000$ $\begin{array}{lll}\mathrm{Eu}-152 & 0.000 \mathrm{E}+00 \quad 0.0000\end{array}$ $\begin{array}{lll}\mathrm{Pu}-238 & 0.000 \mathrm{E}+00 & 0.0000 \\ \mathrm{Pu}-239 & 0.000 \mathrm{E}+00 & 0.0000\end{array}$ $\begin{array}{lll}\mathrm{Sr}-90 & 0.000 \mathrm{E}+00 & 0.0000\end{array}$ $\begin{array}{lll}\mathrm{U}-234 & 0.000 \mathrm{E}+00 & 0.0000 \\ \mathrm{U}-235 & 0.000 \mathrm{E}+00 & 0.0000\end{array}$ $\mathrm{U}-238 \quad 0.000 \mathrm{E}+00 \quad 0.0000$

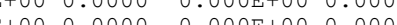
$0.000 \mathrm{E}+000.0000$ 0.000E+00 0.0000 0.000E+00 0.0000 mrem/yr fract. $0.000 \mathrm{E}+00 \quad 0.0000$ $\begin{array}{lllllll}0.000 \mathrm{E}+00 & 0.0000 & 0.000 \mathrm{E}+00 & 0.0000\end{array}$ $\begin{array}{llll}.000 \mathrm{E}+00 & 0.0000 & 0.000 \mathrm{E}+00 & 0.0000\end{array}$ $0.000 \mathrm{E}+00 \quad 0.0000$ $\begin{array}{ll}0.000 \mathrm{E}+00 & 0.0000\end{array}$ $\begin{array}{llll}.0000 & 0.000 \mathrm{E}+00 & 0.0000 & 0\end{array}$ $0.000 \mathrm{E}+00 \quad 0.0000$

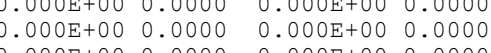
Total $0.000 \mathrm{E}+00 \quad 0.0000$ $0 *$ Sum of all water independent and dependent pathways.
Milk mrem/yr fract. $0.000 \mathrm{E}+00 \quad 0.0000$ $0.000 \mathrm{E}+00 \quad 0.0000$ $0.000 \mathrm{E}+00 \quad 0.0000$ $0.000 \mathrm{E}+00 \quad 0.0000$ $\begin{array}{lll}0.000 E+00 & 0.0000\end{array}$ $0.000 \mathrm{E}+00 \quad 0.0000$ $0.000 \mathrm{E}+00 \quad 0.0000$ $0.000 \mathrm{E}+00 \quad 0.0000$ $0.000 \mathrm{E}+00 \quad 0.0000$
All Pathways* $\begin{array}{lll}1.071 \mathrm{E}+00 & 0.0824\end{array}$ $\begin{array}{lll}3.323 \mathrm{E}+00 & 0.2556\end{array}$ $1.516 \mathrm{E}+00 \quad 0.1167$ $\begin{array}{ll}1.118 \mathrm{E}-02 & 0.0009\end{array}$ 1. $300========$ 
Total Dose Contributions TDOSE (i,p,t) for Individual Radionuclides (i) and Pathways (p) As mrem/yr and Fraction of Total Dose At $t=1.000 \mathrm{E}+01$ years Water Independent
Radon Plant

Milk

\begin{tabular}{|c|c|c|c|c|c|}
\hline \multicolumn{2}{|c|}{ Meat } & \multicolumn{2}{|c|}{ Milk } & \multicolumn{2}{|c|}{ Soil } \\
\hline mrem/yr & fract. & mrem/yr & fract. & rem/yr & frac \\
\hline & & & & & \\
\hline & & & & & \\
\hline & & & & & \\
\hline & 0. & 0 & 0.0 & & \\
\hline & 0.0 & 0 & & 01 & 0 \\
\hline & c & & & & \\
\hline & 0.00 & 0 & 0 & 05 & \\
\hline & 0.00 & & 0.0 & & 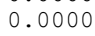 \\
\hline & ( & 0 & 0.0 & 8 & 0 \\
\hline & & & & & \\
\hline & & & & & \\
\hline
\end{tabular}

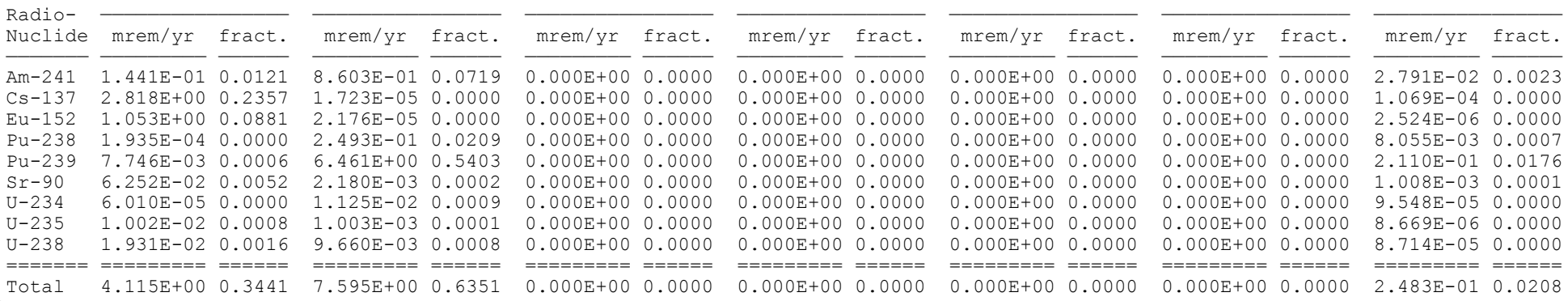
0

Total Dose Contributions TDOSE (i,p,t) for Individual Radionuclides (i) and Pathways (p) As mrem/yr and Fraction of Total Dose At $t=1.000 \mathrm{E}+01$ years

0 Fish Plant

mrem/yr fract. Milk Radio-
Nuclide mrem/yr fract. mrem/yr fract. mrem/yr fract. mrem/yr fract.

$0.000 \mathrm{E}+000.0000$
$\begin{array}{lll}\mathrm{Am}-241 & 0.000 \mathrm{E}+00 & 0.0000\end{array}$ $\begin{array}{lll}C s-137 & 0.000 E+00 & 0.0000 \\ \text { Eu-152 } & 0.000 E+00 & 0.0000\end{array}$ $0.000 \mathrm{E}+00 \quad 0.0000$ $\begin{array}{lllllll}0.000 \mathrm{E}+00 & 0.0000 & 0.000 \mathrm{E}+00 & 0.0000\end{array}$ $\begin{array}{lllllll}0.000 \mathrm{E}+00 & 0.0000 & 0.000 \mathrm{E}+00 & 0.0000\end{array}$ $\begin{array}{lllllllll}0.0000 & 0.000 \mathrm{E}+00 & 0.0000 & 0.000 \mathrm{E}+00 & 0.0000 & 0.000 \mathrm{E}+00 & 0.0000 & 0.000 \mathrm{E}+00 & 0.0000 \\ 0.000 \mathrm{E}+00 & 0.0000 & 0.0000\end{array}$ $\begin{array}{llllllllllll}\mathrm{Pu}-238 & 0.000 \mathrm{E}+00 & 0.0000 & 0.000 \mathrm{E}+00 & 0.0000 & 0.000 \mathrm{E}+00 & 0.0000 & 0.000 \mathrm{E}+00 & 0.0000 & 0.000 \mathrm{E}+00 & 0.0000\end{array}$ Pu-239 $0.000 \mathrm{E}+00 \quad 0.0000$ $\begin{array}{lll}\mathrm{Sr}-90 & 0.000 \mathrm{E}+00 & 0.000\end{array}$ $\begin{array}{llllllll}.000 \mathrm{E}+00 & 0.0000 & 0.000 \mathrm{E}+00 & 0.0000 & 0.000 \mathrm{E}+00 & 0.0000 & 0.000 \mathrm{E}+00 & 0.0000\end{array}$ $\begin{array}{lllllllllllll}-234 & 0.000 \mathrm{E}+00 & 0.0000 & 0.000 \mathrm{E}+00 & 0.0000 & 0.000 \mathrm{E}+00 & 0.0000 & 0.000 \mathrm{E}+00 & 0.0000 & 0.000 \mathrm{E}+00 & 0.0000 & 0.000\end{array}$

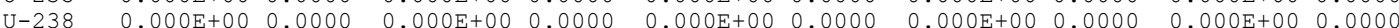
$0.000 \mathrm{E}+00$ 0.0000 $0.000 \mathrm{E}+000.0000$ - $0.000 \mathrm{E}+00$ 0.0000 $=============$ $=============$ All Pathways* mrem/yr fract. $0 *$ Sum of all water independent and dependent pathways. 
Total Dose Contributions TDOSE (i,p,t) for Individual Radionuclides (i) and Pathways (p) As mrem/yr and Fraction of Total Dose At $t=3.000 \mathrm{E}+01$ years Water Independent
Radon Plant

Milk

\begin{tabular}{|c|c|c|c|c|c|}
\hline \multicolumn{2}{|c|}{ Meat } & \multicolumn{2}{|c|}{ Milk } & \multicolumn{2}{|l|}{ Soil } \\
\hline mrem/yr & fract & $\mathrm{mrem} / \mathrm{yr}$ & fract. & $\mathrm{mrem} / \mathrm{yr}$ & frac \\
\hline & & & & & \\
\hline & & & & & \\
\hline & & & & & \\
\hline & & & & & \\
\hline & 0.00 & 0 & 0 & & 0.0210 \\
\hline & 0.00 & & & & \\
\hline$E+00$ & 0.000 & 0.00 & 0.0 & 9. & 0 \\
\hline & 0.00 & & & & \\
\hline 0 & 0. & $0.000 \mathrm{E}+00$ & 0.00 & $8.362 \mathrm{E}-05$ & 0 \\
\hline & & & & & \\
\hline 0.00 & .0000 & $.000 \mathrm{E}+00$ & 0000 & E-01 & \\
\hline
\end{tabular}

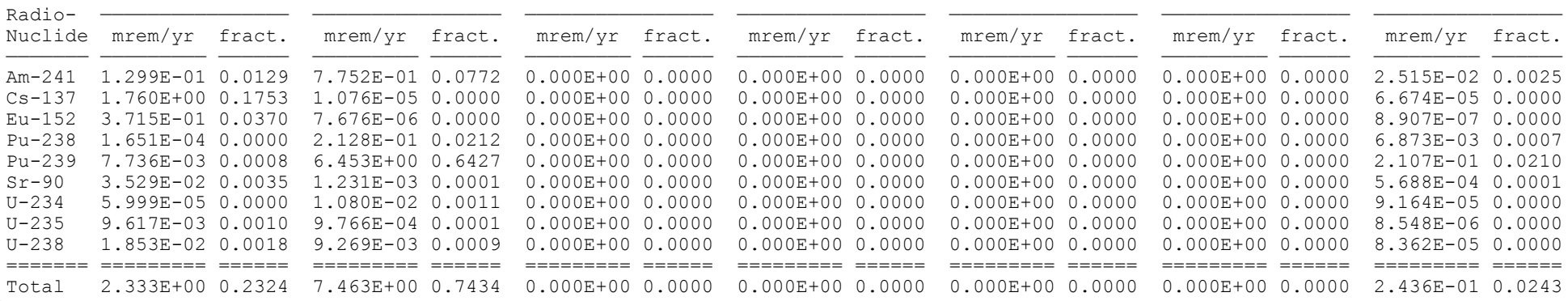

Total Dose Contributions TDOSE (i,p,t) for Individual Radionuclides (i) and Pathways (p) As mrem/yr and Fraction of Total Dose At $t=3.000 \mathrm{E}+01$ years

0 $\begin{array}{ll}\text { Radio- } & \text { Water } \\ \text { Nuclide } & \text { mrem/yr fract. }\end{array}$ Fish Radon Plant

Meat

mrem/yr fract. mrem/yr fract. $0.000 \mathrm{E}+00 \quad 0.0000$ $\begin{array}{lll}0.000 \mathrm{E}+00 & 0.0000 \\ 0.000 \mathrm{E}+00 & 0.0000\end{array}$ $\begin{array}{lll}A m-241 & 0.000 E+00 \quad 0.0000 & 0\end{array}$ $\begin{array}{lll}\mathrm{Cs}-137 & 0.000 \mathrm{E}+00 & 0.0000 \\ \mathrm{Eu}-152 & 0.000 \mathrm{E}+00 & 0.0000 \\ \mathrm{Pu}-238 & 0.000 \mathrm{O}+00 & 0.0000\end{array}$ $\begin{array}{lll}\mathrm{Eu}-152 & 0.000 \mathrm{E}+00 & 0.0000 \\ \mathrm{Pu}-238 & 0.000 \mathrm{E}+00 & 0.0000\end{array}$ Pu-239 $0.000 \mathrm{E}+00 \quad 0.0000$ $\begin{array}{lll}\mathrm{Sr}-90 & 0.000 \mathrm{E}+00 & 0.0000 \\ \mathrm{U}-234 & 0.000 \mathrm{E}+00 & 0.0000\end{array}$ $\begin{array}{lll}\mathrm{U}-235 & 0.000 \mathrm{E}+00 & 0.0000\end{array}$ mrem/yr fract. $0.000 \mathrm{E}+00 \quad 0.0000$ $0.000 \mathrm{E}+00 \quad 0.0000$ $.000 \mathrm{E}+00 \quad 0.0000$ $0.000 \mathrm{E}+00 \quad 0.0000$ $0.000 \mathrm{E}+000.0000$ $0.000 \mathrm{E}+00 \quad 0.0000$

$\begin{array}{llllllll}\text { Total } & 0.000 \mathrm{E}+00 & 0.0000 & 0.000 \mathrm{E}+00 & 0.0000 & 0.000 \mathrm{E}+00 & 0.000\end{array}$ mrem/yr fract. $0.000 \mathrm{E}+000.0000$ $\begin{array}{llllll}0.000 \mathrm{E}+00 & 0.0000 & 0.000 \mathrm{E}+00 & 0.0000\end{array}$ $\begin{array}{llll}0.000 \mathrm{E}+00 & 0.0000 & 0.000 \mathrm{E}+00 & 0.0000\end{array}$ $\begin{array}{llll}0.000 \mathrm{E}+00 & 0.0000 & 0.000 \mathrm{E}+00 & 0.0000\end{array}$ $0.000 \mathrm{E}+00 \quad 0.0000$ $\begin{array}{llll}0.000 \mathrm{E}+00 & 0.0000 & 0.000 \mathrm{E}+00 & 0.0000\end{array}$

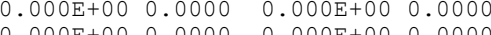
$0.000 \mathrm{E}+00 \quad 0.0000 \quad 0.000 \mathrm{E}+00 \quad 0.0000$ $\begin{array}{ll}0.000 \mathrm{E}+00 & 0.0000\end{array}$ *Sum of all water independent and dependent pathways. mrem/yr fract.

$0.000 \mathrm{E}+00 \quad 0.0000$ $\begin{array}{lll}0.000 \mathrm{E}+00 & 0.0000 \\ 0.000 \mathrm{E}+00 & 0.0000 & 0\end{array}$ $0.000 \mathrm{E}+00 \quad 0.0000$ $0.000 \mathrm{E}+00 \quad 0.0000$ $\begin{array}{lll}0.000 \mathrm{E}+00 & 0.000 \\ 0.000 \mathrm{E}+00 & 0.0000\end{array}$ $0.000 \mathrm{E}+00 \quad 0.0000$ $0.000 \mathrm{E}+00 \quad 0.0000$ $0.000 \mathrm{E}+00 \quad 0.0000$
All Pathways* $9.302 \mathrm{E}-01 \quad 0.0927$ $1.760 \mathrm{E}+00 \quad 0.1753$ $\begin{array}{lll}1.715 \mathrm{E}-01 & 0.0370\end{array}$ $\begin{array}{ll}.709 \mathrm{E}-02 & 0.0037 \\ .095 \mathrm{E}-02 & 0.0011\end{array}$ $\begin{array}{ll}1.060 \mathrm{E}-02 & 0.0011\end{array}$ $1.004 \mathrm{E}+011.0000$ 
Total Dose Contributions TDOSE (i,p,t) for Individual Radionuclides (i) and Pathways (p) As mrem/yr and Fraction of Total Dose At $t=1.000 \mathrm{E}+02$ years Water Independent
Radon Plant

Milk Soil

\begin{tabular}{|c|c|c|c|c|c|c|c|}
\hline & & & & & & & \\
\hline mrem/yr & fract. & mrem/yr & fract. & mrem/yr & fract. & mrem/yr & fract. \\
\hline 1 & 0. & +00 & 0. & +00 & 0. & +00 & 0 . \\
\hline & & +00 & & 00 & 0 & +00 & 0.0000 \\
\hline & 0 . & $000 \mathrm{E}+00$ & 0.0 & 0.00 & 0.0 & 0.00 & \\
\hline $221 \mathrm{E}-01$ & 0.0156 & $0.000 \mathrm{E}+00$ & 0.0000 & $0.000 \mathrm{E}+00$ & 0.0000 & $0.000 \mathrm{E}+00$ & 0.0000 \\
\hline $424 \mathrm{E}+00$ & 0.8225 & $0.000 \mathrm{E}+00$ & 0.0000 & $0.000 \mathrm{E}+00$ & 0.0000 & $0.000 \mathrm{E}+00$ & 0.0000 \\
\hline $663 E-04$ & 0.0000 & $0.000 \mathrm{E}+00$ & 0. & $0.000 \mathrm{E}+00$ & 0.0 & $0.000 \mathrm{E}+00$ & 000 \\
\hline $361 \mathrm{E}-03$ & 0.0012 & $0.000 \mathrm{E}+00$ & 0.0000 & $0.000 \mathrm{E}+00$ & 0.0000 & $0.000 \mathrm{E}+00$ & 0.0000 \\
\hline & 0.0001 & $0.000 \mathrm{E}+00$ & 0.00 & $0.000 \mathrm{E}+00$ & 0.00 & $0.000 \mathrm{E}+00$ & 0.0000 \\
\hline $.021 \mathrm{E}-03$ & 0.0010 & $0.000 \mathrm{E}+00$ & 0.0000 & $0.000 \mathrm{E}+00$ & 0.0000 & $0.000 \mathrm{E}+00$ & 0.0000 \\
\hline
\end{tabular}

mrem/yr fract. $0.000 \mathrm{E}+000.0000$

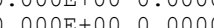
$0.000 \mathrm{E}+000.0000$ $0.000 \mathrm{E}+00 \quad 0.0000$ $0.000 \mathrm{E}+00 \quad 0.000$ $0.000 \mathrm{E}+00 \quad 0.000$ $0.000 \mathrm{E}+00 \quad 0.000$ $0.000 \mathrm{E}+00 \quad 0.0000$

$0.000 \mathrm{E}+00 \quad 0.0000$ mrem/yr fract. $1.747 \mathrm{E}-02 \quad \overline{0.0022}$ $2.324 \mathrm{E}-08 \quad 0.0000$ $3.944 \mathrm{E}-03 \quad 0.0005$ $2.098 \mathrm{E}-01 \quad 0.0269$ $7.686 \mathrm{E}-05 \quad 0.0000$ $7.941 \mathrm{E}-05 \quad 0.0000$ $8.325 \mathrm{E}-06 \quad 0.0000$ $7.236 \mathrm{E}-05 \quad 0.0000$ $\begin{array}{ll}========= & ===== \\ 2.314 \mathrm{E}-01 & 0.0296\end{array}$ Total Dose Contributions TDOSE $(i, p, t)$ for Individual Radionuclides (i) and Pathways (p) As mrem/yr and Fraction of Total Dose At $t=1.000 \mathrm{E}+02$ years

$$
0^{\text {Tota }}
$$

\begin{tabular}{lll}
$1.603 \mathrm{E}-02$ & 0.0021 \\
\hline
\end{tabular}

$\begin{array}{ll}======== & ===== \\ 4.759 \mathrm{E}-01 & 0.0609\end{array}$

Fish Radon Plant

$$
\text { Plant }
$$

Meat

$$
\text { mrem/yr fract. }
$$

mrem/yr fract.

mrem/yr fract.

Nuclide mrem/yr fract.

mrem/yr fract.

$0.000 \mathrm{E}+00 \quad 0.0000$

$\begin{array}{lll}A m-241 & 0.000 E+00 \quad 0.0000\end{array}$

$\begin{array}{lll}\mathrm{Cs}-137 & 0.000 \mathrm{E}+00 & 0.0000 \\ \mathrm{E} u-152 & 0.000 \mathrm{E}+00 & 0.0000\end{array}$

$0.000 \mathrm{E}+00 \quad 0.0000$

$0.000 \mathrm{E}+00 \quad 0.0000$

$\begin{array}{lll}0.000 \mathrm{E}+00 & 0.0000 \\ 0.000 \mathrm{E}+00 & 0.0000\end{array}$

$0.000 \mathrm{E}+00 \quad 0.0000$

$\begin{array}{llllll}0.000 \mathrm{E}+00 & 0.00000 & 0.000 \mathrm{E}+00 & 0.0000\end{array}$

$\begin{array}{ll}\mathrm{Pu}-238 & 0.000 \mathrm{E}+00 \quad 0.000\end{array}$

$\begin{array}{lll}\mathrm{Pu}-239 & 0.000 \mathrm{E}+00 & 0.000\end{array}$

$\begin{array}{lll}\mathrm{Sr}-90 & 0.000 \mathrm{E}+00 & 0.0000 \\ \mathrm{U}-234 & 0.000 \mathrm{E}+00 & 0.0000\end{array}$

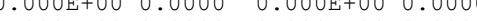

$0.000 \mathrm{E}+000.0000$

$\begin{array}{ll}0.000 \mathrm{E}+00 & 0.0000 \\ 0.000 \mathrm{E}+00 & 0.0000\end{array}$

$0.000 \mathrm{E}+00 \quad 0.0000$

$\begin{array}{ll}0.000 \mathrm{E}+00 & 0.0000 \\ 0.000 \mathrm{E}+00 & 0.0000\end{array}$

$0.000 \mathrm{E}+00$
0.00000

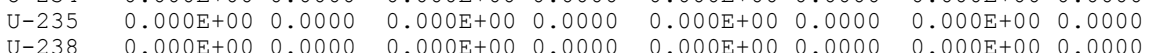

$0.000 \mathrm{E}+00 \quad 0.0000$

$0.000 \mathrm{E}+00 \quad 0.0000$

$0 *$ Sum of all water independent and dependent pathways.
Milk

mrem/yr fract.

$0.000 \mathrm{E}+00 \quad 0.0000$

$0.000 \mathrm{E}+00 \quad 0.0000$

$\begin{array}{ll}0.000 \mathrm{E}+00 & 0.0000 \\ 0.000 \mathrm{E}+00 & 0.0000\end{array}$

$\begin{array}{ll}0.000 \mathrm{E}+00 & 0.0000 \\ 0.000 \mathrm{E}+00 & 0.0000\end{array}$

$0.000 \mathrm{E}+000.0000$

$\begin{array}{ll}0.000 \mathrm{E}+00 & 0.0000 \\ 0.000 \mathrm{E}+00 & 0.0000 \\ 0.0000 \mathrm{E}+00 & 0.0000\end{array}$

$0.000 \mathrm{E}+00 \quad 0.0000$

$0.000 \mathrm{E}+00 \quad 0.0000$

All Pathways*

$\begin{array}{lll}.461 \mathrm{E}-01 & 0.0827\end{array}$ $3.390 \mathrm{E}-01 \quad 0.0434$ $\begin{array}{ll}9.694 \mathrm{E}-03 & 0.0012\end{array}$ $.011 \mathrm{E}-03 \quad 0.0006$
$.516 \mathrm{E}-03$ $\begin{array}{ll}.516 \mathrm{E}-03 & 0.0012\end{array}$ $2.412 \mathrm{E}-02 \quad 0.0031$ $7.810 \mathrm{E}+00 \quad 1.0000$ 
Total Dose Contributions TDOSE (i,p,t) for Individual Radionuclides (i) and Pathways (p) As mrem/yr and Fraction of Total Dose At $t=3.000 \mathrm{E}+02$ years Water Independent
Radon Plant

$\operatorname{Milk}$

\begin{tabular}{|c|c|c|c|c|c|}
\hline \multicolumn{2}{|c|}{ Meat } & \multicolumn{2}{|c|}{ Milk } & \multicolumn{2}{|l|}{ Soil } \\
\hline $\mathrm{mrem} / \mathrm{yr}$ & fract. & mrem/yr & fract. & $\mathrm{m} / \mathrm{yr}$ & frac \\
\hline & & & & & \\
\hline & & & & & \\
\hline & & & & & \\
\hline & & & & & \\
\hline & & & & & \\
\hline 0 & 0.00 & 0 & 0 & 07 & 0.0 \\
\hline & & & & & \\
\hline & & & & & \\
\hline $0.000 \mathrm{E}+00$ & 0.0000 & $0.000 \mathrm{E}+00$ & 0.0000 & $4.787 \mathrm{E}-05$ & 0.0000 \\
\hline+00 & 0000 & +00 & 100 & $E-01$ & 0.03 \\
\hline
\end{tabular}

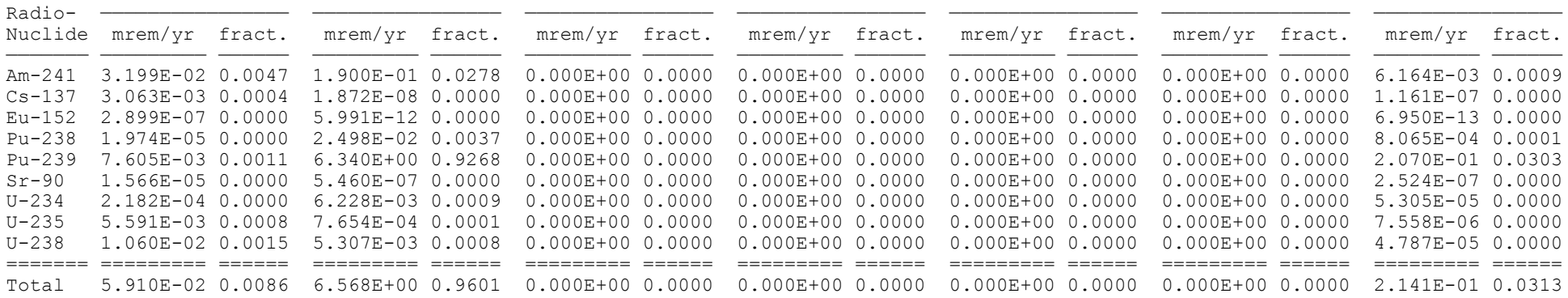
0

Total Dose Contributions TDOSE (i,p,t) for Individual Radionuclides (i) and Pathways (p) As mrem/yr and Fraction of Total Dose At $t=3.000 \mathrm{E}+02$ years

0

Plant$$
\overline{\text { mrem/yr fract. }} \overline{\text { mrem/yr fract. }}
$$$$
\text { Meat }
$$

$\begin{array}{llll}0.000 E+00 & 0.0000 & 0.000 E+00 & 0.0000\end{array}$

Nuclide mrem/yr fract. $\begin{array}{lll}\mathrm{Am}-241 & 0.000 \mathrm{E}+00 & 0.0000 \\ \mathrm{Cs}-137 & 0.000 \mathrm{E}+00 & 0.0000\end{array}$ $\begin{array}{lll}\mathrm{Eu}-152 & 0.000 \mathrm{E}+00 & 0.0000\end{array}$ $\begin{array}{lll}\mathrm{Pu}-238 & 0.000 \mathrm{E}+00 & 0.000\end{array}$ $\begin{array}{lll}\mathrm{Pu}-239 & 0.000 \mathrm{E}+00 & 0.0000 \\ \mathrm{Sr}-90 & 0.000 \mathrm{E}+00 & 0.0000\end{array}$

$\begin{array}{lll}\mathrm{Sr}-90 & 0.000 \mathrm{E}+00 & 0.0000 \\ \mathrm{U}-234 & 0.000 \mathrm{E}+00 & 0.0000\end{array}$

$\mathrm{U}-238 \quad 0.000 \mathrm{E}+00 \quad 0.0000$

mrem/yr fract. $\begin{array}{ll}0.000 \mathrm{E}+00 & 0.0000 \\ 0.000 \mathrm{E}+00 & 0.0000 \\ 0.000 \mathrm{E}+00 & 0.0000\end{array}$ $\begin{array}{lll}0.000 \mathrm{E}+00 & 0.0000 \\ 0.000 \mathrm{O} & 0.000 & 0.0000\end{array}$ $0.000 \mathrm{E}+00 \quad 0.0000$

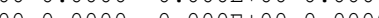
$\begin{array}{lllll}0.000 \mathrm{E}+00 & 0.0000 & 0.000 \mathrm{E}+00 & 0.0000\end{array}$ $0.000 \mathrm{E}+00 \quad 0.0000$ $\begin{array}{ll}.0====== & ===== \\ 0.00 & =0\end{array}$ $0.000 \mathrm{E}+00 \quad 0.0000$

$\begin{array}{llllllllll}\text { Total } & 0.000 \mathrm{E}+00 & 0.0000 & 0.000 \mathrm{E}+00 & 0.0000 & 0.000 \mathrm{E}+00 & 0.0000\end{array}$

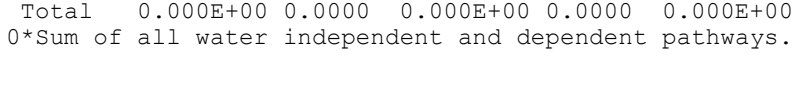

Milk

mrem/yr fract.

$0.000 \mathrm{E}+00 \quad 0.0000$ $\begin{array}{ll}0.000 \mathrm{E}+00 & 0.0000 \\ 0.000 \mathrm{E}+00 & 0.0000 \\ 0.000 \mathrm{E}+00 & 0.0000\end{array}$ $\begin{array}{ll}0.000 \mathrm{E}+00 & 0.0000 \\ 0.000 \mathrm{E}+00 & 0.0000\end{array}$ $\begin{array}{ll}0.000 \mathrm{E}+00 & 0.0000 \\ 0.000 \mathrm{E}+00 & 0.0000\end{array}$ $\begin{array}{ll}0.000 \mathrm{E}+00 & 0.0000 \\ 0.000 \mathrm{E}+00 & 0.0000\end{array}$ $0.000 \mathrm{E}+000.0000$ $0.000 \mathrm{E}+00 \quad 0.0000$ $0.000 \mathrm{E}+00 \quad 0.0000$ $0.000 \mathrm{E}+00 \quad 0.0000$
All Pathways* mrem/yr fract. $\begin{array}{ll}2.281 \mathrm{E}-01 & 0.0334 \\ 3\end{array}$ $3.063 \mathrm{E}-03 \quad 0.0004$ $6.499 \mathrm{E}-03 \quad 0.0010$ $6.841 \mathrm{E}+00 \quad 1.0000$ 
Total Dose Contributions TDOSE (i,p,t) for Individual Radionuclides (i) and Pathways ( $p$ ) As mrem/yr and Fraction of Total Dose At $t=1.000 \mathrm{E}+03$ years Water Independent Plant

Milk

\begin{tabular}{|c|c|c|c|c|c|}
\hline \multicolumn{2}{|c|}{ Meat } & \multicolumn{2}{|c|}{ Milk } & \multicolumn{2}{|l|}{ Soil } \\
\hline mrem/yr & fract. & mrem/yr & fract. & yr & frac \\
\hline & & & & & \\
\hline & & & & & \\
\hline & & & & & \\
\hline & & & & & \\
\hline & & & & & \\
\hline 0 & 0.0 & 0 & 0. & 16 & \\
\hline & & & & & \\
\hline & & & & & \\
\hline $0.000 \mathrm{E}+00$ & 0.0000 & $0.000 \mathrm{E}+00$ & 0.0000 & $1.128 \mathrm{E}-05$ & 0.0000 \\
\hline+00 & 0000 & +00 & 00 & $0 E-01$ & 0.0 \\
\hline
\end{tabular}

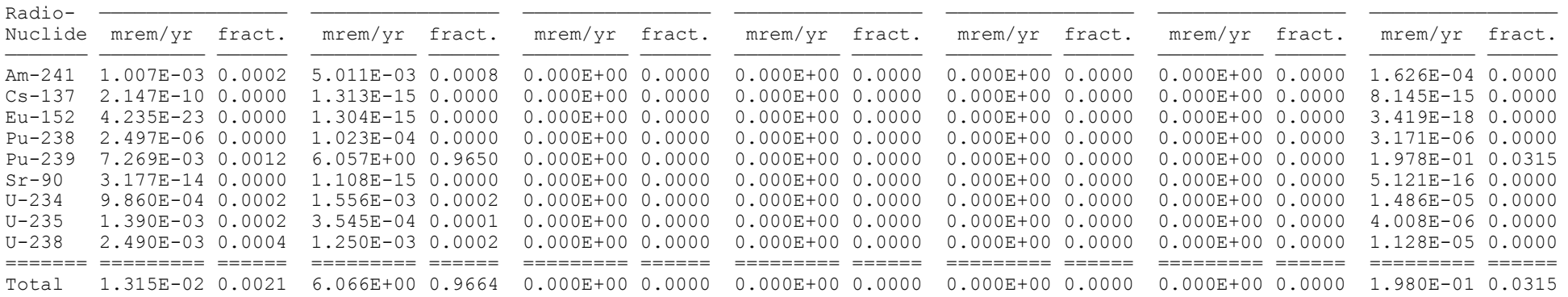
0

Total Dose Contributions TDOSE (i,p,t) for Individual Radionuclides (i) and Pathways (p) As mrem/yr and Fraction of Total Dose At $t=1.000 \mathrm{E}+03$ years

0 Fish Plant

Milk Radio-
Nuclide
mrem/yr fract. mrem/yr fract. mrem/yr fract. mrem/yr fract. mrem/yr fract. mrem/yr fract. $\begin{array}{lll}\text { Am-241 } & 0.000 \mathrm{E}+00 & 0.0000\end{array}$ $\begin{array}{lll}\mathrm{Cs}-137 & 0.000 \mathrm{E}+00 & 0.0000 \\ \mathrm{E} 1 \mathrm{-152} & 0.000 \mathrm{E}+00 & 0.0000 \\ \mathrm{Pu}-238 & 0.000 \mathrm{E}+00 & 0.0000\end{array}$ $0.000 \mathrm{E}+00 \quad 0.0000$ $0.000 \mathrm{E}+00 \quad 0.0000$ $0.000 E+00 \quad 0.0000$ $\begin{array}{lllll}0.000 \mathrm{E}+00 & 0.0000 & 0.000 \mathrm{E}+00 & 0.0000\end{array}$ $\begin{array}{lll}\mathrm{Eu}-152 & 0.000 \mathrm{E}+00 & 0.0000 \\ \mathrm{Pu}-238 & 0.000 \mathrm{E}+00 & 0.0000\end{array}$ $\begin{array}{lll}\mathrm{Pu}-238 & 0.000 \mathrm{E}+00 & 0.0000 \\ \mathrm{Pu}-239 & 0.000 \mathrm{E}+00 & 0.0000\end{array}$ $\mathrm{Sr}-90 \quad 0.000 \mathrm{E}+00 \quad 0.000$ $\begin{array}{llll}0.000 \mathrm{E}+00 & 0.0000 & 0.000 \mathrm{E}+00 & 0.0000\end{array}$ $0.000 \mathrm{E}+00 \quad 0.0000$ $.000 \mathrm{E}+000.0000$ $0.000 \mathrm{E}+00 \quad 0.0000$ $\begin{array}{lllllll}-234 & 0.000 \mathrm{E}+00 & 0.0000 & 0.000 \mathrm{E}+00 & 0.0000 & 0.000 \mathrm{E}+00 & 0.0000\end{array}$ $\begin{array}{lll}0.0000 & 0.000 \mathrm{E}+00 & 0.0000\end{array}$ $\begin{array}{lllll}0.000 \mathrm{E}+00 & 0.0000 & 0.000 \mathrm{E}+00 & 0.0000\end{array}$ $\begin{array}{llll}0.000 \mathrm{E}+00 & 0.0000 & 0.000 \mathrm{E}+00 & 0.0000 \\ 0.0000 \mathrm{E}+00 & 0.0000 & 0.000 \mathrm{E}+00 & 0.0000\end{array}$ $\begin{array}{lllll}0.000 \mathrm{E}+00 & 0.0000 & 0.000 \mathrm{E}+00 & 0.0000 \\ 0.0000 & 0.000 \mathrm{E}+00 & 0.0000 & 0.000 \mathrm{E}+00 & 0.0000\end{array}$ $\begin{array}{lllllll} & 0.000 \mathrm{E}+00 & 0.0000\end{array}$

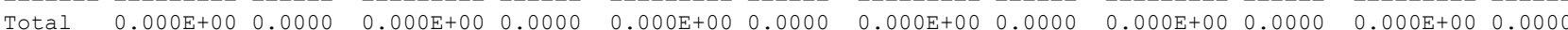
$0.000 \mathrm{E}+00 \quad 0.0000$ $0.000 \mathrm{E}+00 \quad 0.0000$ $0.000 \mathrm{E}+00 \quad 0.000$ $0.000 \mathrm{E}+00 \quad 0.0000$ $0.000 \mathrm{E}+000.0000$ $0.000 \mathrm{E}+00 \quad 0.0000$ (0.000 $0.000 \mathrm{E}+00 \quad 0.0000$ $0 *$ Sum of all water independent and dependent pathways. 
Dose/Source Ratios Summed Over All Pathways

Parent and Progeny Principal Radionuclide Contributions Indicated

OParent Product Branch $\operatorname{DSR}(j, \mathrm{t}) \quad(\mathrm{mrem} / \mathrm{yr}) /(\mathrm{pCi} / \mathrm{g})$

\begin{tabular}{|c|c|c|c|c|c|c|c|c|c|c|}
\hline (i) & (j) & Fraction $* t=$ & $0.000 \mathrm{E}+00$ & $1.000 \mathrm{E}+00$ & 3 & $00 \mathrm{E}$ & & $000 \mathrm{E}+02$ & 3.0 & 1. \\
\hline & & $.000 \mathrm{E}+00$ & $3.6 / 4 \mathrm{E}-0 \mathrm{~L}$ & $3.0 J 3 E-0 Z$ & $3.01 / E-0 Z$ & $3.48 / E-02$ & $3.14 \angle E-0 Z$ & $\angle .18<\mathrm{E}-0 \angle$ & $1.100 \mathrm{E}-03$ & $2.009 E-04$ \\
\hline 241 & -237 & $000 \mathrm{E}+00$ & $2.586 \mathrm{E}-08$ & $7.740 \mathrm{E}-08$ & $796 \mathrm{E}-07$ & $5.287 \mathrm{E}-07$ & $1.455 \mathrm{E}-06$ & $3.993 \mathrm{E}-06$ & $7.464 \mathrm{E}-06$ & $7.869 \mathrm{E}-06$ \\
\hline$n-241$ & -233 & $1.000 \mathrm{E}+00$ & $256 \mathrm{E}-15$ & $576 \mathrm{E}-14$ & $8.292 \mathrm{E}-14$ & $300 \mathrm{E}-13$ & $5.879 \mathrm{E}-12$ & $.455 \mathrm{E}-11$ & $3.201 \mathrm{E}$ & $55 \mathrm{E}-09$ \\
\hline $\mathrm{Am}-241$ & Th-229 & $1.000 \mathrm{E}+00$ & $.813 \mathrm{E}-18$ & $715 \mathrm{E}-17$ & $3.157 \mathrm{E}-16$ & $8.270 \mathrm{E}-15$ & $1.954 \mathrm{E}-13$ & $6.201 \mathrm{E}-12$ & $1.203 \mathrm{E}$ & \\
\hline$A m-241$ & $\operatorname{SDSR}(j)$ & & & & & & & & & \\
\hline $0 \mathrm{Cs}-137$ & $\mathrm{Cs}-137$ & $.000 \mathrm{E}+00$ & $3.566 \mathrm{E}-01$ & $3.483 \mathrm{E}-01$ & $3.323 \mathrm{E}-01$ & $2.818 \mathrm{E}-01$ & $1.760 \mathrm{E}-01$ & & & $2.147 \mathrm{E}-11$ \\
\hline $0 \mathrm{Eu}-152$ & Eu-152 & $7.208 \mathrm{E}-01$ & $5.194 \mathrm{E}-01$ & $4.930 \mathrm{E}-01$ & $4.443 \mathrm{E}-01$ & $3.085 E-01$ & $1.089 \mathrm{E}-01$ & $2.840 \mathrm{E}-03$ & $8.495 \mathrm{E}-08$ & $1.241 \mathrm{E}-23$ \\
\hline & -152 & $2.792 \mathrm{E}-01$ & $2.012 \mathrm{E}-01$ & $1.910 \mathrm{E}-01$ & $1.721 \mathrm{E}-01$ & $195 \mathrm{~F}-01$ & $4.217 \mathrm{E}-02$ & $100 \mathrm{E}-03$ & $3.290 \mathrm{E}-08$ & $.806 \mathrm{E}-24$ \\
\hline $\mathrm{Eu}-152$ & 152 & $2.792 \mathrm{E}-01$ & -17 & -17 & & & & & & \\
\hline $\mathrm{Eu}-152$ & $\operatorname{SDSR}(j)$ & & $2.012 \mathrm{E}-01$ & $1.910 \mathrm{E}-01$ & $1.721 \mathrm{E}-01$ & $1.195 \mathrm{E}-01$ & $4.217 \mathrm{E}-02$ & $1.100 \mathrm{E}$ & $3.290 \mathrm{E}$ & $5.314 \mathrm{E}-16$ \\
\hline $\mathrm{OPu}-238$ & $\mathrm{Pu}-238$ & $.000 \mathrm{E}+00$ & $2.789 \mathrm{E}-02$ & $2.767 \mathrm{E}-02$ & $2.723 \mathrm{E}-02$ & $2.576 \mathrm{E}-02$ & $2.198 \mathrm{E}-02$ & $1.261 \mathrm{E}-02$ & $2.579 \mathrm{E}-03$ & $9.969 \mathrm{E}-06$ \\
\hline $\mathrm{Pu}-238$ & $\mathrm{U}-234$ & $1.000 \mathrm{E}+00$ & $1.307 \mathrm{E}-08$ & $3.904 \mathrm{E}-08$ & $9.020 \mathrm{E}-08$ & $2.613 \mathrm{E}-07$ & $6.877 \mathrm{E}-07$ & $1.617 \mathrm{E}-06$ & $1.988 \mathrm{E}-06$ & $5.614 \mathrm{E}-07$ \\
\hline $\mathrm{Pu}-238$ & $n-230$ & 1.00 & & 6.76 & 3.55 & & & & & \\
\hline $\mathrm{Pu}-\mathrm{-}$ & $\mathrm{R}$ & 1.00 & 5 & 8.1 & 9. & & & & & -07 \\
\hline $\mathrm{Pu}-238$ & $\mathrm{~Pb}-210$ & $1.000 \mathrm{E}+00$ & $1.229 \mathrm{E}-20$ & $3.780 \mathrm{E}-19$ & $9.369 \mathrm{E}-18$ & $6.904 \mathrm{E}-16$ & $4.156 \mathrm{E}-14$ & $2.929 \mathrm{E}-12$ & $7.568 \mathrm{E}-11$ & $8.216 \mathrm{E}-10$ \\
\hline $\mathrm{Pu}-238$ & $\operatorname{SDSR}(j)$ & & $2.789 \mathrm{E}-02$ & $2.767 \mathrm{E}-02$ & $2.723 \mathrm{E}-02$ & $2.576 \mathrm{E}-02$ & $2.198 \mathrm{E}-02$ & $1.261 \mathrm{E}-02$ & $2.581 \mathrm{E}-03$ & $1.080 \mathrm{E}-05$ \\
\hline & & bo & & & & & & & & \\
\hline & & & & & & & & & & \\
\hline $\mathrm{Pu}-239$ & $\mathrm{~Pa}-231$ & 1.00 & $3.909 \mathrm{E}-16$ & $2.734 \mathrm{E}-15$ & $1.442 \mathrm{E}-14$ & $1.279 \mathrm{E}-13$ & $1.053 \mathrm{E}-12$ & $1.052 \mathrm{E}-11$ & $7.447 \mathrm{E}-11$ & $3.870 \mathrm{E}-10$ \\
\hline $\mathrm{Pu}-239$ & $A c-227$ & 1.00 & $1.893 \mathrm{E}-17$ & $2.816 \mathrm{E}-16$ & $3.225 \mathrm{E}-15$ & $8.009 \mathrm{E}-14$ & $1.639 \mathrm{E}-12$ & $3.487 \mathrm{E}-11$ & $3.493 \mathrm{E}$ & $2.069 \mathrm{E}-09$ \\
\hline $\mathrm{Pu}-239$ & $\operatorname{SDSR}(j)$ & & $E-02$ & 2 & 3.06 & 3.06 & 3. & 22 & & \\
\hline & & & & & & & & & & \\
\hline $\mathrm{U}-2$ & & & & & & & & & & \\
\hline $\mathrm{U}-234$ & Th-2 & 1.00 & $1.027 \mathrm{E}-07$ & 3.078 & $7.166 \mathrm{E}-07$ & $2.134 \mathrm{E}-06$ & $6.073 \mathrm{E}-06$ & 1.86 & 05 & $8.614 \mathrm{E}-05$ \\
\hline $\mathrm{U}-234$ & $\mathrm{R}$ & 1.00 & 7 & 09 & 2.8 & 2.50 & 2. & & 1 & $7.761 \mathrm{E}-04$ \\
\hline & -210 & $1.000 \mathrm{E}+00$ & $2.167 \mathrm{E}-14$ & 3.226 & 3.70 & 9.245 & 1.92 & 4.2 & 4 & 2 \\
\hline & & & & & & & & & & \\
\hline OU-235 & $U-235$ & 1.0 & 2 & 2 & -02 & 9.18 & 02 & & & -02 \\
\hline $\mathrm{U}-235$ & $\mathrm{~Pa}-$ & & & & 8. & 05 & 05 & & & -04 \\
\hline $\mathrm{U}-235$ & $\mathrm{Ac}-227$ & 1.0 & 7 & 5.31 & $2.739 \mathrm{E}-06$ & $2.245 \mathrm{E}-05$ & $1.496 \mathrm{E}-04$ & $8.300 \mathrm{E}-04$ & $2.199 \mathrm{E}-03$ & $2.313 \mathrm{E}-03$ \\
\hline & $\$ D S R(j)$ & & $9.377 \mathrm{E}-02$ & $9.358 \mathrm{E}-02$ & $9.320 \mathrm{E}-02$ & $9.190 \mathrm{E}-02$ & $8.835 \mathrm{E}-02$ & $7.728 \mathrm{E}-02$ & $5.304 \mathrm{E}-02$ & $1.457 \mathrm{E}-02$ \\
\hline & 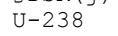 & & & & & & & & & \\
\hline U- & U- & & 8 & 8 & $3 E-08$ & 07 & 7.51 & 2 & 4.23 & 6 \\
\hline $\mathrm{U}-238$ & $\mathrm{Th}$ & & 9 & 3 & 12 & 3.168 & $2.598 \mathrm{E}-10$ & 2.563 & $1.755 \mathrm{E}-08$ & $8.294 \mathrm{E}-08$ \\
\hline & $\mathrm{Ra}-226$ & $1.000 \mathrm{E}+00$ & $5.432 \mathrm{E}-16$ & $8.139 \mathrm{E}-15$ & $9.470 \mathrm{E}-14$ & $2.487 \mathrm{E}-12$ & $5.915 \mathrm{E}-11$ & $1.919 \mathrm{E}-09$ & $3.895 \mathrm{E}$ & $5.759 \mathrm{E}-07$ \\
\hline & & $1.000 \mathrm{E}+00$ & 0 & 9 & 8 & & 14 & & & \\
\hline & & & & & & & & & & \\
\hline
\end{tabular}

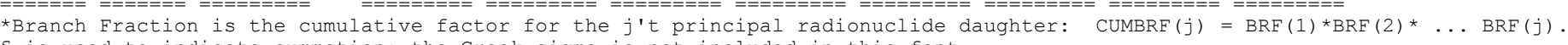
$\$$ is used to indicate summation; the Greek sigma is not included in this font. 
$\mathrm{T}^{1 / 2}$ Limit $=0.5$ year

02/22/2005 17:45 Page 23 Summary : CAU 224250 day/yr worker scenario $10 \mathrm{~m}$ hotspot

Single Radionuclide Soil Guidelines $\mathrm{G}(\mathrm{i}, \mathrm{t})$ in $\mathrm{pCi} / \mathrm{g}$ Basic Radiation Dose Limit $=2.500 \mathrm{E}+01 \mathrm{mrem} / \mathrm{yr}$

ONuclide

\begin{tabular}{|c|c|c|c|c|c|c|c|c|c|}
\hline (i & $t=$ & $0.000 \mathrm{E}+00$ & $1.000 \mathrm{E}+00$ & $3.000 \mathrm{E}+00$ & $1.000 \mathrm{E}+01$ & $3.000 \mathrm{E}+01$ & $1.000 \mathrm{E}+02$ & $3.000 \mathrm{E}+02$ & $1.000 \mathrm{E}+03$ \\
\hline & & $6.805 \mathrm{E}+02$ & $6.840 \mathrm{E}+02$ & $6.912 \mathrm{E}+02$ & $7.169 \mathrm{E}+02$ & $7.955 \mathrm{E}+02$ & $1.145 \mathrm{E}$ & $3.243 \mathrm{E}+03$ & $1.197 \mathrm{E}+05$ \\
\hline & & $7.011 \mathrm{E}+01$ & $7.178 \mathrm{E}+01$ & $7.524 \mathrm{E}+01$ & $8.871 \mathrm{E}+01$ & $1.420 \mathrm{E}+02$ & $7.376 \mathrm{E}+02$ & $8.162 \mathrm{E}+04$ & $5 E+12$ \\
\hline Eu-152 & & $3.469 \mathrm{E}+01$ & $3.655 \mathrm{E}+01$ & $4.056 \mathrm{E}+01$ & $5.841 \mathrm{E}+01$ & $1.655 \mathrm{E}+02$ & $6.344 \mathrm{E}+03$ & $2.121 \mathrm{E}+08$ & $\star 1.765 \mathrm{E}+14$ \\
\hline Pu-238 & & $8.965 E+02$ & $9.036 \mathrm{E}+02$ & $9.181 \mathrm{E}+02$ & $9.705 E+02$ & $1.137 \mathrm{E}+03$ & $1.982 \mathrm{E}+03$ & $9.688 \mathrm{E}+03$ & $2.315 \mathrm{E}+06$ \\
\hline $\mathrm{Pu}-239$ & & $8.153 \mathrm{E}+02$ & $8.154 \mathrm{E}+02$ & $8.155 \mathrm{E}+02$ & $8.159 \mathrm{E}+02$ & $8.169 \mathrm{E}+02$ & $8.207 \mathrm{E}+02$ & $8.314 \mathrm{E}+02$ & $8.703 \mathrm{E}+02$ \\
\hline $\mathrm{Sr}-90$ & & $9.291 \mathrm{E}+03$ & $9.560 \mathrm{E}+03$ & $1.012 \mathrm{E}+04$ & $1.237 \mathrm{E}+04$ & $2.191 \mathrm{E}+04$ & $1.621 \mathrm{E}+05$ & $4.938 \mathrm{E}+07$ & $\star 1.365 \mathrm{E}+14$ \\
\hline $\mathrm{U}-234$ & & $2.705 \mathrm{E}+03$ & $2.710 \mathrm{E}+03$ & $2.722 \mathrm{E}+03$ & $2.761 \mathrm{E}+03$ & $2.876 \mathrm{E}+03$ & $3.310 \mathrm{E}+03$ & $4.847 \mathrm{E}+03$ & $1.232 \mathrm{E}+04$ \\
\hline $\mathrm{U}-235$ & & $2.666 \mathrm{E}+02$ & $2.671 \mathrm{E}+02$ & $2.682 \mathrm{E}+02$ & $2.720 \mathrm{E}+02$ & $2.830 \mathrm{E}+02$ & $3.235 E+02$ & $4.714 \mathrm{E}+02$ & $1.715 \mathrm{E}+03$ \\
\hline $\mathrm{U}-238$ & & $1.020 \mathrm{E}+03$ & $1.022 \mathrm{E}+03$ & $1.026 \mathrm{E}+03$ & $1.041 \mathrm{E}+03$ & $1.085 \mathrm{E}+03$ & $1.254 \mathrm{E}+03$ & $1.896 \mathrm{E}+03$ & $8.063 E+03$ \\
\hline$=======$ & & $=========$ & $=========$ & $=========$ & $=========$ & $=========$ & $=========$ & $=========$ & $=========$ \\
\hline
\end{tabular}

At specific activity limit

Summed Dose/Source Ratios DSR $(i, t)$ in (mrem/yr)/(pCi/g)

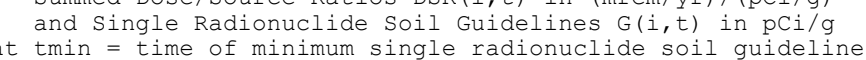
$\begin{aligned} \text { at } \operatorname{tmin} & =\text { time of minimum single radionuclide soil guid } \\ \text { and at } \operatorname{tmax} & =\text { time of maximum total dose }=0.000 \mathrm{E}+00 \text { years }\end{aligned}$

\begin{tabular}{|c|c|c|c|c|c|c|}
\hline $\begin{array}{l}\text { uclide } \\
\text { (i) }\end{array}$ & $\begin{array}{l}\text { Initial } \\
\text { (pCi/g) }\end{array}$ & $\begin{array}{c}\text { tmin } \\
\text { (years) }\end{array}$ & $\operatorname{SSR}(i, \operatorname{tmin})$ & $\begin{array}{c}G(i, \operatorname{tmin}) \\
(\mathrm{pCi} / \mathrm{g})\end{array}$ & $\operatorname{DSR}(i, \operatorname{tmax})$ & $\begin{array}{c}G(i, \operatorname{tmax}) \\
(\mathrm{pCi} / \mathrm{g})\end{array}$ \\
\hline & $2.960 \mathrm{E}+01$ & $0.000 \mathrm{E}+00$ & $3.674 \mathrm{E}-02$ & 6.80 & 3.6 & 6.8 \\
\hline & & & & & & \\
\hline & 2.4 & & & 3 & & \\
\hline & & & & & & \\
\hline & 2. & & & & & 8.1 \\
\hline & & & & & & 9.2 \\
\hline & 1.2 & & & & & \\
\hline & & & & & & 2. \\
\hline & $1.210 \mathrm{E}+00$ & $0.000 \mathrm{E}+00$ & $2.452 \mathrm{E}-02$ & $1.020 \mathrm{E}+03$ & $2.452 \mathrm{E}-02$ & $1.020 \mathrm{E}+03$ \\
\hline$======$ & $=========$ & 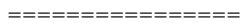 & $========$ & $========$ & $========$ & $=======$ \\
\hline
\end{tabular}


1RESRAD, Version $6.21 \quad T^{1 / 2}$ Limit $=0.5$ year

Tl/2 Iimit $=0.5$ year 02/22/2005 17:45 Page 24

RESRAD, Version $6.210 \mathrm{~T} / \frac{1}{2}$ Limit $=0.5$ year
Summary : CAU $224250 \mathrm{day} / \mathrm{yr}$ worker scenario $10 \mathrm{~m}$ hotspot
File : cau224 $25010 \mathrm{~m}$ parcel. RAD

Individual Nuclide Dose Summed Over All Pathways

Parent Nuclide and Branch Fraction Indicated

ONuclide Parent BRF(i)

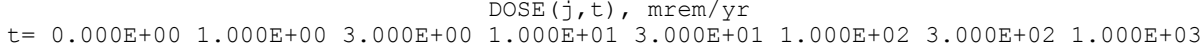

$\overline{\mathrm{Am}-241} \overline{\mathrm{Am}-241} \overline{1.000 \mathrm{E}+00}$ ONp-237 Am-241 $1.000 \mathrm{E}+00$ $00-233$ Am-241 $1.000 \mathrm{E}+00$

$\mathrm{OCs}-137$ Cs $-137 \quad 1.000 \mathrm{~T}+00$

OEu-152 Eu-152 $7.208 \mathrm{E}-01$

Eu-152 Eu-152 2.792E-01

Eu-152 \$DOSE (j)

Eu-152 2.792E-01

$0 \mathrm{Pu}-238 \quad \mathrm{Pu}-238 \quad 1.000 \mathrm{E}+00$

OU-234 $\quad \mathrm{Pu}-238 \quad 1.000 \mathrm{E}+00$

$\mathrm{U}-234 \quad \mathrm{U}-234-1.000 \mathrm{E}+00$

$\mathrm{U}-234 \quad \mathrm{U}-238 \quad 1.000 \mathrm{E}+0$

$\mathrm{U}-234$ SDOSE (j)

Th -230 U $-234 \quad 1.000 \mathrm{E}+00$

Th-230 U $-234 \quad 1.000 \mathrm{E}+00$

Th-230 SDOSE (j)

$\begin{array}{ll}\mathrm{Th}-230 & \text { SDOSE (j) } \\ \mathrm{OR} a-226 & \mathrm{Pu}-238 \\ \mathrm{Ra} & 1.000 \mathrm{E}+00\end{array}$

$\mathrm{Ra}-226 \mathrm{U}-234 \quad 1.000 \mathrm{E}+00$

$\mathrm{Ra}-226 \mathrm{U}-238 \quad 1.000 \mathrm{E}+00$

$\mathrm{Ra}-226$ \$DOSE (j)

$\mathrm{Pb}-210 \mathrm{Pu}-238 \quad 1.000 \mathrm{E}+00$

$\mathrm{Pb}-210 \mathrm{U}-234 \quad 1.000 \mathrm{E}+00$

$\mathrm{Pb}-210 \quad \mathrm{U}-238 \quad 1.000 \mathrm{E}+00$

$\mathrm{Pb}-210$ \$DOSE $(j)$

Pu-239 $\mathrm{Pu}-239 \quad 1.000 \mathrm{E}+00$

$\begin{array}{lll}0 \mathrm{U}-235 & \mathrm{Pu}-239 & 1.000 \mathrm{E}+00\end{array}$

$\mathrm{U}-235$ SDOSE (j)

$\begin{array}{lll}\mathrm{Pa}-231 & \mathrm{Pu}-239 & 1.000 \mathrm{E}+00\end{array}$

$\mathrm{Pa}-231 \mathrm{U}-235 \quad 1.000 \mathrm{E}+00$

Pa-231 \$DOSE (j)

$\begin{array}{llllllll}.087 \mathrm{E}+00 & 1.082 \mathrm{E}+00 & 1.071 \mathrm{E}+00 & 1.032 \mathrm{E}+00 & 9.301 \mathrm{E}-01 & 6.460 \mathrm{E}-01 & 2.279 \mathrm{E}-01 & 5.948 \mathrm{E}-03\end{array}$

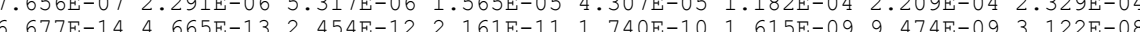

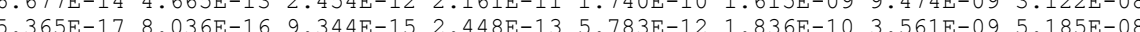
$\begin{array}{llllllll}3.566 \mathrm{E}+00 & 3.483 \mathrm{E}+00 & 3.323 \mathrm{E}+00 & 2.818 \mathrm{E}+00 & 1.760 \mathrm{E}+00 & 3.390 \mathrm{E}-01 & 3.063 \mathrm{E}-03 & 2.147 \mathrm{E}-10\end{array}$ $\begin{array}{lllllllll}1.278 \mathrm{E}+00 & 1.213 \mathrm{E}+00 & 1.093 \mathrm{E}+00 & 7.590 \mathrm{E}-01 & 2.678 \mathrm{E}-01 & 6.988 \mathrm{E}-03 & 2.090 \mathrm{E}-07 & 3.053 \mathrm{E}-23\end{array}$ $\begin{array}{llllllll}4.949 \mathrm{E}-01 & 4.698 \mathrm{E}-01 & 4.233 \mathrm{E}-01 & 2.940 \mathrm{E}-01 & 1.037 \mathrm{E}-01 & 2.707 \mathrm{E}-03 & 8.094 \mathrm{E}-08 & 1.182 \mathrm{E}-23\end{array}$

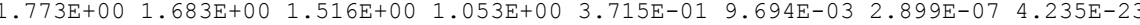
$\begin{array}{llllllll}3.649 \mathrm{E}-17 & 1.070 \mathrm{E}-16 & 2.374 \mathrm{E}-16 & 6.001 \mathrm{E}-16 & 1.132 \mathrm{E}-15 & 1.408 \mathrm{E}-15 & 1.391 \mathrm{E}-15 & 1.307 \mathrm{E}-15\end{array}$

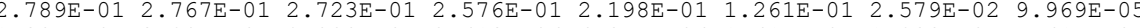

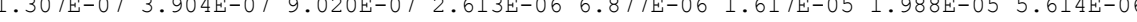

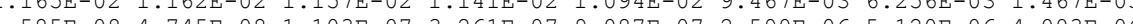

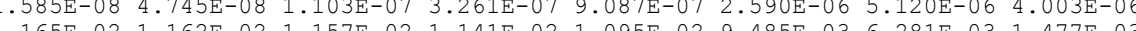

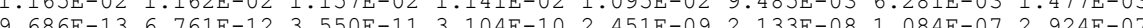

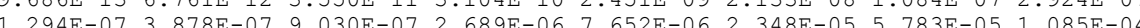
$\begin{array}{llllllll}1.174 \mathrm{E}-13 & 8.206 \mathrm{E}-13 & 4.326 \mathrm{E}-12 & 3.833 \mathrm{E}-11 & 3.144 \mathrm{E}-10 & 3.302 \mathrm{E}-09 & 5.783 \mathrm{E}-05 & 1.085 \mathrm{E}-04 \\ 1.294 \mathrm{E}-07 & 3.878 \mathrm{E}-07 & 9.030 \mathrm{E}-07 & 2.690 \mathrm{E}-06 & 7.655 \mathrm{E}-06 & 2.351 \mathrm{E}-05 & 5.796 \mathrm{E}-05 & 1.004 \mathrm{E}-07\end{array}$ $\begin{array}{llllllll}1.294 \mathrm{E}-07 & 3.878 \mathrm{E}-07 & 9.030 \mathrm{E}-07 & 2.690 \mathrm{E}-06 & 7.655 \mathrm{E}-06 & 2.351 \mathrm{E}-05 & 5.796 \mathrm{E}-05 & 1.089 \mathrm{E}-04\end{array}$ $\begin{array}{llllllll}5.426 \mathrm{E}-15 & 8.119 \mathrm{E}-14 & 9.420 \mathrm{E}-13 & 2.449 \mathrm{E}-11 & 5.661 \mathrm{E}-10 & 1.669 \mathrm{E}-08 & 2.683 \mathrm{E}-07 & 2.391 \mathrm{E}-06\end{array}$

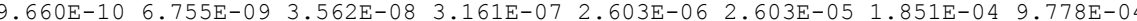

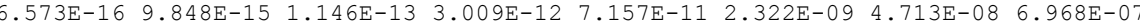

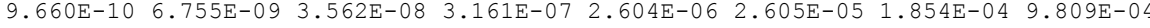

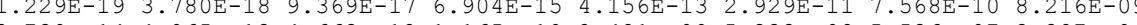

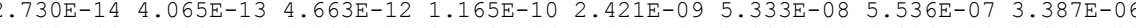
$\begin{array}{llllllll}1.488 \mathrm{E}-20 & 4.583 \mathrm{E}-19 & 1.138 \mathrm{E}-17 & 8.458 \mathrm{E}-16 & 5.214 \mathrm{E}-14 & 3.999 \mathrm{E}-12 & 1.296 \mathrm{E}-10 & 2.359 \mathrm{E}-0\end{array}$

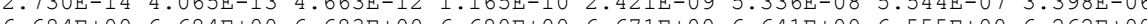

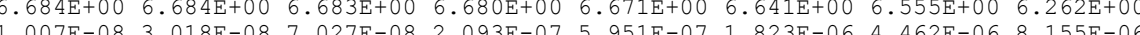

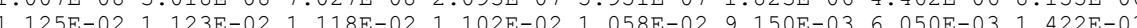

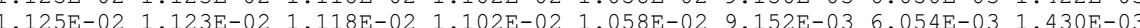
$\begin{array}{llllllll}8.523 \mathrm{E}-14 & 5.960 \mathrm{E}-13 & 3.143 \mathrm{E}-12 & 2.788 \mathrm{E}-11 & 2.295 \mathrm{E}-10 & 2.293 \mathrm{E}-0 & 1.623 \mathrm{E}-08 & 8.437 \mathrm{E}-0\end{array}$ $\begin{array}{llllllll}1.429 \mathrm{E}-07 & 4.279 \mathrm{E}-07 & 9.950 \mathrm{E}-07 & 2.948 \mathrm{E}-06 & 8.266 \mathrm{E}-06 & 2.407 \mathrm{E}-05 & 5.057 \mathrm{E}-05 & 4.943 \mathrm{E}-0\end{array}$

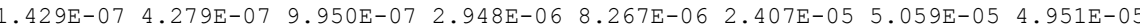


1RESRAD, Version $6.21 \quad T^{\frac{1}{2} / 2}$ Limit $=0.5$ year $\quad 02 / 22 / 2005 \quad 17: 45$ Page 25

Summary : CAU 224250 day/yr worker scenario $10 \mathrm{~m}$ hotspot
File : cau224 $25010 \mathrm{~m}$ parcel. RAD

Individual Nuclide Dose Summed Over All Pathways

Parent Nuclide and Branch Fraction Indicated

Nuclide Parent BRF(i)

$\frac{\text { (j) }}{\mathrm{Ac}-227} \frac{\text { (i) }}{\mathrm{Pu}-239} \frac{}{1.000 \mathrm{E}+00}$

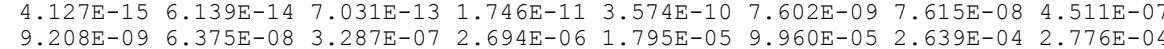

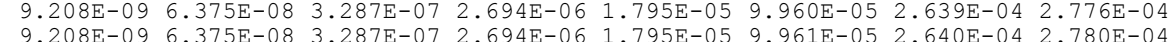

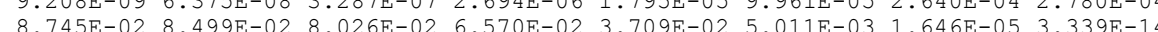

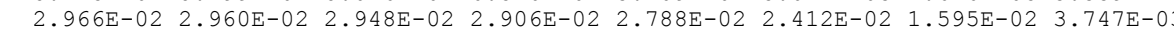

BRF(i) is the branch fraction of the parent nuclide.

is used to indicate summation; the Greek sigma is not included in this font. 
1RESRAD, Version 6.21

$T^{1 / 2}$ Limit $=0.5$ year

02/22/2005 17:45 Page 26

Summary : CAU 224250 day/yr worker scenario $10 \mathrm{~m}$ hotspot
File : cau224 $25010 \mathrm{~m}$ parcel. RAD

Individual Nuclide Soil Concentration

Parent Nuclide and Branch Fraction Indicated

ONuclide Parent BRF(i)

(j) (i)

$\overline{\mathrm{Am}-241} \overline{\mathrm{Am}-241} \overline{1.000 \mathrm{E}+00}$ ONp-237 $\mathrm{Am}-241 \quad 1.000 \mathrm{E}+00$ $0 \mathrm{~T}-2339 \mathrm{Am}-241 \mathrm{1}$

$\mathrm{OCs}-137 \mathrm{Cs}-137 \quad 1.000 \mathrm{E}+00$

OEu-152 Eu-152 $7.208 \mathrm{E}-01$

Eu-152 Eu-152 2.792E-01

$\mathrm{Eu}-152$ \$S $(j)$ :

$\begin{array}{lll}\text { OGd-152 } & \mathrm{Eu}-152 & 2.792 \mathrm{E}-01 \\ \mathrm{OPu}-238 & \mathrm{Pu}-238 & 1.000 \mathrm{E}+00\end{array}$

OU-234 $\quad \mathrm{Pu}-238 \quad 1.000 \mathrm{E}+00$

$\mathrm{U}-234 \mathrm{U}-234,1.000 \mathrm{E}+00$

$\mathrm{U}-234-\mathrm{U}-238-1.000 \mathrm{E}+00$

$\begin{array}{ccc}\mathrm{U}-234 & \$ S(j): & \\ 0 \mathrm{Th}-230 & \mathrm{Pu}-238 & 1.000 \mathrm{E}+00\end{array}$

Th-230 Pu-238 $1.000 \mathrm{E}+00$

Th-230 -234 1.000E+00

Th-230 \$S $(j):$ :

ORa-226 Pu-238 1.000E+00

$\begin{array}{lll}\mathrm{Ra}-226 & \mathrm{U}-234 & 1.000 \mathrm{E}+00 \\ \mathrm{Ra}-226 & \mathrm{U}-238 & 1.000 \mathrm{E}+00\end{array}$

$\begin{array}{lll}\mathrm{Ra}-226 & \$ \mathrm{~S}(\mathrm{j}): & \\ \mathrm{R}-210 & & \\ \mathrm{~Pb}-210 & \mathrm{Pu}-238 & 1.000 \mathrm{E}+00\end{array}$

$\begin{array}{cll}0 \mathrm{~Pb}-210 & \mathrm{Pu}-238 & 1.000 \mathrm{E}+00 \\ \mathrm{~Pb}-210 & \mathrm{U}-234 & 1.000 \mathrm{E}+00\end{array}$

$\begin{array}{lll}\mathrm{Pb}-210 & \mathrm{U}-234 & 1.000 \mathrm{E}+00 \\ \mathrm{~Pb}-210 & \mathrm{U}-238 & 1.000 \mathrm{E}+00\end{array}$

$\begin{array}{lll}\mathrm{Pb}-210 & \$ \mathrm{~S}(j): & \\ 0 \mathrm{Pu}-239 & \mathrm{Pu}-239 & 1.000 \mathrm{E}+00\end{array}$

$\begin{array}{lll}0 \mathrm{Pu}-239 & \mathrm{Pu}-239 & 1.000 \mathrm{E}+0\end{array}$

$\mathrm{OU}-235$ Pu-239 $1.000 \mathrm{E}+00$

$\mathrm{U}-235$ \$S $(j):$

$\begin{array}{rll}0 \mathrm{~Pa}-231 & \mathrm{Pu}-239 & 1.000 \mathrm{E}+0 \\ \mathrm{~Pa}-231 & \mathrm{U}-235 & 1.000 \mathrm{E}+00\end{array}$

$\mathrm{Pa}-231 \$ S(j)$

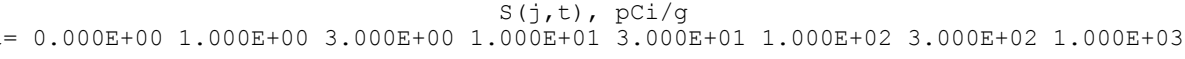

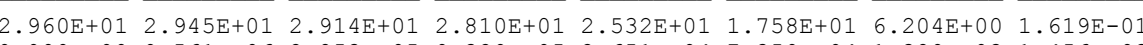

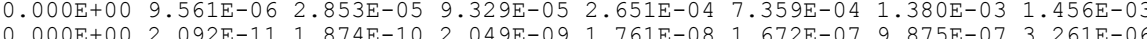

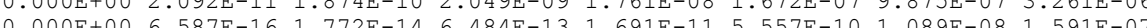
$\begin{array}{llllllll}1.000 \mathrm{E}+01 & 9.767 \mathrm{E}+00 & 9.318 \mathrm{E}+00 & 7.903 \mathrm{E}+00 & 4.936 \mathrm{E}+00 & 9.506 \mathrm{E}-01 & 8.589 \mathrm{E}-03 & 6.024 \mathrm{E}-10\end{array}$ $\begin{array}{llllllll}1.773 \mathrm{E}+00 & 1.683 \mathrm{E}+00 & 1.517 \mathrm{E}+00 & 1.053 \mathrm{E}+00 & 3.716 \mathrm{E}-01 & 9.697 \mathrm{E}-03 & 2.900 \mathrm{E}-07 & 4.242 \mathrm{E}-23\end{array}$ $\begin{array}{llllllll}6.868 \mathrm{E}-01 & 6.520 \mathrm{E}-01 & 5.875 \mathrm{E}-01 & 4.080 \mathrm{E}-01 & 1.440 \mathrm{E}-01 & 3.756 \mathrm{E}-03 & 1.123 \mathrm{E}-07 & 1.643 \mathrm{E}-23\end{array}$

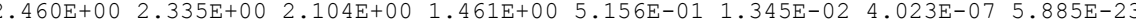

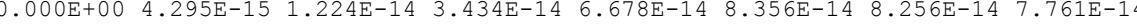

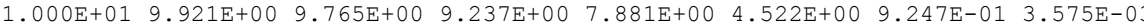
$\begin{array}{llllllll}0.000 \mathrm{E}+00 & 2.821 \mathrm{E}-05 & 8.378 \mathrm{E}-05 & 2.697 \mathrm{E}-04 & 7.329 \mathrm{E}-04 & 1.744 \mathrm{E}-03 & 2.150 \mathrm{E}-03 & 3.575 \mathrm{E}-03\end{array}$

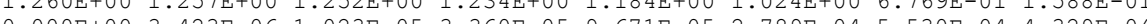

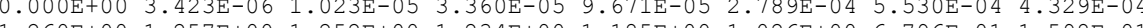

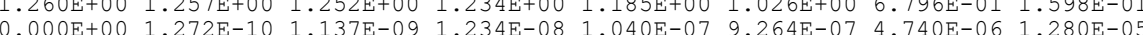
$0.000 \mathrm{E}+00 \quad 1.133 \mathrm{E}-05 \quad 3.392 \mathrm{E}-05 \quad 1.123 \mathrm{E}-04 \quad 3.299 \mathrm{E}-04 \quad 1.024 \mathrm{E}-03 \quad 2.530 \mathrm{E}-03 \quad 4.754 \mathrm{E}-03$ $0.000 \mathrm{E}+00 \quad 1.542 \mathrm{E}-11 \quad 1.384 \mathrm{E}-10 \quad 1.523 \mathrm{E}-09 \quad 1.333 \mathrm{E}-08 \quad 1.346 \mathrm{E}-07 \quad 9.276 \mathrm{E}-03 \quad 4.394 \mathrm{E}-03$

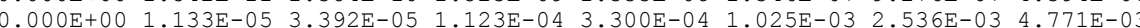

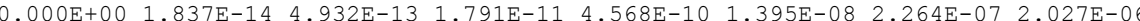

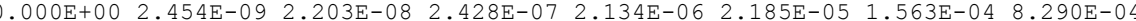

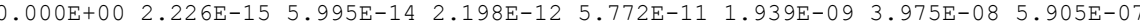

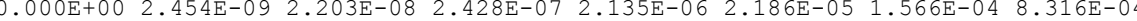

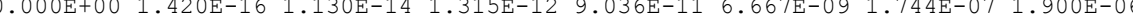

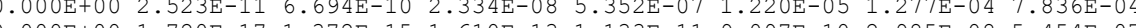
$\begin{array}{llllllll}0.000 \mathrm{E}+00 & 1.720 \mathrm{E}-17 & 1.372 \mathrm{E}-15 & 1.610 \mathrm{E}-13 & 1.133 \mathrm{E}-11 & 9.097 \mathrm{E}-10 & 2.985 \mathrm{E}-08 & 5.454 \mathrm{E}-0\end{array}$

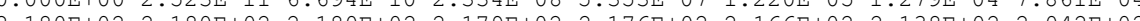

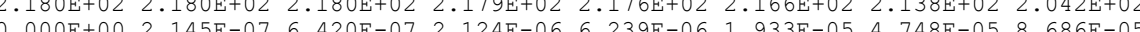

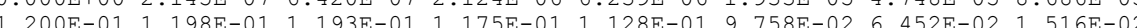

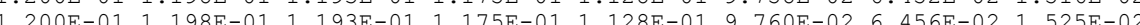
$\begin{array}{llllllll}0.000 \mathrm{E}+00 & 2.269 \mathrm{E}-12 & 2.037 \mathrm{E}-11 & 2.244 \mathrm{E}-10 & 1.972 \mathrm{E}-0 & 2.016 \mathrm{E}-08 & 1.437 \mathrm{E}-0 & 7.485 \mathrm{E}-02\end{array}$ $\begin{array}{llllllll}0.000 \mathrm{E}+00 & 2.535 \mathrm{E}-06 & 7.577 \mathrm{E}-06 & 2.494 \mathrm{E}-05 & 7.223 \mathrm{E}-05 & 2.127 \mathrm{E}-04 & 4.485 \mathrm{E}-04 & 4.389 \mathrm{E}-04\end{array}$ 0.000E+00 2.535E-06 7.577E-06 2.494E-05 7.223E-05 2.128E-04 4.486E-04 4.396E-04 
1RESRAD, Version $6.21 \quad T^{3 / 2}$ Limit $=0.5$ year $02 / 22 / 2005 \quad 17: 45$ Page 27 Summary : CAU 224250 day/yr worker scenario $10 \mathrm{~m}$ hotspot

Individual Nuclide Soil Concentration

Parent Nuclide and Branch Fraction Indicated

ONuclide Parent BRF(i)

(j) $\frac{\text { (i) }}{1.000 E+000}$

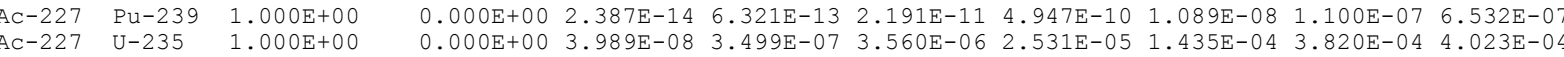

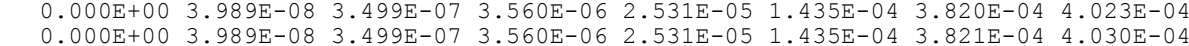

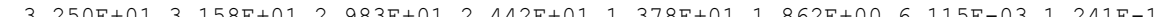

$\begin{array}{llllllllll}1.210 \mathrm{E}+00 & 1.207 \mathrm{E}+00 & 1.203 \mathrm{E}+00 & 1.185 \mathrm{E}+00 & 1.137 \mathrm{E}+00 & 9.839 \mathrm{E}-01 & 6.505 \mathrm{E}-01 & 1.529 \mathrm{E}-01\end{array}$

RF(i) is the branch fraction of the parent nuclide.

$\$$ is used to indicate summation; the Greek sigma is not included in this font.
ORESCALC.EXE execution time $=4.40$ seconds 
Exhibit 4

RESRAD Scenario A Hot Spot $1 \mathrm{~m}^{2}$

(27 Pages) 
1RESRAD, Version $6.21 \quad T^{1 / 2}$ Limit $=0.5$ year

Summary : CAU 224250 day/yr worker scenario $1 \mathrm{~m}$ hotspot

File: cau224 $2501 \mathrm{~m}$ parcel.RAD

$$
\text { Table of Contents }
$$

Part I: Mixture Sums and Single Radionuclide Guidelines

Dose Conversion Factor (and Related) Parar Site-specific Parameter summary .........................

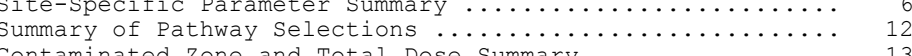
Contaminated Zone and Tota Time $=0.000 \mathrm{E}+00$

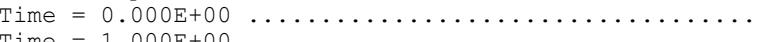

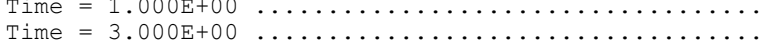

Time $=1.000 \mathrm{E}+01$

Time $=3.000 \mathrm{E}+01$

Time $=3.000 \mathrm{E}+02$

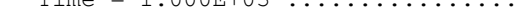

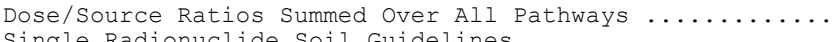

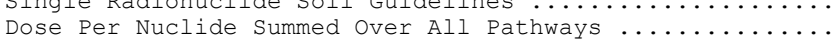

Soil Concentration Per Nuclide... 
Dose Conversion Factor (and Related) Parameter Summary

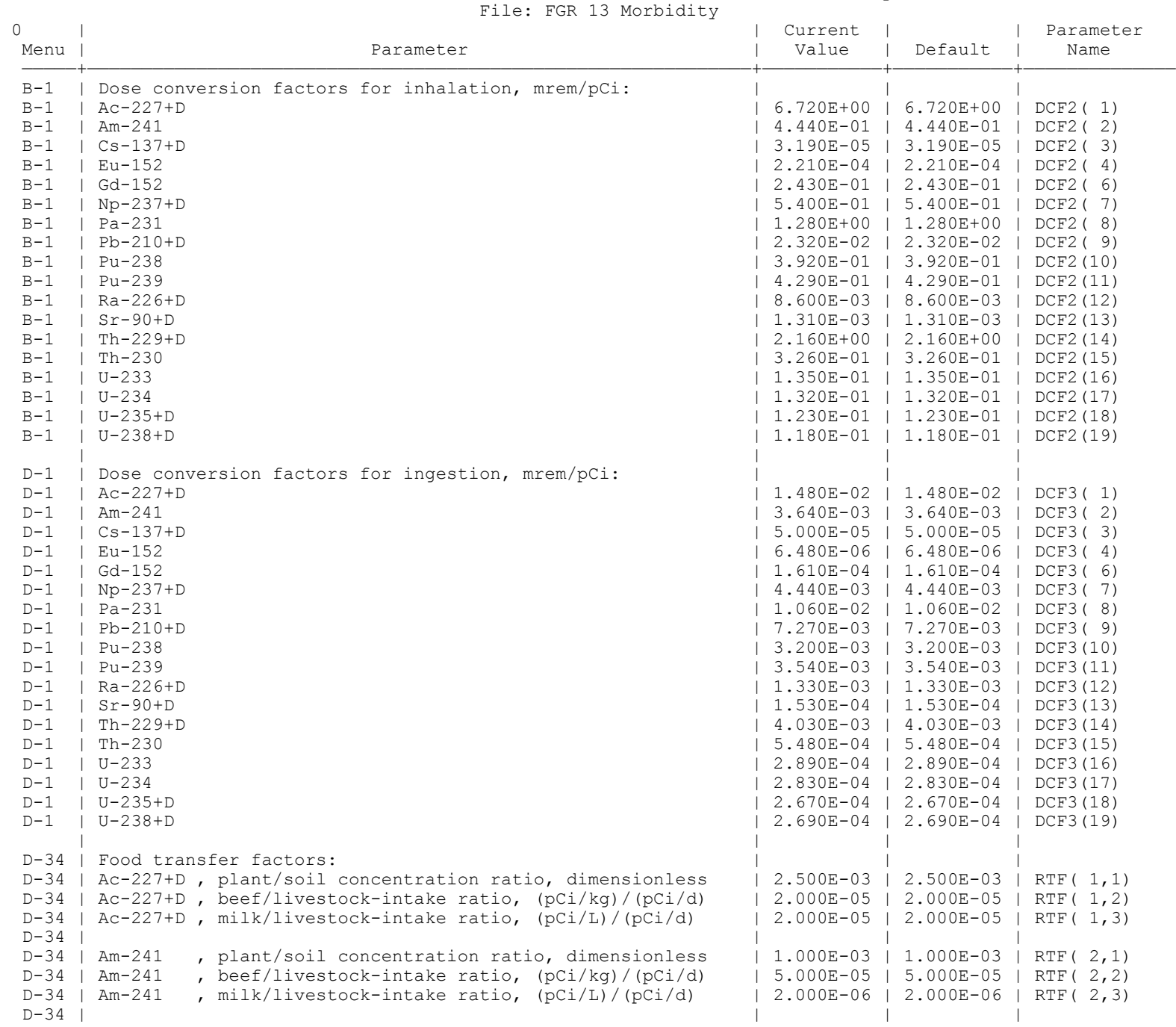


Dose Conversion Factor (and Related) Parameter Summary (continued) File: FGR 13 Morbidity

Menu Paramete

\begin{tabular}{|c|c|c|}
\hline $\begin{array}{l}\text { Current } \\
\text { Value }\end{array}$ & Default & $\begin{array}{l}\text { Parameter } \\
\text { Name }\end{array}$ \\
\hline $4.000 \mathrm{E}-02$ & $4.000 \mathrm{E}-02$ & $\operatorname{RTF}(3,1)$ \\
\hline $3.000 \mathrm{E}-02$ & $3.000 \mathrm{E}-02$ & $\operatorname{RTE}(3,2)$ \\
\hline $8.000 \mathrm{E}-03$ & $8.000 \mathrm{E}-03$ & $\operatorname{RTE}(3,3)$ \\
\hline $2.500 \mathrm{E}-03$ & $2.500 \mathrm{E}-03$ & $\operatorname{RTF}(4,1)$ \\
\hline $2.000 \mathrm{E}-03$ & $2.000 \mathrm{E}-03$ & $\operatorname{RTF}(4,2)$ \\
\hline $2.000 \mathrm{E}-05$ & $2.000 \mathrm{E}-05$ & $\operatorname{RTE}(4,3)$ \\
\hline $2.500 \mathrm{E}-03$ & $2.500 \mathrm{E}-03$ & $\operatorname{RTF}(6,1)$ \\
\hline $2.000 \mathrm{E}-03$ & $2.000 \mathrm{E}-03$ & $\operatorname{RTE}(6,2)$ \\
\hline $2.000 \mathrm{E}-05$ & $2.000 \mathrm{E}-05$ & $\operatorname{RTF}(6,3)$ \\
\hline $2.000 \mathrm{E}-02$ & $2.000 \mathrm{E}-02$ & $\operatorname{RTF}(7,1)$ \\
\hline $1.000 \mathrm{E}-03$ & $1.000 \mathrm{E}-03$ & $\operatorname{RTE}(7,2)$ \\
\hline $5.000 \mathrm{E}-06$ & $5.000 \mathrm{E}-06$ & $\operatorname{RTE}(7,3)$ \\
\hline $1.000 \mathrm{E}-02$ & $1.000 \mathrm{E}-02$ & $\operatorname{RTF}(8,1)$ \\
\hline $5.000 \mathrm{E}-03$ & $5.000 \mathrm{E}-03$ & $\operatorname{RTF}(8,2)$ \\
\hline $5.000 \mathrm{E}-06$ & $5.000 \mathrm{E}-06$ & $\operatorname{RTE}(8,3)$ \\
\hline 1. $000 \mathrm{E}-02$ & & \\
\hline $\begin{array}{l}1.000 \mathrm{E}-02 \\
8.000 \mathrm{E}-04\end{array}$ & $\begin{array}{l}1.000 \mathrm{E}-02 \\
8.00 \mathrm{E}-04\end{array}$ & $\begin{array}{l}\operatorname{RTT}(9,1) \\
\operatorname{RTT}(9,2)\end{array}$ \\
\hline $3.000 \mathrm{E}-04$ & $3.000 \mathrm{E}-04$ & $\operatorname{RTE}(9,3)$ \\
\hline $1.000 \mathrm{E}-03$ & $1.000 \mathrm{E}-03$ & $\operatorname{RTF}(10,1)$ \\
\hline $1.000 \mathrm{E}-04$ & $1.000 \mathrm{E}-04$ & $\operatorname{RTE}(10,2)$ \\
\hline $1.000 \mathrm{E}-06$ & $1.000 \mathrm{E}-06$ & $\operatorname{RTF}(10,3)$ \\
\hline $1.000 \mathrm{E}-03$ & $1.000 \mathrm{E}-03$ & $\operatorname{RTF}(11,1)$ \\
\hline $1.000 \mathrm{E}-04$ & $1.000 \mathrm{E}-04$ & $\operatorname{RTF}(11,2)$ \\
\hline $1.000 \mathrm{E}-06$ & $1.000 \mathrm{E}-06$ & $\operatorname{RTE}(11,3)$ \\
\hline $4.000 \mathrm{E}-02$ & $4.000 \mathrm{E}-02$ & $\operatorname{RTF}(12,1)$ \\
\hline $1.000 \mathrm{E}-03$ & $1.000 \mathrm{E}-03$ & $\operatorname{RTF}(12,2)$ \\
\hline $1.000 \mathrm{E}-03$ & $1.000 \mathrm{E}-03$ & $\operatorname{RTE}(12,3)$ \\
\hline $3.000 \mathrm{E}-01$ & $3.000 \mathrm{E}-01$ & $\operatorname{RTF}(13,1)$ \\
\hline $8.000 \mathrm{E}-03$ & $8.000 \mathrm{E}-03$ & $\operatorname{RTF}(13,2)$ \\
\hline $2.000 \mathrm{E}-03$ & $2.000 \mathrm{E}-03$ & $\operatorname{RTE}(13,3)$ \\
\hline $1.000 F-03$ & & $\operatorname{RTF}(14,1)$ \\
\hline $1.000 \mathrm{E}-04$ & $1.000 \mathrm{E}-04$ & $\operatorname{RTF}(14,2)$ \\
\hline $5.000 \mathrm{E}-06$ & $5.000 \mathrm{E}-06$ & $\operatorname{RTF}(14,3)$ \\
\hline $1.000 \mathrm{E}-03$ & $1.000 \mathrm{E}-03$ & $\operatorname{RTF}(15,1)$ \\
\hline $1.000 \mathrm{E}-04$ & $1.000 \mathrm{E}-04$ & $\operatorname{RTF}(15,2)$ \\
\hline $5.000 \mathrm{E}-06$ & $5.000 \mathrm{E}-06$ & $\operatorname{RTF}(15,3)$ \\
\hline & & \\
\hline
\end{tabular}

D-34 Cs-137+D, plant/soil concentration ratio, dimensionless D-34 Cs-137+D, beef/livestock-intake ratio, (pCi/kg)/(pCi/d)

D-34 | Eu-152, plant/soil concentration ratio, dimensionless $\mathrm{D}-34 \mid \mathrm{Fu}-152$, beef/livestock-intake ratio, $(\mathrm{pCi} / \mathrm{kg}) /(\mathrm{pCi} / \mathrm{d})$ D-34 Eu-152, milk/livestock-intake ratio, (pCi/L)/(pCi/d)

D-34 Gd-152, plant/soil concentration ratio, dimensionless , beef/livestock-intake ratio, $(\mathrm{pCi} / \mathrm{kg}) /(\mathrm{pCi} / \mathrm{d})$ D-34 | Gd-152, milk/livestock-intake ratio, (pCi/L)/(pCi/d) D- 34 D-34 Np-237+D, plant/soil concentration ratio, dimensionless D-34 Np-237+D, beef/livestock-intake ratio, (pCi/kg)/(pCi/d) D-34 I Np-237+D, milk/livestock-intake ratio, (pCi/L)/(pCi/d) D -34

| Pa-231, plant/soil concentration ratio, dimensionless , beef/livestock-intake ratio, $(\mathrm{pCi} / \mathrm{kg}) /(\mathrm{pCi} / \mathrm{d})$ \begin{tabular}{l|l}
$\mathrm{D}-34$ \\
$\mathrm{D}-34$
\end{tabular}

D-34 Pb-210+D, plant/soil concentration ratio, dimensionless $\mathrm{D}-34 \mathrm{~Pb}-210+\mathrm{D}$, beef/livestock-intake ratio, $(\mathrm{pCi} / \mathrm{kg}) /(\mathrm{pCi} / \mathrm{d})$ D-34 Pb-210+D, milk/livestock-intake ratio, (pCi/L)/(pCi/d) D-34 I

D-34 | Pu-238, plant/soil concentration ratio, dimensionless , beef/livestock-intake ratio, $(\mathrm{pCi} / \mathrm{kg}) /(\mathrm{pCi} / \mathrm{d})$ ( D -34

| Pu-239, plant/soil concentration ratio, dimensionless , beef/livestock-intake ratio, (pCi/kg)/(pCi/d) (1) kivestock-intake ratio,

D-34 Ra-226+D, plant/soil concentration ratio, dimensionless D-34 Ra-226+D, beef/livestock-intake ratio, (pCi/kg)/(pCi/d) D-34 Ra-226+D, milk/livestock-intake ratio, (pCi/L)/(pCi/d) D-34 I

D-34 Sr-90+D, plant/soil concentration ratio, dimensionless D-34 Sr-90+D, beef/livestock-intake ratio, (pCi/kg)/(pCi/d) D-34 Sr-90+D , milk/livestock-intake ratio, (pCi/L)/(pCi/d)

D-34 Th-229+D, plant/soil concentration ratio, dimensionless D, milk/livestock-intake ratio, (pCi/L)/(pCi/d)

D-34 Th-230, plant/soil concentration ratio, dimensionless D-34 Th-230, beef/livestock-intake ratio, $(\mathrm{pCi} / \mathrm{kg}) /(\mathrm{pCi} / \mathrm{d})$ D-34 
1RESRAD, Version 6.21 T3/2 Limit $=0.5$ year $02 / 22 / 2005 \quad 17: 50$ Page 4 Summary : CAU 224250 day/yr worker scenario $1 \mathrm{~m}$ hotspot

Dose Conversion Factor (and Related) Parameter Summary (continued)

\begin{tabular}{|c|c|c|c|c|c|}
\hline Menu & & Parameter & $\begin{array}{l}\text { Current } \\
\text { Value }\end{array}$ & Default & $\begin{array}{l}\text { Parameter } \\
\text { Name }\end{array}$ \\
\hline D-34 & $\mathrm{U}-233$ & , plant/soil concentration ratio, dimensionless & $2.500 \mathrm{E}-03$ & $2.500 \mathrm{E}-03$ & $\operatorname{RTF}(16,1)$ \\
\hline D-34 & 33 & intake rati & $3.400 \mathrm{E}-04$ & OOE-04 & $\operatorname{RTF}(16,2)$ \\
\hline$D-34$ & $\mathrm{U}-233$ & , milk/livestock-intake ratio, (pCi/L)/(pCi/d) & $6.000 \mathrm{E}-04$ & $6.000 \mathrm{E}-04$ & $\operatorname{RTF}(16,3)$ \\
\hline D-34 & & & & & \\
\hline$D-34$ & $\mathrm{U}-234$ & , plant/soil concentration ratio, dimensionless & $2.500 \mathrm{E}-03$ & $2.500 \mathrm{E}-03$ & $\operatorname{RTF}(17,1)$ \\
\hline$D-34$ & $\mathrm{U}-234$ & , beef/livestock-intake ratio, $(\mathrm{pCi} / \mathrm{kg}) /(\mathrm{pCi} / \mathrm{d})$ & $3.400 \mathrm{E}-04$ & $3.400 \mathrm{E}-04$ & $\operatorname{RTF}(17,2)$ \\
\hline$D-34$ & $\mathrm{U}-234$ & , milk/livestock-intake ratio, $(\mathrm{pCi} / \mathrm{L}) /(\mathrm{pCi} / \mathrm{d})$ & $6.000 \mathrm{E}-04$ & $6.000 \mathrm{E}-04$ & $\operatorname{RTE}(17,3)$ \\
\hline $\begin{array}{l}D-34 \\
D-34\end{array}$ & $\mathrm{U}-235+\mathrm{D}$ & , plant/soil concentration ratio, dimensionless & $2.500 \mathrm{E}-03$ & $2.500 \mathrm{E}-03$ & $\operatorname{RTF}(18,1)$ \\
\hline D-34 & $\mathrm{U}-235+\mathrm{D}$ & , beef/livestock-intake ratio, $(\mathrm{pCi} / \mathrm{kg}) /(\mathrm{pCi} / \mathrm{d})$ & $3.400 \mathrm{E}-04$ & $3.400 \mathrm{E}-04$ & $\operatorname{RTF}(18,2)$ \\
\hline $\begin{array}{l}D-34 \\
D-34\end{array}$ & $\mathrm{U}-235+\mathrm{D}$ & , milk/livestock-intake ratio, $(\mathrm{pCi} / \mathrm{L}) /(\mathrm{pCi} / \mathrm{d})$ & $6.000 \mathrm{E}-04$ & $6.000 \mathrm{E}-04$ & $\operatorname{RTE}(18,3)$ \\
\hline D-34 & $-238+D$ & , plant/soil concentration ratio, dimensionless & $2.500 \mathrm{E}-03$ & $2.500 \mathrm{E}-03$ & $\operatorname{RTF}(19,1)$ \\
\hline$D-34$ & $-238+D$ & , beef/livestock-intake ratio, (pCi/k & $3.400 \mathrm{E}-04$ & $3.400 \mathrm{E}-04$ & $\operatorname{RTE}(19,2)$ \\
\hline$D-34$ & $\mathrm{U}-238+\mathrm{D}$ & , milk/livestock-intake ratio, $(\mathrm{pCi} / \mathrm{L}) /(\mathrm{pCi} / \mathrm{d})$ & $6.000 \mathrm{E}-04$ & $6.000 \mathrm{E}-04$ & $\operatorname{RTF}(19,3)$ \\
\hline D-5 & ioaccums & ulation factors, fresh water, L/ & & & \\
\hline D-5 & $A c-227+D$ & , fish & $1.500 \mathrm{E}+01$ & $1.500 \mathrm{E}+01$ & $\operatorname{BIOFAC}(1,1)$ \\
\hline $\begin{array}{l}D-5 \\
D-5\end{array}$ & Ac-227+D & , crustacea and mollusks & $1.000 \mathrm{E}+03$ & $1.000 \mathrm{E}+03$ & $\operatorname{BIOFAC}(1,2)$ \\
\hline$D-5$ & $n-241$ & , fish & $3.000 \mathrm{E}+01$ & $3.000 \mathrm{E}+01$ & $\operatorname{BIOFAC}(2,1)$ \\
\hline $\begin{array}{l}D-5 \\
D-5\end{array}$ & $A m-241$ & crustacea and mollusks & $1.000 \mathrm{E}+03$ & $1.000 \mathrm{E}+03$ & $\operatorname{BIOFAC}(2,2)$ \\
\hline$D-5$ & Cs $-137+D$ & , fish & $2.000 \mathrm{E}+03$ & $2.000 \mathrm{E}+03$ & $\operatorname{BIOFAC}(3,1)$ \\
\hline $\begin{array}{l}D-5 \\
D-5\end{array}$ & $\mathrm{Cs}-137+\mathrm{D}$ & , crustacea and mollusks & $1.000 \mathrm{E}+02$ & $1.000 \mathrm{E}+02$ & $\operatorname{BIOFAC}(3,2)$ \\
\hline$D-5$ & Eu-152 & , fish & $5.000 \mathrm{E}+01$ & $5.000 \mathrm{E}+01$ & $\operatorname{BIOFAC}(4,1)$ \\
\hline $\begin{array}{l}D-5 \\
D-5\end{array}$ & $\mathrm{Eu}-152$ & , crustacea and mollusks & $1.000 \mathrm{E}+03$ & $1.000 \mathrm{E}+03$ & $\operatorname{BIOFAC}(4,2)$ \\
\hline D-5 & Gd-152 & fish & $2.500 \mathrm{E}+01$ & $2.500 \mathrm{E}+01$ & $\operatorname{BIOFAC}(6,1)$ \\
\hline$D-5$ & $\mathrm{Gd}-152$ & , crustacea and mollusks & $1.000 \mathrm{E}+03$ & $1.000 \mathrm{E}+03$ & $\operatorname{BIOFAC}(6,2)$ \\
\hline $\begin{array}{l}D-5 \\
D-5\end{array}$ & & & & & \\
\hline $\begin{array}{l}D-5 \\
D-5\end{array}$ & $\begin{array}{l}\mathrm{Np}-237+\mathrm{D} \\
\mathrm{Np}-237+\mathrm{D}\end{array}$ & $\begin{array}{l}\text { fish } \\
\text { crustacea and mollusks }\end{array}$ & $\begin{array}{l}3.000 \mathrm{E}+01 \\
4.000 \mathrm{E}+02\end{array}$ & $\begin{array}{l}3.000 \mathrm{E}+01 \\
4.000 \mathrm{E}+02\end{array}$ & $\begin{array}{l}\operatorname{BIOFAC}(7,1) \\
\operatorname{BIOFAC}(7,2)\end{array}$ \\
\hline D-5 & & & & & \\
\hline D-5 & $\mathrm{Pa}-231$ & , fis & 1.0 & $1.000 \mathrm{~B}$ & $\operatorname{BIOFAC}(8,1)$ \\
\hline $\begin{array}{l}D-5 \\
D-5\end{array}$ & $\mathrm{~Pa}-231$ & , crustacea and mollusks & 1 & +02 & $\operatorname{BIOFAC}(8,2)$ \\
\hline$D-5$ & $\mathrm{~Pb}-210+\mathrm{D}$ & , fish & $3.000 \mathrm{E}+02$ & $3.000 \mathrm{E}+02$ & BIOFAC ( \\
\hline $\begin{array}{l}D-5 \\
D-5\end{array}$ & $\mathrm{~Pb}-210+\mathrm{D}$ & , crustacea and mollusks & 1.0 & $1.000 \mathrm{E}+02$ & $\operatorname{BIOFAC}(9,2)$ \\
\hline D-5 & | Pu-238 & , fi. & 3.0 & 3. & BIOFAC $(10$ \\
\hline $\begin{array}{l}D-5 \\
D-5\end{array}$ & $\mathrm{Pu}-238$ & , crustacea and mollusks & $1.000 \mathrm{E}+02$ & $1.000 \mathrm{E}+02$ & $\operatorname{BIOFAC}(10,2)$ \\
\hline D-5 & Pu-239 & , fis & & & BIC \\
\hline $\begin{array}{l}D-5 \\
D-5\end{array}$ & $\mathrm{Pu}-239$ & acea and mollusks & & $000 \mathrm{E}+02$ & $\operatorname{BIOFAC}(11,2)$ \\
\hline$D-5$ & $\mathrm{Ra}-226+\mathrm{D}$ & , fist & & & BIOFAC ( 12 \\
\hline$D-5$ & $R a-226+D$ & , crustacea and mollusks & $2.500 \mathrm{E}+02$ & $2.500 \mathrm{E}+02$ & $\operatorname{BIOFAC}(12,2)$ \\
\hline
\end{tabular}


1RESRAD, Version 6.21 T13/2 Limit $=0.5$ year $02 / 22 / 2005 \quad 17: 50$ Page 5 Summary : CAU 224250 day/yr worker scenario $1 \mathrm{~m}$ hotspot

Dose Conversion Factor (and Related) Parameter Summary (continued)

\begin{tabular}{|c|c|c|c|c|c|}
\hline Menu & । & Parameter & $\begin{array}{l}\text { Current } \\
\text { Value }\end{array}$ & I Default & $\begin{array}{c}\text { Parameter } \\
\text { Name }\end{array}$ \\
\hline D-5 & $S r-90+D$ & , fish & $6.000 \mathrm{E}+01$ & $6.000 \mathrm{E}+01$ & $\operatorname{BIOFAC}(13,1)$ \\
\hline $\begin{array}{l}D-5 \\
D-5\end{array}$ & $\mathrm{Sr}-90+\mathrm{D}$ & crustacea and mollusks & $1.000 \mathrm{E}+02$ & $1.000 \mathrm{E}+02$ & $\operatorname{BIOFAC}(13,2)$ \\
\hline$D-5$ & Th-229+D & , fish & $1.000 \mathrm{E}+02$ & $1.000 \mathrm{E}+02$ & $\operatorname{BIOFAC}(14,1)$ \\
\hline $\begin{array}{l}D-5 \\
D-5\end{array}$ & Th-229+D & , crustacea and mollusks & $5.000 \mathrm{E}+02$ & $5.000 \mathrm{E}+02$ & $\operatorname{BIOFAC}(14,2)$ \\
\hline D-5 & Th-230 & , fish & $1.000 \mathrm{E}+02$ & $1.000 \mathrm{E}+02$ & $\operatorname{BIOFAC}(15,1)$ \\
\hline $\begin{array}{l}D-5 \\
D-5\end{array}$ & $\operatorname{Th}-230$ & , crustacea and mollusks & $5.000 \mathrm{E}+02$ & $5.000 \mathrm{E}+02$ & $\operatorname{BIOFAC}(15,2)$ \\
\hline$D-5$ & U-233 & , fish & $1.000 \mathrm{E}+01$ & $1.000 \mathrm{E}+01$ & $\operatorname{BIOFAC}(16,1)$ \\
\hline $\begin{array}{l}D-5 \\
D-5\end{array}$ & $\mathrm{U}-233$ & crustacea and mollusks & $6.000 \mathrm{E}+01$ & $6.000 \mathrm{E}+01$ & $\operatorname{BIOFAC}(16,2)$ \\
\hline D-5 & U-234 & , fish & $1.000 \mathrm{E}+01$ & $1.000 \mathrm{E}+01$ & $\operatorname{BIOFAC}(17,1)$ \\
\hline $\begin{array}{l}D-5 \\
D-5\end{array}$ & $\mathrm{U}-234$ & , crustacea and mollusks & $6.000 \mathrm{E}+01$ & $6.000 \mathrm{E}+01$ & $\operatorname{BIOFAC}(17,2)$ \\
\hline$D-5$ & $\mathrm{U}-235+\mathrm{D}$ & , fish & $1.000 \mathrm{E}+01$ & $1.000 \mathrm{E}+01$ & $\operatorname{BIOFAC}(18,1)$ \\
\hline $\begin{array}{l}D-5 \\
D-5\end{array}$ & $U-235+D$ & , crustacea and mollusks & $6.000 \mathrm{E}+01$ & $6.000 \mathrm{E}+01$ & $\operatorname{BIOFAC}(18,2)$ \\
\hline D-5 & $\mathrm{U}-238+\mathrm{D}$ & , fish & $1.000 \mathrm{E}+01$ & $1.000 \mathrm{E}+01$ & $\operatorname{BIOFAC}(19$, \\
\hline$D-5$ & $\mathrm{U}-238+\mathrm{D}$ & , crustacea and mollusks & $6.000 \mathrm{E}+01$ & $6.000 \mathrm{E}+01$ & $\operatorname{BIOFAC}(19,2)$ \\
\hline
\end{tabular}




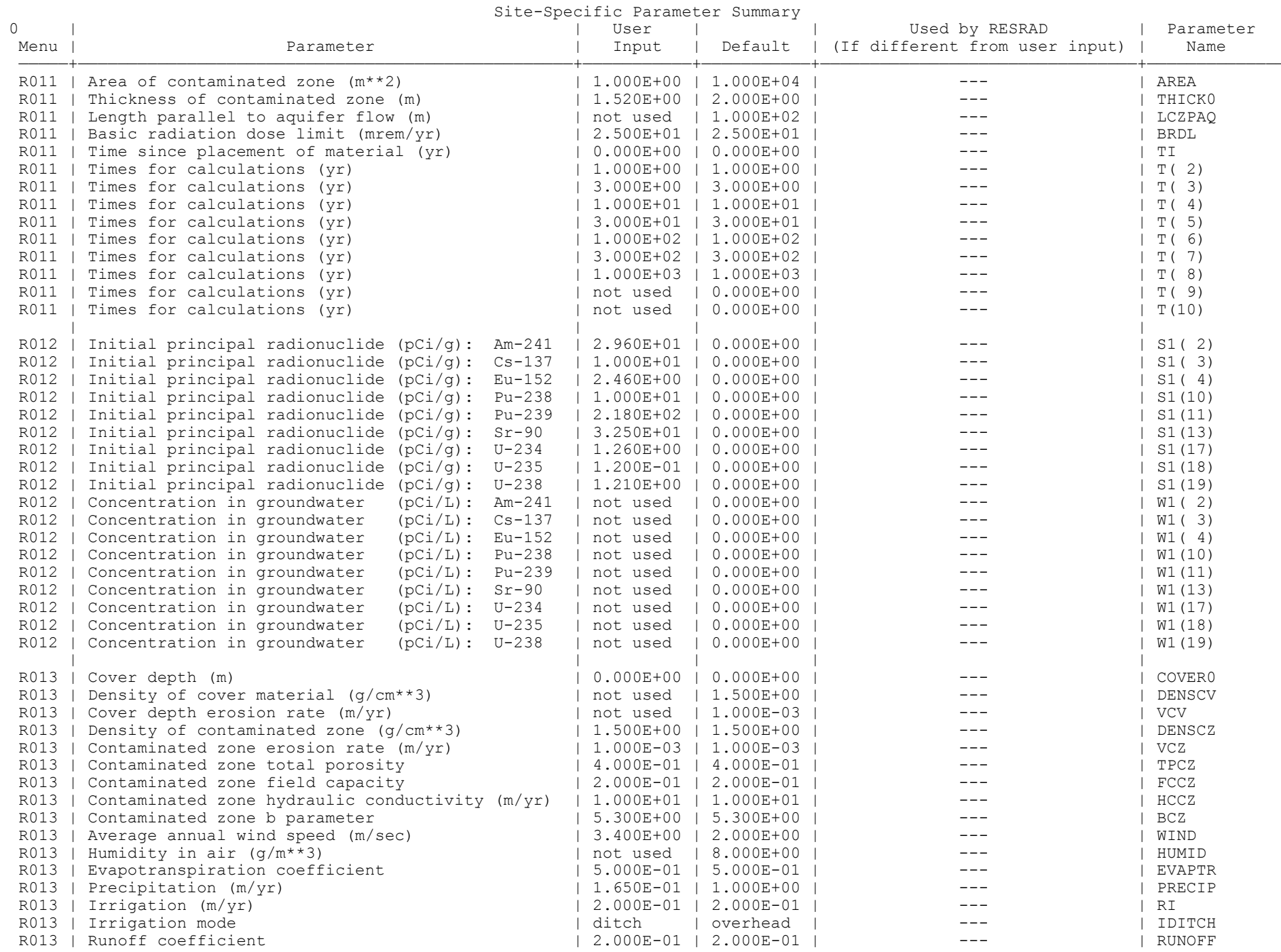


1RESRAD, Version $6.21 \quad T^{3} / 2$ Limit $=0.5$ year

Summary : CAU 224250 day/yr worker scenario $1 \mathrm{~m}$ hotspot

02/22/2005 17:50 Page 7

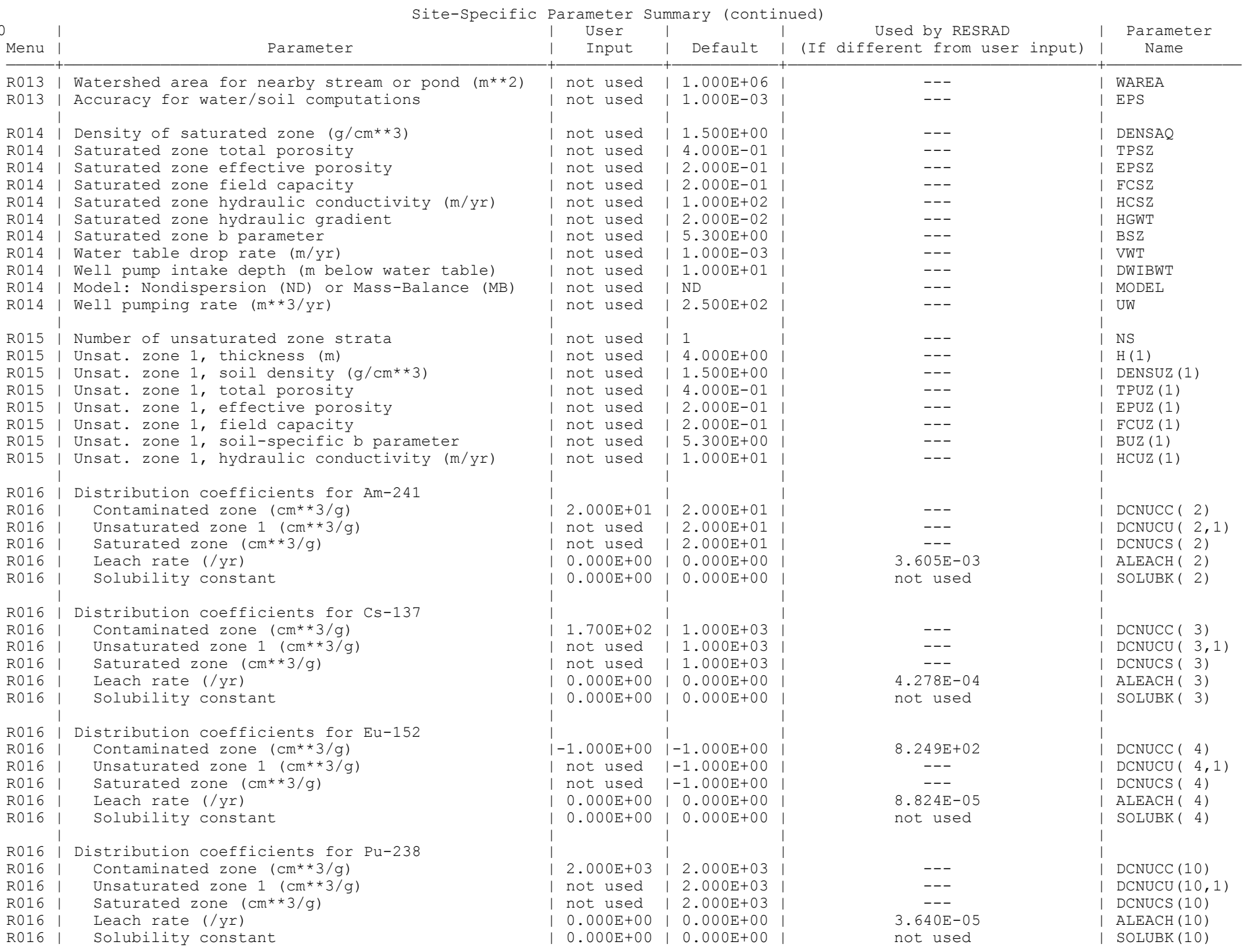


1RESRAD, Version $6.21 \quad T^{3} / 2$ Limit $=0.5$ year

Summary : CAU 224250 day/yr worker scenario $1 \mathrm{~m}$ hotspot

Site-Specific Parameter Summary (continued)

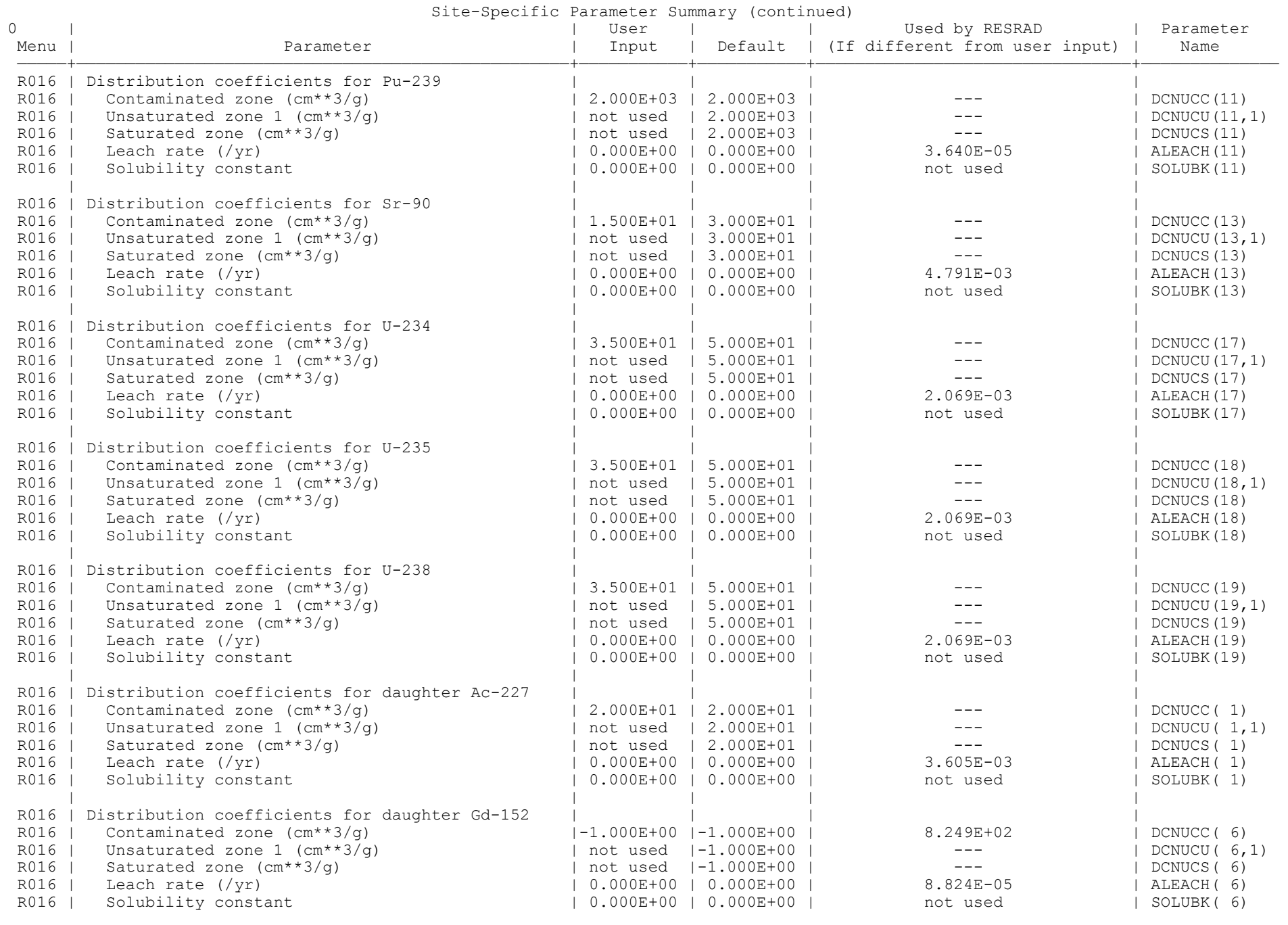


1RESRAD, Version $6.21 \quad T^{3} / 2$ Limit $=0.5$ year

Summary : CAU 224250 day/yr worker scenario $1 \mathrm{~m}$ hotspot

: cau224 $2501 \mathrm{~m}$ parcel.RAD

Site-Specific Parameter Summary (continued)

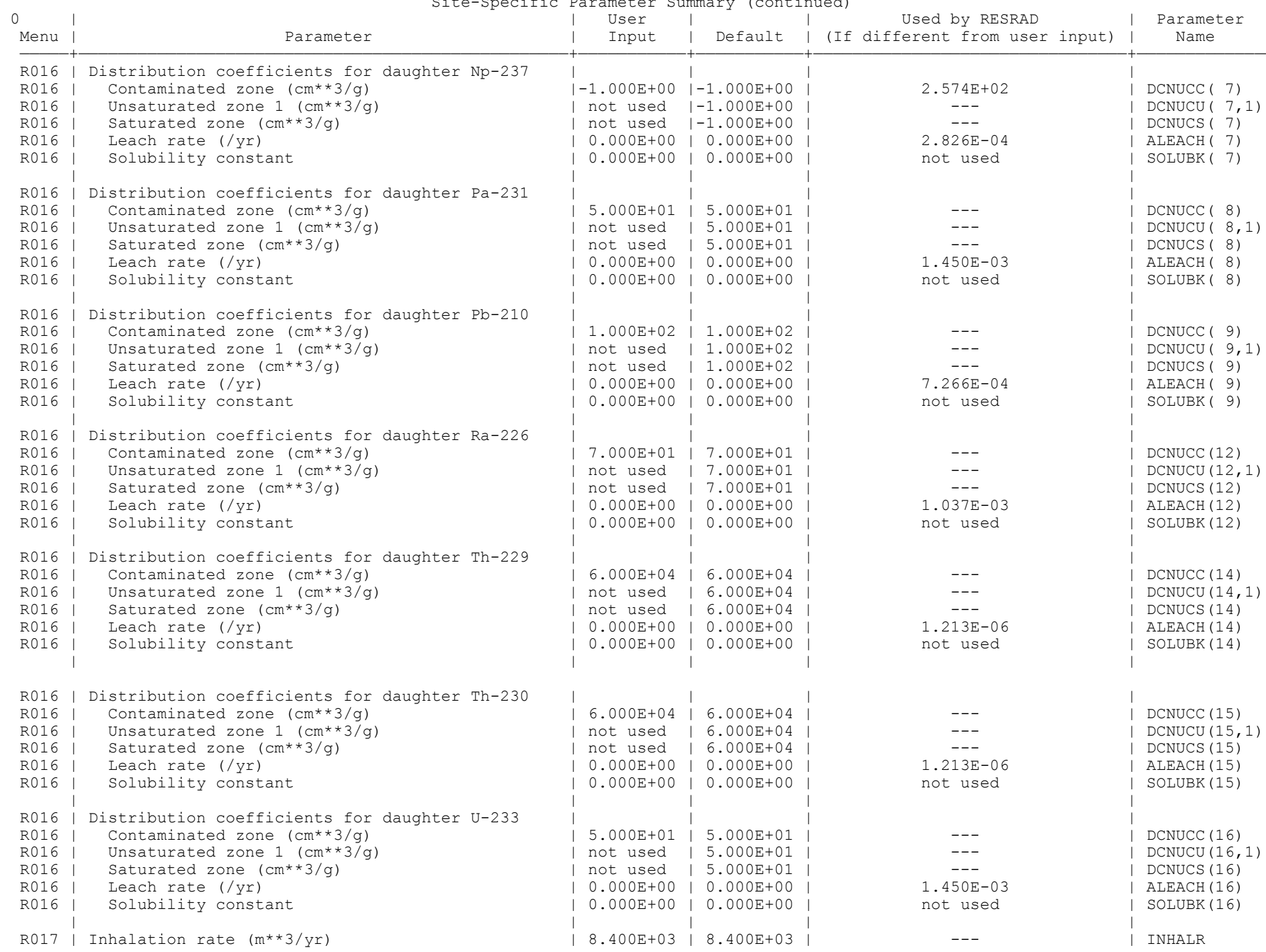




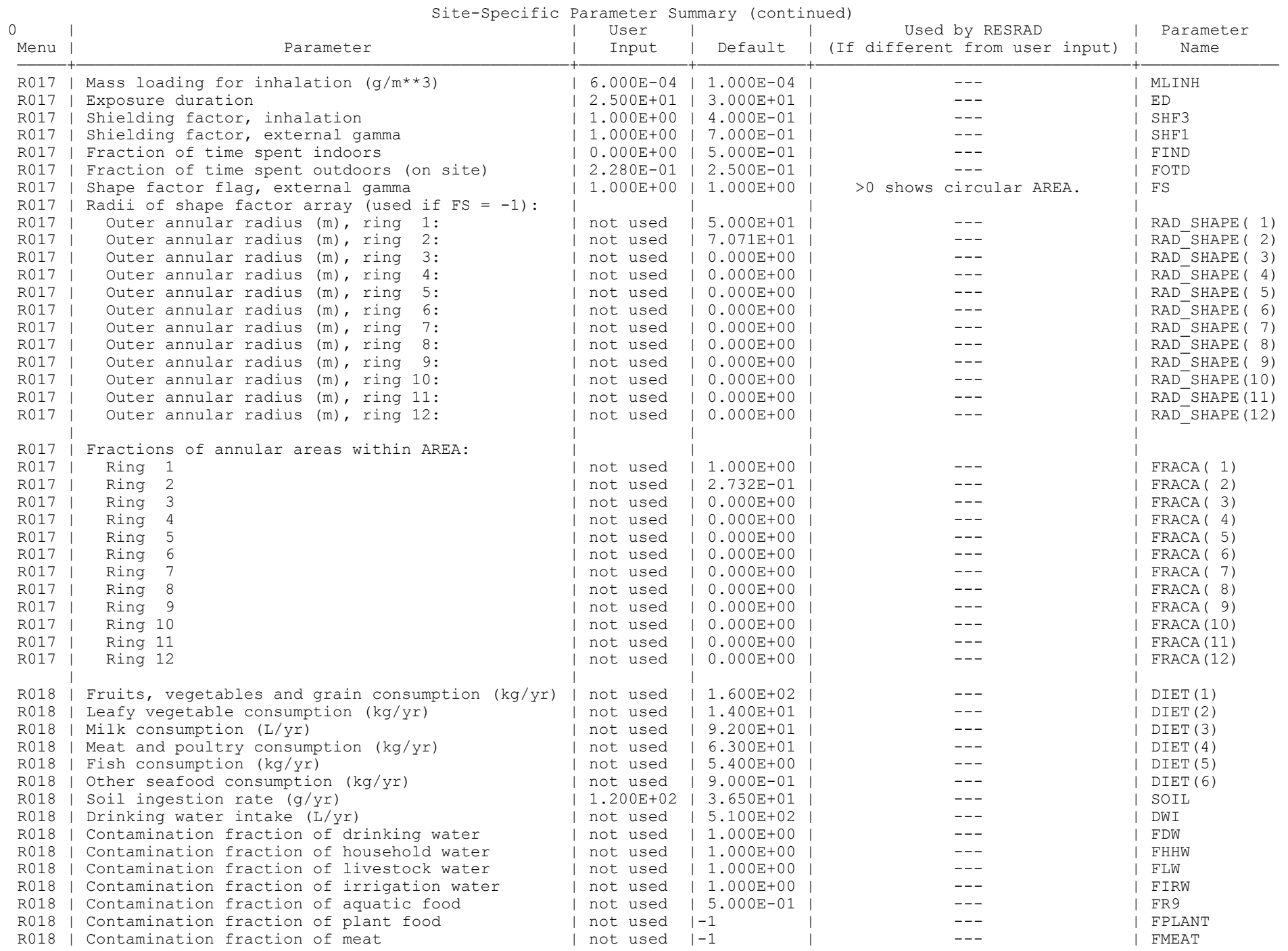


Site-Specific Parameter Summary (continued)

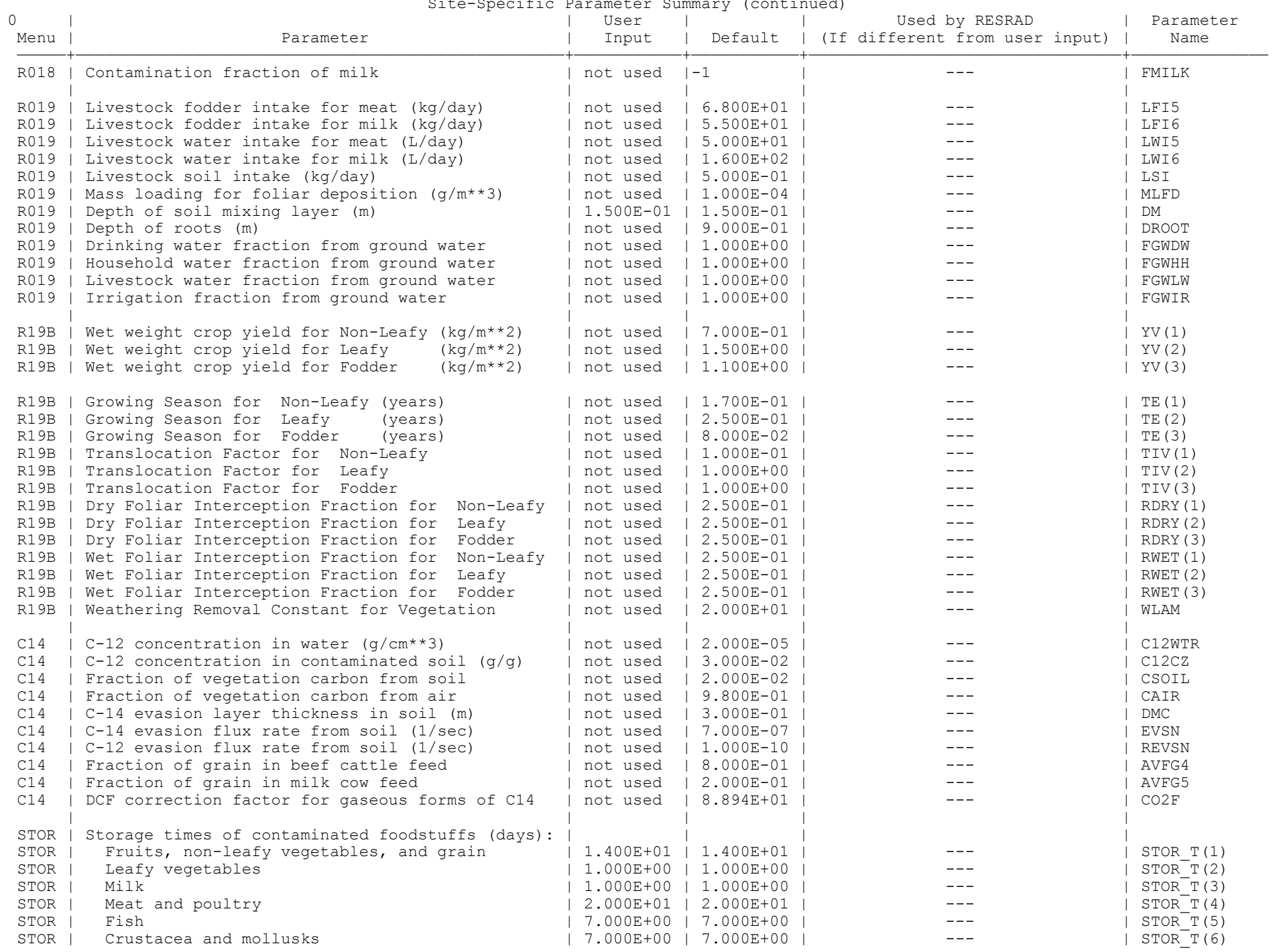


1RESRAD, Version $6.21 \quad$ T3/2 Limit $=0.5$ year Summary : CAU 224250 day/yr worker scenario $1 \mathrm{~m}$ hotspot

02/22/2005 17:50 Page 12

: Cau224 2501 m parcel. RAD

Site-Specific Parameter Summary (continued)

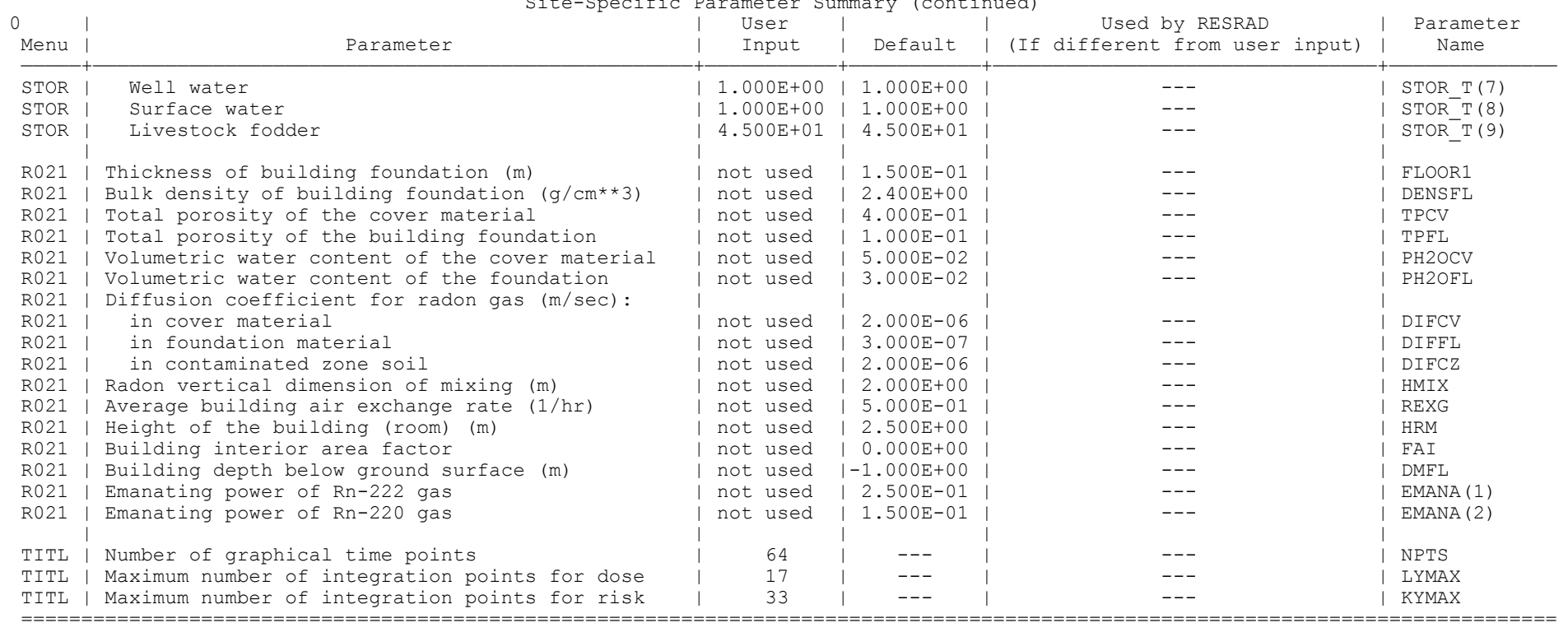

Summary of Pathway Selections

Pathway | User Selection

\begin{tabular}{|c|c|}
\hline & \\
\hline 1 -- external gamma & active \\
\hline 2 -- inhalation (w/o radon) & active \\
\hline 3 -- plant ingestion & suppressed \\
\hline 4 -- meat ingestion & suppressed \\
\hline 5 -- milk ingestion & suppressed \\
\hline $6--$ aquatic foods & suppressed \\
\hline 7 -- drinking water & suppressed \\
\hline 8 -- soil ingestion & active \\
\hline 9 -- radon & suppressed \\
\hline Find peak pathway doses & active \\
\hline
\end{tabular}


1RESRAD, Version 6.21 T13/2 Limit $=0.5$ year $02 / 22 / 2005 \quad 17: 50$ Page 13 Summary : CAU 224250 day/yr worker scenario $1 \mathrm{~m}$ hotspot

Contaminated Zone Dimensions

Area: 1.00 square meters

Cover Depth:

1.52 meter

.00 meters

Total Dose TDOSE(t), mrem/y

\begin{tabular}{cc} 
Initial Soil Concentrations, pCi/g \\
\hline Am-241 & $2.960 \mathrm{E}+01$ \\
$\mathrm{Cs}-137$ & $1.000 \mathrm{E}+01$ \\
$\mathrm{Eu}-152$ & $2.460 \mathrm{E}+00$ \\
$\mathrm{Pu}-238$ & $1.000 \mathrm{E}+01$ \\
$\mathrm{Pu}-239$ & $2.180 \mathrm{E}+02$ \\
$\mathrm{Sr}-90$ & $3.250 \mathrm{E}+01$ \\
$\mathrm{U}-234$ & $1.260 \mathrm{E}+00$ \\
$\mathrm{U}-235$ & $1.200 \mathrm{E}-01$ \\
$\mathrm{U}-238$ & $1.210 \mathrm{E}+00$
\end{tabular}

Basic Radiation Dose Limit $=2.500 \mathrm{E}+01 \mathrm{mrem} / \mathrm{yr}$

Total Mixture Sum $M(t)=$ Fraction of Basic Dose Limit Received at Time $(t)$

$t$ (years): $\begin{array}{llllllll}0.000 \mathrm{E}+00 & 1.000 \mathrm{E}+00 & 3.000 \mathrm{E}+00 & 1.000 \mathrm{E}+01 & 3.000 \mathrm{E}+01 & 1.000 \mathrm{E}+02 & 3.000 \mathrm{E}+02 & 1.000 \mathrm{E}+03\end{array}$

$\begin{array}{llllllll} & & \end{array}$ $\begin{array}{lllllllll}M(t): & 2.886 \mathrm{E}-01 & 2.868 \mathrm{E}-01 & 2.834 \mathrm{E}-01 & 2.732 \mathrm{E}-01 & 2.534 \mathrm{E}-01 & 2.258 \mathrm{E}-01 & 2.054 \mathrm{E}-01 & 1.893 \mathrm{E}-01\end{array}$ 
Total Dose Contributions TDOSE (i,p,t) for Individual Radionuclides (i) and Pathways (p) As mrem/yr and Fraction of Total Dose At $t=0.000 \mathrm{E}+00$ years Water Independent
Radon Plant
Meat

\begin{tabular}{|c|c|c|c|c|c|c|c|c|c|c|c|c|c|c|}
\hline \multirow{2}{*}{$\begin{array}{l}\text { Radio- } \\
\text { Nuclide }\end{array}$} & \multicolumn{2}{|c|}{ Ground } & \multicolumn{2}{|c|}{ Inhalation } & \multicolumn{2}{|c|}{ don } & \multicolumn{2}{|c|}{ Plant } & \multicolumn{2}{|c|}{ Meat } & \multicolumn{2}{|c|}{ Milk } & \multicolumn{2}{|c|}{ Soil } \\
\hline & $\mathrm{rem} / \mathrm{yr}$ & fact. & $\mathrm{m} / \mathrm{yr}$ & ract. & mrem/yr & fract & mrem/yr & fract. & mrem/yr & fract. & hrem/yr & fract & mrem/yr & frac \\
\hline & & & & & & & & & & & & & & \\
\hline & & & & & & & & & & & & & & \\
\hline & & & & & & & & & & & & & & \\
\hline & & & & & & & & & & & & & & \\
\hline & & & & & & & & & & & & & & \\
\hline & & & & & & & & & & & & & & \\
\hline & & & & & & & & & & & & & & \\
\hline & & ( & & & & 0 & & 0 & & & & & & \\
\hline & & & & & & & & & & & & & & \\
\hline & & & & & & & & & & & & & & \\
\hline otal & $1.236 \mathrm{E}+00$ & 0.1714 & $5.953 \mathrm{E}+00$ & 0.8252 & $0.000 \mathrm{E}+00$ & 0.0000 & $0.000 \mathrm{E}+00$ & 0.0000 & $0.000 \mathrm{E}+00$ & 0.0000 & $0.000 \mathrm{E}+00$ & 0.0000 & $2.509 \mathrm{E}-02$ & 0.0035 \\
\hline
\end{tabular}

Total Dose Contributions TDOSE (i,p,t) for Individual Radionuclides (i) and Pathways (p)

0 As mrem/yr and Fraction of Total Dose At $t=0.000 \mathrm{E}+00$ years Radio- $\frac{\text { Water }}{\text { Nuclide }}$ Plant

Meat mrem/yr fract. mrem/yr fract. mrem/yr fract. $\overline{A m-241} \overline{0.000 E+00} \overline{0.0000}$

$\begin{array}{lll}C s-137 & 0.000 E+00 & 0.0000 \\ E u-152 & 0.000 E+00 & 0.0000\end{array}$ $\begin{array}{lll}\mathrm{Eu}-152 & 0.000 \mathrm{E}+00 & 0.0000 \\ \mathrm{Pu}-238 & 0.000 \mathrm{E}+00 & 0.0000\end{array}$ $\begin{array}{lll}\mathrm{Pu}-238 & 0.000 \mathrm{E}+00 & 0.0000 \\ \mathrm{Pu}-239 & 0.000 \mathrm{E}+00 & 0.0000\end{array}$ $\begin{array}{lll}\mathrm{Pu}-239 & 0.000 \mathrm{E}+00 & 0.0000 \\ \mathrm{Sr}-90 & 0.000 \mathrm{E}+00 & 0.0000\end{array}$ $\begin{array}{lll}\mathrm{S}-234 & 0.000 \mathrm{E}+00 & 0.0000\end{array}$ $\mathrm{U}-238 \quad 0.000 \mathrm{E}+00 \quad 0.0000$ $============$

\section{$\begin{array}{llll}.000 \mathrm{E}+00 & 0.0000 & 0.000 \mathrm{E}+00 & 0.0000\end{array}$}

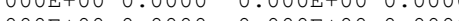
$\begin{array}{ll}0.0000 \mathrm{E}+00 & 0.0000 \\ 0.000 \mathrm{E}+00 & 0.0000\end{array}$ $\overline{0.000 \mathrm{E}+00} \overline{0.0000} \overline{0.000 \mathrm{E}+00} \overline{0.0000}$ $0.000 \mathrm{E}+000.0000 \quad 0.000 \mathrm{E}+00 \quad 0.0000$ $\begin{array}{lllll}0.000 \mathrm{E}+00 & 0.00000 & 0.000 \mathrm{E}+00 & 0.0000\end{array}$ $0.000 \mathrm{E}+00 \quad 0.0000 \quad 0.000 \mathrm{E}+00 \quad 0.0000$ $0.000 \mathrm{t}+00.0 .0000$ $0.000 \mathrm{E}+00 \quad 0.0000$ $\begin{array}{lll}0.000 \mathrm{E}+00 & 0.0000 \\ ========== & 0 \\ 0.000 \mathrm{E}+00 & 0.0000\end{array}$

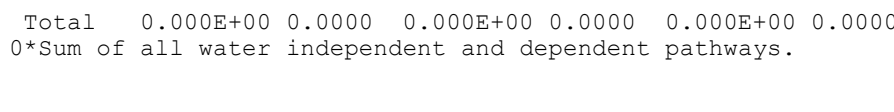

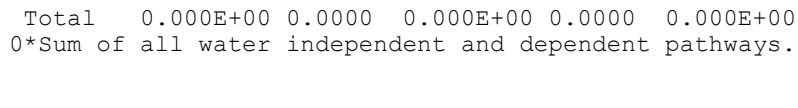
mrem/yr fract.

Milk

mrem/yr fract. $\overline{0.000 \mathrm{E}+00} \overline{0.0000} \overline{7.436 \mathrm{E}-01} \overline{0.1031}$ $0.000 \mathrm{E}+00 \quad 0.0000$ $\begin{array}{llll}0.000 \mathrm{E}+00 & 0.0000 & 0.000 \mathrm{E}+00 & 0.0000\end{array}$ $\begin{array}{llll}0.000 \mathrm{E}+00 & 0.0000 & 0.000 \mathrm{E}+00 & 0.0000\end{array}$ $\begin{array}{lllll}0.000 E+00 & 0.0000 & 7.843 E-01 & 0.1087\end{array}$ $\begin{array}{llll}0.000 \mathrm{E}+00 & 0.0000 & 3.883 \mathrm{E}-01 & 0.0538\end{array}$ $\begin{array}{llll}0.000 \mathrm{E}+00 & 0.0000 & 2.105 \mathrm{E}-01 & 0.0292\end{array}$ $\begin{array}{llll}0.000 \mathrm{E}+00 & 0.0000 \quad 5.043 \mathrm{E}+00 & 0.02990\end{array}$ $\begin{array}{ll}0.000 \mathrm{E}+00 & 0.0000 \quad 8.044 \mathrm{E}-03 \quad 0.0012\end{array}$ $0.000 \mathrm{E}+00 \quad 0.0000 \quad 3.092 \mathrm{E}-03 \quad 0.0004$ $0.000 \mathrm{E}+00 \quad 0.0000 \quad 1.204 \mathrm{E}-02 \quad 0.0017$ $\begin{array}{llll}0.000 \mathrm{E}+00 & 0.0000 & 7.214 \mathrm{E}+00 & 1.0000\end{array}$ 
Total Dose Contributions TDOSE (i,p,t) for Individual Radionuclides (i) and Pathways (p) As mrem/yr and Fraction of Total Dose At $t=1.000 \mathrm{E}+00$ years Water Independent
Radon Plant Meat

\begin{tabular}{|c|c|c|c|c|c|c|c|c|c|c|c|c|c|c|}
\hline \multirow{2}{*}{$\begin{array}{l}\text { Radio- } \\
\text { Nuclide }\end{array}$} & \multicolumn{2}{|c|}{ Ground } & \multicolumn{2}{|c|}{ Inhalation } & \multicolumn{2}{|c|}{ Radon } & \multicolumn{2}{|c|}{ Plant } & \multicolumn{2}{|c|}{ Meat } & \multicolumn{2}{|c|}{ Milk } & \multicolumn{2}{|c|}{ Soil } \\
\hline & $\mathrm{rem} / \mathrm{yr}$ & fract. & mrem/yr & fract. & mrem/yr & fract. & $\mathrm{mrem} / \mathrm{yr}$ & fract. & mrem/yr & fract. & mrem/yr & fract. & $\mathrm{mrem} / \mathrm{yr}$ & fract. \\
\hline-241 & 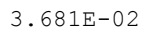 & c & 1 & c & 0 & & 0 & & 0 & & 0 & & 3 & 4 \\
\hline & & & & & & & & & & & & & & \\
\hline & & & & & & & & & & & & & & \\
\hline & & 0.0 & 1 & 0. & & 0.0 & 0 & & & & & & 04 & 0 \\
\hline & & & & & & & & & & & & & & \\
\hline & & & & & & & & & & & & & & \\
\hline & & & & & & & & & & & & & & \\
\hline-2 & & & & & & & & & & & & & & \\
\hline $\mathrm{U}-2$ & & & & & & & & & & & & & & \\
\hline ta & $198 \mathrm{E}+00$ & 1670 & $947 \mathrm{E}+00$ & 0.8295 & $000 \mathrm{E}+00$ & 0000 & $000 \mathrm{E}+00$ & 0000 & $.000 E+00$ & 0000 & $00 \mathrm{E}+00$ & .0000 & $.507 \mathrm{E}-02$ & 1.00 \\
\hline
\end{tabular}
0

Total Dose Contributions TDOSE (i,p,t) for Individual Radionuclides (i) and Pathways (p) As mrem/yr and Fraction of Total Dose At $t=1.000 \mathrm{E}+00$ years

0 Radio-
Nuclide Fish Plant mrem/yr fract. Milk

\section{$\frac{\text { Nuclide }}{\mathrm{Am}-241} \frac{\text { mrem/yr }}{0.000 \mathrm{E}+00} \frac{\text { fract. }}{0.0000}$} mrem/yr fract. mrem/yr fract. mrem/yr fract. mrem/yr fract. $\begin{array}{lll}C s-137 & 0.000 E+00 & 0.0000 \\ \mathrm{E} 11-152 & 0.000 \mathrm{E}+00 & 0.0000 \\ \mathrm{Pu}-238 & 0.000 \mathrm{E}+00 & 0.0000\end{array}$ $0.000 \mathrm{E}+00 \quad 0.0000$ $\begin{array}{lll}\mathrm{Eu}-152 & 0.000 \mathrm{E}+00 & 0.0000 \\ \mathrm{Pu}-238 & 0.000 \mathrm{E}+00 & 0.0000 \\ \mathrm{Pu}-239 & 0.000 \mathrm{E}+00 & 0.0000\end{array}$ $\begin{array}{lll}\mathrm{Pu}-238 & 0.000 \mathrm{E}+00 & 0.0000 \\ \mathrm{Pu}-239 & 0.000 \mathrm{E}+00 & 0.0000\end{array}$ $\begin{array}{lll}\mathrm{Pu} r-90 & 0.000 \mathrm{E}+00 & 0.0000\end{array}$ $0.000 \mathrm{E}+000.0000$ $0.000 \mathrm{E}+00 \quad 0.0000 \quad 0.000 \mathrm{E}+000.0000$ $\begin{array}{lllll}0.000 E+00 & 0.0000 & 0.000 E+00 & 0.0000\end{array}$ $\begin{array}{llll}0.000 \mathrm{E}+00 & 0.0000 & 0.000 \mathrm{E}+00 & 0.0000 \\ 0.000 & 0.000 & 0\end{array}$ $\begin{array}{lllllllll}0.000 \mathrm{E}+00 & 0.0000 & 0.000 \mathrm{E}+00 & 0.0000 & 0.000 \mathrm{E}+00 & 0.0000 & 0.000 \mathrm{E}+00 & 0.0000\end{array}$ 0.000 $\mathrm{U}-234 \quad 0.000 \mathrm{E}+00 \quad 0.0000$ $0.000 \mathrm{E}+000.0000 \quad 0.000 \mathrm{E}+00 \quad 0.0000 \quad 0.000 \mathrm{E}+000.0000 \quad 0.000 \mathrm{E}+000.0000$ $\mathrm{U}-238-0.000 \mathrm{E}+00 \quad 0.0000 \quad 0.000 \mathrm{E}+00 \quad 0.0000 \quad 0.000 \mathrm{E}+00 \quad 0.0000 \quad 0.000 \mathrm{E}+00 \quad 0.0000 \quad 0.000 \mathrm{E}+000.0000$

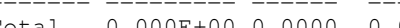
All Pathways* mrem/yr fract. $0 *$ Sum of all water independent and dependent pathways. 
Total Dose Contributions TDOSE (i,p,t) for Individual Radionuclides (i) and Pathways (p) As mrem/yr and Fraction of Total Dose At $t=3.000 \mathrm{E}+00$ years

\begin{tabular}{|c|c|c|c|c|c|c|c|c|c|c|c|c|c|c|}
\hline \multirow{2}{*}{$\begin{array}{l}\text { Radio- } \\
\text { Nuclide }\end{array}$} & \multicolumn{2}{|c|}{ Ground } & \multicolumn{2}{|c|}{ Inhalation } & \multicolumn{2}{|c|}{ Radon } & \multicolumn{2}{|c|}{ Plant } & \multicolumn{2}{|c|}{$\begin{array}{l}\text { Sadon) } \\
\text { Meat }\end{array}$} & \multicolumn{2}{|c|}{ Milk } & \multicolumn{2}{|l|}{ Soil } \\
\hline & $r$ & $\mathrm{f}$ & mrem/yr & fract. & mrem/yr & fract. & mrem/yr & fr & mrem/yr & ra & Irem/yr & $\mathrm{f}$ & $\mathrm{r}$ & $\mathrm{rac}$ \\
\hline & & & & & & & & & & & & & & \\
\hline & & & & & & & & & & & & & & \\
\hline & & & & & & & & & & & & & & \\
\hline & & & & & & & & & & & & & & \\
\hline & & & & & & & & & & & & & & \\
\hline & & 0 & & & & & & & & & & & & \\
\hline & & & & & & & & & & & & & & \\
\hline & & & & & & & & & & & & & & \\
\hline 200 & $345 \mathrm{E}-03$ & 0.0006 & $7.609 \mathrm{E}-03$ & 0.001 & $0.000 \mathrm{E}+00$ & 0.0000 & $0.000 \mathrm{E}+00$ & 0.0000 & $0.000 \mathrm{E}+00$ & 0.0000 & $0.000 \mathrm{E}+00$ & 0.0000 & $8.841 \mathrm{E}-06$ & 0.0000 \\
\hline & $1.125 \mathrm{E}+00$ & 0.1587 & $.936 \mathrm{E}+00$ & 0.8378 & $000 \mathrm{E}+00$ & 0.0000 & $0.000 \mathrm{E}+00$ & 0.0000 & $0.000 \mathrm{E}+00$ & 0.0000 & $0.000 \mathrm{E}+00$ & 0.0000 & $.501 \mathrm{E}-02$ & 0.0035 \\
\hline
\end{tabular}

Total Dose Contributions TDOSE (i,p,t) for Individual Radionuclides (i) and Pathways (p) As mrem/yr and Fraction of Total Dose At $t=3.000 \mathrm{E}+00$ years

0 Radio- $\frac{\text { Water }}{\text { Nuclide }}$ Fish Radon Plant

Meat

mrem/yr fract. $\begin{array}{lll}\text { Am-241 } & 0.000 E+00 & 0.0000\end{array}$ $\begin{array}{lll}\mathrm{CS}-137 & 0.000 \mathrm{E}+00 & 0.0000\end{array}$ $\begin{array}{lll}\mathrm{Eu}-152 & 0.000 \mathrm{E}+00 & 0.0000 \\ \mathrm{Pu}-238 & 0.000 \mathrm{E}+00 & 0.0000\end{array}$ $\begin{array}{lll}\mathrm{Pu}-239 & 0.000 \mathrm{E}+00 & 0.0000\end{array}$ $\begin{array}{lll}\mathrm{Sr}-90 & 0.000 \mathrm{E}+00 & 0.000\end{array}$ $\begin{array}{lll}\mathrm{U}-234 & 0.000 \mathrm{E}+00 & 0.0000 \\ \mathrm{U}-235 & 0.000 \mathrm{E}+00 & 0.0000\end{array}$ mrem/yr fract. $0.000 \mathrm{E}+00 \quad 0.0000$ $0.000 \mathrm{E}+00 \quad 0.0000$ $0.000 \mathrm{E}+00 \quad 0.0000$ $0.000 \mathrm{E}+00 \quad 0.0000$ $0.000 \mathrm{E}+00 \quad 0.0000$ $\begin{array}{lll}0.000 \mathrm{E}+00 & 0.0000 \\ 0.0000 & 0.0000\end{array}$ $0.000 \mathrm{E}+00 \quad 0.0000$ mrem/yr fract. mrem/yr fract. $0.000 \mathrm{E}+00 \quad 0.0000$ $\begin{array}{llllll}0.0000 \mathrm{E}+00 & 0.0000 & 0.000 \mathrm{E}+00 & 0.0000\end{array}$ $0.000 \mathrm{E}+00 \quad 0.0000$ $\begin{array}{ll}0.000 \mathrm{E}+00 & 0.0000 \\ 0.000 \mathrm{E}+00 & 0.000 \\ 0.000 \mathrm{E}+00 & 0.0000\end{array}$ $\begin{array}{llll}0.000 \mathrm{E}+00 & 0.0000 & 0.000 \mathrm{E}+00 & 0.0000\end{array}$ $0.000 \mathrm{E}+00 \quad 0.0000$ $\begin{array}{lll}0.000 E+00 & 0.0000\end{array}$ $\begin{array}{llllllll}\text { Total } & 0.000 \mathrm{E}+00 & 0.0000 & 0.000 \mathrm{E}+00 & 0.0000 & 0.000 \mathrm{E}+00 & 0.0000\end{array}$ $0.000 \mathrm{E}+00 \quad 0.0000$ $0.000 \mathrm{E}+00 \quad 0.00000$ $0.000 \mathrm{E}+00 \quad 0.0000$ * Sum of all water independent and dependent pathways.
Milk

mrem/yr fract.

$0.000 \mathrm{E}+00 \quad 0.0000$ $0.000 \mathrm{E}+00 \quad 0.0000$ $\begin{array}{ll}0.000 \mathrm{E}+00 & 0.0000 \\ 0.000 \mathrm{E}+00 & 0.0000\end{array}$ $0.000 \mathrm{E}+00 \quad 0.0000$ $0.000 \mathrm{E}+000.0000$ $\begin{array}{lll}0.000 \mathrm{E}+00 & 0.0000 \\ 0.000 \mathrm{E}+00 & 0.0000\end{array}$ $0.000 \mathrm{E}+00 \quad 0.0000$ $0.000 \mathrm{E}+00 \quad 0.0000$
All Pathways* mrem/yr fract. $7.321 \mathrm{E}-01 \quad 0.1033$ $7.308 \mathrm{E}-010.1031$ $3.321 \mathrm{E}-010.0469$ $1.196 \mathrm{E}-02 \quad 0.0017$ $7.086 \mathrm{E}+00 \quad 1.0000$ 
Total Dose Contributions TDOSE (i,p,t) for Individual Radionuclides (i) and Pathways (p) As mrem/yr and Fraction of Total Dose At $t=1.000 \mathrm{E}+01$ years Water Independent
Radon Plant Meat

\begin{tabular}{|c|c|c|c|c|c|c|c|c|c|c|c|c|c|c|}
\hline \multirow{2}{*}{$\begin{array}{l}\text { Radio- } \\
\text { Nuclide }\end{array}$} & \multicolumn{2}{|c|}{ Ground } & \multicolumn{2}{|c|}{ Inhalation } & \multicolumn{2}{|c|}{ idon } & \multicolumn{2}{|c|}{ Plant } & \multicolumn{2}{|c|}{ Meat } & \multicolumn{2}{|c|}{ Milk } & \multicolumn{2}{|c|}{ Soil } \\
\hline & $\mathrm{rem} / \mathrm{yr}$ & act. & $\mathrm{m} / \mathrm{yr}$ & ract. & mrem/yr & fract & mrem/yr & fract. & mrem/yr & fract. & hrem/yr & fract & mrem/yr & frac \\
\hline & & & & & & & & & & & & & & \\
\hline & & & & & & & & & & & & & & \\
\hline & & & & & & & & & & & & & & \\
\hline & & & & & & & & & & & & & & \\
\hline & & & & & & & & & & & & & & \\
\hline & & & & & & & & & & & & & & \\
\hline & & & & & & & & & & & & & & \\
\hline & 2 & ( & & 0 & & 0 & & 0 & & & & & & \\
\hline & & & & & & & & & & & & & & \\
\hline & & & & & & & & & & & & & & \\
\hline otal & $9.077 \mathrm{E}-01$ & 0.1329 & $5.897 \mathrm{E}+00$ & 0.8635 & $0.000 \mathrm{E}+00$ & 0.0000 & $0.000 \mathrm{E}+00$ & 0.0000 & $0.000 \mathrm{E}+00$ & 0.0000 & $0.000 \mathrm{E}+00$ & 0.0000 & $2.483 \mathrm{E}-02$ & 0.0036 \\
\hline
\end{tabular}

Total Dose Contributions TDOSE (i,p,t) for Individual Radionuclides (i) and Pathways (p) As mrem/yr and Fraction of Total Dose At $t=1.000 \mathrm{E}+01$ years

0 Radio-
Nuclide Plant

Meat mrem/yr fract. $\mathrm{Am}-241 \quad 0.000 \mathrm{E}+00 \quad 0.0000$ $\begin{array}{lll}\mathrm{Cs}-137 & 0.000 \mathrm{E}+00 & 0.0000 \\ \mathrm{Eu}-152 & 0.000 \mathrm{E}+00 & 0.0000 \\ \mathrm{Pu}-238 & 0.000 \mathrm{O}+00 & 0.0000\end{array}$ $\begin{array}{lll}\mathrm{Pu}-238 & 0.000 \mathrm{E}+00 & 0.0000\end{array}$ $\begin{array}{lll}\mathrm{Pu}-239 & 0.000 \mathrm{E}+00 & 0.0000\end{array}$ $\begin{array}{lll}\mathrm{Sr}-90 & 0.000 \mathrm{E}+00 & 0.0000 \\ \mathrm{U}-234 & 0.000 \mathrm{E}+00 & 0.0000\end{array}$ $\begin{array}{lll}\mathrm{U}-235 & 0.000 \mathrm{E}+00 & 0.0000\end{array}$ mrem/yr fract. mrem/yr fract. mrem/yr fract. $0.000 \mathrm{E}+00 \quad 0.0000$ $\begin{array}{llll}0.000 \mathrm{E}+00 & 0.0000 & 0.000 \mathrm{E}+00 & 0.0000 \\ 0.000 \mathrm{E}+00 & 0.0000 & 0.000 \mathrm{E}+00 & 0.0000\end{array}$ $\begin{array}{lllll}0.000 \mathrm{E}+00 & 0.0000 & 0.000 \mathrm{E}+00 & 0.0000\end{array}$ $\begin{array}{lll}.000 \mathrm{E}+00 & 0.0000 \\ 0.000 & 0.0000\end{array}$

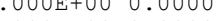

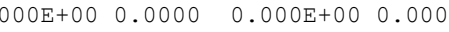
$\begin{array}{ll}0.000 \mathrm{E}+00 & 0.00000 \\ 0.000 & 0\end{array}$ $0.000 \mathrm{E}+00 \quad 0.0000$ $\begin{array}{ll}0.000 \mathrm{E}+00 & 0.0000 \\ 0.000 \mathrm{E}+00 & 0.00000 \\ 0.000 \mathrm{E}+00 & 0.0000\end{array}$ $\begin{array}{lll}\mathrm{J}-238 & 0.000 \mathrm{E}+00 & 0.0000\end{array}$ E $0.000 \mathrm{E}+00 \quad 0.0000$

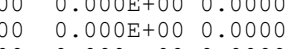
Total $0.000 \mathrm{E}+00 \quad 0.0000$ $000.0000 \quad 0.000 \mathrm{E}+00 \quad 0.0000$ $000 \mathrm{E}+00 \quad 0.0000$ $0.000 \mathrm{E}+00 \quad 0.0000$ $0.000 \mathrm{E}+00 \quad 0.0000$ $0.000 \mathrm{E}+00 \quad 0.0000$ $0.000 \mathrm{E}+00 \quad 0.0000$ $\begin{array}{ll}0.000 \mathrm{E}+00 & 0.0000 \\ .000 \mathrm{E}+00 & 0.0000\end{array}$ $0.000 \mathrm{E}+00 \quad 0.0000$ $0.000 \mathrm{E}+00 \quad 0.0000$ Milk mrem/yr fract. $0.000 \mathrm{E}+00 \quad 0.0000$ $0.000 \mathrm{E}+00 \quad 0.0000$ $0.000 \mathrm{E}+00 \quad 0.0000$ $0.000 \mathrm{E}+00 \quad 0.0000$ $0.000 \mathrm{E}+00 \quad 0.000$ $\begin{array}{lll}0.000 \mathrm{E}+00 & 0.0000 \\ 0.000 \mathrm{E}+00 & 0.0000\end{array}$ $0.000 \mathrm{E}+00 \quad 0.0000$
$0.000 \mathrm{E}+00$ $.000 \mathrm{E}+00 \quad 0.0000$ $0.000 \mathrm{E}+00 \quad 0.0000$ All Pathways* mrem/yr fract. $\begin{array}{ll}7.059 \mathrm{E}-01 & 0.1034\end{array}$ $\begin{array}{lll}6.198 \mathrm{E}-01 & 0.0908\end{array}$ $2.306 \mathrm{E}-01 \quad 0.0338$ $\begin{array}{ll}8.762 \mathrm{E}-03 & 0.0013\end{array}$ $\begin{array}{lll}3.179 \mathrm{E}-02 & 0.0017\end{array}$ $6.830 \mathrm{E}+00 \quad 1.0000$ 
Total Dose Contributions TDOSE (i,p,t) for Individual Radionuclides (i) and Pathways (p) As mrem/yr and Fraction of Total Dose At $t=3.000 \mathrm{E}+01$ years Water Independent
Radon $\frac{\text { Plant }}{\text { mrem/yr fract. }} \frac{\text { Meat }}{\text { mrem/yr fract. }}$

\begin{tabular}{|c|c|c|}
\hline \multirow{2}{*}{$\begin{array}{l}\text { Radio- } \\
\text { Nuclide }\end{array}$} & \multicolumn{2}{|c|}{ Ground } \\
\hline & $\mathrm{mrem} / \mathrm{yr}$ & fract. \\
\hline Am-241 & $3.165 \mathrm{E}-02$ & 0.0050 \\
\hline Cs-137 & $3.871 \mathrm{E}-01$ & 0.0611 \\
\hline $\mathrm{Eu}-152$ & $8.138 \mathrm{E}-02$ & 0.0128 \\
\hline $\mathrm{Pu}-238$ & $4.296 \mathrm{E}-05$ & 0.0000 \\
\hline Pu-239 & $1.840 \mathrm{E}-03$ & 0.0003 \\
\hline $\operatorname{Sr}-90$ & $7.726 \mathrm{E}-03$ & 0.0012 \\
\hline $\mathrm{U}-234$ & $1.451 \mathrm{E}-05$ & 0.0000 \\
\hline $\begin{array}{l}\mathrm{U}-235 \\
\mathrm{U}-238\end{array}$ & $\begin{array}{l}2.162 \mathrm{E}-03 \\
4.109 \mathrm{E}-03\end{array}$ & $\begin{array}{l}0.0003 \\
0.0006\end{array}$ \\
\hline & & \\
\hline Total & $5.161 \mathrm{E}-01$ & 0.0815 \\
\hline
\end{tabular}
Inhalation Radon Milk Soil mrem/yr fract. mrem/yr fract. $\overline{0.000 \mathrm{E}+00} \overline{0.0000} \overline{0.000 \mathrm{E}+00} \overline{0.0000}$

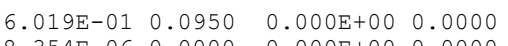
$\begin{array}{llllllll}5.350 \mathrm{E}-06 & 0.0000 & 0.000 \mathrm{E}+00 & 0.0000 & 0.000 \mathrm{E}+00 & 0.0000 & 0.000 \mathrm{E}+00 & 0.0000\end{array}$ $\begin{array}{lllllllll}1.652 \mathrm{E}-01 & 0.0261 & 0.000 \mathrm{E}+00 & 0.0000 & 0.000 \mathrm{E}+00 & 0.0000 & 0.000 \mathrm{E}+00 & 0.0000\end{array}$ $\begin{array}{lllllllll}5.010 \mathrm{E}+00 & 0.7909 & 0.000 \mathrm{E}+00 & 0.0000 & 0.000 \mathrm{E}+00 & 0.0000 & 0.000 \mathrm{E}+00 & 0.0000\end{array}$ $\begin{array}{llllllll}9.555 \mathrm{E}-04 & 0.0002 & 0.000 \mathrm{E}+00 & 0.0000 & 0.000 \mathrm{E}+00 & 0.0000 & 0.000 \mathrm{E}+00 & 0.0000\end{array}$ $\begin{array}{llllllll}8.387 \mathrm{E}-03 & 0.0013 & 0.000 \mathrm{E}+00 & 0.0000 & 0.000 \mathrm{E}+00 & 0.0000 & 0.000 \mathrm{E}+00 & 0.0000\end{array}$ mrem/yr fract. $0.000 \mathrm{E}+00 \quad 0.0000$ $0.000 \mathrm{E}+000.0000$ $0.000 \mathrm{E}+00 \quad 0.0000$ $0.000 \mathrm{E}+00 \quad 0.0000$ $0.000 \mathrm{E}+00 \quad 0.0000$ $0.000 \mathrm{E}+00 \quad 0.0000$ mrem/yr fract. $\begin{array}{ll}2.515 \mathrm{E}-03 & 0.0004\end{array}$ $8.907 \mathrm{E}-08 \quad 0.0000$ $\begin{array}{ll}6.873 \mathrm{E}-04 & 0.0001\end{array}$ $2.107 \mathrm{E}-02 \quad 0.0033$ $5.688 \mathrm{E}-05 \quad 0.0000$ $9.164 \mathrm{E}-06 \quad 0.0000$ $\begin{array}{llllllll}7.583 \mathrm{E}-04 & 0.0001 & 0.000 \mathrm{E}+00 & 0.0000 & 0.000 \mathrm{E}+00 & 0.0000 & 0.000 \mathrm{E}+00 & 0.0000 \\ 7.197 \mathrm{E}-03 & 0.0011 & 0.000 \mathrm{E}+00 & 0.0000 & 0.000 \mathrm{E}+00 & 0.0000 & 0.000 \mathrm{E}+00 & 0.0000\end{array}$

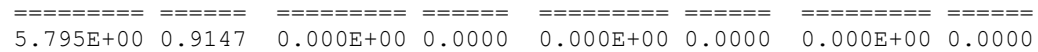
$\begin{array}{llll}0.000 \mathrm{E}+00 & 0.0000 & 8.548 \mathrm{E}-07 & 0.0000 \\ 0.000 \mathrm{E}+00 & 0.0000 & 8.362 \mathrm{E}-06 & 0.0000\end{array}$ $\begin{array}{llll}0.000 \mathrm{E}+00 & 0.0000 & 2.436 \mathrm{E}-02 & 0.0038\end{array}$ Total Dose Contributions TDOSE $(i, p, t)$ for Individual Radionuclides (i) and Pathways ( $p$ )

0
0 As mrem/yr and Fraction of Total Dose At $t=3.000 \mathrm{E}+01$ years Radon $\quad$ Plant Fish

Milk

Radio- $\overline{\text { Nuclide }}$ mrem/yr fract.

mrem/yr fract. $\begin{array}{lll}\mathrm{Am}-241 & 0.000 \mathrm{E}+00 & 0.0000 \\ \mathrm{Cs}-137 & 0.000 \mathrm{E}+00 & 0.0000 \\ \mathrm{E}-152 & 0.000 \mathrm{O}+00 & 0.0000\end{array}$

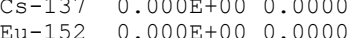
$\begin{array}{lll}\mathrm{Pu}-238 & 0.000 \mathrm{E}+00 & 0.0000\end{array}$ $\begin{array}{ll}\mathrm{Pu}-239 & 0.000 \mathrm{E}+00 \quad 0.0000\end{array}$ $\begin{array}{lll}\mathrm{Sr}-90 & .000 \mathrm{E}+00 & 0.0000 \\ \mathrm{U}-234 & 0.000 \mathrm{E}+00 & 0.0000\end{array}$ $\mathrm{U}-235 \quad 0.000 \mathrm{E}+00 \quad 0.0000$ $\mathrm{U}-238 \quad 0.000 \mathrm{E}+00 \quad 0.0000$ $=============$ mrem/yr fract. mrem/yr fract. mrem/yr fract. $0.000 \mathrm{E}+00 \quad 0.0000$ $\begin{array}{lllll}0.000 \mathrm{E}+00 & 0.0000 & 0.000 \mathrm{E}+00 & 0.0000 \\ 0.000 \mathrm{E}+00 & 0.0000 & 0.000 \mathrm{E}+00 & 0.0000\end{array}$ $\begin{array}{lll}.000 \mathrm{E}+00 & 0.0000 \\ 0.000 \mathrm{E}+00 & 0.0000 & 0\end{array}$ $0.000 \mathrm{E}+000.0000$ $0.000 \mathrm{E}+00 \quad 0.0000$ $0.000 \mathrm{E}+00 \quad 0.0000 \quad 0.000 \mathrm{E}+00 \quad 0.0000$

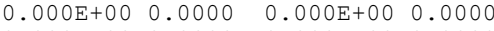
$0.000 \mathrm{E}+00 \quad 0.0000 \quad 0.000 \mathrm{E}+00 \quad 0.0000$ $\begin{array}{llll}0.000 \mathrm{E}+00 & 0.0000 & 0.000 \mathrm{E}+00 & 0.0000\end{array}$

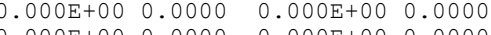
$\begin{array}{llll}0.000 \mathrm{E}+00 & 0.0000 & 0.000 \mathrm{E}+00 & 0.0000 \\ 0.000 \mathrm{E}+00 & 0.0000 & 0.0000 \mathrm{E}+00 & 0.0000\end{array}$ $0.000 \mathrm{E}+00.0000$ $0.000 \mathrm{E}+00$ 0.0000 $0.000 \mathrm{E}+000.0000$ $0.000 \mathrm{E}+00 \quad 0.0000$ $0.000 \mathrm{E}+00 \quad 0.0000$ $0.000 \mathrm{E}+00 \quad 0.0000$ $0.000 \mathrm{E}+00 \quad 0.0000$ $+00 \quad 0.0000$ $0.000 \mathrm{E}+00 \quad 0.0000$ mrem/yr fract. $0.000 \mathrm{E}+00 \quad 0.0000$ $0.000 \mathrm{E}+00 \quad 0.0000$ $0.000 \mathrm{E}+00 \quad 0.0000$ $0.000 \mathrm{E}+00 \quad 0.0000$ $\begin{array}{lll}0.000 \mathrm{E}+00 & 0.0000 \\ 0.000 \mathrm{E}+00 & 0.0000\end{array}$

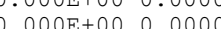
$0.000 \mathrm{E}+00 \quad 0.0000$ $0.000 \mathrm{E}+00 \quad 0.0000$
All Pathways* mrem/yr fract. $\begin{array}{lll}6.360 \mathrm{E}-01 & 0.1004\end{array}$ $\begin{array}{lll}3.871 \mathrm{E}-01 & 0.0611\end{array}$ $\begin{array}{ll}8.138 \mathrm{E}-02 & 0.0128\end{array}$ $\begin{array}{ll}1.659 \mathrm{E}-01 & 0.0262 \\ 5.033 \mathrm{E}+00 & 0.7945\end{array}$ $8.033 \mathrm{E}+000.7945$ 030.0013 $\begin{array}{ll}1.131 \mathrm{E}-02 & 0.0018\end{array}$ $6.335 \mathrm{E}+00 \quad 1.0000$

$0 *$ Sum of all water independent and dependent pathways. 
Total Dose Contributions TDOSE (i,p,t) for Individual Radionuclides (i) and Pathways (p) As mrem/yr and Fraction of Total Dose At $t=1.000 \mathrm{E}+02$ years

\begin{tabular}{|c|c|c|c|c|c|c|c|c|c|c|c|c|c|c|}
\hline \multirow{2}{*}{$\begin{array}{l}\text { Radio- } \\
\text { Nuclide }\end{array}$} & \multicolumn{2}{|c|}{ Ground } & \multicolumn{2}{|c|}{ Inhalation } & \multicolumn{2}{|c|}{$\begin{array}{l}\text { Dender } \\
\text { Rador }\end{array}$} & \multicolumn{2}{|c|}{$\begin{array}{l}\text { Inhal } \\
\text { Plan }\end{array}$} & \multicolumn{2}{|c|}{$\begin{array}{l}\text { S radd } \\
\text { Meat }\end{array}$} & \multicolumn{2}{|c|}{ Milk } & \multicolumn{2}{|l|}{ Soil } \\
\hline & r & f & 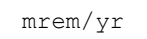 & 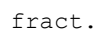 & HII CIII/ Y & Fere & (11il/ yt & Hace. & HIILAI/ Y & Hace. & nemitio & fract. & $\mathrm{cem} / \mathrm{yr}$ & fract. \\
\hline & & & & & & & & & & & & & & \\
\hline & & & & & & & & & & & & & & \\
\hline & & & & & & & & & & & & & & \\
\hline & & & & & & & & & & & & & & \\
\hline & & & & & & & & & & & & & & \\
\hline & & & & & & & & & & & & & & \\
\hline & & & & & & & & & & & & & & \\
\hline & & & & & & & & & & & & & & \\
\hline-23 & $3.555 E-03$ & 0.00 & $E-03$ & 0.00 & 0 & 0.00 & 0 & 0 & 0 & 0. & +00 & 0 & -06 & 000 \\
\hline tal & $\begin{array}{l}======= \\
1.070 \mathrm{E}-01\end{array}$ & & $=00$ & & $\begin{array}{l}==== \\
0= \pm 0\end{array}$ & 2000 & $\begin{array}{l}====- \\
==5 \mathrm{~F}\end{array}$ & 0000 & $000=0+0$ & 0000 & $=======$ & 00000 & $\begin{array}{l}======= \\
314 \mathrm{~F}-02\end{array}$ & \\
\hline & $1.070 \mathrm{E}-01$ & 190 & +00 & 9769 & $E+00$ & 0000 & $\mathrm{E}+00$ & 0.0000 & $.000 \mathrm{E}+00$ & .0000 & $.000 \mathrm{E}+00$ & 0.0000 & $.314 \mathrm{E}-02$ & \\
\hline
\end{tabular}

Total Dose Contributions TDOSE (i,p,t) for Individual Radionuclides (i) and Pathways (p) As mrem/yr and Fraction of Total Dose At $t=1.000 \mathrm{E}+02$ years

0 $\begin{array}{ll}\text { Radio- } & \text { Water } \\ \text { Nuclide } & \text { mrem/yr fract. }\end{array}$ Fish Radon Plant mrem/yr fract. $\frac{\text { Milk }}{\text { mrem/yr fract. }}$ $\frac{1}{\mathrm{Am}-241} \frac{\mathrm{mrem} / \mathrm{yr}}{0.000 \mathrm{E}+00} \frac{\text { ract. }}{0.0000}$ mrem/yr fract. mrem/yr fract. mrem/yr fract. mrem/yr fract. $\begin{array}{lllllllllll}\mathrm{Am}-241 & 0.000 \mathrm{E}+00 & 0.0000 & 0.000 \mathrm{E}+00 & 0.0000 & 0.000 \mathrm{E}+00 & 0.0000 & 0.000 \mathrm{E}+00 & 0.0000 & 0.000 \mathrm{E}+00 & 0.0000\end{array}$

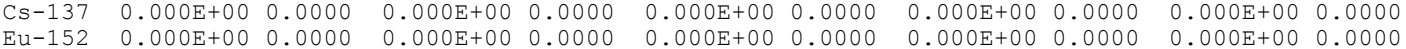

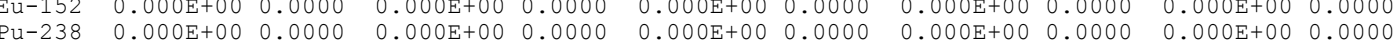
$\begin{array}{lllllllllll}\mathrm{Pu}-239 & 0.000 \mathrm{E}+00 & 0.0000 & 0.000 \mathrm{E}+00 & 0.0000 & 0.000 \mathrm{E}+00 & 0.0000 & 0.000 \mathrm{E}+00 & 0.0000 & 0.000 \mathrm{E}+00 & 0.0000\end{array}$

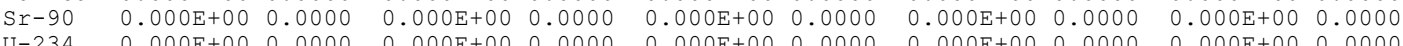

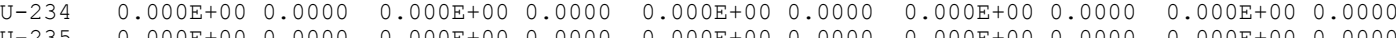

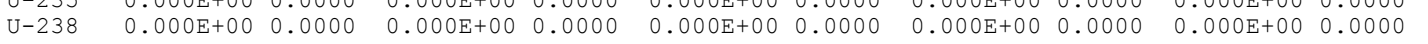
All Pathways* mrem/yr fract. $0.000 \mathrm{tan} 0.0000$ $0.000 \mathrm{E}+00 \quad 0.0000$ $0.000 \mathrm{E}+00 \quad 0.000$ $0.000 \mathrm{E}+00 \quad 0.0000$ $0.000 \mathrm{E}+00 \quad 0.0000$ $0.000 \mathrm{E}+00 \quad 0.000$

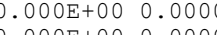
$0.000 \mathrm{E}+00 \quad 0.0000$ $0.000 \mathrm{E}+00 \quad 0.0000$ $0 *$ Sum of all water independent and dependent pathways. 
Total Dose Contributions TDOSE (i,p,t) for Individual Radionuclides (i) and Pathways (p) As mrem/yr and Fraction of Total Dose At $t=3.000 \mathrm{E}+02$ years Water Independent
Radon Plant
Meat

\begin{tabular}{|c|c|c|c|c|c|c|c|c|c|c|c|c|c|c|}
\hline \multirow{2}{*}{$\begin{array}{l}\text { Radio- } \\
\text { Nuclide }\end{array}$} & \multicolumn{2}{|c|}{ Ground } & \multicolumn{2}{|c|}{ Inhalation } & \multicolumn{2}{|c|}{ Radon } & \multicolumn{2}{|c|}{ Plant } & \multicolumn{2}{|c|}{ Meat } & \multicolumn{2}{|c|}{ Milk } & \multicolumn{2}{|c|}{ Soil } \\
\hline & & $\mathrm{f}$ & $\mathrm{em} /$ & $\mathrm{cac}$ & mrem & frad & re & frac & r & frac & re & frac & r & frac \\
\hline & & & & & & & & & & & & & & 1 \\
\hline & & & & & & & & & & & & & & \\
\hline & & & & & & & & & & & & & & \\
\hline & & & & & & & & & & & & & & \\
\hline & & & & & & & & & & & & & & \\
\hline & & c & & & & & & & & & & & & \\
\hline & & & & & & & & & & & & & & \\
\hline & & & & & & & & & & & & & & \\
\hline-2 & 2.35 & 0.00 & 3 & 0.00 & 0 & 0 . & 0 & 0.0 & 0. & 0 & 00 & & 4. & 0 \\
\hline ec & -02 & 1 & 00 & 0 & 00 & 00 & 00 & 00 & $0 E+00$ & 0.0000 & $0 E+00$ & 000 & $.141 \mathrm{E}-02$ & \\
\hline
\end{tabular}
0

Total Dose Contributions TDOSE (i,p,t) for Individual Radionuclides (i) and Pathways (p)

0 As mrem/yr and Fraction of Total Dose At $t=3.000 \mathrm{E}+02$ years Radio- $\frac{\text { Water }}{\text { Nuclide }}$ Radon Plant mrem/yr fract. Milk

\section{$\frac{\text { Nuclide }}{\mathrm{Am}-241} \frac{\text { mrem/yr }}{0.000 \mathrm{E}+00} \frac{\text { fract. }}{0.0000}$} mrem/yr fract. mrem/yr fract. mrem/yr fract. mrem/yr fract. $\begin{array}{lll}C s-137 & 0.000 E+00 & 0.0000 \\ \mathrm{E} 11-152 & 0.000 \mathrm{E}+00 & 0.0000 \\ \mathrm{Pu}-238 & 0.000 \mathrm{E}+00 & 0.0000\end{array}$ $0.000 \mathrm{E}+00 \quad 0.0000$ $\begin{array}{lll}\mathrm{Eu}-152 & 0.000 \mathrm{E}+00 & 0.0000 \\ \mathrm{Pu}-238 & 0.000 \mathrm{E}+00 & 0.0000 \\ \mathrm{Pu}-239 & 0.000 \mathrm{E}+00 & 0.0000\end{array}$ $\begin{array}{lll}\mathrm{Pu}-238 & 0.000 \mathrm{E}+00 & 0.0000 \\ \mathrm{Pu}-239 & 0.000 \mathrm{E}+00 & 0.0000\end{array}$ $\begin{array}{lll}\mathrm{Pu}-\mathrm{S}-90 & 0.000 \mathrm{E}+00 & 0.0000\end{array}$ $0.000 \mathrm{E}+000.0000$ $0.000 \mathrm{E}+00 \quad 0.0000 \quad 0.000 \mathrm{E}+00 \quad 0.0000$ $\begin{array}{llll}0.000 E+00 & 0.0000 & 0.000 E+00 & 0.0000\end{array}$ $\begin{array}{llll}0.000 \mathrm{E}+00 & 0.0000 & 0.000 \mathrm{E}+00 & 0.0000 \\ 0.000 & 0.000 & 0.000\end{array}$ $\begin{array}{llll}0.000 \mathrm{E}+00 & 0.0000 & 0.000 \mathrm{E}+00 & 0.0000 \\ 0.000 \mathrm{E}+00 & 0.0000 & 0.000 \mathrm{E}+00 & 0.0000 \\ 0.000 \mathrm{E}+00 & 0.0000 & 0.000 \mathrm{a}+00 & 0.0000\end{array}$

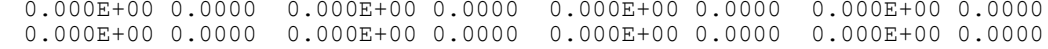

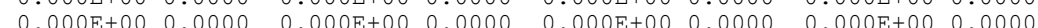

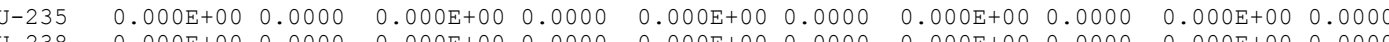
$0.000 \mathrm{E}+00 \quad 0.0000 \quad 0.000$ All Pathways* mrem/yr fract.

U.0.0.0.000 $=============$ $0.000 \mathrm{E}+00 \quad 0.0000$ $0.000 \mathrm{E}+00 \quad 0.000$ $\begin{array}{lll}0.000 \mathrm{E}+00 & 0.0000 \\ 0.000 \mathrm{E}+00 & 0.0000 \\ 0.000 \mathrm{E}+00 & 0.0000\end{array}$ $\begin{array}{ll}0.000 \mathrm{E}+00 & 0.0000 \\ 0.000 \mathrm{E}+00 & 0.0000\end{array}$ $0.000 \mathrm{E}+000.0000$ $0.000 \mathrm{E}+00 \quad 0.0000$ $0.000 \mathrm{E}+00 \quad 0.0000$ $============$ $\begin{array}{lll}1.559 \mathrm{E}-01 & 0.0304\end{array}$ $6.737 \mathrm{E}-04 \quad 0.0001$ $\begin{array}{ll}6.350 \mathrm{E}-08 & 0.0000\end{array}$ $\begin{array}{lll}1.948 \mathrm{E}-02 & 0.0038\end{array}$ $\begin{array}{lll}4.945 \mathrm{E}+00 & 0.9631 \\ 3.877 \mathrm{E}-06 & 0.0000\end{array}$ $3.877 \mathrm{E}-06 \quad 0.0000$ $\begin{array}{ll}4.889 \mathrm{E}-03 & 0.0010 \\ 1.852 \mathrm{E}-03 & 0.0004\end{array}$ $6.476 \mathrm{E}-03 \quad 0.0013$ $\begin{array}{ll}======= & ====== \\ 5.135 \mathrm{E}+00 & 1.0000\end{array}$ $0 *$ Sum of all water independent and dependent pathways. 
Total Dose Contributions TDOSE (i,p,t) for Individual Radionuclides (i) and Pathways (p) As mrem/yr and Fraction of Total Dose At $t=1.000 \mathrm{E}+03$ years Water Independent
Radon Plant Meat

\begin{tabular}{|c|c|c|c|c|c|c|c|c|c|c|c|c|c|c|}
\hline \multirow{2}{*}{$\begin{array}{l}\text { Radio- } \\
\text { Nuclide }\end{array}$} & \multicolumn{2}{|c|}{ Ground } & \multicolumn{2}{|c|}{ Inhalation } & \multicolumn{2}{|c|}{ Radon } & \multicolumn{2}{|c|}{$\mathrm{Plant}$} & \multicolumn{2}{|c|}{ Meat } & \multicolumn{2}{|c|}{ Milk } & \multicolumn{2}{|c|}{ Soil } \\
\hline & mrem/yr & fract. & mrem/yr & fract. & mrem/yr & fract. & $\mathrm{mrem} / \mathrm{yr}$ & fract. & $\mathrm{mrem} / \mathrm{yr}$ & fract. & mrem/yr & fract. & mrem/yr & IIdact. \\
\hline & & & & & & & & & & & & & & \\
\hline & & & & & & & & & & & & & & \\
\hline & & & & & & & & & & & & & & \\
\hline & & & & & & & & & & & & & & \\
\hline & & & & & & & & & & & & & & \\
\hline & & & & & & & & & & & & & & \\
\hline & & & & & & & & & & & & & & \\
\hline & & & & & & & & & & & & & & \\
\hline & & & & & & & & & & & & & & \\
\hline & -03 & 106 & +00 & 952 & 00 & 000 & +00 & 00 & 00 & 00 & 00 & 000 & -02 & \\
\hline
\end{tabular}

Total Dose Contributions TDOSE (i,p,t) for Individual Radionuclides (i) and Pathways (p) As mrem/yr and Fraction of Total Dose At $t=1.000 \mathrm{E}+03$ years

0 $\begin{array}{ll}\text { Radio- } & \text { Water } \\ \text { Nuclide } & \text { mrem/yr fract. }\end{array}$ Fish Plant

Meat mrem/yr fract. mrem/yr fract. mrem/yr fract. $0.000 \mathrm{E}+00 \quad 0.0000$ $0.000 \mathrm{E}+00 \quad 0.0000$ $\begin{array}{lll}A m-241 & 0.000 E+00 \quad 0.0000\end{array}$ $\begin{array}{lll}C s-137 & 0.000 E+00 & 0.0000 \\ \text { Eu-152 } & 0.000 E+00 & 0.0000\end{array}$ $\begin{array}{lll}\text { Eu-152 } & 0.000 E+00 & 0.0000 \\ \text { Pu-238 } & 0.000 E+00 & 0.0000\end{array}$ $\begin{array}{lll}\text { Pu-239 } & 0.000 \mathrm{E}+00 & 0.0000\end{array}$ $\begin{array}{lll}\mathrm{Sr}-90 & 0.000 \mathrm{E}+00 & 0.0000 \\ \mathrm{U}-234 & 0.000 \mathrm{E}+00 & 0.0000\end{array}$ $\begin{array}{lll}\mathrm{U}-234 & 0.000 \mathrm{E}+00 & 0.0000 \\ \mathrm{U}-235 & 0.000 \mathrm{E}+00 & 0.0000\end{array}$ $0.000 \mathrm{E}+00 \quad 0.0000$ $0.000 \mathrm{E}+00 \quad 0.0000$ $\begin{array}{llll}0.000 \mathrm{E}+00 & 0.0000 & 0.000 \mathrm{E}+00 & 0.0000\end{array}$ $\begin{array}{lllll}0.000 \mathrm{E}+00 & 0.0000 & 0.000 \mathrm{E}+00 & 0.0000\end{array}$ $0.000 \mathrm{E}+00 \quad 0.0000$ $\begin{array}{llll}0.000 \mathrm{E}+00 & 0.0000 & 0.000 \mathrm{E}+00 \quad 0.0000\end{array}$

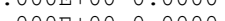
$\begin{array}{lll}0.000 \mathrm{E}+00 & 0.0000 \\ 000 & 0.000 \mathrm{E}+00 & 0.0000\end{array}$ $0.000 \mathrm{E}+00 \quad 0.0000$ $\mathrm{J}-238 \quad 0.000 \mathrm{E}+00 \quad 0.0000$ $0.000 \mathrm{E}+00 \quad 0.0000$ $0.000 \mathrm{E}+00 \quad 0.0000$

$0.000 \mathrm{E}+000.0000$ 0.000E+00 $0.00000 .000 \mathrm{E}+000.0000$ $0.000 \mathrm{E}+00 \quad 0.0000$ $0 *$ Sum of all water independent and dependent pathways.
Milk

mrem/yr fract. $0.000 \mathrm{E}+00 \quad 0.0000$ $0.000 \mathrm{E}+00 \quad 0.0000$ $0.000 \mathrm{E}+00 \quad 0.0000$ $0.000 \mathrm{E}+00 \quad 0.0000$ $0.000 \mathrm{E}+00 \quad 0.0000$ $0.000 \mathrm{E}+000.000$ $0.000 \mathrm{E}+000.0000$ $0.000 \mathrm{E}+00 \quad 0.0000$ $0.000 \mathrm{E}+00 \quad 0.0000$ All Pathways* mrem/yr fract. $\begin{array}{ll}4.149 \mathrm{E}-03 & 0.0009\end{array}$ $\begin{array}{lll}4.722 \mathrm{E}-11 & 0.0000\end{array}$ $\begin{array}{lll}1.013 \mathrm{E}-15 & 0.0000\end{array}$ $\begin{array}{ll}7.866 \mathrm{E}-15 & 0.0000\end{array}$ $\begin{array}{ll}.824 E-03 & 0.0003 \\ 1.523 E-04 & 0.0001\end{array}$ $1.524 \mathrm{E}-03 \quad 0.0003$ $4.733 \mathrm{E}+001.0000$ 
1RESRAD, Version 6.21

$\mathrm{T}^{1 / 2}$ Limit $=0.5$ year

Summary : CAU 224250 day/yr worker scenario $1 \mathrm{~m}$ hotspot

02/22/2005 17:50 Page 22

File : cau224 $2501 \mathrm{~m}$ parcel.RAD

Dose/Source Ratios Summed Over All Pathways

Parent and Progeny Principal Radionuclide Contributions Indicated

(i) (j) Fraction* $t=0.000 \mathrm{E}+00 \quad 1.000 \mathrm{E}+00 \quad 3.000 \mathrm{E}+00 \quad 1.000 \mathrm{E}+01 \quad 3.000 \mathrm{E}+01 \quad 1.000 \mathrm{E}+02 \quad 3.000 \mathrm{E}+02 \quad 1.000 \mathrm{E}+03$

$\overline{\mathrm{Am}-241} \overline{\mathrm{Am}-241} \overline{1.000 \mathrm{E}+00} \quad \overline{2.512 \mathrm{E}-02} \overline{2.499 \mathrm{E}-02} \overline{2.473 \mathrm{E}-02} \overline{2.385 \mathrm{E}-02} \overline{2.149 \mathrm{E}-02} \overline{1.492 \mathrm{E}-02} \overline{5.265 \mathrm{E}-03} \overline{1.374 \mathrm{E}-04}$ $\begin{array}{lllllllllll}\mathrm{Am}-241 & \mathrm{~Np}-237 & 1.000 \mathrm{E}+00 & 9.118 \mathrm{E}-09 & 2.729 \mathrm{E}-08 & 6.333 \mathrm{E}-08 & 1.864 \mathrm{E}-07 & 5.129 \mathrm{E}-07 & 1.408 \mathrm{E}-06 & 2.631 \mathrm{E}-06 & 2.774 \mathrm{E}-0\end{array}$

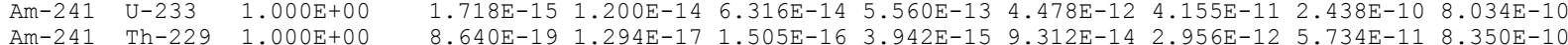

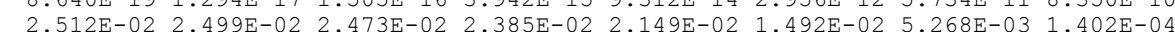

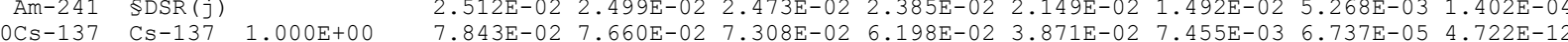

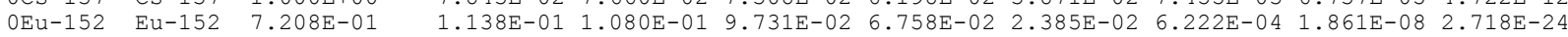

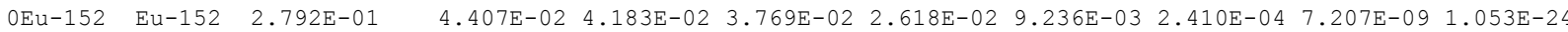

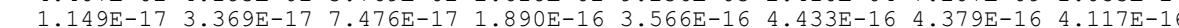
Pu-238 $\mathrm{Pu}-238 \quad 1.000 \mathrm{E}+00$ $\mathrm{Pu}-238 \mathrm{U}-234 \quad 1.000 \mathrm{E}+00$ $\mathrm{Pu}-238 \mathrm{Th}-230 \quad 1.000 \mathrm{E}+00$ $\mathrm{Pu}-238 \mathrm{Ra}-226 \quad 1.000 \mathrm{E}+00$ Pu-238 $\operatorname{Pu}-238$ SDSR ( $j)$

$\mathrm{Pu}-239-1.000 \mathrm{E}+00$ $\mathrm{U}-235 \quad 1.000 \mathrm{E}+00$ $\mathrm{Pu}-239 \mathrm{~Pa}-231 \quad 1.000 \mathrm{E}+00$ $\mathrm{Pu}-239 \quad \mathrm{Ac}-227 \quad 1.000 \mathrm{E}+00$ $\mathrm{Pu}-239$ SDSR (j) $1.000 \mathrm{E}+00$ OU -234 U $-234 \quad 1.000 \mathrm{E}+00$ $\mathrm{U}-234 \quad \mathrm{Th}-230 \quad 1.000 \mathrm{E}+00$ $\mathrm{U}-234 \quad \mathrm{Ra}-226 \quad 1.000 \mathrm{E}+00$ $\mathrm{U}-234$ Po-210 $1.000 \mathrm{E}+0$ $\mathrm{U}-234 \quad \operatorname{SDSR}(j)$

$1.000 \mathrm{E}+00$ $\mathrm{U}-235$ AC-227 $1.000 \mathrm{O}+00$ OU-238 $\mathrm{U}-238 \quad 1.000 \mathrm{E}+00$ $\mathrm{U}-238 \mathrm{U}-234 \quad 1.000 \mathrm{E}+00$ $\begin{array}{lll}\mathrm{U}-238 & \mathrm{Th}-230 & 1.000 \mathrm{E}+00\end{array}$ $\mathrm{U}-238 \mathrm{Ra}-226 \quad 1.000 \mathrm{E}+00$ $\mathrm{U}-238 \quad \mathrm{~Pb}-210 \quad 1.000 \mathrm{E}+00$ $\mathrm{U}-238$ $\operatorname{SDSR}(j)$

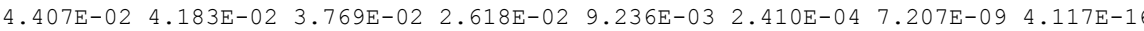

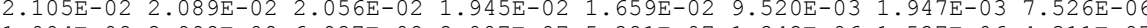

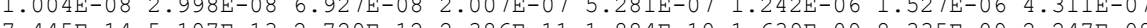

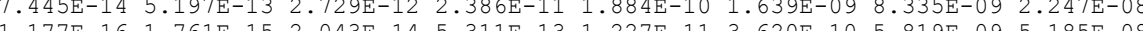

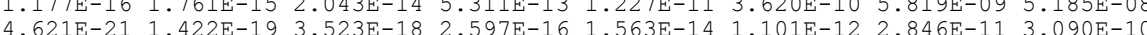

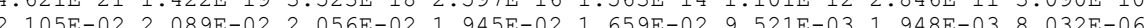

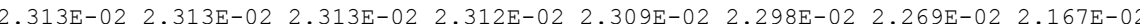
$\begin{array}{llllllll}1.269 \mathrm{E}-11 & 3.803 \mathrm{E}-11 & 8.856 \mathrm{E}-11 & 2.637 \mathrm{E}-10 & 7.500 \mathrm{E}-10 & 2.297 \mathrm{E}-09 & 5.623 \mathrm{E}-09 & 1.028 \mathrm{E}-02\end{array}$ $\begin{array}{lllllllll}2.559 \mathrm{E}-16 & 1.790 \mathrm{E}-15 & 9.438 \mathrm{E}-15 & 8.373 \mathrm{E}-14 & 6.893 \mathrm{E}-13 & 6.885 \mathrm{E}-12 & 4.8235 \mathrm{E}-11 & 2.528 \mathrm{E}-10\end{array}$ $\begin{array}{llllllll}1.127 \mathrm{E}-17 & 1.676 \mathrm{E}-16 & 1.920 \mathrm{E}-15 & 4.768 \mathrm{E}-14 & 9.759 \mathrm{E}-13 & 2.076 \mathrm{E}-11 & 2.080 \mathrm{E}-10 & 1.232 \mathrm{E}-09\end{array}$

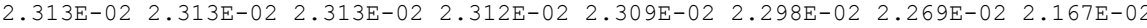

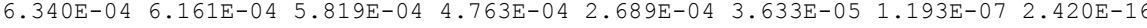

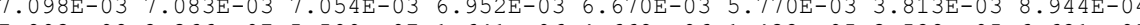

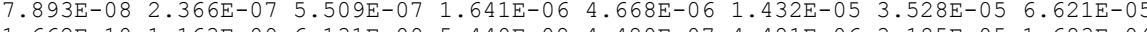
$\begin{array}{llllllll}1.662 \mathrm{E}-10 & 1.163 \mathrm{E}-09 & 6.131 \mathrm{E}-09 & 5.440 \mathrm{E}-08 & 4.480 \mathrm{E}-07 & 4.481 \mathrm{E}-06 & 3.185 \mathrm{E}-05 & 1.683 \mathrm{E}-0\end{array}$

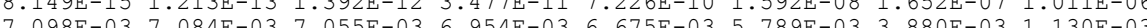

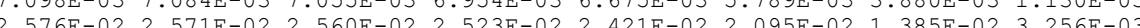

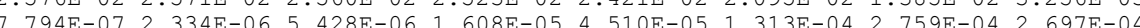
$\begin{array}{lllllllll}4.568 \mathrm{E}-08 & 3.162 \mathrm{E}-07 & 1.630 \mathrm{E}-06 & 1.336 \mathrm{E}-05 & 8.906 \mathrm{E}-05 & 4.941 \mathrm{E}-04 & 1.309 \mathrm{E}-03 & 1.377 \mathrm{E}-03\end{array}$ $\begin{array}{lllllllll}2.576 \mathrm{E}-02 & 2 & 571 \mathrm{E}-02 & 2.561 \mathrm{E}-02 & 2.526 \mathrm{E}-02 & 2.435 \mathrm{E}-02 & 2.157 \mathrm{E}-02 & 1.544 \mathrm{E}-02 & 4.902 \mathrm{E}-03\end{array}$ $\begin{array}{llllllll}9.949 \mathrm{E}-03 & 9.928 \mathrm{E}-03 & 9.887 \mathrm{E}-03 & 9.745 \mathrm{E}-03 & 9.350 \mathrm{E}-03 & 8.090 \mathrm{E}-03 & 5.349 \mathrm{E}-03 & 1.257 \mathrm{E}-03\end{array}$

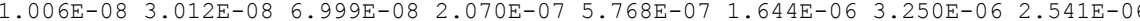

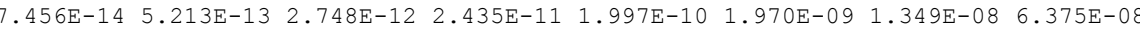
$\begin{array}{llllllll}1.178 \mathrm{E}-16 & 1.765 \mathrm{E}-15 & 2.053 \mathrm{E}-14 & 5.393 \mathrm{E}-13 & 1.283 \mathrm{E}-11 & 4.161 \mathrm{E}-10 & 1.349 \mathrm{E}-08 & 6.375 \mathrm{E}-08\end{array}$

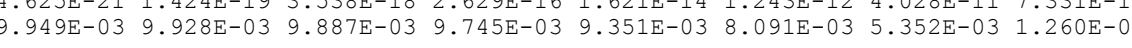

*Branch Fraction is the cumulative factor for the j't principal radionuclide daughter: $\operatorname{CUMBRF}(j)=\operatorname{BRF}(1) * \operatorname{BRF}(2) * \ldots \operatorname{BRF}(j)$. $\$$ is used to indicate summation; the Greek signa is not included in this font. 
File : cau224 $2501 \mathrm{~m}$ parcel.RAD

Single Radionuclide Soil Guidelines $G(i, t)$ in $\mathrm{pCi} / \mathrm{g}$

ONuclide

Basic Radiation Dose Limit $=2.500 \mathrm{E}+01 \mathrm{mrem} / \mathrm{yr}$

\begin{tabular}{|c|c|c|c|c|c|c|c|c|c|}
\hline $\begin{array}{l}\text { li } \\
\text { (i) }\end{array}$ & $t=$ & $0.000 \mathrm{E}+00$ & $1.000 \mathrm{E}+00$ & $3.000 \mathrm{E}+00$ & $1.000 \mathrm{E}+01$ & $3.000 \mathrm{E}+01$ & $1.000 \mathrm{E}+02$ & $3.000 \mathrm{E}+02$ & $1.000 \mathrm{E}+03$ \\
\hline Am & & $9.952 \mathrm{E}+02$ & $1.000 \mathrm{E}+03$ & $1.011 \mathrm{E}+03$ & $1.048 \mathrm{E}+03$ & $1.163 \mathrm{E}+03$ & $1.675 \mathrm{E}+03$ & $4.746 \mathrm{E}+03$ & $1.783 E+05$ \\
\hline & & $3.188 \mathrm{E}+02$ & $3.263 \mathrm{E}+02$ & $3.421 \mathrm{E}+02$ & $4.033 \mathrm{E}+02$ & $6.458 \mathrm{E}+02$ & $3.353 \mathrm{E}+03$ & $3.711 \mathrm{E}+05$ & $5.294 \mathrm{E}+12$ \\
\hline $\mathrm{Eu}-152$ & & $1.584 \mathrm{E}+02$ & $1.669 \mathrm{E}+02$ & $1.852 \mathrm{E}+02$ & $2.666 \mathrm{E}+02$ & $7.557 \mathrm{E}+02$ & $2.896 \mathrm{E}+04$ & $9.685 \mathrm{E}+08$ & $* 1.765 \mathrm{E}+14$ \\
\hline $\mathrm{Pu}-238$ & & $1.188 \mathrm{E}+03$ & $1.197 \mathrm{E}+03$ & $1.216 \mathrm{E}+03$ & $1.286 \mathrm{E}+03$ & $1.507 \mathrm{E}+03$ & $2.626 \mathrm{E}+03$ & $1.283 \mathrm{E}+04$ & $3.113 \mathrm{E}+06$ \\
\hline $\mathrm{Pu}-239$ & & $1.081 \mathrm{E}+03$ & $1.081 \mathrm{E}+03$ & $1.081 \mathrm{E}+03$ & $1.081 \mathrm{E}+03$ & $1.083 \mathrm{E}+03$ & $1.088 \mathrm{E}+03$ & $1.102 \mathrm{E}+03$ & $1.154 \mathrm{E}+03$ \\
\hline $\mathrm{Sr}-90$ & & $3.943 \mathrm{E}+04$ & $4.058 \mathrm{E}+04$ & $4.297 \mathrm{E}+04$ & $5.249 \mathrm{E}+04$ & $9.298 \mathrm{E}+04$ & $6.881 \mathrm{E}+05$ & $2.096 \mathrm{E}+08$ & $* 1.365 \mathrm{E}+14$ \\
\hline $\mathrm{U}-234$ & & $3.522 \mathrm{E}+03$ & $3.529 \mathrm{E}+03$ & $3.544 \mathrm{E}+03$ & $3.595 \mathrm{E}+03$ & $3.745 \mathrm{E}+03$ & $4.319 E+03$ & $6.443 E+03$ & $2.212 \mathrm{E}+04$ \\
\hline $\mathrm{U}-235$ & & $9.704 \mathrm{E}+02$ & $9.723 \mathrm{E}+02$ & $9.762 \mathrm{E}+02$ & $9.895 \mathrm{E}+02$ & $1.027 \mathrm{E}+03$ & $1.159 \mathrm{E}+03$ & $1.620 \mathrm{E}+03$ & $5.100 \mathrm{E}+03$ \\
\hline $\mathrm{U}-238$ & & $2.513 \mathrm{E}+03$ & $2.518 \mathrm{E}+03$ & $2.529 \mathrm{E}+03$ & $2.565 \mathrm{E}+03$ & $2.674 \mathrm{E}+03$ & $3.090 \mathrm{E}+03$ & $4.671 \mathrm{E}+03$ & $1.985 \mathrm{E}+04$ \\
\hline
\end{tabular}

Summed Dose/Source Ratios DSR (i,t) in (mrem/yr)/(pCi/g)

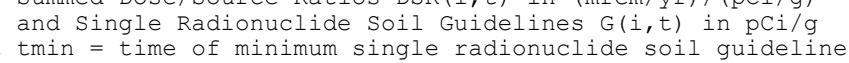
$\begin{aligned} \text { at } \operatorname{tmin} & =\text { time of minimum single radionuclide soil guide } \\ \text { and at tmax } & =\text { time of maximum total dose }=0.000 \mathrm{E}+00 \text { years }\end{aligned}$

\begin{tabular}{|c|c|c|c|c|c|c|}
\hline $\begin{array}{l}\text { Nuclide } \\
\text { (i) }\end{array}$ & $\begin{array}{l}\text { Initial } \\
(\mathrm{pci} / \mathrm{g})\end{array}$ & $\begin{array}{c}\operatorname{tmin} \\
\text { (years) }\end{array}$ & $\operatorname{DSR}(i, \operatorname{tmin})$ & $\begin{array}{c}\mathrm{G}(i, \operatorname{tmin}) \\
(\mathrm{pCi} / \mathrm{g})\end{array}$ & $\operatorname{DSR}(i, \operatorname{tmax})$ & $\begin{array}{c}G(i, \text { tmax }) \\
\quad(\mathrm{pC} i / \mathrm{g})\end{array}$ \\
\hline & $960 \mathrm{E}+01$ & $0.000 \mathrm{E}+00$ & $2.512 \mathrm{E}$ & $9.952 \mathrm{E}+02$ & $2.512 \mathrm{E}-02$ & $9.952 \mathrm{E}+02$ \\
\hline-137 & 1.00 & 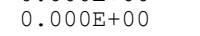 & & & & \\
\hline Eu-152 & $2.460 \mathrm{E}+00$ & - & & 1.5 & & $E+02$ \\
\hline Pu-238 & 1.00 & & & 1.18 & & \\
\hline Pu-239 & $2.180 \mathrm{E}+$ & $\begin{array}{ll}0.0 \\
0\end{array}$ & 2.3 & $1.081 \mathrm{E}+03$ & $2.313 \mathrm{E}-02$ & $1.081 \mathrm{E}$ \\
\hline $\operatorname{sr}-90$ & $3.250 \mathrm{E}+$ & & & $3.943 E+04$ & & \\
\hline $\mathrm{U}-234$ & 1.26 & 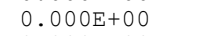 & 3 & 3.52 & 7.09 & $3.522 \mathrm{E}+03$ \\
\hline & 1.2 & $0.000 \mathrm{E}$ & 2.310 & 9.70 & 2.5 & $9.704 \mathrm{E}+02$ \\
\hline $\mathrm{U}-238$ & $1.210 \mathrm{E}+00$ & $0.000 \mathrm{E}+00$ & $9.949 \mathrm{E}-03$ & $2.513 E+03$ & $9.949 \mathrm{E}-03$ & $2.513 \mathrm{E}+03$ \\
\hline
\end{tabular}


1RESRAD, Version 6.21

$T^{1 / 2}$ Limit $=0.5$ year

Summary : CAU 224250 day/yr worker scenario $1 \mathrm{~m}$ hotspot

02/22/2005 17:50 Page 24

File : cau224 $2501 \mathrm{~m}$ parcel. RAD

Individual Nuclide Dose Summed Over All Pathways

Parent Nuclide and Branch Fraction Indicated

ONuclide Parent BRF(i)

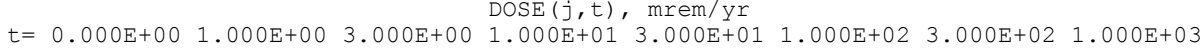

$\overline{\mathrm{Am}-241} \overline{\mathrm{Am}-241} \overline{1.000 \mathrm{E}+00}$

0Np-237 Am-241 $1.000 \mathrm{E}+0$

00

$\mathrm{OCs}-137$ Cs -137 1.000

OEu-152 Eu-152 $7.208 \mathrm{E}-01$

Eu-152 Eu-152 2.792E-01

Eu-152 \$DOSE $(j)$

Eu-152 2.792E-01

$0 \mathrm{Pu}-238 \quad \mathrm{Pu}-238 \quad 1.000 \mathrm{E}+00$

OU-234 $\quad \mathrm{Pu}-238 \quad 1.000 \mathrm{E}+00$

$\mathrm{U}-234 \quad \mathrm{U}-234 \quad 1.000 \mathrm{E}+00$

$\mathrm{U}-234 \mathrm{U}-238 \quad 1.000 \mathrm{E}+0$

$\mathrm{U}-234$ SDOSE (j)

Th -230 U $-234 \quad 1.000 \mathrm{E}+00$

Th-230 U $-234 \quad 1.000 \mathrm{E}+00$

Th -230 SDOSE (j)

ORa-226 Pu-238 1.000E+00

$\mathrm{Ra}-226 \mathrm{U}-234 \mathrm{U}$

$\mathrm{Ra}-226 \mathrm{U}-238 \quad 1.000 \mathrm{E}+00$

$\mathrm{Ra}-226$ \$DOSE (j)

$\mathrm{Pb}-210 \quad \mathrm{Pu}-238 \quad 1.000 \mathrm{E}+00$

$\mathrm{Pb}-210 \mathrm{U}-234 \quad 1.000 \mathrm{E}+00$

$\mathrm{Pb}-210 \quad \mathrm{U}-238 \quad 1.000 \mathrm{E}+00$

$\mathrm{Pb}-210$ \$DOSE $(j)$

OPu-239 $\mathrm{Pu}-239 \quad 1.000 \mathrm{E}+00$

$\begin{array}{lll}0 \mathrm{U}-235 & \mathrm{Pu}-239 & 1.000 \mathrm{E}+00\end{array}$

$\mathrm{U}-235$ SDOSE (j)

$\begin{array}{lll}\mathrm{P} a-231 & \mathrm{Pu}-239 & 1.000 \mathrm{E}+00\end{array}$

$\mathrm{Pa}-231 \mathrm{U}-235 \quad 1.000 \mathrm{E}+00$

$\mathrm{Pa}-231$ \$DOSE $(j)$

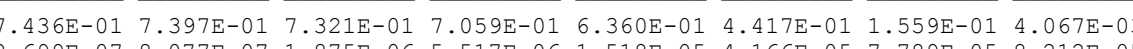

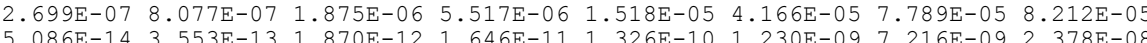

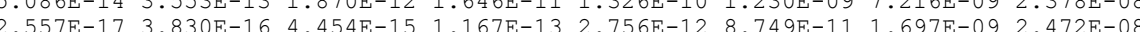
$\begin{array}{lllllllll}7.843 \mathrm{E}-01 & 7.660 \mathrm{E}-01 & 7.308 \mathrm{E}-01 & 6.198 \mathrm{E}-01 & 3.871 \mathrm{E}-01 & 7.455 \mathrm{E}-02 & 6.737 \mathrm{E}-04 & 4.722 \mathrm{E}-11\end{array}$ $\begin{array}{lllllllll}2.799 \mathrm{E}-01 & 2.657 \mathrm{E}-01 & 2.394 \mathrm{E}-01 & 1.663 \mathrm{E}-01 & 5.866 \mathrm{E}-02 & 1.531 \mathrm{E}-03 & 4.577 \mathrm{E}-08 & 6.686 \mathrm{E}-24\end{array}$

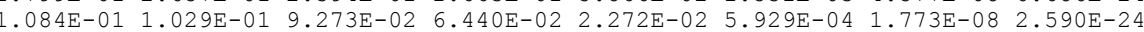

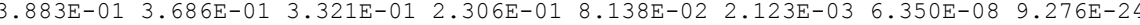

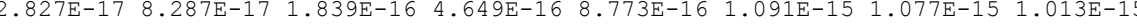

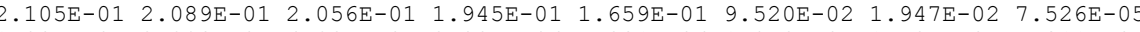
$\begin{array}{llllllll}1.004 \mathrm{E}-07 & 2.998 \mathrm{E}-07 & 6.927 \mathrm{E}-07 & 2.007 \mathrm{E}-06 & 5.281 \mathrm{E}-06 & 1.242 \mathrm{E}-05 & 1.527 \mathrm{E}-05 & 4.326 \mathrm{E}-05\end{array}$

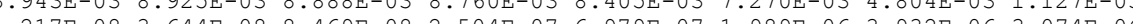

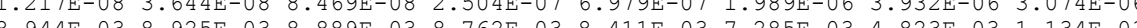

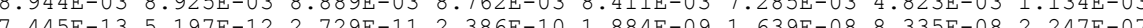

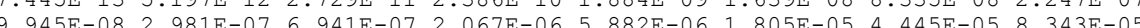
$\begin{array}{lllllllll}9.022 \mathrm{E}-14 & 6.308 \mathrm{E}-13 & 3.325 \mathrm{E}-12 & 2.946 \mathrm{E}-11 & 2.417 \mathrm{E}-10 & 2.384 \mathrm{E}-0 & 1.632 \mathrm{E}-05 & 8.343 \mathrm{E}-05\end{array}$

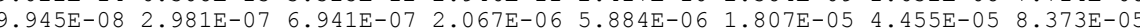
$\begin{array}{llllllll}1.177 \mathrm{E}-15 & 1.761 \mathrm{E}-14 & 2.043 \mathrm{E}-13 & 5.311 \mathrm{E}-12 & 1.227 \mathrm{E}-10 & 3.620 \mathrm{E}-09 & 5.819 \mathrm{E}-08 & 5.185 \mathrm{E}-07\end{array}$

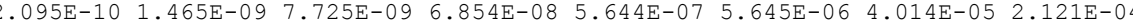
$\begin{array}{llllllll}1.425 \mathrm{E}-16 & 2.135 \mathrm{E}-15 & 2.485 \mathrm{E}-14 & 6.525 \mathrm{E}-13 & 1.552 \mathrm{E}-11 & 5.035 \mathrm{E}-10 & 1.022 \mathrm{E}-08 & 1.511 \mathrm{E}-07\end{array}$ 2.095E-10 1.465E-09 $7.725 \mathrm{E}-09 \quad 6.854 \mathrm{E}-08 \quad 5.646 \mathrm{E}-07 \quad 5.650 \mathrm{E}-06 \quad 4.020 \mathrm{E}-05 \quad 2.127 \mathrm{E}-04$

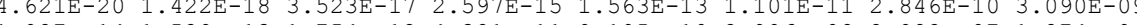
$\begin{array}{llllllll}1.027 \mathrm{E}-14 & 1.529 \mathrm{E}-13 & 1.754 \mathrm{E}-12 & 4.381 \mathrm{E}-11 & 9.105 \mathrm{E}-10 & 2.006 \mathrm{E}-08 & 2.082 \mathrm{E}-07 & 1.274 \mathrm{E}-06\end{array}$ $\begin{array}{llllllll}5.596 \mathrm{E}-21 & 1.724 \mathrm{E}-19 & 4.281 \mathrm{E}-18 & 3.181 \mathrm{E}-16 & 1.961 \mathrm{E}-14 & 1.504 \mathrm{E}-12 & 4.874 \mathrm{E}-11 & 8.871 \mathrm{E}-10\end{array}$ $1.027 \mathrm{E}-141.529 \mathrm{E}-131.754 \mathrm{E}-12 \quad 4.381 \mathrm{E}-11 \quad 9.106 \mathrm{E}-102.007 \mathrm{E}-082.085 \mathrm{E}-07 \quad 1.278 \mathrm{E}-06$

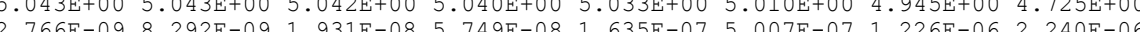

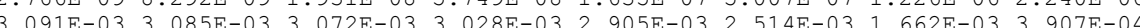
$\begin{array}{lllllllll}3.091 \mathrm{E}-03 & 3.085 \mathrm{E}-03 & 3.072 \mathrm{E}-03 & 3.028 \mathrm{E}-03 & 2.906 \mathrm{E}-03 & 2.514 \mathrm{E}-03 & 1.662 \mathrm{E}-03 & 3.907 \mathrm{E}-04\end{array}$ $\begin{array}{lllllllll}5.579 \mathrm{E}-14 & 3.902 \mathrm{E}-13 & 2.057 \mathrm{E}-12 & 1.825 \mathrm{E}-11 & 1.503 \mathrm{E}-10 & 1.501 \mathrm{E}-0 & 1.063 \mathrm{E}-0 & 5.529 \mathrm{E}-04\end{array}$

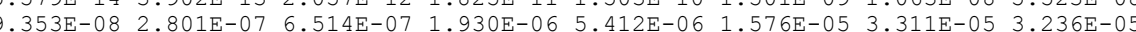
9.353E-08 2.801E-07 6.514E-07 1.930E-06 5.412E-06 $1.576 \mathrm{E}-05 \quad 3.312 \mathrm{E}-05 \quad 3.242 \mathrm{E}-05$ 
$T^{3} / 2$ Limit $=0.5$ year

Summary : CAU 224250 day/yr worker scenario $1 \mathrm{~m}$ hotspot

02/22/2005 17:50 Page 25

File : cau224 $2501 \mathrm{~m}$ parcel. RAD

Individual Nuclide Dose Summed Over All Pathways

Parent Nuclide and Branch Fraction Indicated

ONuclide Parent BRF(i)

$\frac{\text { (j) }}{\mathrm{Ac}-227} \frac{\text { (i) }}{\mathrm{Pu}-239} \frac{}{1.000 \mathrm{E}+00}$

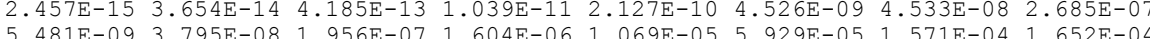

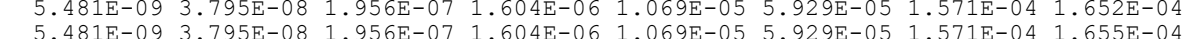

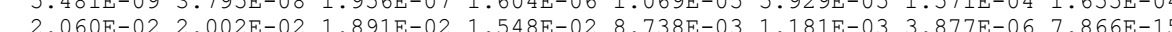

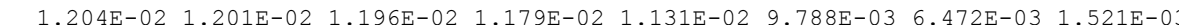

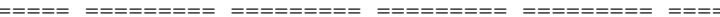

the branch fraction of the parent nuclide.

is used to indicate summation; the Greek sigma is not included in this font. 
1RESRAD, Version 6.21

$T^{1 / 2}$ Limit $=0.5$ year

Summary : CAU 224250 day/yr worker scenario $1 \mathrm{~m}$ hotspot
File : cau224 $2501 \mathrm{~m}$ parcel.RAD

$02 / 22 / 2005 \quad 17: 50$ Page 26

Individual Nuclide Soil Concentration

Parent Nuclide and Branch Fraction Indicated

ONuclide Parent BRF(i)

$\mathrm{t}=0.000 \mathrm{E}+00 \quad 1.000 \mathrm{E}+00 \quad 3.000 \mathrm{E}+00 \quad \mathrm{~S}(j, \mathrm{t}), \mathrm{pCi} / \mathrm{g}$

$\overline{\mathrm{Am}-241} \overline{\mathrm{Am}-241} \overline{1.000 \mathrm{E}+00} \quad \overline{2.960 \mathrm{E}+01} \overline{2.945 \mathrm{E}+01} \overline{2.914 \mathrm{E}+01} \overline{2.810 \mathrm{E}+01} \quad \overline{2.532 \mathrm{E}+01} \overline{1.758 \mathrm{E}+01} \overline{6.204 \mathrm{E}+00} \overline{1.619 \mathrm{E}-01}$ $\begin{array}{lllllllllll}0 \mathrm{~Np}-237 & \mathrm{Am}-241 & 1.000 \mathrm{E}+00 & 0.000 \mathrm{E}+00 & 9.561 \mathrm{E}-06 & 2.853 \mathrm{E}-05 & 9.329 \mathrm{E}-05 & 2.651 \mathrm{E}-04 & 7.359 \mathrm{E}-04 & 1.380 \mathrm{E}-03 & 1.456 \mathrm{E}-03 \\ 0 \mathrm{U}-233 & \mathrm{Am}-241 & 1.000 \mathrm{E}+00 & 0.000 \mathrm{E}+00 & 2.092 \mathrm{E}-11 & 1.874 \mathrm{E}-10 & 2.049 \mathrm{E}-09 & 1.761 \mathrm{E}-08 & 1.672 \mathrm{E}-07 & 9.875 \mathrm{E}-07 & 3.261 \mathrm{E}-0\end{array}$

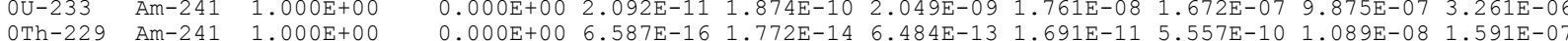

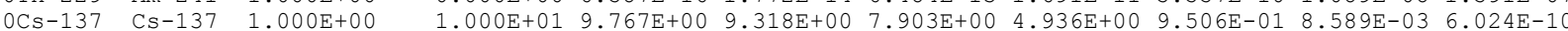

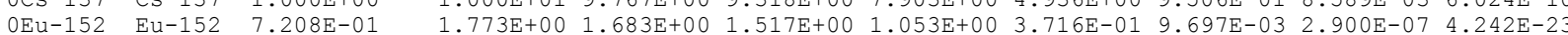
$\begin{array}{llllllllllll}\mathrm{Eu}-152 & \mathrm{Eu}-152 & 2.792 \mathrm{E}-01 & 6.868 \mathrm{E}-01 & 6.520 \mathrm{E}-01 & 5.875 \mathrm{E}-01 & 4.080 \mathrm{E}-01 & 1.440 \mathrm{E}-01 & 3.756 \mathrm{E}-03 & 1.123 \mathrm{E}-07 & 1.643 \mathrm{E}-23\end{array}$ $\mathrm{GGd}-152$ Eu-152 $2.792 \mathrm{E}-01$ $0 \mathrm{Pu}-238 \quad \mathrm{Pu}-238 \quad 1.000 \mathrm{E}+00$ $\mathrm{OU}-234 \quad \mathrm{Pu}-238$ 1.000 1 E+00 $\mathrm{U}-234-\mathrm{U}-234-1.000 \mathrm{E}+00$ $\mathrm{U}-234 \mathrm{U}-238-1.000 \mathrm{E}+00$ $\begin{array}{lll}\mathrm{U}-234 & \$ S(j): & \\ 0 \mathrm{Th}-230 & \mathrm{Pu}-238 & 1.000 \mathrm{E}+00\end{array}$ Th-230 Pu-238 $1.000 \mathrm{E}+00$ Th-230 U-238 $1.000 \mathrm{E}+00$ Th-230 SS(j): $\begin{array}{lll}\mathrm{Th}-230 & \$ S(j): & \\ \mathrm{ORa}-226 & \mathrm{Pu}-238 & 1.000 \mathrm{E}+00\end{array}$ $\mathrm{Ra}-226 \mathrm{U}-234 \quad 1.000 \mathrm{E}+00$ $\mathrm{Ra}-226 \mathrm{U}-238 \quad 1.000 \mathrm{E}+00$ $\mathrm{Ra}-226$ \$S $(j):$ $\begin{array}{lll}0 \mathrm{~Pb}-210 & \mathrm{Pu}-238 & 1.000 \mathrm{E}+00 \\ \mathrm{~Pb}-210 & \mathrm{U}-234 & 1.000 \mathrm{E}+00\end{array}$ $\begin{array}{lll}\mathrm{Pb}-210 & \mathrm{U}-234 & 1.000 \mathrm{E}+00 \\ \mathrm{~Pb}-210 & \mathrm{U}-238 & 1.000 \mathrm{E}+00\end{array}$ $\mathrm{Pb}-210 \mathrm{U}-238,1.000 \mathrm{E}+00$ $\begin{array}{lll}\mathrm{Pu}-239 & \mathrm{Pu}-239 & 1.000 \mathrm{E}+00\end{array}$ $\begin{array}{lll}\mathrm{Pu}-239 & \mathrm{Pu}-239 & 1.000 \mathrm{E}+00 \\ \mathrm{OU}-235 & \mathrm{Pu}-239 & 1.000 \mathrm{E}+00\end{array}$ U-235 $\mathrm{Uu}-235 \mathrm{C}-1.000 \mathrm{E}+00$ $\mathrm{U}-235 \quad \$(j):$ $\begin{array}{ccc}0 \mathrm{~Pa}-231 & \mathrm{Pu}-239 & 1.000 \mathrm{E}+0 \\ \mathrm{~Pa}-231 & \mathrm{U}-235 & 1.000 \mathrm{E}+00\end{array}$ $\mathrm{Pa}-231$ \$S $(j):$

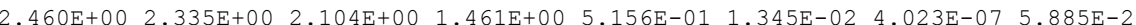

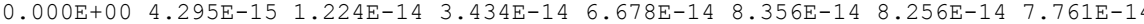

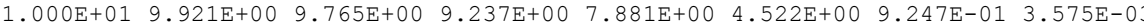

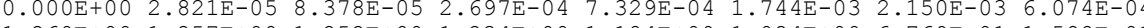

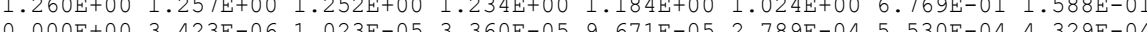

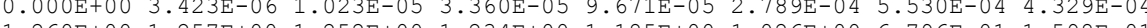

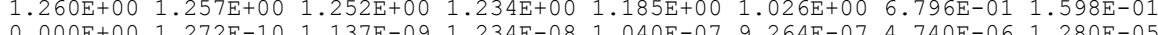

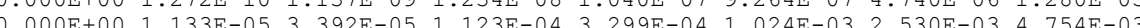

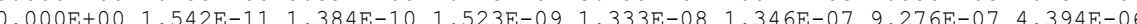

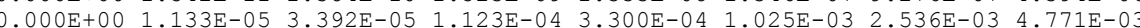

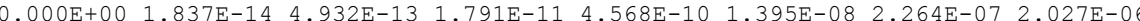

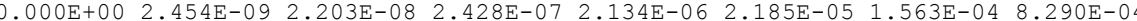

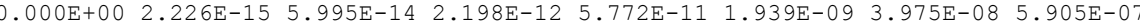

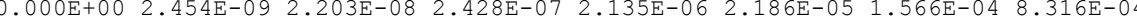

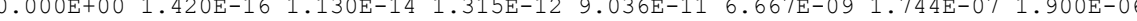
$\begin{array}{llllllll}0.000 \mathrm{E}+00 & 2.523 \mathrm{E}-11 & 6.694 \mathrm{E}-10 & 2.334 \mathrm{E}-08 & 5.352 \mathrm{E}-07 & 1.220 \mathrm{E}-05 & 1.277 \mathrm{E}-04 & 7.836 \mathrm{E}-04\end{array}$

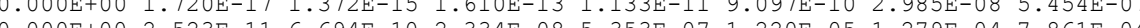

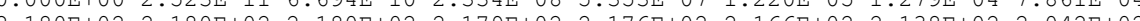

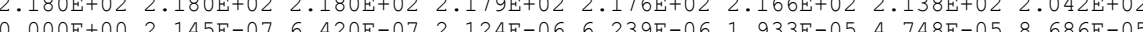

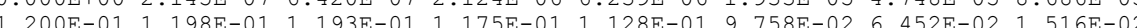

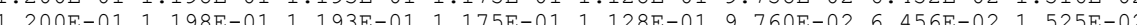

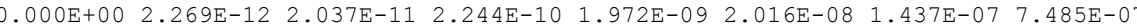

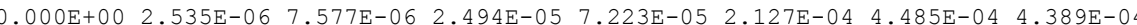

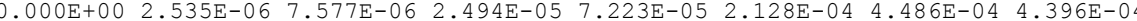


$T^{3 / 2}$ Limit $=0.5$ year

Summary : CAU 224250 day/yr worker scenario $1 \mathrm{~m}$ hotspot

02/22/2005 17:50 Page 27

File : cau224 $2501 \mathrm{~m}$ parcel.RAD

Individual Nuclide Soil Concentration

ONuclide Parent BRF(i)

(j) (i)

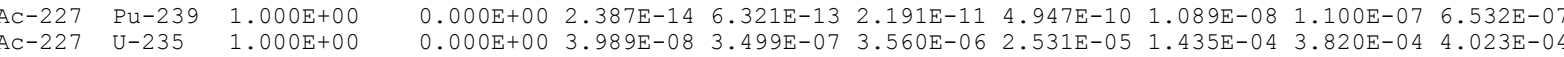

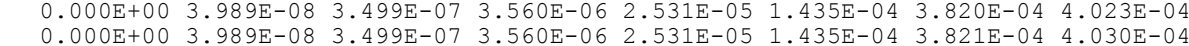

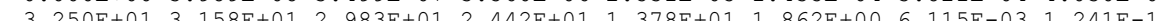

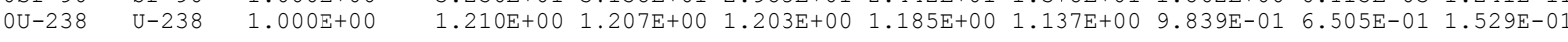
$=========1.52$

BRF(i) is the branch fraction of the parent nuclide.

$\$$ is used to indicate summation; the Greek sigma is not included in this font. 
Appendix $\mathrm{H}$

NDEP Comments 


\section{NEVADA ENVIRONMENTAL RESTORATION PROJECT}

\section{DOCUMENT REVIEW SHEET}

\begin{tabular}{|c|c|c|c|c|c|}
\hline \multicolumn{4}{|c|}{$\begin{array}{l}\text { 1. Document Title/Number: Draft Corrective Action Decision Document for Corrective Action Unit 224: Decon } \\
\text { Pad and Septic Systems, Nevada Test Site, Nevada }\end{array}$} & \multicolumn{2}{|l|}{ 2. Document Date: March 2005} \\
\hline \multicolumn{4}{|c|}{ 3. Revision Number: 0} & \multicolumn{2}{|c|}{ 4. Originator/Organization: Stoller-Navarro } \\
\hline \multicolumn{4}{|c|}{ 5. Responsible NNSA/NV ERP Project Mgr.: Janet Appenzeller-Wing } & \multicolumn{2}{|l|}{ 6. Date Comments Due: April 7, 2005} \\
\hline \multicolumn{6}{|c|}{ 7. Review Criteria: Full } \\
\hline \multicolumn{4}{|c|}{ 8. Reviewer/Organization/Phone No.: Greg Raab, NDEP, 486-2867 } & \multicolumn{2}{|l|}{ 9. Reviewer's Signature: } \\
\hline $\begin{array}{l}\text { 10. Comment } \\
\text { Number/ } \\
\text { Location }\end{array}$ & 11. Type* & 12. Comment & & 13. Comment Response & 14. Accept \\
\hline 1) Acronym List & & $\begin{array}{l}\text { The acronym "FAL" (final action level) is incorrectly defined in } \\
\text { the acronym list in the front of the document as "field action } \\
\text { level." Unless this is a new acronym, correct it. If not, change } \\
\text { the acronym from FAL to something else. }\end{array}$ & $\begin{array}{l}\text { FAL definition cha } \\
\text { Acronym list. }\end{array}$ & iged to "Final Action Level" in the & Yes \\
\hline $\begin{array}{l}\text { 2) Section } 2.3 .2 \\
\text { Leachfield } \\
\text { (CAS 03-05-01) } \\
\text { Pg. } 19 \text { of } 38\end{array}$ & & $\begin{array}{l}\text { Describes a location containing Pu-239 as a "hotspot." This } \\
\text { indicates but does not state that the Pu-239 exceeds a PAL or } \\
\text { FAL. Clarify this.In addition to the foregoing comment, just } \\
\text { because the occurrence of Pu-239 is not consistent with the } \\
\text { release mechanisms of the CAS, it does not preclude } \\
\text { corrective action. If it is a hotspot that is exceeds a PAL or } \\
\text { FAL, then NNSA/NSO must either clean it up under this CAU, } \\
\text { or at the very least, re-assign it to another CAU and address it } \\
\text { there. }\end{array}$ & $\begin{array}{l}\text { Text changed to re } \\
\text { was not a COC sir } \\
\text { the } 25 \mathrm{mrem} / \mathrm{yr} \text { do } \\
\text { action is required. }\end{array}$ & $\begin{array}{l}\text { flect the fact that the isolated Pu-239 hit } \\
\text { ice it is below the FALs established from } \\
\text { se exposure. Therefore no corrective }\end{array}$ & Yes \\
\hline $\begin{array}{l}\text { 3) Section } 3.4 \\
\text { Evaluation and } \\
\text { Comparison of } \\
\text { Alternatives } \\
\text { Pg. } 31 \text { of } 38\end{array}$ & & $\begin{array}{l}\text { CAA is a new acronym used to the first time and not spelled out } \\
\text { either in the text or in the acronym list. }\end{array}$ & $\begin{array}{l}\text { Acronym is called } \\
\text { the definition was }\end{array}$ & $\begin{array}{l}\text { out as "Corrective action alternative" and } \\
\text { added to acronym list. }\end{array}$ & Yes \\
\hline $\begin{array}{l}\text { 4) Section A.2.6 } \\
\text { Geology } \\
\text { Pg. A-11 of A-160 }\end{array}$ & & $\begin{array}{l}\text { SCL is a new acronym used for the first time and not spelled } \\
\text { out either in the text or in the acronym list. }\end{array}$ & $\begin{array}{l}\text { Acronym is called } \\
\text { definition was add }\end{array}$ & $\begin{array}{l}\text { out as Sample collection log and the } \\
\text { ed to acronym list. }\end{array}$ & Yes \\
\hline $\begin{array}{l}\text { 5) Figure A.3-1 } \\
\text { Sample Location Map } \\
\text { Pg. A-16 of A-160 }\end{array}$ & & $\begin{array}{l}\text { The table within the figure has acronyms(e.g. BAP, DiBen, } \\
\text { BAA, BBF, Indeno) and there are no descriptions of what they } \\
\text { mean. Insert a legend like the one in Figure A.3-4, pg. A-46 of } \\
\text { A-64 of CAU536 final CADD. }\end{array}$ & Definitions of term & $s$ added to explanation box in figure. & Yes \\
\hline
\end{tabular}




\section{NEVADA ENVIRONMENTAL RESTORATION PROJECT}

\section{DOCUMENT REVIEW SHEET}

\begin{tabular}{|c|c|c|c|c|}
\hline $\begin{array}{l}\text { 10. Comment } \\
\text { Number/ } \\
\text { Location }\end{array}$ & 11. Type* & 12. Comment & 13. Comment Response & 14. Accept \\
\hline 6) General Comment & & $\begin{array}{l}\text { With the exception of the RESRAD evaluation of Pu-239 in } \\
\text { Appendix G, there is no documentation evaluating the PALs } \\
\text { against the DQOs and finalizing them. NNSA/NSO must } \\
\text { document the finalization of the Action Levels and can include } \\
\text { it in Appendix B, Data Assessment. }\end{array}$ & $\begin{array}{l}\text { As discussed, please refer to Section } 3.1 \text { of the main } \\
\text { document for an explanation of how FALs were determined } \\
\text { and how they meet the criteria of the DQOs. }\end{array}$ & $\overline{\text { Yes }}$ \\
\hline $\begin{array}{l}\text { 7) Figure A.3-1 } \\
\text { Sample Location Map } \\
\text { Pg. A-16 of A-160 }\end{array}$ & & $\begin{array}{l}\text { In the same table of Figure A.3-1, under A09, sample } 224 \mathrm{~A} 018 \\
\text { is replicated three times. }\end{array}$ & $\begin{array}{l}\text { The two duplicates have been removed. Sample 224A020 } \\
\text { added to table. }\end{array}$ & Yes \\
\hline $\begin{array}{l}\text { 8) Section A.4.2.6.1 } \\
\text { Isotopes } \\
\text { Pg. A-34 of A-160 }\end{array}$ & & $\begin{array}{l}\text { Describes a location containing Pu- } 239 \text { exceeds the FAL but } \\
\text { because the occurrence of Pu- } 239 \text { is "not considered to be } \\
\text { associated with the CAS," it does not considered a COC. This } \\
\text { is an unacceptable philosophy. Just because it is not } \\
\text { associated with the CAS, it does not preclude corrective action. } \\
\text { If the Pu- } 239 \text { exceeds the FAL, it is a COC regardless of its } \\
\text { source. However, in this case, although there is a hit, and it } \\
\text { does not exceed the FAL, i.e. no further action is warranted, it } \\
\text { must be rewritten to say that. }\end{array}$ & $\begin{array}{l}\text { Language changed to reflect the fact that the Pu- } 239 \text { hit is } \\
\text { not a COC, since the activity is below the } 25 \mathrm{mrem} / \mathrm{yr} \text { dose } \\
\text { based FAL. }\end{array}$ & Yes \\
\hline $\begin{array}{l}\text { 9) Section B.1.5 } \\
\text { Result } \\
\text { Pg. B-33 of B-34 }\end{array}$ & & $\begin{array}{l}\text { The last sentence in the first paragraph references the isolated } \\
\text { Pu- } 239 \text { hit as not being associated with the CAS, therefore it is } \\
\text { not considered a COC. As noted above, this is an } \\
\text { unacceptable philosophy. Just because it is not associated } \\
\text { with the CAS, it does not preclude corrective action. If the } \\
\text { Pu- } 239 \text { exceeds the FAL, it is a COC regardless of its source. } \\
\text { However, in this case, although there is a hit, and it does not } \\
\text { exceed the FAL, i.e. no further action warranted, it must be } \\
\text { rewritten to say that. }\end{array}$ & $\begin{array}{l}\text { Language changed to reflect the fact that the Pu- } 239 \text { hit is } \\
\text { not a COC, since the activity is below the } 25 \mathrm{mrem} / \mathrm{yr} \text { dose } \\
\text { based FAL. }\end{array}$ & Yes \\
\hline $\begin{array}{l}\text { 10) Appendix G } \\
\text { Cover }\end{array}$ & & $\begin{array}{l}\text { "Deviation" should probably be "Derivation," and pg. } 5 \text { of } 29 \text {, } \\
\text { fifth sentence does not make sense as written: "These } \\
\text { parameters were (identified? analyzed?) through a sensitivity } \\
\text { analysis..." }\end{array}$ & $\begin{array}{l}\text { Title page changed as indicated. Page } 5 \text { sentence changed } \\
\text { to read "A Sensitivity analysis was performed on these } \\
\text { parameters in Scenario A." }\end{array}$ & Yes \\
\hline
\end{tabular}

${ }^{\text {a }}$ Comment Types: $M=$ Mandatory, $S$ = Suggested.

Return Document Review Sheets to NNSA/NV Environmental Restoration Division, Attn: QAC, M/S 505. 


\section{Library Distribution List}

U.S. Department of Energy

National Nuclear Security Administration

Nevada Site Office

Technical Library

P.O. Box 98518, M/S 505

Las Vegas, NV 89193-8518

U.S. Department of Energy

Office of Scientific and Technical Information

P.O. Box 62

Oak Ridge, TN 37831-0062

Southern Nevada Public Reading Facility

c/o Nuclear Testing Archive

P.O. Box 98521, M/S 400

Las Vegas, NV 89193-8521

Manager, Northern Nevada FFACO

Public Reading Facility

c/o Nevada State Library \& Archives

100 N Stewart Street

Carson City, NV 89701-4285
1 (Uncontrolled)

1 (Uncontrolled, electronic copy)

2 (Uncontrolled, electronic copies)

1 (Uncontrolled, electronic copy) 